Andreas Köpfe Justin J.W. Powel

Raphael Zahnd (Hrsg.)

Handbuch

Inklusion international

International Handbook of Inclusive Education 
Handbuch Inklusion international

International Handbook of Inclusive Education 


Andreas Köpfer
Justin J.W. Powell
Raphael Zahnd (Hrsg.)

\section{Handbuch Inklusion international}

Globale, nationale und lokale Perspektiven auf Inklusive Bildung

\section{International Handbook of Inclusive Education}

Global, National and Local Perspectives

Verlag Barbara Budrich Opladen • Berlin • Toronto 2021 
Bibliografische Information der Deutschen Nationalbibliothek

Die Deutsche Nationalbibliothek verzeichnet diese Publikation in der Deutschen Nationalbibliografie; detaillierte bibliografische Daten sind im Internet über

https://portal.dnb.de abrufbar.

(C) 2021 Dieses Werk ist bei der Verlag Barbara Budrich GmbH erschienen und steht unter der Creative Commons Lizenz Attribution 4.0 International

(CC BY 4.0): https://creativecommons.org/licenses/by/4.0/

Diese Lizenz erlaubt die Verbreitung, Speicherung, Vervielfältigung und Bearbeitung unter Angabe der UrheberInnen, Rechte, Änderungen und verwendeten Lizenz.

www.budrich.de

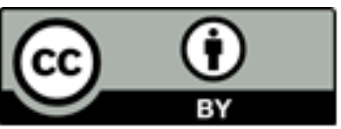

Dieses Buch steht im Open-Access-Bereich der Verlagsseite zum kostenlosen Download bereit (https://doi.org/10.3224/84742446).

Eine kostenpflichtige Druckversion (Print on Demand) kann über den Verlag bezogen werden. Die Seitenzahlen in der Druck- und Onlineversion sind identisch.

$$
\begin{array}{ll}
\text { ISBN } & 978-3-8474-2446-8 \text { (Paperback) } \\
\text { eISBN } & 978-3-8474-1577-0 \text { (eBook) } \\
\text { DOI } & 10.3224 / 84742446
\end{array}
$$

Umschlaggestaltung: Walburga Fichtner, Köln

Satz: Anja Borkam, Jena - kontakt@lektorat-borkam.de

Druck: paper \& tinta, Warschau

Printed in Europe 


\section{Inhalt}

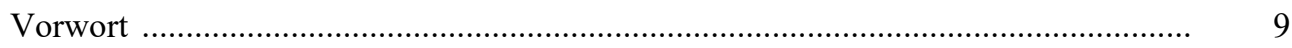

1 Entwicklungslinien internationaler und komparativer Inklusionsforschung ........ 11 Andreas Köpfer/Justin J.W. Powell/Raphael Zahnd

I Grundlagen: Theoretische und methodologische Positionen und zentrale Begriffe

2 What is a Human Right to Inclusive Education? The Promises and Limitations of the CRPD's Inclusion Mandate

Katharina Heyer

3 A Sociology of Special and Inclusive Education: Insights from the UK, US, Germany, and Finland

Sally Tomlinson

4 Inclusion and Equity in Education: Responding to a Global Challenge Mel Ainscow

5 The Universal Value of Teacher Education for Inclusive Education Lani Florian

6 Weltbank: Von ersten Auseinandersetzungen mit Behinderung hin zu inklusiven Perspektiven Raphael Zahnd

7 Disability Studies in Education: Normalität/en im inklusiven Unterricht und im Bildungsbereich hinterfragen Marianne Hirschberg/Swantje Köbsell

8 Die diskursive Konstruktion inklusiver Bildung im südlichen Afrika 147 Myriam Hummel

\section{Komparative Perspektiven zu ausgewählten nationalen, regionalen und} lokalen Bildungskontexten

A Gesetzliche Bedingungen und Governance

9 Der Einfluss der UN-BRK auf inklusive Bildung in Nigeria und Deutschland 
10 Same same but different - Ein Vergleich der Entwicklung inklusiver

Strukturen in zwei Bundesländern in Deutschland

Julia Gasterstädt

11 Maßnahmen inklusiver Bildung - Ein international vergleichender Blick auf

Andalusien (Spanien) und Berlin (Deutschland)

Lea Schäfer

12 Inklusive Bildung als Institution am Beispiel Schweiz

Mathias Mejeh

13 Inklusive Bildung in Österreich - Von den Anfängen der Entwicklung eines rechtlich-normativen Auftrags bis zu den Perspektiven für die Zukunft Gottfried Biewer

14 Einflüsse von Bildungspolitiken auf biographische Verläufe in international vergleichender Perspektive erforschen: Erkenntnisse aus dem Quali-TYDESProjekt

Tobias Buchner

B Schulstrukturen, -kulturen und -praktiken

15 Inklusive Unterstützungsstrukturen in Schulen in Island und Kanada - Eine kulturvergleichende Analyse mit Blick auf schulrechtliche Entwicklungen und In-School-Support

Edda Óskarsdóttir/Andreas Köpfer

16 Vergleichende Perspektiven auf die Kultur(-en) der (schulischen) Inklusion in Addis Abeba und Bangkok

Michelle Proyer/Margarita Bilgeri

17 Exploring Successful Inclusive Practice in China: An Inclusive Public

Kindergarten in Shanghai

Run Tan

18 Inclusive School Development: The First Years of an English Free School Julie Allan/Clara Rübner Jørgensen

19 Steps to Inclusion? The Role of Tiered Intervention in Finland and in the United States

Markku Jahnukainen/Tiina Itkonen

20 Heterogene Lerngruppen in Europa inklusionsorientiert unterrichten:

Inspirierende Praktiken und Erkenntnisse aus Deutschland, Island, Litauen, Luxemburg, Schweden und Spanien Justin J.W. Powell/Kerstin Merz-Atalik \& TdiverS-Projekt 
C Schule, Unterricht und Professionalisierung

21 Schulleitungshandeln in integrations-/inklusionsorientierten

Schulentwicklungsprozessen - Empirische Befunde aus der Schweiz und

Deutschland

Benjamin Badstieber/Bettina Amrhein

22 Konstruktion von (Leistungs-)Differenzen in der Schule - Ein transnationaler

Fallvergleich unterrichtlicher Praxen

Tanja Sturm

23 Teachers' Learning in Schools Moving Towards Inclusion: Experiences from

England and Germany

Elisabeth Plate/Alison Peacock

24 Multilingual Teaching Assistants in the UK: Translators in the Field of Inclusive Education

Bettina Fritzsche/Michalis Kakos

\section{Inklusion, Hochschule und Lebenslanges Lernen}

25 Diversität, Inklusion und Internationalisierung in der Hochschule:

Eine komparative Perspektive

Cristina Allemann-Ghionda

26 Studieren mit Behinderungen im Spannungsfeld zwischen

Behindertenrechtskonvention und Neoliberalismus:

Entwicklungen im europäischen Hochschulkontext

Arthur Limbach-Reich

27 Vulnerabilität als (neues) europäisches bildungspolitisches Deutungsmuster?

Ein Beispiel aus dem Bereich des Lebenslangen Lernens

Marcelo Parreira do Amaral/Jozef Zelinka

28 Shifting Lenses to a Participatory Ethos in Research: Adult Learners with

Disabilities in Germany and South Africa

Silke Schreiber-Barsch/Peter Rule

Autor*innenverzeichnis 



\section{Vorwort}

Das Anliegen des vorliegenden zweisprachigen Handbuchs Inklusion international / International Handbook of Inclusive Education ist es, einen Ein- und Überblick über den vielschichtigen internationalen Diskurs um Inklusive Bildung zu geben. Partizipation, Teilhabe, Ein- und Ausschluss im Bildungssystem sind nicht nur im deutschsprachigen Raum, sondern auch weltweit höchst aktuelle Themen. Es zeigt sich, dass, trotz globaler Entwicklungen und der Diffusion globaler Normen, Vielfalt und individueller Lernfortschritt in Bildungsinstitutionen und -organisationen aufgrund der jeweiligen kulturellen, politischen und sozialen Rahmenbedingungen der Bildungs- und Schulsysteme höchst unterschiedlich bearbeitet und unterstützt wird. Wir nehmen gerade diese Unterschiedlichkeit zum Anlass und versammeln 28 aktuelle Perspektiven in Form von internationalen und interkulturellen Vergleichen zu Fragestellungen Inklusiver Bildung - aus mehreren Disziplinen. Wenngleich sicherlich nicht erschöpfend, so freuen wir uns, mit diesem Band diverse Länderkontexte und Kulturen auf allen Ebenen des Bildungssystems hinsichtlich der Auseinandersetzung mit Fragen von Exklusion und Inklusion in deutscher und englischer Sprache zugänglich und (dank open access) auch überall verfügbar machen zu können.

Dass dieses umfassende Handbuch in dieser Form nun vorliegt, haben wir der Unterstützung vieler zu verdanken. So geht unser Dank zunächst vor allem an die beteiligten Autor*innen. Wir freuen uns, dass so viele der Autor*innen, die sich - im deutschsprachigen wie globalen Kontext - in ihrer Forschung und Lehre intensiv mit Fragen komparativer Inklusionsforschung auseinandersetzen, unserer Einladung gefolgt sind und Beiträge für das Handbuch eingebracht haben.

Darüber hinaus danken wir besonders Julia Biermann (Innsbruck) und Michelle Proyer (Wien) als critical friends für wertvolle fachliche Impulse zur abschließenden Synthetisierung und Strukturierung des Bandes in der folgenden Einleitung. Für die unzähligen redaktionellen Arbeiten, die stets mit Sorgfalt und Zuverlässigkeit vorgenommen wurden, danken wir Flurin Dummermuth (Basel), Franziska Maria Bänsch (Freiburg), Rebecca Starke (Freiburg) und Zane Leadley (Luxemburg). Und abschließend gilt unser besonderer Dank dem Verlag Barbara Budrich, an erster Stelle Miriam von Maydell, für die kompetente und angenehme Begleitung und Kooperation zur Entstehung dieses Handbuchs.

Freiburg, Oxford und Muttenz, im September 2020

Andreas Köpfer, Justin J.W. Powell und Raphael Zahnd 



\title{
1 Entwicklungslinien internationaler und komparativer Inklusionsforschung
}

\author{
Andreas Köpfer, Justin J.W. Powell, Raphael Zahnd
}

In diesem Handbuch wird das Augenmerk auf globale, regionale und lokale Fragestellungen Inklusiver Bildung gerichtet. Inklusive Bildung kann dabei als weltweites Paradigma bezeichnet werden, mit dem Teilhabe und Partizipation sowie Prozesse des Ein- und Ausschlusses in Bildungskontexten und darin eingelagerte Barrieren sowie Diskriminierungen in den Blick genommen werden (Ainscow/Sandill 2010; Richardson/Powell 2011). Politisch-normative Setzungen Inklusiver Bildung - u.a. durch die UN-Behindertenrechtskonvention (UN-BRK) (UN 2006) - werden derzeit in bildungspolitischer Hinsicht national, regional und lokal aufgegriffen und daran anschließend Prozesse der Steuerung in Bildungssystemen initiiert. Inklusion kann dabei als fuzzy concept (Artiles/Dyson 2005: 43) beschrieben werden, das sich dadurch auszeichnet, dass es auf nationaler und regionaler Ebene in differenten historisch entwickelten, kulturell ausgeprägten sowie normativ und rechtlich fundierten Bildungssystemen aufgenommen und in die Praxis übersetzt bzw. transformiert werden muss (Kozleski et al. 2011; Biermann 2021 i.E.). In den deutschsprachigen Bildungs- und Erziehungswissenschaften ist Inklusive Bildung zu einem interdisziplinären Leitbegriff avanciert, der insbesondere in der Schulpädagogik, der Sonderpädagogik und der Allgemeinen Erziehungswissenschaft diskutiert wird (Budde 2018), allerdings auch hier mit unterschiedlichen Vorstellungen (Hackbarth/Martens 2018; Kiel/Esslinger-Hinz/Reusser 2014; Werning 2014).

Trotz globaler Relevanz stellen internationale und komparative Fragestellungen im Kontext Inklusiver Bildung bislang im deutschsprachigen wie internationalen Diskurs ein unzureichend bearbeitetes Forschungsfeld dar (Sturm/Wagner-Willi 2018) - insbesondere mit Blick auf international und interkulturell vergleichende Forschungsarbeiten (Powell [2011] 2016). Vor diesem Hintergrund sind in diesem Handbuch Beiträge versammelt, die das Feld der internationalen, interkulturellen und komparativen Forschung schärfen und Inklusive Bildung - anschließend an die Diskurslinien der Disability Studies - als Prozess der Beseitigung von Barrieren und Benachteiligungen in Bildungssystemen und -organisationen verstehen. Dementsprechend werden Prozesse der Exklusion und Aussonderung sowie der Unterstützung und Inklusion mit besonderer Berücksichtigung komparativer Perspektiven analysiert, wobei unterschiedliche Dimensionen von Heterogenität und deren Intersektionalität, insbesondere mit Blick auf marginalisierte und von Exklusion bedrohter Personengruppen, berücksichtigt werden. Aufgrund der disziplinären Verortung der Autor*innen - v.a. in der Erziehungswissenschaft und Soziologie - und deren Beschäftigung mit Behinderungen und Benachteiligungen, rückt die Differenzlinie Behinderung bzw. „behindert werden“ in den Vordergrund. 


\section{Bedeutung Inklusiver Bildung für formale, non-formale und informelle Bildungsprozesse}

Inklusive Bildung adressiert Bildungsprozesse in ihrer Gesamtheit sowie über die gesamte Lebensspanne, sie bezieht sich auf alle Bereiche des Bildungswesens - frühkindliche Bildung, alle Altersstufen des Schulwesens, die Aus-, Fort- und Weiterbildung sowie die Hochschulen - und betrifft neben formalen auch non-formale und informelle Bildungsangebote (UN 2006; UN CRPD 2016). Allen Menschen soll, unabhängig ihrer Verschiedenheit bezüglich Alter, Geschlecht (Köbsell/Hirschberg 2021 i.d.B.), sozio-ökonomischem Status, Migrationshintergrund (Powell/Wagner 2014), Behinderung (Sturm 2016) und unter Berücksichtigung von Intersektionalität - etwa im Sinne einer „Pädagogik der Vielfalt“ (Prengel 2006; Preuss-Lausitz 2001) - die Teilhabe an gemeinsamen Bildungsprozessen ermöglicht werden. Inklusive Bildung kann in den Merkmalen des Zugangs und der Anwesenheit, der Beteiligung und der Teilhabe aller Lernenden (Qualität der Lernerfahrungen und der sozialen Partizipation aus Sicht der Lernenden) sowie in Bezug auf die Lern-Leistung respektive deren Bewertung und Zertifizierung betrachtet werden.

Mit dem Anliegen, die Lern- und Entwicklungsständen aller Lernenden zu berücksichtigen und damit individualisierte und differenzierte Lernumgebungen zu gestalten, schafft Inklusive Bildung eine Grundlage für erfolgreiche Lernprozesse, die allen zugutekommt (vgl. Eckstein et al. 2013; Werning 2014; Buchner 2018). Gleichzeitig fokussiert sie das bedeutsame Ziel, Lernprozesse als gemeinsame Prozesse aller beteiligten Individuen zu gestalten (vgl. Feuser 2013; Vock/Gronostaj 2017). Die Frage der Gemeinschaft, in Lernprozessen aber auch darüber hinaus, ist gerade deshalb von Relevanz, weil marginalisierte und von Segregation bedrohte Gruppen von Lernenden wie z.B. Schüler*innen mit Behinderungen ein erhöhtes Risiko mangelnder sozialer Partizipation haben (vgl. Pfahl 2011; Garrote/Sermier Dessemontet/Moser Opitz 2017; Henke et al. 2017; Krawinkel/Südkamp/Tröster 2017). Inklusive Bildung adressiert dementsprechend nicht nur die Frage der gemeinsamen Beschulung bezüglich des Ortes und der Zeit, sondern versteht Bildungsprozesse per se als soziale Prozesse, die Lernende miteinander vollziehen. Darüber hinaus hat sie den Anspruch, die Grundlage für die gesellschaftliche Teilhabe aller bereitzustellen. Trotz dieser breiten Perspektive auf Bildungsprozesse ist - insbesondere mit Blick auf die im internationalen Kontext in den letzten Jahren immer relevanter werdenden (vergleichenden) Messungen von Lern-Leistungen (vgl. z.B. Meyer/Benavot 2013) ${ }^{1}$ - darauf hinzuweisen, dass sich inklusiver Unterricht positiv auf die Lernentwicklung aller Lernenden auswirkt (vgl. z.B. Kocaj et al. 2014; Werning 2014; Preuss-Lausitz 2019) und auch bezüglich langfristiger, beruflicher Perspektiven positive Effekte hat (vgl. z.B. Eckhart/Sahli Lozano/Blanc 2011; Werning 2014; Blanck 2020).

1 Dabei ist allerdings festzuhalten, dass Schulleistungsmessungen im Kontext internationaler Studien den Leistungsbegriff engführen, d.h. in der Regel nur Lernzuwachs in Kernfächern überprüfen, und damit die Effekte der Inklusion im Sinne einer breiten Perspektive auf Bildung nicht erfassen können (vgl. Schuelka 2013). 


\section{Inklusive Bildung als Menschenrecht und als globale Norm}

Durch Initiativen wie Education for All (UNESCO 2015), die Zielsetzung des Sustainable Development Goal 4 der Agenda 2030 - ,Ensure inclusive and equitable quality education and promote lifelong learning opportunities for all" (United Nations 2015) - sowie die UNBRK (UN 2006, insbesondere Artikel 24 - Bildung), werden inklusionspädagogische Fragestellungen aufgegriffen. Problemlagen mit Blick auf Teilhabe, Ein- und Ausschluss sowie soziale Ungleichheit werden zunehmend in Bildungspolitik und Bildungspraxis weltweit, oft kontrovers, diskutiert (vgl. z.B. Peters 2007; Richardson/Powell 2011; Budde et al. 2019). Die Ratifizierung der UN-BRK (UN 2008, 2020) durch die Vertragsstaaten hat die Wahrnehmung der Notwendigkeit des Umbaus der Bildungssysteme sowie die Erforschung des Wandels durch Vergleiche unterstrichen. Die Vorgaben der UN-BRK spezifizieren die weltweiten Ideale der Teilhabe und die Normen der Unterstützung jedes*r einzelnen Lernenden im gemeinsamen Unterricht (vgl. Degener 2017). Inklusive Bildung, als Menschenrecht verstanden, ist zugleich Resultat und Quelle dieser Weiterentwicklung von Bildungssystemen im Sinne individualisierter Förderung und Unterstützung. Gleichzeitig aber ist eine Tendenz ersichtlich, dass sich existierende Organisationsformen und affirmative Diskurse verstärken: Professionen, Elternverbände, Behindertenorganisationen haben sich im Anschluss an soziale Bewegungen erfolgreich etabliert und Missstände kritisiert, jedoch bisher nicht gegen die „,institutionelle Logik“ des Bildungswesens, die meistens durch Segregation oder Separation geprägt ist, durchgesetzt (Powell [2011] 2016). Aus dieser Herausforderung begründet sich auch die Relevanz von Forschungsvorhaben, die sich mit (normativen) Diskursen zu Inklusiver Bildung auseinandersetzen (z.B. Biermann 2021 i.d.B.; Hummel 2021 i.d.B.; Zahnd 2021 i.d.B.).

Trotz der unbestreitbaren Erfolge in den Bemühungen, allen Kindern den Zugang zu Bildung zu ermöglichen und somit schulische Exklusion zu reduzieren, ist eine vollständige schulische Inklusion aller Schüler*innen weiterhin eine große Herausforderung. Dies gilt weltweit, wobei sich die Ausgangslagen sehr unterschiedlich darstellen. Selbst in den nordischen Ländern (Dänemark, Finnland, Island, Norwegen und Schweden), denen vergleichsweise fortgeschrittene inklusive Bildungssysteme zugeschrieben werden (vgl. Biermann/ Powell 2014; Barow/Persson/Allan 2016), scheint das Ziel noch nicht erreicht. ${ }^{2}$ So ist eher zutreffend, dass man sich noch auf dem Weg zu einem inklusiven Bildungssystem befindet, obwohl diese Länder zu den egalitärsten sowie wohlhabendsten Regionen der Welt gehören und über demokratische Werte sowie viele Ressourcen verfügen, die Inklusive Bildung begünstigen. Die deutschsprachigen Länder Deutschland, Luxemburg, Österreich und die Schweiz, mit ihren selektiven und stark stratifizierten Bildungssystemen und längeren Traditionen der sonderpädagogischen Förderung in Sonderschulen und -klassen, haben Inklusive Bildung im Anschluss an die internationalen Forderungen zwar ausgebaut, allerdings unter Beibehaltung vieler Sondereinrichtungen (vgl. Powell/Hadjar 2018). ${ }^{3}$

2 Die nordischen Länder werden i.d.B., auch vergleichend, untersucht: Jahnukainen/Itkonen (2021 i.d.B.) sowie Tomlinson (2021 i.d.B.) zu Finnland; Óskarsdóttir/Köpfer (2021 i.d.B.) zu Island; Powell/MerzAtalik et al. (2021 i.d.B.) zu Island und Schweden; Sturm (2021 i.d.B.) zu Norwegen.

3 Viele Kapitel i.d.B. widmen sich den deutschsprachigen Ländern, u.a. Badstieber/Amrhein (2021 i.d.B.), Biewer (2021 i.d.B.), Gasterstädt (2021 i.d.B.), Mejeh (2021 i.d.B.). 
Noch komplexer präsentiert sich die Situation im globalen Süden, dessen Situation hinsichtlich Inklusiver Bildung (allerdings nicht nur diesbezüglich) im globalen Norden kaum (wissenschaftlich) zur Kenntnis genommen wird und die dementsprechend in den wissenschaftlichen Diskursen unterrepräsentiert ist (vgl. Morin 2014; Nguyen 2018; Slee et al. 2019; Hummel 2021 i.d.B.). Dabei wäre die Frage, was Inklusive Bildung gerade in diesen Weltregionen bedeutet, unter den Vorzeichen globaler Disparitäten, die im Süden mitunter prekäre Lebenssituationen und eingeschränkten Zugang zu Bildung zur Folge haben können, äußerst zentral (z.B. Grech 2009; Meekosha/Soldatic 2011). ${ }^{4}$

Die bisherigen Ausführungen zu Inklusiver Bildung sind gerade mit Blick auf ihre Ambivalenz hochrelevant. Denn sie zeigen, dass trotz globalen, normativen Vorgaben und der Ratifizierung der UN-BRK durch eine sehr hohe Anzahl an Vertragsstaaten, die Umsetzung Inklusiver Bildung noch weit von den menschenrechtsbasierten Forderungen entfernt ist. Auch wenn internationale, normative (und rechtliche) Vorgaben sowie wissenschaftliche Befunde die Advokat*innen Inklusiver Bildung nachhaltig stärken, zeigt sich, dass deren Implementierung ein schrittweiser, pfadabhängiger Prozess unterhalb der nationalen Ebene und keine fundamentale Transformation sein wird. So gibt es z.B. gerade in föderalen Ländern wie Deutschland, Österreich, der Schweiz, Spanien, Kanada oder den USA eine Persistenz einzelstaatlicher Disparitäten trotz (inter-)nationaler Ziele, Normen und völkerrechtlicher Verträge (vgl. Biewer 2021 i.d.B.; Blanck 2014, 2020; Blanck/Edelstein/Powell 2013; Gasterstädt 2019, 2021 i.d.B.; Mejeh 2016, 2021 i.d.B.; Schäfer 2021 i.d.B.). Unabhängig von föderalistischen Strukturen vollzieht sich eine Transformation von Exklusion zu Inklusion im Hinblick auf schulisch-unterrichtliche Bildungssettings - wie die Ausweitung des Zugangs zu formaler Bildung insgesamt - bisher in allen Ländern graduell. Dabei wird inklusions- und sonderpädagogische Unterstützung in verschiedenen Organisationsformen angeboten, entlang eines Kontinuums von Segregation (Unterricht in unterschiedlichen Gebäuden), über Separation (Unterricht im selben Schulgebäude aber in unterschiedlichen Räumen) und Integration (teilweise gemeinsamer Unterricht) hin zu Inklusion (umfassender gemeinsamer Unterricht).

Zusammenfassend ergibt sich damit ein zwiespältiges Bild bezüglich der Umsetzung Inklusiver Bildung als Menschenrecht variierend nach Kontext (vgl. Heyer 2021 i.d.B.). Auch wenn sich in Bildungssystemen in einer großen Anzahl von Regionen rudimentäre inklusive Strukturen und Kulturen entwickelt haben und entwickeln, lassen sich derzeit weltweit sehr wenige Bildungssysteme finden, in denen alle Schüler*innen in inklusiven Klassen und Schulen gemeinsam lernen und damit den internationalen, menschenrechtsbasierten Vorgaben entsprechen; hingegen nimmt der Ausbau sonderpädagogischer Förderung seit Jahrzehnten weltweit stetig zu (vgl. Peters 2007; Richardson/Powell 2011; Tomlinson 2017). Die etablierten separierenden und segregierenden sonderpädagogischen Organisationsformen, die verallgemeinert und verteidigt werden, behindern damit den Ausbau Inklusiver Bildung (vgl. Powell [2011] 2016). Zugleich wird so die weiterführende (Aus-)Bildung für Schulabgänger*innen mit Förderbedarf erschwert (vgl. Pfahl 2011; Blanck 2020).

4 In diesem Band werden in einer Reihe von Beiträgen die bildungspolitischen sowie bildungspraktischen Kontexte verschiedener afrikanischer Länder, auch komparatistisch, untersucht: Biermann (2021 i.d.B.) zu Nigeria; Hummel (2021 i.d.B.) zu den Staaten im Süden Afrikas; Proyer/Bilgeri (2021 i.d.B.) zu Äthiopien; Schreiber-Barsch/Rule (2021 i.d.B.) zu Südafrika. 


\section{Historische und vergleichende Ansätze und Studien}

Die bisherigen Ausführungen verweisen unmittelbar darauf, dass im Bereich Inklusiver Bildung viele Gründe bestehen, internationale, interkulturelle und komparative Perspektiven einzubeziehen. Die Stärkung dieses Ansatzes ist somit auch das zentrale Anliegen dieses Handbuches. Seit Mitte des 20. Jahrhunderts hat es eine weltweite Bildungsexpansion gegeben, die kein Land unberührt ließ: Die Institutionalisierung vielfältiger Organisationsformen formaler Bildung schreitet voran, so dass von einer komplett beschulten Gesellschaft gesprochen werden kann (vgl. Baker 2014). Die Sonderpädagogik und sonderpädagogische Einrichtungen haben sich, teilweise vor Einführung der allgemeinen Schulpflicht, etabliert, und somit besonders die Leitprinzipien und Begründungen schulischer Strukturen und pädagogischen Handelns in Bezug auf Heterogenität mitbestimmt (z.B. Hofsäss 1993; Hänsel 2005; Richardson/Powell 2011).

Während internationale und intranationale Vergleiche Kritiken des Status quo begünstigen, unterstreichen auch historische Vergleiche die Kontingenz der Entwicklungen. Seit Jahrhunderten werden Vergleiche als Quelle der wissenschaftlichen Erkenntnis genutzt, durch die Selbstverständlichkeiten hinterfragt werden können (vgl. Schriewer 2003, 2013). Signifikant in unserer globalisierten Welt sind die kontinuierlichen Versuche des ,Lernens von anderen": Ob Schulleitungstests, Benchmarks oder Rankings, kein Land kann sich den internationalen Vergleichsprozessen entziehen, auch nicht im Bereich der sonderpädagogischen Förderung und Inklusiven Bildung (vgl. z.B. OECD 2007; EASNI 2011, 2014; NESSE 2012; UNESCO 2020). Gleichzeitig sammeln Projekte für die unterschiedlichen Ebenen des Schulsystems ,, inspiring practices “, die sie der Praxis, Wissenschaft und Politik zur Verfügung stellen (z.B. Powell/Merz-Atalik et al. 2021 i.d.B.).

Solche Vergleiche gehen von einer grenzüberschreitenden Transnationalisierung aus, gemessen anhand der Verbreitung von Ideen und Leitbildern, von Normen und konkreten Standards oder gar von ganzen Politiken, wobei in allen drei Dimensionen - ideell, normativ und regulativ - die Interpretationen problematisiert und Implementationsprozesse zu analysieren sind (vgl. dazu die theoretischen Ausführungen später im Text). Einerseits fungieren solche Modelle als Orientierung und Legitimation nationaler und lokaler Reformagenden. Andererseits wird in diesen supranationalen Modellen auch etwas Neues konstruiert, basierend auf internationalen Aushandlungsprozessen. Die weltweite Bildungsrevolution (vgl. Meyer 2005; Baker 2014) hat Bildung als Menschenrecht zu einer Selbstverständlichkeit erhoben, wobei die Realisierung in vielen Weltregionen noch aussteht - gerade die Gleichstellung benachteiligter und behinderter Kinder und Jugendlicher stellt eine kontinuierliche Herausforderung für Politik und Praxis dar (Heyer 2015, 2021 i.d.B.). Noch immer lassen sich überall Disparitäten im Zugang, der Lerngelegenheiten und der Abschlüsse finden (UNESCO 2020). Mit den stetig gestiegenen Ansprüchen auf formale Bildung gingen auch verstärkte Messungen und Standardisierungen einher, wie in den OECD-PISA Schulleistungstests ersichtlich wird, wobei auch für diesen Bereich die Exklusion von Schüler*innen mit Förderbedarf problematisiert werden muss - und nicht nur ein Messungsproblem darstellt (vgl. Schuelka 2013). Gleichzeitig bleiben alternative, individualisierte Erfassungen schulischer Leistungen - sowohl quantitativ als auch qualitativ - eine wichtige, aber noch auszubauende, Voraussetzung für Inklusive Bildung.

Vergleichende und institutionelle Analysen liefern Fallstudien der sozialen und politischen Konstruktion von ,Kompetenzen“ oder ,Fähigkeiten“ und ,Förderbedarf‘, von ,Beein- 
trächtigung' oder ,Behinderung' und ,Benachteiligung'. Angesiedelt sind solche Studien an der Schnittstelle von Benachteiligung und Stigmatisierung einerseits, der weltweiten Behindertenbewegung und Stärkung der Menschenrechte andererseits, worauf ihre hohe Bedeutung für Gerechtigkeit und Demokratie basiert. Insbesondere die Entwicklung der Disability Studies erweitert und stärkt diese kritische Perspektive, indem sie einen Paradigmenwechsel von der Defizitorientierung des medizinischen Modells hin zum minderheiten- und menschenrechtsbasierten Verständnis eines sozialen und kulturellen Modells von Behinderung verfolgt - auch im Kontext partizipativer Forschungsansätze (vgl. Nind/Vinha 2014; Buchner 2018, 2021 i.d.B.; Schreiber-Barsch/Rule 2021 i.d.B.). Hier wird der jeweilige Kontext zentral, da das ,behindert werden' v.a. auf Umweltfaktoren zurückzuführen ist (vgl. Cloerkes 2003; Pfahl/Powell 2014, 2016; Köbsell/Hirschberg 2021 i.d.B.; Waldschmidt i.E.). Mit Blick auf die bisher genannten Herausforderungen ergeben sich für komparative Analysen somit einige Herausforderungen.

Wie können Inklusive Bildung und ihre Institutionalisierung im Ländervergleich sinnvoll untersucht werden? Wie werden Merkmale, Differenzen und Vielfalt der Schülerschaft definiert? Welche Kategorien des Förderbedarfs, der Behinderung und der Benachteiligung werden offiziell anerkannt: Wer gilt überhaupt als förder- bzw. unterstützungsbedürftig? Welche schulisch-unterrichtlichen Organisationsformen, also welche Schulen und Klassen, und Unterstützungsdienstleistungen werden in den verschiedenen Bildungssystemen bereitgestellt und welche Standards festgelegt? Neben solchen systembezogenen Fragen ist zudem nach individuellen Bildungs- und Lebensverläufen zu fragen: Welche Lernergebnisse und Schulabschlüsse werden erlangt und wie werden die Übergänge und Zugänge zur Berufsbildung, zur Hochschule und zum Arbeitsmarkt unterstützt? Wie gestalten sich die spätere Teilhabe und die Lebenschancen von Abgänger*innen unterschiedlicher Schulformen?

Aufgrund der relativ geringen, aber wachsenden Anzahl internationaler Vergleiche sonderpädagogischer Fördersysteme wie Inklusiver Bildung im deutschsprachigen Diskurs (siehe z.B. Klauer/Mitter 1987; Albrecht/Bürli/Erdélyi 2006; Biewer/Luciak/Schwinge 2007; Bürli/Strasser/Stein 2009; Bürli 2010, 2020; Erdély/Schmidtke/Sehrbrock 2012; Johnson 2013; Köpfer 2013; Sehrbrock/Erdélyi/Gand 2013; Leonhardt/Müller/Truckenbrodt 2015; Hedderich/Zahnd 2016) gibt es eine ganze Reihe von Forschungslücken. Biewer und Luciak kamen bereits 2010 zu einem heute noch gültigen Schluss: „Trotz der Fülle an Darstellungen von Sonderpädagogik in anderen Ländern, [...mangelt] es nach wie vor sowohl an anspruchsvollen systematischen Arbeiten wie auch an empirischer Forschung mit elaboriertem Methodendesign“ (Biewer/Luciak 2010: o.S.). Wenngleich Forschungsfragen Inklusiver Bildung zunehmend über die Grenzen der Sonderpädagogik hinweg in Kontexten der Erziehungswissenschaften, Migrationspädagogik und angrenzenden, transdisziplinären Feldern bearbeitet werden (vgl. Panagiotopoulou et al. 2020) und häufig mit differenz- und alteritätstheoretischen Fragestellungen verbunden werden (vgl. u.a. Emmerich/Hormel 2013; Budde et al. 2017, 2019), lässt sich konstatieren, dass grundlegende systematische Erkenntnisse bezogen auf internationale Vergleiche und Kulturvergleiche bislang noch ausstehen.

Auch im englischsprachigen Diskurs ist die Forschungslage bezüglich Komparatistik noch ungenügend (vgl. Carrier 1984; gesammelte Länderberichte, z.B. Mazurek/Winzer 1994; Richardson/Powell 2011). So zeigt Brown (2014) in einem Review wissenschaftlicher Beiträge mit Bezug auf das Thema „Behinderung“ in der vergleichenden und internationalen Erziehungswissenschaft in führenden englischsprachigen Zeitschriften, dass v.a. zwei Themen dominieren: die soziale Interpretation von Behinderung sowie Fragen der analytischen Betrachtungsebenen - global, national oder lokal. Die globale Wahrnehmung von Behin- 
derung in verschiedenen Paradigmen - medizinisch-klinisch, sozial-politisch sowie menschenrechtsbasiert - kann anhand von Diskursanalysen internationaler Organisationen (z.B. die Weltbank, siehe Zahnd 2017, 2021 i.d.B.) oder die Übersetzung und Wirkung dessen in konträren Kulturen und Ländern gezeigt werden (z.B. der Vergleich der Umsetzung der UNBRK in Deutschland und Nigeria, siehe Biermann 2021 i.d.B.). Vergleichende und synthetische Literaturstudien sind rar, stellen aber eine wichtige Grundlage komparatistischer Forschung dar, ob international oder regional (z.B. zu den internationalen Disability Studies vgl. Biermann/Powell, 2021 i.E.; Hummel 2021 i.d.B.).

Trotz umfassenden vergleichenden Forschungsbemühungen in den letzten Jahrzehnten, wie die der UNESCO oder der OECD sowie, in Europa, die der European Agency for Special Needs and Inclusive Education (EASNIE) oder der Expertennetzwerke, gibt es viele Wissenslücken zu den Entwicklungen von Unterstützungssystemen wie auch zu den Bestrebungen, inklusive Bildungssysteme zu entwickeln. Die bestehende, vergleichende Forschung bezieht sich zudem v.a. auf westliche Länder, wobei auch hier große Anstrengungen nötig sind, um verlässliche Datengrundlagen zur Implementierung inklusiver Bildungsangebote herzustellen (vgl. MIPIE 2011). Die EASNIE fasst die Zusammenarbeit und Koordination in 31 Mitgliedstaaten in Europa der letzten Dekade so zusammen: „Der Prozess der Planung und Umsetzung der inklusiven Bildung betrifft das gesamte Bildungssystem und alle Lernenden; Gerechtigkeit und Qualität sind miteinander verbunden; inklusive Bildung muss als ein sich ständig entwickelndes Konzept betrachtet werden, in dem Fragen zur Diversität und Demokratie an Bedeutung zunehmen“ (EASNIE 2014: 7). Zudem wird die bedeutsame Rolle von Daten, sowohl hinsichtlich ihrer Vorteile sowie bezogen auf Einschränkungen ihrer Verwendung betont (EASNIE 2014). Ähnlich zeigt der 2020 Global Education Monitoring Report mit dem Titel „Inclusion and Education: All Means All“ (UNESCO 2020) wie wichtig sowohl Datensammlung als auch komparative Mehrebenanalysen sind, die ähnliche wie kontrastierende Dynamiken auf globaler, nationaler und lokaler Ebene untersuchen und vergleichen.

Durch die internationale Reaktion auf die Coronavirus-Pandemie im Jahr 2020 wurde neben der Stillstellung etlicher Zweige der Wirtschaft auch der formale Bildungsbetrieb in Schulen in vielen Ländern kurzfristig unterbunden und es kam zu einer weltweiten Schließung von Schulen, welche bis zu 90 Prozent der weltweiten Schülerpopulation betroffen hat. Dies hat zu einer Krise im Kontext von Bildung beigetragen bzw. krisenhafte Verhältnisse verstärkt, insbesondere mit Blick auf existierende Disparitäten global sowie innerhalb von Ländern (UNESCO 2020; vgl. für Großbritannien, Tomlinson 2021 i.d.B.), die - bereits bestehend - durch die Coronavirus-Pandemie brennglasartig zum Vorschein gebracht wurden bzw. sich intensivieren. Die Stillstellung sowie Digitalisierung von Bildungsorganisationen und die im Zuge der Wiederöffnung verfolgte Priorisierung schulischer Prinzipien (u.a. die leistungsnormierte Qualifikationsfunktion von Schule) können und sollten Anlass für internationale und interkulturelle Diskussionen und Untersuchungen im Kontext Inklusiver Bildung sein. Zukünftige Analysen sollten global verstärkt sowohl zwischen Osten und Westen als auch zwischen Norden und Süden vergleichen, insbesondere aus interkultureller Perspektive, um die Folgen globaler Entwicklungen wie Herausforderungen zu bemessen und zu verstehen (vgl. Biermann et al. 2014). Die gestiegene Relevanz internationaler Organisationen (Zahnd 2021 i.d.B.) sowie Netzwerke der Behindertenbewegung könnten analysiert werden, um die Interpretation globaler Normen wie die der Inklusion auf unterschiedlichen Ebenen zu untersuchen (vgl. Biewer/Luciak 2010; Artiles/Kozleski/Waitoller 2011). 
Damit die aktuellen Grenzen inklusiver Bildung erkannt, verstanden und verändert werden können, sind theoretisch anspruchsvolle und systematische Ländervergleiche von Bildungssystemen sowie die Erforschung internationaler (z.B. neoliberaler oder menschenrechtsbasierter) Strömungen und Entwicklungslinien im Bereich Bildung entscheidend. Um den komplexen Zusammenhang Inklusiver Bildung im globalen Kontext fachlich zu fassen, bieten sich unterschiedliche Ansätze an, die - theoretisch wie empirisch - Analysezugänge und Methoden zur Verfügung stellen, um den postulierten und angestoßenen Transformationsprozess im Bereich Inklusiver Bildung kritisch zu begleiten und international sowie interkulturell zu vergleichen. Entlang der disziplinären Affinität des Handbuches an der Schnittstelle soziologischer und erziehungswissenschaftlicher Betrachtungsweisen, wird im Folgenden ausschnitthaft in hierfür ausgewählte zentrale theoretische wie methodologische Ansätze eingeführt.

\section{Inklusive Bildung als Thema der Weltgesellschaft}

Durch die Platzierung auf der Agenda der Vereinten Nationen und bedeutsamen internationalen Organisationen - z.B. UNESCO (vgl. Kiuppis 2014), Weltbank (vgl. Zahnd 2017, 2021 i.d.B.) oder OECD (2007) - verweisen Inklusion und damit auch Inklusive Bildung implizit auf einen weltweiten Begründungszusammenhang. Das heißt, die sich vollziehenden Entwicklungen können nicht mehr ausschließlich auf Ebene der Nationalstaaten erfasst werden, sondern beziehen sich gleichzeitig auf einen globalen Referenzrahmen. In der Soziologie wird dieser übergeordnete Rahmen seit den 1970er Jahren über den Begriff der „Weltgesellschaft" gefasst (vgl. Greve/Heintz 2005), wobei verschiedene theoretische Konzeptionen existieren wie z.B. der Weltsystemansatz (Wallerstein 2004), der World Polity Ansatz (Baker 2014; Meyer 2005, 2009; Krücken/Drori 2009) oder die Weltgesellschaftstheorie von Peter Heintz (1982). Als Grundlogik teilen sie das Verständnis, dass es zusätzlich zu den Nationalstaaten einen weltweiten Funktionszusammenhang gibt (vgl. Biermann et al. 2014), der sich nicht als Summe nationalstaatlicher Handlungen erklären lässt, sondern eine eigenständige Organisationsform aufweist (vgl. Greve/Heintz 2005).

Weltgesellschaftliche Theorieansätze ${ }^{5}$ sind an dieser Stelle von Relevanz, weil sie dazu anregen, Inklusive Bildung in ihrer globalen Komplexität zu analysieren, und dazu eine Rahmung liefern, die helfen kann, neuralgische Punkte zu identifizieren. So eröffnen die Ansätze von Wallerstein (2004), Baker (2014) oder Heintz (1982) z.B. neue Perspektiven auf die Frage globaler Konvergenz sowie Disparitäten, die für Fragen Inklusiver Bildung hilfreich sind, weil sie ein vertieftes Verständnis für die Zusammenhänge zwischen kulturellem Wandel, Entwicklungspolitik und Bildung ermöglichen. Gerade die Problematik globaler Disparitäten und damit verbundener Entwicklungspolitik, die unmittelbar mit der Frage von Bildung verbunden ist, kann vor dem Hintergrund der (insbesondere auch historisch bedeutsamen) Hegemonie des globalen Nordens als äußerst relevant erachtet werden. Zu thematisieren wären diesbezüglich auch Vorwürfe wie derjenige der latenten Kolonialisierung über Ideen und Konzepte, die trotz global breit abgestützten Debatten im Kontext der Vereinten

5 Es sei darauf hingewiesen, dass Theoriekonzepte mit Fokus auf globale Zusammenhänge nicht nur im Kontext der Soziologie bestehen, sondern auch andere wissenschaftliche Disziplinen solche aufweisen, wie bspw. die Politikwissenschaft sowie Ökonomie. 
Nationen dazu führen, dass Inklusive Bildung teilweise mit Konflikten konfrontiert ist, die in den Ländern des globalen Nordens kaum mitgedacht werden können (vgl. Grech 2011; Meekosha 2011; Biermann et al. 2014). Trotz der globalen Reichweite und des Miteinbezugs aller Mitgliedstaaten sowie die für die Entwicklung der UN-BRK entscheidende Kooperation der weltumspannenden Behindertenbewegung (Charlton 1998) folgen die Menschenrechte einer Konzeption, die wesentlich im globalen Norden entwickelt wurde (Hessel 2010). Dies mindert nicht den Wert daraus entstandener normativer Dokumente und Prozesse, aber es regt dazu an, gerade diese Weltregionen mitzudenken, die ansonsten kaum in hegemonialen, wissenschaftlichen Diskursen auftreten.

Während im Kontext soziologischer Auseinandersetzungen mit der Rahmung Weltgesellschaft bis heute verschiedene Theorieansätze Verwendung finden - die meistrezipierten Zugänge sind dabei der Weltsystemansatz, die World Polity Schule oder der neogramscianische Ansatz (vgl. Herkenrath 2012) - verengt sich die Gesamtperspektive im Rahmen erziehungswissenschaftlicher Forschung deutlich. So finden sich sowohl im Bereich internationaler Bildungsexpertisen (vgl. Bürgi 2017) als auch im Kontext internationaler Studien zum Thema Inklusion häufig neo-institutionalistische Zugänge. Es werden auch in diesem Band Anwendungen in unterschiedlichen Kontexten präsentiert, wobei insbesondere die Beiträge von Biermann (2021 i.d.B.), Mejeh (2021 i.d.B.), Schäfer (2021 i.d.B.) und SchreiberBarsch/Rule (2021 i.d.B.) diese theoretische Grundlage verwenden. Aufgrund seiner relativ hohen Verbreitung und unserer Einschätzung, dass er für die internationale, komparative Forschung eine gewinnbringende theoretische Konzeption beinhaltet, soll dieser Zugang nachfolgend dargestellt werden.

\section{Zur Institutionalisierung sonderpädagogischer Förderung und Inklusiver Bildung}

Der Fokus des historischen sowie soziologischen Neo-Institutionalismus liegt auf der Institutionalisierung diverser Organisationsformen - von Sonderschulen und Sonderklassen bis hin $\mathrm{zu}$ inklusiven Klassen - und bezieht deren historische Entwicklung in verschiedenen Kontexten mit ein. Institutionen bauen auf kognitiven, normativen und regulativen Strukturen und Verhaltensweisen auf. Institutionalisierung, verstanden als Entwicklungsprozess der Verfestigung sozialer Normen und Verhaltensmuster, kann über diese Dimensionen untersucht werden. Nach Scotts (2013) klassischer Darstellung werden drei Säulen oder Dimensionen von Institutionen analysiert, im Fall der Analyse Inklusiver Bildung wären dies: Behinderungsparadigmen und -kategorien als kulturell-kognitive Säule, pädagogische Professionen und Organisationsformen als normative Säule und die Bildungspolitik und Rechtsprechung als regulative Säule (vgl. Powell [2011] 2016; Mejeh 2021 i.d.B.). Diese umfassende Konzeption ermöglicht den kontextualisierten Vergleich fundamentaler Aspekte von Bildungsinstitutionen, die die Entwicklung diverser Bildungsorganisationen rahmen (vgl. Leemann et al. 2016).

Mit Hilfe des Neo-Institutionalismus kann einerseits aufgezeigt werden, dass sowohl sonderpädagogische Förderung als auch schulische Inklusion als Prinzipien - manchmal konflikthaft, jedoch meistens aufeinander bezogen - weltweit diffundieren und zu globalen Normen geworden sind. Andererseits können Barrieren der schulischen Inklusion darauf 
zurückgeführt werden, dass sonderpädagogische Fördersysteme in vielen Ländern vornehmlich als segregierende oder separierende Organisationsformen institutionalisiert wurden, die nur schwer zu verändern sind. Zum einen wird also die zunehmende Rationalisierung und Strukturanpassung (,Isomorphie') einer sich-konstituierenden Weltgesellschaft in solchen Analysen wie auch der breite Einfluss nationaler wie internationaler Institutionen und Organisationen deutlich (vgl. Meyer 2005; Baker 2014). Zum anderen wird immer wieder die Entkoppelung von Umweltanforderungen (etwa die Vorgaben der UN-BRK zur schulischen Inklusion) und das konkrete Handeln in Organisationen (etwa inner-schulische Praxen, die nicht inkludierend wirken) festgestellt (vgl. Hasse/Krücken 2005). Aufgrund solcher Spannungsverhältnisse sind große Unterschiede in den institutionellen Strukturen und Organisationsformen der sonderpädagogischen Förderung und den daran geknüpften Schülerkarrieren sowohl zwischen als auch innerhalb von Ländern zu finden (vgl. Powell [2011] 2016). Wie es zu dieser Varianz kommt und inwiefern persistente Disparitäten auf der Trägheit der institutionalisierten Bildungssysteme beruhen, wird mit Ansätzen des Neo-Institutionalismus analysierbar. Sonderpädagogische Fördersysteme bieten in Bezug auf Entstehung, Expansion und Persistenz ein paradigmatisches Beispiel für die eigendynamische institutionelle Verstärkung existierender Organisationsformen im Bildungswesen, dies zeigt sich z.B. daran, dass derzeit immer noch die überwiegende Mehrheit aller Schüler*innen mit SPF in Deutschland segregativ beschult wird, trotz Ratifizierung der UN-BRK (vgl. Blanck/Edelstein/Powell 2013; Powell [2011] 2016; Gasterstädt 2019, 2021 i.d.B.).

Das Erreichen institutionell bestimmter Ziele erfolgt in hohem Maße durch Organisationen. Um die vielfältigen, sich wandelnden Organisationsstrukturen pädagogischer Angebote sowie sonderpädagogischer Fördersysteme zu verstehen, sind Analysen notwendig, die die komplexen und langfristigen sozialen Prozesse der Institutionalisierung als empirisch analysierbare Dimensionen fassbar machen. Kennzeichnend für den soziologischen Neo-Institutionalismus sind folgende Annahmen (vgl. Hasse/Krücken 2005: 18f.): Erstens bestimmen nicht nur formale Vorgaben, sondern auch unhinterfragte Ideale und Grundüberzeugungen das Handeln. Die Wirkung solcher Annahmen und ihrer Hartnäckigkeit - etwa das Ideal einer der ,Begabung ' entsprechenden Beschulung - zeigt sich in den derzeitigen Debatten um schulische Inklusion und Schulstrukturreformen im Allgemeinen. Zweitens bleiben in der Praxis, die oft durch Routinen und Angemessenheitskriterien geleitet wird, Freiräume bestehen. Die entstandenen Förderbedarfe spiegeln die politisch gesetzten Rahmenbedingungen ebenso wider wie persönliche Erfahrungen und Präferenzen, aus denen große räumliche Disparitäten in der Identifikation von Förderbedarfen resultieren, etwa wenn Finanzierungsmodi erhöhte Förderquoten mit zusätzlichen Ressourcen belohnen (vgl. Kottmann 2006). Drittens bestimmen nicht nur allein die Normen, sondern auch die Orientierungen der Akteur*innen die Erwartungen und das Handeln. Gemeinsamer Unterricht wird oft von Lehrer*innen sowie Familien gegen allgemeine Normen im Bildungswesen und in der Gesellschaft durchgesetzt. Viertens haben institutionelle Vorgaben oft nicht-intendierte Folgen und erfüllen nicht immer die gewünschten positiven Funktionen. Trotz der Annahme, dass zusätzliche Ressourcen für Förderung Schüler*innen helfen, zeigt sich, dass Stigmatisierungen sowie negative Folgen von Separation und Segregation die positiven Aspekte oft deutlich übersteigen (vgl. Pfahl 2011). Fünftens muss davon ausgegangen werden, dass Institutionen, von ihrer Begründung über die Genese bis hin zur Erosion, von langfristigen Prozessen und verschiedenen Entwicklungsphasen bestimmt werden. In solchen Analysen können Organisationsformen und -praxen auf der Basis von institutionellen Mechanismen der Nachahmung, des normativen Drucks und des Zwangs untersucht werden (vgl. Scott 2013). Die Entwicklung von Bildungs- 
systemen erfolgt ,pfadabhängig', d.h. schrittweise verstärkend, denn jegliche Reform muss sich auf die schon existierenden Institutionen und Organisationen beziehen, die aufgrund bereits erfolgter Investitionen schwer veränderbar sind (vgl. Blanck/Edelstein/Powell 2013): Dies zeigt sich darin, dass Sonderschulen und -klassen bis heute und aufgrund der institutionellen Reproduktion die Hauptorganisationsformen sonderpädagogischer Förderung im deutschsprachigen Raum - und darüber hinaus - geblieben sind (vgl. Richardson/Powell 2011; Mejeh 2016).

Diese Analyseperspektive fokussiert also die Bedeutung von Ideen für die Entwicklung von Institutionen und Organisationen und unterstreicht dabei die kulturellen und strukturellen Kontextfaktoren, die inklusive Bildung erschweren oder ermöglichen (vgl. Biermann 2021 i.d.B., i.E.). Wechselwirkungen zwischen Behinderungsparadigmen sowie Kategorien des Förderbedarfs einerseits und der Entwicklung sonderpädagogischer Organisationsformen andererseits werden sichtbar, wenn rechtlich kodifizierte, disziplinär abgesicherte und kulturell geprägte Klassifizierungsprozesse in sonderpädagogischen Fördersystemen symbolische und soziale Grenzen ziehen, die weitreichende Auswirkungen auf die individuellen Entwicklungsmöglichkeiten und Lebenschancen von Schüler*innen haben (vgl. Pfahl/Powell 2016).

Die aktuelle Verfasstheit der Fördersysteme und die Debatten um deren Reform sind nur zu verstehen, wenn die historischen Legitimitätsbestrebungen und Interessenlagen bekannt sind, die zu diesen hochgradig institutionalisierten Organisationsformen geführt haben. Neoinstitutionalistische Ansätze unterstreichen die Wirkmächtigkeit historisch überlieferter Ideen - etwa medizinischer Modelle von Behinderung - sowie die Persistenz der auf deren Basis legitimierten schulischen Organisationsformen - wie Sonderschulen oder -klassen. Wesentliche Barrieren für die heutige schulische Integration respektive Inklusion, v.a. in westlichen Wohlfahrtsstaaten, liegen in der langfristigen Institutionalisierung sonderpädagogischer Fördersysteme sowie der Stratifizierung des Bildungswesens. Die unterschiedlichen Bildungssysteme wurden durch sich verändernde Behinderungsparadigmen und kulturelle Leitideen, insbesondere in Bezug auf Bildung, und Gleichheitsprinzipien sowie politische und gesellschaftliche Bewegungen geprägt. Die Förderbedürfnisse behinderter Kinder und Jugendlicher lassen sich nicht allein durch ihre Diversität, ihre Bildungswege und Lern(miss)erfolge erklären; der Umgang mit Vielfalt wird langfristig im Bildungssystem entwickelt (vgl. Schäfer 2019, 2021 i.d.B.).

Aktuell wird weltweit über Leistungsstandards und individuelle Kompetenzen sowie die vielerorts angestrebte ,Schule für alle' diskutiert (Moser 2012). Dennoch, wenn schulische Inklusion, in welcher alle Schüler*innen in der Erreichung ihrer individuellen Lernziele unterstützt werden, nicht nur eine rhetorische Forderung bleiben soll, muss sich diese gegen kognitive, normative und rechtliche Beharrungskräfte der institutionalisierten Organisationsformen durchsetzen, die für Schüler*innen mit attestiertem Förderbedarf entwickelt worden sind. Hier kann v.a. zwischen graduellem und transformativem Wandel unterschieden werden, exemplarisch zeigt sich diese Differenz z.B. im stetigen Ausbau des Sonderschulwesens seit den 1960er-Jahren bis heute in Westdeutschland im Vergleich zur Transformation des Bildungswesens in den neuen Bundesländern nach der Wiedervereinigung (mit einer erstaunlich starken Erhöhung der Sonderbeschulung in den neuen Bundesländern, vgl. Autorengruppe Bildungsbericht 2014, 2020).

Insbesondere in föderalen politischen Systemen wie Deutschland (vgl. z.B. Blanck 2014, 2020; Gasterstädt 2019, 2021 i.d.B.), Österreich (vgl. Biewer 2021 i.d.B.), der Schweiz (vgl. Mejeh 2016, 2021 i.d.B.), Kanada (vgl. Köpfer 2013; Óskarsdóttir/Köpfer 2021 i.d.B.), oder den USA (vgl. z.B. Johnson 2013; Powell [2011] 2016) darf die Analyse von Disparitäten 
nicht auf der Nationalebene stehen bleiben, da es auch große regionale Varianzen gibt; es sind deshalb auch intranationale Vergleiche von Nöten. Vor diesem Hintergrund sind in diesem Handbuch spezifisch für Deutschland (vgl. Gasterstädt 2021 i.d.B.; Schäfer 2021 i.d.B.), Österreich (vgl. Biewer 2021 i.d.B.) und die Schweiz (vgl. Mejeh 2021 i.d.B.) intranationale Vergleichsperspektiven eingebracht worden.

\section{Ethnographische und kulturvergleichende Perspektiven auf Inklusive Bildung}

Weitere Forschungs- und Analysezugänge, die sich vergleichend mit Fragestellungen (Inklusiver) Bildung im internationalen bzw. interkulturellen Kontext - insbesondere mit Blick auf Prozesse und Praktiken in Bildungsorganisationen - beschäftigen, greifen ethnographische und kulturvergleichende Verfahren auf (vgl. Hummrich et al. 2016; Nohl 2019). Hier steht im Zentrum, wie sich - vor dem Hintergrund sozialer, kultureller, politischer Rahmenbedingungen in entsprechenden Ländern bzw. Regionen - Praktiken bei Akteur*innen in Bildungsorganisationen entwickelt haben, in denen Partizipation, Nichtaussonderung, Gemeinsames Lernen und ein wertschätzender Umgang mit Verschiedenheit zum Ausdruck kommen. Insbesondere durch internationale Policies, wie z.B. die Salamanca-Erklärung (UNESCO 1994) oder die menschenrechtliche Fundierung von Inklusiver Bildung durch die UNBRK, werden in globaler Hinsicht Normen vereinbart und formalisiert, die sich in den spezifischen Länderkontexten diffundieren und materialisieren sollen.

Ethnographische und kulturvergleichende Zugänge ermöglichen hierbei, an der Scharnierstelle von Norm und Praxis Erkenntnisse zur Umsetzung und (Re-)Kontextualisierung Inklusiver Bildung zu generieren (vgl. Köpfer 2020a). So interessieren sich Vertreter*innen dieser Ansätze dafür, mitunter ambivalente Aushandlungsprozesse und - im strukturtheoretischen Sinne - antinomische Beziehungen des Umgangs mit veränderten formalen Bedingungen (z.B. Fragen von Professionalisierung und Expertise im Rahmen inklusionsorientierter Lehrer*innenbildung, Aushandlungen von formalen Rollen und veränderten Zuständigkeiten im schulischen Kontext, etc.) abzubilden und diese spannungsbeladenen Zusammenhänge aufzudecken. Dabei wird davon ausgegangen, dass durch Inklusive Bildung bereits bestehende Ambivalenzen im Rahmen von institutionalisierten Bildungs-, Erziehungs- und Sozialisationsprozessen (z.B. Förderung vs. Selektion, Nähe vs. Distanz) re-aktualisiert und hinsichtlich ihrer Spannungsbeladenheit - besonders bezogen auf Fragen von Ein- und Ausschluss, Partizipation und Aussonderung - neu und kritisch verhandelt werden (Slee 2001; Allan 2008).

Diese häufig wissenssoziologisch und kulturtheoretisch informierten Zugänge gehen ähnlich zum oben aufgeführten neo-institutionalistischen Ansatz - von der Annahme aus, dass institutionalisierte Bildungsorganisationen globale Ähnlichkeiten aufweisen, sich aber gleichzeitig in unterschiedlichen Länderkontexten, Regionen, Kulturen different und in Relation zu vorherrschenden sozialen, politischen und religiösen Traditionslinien entwickelt haben. Diese Vorstellungen von Bildung und Erziehung haben sich im Bildungssystem materialisiert und drücken sich u.a. in der Struktur des Schulsystems, z.B. höchst leistungsselektiv und -stratifiziert in den deutschsprachigen Ländern, aus. 
So haben sich fortlaufend in unterschiedlichen Länderkontexten Bildungs-, Schul- und Lernkulturen entwickelt, die sich sowohl in der institutionalisierten Struktur sowie in den Bildungspraktiken der Akteur*innen widerspiegeln. Kultur wird dabei nicht anthropologisch als essentialistische Entität verstanden, sondern als relationales Interaktionsmuster, das sich in Organisationen entwickeln und materialisieren kann (vgl. Dietz 2007: 21). Daher können es höchst unterschiedliche Fragen von Ein- und Ausschluss in Bildungsorganisationen sein, die in den jeweiligen kulturellen und länderspezifischen Kontexten artikuliert werden (vgl. Hall 1985; Köpfer 2020b). Während in Deutschland Inklusive Bildung im Kontext Schule primär als Gegenstand des gemeinsamen Lernens von Schüler*innen mit zugewiesenem sonderpädagogischen Unterstützungsbedarf artikuliert wird, wird sie in anderen Ländern zum Beispiel mit Fragen und Diskursen demokratischer Schulentwicklung (Island; vgl. Óskarsdóttir/Köpfer 2021 i.d.B.) oder Bildung für Alle (Nigeria; vgl. Biermann 2021 i.d.B.) verbunden. So wird deutlich, dass die UN-BRK zwar als Resultat einer internationalen Emanzipationsbewegung für mehr gesellschaftliche Partizipation für Menschen mit zugeschriebenen Behinderungen erachtet werden kann, die in einer menschenrechtsbasierten Fundierung von Inklusiver Bildung mündete (vgl. Heyer 2021 i.d.B.). Der dadurch angestoßene Umsetzungsprozess - zwischen formal-programmatischer Politiken und habitueller Praxis/Kultur in Bildungsorganisationen (vgl. Sturm/Wagner-Willi 2018) - nimmt in interkultureller Hinsicht aber differente Ausprägungsformen an.

Unter den Vorzeichen des oben eingeführten Kulturbegriffs können ethnographische und kulturvergleichende Zugänge dazu dienen, vertiefte empirische Analysen von Behinderungen und Benachteiligungen in Bildungsorganisationen zu prozessieren, die sich letztlich in Form von Ein- und Ausschluss für spezifische Personenkreise ausdrücken. Werden Behinderungen - in einem sozial-konstruktivistischen Verständnis - nicht als naturalisierte und personenbezogene Defizite betrachtet, sondern, im Anschluss an die International Classification of Functioning, Disability and Health (ICF) (vgl. WHO 2005; Hirschberg 2009) als Wechselwirkung von systemischen Rahmenbedingungen bzw. Kontextfaktoren und personenbezogenen Dispositionen, so können im Kontext internationaler Inklusionsforschung ebensolche Rahmenbedingungen vergleichend in den Blick genommen werden, die Behinderungen und Benachteiligungen hervorbringen. Dieses Moment der Hervorbringung wird nicht zuletzt durch die Vorarbeiten von Tony Booth und Mel Ainscow (2002) zum Ausdruck gebracht, in dem sie in ihrem systemisch angelegten Inklusionsverständnis (Index for Inclusion) auf Strukturen, Kulturen und Praktiken von Bildungsorganisationen fokussieren, die potenzielle Barrieren beinhalten und die es kritisch zu reflektieren und bezogen auf die Partizipation aller weiterzuentwickeln gilt. Bei Inklusion im schulischen Kontext steht also nicht die Integration spezifischer Gruppen im Vordergrund, sondern die Weiterentwicklung von Schule im Sinne eines Schulentwicklungsprozesses zur Vermeidung von Aussonderung (vgl. Ainscow 2021 i.d.B.; Allan 2008; Messiou 2017). Abgehend von einer Naturalisierung sozialer Differenzen (vgl. Bourdieu 1987; Rieger-Ladich 2019) - kann so der Blick auf Strukturen, Kulturen und Praktiken in Bildungsorganisationen gelenkt werden, die in der Herstellung von Differenz oder Gemeinschaftlichkeit münden und im internationalen Vergleich bzw. Kulturvergleich adressiert werden können, z.B. in schulischen Leistungserwartungen (vgl. Sturm 2021 i.d.B.), in Behinderungs- und Inklusionsverständnissen von Akteur*innen (vgl. Proyer/ Bilgeri 2021 i.d.B.), in innerschulischen Unterstützungs- und Assistenzpraktiken für Vielfalt (vgl. Fritzsche/Kakos 2021 i.d.B.; Óskarsdóttir/Köpfer 2021 i.d.B.; Tan 2021 i.d.B.), im Schulleitungshandeln mit Blick auf den Reformauftrag Inklusion (vgl. Badstieber/ 
Amrhein 2021 i.d.B.) oder in Unterrichtspraktiken zum Umgang mit heterogenen Lerngruppen (vgl. Powell/Merz-Atalik et al. 2021 i.d.B.).

So können vergleichend in den jeweiligen Ländern und Regionen vor dem Hintergrund spezifischer kultureller Rahmenbedingungen „Inklusionsbedingungen“ (Weisser 2017: 146; Köpfer et al. 2019) und Exklusionsmechanismen sichtbar gemacht werden, die sich in den Kulturen, Strukturen und Praktiken der Bildungsorganisationen verankert haben und als Barrieren fortlaufend an die Schüler*innen gerichtet werden und über deren Teilhabe am und im System entscheiden (vgl. Graf 2015).

Ethnographische und kulturvergleichende Zugänge bieten sich für eine solche international und interkulturell vergleichende Inklusionsforschung in besonderem Maße an, da implizite und habitualisierte Prozesse der Herstellung von Behinderungen und Benachteiligungen in und durch Bildungsstrukturen und -kulturen fokussiert werden können - die sozusagen als Beobachtung zweiter Ebene sichtbar werden (vgl. Weisser 2017, in Rekurs auf Luhmann). Diese sind den Akteur*innen häufig nicht vorbewusst zugänglich, sondern in habitualisierten Praktiken verankert. Gerade im Vergleich und in der Möglichkeit, das Proprium als Drittes (Tertium Comparationis) über den Vergleich zu erkennen, können diese Praktiken sichtbar gemacht werden. Fuchs und Berg (1999: 7) drücken diese Alteritätsperspektive wie folgt aus: „Das Andere der eigenen Kultur, dem wir im Fremden begegnen, bildet eine Herausforderung, die den Horizont der eigenen Lebensweise transzendiert und für andere Möglichkeiten der Existenz öffnet, die eigene Lebensweise aber auch in Frage stellt." Dies hat zur Konsequenz, dass über Forschung einerseits eine Nähe zum Feld aufgebaut wird, um vertieft und mikroanalytisch die Regeln, Strukturen und Praktiken des Feldes kennenzulernen - gleichsam aber auch eine forschungsstrukturierende Distanz gewährt wird. Für eine solche ,dichte Beschreibung“ (Geertz 1987) im internationalen und interkulturellen Vergleich werden daher häufig Methoden aus der Ethnomethodologie, wie z.B. Teilnehmende Beobachtungen, Feldprotokolle, Interviews, Gruppendiskussionen oder videographische Verfahren herangezogen. Durch diese können auf reflexive Art und Weise die Praxis des Feldes beobachtet und Narrative kollektiviert werden, die Rückschlüsse auf Orientierungen und habitualisierte Praktiken von Akteur*innen, z.B. auf Vorstellungen von Behinderungen und deren Bearbeitung und Aushandlung vor dem Hintergrund formaler Rahmenbedingungen geben.

Weiter zeichnet sich die Tendenz einer zunehmenden Etablierung kulturvergleichender Zugänge in der Inklusionsforschung ab (vgl. Hummrich et al. 2016; Hummrich/Pfaff 2018), in denen im Vordergrund steht, die oben genannten habitualisierten Praktiken des Feldes mittels rekonstruktiver Methoden zu adressieren. Unter Rekonstruktion kann hierbei verstanden werden, ,nicht auf der Ebene der Selbstrepräsentanz der sozialen Praxis stehen zu bleiben, sondern diese selbst als hervorgebracht zu entwerfen und das Prinzip ihrer Hervorbringung freizulegen“ (Hummrich/Kramer 2016: 66f.). So wird intendiert, Prozesse von Ein- und Ausschluss nicht als statisches Phänomen zu erfassen und international zu vergleichen, sondern die Kulturen der spezifischen Regionen und deren Bildungsorganisationen als performatives Phänomen zu adressieren. Hierdurch kann, so Hummrich und Rademacher (2016: 10) ein ,analytischer Nachvollzug[s] kultureller Bedeutungskonstruktionen in Sozialisation, Erziehung und Bildung“" am Gegenstand der Entwicklung inklusiver Bildung festgemacht werden. Wenngleich weitreichende Forschungsbefunde und eine ausgearbeitete Methodologie des Kulturvergleichs und der Rekonstruktion in der international und interkulturell vergleichenden Inklusionsforschung noch ausstehen, scheinen hier Möglichkeitsräume gegeben, um soziale Konstruktionen, wie z.B. die Zuschreibung eines sonderpädagogischen Förderbedarfs oder die Etablierung einer paraprofessionellen Assistenzrolle einer Schulbegleitung 
(vgl. Fritzsche/Köpfer 2019), vergleichend, aber in Relation zu den schulkulturellen Ausgangslagen der jeweiligen Regionen und mit Blick auf deren Hervorbringung zu analysieren. Dabei geht es weniger darum, Gelingensbedingungen herauszuarbeiten, sondern vielmehr durch Kulturvergleiche komplexe Strukturen, Kulturen und Praktiken von Ein- und Ausschluss offenzulegen und hierdurch Erkenntnisse zu internationalen Zusammenhängen und Regelhaftigkeiten hervorzubringen (Artiles/Dyson 2005). So können gerade komparative Analysen mittels qualitativer-rekonstruktiver Forschung dazu beitragen, apodiktische Annahmen zu hinterfragen (Arnove 2012; Parreira do Amaral 2015), transzendentale Perspektiven zu den jeweiligen Bildungsstrukturen, -kulturen und -praktiken zu entwickeln (Adick 2015; Dale 2015) und Erkenntnisse zu historisch und kulturell geprägten Vorstellungen von Inklusiver Bildung zu liefern (D’Alessio/Cowan 2013).

\section{Resümee - Perspektiven und Spannungsfelder international und interkulturell vergleichender Inklusionsforschung}

Die Entwicklung Inklusiver Bildung im Anschluss an menschenrechtliche Forderungen stellt uns insgesamt vor etliche Herausforderungen. Dies gilt auch für deren Erforschung in lokalen Kontexten. Mit Blick auf die Entwicklung des Forschungsfeldes zu internationaler und komparativer Inklusionsforschung ist es jedoch relevant, die verschiedenen Ebenen - Entwicklung Inklusiver Bildung, Inklusionsforschung im Allgemeinen und internationale und komparative Forschung zu Inklusiver Bildung im Spezifischen - nicht miteinander zu vermischen. Das Handbuch unternimmt den Versuch, unter Anerkennung der Komplexität kulturell diverser Ausgangsformen, im Vergleich unterschiedliche Beispiele der Umsetzung und Verhandlung Inklusiver Bildung im internationalen Kontext abzubilden - mit Betonung von Behinderung und Benachteiligung im Bildungssystem. Aus den bisherigen Ausführungen werden an dieser Stelle resümierend zentrale Perspektiven, Problemstellungen und Forschungsdesiderate international und komparativer Inklusionsforschung angedeutet, die im Rahmen des Handbuchs Vertiefung finden und nachfolgend als Fragestellungen/Postulierungen ausformuliert sind. Sie beziehen sich auf die theoretische Rahmung globaler/internationaler Forschung, die Methodik der Komparatistik und die Wahl des Forschungsgegenstandes:

- Welche theoretischen Rahmungen (auch über die dargestellten hinaus) bieten sich an, um die Komplexität internationaler und komparativer Forschung zu fassen?

- Insbesondere gilt es - im Sinne von Verhältnisbestimmungen - aufzuzeigen, wie globale, nationale und lokale Strukturen (Internationale Organisationen, nationale Regierungen, lokale Netzwerke usw.) zusammenhängen und wie sich dies zwischen verschiedenen Ländern unterscheidet (bspw. zwischen föderalistischen und zentralistischen Staaten).

- Wie tangieren globale Einflussgrößen im Sinne weltgesellschaftlicher Diskurse Inklusive Bildung und wie können diese erfasst werden?

- Wie können Bildungs- und Erziehungsverhältnisse in den jeweiligen Länderkontexten und Kulturkreisen in ihrer Komplexität bei Vergleichen Berücksichtigung finden? Damit zusammenhängend: Welche Tertium Comparationis werden für die inter-, transnationalen und kulturvergleichenden Analysen zu Inklusiver Bildung ausgewählt? 
- Wie werden inter-/transnationale und interkulturelle Vergleiche durch Daten abgebildet, zugänglich gemacht, vorenthalten, und in die bildungspolitischen Entwicklungen einbezogen?

- Welche Stimmen und Narrative sowie Daten und Indikatoren werden im Vergleich von den Forschenden als relevant erachtet - und welche fehlen? Welche Artikulationen werden gehört, welche überhört? Inwieweit werden Partizipative Forschungsansätze berücksichtigt und ermöglicht?

- Liegt der Fokus bei Inklusiver Bildung auf allen am Bildungsprozess Beteiligten oder werden einige (insbes. Schüler*innen mit Förderbedarf) askriptiv in den Blick genommen? Wie können hierbei Aspekte von Intersektionalität in ihrer komplexen Hervorbringung und pädagogischen Bearbeitung Eingang in Forschung finden?

Unter Berücksichtigung des bisherigen Forschungsstandes in der internationalen und komparativen Inklusionsforschung kann dieser Band dem Anspruch eines weltumfassenden Handbuches nur bedingt Folge leisten. Allerdings beinhalten die Beiträge weitreichende Perspektiven, die wir in den nachfolgenden Ausführungen systematisieren möchten. Die so entstehende Gesamtschau bildet unserer Ansicht nach einen geeigneten Ausgangspunkt, um das Forschungsfeld einen Schritt weiter zu bringen. Die oberhalb abgeleiteten Perspektiven und Spannungsfelder werden derzeit auch im Rahmen des DFG wissenschaftlichen Netzwerks „Inklusive Bildung im internationalen Vergleich“ (2020-2023), dem Autor*innen und die Herausgeber dieses Handbuches angehören, theoretisch wie methodologisch bearbeitet.

\section{Disziplinäre, theoretische und methodologische Zugänge in diesem Handbuch}

Die in diesem Band versammelten theoretischen Perspektiven sind vielfältig, können jedoch überwiegend als Ansätze der Erziehungswissenschaft und Soziologie ausgewiesen werden. Ein Großteil der Beiträge, die unterhalb skizziert werden, stammt von Wissenschaftler*innen, die in den Erziehungswissenschaften ausgebildet und in diesem breiten Feld in Forschung und Lehre tätig sind und in den vergangenen Jahren einschlägige Forschungsarbeiten im Bereich international/interkulturell vergleichender Inklusionsforschung vorgelegt haben. Neben erziehungswissenschaftlichen, sonderpädagogischen und soziologischen sind es zudem politik- und rechtswissenschaftliche Ansätze, die den Dialog um die Bedingungen, Prozesse, Konsequenzen und Umsetzungsformen Inklusiver Bildung in den Beiträgen fundieren. Einerseits bauen die Autor*innen Brücken zwischen Disziplinen, beispielsweise in der multidisziplinären Bildungssoziologie, der kultur- und sozialwissenschaftlich informierten Erziehungswissenschaft oder den zunehmend sich verbreitenden und internationalisierenden Disability Studies. Andererseits beleuchten die Autor*innen ihre zu analysierenden Gegenstände zunehmend aus spezifizierten Fachperspektiven der Erziehungswissenschaft, und nehmen unterschiedliche Foki, z.B. Lehrer*innenbildung oder Hochschulforschung in den Blick. So zeigt sich eine Pluralität und Interdisziplinarität von theoretischen Perspektiven, die einerseits in diesem Band aufgenommen, aber auch kritisch diskutiert und weiterentwickelt werden.

Jeweils ein Sechstel der Beiträge sind explizit den Disability Studies (insbesondere mit der Fokussierung auf Bildung) oder dem Neo-Institutionalismus (konzentriert auf Fragen des 
institutionellen Wandels, der Governance sowie Bildungsorganisationen) zuzuordnen. Theoretische Ansätze von Inklusion als Menschenrecht, philosophisch oder rechtlich gefasst, sowie Fragen gesellschaftlicher Werte der Gleichheit und Gerechtigkeit sind in einem Fünftel der Beiträge zentral, auch wenn diese von allen Autor*innen des Handbuchs in der einen oder anderen Weise angesprochen werden. In Anlehnung dazu werden Fragen von Gemeinschaft und Gesellschaft sowie die der Diskriminierung, Benachteiligung und Vulnerabilität diskutiert. (Aus)Bildungssysteme, vor allem Schulen, Hochschulen sowie Lebenslanges Lernen und Weiterbildung in diversen Settings, werden analysiert. Dies spiegelt auch die Forderungen der UN-BRK wider, die zum Ausdruck bringen, dass Inklusive Bildung in allen Lebensphasen und Bildungsetappen garantiert werden soll. Entwicklungen in formalen Bildungsstrukturen werden mit Hilfe von Ansätzen der Internationalisierung und Europäisierung (Hochschule) gedeutet, während Fragen der Schulentwicklung vor allem mit Professionalisierungs- und Organisationstheorien hinsichtlich der Perspektive des Abbaus von Barrieren sowie des Aufbaus förderlicher Faktoren und Unterstützungsstrukturen für erfolgreiche Inklusion bearbeitet werden. Lebenslanges Lernen wird in Konzepten des Lebens(ver)laufs eingebettet, um die kumulative Benachteiligung von Kindern und Jugendlichen mit Förderbedarf und Menschen mit zugeschriebenen Behinderungen sowie anderen Minderheiten in formellen und informellen Bildungsprozessen zu verdeutlichen. Der starke Fokus auf Diversität wird in Rekurs auf Differenz-, Intersektionalitäts-, Sozialisations- und Normalismustheorien gedeutet. Auf der Ebene sozialer Interaktion werden u.a. Ansätze des Multilingualismus sowie die der sonderpädagogischen Förderung (u.a. auf Basis der Psychometrie) angewandt. Mit diesen kontrastreichen Ansätzen - und komparativ - werden die persistenten Barrieren aber auch Möglichkeiten zur Stärkung des Prozesses der Inklusion auf verschiedenen Ebenen aufgezeigt und analysiert.

Methodologisch und methodisch werden, gerade aufgrund der Stärke des Vergleichs bei Analysen auf Makroebene, in einem Drittel der Beiträge Arten von Policy-Analysen vorgenommen. Die Policy-Forschung versucht, im Sinne der klassischen Vergleichenden Erziehungswissenschaften, von den Erfahrungen und Entwicklungsdynamiken anderer Länder zu lernen. Diese Zielsetzung ist gerade bei der Herausforderung einer Transformation hin zu Inklusiver Bildung hilfreich und nötig, insbesondere weil es wenige Länder gibt, die flächendeckend inklusive Schulen etabliert haben. Während diese Beiträge Policy-Relevanz anstreben, werden auch die Methoden der vergleichenden Institutionen- sowie Diskursanalyse genutzt, um grundlegende Einblicke und Erkenntnisse über Grenzen hinweg sichtbar zu machen und kritisch zu reflektieren. Teilweise geschieht dies auch im historischen Sinne, sowohl qualitativ als auch quantitativ. Auch sich in den letzten Jahren im deutschsprachigen Raum verstärkende Ansätze wie die wissenssoziologische Diskursanalyse kommen hier zur Geltung. Mindestens ein Fünftel der Beiträge ziehen einen explizit historischen Ansatz heran, um Entwicklungen auf verschiedenen Ebenen abzubilden, seien es Institutionen, Bildungspolitiken oder Schulen. Dokumentenanalysen und statistische Zeitreihen sowie Literatursynthesen verdeutlichen, wie sich der Übergang aber auch die gegenseitige Beeinflussung sonderpädagogischer Fördersysteme und Inklusiver Bildung - insbesondere in den Organisationen und Pädagogiken sichtbar - historisch verändert. Literatur aus verschiedenen Ländern wird hier zusammengeführt, was eine bedeutsame Übersetzungsleistung darstellt. Die Herausgeber haben sowohl deutschsprachige als auch englischsprachige Beiträge gesammelt, um die Horizonte zu erweitern; die Mehrsprachigkeit des Bandes spiegelt auch die internationale Dimension des wissenschaftlichen Diskurses zu Inklusiver Bildung wider. Darüber hinaus bieten einige Beiträge Metaanalysen oder Metavergleiche schulethnographischer Studien 
und dokumentarische Kulturvergleiche, um durch komparative Analysen fallübergreifende Muster bzw. Orientierungen aufzuzeigen. Hierbei kommen Methoden wie Interviewführung, Biographieforschung, Teilnehmende Beobachtungen und Klassifikationsanalyse zum Einsatz, um die Handlungen und Perspektiven von Akteur*innen in (nicht)inklusiven Settings aufzuzeigen, unter anderem auch im Sinne partizipativer Forschung, in der betroffene Akteur*innen selbst zu Wort kommen und die Forschung mitentwickeln.

Mit Blick auf die Ebene des Vergleichs bieten die Beiträge vielfältige komparatistische Dimensionen. Ein Viertel der Beiträge nimmt vor allem eine globale Perspektive ein, entweder in allumfassender Perspektive oder die Relationen im Vergleich zwischen globalem Süden und globalem Norden. Perspektive oder die Relationen im Vergleich zwischen globalem Süden und globalem Norden. Unter den Weltregionen werden vor allem Entwicklungen in Europa analysiert, aber auch Fachdiskurse in und zwischen Nordamerika und Afrika werden verglichen. Viele Beiträge nutzen die in unserer Zeit des Wettbewerbs sehr starke international vergleichende Perspektive, beziehen in ihren Analysen aber auch oft intranationale oder lokale Entwicklungen mit ein. Solche Mehrebenanalysen tragen den Realitäten in Bildungspolitik und -systemen - gerade in föderalen Ländern - sowie den Verwobenheiten in der Governance und den wesentlichen Verflechtungen unterschiedlicher Ebenen Rechnung. Zwei Fünftel der Beiträge thematisieren vor allem die einflussreiche nationale Ebene. Oft werden dabei die Varianz und die Disparitäten sowie die regionalen und lokalen Besonderheiten berücksichtigt. Die Gegenstände der Komparatistik sind ebenfalls vielfältig und erstrecken sich auf alle Ebenen.

Fragen der Umsetzung der UN-BRK bestimmen große Teile der fachlichen und allgemeinen Diskussion über die Inklusive Bildung seit über einem Jahrzehnt. Dies zeigt sich auch in diesem Handbuch, denn in mehreren Beiträgen werden vergleichend die Umsetzungsversuche der UN-BRK dargestellt, nachverfolgt und kritisiert - auf unterschiedlichen Ebenen, von Weltregionen über Nationalstaaten und Bundesländern bis hin zur lokalen Ebene. Weitere Beiträge nehmen fachliche Orientierungen und Entwicklungen in den Blick, indem sie die Disability Studies (in Education) global sowie Diskurse um Inklusive Bildung oder die einflussreiche internationale Organisation der Weltbank und deren Programme in Bezug auf Behinderung rekonstruieren. Vergleiche der Kulturen der Inklusion, der Bildungspolitiken sowie die Umsetzungsprozesse inklusiver Bildungsreformen und deren Einfluss auf Individuen sind weitere Gegenstände vergleichender Analysen. Vergleiche unterschiedlicher Policies und Strukturen zeigen auf, wie sich der Aufbau inklusiver Hochschul- und Erwachsenenbildung in verschiedenen Kontexten entwickelt. Policy-Analysen sind nicht nur auf Bildungssysteme fokussiert, sondern thematisieren auch benachteiligte Gruppen und deren Exklusion vom oder Marginalisierung im bzw. Exklusion vom Arbeitsmarkt. Schulentwicklung spielt in den Vergleichen eine bedeutsame Rolle, indem Fragen von Leitung, Handlungspraktiken oder auch Bewertungen von Leistungsdifferenzen durch Lehrpersonen diskutiert werden. Hierfür werden auch Unterschiede in der Lehrer*innenbildung, der Assistenz oder auch die innerschulischen Unterstützungsmaßnahmen vergleichend herausgearbeitet. Auf Schulebene wie in Kindergärten oder Universitäten werden der Aufbau und die Etablierung von Unterstützungssystemen in Bezug auf Inklusive Bildung verglichen. An dieser Schnittstelle werden auch Lehrerbildungspolitiken wie -programme komparatistisch behandelt.

Gesamthaft bilden die Handbuchbeiträge den fortlaufenden Entwicklungsprozess im Kontext Inklusiver Bildung und damit einhergehende Forschungsdesiderate im Bereich internationaler, komparativer Forschung ab. Wenngleich sicherlich weiterhin viele Leerstellen 
vorhanden sind, vermögen sie aufzuzeigen, wo in theoretischer, methodologischer und praktischer Hinsicht angesetzt werden kann und Schnittstellen weiter bearbeitet werden können.

\section{Zu den Beiträgen}

Nun zu den in diesem Band versammelten Autor*innen und ihren Erkenntnissen kommend, werden die einzelnen Beiträge kurz resümiert. Darüber hinaus werden exemplarische Verweise zu grundlegenden Analysen und Vorarbeiten der Autor*innen - im Stil von Quellennachweisen - aufgeführt, die das Feld strukturiert und geprägt haben und in die Beiträge dieses Handbuchs eingeflossen sind.

Im ersten Teil „Grundlagen: Theoretische und methodologische Positionen und zentrale Begriffe "werden Grundlagentexte als Rahmen präsentiert. Katharina Heyer (2015) verbindet menschenrechtliche, juristische und politikwissenschaftliche Perspektiven und untersucht damit die weltweite Ratifizierung der UN-BRK sowie die nationalen Unterschiede in der Umsetzung, insbesondere in den Ländern Deutschland, Japan und den USA. Sally Tomlinson (2017) skizziert in ihrem Beitrag eine Soziologie der sonderpädagogischen bzw. auch Inklusiven Bildung, in dem sie Exklusion und Inklusion, die Expansion sonderpädagogischer Maßnahmen und segregierender Settings (in der Schule und Berufsausbildung) sowie Übergänge von Schule in den Beruf in vier Ländern darstellt. Außerdem skizziert sie aktuelle Herausforderungen der Coronavirus Pandemie. Mel Ainscow (Ainscow et al. 2006) eröffnet die Diskussion um Inklusive Bildung mit einem grundlegenden Beitrag über Werte als zentralen Aspekt für Schulentwicklungen, von Inklusiver Bildung als Menschenrecht, als hochwertigem Unterricht und als sozial gerecht. Lani Florian (2014) geht in ihrem Beitrag den Ähnlichkeiten und Disparitäten von Inhalt und Qualität in der Lehrer*innenbildung in unterschiedlichen Kontexten auf den Grund. Raphael Zahnd (2017) untersucht die Programme der Weltbank und zeichnet die vielfältigen Diskursstränge nach, die den graduellen Wandel in den Behinderungsparadigmen dieser mächtigen internationalen Organisation ausmachen. Swantje Köbsell (2006) und Marianne Hirschberg (2009) bereiten synthetisch die vielen Denkanstöße des Diskurses Disability Studies in Education aus dem angloamerikanischen für den deutschsprachigen Raum auf. Myriam Hummel (2018) zeigt diverse diskursive Konstruktionen Inklusiver Bildung im südlichen Afrika auf: in einigen Ländern und inhaltlichen Aspekten werden Diskurse des globalen Nordens ohne Kontextualisierung übernommen, wohingegen sich in anderen Ländern eigene Diskurse herausbilden.

Im zweiten Teil „Komparative Perspektiven zu ausgewählten nationalen, regionalen und lokalen Bildungskontexten" werden zunächst gesetzliche Bedingungen und Fragestellungen von Governance diskutiert. Julia Biermann (Biermann 2021 i.E.) verbindet eine menschenrechtsbasierte globale Analyse des Artikels 24 (Bildung) der UN-BRK und der weltweiten Ratifizierung mit den nationalen Disparitäten bezüglich deren diskursiven Übersetzung und (Nicht-)Umsetzung inklusiver Bildung in Deutschland und Nigeria. Julia Gasterstädt (2019) zeigt für Deutschland, wie Inklusion mit verschiedenen Konzepten verbunden wird: eine globale Norm wird auch innerhalb von Nationalstaaten sehr unterschiedlich interpretiert, mit Konsequenzen für den institutionellen Wandel. Lea Schäfer (2019) untersucht die beiden föderalen Staaten Spanien und Deutschland - spezifisch die Autonome Region Andalusien und das Land Berlin -, um Gemeinsamkeiten und Unterschiede der politischen Vorgaben hinsichtlich organisationaler Maßnahmen zur Umsetzung des Umgangs mit Diversität 
festzustellen. Mathias Mejeh (2016) zeigt innerhalb der Schweiz die persistente Separation der Schüler*innen mit sonderpädagogischem Förderbedarf sowie interkantonale Disparitäten trotz exogenen Drucks globaler Inklusionsreformen. Gottfried Biewer (2006) analysiert für Österreich die Bedeutung von Elternbewegungen sowie Lehrer*innenbildung als nachhaltige Faktoren für die Stärkung Inklusiver Bildung. Tobias Buchner (2018) findet in seinem Beitrag Gemeinsamkeiten zwischen europäischen Ländern in der fehlenden Umsetzung von Politiken Inklusiver Bildung sowie Mängel in der Umsetzung auf der Ebene des Individuums.

Weiter werden Schulstrukturen, -kulturen und -praktiken in den Blick genommen. Edda Óskarsdóttir (2017) und Andreas Köpfer (2013) vergleichen den langfristigen ganzheitlichen Aufbau von professionellen Unterstützungs- und Organisationsstrukturen für alle Schüler*innen in Island und Kanada. Michelle Proyer (2014) und Margarita Bilgeri (Schiemer 2017) berichten in ihrem Vergleich von Schulkulturen in Addis Abeba, Äthiopien, und Bangkok, Thailand, aus dem interkulturellen Projekt CLASDISA, um die Verbindungen westlicher Ideen von Inklusion mit lokalen Kulturen aufzuzeigen. Run Tan (2020) untersucht die außerordentlich wichtige Rolle der Leitung und der Gemeinschaft in der Schaffung inklusiver Bildung in einem Kindergarten in Schanghai, China. Julie Allan (2008) und Clara Rübner Jørgensen (2015) zeigen in einer englischen Fallstudie anhand von Bildern, wie Schüler*innen die Vorteile von Vielfalt für ihr schulisches Lernen erkennen. Markku Jahnukainen (2015) und Tiina Itkonen (2010) nehmen einen Vergleich des finnischen und des US-amerikanischen Schulsystems hinsichtlich der Schulstruktur und der Implementierung von Förderstrukturen vor. Justin Powell (2014), Kerstin Merz-Atalik (2001) und die Teammitglieder des TdiverS-Projekts untersuchen in sechs europäischen Ländern (Deutschland, Luxemburg, Island, Litauen, Schweden und Spanien) diverse Entwicklungsprozesse hin zu ,inspirierenden Praktiken“ inklusiver Bildung, auch unabhängig von nationalen Politiken, durch Schulleitungen, Einbettung in der Gemeinschaft sowie didaktische Innovationen.

Darauffolgend wird die Rolle der Schule, des Unterrichts und der Professionalisierung beleuchtet. Benjamin Badstieber (Badstieber et al. 2018) und Bettina Amrhein (2011) untersuchen Schulleitungen aus Deutschland und der Schweiz und nehmen deren Handlungspraktiken und -spielräume in den Blick, um Inklusion sinnstiftend zu bearbeiten und zu fördern dabei geht es vor allem um die Entscheidung zwischen Systemkonformität oder Transformation. Tanja Sturm (2019) verdeutlicht im Vergleich von norwegischen und deutschen Datensequenzen, wie inklusive Haltungen durch bestehende schulische Strukturen sowie Bewertungsmodi erschwert werden. Elisabeth Plate (2012) und Alison Peacock (2016) argumentieren mit Blick auf Erfahrungen aus dem deutschen und englischen Bildungssystem, dass Phasen der professionellen Weiterbildung stärker als fortwährender Prozess gesehen werden müssen - mit Inklusion als grundlegender Orientierung. Bettina Fritzsche (2014) und Michalis Kakos (Kakos/Fritzsche 2017) zeigen empirisch auf, mit welchen Chancen und Herausforderungen mehrsprachige Lehrassistenten in England als Übersetzer in der inklusiven Schule konfrontiert sind.

Abschließend werden im dritten Teil „Inklusion in Hochschule und im Kontext lebenslangen Lernens “ fokussiert. Cristina Allemann-Ghionda (2013) analysiert die Europäisierung von Hochschulen und zeigt mit Beispielen aus Deutschland und den USA die allgemeine Herausforderung von Diversity sowie Fächerunterschiede in der curricularen Berücksichtigung von Barrierefreiheit, Diversity und Inklusion auf. Arthur Limbach-Reich (2016) kontrastiert soziale und ökonomische Perspektiven, indem er nach den Entwicklungen inklusiver Hochschulen fragt und Disparitäten aufzeigt. Trotz der Vorgaben der UN-BRK sowie europäischer Initiativen zeigt sich, dass Hochschulen hinter Schulen in der Umsetzung von 
Inklusion zurückbleiben. Marcelo Parreira do Amaral (2011) und Jozef Zelinka (2019) untersuchen Politiken des Lebenslangen Lernens und Erwartungen gegenüber jungen Erwachsenen in Europa, um zu zeigen, wie ,Vulnerabilität' als gesellschaftspolitischer Interpretationsrahmen den ,normalen“ (linearen) Lebenslauf von Schule in den Arbeitsmarkt herausfordert. Im letzten Kapitel des Buches unterstreichen Silke Schreiber-Barsch (2015) und Peter Rule (2004) im Vergleich deutscher und südafrikanischer Bildungsorganisationen die enorme Bedeutung partizipativer Zugänge und deren Ethik, um Machtunterschiede zwischen behinderten und nicht-behinderten Forschenden konstruktiv zu begegnen - und damit zugleich die partizipative Zukunft des Feldes.

\section{Literatur}

Adick, Christel (2015): Das Potenzial der Vergleichenden Erziehungswissenschaft zwischen elaborierter Vergleichsmethodik und praktischer Bedeutsamkeit. In: Tertium Comparationis 21, 1, S. 105-129.

Ainscow, Mel (2021 i.d.B.): Inclusion and Equity in Education. In: Köpfer, Andreas/Powell, Justin J.W./Zahnd, Raphael (Hrsg.): Handbuch Inklusion international. Opladen: Budrich, S. 7587.

Ainscow, Mel/Sandill, Abha (2010): Developing Inclusive Education Systems: The Role of Organizational Cultures and Leadership. In: International Journal of Inclusive Education 14, 4, S. 401-416.

Ainscow, Mel/Dyson, Alan/Booth, Tony (2006): Improving Schools, Developing Inclusion. Routledge: London.

Albrecht, Friedrich/Bürli, Alois/Erdélyi, Andrea (Hrsg.) (2006): Aspekte internationaler Heil- und Sonderpädagogik. Bad Heilbrunn: Klinkhardt.

Allan, Julie (2008): Rethinking Inclusive Education: The Philosophers of Difference in Practice. Heidelberg: Springer VS.

Allemann-Ghionda, Cristina (2013): Bildung für alle, Diversität und Inklusion: Internationale Perspektiven. Bad Heilbrunn: Klinkhardt.

Amrhein, Bettina (2011): Inklusion in der Sekundarstufe. Bad Heilbrunn: Klinkhardt.

Arnove, Robert F. (2012): Introduction: Reframing Comparative Education: The Dialectic of the Global and the Local. In: Arnove, Robert F./Torres, Carlos A./Franz, Stephen (Hrsg.): Comparative Education: The Dialectic of the Global and the Local. Lanham: Rowman Littlefield Publishers, S.1-26.

Artiles, Alfredo J./Dyson, Alan (2005): Inclusive Education in the Globalization Age: The Promise of Comparative Cultural Historical Analysis. In: Mitchell, David (Hrsg.): Contextualizing Inclusive Education. London: Routledge, S. 37-62.

Artiles, Alfredo J./Kozleski, Elizabeth B./Waitoller, Federico R. (Hrsg.) (2011): Inclusive Education: Examining Equity on Five Continents. Cambridge, MA: Harvard Education Press.

Autorengruppe Bildungsberichterstattung (2014, 2020): Bildung in Deutschland 2014, 2020. Bielefeld: Bertelsmann.

Badstieber, Benjamin/Amrhein, Bettina (2021 i.d.B.): Handlungspraktiken von Schulleitenden im Kontext integrations-/inklusionsorientierter Schulentwicklungsprozesse. In: Köpfer, Andreas/Powell, Justin J.W./Zahnd, Raphael (Hrsg.): Handbuch Inklusion international. Opladen: Budrich, S. 383-406. 
Badstieber, Benjamin/Köpfer, Andreas/Amrhein, Bettina (2018): Schulleitungen im Kontext inklusiver Bildungsreformen. In: Sturm, Tanja/Wagner-Willi, Monika (Hrsg.): Handbuch schulische Inklusion. Opladen: Budrich, S. 235-249.

Baker, David P. (2014): The Schooled Society: The Educational Transformation of Global Culture. Stanford: Stanford University Press.

Barow, Thomas/Persson, Bengt/Allan, Julie (2016): Inclusive Education in Großbritannien und Skandinavien. In: Hedderich, Ingeborg/Biewer, Gottfried/Hollenweger, Judith/Markowetz, Reinhard (Hrsg.): Handbuch Inklusion und Sonderpädagogik. Stuttgart: UTB, S. 189-193.

Biermann, Julia (2021, i.E.): Translating Human Rights in Education: The Influence of Article 24 UN CRPD in Nigeria and Germany. Ann Arbor: University of Michigan Press.

Biermann, Julia (2021 i.d.B.): Der Einfluss der UN-BRK auf inklusive Bildung in Nigeria und Deutschland. In: Köpfer, Andreas/Powell, Justin J.W./Zahnd, Raphael (Hrsg.): Handbuch Inklusion international. Opladen: Budrich, S. 167-178.

Biermann, Julia/Graf, Erich Otto/Proyer, Michelle/Reisenbauer, Simon/Zahnd, Raphael (2014): Über die Grenzen der ,nationalen und Grenzen überschreitenden europäischen Heil- und Sonderpädagogik' und darüber hinaus. Vierteljahresschrift für Heilpädagogik und ihre Nachbargebiete 20,3, S. 259-263.

Biermann, Julia/Powell, Justin J.W. (2014): Institutionelle Dimensionen inklusiver Schulbildung. Zeitschrift für Erziehungswissenschaft 17, 4, S. 679-700.

Biermann, Julia/Powell, Justin J.W. (2021): Internationale Disability Studies. In: Waldschmidt, Anne (Hrsg.): Handbuch Disability Studies. Heidelberg: Springer VS, i.E.

Biewer, Gottfried (2021 i.d.B.): Inklusive Bildung in Österreich. In: Köpfer, Andreas/Powell, Justin J.W./Zahnd, Raphael (Hrsg.): Handbuch Inklusion international. Opladen: Budrich, S. 239246.

Biewer, Gottfried (2006): Schulische Integration in Deutschland und Österreich im Vergleich. Erziehung und Unterricht - Österreichische Pädagogische Zeitschrift 156, 1-2, S. 21-28.

Biewer, Gottfried/Luciak, Mikael (2010): Der internationale Vergleich in der Sonderpädagogik. In: Enzyklopädie Erziehungswissenschaft online 2010, S. 1-19. http://www.erzwissonline.de/ [Zugriff: 15.08.2020]

Biewer, Gottfried/Luciak, Mikael/Schwinge, Mirella (Hrsg.) (2007): Begegnungen und Differenz: Menschen - Länder - Kulturen. Beiträge zur Heil- und Sonderpädagogik. Bad Heilbrunn: Klinkhardt.

Blanck, Jonna M. (2014): Organisationsformen schulischer Integration und Inklusion. Eine vergleichende Betrachtung der 16 Bundesländer. Discussion Paper SP I 2014-501. Berlin: WZB.

Blanck, Jonna M. (2020): Übergänge nach der Schule als »zweite Chance«? Eine quantitative und qualitative Analyse der Ausbildungschancen von Schülerinnen und Schülern aus Förderschulen »Lernen«. Weinheim: Beltz Juventa.

Blanck, Jonna M./Edelstein, Benjamin/Powell, Justin J.W. (2013): Persistente schulische Segregation oder Wandel zur inklusiven Bildung? Die Bedeutung der UN-Behindertenrechtskonvention für Reformprozesse in den deutschen Bundesländern. In: Schweizerische Zeitschrift für Soziologie 39, 5, S. 267-292.

Booth, Tony/Ainscow, Mel (2002): Index for Inclusion: Developing Learning and Participation in Schools. Bristol, UK: Centre for Studies on Inclusive Education.

Bourdieu, Pierre (1987): Sozialer Sinn. Kritik der theoretischen Vernunft. Frankfurt am Main: Suhrkamp Wissenschaft.

Brown, Alisha M.B. (2014): Situating Disability Within Comparative Education. In: Global Education Review 1, 1, S. 56-75.

Buchner, Tobias (2021 i.d.B.): Einflüsse von Bildungspolitiken auf biographische Verläufe in international vergleichender Perspektive erforschen. In: Köpfer, Andreas/Powell, Justin J.W./ Zahnd, Raphael (Hrsg.): Handbuch Inklusion international. Opladen: Budrich, S. 247-273. 
Buchner, Tobias (2018): Die Subjekte der Integration. Bad Heilbrunn: Klinkhardt.

Budde, Jürgen (2018): Erziehungswissenschaftliche Perspektiven auf Inklusion und Intersektionalität. In: Sturm, Tanja/Wagner-Willi, Monika (Hrsg.): Handbuch schulische Inklusion. Opladen: Verlag Barbara Budrich, S. 45-60.

Budde, Jürgen/Dlugosch, Andrea/Herzmann, Petra/Rosen, Lisa/Panagiotopoulou, Argyro/Sturm, Tanja/Wagner-Willi, Monika (Hrsg.) (2019): Inklusionsforschung im Spannungsfeld von Erziehungswissenschaft und Bildungspolitik. Opladen: Verlag Barbara Budrich.

Budde, Jürgen/Dlugosch, Andrea/Sturm, Tanja (Hrsg.) (2017): (Re-)Konstruktive Inklusionsforschung. Opladen: Verlag Barbara Budrich.

Bürgi, Regula (2017): Die OECD und die Bildungsplanung der freien Welt. Denkstile und Netzwerke einer internationalen Bildungsexpertise. Opladen: Verlag Barbara Budrich.

Bürli, Alois (2010): Wie hast du's, Europa, mit der Integration Behinderter? Zeitschrift für Inklusion 2/2010. http://www.inklusion-online.net/index.php/inklusion-online/article/view/137/137 [Zugriff: 10.07.2020].

Bürli, Alois (2020): Behindertenpädagogik international. Grundlagen - Perspektiven - Beispiele. Stuttgart: Kohlhammer.

Bürli, Alois/Strasser, Urs/Stein, Anne-Dore (Hrsg.) (2009): Integration/Inklusion aus internationaler Sicht. Bad Heilbrunn: Klinkhardt.

Carrier, James G. (1984): Comparative Special Education: Ideology, Differentiation and Allocation in England and the United States. In: Barton, Len/Tomlinson, Sally (Hrsg.): Special Education and Social Interests. London: Croom Helm, S. 35-64.

Charlton, James I. (1998): Nothing About Us Without Us: Disability Oppression and Empowerment. Berkeley, CA: University of California Press.

Cloerkes, Günther (Hrsg.) (2003): Wie man behindert wird: Texte zur Konstruktion einer sozialen Rolle und zur Lebenssituation betroffener Menschen. Heidelberg: Universitätsverlag Winter.

D'Alessio, Simona/Cowan, Steven (2013): Cross-Cultural Approaches to the Study of "Inclusive" and "Special Needs" Education. In: Wiseman, Alexander W./Anderson, Emily (Hrsg.): Annual Review of Comparative and International Education. Bingley, UK: Emerald, S. 227-261.

Dale, Roger (2015): Globalisierung in der Vergleichenden Erziehungswissenschaft re-visited: Die Relevanz des Kontexts des Kontexts. In: Parreira do Amaral, Marcelo/Amos, S. Karin (Hrsg.): Internationale und Vergleichende Erziehungswissenschaft. Geschichte, Theorie, Methode und Forschungsfelder. Münster: Waxmann, S. 171-188.

Degener, Theresia (2017): Grundlagen der Inklusion aus völkerrechtlicher Perspektive nach der Behindertenrechtskonvention der Vereinten Nationen (UN-BRK) In: Institut für Bildungsrecht und Bildungsforschung e.V. / DIPF (Hrsg.) Auf dem Weg zur Inklusiven Schule. Baden-Baden: Nomos, S. 11-24.

Deutsche Forschungsgemeinschaft (DFG) (2020-2023): Wissenschaftliches Netzwerk "Inklusive Bildung im internationalen Vergleich”, Projektnummer 427476584.

Dietz, Gunther (2007): Keyword: Cultural Diversity. In: Zeitschrift für Erziehungswissenschaft 10, 1, S. 7-30.

EADSNE European Agency for Development in Special Needs Education (2011): Mapping the Implementation of Policy for Inclusive Education. Odense: EADSNE.

EASNIE European Agency for Special Needs and Inclusive Education (2014): Country Information. Odense: EASNIE. http://www.european-agency.org/country-information. [Zugriff: 10. 07.2020].

Eckhart, Michael/Sahli Lozano, Caroline/Blanc, Philippe (2011): Langzeitwirkungen der schulischen Integration: Eine empirische Studie zur Bedeutung von Integrationserfahrungen in der Schulzeit für die soziale und berufliche Situation im jungen Erwachsenenalter. Bern: Haupt.

Eckstein, Boris/Reusser, Kurt/Stebler, Rita/Mandel, Debbie (2013): Umsetzung der integrativen Volksschule - Was Lehrpersonen optimistisch macht. Eine Analyse der Überzeugungen von 
Klassenlehrpersonen im Kanton Zürich. In: Schweizerische Zeitschrift für Bildungswissenschaften, 35, 1, S. 91-112.

Emmerich, Marcus/Hormel, Ulrike (2013): Heterogenität - Diversity - Intersektionalität: Zur Logik sozialer Unterscheidungen in pädagogischen Semantiken der Differenz. Wiesbaden: VS Verlag für Sozialwissenschaften.

Erdélyi, Andrea/Schmidtke, Hans-Peter/Sehrbrock, Peter (Hrsg.) (2012): International vergleichende Heil- und Sonderpädagogik weltweit: Grundlagen - Migration - «Dritte Welt» - Europa. Bad Heilbrunn: Klinkhardt.

Feuser, Georg (2013): Die Kooperation am Gemeinsamen Gegenstand. In: behinderte menschen 21,3 , S. 16-35.

Florian, Lani (Hg.) (2014): The SAGE Handbook of Special Education (second edition). London: SAGE.

Fritzsche, Bettina (2014): Inklusion als Exklusion. Differenzproduktionen im Rahmen des schulischen Anerkennungsgeschehens. In: Tervooren, Anja/Engel, Nicolas/Göhlich, Michael/ Miethe, Ingrid/Reh, Sabine (Hrsg.): Ethnographie und Differenz in pädagogischen Feldern. Internationale Entwicklungen erziehungswissenschaftlicher Forschung. Bielefeld: transcript Verlag, S. 329-345.

Fritzsche, Bettina/Kakos, Michaelis (2021 i.d.B.): Multilingual Teaching Assistants in the UK. In: Köpfer, Andreas/Powell, Justin J.W./Zahnd, Raphael (Hrsg.): Handbuch Inklusion international. Opladen: Budrich, S. 453-469.

Fritzsche, Bettina/Köpfer, Andreas (2019): (Para-)Professionalität im Umgang mit Ungewissheitsstrukturen - eine kulturvergleichende Rekonstruktion von Interviews mit Assistenzkräften im inklusionsorientierten Unterricht. In: Donlic, Jasmin /Jacksche-Hoffmann, Elisabeth /Peterlini, Hans Karl (Hrsg.): Ist inklusive Schule möglich? Nationale und internationale Perspektiven. Bielefeld: transcript Verlag, S. 133-160.

Fuchs, Martin/Berg, Eberhard (1999): Phänomenologie der Differenz. Reflexionsstufen ethnographischer Repräsentation. In: Fuchs, Martin/Berg, Eberhard (Hrsg.): Kultur, soziale Praxis, Text. Frankfurt am Main: Suhrkamp, S. 11-108.

Garrote, Ariana/Sermier Dessemontet, Rachel/Moser Opitz, Elisabeth (2017): Facilitating the social participation of pupils with special educational needs in mainstream schools: A review of school-based interventions. In: Educational Research Review 20 (Supplement C), S. 12-23.

Gasterstädt, Julia (2019): Der Komplexität begegnen und Inklusion steuern. Eine Situationsanalyse zur Umsetzung von Artikel 24 der UN-BRK in zwei Bundesländern. Wiesbaden: Springer VS.

Gasterstädt, Julia (2021 i.d.B.): Same same but different. In: Köpfer, Andreas/Powell, Justin J.W./Zahnd, Raphael (Hrsg.): Handbuch Inklusion international. Opladen: Budrich, S. 179196.

Geertz, Clifford (1987): Dichte Beschreibung. Frankfurt am Main: Suhrkamp.

Graf, Erich O. (2015): Der Fremde bin ich selbst. In: Graf, Erich O. (Hrsg.): Globale Perspektiven auf Behinderung. Berlin: epuli, S. 19-59.

Grech, Shaun (2009): Disability, Poverty and Development: Critical Reflections on the Majority World Debate. In: Disability \& Society 24, 6, S. 771-784.

Grech, Shaun (2011): Recolonising Debates or Perpetuated Coloniality? Decentring the Spaces of Disability, Development and Community in the Global South. In: International Journal of Inclusive Education 15, 1, S. 87-100.

Greve, Jens/Heintz, Bettina (2005): Die «Entdeckung» der Weltgesellschaft. Entstehung und Grenzen der Weltgesellschaftstheorie. In: Zeitschrift für Soziologie, Sonderheft «Weltgesellschaft», S. 89-119. 
Hackbarth, Anja/Martens, Matthias (2018): Inklusiver (Fach-)Unterricht: Befunde - Konzeptionen - Herausforderungen. In: Sturm, Tanja/Wagner-Willi, Monika (Hrsg.): Handbuch schulische Inklusion. Opladen: Verlag Barbara Budrich, S. 191-205.

Hänsel, Dagmar (2005): Die Historiographie der Sonderschule: Eine kritische Analyse. In: Zeitschrift für Pädagogik 51, 1, S. 101-115.

Hall, Stuart ([1985] 2000): Postmoderne und Artikulation. In: Räthzel, Nora (Hrsg.): Cultural Studies. Ein politisches Theorieprojekt. Hamburg: Argument Verlag, S. 52-77.

Hasse, Raimund/Krücken, Georg (2005): Neo-Institutionalismus. Bielefeld: transcript Verlag.

Hedderich, Ingeborg/Zahnd, Raphael (Hrsg.) (2016): Teilhabe und Vielfalt: Herausforderungen einer Weltgesellschaft. Bad Heilbrunn: Klinkhardt.

Heintz, Peter (1982): Die Weltgesellschaft im Spiegel von Ereignissen. Zürich: Rüegger.

Henke, Thorsten/Bogda, Katja/Lambrecht, Jennifer/Bosse, Stefanie, Koch, Helvi/Maaz, Kai/Spörer, Nadine (2017): Will you be my friend? A Multilevel Network Analysis of Friendships of Students with and without Special Educational Needs Backgrounds in Inclusive Classrooms. In: Zeitschrift für Erziehungswissenschaft 20, 3, S. 449-474.

Herkenrath, Marc (2012): Macht, Herrschaft und die Rolle oppositioneller Akteure im Weltsystem. In: Imbusch, Peter (Hrsg.): Macht und Herrschaft: Sozialwissenschaftliche Theorien und Konzeptionen. (2. vollständig überarbeitete Auflage). Wiesbaden: VS Verlag, S. 451-472.

Hessel, Stéphane (2010): Empört Euch! Berlin: Ullstein.

Heyer, Katharina (2015): Rights Enabled. The Disability Revolution, from the US, to Germany and Japan, to the United Nations. Ann Arbor: University of Michigan Press.

Heyer, Katharina (2021 i.d.B.): What is a Human Right to Inclusive Education? In: Köpfer, Andreas/Powell, Justin J.W./Zahnd, Raphael (Hrsg.): Handbuch Inklusion international. Opladen: Budrich, S. 45-57.

Hirschberg, Marianne (2009): Behinderung im internationalen Diskurs: Die flexible Klassifizierung der Weltgesundheitsorganisation. Frankfurt am Main: Campus.

Hofsäss, Thomas R. (1993): Die Überweisung von Schülern auf die Hilfsschule und die Schule für Lernbehinderte. Berlin: Marhold.

Hummel, Myriam (2018): Inclusive Education in Situated Contexts. Dissertation. Hannover, Germany: Gottfried Wilhelm Leibniz Universität.

Hummel, Myriam (2021 i.d.B.): Die diskursive Konstruktion inklusiver Bildung im südlichen Afrika. In: Köpfer, Andreas/Powell, Justin J.W./Zahnd, Raphael (Hrsg.): Handbuch Inklusion international. Opladen: Budrich, S 147-163.

Hummrich, Merle/Kramer, Rolf-Thorsten (2016): Rekonstruktion und Kritik. In: Schippling, Anne/Grunert, Cathleen/Pfaff, Nicolle (Hrsg.): Kritische Bildungsforschung. Standortbestimmungen und Gegenstandsfelder. Opladen: Verlag Barbara Budrich, S. 64-71.

Hummrich, Merle/Pfaff, Nicolle (2018): Transnationalisierung. Tertium Comparationis 24, 2, S. 143-150.

Hummrich, Merle/Pfaff, Nicolle/Dirim, Inci/Freitag, Christine (Hrsg.) (2016): Kulturen der Bildung. Wiesbaden: Springer VS.

Hummrich, Merle/Rademacher, Sandra (Hrsg.) (2016): Kulturvergleich in der qualitativen Forschung: Erziehungswissenschaftliche Perspektiven und Analysen. Wiesbaden: Springer VS.

Itkonen, Tiina/Jahnukainen, Markku (2010): Disability or Learning Difficulty? Politicians or Educators? Constructing Special Education in Finland and the United States. In: Comparative Sociology, 9, 2, S. 182-201.

Jahnukainen, Markku (2015): Inclusion, Integration, or What? A Comparative Study of the School Principals' Perceptions of Inclusive and Special Education in Finland and in Alberta, Canada. In: Disability \& Society 30, 1, S. 59-72. 
Jahnukainen, Markku/Itkonen, Tiina (2021 i.d.B.): Steps to Inclusion? In: Köpfer, Andreas/Powell, Justin J.W./Zahnd, Raphael (Hrsg.): Handbuch Inklusion international. Opladen: Budrich, S. 345-356.

Johnson, Magdalena (2013): Schulische Inklusion in den USA. Bad Heilbrunn: Klinkhardt.

Jørgensen, Clara Rübner (2015): Three Advantages of Cross-national Comparative Ethnography Methodological Reflections from a Study of Migrants and Minority Ethnic Youth in English and Spanish Schools. In: Ethnography and Education 10, 1, S. 1-16.

Kakos, Michalis/Fritzsche, Bettina (2017): A Meta-ethnography of Two Studies on Interactions in Schools: Reflections on the Process of Translation. In: Ethnography and Education 12, 2, S. 228-242.

Kiel, Ewald/Esslinger-Hinz, Ilona/Reusser, Kurt (2014): Einführung in den Thementeil „Allgemeine Didaktik für eine inklusive Schule“. In: Zierer, Klaus (Hrsg.): Jahrbuch für Allgemeine Didaktik. Baltmannsweiler: Schneider Verlag Hohengehren, S. 9-15.

Kiuppis, Florian (2014): Heterogene Inklusivität, inklusive Heterogenität. Münster: Waxmann.

Klauer, Karl J./Mitter, Wolfgang (Hrsg.) (1987): Vergleichende Sonderpädagogik. Berlin: Marhold.

Kocaj, Aleksander/Kuhl, Poldi/Kroth, Anna J./Pant, Hans Anand/Stanat, Petra (2014): Wo lernen Kinder mit sonderpädagogischem Förderbedarf besser? Ein Vergleich schulischer Kompetenzen zwischen Regel- und Förderschulen in der Primarstufe. In: Kölner Zeitschrift für Soziologie und Sozialpsychologie 66, 2, S. 165-191. https://doi.org/10.1007/s11577-014-0253-x

Köbsell, Swantje (2006): Towards Self-Determination and Equalization: A Short History of the German Disability Rights Movement. In: Disability Studies Quarterly 26, 2. https://dsqsds.org/article/view/692/869 [Zugriff: 14.07.2020].

Köbsell, Swantje/Hirschberg, Marianne (2021 i.d.B.): Disability Studies in Education. In: Köpfer, Andreas/Powell, Justin J.W./Zahnd, Raphael (Hrsg.): Handbuch Inklusion international. Opladen: Budrich, S. 127-146.

Köpfer, Andreas (2013): Inclusion in Canada: Analyse inclusiver Unterrichtsprozesse, Unterstützungsstrukturen und Rollen am Beispiel kanadischer Schulen in den Provinzen New Brunswick, Prince Edward Island und Québec. Bad Heilbrunn: Klinkhardt.

Köpfer, Andreas (2020a): Herausforderungen international vergleichender Inklusionsforschung im Kontext von Behinderung und Benachteiligung - ethnographische und kulturvergleichende Perspektiven. In: Zeitschrift Behinderung und Internationale Entwicklung/Journal for Disability and International Development, Schwerpunktheft „Inclusive Education“ 31, 2, S. 5-11.

Köpfer, Andreas (2020b): Artikulationen Inklusiver Bildung zwischen Strukturkritik und funktionaler Integration - Erkundungen mit Stuart Hall. In: Vierteljahresschrift für wissenschaftliche Pädagogik 93, 2, S. 296-310.

Köpfer, Andreas/Papke, Katharina/Gerdes, Jürgen (2019): Rekonstruktionen zum Verhältnis von Inklusionsverständnissen und -bedingungen praktizierender Lehrkräfte und dessen Bedeutung für die modulare Entwicklung von Fort- und Weiterbildungen. In: Journal für Psychologie 27, 2, S. 170-191.

Kottmann, Brigitte (2006): Selektion in die Sonderschule. Bad Heilbrunn: Klinkhardt.

Kozleski, Elizabeth B./Artiles, Alfredo J./Waitoller, Federico R. (2011): Equity in Inclusive Education. Historical Trajectories and Theoretical Commitments. In: Artiles, Alfredo J./Kotzleski, Elizabeth B./Waitoller, Federico R. (Hrsg.): Inclusive Education. Examining Equity on Five Continents. Cambridge, MA: Harvard Education Press, S. 1-14.

Krawinkel, Stefanie/Südkamp, Anna Sarah/Tröster, Heinrich (2017): Soziale Partizipation in inklusiven Grundschulklassen: Bedeutung von Klassen- und Lehrkraftmerkmalen. In: Empirische Sonderpädagogik 9, 3, S. 277-295.

Krücken, Georg/Drori, Gili S. (Hrsg.) (2009): World Society: The Writings of John W. Meyer. Oxford: Oxford University Press. 
Leemann, Regula Julia/Imdorf, Christian/Powell, Justin J.W./Sertl, Michael (Hrsg.) (2016): Die Organisation von Bildung. Soziologische Analysen zu Schule, Berufsbildung, Hochschule und Weiterbildung. Weinheim: Beltz Juventa.

Leonhardt, Annette/Müller, Katharina/Truckenbrodt, Tilly (Hrsg.) (2015): Die UN-Behindertenrechtskonvention und ihre Umsetzung. Beiträge zur Interkulturellen und International-vergleichenden Heil- und Sonderpädagogik. Bad Heilbrunn: Klinkhardt.

Limbach-Reich, Arthur (2016): Inklusion. Analyse eines aktuellen Leitbegriffs sozial- und bildungspolitischer Auseinandersetzungen. Konzeptionelle Ankerpunkte und empirische Antworten. In: Bliemetsrieder, Sandro/Bäuml-Rossnagl, Maria-Anna (Hrsg.): Inklusion im interdisziplinären Diskurs. München: Utz, S. 191-233.

Mazurek, Kas/Winzer, Margret (Hrsg.) (1994): Comparative Studies in Special Education. Washington, DC: Gallaudet University Press.

Meekosha, Helen (2011): Decolonising Disability: Thinking and Acting Globally. In: Disability \& Society 26, 6, S. 667-682.

Meekosha, Helen/Soldatic, Karen (2011): Human Rights and the Global South: The case of disability. In: Third World Quarterly 32, 8, S. 1383-1397.

Mejeh, Mathias (2016): Absicht und Wirklichkeit integrativer Bildung. Wiesbaden: VS.

Mejeh, Mathias (2021 i.d.B.): Inklusive Bildung als Institution am Beispiel Schweiz. In: Köpfer, Andreas/Powell, Justin J.W./Zahnd, Raphael (Hrsg.): Handbuch Inklusion international. Opladen: Budrich, S. 221-238.

Messiou, Kyriaki (2017): Research in the Field of Inclusive Education: Time for a Rethink? In: International Journal of Inclusive Education 21, 2, 146-159.

Meyer, Heinz-Dieter/Benavot, Aaron (Hrsg.) (2013): PISA, Power, and Policy. The Emergence of Global Educational Governance. Oxford: Symposium Books.

Meyer, John W. (2005): Weltkultur: Wie die westlichen Prinzipien die Welt durchdringen. Frankfurt am Main: Suhrkamp.

Meyer, John W. (2009): Reflections: Institutional Theory and World Society. In: Krücken, Georg/Drori, Gili S. (Hrsg.): World Society: The Writings of John W. Meyer. Oxford: Oxford University Press, S. 36-63.

Merz-Atalik, Kerstin (2001): Interkulturelle Pädagogik in Integrationsklassen. Subjektive Theorien von Lehrern im gemeinsamen Unterricht von Kindern mit und ohne Behinderungen. Opladen: Leske und Budrich.

MIPIE Mapping the Implementation of Policy for Inclusive Education (2011): Die Entwicklung von Datengrundlagen zur Implementierung inklusiver Bildung. Odense: EASNIE.

Morin, Edgar (2014): Für ein Denken des Südens. Berlin: Matthes \& Seitz.

Moser, Vera (Hrsg.) (2012): Die inklusive Schule: Standards für die Umsetzung. Stuttgart: Kohlhammer.

NESSE Network of Experts in Social Sciences of Education and Training (2012): Education and Disability/Special Needs Policies and Practices in Education, Training and Employment for Students with Disability and Special Educational Needs in the EU. Brussels: EC.

Nguyen, Xuan Thuy (2018): Critical Disability Studies at the Edge of Global Development: Why Do We Need to Engage with Southern Theory? In: Canadian Journal of Disability Studies 7, 1, S. 1-25.

Nind, Melanie/Vinha, Hilra (2014): Doing Research Inclusively: Bridges to Multiple Possibilities in Inclusive Research. In: British Journal of Learning Disabilities 42, 2, S. 102-109.

Nohl, Arnd-Michael (2019): Country Comparison and Multi-Level Analysis in Qualitative Research - Methodological Problems and Practical Solutions. In: Child Indicators Research 12, 2, S. 409-423.

OECD (2007): Students with Disabilities, Learning Difficulties and Disadvantages: Policies, Statistics and Indicators. Paris: OECD. 
Óskarsdóttir, Edda (2017): Constructing Support as Inclusive Practice: A Self-Study. Dissertation, University of Iceland.

Óskarsdóttir, Edda/Köpfer, Andreas (2021 i.d.B.): Inklusive Unterstützungsstrukturen in Schulen in Island und Kanada. In: Köpfer, Andreas/Powell, Justin J.W./Zahnd, Raphael (Hrsg.): Handbuch Inklusion international. Opladen: Budrich, S. 277-295.

Panagiotopoulou, Julie A./Rosen, Lisa/Strzykala, Jenna (Hrsg.) (2020): Inclusion, Education, and Translanguaging: How to Promote Social Justice in (Teacher) Education? Wiesbaden: Springer VS.

Parreira do Amaral, Marcelo (2011): Emergenz eines internationalen Bildungsregimes? International Educational Governance und Regimetheorie. Münster: Waxmann.

Parreira do Amaral, Marcelo (2015): Methodologie und Methode in der International Vergleichenden Erziehungswissenschaft. In: Parreira do Amaral, Marcelo/Amos, S. K. (Hrsg.): Internationale und Vergleichende Erziehungswissenschaft. Geschichte Theorie Methode und Forschungsfelder. Münster: Waxmann, S. 107-130.

Parreira do Amaral, Marcelo/Zelinka, Jozef (2019): Lifelong Learning Policies Shaping the Life Courses of Young Adults. An Interpretative Analysis of Orientations, Objectives and Solutions. In: Comparative Education 55, 3, S. 404-421.

Peacock, Alison (2016): Assessment for Learning without Limits. London: Open University Press.

Peters, Susan B. (2007): Education for All? A Historical Analysis of International Inclusive Education Policy and Individuals with Disabilities. In: Journal of Disability Policy Studies 18, 2, S. 98-108.

Pfahl, Lisa (2011): Techniken der Behinderung: Der deutsche Lernbehinderungsdiskurs, die Sonderschule und ihre Auswirkungen auf Bildungsbiografien. Bielefeld: transcript Verlag.

Pfahl, Lisa/Powell, Justin J.W. (2014): Subversive Status: Disability Studies in Germany, Austria and Switzerland. In: Disability Studies Quarterly 34, 2, https://dsq-sds.org/article/view/4256 (Zugriff 19.20.2020)

Pfahl, Lisa/Powell, Justin J.W. (2016): „Ich hoffe sehr, sehr stark, dass meine Kinder mal eine normale Schule besuchen können." Pädagogische Klassifikationen und ihre Folgen für die (Selbst-)Positionierung von Schüler/innen. In: Zeitschrift für Pädagogik, Beiheft 62, S. 58-74.

Plate, Elisabeth (2012): Staff Support for Inclusion - An International Study. Dissertation. Canterbury: University of Kent/Canterbury Christ Church University.

Powell, Justin J.W. (2014): Comparative and International Perspectives on Special Education. In: Florian, Lani (Hrsg.): The SAGE Handbook of Special Education, second edition. Los Angeles, CA: SAGE, S. 335-349.

Powell, Justin J.W. ([2011] 2016): Barriers to Inclusion: Special Education in the United States and Germany. Abingdon: Routledge.

Powell, Justin, J.W. (2018): Inclusive Education: Entwicklungen im internationalen Vergleich. In: Sturm, Tanja/Wagner-Willi, Monika (Hrsg.): Handbuch schulische Inklusion. Opladen/Toronto: Verlag Barbara Budrich, S. 127-142.

Powell, Justin J.W./Hadjar, Andreas (2018): Schulische Inklusion in Deutschland, Luxemburg und der Schweiz: Aktuelle Bedingungen und Herausforderungen [Inclusive Schooling in Germany, Luxembourg and Switzerland]. In: Rathmann, Katharina/Hurrelmann, Klaus (Hrsg.): Leistung und Wohlbefinden in der Schule: Herausforderung Inklusion [Performance and Wellbeing in School]. Weinheim: Beltz Juventa, S. 46-64.

Powell. Justin, J.W./Merz-Atalik, Kerstin et al. (2021 i.d.B.): Heterogene Lerngruppen inklusionsorientiert unterrichten. In: Köpfer, Andreas/Powell, Justin J.W./Zahnd, Raphael (Hrsg.): Handbuch Inklusion international. Opladen: Budrich, S. 357-379.

Powell, Justin J.W./Wagner, Sandra J. (2014): An der Schnittstelle Ethnie und Behinderung benachteiligt: Jugendliche mit Migrationshintergrund an deutschen Sonderschulen weiterhin 
überrepräsentiert. In: Wansing, Gudrun/Westphal, Manuela (Hrsg.): Behinderung und Migration. Wiesbaden: VS, S. 177-199.

Prengel, Annedore (2006): Pädagogik der Vielfalt. Verschiedenheit und Gleichberechtigung in Interkultureller, Feministischer und Integrativer Pädagogik. Wiesbaden: VS Verlag.

Preuss-Lausitz, Ulf (2001): Gemeinsamer Unterricht Behinderter und Nichtbehinderter. In: Zeitschrift für Erziehungswissenschaft 4, 2, S. 209-224.

Preuss-Lausitz, Ulf (2019). Ergebnisse der Inklusions- und Separationsforschung nach zehn Jahren UN-Konvention über die Rechte von Menschen mit Behinderungen. Bilanz und Perspektiven. In: Zeitschrift für Heilpädagogik 70, 10, S. 468-483.

Proyer, Michelle (2014): Educational Environments of Children with Disabilities in Greater Bangkok. Accepting Disability and Leading a Happy Life in a Transiting Urban Area. Dissertation. Wien: Universität Wien.

Proyer, Michelle/Bilgeri, Margarita (2021 i.d.B.): Vergleichende Perspektiven auf die Kultur(-en) der (schulischen) Inklusion in Addis Abeba und Bangkok. In: Köpfer, Andreas/Powell, Justin J.W./Zahnd, Raphael (Hrsg.): Handbuch Inklusion international. Opladen: Budrich, S. 297312.

Richardson, John G./Powell, Justin J.W. (2011): Comparing Special Education: Origins to Contemporary Paradoxes. Stanford, CA: Stanford University Press.

Rieger-Ladich, Markus (2019): Bildungstheorien. Hamburg: Junius Verlag.

Rule, Peter (2004): Dialogic Spaces: Adult Education Projects and Social Engagement. In: International Journal of Lifelong Education 23, 4, S. 319-334.

Schäfer, Lea (2021 i.d.B.): Maßnahmen inklusiver Bildung. In: Köpfer, Andreas/Powell, Justin J.W./Zahnd, Raphael (Hrsg.): Handbuch Inklusion international. Opladen: Budrich, S. 197220.

Schäfer, Lea (2019): Institutioneller Wandel und Steuerung von „Förderbedürftigkeit“ im Zuge inklusiver Bildung: Ein qualitativer Mehrebenenvergleich am Fallbeispiel von Sekundarschulen in Andalusien. Dissertation, Humboldt Universität zu Berlin.

Schiemer, Margarita (2017): Education for Children with Disabilities in Addis Ababa, Ethiopia. Developing a Sense of Belonging. Cham, Switzerland: Springer VS.

Schreiber-Barsch, Silke (2015): Von Sonder-Räumen zu inklusiven Lernorten. Raumordnungen in der Erwachsenenbildung. In: Bernhard, Christian/Kraus, Katrin/Schreiber-Barsch, Silke/Stang, Richard (Hrsg.): Erwachsenenbildung und Raum. Theoretische Perspektiven professionelles Handeln - Rahmungen des Lernens. Bielefeld: WBV, S. 193-204.

Schreiber-Barsch, Silke/Rule, Peter (2021 i.d.B.): Shifting Lenses to a Participatory Ethos in Research. In: Köpfer, Andreas/Powell, Justin J.W./Zahnd, Raphael (Hrsg.): Handbuch Inklusion international. Opladen: Budrich, S. 547-572.

Schriewer, Jürgen (2003): Problemdimensionen sozialwissenschaftlicher Komparatistik. In: Schriewer, Jürgen (Hrsg.): Discourse Formation in Comparative Education. Bern: Peter Lang, S. 9-52.

Schriewer, Jürgen (2013): Vergleichende Erziehungswissenschaft als Forschungsfeld. In: Hummrich, Merle/Rademacher, Sandra (Hrsg.): Kulturvergleich in der qualitativen Forschung. Wiesbaden: Springer VS, S. 15-41.

Schuelka, Matthew (2013): Excluding Students with Disabilities from the Culture of Achievement. In: Journal of Education Policy 28, 2, S. 216-230.

Scott, W. Richard (2013): Institutions and Organizations. Thousand Oaks, CA: SAGE.

Sehrbrock, Peter/Erdélyi, Andrea/Gand, Sina (Hrsg.) (2013): Internationale und Vergleichende Heil- und Sonderpädagogik und Inklusion: Individualität und Gemeinschaft als Prinzipien Internationaler Heil- und Sonderpädagogik. Bad Heilbrunn: Klinkhardt.

Slee, Roger/Allan, Julie (2001): Excluding the Included: A Reconsideration of Inclusive Education. In: International Studies in Sociology of Education 11, 2, S. 173-192. 
Slee, Roger/Corcoran, Tim/Best, Marnie (2019): Disability Studies in Education - Building Platforms to Reclaim Disability and Recognise Disablement. In: Journal of Disability Studies in Education, 2019, S. 1-11.

Sturm, Tanja (2016): Lehrbuch Heterogenität in der Schule. 2. überarb. Aufl., München: Reinhardt (UTB).

Sturm, Tanja (2019): Constructing and Addressing Differences in Inclusive Schooling - Comparing Cases from Germany, Norway and the United States. In: International Journal of Inclusive Education 23, 6, S. 656-669.

Sturm, Tanja (2021 i.d.B.): Konstruktion von (Leistungs-)Differenzen in der Schule. In: Köpfer, Andreas/Powell, Justin J.W./Zahnd, Raphael (Hrsg.): Handbuch Inklusion international. Opladen: Budrich, S. 407-422.

Sturm, Tanja/Wagner-Willi, Monika (Hrsg.) (2018): Handbuch schulische Inklusion. Opladen \& Toronto: Verlag Barbara Budrich.

Tan, Run (2020): Promoting Peer Interactions in Chinese Inclusive Preschool Classrooms: Strategies Teachers Apply for Children with Special Educational Needs. Dissertation. Bielefeld: Universität Bielefeld.

Tan, Run (2021 i.d.B.): Exploring Successful Inclusive Practice in China. In: Köpfer, Andreas/ Powell, Justin J.W./Zahnd, Raphael (Hrsg.): Handbuch Inklusion international. Opladen: Budrich, 313-328.

Tomlinson, Sally (2017): A Sociology of Special and Inclusive Education: Exploring the Manufacture of Inability. Abingdon: Routledge.

Tomlinson, Sally (2021 i.d.B.): Sociology of Special and Inclusive Education. In: Köpfer, Andreas/Powell, Justin J.W./Zahnd, Raphael (Hrsg.): Handbuch Inklusion international. Opladen: Budrich, S. 59-73.

UNESCO (1994): Die Salamanca Erklärung und der Aktionsrahmen zur Pädagogik für besondere Bedürfnisse. URL: https://www.unesco.de/sites/default/files/2018-03/1994_salamanca-erklae rung.pdf [Zugriff: 20.12.2018].

UNESCO (2015): Education for All 2000-2015: Achievements and Challenges. Paris: United Nations Educational, Scientific, and Cultural Organization.

UNESCO (2020): Global Education Monitoring Report 2020: Inclusion and Education - All Means All. Paris: United Nations Educational, Scientific, and Cultural Organization.

UN Committee on the Rights of Persons with Disabilities (CRPD) (2016): General comment No. 4 (2016), Article 24: Right to inclusive education, 2 September 2016, CRPD/C/GC/4 https:// www.refworld.org/docid/57c977e34.html [Zugriff 19.08.2020]

United Nations (2006): Convention on the Rights of Persons with Disabilities. A/RES/61/106, Annex I. http://www.un.org/disabilities/default.asp?id=150 [Zugriff 24.08.2020]

United Nations (2013): Thematic Study on the Right of Persons with Disabilities to Education. New York: General Assembly, Human Rights Council: A/HRC/25/29.

United Nations (2015): Transforming our World: The 2030 Agenda for Sustainable Development. New York: UN.

United Nations (2018): Sustainable Development Goals. URL: https://www.un.org/sustainable [Zugriff 24.08.2020]

Vock, Miriam/Gronostaj, Anna (2017): Umgang mit Heterogenität in Schule und Unterricht. Berlin/Bonn: Friedrich-Ebert-Stiftung.

Waldschmidt, Anne (Hrsg.) (im Erscheinen): Handbuch Disability Studies. Heidelberg: Springer VS.

Wallerstein, Immanuel (2004): World Systems Analysis. Durham, NC: Duke University Press.

Weisser, Jan (2015): Doing international disability studies and coping with different thought styles. In: Graf, Erich O. (Hrsg.): Globale Perspektiven auf Behinderung. Berlin: epubli, S. 1517. 
Weisser, Jan (2017): Konfliktfelder schulischer Inklusion und Exklusion im 20. Jahrhundert. Weinheim: Beltz Verlag.

Werning, Rolf (2014): Stichwort: Schulische Inklusion. In: Zeitschrift für Erziehungswissenschaft 17,4 , S. 601-623.

WHO (2003): International Klassifikation der Funktionsfähigkeit, Behinderung und Gesundheit. DIMDI.

Zahnd, Raphael (2017): Behinderung und sozialer Wandel. Eine Fallstudie am Beispiel der Weltbank. Bad Heilbrunn: Klinkhardt.

Zahnd, Raphael (2021 i.d.B.): Weltbank. In: Köpfer, Andreas/Powell, Justin J.W./Zahnd, Raphael (Hrsg.): Handbuch Inklusion international. Opladen: Budrich, S. 107-126. 



\section{I \\ Grundlagen: Theoretische und methodologische Positionen und zentrale Begriffe}





\title{
2 What is a Human Right to Inclusive Education? The Promises and Limitations of the CRPD's Inclusion Mandate
}

\author{
Katharina Heyer
}

\begin{abstract}
Article 24 of the UN Convention on the Rights of Persons with Disabilities is universally celebrated for its comprehensive and visionary embrace of the inclusion principle. Not only does it spell out the importance of education rights for affirming the dignity and self-worth of persons with disabilities and their right to full participation in society, but it also places affirmative duties on state parties to provide the kinds of accommodations necessary to fully include students with disabilities in all levels of the general education system. As such, it calls for state parties to enact education reforms that fundamentally rethink the nature and provision of disability education rights. This chapter follows the development of the inclusion principle in international human rights instruments and spells out the theoretical foundations of inclusive education within the larger construction of disability right as human rights. It examines the tensions within the inclusive education mandate brought about by the progressive realization of education rights, the distinction between inclusion and integration, and the implementation of Article 24 within systems with established special schools.
\end{abstract}

\section{Disability Rights as Human Rights}

The 2006 United Nations Convention on the Rights of Persons with Disabilities (CRPD) is the first international convention to recognize disability as a human rights issue (UN 2006). It frames disability as a social construction that results from "the interaction between persons with impairments and attitudinal and environmental barriers that hinders their full and effective participation in society on an equal basis with others" (Article 1). The CRPD calls for the removal of "all barriers to full participation and inclusion in society" and thus locates the need for change in social institutions, not in individuals with disabilities. As such, it reflects the well-documented paradigm shift from the medical model to the social model of disability and also "brings a human rights dimension to disability issues" (De Beco 2014: 269).

The CRPD is rightfully called a ground-breaking treaty that has the potential to become a transformative legal instrument, promoting shifts in attitudes and signalling new values towards disability (Lewis 2010). It powerfully affirms people with disabilities as "part of human diversity and humanity" (CRPD Preamble) and demands that states raise awareness, foster respect, and combat stereotypes about disability. It is the first international treaty mandating reasonable accommodations and the accessibility of physical environments and services. The CRPD establishes a rigorous framework for monitoring the implementation of the 
Convention (Article 33) and requires that a UN Committee responsible for evaluating state party reports includes experts with disabilities (Article 34). State parties must also set up independent monitoring mechanisms at the national level, usually in the form of independent human rights institutions, to ensure the greatest level of civil society participation in the monitoring process. Finally, the CRPD's Optional Protocol allows for individual complaints to be brought before the CRPD Committee in cases when CRPD rights have been violated by state parties that have ratified the Optional Protocol.

The participation of civil society advocates, including disabled peoples' organizations, is especially important since the CRPD mandates the elimination of discrimination by both public and private entities, thus taking human rights law into the private sphere for the first time. The CRPD broke human rights records for involving the greatest level of participation by civil society advocates in all parts of the treaty process: it was developed directly with people with disabilities and is monitored by a Committee composed by a majority of people with disabilities. The Committee is thus a body both of and for people with disabilities, which adds to the legitimacy of its findings (Byrne 2019: 6). The CRPD is also the longest and most detailed status-based human rights treaty to date, outlining the specifics of what it means to promote disability rights as rights of inclusion.

\section{Disability Rights as Rights of Inclusion}

The CRPD's normative shift towards inclusion is based on the recognition of a painful history of segregation and marginalization in all areas of society. This recognition was especially important in the framing of disability education rights of the CRPD's Article 24, an extensive and detailed Article in the Convention that responds to the history of purposive exclusion and marginalization of students with disabilities by guaranteeing the right to equality and nondiscrimination in the state provision of education rights (Arnardóttir 2011). Specifically, it frames education rights as rights of inclusion, mandating an ,, inclusive education system at all levels and lifelong learning" (Article 24 (1)). This was the first global recognition of disability education rights as rights to an inclusive education, from pre-school all the way to secondary and adult education, with profound implications to state parties, education policy makers, school administrators, and children with disabilities around the globe.

There is a stark gap between the CRPD's aspirational text and the realities on the ground (see Biermann 2021 in this volume on the paradoxical effects on Germany and Nigeria). The CRPD's promise of inclusive education is set against a global picture of exclusion, rather than inclusion. In 2011 the World Health Organization and the World Bank published the World Disability Report which provides the most comprehensive account of the global position of people with disabilities to date (see Zahnd 2021 in this volume on disability in the programs of the World Bank). The report revealed that for many persons with disabilities around the world, education is still available only in segregated settings, where they are isolated from their peers and where the education they receive is of an inferior quality. Children with disabilities continue to face significant barriers to inclusive education, ranging from complete exclusion, to segregated education, low rates of school completion, lack of educational accommodations, poor teacher and curricular supports, and physical barriers to school buildings and transport. Children with disabilities continue to face disproportionate amounts of bullying and violence in their schools and communities (WHO 2011: 22). 
The CRPD's inclusion mandate is set against this background as well as a result of a growing international disability advocacy movement that has been instrumental in the framing, passing, and monitoring of the Convention (Sabatello/Schulze 2013). In international educational advocacy, there is near universal agreement about the stigmatizing effect of segregated education, especially when separate schools are the only choice provided to children with disabilities. An important exception here is the advocacy of the World Deaf Congress that has consistently called for an exception for the education of children who are native users of sign language, and who occupy a dual identity as both disabled (and therefore covered by disability rights) and as part of a linguistic minority, and are therefore entitled to an education with other native signers (Murray et al. 2015). Inclusive education advocates locate the damaging effect of school segregation not only in those excluded, but in all children, who are deprived of the kinds of social interactions and awareness of the diversity of learners in their communities. The experience of racially segregated education in the United States has inspired this movement on a global scale: many refer to the U.S. Supreme Court's eloquent ruling that "separate education is inherently unequal" because of the stigma, or "badge of inferiority" it imposes (U.S. Supreme Court opinion: Brown v. Board of Education of Topeka, 347 U.S. 483 (1954)). Even though the analogy between race and disability discrimination is troubled, it has generated powerful arguments challenging the "natural" or "inevitable" separation of children on the basis of disability as a form of bias and discrimination (Heyer 2015).

\section{From Education Rights to Inclusion Rights}

Education occupies a special place in the human rights canon. It is important in itself, aiding in the full development and dignity of each person and the ability to fully participate in society. It is also considered a multiplier, as it impacts the ability to access other human rights, such as the right to work, the right to vote, or freedom of speech, none of which are possible without an education. The importance of education as a fundamental human right is recognized in the Universal Declaration of Human Rights, the founding document in the human rights canon, that proclaimed a universal right to an education that is compulsory and free (Article 26).

This right was made binding by the International Covenant on Economic, Social and Cultural Rights (ICESCR), which spells out the "progressive realization" of education rights. The idea of progressive realization of social and economic rights acknowledges that states will need to "take steps" to use resources to the "maximum of their availability" in the realization of these rights, and that this will take time (Article 2, para 1). The Committee on Economic, Social and Cultural Rights, which monitors the implementation of the ICESCR, has recognized that education rights can be hampered by a lack of resources and that their full realization can generally not be achieved in a short period of time. The mandate towards "maximum available resources" includes a host of financial, human, and technological resources as well as potential land or tax reforms: state parties may therefore delay their obligation towards these rights to a certain extent (Robertson 1994). In contrast, civil and political rights can be realized immediately, as they require states to refrain from acting in ways that limit individual freedoms. In the corresponding International Covenant on Civil and Political Rights, then, education rights are framed in the mandate for states to respect the rights of 
parents to choose their children's education in accordance to religious and cultural convictions, along with the right to establish private schools. Education is thus both a negative right, protecting parental choice and limiting state control, and a positive right that children can demand from the state for the full enjoyment of their human rights.

This distinction between positive and negative state obligations and the incremental realization of education rights through resource allocation have powerful implications for the education rights of children with disabilities as well. The 1989 Convention on the Rights of the Child (CRC) is the first international treaty to mention disability as a protected category, and spells out their right to access an education "in a manner conducive to the child's achieving the fullest possible social integration and individual development" (Article 23). Thus, children not only have the right to an education without discrimination, but also a right to the kind of assistance that makes that right effective. The provision of this assistance, however, is contingent on "available resources" (Art. 23-3), a phrasing which has been strongly challenged by Disabled People's Organizations. Neither does the CRC specify what kind of education children with disabilities should receive regarding the level of inclusion into regular education systems.

Thus, the very first expression of disability education rights incorporated two fundamental tensions in international law: first, that the progressive realization of disability education rights might demand more state resources than the realization of so-called regular education rights and thus requires a large degree of discretion in the allocation of resources. This allows states to make gestures towards compliance while not committing actual resources. Secondly, it points to the variety of approaches to disability education rights and the terminology to describe them, ranging from segregated education in separate schools, to integration which provides access to general education, and to inclusion, which requires fundamental changes in and of the education system. There are real implications for the terminology used to describe the ways in which children with disabilities are to activate their education rights, and one of the challenges of international human rights treaty was to clarify and streamline these terms.

The 1993 Standard Rules on the Equalization of Opportunities for Persons with Disabilities took steps towards integration into mainstream schools by prompting states to include the needs of students with disabilities in all parts of education planning, curricular development and school organization. A mainstream education "presupposes the provision of interpreter and other appropriate support services [...] designed to meet the needs of persons with different disabilities" (Rule 6 (2)). However, the Standard Rules conceded that "in situations where the general school system does not yet adequately meet the needs of all persons with disabilities, special education may be considered" but "should be aimed at preparing students for education in the general school system" (Rule 6 (3)). The choice of the term "not yet" points to the progressive realization of disability education rights, while also justifying a dual system in which some children who can easily be supported can be integrated, while others with more profound needs will remain separate.

A year later, the Salamanca Statement resulting from the UNESCO World Conference on Special Needs Education modified this requirement by declaring what many consider the first articulation of inclusive education in the international human rights discourse. It affirmed the importance for all children to learn together regardless of their different styles and rates of learning. This was qualified with the phrase "wherever possible," but the emphasis was on regular schools needing to accommodate students with special educational needs with a "child-centred pedagogy capable of meeting these needs" (1994 Salamanca Statement and 
Framework for Action on Special Needs Education, paragraphs 2 and 3). Importantly, the Salamanca Statement recognized regular schools with this inclusive orientation as "the most effective means of combating discriminatory attitudes, creating welcoming communities, building an inclusive society and achieving education for all" (ibid.). Like the Standard Rules, the Salamanca Statement was an important step towards articulating the need for inclusive education rights in international instruments, but it did not have the treaty power of a Convention to hold states accountable to implement these changes. It was the 2006 Convention on the Rights of Persons with Disabilities that ultimately gave legally binding status to the concept of "inclusive education," essentially deciding that, by definition, the right to education is to be thought of a right to inclusive education.

\section{Inclusion is not Integration: CRPD Article 24}

Accordingly, Article 24 (1) mandates that state parties "ensure an inclusive education system at all levels and lifelong learning." Children with disabilities may not be excluded from or denied an education on the basis of disability and must be able to access an "inclusive, quality and free primary education and secondary education on an equal basis with others in the communities in which they live" (Article 24, para 2 (a) and (b)). This includes the education provided by private schools that are prohibited from discrimination because they provide a public service (Cera 2015: 92). While Article 24 does not explicitly mention private education, the CRPD Committee has specified the right to equal access to both public and private schools (CRPD Committee General Comment No. 4, para 24.). In realizing inclusive education rights, state parties must provide for reasonable accommodations of the individual's needs, as well as general school supports, to facilitate their effective education (Article 24, para 2 (c) and (d)). Another way of realizing these rights is through the provision of teachers, including teachers with disabilities, who are trained in sign language or Braille, and to offer training that promotes disability awareness and the use of appropriate augmentative and alternative modes, means and formats of communication, educational techniques, and materials to support persons with disabilities (Article 24, para 4).

It is significant that the CRPD does not offer a definition of the term inclusion in Article 24 but rather describes the purpose and goal of an inclusive education in human rights terms. These are located in the universal goals of human rights education, leading to (a) the full development of human potential and sense of dignity and self-worth, and the strengthening of respect for human rights, fundamental freedoms, and human diversity; (b) the development by persons with disabilities of their personality, talents, and creativity, as well as their mental and physical abilities, to their fullest potential; and (c) enabling persons with disabilities to participate effectively in a free society (Article 24, para 1).

Prior to the passage of the CRPD, the Office of the UN High Commissioner for Human Rights, in its Thematic Study on the Right of Persons with Disabilities to Education of 2013, had defined inclusion as a "process" that recognizes not only the elimination of barriers to participation, but also the "need to change culture, policy and practice of the mainstream schools to accommodate the needs of all students, including those with impairments" (para. 7). Ten years after the passage of the CRPD, the CRPD Committee took up the charge to qualify its understanding of inclusive education with the publication of its General Comment No. 4 on the Right to Inclusive Education. This 21-page document responded to central issues 
in the implementation of the inclusive education mandate the Committee had recognized in its work with the state parties. Importantly, it offered a definition of inclusion that re-iterated the notion of process suggested earlier by the High Commissioner on Human Rights. The CRPD Committee defined inclusion as "a process of systemic reform embodying changes and modifications in content, teaching methods, approaches, structures and strategies in education to overcome barriers with a vision serving to provide all students of the relevant age range with an equitable and participatory learning experience and the environment that best corresponds to their requirements and preferences" (CRPD Committee General Comment No. 4 on the normative content of Article 24. 2016, para 11).

The Committee also clarified what inclusion is not:

placing students with disabilities within mainstream classes without accompanying structural changes to, for example, organization, curriculum and teaching and learning strategies, does not constitute inclusion (ibid).

There are important distinctions between inclusion and integration, two terms that are often confused by state parties. Integration is seen as ,the process of placing persons with disabilities in existing mainstream educational institutions with the understanding that they can adjust to the standardized requirements of such institutions" (ibid.). Inclusion, in contrast, does not demand the student to adjust to the mainstream, but rather calls for fundamental reforms of classrooms that support and welcome diversity among all learners (on teaching diverse learners, see Powell et al. 2021 in this volume). The shift from integration to inclusion moves the locus of change from the individual to the institution. Integration offers individualized supports to allow students to access the curriculum, while inclusion looks towards changes in the curriculum that could make individual supports unnecessary.

Inclusion, then, is more than just placement of children with disabilities in regular classrooms. It is the transformation of that classroom into a place where all students' educational needs are recognized and met in ways that don't require them to be labelled by disability. In that sense, inclusion is aimed at all children, not just those that were traditionally excluded from mainstream classrooms on the basis of disability. The goal is to eliminate all social exclusion that result from attitudes and policy responses to diversity in race, class, ethnicity, religion, or gender, in addition to ability. By acknowledging the diversity of learners in a classroom, inclusion seeks to de-stigmatize disability difference, create welcoming communities, and improve the quality and effectiveness of the education system as a whole. The idea is that all children benefit, not just those for whom educational accommodations are designed. Inclusion advocates see it as not only a human right, but also as a foundation of a just society (Slee 2011).

\section{Accessing Inclusion: Individualized Supports or System Change?}

The focus on systemic change to accommodate all learners is a central contribution of the philosophy of Universal Design, which the CRPD has adopted for education, just as it has 
for access to environments and services. ${ }^{1}$ The CRPD defines universal design as the "design of products, environments, programmes and services to be usable by all people, to the greatest extent possible, without the need for adaptation or specialized design" (CRPD Article 2). The CRPD is quick to note that a commitment to universal design does not exclude assistive devices for individuals when needed, even though in its idealized form universal design seeks to make individual adaptations largely unnecessary when environments are designed from the start for universal use.

Article 24 incorporates this tension between individual supports and system change by providing for both reasonable accommodations, geared towards individual needs, and support measures within the general education system (Article 24, para 2 (c) and (d)). Both levels of supports are deemed necessary for accessing inclusive education rights and making that education effective. There is considerably more attention paid to individualized supports in Article 24, however, and less attention to the ways states should eradicate structural barriers that hinder the effective inclusion of children within the mainstream education system. This has led some observers to criticize Article 24 for making inclusion contingent upon the extent of individual rather than institutional deficits. Byrne (2013: 242) suggests that focusing on individual supports clearly is politically "safe" and acceptable while legitimizing less than inclusive practices.

The focus on individualized support in the form of reasonable accommodations is also a reflection of the Convention's distinction between rights that are subject to progressive realization, and those that are to be realized immediately. The duty to provide reasonable accommodations occupies a large part of the Convention, as it is seen as a central path towards equalized opportunities and full participation. Since the denial of reasonable accommodation is identified as a form of discrimination, this is an obligation that must be fulfilled immediately. In education, the reasonable accommodation mandate covers both physical access to buildings and classrooms, transportation, and communication technologies. Educational accommodations are a response to an individual's needs, so they are negotiated between student and school on a case by case basis, including the determination of whether the accommodations are, indeed, reasonable, or whether they provide an undue burden to the school.

In contrast, the support measures outlined in Article 24 are geared towards adapting the general education system to make it more accessible to learners with disabilities. Specifically, Article 24 (2) (d) mandates that state parties guarantee that "persons with disabilities receive the support required, within the general education system, to facilitate their effective education". Because they are geared toward the systemic change of the education system, support measures are subject to progressive realization. Examples of general supports include the provision of materials in Braille, or sign language, as outlined in Article 24 (3). Other examples include alterations in the physical environment, such as the design of hallways and classrooms, desks, widening entrances, building ramps, installation of elevators, altering or reconsidering geographical locations, adapting rules and admission standards (Special Rapporteur on the Right to Education, OHCHR (2007), para 14, as cited in De Beco (2014: 282). Personal factors entail the provision of supplementary classes, alternative or additional forms of communication, special tutors or support staff, and nutritious meals (ibid.). The support and training given to teachers and school staff is an essential form of general support as well (CRPD Article 24 (4)).

1 There are now well-developed standards for Universal Design for Learning (UDL) and Universal Design for Instruction (UDI), see, e.g., Rose (2000) and Burgstahler (2008). 
Reasonable accommodations and general supports are closely interrelated in the provision of an inclusive education system. General supports adapt the education system to make it more accessible to students with disabilities, whereas reasonable accommodations enable these students to be educated in these schools. The two are bound to overlap in the sense that the more supports are given to make schools accessible, the less individual accommodations will be necessary (De Beco 2014: 283). There is an important difference, however, in the urgency with which the Convention frames these two principles, based on the demands of the progressive realization principle. The duty to provide reasonable accommodations is an immediate obligation, as their denial is defined as a form of discrimination in the Convention (Art. 2). These accommodations are individualized to the student's need to be educated in mainstream schools, but they do not aim to achieve inclusive education by targeting the general education system. When it comes to the provision of inclusive education, the Convention specifies a progressive, rather than an immediate realization, that allows state parties to choose how they will achieve this goal. The notion of progressive realization is key to understanding the varied response to the inclusive education mandate.

\section{Progressive Realization of Inclusive Education Rights}

Because human rights doctrine posits that education rights are subject to progressive realization, the CRPD recognizes that the right to education of persons with disabilities may be achieved over a certain period of time (See UN Committee on Economic, Social and Cultural Rights, general comment No. 3 (1990) on the nature of States parties' obligations, para 9). In fact, Article 4 (2), of the CRPD provides that economic, social, and cultural rights have to be progressively realized within the maximum amount of available resources. The CRPD Committee is very clear, however, that the progressive realization of the right to education of persons with disabilities does not mean that inclusive education can just be postponed. State Parties to the Convention need to move "as expeditiously and effectively as possible" towards the full realization of Article 24 (CRPD Committee General Comment No. 4 (2016) para 40). This call towards full realization should be taken as an invitation by both disability activists and comparative education researchers to follow implementation trajectories, and to hold their governments accountable. The CRPD Committee foresees that State Parties take steps to this end, even though the objective does not have to be reached right away, and put in place their resources with a view to fully realize the right to inclusive education by providing reasonable accommodations and adopting support measures.

Importantly, the Committee sees this mandate as incompatible with sustaining two systems of education: a mainstream education system and a special or segregated education system. The assumption is that states with established special education systems will move towards inclusive education systems and adjust their budget allocations accordingly. State education budgets should prioritize allocation towards inclusion measures, which includes "transferring part of their budgets to the development of inclusive education" (ibid.). Even in times of education budget cuts, learners with disabilities must not be disproportionately targeted.

This comment reflects a recognition that shortage of resources is one of the more commonly cited reasons for states' failure to implement inclusive education. It also points to the problem that permitting dual systems, even in the short term during a budget crisis, will 
inevitably divert resources from inclusion and prohibit its development to maximum effect. Civil society groups advocating for inclusion emphasized this point during the drafting of the CRPD suggesting that "economically, it is far more efficient to target resources towards a single inclusive education system from the outset than to develop a dual system of separate education for disabled and non-disabled persons and then have to work towards bringing about inclusive education" (Center for Studies in Inclusive Education, Ad Hoc Committee (2005), as cited in Kayess (2019: 137). This is based on a common history in most Western education systems moving from exclusion to separation, and then to integration before arriving at inclusion (see, however, Biermann's chapter, in this volume, on Nigeria that does not move directly from exclusion to inclusion). There is a growing consensus in the human rights community that inclusive education systems are not more expensive than segregated education systems. In fact, "countries are now increasingly realizing the inefficiency of multiple systems of administration, organizational structures and services, and that it is the option of special schools which is financially unrealistic" (UNICEF 2012 as cited in Cera (2015: 90). The suggestion is that inclusive education may bring additional costs in the short term only to become profitable in the long term. This form of human rights advocacy based on cost effectiveness might find traction in neoliberal regimes, but it violates central premises behind human rights as universal, unalienable, and not valued by the practicality of their implementation.

\section{Inclusion versus Special Education and Special Schools}

The question remains of what is to be done with special schools. The inclusion mandate and the question of special education and special schools generated considerable debate during the drafting of the CRPD. The drafters of Article 24 did not immediately agree on the right to inclusive education. In fact, the Ad Hoc Committee initially left options for special education as a choice for students when the general education system did not adequately meet their needs (see Cera 2015: 90). ${ }^{2}$ The intention was to create a choice towards inclusive education, while also allowing students with disabilities the choice to opt out of the general education system if it didn't meet their needs (De Beco 2014: 273). In that sense, the framers of the CRPD defined "inclusion" by its opposite, "special education". Some committee members wanted both choices towards inclusion and special education equally available, while others wanted to emphasize inclusion as the norm and special education as the exception (Cera 2015: 89).

In the meantime, the UN Committee on the Rights of the Child (UN CRC 2006) had clarified its stance towards disability education rights in its General Comment No. 9, stating that inclusive education should be the main objective of education rights for children with disabilities. The CRC Committee had left some room for a continuum of services and programs that should be maintained in cases where inclusive education was not "feasible to achieve in the immediate future" (UN CRC 2006: para 66). The international consensus was thus moving towards inclusion, while maintaining doubts about the future of special

22004 Draft articles for a Comprehensive and Integral International Convention on the Protection and Promotion of the Rights and Dignity of Persons with Disabilities https://www.un.org/esa/socdev/enable/rights/ahcwgreportax1.htm [Accessed: 17.10.2019]. 
education. Within the CRPD Committee, the advocates for full inclusion won out and any reference to special education was deleted in the final version of Article 24. The article does not expressly prohibit the provision of special education services, however, as was suggested by the Centre for Studies on Inclusive Education (De Beco 2014: 274). This leaves the question of whether special schools should continue to exist, or how long they can be maintained parallel to the general education system moving towards inclusion.

The only exception to the exclusion of special education was made for the inclusion of sign language and Braille. This so-called "sensory exception" came in response to intense advocacy from the World Federation of the Deaf, the World Blind Union, and the World Federation of the Deaf-Blind during the drafting of Article 24. Deaf-led organizations have traditionally resisted the mandate of full inclusion for deaf and deafblind students because they see the individual placement in local schools as linguistically and socially isolating. The World Federation of the Deaf was actively involved in the entire CRPD negotiations and promoted language rights for children as a cultural-linguistic minority. They argued that inclusive settings could "create de facto segregation in public schools where children who are Deaf, Blind, and Deaf-Blind would be physically present but mentally and socially absent" (Murray et al. 2018: 45). In that sense the WFD argued that bilingual education in sign language should not be seen as "special education" or "segregated education," as the disability movement understands it, but as a form of education within the inclusive education system (Murray et al. 2018: 44).

The advocacy resulted in the compromise of Article 24 (3), mandating that the "education of persons, and in particular children, who are blind, deaf or deafblind, is delivered in the most appropriate languages and modes and means of communication for the individual, and in environments which maximize academic and social development." The Deaf community has interpreted the term "environments" to include education in separate classes or even schools to ensure deaf children learn sign language and deaf culture from teachers who are fluent signers and in a community of their peers.

\section{The CRPD Committee: It's about Inclusion, not Integration}

It should come as no surprise that state parties have interpreted the CRPD's inclusion mandate in vastly different ways. This interpretation is largely dependent on whether state parties have established separate education systems that they are invested in maintaining, rather than fundamentally reforming their general education systems. In its Concluding Observations on country reports, the CRPD Committee repeatedly criticized the lack of access to inclusive education and continuing placement into segregated schools, even in, or especially in countries with highly developed special education systems, such as Germany, Austria, Portugal, and Australia. ${ }^{3}$ Similarly, the Committee has commented on a tendency in country reports to use the terms "inclusion" and "integration" interchangeably when reporting on their implementation of the inclusive education mandate. For example, in its response to Austria's 2013 report on its implementation of the CRPD, the Committee noted that, "there are some con-

3 https://www.ohchr.org/EN/HRBodies/CRPD/Pages/CRPDIndex.aspx [Accessed: 17.10.2019]. 
fusions between "inclusive education" and "integrated education" in that State". 4 The Committee made similar observations on Poland's 2018 report.

In her analysis of all the Concluding Observations by the CRPD Committee, Byrne (2019) mentions the politics of educational placement, from segregation to integration, and to inclusion, that occupied the majority of the Committee's responses. She interprets this as an example of the ongoing challenge of implementing meaningful inclusion in a global educational setting that is still very much segregated. As an example, consider the response to Poland in 2016 where the Committee commented on Poland's establishment of "model schools" for deaf, blind, deaf-blind, visually impaired, and autistic students, but then stated that these were a form of discrimination (Byrne 2019: 11). While the Concluding Observations do not have the force of law, they still carry powerful weight in the international community in promoting norms of inclusion that prohibit the establishment of special schools.

\section{Radical Rights to Inclusion}

This interpretation of the CRPD inclusive education mandate has prompted some critics to declare the CRPD Committee's interpretation of inclusion as a "radical" right to inclusive education without qualification (Kauffman et al. 2018) that demonstrates "hostility" toward all special education settings that are not fully inclusive classrooms (Anastasiou et al. 2018). The emphasis on a single educational space (the general education classroom), the lack of discussion of special education teachers, and the general silence on the need for special education services in Comment No. 4 led these critics to wonder whether Article 24 misses the mark and shows indifference to the "real world" needs of students with special educational needs. They point to a different, less narrow way of interpreting the antidiscrimination mandate of the CRPD, which they see as too narrowly prioritizing equality and sameness over attention to disability difference, especially when it comes to students with cognitive, learning, or behavioural and emotional disabilities.

In general, this critique of inclusion questions the wisdom of applying this philosophy for educational practice since inclusion is more a question of placement than of instruction, which they believe should be of primary concern for the education of students with disabilities. They suggest that inclusion will overburden general education teachers who do not have the appropriate training and resources to address the range of learning needs when students with special education needs are in their classrooms. A related critique is made by disability law professor Ruth Colker (2008) who has challenged the integration presumption in the U.S. context by asking, "when is separate unequal"?

While the inclusion debate continues in the education literature, legal scholars point to the expressive, educational and proactive role of human rights instruments (Fredman 2008). The CRPD has "expressive value" by encouraging state parties to rethink assumptions, evaluate policies, and shift social norms surrounding disability (Lewis 2010: 98). Thus, while the Convention's monitoring mechanism does not have the force of law, the CPRD Committee's reports, such as the influential Comment No. 4 on inclusive education, are recognized as authoritative interpretations of the Convention and thus "express" an international

4 Concluding observations to the 2013 report of Austria (2013), paragraph 40. https://www.ohchr.org/EN/ HRBodies/CRPD/Pages/CRPDIndex.aspx [Accessed: 17.10.2019], as cited in De Beco (2014: 275). 
consensus of the value of inclusion. It is doubtful that Article 24 will ever fulfil its radical potential, especially as it is mitigated through the processes and limitations of progressive realization in international law. And yet, the CRPD made inclusive education a powerful goal to be achieved for people with disabilities, recognizing that disability is merely one aspect of the human condition and that people with disabilities should participate fully in their communities. Arlene Kanter (Kanter et al. 2014: 24) suggests that "ideally, countries will use Article 24 as a model in revising their own domestic laws to ensure greater access to inclusive education, greater hope for increased productivity, lower rates of poverty, and improved literacy rates - all of which are factors in achieving equality of educational opportunities for people with disabilities." The realization of the CRPD's promise thus lies in the continuous engagement with the values it expresses, and in the fostering of disability advocacy movements that hold state parties accountable for their international obligations. By framing disability rights as rights to inclusion, Article 24 points to the promise of human rights law enabling children to be educated together, share common experiences, and have the opportunity to form lifelong friendships and connections.

\section{References}

Anastasiou, Dimitris/Gregory, M./Kauffman, James M. (2018): Commentary on Article 24 of the CRPD. The Right to Education. Commentary on the UN Convention on the Rights of Persons with Disabilities. New York: Oxford University Press.

Arnardóttir, Oddný Mjöll (2011): The right to inclusive education for children with disabilities Innovations in the CRPD. In: Eide, Asbjørn/Möller, Jakob Th./Zimele, Inete (Eds.): Making Peoples Heard. Essays on Human Rights in Honour of Gudmundur Alfredsson. Leiden, NL: Brill Nijhoff, pp. 197-227.

Biermann, Julia (2021 in this volume): Der Einfluss der UN-BRK auf inklusive Bildung in Nigeria und Deutschland. In: Köpfer, Andreas/Powell, Justin J.W./Zahnd, Raphael (Eds.): Handbuch Inklusion international. Opladen: Verlag Barbara Budrich, pp. 167-178.

Burgstahler, Sheryl (2008): Equal Access: Universal Design of Instruction. Seattle: University of Washington.

Byrne, Bronagh (2013): Hidden contradictions and conditionality. Conceptualisations of inclusive education in international human rights law. In: Disability \& Society 28, 2, pp. 232-244.

Byrne, Bronagh (2019): How inclusive is the right to inclusive education? An assessment of the UN convention on the rights of persons with disabilities' concluding observations. In: International Journal of Inclusive Education, pp. 1-18.

Cera, Rachele (2015): National legislations on inclusive education and special educational needs of people with autism in the perspective of Article 24 of the CRPD. In: Della Fina, Valentina/Rachele Cera (eds.): Protecting the Rights of People with Autism in the Fields of Education and Employment. Heidelberg: Springer Open, pp. 79-108.

Colker, Ruth (2008): When is Separate Unequal? A Disability Perspective. New York: Cambridge University Press.

De Beco, Gauthier (2014): The right to inclusive education according to Article 24 of the UN Convention on the rights of persons with disabilities: Background, requirements and (remaining) questions. In: Netherlands Quarterly of Human Rights 32, 3, pp. 263-287.

Fredman, Sandra (2008): Human Rights Transformed: Positive Rights and Positive Duties Oxford: Oxford University Press. 
Heyer, Katharina (2015): Rights Enabled. The Disability Revolution, from the US, to Germany and Japan, to the United Nations. Ann Arbor: University of Michigan Press.

Kanter, Arlene S./Damiani, Michelle L./Ferri, Beth A. (2014): The right to inclusive education under international law: Following Italy's lead. In: Journal of International Special Needs Education 17, 1, pp. 21-32.

Kauffman, James M./Felder, M./Ahrbeck, B./Badar, J./Schneiders, K. (2018): Inclusion of All students in general education? International appeal for a more temperate approach to inclusion. In: Journal of International Special Needs Education 21, 2, pp. 1-20.

Kayess, Rosemary (2019): Drafting Article 24 of the Convention on the Rights of Persons with Disabilities. In: De Beco, Gauthier/Quinlivan, Shivaun/Lord, Janet (eds.): The Right to Inclusive Education in International Human Rights Law. Cambridge, UK: Cambridge University Press, pp. 122-140.

Lewis, Oliver (2010): The expressive, educational and proactive roles of human rights. An analysis of the United Nations Convention on the Rights of Persons with Disabilities. In: McSherry, Bernadette/Weller, Penny (eds.): Rethinking Mental Health Laws. Oxford, UK: Hart Publishing, pp. 97-128.

Murray, Joseph J./De Meulder, Maartje/le Maire, Delphine (2018): An education in sign language as a human right? The sensory exception in the legislative history and ongoing interpretation of Article 24 of the UN Convention on the Rights of Persons with Disabilities. In: Human Rights Quarterly 40, 1, pp. 37-60.

Powell, Justin, J.W./Merz-Atalik, Kerstin, et al. (2021 in this volume): Heterogene Lerngruppen inklusionsorientiert unterrichten. In: Köpfer, Andreas/Powell, Justin J.W./Zahnd, Raphael (eds.): Handbuch Inklusion international. Opladen: Verlag Barbara Budrich, pp. 357-379.

Robertson, Robert (1994). Measuring state compliance with the obligation to devote the maximum available resources to realizing economic, social, and cultural rights. In: Human Rights Quarterly 16, pp. 693-714.

Rose, David (2000), Universal design for learning. In: Journal of Special Education Technology 15,3 , pp. 45-49.

Sabatello, Maya/Schulze, Marianne (eds.) (2013): Human Rights and Disability Advocacy. Philadephia: University of Pennsylvania Press.

Slee, Roger (2011): The Irregular School. Exclusion, Schooling, and Inclusive Education. London: Routledge.

UN (2006): The Convention on the Rights of Persons with Disabilities and its Optional Protocol (A/RES/61/106). New York: United Nations.

UN CRC (2006): General Comment No. 9: The rights of children with disabilities. New York: United Nations Committee on the Rights of the Child.

UNICEF (2012): The Right of Children with Disabilities to Education: A Rights-Based Approach to Inclusive Education. Geneva: UNICEF Regional Office for Central and Eastern Europe and the Commonwealth of Independent States (CEECIS).

World Health Organization: (2011) World Disability Report. https://www.who.int/disabilities/ world_report/ 2011/accessible_en.pdf [Accessed: 17.07.2020].

Zahnd, Raphael (2021 in this volume): Weltbank. In: Köpfer, Andreas/Powell, Justin J.W./Zahnd, Raphael (eds.): Handbuch Inklusion international. Opladen: Verlag Barbara Budrich, pp.107126. 



\title{
3 A Sociology of Special and Inclusive Education: Insights from the UK, US, Germany, and Finland
}

\author{
Sally Tomlinson
}

\begin{abstract}
This chapter introduces some sociological understandings of special and inclusive education, especially where social class, race and 'ability' is concerned. It discusses the situation of lower attainers, disabled youth, and any who have experienced a special education, using England, the USA, Germany, and Finland as examples. It notes that in countries with developed or developing mass education, these systems were never intended to incorporate all young people equally; they are hierarchical in terms of wealth, status and ideologies of "normality'. They differ in how they educate lower attainers or disabled pupils. Comparatively, it should be noted that while there is some global convergence of educational governance, through market competition and assessment, countries do differ in their histories and economies. With the Covid-19 pandemic beginning in 2020, national economies have entered a period of insecurity and the future of those with special educational needs and disabilities looks even more uncertain.
\end{abstract}

\section{Introduction to Sociology of Special and Inclusive Education}

Special education is permeated with an ideology of benevolent humanitarianism, which provides a moral framework within which professionals and practitioners work. But it is important to recognise that... the treatment of children who have at various times been defined as defective, handicapped or having special needs... is a social categorisation of weaker social groups (Tomlinson 1982: 5).

Sociology is a subject whose insights should be available to all members of a society to help them understand the mystification and untruths about their social reality which are continually offered to them by those with power and influence. It is not popular with governments because, as a critical exercise, it questions their value assumptions, policy orientations and self-interest. What passes for social reality is mainly a version provided by powerful political elites and much of what happens in social life is a product of their decisions and vested interests. The separation of children and young people from a society's mainstream education and skill system, whatever their special needs, learning difficulties or disabilities, is no exception to this. The history of special education through various kinds of segregation to contested notions of inclusion demonstrates the power and the conflicting interests of political and policy groups, professionals, practitioners, administrators and others to substantially affect the lives of young people. The expansion of subsystems and labels for those regarded as having 
special needs, defects, or disabilities requires study and explanation beyond the individual characteristics of the children.

In particular the contorted and contradictory language intended to mystify people should be examined. In 2018, for example, children in some German schools "diagnosed" with Attention Deficit Hyperactivity Disorder (ADHD) were required to wear vests filled with sand, referred to as compression vests or squeeze jackets, to keep them in their seats in class. The Head of the Inclusion Unit at Grumbrechtstrasse school in Hamburg was enthusiastic for these vests. Weighing children down with sand, she thought, was a gentler form of therapy than drugs (Connolly 2018). The children clothed in sand were already in an Inclusion Unit. So a sociologist might question whether drugs and sand are acceptable ways of "including" young people? Or as Ulrich Beck wrote in a posthumous publication, they might consider why "the world is unhinged...it has gone mad" (Beck 2012: 1). How has the language of inclusion, so popular over the past forty years with policymakers, practitioners and some academics, become a contortion of what is really happening?

In countries with developed or developing mass education systems, these systems have never included all children. They are hierarchical, differential in terms of wealth and status, dependent on ideological assumptions of normality and ability and thus inevitably exclusive however much this is disguised. The expansion of education, with organisation, resources and funding for groups previously excluded or offered only minimal education, was historically a positive move, but it meant that systems now incorporated large numbers of young people who had difficulty with constantly raised expectations, more and more tests and examinations, and who lacked the necessary cultural capital that middle and upper class homes could provide. Education systems also included immigrant and minority children who have over the years consistently been over-represented in special and inclusive education arrangements. However, national governments, believing that success in a competitive global economy depended on higher levels of education and skill training, were increasingly concerned that all young people should attain beyond minimal levels. Special education programmes expanded, many eventually becoming subsumed under notions of inclusive education, giving rise to a whole "inclusion" industry, writing, explaining, researching, prescribing and arguing about meanings and teaching programmes. The whole edifice of mass education was quickly underpinned by expanded provision for those regarded as having learning difficulties, disabilities, or acquiring any of the many labels of special educational need. But whatever setting the provision is offered in - separate schools, units, classes, or groups - it affects spatial segregation, not leading to inclusion.

In addition, governments have always been anxious that as many people as possible should work, and not be a burden on taxpayers, and the training and employment of young people with learning difficulties and disabilities has always been regarded as a problem. In the 21 st century the question as to how local, regional and national economies could create jobs and employ all the young people became crucial. The Great Recession of 2008 put enormous pressure on education and social systems world-wide. In 2019 Elizabeth Schumacher despaired that Germany and indeed the whole of the European Union was failing to provide disabled students with jobs (Schumacher 2019). By the spring of 2020 much of the global economy was shutting down due to the global pandemic of a corona virus, and the futures and life chances of those with special educational needs and disabilities (SEND) was looking even more precarious.

This chapter considers some sociological understandings of special and inclusive education, especially where class, race, and ideas of "ability" are concerned, notes the place of the 
lower attainers and disabled in what was a developing global economy, and briefly overviews the education and training offered these young people in England, the USA, Germany and Finland. After a global pandemic of the corona virus in 2020, national economies and whole societies entered a period of uncertainty, and governments could no longer be sure what their plans for education and training would be. The signs were that many education systems might become more hierarchical and segregated, with expanded methods of exclusion from mainstream education, and more unemployment among the vulnerable as businesses struggled to regain their place in a global economy.

\section{Some Sociological Understandings}

Whether sociological insights can change social situations and structures is debatable, but sociologists can make clearer on what basis and on whose values and judgments policy and practice takes place. The great sociologists of the 19th and 20th century can help provide a critique of the expanded subsystems that comprise special and inclusive education. Max Weber excelled in explaining the growth of bureaucracy, which was necessary in mass social institutions, but could lead to inhuman routines and judgements (Gerth/Mills 1948). Parents and teachers, trying to deal with the legalities and complexities of administration in the SEND area would recognise Weber's insights in the sometimes heartless judgments about their children. He would have understood why the expansion of medical, social and administrative labels given to children expanded, and the way in which the embrace of inclusion by governments after the 1994 Salamanca statement (UNESCO 1994) continued to use the terminology of "special educational needs". Subsequently, many legislative frameworks in most countries began to use a language of inclusion to continue exclusionary practices. ${ }^{1}$ Karl Marx noted that as a capitalist mode of production developed, surplus labour could be used or discarded, a situation that could be applied to many young people after their special inclusive education. Emile Durkheim wrote about organic solidarity and the morality of co-operation. Social exclusion can lead to alienation, and the "cult of the individual" to social disintegration. This would certainly apply to the rise in mental health problems and suicides in young people forced to compete endlessly in modern education systems and given limited supports in transitioning from school to work.

In the 20th century, the American sociologist Talcott Parsons provided a model for an accepting understanding of special education. His functionalist approach was concerned with order, balance and stability in society (Parsons 1952). For him, those considered defective and disruptive were "not normal" according to the definitions of normality applied by society. Fitting in the "not normal" category demanded data collection, surveys, counting, labelling and then exclusion from mainstream education and in some cases from society, and establishing or expanding separate provisions. His approach was taken up in a sociology of deviance, in which young people who disrupt acceptable normal life were discussed as in need of social control. As an antidote to this view, Richardson and Powell (2011) and Richardson et al (2017) have described the paternal and punitive benevolence, which are forms of social and cultural control of the poor and marginal, that led to the marginalisation of many working

1 See Roger Slee (2018: 22-25) for lists of the international conventions, acts, and reports which followed a notional embrace of inclusion by 92 governments. 
class and minority young people, many ending up in a "school to prison pipeline". Another well-known American sociologist, C. Wright Mills argued that there is a need for a sociological imagination to understand how social structures and historical developments give rise to individual personal troubles (Mills 2000), but many policy makers and practitioners still prefer individual functionalist explanations for those whose development or behaviour violates accepted norms. It took a global Disability Movement from the 1970s and a number of disabled Professors of Sociology to develop a social model of disability by which it is understood that people with impairments are disabled by a society's failure to accommodate to their needs (see, e.g., Oliver 1990). But the 21 st century has seen a resurgence of eugenic theories in which developments in neuroscience and genetics are used to support individualistic views of those regarded as defective or deviant (Tomlinson 2017).

\section{Class, Race and Ability}

In class-based and racist societies where education systems were designed to educate the (white) middle and upper classes, the incorporation of working class, immigrant and ethnic minority groups previously excluded or given only minimal schooling created severe conflicts of interest. In pre-industrial societies, divisions between the skilled and unskilled were of less importance as it was accepted that all activity, whether manual or mental. required some form of skill. In industrial society, with a division of labour and hierarchies of schooling, contrasts between intellectual and manual labour developed, and separate schooling for disabled and 'feeble-minded' youth led inexorably to their relegation to lower level schooling, vocational training and work.

This situation has persisted globally and perhaps especially in England, where vocational education and training is associated with second class provision. Governments have an interest in making all young people economically productive if possible, but those who interfere with aims of "raising standards" need to be separated out. Theories of human capital are useful to urge all young people to invest in themselves and enhance their own human capital but those who are unable or unwilling to reach higher levels of education and skill still need special provision. The development of intense testing regimes to sort out the "successful" meant that not only were working class, immigrants and minority children more likely to fail, but many middle-class parents also feared for those of their children who had difficulties in learning in competitive school environments. It was inevitable that an expanding and expensive subsystem - a "SEN Industry" which gradually turned into an "Inclusion Industry" would develop to deal with different kinds of disabled, difficult and deviant young people.

The Platonic myth that children are born as gold, silver, iron or brass has long served elite groups who need rationalisations and ideological justification for treating children differently by social class, race and ethnicity and disability. This myth remains behind beliefs held in schools and classrooms where children are assumed to be born able, average or less able and disabled. The myth is sustained by the notion that "intelligence" can be measured by IQ tests developed from the early 20th century with scores fixed along a bell curve. The curve was useful in that it appeared to demonstrate that some children could and should be labelled as idiots or imbeciles at the lower end and gifted or high ability at the other end. Eugenic theories of genetic inheritance and psychometric theories of measurable intelligence continue to influence education around the world (Dorling 2015; Tomlinson 2017), and 
popular psychology invites the general public to test their own IQ. Karl Pearson, English mathematician and London University Professor who was credited with developing the bell curve and other statistical methods (including correlation and chi square), had strong views as to the inferiority and possible degeneracy of lower classes and alien races. He wrote in 1892 that "no degenerate or feeble-minded stock can be converted into healthy and sound stock by the efforts of education" (Pearson 1892). Following a somewhat dubious study of Jewish and Russian immigrant children in a London school, he wrote two articles in the $\mathrm{An}$ nals of Eugenics Journal on "The problem of alien immigration into great Britain" (Pearson/Mold 1925). He thought that Jewish people, despite the occasional Einstein, were inferior, and if oppressed in Eastern Europe, they should go to some country other than Britain. He conceded that if immigration was necessary, Jewish people should go to Yorkshire to work in agriculture. He seemed to have been unaware that two young Jewish migrants in the 1880s, Michael Marks and Thomas Spencer, had travelled to Leeds in Yorkshire and set up what became a very successful retail store, still in business 150 years later!

Intelligence tests have remained a popular way of separating out children into different schools. In England, most secondary schools became comprehensive, but 164 grammar schools remained. There is still a test at age $11+$ for these schools and their retention persists, the Conservative government in 2018 giving extra money to them. Whereas in the 1940s selecting out the "able" had justified selecting out the less able for special schooling, Gorard and Siddiqui (2018) pointed out that as an inclusive schools movement was intended to bring most children back into comprehensive schools, separate schools for the "more able" made little sense. But the point of such separation is political, not educational, as governments use a rhetoric of social mobility to persuade people that in a class-based society a few children can be separated for superior schooling and life chances. In fact, in England, the ultimate privileged schooling can still be bought at the expensive "public" schools, which up to the present day educate elite politicians, especially at Eton and then Oxford University (Green/Kynaston 2019).

In the 21 st century, debates on intelligence have been infused with new life by the completion of the human genome project, DNA biobanks, behavioural genetics and neurosciences. Robert Plomin, an American geneticist working at Kings College London was introduced to the then Education Minister Michael Gove by his adviser Dominic Cummings. Plomin claimed that the largest factor in school performance was genetic inheritance, and eventually all children could be tested and then "chips" inserted in them (as in heart medicine) to allow teachers to teach to their levels (Asbury/Plomin 2014: 12). In the USA, biological determinism and attempts to prove the inferiority of African-American citizens persist. There was a furore over the publication in 1994 of The Bell Curve (Herrnstein/Murray 1994), which claimed to document the intellectual inferiority of social and racial groups, especially black single mothers. The book had a large popular and some academic support and in 2007 Nobel prize-winning geneticist James Watson asserted that "all our social politics are based on the fact that their African intelligence is the same as ours... whereas all the testing says not really.... And people who have to deal with black employees find this is the case" (HunteGrubbe 2007). Although Watson took a forced retirement from his post for such falsehoods, a movement persists on both sides of the Atlantic searching for biological underpinnings for class and race "intelligence". It is in societies where elites need excuses for the unequal social and educational treatment of lower class and minority groups that assumptions of lower intelligence offer a perfect rationalisation. 


\section{Policy-driven Inclusion and Exclusion in England}

Segregated special schooling in England in 1945 placed children into designated "categories of handicap" with special schools and classes for the various conditions. Post-1981 statutory categories notionally disappeared and a variety of other categories appeared under the wider description of "special educational needs" (SEN). As in other countries, dyslexia, autism or autistic spectrum disorder became popular labels for literacy and behavioural problems, although as more middle-class parents and students claimed resources and funding for these conditions it was recognised that becoming literate and accommodating to an arbitrary norm of behaviour should not necessarily lead to punitive treatment. From the 1980s policy and practice encouraged what was first known as integration, then inclusion. Mainstream schools were legally required to appoint a special educational needs coordinator (SENCO), and the 1995 Act added Disability to the all-encompassing label of SEND. Young people leaving school at 16 with lower attainments, disabilities and special needs, who were "not in education, training or employment," were labelled as NEETS, and were the focus for a plethora of youth training schemes (Tomlinson 2013). In England children with more severe physical, sensory or learning difficulties were legally required to have a Statement of their needs with a school placement or resources and funding decided by the local authority after consultation with professionals. Mainstream schools were notionally funded for the other children "at risk" of needing special educational help, although these were "included" through attention from special educational needs departments, units or classes.

In 2014 the Statement became an Education, Health and Care Plan (EHCP), and there was intense competition for a Plan which brought funding and resources. Local Authorities were responsible for assessing children and young people for Plans but were not given sufficient money from central government to pay for assessments and placements. Funding for education was drastically reduced from 2010 with money for SEND having been steadily cut annually by central government. Local Authorities developed strategies to deny parents and students a Plan if possible, leading to much anger and litigation as parents employed lawyers to claim the resources. Campaigns against the cuts grew, including Special Needs Jungle, a campaign led by lawyers. By 2018 some $15 \%$ of all children aged 5-16 were recorded as in need of some kind of special provision, with moderate learning difficulties and mental health needs being prominent, but funding and resources for children both with and without Plans was limited. Local Authorities were legally responsible for young people with severe disabilities up to age 25 .

Segregated special schools, which had steadily been decreasing in numbers, began to appear again, helped on by a Conservative policy from 2010 to allow "Free schools" to develop, essentially tax-payer funded schools set up by parental, community or religious groups (see Allan/Jørgensen 2021 in this volume). The pressure on mainstream schools to achieve higher test scores led to an increase in school exclusions not only into special schools and units, but also by actual exclusion from all types of state schooling, usually for behavioural reasons. In 2015 over 300,000 children were temporarily or permanently excluded from primary, secondary and even special schools. Head teachers could exclude children from their schools without using special education procedures, and working-class students (mainly boys), African-Caribbean and Roma children continued to have the highest rates of school exclusion. In March 2020 Parliament passed a Corona Virus Act, which included clauses removing the legal duties of Local Authorities from naming the schools children with a Plan 
should attend, which was intended to solve the problem of lack of central funding given to the local authorities. Mainstream schools continued to struggle with less funding for "included" children. Ironically, parents could still legally be prosecuted if their children truanted and failed to attend school.

The diversity of schools which had fragmented the English state system in the $21 \mathrm{st}$ century included Pupil Referral Units (PRUs) where schools could send their excluded pupils who remained on the original school roll. Academy schools, banding together in MultiAcademy Trusts (MATs) gradually created Alternative Provision schools, some of them existing PRUs. This was all intended to remove problematic young people from mainstream schools which could then get on with the core business of credentialing the students who did not take up teacher time with too many problems. In addition, state special schooling was increasingly supplemented by many fee-charging private schools, a market intended to capture parents with enough money to pay for their children or who could argue, usually with legal help, that the local authority should pay. Private agencies offered guidance to find schools "relevant to the condition of your child". A private American business, The Priory Group claimed they could place children with some seventy conditions, an elaborate mix of medical and social "conditions" through which children and young people could be "treated" for payment. ${ }^{2}$ This was one solution to the competitive test-driven economy in which many children were set to fail, but provided for if their parents could afford it. It was an elaborated way of excluding a large number of young people through a language of defect, inability and disability but still using a language of benevolent inclusion. This was a situation noted by Slee (2018) in his entertaining book - Inclusive Education Isn't Dead, It Just Smells Funny which pointed out that efforts to dismantle exclusion have often actually sustained it. Sociologists try to explain how elites sustain their power using theories of symbolic violence and elite power (see Boronski/Hassan 2020).

\section{Vocational Initiatives in England}

Those who have been through a special education programme or included at lower levels of schooling will mainly progress to some kind of vocational education although in England concern with education and training for a majority of young people post-14 is relatively recent. Up to 1939 over $88 \%$ had left school by 14, the school leaving age raised to 15 in 1945 , 16 in 1972 and 18 in 2013, providing the young person remained in full or part time education or work-based learning. Governments did not take much interest in vocational education and training until 1973 when a global oil crisis led to a collapse of the youth labour market. This led to a variety of Youth Training schemes, created and abandoned over the years, with civil servants and officials often moving from one scheme to another. A Manpower Services Commission (MSC) was set up in 1983 which oversaw a Youth Opportunities Programme

2 Some medical and social 'conditions' the Priory Group claimed they could find placements for included: Moderate Learning Difficulties; Profound and Multiple Learning Difficulties; Severe Learning Difficulties; Dyslexia, Disgraphia, Dyscalculia, and Dyspraxia; Asperger's Syndrome; Epilepsy; Behavioural/social/emotional Difficulties; Cerebral Palsy; Down Syndrome; Complex Health Needs; Fragile X Syndrome; Hearing impaired; Physical impairment; Neurological Dysfunction/Brain injury, Rett syndrome; Speech, language and communication difficulties; Tourette's Syndrome; Gifted children; Sexual/emotional or physical abuse; and Fostered children. 
(YOPS) which became a Youth Training Scheme (YTS), and a Technical and Vocational Educational Initiative (TVEI) was piloted in schools, and later ended. A National Council for Vocational Qualifications (NCVQ) was set up in 1986 which lasted into the 1990s, when most youth training schemes were handed over to a Training and Enterprise Council (TEC) which gave way to a Young Peoples Learning Agency (YPLA) in 2002. This lasted only two years before its funding was transferred back to an Agency in the Department for Education. Colleges of Further Education, which actually took in the majority of young people who did not go to University, were progressively underfunded during the 2000 s.

The English policymakers, unlike in Germany (see below), could never decide whether it was employers, central or local education authorities, or private agencies, who should control vocational education and training. Unions lost most of their influence and were seldom consulted. Alison Wolf, commissioned to carry out a review of vocational education, reported in 2011 that two and a half million young people were on some kind of vocational course, many of these having no value in the labour market. She recommended that young people should not be tracked irreversibly into vocational courses and employers should take more responsibility (Wolf 2011). Under David Cameron's Conservative government, apprenticeships were to be paid for by a levy (tax) on business and there was an increase in apprenticeships of all kinds. Unfortunately, some employers tended to rename existing workers as apprentices and few school leavers were taken on. Eventually government increased money for apprenticeships and provided more funding for vocational courses in the Colleges of Further Education. Disability Inclusion Consultancies sprang up, but employers continued to be reluctant to take on those with disabilities or lower school attainments. Ironically, in the panic created by the 2020 corona virus global emergency, jobs that had been considered low status - care-workers, nursery assistants, street cleaners, van drivers - suddenly attained visibility as of great importance to the functioning of society.

It was also of note that, although vocational training was still the destination for the majority of young people, especially those who had been excluded from mainstream education due to their disabilities, or lower attainments, there was an increase in disabled young people entering higher education (in this volume, see Allemann-Ghionda 2021; Limbach-Reich 2021). In the 1980s numbers of students with disabilities in universities were not worth separate enumeration tables but were counted among the "disadvantaged". Under the 2004 Higher Education Act (part 3) institutions could set their own fees ( $£ 3000$ to this point, $£ 9250$ by 2017) but had to submit plans to promote equal opportunities. This encouraged universities to seek out those who qualified for university entry and make some provisions for them. The Office for Students reported in 2017 that $13.2 \%$ of students in English universities claimed they had some kind of disability (OFS 2019).

\section{Contrasts with the USA}

A major difference with the UK is that although in the USA there is an official leaving age of 16/17 most students stay in public high schools until 18/19 with expectations that, apart from drop-outs and those completely excluded, all would progress to a four-year university course or at least a two-year course in a Community College. The decentralised structures in the USA allowed the Federal government to set a framework in which 50 states function, but educational control is largely delegated to states, local school districts and boards, as well as 
thousands of autonomous higher education institutions. The history and treatment of those falling into categories of disability and special educational needs has been similar to England. Economic, political and social factors, especially pressures to ramp up testing to higher and higher levels, led to an expanding special education sector, and then an expanding "inclusion" sector. Powell (2011) illustrated the way the categories of special education developed in the USA were similar to the English categories and were heavily dependent on medical and psychological ideas and strong professional influence, with Learning Disability becoming a popular label for middle-class families wanting extra resources. Richardson and Powell (2011) noted that the whole edifice of special education and its ever-changing population of young people could not be understood without reference to vocational education and labour markets, and also to disparities in participation rates by race and ethnicity and delinquency. As in England, class and race have been influential in deciding who is likely to be in special or inclusive educational settings, although in the USA there are stronger persistent beliefs that racial minorities are more likely to be less educable, be lower attainers and more likely to be delinquent and candidates for the prevalent school to prison pipeline. The limited welfare state provides some social assistance, an inadequate response to widespread labour market marginalisation.

Although provision for vocational education varies between states, more attention has been given to the sector than in England. The Smith-Hughes Act in 1917 made the Federal government responsible for providing funding for vocational courses, and these were to be primarily in high schools to prevent an academic-vocational split. There were early and repeated attempts to introduce German-style apprenticeships, but there was never a similar harmony between, employers, unions and educationalists. The 1973 Vocational Rehabilitation Act prohibited discrimination against disabled students and over the years Federal money has supported States in developing a variety of school-to-work programmes and vocational courses. By the 1990s there was more recognition of changing workplace practices centred round the production of "flexible workers" and adapting to a split labour market of high and low skill jobs. One study in 2003 reported that learning disabled, and many black students, were being prepared for the "five F's": low-paid, low-skill work in Food (cafes, fast food outlets), Filth (cleaning in streets and offices), Folding (laundry work), Fetching (messenger work) and Filing (low level office work) (Moxley/Finch 2003).

By the later 2000s technical and trade schools were educating more students post-16 and there were more apprenticeships offered by employers, although unions usually wanted to limit the number of apprenticeships. It was the two-year state Community Colleges that provided around $30 \%$ of the country's vocational training after high school, with private Colleges also offering post-secondary vocational and technical training, including military training. There had been suggestions, as more recently in England, that the dream of a College degree for all may be less necessary in a changed economic climate, with entry level employment or an apprenticeship offering a better rate of return for young people. In response, Lipman (2004) pointed out that in economies where vocational education was not high status "in the information economy one's education is a key determinant as to whether one is a highly paid knowledge worker, or part of the downgraded sector of labour" (Lipman 2004: 63). She suggested that, in the USA, links between education and the economy had created a system that was less inclusive, more selective, and reproducing a differentiated workforce with lower class and racial minorities being in low status low wage jobs or bearing the brunt of unemployment. 


\section{Transition to Work in Germany}

As in the USA, Germany has a decentralised political system in which 16 states or Länderfive of them former East German (GDR) states - function under the wider federal government. Despite the world economic crisis of 2008 and the eurozone debt crisis, Germany's economy continued to perform well. Much of the past success and also especially dealing with the 2020 global pandemic and economic crisis was credited to Chancellor Angela Merkel and her leadership (Chazan 2020). But it was also due to a strong manufacturing sector which for years had benefited from a highly-skilled workforce trained in the dual system of apprenticeships in the industrial and commercial areas where the majority of young people were offered good vocational training.

Although the education system remained among the most social stratified in Europe and largely selective from age 10, with the Gymnasium (academic grammar school) still the most sought-after type of school, there has been good schooling for young people in the Realschule (technical school), less so in the Hauptschule (basic general and vocational school) or Förderschule or Sonderschule (special school). Some Länder have joined the lower secondary school types or even developed the Gesamtschule (comprehensive schools) that teach multiple ability groups but are not necessarily inclusive of children with special educational needs. There has been considerable criticism of this stratification and early selection, but the system is guarded by teachers, heads of school and parental choice. There is limited permeability, with the possibility of moving between schools mainly downward. Around $6 \%$ of all children and youth are assessed for Förderschule and there remained a strong resistance to including children in mainstream school from the special education profession. Pfahl and Powell (2011) noted that the professional association - the Association of German Special Education (Verband Deutscher Sonderpädagogik) - has been highly influential in constructing differences and maintaining a classification system that underpins special schools. As in the UK, the category of Learning Disability (Lernbehinderung) represents the largest group receiving special educational support, with children of the lower social classes and migrant groups mainly given the LD label and middle-class children tending to be described as dyslexic.

But the relationship between vocational education, the dual system and the labour market, continued to be more inclusive of low attaining and some disabled students. The Federal government regulated apprentice training and there were around 350 state-regulated occupations that make up all areas of economic and commercial life, with some $45 \%$ of young people over 16 being in the dual system at any one time. While manufacturing and industrial jobs continued to be important, jobs in new technologies and digital communications became more necessary. In all the European and other developed countries, there was a need for highly skilled workers, unskilled workers were less likely to be needed and low attainers who were not in the dual system or even in a "transition" system while seeking training were more likely to be excluded from the economy. While middle-class students usually aimed for university, and higher education (with very low fees of around $€ 400$ per year) has been expanding rapidly, many still opt for good apprenticeships, especially those dual study programs combining apprenticeships and post-secondary education, thus further disadvantaging lower attainers. The arrival of many more migrants in Germany in the refugee crisis of 2015/16 added to pressures on vocational training systems and on employment possibilities. Similarities with the English system exist in that Colleges provide a variety of low-level transition 
courses without specific employment aims but that offer some kind of certification, which may allow progression to other courses or even employment later.

\section{Finland: A Model for other Countries?}

Comparing education and training systems, especially arrangements for lower attainers and those with disabilities, is of permanent interest to governments. As international comparative tests of student performance have become accepted around the world, there has been much anxiety among governments globally about where their students place in testing global league tables. Finland, with students consistently performing better than those in other countries, became a place where policy makers and educationalists of all kinds visited to find out how good results were achieved. One lasting feature of the Finnish test results was that the lowest quartile of student achievers - mainly those with special educational needs or disabilities obtained higher scores than similar groups in other countries. A second feature is that the education and training system is one of the most inclusive in the world, with almost all students regarded as having special educational needs. Apart from a small group of students with more severe impairments who go on after 16 to special vocational colleges and training centres, over $40 \%$ of students attend well-resourced vocational colleges leading to recognised qualifications and employment. Some $55 \%$ go on to university and employment. So, the question was raised that if Finland could prepare almost all young people, including those with special needs and disabilities for higher education or good vocational training and employment, should not Finland be a model for systems around the world?

Pasi Sahlberg, at Helsinki University, who has studied education and training systems globally and advised governments, has noted that overall Finland has good governance, a respected education system, and a broad consensus between policy makers, educationalists and the public on the future direction of the Finnish economy and society as a whole (Sahlberg 2015). After a contested development over many years towards a fully comprehensive and inclusive schooling system, education for all was accepted as a public good. The education and skill training system is based on the belief that all young people should have free and equal access to education to the highest possible levels. Excellent and lengthy teacher training and a culture of trust between political leaders, education authorities, teachers and parents developed, and all children, whatever their special needs or disabilities have a right to attend their local school. A Basic Education Act in 1998 required an education plan for all those who needed extra support and at any time around $30 \%$ of children are receiving support. As elsewhere, a common claim is for dyslexia, but all children are also required to learn another language. There is no national testing and children are given regular formative tests to check on their progress. Students go on at $15 / 16$ to upper secondary study for university or, as in Germany and other Nordic countries, to well organised vocational courses leading to employment for most, though those with SEN remain marginalised.

No system is perfect, and success in education systems does not always translate easily into success in the economy. Finland, claimed in the early 21 st century to be the fourth most successful "knowledge economy" in the world, had problems in 2012, especially with the collapse of the telecommunications industry and Nokia, its global champion, and although there are a range of other occupations, in 2019 there was a $16 \%$ unemployment rate, with only $20 \%$ of disabled young people in permanent employment and $50 \%$ in temporary or part- 
time work. In addition, those in low income households and many immigrant children faced more challenges in learning and working. But Finland remains one of the most equal countries by income in the world and has high rates of freedom to make life choices irrespective of social origins.

\section{Comparing Systems}

While research and literature comparing education systems has expanded over the past forty years, especially now that diverse data from national systems can be collected and compared, the dangers of basing universal policies on what seems to improve education in any particular country are becoming clearer. Dangers are that the histories, economies, politics and previous social policies are ignored or minimised in discussion of what is regarded as successful education. The four countries noted above all participate in the OECD Programme for International Student Assessment (PISA). The national governments in England, the USA and Germany, have all been anxious to the point of panic when the scores of their 15-year-olds in these international tests are low and the country falls in the international league tables. Finland, whose scores are either always top or very near, exhibits no such panic. Thus, over the years, many people have visited Finland to examine how their success is achieved. Commentators have pointed to the egalitarian assumption in Finland that all young people are to be valued equally whatever their learning problems, the funding and organisation of the system, the excellent teacher preparation, and the absence of competition between schools (Meyer/Benavot 2013; Tomlinson 2013). Meyer and Benavot (2013) have also criticised the model of global educational governance, which the PISA organisers advance, driven by competition and market demands and in which success in passing tests is prized over other educational aims. They expressed concern that the OECD, through its PISA programme, was attempting to institute new governance in which countries reduce their school curricula to a narrow range of subjects with the aim of improving individual and economic competitiveness and national economic growth. While the effects of the Covid-19 pandemic increase economic insecurities worldwide, notions of economic growth, and preparation of young people for shrinking economies are likely to be reconsidered in the coming years. The incorporation of market goals and competition in schooling, which the German Federal government is planning to adopt, may have to be reconsidered as well. Gomolla (2020) has provided a thorough description of the Germany's political discourse on education from 1945 to the present, noting the impact of the "PISA shock" - when the government found students performed less well than in some other European countries. She suggests that copying the market models of England and the USA might not be the best direction for school reform and educational governance at the present time.

\section{Coda}

In 2020 accepted or developing ideas about preparing young people in inclusive education systems for a global economy were thrown into chaos. A global pandemic caused by Covid- 
19, a corona virus that appeared first in China, rapidly spread to countries around the world, killing hundreds of thousands. As Elliot wrote at the end of March 2020 "Europe is being ravaged by the corona virus", border controls reappeared between countries, many of which had "locked down" their citizens in home isolation, national economies were threatened and employment of all kinds came to a halt. International organisations such as the IMF (International Monetary Fund), the United States Federal Reserve, the European Central Bank and the Bank of England all attempted to prevent a global recession, which seemed inevitable. In England 1.5 million people were already at risk of losing jobs to automation, and as the crisis progressed the government attempted to find ways of paying workers without work. In the USA some 3.5 million people filed for unemployment benefits per month. Wealthy people attempted to protect themselves and their wealth and there was no bureaucratic benevolence apparent during the crisis. Schools, including special schools, colleges and universities in affected countries closed down and students were expected to study on-line with parental help. The social and educational inequalities implicit in this were immediately obvious. Low income, disadvantaged households, migrants and refugee children and those with the variety of special educational needs and disabilities were at most risk educationally and socially.

The task of sociologists in the future will be to analyse and explain what has and is happening to special and inclusive education systems in what will be a changed global world and a changed economy. It may be that the survival of whole populations now depends on understanding the social structures and economic constraints which develop, and whether the same elite groups who are in governments will try to maintain their ideological and power structures. While it is unlikely that social breakdown in European societies will occur, Talcott Parson's claim that order and stability is a major goal in societies is certainly challenged when pandemics beyond the control of governments develop.

\section{References}

Allan, Julie/Jørgensen, Clara Rübner (2021 in this volume): Inclusive School Development. In: Köpfer, Andreas/Powell, Justin J.W./Zahnd, Raphael (Eds.): Handbuch Inklusion international. Opladen: Verlag Barbara Budrich, pp. 329-344.

Allemann-Ghionda, Cristina (2021 in this volume): Diversität, Inklusion und Internationalisierung in der Hochschule. In: Köpfer, Andreas/Powell, Justin J.W./Zahnd, Raphael (Eds.): Handbuch Inklusion international. Opladen: Verlag Barbara Budrich, pp. 473-498.

Asbury, Kathryn/Plomin, Robert (2014): G is for Genes. Chichester: Wiley.

Beck, Ulrich (2012): The Metamorphosis of the Modern World. Cambridge: Polity Press.

Boronski, Thomas/Hassan, Nasima (2020): Sociology of Education. London: Sage.

Chazan, Guy (2020): Germany's Crisis Manager is Back. Financial Times Weekend, 28 March 2020.

Connolly, Kate (2018): Use of sand vests to calm children with ADHD sparks controversy in Germany. The Guardian, 20 January 2018. https://www.theguardian.com/world/2018/jan/20/ use-of-sand-vests-to-calm-children-with-adhd-sparks-concern

Dorling, Danny (2015): Injustice: Why Social Inequality Still Persists. Bristol: Policy Press.

Elliot, Larry (2020): Solidarity? Covid-19 Crisis has Exposed the EU's Weakness. The Guardian, 30 March 2020. 
Gerth, H.H./Mills, C. Wright (1948): From Max Weber: Essays in Sociology. London: Routledge and Kegan Paul.

Gorard, Stephen/Siddiqui, Nadia (2018): Grammar Schools in England: A New Analysis of Segregation and Academic Outcomes" In: British Journal of Sociology of Education 39, 7, S. 909924.

Gomolla, Mechtild (2020): School reform, educational governance and discourses on social justice and democratic education in Germany. In: Pink, William T. (ed.): Oxford Encyclopedia of School Reform. New York: Oxford University Press.

Green, Francis/Kynaston, David (2019): Engines of Privilege: Britain's Private School Problem. London: Bloomsbury.

Herrnstein, Richard J./Murray, Charles (1994): The Bell Curve. New York: The Free Press.

Hunte-Grubbe, Charlotte (2007): The Elementary DNA of Dr Watson. The Sunday Times, 14 October 2007.

Limbach-Reich, Arthur (2021 in this volume): Studieren mit Behinderungen im Spannungsfeld zwischen Behindertenrechtskonvention und Neoliberalismus. In: Köpfer, Andreas/Powell, Justin J.W./ Zahnd, Raphael (Eds.): Handbuch Inklusion international. Opladen: Budrich, pp. 499522.

Lipman, Pauline (2004): High Stakes Education: Inequality, Globalization, and Urban School Reform. New York: RoutledgeFalmer.

Meyer, Heinz-Dieter/Benavot, Aaron (eds.) (2013): PISA, Power, and Policy. The Emergence of Global Educational Governance. Oxford: Symposium Books.

Mills, C. Wright (2000): The Sociological Imagination. Harmondsworth: Penguin.

Moxley, David P./Finch, John R. (2003): Sourcebook of Rehabilitation and Mental Health Practices. New York: Plenum Books.

OFS (2019) Beyond the Bare Minimum: Are Universities and Colleges Doing Enough for Disabled Students. Insight no 4. London: Office for Students.

Oliver, Michael (1990): The Politics of Disablement: A Sociological Approach. Basingstoke: Palgrave Macmillan.

Parsons, Talcott (1952): The Social System. New York: The Free Press.

Pearson, Karl (1892): The Grammar of Science. London: Walter Scott.

Pearson, Karl/Moul, Margaret (1925): The problem of Alien Immigration into Great Britain, illustrated by an examination of Russian and Polish Jewish children. In: Annals of Eugenics 1, 2, pp. 5-54.

Pfahl, Lisa/Powell Justin J.W. (2011): Legitimating School Segregation: The Special Education Profession and the Discourse of Learning Disability in Germany. In: Disability and Society 26, 4, pp. 449-462.

Powell, Justin J.W. (2011/2016): Barriers to Inclusion: Special Education in the US and Germany. Abingdon: Routledge.

Richardson, John G./Powell, Justin J.W. (2011): Comparing Special Education: Origins to Contemporary Paradoxes. Stanford: Stanford University Press.

Richardson, John G./Wu, Jinting/Judge, Douglas M. (2017) The Global Convergence of Vocational and Special Education. London: Routledge.

Sahlberg, Pasi (2015): Finnish Lessons 2.0. New York: Teachers College Press.

Schumacher, Elizabeth (2019): How Germany is failing disabled and special needs students. In: Deutsche Welle (DW) 11 March 2019.

Slee, Roger (2018): Inclusive Education Isn't Dead, It Just Smells Funny. London: Routledge.

Tomlinson, Sally (1982/2012): A Sociology of Special Education. London: Routledge.

Tomlinson, Sally (2013): Ignorant Yobs? Low Attainers in a Global Knowledge Economy. London: Routledge.

Tomlinson, Sally (2017): A Sociology of Special and Inclusive Education. London: Routledge. 
UNESCO (1994): Salamanca Statement and Framework for Action on Special Educational Needs. Paris: United Nations Educational, Scientific and Cultural Organization.

Wolf, Alison (2011): Review of Vocational Education: The Wolf Report. London: Department for Education. 



\title{
4 Inclusion and Equity in Education: Responding to a Global Challenge
}

\author{
Mel Ainscow
}

\begin{abstract}
This chapter traces the development of the United Nations' policy Education for All since 1990, noting the struggles that have gone on to ensure that it is, in fact, concerned with all children, whatever their characteristics and circumstances. In so doing, it points to the need to focus particular efforts on the most disadvantaged learners to ensure that no one is overlooked. In addressing this global challenge, the chapter draws on findings from the author's own research in order to suggest an overall agenda for change, focusing on national policies for promoting equity and the development of inclusive school practices.
\end{abstract}

\section{The International Policy Context}

Over the last 30 years there have been major international efforts to encourage inclusive educational developments. In particular, the United Nation's Education for All (EFA) movement has worked to make quality basic education available to all learners. Agreed in 1990, the EFA Declaration sets out an overall vision, which is about being proactive in identifying the barriers some learners encounter in attempting to access educational opportunities. It also involves the identification of resources available at national and community level and bringing them to bear on overcoming those barriers.

This vision was reaffirmed by the World Education Forum in Dakar in 2000, held to review the progress made in the previous decade. The Forum declared that EFA must take particular account of the needs of the poor and the disadvantaged, including working children, remote rural dwellers and nomads, and ethnic and linguistic minorities, children, young people affected by conflict, HIV/AIDS, hunger and poor health, and those with special learning needs.

Meanwhile, further impetus for inclusive education was given by the World Conference on Special Needs Education in 1994. More than 300 participants representing 92 governments and 25 international organizations met in Salamanca, Spain to further the objective of Education for All by considering the fundamental policy shifts required to promote the approach of inclusive education, namely enabling schools to serve all children (UNESCO 1994). Although the immediate focus of the Salamanca conference was on what was termed special needs education, its conclusion was that:

Special needs education - an issue of equal concern to countries of the North and of the South cannot advance in isolation. It has to form part of an overall educational strategy and, indeed, of 
new social and economic policies. It calls for major reform of the ordinary school (UNESCO 1994: iii-iv).

The aim, then, is to reform education systems. This can only happen, it is argued, if mainstream schools become capable of educating all children in their local communities. The Salamanca Statement concluded that:

Regular schools with [an] inclusive orientation are the most effective means of combating discriminatory attitudes, creating welcoming communities, building an inclusive society and achieving education for all; moreover, they provide an effective education to the majority of children and improve the efficiency and ultimately the cost-effectiveness of the entire education system (UNESCO 1994: ix).

As this key passage indicates, the move towards inclusive schools can be justified on a number of grounds. There is an educational justification: the requirement for inclusive schools to educate all children together means that they have to develop ways of teaching that respond to individual differences and that therefore benefit all children; a social justification: inclusive schools are able to change attitudes to difference by educating all children together, and form the basis for a just and non-discriminatory society; and an economic justification: it is likely to be less costly to establish and maintain schools which educate all children together than to set up a complex system of different types of school specializing in particular groups of children.

These developments were further strengthened in 2008 by the 48th session of the IBEUNESCO International Conference on Education, with its theme "Inclusive Education: The Way of the Future" (Opertti et al. 2014). The long-term objective of this event was to support UNESCO member states in providing the social and political conditions that every person needs in order to exercise their human right to access, take an active part in, and learn from educational opportunities. During the conference, ministers, government officials and representatives of organizations discussed the importance of broadening the concept of inclusion to reach all children, under the assumption that every learner matters equally and has the right to receive effective educational opportunities.

Moving forward, the year 2016 was particularly important in relation to the future of the EFA movement and, indeed, the legacy of Salamanca. Building on the Incheon Declaration agreed at the World Forum on Education in May 2015 (UNESCO 2015), it saw the publication by UNESCO of the "Education 2030 Framework for Action," which emphasizes inclusion and equity as laying the foundations for quality education. It also stresses the need to address all forms of exclusion and marginalization; disparities and inequalities in access and participation; and learning processes and outcomes. In this way, it is made clear that the international EFA agenda really has to be about "all". The importance of including disabled children is an essential strand within this new international policy agenda. This is stressed in the United Nations' Convention on the Rights of Persons with Disabilities (UN CRPD) (UN 2006), which states in General Comment No 4:

The right to inclusive education encompasses a transformation in culture, policy and practice in all educational environments to accommodate the differing requirements and identities of individual students, together with a commitment to remove the barriers that impede that possibility.

The Convention defines non-inclusion as the education of students with disabilities in separate environments (i.e. in separate special schools, or in special education units located with regular schools). It commits to ending segregation within educational settings by ensuring inclusive classroom teaching in accessible learning environments with appropriate support. 
This means that education systems must provide a personalized educational response, rather than expecting the student to fit the system.

Like all major policy changes, progress in relation to inclusion and equity requires an effective strategy for implementation. In particular, there is a need to move away from ways of thinking that focus on what certain individual learners lack, towards a focus on the contextual barriers that are limiting the participation and progress of many children. The implication is that overcoming such barriers is the most important means of development of forms of education that are effective for all children. In this way, inclusion becomes a way of achieving the overall improvement of education systems.

In what follows, I use a framework that has emerged from research I have carried out with colleagues in many countries to illustrate what this involves (e.g. Ainscow et al. 2006; Harris et al. 2017).

\section{System Change}

Education policies are intended to make a difference to thinking and action in the field. They must, therefore, be concerned with processes of change. With this as the focus, a policy for promoting inclusion and equity must determine changes that are needed, create and implement action plans, and monitor their implementation.

Drawing on international research regarding system change (e.g. Ainscow et al. 2020; Fullan 2007), policies should also take account of the following assumptions:

- Policy is made at all levels of a national education system, not least at the school and classroom levels;

- Educational change is technically simple but socially complex;

- Clarity of purpose is essential in order to mobilize widespread support; and

- Evidence of various kinds is the catalyst for successful change processes.

Relevant to these ideas are a number of international documents that can be used to review policies within countries. ${ }^{1}$

A good example of a country that has recently implemented a strong policy for promoting inclusion is Portugal. There, a new School Inclusion Law was developed over an 18-month consultation period involving the public and private school sectors, teachers' associations and unions, academic organizations, parent associations and disability representative associations. At the centre of the Portuguese policy is the need for all schools to recognize the added value of the diversity of its students, finding ways to deal with differences, adjusting teaching processes to the individual characteristics and conditions of each student, mobilizing the means at disposal so that everyone learns and participates in the life of the educational community.

1 UN Girls' Education Initiative (2010): Equity and inclusion in education. A guide to support education sector planning, preparation and appraisal. Washington, DC: United Nations EFA Fast Track Initiative. UNESCO-IBE (2016): Reaching out to all learners. A Resource Pack for Supporting Inclusive Education. Geneva: UNESCO International Bureau of Education. UNESCO (2017): A guide for ensuring inclusion and equity in education. UNESCO: Paris. 
Figure 1: Framework to promote inclusion and equity within education systems.

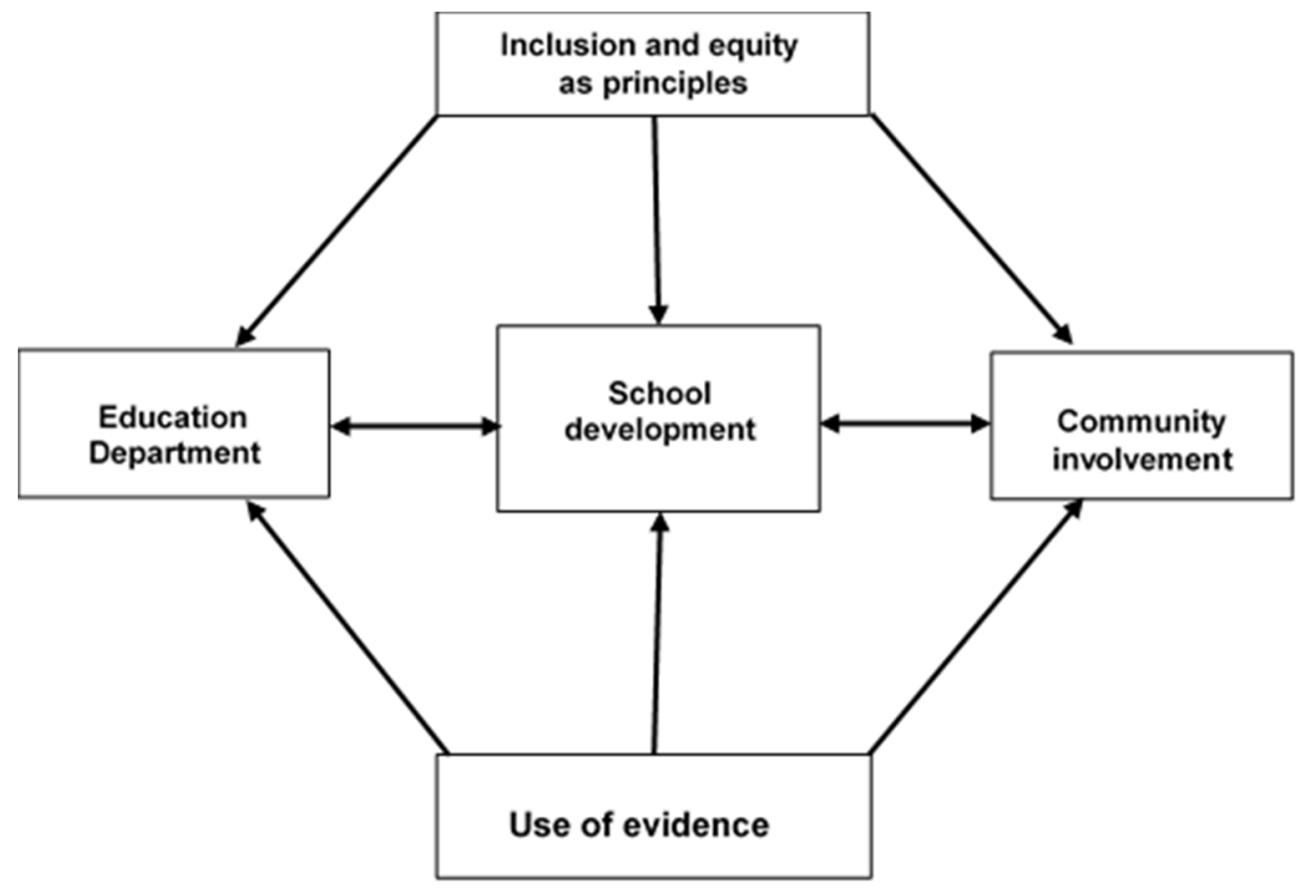

Source: own diagram

My own international experiences have led me to formulate a framework for thinking about how to promote inclusion and equity within education systems (see Figure 1). This framework emerged from efforts to move education systems in a more inclusive direction. Based on the adage "the best way to understand an organisation is by trying to change it," these experiences have thrown light on the factors that can facilitate or limit the progress of such initiatives. More recently, the framework has been used as part of improvement initiatives in countries as diverse as Colombia, Oman, Sweden and Uruguay.

Amended from an earlier version (see Ainscow 2005), the framework focuses attention on five interrelated factors. Together, they can help in determining "levers for change". Senge (1989) sees "levers" as actions that can be taken in order to change the behaviour of an organisation and those individuals within it. He goes on to argue that those who wish to encourage change within an organisation must be smart in determining where the high leverage lies. Too often, he suggests, approaches used to bring about large-scale changes in organisations are "low leverage". That is to say, they tend to change the way things look but not the way they work. Possible examples of low leverage activity in the education field include: the production of policy documents, conferences and in-service courses. Whilst such initiatives may make a contribution, by and large they do not lead to significant changes in thinking and practice. Our aim, therefore, must be to identify what may turn out to be more subtle, less obvious and higher leverage efforts to bring about change in schools. 


\section{Reviewing Inclusion and Equity within Education Systems}

As can be seen, the framework places schools at the centre of the analysis. This reinforces the point that moves towards inclusion must be focussed on increasing the capacity of local neighbourhood mainstream schools to support the participation and learning of an increasingly diverse range of learners. This is the paradigm shift implied by the Salamanca statement, which I have previously described as an "inclusive turn" (Ainscow 2007). It argues that moves towards inclusion are about the development of schools, rather than attempts to integrate vulnerable groups of students into existing arrangements.

At the same time, the framework draws attention to a range of contextual influences that bear on the way schools carry out their work. These influences are: the principles that guide policy priorities within an education system; the criteria that are used to evaluate the performance of schools; and the views and actions of others within the local context, including members of the wider community that the schools serve and the staff of the departments that have responsibility for the administration of the education system. As I will explain, these influences may provide support and encouragement to those in schools who are wishing to move in an inclusive direction. However, the same factors can also act as obstacles to progress.

In what follows each of the five factors is explained, leading to a series of key ideas that need to be considered in developing future policies. These ideas are guided by a belief that inclusion and equity should not be seen as separate policies. Rather, they should be viewed as principles that inform all national policies, particularly those that deal with the curriculum, assessment, supervision, school evaluation, teacher education and budgets. They must also inform all stages of education, from early years through to higher education.

Principles. Terms such as "equity" and "inclusion" can be confusing, since they may mean different things to different people. This is a particular problem when trying to move forward with others - particularly in schools, where everybody is so busy. Put simply, if there is not a shared understanding of the intended direction, progress will be more difficult. There is, therefore, a need to agree definitions of these concepts.

In some countries, inclusive education is still thought of as an approach to serving children with disabilities within general education settings. Internationally, however, it is increasingly seen more broadly as a principle that supports and welcomes diversity amongst all learners (UNESCO 2017). It presumes that the aim is to eliminate social exclusion that is a consequence of attitudes toward and responses to diversity of social class, race, ethnicity, religion, gender and ability. As such, it starts from the belief that education is a basic human right and the foundation for a more just society. Hence, the emphasis on equity, which implies a concern with fairness.

In establishing a definition of inclusion for strategic purposes, our earlier research led us to suggest the following elements (Ainscow/Booth/Dyson 2006):

- Inclusion is a process. That is to say, inclusion has to be seen as a never-ending search to find better ways of responding to diversity. It is about learning how to live with difference and learning how to learn from difference. In this way differences come to be seen more positively as a stimulus for fostering learning, amongst children and adults.

- Inclusion is concerned with the identification and removal of barriers. Consequently, it involves collecting, collating and evaluating information from a wide variety of sources 
in order to plan for improvements in policy and practice. It is about using evidence of various kinds to stimulate creativity and problem-solving.

- Inclusion is about the presence, participation and achievement of all students. Here, ,presence' is concerned with where children are educated, and how reliably and punctually they attend; ,participation' relates to the quality of their experiences whilst they are there and, therefore, must incorporate the views of the learners themselves; and ,achievement" is about the outcomes of learning across the curriculum, not merely test or examination results.

- Inclusion involves a particular emphasis on those groups of learners who may be at risk of marginalisation, exclusion or underachievement. This indicates the moral responsibility to ensure that those groups that are statistically most at risk are carefully monitored, and that, where necessary, steps are taken to ensure their presence, participation and achievement within the education system.

Our experience is that a well-orchestrated debate about these elements can lead to a wider understanding of the principle of inclusion. Furthermore, such a debate, though by its nature slow and, possibly, never ending, can have leverage in respect to fostering the conditions within which schools can feel encouraged to move in a more inclusive direction. Crucially, this must seek to involve all stakeholders, including families, political and religious leaders, and the media. It must also involve those within local education district offices.

The use of evidence. We have argued that evidence is the life-blood of inclusive development (Ainscow/Booth/Dyson 2006). Therefore, deciding what kinds of evidence to collect and how to use it requires care, since, within education systems, "what gets measured gets done". This is widely recognised as a double-edged sword precisely because it is such a potent lever for change. On the one hand, data are required in order to monitor the progress of children, evaluate the impact of interventions, review the effectiveness of policies and processes, plan new initiatives, and so on. On the other hand, if effectiveness is evaluated on the basis of narrow, even inappropriate, performance indicators, then the impact can be deeply damaging. Whilst appearing to promote the causes of accountability and transparency, the use of data can, in practice, conceal more than they reveal; invite misinterpretation; and, worst of all, have a perverse effect on the behaviour of professionals. The challenge is, therefore, to harness the potential of evidence as a lever for change, whilst avoiding these potential problems.

The starting point for making decisions about the evidence to collect at the system level should be with an agreed definition of inclusion. In other words, we must "measure what we value", rather than is often the case, "valuing what we can measure". In line with the suggestions made earlier, then, evidence collected within an education system needs to relate to the "presence, participation and achievement" of all students, with an emphasis placed on those groups of learners regarded to be "at risk of marginalisation, exclusion or underachievement".

An engagement with evidence is also crucial at the level of the school, where it can provide the stimulus for professional learning. This requires the sharing of existing approaches through collaboration amongst staff, leading to experimentation with new practices that will reach out to all students (Ainscow 2016b). Through processes of social learning within a school, this can facilitate the development of a common language with which colleagues can talk to one another and, indeed, to themselves, about detailed aspects of their practice. Without such a language, teachers find it very difficult to experiment with new possibilities (Huberman 1993). 
The use of evidence to study teaching within a school can help in generating such a language of practice by stimulating dialogue amongst teachers (Ainscow/Booth/Dyson 2006). This, in turn, can help to foster the development of practices that are more effective in reaching learners. Specifically, it can create spaces for rethinking by interrupting existing discourses. Particularly powerful techniques in this respect involve the use of mutual lesson observation, sometimes through video recordings, and evidence collected from students about teaching and learning arrangements within a school (Ainscow/Messiou 2017).

Leadership is a crucial factor in making this form of school-based professional development possible. This should focus on making time, promoting trust and encouraging an inquiring stance amongst staff members. The evidence needed to create this stimulation can take many forms and involves a variety of techniques. What is common among them is the way they create "interruptions" in the busy day of teachers that lead to the sharing of practices and the generation of new ways of working (Ainscow/Booth/Dyson 2006). Much of our own work involves us collaborating with teams of staff within schools in order to learn more about how to make this work within current policy contexts (Ainscow et al. 2016b).

In terms of evidence, the obvious starting point is usually with the statistical information available in schools regarding attendance, behaviour and student progress. In recent years the extent and sophistication of such data have improved in many countries, so much so that the progress of groups and individuals can now be tracked in considerable detail, giving a much greater sense of the value that a school is adding to its students. If necessary, further relevant statistical material can be collected through questionnaire surveys of the views of students, staff members and, where relevant, parents and carers. However, statistical information alone tells us very little. What brings such data to life is when "insiders" start to scrutinise and ask questions together as to their significance, bringing their detailed experiences and knowledge to bear on the process of interpretation.

An effective approach for introducing these techniques is lesson study, a systematic procedure for the development of teaching that is well established in Japan and some other Asian countries (Lewis et al. 2006). The goal of lesson study is to improve the effectiveness of the experiences that teachers provide for all of their students. The focus is on a particular lesson or activity, which is then used as the basis for gathering evidence on the quality of experience that students receive. These lessons are called research lessons and are used to examine the responsiveness of students to the planned activities. In using this approach, we have taken a further step forward by incorporating the views of students. Our research suggests that it is this factor, more than anything else, that makes the difference as far as responding to learner diversity is concerned (Ainscow/Messiou 2017).

A current study in primary schools in five European countries is taking this idea forward, using a new model for developing learning and teaching that we call "Inclusive Inquiry".2 This uses dialogue involving teachers and students in order to explore possibilities for making lessons more inclusive.

School development. There is not one single model of what an inclusive school looks like. What is common to highly inclusive schools, however, is that they are welcoming and supportive places for all of their students, not least for those with disabilities and others who experience difficulties. This does not prevent these schools from also being committed to improving the achievements of all of their students. Indeed, they tend to have a range of strategies for strengthening achievement that are typical of those employed by all effective

2 Details of this project, including the materials used, can be found in various languages at: https://reachingthehardtoreach.eu/ 
schools, and the emphasis on supporting vulnerable students does not appear to inhibit these strategies. A key factor is the emphasis placed on monitoring and supporting the progress of all students.

A problem reported from a number of countries is that despite national policies emphasizing the equal rights of children with disabilities to attend mainstream settings, there has been evidence over many years of significant increases in the proportions of students being categorized in order that their schools can earn additional resources (Fulcher 1989). This has led to dissatisfaction with progress toward integration and brought demands for more radical changes. One of the concerns of those who adopt this view is with the way in which students come to be designated as "having special educational needs" (e.g. Slee 2010). They see this as a social process that needs to be continually challenged. More specifically, they argue that the continued use of what is sometimes referred to as a ,medical model' of assessment within which educational difficulties are explained solely in terms of a child's deficits - prevents progress in the field, not least because it distracts attention from questions about why schools fail to teach so many children successfully.

The implication is that schools need to be reformed and practices need to be improved in ways that will lead them to respond positively to student diversity - seeing individual differences not as problems to be fixed, but as opportunities for enriching learning. Within such a conceptualization, a consideration of difficulties experienced by students can provide an agenda for change and insights as to how such changes might be brought about. Moreover, it has been argued that this kind of approach is more likely to be successful in contexts where there is a culture of collaboration that encourages and supports problem-solving (Ainscow 2016b; Skrtic 1991). According to this view, the development of inclusive practices is seen as involving those within a particular context in working together to address barriers to education experienced by some learners.

The theme of school development is further supported by a review of international research literature that examines the effectiveness of school actions in promoting inclusion (Dyson et al. 2004). In summary, this suggests that some schools are characterized by an "inclusive culture". Within such schools, there is a degree of consensus among adults around values of respect for difference and a commitment to offering all students access to learning opportunities. The extent to which such inclusive cultures lead directly and unproblematically to enhanced student participation is not clear. Some aspects of these cultures, however, can be seen as participatory by definition. For instance, respect for diversity from teachers may itself be understood as a form of participation by children within a school community. Moreover, schools with such cultures are also likely to be characterized by forms of organization (such as specialist support being made within the ordinary classroom, rather than by withdrawal for separate attention) and practices (such as cooperative group work) which could be regarded as participatory by definition.

All of this means that attempts to develop inclusive schools should pay attention to the building of consensus around inclusive values within school communities. This implies that school leaders should be selected in the light of their commitment to inclusive values and their capacity to lead in a participatory manner (Riehl 2000). Finally, the external policy environment should be compatible with inclusive developments in order to support rather than to undermine schools' efforts.

The role of education departments. As I have suggested, policy is made at all levels of an education system, not least at the school and classroom levels. Furthermore, the promotion of equity and inclusion is not simply a technical or organizational change - it is a movement 
in a clear philosophical direction. Moving to more inclusive ways of working therefore requires changes across an education system. These span from shifts in policy-makers' values and ways of thinking, which enable them to provide a vision shaping a culture of inclusion, to significant changes within schools and classrooms.

A culture of inclusion in education requires a shared set of assumptions and beliefs among senior staff at the national, district and school levels that value differences, believe in collaboration, and are committed to offering educational opportunities to all students. However, changing the cultural norms that exist within an education system is difficult to achieve, particularly within a context that is faced with so many competing pressures and where practitioners tend to work alone in addressing the problems they face. Therefore, leaders at all levels, including those in civil society and other sectors, have to be prepared to analyse their own situations, identify local barriers and facilitators, plan an appropriate development process, and provide leadership for inclusive practices and effective strategies for monitoring equity in education.

National and district administrators have important roles in promoting inclusive ways of managing schools and education processes. In particular, they need to establish the conditions for challenging non-inclusive, discriminatory educational practices. They also need to establish the conditions that build consensus and commitment towards putting the universal value of inclusion into practice. Particular forms of leadership are known to be effective in promoting inclusion in education (Riehl 2000). These approaches focus attention on teaching and learning; they create strong supportive communities of students, teachers and parents; they nurture the understanding of a culture of education among families; and, they foster multiagency support.

There is also evidence that school-to-school collaboration can strengthen the capacity of individual organizations to respond to learner diversity (Ainscow 2016a; Muijs et al. 2011). Specifically, collaboration between schools can help to reduce the polarization of schools, to the particular benefit of those students who are marginalized at the edges of the system. In addition, there is evidence that when schools seek to develop more collaborative ways of working, this can have an impact on how teachers perceive themselves and their work. Specifically, comparisons of practices in different schools can lead teachers to view underachieving students in a new light. In this way, learners who cannot easily be educated within the school's established routines are not seen as "having problems", but as challenging teachers to re-examine their practices in order to make them more responsive and flexible.

All of this points to the importance of the ways financial resources are allocated within education systems. This can be crucial in creating the flexibility within schools to encourage the sorts of experimentation I have described. Alternatively, it can lead to further segregation, with resources used to provide separate attention for some students - within the school or in separate special schools or classes. In this sense, finance is another powerful lever for change (Meijer 1999).

Involving the wider community. In order to foster inclusion and equity in education, governments need to mobilise human and financial resources, some of which may not be under their direct control. Forming partnerships among key stakeholders who can support and own the process of change is therefore essential. These stakeholders include: parents/caregivers; teachers and other education professionals; teacher trainers and researchers; national, local and school-level administrators and managers; policy-makers and service providers in other sectors (e.g. health, child protection and social services); civic groups in the community; and members of minority groups that are at risk of exclusion. 
Family involvement is particularly crucial. In some countries, parents and education authorities already cooperate closely in developing community-based programmes for certain groups of learners, such as those who are excluded because of their gender, social status or impairments (Miles 2002). A logical next step is for these parents to become involved in supporting change for developing inclusion in schools. Where parents lack the confidence and skills to participate in such developments, it might be necessary to engage and build capacity and networks. This could include the creation of parent support groups, training parents to work with their children, or building the advocacy skills of parents to negotiate with schools and authorities. Here, it is worth adding that there is evidence that the views of families, including children themselves, can be helpful in bringing new thinking to the efforts of schools to develop more inclusive ways of working.

All of this means changing how families and communities work and enriching what they offer to children. In this respect, there are many encouraging examples of what can happen when what schools do is aligned in a coherent strategy with the efforts of other local players employers, community groups, universities and public services (Kerr et al. 2014). This does not necessarily mean schools doing more, but it does imply partnerships beyond the school, where partners multiply the impacts of each other's efforts.

With this argument in mind, my Manchester colleagues Alan Dyson and Kirstin Kerr have been exploring the idea of area-based initiatives modelled on the principles underpinning the highly acclaimed Harlem Children's Zone in the USA (Dyson/Kerr 2013). This work is attempting to improve outcomes for children and young people in areas of disadvantage through an approach that they characterize as being "doubly holistic". That is to say, they seek to develop coordinated efforts to tackle the factors that disadvantage some children and enhance the factors which support them, across all aspects of their lives, and across their life spans, from conception through to adulthood.

All of this has implications for the various key stakeholders within education systems. In particular, teachers, especially those in senior positions, have to see themselves as having a wider responsibility for all children, not just those that attend their own schools. They also have to develop patterns of internal organization that enable them to have the flexibility to cooperate with other schools and with stakeholders beyond the school gate. It means, too, that those who administer school systems have to adjust their priorities and ways of working in response to improvement efforts that are led from within schools.

\section{Conclusion}

In summary, then, the ideas that have emerged from this review of international experiences, research and policy documents regarding ways of promoting equity and inclusion are as follows:

- Policies should be based on clear and widely understood definitions of what the terms equity and inclusion mean.

- Strategies should be informed by evidence regarding the impact of current practices on the presence, participation and achievement of all students.

- There should be an emphasis on whole-school approaches in which teachers are supported in developing inclusive practices. 
- Education departments, locally and nationally, must provide leadership in the promotion of equity and inclusion as principles that guide the work of teachers in all schools.

- Policies should draw on the experience and expertise of everybody who has an involvement in the lives of children, including the children themselves.

These ideas indicate that the promotion of inclusion and equity in education is less about the introduction of particular techniques or new organisational arrangements, and much more about processes of social learning within particular contexts. As such, it involves a movement in a clear philosophical direction, requiring a culture of inclusion to permeate the education system.

As I have explained, the use of evidence as a means of stimulating experimentation and collaboration is seen as a central strategy. Copland (2003) suggests that inquiry can be the "engine" to enable the distribution of leadership that is needed in order to foster participation in learning, and the ,glue" that can bind a community together around a common purpose.

All of this has major implications for leadership practice within schools. In particular, it calls for coordinated and sustained efforts around the idea that changing outcomes for vulnerable groups of students is unlikely to be achieved unless there are changes in the behaviours of adults. Consequently, the starting point must be with staff members: in effect, enlarging their capacity to imagine what might be achieved, and increasing their sense of accountability for bringing this about. This may also involve tackling taken-for-granted assumptions, most often relating to expectations about certain groups of students, their capabilities and behaviours.

Such an argument is based on an assumption that schools already have much of the expertise that is needed to support the learning of all of their students. If this is true, the logical starting point for inclusive development within a school is through a detailed analysis of existing practice and with the sharing of expertise amongst staff members. It also requires joint practice development aimed at the development of more inclusive ways of working.

Those involved in taking this challenging agenda for change forward may find it helpful to use the resource pack, "Reaching Out to All Learners", that I developed with colleagues at the International Bureau of Education-UNESCO. ${ }^{3}$ Drawing on international research evidence, these materials are intended to influence and support inclusive thinking and practices at all levels of an education system. Consequently, they are designed to be relevant to teachers, school leaders, district level administrators, teacher educators and national policy makers.

The resource pack is intended to be used flexibly in response to contexts that are at different stages of development and where resources vary. With this in mind, it emphasises active learning processes within which those who use the materials are encouraged to work collaboratively, helping one another to review and develop their thinking and practices. Extensive use is made of examples from different parts of the world to encourage the development of new ways to "reach out to all learners".

3 Available free of charge at: http://www.ibe.unesco.org/en/document/training-tools-curriculum-development-reaching-out-all-learners-resource-pack-supporting (Accessed 22.11.2020). 


\section{References}

Ainscow, Mel (2005): Developing inclusive education systems. What are the levers for change? In: Journal of Educational Change 6, 2, pp. 109-124.

Ainscow, Mel (2007): Taking an inclusive turn. In: Journal of Research in Special Educational Needs 7, 1, pp. 3-7.

Ainscow, Mel (2016a): Struggles for equity in education. The selected works of Mel Ainscow. World Library of Educationalists series. London: Routledge.

Ainscow, Mel (2016b): Collaboration as a strategy for promoting equity in education. Possibilities and barriers. In: Journal of Professional Capital and Community 1, 2, pp.159-172.

Ainscow, Mel/Booth, Tony/Dyson, Alan (2006): Improving Schools, Developing Inclusion. London: Routledge.

Ainscow, Mel/Chapman, Chris/Hadfield, Mark (2020): Changing education systems: A researchbased approach. London: Routledge.

Ainscow, Mel/Dyson, Alan/Goldrick, Sue/West, Mel (2012): Developing equitable education systems. London: Routledge.

Ainscow, Mel/Messiou, Kyriaki (2017): Engaging with the views of students to promote inclusion in education. In: Journal of Educational Change 19, 1, pp. 1-17.

Copland, Michael A. (2003): Leadership of inquiry. Building and sustaining capacity for school improvement. In: Educational Evaluation and Policy Analysis 25, 4, pp. 375-395.

Dyson, Alan/Howes, Andrew/Roberts, Barbara (2004): What do we really know about inclusive schools? A systematic review of the research evidence. In: Mitchell, David (ed.): Special Educational Needs and Inclusive Education. Major Themes in Education. London: Routledge.

Dyson, Alan/Kerr, Kirstin (2013): Developing children's zones for England. What's the evidence? London: Save the Children.

Fulcher, Gillian (1989): Disabling Policies? A Comparative Approach to Education Policy and Disability. London: Falmer.

Fullan, Michael (2007): The New Meaning of Educational Change. New York: Teachers College Press.

Harris, Jess/Carrington, Suzanne/Ainscow, Mel (2017): Promoting equity in schools. Collaboration, inquiry and ethical leadership. London: Routledge

Huberman, Michael (1993): The model of the independent artisan in teachers' professional relationships. In: Little, Judith W./McLaughlin, Milbrey W. (eds.): Teachers' Work. Individuals, Colleagues and Contexts. New York: Teachers College Press.

Kerr, Kirstin/Dyson, Alan/Raffo, Carlo (2014): Education, disadvantage and place. Making the local matter. Bristol: Policy Press.

Lewis, Catherine/Perry, Rebecca/Murata, Ari (2006): How Should Research Contribute to Instructional Improvement? The Case of Lesson Study. In: Educational Researcher 35, 3, pp. 314.

Meijer, Cor J.W. (Ed.) (1999): Financing of Special Needs Education. A seventeen-country Study of the Relationship between Financing of Special Needs Education and Inclusion. Middelfart, DK: European Agency for Development in Special Needs Education.

Miles, Susie (2002): Family action for inclusion in education. Manchester: Enabling Education Network.

Muijs, Daniel/Ainscow, Mel/Chapman, Chris/West, Mel (2011): Collaboration and networking in education. London: Springer. 
Opertti, Renato/Walker, Zachary/Zhang, Yi (2014): Inclusive Education. From targeting groups and schools to achieving quality education as the core of EFA, In: Florian, Lani (ed.): The SAGE Handbook of Special Education. London: SAGE.

Riehl, Carolyn J. (2000): The principal's role in creating inclusive schools for diverse students. A review of normative, empirical, and critical literature on the practice of educational administration. In: Review of Educational Research 70, 1, pp. 55-81.

Senge, Peter M. (1989): The fifth discipline. The art and practice of the learning organisation. London: Century.

Skrtic, Thomas M. (1991): Behind special education. A critical analysis of professional culture and school organization. Denver: Love.

Slee, Roger (2010): The irregular school. Exclusion, schooling and inclusive education. London: Routledge.

UN (2006): Convention on the Rights of Persons with Disabilities. New York: United Nations.

UNESCO (1994): Final Report. World conference on special needs education: Access and quality. Paris: UNESCO.

UNESCO (2015): Incheon Declaration and Framework for Action for the implementation of Sustainable Development Goal 4. Paris: UNESCO.

UNESCO (2017): A guide for ensuring inclusion and equity in education. Paris: UNESCO. 



\title{
5 The Universal Value of Teacher Education for Inclusive Education
}

\author{
Lani Florian
}

\begin{abstract}
While it is axiomatic that contextual differences between educational jurisdictions and regions means that inclusive education will be defined and enacted in distinctive ways in different arenas, the principle of education as a human right, a universal entitlement that belongs to each and every person without discrimination, reflects a universal value that transcends contextual differences. This chapter considers the role that teacher education can play to ensure that teachers in different jurisdictions can meet the challenges of inclusive education despite the distinctive barriers imposed by structural differences between national systems that can obscure commonalities.

Regardless of weather,

The moon shines the same;

It is the drifting clouds

That make it seem different

On different nights

(attributed to Basho, 17th century).
\end{abstract}

\section{Introduction}

Inclusive education is an approach to education for all that focuses on reducing barriers to participation in education for marginalized individuals and groups (UNESCO 2017). It emerged in the Western world in the 1980s in response to concerns about the exclusion of some learners from that which was provided to most others based on education policies that provided different forms of education for different types of learners, notably special education for learners with special educational needs. At the time, a growing awareness of the tautological nature of the concept of 'special needs' along with difficulties in identification of special educational needs and poor outcomes for learners so identified led to a view of special needs education as a discriminatory practice reliant on a process of sorting and separating some learners from that which is available to others (Florian 2014, 2019; Thomas/Loxley 2001; Tomlinson 1982; 2017). As an alternative, inclusive education, based on the idea that local schools should provide for all learners on the grounds that education is a human right that must be provided without discrimination emerged as a strategy to offset what was considered the exclusionary nature of separate forms of provision for some learners (Peters 2007). 
The rights-based anti-discriminatory idea of inclusive education aligned well with $E d u$ cation for All (EFA), the global movement initiated by the international community in 1990 to ensure that the benefits of education are available to everyone. Although the concept of inclusive education had initially emerged in response to the exclusion of learners with special educational needs, this focus broadened over time, as the EFA agenda broadened. Today, the alignment of EFA and inclusive education is expressed clearly by the United Nations Sustainable Development Goal (SDG) 4: Ensure inclusive and equitable quality education and promote lifelong learning for all. As the accompanying World Education Forum vision for Education 2030 states:

Inclusion and equity in and through education is the cornerstone of a transformative education agenda, and we therefore commit to addressing all forms of exclusion and marginalization, disparities and inequalities in access, participation and learning outcomes (UNESCO 2015, iv).

This view reflects the ongoing commitment of the international community to ensure basic education for everyone. However, the significant disparities reflected in global measures of inequality mean that what is needed to achieve this vision will vary. Consequently, it is inevitable that inclusive education will be defined and enacted in distinctive ways in different areas depending on how national systems of schooling are organized and who has access to them (Ainscow/Miles 2008). How can a human right entitlement such as education be provided to everyone without discrimination when education is defined and enacted in distinctive ways in different countries and world regions? This chapter considers this question with a specific focus on the role that teacher education can play to ensure that teachers in different jurisdictions can meet the challenges of inclusive education regardless of the distinctive barriers imposed by structural differences between national systems.

\subsection{Background}

For many years, the contextual differences between various educational jurisdictions (local, national, and regional) led researchers and advocates of inclusion to argue that it could only be understood in the context in which it occurred (Booth/Ainscow 1998). A long period of research provided a rich literature that documented the many different ways in which policies of inclusive education were enacted throughout the world. A recent review (Göransson/Nilholm 2014: 265) identified four distinct understandings of inclusive education: (a) inclusion as the placement of pupils with disabilities in mainstream classrooms, (b) inclusion as meeting the social/academic needs of pupils with disabilities, (c) inclusion as meeting the social/academic needs of all pupils and (d) inclusion as creation of communities.

These understandings reflect two interrelated issues. The first is a legacy issue. That is, the association of inclusive education with special needs education. However, who gets identified as having special educational needs varies within and between countries. Moreover, socio-economic disadvantage and gender have strong associations with the identification of special educational needs. There are also concerns with ethnic disproportionality in special needs statistics that varies across both categories of special need and minority ethnic group. In some countries, the concept of special educational needs extends beyond those who may be included in disability categories to cover those who are failing in school for a variety of reasons. Consequently, the very idea of categorical descriptions of special need has been 
challenged in favour of a broader understanding that anyone may experience difficulties or encounter barriers to learning during the school years. This broader understanding reframed inclusion as an equity issue.

As researchers have explored these different understandings of inclusive education, the idea that the meaning of inclusion would take different forms in various places depending on the situation was increasingly reflected in definitions that emphasized inclusive education as: "a process" (Booth/Ainscow 2002); or, "an approach to education embodying particular values" (Ainscow et al. 2006, p. 5, emphasis added) that is located in a broader policy context of standards-based educational reforms that promote academic achievement and competition between schools and jurisdictions as a measure of effectiveness (Rouse/Florian 1997; Florian/Rouse 2014). Such understandings raise important questions about how teachers can and should be prepared for the demands of inclusive education. These questions are important not only because inclusive education is a rights-based approach, but because it is a complex and sometimes contested concept that is enacted within larger national systems of education that creates multiple and sometimes conflicting demands on teachers in schools that are increasingly diverse (Florian/Pantic 2017).

Today, the rights-based principle of inclusion encapsulated in SDG 4, epitomizes the principle of education as a human right, a universal entitlement that belongs to each and every person without discrimination (ICRC 2015: 11). The UNESCO (2015) Framework for Action on Inclusive and Equitable Education which guides the implementation of SDG 4 with a fifteen-year vision for education is reinforced by the Brussels Declaration, an outcome of the 2018 Global Education Meeting, which defined inclusive education as:

$[\ldots]$ the right to safe, quality education and learning throughout life $[\ldots]$ that requires particular attention be given to those in vulnerable situations, persons with disabilities, indigenous peoples, those in remote rural areas, ethnic minorities, the poor, women and girls, migrants, refugees, and displaced persons whether as a result of conflict or natural disaster.

As can been seen by the range of people and circumstances considered to be vulnerable, inclusive education is about everyone. Accordingly, responding to issues of diversity becomes a central imperative of teaching practice rather than a secondary consideration to be dealt with separately.

Over the past decade, since the 48th International Conference on Education (ICE), Inclusive Education: The Way of the Future (UNESCO 2008: 5), concluded with six recommendations specific to teacher education and development, there has been growing international recognition of the importance of preparing classroom teachers for inclusive education. The six recommendations were to:

(1) Reinforce the role of teachers by working to improve their status and their working conditions, and develop mechanisms for recruiting suitable candidates, and retain qualified teachers who are sensitive to different learning requirements;

(2) Train teachers by equipping them with the appropriate skills and materials to teach diverse student populations and meet the diverse learning needs of different categories of learners through methods such as professional development at the school level, pre-service training about inclusion, and instruction attentive to the development and strengths of the individual learner;

(3) Support the strategic role of tertiary education in the pre-service and professional training of teachers on inclusive education practices through, inter alia, the provision of adequate resources; 
(4) Encourage innovative research in teaching and learning processes related to inclusive education;

(5) Equip school administrators with the skills to respond effectively to the diverse needs of all learners and promote inclusive education in their schools; and

(6) Take into consideration the protection of learners, teachers, and schools in times of conflict.

These recommendations reflect the complex terrain of teacher education. They serve as a reminder that inclusion in education occurs within a broader socio-political context of education that depends on teachers who are adequately prepared to understand and respond to the many different kinds of barriers that learners might encounter during schooling. From an international perspective, the focus on removing barriers is considered to be of particular relevance in creating educational opportunities for all school age children and youth to attend local schools. While these barriers will inevitably reflect differences in context that will be important to address, this chapter argues that these should not obscure important commonalities. For example, there is growing awareness throughout the world that the traditional systemic responses of providing special needs education as a strategy to ensure education for all can be a barrier to participation because it excludes some pupils from the opportunities that are available to others. And although inclusive education challenges the concept of special needs education as "different from" or "additional to" that which is provided for the majority of learners as a discriminatory practice, tensions arise when national policies of inclusive education rely on special needs practices, such as individualized education plans (IEPs) because these practices risk marginalizing students within education systems by focusing attention on their problems (Florian, 2019). Awareness of the structural problems of special education has given way to inclusive education as an alternative but the traditional mechanism for accommodating the increasing diversity of an expanding education system on the grounds that something different (for some learners) to that which is available to others of similar age (most learners) is deeply embedded, as the four meanings of inclusion identified by Göransson and Nilholm (2014) made clear.

\section{The Complex Terrain of Teacher Education for Inclusive Education}

\subsection{Structural Barriers}

Structurally, teacher education programs are often based on ideas of different types of teachers for different groups of learners: for example, "primary", "secondary", or "subject specialists", "general" or "special" education teachers. While these programmes may include courses addressing various aspects of diversity or difference, they are often offered as freestanding courses or options within programmes rather than integrated elements of teacher preparation. Even in countries where there are fewer types of programmes leading to qualified teacher status, specialist "options" are often available to enable teachers to undertake further education or higher degree study in particular areas such as special educational needs. However, Winn and Blanton (2005) have noted that separate teacher education programs 
serve as a barrier to inclusion because they reinforce the idea that different types of courses and qualifications are needed to prepare teachers to teach different types of students. And Young (2008) noted that it perpetuates the problem of teachers feeling inadequately prepared to teach increasingly diverse student groups (Young, 2008). Yet,

Equipping teachers for full range of diversity of students in today's schools has long been a shared concern among teacher educators. Bilingual education, special education, culturally responsive teaching, social justice education, and urban education are among the several responses to this fundamental educational goal. These responses, however, while critical for moving the project of educational equity forward, have tended to produce conversations within diversity communities in teacher education rather than across them (Pugach, et al. 2012: 235).

It is unsurprising that inclusive education, with its origins as a response to what was widely viewed as a key problem associated with special needs education, has predominantly focused on preparing teachers to teach learners with special educational needs, and/or reforms to the preparation of special education teachers. While this focus aligns with the conventional view that specialised knowledge is needed to teach particular groups, Cochran-Smith and DudleyMarling (2012) have suggested that presenting issues of diversity as distinct and separate content marginalises them within teacher education programs. Moreover, a focus on unitary markers of identity such as special educational needs status does little to address the transformation of education, called for by the Education 2030 agenda of inclusion and equity in and through education by addressing all forms of exclusion and marginalization, disparities and inequalities in access, participation and learning outcomes (UNESCO 2015: iv).

In the United States, for example, the emphasis on the different types of teacher education programs with different curricula focused on knowledge and skills unique to underpinning disciplinary perspectives have prompted reform efforts that focus on collaboration between sectors such as collaborative teacher education programs where preservice teachers can obtain qualifications as both general and special education teachers (Blanton/Pugach 2007, 2011, 2012). In other countries, such as those of the United Kingdom where nationally mandated qualifications for teachers of pupils with special educational needs have been eliminated (with the exception of teachers of blind and deaf pupils), in favour of professional development and advanced qualifications for experienced teachers, integrating knowledge about inclusion through additional courses about special educational needs and other options remains problematic. In both situations, limiting content knowledge about the concept of inclusive education to addressing special educational needs runs the risk of reproducing content that focuses on how to accommodate differences between learners rather than on more holistic educational approaches that work for everyone. To date, there has been very little research on whether and how these integrated elements work together in a coherent manner. In which ways does the diversified nature of teacher education affect the capacity of teacher education to prepare teachers to deliver on the promise of inclusive education? How can programmes of teacher education respond to the ongoing concerns of teachers who report that they do not have the knowledge or skills to teach diverse learner groups because they do not have the specialized knowledge that is needed to teach particular groups? The following sections consider some of the key issues that need to be addressed in order to answer these questions as well as some of the efforts that have been made to do so. 


\subsection{Content Knowledge}

Cochran-Smith and Lytle $(1993,1999)$ suggest that the most effective teachers combine practical skills with the ability to understand and use research and other evidence for developing their teaching and university-based providers of teacher education are steeped in this tradition. But what do teachers need to know and be able to do if they are to teach in inclusive schools? The answers to this question reflect the legacy and equity issues discussed above. There are two distinct views about the nature of content knowledge. One holds that additional information about special educational needs and other differences is essential. The other emphasizes critical analysis of the structure of schooling that is thought to produce individual needs (Skrtic 1991; Tomlinson 1992, 2017).

Consequently, some recommend more content knowledge about different types of disabilities and difficulties (e.g. Hodkinson 2005; Jones 2006) be "infused" into existing courses, or "topped up" with specialist courses. These responses are now widespread in many countries. In a cross regional study (Australia, Hong Kong, Canada, and Singapore), Sharma, Forlin, and Lorman (2008) reported that although both add-on courses and infusion models of embedding content knowledge into teacher education courses were effective in promoting positive attitudes about inclusive education, it was the pedagogical approach taken within the model, rather than the model itself, that appeared to have the greatest impact on attitudes. However, there is little evidence that attempts to "infuse" additional knowledge into existing courses, or to "top up" with specialist courses have led to changes in teaching practice. Many teachers still report feeling unprepared for inclusive education.

Others (e.g. Slee 2007) have argued that special education content knowledge is not adequate to improve inclusive practice in schools because it is not sufficiently linked to the broader pedagogical and curriculum imperatives that trainee teachers have to learn and be able to apply. It is thought that the additional special education knowledge that is added-on to the teacher education course may not be sufficient for student teachers to act upon when teaching. In the 1990s, the UNESCO Special Needs in the Classroom project was introduced to an international audience of practicing special and regular education teachers (Ainscow 1994). Rather than a focus on disability categories per se, this project emphasized removing the barriers to learning and participation as they are encountered by learners. Informed by a social model of disability, this project followed an approach to preparing teachers, which does not depend on the identification of particular forms of disability or difficulty (Allan 2006; Booth et al. 2003; Gabel 2005). However, this approach has also been criticized insofar as some knowledge about human differences is important - a student who is an English language learner is different from a student with Down Syndrome, and so forth. However, in a study of teacher development for inclusive education in the Western Balkans (Albania, Bosnia and Herzegovina, Croatia, the former Yugoslav Republic of Macedonia, Kosovo, Montenegro, and Serbia), Pantić, Closs and Ivošević (2011) found narrow conceptualizations of inclusive education focused only on children with disabilities to be a barrier to social and educational inclusion.

While there is considerable consensus that teachers need to be well prepared to work with diverse student groups and broad acknowledgement that teachers are not adequately prepared, the different perspectives on which contents should inform teacher education and professional development suggests a fragmented and partial literature that reflects ongoing debates about the concept of inclusive education rather than a solid knowledge base. Blanton and Pugach (2012) undertook a content analysis of a sample of collaborative programmes of 
teacher education in the US but found that although the programmes espoused a commitment to the values of inclusion, the content was generally "additive" in that special education content knowledge was added to programmes as opposed to programmes reflecting content that was transformational in rethinking issues of diversity and the responses to it. A recent study in Ireland (Hick et al. 2018) reported a similar finding.

\subsection{Teacher Education Practice}

Thus, despite various efforts that have been made to address issues of diversity and difference in teacher education in diverse contexts, it is important to note that the knowledge base about these issues remains fragmented. As Cochran-Smith and her colleagues (2015) have noted, one of the biggest gaps in evidence about how successful different teacher education programs are in preparing students for dealing with increasing diversity of school populations is the impact on student teachers' practices. This is in part because research on teacher education has not had the level of sustained investment that has enabled other areas of educational research to establish the kind of robust knowledge that could confidently answer key questions about what teachers need to believe, know and do to support inclusive and equitable education for everyone.

Nevertheless, there are some strong arguments in favour of Slee's (2007) argument that special education content knowledge is not adequate to improve inclusive practice in schools because it is insufficiently linked to the broader pedagogical and curriculum imperatives that trainee teachers have to learn and be able to apply. A growing awareness of a gap between what teachers know as a result of their courses, and what they do in their classrooms, clearly suggests that new approaches to teaching about how to effectively and equitably respond to human difference and diversity are needed. To this end, there have been some cross-national and regional efforts to develop teacher competences for inclusive education, and a number of research and development projects (e.g., Allan 2011; Deppeler 2017; EADSNE 2012; Oyler 2006; Rouse/Florian 2012) have moved in this direction. Florian and Pantić (2017: 3) synthesized these projects within the broader literature about the knowledge, skills and values teachers need to be effective with diverse groups of students, as encapsulating:

- integration of theoretical and practical knowledge and skills;

- building relationships for improved learning outcomes;

- being able to develop a pedagogy that is inclusive of all;

- collaborative skills and attitudes;

- recognizing the importance of home environment and working with diverse families;

- broader understanding of educational change and how it affects the conditions for learning in contexts of exclusion and disadvantage; and

- capacity for reflection and inquiry accounting for moral values and commitment to education for all.

Inclusive education requires that all teachers should be prepared in ways that support a view of difference as to be expected within the remit of classroom teaching. Such a view is predicated on the premise that there is an inherent bias in education systems that are designed for most students, on the grounds that something different can be provided to some as a means of ensuing access for all (e.g. Fendler/Muzaffar 2008; Hart 1998). The idea that all learners 
are unique individuals and differences are to be expected as an ordinary aspect of human development is an essential first step in breaking down the barriers erected following the idea that different types of teachers are needed to teach different types of learners.

\subsection{The Diversity Construct}

Menter (2017) maintains that diversity of the teaching workforce links to how issues of learner diversity are understood. He noted that while the range of people entering the teaching workforce should be representative of the social spectrum of the population, in many jurisdictions this is not the case. Teachers tend to be trained locally for jobs in their home countries where teaching qualifications are regulated within national systems of education. However, in many jurisdictions, increasing mobility and migration mean that national populations are more diverse than ever before. This has led to a "diversity gap" between the demographics of the teaching workforce and student populations. This gap reflects differences in languages spoken, socio-economic status, religion, prior cultural experiences and so forth, leading to misunderstandings that can create disadvantages affecting teaching and learning. This is commonly acknowledged in the idea that teachers need to be prepared to meet the challenges of "diversity". However, this idea positions diversity as a problem to be addressed rather than as a fact of demographic change (Florian 2017).

While there have been attempts in some counties to diversify routes into teaching, these efforts may or may not be aimed at diversifying the teaching workforce. Diversification serves many agendas, such as increasing the number of qualified candidates selecting teaching as a career option (Murray/Mutton 2016). Reforms that aim to address problems of teacher shortage rather than diversification of the workforce may or may not be helpful to reach this goal.

\subsection{Global Disparities}

The UNESCO Global Education Monitoring (GEM) Reports, together with the international literature, provide an overview of many global disparities in educational provision and differences in approaches to teacher education and teacher qualifications in different regions of the world.

As a consequence, issues of teacher preparation and development as part of the Education 2030 agenda must attend to more than noting disparities in teacher quality, qualifications, supply, and deployment. Projects that examine teachers' work, working conditions and career pathways are also needed (e.g. Robertson/Sorenson 2017). The challenge for teacher education is to prepare teachers who can respond when students encounter barriers to learning because in all countries there are students who are identified as having some kind of special or additional support needs. Consequently, there is much to be learned from experiences elsewhere. Comparative work on teacher education can help to answer questions about whether legacy or equity approaches to equipping teachers with the knowledge and skills to teach diverse learner groups are most effective under different conditions. 
The Global Education Monitoring Report (GMR) provides an annual summary on progress towards the education targets in the United Nations Sustainable Development Goals (SDGs). The concept note for the forthcoming 2020 Global Education Monitoring Report on Inclusion (UNESCO 2018) reflected international concern about the preparedness of teachers, school leaders and education support personnel to ensure that they are prepared to accommodate students of all abilities and backgrounds.

However, in countries where the teachers themselves are products of poor education systems and their professional training is meager, improving teacher education is a tall order. Global disparities in teacher qualifications and teacher education that exist in different parts of the world are compounded by other differences that reflect regional and national conditions.

\subsubsection{Regional Differences}

Pantić, Closs and Ivošević (2011) identified systemic barriers in the education system, unconnected professional education for different levels of school staff, lack of competences for inclusive education, and concern that faculty-based teacher educators lack the knowledge needed to develop effective pre-service programs as challenges for the development of teacher education for inclusion in the Western Balkans. Walton and Rusznyak (2019a: 103) note that standards for teacher education in South Africa "reflect a codified but abstracted vision of inclusive teaching" that is shown in substantial variation across university providers of teacher education in South Africa. This concurs with research in Europe (EADSNE 2011) that has documented a wide range of initial teacher education courses of varying lengths and contents with fewer than one in ten European countries offering a specialization in special education during initial teacher education. Where this qualification does exist, it is generally offered at the post-graduate level for experienced teachers. Where teacher education is not a university-based profession, or where the demand for teachers far outstrips supply, countries are forced to rely on unqualified or under-qualified teachers. In such cases, non-governmental organisations play an important role in providing professional development opportunities to teachers.

Clearly these issues are of broad relevance across regions and the regional and intraregional responses that are developed will reflect cultural and socioeconomic differences as well as differences in national teaching qualifications and preparation of teachers. In some countries teaching is a high-status profession with good salaries; in others, teaching is undertaken in extremely difficult circumstances and salaries are barely enough to subsist. Yet despite huge variations, there is considerable consistency in terms of the issues identified.

\subsubsection{National Differences}

In recent years, a growing number of teacher educators around the world have begun to describe some initiatives and articulate their ideas about how teacher education for inclusion might progress. McIntyre (2009) cautioned that the discontinuity between what pre-service teachers learn in university and what they learn in school, especially in contexts where practice is not well established could create barriers to preparing teachers for inclusion. He suggested that teacher educators work collaboratively with colleagues in schools if they aim to do more than promote a theoretical idea. To this end, some promising examples have been 
described in the literature. For example, in Iceland, research has focused on university-school partnerships in teacher education and professional learning and how these partnerships might develop to support inclusive teacher education (Sigurdardóttir 2010). A paper from Cyprus (Symeonidou/Phtiaka 2009) describes how practicing teachers' prior knowledge could be used to develop relevant university courses for teachers interested in developing inclusive practice. Recently, Walton and Rusznyak (2019b) proposed a framework for bridging coursework and practicum experiences as a way to strengthen the synergies between them.

Within countries, a range of approaches have been described. For example, Bartolo (2010) explained how a rights-based approach to initial teacher education in Malta in which courses formerly focused on "handicaps in learning" were replaced with those that focus on removing barriers to learning and responding to student diversity. In the United States, efforts to move teacher education towards a more unified or holistic approach to preparing all teachers has focused on the development of collaborative approaches that increase the special education knowledge of general education teachers and ensure that special education teachers have sufficient background in academic content knowledge (Pugach/Blanton 2009).

However, studies of the impact of national changes to initial teacher education for inclusion suggest that these models have yet to be embedded systematically despite policy reforms of teacher education to ensure that teachers are prepared and supported to meet the goals of inclusive education. For example, in Ireland, a study (Hick et al. 2018) found that providers implemented reforms in different ways across universities. Another recent report from Iceland (Guðjónsdóttir/Óskarsdóttir 2019) found different views on how to prepare teachers for inclusive education within universities.

Clearly, understanding how well teachers are prepared for inclusive education needs to take account of regional and national disparities in teacher qualifications, supply and deployment, and broader issues regarding the quality and content of teacher preparation. But a knowledge base for teacher education for inclusion also needs to be established (EADSNE 2012; Florian/Young/Rouse 2010). While the form and structure of teacher education varies, the common issues and challenges of meeting SDG 4 create an agenda for teacher development at all levels. Teacher education offers a good case to consider how surface issues can be different but underpinning similar values.

\section{Values-based Approaches Offer a Point of Convergence}

While it is reasonable to expect that there will be differences that reflect particular situational and contextual aspects of vulnerability for marginalised groups, the values-based notion of inclusive education offers a point of convergence whereby it is possible that practices that may appear different can be constitutive of inclusion and some practices thought to support one group also benefit others, whether expected or not. Teacher education for inclusive education is a complex task located within persistent questions about how diverse groups of learners can be supported, and strong views about the particular needs of different vulnerable groups with disagreements about how to respond in terms of both the form and content of provision. In addition, the entrenched idea that specialist interventions are needed to offset disadvantage - the positioning of difference as a problem - reinforces the idea that teachers are not equipped to teach all students because separate teacher education programs reinforce implicit assumptions about what teachers need to know to teach different groups of students. 
A response to this problem was developed by Scotland's Inclusive Practice Project (IPP), a research and development project funded by the Scottish Government between 2006 and 2010, to develop an approach to initial teacher education that would ensure that all teachers had greater awareness, understanding and skill in responding to the many problems that can affect children's learning (Rouse/Florian 2012). In order to ensure that the approach was relevant to all university-based providers of initial teacher education, the Scottish Council of Deans, set up a working group consisting of teacher education course directors and inclusion specialists from each university to develop collaboratively a Framework to guide the development of practice (Barrett et al. 2015). The remit of the group was to identify: the values and beliefs; the professional knowledge and understanding; and the skills and abilities expected of student teachers and of qualified teachers throughout their careers. In other words, to specify the habits of the head, the hand, and the heart that constitute professional learning (Shulman 2007).

The resulting Framework for Inclusion built upon the curricular approach to teacher education developed by the IPP based on the concept of inclusive pedagogy, an approach to teaching diverse groups of learners that was derived from studying the practices of expert teachers who have been able to be inclusive of a wide range of pupils in their classrooms and get good attainment results for everyone (Black-Hawkins et al. 2007; Florian/Black-Hawkins 2011). The principles underpinning this approach assume that:

- Teachers view differences between learners as an ordinary aspect of human development;

- they view difficulties in learning as dilemmas for teaching rather than problems of learners; and

- they actively seek support to ensure that individual needs are catered for in ways that do not marginalize learners or exclude them form the opportunities that are available to others.

In other words, as teachers they value the dignity of each and every learner. They value their professional role in ensuring that learners are treated fairly, without discrimination; and they value the opportunity to collaborate with others to ensure that everyone is able to participate and within the community of the classroom. This includes other professionals, families and learners themselves. It often finds expression in the activities that teachers engage with outside of the classroom, for example, through professional development or extra-curricular activities.

The Scottish working group used the General Teaching Council Scotland (GTCS) Standards as a context for exploring the implications for inclusion as an aspect of teacher education. Working through a process of building consensus, they identified the Standards they considered most relevant for developing the habits of the head, hand and heart linked to the core values of inclusive pedagogy. The group then generated a series of questions intended to assist student teachers, teachers and teacher educators to examine the implications of the Standards for the development of inclusive practice. In this way, the Framework for Inclusion provided a map that linked core values associated with inclusive practice to the national teaching standards. The questions enabled each program team to generate distinctive answers that were not prescriptive of practice. As an approach to developing teaching competencies, the Framework proved useful because the values that underpin it are shared. Subsequently, the principles of inclusive pedagogy were elaborated to include the possible ways in which it might be expected to manifest in practice (Florian/Spratt 2013; Spratt/Florian 2015). Determining if, when and how student teachers enact the values underpinning an inclusive pedagogical approach as promoted by their programs requires evidence of the enactment of 
those values in the practices of teachers, knowing that practice will always vary according to the school context and the individuality of the learners in each class. Similar responses to individual differences between learners in a classroom may or may not be inclusive depending on the context of teachers' actions, their planning, their reasoning or the history behind a particular interaction. Rather than comparing the behavior of teachers in different contexts, it is important to look for evidence that practice aligns with the principles and values of inclusion. In this way it is possible to make meaningful comparisons about practice despite inevitable variation in context. Inequalities and discrepancies associated with context are important, but they can obscure rather than drive the development of practice.

\section{Conclusion}

This chapter traced the origins of inclusive education and its development as a key component of SDG4. It reviewed how issues of diversity and difference have long been, and in many contexts still are, considered specialist knowledge with the task of preparing teachers for inclusive education predominately undertaken by special educators within university departments of special education. Consequently, its reach has been confined mainly to special education teachers. However, a growing awareness of the increasing diversity of school populations throughout the world has prompted calls for all teachers to be prepared for inclusive education. These calls are reflected in UNESCO (2017) policy guidance on inclusive education and in recommendations of supranational bodies such as the European Commission (2018). In answering this call, it is important to take stock of current knowledge and efforts that address the preparation of teachers. The four distinct understandings of inclusive education identified by Göransson and Nilholm's (2014) review of inclusion as: the placement of pupils with disabilities in mainstream classrooms; meeting the social/academic needs of pupils with disabilities; meeting the social/academic needs of all pupils; and creation of communities all have implications for teacher education and these were discussed in terms of legacy and equity issues that have address longstanding and current concerns of the field.

The chapter argued that preparing teachers for inclusive education in different parts of the world requires consensus about the values that can guide the development of practice. Such a consensus would provide a framework within which comparative work that tests the efficacy of the different approaches to teacher education discussed in this chapter can be undertaken. This work is essential to meet the international commitments to achieving SDG4 and ensuring that variation in the form and structure of teacher education within and between countries does not become an impediment to preparing teachers to implement a principled approach to inclusive education. Addressing inequalities and discrepancies in context is important but a principled approach is needed if the barriers to opportunity that exist in a globalized world where there is so much variation in context are to be overcome. A principled approach allows for variation in context by specifying the knowledge, skills and values of inclusive education understood as a human right. A project in Scotland provided a model of how a national approach enabled providers to develop distinctive programmes based on an agreement about foundational knowledge, skills and values. By demonstrating how providers worked collaboratively to embed foundational knowledge, skills and values into distinctive programmes of teacher education, the chapter suggests that agreement about universal values 
can play an important role in transcending contextual differences (e.g. EADSNE 2011, 2012; Oyler 2006).

However, this is not to say that variation in content and quality of teacher education across the developed and developing world is unimportant. Variation challenges the creation of a universal approach because there are discrepancies in how inclusive education is enacted in different regions. The 2020 Global Monitoring Report (UNESCO 2020) encapsulates these challenges by documenting multiple discrepancies that (re)produce inequalities. While the form, content and balance of teacher education and professional development courses are developed in response to directives from national governments or accreditation and professional registration agencies, there are many differences of opinion about the nature of the content knowledge student teachers should learn, as well as what and how they should learn about diversity and difference - and adequate responses to those differences. The Education 2030 agenda requires that all jurisdictions reconsider how teachers can be prepared to work in schools where differences between learners are to be expected, and new ways of thinking about diversity are needed.

While considerations about what inclusive education means in different regions as well as the implications for preparing teachers in different parts of the world matter, variation in the form and structure of teacher education within and between countries needs not be an impediment to supporting all teachers to implement a principled approach to inclusive education. The examples presented in this chapter illustrate how it is possible to allow for variation in context by specifying the knowledge, skills and values of inclusive education. In countries where specialist pedagogues or special education teachers are part of the general education system, they should be prepared to work in support of efforts to ensure that students who are experiencing difficulties are meaningfully engaged in classroom activities. In countries where specialist training does not exist, the challenge is to ensure that children with disabilities and other difficulties are not excluded by a culture of silence about their learning needs. Bringing about this culture shift in thinking about teaching all students is necessary work for those who prepare teachers.

\section{References}

Ainscow, Mel (1994): Special Needs in the Classroom: A teacher education guide. Paris: United Nations Educational, Scientific, and Cultural Organization (UNESCO).

Ainscow, Mel (2015): Toward self-improving school systems: Lessons from a city challenge. London: Routledge.

Ainscow, Mel/Booth, Tony/Dyson, Alan (2006): Improving schools, developing inclusion. London: Routledge.

Ainscow, Mel/Miles, Susie (2008): Making Education for All inclusive: Where next? Prospects 37, 1, pp. 15-34.

Allan, Julie (2011): Responsibly Competent: Teaching, Ethics and Diversity. In: Policy Futures in Education 9, 1, pp. 130-137.

Barrett, Louise/Beaton, Mhairi/Head, George/McAuliffe, Lisa/Moscardini, Lio/Spratt, Jennifer/ Sutherland, Margaret (2015): Developing inclusive practice in Scotland: the National Framework for Inclusion. In: Pastoral Care in Education 33, 3, pp. 180-187. 
Bartolo, Paul A. (2010): The "Process" of Teacher Education for Inclusion: The Maltese Experience. In: Journal of Research in Special Educational Needs 10, 1, pp. 139-148.

Black-Hawkins, Kristine/Florian, Lani/Rouse, Martyn (2007): Achievement and Inclusion in Schools. London: Routledge Falmer.

Blanton, Linda P./Pugach, Marlene C. (2007): Collaborative programs in general and special teacher education: An action guide for higher education and state policymakers. Washington, DC: Council of Chief State School Officers. Retrieved from http://www.aacte.org/index.php?/Traditional-Media/Resources/ [Accessed: 15.09.2020].

Blanton, Linda P./Pugach, Marlene C. (2011): Using a classification system to probe the meaning of dual licensure in general and special education. In: Teacher Education and Special Education 34, 3, pp. 219-234.

Booth, Tony/Ainscow, Mel (1998): From them to us: An international study of inclusion in education. London: Routledge.

Booth, Tony/Ainscow, Mel (2002): The Index for Inclusion: Developing Learning and Participation in Schools. Bristol: CSIE.

Booth, Tony/Nes, Kari/Strømstad, Marit (2003): Developing Inclusive Teacher Education. London: Routledge.

Cochran-Smith, Marilyn/Dudley-Marling, Curt (2012): Diversity in Teacher Education and Special Education: The Issues That Divide. In: Journal of Teacher Education 63, 4, pp. 237-244.

Cochran-Smith, Marilyn/Lytle, Susan L. (1993): Inside/Outside Teacher Research and Knowledge. New York: Teachers College Press.

Cochran-Smith, Marilyn/Villegas, Ana M./Abrams, Linda/Chavez-Moreno, Laura/Mills, Tammy/ Stern, Rebecca (2015): Critiquing Teacher Preparation Research: An overview of the field, Part II. In: Journal of Teacher Education 66, 2, pp. 109-121.

Deppeler, Joanne (2017): Navigating the Boundaries of Difference: Using Collaboration in Inquiry to Develop Teaching and Progress Student Learning. In: Florian, L./Pantić, N. (eds.): Teacher education for the changing demographics of schooling. Dordrecht: Springer, pp. 168187.

Englebrecht, Petra. (ed.) (2013): Teacher education for inclusion: International Perspectives. In: European Journal of Special Needs Education 28, 2, pp. 115-118.

EADSNE (2011): Teacher education for inclusion across Europe: A synthesis of policy and practice in 25 countries. European Agency for Development in Special Needs Education. Denmark: Odense.

EADSNE (2012): Teacher Education for Inclusion. Profile of Inclusive Teachers. European Agency for Development in Special Needs Education. Denmark: Odense. https://www.european-agency.org/projects/te4i/profile-inclusive-teachers [Accessed: 15.09.2020].

European Commission (2018): Council Recommendation on promoting common values, inclusive education, and the European dimension of teaching. https://ec.europa.eu/transparency/regdoc/rep/1/2018/EN/COM-2018-23-F1-EN-MAIN-PART-1.PDF [Accessed: 31 October 2019].

Fendler, Lynn/Muzaffar, Irfan (2008): The history of the bell curve: sorting and the idea of normal. In: Educational Theory 58, 1, pp. 63-82.

Florian, Lani (2014): Reimagining special education: Why new approaches are needed. In: Florian, Lani (ed.): The Sage Handbook of Special Education, second edition. London: Sage, pp. 9-22.

Florian, Lani (2017): Teacher Education for the Changing Demographics of Schooling: Inclusive Education for Each and Every Learner. In: Florian, Lani/Pantić, Natasha (eds.): Teacher education for the changing demographics of schooling. Dordrecht: Springer, pp. 9-20. 
Florian, Lani (2019): On the necessary co-existence of special and inclusive education. In: International Journal of Inclusive Education 23, 7-8, pp. 691-704. DOI: 10.1080/13603116.2019. 1622801

Florian, Lani/Black-Hawkins, Kristine/Rouse, Martyn (2017): Achievement and Inclusion in Schools, second edition. London: Routledge.

Florian, Lani/Pantić, Nataša (eds.) (2017): Teacher Education for the Changing Demographics of Schooling. Dordrecht: Springer.

Florian, Lani/Rouse, Martyn (2014): International perspectives on effective inclusive schools: What can be known about effective inclusive schools. In: McLeskey, James/Waldron, N. L./Spooner, F./Algozzine, B. (eds.): Handbook of Research and Practice for Effective Inclusive Schools. New York: Routledge, pp. 507-520.

Florian, Lani/Spratt, Jennifer (2013): Enacting inclusion: A framework for interrogating inclusive practice. In: European Journal of Special Needs Education 28, 2, pp. 117-135.

Florian, Lani/Young, Kathryn/Rouse, Martyn (2010): Preparing Teachers for Inclusive and Diverse Educational Environments: Studying Curricular Reform in an Initial Teacher Education Course. In: International Journal of Inclusive Education 14, 7, pp. 709-722.

Gabel, Susan (ed.) (2005): Disability studies in education: Readings in theory and method. New York: Peter Lang.

Göransson, Kerstin/Nilholm, Claes (2014): Conceptual diversities and empirical shortcomings a critical analysis of research on inclusive education. In: European Journal of Special Needs Education 29, 3, pp. 265-280.

Guðjónsdóttir, Hafdis/Óskarsdóttir, Edda (2019): Dealing with diversity: Debating the focus of teacher education for inclusion. In: European Journal of Teacher Education 43, 1, pp. 95-109.

Hart, Susan (1998): A sorry tail: Ability, pedagogy and educational reform. In: British Journal of Educational Studies 46, 12, pp. 153-168.

Hick, Peter/Solomon, Yvette/Mintz, Joseph/Matziari, Aikaterini/Finn, Ó Murchú/Hall, Kathy/Cahill Kevin/Curtin, Catriona/Margariti, Despoina (2018): Initial Teacher Education for Inclusion Phase 1 and 2 Report: NCSE Research Report No. 26. Meath Ireland: National Council for Special Education, Ireland.

Hodkinson, Alan J. (2005): Conceptions and misconceptions of inclusive education: A critical examination of final year teacher trainees' knowledge and understanding of inclusion. In: International Journal of Research in Education 73, 1, pp. 15-28.

ICRC (2015): International rules and standards for policing. Geneva: International Committee of the Red Cross.

Jones, Phyllis (2006): They are not like us and neither should they be: Issues of teacher identity for teachers of pupils with profound and multiple learning difficulties. In: Disability \& Society 19, 2, pp. 159-169.

McIntyre, Donald (2009): The difficulties of inclusive pedagogy for initial teacher education and some thoughts on the way forward. In: Teaching and Teacher Education 25, 4, pp. 602-608.

Menter, I. (2017): Diversity, development, devolution: The 3 D's of UK teacher education and professional development in the $21^{\text {st }}$ century. In: Florian, L./Pantić, N. (eds.): Teacher education for the changing demographics of schooling. Dordrecht: Springer, pp. $42-59$.

Murray, Jean/Mutton, Trevor (2016): Teacher education in England: change in abundance, continuities in question. In: Teacher Education Group (eds.): Teacher Education in Times of Change. Bristol: Policy Press, pp. 57-74.

Oyler, C. (2006): Learning to teach inclusively. Mahwah, NJ: Lawrence Erlbaum Associates.

Pantić, Nataša/Closs, Alison/Ivošević, Vesna (2011): Teachers for the future: Teacher development for inclusive education in the Western Balkans. Turin: European Training Foundation.

Peters, Susan (2007): Inclusion as a strategy for achieving education for all. In: Florian, Lani (ed.): The Sage Handbook of Special Educational Needs. London: Sage, pp. 117-130. 
Pugach, Marlene/Blanton, Linda (2009): A framework for conducting research on collaborative teacher education. In: Teaching and Teacher Education 25, 4, pp. 575-582.

Pugach, Marlene C./Blanton, Linda P./Florian, Lani (2012): Unsettling conversations: Diversity and Disability in Teacher Education. In: The Journal of Teacher Education 63, 4, pp. 235-236.

Robertson, Susan L./Sorensen, Tore B. (2017): Global transformations of the state, governance and teachers' labour: Putting Bernstein's conceptual grammar to work. In: European Educational Research Journal 14, 4, pp. 470-488.

Rouse, Martyn/Florian, Lani (1997): Inclusive education in the marketplace. In: International Journal of Inclusive Education 1, 4, pp. 323-336.

Rouse, Martyn/Florian, Lani (2012): Inclusive Practice Project: Final Report. Aberdeen: University of Aberdeen. https://www.academia.edu/4432038/The_Inclusive_Practice_Project_Final_Report [Accessed: 15.09.2020]

Sharma, Umesh/Forlin, Chris/Loreman, Tim (2008): Impact of training on pre-service teachers' attitudes and concerns about inclusive education and sentiments about persons with disabilities. In: Disability \& Society 23, 7, pp. 773-785.

Shulman, Lee (2007): Keynote lecture to American Association of Colleges for Teacher Education annual conference. New Orleans, February 2007.

Skrtic, Thomas M. (1991): Behind special education: A critical analysis of professional culture and school organization. Denver. CO: Love Publishing.

Sigurdardóttir, Anna Kristín (2010): School-university partnership in teacher education for inclusive education. In: Journal of Research in Special Educational Needs 10, 1, pp. 149-156.

Spratt, Jennifer/Florian, Lani (2015): Respecting the dignity of the individual child in the learning community of the classroom: From learning about inclusive pedagogy to action. In: Teaching and Teacher Education 49, pp. 89-96.

Symeonidou, Simoni/Phtiaka, Helen (2009): Using teachers' prior knowledge, attitudes and beliefs to develop in-service teacher education courses for inclusion. In: Teaching and Teacher Education 25, 4, pp. 543-550.

Slee, Roger (2007): Inclusive schooling as a means and end of education? In: Florian, L. (ed.): The Sage Handbook of Special Education. London: Sage Publications, pp. 160-174.

Thomas, Gary/Loxley, Andrew (2001): Deconstructing special education and constructing inclusion. Buckingham: Open University Press.

Tomlinson, Sally (1982): A sociology of special education. London: Routledge \& Kegan Paul.

Tomlinson, Sally (2017): A sociology of special and inclusive education: Exploring the manufacture of inability. London: Routledge.

UNESCO (2008): Conclusions and Recommendations of the 48th Session of the ICE. http:// www.ibe.unesco.org/fileadmin/user_upload/Policy_Dialogue/48th_ICE/CONFINTED_485_Conclusions_english.pdf [Accessed: 25 October 2016].

UNESCO (2017): A guide for ensuring inclusion and equity in education. Paris. https://unesdoc.unesco.org/ark:/48223/pf0000248254 [Accessed: 15.09.2020].

UNESCO (2018a): Global Education Meeting, Brussels Declaration, document ED-2018/GEM/1. Paris. https://unesdoc.unesco.org/ark:/48223/pf0000366394?posInSet=1\&queryId=f00bbeb5caf0-495d-9782-e4caad1e9e0f [Accessed: 10 April 2019].

UNESCO (2018b): Concept note for the 2020 Global Education Monitoring Report on Inclusion ED/GEM/MRT/2018/CN/2020. Paris. https://unesdoc.unesco.org/ark:/48223/pf0000265329/ PDF/265329eng.pdf.multi, [Accessed: 11 November 2019].

UNESCO (2020): Global Monitoring Report on Inclusive Education. Paris.

Walton, Elizabeth/Rusznyak, Lee (2019a): Developing standards for inclusive teaching in South Africa: A dilemma analysis. In: Southern Africa Review of Education 25, 1, pp. 89-106. 
Walton, Elizabeth/Rusznyak, Lee (2019b): Cumulative knowledge building for inclusive education in initial teacher education. In: European Journal of Teacher Education, online first. doi: 10.1080/02619768.2019.1686480

Winn, Judith/Blanton, Linda P. (2005): The call for collaboration in teacher education. In: Focus on Exceptional Children 38, 2, pp. 1-10.

Young, Kathryn (2008): “I don't think I'm the right person for that": theoretical and institutional questions about a combined credential program. In: Disability Studies Quarterly 28, 4, pp. 116. doi: $10.18061 /$ dsq.v28i4 



\title{
6 Weltbank: Von ersten Auseinandersetzungen mit Behinderung hin zu inklusiven Perspektiven
}

\author{
Raphael Zahnd
}

\begin{abstract}
Ausgehend von einer Verortung im Rahmen von Weltgesellschaftstheorien zeigt dieser Beitrag auf, wie Behinderung innerhalb der Weltbank in unterschiedlichen politischen, ökonomischen und gesellschaftlichen Zusammenhängen diskursiv konstruiert wird. Anhand der drei dominantesten diskursiven Muster, die sich in den Dokumenten des Weltbankarchivs abbilden, wird im Sinne einer Gesamtperspektive aufgezeigt, wie sich die Bearbeitung des Themas Behinderung in der Weltbank über die Zeit verändert.
\end{abstract}

\section{Einleitung}

Wenn es um Fragen der internationalen Entwicklungspolitik geht, gehört die Weltbank zu denjenigen Organisationen, die man im Blick haben sollte. Sie ist weltweit einer der dominantesten Akteure in diesem Feld und verfügt über einen umfangreichen Forschungsapparat. Mit Blick auf die globalen Disparitäten verfolgt die Weltbank ein redistributives Programm, ihr Mandat besteht vereinfacht ausgedrückt darin, ,gestützt auf Ressourcen aus den entwickelten Staaten aus dem Norden, die Entwicklung der weniger entwickelten Staaten vor allem aus dem Süden zu unterstützen" (Rittberger et al. 2013: 206). Vor dem Hintergrund dieses Mandats richtet sie ihre entwicklungspolitische Aufmerksamkeit bereits seit längerem auf den globalen Süden und damit auch auf denjenigen Teil der Welt, in dem gemäß Schätzungen der Weltgesundheitsorganisation (2018) ca. 80\% aller Menschen mit Behinderung leben. Diese Ausgangslage macht die Untersuchung der Mechanismen und Diskurse der Weltbank bedeutsam für internationale Fragestellungen im Kontext von Inklusion.

Inwiefern das Handeln der Weltbank zum Abbau globaler Entwicklungsdisparitäten beiträgt, lässt sich auf einer gesicherten empirischen Basis bisher nicht abschließend beurteilen und die Organisation steht aufgrund ihres diesbezüglichen Vorgehens aus unterschiedlichsten Gründen in der Kritik (vgl. Rittberger et al. 2013: 213). Die Kritik bezieht sich zuweilen auch auf den Umgang mit dem Thema Behinderung. So wurde bspw. bemängelt, dass sich die Weltbank sachlich nicht angemessen mit Behinderung auseinandersetze, das Thema zu wenig berücksichtige oder Menschen mit Behinderung nicht in Entscheidungsprozesse miteinbeziehe (vgl. Barnes/Sheldon 2010; Grech 2009; Metts 2000; Soldatic 2013; Stienstra 2002). Diese Kritik ist vor dem Hintergrund der obengenannten Schätzung der Weltgesundheitsorganisation hochrelevant, denn sie verweist darauf, dass das Thema Behinderung genau dort zu wenig Beachtung erfährt, wo die meisten Menschen mit Behinderung leben. Als Ergänzung zu diesen Kritikpunkten, die in der Regel mit Blick auf spezifische Maßnahmen 
oder Dokumente der Weltbank geäußert wurden, ist darauf hinzuweisen, dass sich über die Zeit deutliche Veränderung bezüglich der Bearbeitung des Themas Behinderung erkennen lassen (vgl. Zahnd 2017). Während erste Auseinandersetzungen aus einer medizinischen Perspektive erfolgten und eine deutliche Abwertung von Menschen mit Behinderung beinhalteten, finden sich ab den 1990er Jahren auch erste Zugänge auf Ebene des sozialen Modells der Behinderung (vgl. Köbsell/Hirschberg 2021 i.d.B.), die im weiteren Verlauf an Relevanz gewinnen. Der Wandel ist dabei vergleichbar mit dem von Degener (2005: 889) beschriebenen Umdenken in (europäischen) Nationalstaaten, das ca. ab den 1990er Jahren in einem breiteren Ausmaß beobachtbar ist: „Statt Behinderung als individuelles Problem zu verstehen, wurde mit dem sozialen Modell von Behinderung der Blick auf die Umwelt und die Gesellschaft und deren Umgang mit Differenz gerichtet.“ Dieser Veränderungsprozess wird, mit allen seinen Facetten, auch in den Positionen der Weltbank ersichtlich. Die Einführung des sozialen Modells ist allerdings nicht gleichzusetzen mit der Ablösung des medizinischen Modells bzw. Behinderungsverständnisses, denn die unterschiedlichen Perspektiven auf Behinderung bleiben (nicht nur) in der Weltbank langfristig erhalten (vgl. Zahnd 2017).

Dieser Handbuchbeitrag greift die Veränderungen in Bezug auf die Bearbeitung des Themas Behinderung auf und hat zum Ziel, einen allgemeinen Überblick über die diskursiven Konstruktionen von Behinderung und die behinderungsbezogenen Handlungsfelder der Weltbank zu geben. Aus theoretischer Perspektive wird die Weltbank in den nachfolgenden Ausführungen nicht als monolithische Organisation, sondern als offenes System verstanden, das Aushandlungsprozesse (in einem vorgegebenen Rahmen) zulässt. Ausgehend von dieser Prämisse und einer theoretischen Verortung im Rahmen von Weltgesellschaftstheorien wird aufgezeigt, wie Behinderung innerhalb der Weltbank in unterschiedlichen politischen, ökonomischen und gesellschaftlichen Zusammenhängen diskursiv konstruiert wird. Über die Darstellung der das Thema Behinderung formenden Diskursstränge wird zudem aufgezeigt, ab wann das Thema inklusive Bildung innerhalb der Weltbank auch für Menschen mit Behinderung als relevant anerkannt wurde. Obwohl sich die dargestellten Diskurse im Sinne der Komparatistik auch mit denjenigen anderer internationalen Organisationen oder auch mit Länderperspektiven vergleichen lassen würden, liegt der Schwerpunkt dieses Beitrags nicht darauf. Vielmehr soll er aufzeigen, wie die verschiedenen Diskurse überhaupt erst im Rahmen der Weltbank entstehen konnten. Dieses Vorgehen bietet die Grundlage, um die Entstehung derjenigen Diskurse besser nachzuvollziehen, die nicht auf Länderebene zu verorten sind, sondern supranational auf Ebene Weltgesellschaft.

\section{Theoretische Verortung und analytischer Zugang}

Theoretisch sind die nachfolgenden Ausführungen zur Weltbank im Rahmen der Weltgesellschaftstheorien (vgl. Greve/Heintz 2005) verortet. Ausgehend von dieser Rahmung soll deshalb zunächst geklärt werden, auf welchen theoretischen Grundlagen dieses Kapitel basiert und welche methodische Perspektive eingenommen wurde. 


\subsection{Weltgesellschaftstheorien}

Die sogenannten Weltgesellschaftstheorien sind keine erziehungswissenschaftliche Entdeckung, sondern, wie der Begriff Gesellschaft nahelegt, im Rahmen soziologischer Theoriebildung entstanden. Die Ausweitung des soziologischen Gesellschaftsbegriffs auf eine globale Perspektive geht auf die 1970er Jahre zurück und wurde zugleich von mehreren Wissenschaftlern, jeweils mit unterschiedlichen theoretischen Konzeptionen, vollzogen (vgl. Greve/Heintz 2005). In der international vergleichenden Erziehungswissenschaft bzw. auch Sonderpädagogik ist im Kontext der Weltgesellschaftstheorien vor allem John Meyers World Polity Ansatz verbreitet (vgl. bspw. Kiuppis 2014; Powell 2016), da er unter Anderem zu erklären versucht, wie internationale Policies die Entwicklungen von Nationalstaaten beeinflussen können (vgl. Mejeh 2021 i.d.B.). Neben Meyers Ansatz gibt es aber auch noch weitere, in der Erziehungswissenschaft bzw. auch Sonderpädagogik weniger beachtete Weltgesellschaftstheorien, wie bspw. den Weltsystemansatz, den neogramscianischen Ansatz (vgl. Herkenrath 2012) oder die theoretischen Perspektiven von Peter Heintz und Niklas Luhmann (vgl. Greve/Heintz 2005).

Im Sinne der genannten Theorieansätze folgt die Auseinandersetzung mit internationalen Organisationen - und damit auch mit der Weltbank - einer anderen Logik, als die klassisch in der international vergleichenden Forschung vorzufindende Komparatistik. Die Weltgesellschaftstheorien führen eine neue Beobachtungsebene ein, weil sie statuieren, dass die Weltgesellschaft „,mehr und etwas anderes als die Summe der Nationalstaaten und deren Beziehungen“ (ebd.: 109) ist. Sie gehen davon aus, dass ,im Laufe der historischen Entwicklung ein globaler Zusammenhang entstanden ist, der als umfassendstes System die Randbedingungen für alle anderen sozialen Einheiten und Prozesse vorgibt" (ebd.: 109). Die Weltbank ist Teil dieses Systems, weil sie letztendlich dazu beiträgt, überstaatliche Randbedingungen zu konstruieren und diese bis zu einem gewissen Grad auch durchzusetzen (vgl. Rittberger et al. 2013). Aus dieser Perspektive wird sie auch nachfolgend betrachtet, um einen Beitrag zum Verständnis der Ebene Weltgesellschaft beizusteuern.

\subsection{Die Weltbank als Organisation}

Für eine Auseinandersetzung mit der Weltbank ist von Bedeutung, dass sich ihre Funktion im Verlaufe der Zeit verändert hat. Während sie im Anschluss an den 2. Weltkrieg mit Nachkriegsrekonstruktionen und Entwicklung beschäftigt war, ist sie heute eine Entwicklungsorganisation, die ihr primäres Ziel in der weltweiten Armutsreduktion sieht (vgl. Rittberger et al. 2013: 206ff.). Als Gesamtkonstrukt handelt es sich bei der Weltbank um eine komplexe Organisation und es ist nicht immer geklärt, was oder wer gemeint ist, wenn sie als Organisation genannt wird. Die Weltbankgruppe beschäftigt insgesamt über 10`000 Mitarbeitende, an mehr als 120 Standorten (vgl. The World Bank 2019d) und besteht aus vier Organisationen: Die ,International Bank for Reconstruction and Development ${ }^{6}$ - als Weltbank bezeichnet - die ,International Finance Corporation', die ,International Development Association“ und die ,Multilateral Investment Guarantee Agency“ (vgl. Rittberger et al. 2013: 207).

Trotz dieser Größe und Komplexität wird die Weltbank(-gruppe) oft als Akteur wahrgenommen, der eine klare und einheitliche Position vertritt. Es ist aber darauf hinzuweisen, dass 
ihr die dieser Darstellung zugrundeliegende Vorstellung einer Organisation, in der alle Mitarbeitenden und beigezogenen Expert*innen kohärente und kongruente Ziele verfolgen, keinesfalls gerecht wird. Auch wenn sie als Organisation logischerweise Ziele verfolgt, funktioniert sie nicht als ideologisch angetriebener Think Tank (vgl. Pereira 2016). Ihr Agieren kann besser verstanden werden, wenn sie theoretisch als offenes System gefasst wird, das jeweils auf lokale Bedingungen und Einflüsse von verschiedensten Gruppen und Personen eingehen muss (vgl. Béland/Orenstein 2013; Koch 2008). Dies gilt aber nicht nur im Rahmen von lokalen Projekten, sondern auch im übergeordneten, weltgesellschaftlichen Kontext, denn die Weltbank orientiert sich bspw. auch am Agenda-Setting anderer internationaler Organisationen und ist nicht per se Taktgeber (vgl. Brandecker 2007: 7). Das negiert natürlich nicht die Tatsache, dass sie als Gesamtorganisation in einer machtvollen Position ist. Es ist aber ein Hinweis darauf, dass die Weltbank intern nicht so homogen ist, wie sie gegen außen bei der Umsetzung von Projekten zuweilen auftreten mag.

\subsection{Diskursanalytischer Zugang}

Vor dem Hintergrund der Annahme, dass die Weltbank als offenes System im Rahmen der Weltgesellschaft funktioniert, bietet es sich an, ihre Auseinandersetzungen in einer diskursanalytischen Perspektive zu betrachten. Diskursanalysen beziehen sich jeweils auf einen Dokumentenkorpus (vgl. Gasteiger 2008: 42), das gilt auch für die nachfolgenden Darstellungen, die in wesentlichen Teilen auf Dokumente aus dem Korpus einer Studie abstützt (Zahnd 2017). Es handelt sich dabei um insgesamt 4844 Dokumente aus dem Archiv der gesamten Weltbankgruppe, die im Zeitraum von 1948-2012 erstellt wurden und einen Bezug zum Thema Behinderung haben. Der Korpus wurde zudem teilweise durch neuere Dokumente ergänzt, um den Bezug zu aktuellen Debatten herzustellen

Analytisch liegt der Fokus der nachfolgenden Ausführungen auf der Frage, in welchem Zusammenhang (bzw. in welchen Themenfeldern) Behinderung in den Dokumenten der Weltbank wie konstruiert wird (vgl. Naue 2011; Weisser 2004). Gemäß Ullrich (2008: 24) gilt es in einer diskursanalytischen Perspektive dabei nach Folgendem zu fragen: ,[...] Welche semantischen Elemente strukturieren den Diskurs? Welche Praktiken, welche Art von Äußerungen geben ihm seine spezifische Gestalt? Wie ist der Diskurs gesellschaftlich eingebettet?" Die Beschreibung des Diskurses innerhalb der Weltbank orientiert sich an diesen Fragen und ist angelehnt an die genannte empirische Studie. Limitierend sei darauf hingewiesen, dass es sich bei den nachfolgenden Passagen um Beschreibungen von Diskurssträngen handelt, die an sich noch nichts über das tatsächliche Handeln der Weltbank oder die Lebensrealität von Menschen mit Behinderung aussagen (vgl. Ullrich 2008: 28). Dennoch bilden sie ab, wie sich die Weltbank im Verlaufe der Zeit mit dem Thema Behinderung auseinandersetzt. 


\section{Der Behinderungsdiskurs der Weltbank}

Die Darstellung des Behinderungsdiskurses der Weltbank ist in drei Diskursstränge unterteilt, die jeweils eigene Themenfelder repräsentieren. Für jedes dieser Themenfelder ist relevant, dass es sich ursprünglich entlang einer (entwicklungspolitischen) Problemformulierung ausgebildet hat. Die ersten beiden Diskursstränge unterscheiden sich insofern vom dritten, als das Thema Behinderung in diesen nicht im Zentrum der Auseinandersetzung steht. Die Dokumente fokussieren vielmehr die Relevanz medizinischer Interventionen für die Entwicklung(-spolitik) (Diskursstrang 1) bzw. die Ausgestaltung der sozialen Absicherung (Diskursstrang 2). Behinderung wird in beiden Themenfeldern eher beiläufig zum Thema. Im letzten Diskursstrang (3) wird Behinderung hingegen als eigenständiges Thema diskutiert. Obwohl diese drei Diskursstränge zu unterschiedlichen Zeitpunkten entstehen, lösen sie sich nicht ab. Vielmehr existiert ein bereits bestehender Diskursstrang auch dann weiter, wenn ein neuer entsteht. In allen lässt sich aufzeigen, dass unterschiedliches Wissen über Behinderung vorhanden ist und welche Konsequenz dies für die Konstruktion von Behinderung hat (vgl. Weisser 2005). Für die nachfolgenden Ausführungen ist zu beachten, dass die drei Diskursstränge zunächst mit starkem Fokus auf ihren Entstehungskontext und den dazugehörigen Zeitraum beschrieben werden. Eine Darstellung der weiterführenden Entwicklung und das Aufgreifen der Frage, wie sie sich allenfalls beeinflussen, erfolgt erst im Anschluss.

\subsection{Diskursstrang 1: Behinderung im Kontext medizinischer Interventionen}

Behinderung wird in den Dokumenten der Weltbank während den 1970er Jahren ein erstes Mal im Kontext einer Auseinandersetzung mit medizinischen Interventionen in den sogenannten Entwicklungsländern zum Thema. Am Ursprung der Debatte um medizinische Interventionen in diesen Ländern steht die Auseinandersetzung mit der Frage, wie ökonomische Entwicklung gefördert werden kann. Bereits in den 1960er Jahren beginnen innerhalb der Weltbank Diskussionen darüber, ob die bis dahin verfolgte Entwicklungspolitik, die davon ausgeht, dass Investitionen in Infrastrukturprojekte Entwicklung ankurbeln kann, noch sinnvoll ist (vgl. Rittberger et al. 2013: 209). Die wesentlichen Erkenntnisse dieser Auseinandersetzung werden anschließend im ,Pearson Report“ (Pearson 1970) festgehalten. Mit den Ölkrisen der 1970er Jahre wird das Hinterfragen der Entwicklungspolitik nochmals zusätzlich befeuert. Robert McNamara, damals Präsident der Weltbank, verweist in diesem Zusammenhang auf die verheerenden Auswirkungen der Ölkrisen in den sogenannten Entwicklungsländern und den daraus resultierenden dringenden Unterstützungsbedarf (vgl. The World Bank Group 2019: 183). Die Auseinandersetzung mit der Entwicklungspolitik - und damit auch die Suche nach neuen Ideen und Konzepten - führt dazu, dass sich die Weltbank dem Thema Armut und den Grundbedürfnissen von Menschen in Entwicklungsländern zuwendet (vgl. The World Bank 2019b). Was dies inhaltlich bedeutet, zeigt sich exemplarisch in einem Buch von Andrew Kamarck (1976), damals Leiter des ,Economic Development Institute' der Weltbank. In ,The Tropics and Economic Development: A Provocative Inquiry into the Poverty of Nations' befasst sich dieser mit der Frage, welche Bedingungen die 
Entwicklung in tropischen Regionen erschweren und zeigt auf, dass bspw. das Klima, die Bodenbeschaffenheit, Schädlinge oder auch Krankheiten ein Hindernis für ökonomische Entwicklung darstellen können. Ab Mitte der 1970er Jahre wird dann Gesundheit (neben anderen Themen) ein Bereich der Entwicklungspolitik der Weltbank, was sich auch in der Veröffentlichung einer ersten Policy mit dem prägnanten Titel ,Health“ (The World Bank 1975) äußert.

Mit dem Aufgreifen des Themas Gesundheit wird auch Behinderung in den Dokumenten adressiert. Sie wird aber nicht als eigenständiges Thema aufgegriffen, sondern als zu verhindernde Folge von Mangelernährung oder Krankheiten. Die Ausführungen schließen sich dabei an der Logik eines Krankheitsfolge-Modells an (vgl. Tab. 1), d.h. Behinderung wird ausschließlich als mögliche Folgeerscheinung eines medizinischen Problems diskutiert. Da die auf Gesundheit bezogenen Interventionen das Ziel verfolgen, ökonomische Entwicklung zu indizieren, wird Behinderung zudem auch diesbezüglich diskutiert. Dies im Sinne dessen, dass sie als hinderlich für die ökonomische Produktivität betrachtet wird (vgl. Tab. 1).

Tabelle 1: Behinderung als Konsequenz von Mangelernährung oder Krankheiten und die Verbindung zur Frage der ökonomischen Produktivität

\begin{tabular}{lll}
\hline & Behinderung als Folge von... & Ökonomische Konsequenzen von... \\
\hline $\begin{array}{l}\text { Mangel- } \\
\text { ernährung }\end{array}$ & $\begin{array}{l}\text { "Considerable evidence further indica- } \\
\text { tes a relation between malnutrition of } \\
\text { children [...] and mental retardation." (S. } \\
\text { 29) }\end{array}$ & $\begin{array}{l}\text { "If infant malnutrition does impact on mental ca- } \\
\text { pacity later in life, this would be still another im- } \\
\text { portant economic effect of this disease." S. 68) }\end{array}$ \\
\hline Krankheit & $\begin{array}{l}\text { "River blindness causes partial or total } \\
\text { loss of sight.“ (S. 69) }\end{array}$ & $\begin{array}{l}\text { "In addition to the cost to the community and the } \\
\text { individual resulting from total blindness, the vari- } \\
\text { ous degrees of impairment of vision suffered by } \\
\text { others must also have a substantial impact on } \\
\text { productivity." (S. 71) }\end{array}$ \\
\hline
\end{tabular}

Quelle: Eigene Darstellung, basierend auf Kamarck (1976)

Mit der Anerkennung der Relevanz des Themas Gesundheit für die ökonomische Entwicklung stellt man sich in der Weltbank auch die Frage der Kosteneffizienz von diesbezüglichen Interventionen. Während in der oben beschriebenen, medizinisch orientierten Perspektive das Ziel ist, Behinderung zu verhindern, wird die damit einhergehende Wertung von Menschen mit Behinderung in den Ausführungen zur Frage der Kosteneffizienz nochmals deutlicher.

The argument is that complete disability is equivalent to economic death, in the sense that the completely disabled person is totally non-productive, and therefore may be regarded the same as premature death, which has the same result. However, some would argue that complete disability is worse than premature death [...]. The principal reason is that, in addition to foregoing the output that would have been produced by a blind worker if he was not disabled, the other members of society are imposed with the burden of sharing the remaining total output with the disabled worker [...] (Prescott/Prost 1983: 12f.).

Das exemplarische Zitat zeigt auf, dass die Frage nach Kosteneffizienz medizinischer Interventionen zu einer totalen Abwertung von Menschen mit Behinderung führt. Dies wird zwar teilweise kritisiert (vgl. bspw. Barlow/Grobar 1986: 10), verbleibt aber schlussendlich ein wichtiges Argumentationsmuster. 


\subsection{Diskursstrang 2: Behinderung im Kontext sozialer Absicherung}

Ab den späten 1980er Jahren entwickelt sich in der Weltbank ein zweiter Diskursstrang, in dem Behinderung adressiert wird. Mit dem neo-konservativen finanzpolitischen Klima in den angelsächsischen Ländern während der Regierungszeit von Margaret Thatcher in Großbritannien und Ronald Reagan in den Vereinigten Staaten beginnt auch die Weltbank einen (neo-)liberalen Kurs zu verfolgen (vgl. Pereira 2016: 2; Rittberger et al. 2013: 209). Die wirtschaftspolitische Orientierung dieser Zeit wird im ,Washington Consensus ‘ festgehalten, der im Übergang von den 1980er zu den 1990er Jahren veröffentlicht wird (vgl. Williamson 2000) und eine Zusammenfassung der britischen und amerikanischen Idee des Neoliberalismus beinhaltet (vgl. Harvey 2007: 93). Er propagiert Marktliberalisierung, Deregulierung und Privatisierung als zentrale Konzepte (vgl. Stiglitz 1998). Dieser Logik folgen bspw. auch die von der Weltbank erstmals zu Beginn der 1980er Jahre vergebenen StrukturanpassungsKredite (vgl. Rittberger et al. 2013: 209; The World Bank Group 2019: 206; Ziai 2006: 62). Neben den politischen Entwicklungen in den angelsächsischen Ländern und den damit in Verbindung stehenden finanzpolitischen Konzepten ist zudem von Bedeutung, dass der sowjetische Block in den 1980ern zusammenbricht und die Weltbank schnell vor Ort ist (vgl. The World Bank 2019a). Das einzige „Gegenprojekt“ zum Kapitalismus ist damit gescheitert und die Weltbank verweist in diesem Zusammenhang sowohl auf neue Möglichkeiten, als auch auf Gefahren der anstehenden Entwicklung in den ehemals sozialistischen Ländern (vgl. Andrews/Rashid 1996).

Eingebettet in den Zeitraum dieser Veränderungen, beginnt die Weltbank das Thema der sozialen Absicherung zu fokussieren. Die in den Dokumenten ab den 1980er Jahren sichtbare Auseinandersetzung bleibt zunächst auf Lateinamerika beschränkt (vgl. McGreevey 1990: 1). Ausgangspunkt ist dabei Chile, wo zum ersten Mal ein Sozialversicherungssystem im Sinne eines neoliberalen Konzepts umgestaltet wird (vgl. Zahnd 2017: 130). Dies ist für die entstehende Debatte zwar nicht ein grundlegendes aber dennoch interessantes Detail, weil die neoliberale Denkweise für den Diskursstrang zum Thema der sozialen Absicherung in den 1990er Jahren prägend ist, auch wenn die ersten Empfehlungen in diesem Bereich zunächst nicht diese Stoßrichtung aufweisen (vgl. Orenstein 2008: 76f.). Entscheidend dafür, dass sich das Thema der sozialen Absicherung ab den 1990ern als wichtiges und globales Thema innerhalb der Bank festsetzen kann, ist die sogenannte ,Old Age Crisis' (vgl. The World Bank 1994). Aufgrund des weltweiten demografischen Wandels sieht man die finanzielle Stabilität der Systeme der sozialen Absicherung unter Druck (vgl. Orenstein 2008: 77).

In der Auseinandersetzung mit der finanziellen Stabilität der Rentensysteme, werden auch Renten für Menschen mit Behinderung zum Thema. Obwohl es durchaus Autor*innen gibt, die darauf hinweisen, dass eine Auseinandersetzung mit Versicherungssystemen nicht nur auf deren Kosten beruhen sollte, sondern auch die Qualität der erbrachten Leistungen erhoben werden müsste (bspw. Valdés-Prieto 1994), dominieren Argumentationslinien, die ausschliesslich auf die Frage der Kosten ausgerichtet sind. Dies zeigt sich auch in der Auseinandersetzung mit den Behindertenrenten. Die realen Lebenssituationen bzw. der diesbezügliche Nutzen der Renten werden in den Dokumenten kaum verhandelt. Vielmehr erscheint Behinderung als administrative Kategorie und mathematische Variable im Diskurs und wird auf einer sehr abstrakten Ebene verhandelt, was sich im nachfolgenden Zitat exemplarisch zeigt: 
The net increase in disability pensioners is calculated for each year. The baseline assumption for net new disability pensioners is based on the experience in between 1992 and 1994, a period during which the net increase in disability pensioners rose by approximately 30,000 each year. The rate of new disability claims has doubled since the mid-1980s and is the result of policy choices, not demographic changes (The World Bank 1995: 139).

Um die langfristige finanzielle Stabilität der Rentensysteme zu sichern, werden auch die Behindertenrenten kritisch begutachtet bzw. als problematischer Teil des Systems identifiziert: „Early retirement and lax eligibility for disability are [...] bad for the system because they impose a heavy financial burden that makes it nonsustainable" (Demirguc-Kunt/Schwarz 1995: 8). Aufgrund dieser Problemdefinition sucht man nach Möglichkeiten der Kostensenkung und findet sie in der Logik der Verengung des Zugangs zu den Renten, was sich auch hier an einem exemplarischen Zitat aufzeigen lässt:

In addition, the criteria for receipt of a disability benefit could probably be tightened [...] Access to disability pensions is relatively easy and there is no maximum duration of benefit. It is not known whether there exists a system of monitoring the decisions of the local commissions, but evidence from other countries suggests that monitoring is necessary to prevent abuses (particularly in times of labor surplus) (The World Bank 1993a: 72).

Neben einer Lösung für das wahrgenommene Problem zeigt sich in diesem Zitat, dass über die Verwendung der Renten wenig bis kein Wissen vorhanden zu sein scheint, da nicht einmal klar ist, ob ein Monitoring der Entscheidungsfindung existiert. Dennoch wird Behinderung über die Verbindung mit der Zugangsverengung zur regulierbaren Variable. Die gesamte Auseinandersetzung beinhaltet zudem ein grundlegendes Misstrauen gegenüber der Kategorie Behinderung, dies im Sinne dessen, dass man hinterfragt, ob die richtigen Personen mit dieser Kategorie erfasst sind (bspw. Demirguc-Kunt/Schwarz 1995; Valdés-Prieto 1994; Vittas 1995).

Die in den Dokumenten vorzufindenden Definitionen von Behinderung sind in den meisten Fällen eine schlichte Wiedergabe der bisherigen, medizinisch orientierten Behinderungskonzeptionen in den verschiedenen Nationalstaaten (bspw. Barr 1992; The World Bank 1991, 1993a, 1993b). Da die grundlegende Struktur des Diskurses auf einer abstrakten, administrativen Ebene verbleibt, findet in der Regel auch keine Auseinandersetzung mit diesen Konzeptionen oder dem Thema Behinderung an sich statt. Trotz dieses allgemeinen Musters gibt es allerdings auch eine Ausnahme. Im Zusammenhang mit der Regulierung des Zugangs zu den Behindertenrenten, und dem geäußerten Misstrauen gegenüber der Kategorie, zeigt sich in wenigen Publikationen, dass sich eine neue Konzeption von Behinderung anbahnt. Diese weicht zwar nicht grundsätzlich von einer medizinischen Logik ab, aber sie setzt eine medizinisch diagnostizierte Behinderung nicht mehr mit Arbeitsunfähigkeit gleich. Ausgangspunkt dieses Umdenkens ist die Feststellung, dass eine körperliche Schädigung nicht zwingend mit einer Arbeitseinschränkung einher geht: „An individual who (say) loses a limb, but whose long-run health is otherwise unimpaired and whose working capacity is unaffected, will receive a Group 3 invalidity pension whilst continuing to do his old job and receiving a full wage“ (The World Bank 1991: 49) (vgl. auch Barr 1992; Braithwaite 1995; The World Bank 1993b). Gleichzeitig findet sich in diesem Zeitraum auch der Vorschlag, Behinderung nicht mehr über die Ursache zu erfassen und Menschen dichotom in solche mit und ohne Behinderung einzuteilen, sondern einen Einschränkungsgrad zu bestimmen: „In particular, many policy analysts recommend that countries move toward unified systems of disability benefits based on degree rather than on cause of disability“ (Nguyen 1994: 13). Damit einher geht eine Logik, die darauf aufbaut Behinderung zwar nach wie vor anhand einer medizi- 
nischen Prüfung zu bestimmen, aber diese regelmäßig zu wiederholen und eine Arbeitsmarktintegration anzustreben. Es zeichnet sich damit eine Entwicklung hin zu einem Modell ab, das theoretisch unter dem Begriff „Workfare“ gefasst wird (vgl. Wyss 2007). ${ }^{1}$

\subsection{Diskursstrang 3: Behinderung als eigenständiges Thema der Entwicklungspolitik}

Eine Anerkennung des Themas Behinderung als eigenständiges Thema der Entwicklungspolitik erfolgt erst mit der Jahrtausendwende. Dies zeigt sich im dritten Diskursstrang, der auf inhaltlicher und semantischer Ebene einen komplett neuen Zugang zu Behinderung mit sich bringt und dermaßen radikal mit dem bisherigen bricht, dass Colin Barnes (2009: 17) die Veränderung auch schon als „sea change“ bezeichnete. Obwohl sich dieser Diskursstrang erst nachhaltig ab der Jahrtausendwende durchsetzt, zeigen sich erste Anzeichen für eine steigende Relevanz des Themas Behinderung - und eine damit verbundene neue diskursive Konstruktion - bereits in den 1990er Jahren. Auch diese Entwicklung lässt sich besser nachvollziehen, wenn sie im einbettenden Kontext betrachtet wird. Im Jahr 1995 wird James D. Wolfensohn vom damaligen US-Präsidenten Bill Clinton zum neuen Präsidenten der Weltbank ernannt (vgl. The World Bank Group 2019: 298). Wolfensohn übernimmt die Bank in einer turbulenten Zeit. Dies gilt einerseits für die globale Ökonomie, die Mitte der 1990er mit der mexikanischen Strukturkrise und der asiatischen Finanzkrise durchgerüttelt wird. Andererseits steht die Weltbank aber auch aufgrund ihrer Projektaktivitäten in der Kritik und ein Jahr vor der Amtsübernahme durch Wolfensohn wird die Jahresversammlung von Protestaktionen gestört (vgl. The World Bank 2019b). Zwei Jahre nach Wolfensohn wird Joseph E. Stiglitz Vizepräsidenten und Chef-Ökonom der Weltbank, verbleibt aber nur bis zur Jahrtausendwende (vgl. Brandecker 2007: 19). Unter der Führung der beiden vollzieht die Weltbank einen Kurswechsel, der zuvor kaum möglich schien und sich im Weltentwicklungsbericht (Chhibber et al. 1997) von 1997 abbildet. Unter der neuen Leitung möchte die Weltbank vom bisher praktizierten Liberalismus abweichen und sich vom Marktfundamentalismus des Internationalen Währungsfonds distanzieren (vgl. Pereira 2016: 8). Der bis dahin immer noch zentrale ,Washington Consensus' wird zunächst verworfen. Joseph Stiglitz vertritt dabei die Position des sogenannten ,Post-Washington Consensus', gemäß seiner Idee sollte eine breitere Perspektive auf die Entwicklungsfrage eingenommen werden: „We seek increases in living standards - including improved health and education - not just increases in measured GDP“ (Stiglitz 1998: 31). Obwohl der ,Post-Washington Consensus“ die Ideen des ,Washington Consensus ' nicht komplett verwirft, verbindet er dessen marktliberale Konzeption mit Strategien zur Armutsbekämpfung und Themen wie Bildung und Gesundheit (vgl.

1 Der Wechsel des wohlfahrtsstaatlichen Modells (von Welfare zu Workfare) zeigt sich in den 1990er Jahren auch in zahlreichen Nationalstaaten. Exemplarisch sei hierzu auf Maschke (2008: 271) verwiesen, der für die Behindertenpolitik der Europäischen Union festhält: „Unter der politischen Vorgabe, die gesellschaftliche Teilhabe behinderter Menschen zu sichern und eine weitere ,Kostenexplosion“ zu verhindern, wurden sowohl die Effektivität als auch Effizienz der Behindertenpolitik auf den Prüfstand gestellt. Thematisch an die Diskussion zur Aktivierung von Arbeitslosen angelehnt, wurde die Überbetonung monetärer Transfers im Verhältnis zu aktivierenden Maßnahmen debattiert und schließlich in mehreren Ländern der Zugang zu Erwerbs- und Berufsunfähigkeitsrenten erschwert sowie die Anreize für die Aufnahme von Arbeit erhöht.“ 
Brandecker 2007: 20ff.; Pereira 2016: 10f.). Allerdings ist anzumerken, dass sich innerhalb der Weltbank durchaus nicht alle mit Stiglitz' Position identifizieren können, auch wenn insgesamt Einigkeit darüber herrscht, dass es ergänzende Maßnahmen braucht (vgl. Pereira 2016: 10). Auch wenn Stiglitz schlussendlich die Weltbank wieder verlassen muss, führt die interne Auseinandersetzung zu wichtigen Veränderungen. Zudem ist für die Weltbank relevant, dass 1995 der ,World Summit for Social Development‘ stattfindet, der das Thema Armut ins Zentrum der Aufmerksamkeit rückt und die Vereinten Nationen den Zeitraum von 1997-2006 als Dekade der Armutsbekämpfung festlegen (vgl. Brandecker 2007: 19). Die Bekämpfung von Armut findet sich dann auch wieder als zentrales Ziel innerhalb der ,Millennium Development Goals', deren sich die Weltbank verpflichtet (vgl. The World Bank 2019c). ${ }^{2}$

Im Kontext der oben beschriebenen Entwicklungen zeigt sich, dass die Weltbank versucht die sozialen Nebenwirkungen des Neoliberalismus zu lindern und ein Entwicklungsparadigma zu entwerfen, das soziale Aspekte miteinbezieht (vgl. Pereira 2016: 11f.). Ab den 1990er Jahren gewinnt im Zuge dieser Entwicklung das Thema Behinderung langsam an Bedeutung. Dies zeigt sich beispielsweise in der Auseinandersetzung mit Community-basierten Dienstleistungen für Menschen mit Behinderung (bspw. Lynch 1994; The World Bank 1997; Tobis 2000) und der Auseinandersetzung mit Behinderung als Folge von kriegerischen Auseinandersetzungen (bspw. Colletta et al. 1996; The World Bank 1999), in denen erstmals im Rahmen von Weltbank-Dokumenten spezifisch über Hilfe für Menschen mit Behinderung verhandelt wird.

Kurz nach der Jahrtausendwende wird innerhalb der Weltbank, im Anschluss an die Umsetzung der ,Social Protection Strategy“ im Jahr 2001, ein ,Disability and Development Team‘ geschaffen (vgl. Braithwaite et al. 2008: 1f.). In diesem Zusammenhang ist auch darauf zu verweisen, dass Judith E. Heumann, eine wichtige Exponentin der Behindertenbewegung, im Jahr 2002 als erste Beraterin der Weltbank für das Thema ,Disability and Development' ernannt wird (vgl. The World Bank Group 2016). Damit entsteht ab den 2000ern eine Strategie im Umgang mit Behinderung, die eine komplett neue Perspektive im Diskurs auftauchen lässt:

Disability should be broadly understood and conceptualized as a social construct in much the same way that gender, race and ethnicity are socially constructed. This construction originates in an almost exclusively negative interaction of a person with his or her environment. Some examples of the interaction are discrimination and social isolation, as well as attitudinal and structural barriers (Guernsey et al. 2006: 3).

Obwohl mit dem radikalen Wandel auch das soziale Modell der Behinderung in einem zentralen Dokument platziert wird (Guernsey et al. 2006), setzt sich in diesem Diskursstrang, im Anschluss an die ,International Classification of Functioning Disability and Health' (World Health Organization 2001), längerfristig ein relationales Behinderungsverständnis durch (bspw. Guernsey et al. 2007; World Health Organization/The World Bank 2011). Behinderung wird als Ergebnis der Interaktion von Individuum und Umwelt verstanden und dabei das soziale Modell mit einer medizinischen Sichtweise kombiniert. Dennoch verbleiben

2 Die ,Millenium Development Goals“ sind in gewisser Weise „Vorgänger“ der ,Sustainable Development Goals', die 2015 im Rahmen der Agenda 2030 von den Vereinten Nationen beschlossen wurden. Es handelt sich um übergeordnete Entwicklungsziele, die u.A. der Armutsbekämpfung, Wahrung der Menschenrechte, Sicherung des Friedens und einem nachhaltigen Umgang mit ökologischen Ressourcen dienen sollen. 
zentrale Argumentationslinien im Anschluss an das soziale Modell - und auch dessen emanzipatorische Seite - im Diskurs, wie bspw. der Fokus auf Barrieren oder auch der Verweis auf die soziale Konstruktion von Behinderung (vgl. Zahnd 2017: 168). Gleichzeitig wird ein komplett neues Bild von Menschen mit Behinderung geschaffen, das diese als aktive Mitglieder der Gesellschaft und relevanten Teil der Ökonomie positioniert. Dabei werden auch bisherige Aktivitäten und Ansätze im Zusammenhang mit dem Thema Behinderung kritisiert. Die Kritik wird ein erstes Mal bereits im Jahr 2000 von Robert Metts (2000) im von ihm geschriebenen Dokument ,Disability Issues, Trends and Recommendations for the World Bank' geäussert. Auch in nachfolgenden Dokumenten finden sich zudem kritische Kommentare (bspw. Guernsey et al. 2006; World Health Organization/The World Bank 2011). Exemplarisch hervorzuheben ist dabei Metts' Beurteilung des DALY-Konzepts ${ }^{3}$, weil sie explizit eine Argumentationslinie kritisiert, die im oben dargestellten, ersten Diskursstrang prägend ist.

The first false assumption renders the DALY inadequate for measuring the global burden of disease because it leaves the system with no mechanism for evaluating and measuring the roles played by environmental factors in determining the severity of disabilities; the second renders the DALY inadequate for measuring the effectiveness of health interventions because it leads to the erroneous conclusion that such interventions can have no effect; and together they render the DALY dangerously misleading because they inaccurately suggest that the prevention of impairments is the only available strategy for reducing the negative consequences of disability (Metts 2000: 1).

Um die neue Denkweise - und die darin liegende Kritik am bisherigen Vorgehen - auch für das Handeln der Weltbank fruchtbar zu machen, setzt man auf einen sogenannten ,TwinTrack Approach“: Erstens soll die Auseinandersetzung mit Behinderung (nach der neuen Logik) in alle bestehenden Entwicklungsprogramme integriert werden und zweitens sollen spezifische Programme entwickelt werden, wo dies notwendig ist (vgl. Guernsey et al. 2006: 10). Im Anschluss zeigt sich dann, dass Behinderung in den Dokumenten der Weltbank als bereichsübergreifendes Thema bearbeitet wird (vgl. Tab. 2).

Behinderung wird damit innerhalb der Weltbank nicht nur komplett neu situiert, sondern auch auf vielseitige Weise bearbeitet. Mit Blick auf die Perspektive des Handbuchs sei an dieser Stelle kurz die Verbindung des Themas Behinderung mit dem Thema Bildung erläutert, die sich erstmals im Jahr 1994 finden lässt (vgl. Tab. 2). Dies ist insofern ein später Zeitpunkt, als das Thema Bildung bereits ab den 1960ern ein erstes Mal von der Weltbank aufgegriffen wurde, aber dennoch nicht überraschend, da Bildung (wie auch Behinderung) erst ca. ab der Jahrtausendwende als eigenständiges Ziel der Entwicklungszusammenarbeit verstanden wird (vgl. Brandecker 2007; Zahnd 2017). Die zuvor fehlende Koppelung wird auch im Dokument ,Provision for Children with Special Educational Needs in the Asia Region“ (Lynch 1994: vii) hervorgehoben: „Although the Bank's central policy unit has not focused on the issues of children with special educational needs, some work has been undertaken in the Asia region of the Bank." Auch wenn dieses Dokument auf eine erste Auseinandersetzung verweist und dabei gleich in Richtung inklusiver Bildung tendiert, folgt eine intensivere Debatte erst mit dem Jahrtausendwechsel. Die Argumentationslinie ist hier ähnlich radikal wie bezüglich Behinderung im Allgemeinen. So basiert das Dokument ,Inclusive

3 DALY ist ein Maß, das nach folgender Logik berechnet wird: ,measure of the gap in healthy years of life lived by a population as compared with a normative standard. More formally, DALYs are a time based measure which adds together years of life lost due to premature mortality with the equivalent number of years of life lived with disability or illness“" (Jamison et al. 2006:1353). 
Education: Achieving Education for All by Including those with Disabilities and Special Education Needs' (Peters 2003) auf einer Grundorientierung, die an das ,Salamanca Statement' (UNESCO 1994) anschließt bzw. auch auf dieses verweist und ein Schulsystem anstrebt, in dem alle Schüler*innen maximale Entfaltungsmöglichkeiten für ihre Potentiale erhalten.

Tabelle 2: Exemplarische Beispiele der thematischen Bearbeitung von Behinderung durch die Weltbank

\begin{tabular}{|c|c|}
\hline Thema & Exemplarische Dokumente mit Report Number aus dem World Bank Archive \\
\hline \multirow[t]{3}{*}{ Armut } & Elwan, A. (1999): Poverty and disability. A survey of the literature (Nr. 21315). \\
\hline & $\begin{array}{l}\text { Dudzik, P./McLeod, D. (2000): Including the most vulnerable. Social funds and people } \\
\text { with disabilities (Nr. 23143). }\end{array}$ \\
\hline & $\begin{array}{l}\text { The World Bank (2004): Poverty reduction strategies: Their importance for disability (Nr. } \\
\text { 69731). }\end{array}$ \\
\hline \multirow[t]{3}{*}{ Gesundheit } & $\begin{array}{l}\text { Wiman, R./Helander, E./Westland, J. (2002): Meeting the needs of people with } \\
\text { disabilities - new approaches in the health sector (Nr. 26692). }\end{array}$ \\
\hline & Groce, N. (2004): HIVIAIDS and disability. Capturing hidden voices (Nr. 34313). \\
\hline & $\begin{array}{l}\text { Melte, C. (2008). Economic implications of chronic illness and disability. In Eastern } \\
\text { Europe and the former Soviet Union (Nr. 42851). }\end{array}$ \\
\hline \multirow[t]{3}{*}{$\begin{array}{l}\text { Soziale } \\
\text { Absicherung }\end{array}$} & $\begin{array}{l}\text { Hoogeveen, J. G. (2004): Measuring welfare for small but vulnerable groups. Poverty } \\
\text { and disability in Uganda (Nr. 30168). }\end{array}$ \\
\hline & Mitra, S. (2005): Disability and social safety nets in developing countries (Nr. 32740). \\
\hline & $\begin{array}{l}\text { The World Bank (2008): Building resilient communities. Risk management and response } \\
\text { to natural disasters through social funds and community-driven development operations } \\
\text { (Nr. 48723). }\end{array}$ \\
\hline \multirow[t]{3}{*}{ Arbeit } & Hoopengardner, T. (2001): Disability and work in Poland (Nr. 23145). \\
\hline & Mont, D. (2004): Disability employment policy (Nr. 30162). \\
\hline & $\begin{array}{l}\text { Roggero, P. et al. (2005): „Employment and Youth with Disabilities. Sharing Knowledge } \\
\text { and Practices“ - report of the e-discussion on youth and disabilities (Nr. 37274). }\end{array}$ \\
\hline \multirow[t]{3}{*}{ Bildung } & $\begin{array}{l}\text { Lynch, J. (1994): Provision for children with special educational needs in the Asia } \\
\text { region (Nr. WTP261). }\end{array}$ \\
\hline & $\begin{array}{l}\text { Peters, S. J. (2003): Inclusive education. Achieving education for all by including those } \\
\text { with disabilities and special education needs (Nr. 26690). }\end{array}$ \\
\hline & Peters, S. (2004): Inclusive education. An EFA strategy for all children (Nr. 31195). \\
\hline
\end{tabular}

Quelle: Eigene Darstellung

\subsection{Ausblick: Stabilität und Wandel innerhalb der Diskursstränge}

Mit der Vorgehensweise des ,Twin-Track Approaches' und der radikalen Neukonstruktion des Zugangs zum Thema Behinderung stellt sich die Frage, ob die gewählte Strategie innerhalb der Weltbank auch erfolgreich ist und die zuvor entstandenen Auseinandersetzungen beeinflusst. Dabei ist zunächst grundsätzlich darauf zu verweisen, dass sich in der Auseinandersetzung mit medizinischen Interventionen und den Systemen der sozialen Absicherung im Verlaufe der Zeit einige Änderungen ergeben. So findet bspw. der Ansatz der Privatisierung von Rentensystemen 2004 sein Ende (vgl. Béland/Orenstein 2013: 130; Zahnd 2017: 208) und die durch eine allgemeine Verbesserung der medizinischen Versorgung im globalen Süden höher werdende Lebenserwartung bringt mit sich, dass zusätzliche medizinische 
Probleme an Relevanz gewinnen, dazu gehören insbesondere Erkrankungen des Herz-Kreislaufsystems und der Psyche (vgl. Jamison et al. 1993: xi; Zahnd 2017: 195). Obwohl diese Veränderungen auch eine veränderte Semantik (durchaus auch bezüglich Behinderung) mit sich bringen, scheint die grundlegende Argumentationslogik innerhalb der Diskursstränge 1 \& 2 unverändert zu bleiben und damit insbesondere die für das Thema Behinderung bedeutenden Strukturen. ${ }^{4}$

Im Fall der medizinischen Interventionen sind die problematischen Strukturen insbesondere darin zu sehen, dass eine medizinische Perspektive auf Behinderung dominiert und Behinderung über die Frage der Kosteneffizienz medizinischer Interventionen komplett abgewertet wird. Mit Blick auf die längerfristige Entwicklung des Diskursstrangs ist darauf hinzuweisen, dass sich radikale Äußerungen, die eine komplette Abwertung von Behinderung explizit vornehmen, längerfristig nicht mehr finden bzw. nur noch in kaschierter Form, wie im Zusammenhang mit der Abwägung von Kosteneffizienz pränataler Diagnostik (vgl. Tab. $3) .^{5}$

Tabelle 3: Beispiele der Abwertung von Behinderung im Bereich der medizinischen Interventionen

\begin{tabular}{ll}
\hline Jahr & Zitat \\
\hline 1983 & $\begin{array}{l}\text { "The argument is that complete disability is equivalent to economic death, in the sense that the } \\
\text { completely disabled person is totally non-productive, and therefore may be regarded the same } \\
\text { as premature death, which has the same result." (Prescott/Prost 1983:12f.) }\end{array}$ \\
\hline 1993 & $\begin{array}{l}\text { "Also, by definition, the measure assumes that one year of complete disability is equivalent to } \\
\text { one year of premature death. It could be argued that meeting the needs of a disabled person } \\
\text { places a heavier burden on the community.“ (Prost/Jancloes 1993:743) }\end{array}$ \\
\hline $\begin{array}{l}\text { "Because costs of care will vary widely across and within countries, cost estimates should be } \\
\text { done for individual regions. Lower costs of care will reduce cost effectiveness of prenatal scree- } \\
\text { ning for DS [Down Syndrome; R.Z.]. However, even after the cost adjustment, it is unlikely that } \\
\text { the benefits will completely go away, because of the large difference between a relatively cheap } \\
\text { screening program and high burden of disease of DS." (Durkin et al. 2006: 943) }\end{array}$ \\
\hline
\end{tabular}

Quelle: Eigene Darstellung

Mit einer längerfristigen Perspektive zeigt sich aber insbesondere in der Weiterverwendung der DALYs, dass problematische Grundstrukturen erhalten bleiben (bspw. Saleh et al. 2018; Walters et al. 2018). Auch wenn bezüglich der Verwendbarkeit des Konzepts Diskussionen sichtbar sind, verbleibt es ein relevantes Maß in den Publikationen (vgl. Jamison et al. 2017), das eben gerade nicht den notwendigen, umfassenden Blick auf Behinderung ermöglicht.

Auch im Diskursstrang zum Thema der sozialen Absicherung bleibt die problematische Argumentationsstruktur erhalten. Sie findet sich hier in der abstrahierten Abhandlung bezüglich der finanziellen Stabilität der Rentensysteme und der Verengung des Zugangs (bspw. Holzmann/Palmer 2006; The World Bank 1998) ohne Berücksichtigung der Lebensbedingungen der davon betroffenen Personen (vgl. Tab. 4).

4 Bezüglich dieser Einschätzung muss einschränkend angemerkt werden, dass auf empirischer Basis nicht abschließend geklärt ist, ob die Entwicklung einen nachhaltigen Einfluss auf die Weiterentwicklung der Gesamtorganisation hat. Grund dafür ist, dass die in der zugrundeliegenden Studie verwendeten Daten nur bis in das Jahr 2012 reichen. Mit Blick auf die Weiterentwicklung der dargestellten Diskursstränge kann aber festgehalten werden, dass ein Wandel, falls er stattfindet, nur zögerlich erfolgt und es eher zu bezweifeln ist, dass sich die Diskursmuster grundlegend verändern.

5 Diese Problematik findet sich in vergleichbarer Weise auf Ebene der Nationalstaaten, wie bspw. bei Baldus (2016) exemplarisch nachzulesen ist. 
Tabelle 4: Beispiele der abstrakten Abhandlung von Behinderung im Kontext der sozialen Absicherung

\begin{tabular}{ll}
\hline Jahr & Zitat \\
\hline 1998 & $\begin{array}{l}\text { "The financial problems of the RGPS are the result of: [...] (vii) generous replacement rates for } \\
\text { disability benefits of } 100 \text { percent, and lenient eligibility rules, which result in a high percentage } \\
\text { of pensioners receiving disability pensions." (The World Bank 1998: 4) }\end{array}$ \\
\hline 2009 & $\begin{array}{l}\text { "Benefit eligibility requires an individual to have lost at least 30-67 percent of his or her working } \\
\text { capacity, depending on the country. Some countries provide both partial and full disability pensi- } \\
\text { ons, while others provide only full disability pensions." (Holzmann/Guven 2009: 27) }\end{array}$ \\
\hline
\end{tabular}

Quelle: Eigene Darstellung

Mit den Zitaten in Tabelle 4 zeigt sich aber - und dies gilt auch für den Diskursstrang der medizinischen Interventionen -, dass trotz gleichbleibender diskursiver Strukturen Behinderung längerfristig in einem relationalen Sinne gefasst wird und es diesbezüglich durchaus Veränderungen gibt, die sich auch im Kontext neuer Debatten um Behinderung einordnen lassen. So verweisen Durkin et al. (2006: 937) in einem Dokument aus dem Themenbereich der medizinischen Debatte auf Folgendes: „Disability is context specific and may vary from culture to culture." Mit Blick auf das Thema der sozialen Absicherung zeigt sich die Veränderung bspw. im Dokument ,For Protection and Promotion: The Design and Implementation of Effective Safety Nets“ (Grosh et al. 2008: 508):

Disability. A physical, mental, or psychological condition that limits a person's activities. The social model of disability emphasizes people's ability to function in their particular physical and social environment. Disability therefore arises when barriers prevent people with functional limitations caused by age, disease, injury, or other causes from participating fully in society.

Von Interesse ist hier, dass Behinderung dennoch zunächst als ein medizinisches Problem festgehalten wird, um anschließend eine Perspektive zu ergänzen, die stark am sozialen Modell der Behinderung orientiert ist. Trotz der Tatsache, dass Behinderung damit immer auch in einem relationalen Sinne verstanden wird, verbleibt eine medizinische Grundorientierung in beiden Diskurssträngen erhalten. Diese ist für den Erhalt der jeweiligen diskursiven Strukturen allerdings auch notwendig, da im Bereich der sozialen Absicherung ein Defizit, das einen Rentenanspruch mit sich bringt, jeweils am Individuum festgemacht wird und der Diskursstrang der medizinischen Interventionen sowieso grundsätzlich auf einer medizinischen Argumentation aufbaut. Nicht verhandelt werden dabei die eigentlichen Widersprüche, die sich in der Auseinandersetzung mit dem dritten Diskursstrang ergeben würden.

\section{Diskussion}

Die Ausführungen entlang dreier Diskursstränge, die sich in den Dokumenten der Weltbankgruppe finden und Bezug auf das Thema Behinderung nehmen, zeigen eindrücklich, wie sich neue diskursive Muster entlang von auftauchenden Problemstellungen entwickeln. Problemstellungen und Lösungen sind immer auch mit den Personen verbunden, die die Fragen stellen bzw. die Lösungen liefern, dies gilt insbesondere auch für Problemstellungen im Zusam- 
menhang mit Behinderung (vgl. Reynolds Whyte/Ingstad 2007; Weisser 2007). ${ }^{6}$ Im Kontext der Weltbank wird dies bspw. besonders deutlich, als mit Judith E. Heumann eine Exponentin der Behindertenbewegung ihr Wissen in die Auseinandersetzung miteinbringt und damit einen radikalen Bruch mit dem bisherigen Problemformulierungen im Kontext von Behinderung erzeugt.

Als besonders problematisches Muster in der Auseinandersetzung mit Behinderung zeigt sich - sowohl in den Debatten zu medizinischen Interventionen als auch denjenigen zur sozialen Absicherung - ein medizinisch orientiertes Verständnis von Behinderung, das wenig Raum für die Diskussion der eigentlichen Lebensverhältnisse von Menschen mit Behinderung lässt. Im Themenbereich der medizinischen Interventionen wird das an sich schon problematische Muster noch deutlich problematischer, wenn Behinderung im Rahmen von Kosteneffizienzberechnung eine totale Abwertung erfährt. Im Themenbereich der sozialen Absicherung ist hingegen eher zu problematisieren, dass eine komplett abstrahierte Abhandlung in Kombination mit einem starken Misstrauen gegenüber der Kategorie Behinderung zu ungünstigen Aushandlungsprozessen führt.

Erfreulicherweise entsteht im Übergang zum neuen Jahrtausend innerhalb der Weltbank dann eine neue Auseinandersetzung, die sich teilweise sogar an einem emanzipatorischen Verständnis orientiert und auch zum Ziel hat, die (diskursiven) Strukturen der gesamten Organisation zu verändern (auch wenn sie die ökonomische Orientierung nicht in Frage stellt). Obwohl die empirischen Daten nur einen beschränkten Rückschluss darauf zulassen, wie erfolgreich die neue Strategie der Weltbank mit der Implementation eines ,Disability \& Development Teams' längerfristig ist - sie ist zumindest empirisch noch nicht abschliessend geklärt -, kann bisher davon ausgegangen werden, dass sich die grundlegenden Muster und Strukturen der Diskursstränge nicht verändern. D.h. es lässt sich in den zu problematisierenden diskursiven Strukturen eine gewisse Stabilität erkennen.

Vor dem Hintergrund der komplexen Struktur und der Organisationsgrösse der Weltbank ist abschließend zu betonen, dass die Ausführungen zu deren Behinderungsdiskurs als Ausgangspunkt für weiterführende Forschung und nicht als abschließende Beschreibung zu verstehen sind. Dies gilt aber zugleich nicht nur für die Untersuchung der Weltbank, sondern ist im Bereich der international (vergleichenden) Forschung zu den Themen Inklusive Bildung, Behinderung oder auch Sonderpädagogik eher die Regel als die Ausnahme (vgl. Biermann et al. 2015). Für die Weltbank bieten sich, neben einer Weiterverfolgung der diskursiven Konstruktion von Behinderung und dem Vergleich mit anderen internationalen Organisationen oder Ländern, unterschiedlichste Zugänge für Forschung an. Insbesondere stellt sich die Frage, welche Auswirkungen die Projekte und die dahinterstehenden diskursiven Konstruktionen in konkreten Lebenssituationen von Menschen mit Behinderung haben. Oder auch die Frage, wo und aus welchem Grund eigentlich welche Projekte umgesetzt werden, wer davon profitiert und wer nicht mitberücksichtigt wird. D.h. es geht hier um Forschungsvorhaben, die auch über die klassische Komparatistik hinausgehen und sich mit Fragen beschäftigen, wie sich verschiedene Akteure in einer komplexen Weltgesellschaft eigentlich beeinflussen und wie ihr agieren gekoppelt ist.

Für die Frage des Policy-Making im Kontext von Inklusion zeigt die Analyse der Weltbank zwei Dinge sehr deutlich auf, die an sich nicht überraschend aber dennoch zentral sind. (1) Es ist entscheidend, dass die richtigen Personen (dabei insbesondere Menschen mit Behinderung) in Entscheidungsprozesse nicht nur miteinbezogen werden, sondern auch in zen-

6 Dies gilt auch für die konkreten Aushandlungen der jeweiligen Problemlösungen, was sich ebenfalls in den Dokumenten der Weltbank zeigt, hier aus Platzgründen aber nicht dargestellt werden konnte. 
tralen Positionen platziert sind. (2) Eine zentrale Herausforderung für die Umsetzung von Inklusion ergibt sich in Themenfeldern, deren grundlegende Struktur bzw. auch deren diskursive Muster die Logik des Themas Inklusion nicht von Grund auf mitberücksichtigen bzw. diese sogar negieren. Zieht man die Erkenntnisse aus der Analyse des Weltbank-Diskurses zu den Themenfeldern „,medizinische Interventionen“ und „soziale Absicherung“ bei, muss man unweigerlich zum Schluss kommen, dass ein grundlegendes Neudenken dieser Themenfelder anstehen würde.

\section{Literatur}

Andrews, Emily S./Rashid, Mansoora (1996): The Financing of Pension Systems in Central and Eastern Europe. An Overview of Major Trends and their Determinants, 1990-1993. Washington, DC: The World Bank.

Baldus, Marion (2016): Laute Inklusionsrhetorik - stille Selektion. In: Hedderich, Ingeborg/ Zahnd, Raphael (Hrsg.): Teilhabe und Vielfalt: Herausforderungen einer Weltgesellschaft. Bad Heilbrunn: Klinkhardt, S. 379-387.

Barlow, Robin/Grobar, Lisa M. (1986): Cost and benefits of controlling parasitic diseases. Population, Health and Nutrition Department technical note. PHN 8517. Washington, DC: The World Bank.

Barnes, Colin (2009): Disability in a Majority World Context. A materialist account. Manchester: Metropolitan University.

Barnes, Colin/Sheldon, Alison (2010): Disability, politics and poverty in a majority world context. In: Disability \& Society 25, 7, S. 771-782.

Barr, Nicholas (1992): Russian Federation - Income Transfers and Social Safety Net in Russia. 11168. Washington, DC: The World Bank.

Béland, Daniel/Orenstein, Mitchell A. (2013): International organizations as policy actors: An ideational approach. In: Global Social Policy 13, 2, S. 125-143.

Biermann, Julia/Graf, Erich Otto/Kramann, Michaela/Proyer, Michelle D./Reisenbauer, Simon/ Schiemer, Margarita/Wlodarczyk, Sandra/Zahnd, Raphael (2015): ,Behinderungsforschung in globaler Perspektive“ - Bericht aus der Arbeit der agru_int. In: Leonhardt, Annette/Müller, Katharina/Truckenbrodt, Tilly (Hrsg.): Die UN-Behindertenrechtskonvention und ihre Umsetzung. Beiträge zur Interkulturellen und International vergleichenden Heil- und Sonderpädagogik. Bad Heilbrunn: Klinkhardt, S. 224-231.

Braithwaite, Jeanine (1995): The Old and New Poor in Russia. Trends in Poverty. 21227. Washington, DC: The World Bank.

Braithwaite, Jeanine/Carroll, Richard/Mont, Daniel/Peffley, Karen (2008): Disability and Development in the World Bank: FY2000-2007. 45178. Washington, DC: The World Bank.

Brandecker, Nora (2007): Der Wandel der Bildungspolitischen Ansichten der Weltbank. Working Paper. Nr. 82. Mainz: Institut für Ethnologie und Afrikastudien, Johannes-Gutenberg-Universität.

Chhibber, Ajay/Commander, Simon John/Evans, Alison Margaret/Fuhr, Harald L./Kane, Cheikh T./Leechor, Chad/Levy, Brian David/Pradhan, Sanjay/Weder, Beatrice Silvia (1997): World Development Report 1997: The state in a changing world. 17300. Washington, DC: The World Bank. 
Colletta, Nat J./Kostner, Markus/Wiederhofer, Ingo (1996): Case Studies in War-to-Peace Transition. The Demobilization and Reintegration of Ex-Combatants in Ethiopia, Namibia, and Uganda. WDP331. Washington, DC: The World Bank.

Degener, Theresia (2005): Antidiskriminierungsrechte für Behinderte: Ein globaler Überblick. In: Zeitschrift für ausländisches öffentliches Recht und Völkerrecht 65, 4, S. 887-935.

Demirguc-Kunt, Asli/Schwarz, Anita (1995): Costa Rican Pension System: Options for Reform. WPS1483. Washington, DC: The World Bank.

Durkin, Maureen S./Schneider, Helen/Pathania, Vikram S. /Nelson, Karin B./Solarsh, Geoffrey C./Bellows, Nicole/Scheffler, Richard M./Hofman, Karen J. (2006): Learning and Developmental Disabilities. In: Jamison, Dean T./Breman, Joel G./Measham, Anthony R./Alleyne, George/Claeson, Mariam/Evans, David B./Jha, Prabhat/Mills, Ann/Musgrove, Philipp (Hrsg.): Disease control priorities in developing countries, second edition. New York: Oxford University Press, S. 933-952.

Gasteiger, Ludwig (2008): Michel Foucaults interpretative Analytik und das unbestimmte Ethos der Kritik. In: Freikamp, Ulrike/Leanza, Matthias/Mende, Janne/Müller, Stefan/Ullrich, Peter/Voß, Heinz-Jürgen (Hrsg.): Kritik mit Methode? Forschungsmethoden und Gesellschaftskritik. Berlin: Karl Dietz Verlag, S. 19-31.

Grech, Shaun (2009): Disability, poverty and development: critical reflections on the majority world debate. In: Disability \& Society 24, 6, S. 771-784.

Greve, Jens/Heintz, Bettina (2005): Die ,Entdeckung' der Weltgesellschaft. Entstehung und Grenzen der Weltgesellschaftstheorie. Zeitschrift für Soziologie, Sonderheft ,Weltgesellschaft', S. 89-119.

Grosh, Margaret/del Ninno, Carlo/Tesliuc, Emil/Ouerghi, Azedine (2008): For Protection and Promotion. The Design and Implementation of Effective Safety Nets. 45496. Washington, DC: The World Bank.

Guernsey, Katherine/Nicoli, Marco/Ninio, Alberto (2006): Making Inclusion Operational. Legal and Institutional Resources for World Bank Staff on the Inclusion of Disability Issues in Investment Projects. 39475. Washington, DC: The World Bank.

Guernsey, Katherine/Nicoli, Marco/Ninio, Alberto (2007): Convention on the Rights of Persons with Disabilities. Its Implementation and Relevance for the World Bank. 39983. Washington, DC: The World Bank.

Harvey, David (2007): A Brief History of Neoliberalism. New York: Oxford University Press.

Herkenrath, Marc (2012): Macht, Herrschaft und die Rolle oppositioneller Akteure im Weltsystem. In: Imbusch, Peter (Hrsg.): Macht und Herrschaft: Sozialwissenschaftliche Theorien und Konzeptionen. Wiesbaden: VS Verlag für Sozialwissenschaften, S. 451-472.

Holzmann, Robert/Guven, Melis U. (2009): Adequacy of Retirement Income after Pension Reforms in Central, Eastern, and Southern Europe: Eight Country Studies. 47812. Washington, DC: The World Bank.

Holzmann, Robert/Palmer, Edward (2006): Pension Reform - Issues and Prospects for Non-Financial Defined Contribution (NDC) Schemes. 35347. Washington, DC: The World Bank.

Jamison, Dean T./Breman, Joel G./Measham, Anthony R./Alleyne, George/Claeson, Mariam/ Evans, David B./Jha, Prabhat/Mills, Ann/Musgrove, Philip (2006): Disease Control Priorities in Developing Countries, Second Edition. 41460. Washington, DC: The World Bank.

Jamison, Dean T./Gelband, Hellen/Horton, Susan E./Jha, Prabhat K./Laxminarayan, Ramanan/ Mock, Charles N./Nugent., Rachel (2017): Disease Control Priorities. Improving Health and Reducing Poverty. 121615. Washington, DC: The World Bank.

Jamison, Dean T./Mosley, W. Henry/Measham, Anthony R./Bobadilla, José Luis (1993): Disease Control Priorities in Developing Countries. 12384. New York: The World Bank.

Kamarck, Andrew M. (1976): The Tropics and Economic Development. A Provocative Inquiry into the Poverty of Nations. Baltimore and London: The Johns Hopkins University Press. 
Kiuppis, Florian (2014): Heterogene Inklusivität, inklusive Heterogenität. Fallstudie über den Bedeutungswandel imaginierter pädagogischer Konzepte im Kontext internationaler Organisationen. Münster: Waxmann Verlag.

Koch, Martin (2008): Verselbständigungsprozesse internationaler Organisationen. Wiesbaden: VS Verlag für Sozialwissenschaften.

Köbsell, Swantje/Hirschberg, Marianne (2021 i.d.B.): Disability Studies in Education. In: Köpfer, Andreas/Powell, Justin J.W./Zahnd, Raphael (Hrsg.): Handbuch Inklusion international. Opladen: Budrich, S. 127-146.

Lynch, James (1994): Provision for Children with Special Educational Needs in the Asia Region. WTP261. Washington, DC: The World Bank.

Maschke, Michael (2008): Behindertenpolitik in der Europäischen Union. Lebenssituation behinderter Menschen und nationale Behindertenpolitik in 15 Mitgliedstaaten. Wiesbaden: VS Verlag für Sozialwissenschaften.

McGreevey, William (1990): Social Security in Latin America. Issues and Options for the World Bank. WDP110. Washington, DC: The World Bank.

Mejeh, Mathias (2021 i.d.B.): Inklusive Bildung als Institution am Beispiel Schweiz. In: Köpfer, Andreas/Powell, Justin J.W./Zahnd, Raphael (Hrsg.): Handbuch Inklusion international. Opladen: Budrich, S. 221-238.

Metts, Robert L. (2000): Disability Issues, Trends and Recommendations for the World Bank. Washington, DC: The World Bank.

Naue, Ursula (2011): Behindertenpolitik heute. Zwischen alten Inhalten und neuen Möglichkeiten. Eine vergleichende Studie. Saarbrücken: VDM Verlag.

Nguyen, Nguyen X. (1994): Social Security Issues and Elements of Reform. 13004. Washington, DC: The World Bank.

Orenstein, Mitchell A. (2008): Privatizing Pensions. The Transnational Campaign for Social Security Reform. Princeton, NJ: Princeton University Press.

Pearson, Lester Bowles (1970): Partners in development. Report of the Commission on International Development. London: Pall Mall Press.

Pereira, João Márcio Mendes (2016): Recycling and expansion. An analysis of the World Bank agenda (1989-2014). In: Third World Quarterly, S. 1-22.

Peters, Susan J. (2003): Inclusive Education. Achieving Education for All by Including Those with Disabilities and Special Education Needs. 26690. Washington, DC: The World Bank.

Powell, Justin J. W. (2016): Neo-Institutionalismus. In: Hedderich, Ingeborg/Biewer, Gottfried/ Markowetz, Reinhard/Hollenweger, Judith (Hrsg.): Handbuch Inklusion und Sonderpädagogik. Bad Heilbrunn: Julius Klinkhardt, S. 680-684.

Prescott, Nicholas/Prost, Andre (1983): The Cost-Effectiveness of Blindness Prevention by the Onchocerciasis Control Program in Upper Volta. Working Paper (Numbered Series). RES7. Washington, DC: The World Bank.

Prost, André/Jancloes, Michel (1993): Rationales for Choice in Public Health. The Role of Epidemiology. In: Jamison, Dean T./Mosley, W. Henry/Measham, Anthony R./Bobadilla, José Luis (Hrsg.): Disease Control Priorities in Developing Countries. New York: Oxford University Press, S. 741-746.

Reynolds Whyte, Susan/Ingstad, Benedicte (2007): Introduction. Disability Connections. In: Reynolds Whyte, Susan/Ingstad, Benedicte (Hrsg.): Disability in Local and Global Worlds. Berkeley, Los Angeles, London: University of California Press, S. 1-29.

Rittberger, Volker/Zangl, Bernhard/Kruck, Andreas (2013): Internationale Organisationen. Wiesbaden: Springer.

Saleh, Karima/Bitran, Ricardo/Couttolenc, Bernard F. (2018): The Financial Sustainability of HIV/AIDS and Universal Health Coverage Programs in Sub-Saharan Africa. AUS0000397. Washington, DC: The World Bank. 
Soldatic, Karen (2013): The transnational sphere of justice. Disability praxis and the politics of impairment. In: Disability \& Society 28, 6, S. 744-755.

Stienstra, Deborah (2002): DisAbling Globalisation. Rethinking Global Political Economy with a Disability Lens. In: Global Society 16, 2, S. 109-121.

Stiglitz, Joseph E. (1998): More Instruments and Broader Goals. Moving toward the PostWashington Consensus. Helsinki: UNU/WIDER.

The World Bank (1975): Health. Policy Paper. 11019. Washington, DC: The World Bank.

The World Bank (1991): Poland - Income Support and the Social Safety Net. Policies for the Transition. 9661. Washington, DC: The World Bank.

The World Bank (1993a): Kyrgyzstan - Social Protection in a Reforming Economy. 12362. Washington, DC: The World Bank.

The World Bank (1993b): Ukraine - Employment, Social Protection, and Social Spending in the Transition to a Market Economy. 11176. Washington, DC: The World Bank.

The World Bank (1994): Averting the Old Age Crisis. Policies to Protect the Old and Promote Growth. 13584. Washington, DC: The World Bank.

The World Bank (1995): Hungary - Structural Reforms for Sustainable Growth. 13577. Washington, DC: The World Bank.

The World Bank (1997): Lithuania - Social Policy and Community Social Services Development Project. 15376. Washington, DC: The World Bank.

The World Bank (1998): Brazil - Social Security Special Sector Adjustment Loan Project. P7277. Washington, DC: The World Bank.

The World Bank (1999): Bosnia and Herzegovina - Emergency Recovery Project. 19481. Washington, DC: The World Bank.

The World Bank (2019a): Barber Conable. http://www.worldbank.org/en/about/archives/history/ past-presidents/barber-conable [Zugriff 19.07.2019].

The World Bank (2019b): History. http://www.worldbank.org/en/about/history [Zugriff 19.07. 2019].

The World Bank (2019c): James David Wolfensohn. http://www.worldbank.org/en/about/archives/history/past-presidents/james-david-wolfensohn [Zugriff 19.07.2019].

The World Bank (2019d): What We Do. http://www.worldbank.org/en/about/what-we-do [Zugriff 19.07.2019].

The World Bank Group (2016): News \& Broadcast - Mainstreaming Disability - World Bank Appoints New Disability Advisor. http://web.worldbank.org/WBSITE/EXTERNAL/NEWS/ 0,,contentMDK:20048666 menuPK:34463 pagePK:64003015 piPK:64003012 theSitePK: 4607,00.html [Zugriff 19.07.2019].

The World Bank Group (2019): World Bank Group Archivists' Chronology 1944-2013. Washington, DC: The World Bank.

Tobis, David (2000): Moving from Residential Institutions to Community-Based Social Services in Central and Eastern Europe and the Former Soviet Union. 20943. Washington, DC: The World Bank.

Ullrich, Peter (2008): Diskursanalyse, Diskursforschung, Diskurstheorie. Ein- und Überblick. In: Freikamp, Ulrike/Leanza, Matthias/Mende, Janne/Müller, Stefan/Ullrich, Peter/Voß, HeinzJürgen (Hrsg.): Kritik mit Methode? Forschungsmethoden und Gesellschaftskritik. Berlin: Karl Dietz Verlag, S. 19-31.

UNESCO (1994): The Salamanca Statement and Framework for Action on Special Needs Education. Paris: UNESCO.

Valdés-Prieto, Salvador (1994): Administrative Charges in Pensions in Chile, Malaysia, Zambia, and the United States. WPS1372. Washington, DC: The World Bank.

Vittas, Dimitri (1995): Sequencing Social Security, Pension, and Insurance Reform. WPS1551. Washington, DC: The World Bank. 
Walters, Dylan David/Eberwein, Julia Dayton/Schultz, Linda Brooke/Kakietek, Jakub Jan/ Ahmadzai, Habibullah/Mustaphi, Piyali/Saeed, Khwaja Mir Ahad/Zawoli, Mohammad Yonus/Shekar, Meera (2018): An Investment Framework for Nutrition in Afghanistan: Estimating the Costs, Impacts, and Cost-Effectiveness of Expanding High-Impact Nutrition Interventions to Reduce Stunting and Invest in the Early Years. 126287. Washington, DC: The World Bank.

Weisser, Jan (2004): Was leistet die Diskursanalyse in der Sonderpädagogik? In: Heilpädagogik online 3, 4, S. 23-45.

Weisser, Jan (2005): Sonderpädagogische Probleme formulieren: Konzepte und die Logik sonderpädagogischen Wissens. In: Horster, Detlef/Hoyningen-Süess, Ursula/Liesen, Christian (Hrsg.): Sonderpädagogische Professionalität. Beiträge zur Entwicklung der Sonderpädagogik als Disziplin und Profession. Wiesbaden: VS Verlag für Sozialwissenschaften, S. 97-115.

Weisser, Jan (2007): Für eine anti-essentialistische Theorie der Behinderung. In: Behindertenpädagogik, 46, 3/4, S. 237-249.

Williamson, John (2000): What Should the World Bank Think about the Washington Consensus? In: World Bank Research Observer 15, 2, S. 251-64.

World Health Organization (2001): International Classification of Functioning, Disability and Health: ICF. Geneva: World Health Organization.

World Health Organization (2018): Disability and Health. https://www.who.int/news-room/factsheets/detail/disability-and-health [Zugriff: 10.07.2019]

World Health Organization/The World Bank (2011): World Report on Disability. Bd. 1. Washington, DC: World Health Organization.

Wyss, Kurt (2007): Workfare. Sozialstaatliche Repression im Dienst des globalisierten Kapitalismus. Zürich: Edition 8.

Zahnd, Raphael (2017): Behinderung und sozialer Wandel. Eine Fallstudie am Beispiel der Weltbank. Bad Heilbrunn: Klinkhardt.

Ziai, Aram (2006): Zwischen Global Governance und Post-Development. Entwicklungspolitik aus diskursanalytischer Perspektive. Münster: Westfälisches Dampfboot. 


\title{
7 Disability Studies in Education: Normalität/en im inklusiven Unterricht und im Bildungsbereich hinterfragen
}

\author{
Marianne Hirschberg, Swantje Köbsell
}

\begin{abstract}
Disability Studies und Pädagogik haben lange getrennte Diskurse gepflegt, wobei sich die Disability Studies von der traditionellen (Sonder-)Pädagogik eindeutig abgegrenzt haben. Ausgehend von den USA etabliert sich jedoch in den letzten Jahren mit den Disability Studies in Education (DSE) ein Diskursstrang, der das Behinderungsverständnis der Disability Studies in einen kritischen pädagogischen Diskurs einbringt, um so der gemeinsamen Bildung behinderter und nicht behinderter Schüler*innen eine emanzipatorische Basis zu geben, die auch intersektionale Aspekte berücksichtigt.
\end{abstract}

\section{Einleitung}

Vom deutschen Inklusionsdiskurs weitgehend unbemerkt, hat sich in den USA ein Diskursstrang entwickelt, der sich auf Basis der theoretischen Grundlagen der Disability ${ }^{1}$ Studies kritisch mit dem vorherrschenden Pädagogik- und Behinderungsverständnis der (Sonder-)Pädagogik auseinandersetzt. Ein zentraler Aspekt ist hierbei die kritische Reflexion von Ableism in pädagogischen Kontexten allgemein sowie im Hinblick auf Einstellungen, Vorurteile und Umgangsweisen damit von Lehrer*innen.

Das Anliegen der Disability Studies in Education (DSE) kann mit ,change from within“ beschrieben werden: Indem sich Wissenschaftler*innen und Praktiker*innen im Austausch miteinander in ihren kritischen Haltungen bestärken, bekommen sie „Rüstzeug“ für die pädagogische Praxis, das dabei hilft, diese von innen heraus zu verändern.

Der Beitrag wird zunächst die Disability Studies und ihr Verständnis von Behinderung darstellen. Hierfür zentral ist die Auseinandersetzung mit der Konstruktion von (physischer, sensorischer, psychischer und kognitiver) Normalität, weil auf dieser der im Konstruktionsprozess von Behinderung wirkmächtige Ableism fußt. Daran anschließend wird der Ansatz der Disability Studies in Education (DSE), seine Geschichte und seine Potenziale geschildert,

1 Anders als bei anderen konstruierten Kategorien gesellschaftlicher Ungleichheit wie gender, class, race, gibt es im Kontext von Behinderung keinen Begriff, der behinderte und (noch) nicht behinderte Menschen ,unter einem Dach“ zusammenführt und darüber verdeutlicht, dass es das eine ohne das andere nicht gibt (Linton 2005: 519f.). Um hierauf zu verweisen, setzt sich immer mehr die Schreibweise dis/ability durch, die darüber hinaus auch die „Flüssigkeit“ der Kategorie (wer gehört wann/warum noch/nicht dazu?) aufzeigt; die Schreibweise dis_ability, im Sinne eines ,,ability gap“, weist noch stärker auf die fließende Grenze zwischen Nicht_behinderung hin. 
der den Inklusionsdiskurs um eine kritische Perspektive auf Nicht_behinderung und den Umgang mit Verschiedenheit bereichert, wie insbesondere der neue Ansatz DisCrit zeigt, der Disability Studies in Education mit Critical Race Theory verbindet. Abschließend nehmen wir eine Einordnung von DSE im Hinblick auf ihr Potenzial für die schulische Inklusion vor.

\section{Disability Studies}

Die Disability Studies wurden in den 1970er Jahren nahezu zeitgleich, jedoch weitgehend unabhängig voneinander, in den USA und Großbritannien entwickelt. Die Entwicklung ging in beiden Ländern von einer - durch Frauenbewegung, Bürgerrechtsbewegung der Afroamerikaner*innen und durch die Student*innenbewegung inspirierten - politischen Behindertenrechtsbewegung aus. Hintergrund dieser Entwicklung wiederum war die sich zunehmend breiter durchsetzende Erkenntnis bei behinderten Menschen selbst, dass es keinen zwangsläufigen, quasi-natürlichen Zusammenhang zwischen der individuellen Abweichung von gesellschaftlichen Normalitätserwartungen und gesellschaftlichem Ausschluss gab. Vielmehr handelte es sich dabei um eine mögliche gesellschaftliche Antwort auf eben diese Abweichungen, die jedoch auch anders ausfallen bzw. verändert werden könnte (Köbsell 2012b).

Diese Erkenntnis wurde 1976 von der britischen Union of the Physically Impaired against Segregation (UPIAS) folgendermaßen auf den Punkt gebracht:

In our view it is society which disables physically impaired people. Disability is something imposed on top of our impairments by the way we are unnecessarily isolated and excluded from full participation in society (Oliver 1996: 33, Hervorh. MH/SK).

Die Behinderungsdefinition der UPIAS bildete die Grundlage des sozialen Modells von Behinderung, das in Großbritannien durch den kürzlich verstorbenen „Vater der britischen Disability Studies“" Mike Oliver ausformuliert wurde.

Auch in den USA bildete die Sichtweise von Behinderung als sozialem Tatbestand, als gesellschaftlich hergestellt, die Grundlage der Entwicklung der Disability Studies. Diese Perspektive stand in beiden Ländern diametral zum traditionellen Modell von Behinderung - als individuelles bzw. medizinisches Modell bezeichnet -, das nicht zwischen Behinderung und Beeinträchtigung differenzierte und Behinderung im Körper der betroffenen Menschen verortete. Diese Sichtweise hatte für die aus dieser Warte als „,behindert“ Angesehenen weitreichende Folgen: Sie galten als hilflose, bedürftige, abhängige und geschlechtslose Gruppe, die nicht für sich selbst sprechen konnte (und durfte). Deswegen wurden Expert*innen benötigt, die für „die Behinderten“ sprachen, und entsprechend groß war die Dominanz von Behinderungsspezialist*innen im Diskurs zu Behinderung. Dies bedeutete für behinderte Frauen und Männer, dass sie nicht als Träger*innen von Rechten angesehen wurden, ihnen keinerlei Wege der politischen Selbstvertretung zur Verfügung standen und somit ihr Leben von weitreichender Fremdbestimmung in allen Bereichen gekennzeichnet und gesellschaftliche Teilhabe kaum möglich war.

Die Erkenntnis, dass Behinderung durch gesellschaftliche Prozesse der Nicht-Anerkennung und Ausgrenzung hergestellt wird, und dass die damalige Situation durch massive Menschenrechtsverletzungen an behinderten Menschen geprägt war (Köbsell 2012b: 15), ermög- 
lichte beeinträchtigungsübergreifende Solidarität ${ }^{2}$ zur aktiven Anprangerung und Bekämpfung dieses Zustandes. Wichtig war die geteilte Erfahrung des Behindert-Werdens, nicht die zugeschriebene Diagnose. Diese neue Sicht führte auch zu einer veränderten Selbstsicht, einem neuen Selbstbewusstsein (nicht ich bin ,falsch“, sondern die Art, wie gesellschaftlich mit Menschen wie mir umgegangen wird), das erstmalig den Aufbau einer positiven Identität als ,behindert“ ermöglichte (Gottschalk 1999).

Die in der neuen Sichtweise auf Behinderung vollzogene Trennung von körperlicher (Beeinträchtigung/impairment) und gesellschaftlicher Ebene (Behinderung/disability) ermöglichte es, Behinderung zu entnaturalisieren, den Prozess der Konstruktion von Behinderung zu analysieren und für gesellschaftliche Veränderungen wie rechtliche Gleichstellung und gesellschaftliche Teilhabe zu kämpfen. Durch die Erkenntnis, dass es sich bei Behinderung nicht um eine objektiv medizinisch-biologisch definierte Tatsache, sondern um eine gesellschaftlich hergestellte Ungleichheitsdimension handelt, rückten zunehmend Fragen nach den historischen und kulturellen Dimensionen von Behinderung in den Fokus.

Die interdisziplinäre Untersuchung dieser Fragen beschreibt das Tätigkeitsfeld der Disability Studies:

Allen Vertreterinnen und Vertretern der Disability Studies geht es um den Versuch, Behinderung als soziale Konstruktion zu konzeptionalisieren, d.h. gesundheitsrelevante Differenz wird nicht als (natur)-gegeben verstanden, im Sinne einer vermeintlich objektiv vorhandenen, medizinisch-biologisch definierbaren Schädigung oder Beeinträchtigung, sondern als historisches, kulturelles und gesellschaftliches Differenzierungsmerkmal (Waldschmidt 2009: 130).

Dies bedeutet auch einen vielfältigen Perspektivenwechsel in der Forschung zu Behinderung: Zum einen wird hier statt wie bisher über, nun von und mit behinderten Menschen geforscht, die so vom Forschungsobjekt zum aktiven Subjekt der Forschung werden. Auch die Blickrichtung insgesamt ändert sich: „Die Mehrheitsgesellschaft wird aus Sicht der ,Behinderung“ untersucht, und nicht umgekehrt, wie es eigentlich üblich ist" (Waldschmidt 2003: 16).

Hierbei beziehen sich Disability Studies Vertreter*innen international häufig auf Michel Foucaults Theorie, speziell auf seine Untersuchungen zur Konstruktion von Normen bzw. Normalität in der Medizin (Waldschmidt 2007; Hirschberg 2009). Ebenso wie auf Foucaults Forschungsarbeiten rekurrieren die Disability Studies auch auf die Stigmatheorie Erving Goffmans (Goffman 1967/2010) oder auf die marxistische Analyse der gesellschaftlichen Produktionsverhältnisse (Turner 2001: 255f.; Williams 2001: 129; Thomas 2004: 22). Letztere kann zeigen, dass seit der Entstehung des industriellen Kapitalismus der Standardkörper für die Fließbandproduktion in den Fabriken erforderlich wurde, wohingegen abweichende (unproduktive) Körper in Wohlfahrtseinrichtungen verwiesen wurden (Thomas 2002: 46f.). Der historisch-materialistische Hintergrund wird auch daran deutlich, dass das Soziale Modell von Behinderung als ,social oppression theory“ verstanden wird (ausführlich Oliver 1996: 30ff.). Behinderung ist dementsprechend jeweils vor dem zeitgeschichtlichen, räumlichen, kulturellen und ökonomischen Kontext zu analysieren.

2 Vor diesem Hintergrund organisierten sich zunächst Menschen mit körperlichen und Sinnesbeeinträchtigungen; Menschen mit Lernschwierigkeiten/kognitiven Beeinträchtigungen wurden vom Anspruch her mitvertreten, als Selbstvertreter*innen (z.B. im Rahmen von People First), traten sie erst später in Erscheinung. 


\section{Gegenkräfte: Normalität \& Ableism}

Wer sich mit dem Thema Behinderung beschäftigt, kommt nicht umhin, sich ebenfalls mit Normalität zu beschäftigen. Normalität ist „ohne komplementäre Anormalität schlechthin undenkbar" (Link 1999: 355) und Behinderung wird im Bereich der Anormalität verortet:

Konstitutionell anormal sind unter anderem sämtliche Phänomene, die wir heute unter dem Begriff der Behinderung zusammenfassen (Link 2004a: 135).

Was jedoch ist Normalität? Versucht man sich dem Begriff zu nähern, wird deutlich, dass er mit verschiedensten Bedeutungen gefüllt werden kann. Da ist zum einen die Gleichsetzung von Normalität mit Durchschnittlichkeit, die oftmals als langweilig und damit nicht erstrebenswert empfunden wird, was der oft in diesem Zusammenhang angeführte Spruch ,lieber lebendig als normal" verdeutlicht. Auf der anderen Seite hat Normalität eine große Anziehungskraft, was Buchtitel wie „Ich will doch nur normal sein“ (J. 2009) oder „Mein Kind ist fast ganz normal“ (Miller 1997) zum Ausdruck bringen. So betrachtet bedeutet Normalsein nicht aufzufallen, dazuzugehören. Nicht Normalsein aufgrund eigener Entscheidungen, sich beispielsweise durch Kleidungs- und Lebensstil bewusst vom Durchschnitt abzusetzen ist etwas ganz anderes, als aufgrund angeborener oder später erworbener als negativ bewerteter Merkmale aus der Normalität „herauszufallen“ und aufgrund dessen stigmatisiert oder in anderer Weise benachteiligt zu werden.

Hier interessieren jedoch die Vorstellungen von Normalität, die sich darauf beziehen, wie Menschen zu sein bzw. sich zu verhalten haben. Diesbezügliche Normalitätsvorstellungen bzw. -anforderungen sind nicht ,,in Stein gemeißelt“", sondern im Laufe der Geschichte zum Teil erheblichen Veränderungen unterworfen gewesen. So waren bis in die 1960er Jahre die Normalitätsgrenzen starr gesetzt. Es gab Dinge, die tat „man“ einfach nicht - wer sich über diese Grenzen hinwegsetzte musste mit Sanktionen rechnen. Bedeutete dies z.B. im Hinblick auf Ehescheidung und außereheliche Geburt „nur“ die soziale Ächtung, war Homosexualität ein Straftatbestand, der mit Gefängnisstrafen belegt war. ${ }^{3}$ Heute gibt es Politiker*innen und andere Menschen des öffentlichen Lebens, die in gleichgeschlechtlichen Beziehungen leben; und geschieden zu sein oder nicht verheiratete Eltern zu haben, ist längst keine Schande mehr. Das heißt jedoch nicht, dass es keine Normalitätsanforderungen mehr gäbe. Allerdings sind diese weniger offensichtlich.

Lennard Davis, ein Vertreter der amerikanischen Disability Studies, postuliert, dass Normalität in postmodernen, und wesentlich stärker noch in neoliberalen, Zeiten an Bedeutung verlöre und als Begriff durch Diversität zu ersetzen sei (Davis 2016: 3). Dennoch bliebe die Orientierung an einem geltenden, ökonomisch verwertbaren Maßstab der Leistungsfähigkeit erhalten und damit auch die Kategorie Behinderung (Davis 2016: 14). Das Normale gilt als das gesellschaftlich Erwünschte, auch wenn dies sich unterschiedlich gestalten kann (Buchner et al. 2015: 6). Entscheidend ist die eigene Positionierung innerhalb eines gesellschaftlich anerkannten Normalitätsspektrums; Buchner et al. bezeichnen dies als einen ,,medizinischbiologischen, aber auch pädagogisch definierten Normalitätskorridor" (2015: 6). Wie sich diese Positionierungen vollziehen und auf welche unterschiedlich konstruierten und konturierten Normalitäten rekurriert wird, soll im Folgenden näher ausgeführt werden.

3 Der diesen Strafen zugrundeliegende Paragraf 175 StGB wurde in der DDR 1988, in der Bundesrepublik jedoch erst 1994 endgültig aufgehoben. 


\subsection{Analyse von Normalitäten - Normalismustheorie}

Die behindertenpädagogische Tradition hat die Bedeutung der Kategorie Normalität lange ignoriert. Obwohl das Verhältnis von Behinderung und Normalität bereits Ende des 19. Jahrhunderts durch Georgens und Deinhardt thematisiert wurde, erlangte es erst ab Ende der 1970er Jahre verstärkte Aufmerksamkeit (zum Überblick s. Weinmann 2003).

Hierbei nahm die Frauenforschung in der Behindertenpädagogik eine führende Funktion ein. Ausgangspunkt war der Themenkomplex Normalität, Krankheit und Behinderung (ausführlich Schildmanns ${ }^{4}$ Veröffentlichungen seit 1983). Hinsichtlich der geringen Auseinandersetzung mit der Kategorie Normalität unterstreicht Schildmann, dass (vor der Linkschen Erarbeitung der Normalismustheorie) ein Dilemma vorlag, da Normalität im engeren Sinn nicht definiert war (Schildmann 2000: 92). Zur Kategorie Normalität führt sie - in Anlehnung an Link - aus:

Vielmehr erfassen wir ihre Bedeutung und ihre Grenzen über das, was eine jeweilige Gesellschaft unter ,abweichend' versteht (im negativen, wie im positiven, idealisierenden Sinne). Die Strukturen von Normalität lernen wir darüber kennen, wie Normalität funktioniert und wie sie produziert wird (Schildmann 2000: 92).

\subsubsection{Normalität und Normativität}

Gemäß der Normalismustheorie ist der normalistische Diskurskomplex im Gegensatz zum normativen Diskurskomplex ein Kennzeichen der Moderne. Normativ seien speziell ethische oder juristische Konzepte, normalistisch dagegen alle auf einem statistischen Wert basierenden Phänomene (Link 1999: 443). Während sich Normalität am Durchschnitt orientiere und daher deskriptiv und postskriptiv sei, sei Normativität präskriptiv. Ein Durchschnitt werde erst nachträglich festgestellt, nicht ,im Vorhinein“ wie bei normativen Normen (Link 1999: 443). Verdatung und statistische Größen stellen also ein Kennzeichen von Normalitäten dar. Exemplarisch lässt sich dies an Tableaus von Krankheits-, Behinderungs-, Geburts- oder Todesfällen erkennen, die Häufigkeiten angeben, anhand derer konstituiert wird, wie normal eine Krankheit oder Todesursache ist. Dementsprechend ist von der theoretischen Grundannahme auszugehen, dass Normalitäten produziert werden. Normalität ist daher als dynamische und nicht als statische Kategorie zu fassen.

Die Definition über Abweichungen bildet die Basis für Normalitätskonzeptionen (Link 2009: 333). Relevant für Behinderung ist das Basis-Normalfeld Leistung, da in diesem Behinderung und generell Einschränkungen der Leistungsfähigkeit definiert werden (Schildmann 2004a: 20; 2004b). Schildmann belegt dies durch zwei Beispiele des gesellschaftlich gewohnten Sprachgebrauchs:

Insbesondere die normale Entwicklung des Kindes und die durchschnittliche Arbeitsleistung des erwachsenen Menschen (gemessen an männlicher Erwerbstätigkeit) basieren auf statistischen

4 Ulrike Schildmann hat als erste behindertenpädagogische Forscherin Normalität als analytische Kategorie zur Analyse gesellschaftlicher Ungleichheiten genutzt, wie beispielsweise für die Analyse gesellschaftlicher Prozesse des Behindertwerdens. Hierbei griff sie Links Normalismustheorie auf und begründete damit deren Relevanz für die Behindertenpädagogik und pädagogische Analysen generell. 
Vereinheitlichungen und stellen die Grundlagen dafür dar, was als gesellschaftlich abweichend definiert und behandelt wird (Schildmann 2004a: 26).

Ebenso wie die Kindesentwicklung und die Erwerbstätigkeit am Basis-Normalfeld Leistung gemessen werden, werden Lernbeeinträchtigungen und die Fähigkeit zur selbständigen Lebensführung durch das Normalfeld Intelligenz bestimmt (Schildmann 2000: 92).

Mit der Normalismustheorie hat Link die „Basis eines analytisch-deskriptiven Instrumentariums“ geschaffen, das einer „,möglichst präzisen historischen Konkretisierung dienen soll“" (Link 1999: 444). In diesem theoretischen Konzept definiert er Normalismus als

die Gesamtheit aller sowohl diskursiven wie praktisch-intervenierenden Verfahren, Dispositive, Instanzen und Institutionen [...], durch die in modernen Gesellschaften Normalitäten produziert und reproduziert werden. Konstitutiv sind dabei insbesondere die Dispositive der massenhaften Verdatung, d. h. die statistischen Dispositive im weitesten Sinne: auf der Ebene der Datenerfassung, einschließlich der Befragungen, auf der Ebene der Auswertung einschließlich der mathematisch-statistischen Verteilungstheorien, auf der Ebene der praktischen Intervention einschließlich aller sozialen Um-Verteilungs-Dispositive (sic!) (Link 2004b: 223).

Normalitäten existieren also nicht per se - etwa als natürliches Phänomen -, sondern werden durch Diskurse, Institutionen oder Verfahren erst hergestellt. Zudem funktioniert der Normalismus nicht ohne Subjekte, da ein reflexives Verhältnis zwischen dem Normalismus und Subjekten besteht. Subjekte werden in ihrer Handlungsweise von Normalitäten beeinflusst, begründen diese jedoch ebenfalls, indem sie Strategien zu deren Konstitution einsetzen (Link 1999: 25).

\subsubsection{Zwei Spielarten oder Strategien der Normalität: Protonormalismus und flexibler Normalismus}

Sowohl historisch als auch systematisch lassen sich zwei entgegengesetzte Strategien des Normalismus unterscheiden: der Protonormalismus und der flexible Normalismus. Beide basieren auf dem Prinzip, dass Übergänge zwischen Normalität und Anormalität vorhanden sind. Die Grenze zwischen diesen beiden Normalismusstrategien kann von beiden Seiten überschritten werden (Link 2004a: 134).

Der Bezugspunkt der flexibel-normalistischen Strategie liegt ebenso wie bei der protonormalistischen Strategie in der Gegenüberstellung von Normalitätszone und Anormalität. Anormalitäten begrenzen die Normalitätszone zu beiden Seiten. Beide Strategien sind an die Gaußsche Verteilung angelehnt, mit der Normalität und Abweichung als statistisch basierte Verteilungen konstruiert werden (Link 2004a: 135). Die Normalitätszone tendiert bei der protonormalistischen Strategie dazu, sich so eng wie möglich zusammenzuziehen; bei der flexibelnormalistischen Strategie, sich so weit wie möglich auszudehnen. Auch bei der jüngeren, flexibel-normalistischen, Strategie werden die Pole von normal und anormal nicht aufgelöst, es handelt sich lediglich um eine verbreiterte Normalitätszone, die - sofern sie bis zum äußersten Rand ausgeweitet wird - ebenso wie bei der protonormalistischen Strategie an die Grenze zur Anormalität stößt (Link 2004a; 1998: 268). Insofern sind beide Strategien nicht vollständig voneinander zu trennen, sondern operieren ,zwar in großenteils entgegengesetzte[n] Richtungen, aber in ein und demselben Normalitäts-Feld“" (Link 2009: 125f.). Beide verfolgen die Absicht, Normalitäten herzustellen und aufrecht zu erhalten, Ereignisse oder Informationen anhand eines Durchschnitts vergleichen zu können. Sie unterscheiden 
sich in ihrer Schwerpunktsetzung, basieren allerdings beide auf einer statistischen Erfassung von Handlungen. Link beschreibt die Verbindung beider Strategien als ,enge gegenseitige Abhängigkeit“ (ebd.: 59), da sich beide gegenseitig stabilisieren.

Bei der protonormalistischen Strategie wird die Normalitätsgrenze durch mehrere Verfahren manifestiert. So wird die Normalitätszone stark komprimiert und dementsprechend der Bereich der Anormalitäten ausgeweitet. Link bezeichnet die Normalitätsgrenze in dieser Strategie treffender Weise als „Stigmagrenze“ (Link 2009: 57). Durch diese Grenze werden Auffälligkeiten negativ bewertet und aus dem Normalitätsspektrum ausgeschlossen (Exklusion). Wie bereits der Begriff Protonormalismus andeutet, enthält diese normalistische Strategie eine eher starre Normalitätsvorstellung, die sich an einem Prototyp von Normalität orientiert und an ,vornormalistische Ideologeme [wie] Natur, Gesundheit, Charakter, Industrienorm[en]“, an normative Konzepte oder an „Medizin/Psychiatrie, Biologie [und] Industrialismus" als Leitdiskurse angelehnt ist (Link 2009: 58). Das Individuum wird bei der protonormalistischen Strategie von außen gelenkt oder manipuliert und als Subjekt auf eine enge Normalität ausgerichtet. Dies kann allerdings dazu führen, dass das Subjekt den Schein der Normalität wahrt, jedoch von der Normalität abweichende Praktiken im Geheimen ausübt. Link bezeichnet diese Folge der protonormalistischen Subjekttaktiken als „Fassaden-Normalität" (ebd.: 58).

Während die protonormalistische Strategie auf den meisten gesellschaftlichen Feldern historisch zu Beginn des Normalismus dominierte, ist seit den 1950er Jahren die flexibelnormalistische Variante des Normalismus verbreiteter (Weinmann 2003: 22; Link 2004b: 224). Im Gegensatz zur protonormalistischen Strategie wird die Normalitätszone bei der flexibel-normalistischen Strategie stark ausgeweitet, und vielfältige - protonormalistisch als anormal beurteilte - Phänomene werden integriert (Inklusion). Die Grenzen zwischen Normalität und Anormalität sind fließend und variieren dynamisch, so dass eher Risikozonen als starre Grenzen bestehen (Link 2009: 57). Wie diese locker gestalteten und offenen Grenzen verdeutlichen, orientiert sich die flexibel-normalistische Strategie nicht an normativen Konzepten, sondern eher an der Ökonomie (Link 2009: 58), was sich in Wirtschaftlichkeit und Effizienz auch im pädagogischen Handeln zeigt. Das Individuum unterliegt keinem direkten, sondern einem indirekten Anpassungsdruck zur Konformität und tendiert zu selbstnormalisierenden Verhaltensweisen, indem es Risiko und Chance der Veränderung gegeneinander abwägt. Diese Subjekttaktik ermöglicht es dem Individuum, einerseits authentisch seine eigenen Präferenzen zu zeigen, andererseits neigt das Subjekt dazu, sich selbst an eine Normalität anzupassen und hierbei normalitätsbezogene Risiken zu berücksichtigen.

Weder bei der proto- noch der flexibel-normalistischen Strategie wird die Polarität von Normalität und Anormalität aufgelöst. Dementsprechend kann eine Normalität, in der das Individuum selbst und nicht ein Durchschnitt als Maßstab fungiert, nicht durch diese Strategien beschrieben werden.

\subsubsection{Normalitätsstrategien im pädagogischen Feld}

Die Ausweitung des Normalbereiches erfolgt, indem mehr und mehr protonormalistische Anomalitäten in diesen Bereich hineingenommen werden (Link 2004a: 135). Im Kontext von Behinderung waren es vor allem die Strategien der Integration (1970er bis frühe 2000er) und der Inklusion, die eine zunehmende Teilhabe behinderter Menschen am gesellschaftlichen Leben bewirkten und damit auch die Verschiebung der diesbezüglichen Normalitätsgrenzen. 
War es bis in die 1980er Jahre normal, dass behinderte Menschen in großen Einrichtungen am Stadtrand leben mussten und im Alltag der meisten Menschen gar nicht auftauchten, wurde es - durch zunehmendes Selbstbewusstsein und erkämpfte Barrierefreiheit im öffentlichen Raum - normaler, Menschen mit Beeinträchtigungen in allen Bereichen gesellschaftlichen Lebens zu begegnen.

Im Zuge des Diskurses um früher integrative, jetzt inklusive Bildung ist es auch selbstverständlicher geworden, dass als behindert geltende Kinder in Regelschulklassen unterrichtet werden. Link zufolge kann dies jedoch nicht unbegrenzt funktionieren: „Es gibt keinen Normalismus ohne Gradualismus, das heißt ohne die Annahme, dass eine bestimmte isolierbare Dimension [...] graduell und kontinuierlich ab- und zunimmt und dass diese Grade irgendwie messbar sind" (Link 2004a: 136). Im Kontext von Behinderung findet diese Messung auf der Basis der Schweregrade von Behinderung statt; in der Folge seien Menschen mit „leichteren“ Graden der Behinderung einfacher zu integrieren als solche mit „,komplexeren“ Behinderungen. Auch könne die Grenze zwischen Normalität und Anormalität nicht völlig zum Verschwinden gebracht werden, denn ,(i)rgendwo stößt auch der flexibelste Normalismus schließlich an seine Grenze“ (ebd.).

Diese Einschätzung könnte dahingehend interpretiert werden, dass Inklusion - also eine Gesellschaft, in der alle Menschen gesellschaftlich teilhaben können - in letzter Konsequenz nicht umsetzbar sei. Einen belastbaren Grund dafür gibt es allerdings nicht, sodass die Frage bleibt, wie die gesellschaftlichen Verhältnisse gestaltet sein müssten, um Inklusion zu ermöglichen (Hirschberg/Köbsell 2016). Diese Position vertreten Vertreter*innen der Integrations-/Inklusionspädagogik im Anschluss an Links Normalismustheorie. Wie eine derartige Normalitätsform, die transnormalistische Strategie, ausgeformt ist, führt Lingenauber (2003) auf der Basis der Linkschen Theorie aus. Diese Strategie basiert auf einem individuellen Maßstab, der vom statistischen Durchschnitt losgelöst ist und sich nicht auf die Polarität zwischen Behinderung und Normalität stützt (Lingenauber 2003: 177). Stattdessen wird jeder Mensch als seine je eigene Normalität angesehen und nicht zu anderen ins Verhältnis gesetzt. In transnormalistischer Perspektive wird somit eine neue, nicht ausgrenzende, Normalität hervorgebracht und Inklusion vorstellbar.

\subsection{Ableism}

Normalität wird im Kontext von Behinderung vor allem im Hinblick auf das (Nicht)Vorhandensein jeweils als „normal“ bzw. ,typisch menschlich“ (Maskos 2010: o.S.) gesetzter Fähigkeiten verhandelt. Diese Bewertungsstruktur entlang der Differenzierung nicht/fähig wird auch im deutschen Diskurs zunehmend unter dem Begriff Ableism adressiert. Dass hier die menschlichen Fähigkeiten im Fokus stehen, wird durch das vorangestellte ,able“, also „fähig“ verdeutlicht. Die Endung ,ism“ steht, wie auch bei Sexism, Racism, Classism etc. für ein gesellschaftliches Machtverhältnis, innerhalb dessen Menschen aufgrund bestimmter Merkmale diskriminiert oder privilegiert werden. Auch bei Ableism handelt es sich um gesellschaftliche Annahmen und Praktiken, mit denen die unterschiedliche und ungleiche Behandlung von Menschen mit offensichtlichen oder angenommenen Unterschieden begründet wird, indem bestimmte Arten von Selbst und Körper als perfekt, arttypisch und für das Menschsein zentral gesetzt werden. Im Gegenzug werden Menschen mit „abweichenden“ Arten von Selbst und Körper als weniger wert betrachtet. Wohin diese Sichtweise im 
Extremfall führen kann, belegen sowohl das Tötungsprogramm der Nationalsozialisten, verharmlosend „Euthanasie“ genannt, als auch der präferenzutilitaristische Ansatz des australischen Philosophen Peter Singer, der die Tötung schwerstbehinderter Neugeborener für das Gemeinwohl proklamiert (vgl. Singer 1984).

Ableistisches Denken ist und wird in uns alle hineinsozialisiert. Es beeinflusst die Einstellungen und Vorstellungen von Menschen, die sich in ableistischen Haltungen und Umgangsweisen niederschlagen, die auch Auswirkungen auf Maßnahmen und Programme für als behindert geltende Menschen haben. Ableism ist somit Teil der Dominanzkultur, die Rommelspacher als ,Ensemble gesellschaftlicher Praxen und gemeinsam geteilter Bedeutungen“" definiert, das durch „,spezifische Kategorien von Über- und Unterordnung“ gekennzeichnet ist (Rommelspacher 1995: 22f.). Ableism ist in markanter Weise an der Konstruktion von Behinderung beteiligt und ist Grundlage der Ungleichbehandlung von Menschen auch seitens Professioneller - was für die als ,behindert“ Angesehenen eingeschränkte Teilhabe in vielen gesellschaftlichen Bereichen zur Folge hat:

An ableist society is said to be one that treats non-disabled individuals as the standard of "normal living', which results in public and private places and services, education, and social work that are built to serve 'standard" people, thereby inherently excluding those with various disabilities (stopableism.org, o.J.: o.S.).

Ableism ist die Grundlage dessen, was im Deutschen als „Behindertenfeindlichkeit“ und von Wolbring (2009: 30) als „Disableism“ bezeichnet wird:

diskriminierende[s] unterdrückende[s] oder beleidigende[s] Verhalten, das aus dem Glauben entsteht, dass Menschen ohne diese ,essentiellen' Fähigkeiten anderen unterlegen seien.

Anders als der Begriff der Behindertenfeindlichkeit deckt Ableism ein ungleich umfassenderes Themenspektrum ab: Während „Behindertenfeindlichkeit“ nur das Negative fokussiert, das behinderten Menschen widerfährt, verweist Ableism nicht nur auf Ungerechtigkeiten, Ausgrenzungen und Menschenrechtsverletzungen gegenüber behinderten Menschen, sondern bezieht die gegenseitige Bedingtheit von Behinderung und Normalität genauso mit ein wie die Tatsache, dass es sich bei Ableism - wie bei Rassismus und Sexismus - um ein gesellschaftliches Machtverhältnis handelt, das alle Menschen, und nicht nur die als „,behindert“ markierten, betrifft (vgl. Köbsell 2015). Wie bei den anderen ,--ismen“ werden Unterschiede naturalisiert, Menschen in Gruppen homogenisiert, in der Zuordnung „behindert-nichtbehindert" polarisiert und der Erfüllung erwarteter Fähigkeiten entsprechend in eine hierarchische Ordnung gebracht (vgl. Rommelspacher 2009: 29). Auch hier handelt es sich um ein gesellschaftliches (Macht-)Verhältnis und nicht lediglich um individuelle Vorurteile (vgl. ebd.).

Ableism ist kulturell tief verankert und beeinflusst den individuellen wie auch gesellschaftlichen Umgang mit behinderten Menschen (Valle/Connor 2011: 18). Obwohl allgegenwärtig, wird er nur selten bemerkt und kaum infrage gestellt, denn: „Wer die Normalität auf seiner Seite weiß, hinterfragt sie nicht“ (Sierck 2013: 11). Selbst die Betroffenen nehmen Ableism und seine Auswirkungen oftmals nicht wahr, so verinnerlicht und damit „normal“ sind auch für sie die zugeschriebene gesellschaftliche Positionierung und die daraus resultierenden Umgangsweisen der alltäglichen Abwertungen und Diskriminierungen.

Fester Bestandteil von Ableism ist „,compulsory able-bodiedness“, ein Konzept, das 2003 von Alison Kafer ausgeführt wurde. Orientiert an einem wegweisenden Text von Adrienne Rich (1980), die darin das Konzept der „,compulsory heterosexuality“ entwickelte, zeigt Kafer auf, dass alle gesellschaftlichen Felder nicht nur an der verpflichtenden Heterosexualität, sondern auch an einer verpflichtenden ,able-bodiedness“ ausgerichtet sind. Sie ver- 
deutlicht dies an zahlreichen Parallelen zwischen beiden Konzepten: Sowohl Heterosexualität wie auch "able-bodiedness" werden gesellschaftlich als unpolitisch, als universelle Idee und die normale Art zu leben angesehen (Kafer 2003: 79).

Compulsory able-bodiedness is pervasive, built on the foundations of our culture, $[\ldots]$ elucidating the ableism and able-centrism of our culture (ebd.: 81).

Kafer beschreibt verpflichtende ,able-bodiedness“ als eine politische Institution, zu deren Durchsetzung nicht nur gewaltförmige Handlungen wie etwa Zwangssterilisationen, Pränataldiagnostik, ,Euthanasie“ und gewaltsame Übergriffe auf behinderte Menschen gehören:

Compulsory able-bodiedness is also instituted and maintained through less physical - although no less coercive - means, through 'verbal and nonverbal messages'. Perhaps the most basic manifestation of this system is the cultural presumption of able-bodiedness (ebd.: 80).

Aufbauend auf Kafers Ansatz verdeutlicht Robert McRuer den Bezug von verpflichtender able-bodiedness zur Erfüllung gesellschaftlicher Normalitätserwartungen bezüglich ökonomisch verwertbarer Fähigkeiten:

first, to be able-bodied is to be 'free from physical disability', just as to be heterosexual is to be 'the opposite of homosexual'. Second [...] being able-bodied means being capable of the normal physical exertions required in a particular system of labor. It is here as well that we can begin to understand the compulsory nature of able-bodiedness: in the emergent industrial capitalist system, free to sell one's labor but not free to do anything else effectively meant free to have an able body but not particularly free to have anything else (McRuer 2006: 303).

Fiona Kumari Campbell, die maßgeblich zur theoretischen Fundierung von Ableism beigetragen hat, sieht dessen Funktion darin, diese scheinbar natürliche, naturalisierte Norm der Nichtbehinderung aufrecht zu erhalten, die sich als „ethos of compulsory ablebodiedness“ (Campbell 2009: 6) durch alle gesellschaftlichen Bereiche zieht. Hierüber werde die Trennlinie hergestellt, entlang der Körper und Identitäten in einer binären Dynamik als behindert bzw. nichtbehindert markiert und positioniert werden. Ableism erzwinge eine grundlegende Trennung ('constitutional divide') zwischen

perfected naturalised humanity and the aberrant, the unthinkable, quasi-human hybrid and therefore non-human. This constitution provides the layout, the blueprint for the scaling and marking of bodies and the ordering of their terms of relation. It is not possible to have a concept of difference without ableism (ebd.).

Damit beschreibt Ableism weit mehr als die Markierung von Menschen als nicht-/behindert: Es geht vielmehr um das Markieren und Positionieren aller menschlichen Körper in Abhängigkeit vom Grad der Erfüllung bzw. der Abweichung von der ableistischen Norm.

Indem Ableism die vermeintlichen Defizite von Menschen fokussiert und Soziales biologisiert, bzw. gesellschaftliche Dimensionen ignoriert, stützt er das im akademischen und (menschen)rechtlichen Diskurs inzwischen überholte medizinische bzw. individuelle Modell von Behinderung und trägt dazu bei, es im gesellschaftlichen und institutionellen Alltag fortzuschreiben. Und so verwundert es nicht, dass sich Ableism auch in der Sprache niederschlägt. Dies zeigt sich an Schimpfwörtern wie „Spasti“ und „Mongo“, aber auch an scheinbar harmlosen Formulierungen, die dennoch die ableistischen Vorstellungen von Behinderung bzw. dem Behindertsein fortschreiben und verstärken, da auch die Medien sich dieser Art von Sprache gern bedienen. Da sind dann Menschen ,an den Rollstuhl gefesselt“, 
„leiden“ immer an ihrer Beeinträchtigung und tun, was sie tun, entweder „wegen“ oder „trotz" ihrer Beeinträchtigung (leidmedien.de o.J.: o.S.).

Dadurch, dass Ableism allgegenwärtig bei gleichzeitiger „Unauffälligkeit“ ist, beeinflusst er - wie beschrieben - das Denken über und den Umgang mit als „,behindert“ angesehenen Menschen in allen gesellschaftlichen Bereichen. Die wichtige Sozialisierungs- und Bildungsinstitution Schule bildet da keine Ausnahme. Auch hier wird Ableism auf den unterschiedlichsten Ebenen wirksam: In der Interaktion zwischen Schüler*innen und Lehrer*innen sowie zwischen den Schüler*innen, aber auch in der Auswahl von Lehrinhalten, -methoden und -materialien. Lehrer*innen und Schüler*innen sind genau wie Lehrbuchautor*innen und Verantwortliche in Verlagen für Lehrmaterialien in eine ableistische Gesellschaft hineinsozialisiert worden, in der Regel ohne dies jemals hinterfragt zu haben. Somit stellt sich für eine gelingende - gelingend im Sinne von individuelle Teilhabe wie auch gesellschaftliche Veränderung bewirkende - Inklusion in der Schule die Aufgabe, sowohl in der Institution Schule wie auch im Unterricht ableistische Diskurse, Strukturen und Praktiken zu identifizieren, kritisch zu reflektieren, sie anzusprechen und zu verändern und damit eine der wichtigsten ,Barrieren in den Köpfen' abzubauen. Genau dies ist eines der zentralen Anliegen der Disability Studies in Education.

\section{Disability Studies in Education}

\subsection{Entstehung der Disability Studies in Education}

Disability Studies in Education (DSE) entstanden im Kontext des Inklusionsdiskurses der USA durch kritische Sonderpädagog*innen, die Behinderung anders denken und konzeptionalisieren wollten als innerhalb der defizitorientierten Sonderpädagogik üblich und hiermit auch insgesamt zu einem veränderten Denken über Behinderung beitragen wollten bzw. wollen (Connor 2014). Kritisiert wurde der Konservatismus der US-amerikanischen Sonderpädagogik, die aktuelle Diskurse im Themenfeld Behinderung ignorierte. Als Folge dieses Konservativismus' fand weder eine kritische Auseinandersetzung mit dem Themenkomplex Behinderung und Normalität statt (Valle/Connor 2011: 43ff.) noch die Hinterfragung der eigenen Rolle als Sonderpädagog*innen (Connor 2014).

Die Disability Studies, die sich zwar oft mit Inklusion (Barton/Armstrong 2001), wenn auch wenig mit pädagogischen Fragen befassen, boten für die Kritiker*innen eine neue geistige Heimat: Hier fanden sie Wissenschaftler*innen und Praktiker*innen, die Behinderung als soziale Konstruktion begriffen. Vor diesem Hintergrund üben die Disability Studies grundlegende Kritik an Sonderbeschulung und Sonderpädagogik, die als zentrale Akteure des Be-hinderungsprozesses angesehen werden. Die tradierte Praxis des schulischen „Besonderns" wird dort als zentral für die Hervorbringung separierender Denk- und Handlungsstrukturen und deren fortlaufende Rechtfertigung und Fortschreibung angesehen (Goodley 2011: 138). Der Fokus der Disability Studies liegt hier somit auf der Analyse der Strukturen und Bedingungen, die Behinderung im Bildungswesen bzw. -prozess hervorbringen und weniger auf den pädagogischen Aspekten der inklusiven Bildung (Köbsell 2012a).

Vertreter*innen der Kritiker*innen nahmen Kontakt mit der US-amerikanischen Society für Disability Studies auf, in die sie ab den 1990ern ihre Themen aktiv einbrachten. Leitend 
war hierbei die Frage, wie Behinderung - insbesondere im Kontext Schule - konstruiert wird und wie ein Verständnis von Behinderung im Sinne der Disability Studies pädagogische Theorie, Forschung und Lehre sowie pädagogische Interaktionen beeinflussen kann (Baglieri et al. 2011: 268). Ergebnis dieser inhaltlichen Auseinandersetzungen war im Jahr 2000 die Gründung der „Special Interest Group (SIG) Disability Studies in Education“ innerhalb der American Educational Research Association (AERA). Seitdem organisiert die Gruppe jedes Jahr an wechselnden Orten eine Tagung, die Gelegenheit zu Austausch und inhaltlicher Vertiefung bietet. Auch werden jeweils herausragende Wissenschaftler*innen, die durch kritische Beiträge zum Verständnis von Behinderung beigetragen, ebenso ausgezeichnet wie Nachwuchswissenschaftler*innen in diesem Bereich.

Auf Interdisziplinarität und Pluralität wird großer Wert gelegt und so versammelt sich unter dem Dach der DSE ein breites Spektrum an Akademiker*innen und Praktiker*innen unterschiedlichster fachlicher Herkunft und theoretischer Prägung:

Scholarships in these areas include social constructionist or interpretist, materialist, postmodernist, poststructuralist, legal and even structural-functionalist perspectives and draws on disciplines as diverse as sociology, literature, critical theory, economics, law, history, art, philosophy, and others (Taylor 2006, zit. n. Baglieri et al. 2011: 270).

Über diese „Vielstimmigkeit“ soll sichergestellt werden, dass man sich außerhalb der traditionellen sonderpädagogischen „Pfade“ bewegt. Gemeinsame Grundlage ist ein Verständnis von Behinderung als soziale Konstruktion und die getrennte Betrachtung von individueller und gesellschaftlicher Ebene. Theoretischer Bezugspunkt ist das Soziale Modell britischer Prägung, wie in vielen Äußerungen deutlich wird: „Furthermore, we make a distinction between impairment and disability” (Baglieri et al. 2011: 270), denn „,[s]ocial models of disability $[. .$.$] provide theories of practice that disrupt much that we have taken for granted in$ education“"(ebd.: 273). Anders als in der Sonderpädagogik wird Behinderung nie ,an sich“ betrachtet, sondern in einen politischen, historischen und gesellschaftlichen Kontext gestellt. Die Interessen, Erfahrungen, Ansichten und Stimmen behinderter Menschen i.S. von „Expert*innen in eigener Sache“ dienen als wichtiger Bezugspunkt von Forschung und Lehre. Ferner spielt die kritische Auseinandersetzung mit Ableism und seinen Auswirkungen für die Konstruktion von Behinderung im schulischen Kontext eine zentrale Rolle (Gabel o.J.). Dies alles soll Defizitmodelle von Behinderung verdrängen und Gerechtigkeit, Bildungsgerechtigkeit sowie die Menschenrechte und die volle gesellschaftliche Teilhabe behinderter Menschen fördern.

\subsection{Hauptanliegen der Disability Studies in Education}

Für die bisher genannten und weitere, damit zusammenhängende Fragen möchte DSE einen Organisationsrahmen bieten, der Zusammenarbeit und Austausch ermöglicht, innerhalb dessen Fragen von sozialer Gerechtigkeit im Bildungswesen allgemein, aber vor allem im Hinblick auf den Umgang mit Verschiedenheit und insbesondere mit Behinderung im inklusiven Unterricht kritisch diskutiert werden. Hierzu gehört:

disseminating information about disability studies, encouraging educational researchers to become interested in and use disability studies, and providing alternative ways to think and talk about disability in educational research (Gabel o.J.). 
Anliegen ist, den Einfluss und die Sichtbarkeit von DSE im Inklusionsdiskurs zu erhöhen und in der Folge DSE in die Curricula der Lehrer*innenausbildung zu bringen, damit dort Behinderung nicht mehr als Defizit, sondern als Teil menschlicher Vielfalt wahrgenommen wird. Dazu gehört auch die Thematisierung von Ableism in seinen zahlreichen Erscheinungsformen, das generelle Hinterfragen von Normalitätskonzepten sowie das kritische Reflektieren der eigenen Rolle als in der Regel nicht beeinträchtigte Lehrperson, die in eine ableistischen Welt sozialisiert wurde. Dies sei "crucial because special education enculturates all educators who work within it to be unquestioning of its own practices and the status quo it helps maintain“ (Connor 2014: o.S). Über die Reflexion der eigenen Rolle hinaus - auch im Hinblick auf die Vorbildfunktion als Lehrer*in - bedürfe es jedoch auch einer kritischen Auseinandersetzung mit institutionalisierter Bildung und ihren Verwobenheiten mit gesellschaftlichen Machtstrukturen. So sollen zukünftige Lehrer*innen dazu befähigt werden, Schüler*innen mit Beeinträchtigungen bei der Entwicklung einer positiven ,disability identity“ zu unterstützen.

Der Ansatz der DSE fokussiert nicht nur Theoriebildung, Ausbildung und das praktische Unterrichtsgeschehen im Klassenraum, sondern möchte ebenso die Forschung innerhalb der (Sonder)Pädagogik verändern. Getreu dem Grundsatz „Nothing about us without us“ sollen Menschen mit Beeinträchtigungen in Forschungsprozessen zum Themenfeld „Behinderung“ eine zentrale Rolle einnehmen, denn ,disabled people are the experts on disability and that their leadership and involvement in the research process is necessary for any research about them. [...] to the greatest extent possible" (Gabel o.J.: o.S.). Angeregt wird weiter u.a. die Qualität und Bedeutung von Peer-Kontakten oder auch die Hintergründe für negative Einstellungen gegenüber behinderten Schüler*innen zu erforschen.

Zusammenfassend geht es darum, den kritischen Behinderungsdiskurs der Disability Studies in die (sonder)pädagogischen Diskurse hineinzutragen und die dort immer noch stark vom individuellen Modell von Behinderung dominierten Vorstellungen und Haltungen zu hinterfragen und zu verändern. Die Vertreter*innen der DSE setzen sich vor diesem Hintergrund für einen Bildungsdiskurs ein, der Behinderung als Konstruktion begreift, intersektional betrachtet und Normalisierungstendenzen widersteht. Durch ein verändertes Rollenverständnis von Sonder- und Regelpädagog*innen und die Entwicklung von klassenzimmertauglichen Unterrichtsbeispielen soll eine veränderte, tatsächlich inklusive Unterrichtspraxis hervorgebracht werden, die alle Schüler*innen respektvoll behandelt.

\subsection{DisCrit}

Innerhalb der DSE wird immer wieder darauf aufmerksam gemacht, dass es nicht ausreiche, Forschung ausschließlich auf Behinderung zu fokussieren, vielmehr müsse sie im Hinblick darauf erforscht werden, wie sie mit race 5 , gender, class und sexueller Orientierung

5 Im Gegensatz zu den USA wird im deutschsprachigen Raum der Begriff race (Rasse) möglichst vermieden, da er automatisch mit dem Biologismus der Rassenideologie und -hygiene der Nationalsozialist*innen und deren tödlichen Folgen assoziiert wird. Er wird, wenn nicht der englische Begriff verwendet wird, in Anführungsstriche gesetzt und oftmals mit „Ethnizität“ gepaart oder dadurch ersetzt. Allerdings ist es ohne „Rasse“ zu benennen schwierig, Rassismus zu adressieren (vgl. Lutz et al. 2010: 19ff.). 
zusammenwirke (Connor 2014). Dieser intersektionalen Betrachtung menschlicher Diversität wird für die Veränderung der (schul-)pädagogischen Praxis große Bedeutung beigemessen:

[...] our intention is to question conventional and naturalized ways of thinking about difference to bring greater balance to the intellectual grounding for understanding and responding to school failure. We are interested in generating knowledge about how macrolevel processes - such as societal attitudes about diversity - intersect with disability issues as well as how better to understand the ways that race, class, gender, language, culture, and sexual orientation shape the experience of disability (Baglieri et al. 2011: 270).

Dieses Anliegen führte zur Entwicklung von DisCrit; hier werden DSE und Critical Race Theory zusammengeführt, um das Zusammenwirken von race und Dis/ability, bzw. von Rassismus und Ableism genauer zu untersuchen: „DisCrit explores ways in which both race and ability are socially constructed and interdependent" (Annamma et al. 2016: 14). Diese Entwicklung erfolgte vor dem Hintergrund, dass in den USA Schwarze Schüler*innen deutlich öfter das US-amerikanische Äquivalent sonderpädagogischen Förderbedarfs zuerkannt wird als weißen Schüler*innen (ebd.: 11), und dass die Etikettierung für sie drastischere Folgen hat: Sie werden öfter in Sondereinrichtungen überwiesen, sind eher in Gefahr, ganz von der Schule verwiesen oder sogar in der Schule verhaftet zu werden. Auch werden Schwarze Schüler*innen, denen ein schulischer Förderbedarf attestiert wurde, wesentlich häufiger in Sondereinrichtungen beschult als weiße Schüler*innen mit dem gleichen attestierten Förderbedarf. ,This [...] is evidence that race and perceived ability (or lack thereof) are still connected within educational structures and practices today" (ebd.: 11).

Trotz dieses offensichtlichen Zusammenhangs, der seine Wurzeln in historisch gewachsenen Zuschreibungen einer „niedrigeren Entwicklungsstufe“ als Mensch und einem demzufolge geringerem Intellekt bei Schwarzen Menschen hat, gebe es kaum Untersuchungen dazu, wie race und Dis/ability miteinander interagieren. Die Disability Studies kümmerten sich nur selten um den Aspekt race, die Critical Race Studies wiederum ignorierten Dis/ability weitgehend (ebd.). Aufgrund der Fakten sei es jedoch offensichtlich, dass es einen Zusammenhang zwischen race und Dis/ability gebe. Diese Lücke, die offen zu lassen als unverantwortlich angesehen wird, möchte DisCrit nun schließen.

Neben dem Infragestellen jeglicher Normalitätskonstruktionen sind dabei sieben Grundsätze leitend: Fokussierung des Zusammenspiels von race und Disability (1), Wertschätzung multidimensionaler Identitäten (2), Betonung der sozialen Konstruktion sowohl von race als auch Dis/ability (3), Zentralsetzung der Stimme betroffener Menschen im Sinne von „Nothing about us without us“ (4), Betrachtung der rechtlichen und historischen Entwicklungen im Hinblick darauf, wie diese dazu genutzt wurden und werden, bestimmten Menschen ihre Rechte vorzuenthalten (5), kritische Reflexion der Errungenschaften der (weißen) Behindertenbewegung (6) sowie die Forderung nach und Unterstützung von Aktivismus und Protest (7) (ebd: 19).

In den Ausführungen wird deutlich, dass auch class in diesem Gefüge von Ungleichheitsdimensionen eine zentrale Rolle spielt, da Schwarze Menschen in den USA strukturell stärker von Armut betroffen sind als weiße (Davis 2008: 20). Weniger berücksichtigt wird dagegen gender, obwohl davon auszugehen ist, dass dies ebenfalls eine Rolle im Hinblick darauf spielt, wer welchem Förderschwerpunkt zugeordnet wird - führen z.B. zu ausgeprägte männliche Verhaltensweisen eher dazu, als ,,verhaltensgestört“ gelabelt zu werden.

Der Ansatz von DisCrit, das Zusammenwirken von Rassismus und Ableism zu untersuchen, hat auch für den deutschen Sprachraum Relevanz. So wird hier zwar nur von race oder 
gar Rassismus, sondern scheinbar neutral vom Migrationshintergrund gesprochen. Es ist jedoch auch hier offensichtlich, dass es einen Zusammenhang zwischen race/Migrationshintergrund und Behinderung gibt, denn ,das Vorliegen eines Migrationshintergrundes [erhöht] die Wahrscheinlichkeit, dass ein sonderpädagogischer Förderbedarf zugeschrieben wird“ (Wansing/Köbsell 2016: 476, s. auch Powell 2011/2016). Dieser Zusammenhang ist bereits seit vielen Jahren bekannt; auch, dass diese Kinder vor allem dem Förderschwerpunkt Lernen zugewiesen werden. Ebenso lässt sich ein deutlicher Einfluss des Faktors Geschlecht feststellen:

Ist es im Hinblick auf fehlende Schulabschlüsse das weibliche Geschlecht, das schlechter abschneidet, ist es im Hinblick auf die Zuschreibung von sonderpädagogischem Förderbedarf das männliche Geschlecht, das die Wahrscheinlichkeit dieser Zuschreibung erhöht (Wansing/Köbsell 2016: 476).

Auch in Deutschland ist es so, dass deutsche Kinder mit Förderbedarf eher inklusiv beschult werden, Schüler*innen mit Förderbedarf und Migrationshintergrund hingegen eher sonderbeschult werden:

Während etwa die Hälfte der deutschen Schülerinnen und Schüler mit sonderpädagogischem Förderbedarf im Förderschwerpunkt Lernen in inklusiven Schulformen unterrichtet werden, besucht die Mehrheit (drei Viertel) der ausländischen Schülerinnen und Schüler im selben Förderschwerpunkt Förderschulen (ebd.).

Viele der mit dem DisCrit-Ansatz abgesprochenen Problemlagen und Fragestellungen sind somit auch für den deutschen Kontext relevant; aber auch hier muss das Rad nicht neu erfunden werden. Annedore Prengel hat bereits 1993 in ihrer „Pädagogik der Vielfalt“, die inzwischen mehrfach unverändert neu aufgelegt wurde (zuletzt 2019), die pädagogischen Diskurse der feministischen, Migrations- und Integrationspädagogik zusammengeführt. Das Buch entstand, bevor der Begriff Intersektionalität Einzug in den Diskurs gehalten hat. Prengel verwies jedoch bereits auf ,heterogene Persönlichkeitsanteile“ und ,widersprüchliche Lebenslagen“ (2019: 199), die in Bildungsprozessen zu berücksichtigen seien und auf das „Defizit an antirassistischen Ansätzen“" (ebd.: 176). Es scheint somit durchaus lohnend, die Pädagogik der Vielfalt mit einer durch DSE und DisCrit neu geschärften „Brille“ neu zu lesen.

\section{Ausblick: DSE als kritische Begleiterin der Inklusion}

Der Diskurs zu Disability Studies in Education nimmt langsam auch im deutschen Sprachraum Fahrt auf, wie jüngere Veröffentlichungen vermuten lassen (Weisser 2013; Kremsner et al. 2020; Reisenauer/Gerhartz-Reiter 2020). Dies ist in Anbetracht der Tatsache, dass der Alltagsdiskurs zu Behinderung auch in vielen Lehrer*innenköpfen immer noch stark vom medizinischen Modell von Behinderung beeinflusst wird, außerordentlich zu begrüßen. Auswirkungen von Ableism in Schule und Klassenzimmer, in der Interaktion zwischen Schüler*innen, Lehrer*innen und anderen Mitarbeiter*innen können mittels DSE erkannt, problematisiert oder produktiv hinterfragt werden.

Behinderung im Sinne des Sozialen Modells bzw. der UN-BRK wird durch den zunehmenden Abbau von Teilhabebarrieren weniger werden. Beeinträchtigungen unterschiedlichster Art wird es aber trotz dieser Enthinderungsmaßnahmen weiterhin geben, und auch der 
kulturell tiefsitzende Ableism mit seinen Normalisierungsanforderungen wird uns noch lange erhalten bleiben, weshalb es, wenn Inklusion gewollt ist, mehr denn je notwendig ist, sich mit Beeinträchtigungs-, Behinderungs- und Normalitätskonstruktionen auseinanderzusetzen. Nur so wird es möglich sein, Aufmerksamkeit für Ableism und seine Auswirkungen zu entwickeln.

Da Beeinträchtigungen durch Inklusion nicht verschwinden, bedarf es einer Sprache, die aktiv mit Beeinträchtigung und Behinderung umgehen kann ohne zu bemitleiden, zu stigmatisieren oder zu bevormunden. Kinder mit Beeinträchtigungen müssen mit diesen ernst genommen werden - dies heißt aber, dass darüber gesprochen werden muss. Mit einer Beeinträchtigung aufzuwachsen bedeutet auch, hiermit verbundene Entwicklungsaufgaben bewältigen zu müssen, die sehr unterschiedlich sein können: Manche Kinder müssen lernen mit Schmerzen umzugehen, andere ermüden leicht, wieder andere sind bei alltäglichen Verrichtungen auf Hilfe angewiesen, brauchen mehr Zeit für viele Dinge, können aufgrund eines höheren Organisationsaufwandes nicht so spontan sein wie andere und vieles anderes mehr muss ggf. in respektvoller, anerkennender Weise angesprochen werden, um die Entwicklung zu einem selbstbewussten Menschen, der mit einer Beeinträchtigung lebt, zu unterstützen. Kurzum: „Inclusion cannot be realised through the denial of disability“ (Cook et al. 2001: 308). Vielmehr werden durch Verschweigen/Negieren von Beeinträchtigung und Behinderung gerade die Bilder transportiert, die Inklusion eigentlich überwinden will:

Why is there silence around disability? If inclusion is about embracing diversity, why are we afraid to talk about disability in the classroom? It is sobering to acknowledge just how much our students reflect what we project as teachers. Children get messages about disability from somewhere, and that somewhere includes teachers. If we are uncomfortable about disability, it stands to reason that students with and without disabilities will be too (Valle/Connor 2011: 191).

Die Disability Studies bzw. DSE können für inklusive Prozesse als kritische Begleiterin agieren (Danforth/Gabel 2008: 1) und dafür sorgen, dass für gelingende Inklusion zentrale Themen und Fragen tatsächlich berücksichtigt werden, die sonst im (Schul)Alltag unter dem Druck der Alltagsverhältnisse schnell ,unter den Tisch fallen“ oder nur oberflächlich ,abgehandelt“ werden. „DSE reminds us to always be vigilant to the pull of powerful normalizing discourses" (Morton 2012, zit. n. Connor 2014: o.S.). Schule als wichtige Sozialisierungsinstanz und zentraler gesellschaftlicher Ort kann zur Veränderung z.B. von ableistischen Sicht- und Verhaltensweisen beitragen, wenn die scheinbare Naturhaftigkeit von Behinderung ebenso wie Normalitätskonstruktionen hinterfragt, Ableism entlarvt und Umgangsweisen entsprechend verändert werden. Um dieses Ziel zu erreichen, muss in Ausbildungscurricula für angehende Lehrer*innen Raum für die Behandlung damit verbundener Themenstellungen geschaffen werden. Dazu gehören z.B. das Hinterfragen der eigenen Rolle, der eigenen Privilegien als nichtbehinderte*r Akteur*in im Bildungssystem. Die Konstruktion von Nicht/Behinderung im Bildungssystem müsste ebenso aufgenommen werden wie die Fragen danach, wie Behinderung und Ableism von den Betroffenen erlebt werden und schließlich auch, wie das veränderte Denken über Behinderung sich in pädagogische Handlungen im Klassenzimmer überführen lässt.

In diesen Fragen sind DSE der deutschsprachigen Diskussion momentan um einiges voraus. Das dort bereits Erarbeitete könnte für einen deutschsprachigen Diskurs wertvolle Denkanstöße geben. Darüber hinaus könnte eine Auseinandersetzung mit den Disability Studies dabei helfen, exkludierende Praktiken, die sich unter dem Begriff ,Inklusion“ ein fortschrittliches Mäntelchen umhängen wollen, zu entlarven und so dazu beizutragen, dass inklusive Bildungsangebote erfolgreich im Sinne der gelungenen Berücksichtigung der Be- 
dürfnisse beeinträchtigter Schüler*innen - ohne die Reproduktion behindernder Strukturen und Verhaltensweisen oder gar neuer Ausgrenzungserfahrungen - sein können.

\section{Literatur}

Annamma, Subini/Connor, David/Ferri, Beth (2016): Dis/ability Critical Race Studies (DisCrit). Theorizing at the Intersections of Race and Dis/ability. In: Connor, David/Ferri, Beth/Annamma, Subini (Hrsg.): DisCrit. Disability Studies and Critical Race Theory in Education. New York: Teachers College Press, S. 9-32.

Baglieri, Susan/Valle, Jan/Connor, David/Gallagher, Rory (2011): Disability Studies in Education. The need for a Plurality of Perspectives on Disability. In: Remedial and Special Education 32, 4, S. 267-278.

Barton, Len/Armstrong, Felicity (2001): Disability, Education and Inclusion. Cross-cultural Issues and Dilemmas. In: Albrecht, Gary/Seelman, Katherine/Bury, Michael (Hrsg.): Handbook of Disability Studies. London: Sage Publications, S. 693-710.

Buchner, Tobias/Pfahl, Lisa/Traue, Boris (2015): Zur Kritik der Fähigkeiten: Ableism als neue Forschungsperspektive der Disability Studies und ihrer Partner_innen, In: Zeitschrift Für Inklusion, (2). https://www.inklusion-online.net/index.php/inklusion-online/article/view/273, [22.11.2019].

Campbell, Fiona Kumari (2009): Contours of Ableism. The Production of Disability and Abledness. Houndmills/Basingstoke: Palgrave McMillan.

Connor, David (2014): The Disability Studies in Education Annual Conference. Explorations of Working Within and Against, Special Education. In: Disability Studies Quarterly 34, 2, http://dsq-sds.org/article/view/4257/3597 [Zugriff: 10.11.2019].

Cook, Tina/Swain, John/French, Sally (2001): Voices from Segregated Schooling. Towards an inclusive education system. In: Disability \& Society 16, 2, S. 293-310.

Danforth, Scot/Gabel, Susan (Hrsg.) (2008): Vital Questions Facing Disability Studies in Education. New York etc.: Peter Lang.

Davis, Kathy (2008): Intersectionality in Transatlantic Perspective. In: Klinger, Cornelia/Knapp, Gudrun-Axeli (Hrsg.): ÜberKreuzungen. Fremdheit, Ungleichheit, Differenz. Münster: Westfälisches Dampfboot, S. 19-35.

Davis, Lennard (2016): The End of Normal. In: Davis, Lennard (Hrsg.): The End of Normal. Identity in a Biocultural Era. Ann Arbor: University of Michigan Press, S. 1-14.

Gabel, Susan (o.J.): Disability Studies in Education, http://edr1.educ.msu.edu/dseconf/SusanGabel.htm [Zugriff:19.06.2019, inzwischen nicht mehr abrufbar].

Goffman, Erving (1967/2010): Stigma. Über Techniken der Bewältigung beschädigter Identität. Frankfurt/M.: Suhrkamp.

Goodley, Dan (2011): Disability Studies. An interdisciplinary introduction. London: Sage Publications.

Gottschalk, Ulrike (1999): „Sie haben Probleme mit Macht“. Therapieerfahrungen aus 40 Jahren. In: Rommelspacher, Birgit (Hrsg.): Behindertenfeindlichkeit. Ausgrenzungen und Vereinnahmungen. Göttingen: Lamuv, S. 97-121.

Hirschberg, Marianne (2009): Behinderung im internationalen Diskurs. Die flexible Klassifizierung von Behinderung. Frankfurt/Main: Campus.

Hirschberg, Marianne/Köbsell, Swantje (2016): Grundbegriffe und Grundlagen. Disability Studies, Diversity und Inklusion. In: Hedderich, Ingeborg/Biewer, Gottfried/ Hollenweger, Judith, 
Markowetz, Reinhard (Hrsg.): Handbuch Inklusion und Sonderpädagogik. Bad Heilbrunn: Verlag Julius Klinkhardt, S. 555-568.

J., Tina (2009): Ich will doch nur normal sein! Leipzig: Engelsdorfer.

Kafer, Alison (2003): Compulsory Bodies. Reflections on Heterosexuality and Able-Bodiedness. In: Journal of Women's History 15, 3, S. 77-89.

Köbsell, Swantje (2012a): Integration/Inklusion aus Sicht der Disability Studies. Aspekte aus der internationalen und der deutschen Diskussion. In: Rathgeb, Kerstin (Hrsg.): Disability Studies. Kritische Perspektiven für die Arbeit am Sozialen. Wiesbaden: Springer VS, S. 39-54.

Köbsell, Swantje (2012b): Wegweiser Behindertenbewegung. Neues (Selbst)-Verständnis von Behinderung. Neu-Ulm: AG SPAK.

Köbsell, Swantje (2015): Ableism - neue Qualität oder ,alter Wein in neuen Schläuchen“? In: Attia, Iman/Köbsell, Swantje/Prasad, Nivedita (Hrsg.): Dominanzkultur reloaded. Neue Texte zu gesellschaftlichen Machtverhältnissen und ihren Wechselwirkungen. Bielefeld: transcript, S. 21-34.

Kremsner, Gertraud/Zahnd, Raphael/Proyer, Michelle/Paukner, Andreas/Nemestothy, Barbara/ Prummer, Susanne/Klement, Alexander (2020): Kategorisierungsmanie und Wissenshysterie in der Lehrer*innenbildung. In: Brehme, David/Fuchs, Petra/Köbsell, Swantje/Wesselmann, Carla (Hrsg.): Disability Studies - zwischen Emanzipation und Vereinnahmung. Weinheim: Beltz Juventa.

Leidmedien.de (o.J.): https://leidmedien.de/statements/ [Zugriff: 22.11.2019].

Lingenauber, Sabine (2003): Integration, Normalität und Behinderung. Eine normalismustheoretische Analyse der Werke (1970-2000) von Hans Eberwein und Georg Feuser. Wiesbaden: VS Verlag für Sozialwissenschaften.

Link, Jürgen (1998): Von der ,Macht der Norm‘ zum ,flexiblen Normalismus‘. Überlegungen nach Foucault. In: Jurt, Joseph (Hrsg.): Zeitgenössische französische Denker. Eine Bilanz. Freiburg/Breisgau: Rombach, S. 251-268.

Link, Jürgen (1999): Versuch über den Normalismus. Wie Normalität produziert wird. 2. Aktualisierte und erweiterte Auflage. Opladen/Wiesbaden: Westdeutscher Verlag.

Link, Jürgen (2004a): „Irgendwo stößt die flexibelste Integration schließlich an eine Grenze“ Behinderung zwischen Normativität und Normalität. In: Graumann, Sigrid/Grüber, Katrin/Nicklas-Faust, Jeanne/Schmidt, Susanna/Wagner-Kern, Michael (Hrsg.): Ethik und Behinderung. Ein Perspektivwechsel. Frankfurt/New York: Campus, S. 132-141.

Link, Jürgen (2004b): Die Entdeckung des ,normal sex“ durch Raymond Pearl im Jahr 1925. Zu einem Kapitel aus der Inkubationszeit des flexiblen Kapitalismus in den USA. In: Bröckling, Ulrich/Bühler, Benjamin/Hahn, Marcus/Schöning, Matthias/Weinberg, Manfred (Hrsg.): Disziplinen des Lebens. Zwischen Anthropologie, Literatur und Politik. Tübingen: Narr Francke Attempto, S. 223-234

Link, Jürgen (2009): Versuch über den Normalismus. Wie Normalität produziert wird. 4. Aktualisierte und erweiterte Auflage. Göttingen: Vandenhoeck \& Ruprecht.

Linton, Simi (2005): What is Disability Studies? In: PMLA 120, 2, S. 518-522. https://www.jstor. org/stable/25486177?origin=JSTOR-pdf\&seq=1\#page_scan_tab_contents [Zugriff: 15.05 . 2019].

Lutz, Helma/Herrera Vivar, Maria Teresa/ Supik, Linda (2010): Fokus Intersektionalität - Eine Einleitung. In: Lutz, Helma/Herrera Vivar, Maria Teresa/ Supik, Linda (Hrsg.): Fokus Intersektionalität. Bewegungen und Verortungen eines vielschichtigen Konzeptes. Wiesbaden: VS Verlag, S. 9-30.

Maskos, Rebecca (2010): Was heißt Ableism? Überlegungen zu Behinderung und bürgerlicher Gesellschaft. http://arranca.org/43/was-heisst-ableism [Zugriff: 10.06.2019]. 
McRuer, Robert (2006): Compulsory Able-Bodiedness and Queer/disabled Existence. In: Davis, Lennard J. (Hrsg.): The Disability Studies Reader. Abingdon/New York: Routledge Chapman \& Hall, S. 301-308.

Miller, Nancy B. (1997): Mein Kind ist fast ganz normal. Leben mit einem behinderten oder verhaltensauffälligen Kind - Wie Familien gemeinsam den Alltag meistern. Stuttgart: TRIAS.

Oliver, Michael (1996): Understanding Disability. From Theory to Practice. London: Palgrave.

Powell, Justin (2011/2016): Barriers to Inclusion. Special Education in the United States and Germany. New York: Routledge.

Prengel, Annedore (2019): Pädagogik der Vielfalt. Verschiedenheit und Gleichberechtigung in Interkultureller, Feministischer und Integrativer Pädagogik. Wiesbaden: Springer VS.

Reisenauer, Catrin/Gerhartz-Reiter, Sabine (2020): Disability Studies als kritische Instanz der Schulpädagogik - Überlegungen zu Chancen und Spannungsfeldern am Beispiel pädagogischer Diagnostik. In: Brehme, David/Fuchs, Petra/Köbsell, Swantje/Wesselmann, Carla (Hrsg.): Disability Studies - zwischen Emanzipation und Vereinnahmung. Weinheim: Beltz Juventa, S. 239-245.

Rich, Adrianne (1980): Compulsory Heterosexuality and Lesbian Existence. In: Signs 5, 4, S. 631-660.

Rommelspacher, Birgit (1995): Dominanzkultur. Berlin: Orlanda.

Rommelspacher, Birgit (2009): Was ist eigentlich Rassismus? In: Melter, Claus/Mecheril, Paul (Hrsg.): Rassismuskritik. Bd. 1. Schwalbach/Taunus: Wochenschau-Verlag, S. 25-38.

Schildmann, Ulrike (1983): Lebensbedingungen behinderter Frauen. Aspekte gesellschaftlicher Unterdrückung. Gießen: Focus.

Schildmann, Ulrike (2000): Forschungsfeld Normalität. Reflexionen vor dem Hintergrund von Geschlecht und Behinderung. In: Zeitschrift für Heilpädagogik 51, 3, S. 90-94.

Schildmann, Ulrike (2004a): Normalismusforschung über Behinderung und Geschlecht. Eine empirische Untersuchung der Werke von Barbara Rohr und Annedore Prengel. Opladen: Leske und Budrich.

Schildmann, Ulrike (2004b): Geschlecht und (geistige) Behinderung. In: Wüllenweber, Ernst (Hrsg.): Soziale Probleme von Menschen mit geistiger Behinderung. Fremdbestimmung, Benachteiligung, Ausgrenzung und soziale Abwertung. Stuttgart: Kohlhammer, S. 36-45.

Sierck, Udo (2013): Budenzauber Inklusion. Neu-Ulm: AG SPAK.

Singer, Peter (1984): Praktische Ethik. Stuttgart: Reclam.

stopableism.org (o.J.): http://www.stopableism.org/p/what-is-ableism.html [Zugriff: 16.11.2019].

Thomas, Carol (2002): Disability Theory. Key Ideas, Issues, and Thinkers. In: Barnes, Colin/Oliver, Marc/Barton, Len (Hrsg.): Disability Studies Today. Cambridge: Polity Press, S. 38-57.

Thomas, Carol (2004): Disability and impairment. In: Swain, John/French, Sally/Barnes, Colin/ Thomas, Carol (Hrsg.): Disabling Barriers - Enabling Environments. London: Sage, S. 21-27.

Turner, Bryan (2001): Disability and the Sociology of the Body. In: Albrecht, Gary/Seelman, Katherine/Bury, Michael (Hrsg.): Handbook of Disability Studies. Thousand Oaks: Routledge, S. 252-266.

Valle, Jan/Connor, David (2011): Rethinking Disability. A Disability Studies Approach to Inclusive Practices. New York: McGraw Hill.

Waldschmidt, Anne (2003): ,Behinderung' neu denken. Kulturwissenschaftliche Perspektiven der Disability Studies. Tagungsdokumentation, Kassel: bifos.

Waldschmidt, Anne (2007): Macht - Wissen - Körper: Anschlüsse an Michel Foucault in den Disability Studies. In: Waldschmidt, Anne/Schneider, Werner (Hrsg.): Disability Studies, Kultursoziologie und Soziologie der Behinderung. Erkundungen in einem neuen Forschungsfeld. Bielefeld: transcript, S. 55-78.

Waldschmidt, Anne (2009): Disability Studies. In: Dederich, Markus/Jantzen, Wolfgang (Hrsg.): Behinderung und Anerkennung. Stuttgart: Kohlhammer, S. 125-133. 
Wansing, Gudrun/Köbsell, Swantje (2016): Kommentar des wissenschaftlichen Beirats zu Menschen mit Beeinträchtigungen und Migrationshintergrund. In: ISG Institut für Sozialforschung und Gesellschaftspolitik GmbH. Teilhabebericht der Bundesregierung über die Lebenslagen von Menschen mit Beeinträchtigungen. Berlin: BMAS, S. 471-491.

Weinmann, Ute (2003): Normalität und Behindertenpädagogik. Historisch und Normalismustheoretisch rekonstruiert am Beispiel repräsentativer Werke von Jan Daniel Georgens, Heinrich Marianus Deinhardt, Heinrich Hanselmann, Linus Bopp und Karl Heinrichs. Opladen: Leske und Budrich.

Weisser, Jan (2013): DisabiLiTy STudies in EducaTion. Kritische Wissensaktivitäten entfalten. In: Dederich, Markus/Greving Heinrich/Mürner, Christian/Rödler, Peter (Hrsg.): Behinderung und Gerechtigkeit. Heilpädagogik als Kulturpolitik. Gießen: Psychosozial-Verlag, S. 261-290.

Williams, Gareth (2001): Theorizing Disability. In: Albrecht, Gary/Seelman, Katherine/Bury, Michael (Hrsg.): Handbook of Disability Studies. Thousand Oaks: Sage, S. 123-144.

Wolbring, Gregor (2009): Die Konvergenz der Governance von Wissenschaft und Technik mit der Governance des „Ableism“. In: Technikfolgenabschätzung - Theorie und Praxis 18, 2, S. 29-35. 


\title{
8 Die diskursive Konstruktion inklusiver Bildung im südlichen Afrika
}

\author{
Myriam Hummel
}

\begin{abstract}
Schon 1999 stellte Dyson heraus, dass die unterschiedlichen Verständnisse von inklusiver Bildung in unterschiedlichen theoretischen Diskursen begründet liegen (Dyson 1999). Ohne es explizit zu benennen, scheint Dyson hierbei von den wissenschaftlichen Diskursen des globalen Nordens, die von Wissenschaftler*innen aus Nordamerika, Europa und Australasien geprägt sind, zu sprechen. Aufgrund globaler Deklarationen ist inklusive Bildung derzeit ein omnipräsentes Konzept in Bildungssystemen weltweit. Dieser Beitrag untersucht anhand eines systematischen Literaturreviews (Hart 1998) den wissenschaftlichen Diskurs zu inklusiver Bildung im südlichen Afrika unter besonderer Berücksichtigung der verwendeten Definitionen von inklusiver Bildung und der geführten Begründungsdiskurse. Die Ergebnisse zeigen, dass in einigen Ländern inhaltliche Aspekte des nördlichen Diskurses ohne Kontextualisierung übernommen werden, wohingegen in anderen Ländern und hinsichtlich bestimmter Themen sich eigene Diskurse herausbilden.
\end{abstract}

\section{Einleitung}

Das Konzept der inklusiven Bildung, das durch internationale Erklärungen wie die Salamanca-Erklärung (UNESCO 1994) und die UN-Konvention über die Rechte von Menschen mit Behinderungen (UN 2006) getragen wird, wurde von Wissenschaftler*innen in den letzten Jahrzehnten als globale Agenda (Pijl et al. 1997) und internationale Bewegung (Artiles/Dyson 2005: 43) bezeichnet. Es kann konstatiert werden, dass derzeit viele unterschiedliche Definitionen von inklusiver Bildung existieren und verwendet werden (Artiles et al. 2006; Ainscow et al. 2006; Slee 2011). Diese unterschiedlichen Definitionen entstehen aus verschiedenen Diskursen über die Begründung und die Umsetzung von inklusiver Bildung (Dyson 1999). Da inklusive Bildung derzeit in Bildungssystemen weltweit implementiert wird, ist es von Bedeutung auch Diskurse jenseits des globalen Nordens (Nordamerika, Europa und Australasien) zu beachten und zu untersuchen, wie diese verschiedenen räumlichen Diskurse miteinander verwoben sind oder sich gegenseitig beeinflussen. Daher beabsichtigt dieser Beitrag die Hauptstränge des wissenschaftlichen Diskurses über inklusive Bildung im südlichen Afrika ${ }^{1}$ durch ein systematisches Literaturreview darzustellen. In den Ergebnissen

1 Die Region südliches Afrika wird für den Zweck dieses Beitrags definiert als die 15 Mitgliedstaaten der Entwicklungsgemeinschaft des südlichen Afrika (SADC), nämlich: Angola, Botswana, Demokratische Republik Kongo, Lesotho, Madagaskar, Malawi, Mauritius, Mosambik, Namibia, Seychellen, Südafrika, Swasiland, Tansania, Sambia und Simbabwe (SADC 2012). 
werden der Begründungsdiskurs zu inklusiver Bildung, die verwendeten Definitionen von inklusiver Bildung in den Diskursen des südlichen Afrikas und die Beziehungen von Diskursen untereinander dargestellt.

\section{Aktuelle Inklusionsdiskurse}

Zahlreiche Wissenschaftler*innen haben wiederholt die „multiple Bedeutung“ (Artiles et al. 2006: 69) des Begriffs Inklusion betont, was zu großer Unsicherheit in der Verwendung des Begriffs führt (z.B. Ainscow et al. 2006; Armstrong et al. 2011; Slee 2011). Inklusion und inklusive Bildung werden als eine ehrwürdige Idee (Artiles/Kozleski 2016: 1), als ein heikles Thema (Hardy/Woodcock 2015: 141) und als höchst umstritten (Armstrong et al. 2011: 29) beschrieben. Sowohl Ainscow, Booth und Dyson (2006) als auch Göransson und Nilholm (2014) erarbeiteten Typologien von Inklusionsdefinitionen, die die Vielfalt der Bedeutungen darstellen. Die unterschiedlichen Typen von Inklusionsdefinitionen lassen sich zwischen den Polen weit und eng in Hinblick auf das Inklusionsverständnis verorten. Ein enges Inklusionsverständnis fokussiert Menschen mit Behinderung bzw. sonderpädagogischem Förderbedarf und adressiert vorrangig Fragen der Platzierung und Förderung. Einem weiten Inklusionsverständnis nach bezieht sich Inklusion auf die Gesellschaft im Allgemeinen, thematisiert Machtstrukturen und umfasst Menschen in ihren unterschiedlichen Heterogenitätsdimensionen.

Angesichts dieser Vielfalt von Definitionen, die zu konzeptioneller Unklarheit von inklusiver Bildung führt, weist Dyson (1999: 36) darauf hin, dass es angebracht ist, über „Inklusionen“" zu sprechen. Des Weiteren stellt er heraus, dass diese Mehrdeutigkeiten aus verschiedenen Diskursen resultieren, durch die unterschiedliche theoretische Vorstellungen von Inklusion konstruiert werden (ebd.). Dyson zufolge wird die Begründung von Inklusion von zwei verschiedenen Orientierungen getragen: dem Rechts- und Ethikdiskurs einerseits und dem Wirksamkeitsdiskurs andererseits. Der Rechts- und Ethikdiskurs befasst sich mit dem Zusammenhang von Inklusion und sozialer Gerechtigkeit. Der Wirksamkeitsdiskurs fokussiert hingegen die Frage, ob inklusive Schulen pädagogisch wirksamer und kostengünstiger sind als ein System von Regel- und Förderschulen. Die andere Dimension, in der sich die Diskurse über inklusive Bildung kategorisieren lassen, betrifft die Realisierung inklusiver Bildung. Während im politischen Diskurs der Übergang von einem segregierten zu einem inklusiven System als politischer Kampf angesehen wird, beschäftigt sich der pragmatische Diskurs mit der Frage, wie inklusive Bildung in der Praxis gestaltet werden kann. Dyson (1999: 39) betrachtet diese Diskurse nicht als konkurrierende Paradigmen, sondern als Pole entlang einer Dimension, da sie häufig interagieren.

Diskurse über inklusive Bildung werden in verschiedenen räumlichen Kontexten, aber auch innerhalb und zwischen verschiedenen Akteur*innengruppen geführt. Dyson bezieht sich in der genannten Kategorisierung der Diskurse auf Skrtic (1991) und greift in seiner Darstellung ausschließlich auf Wissenschaftler*innen aus Nordamerika, Europa und Australasien zurück. Ohne dies ausdrücklich zu erwähnen, beschreibt er damit die Diskurse über inklusive Bildung im globalen Norden. 


\section{Die Entwicklung von Inklusion in unterschiedlichen regionalen Kontexten}

Wie bereits ausgeführt, kann Inklusion als ein globales Thema bezeichnet werden. Dabei ist $\mathrm{zu}$ betonen, dass inklusive Bildung in den verschiedenen Regionen und Ländern der Welt über unterschiedliche Entstehungsgeschichten verfügt. Im globalen Norden basieren die aktuellen Inklusionsdiskurse auf einer Entwicklung vom vollständigen Ausschluss aus der formalen Bildung von Schülerinnen und Schülern, die als außerhalb der Norm wahrgenommen wurden, hin zur Beschulung dieser Schülerinnen und Schüler in Förderschulen. Dieses System der Separation wurde durch Integrationsinitiativen kritisiert, die bestimmte Lernende aus Förderschulen unter bestimmten Bedingungen in den Regelschulen platzieren, aber nicht davon ausgingen, dass alle Lernenden ein unbestrittenes Recht darauf haben, eine Regelschule zu besuchen (Engelbrecht/Green 2018: 5). Hingegen wurde Inklusive Bildung im globalen Süden zunächst als Instrument betrachtet, um den großen Zahlen von Kindern zu begegnen, die nicht zur Schule gehen, und Bildung für Alle (UNESCO 1990) zu erreichen. Ein differenziertes und etabliertes Förderschulsystem existierte kaum.

Die Erfahrung des globalen Nordens prägte das globale Streben nach Inklusion, das in internationalen Dokumenten wie der Salamanca-Erklärung (UNESCO 1994) und der UNKonvention über die Rechte von Menschen mit Behinderungen (UN 2006) postuliert wurde. Dies führte dazu, dass nördliche Werte, Erfahrungen und Politiken ohne konzeptionelle Anpassungen auf andere Kontexte übertragen wurden (Kalyanpur 2016). Da Diskurse das Verständnis von Inklusion prägen, lohnt es sich, den Blick auch auf Diskurse abseits des globalen Nordens zu lenken. Daher ist die Darstellung der wissenschaftlichen Diskurse über inklusive Bildung im südlichen Afrika als eine spezifische Region des globalen Südens das Ziel dieses Beitrags. Die Diskurse über Inklusion im globalen Süden werden sowohl von Forschenden aus dem globalen Norden als auch Süden geführt. Zudem gilt es zu beachten, dass Forschende selbstverständlich über Landesgrenzen hinweg zusammenarbeiten, wodurch es unmöglich wird, eine klare Trennlinie zwischen den Diskursen des Nordens und des Südens vorzunehmen. Diese Analyse fokussiert sich daher auf die von Forschenden aus dem südlichen Afrika geführten Diskurse.

\section{Methodischer Ansatz}

Die Analyse verwendet eine Forschungsperspektive, die auf dem sozialkonstruktivistischen Ansatz von Berger und Luckmann (1966) basiert. Keller (2001) ergänzt diesen Ansatz mit Grundlagen der von Foucault skizzierten Diskurstheorie, indem er die Perspektive auf institutionelle Produktion, Objektivierung und Verbreitung von Wissen erweitert. Die Wissenssoziologische Diskursanalyse (WDA) beschäftigt sich somit mit der Rekonstruktion von Prozessen der sozialen Konstruktion, Objektivierung, Kommunikation und Legitimation von Bedeutungsstrukturen (d.h. Interpretations- und Handlungsstrukturen) auf der Ebene von Institutionen und Organisationen oder sozialen (kollektiven) Akteuren und mit der Analyse der sozialen Auswirkungen solcher Prozesse (Keller 2013: 61f). Diskurse im foucaultschen Sinne sind Praktiken, die systematisch die Objekte bilden, von denen sie sprechen (Foucault 
2010: 49). Demzufolge wird die Bedeutung von Inklusion durch Diskurse konstruiert und diese Diskurse beeinflussen die Implementierung von Inklusion. Durch wissenschaftliche Diskurse z.B. in Fachzeitschriften wird Wissen produziert, objektiviert und verbreitet. Keller konzentriert sich auf Institutionen und Instanzen, die zwischen legitimen und illegitimen Sprecher*innen unterscheiden und daher über die Aufnahme oder den Ausschluss von Sprecher*innen in einen bestimmten Diskurs entscheiden (Keller 2001; Foucault 1978). In diesem Beitrag sind dies die Zeitschriften (vertreten durch Redakteur*innen und Gutachter*innen) und Datenbanken, die über die Aufnahme oder den Ausschluss von Beiträgen entscheiden.

In einer Studie, in der eine weiße $e^{2}$, europäische Forscherin die Diskurse südafrikanischer Forschender analysiert, ist es von Bedeutung, sich über die eigene Position in Bezug auf Insider/Outsider-Status und Machtstrukturen (im englischsprachigen Raum „Positionality“, Merriam et al. 2001) bewusst zu werden. Die Analyse der Diskurse des südlichen Afrikas und z.B. die Formulierung von Ein- und Ausschlusskriterien erfordert eine kritische Reflexion über die eigene Position und deren Auswirkungen auf das Forschungsvorhaben. Da diese Forschung eine sozial-konstruktivistische Perspektive als epistemologischen Ansatz anwendet, sind die Ergebnisse und die daraus resultierenden Aussagen als Konstruktionen zu verstehen, die keineswegs behaupten, die „einzige wahre Realität“ zu sein.

Diese Analyse untersucht, welche Begründungsdiskurse zu inklusiver Bildung von Wissenschaftler*innen im südlichen Afrika geführt werden und welche Definitionen von Inklusion angewendet werden. Da der Gegenstand der Analyse wissenschaftliche Diskurse über inklusive Bildung im südlichen Afrika sind, wurde ein systematisches Literaturreview (Hart 1998; Cooper 1988) von Publikationen in wissenschaftlichen Fachzeitschriften durchgeführt. Es wurde eine qualitative Textanalyse mit offener (Strauss 1994; Strauss/Corbin 1996) und thematischer (Flick 2007; Gibbs 2012) Kodierung durchgeführt.

Forschende sind meist über Ländergrenzen hinweg mobil und tauschen sich international aus, zitieren grenzüberschreitend und veröffentlichen in internationalen Autor*innenteams oder in internationalen Zeitschriften. Daher ist es nicht möglich, räumliche Diskurse klar voneinander abzugrenzen. Es geht hierbei nicht darum, internationale wissenschaftliche Arbeit zu nationalisieren oder zu kulturalisieren, sondern sich auf eine bestimmte unterrepräsentierte Region zu konzentrieren, um ein Verständnis für Besonderheiten und das Verhältnis von räumlichen Diskursen zu gewinnen. Zentral ist nicht der Vergleich von Orten, sondern der Vergleich von Diskursen, die in sozio-historischen und räumlichen Kontexten existieren. Für den Zweck dieser Analyse werden Publikationen mit mindestens einem Autor oder einer Autorin, der bzw. die eine institutionelle Zugehörigkeit zu einer Universität im südlichen Afrika hat, als Fragment der Diskurse des südlichen Afrikas verstanden. Dazu gehören Fachartikel, die sowohl in regional ansässigen Zeitschriften als auch in internationalen Zeitschriften veröffentlicht wurden. Dies soll lediglich als eine Arbeitsdefinition für den Kontext dieser Analyse verstanden werden.

Zwei verschiedene Ansätze zur Identifizierung von Publikationen wurden verwendet: einerseits die Verwendung der Suchmaschine der ERIC-Datenbank durch ProQuest und andererseits eine direkte Suche in acht identifizierten Fachzeitschriften aus der Region, nämlich Acta Academica, African Educational Research Journal, African Journal of Teacher Education, Critical Studies of Teaching and Learning, Perspectives in Education, South African Journal of Education, The African Symposium und Zimbabwe Journal of Educational Research. Nur eine dieser genannten Zeitschriften (Perspectives in Education) ist in der ERIC-

2 Die Kursivsetzung soll verdeutlichen, dass es sich hierbei um eine politische Beschreibung und keine Farbbezeichnung handelt (vgl. Ogette 2017: 14). 
Datenbank aufgenommen. Es wurden Zeitschriftenartikel aufgenommen, die zwischen 2006 und 2016 in englischer Sprache veröffentlicht wurden. Folgende Suchbegriffe wurden kombiniert: inclusi* und jeder der 15 Ländernamen oder Afrika. Es wurden ausschließlich englischsprachige Publikationen berücksichtigt. Diese Suche ergab insgesamt 156 Treffer; 127 Ergebnisse aus der ERIC-Datenbank und 29 direkt aus den Zeitschriften.

In einem zweiten Schritt wurden die gefundenen Referenzen weiter untersucht und Publikationen ausgeschlossen, die die folgenden Kriterien nicht erfüllten:

- Inclusion/inclusiv als Schlagwort: zweifelsohne können Publikationen mit anderen Schlagwörtern ebenfalls zu den Diskursen über Inklusion gerechnet werden. Doch durch die Einbeziehung anderer Schlagworte würde eine Setzung vorgenommen werden, was unter Inklusion gefasst wird und was nicht. Da diese Analyse die von Wissenschaftler*innen aus dem südlichen Afrika verwendeten Definitionen untersuchen soll, wurden nur Artikel berücksichtigt, die von den Autor*innen selbst mit den Schlagworten inclusion/inclusive gekennzeichnet wurden.

- Gegenstandsrelevanz: Nur Artikel, die sich auf den Bildungsbereich beziehen, wurden in die Analyse einbezogen.

- Institutionelle Zugehörigkeit: Für die Zwecke dieser Analyse wurde festgelegt, dass mindestens ein*e Autor*in über eine institutionelle Zugehörigkeit zu einem Land im südlichen Afrika verfügt.

Nach Ausschluss von Artikeln, die nicht alle diese Kriterien erfüllten oder trotz intensiver Recherche nicht als Volltexte verfügbar waren, stand der endgültige Datenkorpus mit 66 Publikationen fest. 51 Publikationen stammten aus Zeitschriften, die über ERIC gefunden wurden, und 15 Publikationen direkt aus Fachzeitschriften aus dem südlichen Afrika.

\section{Beschreibung des Datenkorpus}

Die institutionelle Zugehörigkeit der Autor*innen der 66 Publikationen, die den endgültigen Korpus für die Analyse bilden, ist wie folgt verteilt: Südafrika (47), Botswana (7), Simbabwe (4), Swasiland (2), Sambia (2), Lesotho (1), Malawi (1), Namibia (1) und Tansania (1). Mit den oben beschriebenen Kriterien wurden keine Publikationen von Autor*innen mit institutioneller Zugehörigkeit in Angola, der Demokratischen Republik Kongo, Madagaskar, Mauritius, Mosambik oder den Seychellen gefunden. Dabei muss beachtet werden, dass die meisten dieser genannten Länder nicht Englisch als Amtssprachen haben. Eine Erweiterung der Suche auf andere Sprachen würde zweifelsohne zu anderen Ergebnissen führen.

Diese Verteilung zeigt, dass die überwiegende Mehrheit der an den Inklusionsdiskursen teilnehmenden Wissenschaftler*innen institutionell in Südafrika verankert sind. Die Teilnahme von Wissenschaftler*innen aus anderen Ländern der Region ist marginal bis zu nicht vorhanden. 19 der 66 Artikel wurden von internationalen Autor*innenteams veröffentlicht. In all diesen Fällen der internationalen Autor*innenschaft war jeweils nur ein Land des südlichen Afrika vertreten. Autor*innenteams aus zwei oder mehr Ländern des südlichen Afrikas waren nicht Teil des finalen Datenkorpus. 


\section{Zentrale Ergebnisse}

\subsection{Begründungsdiskurs: Entstehung eines eigenständigen Diskurses in Südafrika}

Dyson (1999) betrachtet den Rechts- und Ethikdiskurs und den Diskurs über die Wirksamkeit als zwei Pole innerhalb des Begründungsdiskurses zu inklusiver Bildung. Im analysierten Datenkorpus ist der Diskurs bezogen auf rechtliche und ethische Argumentationen deutlich stärker erkennbar als der Diskurs über die Bildungseffektivität oder Kosteneffizienz von inklusiven Schulen. In den meisten Publikationen bilden soziale Gerechtigkeit, Gleichheit und Menschenrechte die Hauptbezugspunkte für die Begründung von Inklusion. Im Hinblick auf den Rechts- und Ethikdiskurs wird ein wesentlicher Unterschied zwischen Veröffentlichungen südafrikanischer Wissenschaftler*innen und Veröffentlichungen von Wissenschaftler*innen aus anderen Ländern des südlichen Afrikas sichtbar. Der Inklusionsdiskurs in Südafrika zeichnet sich dadurch aus, dass darin häufig Bezüge zu den Konzepten Freiheit, Demokratie und (Staats-)Bürgerschaft hergestellt werden.

Das Jahr 1994 stellt einen wichtigen Meilenstein sowohl in der Entwicklung von inklusiver Bildung (Salamanca-Erklärung) als auch in der Geschichte des Landes Südafrika (erste demokratische Wahlen und Ende der Apartheid) dar. In den darauffolgenden Jahren wurde die Frage, wie eine diskriminierungsfreie, gerechte und inklusive Gesellschaft entwickelt werden kann, zum Kern der sozialen und politischen Entwicklung in Südafrika. In vielen Publikationen aus Südafrika wird der historische Zusammenhang zwischen der Entwicklung von inklusiver Bildung auf der internationalen Ebene und dem Ü̉bergang von der Apartheid zur Demokratie in Südafrika betont. Forschende aus Südafrika beschreiben, wie die Etablierung von inklusiver Bildung in Südafrika und der Aufbau einer demokratischen Gesellschaft mit der Achtung der Menschenwürde, Freiheit und Gleichheit vor dem Gesetz - Prinzipien, die seit 1994 in der südafrikanischen Verfassung verankert sind - zeitgleich verlief. Inklusive Bildung im südafrikanischen Kontext wurde als Bildungskonzeption angesehen, die einer demokratischen und gerechten Gesellschaft entspricht (Du Toit/Forlin 2009: 646). Die verwendete Terminologie zeigt die Verflechtung staatlicher Entwicklung und der Entwicklung von inklusiver Bildung in Südafrika. Dieser spezifische historische Zusammenhang wird in einer Publikation von Zembylas et al. (2009) besonders sichtbar, in der am Beispiel von vier Nationen mit einer jüngeren Geschichte von Gewalt und kollektivem Trauma die dynamischen Wechselwirkungen zwischen Versöhnung und Inklusion diskutiert werden.

Der Rechts- und Ethikdiskurs in den anderen Ländern des südlichen Afrikas ist nicht von dieser spezifischen Terminologie geprägt, sondern bezieht sich auf Prinzipien des Zugangs, der Partizipation und der Menschenrechte. Dies folgt einer ähnlichen Argumentationslinie, jedoch mit anderer Akzentuierung. Dieser Diskurs bezieht sich nicht auf bestimmte nationale, historische, politische oder gesellschaftliche Entwicklungen. Autor*innen aus diesen Ländern beziehen sich in ihrer Argumentation ausschließlich auf wissenschaftliche Quellen des globalen Nordens, während südafrikanische Autor*innen entweder südafrikanische Kolleg*innen oder Quellen aus dem globalen Norden heranziehen.

Es lässt sich zusammenfassend festhalten, dass der Begründungsdiskurs zu inklusiver Bildung in Südafrika aufgrund seines spezifischen historischen Kontextes einen eigenen Entwicklungspfad eingeschlagen hat, indem internationale Diskurse rekontextualisiert wurden. 
Die Diskurse in den anderen Ländern des südlichen Afrikas bleiben ohne kontextspezifische Veränderungen eng mit den nördlichen Diskursen verbunden.

\subsection{Wirksamkeitsdiskurs: Geringe Kontextualisierung}

Elf Artikel des gesamten Korpus beziehen sich auf den Wirksamkeitsdiskurs. Urwick und Elliott (2010) stimmen der Argumentation nicht zu, dass inklusive Bildung kostengünstiger und pädagogisch wirksamer sei. Otukile-Mongwaketse, Mangope und Kuyini (2016) führen Vor- und Nachteile von Inklusion auf und belegen, dass das Argument der Wirksamkeit sowohl von Befürwortern als auch Gegnern von Inklusion verwendet wird. Das unter der Apartheid entwickelte Bildungssystem Südafrikas wird nicht nur als ungerecht, sondern auch als wenig effektiv beschrieben (McKinney/Swartz 2016; Greyling 2009). Andere Autor*innen, die sich auf Wirksamkeit von inklusiver Bildung beziehen, folgen der Argumentation, dass für Inklusion pädagogische Gründe sprechen. Hierbei (z.B. Walton 2011) wird insbesondere auf die Salamanca-Erklärung Bezug genommen, in der formuliert ist, dass ,,inklusive Schulen eine effektive Bildung für den Großteil aller Kinder [gewährleisten] und [...] die Effizienz und schließlich das Kosten-Nutzen-Verhältnis des gesamten Schulsystems [erhöhen]" (UNESCO 1994). Die Wirksamkeit von inklusiver Bildung wird in diesem Fall mit einer normativen Erklärung begründet.

Andere Publikationen des Korpus beziehen sich im Wirksamkeitsdiskurs auf empirische Ergebnisse. Bornman und Donohue (2013: 86) argumentieren, dass inklusive Bildung die schulischen Leistungen und sozialen Kompetenzen von Schüler*innen mit sonderpädagogischem Förderbedarf verbessern und Schüler*innen ohne sonderpädagogischem Förderbedarf zur Empathieentwicklung anregen. Dies belegen die Autoren mit einer US-amerikanischen Längsschnittstudie über die Leistungen von Schüler*innen mit sonderpädagogischem Förderbedarf in der Primar- und Sekundarstufe (Blackorby et al. 2005). Studien wie PISA (OECD 2011) und von Barber and Mourshed (2007) werden als weitere empirische Evidenz angeführt, um aufzuzeigen, dass die besten Schulsysteme weltweit auch bei der Inklusion von potenziell marginalisierten Schüler*innengruppen erfolgreich waren (Malinen et al. 2013). Eine weitere Argumentation bezieht sich auf nationale Politikpapiere von Botswana (Mukhopadhyay 2013) und Südafrika (Daniels 2010), in denen inklusive Bildung als kosteneffizient bezeichnet wird.

Es lässt sich festhalten, dass der Wirksamkeitsdiskurs von Wissenschaftler*innen aus dem südlichen Afrika auf empirische Ergebnisse aus dem globalen Norden, die normative Salamanca-Erklärung und nationale Politikpapiere zurückgreift. Bis auf eine Publikation (Engelbrecht et al. 2016) bezieht sich kein Artikel auf empirische Evidenz aus einem der Länder des südlichen Afrikas. In der genannten Publikation wird die Umsetzung von inklusiver Bildung in Südafrika analysiert. Es werden mehrere empirische, aber auch theoretische Beiträge von südafrikanischen Wissenschaftler*innen zur Argumentation herangezogen. Die Autor*innen kommen zu dem Schluss, dass eine wesentliche Lücke zwischen der idealistischen Konzeptualisierung von inklusiver Bildung und ihrer Umsetzung in Südafrika existiert (Engelbrecht et al. 2016: 532).

Die Frage der Effizienz muss als eine relationale Frage betrachtet werden. Dyson beschreibt den (nördlichen) Effizienzdiskurs in Bezug auf den Vergleich eines Förderschulsystems und eines inklusiven Schulsystems. Da die historische Entwicklung der Schulsysteme 
und insbesondere die Rolle der Förderschulen zwischen dem globalen Norden und dem Süden sehr unterschiedlich verlaufen ist (vgl. Biermann 2021 i.d.B.), bezieht sich die relationale Frage, welches System effizienter ist, auf unterschiedliche Bezugspunkte in den jeweiligen Kontexten. Wenn in den Artikeln des Korpus auf empirische Ergebnisse aus dem globalen Norden Bezug genommen wird, wird nicht diskutiert, ob und wie diese Erkenntnisse für das südliche Afrika übertragbar sind. In zwei Publikationen (Mukhopadhyay 2013; Walton 2011) wurde ein Zusammenhang zwischen dem Wirksamkeitsdiskurs und den spezifischen Kontextfaktoren der Länder des südlichen Afrikas hergestellt, indem argumentiert wurde, dass inklusive Bildung eine kosteneffektive Strategie zur Erreichung von Entwicklungszielen wie Bildung Für Alle (UNESCO 1994) und den Millennium Entwicklungszielen (UN 2000) sei. Angesichts der finanziellen Bedingungen in den Ländern des südlichen Afrikas wäre gerade eine Auseinandersetzung mit der Perspektive der Kosteneffizienz von hoher kontextueller Bedeutung für die Region.

Die geringe empirische Grundlage aus den Ländern des südlichen Afrikas und der Bezug auf Ergebnisse aus dem globalen Norden wird auch innerhalb der Diskurse von Wissenschaftler*innen aus dem südlichen Afrika thematisiert. Gous, Eloff und Moen (2014) führen an, dass die meisten Forschungsergebnisse und wissenschaftliche Literatur aus dem globalen Norden stammen und dass es an „eigener“ Forschung zur Thematik mangelt. Der Import nördlichen Wissens über Inklusion wird von Urwick und Elliott (2010) als indoktrinierende Orthodoxie beschrieben, die koloniale Vorstellungen von der Überlegenheit des Nordens über den Süden verstärkt (Kaplan et al. 2007: 23). Chataika et al. (2012) kritisieren, dass lokale Formen der inklusiven Bildung in der Literatur nicht ausreichend dargestellt und anerkannt werden.

\subsection{Definitionen im Gebrauch: Zwischen weitem und engem Verständnis von Inklusion}

Bezüglich der Definition von inklusiver Bildung verweisen die meisten Publikationen von Autor*innen aus Südafrika auf das Education White Paper 6 der südafrikanischen Regierung (DoE 2001). In diesem zentralen Politikpapier wird formuliert: ,all learners are different in some way and have different learning needs" (DoE 2001: 16). In diesem Grundsatzdokument verwendet die Regierung die Terminologie „Barrieren im Lernen und in der Entwicklung“ und verleiht so dem Verständnis Ausdruck, dass die Barrieren innerhalb des Systems bestehen (ebd.: 12). Diese Definition und Terminologie spiegeln sich im weiteren südafrikanischen Diskurs wider, da die meisten Autor*innen auf dieses grundlegende Dokument verweisen und die Terminologie übernehmen.

In allen anderen Ländern des südlichen Afrikas werden sehr unterschiedliche Definitionen verwendet. Gelegentlich wird der Begriff Barrieren in einem ähnlichen Verständnis wie in Südafrika verwendet (Urwick/Elliott 2010; Mutepfa et al. 2007), häufiger jedoch werden die Begriffe Kinder mit Behinderungen oder Kinder mit sonderpädagogischem Förderbedarf genutzt (z.B. Okkolin et al. 2010; Haihambo/Lightfoot 2010; Kuyini/Mangope 2011).

Während in Publikationen aus Südafrika in der Regel eine weite Definition von Inklusion (vgl. Ainscow et al. 2006) angewendet wird, bei der alle Lernenden in ihrer Individualität im Fokus stehen, sind die in den Publikationen aller anderen Länder des südlichen Afrikas verwendeten Definitionen sehr unterschiedlich und reichen von einem Fokus auf Kinder mit 
Behinderungen oder sonderpädagogischem Förderbedarf (z.B. Okkolin et al. 2010; Kuyini/Mangope 2011) über unterschiedliche Differenzkategorien (z.B. HapanyengwiChemhuru 2013) hin zu allen Lernenden (z.B. Mudyahoto/Dakwa 2012; Kaplan et al. 2007). In zwei Publikationen werden Bezüge zur Definition aus Südafrika hergestellt (Hapanyengwi-Chemhuru 2013; Pather/Nxumalo 2013). In allen anderen Fällen beziehen sich die Wissenschaftler*innen aus Ländern des südlichen Afrikas entweder auf Publikationen aus ihren Ländern oder auf Quellen aus dem globalen Norden, nicht aber auf Kolleg*innen aus anderen Ländern der Region.

\subsection{Differenzkategorien: Spezifische Kontexte werden sichtbar}

Die im südafrikanischen Education White Paper 6 genannten Differenzkategorien sind Alter, Geschlecht, ethnische Zugehörigkeit, Sprache, Klasse, Behinderung und HIV-Status (DoE 2001: 6). Weitere Dimensionen der Differenz, wie Religion, sexuelle Orientierung, bevorzugte Lernstile und sozioökonomischer Hintergrund, werden von Gous, Eloff und Moen (2014), Walton (2011) und Mitchell, Lange und Thuy (2008) erwähnt. Zudem werden Waisen als von Benachteiligung gefährdete Gruppe genannt. Hapanyengwi-Chemhuru (2013: 206) listet Kinder, die auf der Straße leben, die Minderheiten angehören, verwaist sind, in Institutionen untergebracht sind, obdachlose und geflüchtete Kinder und Kinder, die von HIV und AIDS betroffen sind, Kinder in Armut und Einwandererkinder als Gruppen auf, die im Schulsystem marginalisiert sein können. Bei der Benennung von Exklusion bedrohter Gruppen wird eine klare Kontextualisierung zu den Bedingungen der Länder des südlichen Afrikas sichtbar.

\subsection{Internationale Beziehungen: Geringe diskursive Interaktion im südlichen Afrika}

In der Beschreibung des Datenkorpus wird deutlich, dass keine der Publikationen im Korpus von Wissenschaftler*innen aus zwei oder mehr verschiedenen Ländern des südlichen Afrikas verfasst wurde. Internationale Autor*innenteams bestehen aus Autor*innen mit einer Zugehörigkeit zu einem Land im südlichen Afrika und Ländern außerhalb des südlichen Afrikas. Darüber hinaus zitieren Autor*innen in der Regel nicht Autor*innen aus anderen Ländern des südlichen Afrikas. Dies kann als Indikator für eine geringe Zusammenarbeit zwischen Wissenschaftler*innen aus verschiedenen Ländern des südlichen Afrikas und separierte Diskurse innerhalb der Region angesehen werden, die sich kaum gegenseitig beeinflussen. Alle einzelnen isolierten Diskurse orientieren sich an den nördlichen Diskursen, auch wenn dies innerhalb dieser Diskurse gleichzeitig kritisiert wird. 


\section{Fazit}

Die vorgestellte Analyse ist durch die Begrenzung auf englischsprachige Artikel in ihrer Aussagekraft deutlich limitiert. Weitere Forschungsvorhaben sind erforderlich, um die dargestellten Ergebnisse zu ergänzen und zu erweitern. Zudem bildet eine stärkere Kontextualisierung in die Wissenschaftsgeschichte der Region, die eng mit der Kolonialgeschichte verbunden ist, und die Interpretation der Ergebnisse vor diesem Hintergrund eine bereichernde Perspektive für die weitere Bearbeitung des dargestellten Themas.

Im Verständnis der Wissenssoziologischen Diskursanalyse haben Diskurse eine produktive Wirkung und konstruieren daher in diesem Fall die Bedeutung von inklusiver Bildung. Die Analyse zeigt, dass sich ein eigener südafrikanischer Diskurs zur Begründung von Inklusion mit einer gemeinsamen Terminologie und gemeinsamen Bezugspunkten entwickelt hat. Die Entwicklung dieses speziellen, nationalen Diskurses wird nicht nur durch die gemeinsame Terminologie, sondern auch durch die Anzahl der Publikationen mit institutioneller Zugehörigkeit im Land sichtbar. In Südafrika wird Inklusion als Gegenpol zur Apartheid konstruiert und hat daher ein besonderes gesellschaftspolitisches Gewicht. In diesem Diskurs wird Inklusion so konstruiert, dass sie über den Bildungssektor hinaus auf breitere gesellschaftliche Ebenen reicht. Die diskursiven Konstruktionen des Begriffs der Inklusion in Südafrika sind von der nationalen Politik beeinflusst und beziehen sich auf das, was als ein breites Verständnis von Inklusion bezeichnet wird. Der Wirksamkeitsdiskurs ist im Gegenzug weniger ausgeprägt und es zeigt sich, dass der nördliche Wirksamkeitsdiskurs nicht kontextualisiert wird. Die Notwendigkeit, Fragen der inklusiven Bildung durch eine eigene empirische Perspektive zu bearbeiten, wird dargestellt und könnte ein zukünftiges Arbeitsfeld für Forscher*innen sein.

Innerhalb des dargestellten Datenkorpuses sind die Inklusionsdiskurse in den anderen Ländern des südlichen Afrikas fragmentiert und geringer ausgeprägt. Konstruktionen von Inklusion sind hauptsächlich Nachbildungen von nördlichen Konstruktionen. Dies wird selbst von den Akteur*innen der Diskurse kritisiert und ein Bedarf an eigenen Konstruktionen postuliert. Die Verflechtung zwischen Inklusion und der Entwicklung von Staat und Gesellschaft ist aufgrund ihres spezifischen historischen und politischen Kontexts nicht so offensichtlich wie in Südafrika.

Es fällt auf, dass es innerhalb der Region keine Zusammenarbeit bei Publikationen über Ländergrenzen hinweg existiert. Eine starke Orientierung an den nördlichen Diskursen zeigt sich insbesondere bei empirischen Bezügen. Gleichzeitig wird diese nördliche Dominanz in den gleichen Diskursen von mehreren Autor*innen aus verschiedenen Ländern diskutiert und kritisiert.

Aus postkolonialer Sicht (Mills 2004) ist es notwendig, die in und durch Diskurse ausgeübte Macht in den vorgestellten Analysen weiter zu reflektieren. Foucault (1994: 39) sagt hierzu: „Eher ist wohl anzunehmen, daß [sic!] die Macht Wissen hervorbringt (und nicht bloß [sic!] fördert, anwendet, ausnutzt); daß [sic!] Macht und Wissen einander unmittelbar einschließen [sic!]; daß [sic!] es keine Machtbeziehungen gibt, ohne daß [sic!] sich ein entsprechendes Wissensfeld konstituiert". Die Betrachtung der Wechselbeziehungen und -wirkungen verschiedener räumlicher Diskurse mit einem besonderen Fokus auf Macht-Wissen-Beziehungen kann eine fruchtbare Perspektive für die weitere Forschung bilden. 


\section{Literatur}

Die mit einem Stern gekennzeichneten Referenzen kennzeichnen Publikationen, die Teil des Literaturreviews sind.

Ainscow, Mel/Booth, Tony/Dyson, Alan (2006): Improving Schools, Developing Inclusion. Oxon: New York.

Armstrong, Derrick/Armstrong, Ann C./Spandagou, Ilektra (2011): Inclusion. by Choice or by Chance? In: International Journal of Inclusive Education 15, 1, S. 29-39. doi: 10.1080/13603 116.2010.496192.

Artiles, Alfredo J./Dyson Alan (2005): Inclusive Education in the Globalization Age. The Promise of Comparative Cultural Historical Analysis. In: Mitchell, David (Hrsg.): Contextualizing Inclusive Education. Evaluating Old and New International Paradigms. London: Routledge, S. 37-62.

Artiles, Alfredo J./Koslezki, Elisabth B. (2016): Inclusive Education's Promises and Trajectories: Critical Notes about Future Research on a Venerable Idea. In: Education Policy Analysis Archives 24, S. 43. doi: 10.14507/epaa.24.1919.

Artiles, Alfredo J./Kozleski, Elisabth B./Dorn, Sherman/Christensen, Carol A. (2006): Learning in inclusive education research. Re-mediating theory and methods with a transformative agenda. In: Review of Research in Education 30, 1, S. 65-108.

Barber, Michael/Mourshed, Mona (2007): How the world's best-performing schools systems come out on top. http://mckinseyonsociety.com/downloads/reports/Education/Worlds School Systems Final.pdf [Zugriff: 9.10.2017].

Berger, Peter L./Luckmann, Thomas (1966): The Social Construction of Reality. A Treatise in the Sociology of Knowledge. Garden City, NY: Anchor Books.

*Beyers, Christa/Hay, Johnnie (2007): Can Inclusive Education in South(ern) Africa Survive the HIV and AIDS Pandemic? In: International Journal of Inclusive Education 11, 4, S. 387-399. doi:10.1080/13603110701391360.

Blackorby, Jose/Wagner, Mary/Cameto, Renée/Davies, Elizabeth/Levine, Phyllis/Newman, Lynn (2005): Engagement, academics, social adjustment, and independence. Palo Alto, CA: SRI.

Biermann, Julia (2021 i.d.B.): Der Einfluss der UN-BRK auf inklusive Bildung in Nigeria und Deutschland. In: Köpfer, Andreas/Powell, Justin J.W./Zahnd, Raphael (Hrsg.): Handbuch Inklusion international. Opladen: Budrich, S. 167-178.

*Bornman, Juan/Donohue, Dana K. (2013): South African Teachers' Attitudes toward Learners with Barriers to Learning. Attention-Deficit and Hyperactivity Disorder and Little or No Functional Speech. In: International Journal of Disability, Development and Education 60, 2, S. 85104. doi:10.1080/1034912X.2013.786554.

*Charema, John (2007): From Special Schools to Inclusive Education. The Way forward for Developing Countries South of the Sahara. In: Journal of the International Association of Special Education 8, 1, S. 88-97.

*Charema, John (2010): Inclusive Education in Developing Countries in the Sub Saharan Africa. From Theory to Practice. In: International Journal of Special Education 25, 1, S. 87-93.

*Chataika, Tsitsi/Mckenzie, Judith A./Swart, Estelle/Lyner-Cleophas, Marcia (2012): Access to Education in Africa. Responding to the United Nations Convention on the Rights of Persons with Disabilities. In: Disability \& Society 27, 3, S. 385-398. doi:10.1018/09687599.2012. 654989. 
*Cockcroft, Kate/Dhana-Dullabh, Hansini (2013): Deaf Children and Children with ADHD in the Inclusive Classroom. Working Memory Matters. In: International Journal of Inclusive Education 17, 10, S. 1023-1039. doi:10.1080/13603116.2012.728252.

Connor, David J./Ferri, Beth A. (2007): The Conflict Within. Resistance to Inclusion and other Paradoxes in Special Education. In: Disability \& Society 22, 1, S. 63-77. doi: 10.1080/096875 90601056717.

Cooper, Harris M. (1988): Organizing Knowledge Syntheses. A Taxonomy of Literature Reviews. In: Knowledge in Society 1, 1, S. 104-126. doi: 10.1007/BF03177550.

*Daniels, Berenice. (2010): Developing Inclusive Policy and Practice in Diverse Contexts. A South African Experience. In: School Psychology International 31, 6, S. 631-643. doi:10.1177/ 0143034310386536.

Department of Education (DoE) (2001): Education White Paper 6 (Special Needs Education). Building an Inclusive Education and Training System. Pretoria: Government Printer.

*Donohue, Dana K./Bornman, Juan (2014): The Challenges of Realising Inclusive Education in South Africa. In: South African Journal of Education 34, 2, S. 1-14.

*Donohue, Dana K./Bornman, Juan (2015): South African Teachers' Attitudes toward the Inclusion of Learners with Different Abilities in Mainstream Classrooms. In: International Journal of Disability, Development and Education 62, 1, S. 42-59. doi:10.1080/1034912X.2014. 985638.

*Du Toit, Petrusa/Forlin, Chris (2009): Cultural Transformation for Inclusion, What Is Needed? A South African Perspective. In: School Psychology International 30, 6, S. 644-666. doi:10.1177/0143034309107081.

Dunne, Linda (2009): Discourses of Inclusion. A Critique. In: Power and Education 1, 1, S. 4256. doi: 10.2304/power.2009.1.1.42.

Dyson, Alan (1999): Inclusion and Inclusions. Theories and Discourses in Inclusive Education. In: Daniels, Harry/Garner, Philip (Hrsg.): World Yearbook of Education 1999. Inclusive Education. London: Routledge, S. 36-53.

Engelbrecht, Petra/Artiles, Alfredo J. (2016): Inclusive Education. Contextualizing the History of a Global Movement. In: Werning, Rolf/Artiles, Alfredo J./Engelbrecht, Petra/Hummel, Myriam/Caballeros, Martha/Rothe, Antje (Hrsg.): Keeping the Promise? Contextualizing Inclusive Education in Developing Countries. Bad Heilbrunn: Klinkhardt, S. 15-28.

Engelbrecht, Petra/Green, Lena (2018): Contextualising inclusive education in southern Africa. In: Engelbrecht, Petra/Green, Lena (Hrsg.): Responding to the challenges of inclusive education in southern Africa. Pretoria: Van Schaik Publishers, S. 3-11.

*Engelbrecht, Petra/Nel, Mirna/Nel, Norma/Tlale, Dan (2015): Enacting Understanding of Inclusion in Complex Contexts. Classroom Practices of South African Teachers. In: South African Journal of Education 35, 3, S.1-10. doi:10.15700/saje.v35n3a107.

*Engelbrecht, Petra/Oswald, Marietjie/Forlin, Chris (2006): Promoting the Implementation of Inclusive Education in Primary Schools in South Africa. In: British Journal of Special Education 33, 3, S. 121-129.

*Engelbrecht, Petra/Nel, Mirna/Smit, Suegnet/van Deventer, Marichelle (2016): The Idealism of Education Policies and the Realities in Schools. The Implementation of Inclusive Education in South Africa. In: International Journal of Inclusive Education 20, 5, S. 520-535. doi:10.1080/13603116.2015.1095250.

Flick, Uwe (2007). Qualitative Sozialforschung. Eine Einführung. Reinbek bei Hamburg: Rowohlt.

Foucault, Michel (1978): Dispositive der Macht. Über Sexualität, Wissen und Wahrheit. Berlin: Merve.

Foucault, Michel (1994): Überwachen und Strafen. Die Geburt des Gefängnisses. Frankfurt a. M.: Suhrkamp. 
Foucault, Michel (2010): The Archaeology of Knowledge and the Discourse on Language. New York: Vintage Books.

*Geldenhuys, Johanna L./Wevers, N. E. J. (2013): Ecological Aspects Influencing the Implementation of Inclusive Education in Mainstream Primary Schools in the Eastern Cape, South Africa. In: South African Journal of Education 33, 3, S. 1-18.

Gibbs, Graham R. (2012): Analyzing Qualitative Data. London: SAGE.

Göransson, Kerstin/Nilholm, Claes (2014): Conceptual Diversities and Empirical Shortcomings a Critical Analysis of Research on Inclusive Education. In: European Journal of Special Needs Education 29, 3, S. 265-280. doi: 10.1080/08856257.2014.933545.

*Gous, Jennifer G./Eloff, Irma F./Moen, Melanie C. (2014): How Inclusive Education Is Understood by Principals of Independent Schools. In: International Journal of Inclusive Education 18, 5, S. 535-552. doi:10.1080/13603116.2013.802024.

*Greyling, Johanna A. (2009): Reaching for the Dream: Quality Education for All. In: Educational Studies 35, 4, S. 425-435. doi:10.1080/03055690902876529.

Gunnpórsdóttir, Hermína/Jóhannesson, Ingólfur. Á. (2014): Additional Workload or a Part of the Job? Icelandic Teachers' Discourse on Inclusive Education. In: International Journal of Inclusive Education 18, 6, S. 580-600. doi: 10.1080/13603116.2013.802027.

*Haihambo, Cynthy/Lightfoot, Elizabeth (2010): Cultural Beliefs regarding People with Disabilities in Namibia: Implications for the Inclusion of People with Disabilities. In: International Journal of Special Education 25, 3, S. 76-87.

*Hall, Anna-Marié/Theron, Linda (2016): How School Ecologies Facilitate Resilience among Adolescents with Intellectual Disability: Guidelines for Teachers. In: South African Journal of Education 36, 2, S. 33-42. doi:10.15700/saje.v36n2a1154.

*Hapanyengwi-Chemhuru, Oswell (2013): Inclusion or Integration: Towards Conceptual Clarity in the Provision of Special Needs Education in Zimbabwe. In: Zimbabwe Journal of Educational Research 25, 3, S. 196-209.

Hardy, Ian/Woodcock, Stuart (2015): Inclusive Education Policies: Discourses of Difference, Diversity and Deficit. In: International Journal of Inclusive Education 19, 2, S. 141-164. doi: 10.1080/13603116.2014.908965.

Hart, Chris (1998): Doing a Literature Review. Releasing the Social Science Research Imagination. London, Thousand Oaks, New Delhi: SAGE.

*Helldin, Rolf/Backman, Orjan/Dwyer, Helen/Skarlind, Anders/Hugo, Anna J./Nel, Norma/Muller, Helene (2011): Opportunities for a Democratic Pedagogy. A Comparative Study of South African and Swedish Teachers' Attitudes to Inclusive Education. In: Journal of Research in Special Educational Needs 11, 2, S. 107-119. doi:10.1111/j.1471-3802.2010.01173.x.

*Hughes, Elizabeth/Chitiyo, Morgan/Itimu-Phiri, Ambumulire/Montgomery, Kristen (2016): Assessing the Special Education Professional Development Needs of Northern Malawian Schoolteachers. In: British Journal of Special Education 43, 2, S. 159-177.

*Jafthas, Joan A. (2008): New Role of Special Schools. Empowering Mainstream Teachers to Enhance Inclusive Education in Western Cape, South Africa. In: Journal of the International Association of Special Education 9, 1, S. 105-108.

Kalyanpur, Maya (2016): Inclusive education policies and practices in the context of international development: Lessons from Cambodia. In: ZEP - Zeitschrift für international Bildungsforschung und Entwicklungspädagogik, 39(3), 16-21.

*Kaplan, Ian/Lewis, Ingrid/Mumba, Paul (2007): Picturing Global Educational Inclusion? Looking and Thinking across Students' Photographs from the UK, Zambia and Indonesia. In: Journal of Research in Special Educational Needs 7, 1, S. 23-35. doi:10.1111/j.1471-3802.2007. 00078.x. 
Keller, Reiner (2001): Wissenssoziologische Diskursanalyse. In: Keller, Reiner/Hirseland, Andreas/Schneider, Werner/Viehöver, Willy (Hrsg.): Handbuch Sozialwissenschaftliche Diskursanalyse. Theorien und Methoden, Band I. Opladen: Leske + Budrich. S. 113-144.

Keller, Reiner (2013): Doing Discourse Research. An Introduction for Social Scientists. Los Angeles, London, New Delhi, Singapore, Washington DC: SAGE.

*Krüger, Deirdré/Yorke, Claire (2010): Collaborative Co-Teaching of Numeracy and Literacy as a Key to Inclusion in an Independent School. In: South African Journal of Education 30, 2, S. 293-306.

*Kuyini, Ahmed B./Mangope, Boitumelo (2011): Student Teachers' Attitudes and Concerns about Inclusive Education in Ghana and Botswana. In: International Journal of Whole Schooling 7, 1, S. 20-37.

Liasidou, Anastasia (2008): Critical Discourse Analysis and Inclusive Educational Policies. The Power to Exclude. In: Journal of Education Policy 23, 5, S. 483-500. doi: 10.1080/02680 930802148933.

*Makhalemele, Thabo/Nel, Mirna (2016): Challenges Experienced by District-Based Support Teams in the Execution of Their Functions in a Specific South African Province. In: International Journal of Inclusive Education 20, 2, S. 168-184. doi:10.1080/13603116.2015.1079270.

*Malinen, Olli-Pekka/Savolainen, Hannu/Engelbrecht, Petra/Xu, Jiacheng/Nel, Mirna/Nel, Norma/Tlale, Dan (2013): Exploring Teacher Self-Efficacy for Inclusive Practices in Three Diverse Countries. In: Teaching and Teacher Education: An International Journal of Research and Studies 33, S. 34-44.

*Mangope, Boitumelo/Mannathoko, Magdeline C./Kuyini, Ahmed B. (2013): Pre-Service Physical Education Teachers and Inclusive Education. Attitudes, Concerns and Perceived Skill Needs. In: International Journal of Special Education 28, 3, S. 82-92.

*Mapuranga, Barbra/Dumba, Oswald/Musodza, Blessing (2015): The Impact of Inclusive Education (IE) on the Rights of Children with Intellectual Disabilities (IDs) in Chegutu. In: Journal of Education and Practice 6, 30, S. 214-223.

*McKay, Veronica I./Romm, Norma R. (2015): Narratives of Agency. The Experiences of Braille Literacy Practitioners in the "Kha Ri Gude" South African Mass Literacy Campaign. In: International Journal of Inclusive Education 19, 4, S. 435-456. doi:10.1080/13603116.2014. 940066.

*Mckenzie, Judith A./Macleod, Catriona I. (2012): The Deployment of the Medico-Psychological Gaze and Disability Expertise in Relation to Children with Intellectual Disability. In: International Journal of Inclusive Education 16, 10, S. 1083-1098. doi:10.1080/13603116.2010. 540042.

*McKinney, Emma L./Swartz, Leslie (2016): Life in Special Schools in South Africa. Voices of Former Students. In: International Journal of Disability, Development and Education 63, 3, S. 309-321. doi:10.1080/1034912X.2015.1089980.

*Mdikana, Andile/Ntshangase, Subusiso/Mayekiso, Tokozile (2007): Pre-Service Educators' Attitudes towards Inclusive Education. In: International Journal of Special Education 22, 1, S. 125-131.

*Meltz, Adrienne/Herman, Chaya/Pillay, Venitha (2014): Inclusive Education. A Case of Beliefs Competing for Implementation. In: South African Journal of Education 34, 3, S. 1-8.

Merriam, Sharan B./Johnson-Baily, Juanita/Ming-Yeh, Lee/Youngwha, Kee/Gabo, Ntseane/Mazanah, Muhamad (2001): Power and Positionality. Negotiating Insider/Outsider Status within and across Cultures. In: International Journal of Lifelong Education 20, 5, S. 405-416. doi: $10.1080 / 02601370120490$.

Mills, Sara (2004): Discourse. New critical idiom. London, New York: Routledge. 
*Mitchell, Claudia/de Lange, Naydene/Xuan Thuy, Nguyen-Thi (2008): “Let's Not Leave This Problem": Exploring Inclusive Education in Rural South Africa. In: Prospects: Quarterly Review of Comparative Education 38, 1, S. 99-112. doi:10.1007/s11125-008-9057-y.

*Mohangi, Kamleshie/Archer, Karen (2015): Mothers' Reflections on the Role of the Educational Psychologist in Supporting their Children with Attention Deficit Hyperactivity Disorder. In: South African Journal of Education 35, 1, S. 1-9.

*Mudyahoto, Tapiwa/Dakwa, Francis E. (2012): An Analysis of the Level of Participation in Sport by Learners with Disabilities in Inclusive Settings. In: Zimbabwe Journal of Educational Research 24, 3, S. 308-315.

*Mukhopadhyay, Sourav (2013): Inclusive Education for Learners with Special Educational Needs in Botswana: Voices of Special Educators. In: Journal of the International Association of Special Education 14, 1, S. 41-49.

*Mukhopadhyay, Sourav (2014): Botswana Primary Schools Teachers' Perception of Inclusion of Learners with Special Educational Needs. In: Journal of Research in Special Educational Needs 14, 1, S. 33-42. doi:10.1111/j.1471-3802.2012.01269.x.

*Mutepfa, Magen M./Mpofu, Elias/Chataika, Tsitsi (2007): Inclusive Education in Zimbabwe: Policy, Curriculum, Practice, Family, and Teacher Education Issues. In: Childhood Education 83, 6, S. 342-346.

*Naicker, Sigamoney (2007): From Policy to Practice: A South-African Perspective on Implementing Inclusive Education Policy. In: International Journal of Whole Schooling 3, 1, S. 1-6.

*Nel, Mirna/Engelbrecht, Petra/Nel, Norma/Tlale, Dan (2014): South African Teachers' Views of Collaboration within an Inclusive Education System. In: International Journal of Inclusive Education 18, 9, S. 903-917. doi:10.1080/13603116.2013.858779.

*Ngcobo, Jabulani/Muthukrishna, Nithi (2011): The Geographies of Inclusion of Students with Disabilities in an Ordinary School. In: South African Journal of Education 31, 3, S. 357-368.

*Nkoane, Milton (2011): Critical Liberatory Inclusive Pedagogy: Arguing for a Zero-Defect Discourse. In: Acta Academica 43, 4, S. 111-126.

*Ntombela, Sithabile (2011): The Progress of Inclusive Education in South Africa. Teachers' Experiences in a Selected District, Kwazulu-Natal. In: Improving Schools 14, 1, S. 5-14. doi:10.1177/1365480206061994.

*Ntshangase, Sibusiso/Mdikana, Andile/Cronk, Candice (2008): A Comparative Study of the Self-Esteem of Adolescent Boys with and without Learning Disabilities in an Inclusive School. In: International Journal of Special Education 23, 2, S. 75-84.

OECD (2011): Lessons from PISA for the United States, strong performers and successful reformers in education. Paris, France: OECD Publishing.

Ogette, Tupoka (2017): exit RACISM. Rassismuskritisch denken lernen. Münster: Unrast Verlag.

*Okkolin, Mari-Anne/Lehtomaki, Elina/Bhalalusesa, Eustella (2010): The Successful Education Sector Development in Tanzania - Comment on Gender Balance and Inclusive Education. In: Gender and Education 22, 1, S. 63-71. doi:10.1080/09540250802555416.

*Oswald, Marietjie/Swart, Estelle (2011): Addressing South African Pre-Service Teachers' Sentiments, Attitudes and Concerns Regarding Inclusive Education. In: International Journal of Disability, Development and Education 58, 4, S. 389-403. doi:10.1080/1034912X.2011. 626665.

*Oswald, Marietjie/de Villiers, Jeanne-Marie (2013): Including the Gifted Learner. Perceptions of South African Teachers and Principals. In: South African Journal of Education 33, 1, S. 121.

*Oswald, Marietjie/Engelbrecht, Petra (2013): Leadership in Disadvantaged Primary Schools. Two Narratives of Contrasting Schools. In: Educational Management Administration \& Leadership 41, 5, S. 620-639. doi:10.1177/1741143213488377. 
*Otukile-Mongwaketse, Mpho/Mangope, Boitumelo/Kuyini, Ahmed B. (2016): Teachers' Understandings of Curriculum Adaptations for Learners with Learning Difficulties in Primary Schools in Botswana. Issues and Challenges of Inclusive Education. In: Journal of Research in Special Educational Needs 16, 3, S. 169-177. doi:10.1111/1471-3802.12069.

*Pather, Sulochini/Nxumalo, Cebsile P. (2013): Challenging Understandings of Inclusive Education Policy Development in Southern Africa through Comparative Reflection. In: International Journal of Inclusive Education 17, 4, S. 420-434. doi:10.1080/13603116.2011.651821.

*Perold, Mariechen/Louw, Charmaine/Kleynhans, Sandra (2010): Primary School Teachers' Knowledge and Misperceptions of Attention Deficit Hyperactivity Disorder (ADHD). In: South African Journal of Education 30, 3, S. 457-473.

Pijl, Sip J./Meijer, Cor J. W./Hegarty, Seamus (1997): Inclusive Education. A Global Agenda. London: Psychology Press.

*Pillay, Jace/Di Terlizzi, Marisa (2009): A Case Study of a Learner's Transition from Mainstream Schooling to a School for Learners with Special Educational Needs (LSEN). Lessons for Mainstream Education. In: South African Journal of Education 29, 4, S. 491-509.

Purdue, Kerry/Ballard, Keith/MacArthur, Jude (2001): Exclusion and Inclusion in New Zealand Early Childhood Education. Disability, Discourses and Contexts. In: International Journal of Early Years Education 9, 1, S. 37-49. doi: 10.1080/09669760120044178.

*Robson, Sue/Kanyanta, Sylvester B. (2007): Moving Towards Inclusive Education Policies and Practices? Basic Education for AIDS Orphans and Other Vulnerable Children in Zambia. In: International Journal of Inclusive Education 11, 4, S. 417-430. doi:10.1080/136031107013913 86.

*Savolainen, Hannu/Engelbrecht, Petra/Nel, Mirna/Malinen, Olli-Pekka. (2012): Understanding Teachers' Attitudes and Self-Efficacy in Inclusive Education: Implications for Pre-Service and In-Service Teacher Education. In: European Journal of Special Needs Education 27, 1, S. 5168. doi:10.1080/08856257.2011.613603.

Singal, Nidhi/Muthukrishna, Nithi (2014): Education, Childhood and Disability in Countries of the South - Re-positioning the Debates. In: Childhood 21, 3, S. 293-307.

Skrtic, Thomas M. (1991): Behind Special Education. A Critical Analysis of Professional Culture and School Organization. Denver: Love.

Slee, Roger (2011): The Inclusion Paradox: The Cultural Politics of Difference. In: Apple, Michael W./Au, Wayne/Gandin, Luís A. (Hrsg.): The Routledge International Handbook of Critical Education. New York and Abingdon: Routledge, S. 177-189.

Southern African Development Community (SADC) (2012): Member States. http://www.sadc. int/member-states/ [Zugriff: 23.02.2017].

Srivastava, Meenakshi/de Boer, Anke/Pijl, Sip J. (2015): Inclusive Education in Developing Countries. A Closer Look at its Implementation in the Last 10 Years. In: Educational Review 67, 2, S. 179-195. doi: 10.1080/00131911.2013.847061.

Strauss, Anselm L. (1994): Grundlagen qualitativer Sozialforschung. München: Fink.

Strauss, Anselm L./Corbin, Juliet (1996): Grounded Theory. Grundlagen qualitativer Sozialforschung. Weinheim: Psychologie Verlags Union.

*Thwala, S'lungile K./Ntinda, Kayi/Hlanze, Buyisile (2015): Lived Experiences of Parents of Children with Disabilities in Swaziland. In: Journal of Education and Training Studies 3, 4, S. 206-215. doi:10.11114/jets.v3i4.902.

United Nations (UN) (2006): Convention on the Rights of Persons with Disabilities and Optional Protocol. New York.

United Nations (UN) (2000). United Nations Millennium Declaration. New York.

United Nations Educational, Scientific, and Cultural Organization (UNESCO) (1990): World declaration on Education for All and framework for action to meet basic learning needs. New York. 
United Nations Educational, Scientific and Cultural Organization (UNESCO) (1994): Die Salamanca Erklärung und der Aktionsrahmen zur Pädagogik für besondere Bedürfnisse. http://bidok.uibk.ac.at/library/unesco-salamanca.html [Zugriff: 04.07.2019].

United Nations Statistics Division (UNSD) (2016): Composition of Macro Geographical (Continental) Regions, Geographical Sub-Regions, and Selected Economic and Other Groupings. https://unstats.un.org/unsd/methods/m49/m49regin.htm [Zugriff: 23.02.2017].

*Urwick, James/Elliott, Julian (2010): International Orthodoxy versus National Realities. Inclusive Schooling and the Education of Children with Disabilities in Lesotho. In: Comparative Education 46, 2, S. 137-150. doi:10.1080/03050061003775421.

*Walton, Elizabeth (2011): Getting Inclusion Right in South Africa. In: Intervention in School and Clinic 46, 4, S. 240-245. doi:10.1177/1053451210389033.

*Walton, Elizabeth (2013): Inclusion in a South African High School? Reporting and Reflecting on What Learners Say. In: International Journal of Inclusive Education 17, 11, S. 1171-1185. doi:10.1080/13603116.2012.742577.

*Walton, Elizabeth (2015): Global Concerns and Local Realities. The "Making Education Inclusive" Conference in Johannesburg. In: Intervention in School and Clinic 50, 3, S. 173-177. doi:10.1177/1053451214542039.

*Walton, Elizabeth/Lloyd, Gillian (2011): An Analysis of Metaphors Used for Inclusive Education in South Africa. In: Acta Academica 43, 3, S. 1-31.

*Walton, Elizabeth/Rusznyak, Lee (2014): Affordances and Limitations of a Special School Practicum as a Means to Prepare Pre-Service Teachers for Inclusive Education. In: International Journal of Inclusive Education 18, 9, S. 957-974. doi:10.1080/13603116.2013.872203.

*Walton, Elizabeth/Rusznyak, Lee (2016): Approaches to Assessing Preservice Teachers' Learning in Authentic and Rigorous Ways. The Case of an Inclusive Education Module. In: Perspectives in Education 34, 1, S. 84-101.

*Yssel, Nina/Engelbrecht, Petra/Oswald, Marietjie/Eloff, Ima/Swart, Estelle (2007): Views of Inclusion. A Comparative Study of Parents' Perceptions in South Africa and the United States. In: Remedial and Special Education 28, 6, S. 356-365.

*Zembylas, Michalinos/Bekerman, Zvi/McGlynn, Claire/Ferreira, Ana (2009): Teachers' Understanding of Reconciliation and Inclusion in Mixed Schools of Four Troubled Societies. In: Research in Comparative and International Education 4, 4, S. 406-422 http://dx.doi.org/10.2304/ rcie.2009.4.4.406 



\section{II \\ Komparative Perspektiven zu ausgewählten nationalen, regionalen und lokalen Bildungskontexten}

\section{A \\ Gesetzliche Bedingungen und Governance}





\title{
9 Der Einfluss der UN-BRK auf inklusive Bildung in Nigeria und Deutschland
}

\author{
Julia Biermann
}

\begin{abstract}
Welchen Einfluss hat Artikel 24 der UN-Behindertenrechtskonvention (UN-BRK) auf die Entwicklung inklusiver Schulsysteme im globalen Süden und Norden? Diese Frage wird im Beitrag basierend auf der Rekonstruktion bildungspolitischer Diskurse in Nigeria und Deutschland untersucht - zwei Vertragsstaaten, deren Schulsysteme gleichermaßen, wenn auch höchst unterschiedlich, durch das Recht auf inklusive Bildung herausgefordert sind. Der Vergleich wird zeigen, dass in beiden Diskursen die Auffassung vorherrscht, dass die Entwicklung eines inklusiven Schulsystems von einem sonderpädagogischen Fördersystem abhängt: in Nigeria aufgrund seines Mangels und in Deutschland aufgrund des hohen Grades seiner Institutionalisierung. Somit kann ein entscheidender Faktor, welcher die Umsetzung von Artikel 24 UN-BRK weltweit behindert, enthüllt werden; und das ist die diskursiv-institutionelle Macht sonderpädagogischer Fördersysteme, den erreichten oder ausbleibenden Fortschritt eines Landes bei der Realisierung einer ,Bildung für Alle‘ zu belegen.
\end{abstract}

\section{Globaler Kontext: Von der Exklusion zur Inklusion in Bildung}

Artikel 24 des Übereinkommens der Vereinten Nationen über die Rechte von Menschen mit Behinderungen (kurz: UN-Behindertenrechtskonvention, UN-BRK) etabliert inklusive Bildung als globale Menschenrechtsnorm, welche Vertragsstaaten völkerrechtlich zum Aufbau inklusiver Bildungssysteme verpflichtet (Della Fina 2017). Im Allgemeinen Kommentar zu Artikel 24 definiert der Ausschuss der Vereinten Nationen für die Rechte von Menschen mit Behinderungen, dass dies Systeme sind, welche Kinder und Jugendliche nicht auf Basis einer Behinderung diskriminieren, sondern gleiche Chancen für alle verwirklichen (CRPD 2016). Die Realisierung des Rechts auf inklusive Bildung verlangt daher nach einer kritischen Reflexion der strukturellen und professionellen Ausrichtung von Schulsystemen.

Die globale Tragweite der Herausforderung zu Reformen wird deutlich angesichts der unterschiedlichen Art und Weise, in welcher Schulsysteme für die Bildung von Kindern mit Behinderungen sorgen (Richardson/Powell 2011). In den Ländern des globalen Südens ist der universelle Zugang zu Grundschulbildung ein noch zu erreichendes Ziel (Mizunoya 2016). Die Zahl schulabstinenter Kinder ist besonders hoch in Subsahara-Afrika; hier leben die Hälfte aller Kinder, welche weltweit keinen Zugang zu formaler Schulbildung haben (Omoeva et al. 2013: 11). Die weit verbreitete Realität von Bildungsausschluss, von welchem insbesondere Kinder und Jugendliche mit Behinderungen betroffen sind, stellt daher eine 
zentrale Herausforderung für die Realisierung des Rechts auf inklusive Bildung dar (WHO/World Bank 2011; UNICEF 2013). In den Ländern des globalen Nordens, wo Massenschulsysteme bereits realisiert sind, bestehen ebenso behinderungsbasierte Ungleichheiten. Diese beziehen sich allerdings nicht auf den absoluten Ausschluss von formaler Bildung, sondern sind verbunden mit der Institutionalisierung sonderpädagogischer Fördersysteme. Diese weisen, unabhängig von den Organisationsformen und Klassifikationsmodi, eine Überrepräsentation von männlichen Schülern, sozial benachteiligten Kindern und ethnischen Minderheiten auf (Berhanu/Dyson 2012; Gabel et al. 2009). Die Realisierung des Rechts auf inklusive Bildung ist hier vor allem durch die weit verbreitete Realität von Segregation und Separation herausgefordert (Beco 2016; Biermann/Powell 2014).

Welchen Einfluss hat die UN-BRK auf die Entwicklung inklusiver Schulsysteme im globalen Süden und Norden? Wie können sich die in Artikel 24 verankerten Menschenrechtsideen gegen die institutionellen Kräfte durchsetzen, die bisher für die Exklusion und Segregation von Kindern und Jugendlichen mit Behinderungen sorgen? Um diese Fragen zu beantworten, wird vergleichend untersucht, welche institutionellen Wirkungen die bildungspolitischen Diskurse um inklusive Bildung in Nigeria und Deutschland haben; zwei UN-BRK Vertragsstaaten mit stark kontrastierenden Schulsystemen. Nigeria ist das Land mit der weltweit höchsten Exklusionsquote, wo die meisten behinderten und benachteiligten Kinder und Jugendlichen überhaupt keinen Zugang zu Bildung haben (FME 2015). Deutschland ist das Land mit einem der differenziertesten sonderpädagogischen Fördersysteme weltweit, in dem Kinder mit Behinderungen hauptsächlich in Sonderschulen und damit getrennt von ihren Peers lernen (Powell/Pfahl 2012; KMK 2016).

Die Ergebnisse der Analyse werden zeigen, dass Artikel 24 UN-BRK sowohl in Nigeria als auch in Deutschland die Verhandlungen über inklusive Bildung beeinflusst und dass diese Verhandlungen Diskurse hervorbringen, die gleichermaßen von einer ,Sonderpädagogisierung der Inklusion“ gekennzeichnet sind - nicht trotz, sondern wegen der großen Unterschiede, die zwischen beiden Schulsystemen bestehen. Auf diese Weise wird in beiden Ländern zwar auf menschenrechtliche Vorgaben reagiert, diese werden jedoch aufgrund kontextueller Besonderheiten umgedeutet und können daher letztlich nicht realisiert werden (Biermann 2018; 2019).

\section{Inklusive Bildung als globale Menschenrechtsnorm: Die Reformherausforderung von Artikel 24 UN-BRK}

Artikel 24 UN-BRK verankert inklusive Bildung als globale Menschenrechtsnorm und stellt damit den vorläufigen Höhepunkt eines jahrzehntelangen Bemühens zur systematischen Stärkung des Rechts auf Bildung für Menschen mit Behinderungen dar (Biermann 2015).

Seit Verabschiedung der UN-BRK ist inklusive Bildung nun nicht mehr länger ,nur` eine Strategie, die dazu beitragen soll, dass das globale Entwicklungsziel ,Bildung für alle ' $(E d u$ cation for All) erreicht wird, wie dies z.B. in der Salamanca-Erklärung von 1994 festgehalten wurde (World Conference on Special Needs Education 1994); inklusive Bildung ist fortan ein Menschenrecht (Degener 2012; de Beco 2014). Der Ausschuss der Vereinten Nationen für die Rechte von Menschen mit Behinderungen definiert inklusive Bildung in seinem Allgemeinen Kommentar zu Art. 24 UN-BRK als ein fundamentales Menschenrecht aller Ler- 
nenden, dessen Realisierung den kontinuierlichen Abbau von Barrieren verlangt, welche den Zugang zu und die gleichberechtigte Teilhabe Aller behindern (CRPD 2016: 10, lit. a, d).

Mit der Ratifizierung der UN-BRK sind die Vertragsstaaten nun also aufgefordert, dafür zu sorgen, dass Kinder und Jugendliche nicht aufgrund von einer Behinderung von Bildung ausgeschlossen werden, sondern gemeinsam mit ihren Peers in wohnortnahen Schulen lernen können (UN OHCHR 2013: 3f.). Perspektivisch impliziert diese Verpflichtung die Überwindung sowohl von Exklusion (der Verweigerung jeglichen Zugangs zu Bildung) als auch von Segregation (der Trennung von Lernumwelten von Kindern und Jugendlichen aufgrund von Behinderungen) (CRPD 2016: lit. 11).

Artikel 24 UN-BRK enthält also einen Reformauftrag - das ist die Vorgabe der Sicherstellung inklusiver Bildungssysteme - und eine Reformagenda - das ist das angestrebte Ergebnis der Verfügbarkeit und Zugänglichkeit aller Schulen für alle Kinder und Jugendlichen. Zusammen bilden Reformauftrag und Reformagenda eine ,Programmatik des Wandels', welche den Zweck und das Ziel der Realisierung des Rechts auf inklusive Bildung festhält und Bildungswandel in den Vertragsstaaten anleiten soll (Biermann 2018: 17f.). Diese ,Programmatik des Wandels' stellt Vertragsstaaten also vor die gleiche Reformaufgabe, fordert sie jedoch in unterschiedlicher Art und Weise heraus, je nachdem welche Schulen für Kinder und Jugendliche mit Behinderungen verfügbar und zugänglich sind. Über die Verfügbarkeit und Zugänglichkeit von Bildung entscheiden die Regeln, Normen und Überzeugungen, welche Schulsysteme als Institutionen stabilisieren (Scott 2008); sie bringen die Bildungsrealitäten hervor, welche gleichberechtigte Teilhabe behindern, und sind folglich die ,Objekte des Wandels‘ (Biermann 2018: 24ff.).

\section{Methodologie: Bildungswandel und Diskurse}

Für die Frage nach dem Einfluss von Artikel 24 UN-BRK auf die Entwicklung inklusiver Schulsysteme ist es zentral zu verstehen, wie die in ihm enthaltenen Menschenrechtsideen in den institutionellen Wandel von Schulsystemen übersetzt werden. Dafür ist es notwendig, die kontextspezifischen Diskurse um inklusive Bildung zu analysieren.

Diese Annahme basiert auf einem wissenssoziologischen Zugang zur sozialen Konstruktion von Wirklichkeit (Keller et al. 2005). Demnach verleihen Akteure durch ihre (kommunikativen) Handlungen der Welt Sinn, welcher als Wissen gespeichert wird (Keller 2008: 235). Im Verlauf der Zeit verfestigt sich dieses Wissen zu Institutionen; d.h. es wird objektiviert in Regeln, Normen und Überzeugungen (Scott 2008: 48). Diese Objektivierung geschieht in und durch Diskurse - die regulierten Praktiken der Wissensproduktion, durch welche die Welt gewusst werden kann (Foucault 2002: 49f., 201). Daraus folgt, dass, wenn das Versprechen, inklusive Bildungssysteme sicherzustellen, eingelöst werden soll, sich das in institutionelle Regeln, Normen und Überzeugungen eingelassene Wissen ändern muss, welches bisher in nationalen Schulsystemen behinderungsbasierte Exklusion und Segregation legitimiert. Einer der Orte, an welchem dieses Wissen herausgefordert wird, sind die Debatten bildungspolitischer Akteure - das ist die Gruppe staatlicher und zivilgesellschaftlicher Organisationen, die über die Entwicklung eines inklusiven Schulsystems sprechen und/oder mit Bezug darauf handeln (Schmidt 2010). In diesen Debatten wird sowohl verhandelt, welche Bedeutung Artikel 24 UN-BRK für Bildungswandel im jeweiligen Landeskontext hat, als auch, welche Richtung dieser Wandel einschlägt. 
Die empirische Untersuchung setzt also an den bildungspolitischen Debatten über inklusive Bildung in Nigeria und Deutschland auf Bundesebene an; die Ebene, auf der die BRK in Deutschland 2009 und in Nigeria 2010 ratifiziert wurde (UN 2020). ${ }^{1}$ Das Ziel ist, die Wissenskonfigurationen zu rekonstruieren, welche die Diskurse um inklusive Bildung in beiden Ländern bis 2015 tragen. $^{2}$ Damit wird ein Einblick in die Verhandlungen kurz nach Ratifizierung der UN-BRK gegeben, nicht jedoch in die materiellen Aspekte dieses Wandels oder konkrete Umsetzungen; diese können zeitverzögert zwar eintreten, müssen es aber nicht.

\section{Fallstudien: Diskurse um inklusive Bildung}

Wie wird die in Artikel 24 UN-BRK enthaltene ,Programmatik des Wandels‘ in Nigeria und Deutschland verhandelt? Welche Wandlungsprozesse werden entworfen und in welchem Verhältnis stehen diese zur globalen Menschenrechtsnorm? Um diese Fragen zu beantworten, werden im Folgenden die Diskurse um inklusive Bildung vor dem Hintergrund der historisch entstandenen Spezifika des jeweiligen Schulsystems vorgestellt.

\subsection{Nigeria: Institutionalisierung eines sonderpädagogischen Fördersystems zur Überwindung von Exklusion und Realisierung von Inklusion}

Charakteristisch für das formale nigerianische Schulsystem ist der Mangel an Bildungsangeboten, welche es allen Kindern und Jugendlichen ermöglichen würden, Zugang zu und Teilhabe an formaler Bildung zu erhalten. Dieses exkludierende System findet seinen Ursprung in der Einführung westlicher Bildung durch Missionare in den 1840 Jahren. Diese verstanden Bildung als Mittel der Evangelisierung und beschränkten sie auf rudimentäre Lese- und Schreibfähigkeiten für sehr wenige Personen (Fafunwa 1991: 127). Das Engagement der britischen Kolonialregierung seit den 1880er Jahren trug in den folgenden Jahrzehnten - dem Konzept der ,indirect rule' folgend (Falola/Heaton 2008: 110) - dann zu einer starken Regionalisierung des sehr exklusiven Schulsystems bei (Fafunwa 1991: 164ff.).

Als Nigeria im Jahr 1960 seine Unabhängigkeit erlangte, versuchte die Regierung das uneinheitliche Bildungssystem zu reformieren, was jedoch aufgrund massiver politischer

1 Trotz weitreichender Zuständigkeit der Bundesstaaten in Nigeria und der Bundesländer in Deutschland für Bildungsgesetzgebung ist es möglich, Verhandlungen über die damit verbundene Reformherausforderung gerade unter bundespolitischen Akteure zu beobachten. Sie sind in Übereinstimmung mit Art. 33 UN-BRK damit beauftragt, den internationalen Menschenrechtsvertrag in innerstaatliches Recht zu überführen, einen nationalen Aktionsplan zu entwickeln und der UNO den ersten Staatenbericht vorzulegen.

2 Das Textkorpus besteht in beiden Fällen aus Dokumenten bildungspolitischer Akteure; dazu zählen sowohl offizielle Texte der Bundesregierungen und Bildungsministerien als auch Texte, die von zivilgesellschaftlichen Organisationen, wie z.B. Professionsverbänden und Behindertenrechtsorganisationen, verfasst wurden. In Nigeria wurde zudem die Gruppe der internationalen Organisationen einbezogen, da diese aktiv an der Gestaltung des Bildungswesens beteiligt sind. 
Umwälzungen und eines Bürgerkriegs (1967 -1970) scheiterte (Falola 1999: 95ff.). Um die zerrüttete nationale Einheit zu fördern, verabschiedete die Regierung dann im Jahr 1977 die National Policy on Education, welche bis heute in überarbeiteter Form in Kraft ist, und führte ein Universal Primary Education Programme ein (Fafunwa 1991: 218f.). Aufgrund des wirtschaftlichen Abschwungs, der Ölkrise in den 1980er Jahren und politischer Unruhen fielen die durch das Primarbildungsprogramms gestiegenen Einschulungsraten massiv, und das Bildungssystem stand zu Beginn der 1990er Jahre kurz vor dem Zusammenbruch (Garuba 1996: 77; FME 2003: 11f). Das Engagement im Bildungssektor nahm dann ab dem Jahr 2000 unter Eindruck der Millennium-Entwicklungsziele und der erneuten Verpflichtung auf die Ziele einer ,Bildung für Alle“ - wieder massiv zu. So wurde ein zu Beginn der 1990er Jahre neu aufgelegtes Primarbildungsprogramm wiederbelebt und zu einem Grundbildungsprogramm ausgebaut (FME 2003: 39). Im Jahr 2004 trat dann das landesweit geltende Grundbildungsgesetz, der Compulsory, Free Universal Basic Education Act, in Kraft, welcher jedem Kind das Recht auf neun Jahre Grundbildung garantiert.

Trotz dieser Anstrengungen verzeichnet Nigeria die weltweit höchste Anzahl schulabstinenter Kinder; 10.5 Millionen im Jahr 2010, das sind ein Drittel aller schulpflichtigen Kinder (UNICEF/UIS 2012). ${ }^{3}$ Das größte Risiko für Bildungsausschluss stellt eine Behinderung dar; während die Hälfte aller Kinder mit körperlicher oder kognitiver Beeinträchtigung nicht zur Schule geht, gilt dies nur für 20 Prozent der Kinder ohne Behinderung (Mizunoya et al. 2016: 22). Dies liegt vor allem daran, dass es für Kinder mit Behinderungen kaum Vorkehrungen im öffentlichen System gibt (IOM 2014: 42; Obiakor et al. 2012). Diese Zahlen unterstreichen die aktuelle Realität der institutionellen Exklusion in Nigeria. Wie bildungspolitische Akteure die Forderungen des Artikels 24 UN-BRK vor diesem Hintergrund verhandeln, ist eine empirische Frage, die im Folgenden beantwortet werden soll.

In Nigeria wird die Entwicklung eines inklusiven Schulsystems diskursiv mit der Implementierung des 2004 erlassenen Grundbildungsgesetzes verbunden. Die Implementierung dieses Gesetzes zielt auf die Realisierung des Entwicklungsziels einer ,Bildung für Alle'. Um dieses Ziel zu erreichen, schlagen politische und zivilgesellschaftliche Akteure vor, den Ausschluss besonders marginalisierter Gruppen durch die Einführung von Sonderbildungsprogrammen zu überwinden. Dazu zählen neben Alphabetisierungsprogrammen (mass literacy programmes) und nomadischer Bildung (nomadic education) auch integrierter Koranunterricht (integrated Qur'anic education) und sonderpädagogische Förderung (special needs education) (Biermann/Pfahl 2018).

Wenn es um sonderpädagogische Förderung geht, dann stehen Kinder mit Behinderungen und ihr Recht auf Grundbildung im Zentrum der Debatten. Internationale Organisationen und staatliche Akteure fokussieren in diesem Zusammenhang vor allem auf Bildungsexpansion, welche durch die Inklusion von Menschen mit Behinderungen in das formale Bildungssystem erreicht werden soll. Zivilgesellschaftliche Akteure, allen voran Behindertenrechtsorganisationen, stellen dahingegen den Bedarf an angemessenen Unterstützungssystemen ins Zentrum ihrer Argumente. Von beiden Gruppen wird daher letztlich das Fehlen eines sonderpädagogischen Fördersystems als der Hauptgrund für behinderungsbasierte Ausschlüsse identifiziert. Die Schaffung eines solchen Systems gilt daher sowohl als wichtiger Schritt, um das Recht auf Grundbildung von Menschen mit Behinderungen zu realisieren als auch, um das Entwicklungsziel ,Bildung für Alle‘ zu erreichen.

3 Diese Zahl wurde im Jahr 2014 auf 8.7 Millionen korrigiert (Ajikobi 2017). Es gibt mittlerweile Hinweise, dass die Zahl schulabstinenter Kinder wieder gestiegen ist (Adedigba 2018). 
Tabelle 1: Dimensionen des Bildungswandels in Nigeria

\begin{tabular}{ll}
\hline Ziel des Wandels & Entwicklung eines inklusiven Schulsystems \\
\hline Grund des Wandels & Implementierung des Grundbildungsgesetzes \\
\hline Problem des Wandels & Mangel an Bildungsangeboten für Kinder mit Behinderungen \\
\hline Lösungen für den Wandel & $\begin{array}{l}\text { Strukturell und professionell: spezielle Vorkehrungen und Unterstützungs- } \\
\text { systeme für Kinder mit Behinderungen }\end{array}$ \\
\hline Angestrebtes Ergebnis & $\begin{array}{l}\text { Bildungsexpansion durch Bereitstellung eines sonderpädagogischen } \\
\text { Fördersystems }\end{array}$ \\
\hline
\end{tabular}

Quelle: Eigene Darstellung

Mit diesen Inhalten wird im nigerianischen Diskurs der Reformauftrag zur Entwicklung eines inklusiven Schulsystems anerkannt; alle Akteure erklären ihn zum Ziel des Wandels, um das Recht auf Grundbildung für marginalisierte Gruppen zu verwirklichen. Jedoch wird das Reformmandat ,alle Schulen für alle' abgelehnt, da die Realität von Exklusion dazu zwinge, sich zunächst auf die Bereitstellung von Sonderbildungsangeboten für marginalisierte Gruppen zu konzentrieren, was für Menschen mit Behinderungen die Bereitstellung eines sonderpädagogischen Fördersystems verlange. Damit wird die Entwicklung eines inklusiven Schulsystems zu einem Anliegen der Institutionalisierung eines sonderpädagogischen Fördersystems, sowohl um Exklusion zu überwinden als auch Inklusion zu realisieren.

\subsection{Deutschland: Verteidigung des hoch professionalisierten sonderpädagogischen Fördersystems}

Deutschland verfügt, weltweit gesehen, über ein sehr gut ausgebautes, aber zugleich stark segregierendes Schulsystem. Dieses zeichnet sich durch die professionelle und strukturelle Trennung von regel- und sonderpädagogischen Bildungsangeboten aus und geht historisch auf die Etablierung von Hilfs- und Sonderschulen zurück. Diese Schulen ebneten seit Mitte des 19. Jahrhunderts sowohl körperlich und geistig beeinträchtigten als auch sozial benachteiligten Kindern den Weg ins formale Bildungssystem (Pfahl 2011: 116).

Im Zuge der Etablierung der Heilpädagogik entwickelten sich Hilfsschulen in den 1920er Jahren zu einem eigenständigen Schultyp für Kinder, die in den Volksschulen scheiterten (Hänsel 2005: 105; Powell 2011: 153). Der Status von Hilfsschulen als ,bedeutende subsidiäre Schulform' wurde in den darauffolgenden Jahrzehnten gestärkt (Powell 2011: 155); während der Nazi-Herrschaft wurden sie mit der Aufgabe betraut, „,bildungsunfähige“ Kinder zu identifizieren (Hänsel 2006: 145ff.; Powell 2011: 157f.). Nach dem Zweiten Weltkrieg erfolgte der Wiederaufbau des zerstörten Schulsystems dann in zwei ideologisch gegensätzlichen Staaten. In der DDR wurde ein sozialistisches Schulsystem eingeführt, welches über ein weitreichendes sonderpädagogisches System verfügte (Poore 2009: 257). Auch in der Bundesrepublik erfolgte der institutionelle Ausbau sonderpädagogischer Förderung; so entwickelte sich die Hilfsschule in eine Sonderschule für Lernbehinderte und in eine separate Sonderschule für geistig Behinderte (Powell 2011: 82, 84). Bestrebungen zur Integration behinderter Kinder in Regelschulen wurden in den 1970er Jahren durch die Einführung von Gesamtschulen in einigen Bundesländern beflügelt. Angesichts des heftigen politischen Widerstands gegen diese konnte Integration jedoch nicht flächendeckend realisiert werden 
(Ellger-Rüttgardt 2016: 22). In der DDR war Integration keine Option, da jede private Initiative von Eltern oder Behindertenrechtsgruppen unterdrückt wurde (Poore 2009: 48).

Mit der Wiedervereinigung Deutschlands im Jahr 1990 wurde das westdeutsche Schulsystem in weiten Teilen von den neu geschaffenen östlichen Bundesländern übernommen, wobei die Unabhängigkeit des Sonderschulwesens erhalten geblieben ist. Im Jahr 1994 wurde das gesamtdeutsche sonderpädagogische Fördersystem durch Einführung eines neuen Klassifikationssystems durch die KMK reformiert (KMK 1994); dieses fokussiert nun auf individuelle Förderbedarfe, orientiert sich aber weiter an den verschiedenen Sonderschultypen (Pfahl 2011: 23f.). Dieses System wurde im Jahr 2011 mit den Empfehlungen der KMK zur inklusiven Bildung von Kindern und Jugendlichen mit Behinderung bestätigt (KMK 2011).

Aktuell lernen zwei Drittel aller Schüler*innen mit einem diagnostizierten sonderpädagogischen Förderbedarf in Sonderschulen; das sind sieben Prozent der Gesamtschüler*innenschaft. Die Zahl ist über die letzten Jahre relativ stabil geblieben, trotz eines steigenden Anteils an Schüler*innen mit sonderpädagogischem Förderbedarf, die in Regelschulen lernen (Powell/Pfahl 2012: 723f.; KMK 2016: XIV, XVIf.).

In Deutschland ist die Entwicklung eines inklusiven Schulsystems eng mit der Ratifizierung der UN-BRK verbunden, welche eine politische Debatte zwischen staatlichen und zivilgesellschaftlichen Akteuren auf Bundesebene angestoßen hat. In diesen Debatten sind sich die politischen Akteure einig, dass mit dem Wandel die Ausweitung des gemeinsamen Lernens von Kindern mit und ohne Behinderungen in Regelschulen angestrebt wird; ein Prozess, welcher den Transfer von sonderpädagogischen Ressourcen und Fachwissen in Regelschulen erfordere. Jedoch wird kontrovers debattiert, was dies für Sonderschulen als segregierte Lernräume bedeutet. Staatliche Akteure und Professionsverbände argumentieren für den Erhalt von Sonderschulen als historische Errungenschaft. Mehr noch, sie identifizieren Sonderschulen als Garant für die Realisierung des Rechts auf Bildung von Kindern mit Behinderungen. Zivilgesellschaftliche Akteure und Behindertenrechtsorganisationen sehen die Schulform dahingegen als das Haupthindernis für die Entwicklung eines inklusiven Schulsystems, da sie den Zugang zu Regelschulen behindere. Aus diesem Grund besteht Uneinigkeit darüber, ob der Ausbau des gemeinsamen Lernens eine grundlegende Transformation der segregierten Schulstrukturen verlangt oder nur einige Reformen.

Tabelle 2: Dimensionen des Bildungswandels in Deutschland

\begin{tabular}{ll}
\hline Ziel des Wandels & Entwicklung eines inklusiven Schulsystems \\
\hline Grund des Wandels & Umsetzung der UN-BRK \\
\hline Problem des Wandels & Organisation sonderpädagogischer Förderung \\
\hline Lösungen für den Wandel & $\begin{array}{l}\text { Strukturell: Beibehaltung vs. Schließung von Sonderschulen } \\
\text { Professionell: Transfer sonderpädagogischer Förderung in Regelschulen }\end{array}$ \\
\hline Angestrebtes Ergebnis & $\begin{array}{l}\text { Ausbau des gemeinsamen Lernens von Kindern mit und ohne } \\
\text { Behinderungen in Regelschulen (bei bestehendem Dissens über die Zukunft } \\
\text { der Sonderschulen) }\end{array}$ \\
\hline
\end{tabular}

Quelle: Eigene Darstellung

Mit diesen Inhalten wird im deutschen Diskurs der Reformauftrag zur Entwicklung eines inklusiven Schulsystems anerkannt; alle Akteure erkennen ihn als Ziel des Bildungswandels an, um gemeinsames Lernen in Regelschulen zu verwirklichen. Die Reformagenda , alle Schulen für alle' bleibt dagegen umstritten; sie wird im Diskurs aufgrund des argumentativen 
Konflikts über die Zukunft der Sonderschulen weder vollkommen anerkannt noch komplett zurückgewiesen. So geht es mit der Entwicklung eines inklusiven Schulsystems letztlich darum, mehr, aber nicht allen, Schüler*innen mit Behinderungen den Zugang zu Regelschulen zu ermöglichen. Damit wird die Entwicklung eines inklusiven Schulsystems zu einem Anliegen der Beibehaltung sonderpädagogischer Professionalität und Ressourcen, so dass das sonderpädagogische Fördersystem zunehmend aus den Sonderschulen in die Regelschulen transferiert wird.

\section{Vergleich: Die „Sonderpädagogisierung der Inklusion“ als globale Logik des Bildungswandels}

In beiden Diskursen wird auf die Herausforderung inklusiver Bildung mit mehr und besserer sonderpädagogischer Förderung reagiert; in Nigeria, um das Entwicklungsziel ,Bildung für Alle' zu erreichen, und in Deutschland, um gemeinsames Lernen zu realisieren. Das sonderpädagogische Fördersystem wird damit zum Bezugspunkt, um kontextuelle Herausforderungen, die mit der Entwicklung eines inklusiven Systems einhergehen, ausdrücken zu können in Nigeria ist das eine von Exklusion geprägte und in Deutschland eine von Segregation geprägte Bildungsrealität. In diesem Sinne resultieren beide Diskurse in einer ,Sonderpädagogisierung der Inklusion " - nicht trotz, sondern gerade wegen der ungleichen schulischen Ausgangsbedingungen (Biermann 2018: 177ff.; Biermann 2019). Das heißt, in beiden Diskursen wird die Auffassung befördert, dass die Entwicklung eines inklusiven Schulsystems von einem sonderpädagogischen Fördersystem abhängt - in Nigeria aufgrund seines Fehlens und in Deutschland aufgrund seines hohen Institutionalisierungsgrades.

Mit der ,Sonderpädagogisierung der Inklusion“ wird folglich die Richtung des Wandels vorgegeben, welcher die materiellen Aspekte des Bildungswandels in beiden Ländern anweist; das ist in Nigeria der Aufbau und in Deutschland der Erhalt eines sonderpädagogischen Fördersystems. Die Hauptverantwortung für die Bildung von Kindern mit Behinderung wird somit in die Hände der sonderpädagogischen Institutionen und Professionen gelegt; diese müssen die Probleme bewältigen, die entstehen, wenn Kinder mit Behinderung Zugang zum Schulsystem oder zu Regelschulen erhalten. Trotz der rhetorischen und rechtlichen Bekenntnisse zu inklusiver Bildung wird damit in beiden Diskursen letztlich die Legitimität von Segregation auf Basis von Behinderung aufrechterhalten.

Dieser Akt des Abweichens von den in Artikel 24 verankerten Prinzipien aus kontextuellen Gründen - so unterschiedlich diese auch sind - basiert auf der geteilten Überzeugung, dass Schulsysteme, welche sonderpädagogische Fördersysteme vorhalten, am besten in der Lage sind, das Recht auf Bildung für Menschen mit Behinderungen zu realisieren. Diese Überzeugung wird durch zirkuläre Annahmen gestützt, die sich in den Verhandlungen um inklusive Bildung gegenseitig verstärken: erstens, dass Menschen mit Behinderungen besondere Bedürfnisse haben, die ihre Teilhabe am Regelunterricht verhindern, und zweitens, dass Schulsysteme dementsprechend sonderpädagogische Angebote vorhalten müssen.

Auch wenn sonderpädagogische Fördersysteme historisch betrachtet dazu beigetragen haben, die Exklusion von beeinträchtigen und benachteiligten Schüler*innen in vielen Bildungssystemen weltweit zu überwinden (Richardson/Powell 2011; Richardson/Wu/Judge 2017), entspricht diese Form der Bildungsteilhabe nicht den Anforderungen des Menschen- 
rechts auf inklusive Bildung, da sie einen diskriminierungsfreien Zugang zu wohnortnahen allgemeinen Schulen behindern (CRPD 2016).

\section{Zusammenfassung und Ausblick}

Die vergleichende Analyse zeigt, dass die diskursive Aneignung der in Artikel 24 UN-BRK enthaltenen Menschenrechtsideen sowohl in Nigeria als auch in Deutschland erfolgreich ist. Die Diskurse unterstützen bislang aber (noch) nicht die Umsetzung der global angestrebten Reformagenda, wie im Allgemeinen Kommentar zu Artikel 24 verankert. Der Grund dafür ist, dass die Verhandlungen in beiden Ländern Diskurse hervorgebracht haben, die von einer ,Sonderpädagogisierung der Inklusion“ gekennzeichnet sind, und dies gleichermaßen angesichts der unterschiedlichen Möglichkeiten zur Bereitstellung sonderpädagogischer Förderung. Somit wird ein Paradox erzeugt: die globale Norm inklusiver Bildung wird durch die Institutionalisierung oder Aufrechterhaltung fähigkeitsselektiver Systeme verfolgt, die Kinder und Jugendliche auf Basis einer Behinderung von ihren Peers trennen, anstatt ein solches System auf menschenrechtlicher Basis herauszufordern.

Ein entscheidender Faktor, welcher zur Nicht-Realisierung von Artikel 24 UN-BRK beiträgt, ist also die diskursiv-institutionelle Macht sonderpädagogischer Fördersysteme, den erreichten oder ausgebliebenen Fortschritt eines Landes bei der Realisierung einer ,Bildung für Alle' zu bestätigen. Die Unterscheidung zwischen Ländern, welche formale Massenschulbildung mit Hilfe sonderpädagogischer Angebote bereits verwirklicht haben oder eben noch nicht, bewirkt, dass die Entwicklung inklusiver Schulsysteme im globalen Kontext zu einem evolutionären und linearen Prozess der Bildungsexpansion wird. Dieser trägt zwar zur Inklusion von Menschen mit Behinderungen in formale Systeme der Massenschulbildung bei, bewirkt aber auch ihren beständigen Ausschluss von wohnortnahen Regelschulen.

Die globalen Politiken der formalen Massenschulbildung tragen also auch dazu bei, dass behinderungsbasierte Diskriminierungen aufrechterhalten werden. Für vergleichende Inklusionsforscher*innen impliziert dies, die fähigkeitsselektiven und entwicklungsbasierten Rationalitäten, welche der ,Sonderpädagogisierung der Inklusion' zugrunde liegen, kritisch und für die eigene Arbeit zu reflektieren. Denn wenn es etwas gibt, was Schulsysteme im globalen Süden und Norden in Bezug auf die Realisierung des Menschenrechts auf inklusive Bildung gleichermaßen herausfordert, dann ist es die Erkenntnis, dass sonderpädagogische Fördersysteme komplizierter sind, als nur den Zugang zu formaler Bildung zu ermöglichen.

\section{Literatur}

Adedigba, Azeezat (2018): Nigeria now has 13.2 million out of school children - UBEC. https:// www.premiumtimesng.com/news/top-news/288344-nigeria-now-has-13-2-million-out-ofschool-children-ubec.html [Zugriff: 27.05.2020].

Ajikobi, David (2017): Does Nigeria have the world's most girls out-of-school, as Malala claimed? https://africacheck.org/reports/nigeria-girls-school-worldwide-activist-malala-claimed/ [Zugriff: 27.05.2020]. 
Beco, Gauthier de (2016): Transition to Inclusive Education Systems According to the Convention on the Rights of Persons with Disabilities. In: Nordic Journal of Human Rights 34, S. 40-59.

Beco, Gauthier de (2014): The Right to Inclusive Education according to Article 24 of the UN Convention on the Rights of Persons with Disabilities: Background, Requirements and (remaining) Questions. In: Netherlands Quarterly of Human Rights 32, 3, S. 263-287.

Berhanu, Girma/Dyson, Alan (2012): Special Education in Europe, Overrepresentation of Minority Students. In: Bank, James A. (Hrsg.): Encyclopedia of Diversity in Education. London: Sage, S. 2070-2073.

Biermann, Julia (2015): Historische Entwicklung des Rechts auf Bildung im UN-System, mit besonderem Fokus auf Menschen mit Behinderungen. Kommentierte Dokumentensammlung erstellt im Auftrag von Prof. Dr. Lisa Pfahl. digitale Bibliothek bidok - behinderung bildung dokumentation.

bidok.uibk.ac.at/projekte/sammlung-un-dokumente/download-un-dokumente/bidok_un-dokumente_uebersicht2.pdf [Zugriff: 27.05.2020].

Biermann, Julia (2018): Comparing Article 24 UN CRPD's Influence on Inclusive Education in Nigeria and Germany: Institutional Change in Educational Discourses. Berlin: Humboldt-Universität zu Berlin.

Biermann, Julia (2019): Sonderpädagogisierung der Inklusion. Artikel 24 UN-BRK und die Diskurse über die Entwicklung inklusiver Schulsysteme in Nigeria und Deutschland. In: Aus Politik und Zeitgeschichte 69, 6-7, S. 19-23.

Biermann, Julia/Pfahl, Lisa (2018): Wissen um Behinderung und das Recht auf Bildung: ein nigerianisch-deutscher Vergleich sonderpädagogischer Klassifikationssysteme und schulischer Inklusion. In: Bildung und Erziehung 71, 4, S. 432-448.

Biermann, Julia/Powell, Justin J.W. (2014): Institutionelle Dimensionen inklusiver Schulbildung - Herausforderungen der UN-Behindertenrechtskonvention für Deutschland, Island und Schweden im Vergleich. In: Zeitschrift für Erziehungswissenschaft 17, 4, S. 679-700.

CRPD [Committee on the Rights of Persons with Disabilities] (2016): General Comment on Article 24 UN CRPD. United Nations Document CRPD/C/GC/4.

Degener, Theresia (2012): Das Recht auf inklusive Bildung als Menschenrecht. In: Kritische Justiz 45, 4, S. 405-419.

Della Fina, Valentina (2017): Article 24 [Education]. In: Della Fina, Valentina/Cera, Rachele/Palmisano, Giuseppe (Hrsg.): The United Nations Convention on the Rights of Persons with Disabilities. A Commentary. Cham: Springer International, S. 439-470.

Ellger-Rüttgardt, Sieglind (2016): Historischer Überblick. In: Hedderich, Ingeborg/Biewer, Gottfried/Hollenweger, Judith/Markowetz, Reinhard (Hrsg.): Handbuch Inklusion und Sonderpädagogik. Bad Heilbrunn: Klinkhardt, S. 17-27.

Fafunwa, A. Babs (1991): History of Education in Nigeria. Ibadan: NPS.

Falola, Toyin (1999): The History of Nigeria. Westport, CT: Greenwood.

Falola, Toyin/Heaton, Matthew M. (2008): A History of Nigeria. Cambridge: Cambridge University Press.

FME [Federal Ministry of Education] (2003): Education Sector Status Report. Abuja: FME. https://planipolis.iiep.unesco.org/sites/planipolis/files/ressources/nigeria_ed_sector_status_may_2003.pdf [Zugriff: 27.05.2020].

FME [Federal Ministry of Education] (2015): Education for All - A Collective Responsibility. Nigeria EFA Review Report 2000-2014 Strides and Milestones. Abuja: FME. http://unesdoc.unesco.org/images/0023/002310/231081e.pdf [Zugriff: 27.05.2020].

Foucault, Michel (2002): The Archaeology of Knowledge. London: Routledge.

Gabel, Susan L./Curcic, Svjetlana/Powell, Justin J.W./Khader, Khaled/Albee, Lynn (2009): Migration and Ethnic Group Disproportionality in Special Education. In: Disability \& Society 24, 5, S. 625-639. 
Garuba, Ayo (1996): Basics of Special Education. Oyo: Educational and Managment Services EMS.

Hänsel, Dagmar (2005): Die Historiographie der Sonderschule. Eine kritische Analyse. In: Zeitschrift für Pädagogik 51, 1, S. 101-115.

Hänsel, Dagmar (2006): Die NS-Zeit als Gewinn für Hilfsschullehrer. Bad Heilbrunn: Klinkhardt.

IOM [International Organization for Migration] (2014): Needs Assessment of Nigerian Education Sector. Abuja: IOM. https://publications.iom.int/system/files/pdf/needs_assessment_nigerianeducsector.pdf [Zugriff: 27.05.2020].

Keller, Reiner/Hirseland, Andreas/Schneider, Werner/Viehöfer, Willy (2005): Die diskursive Konstruktion von Wirklichkeit. Einleitende Bemerkungen zum Verhältnis von Wissenssoziologie und Diskursforschung. In: Keller, Reiner/Hirseland, Andreas/Schneider, Werner/Viehöfer, Willy (Hrsg.): Die diskursive Konstruktion der Wirklichkeit. Konstanz: UVK, S. 7-21.

Keller, Reiner (2008): Wissenssoziologische Diskursanalyse. Grundlegung eines Forschungsprogramms. Wiesbaden: VS Verlag für Sozialwissenschaften.

KMK [Ständige Konferenz der Kultusminister der Länder in der Bundesrepublik Deutschland] (1994): Empfehlungen zur Sonderpädagogischen Förderung in den Schulen in der Bundesrepublik Deutschland. Bonn: KMK. http://www.kmk.org/fileadmin/veroeffentlichungen_beschluesse/1994/1994_05_06-Empfehlung-sonderpaed-Foerderung.pdf [Zugriff: 27.05.2020].

KMK [Ständige Konferenz der Kultusminister der Länder in der Bundesrepublik Deutschland] (2011): Inklusive Bildung von Kindern und Jugendlichen mit Behinderungen in Schulen. Berlin: KMK. https://www.kmk.org/fileadmin/veroeffentlichungen_beschluesse/2011/2011_10_ 20-Inklusive-Bildung.pdf [Zugriff: 27.05.2020].

KMK [Ständige Konferenz der Kultusminister der Länder in der Bundesrepublik Deutschland] (2016): Sonderpädagogische Förderung in Schulen 2005 bis 2014. Berlin: KMK. https:// www.kmk.org/fileadmin/Dateien/pdf/Statistik/Dokumentationen/Dok_210_SoPae_2014.pdf [Zugriff: 27.05.2020].

Mizunoya, Suguru/Mitra, Sopie/Yamasaki, Izumi (2016): Towards Inclusive Education. The impact of disability on school attendance in developing countries, Innocenti Working Paper No.2016-03. Florence: UNICEF. https://www.unicef-irc.org/publications/845-towards-inclusive-education-the-impact-of-disability-on-school-attendance-in-developing.html [Zugriff: 27.05.2020].

Obiakor, Festus E./Eskay, Michael/Afolayan, Michael O. (2012): Special Education in Nigeria. In: Mutua, Kagendo/Szymanski Sunal, Cynthia (Hrsg.): Advances in Research and Praxis in Special Education in Africa, Carribean, and the Middle East. Charlotte, NC: Information Age, S. 23-36.

Omoeva, Carina/Sylla, Benjamin/Hatch, Rachel/Gale, Charles (2013): Out of School Children. Data Challenges In Measuring Access to Education. Washington, DC: Education Policy and Data Center.

Pfahl, Lisa (2011): Techniken der Behinderung. Der deutsche Lernbehinderungsdiskurs, die Sonderschule und ihre Auswirkungen auf Bildungsbiografien. Bielefeld: transcript.

Poore, Carol (2009): Disability in Twentieth-Century German Culture. Ann Arbor, MI: University of Michigan Press.

Powell, Justin J.W. (2011): Barriers to Inclusion. Special Education in the United States and Germany. Boulder, CO: Paradigm.

Powell, Justin J.W./Pfahl, Lisa (2012): Sonderpädagogische Fördersysteme. In: Bauer, Ullrich/Bittlingmayer, Uwe H./Scherr, Albert (Hg.): Handbuch Bildungs- und Erziehungssoziologie. Wiesbaden: VS Verlag, S. 721-739.

Richardson, John G./Powell, Justin J.W. (2011): Comparing Special Education. Origins to Contemporary Paradoxes. Stanford, CA: Stanford University Press. 
Schmidt, Vivien A. (2010): Taking ideas and discourse seriously. In: European Political Science Review 2, 1, S. 1-25.

Scott, W. Richard (2008): Institutions and Organizations. 3. Aufl. Thousand Oaks, CA: Sage.

UN [United Nations] (2020): UN Treaty Collection: Convention on the Rights of Persons with Disabilities. https://treaties.un.org/Pages/ViewDetails.aspx?src=TREATY\&mtdsg_no=IV-15 \&chapter=4\&clang=_en [Zugriff: 27.05 .2020 ].

UN OHCHR [United Nations Office of the High Commissioner for Human Rights] (2013): Thematic study on the right of persons with disabilities. United Nations Document A/HRC/25/29.

UNICEF [United Nations Children's Fund] (2013): The State of the World's Children 2013: Children with Disabilities. New York, NY: UNICEF. https://www.unicef.org/publications/index_69379.html [Zugriff: 27.05.2020].

UNICEF [United Nations Children's Fund]/UIS [UNESCO Institute for Statistics] (2012): All Children in School by 2015. Nigeria Country Study. Abuja: UNICEF. http://uis.unesco.org/sites/default/files/documents/out-of-school-children-nigeria-country-study-education-2012en.pdf [Zugriff: 27.05.2020].

WHO [World Health Organization]/World Bank (2011): World Report on Disability. Geneva: WHO. https://www.who.int/publications-detail/world-report-on-disability [Zugriff: 27.05. 2020].

World Conference on Special Needs Education (1994): The Salamanca Statement and Framework for Action on Special Needs Education. Paris: United Nations Educational, Scientific and Cultural Organization. https://unesdoc.unesco.org/ark:/48223/pf0000098427 [Zugriff: 27.05. 2020]. 


\title{
10 Same same but different - Ein Vergleich der Entwicklung inklusiver Strukturen in zwei Bundesländern in Deutschland
}

\author{
Julia Gasterstädt
}

\begin{abstract}
Der Beitrag geht der Frage nach, wie das ,fuzzy concept “ (Artiles/Dyson 2005: 43) Inklusion auf regionaler Ebene umgesetzt und wie dieser Umsetzungsprozess gesteuert wird. Aufgezeigt wird die Komplexität dieser Prozesse am Beispiel von zwei Regionen in zwei Bundesländern. Dabei wird der Perspektive der Educational Governance-Forschung auf Steuerungsprozesse gefolgt und mit der Situationsanalyse (Clarke 2012) ein theoretischer und methodologischer Vorschlag gemacht, um solche Steuerungsprozesse rekonstruieren zu können.
\end{abstract}

\section{Einleitung}

Es liegt nahe, dass die Umsetzung einer globalen Norm, wie sie in Bezug auf die UN-Konvention über die Rechte von Menschen mit Behinderungen mit dem Begriff Inklusion gefasst ist (Brüggemann 2016), letztlich auf regionaler Ebene sowie auf der Interaktions- und Praxisebene erfolgen muss. Schriewer (2013) beschreibt dabei ein dialektisches Verhältnis zwischen der ,globale[n] Durchsetzung transnationaler Programme und Modelle auf der einen und [der] überraschenden Beharrungskraft variierender sozial-kultureller Interrelations-Gefüge auf der anderen Seite“ (Schriewer 2013: 35). Im Folgenden soll es um die Fragen gehen, wie die Aufforderung zur Entwicklung inklusiver Bildungssysteme aus der Konvention der Vereinten Nationen über die Rechte von Menschen mit Behinderungen (UN-BRK) in zwei deutschen Bundesländern umgesetzt und wie dieser Umsetzungsprozess gesteuert wird. Im Fokus steht dabei die Perspektive von an diesen Steuerungsprozessen beteiligten Akteur*innen, die Ausdruck davon ist, wie das „fuzzy concept“ (Artiles/Dyson 2005: 43) Inklusion regional verstanden und umgesetzt wird. Dabei geht der Beitrag davon aus, dass an Steuerungsprozessen sehr unterschiedliche Akteur*innen beteiligt sind und eine Vorstellung von Steuerung als hierarchisch strukturiert zu kurz greift. Gefolgt wird damit der Leitfragestellung der Educational Governance-Perspektive, die danach fragt, wie „,Spezialisten“ wie LehrerInnen, die Schulleitung, die Schulverwaltung, neue Schulinspektion, externe BeraterInnen, SchülerInnen, Eltern und die Bildungspolitik innerhalb ihrer jeweils spezifischen Sichtweise auf die Schule ein ,kollektives Gut" wie die schulische Bildung auch nur einigermaßen zielgerichtet herstellen“ (Kussau/Brüsemeister 2007: 25) können. Daher wird der Beitrag zuerst auf den Forschungsstand zur Entwicklung inklusiver Strukturen in Deutschland eingehen. Anschließend wird die Educational Governance-Perspektive auf Steuerungsprozesse sowie Studien zu Inklusion mit dieser Fokussierung vorgestellt und aufgezeigt, warum es einer 
rekonstruktiven Perspektive auf solche Prozesse bedarf. Eine solche wird sodann mit dem „theory/methods package“ (Clarke/Star 2008) der Situationsanalyse vorgestellt. Danach werden in einer ersten Annäherung an die Situationen jene Entwicklungen zusammenfassend vorgestellt, die Akteur*innen in den Situationen als zentral für die Entwicklung inklusiver Strukturen markiert haben. In einer zweiten Annäherung werden sodann die Aspekte zur Frage der Steuerung und der diskursiven Rahmung von Inklusion in den Situationen vergleichend dargestellt. Abschließend wird diskutiert, welche Schlussfolgerungen aus diesem Vergleich gezogen werden können, auch hinsichtlich der Frage, inwiefern die regionale Ebene als Vergleichshorizont international vergleichender Studien geeignet erscheint.

\section{Zur Entwicklung inklusiver Bildungssysteme in Deutschland}

Für die Entwicklung inklusiver Bildungssysteme in Deutschland kann konstatiert werden, dass diese an eine langjährige Tradition der Entwicklung integrativer Schulen anschließt (Müller 2018). Diese hat sich 1994 auch auf bildungspolitischer Ebene in einer Empfehlung der Kultusministerkonferenz niedergeschlagen. Gemäß Ellger-Rüttgardt (2012) zielte diese KMK-Empfehlung auf die Flexibilisierung von Förderorten für Schüler*innen mit sonderpädagogischem Förderbedarf und die Entwicklung auch schulsystemischer Strukturen zur Integration dieser Schüler*innen. Im Gegensatz zu anderen Staaten (z.B. Slee/Allan 2001) lässt sich allerdings in Deutschland beobachten, dass erst mit der Ratifizierung der UN-BRK 2009 schulsystemische Veränderungen unter dem Label ,Inklusion“ vorgenommen wurden. So machen Heinrich et al. (2013) deutlich, dass mit der UN-BRK im deutschen Schulsystem entwickelte Handlungsmodi, insbesondere die Trennung zwischen Regel- und Förderschule ${ }^{1}$, in Frage gestellt wurden. Auch Blanck et al. (2013) kommen am Beispiel zweier Bundesländer zu dem Schluss, dass die UN-BRK als exogener Schock die „vorherrschende Selbstverständlichkeit der Sonderschule nachhaltig erschüttert“ habe (Blanck et al. 2013: 287). Der Bezug von Inklusion auf Fragen der Beschulung von Schüler*innen mit sonderpädagogischem Förderbedarf wird dabei als enges Verständnis von Inklusion gekennzeichnet und von Heinrich et al. (2013) von einem weiten Verständnis abgegrenzt, dass als regulative Idee mit Ainscow et al. (2006) auf ein Maximum an Teilhabe und ein Minimum an Diskriminierung zielt. Ganz ähnlich formuliert Degener (2009) für die UN-BRK, dass diese nicht das Ziel hatte, besondere Menschenrechte zu schaffen, sondern den ,universal anerkannte[n] Menschenrechtskatalog auf den Kontext Behinderung" (Degener 2009: 207) auszulegen. In Reaktion auf die Ratifizierung der UN-BRK haben alle Bundesländer in Deutschland Änderungen der Schulgesetzgebung vorgenommen. Mißling und Ückert (2014) stellen allerdings fest, dass bis dato kein Bundesland die Anforderungen der UN-BRK erfüllt. Auch die internationale Staatenprüfung drückt Besorgnis darüber aus, dass in Deutschland der größte Teil der Schüler*innen mit Behinderung an Förderschulen unterrichtet wird und empfiehlt unter anderem ,[to] scale down segregated schools to facilitate inclusion“ (UN 2015: 8). Blanck (2015) hat insgesamt 80 verschiedene Varianten der Organisation von Inklusion in den schulrechtlichen Regelungen der Bundesländer gezählt. Dabei geben die unterschiedlichen

1 Der Terminus 'Förderschule' wird hier als Feldbegriff, der mit der KMK-Empfehlung von 1994 eingeführt wurde, übernommen. Zur Kritik daran siehe unter anderem Bleidick et al. (1995). 
Segregationsquoten der Bundesländer - vorsichtig formuliert ${ }^{2}$ (Tegge/Brüggemann 2016) Hinweise darauf, dass diese unterschiedlichen Struktursetzungen dem Ziel eines inklusiven Schulsystems mehr oder weniger entsprechen. Und Powell (2017) macht im Hinblick auf international und national vergleichende Studien deutlich, dass in Bezug auf die UN-BRK in Bildungssystemen mit langer Tradition segregierender Strukturen die „Expansion und Persistenz der schulischen Segregation, anstatt der Ausweitung der Inklusion“ (Powell 2017: 35) zu beobachten und insbesondere in föderalen Systemen ein „langsamer Wandel“ (Powell 2017: 35) zu erwarten ist.

Im wissenschaftlichen Diskurs um die Frage, wie ein inklusives Schulsystem entwickelt werden kann, werden vor allem Aspekte, wie die Ressourcenverteilung (z.B. Moser/Dietze 2015), die Weiterentwicklung der Organisation sonderpädagogischer Förderung (z.B. Reiser et al. 2007), die Auslegung und Konsequenzen der UN-BRK (z.B. Siehr/Wrase 2014; Wrase 2015, 2016) und aktuell auch die Organisation und der Einsatz von Schulbegleitungen diskutiert (z.B. Rohrmann/Weinbach 2017). Einige wenige Studien thematisieren aus Perspektive der Educational Governance (EG)-Forschung die Frage der Steuerung von Inklusion. Kern der EG-Perspektive ist die Einsicht, dass die Vorstellung von Steuerung als autoritativhierarchischer Vorgang unterkomplex sei. Entsprechend wird in der EG-Perspektive davon ausgegangen, dass Steuerung als Governance in Akteurskonstellationen zu verstehen ist, in der individuelle und kollektive Akteur*innen in Abhängigkeit zueinander stehen und mit ihren jeweiligen Handlungslogiken mit anderen Akteur*innen handelnd zusammenwirken (z.B. Kussau/Brüsemeister 2007). So diskutieren Altrichter und Feyerer (2011) sowie Rürup (2011) und Preuß (2018) mögliche Entwicklungen im österreichischen und deutschen Schulsystem. Dlugosch und Langner (2016) sowie Hinz und Kruschel (2012) fragen empirisch nach konkreten Akteurskonstellationen. Während diese Zugänge die Kategorien der EG-Perspektive zur Analyse von Steuerung anwenden, plädieren Dietrich und Heinrich (2014) für eine rekonstruktive Perspektive auf Governance. Im Anschluss daran fokussieren Studien auf Mikro-Ebene die Konstellation zwischen Akteur*innen z.B. in Hinblick auf Schulbegleitungen (Heinrich et al. 2019). An diese Perspektive auf Governance schließt dieser Beitrag an und fragt, wie auf Ebene der Bildungsadministration versucht wird, die Aufforderung zur Entwicklung inklusiver Bildungssysteme umzusetzen, und wie dieser Umsetzungsprozess gesteuert wird.

Für die Rekonstruktion und Analyse komplexer Steuerungssituationen erweisen sich die Kategorien der EG-Perspektive allerdings als weniger hilfreich (Gasterstädt 2019a): Ihnen liegt implizit eine bestimmte Vorstellung von Steuerung zu Grunde (Lindblad/Popkewitz 2000; Dietrich 2018), die Ebene individueller Akteur*innen wird nicht ausreichend beachtet (Benz et al. 2007), kollektive Akteure werden theoretisch unzureichend hergeleitet und gesamtgesellschaftliche Entwicklungen vernachlässigt (Bormann 2014). Entsprechend wurde zwar die Perspektive auf Akteurskonstellationen der EG-Forschung beibehalten, aber dem „theory/methods package“ (Clarke/Star 2008) der Situationsanalyse gefolgt, um die komplexe Steuerungssituation aus der Perspektive von Akteur*innen der Bildungsadministration rekonstruieren zu können.

2 Zur Kritik an Segregationsquoten und der schulstatistischen Erfassung von inklusiver Beschulung und sonderpädagogischem Förderbedarf siehe Tegge und Brüggemann (2016). 


\section{Wie können komplexe Steuerungssituationen analysiert werden?}

Die Situationsanalyse gilt als eine Weiterentwicklung der Grounded Theory Methodologie (GTM) nach Strauss und Corbin (1996) und verfolgt das Anliegen, die Komplexität von Situationen in den Mittelpunkt der Analyse zu stellen: „Die Bedingungen der Situation sind in der Situation enthalten“ (Clarke 2012: 73). Um solche komplexen Situationen analysieren zu können, schlägt Clarke drei zentrale theoretische Bezüge und daran anschließende methodische Weiterentwicklungen (Mapping-Strategien) vor: die Konzepte der Theorie sozialer Welten und Arenen, die Analyse von Macht/Wissen-Konstellationen und Diskursen im Anschluss an Foucault sowie den Einbezug der Handlungsmacht nicht-menschlicher Elemente mit Latour und Haraway (Clarke 2012). Während sich letzterer Bezug bei der Analyse von Steuerungsprozessen als weniger bedeutsam herausgestellt hat, ermöglichen die Konzepte der Theorie sozialer Welten und Arenen die Rekonstruktion kollektiver Akteur*innen als soziale Welten, deren Abgrenzungen zu anderen kollektiven Akteur*innen mit Hilfe des Konzepts der „,boundary objects“ (Bowker/Star 1999) sowie die Rekonstruktion von Koordinations- bzw. Aushandlungsprozessen in sozialen Arenen. So können die in der Educational Governance-Forschung fokussierten kollektiven Akteur*innen und die spezifische Ausgestaltung von Akteurskonstellationen im Bildungssystem rekonstruiert werden. Des Weiteren ermöglicht der Bezug auf die Analyse von Macht/Wissen-Konstellationen und Diskurse den Fokus auf jene ,großflächige[n] Formationen und Komplexe von Aussagen, Praktiken und Techniken der Bedeutungsgenerierung [zu legen, J.G.], die eine steuernde Wirkung entfalten, ohne dabei ausschließlich intentional oder rational zu sein“ (Bormann 2014: 159). So stellt sich in Bezug auf das „fuzzy concept“ Inklusion z.B. die Frage, wie dieser Begriff gefüllt oder auch genutzt wird, um Entwicklungen in Schulsystemen zu legitimieren.

Forschungsmethodisch wurden diese theoretischen Bezüge mittels der Methoden der klassischen GTM (Strauss/Corbin 1996) sowie mit Hilfe der spezifischen Mapping-Strategien der Situationsanalyse umgesetzt (Clarke 2012; Gasterstädt 2019a). Die schon in der GTM zentrale Strategie des ständigen Vergleichs bleibt dabei sowohl in Bezug auf die Sampling- als auch auf die Auswertungsstrategien erhalten. Im vorliegenden Projekt wurden so zwei Situationen (Region A in Bundesland A und Region B in Bundesland B) ${ }^{3}$ verglichen, die auf Grundlage eines maximalen Kontrastes der schulgesetzlichen Vorgaben zu sonderpädagogischen Organisationsformaten und ,Inklusion“ sowie der Segregationsquote ausgewählt wurden. Verglichen wurde so Situation A, ein Bundesland mit anhaltend sinkender Segregationsquote, in dem Förderschulen zu Förderzentren umgewandelt wurden, die sowohl die Aufgabe haben, notwendige Sonderklassen vorzuhalten als auch sonderpädagogische Expertise in die allgemeinen Schulen zu tragen, mit Situation B, einem Bundesland mit hoher und auch zum Zeitpunkt der Datenerhebung noch steigender Segregationsquote, in dem Förderschulen insofern weiterentwickelt wurden, als dass sie um eine innerschulische Einheit erweitert wurden, aus der heraus Sonderpädagog*innen Beratung und Förderung an allgemeinen Schulen vornehmen.

Um Steuerungsprozesse in Bezug auf die Anforderung der Entwicklung inklusiver Strukturen analysieren zu können, wurden in beiden Regionen/Bundesländern Dokumente sowie leitfadengestütze Interviews mit Expert*innen erhoben. Dokumente wurden dabei als Arte-

3 Aus Gründen der Anonymisierung werden die Bundesländer hier nicht genannt. 
fakte vergangener Aushandlungsprozesse und Entwicklungen in den Situationen verstanden (Prior 2003). So wurden schulgesetzliche Dokumente und Selbstdarstellungen z.B. Websites als ,standardisierte Artefakte“ (Wolff 2012 o.S.) sowohl hinsichtlich struktur- und prozessgestaltender Vorgaben als auch „,institutionalisierte[r] Spuren“ (Wolff 2012 o.S.) über „Aktivitäten, Absichten und Erwägungen ihrer Verfasser" (Wolff 2012 o.S.) analysiert.

Expert*inneninterviews wurden mit Akteur*innen der Bildungsadministration der beiden Regionen/Bundesländer geführt. Ausgehend von der Überlegung, dass Expert*innen unter anderem über Deutungsmacht verfügen, die „die Handlungsbedingungen anderer Akteur*innen in [ihrem] Aktionsfeld in relevanter Weise mit [strukturiert, J.G.] “ (Bogner/Menz 2009: 73), waren jene Akteur*innen von besonderem Interesse, die an Funktionsstellen an der Bearbeitung von Entscheidungs- und Problemlösungsprozessen beteiligt sind (Meuser/ Nagel 1994: 180f.). Im Sinne eines minimalen Samplings ging es entsprechend um jene Akteur*innen, die im engeren Sinne Entwicklungen in Schulsystemen steuern können und entlang der hierarchischen Gliederung der Entscheidungsgewalt im Mehrebenensystem Schule verortet sind. Insgesamt wurden so 13 Einzel- oder Doppelinterviews geführt, wobei fast alle Interviewpartner*innen dem Bereich der Sonderpädagogik zuzuordnen sind. Die Auswahl dieser konkreten Interviewpartner*innen folgte Hinweisen aus dem Forschungsfeld auf geeignete Expert*innen zum Thema Inklusion. Hier zeigen sich Hinweise auf die „Sonderpädagogisierung der Inklusion“ (Boban/Hinz 2009) in beiden Bundesländern.

Die Auswertung der so erhobenen Daten erfolgte teilweise parallel zur Datenauswertung. Neben den klassischen Kodierstrategien wurden vor allem die von Clarke (2012) vorgeschlagenen Mapping-Strategien als analytische Übungen verwandt, um die Komplexität der Steuerungssituation rekonstruieren zu können. Im Folgenden wird nun - stark zusammengefasst - aufgezeigt, wie unterschiedlich der Auftrag zur Entwicklung inklusiver Bildungssysteme regional interpretiert und daran anschließend umgesetzt wird. Dabei sind die beiden Rekonstruktionen als Beispiele für die Breite möglicher Umsetzungsvarianten zu sehen. Zuerst werden jene Aspekte dargestellt, die sich in der Rekonstruktion als zentral für die Entwicklung inklusiver Strukturen herausgestellt haben. Anschließend werden dann zentrale Erklärungsmomente dieser Differenzen im Vergleich vorgestellt und diskutiert. Dabei werden vor allem Aspekte der Steuerungsmodelle und der diskursiven Rahmung von ,Inklusion“ in den beiden Situationen thematisiert. Die Darstellung bleibt im Rahmen dieses Handbuchs notwendig unvollständig. Die detaillierten Rekonstruktionen sind in Gasterstädt (2019a) nachzulesen.

\section{Wie wird die Entwicklung inklusiver Strukturen in Schulsystemen gesteuert?}

\subsection{Die Situationen im Vergleich}

\subsubsection{Beispiel A}

In Situation A wird die Verantwortung für die Entwicklung inklusiver Schulen insbesondere in der allgemeinen Schule gesehen. Dieser Prozess wird in Region A durch das Förder- 
zentrum mit den Schwerpunkten Lernen, emotionale und soziale Entwicklung und Sprache sowie den*die Schulrat*rätin begleitet. In der Region haben sich Sonderpädagogik und allgemeine Schule darauf geeinigt, die Lehrer*innenstunden für Sonderpädagogik in die allgemeinen Schulen zu verlagern. Die Verteilung der Ressourcen aller Förderzentren (Lehrer*innenstunden für Sonderpädagogik) wird in Region A gemeinsam durch die Leitungen der Förderzentren bestimmt und erfolgt kriteriengeleitet. Dieses Modell wird durch die Akteur*innen als Reaktion auf das Etikettierungs-Ressourcen-Dilemma ${ }^{4}$ (Füssel/Kretschmann 1993) diskutiert. Mit dieser Verteilung wird auch das Ziel verbunden, Anreize für , inklusive' Schulentwicklungsprozesse in den allgemeinen Schulen zu setzen, die durch die Akteur*innen der Sonderpädagogik als sinnvoll eingeschätzt werden. Der Sonderpädagogik kommt daher in Region A eine besonders machtvolle Position zu (Gasterstädt 2019a, b).

Thematisierte Handlungsformate von Sonderpädagog*innen beziehen sich dabei vor allem auf Prävention, Beratung, Begleitung von Schul- und Unterrichtsprozessen, gemeinsame Unterrichtsvorbereitung und gemeinsames Unterrichten sowie die Entwicklung von Netzwerken und externer Beratung in Tandems mit Sozialarbeiter*innen. Mit dem Fokus auf Prävention wurden in Region A in Zusammenarbeit mit dem Träger der Eingliederungshilfe sogenannte Präventionsklassen in der Schuleingangsphase geschaffen, die mit deutlich mehr Ressourcen ausgestattet werden und so Schüler*innen mit hohem Assistenzbedarf ein Ankommen in der Schule ermöglichen sollen. Auf die Feststellung von sonderpädagogischem Förderbedarf im Bereich soziale und emotionale Entwicklung wird verzichtet, gleiches ist für den Förderschwerpunkt Lernen geplant. Dabei wird argumentiert, dass die formale Feststellung des Förderbedarfs eine negative Zuschreibung für die Schüler*innen darstelle und dessen ressourcensteuernde Funktion mit der systembezogenen Zuweisung von Ressourcen aufgehoben werde.

\subsubsection{Beispiel B}

In Situation B wird die Verantwortung für die Entwicklung inklusiver Beschulung bei der allgemeinen Schule gesehen. Dazu sollen die an Förderschulen angegliederten Beratungszentren mit allgemeinen Schulen Kooperationsverträge schließen, die die Bedingungen für den Einsatz der Sonderpädagog*innen an den allgemeinen Schulen regeln. Unter inklusiver Schulentwicklung wird durch das in Region B verantwortliche sonderpädagogische Beratungszentrum insbesondere die Implementierung von sogenannten Lerninseln verstanden, die in der allgemeinen Schule einen Rahmen für individuelle sonderpädagogische Förderung sicherstellen sollen. Die Verteilung der Ressourcen (Lehrer*innenstunden für Sonderpädagogik) wird in der Region über verschiedene Wege vorgenommen. Während Förderschulen mit Schwerpunkt geistige Entwicklung Ressourcen durch das Kultusministerium zugewiesen bekommen, werden Ressourcen für die Förderschwerpunkte Lernen, emotionale und soziale Entwicklung für den Bereich der Grundschule durch das jeweils zuständige Beratungs-

4 Vom Etikettierungs-Ressourcen-Dilemma wird dann gesprochen, wenn zusätzliche Ressourcen an die formale Feststellung von entsprechenden Bedarfen geknüpft sind, wenn also sich inklusiv entwickelnde Schulen erst dann Lehrkräftestunden für Sonderpädagogik zur Verfügung gestellt bekommen, wenn auch bei Kindern formal ein sonderpädagogischer Förderbedarf festgestellt worden ist. Dieser Zusammenhang wird kritisiert, weil angenommen wird, dass Schulen so dazu angeregt werden, mehr Schüler*innen mit dem Etikett versehen zu wollen, um mehr Ressourcen in der Schule zur Verfügung $\mathrm{zu}$ haben. 
zentrum systembezogen zugewiesen. Im Bereich der Sekundarstufe erfolgt diese Zuweisung aufgrund der freien Schulwahlentscheidung wiederum anhand der Feststellung des sonderpädagogischen Förderbedarfs.

Sonderpädagogisches Handeln wird vor allem im Bereich der Förderung der Schüler*innen mit festgestelltem sonderpädagogischem Förderbedarf gesehen. Ihr Handlungsfeld wird ausdifferenziert in die Bereiche Diagnostik, Förderung im Setting des gemeinsamen Unterrichts, Beratung und Entwicklung sowie Implementation von Förderkonzepten in der allgemeinen Schule. Dabei wird die Bedeutung der Ausdifferenzierung der sonderpädagogischen Fachlichkeit in den einzelnen Förderschwerpunkten betont.

Die Feststellung von sonderpädagogischem Förderbedarf wird als ,,pädagogisch“ notwendig markiert. Im Rahmen von Förderausschüssen, an denen hauptsächlich Akteur*innen aus dem Bereich der Förderschulen sowie der allgemeinen Schule und die Erziehungsberechtigten der betroffenen Schüler*innen beteiligt sind, wird der individuelle Anspruch auf sonderpädagogische Förderung sowie der geeignete Schulort festgelegt. Das Wahlrecht der Eltern auf die Wahl der Förderschule wird dabei betont.

\subsection{Wie lassen sich diese unterschiedlichen Entwicklungen erklären?}

\subsubsection{Diskursive Rahmung: Inklusion?}

Im Diskurs um Inklusion wird kritisiert, dass der Begriff der Inklusion zu einem „Modebegriff“ (Hinz 2013: o.S.) geworden sei, mit dem ,inzwischen nahezu alles als Inklusion deklariert wird, was sich positiv und fortschrittlich darstellen möchte" (ebd.: o.S.). Katzenbach (2015) spricht von einer „regelrechten Verwahrlosung des Begriffs“ (Katzenbach 2015: 19). Dies begründet er nicht nur mit der gesellschaftlichen und bildungspolitischen Debatte im Anschluss an die UN-BRK, sondern auch mit den inzwischen vielfältigen Theorieangeboten, die mit dem Begriff der Inklusion verbunden werden. Angesichts der englischsprachigen Literatur zu ,Inclusive Education' überrascht diese Entwicklung wenig. So haben Slee und Allan (2001) beispielweise in Bezug auf Erfahrungen mit ,Inclusive Education“ in Australien darauf hingewiesen, dass ,the subversion of inclusion from an emancipatory to a conservative project is ineluctable given the application of traditional epistemologies of special education to new times" (Slee/Allan 2001: 174). Auch Kiuppis (2014) verdeutlicht für den Kontext internationaler Organisationen, dass der Begriff ,Inclusive Education ‘ bei verschiedenen Akteur*innen durchaus unterschiedlich konzeptuell hinterlegt ist. Dies sei aber nicht nur als „Schwäche“ (Kiuppis 2014: 209) des Begriffs zu verstehen. Vielmehr läge darin auch eine „Stärke von Inclusive Education“, „nämlich seine Akzeptanz als humanistische, fortschrittliche und mit den Zielen einer verbesserten Umsetzung von Menschen- und Teilhaberechten kompatiblen Programmatik“ (Kiuppis 2014: 209).

Vor diesem Hintergrund stellte sich die Frage, wie der Begriff Inklusion interpretiert wird. Im Folgenden werden dazu beispielhaft die Diskurspositionen in den rekonstruierten Situationen dargestellt und anschließend diskutiert, welche Rolle der jeweils spezifische Begriff von Inklusion sowie der Bezug auf die UN-BRK dabei spielen. Darin verortet wird auch darauf Bezug genommen, welche Relevanz die Kategorie ,sonderpädagogischer Förderbedarf" hat. 
In Situation A spricht die dominante Position vor allem Diskurselemente an, die Veränderungen in Richtung der Entwicklung einer Schule für Alle thematisieren. Dieses Leitbild findet sich sowohl auf Ebene des Landes als auch der Region. Vor allem regionale Akteur*innen lehnen dabei den Begriff der Inklusion ab und ersetzen diesen durch den des Umgangs mit Vielfalt oder Heterogenität. Damit wird zumindest sprachlich an inklusionspädagogische Entwürfe, wie die „Pädagogik der Vielfalt“ (Prengel 2006), angeschlossen. Ein Bezug zur UN-BRK wird dabei nicht hergestellt. Diese Position bezieht Inklusion bzw. den Umgang mit Vielfalt auf die Ebene der Schulstrukturen und der Entwicklung der Einzelschule sowie des Unterrichts. Die daran anschließenden Strukturentwicklungen in Region A beziehen sich allerdings vor allem auf den Verantwortungsbereich des Förderzentrums Lernen, Sprache und emotionale und soziale Entwicklung. Akteur*innen, die dieser Position zuzuordnen sind, betonen auch, dass segregative Angebote für den Förderschwerpunkt geistige Entwicklung weiterhin bereitgestellt werden müssen. Eine zweite Position in Situation A argumentiert, dass aufgrund der geringen Ressourcen für Sonderpädagogik eine Fokussierung der Sonderpädagog*innen auf Kinder mit festgestelltem Förderbedarf notwendig sei, um im Sinne der UN-BRK angemessene Vorkehrungen für deren individuelle Beschulung bereit zu stellen.

In Situation B werden von der dominanten Position Diskurselemente angesprochen, die mit Inklusion die sogenannte ,Inklusive Beschulung“ von Schüler*innen mit sonderpädagogischem Förderbedarf thematisieren und als räumliche Ausweitung der vor der Ratifizierung der UN-BRK praktizierten integrativen Beschulung von Schüler*innen mit sonderpädagogischem Förderbedarf in allen Schulen zu verstehen sei. Davon ausgehend argumentiert eine zweite Position, dass man in Bezug auf Inklusion auch andere Heterogenitätsdimensionen einbeziehen müsse, während eine dritte Position kritisiert, dass ein solches Verständnis von Inklusion und der UN-BRK systemische Aspekte vernachlässige.

An diese Positionen knüpfen in beiden Situationen Legitimationsfiguren für Strukturentwicklungen an. Mit Bormann (2014) kann diesen so eine steuernde Wirkung zugesprochen werden. Im Vergleich wird deutlich, dass sich Positionen finden, die mit Inklusion auf die Idee der Entwicklung einer Schule für Alle Bezug nehmen. Bemerkenswert ist allerdings, dass sich Akteur*innen dabei explizit vom Wort Inklusion abgrenzen und sich nicht auf die UN-BRK beziehen. So ist auch zu erklären, dass ein solches Verständnis von Inklusion nicht im Widerspruch dazu gesehen wird, die Möglichkeit der Integration von Schüler*innen mit Förderbedarf im Bereich der geistigen Entwicklung zu bezweifeln. Der Forderung nach einer Schule für Alle wird die Auslegung individueller Rechte, zum Beispiel auf sonderpädagogische Förderung, gegenübergestellt. Inklusion erscheint hier bezogen auf die Differenzlinie Behinderung/Beeinträchtigung und dabei teilbar.

Weiter kann festgehalten werden, dass im Gegensatz zur Feststellung von Blanck et al. (2013) die UN-BRK in keiner der beispielhaft analysierten Situationen die ,Selbstverständlichkeit der Sonderschule nachhaltig erschüttert“ (Blanck et al. 2013: 287) hat. Im Vergleich wird deutlich, dass die UN-BRK als Legitimation von Strukturentwicklungen zur Integration von Schüler*innen mit Behinderungen und Beeinträchtigungen in die allgemeinen Schulen genutzt wird. So wird insbesondere in Situation B Inklusion in Bezug auf die UN-BRK als räumliche Erweiterung der Integration von Schüler*innen mit Anspruch auf sonderpädagogische Förderung auf alle allgemeinen Schulen interpretiert. Damit einher geht eine Ausweitung des Handlungsbereichs der Sonderpädagogik in die allgemeine Schule hinein. Beispielsweise versucht das verantwortliche Förderzentrum in Situation B über die Implementation von Lerninseln an allgemeinen Schulen, an denen sonderpädagogische Förderung stattfinden soll, die Entwicklung, inklusiver' Schulen weiter zu bringen (Gasterstädt 2019a). Dabei 
bedarf es der Kategorisierung von Schüler*innen mit sonderpädagogischem Förderbedarf. So ist zu erklären, warum in dieser Region zwar ein systemisches Modell der Ressourcenzuweisung entwickelt wurde, aber der Feststellung von sonderpädagogischem Förderbedarf weiterhin eine hohe Relevanz zugewiesen wird. Der sonderpädagogische Förderbedarf stabilisiert hier als Grenzobjekt (vgl. folgendes Kapitel) die Trennung zwischen Regel- und Sonderschulpädagogik und die damit einhergehende Delegation von Verantwortung (Katzenbach et al. 2006). Inklusion, verstanden als ,Inklusive Beschulung', wird so auf der Ebene des/der einzelnen Schüler*in verhandelt und in Bezug auf die UN-BRK legitimiert.

Dieses Verständnis von Inklusion erinnert einerseits an die amtliche deutschsprachige Übersetzung der UN-BRK, in der ,,inclusive“ mit ,integrativ“ übersetzt wurde. Andererseits lässt sich damit ein spezifischer Modus der Reinterpretation ${ }^{5}$ aufzeigen, der auf die Pfadabhängigkeit solcher Entwicklungen verweist (Mahoney 2000). Dieser besteht darin, die Aufforderung der UN-BRK mit den bildungspolitischen Debatten und Entwicklungen in den Bundesländern, hier jeweils der Integrationsdebatte und den damit verbundenen Erfahrungen, zu verknüpfen. ${ }^{6}$ So bestanden in beiden Bundesländern langjährige Erfahrungen mit der Integration von Schüler*innen mit sonderpädagogischem Förderbedarf im sogenannten gemeinsamen Unterricht an spezifischen Schulen. An diese Entwicklung schließen die Akteur*innen an, wenn sie auf die UN-BRK Bezug nehmen und die Aufforderung der UN-BRK als ,Inklusive Beschulung' von Schüler*innen mit Behinderungen und Beeinträchtigungen interpretieren.

Ein solche Interpretation ist abgrenzbar von einem menschenrechtlich fundierten Verständnis von Inklusion im Anschluss an die UN-BRK. So argumentiert auch die Monitoringstelle zur Umsetzung der UN-BRK in Deutschland in einer Stellungnahme, dass ,die allgemeine Schulbildung nur dann diskriminierungsfrei stattfinden kann, wenn der Schulbetrieb dem Inklusionsansatz folgt" (Aichele 2010: 10) und angemessene Vorkehrungen zur Beschulung und Förderung innerhalb eines inklusiven Regelschulsystems bereitgestellt werden. Entgegen dieser Argumentation bleibt die Parallelität von ,Inklusiver Beschulung' und segregativer Beschulung in beiden Situationen aufrechterhalten.

Die Beobachtung, dass UN-BRK und Inklusionsbegriff gleichgesetzt werden, kann im Übrigen für viele Studien zum Thema Inklusion gelten, die häufig mit dem Verweis auf die UN-BRK beginnen, die dann argumentativ zur Legitimation der Forschung und eines spezifischen, normativen inklusionspädagogischen Ideals einer heterogenitätssensiblen Schulpraxis, an die sich entsprechende Konzepte anschließen, genutzt wird. Hier wird eine Differenz zwischen wissenschaftlichem und bildungspolitischem Diskurs deutlich, die ebenso wie das Verständnis der UN-BRK, an ein spezifisches Verständnis von Integration anschließt. Während im pädagogischen Diskurs um Integration zumeist eine Schulentwicklungsperspektive mitgedacht und Integration eben nicht auf die Beschulung von Schüler*innen mit sonderpädagogischem Förderbedarf beschränkt wurde, scheint aber gerade dies insbesondere in der bildungspolitischen Praxis, zum Beispiel des sogenannten gemeinsamen Unterrichts, in Bundesland B der Fall zu sein.

5 Hier wird nicht Fends Begriff der Rekontextualisierung genutzt, weil dieser in der hierarchischen Struktur eines Mehrebenensystems verankert ist (Fend 2008: 35ff.). In der Analyse zeigte sich aber an vielen Stellen, dass Akteur*innen nicht nur Vorgaben der nächst höheren Ebene rekontextualisieren, sondern sehr unterschiedliche Bezüge nutzen. Damit wird betont, dass die Reinterpretation von sehr unterschiedlichen Einflüssen in die Handlungslogiken ein subjektiv geprägter Vorgang ist.

6 Damit sei nicht gesagt, dass Vertreter*innen der Integrationspädagogik diese auf die Kategorie der Behinderung eng geführt hätten (diskutiert z.B. bei Wocken 2009). 
Es wird damit deutlich, dass die spezifische Reinterpretation der UN-BRK nicht die von Hopf und Kronauer (2016) angesprochene Hoffnung erfüllt, eine Debatte über den „Zustand permanenter Überforderung“ (Hopf/Kronauer 2016: 18) des Bildungssystems zwischen Integrationsanforderung, Leistungsorientierung und der Kritik an Selektionsmechanismen anzustoßen. So wird in Situation A die Rede um die Entwicklung inklusiver Strukturen von Akteur*innen in Bezug auf die Entwicklung einer Schule für Alle gesetzt und mit Strukturreformen z.B. zur umgreifenden Etablierung von Gesamtschulen im Sekundarbereich in Verbindung gebracht und dabei auch der Bildungs- und Erziehungsauftrag von Schule thematisiert. In Situation B hingegen wird die Aufforderung der UN-BRK zur Entwicklung eines inklusiven Schulsystems als zusätzliche und überfordernde Anforderung wahrgenommen, die im Widerspruch zur Leistungsorientierung innerhalb des Bildungssystems in dieser Situation steht. Im Vergleich lassen diese Hinweise darauf schließen, dass deutlich unterschiedliche Konzepte und daran anschließende Strategien zu Fragen der Bildungsgerechtigkeit vertreten werden. Dabei werden zwar Bezüge zu grundlegenden Überzeugungen über die gesellschaftlichen Funktionen des Bildungssystems (vgl. Heinrich et al. 2013: 55, in Auseinandersetzung mit Fends (1980) Bestimmung der Funktion von Schule) oder das Verständnis von Bildungsgerechtigkeit hergestellt (vgl. Lindmeier 2011a, 2011b; Stojanov 2011). Hinweise auf eine veränderte Funktion von Schulen und einen „erweiterten Begriff von gelingender Schulbildung“ (Heinrich et al. 2013: 75) lassen sich allerdings nicht finden.

Zusammenfassend können aus dem Vergleich der diskursiven Elemente und Positionen drei Aspekte festgehalten werden. Erstens knüpfen Akteur*innen mit den verschiedenen Elementen im Diskurs um Inklusion an die bisherige Entwicklung in den beiden Bundesländern an. Daran anschließend wird zweitens das spezifische Verständnis von Inklusion dafür genutzt, (anstehende) Entwicklungen und Erwartungshaltungen an andere Akteur*innen zu legitimieren. Und drittens wird die UN-BRK als inklusive Beschulung von Schüler*innen mit Behinderungen und Beeinträchtigungen reinterpretiert.

\subsubsection{Steuerungsmodelle: Rahmen, Gestaltungsspielräume und Kommunikationsformate}

Benz und Dose (2010) verstehen unter Steuerung die „bewusste Intervention in Handlungsfelder bzw. [...] die Lenkung des Verhaltens von Akteuren, um Änderungen in Richtung festgelegter Ziele zu erreichen“ (Benz/Dose 2010: 26). Die EG-Perspektive ergänzt diesen Steuerungsbegriff um die Dimension der Koordination zwischen Akteur*innen. In den Rekonstruktionen der Steuerungssituationen lag der Fokus allerdings zuerst auf der Frage, welche individuellen und kollektiven Akteur*innen relevant sind, wie diese sich in mehr oder weniger direkten Interaktionen abgrenzen und Themen verhandeln bzw. Entwicklungsstrategien aushandeln. Steuerung findet demnach innerhalb eines durch schulgesetzliche Vorgaben und Ressourcen gesteckten Rahmens statt. Fraglich ist allerdings, welche Gestaltungsspielräume Akteur*innen innerhalb dieses Rahmens wahrnehmen.

In Situation A zielen Akteur*innen der hierarchisch höheren Ebene des Kultusministeriums darauf, dass langfristige Entwicklungsziele durch Akteur*innen hierarchisch niedrigerer Ebenen umgesetzt werden. Verantwortung für die Entwicklung konkreter Strukturen und Konzepte liegt hier vor allem bei Akteur*innen der regionalen Ebene. Steuerung in Situation A erinnert an das Modell Neuer Steuerung in Bildungssystemen (Dedering 2012: 57), allerdings spielt die „Messung von Erfolgskriterien“ (ebd.), die als zentraler Aspekt Neuer Steue- 
rung mit dem Terminus evaluationsbasierter Steuerung verbunden ist (Altrichter/Maag Merki 2010: 35ff.; Fend 2014), in Situation A keine Rolle. In dieser Situation werden Kooperationsbeziehungen entwickelt und Prozesse des Wandels insbesondere auch über Problemlösungen nicht nur in der Einzelschule, sondern auch in der Region initiiert. Entwicklungen finden in Situation A inkrementell und bottom-up statt (Rürup 2013).

In Situation B zielt Steuerung auf die Kommunikation und Umsetzung konkreter Entwicklungsvorgaben innerhalb formaler Ebenen des Systems ab und kann als Beispiel autoritativ-hierarchischer Steuerung interpretiert werden. Verantwortung für die Entwicklung von Strukturen und Konzepten wird hier vor allem bei hierarchisch höher angesiedelten Akteur*innen gesehen, während Akteur*innen ,unten' diese umsetzen sollen. Mit Dedering (2012) lässt sich dies auch als Variante klassischer Steuerung verstehen, ,in der ein (staatliches) Steuerungssubjekt mit deutlich ausgeprägter Lenkungsabsicht über zentralistisch-hierarchische Verfahren der Detailsteuerung tätig wird“ (Dedering 2012: 56), wobei dieser Modus durch Evaluationsverfahren, z.B. landesweite Vergleichstests im dritten Schuljahrgang, ergänzt wird. In beiden Situationen werden Gesetze und Verordnungen als Steuerungsinstrumente beschrieben. Diese können als Grenzobjekte (Bowker/Star 1999) verstanden werden, an die verschiedene Akteur*innen anschließen und diese im Sinne eigener Handlungslogiken reinterpretieren, so Handlungslogiken stabilisieren und sich dabei von anderen Akteur*innen abgrenzen.

In Systemen, in denen regionale Akteur*innen Gestaltungsspielräume wahrnehmen, können daneben in der Region Grenzobjekte ausgehandelt werden, die Ausdruck gemeinsamer Zielorientierungen der beteiligten regionalen Akteur*innen sind (z.B. aus dem Bereich der Schule und der Kinder- und Jugendhilfe) und andererseits durch die beteiligten Akteur*innen (re)interpretiert werden (müssen). Diese regionalen Grenzobjekte können als regionale Steuerungsinstrumente verstanden werden und verdeutlichen z.B. in Situation A die machtvolle Position der Region. Die dort regional ausgehandelten Grenzobjekte ermöglichen Verlässlichkeit über die Handlungsorientierung anderer regionaler Akteur*innen, sofern davon auszugehen ist, dass diese sich mit den Inhalten des Grenzobjektes identifizieren. Auch in Situation B finden sich weitere relevante Grenzobjekte. So können die Feststellung von sonderpädagogischem Förderbedarf und die mit diesem Verfahren zusammenhängende Dokumentation sowie die Kooperationsverträge zwischen Förderzentrum und allgemeiner Schule als Grenzobjekte verstanden werden, an die die jeweils beteiligten Professionen anschließen und diese in eigene Handlungslogiken übersetzen. Während die in Situation A rekonstruierten Grenzobjekte geteilte Zielorientierungen enthalten, die in regionalen Kooperationen ausgehandelt wurden, markieren regionale Grenzobjekte in Situation B Verantwortungsbereiche und Zuständigkeiten der Sonder- und Regelschulpädagogik. Dies könnte erklären, warum in Situation B an der Feststellung des Anspruchs auf sonderpädagogische Förderung festgehalten und dieser als pädagogisch notwendig legitimiert wird.

Mit Pijl und Frissens (2009) könnte im Vergleich der Steuerungsmodelle auch danach gefragt werden, ob Akteur*innen im Rahmen von Steuerung eher im Sinne einer ,professional bureaucracy“ oder einer „machine bureaucracy“ angesprochen werden. Mit ersterer verbinden die Autoren die Einschätzung, dass Veränderungen auf Ebene der Einzelschule möglich werden, wenn Akteur*innen als professionelle Akteur*innen adressiert werden und mit entsprechenden Handlungsspielräumen ausgestattet sind. Dies zeigt sich am Beispiel von Situation A auch als zutreffend für die Ebene der Bildungsadministration. Akteur*innen in Situation A nutzen Gestaltungsspielräume für inkrementelle Aushandlungsprozesse und reflektieren dabei Entwicklungen in der Region. Ähnlich konstatiert Düring (2011), dass innerhalb 
von Sozialraumteams der Kinder- und Jugendhilfe „,kooperatives Steuerungshandeln zu einer erhöhten (Selbst)Reflexivität des professionellen Hilfesystems qua organisierter Reflexionsschleifen führt“ (Düring 2011: 156). Mit „,machine bureaucracy“ verbinden Pijl und Frissen (2009) einen Steuerungsmodus, in dem Akteur*innen der Bildungsadministration „opt for centralized boards, ask for rational planning, analyze assessment data, require reports, want expenses to be accounted using formal systems, etc" (Pijl/Frissen 2009: 368). So versuchen Akteur*innen z.B. in Situation B Vorgaben zu entsprechen und Prozesse der Aushandlung lassen sich eher als formale Zustimmung und informeller Widerstand beschreiben. Mit dieser Form der Steuerung sind, Pijl und Frissen (2009) zu Folge, aber eher keine Prozesse des Wandels verbunden. Stattdessen trägt dieser Steuerungsmodus zur Stabilisierung entwickelter Strukturen bei: „Externally everything is in order; internally nothing has changed“ (Pij1/Frissen 2009: 370).

In ähnlicher Weise wie die hier vorgenommene Konzeption von Rahmen und Gestaltungsspielräumen thematisiert das Konzept der Verfügungsrechte der EG-Perspektive die Legitimation von Entscheidungsbefugnissen von Akteur*innen und damit die Grundlage der Handlungsfähigkeit von Akteur*innen, um Situationen im Benz'schen Sinne zu steuern. Allerdings bezieht sich die EG-Perspektive vor allem auf institutionalisierte Regeln und Normen. Vernachlässigt wird, dass insbesondere die subjektive Wahrnehmung von Gestaltungsspielräumen und der dem vorausgesetzten subjektiven Einschätzung des Rahmens und der Gestaltungsspielräume zentrale Bedeutung zukommt. So wird beispielsweise das Schulgesetz in Situation A interpretiert, während es in Situation B Rechts- und Verfahrenssicherheit zusichert (Rürup 2007) und Akteur*innen diese auch verlangen.

Zusammenfassend lässt sich aus dem Vergleich der Steuerungsmodelle in Situation A und B ein empirisch fundiertes Verständnis von Steuerung entwickeln. Mit Bezug auf das Konzept des prozessualen Ordnens (Strauss 1993) lässt sich Steuerung als Strukturierung der Aushandlungsprozesse zwischen den am Steuerungsprozess beteiligten Akteur*innen verstehen, wobei die konkrete Form der Strukturierung ebenfalls in inkrementellen Aushandlungsprozessen entstanden ist und in diesen stabilisiert oder auch verändert wird. Im Verlauf dieser Aushandlungsprozesse werden bestimmte Formen der Koordination interdependenter Akteur*innen als routinisierte Formen der Aushandlung entwickelt, innerhalb derer Akteur*innen versuchen im Benz'schen Sinne intentional zu steuern. Akteur*innen gehen dabei mit den Ergebnissen früherer Steuerungs- bzw. Aushandlungs- und Ordnungsprozesse um und nehmen diese als Strukturen war. Der Vergleich der Kommunikationsformate zeigt, dass die spezifische Konstellation von Ebenen und, damit verbunden, der Akteur*innen in Situation A und B deutlich unterschiedlich ist. Das betrifft zum einen die Frage, worüber sich verschiedene Akteur*innen in den beiden Situationen abgrenzen und zum anderen, welche Rolle die regionalen Kooperationsformate in den beiden Situationen insbesondere für die Entwicklung inklusiver Strukturen spielen.

Mit der Beobachtung von Grenzobjekten und Kooperationsformaten wird am Beispiel der rekonstruierten Situationen auch deutlich, dass die relevanten Ebenen nicht unbedingt den formalen Ebenen des Bildungswesens entsprechen und im Gegensatz zu Fends Bestimmung des Begriffs der Rekontextualisierung, die Vorgaben, die durch Akteur*innen in je eigenen Handlungslogiken interpretiert werden, nicht nur auf der jeweils hierarchisch höheren Ebene gesetzt werden. Es wurde aufgezeigt, dass grenzüberschreitende Kommunikation zwar in beiden Situationen stattfindet, in Situation A aber vor allem horizontal als Aushandlungsprozess, in B hingegen vertikal als Informations- und Vermittlungsprozess angelegt ist. Und es konnte gezeigt werden, dass die Existenz einer Arena in Region A von zentraler 
Bedeutung für die Entwicklung inklusiver Strukturen in Situation A ist, weil hier Akteur*innen geteilte Verpflichtungen aushandeln, die mit Handlungssicherheit verbunden sind. Hingegen wird in Situation B Handlungssicherheit mit Bezug auf die beschriebenen Grenzobjekte - insbesondere Schulgesetz und Verordnungen - hergestellt.

\section{Fazit: Same same but different}

Der Beitrag stellte die Frage nach dem dialektischen Verhältnis der Durchsetzung einer globalen Norm und der ,überraschenden Beharrungskraft variierender sozial-kultureller Interrelations-Gefüge" (Schriewer 2013: 35) insbesondere auf regionaler Ebene. Mit der Situationsanalyse in zwei Regionen/Bundesländern in Deutschland konnten sowohl diese Gefüge auf Ebene der Bildungsadministration als auch die Reinterpretation der globalen Norm Inklusion rekonstruiert werden. So wurde aufgezeigt, dass sich die Strukturentwicklungen unter dem Anspruch von Inklusion oder der UN-BRK in beiden Bundesländern nur graduell unterscheiden - beispielsweise wurden in beiden Regionen Modelle systembezogener Ressourcenzuweisung entwickelt. Nur in einer der Situationen führte die Entwicklung eines solchen Ressourcensteuerungsmodells allerdings zu der in der Literatur vorzufindende Hoffnung, so das Etikettierungs-Ressourcen-Dilemma bearbeiten zu können (Situation A). Die Situation in den Bundesländern erscheint ,same same but different'. Zur Erklärung solcher Differenzen, hat sich die Rekonstruktion des Steuerungsmodells und der diskursiven Rahmung von Inklusion auf Ebene des Bundeslandes und der Region als hilfreich erwiesen. Diese Rekonstruktionen zeigen auf, wie unterschiedlich die Situationen sind, auf die die Anforderungen der globalen Norm Inklusion treffen. Für die Praxis der Bildungsadministration ergibt sich daraus die Anforderung, entgegen der starken Tendenzen zur Herstellung von Vergleichbarkeit zwischen regionalen Entwicklungen, Gestaltungsräume für regionale Entwicklungen zu öffnen. Um inklusive Strukturen in Bildungssystemen zu entwickeln, müssen vor Ort Lösungen für konkrete Probleme ausgehandelt werden, aus denen sich mittel- und langfristig nachhaltige Strukturentwicklungen ergeben können.

Für international vergleichende Forschung zu Inklusion sei abschließend auf eine Beobachtung von Meijer und Watkins (2016) hingewiesen. Die Autoren stellen fest, dass in den europäischen Staaten mit dem Begriff Inklusion verschiedene Konzepte hinterlegt sind und sich die daran anschließende Gesetzgebung unterscheidet. Gezeigt werden konnte nun, dass der Bezug auf die nationalstaatliche Ebene allerdings verschleiert, dass hier vor allem die Ebene der Bundesländer und Regionen relevant ist, weil dort z.B. die sehr unterschiedlichen Begriffe von Inklusion entwickelt werden, die Strukturentwicklungen legitimieren. Damit wird allerdings fraglich, wozu Forschungen, die das deutsche Schulsystem oder Entwicklungen darin mit dem anderer Nationalstaaten vergleichen, Aussagen treffen, denn nicht nur die 16 Bundesländer sondern auch die Regionen stellen eine notwendige Vergleichsebene dar. Eine international vergleichende Forschung, die Phänomene im Kontext der Entwicklung inklusiver Bildungssysteme auf regionaler Ebene fokussiert und eingebettet in spezifisch ausgebildete Situationen versteht, hat dabei das Potential differente Möglichkeitsräume für die Umsetzung von Inklusion auf Ebene der Einzelschule oder der Interaktion im Unterricht aufzuzeigen (Gasterstädt 2019c) und so zum Beispiel auch Praktiken der Differenzerzeugung weitergehend analysieren zu können (Gasterstädt/Rüger, i.E.). Der internationale Vergleich 
ermöglicht dabei, vermeintliche Selbstverständlichkeiten zu irritieren und so Raum für weiterführende Erkenntnismöglichkeiten zu öffnen.

\section{Literatur}

Aichele, Valentin (2010): Stellungnahme der Monitoring-Stelle zur UN-Behindertenrechtskonvention zur Stellung der UN-Behindertenrechtskonvention innerhalb der deutschen Rechtsordnung und ihre Bedeutung für behördliche Verfahren und deren gerichtliche Überprüfung, insbesondere ihre Anforderungen im Bereich des Rechts auf inklusive Bildung nach Artikel 24 UN-Behindertenrechtskonvention. Gleichzeitig eine Kritik an dem Beschluss des Hessischen Verwaltungsgerichtshofs vom 12. November 2009 (7 B 2763/09): Deutsches Institut für Menschenrechte.

Ainscow, Mel/Booth, Tony/Dyson, Alan (2006): Improving Schools, Developing Inclusion. London u.a.: Routledge.

Altrichter, Herbert/Feyerer, Ewald (2011): Auf dem Weg zu einem inklusiven Schulsystem? Die Umsetzung der UN-Konvention in Österreich aus der Sicht der Governance-Perspektive. In: Zeitschrift für Inklusion, 4, http://www.inklusion-online.net/index.php/inklusion-online/article/view/73/73 [Zugriff: 10.06.2019].

Altrichter, Herbert/Maag Merki, Katharina (2010): Steuerung der Entwicklung des Schulwesens. In: Altrichter, Herbert/Maag Merki, Katharina (Hrsg.): Handbuch Neue Steuerung im Schulsystem. Wiesbaden: VS Verlag für Sozialwissenschaften, S. 15-39.

Artiles, Alfredo/Dyson, Alan (2005): Inclusive education in the globalization age. In: Mitchell, David (Hrsg.): Contextualizing inclusive education. London: Routledge, S. 37-62.

Benz, Arthur/Dose, Nicolai (2010): Governance - Modebegriff oder nützliches sozialwissenschaftliches Konzept? In: Benz, Arthur/Dose, Nicolai (Hrsg.): Governance - Regieren in komplexen Regelsystemen. Wiesbaden: Springer, S. 13-36.

Benz, Arthur/Lütz, Susanne/Schimank, Uwe/Simonis, Georg (2007): Einleitung. In: Benz, Arthur/ Lütz, Susanne/Schimank, Uwe/Simonis, Georg (Hrsg.): Handbuch Governance. Theoretische Grundlagen und empirische Anwendungsfelder. Wiesbaden: VS Verlag für Sozialwissenschaften, S. 9-25.

Blanck, Jonna M. (2015): Schulische Integration und Inklusion in Deutschland. In: Kuhl, Poldi/Stanat, Petra/Lütje-Klose, Birgit/Gresch, Cornelia/Pant, Anand Hans/Prenzel, Manfred (Hrsg.): Inklusion von Schülerinnen und Schülern mit sonderpädagogischem Förderbedarf in Schulleistungserhebungen. Wiesbaden: Springer VS, S. 153-177.

Blanck, Jonna M./Edelstein, Benjamin/Powell, Justin W. (2013): Persistente schulische Segregation oder Wandel zur inklusiven Bildung? Die Bedeutung der UN-Behindertenrechtskonvention für Reformprozesse in den deutschen Bundesländern. In: Wandel und Reformen in Bildungssystemen und Bildungsorganisation. Sonderband der Schweizerischen Zeitschrift für Soziologie 39, 2, S. 267-292.

Bleidick, Ulrich/Rath, Waldtraut/Schuck, Karl Dieter (1995): Die Empfehlungen der Kultusministerkonferenz zur sonderpädagogischen Förderung in den Schulen der Bundesrepublik Deutschland. In: Zeitschrift für Pädagogik 41, 2, S. 247-264.

Boban, Ines/Hinz, Andreas (2009): Inklusive Pädagogik zwischen allgemeinpädagogischer Verortung und sonderpädagogischer Vereinnahmung - Anmerkungen zur internationalen und zur deutschen Debatte. In: Börner, Simone/Glink, Andrea/Jäpelt, Birgit/Sanders, Dietke/Sasse, Ada (Hrsg.): Integration im vierten Jahrzehnt. Bilanz und Perspektiven. Bad Heilbrunn: Klinkhardt, S. 220-228. 
Bogner, Alexander/Menz, Wolfgang (2009): Das theoriegenerierende Experteninterview. Erkenntnisinteresse, Wissensformen, Interaktion. In: Bogner, Alexander/Littig, Beate/Menz, Wolfgang (Hrsg.): Das Experteninterview. Theorie, Methode, Anwendungsfelder. Wiesbaden: VS Verlag für Sozialwissenschaften, S. 61-90.

Bormann, Inka (2014): Diskursanalyse als Verfahren einer wissensorientierten Governance-Forschung. In: Maag Merki, Katharina/Langer, Roman/Altrichter, Herbert (Hrsg.): Educational Governance als Forschungsperspektive: Strategien. Methoden. Ansätze. Wiesbaden: Springer VS, S. 153-176.

Bowker, Geoffrey C./Star, Susan L. (1999): Sorting things out. Classification and its consequences. London: MIT Press.

Brüggemann, Christian (2016): Inklusive Bildung als globale Norm. In: Zeitschrift für Internationale Bildungsforschung und Entwicklungspädagogik 39, 3, S. 4-9.

Clarke, Adele E. (2012): Situationsanalyse. Grounded Theory nach dem Postmodern Turn. Interdisziplinäre Diskursforschung. Wiesbaden: Springer VS.

Clarke, Adele E./Star, Susan L. (2008): The Social Worlds Framework. A Theory/Methods Package. In: Hackett, Edward J./Bijker, Wiebe E./Amsterdamska, Olga/Wajcman, Judy/Lynch, Michael (Hrsg.): The Handbook of Science \& Technology Studies. Cambridge: MIT Press, S. 113-137.

Dedering, Kathrin (2012): Steuerung und Schulentwicklung Bestandsaufnahme und Theorieperspektive. Wiesbaden: Springer VS.

Degener, Theresia (2009): Die UN-Behindertenrechtskonvention als Inklusionsmotor. In: Recht der Jugend und des Bildungswesens 57, 2, S. 200-219.

Dietrich, Fabian (2018): Konturen einer Rekonstruktiven Governanceforschung. In: Heinrich, Martin/Wernet, Andreas (Hrsg.): Rekonstruktive Bildungsforschung. Zugänge und Methoden. Wiesbaden: Springer Fachmedien Wiesbaden, S. 73-94.

Dietrich, Fabian/Heinrich, Martin (2014): Kann man Inklusion steuern? Perspektiven einer rekonstruktiven Governanceforschung. In: Lichtblau, Michael/Blömer, Daniel/Jüttner, Ann-Kathrin/Koch, Katja/Krüger, Michaela/Werning, Rolf (Hrsg.): Forschung zu inklusiver Bildung. Gemeinsam anders lehren und lernen. Bad Heilbrunn: Klinkhardt, S. 26-47.

Dlugosch, Andrea/Langner, Anke (2016): Koordination von ,Inklusion“. Erste Ergebnisse einer explorativen Studie im Bundesland Tirol. In: Moser, Vera/Lütje-Klose, Birgit (Hrsg.): Schulische Inklusion. 62. Beiheft der Zeitschrift für Pädagogik. Weinheim u.a.: Beltz Juventa, S. 124139.

Düring, Diana (2011): Kooperation als gelebte Praxis: Steuerungshandeln in Sozialraumteams der Kinder- und Jugendhilfe. Wiesbaden: Springer VS.

Ellger-Rüttgardt, Sieglind Luise (2012): Geschichte der heil- und sonderpädagogischen Institutionen im schulischen Bereich. In: Heimlich, Ulrich/Kahlert, Joachim (Hrsg.): Inklusion in Schule und Unterricht. Wege zur Bildung für alle. Stuttgart: Kohlhammer, S. 27-79.

Fend, Helmut (1980): Theorie der Schule. München: Urban \& Schwarzenberg.

Fend, Helmut (2008): Schule gestalten. Systemsteuerung, Schulentwicklung und Unterrichtsqualität. Wiesbaden: Springer VS.

Fend, Helmut (2014): Die Wirksamkeit der neuen Steuerung Educational Governance als Forschungsperspektive. In: Maag Merki, Katharina/Langer, Roman/Altrichter, Herbert (Hrsg.): Educational Governance als Forschungsperspektive. Strategien. Methoden. Ansätze. Wiesbaden: Springer VS, S. 27-50.

Füssel, Hans-Peter/Kretschmann, Rudolf (1993): Gemeinsamer Unterricht für behinderte und nichtbehinderte Kinder. Pädagogische und juristische Voraussetzungen; Gutachten im Auftrag der Max-Traeger-Stiftung. Wehle. 
Gasterstädt, Julia (2019a): Der Komplexität begegnen und Inklusion steuern. Eine Situationsanalyse der Umsetzung von Artikel 24 der UN-BRK in zwei Bundesländern in Deutschland. Wiesbaden: Springer VS.

Gasterstädt, Julia (2019b): Von der (Schul-)Leitung zur regionalen Steuerung? Zur veränderten Rolle der Leitung von sonderpädagogischen Förder- und Kompetenzzentren im Kontext der Entwicklung inklusiver Schulsysteme. In: Graß, Doris/Altrichter, Herbert/Schimank, Uwe (Hrsg.): Governance und Arbeit im Wandel: Bildung und Pflege zwischen Staat und Markt. Wiesbaden: Springer VS, S. 111-127.

Gasterstädt, Julia (2019c): Möglichkeitsräume regionaler Kooperationen in Steuerungssituationen zur Entwicklung inklusiver Strukturen. In: Ricken, Gabi/Degenhardt, Sven (Hrsg.): Vernetzung, Kooperation, Sozialer Raum - Inklusion als Querschnittaufgabe. Bad Heilbrunn: Klinkhardt.

Gasterstädt, Julia/Rüger, Stella (i.E.): Differenzsetzung situieren? Möglichkeiten und Grenzen der Situationsanalyse am Beispiel der empirischen Auseinandersetzung mit Differenz. In: Gabriel, Sabine/Kotzyba, Katrin/Leinhos, Patrick/Matthes, Dominique/Meyer, Karina/Völcker, Matthias (Hrsg.): Soziale Differenz und Reifizierung - Theoretische Zugänge und forschungspraktische Bearbeitung. Studien zur Schul- und Bildungsforschung. Wiesbaden: Springer VS.

Heinrich, Martin/Blasse, Nina/Budde, Juergen/Demmer, Christine/Gasterstädt, Julia/Lübeck, Anika/Urban, Michael/Wolf, Juliane (2019): Professionalisierung durch Fallarbeit für die inklusive Schule. In: Ricken, Gabi/Degenhardt, Sven (Hrsg.): Vernetzung, Kooperation, Sozialer Raum - Inklusion als Querschnittaufgabe. Bad Heilbrunn: Klinkhardt.

Heinrich, Martin/Urban, Michael/Werning, Rolf (2013): Grundlagen, Handlungsstrategien und Forschungsperspektiven für die Ausbildung und Professionalisierung von Fachkräften für inklusive Schule. In: Döbert, Hans/Weishaupt, Horst (Hrsg.): Inklusive Bildung professionell gestalten. Münster u.a.: Waxmann, S. 69-133.

Hinz, Andreas (2013): Inklusion - von der Unkenntnis zur Unkenntlichkeit!? Kritische Anmerkungen zu einem Jahrzehnt Diskurs über schulische Inklusion in Deutschland. In: Zeitschrift für Inklusion, 1. https://www.inklusion-online.net/index.php/inklusion-online/article/view/26 [Zugriff: 13.12.2019].

Hinz, Andreas/Kruschel, Robert (2012): Educational Governance als ,Diagnose-Instrument ‘ für die Analyse eines Projekts zur Etablierung inklusiver Entwicklungen. In: Zeitschrift für Inklusion, 3. https://www.inklusion-online.net/index.php/inklusion-online/article/view/55/55 [Zugriff 12.05.2020].

Hopf, Wulf/Kronauer, Martin (2016): Welche Inklusion? In: Moser, Vera/Lütje-Klose, Birgit (Hrsg.): Schulische Inklusion. 62. Beiheft der Zeitschrift für Pädagogik. Weinheim u.a.: Beltz Juventa, S. 14-26.

Katzenbach, Dieter (2015): Zu den Theoriefundamenten der Inklusion. Eine Einladung zum Diskurs aus der Perspektive der kritischen Theorie. In: Schnell, Irmtraud (Hrsg.): Herausforderung Inklusion. Theoriebildung und Praxis. Bad Heilbrunn: Klinkhardt, S. 19-33.

Katzenbach, Dieter/Olda, Valeska/Rinck-Muhler, Stefanie (2006): Zwischenbericht zur Evaluation von Beratungs- und Förderzentren. Frankfurt a.M.: Arbeitsstelle für Sonderpädagogische Schulentwicklung und Projektbegleitung.

Kiuppis, Florian (2014): Heterogene Inklusivität, inklusive Heterogenität. Bedeutungswandel imaginierter pädagogischer Konzepte im Kontext internationaler Organisationen. Münster: Waxmann.

KMK (1994): Empfehlung zur sonderpädagogischen Förderung in den Schulen in der Bundesrepublik Deutschland. Beschluß der Kultusministerkonferenz vom 06.05.1994. Nienburg: Schulze.

Kussau, Jürgen/Brüsemeister, Thomas (2007): Educational Governance: Zur Analyse der Handlungskoordination im Mehrebenensystem der Schule. In: Altrichter, Herbert/Brüsemeister, 
Thomas/Wissinger, Jochen (Hrsg.): Educational Governance. Wiesbaden: Springer VS, S. 1554.

Lindblad, Sverker/Popkewitz, Thomas (2000): Educational Governance and Social Inclusion and Exclusion: Some conceptual difficulties and problematics in policy and research. In: Discourse 21, 1, S. 5-44.

Lindmeier, Christian (2011a): Bildungsgerechtigkeit im schulbezogenen sonder-, integrationsund inklusionspädagogischen Diskurs. In: Dederich, Markus/Schnell, Martin W. (Hrsg.): Anerkennung und Gerechtigkeit in Heilpädagogik, Pflegewissenschaft und Medizin. Auf dem Weg zu einer nichtexklusiven Ethik. Bielefeld: Transcript, S. 159-186.

Lindmeier, Christian (2011b): Bildungsgerechtigkeit und Inklusion. In: Zeitschrift für Heilpädagogik 62, 4, S. 124-135.

Mahoney, James (2000): Path dependence in historical sociology. In: Theory and society 29, 4, S. 507-548.

Meijer, Cor/Watkins, Amanda (2016): Changing conceptions of inclusion underpinning education policy. In: Watkins, Amanda/Meijer, Cor (Hrsg.): Implementing inclusive education: issues in bridging the policy-practice gap. Bingley: Emerald, S. 1-16.

Meuser, Michael/Nagel, Ulrike (1994): ExpertInnenwissen und ExpertInneninterview In: Hitzler, Ronald/Honer, Anne/Maeder, Christoph (Hrsg.): Expertenwissen. Die institutionalisierte Kompetenz zur Konstruktion von Wirklichkeit. Opladen: Westdeutscher Verlag, S. 180-192.

Mißling, Sven/Ückert, Oliver (2014): Inklusive Bildung: Schulgesetze auf dem Prüfstand. Berlin: Deutsches Institut für Menschenrechte.

Moser, Vera/Dietze, Torsten (2015): Perspektiven sonderpädagogischer Unterstützung. In: Kuhl, Poldi/Stanat, Petra/Lütje-Klose, Birgit/Gresch, Cornelia/Pant, Hans Anand/Prenzel, Manfred (Hrsg.): Inklusion von Schülerinnen und Schülern mit sonderpädagogischem Förderbedarf in Schulleistungserhebungen. Wiesbaden: Springer VS, S. 75-99.

Müller, Frank J. (Hrsg.) (2018): Blick zurück nach vorn - WegbereiterInnen der Inklusion. Gießen: Psychosozial-Verlag.

Pijl, Sip Jan/Frissen, Paul H. A. (2009): What Policymakers Can Do to Make Education Inclusive. In: Educational Management Administration \& Leadership 37, 3, S. 366-377.

Powell, Justin J.W. (2017): Räumliche Vielfalt der Inklusiven Bildung und sonderpädagogische Fördersysteme im Vergleich. In: Gercke, Magdalena/Opalinski, Saskia/Thonagel, Tim (Hrsg.): Inklusive Bildung und gesellschaftliche Exklusion: Zusammenhänge - Widersprüche - Konsequenzen. Wiesbaden: Springer VS, S. 25-38.

Prengel, Annedore (2006): Pädagogik der Vielfalt. Verschiedenheit und Gleichberechtigung in Interkultureller, Feministischer und Integrativer Pädagogik. Wiesbaden: VS Verlag für Sozialwissenschaften.

Preuß, Bianca (2018): Inklusive Bildung im schulischen Mehrebenensystem. Behinderung, Flüchtlinge, Migration und Begabung. Wiesbaden: Springer VS.

Prior, Lindsay (2003): Using Documents in Social Research. London u.a.: Sage.

Reiser, Helmut/Willmann, Marc/Urban, Michael (2007): Sonderpädagogische Unterstützungssysteme bei Verhaltensproblemen in der Schule: Innovationen im Förderschwerpunkt emotionale und soziale Entwicklung. Bad Heilbrunn: Klinkhardt.

Rohrmann, Albrecht/Weinbach, Hanna (2017): Inklusive Bildung durch Schulbegleitung? Zur Verstetigung von Schulbegleitung durch das Bundesteilhabegesetz. In: Sozial extra 41, 4, S. 39-42.

Rürup, Matthias (2007): Recht als Quelle - Zur vergleichbaren Erfassung schulpolitischer Entwicklungen im deutschen Bundesstaat. In: Böhm-Kasper, Oliver/Schuchart, Claudia/Schulzeck, Claudia (Hrsg.): Kontexte von Bildung. Erweiterte Perspektiven in der Bildungsforschung. Münster u.a.: Waxmann, S. 197-216. 
Rürup, Matthias (2011): Inklusive Bildung als Reformherausforderung. Zur Perspektive der Educational Governance Forschung. In: Zeitschrift für Inklusion, 4, o.S. https://www.inklusiononline.net/index.php/inklusion-online/article/view/74 [Zugriff: 19.05.2020]

Rürup, Matthias (2013): Graswurzelbewegungen der Innovation - Zur Innovativität von Schulen und Lehrkräften „At-the-Bottom“ der Schullandschaft. In: Rürup, Matthias/Bormann, Inka (Hrsg.): Innovationen im Bildungswesen. Wiesbaden: Springer VS, S. 269-301.

Schriewer, Jürgen (2013): Vergleichende Erziehungswissenschaft als Forschungsfeld. In: Hummrich, Merle/Rademacher, Sandra (Hrsg.): Kulturvergleich in der qualitativen Forschung: Erziehungswissenschaftliche Perspektiven und Analysen. Wiesbaden: Springer Fachmedien Wiesbaden, S. 15-41.

Siehr, Angelika/Wrase, Michael (2014): Das Recht auf inklusive Schulbildung als Strukturfrage des deutschen Schulrechts - Anforderungen aus Art. 24 BRK und Art. 3 Abs. 3 S. 2 GG. In: Recht der Jugend und des Bildungswesens 62, 2, S. 161-182.

Slee, Roger/Allan, Julie (2001): Excluding the included. A reconsideration of inclusive education. In: International Studies in Sociology of Education 11, 2, S. 173-192.

Stojanov, Krassimir (2011): Bildungsgerechtigkeit. Rekonstruktionen eines umkämpften Begriffs. Wiesbaden: Springer VS.

Strauss, Anselm L. (1993): Continual permutations of action. New York: de Gruyter.

Strauss, Anselm L./Corbin, Juliet (1996): Grounded Theory. Grundlagen qualitativer Sozialforschung. Weinheim: Beltz, Psychologie-Verlag-Union.

Tegge, Dana/Brüggemann, Christian (2016): Kennziffern in der Kritik. Anmerkungen zur indikatorengestützten Darstellung von Inklusion in der internationalen Bildungsberichterstattung. In: Zeitschrift für Inklusion, 1. https://www.inklusion-online.net/index.php/inklusion-online/article/view/344/288 [Zugriff: 19.05.2020]

United Nations, Committee on the Rights of Persons with Disabilities (2015): Concluding observations on the initial report of Germany. https://www.institut-fuer-menschenrechte.de/filead$\mathrm{min} /$ user_upload/PDF-Dateien/Pakte_Konventionen/CRPD_behindertenrechtskonvention/CRPD_Concluding_observations_on_the_initial_report_of_Germany_May_2015.pdf [Zugriff: 12.05.2020].

Wocken, Hans (2009): Inklusion \& Integration. Ein Versuch, die Integration vor der Abwertung und die Inklusion vor Träumereien zu bewahren. In: Stein, Anne-Dore/Krach, Stefanie/Niediek, Imke (Hrsg.): Integration und Inklusion auf dem Weg ins Gemeinwesen. Möglichkeitsräume und Perspektiven. Bad Heilbrunn: Klinkhardt, S. 204-234.

Wolff, Stephan (2012): Dokumenten- und Aktenanalyse. In: Flick, Uwe/Kardorff, Ernst von/ Steinke, Ines (Hrsg.): Qualitative Forschung: Ein Handbuch. Reinbek: Rohwolt, S. 502-514.

Wrase, Michael (2015): Die Implementation des Rechts auf inklusive Schulbildung nach der UNBehindertenrechtskonvention und ihre Evaluation aus rechtlicher Perspektive. In: Kuhl, Poldi/Stanat, Petra/Lütje-Klose, Birgit/Gresch, Cornelia/Pant, Anand Hans/Prenzel, Manfred (Hrsg.): Inklusion von Schülerinnen und Schülern mit sonderpädagogischem Förderbedarf in Schulleistungserhebungen. Wiesbaden: Springer VS, S. 41-74.

Wrase, Michael (2016): Auflösung der Förderschulen: Die UN-Behindertenkonvention verlangt die Inklusion von Kindern mit Behinderung an Regelschulen. In: WZ-Brief Bildung No. 33. http://nbn-resolving.de/urn:nbn:de:101:1-201607078339 [Zugriff: 19.05.2020]. 


\title{
11 Maßnahmen inklusiver Bildung - Ein international vergleichender Blick auf Andalusien (Spanien) und Berlin (Deutschland)
}

\author{
Lea Schäfer
}

\begin{abstract}
Inklusive Bildung wird in Spanien im Kontext der Realisierung eines demokratischen Bildungssystems und als Möglichkeit der Garantie von Chancengerechtigkeit durch eine Schule für alle verstanden. Die bildungspolitisch vorgegebenen Maßnahmen zur Berücksichtigung von Diversität zielen auf inklusive Bildung, wobei Behinderung bzw. sonderpädagogischer Förderbedarf - anders als in den rechtlichen Vorgaben der deutschen Bundesländer - explizit nur ein Teil der Zielgruppe inklusiver Bildung darstellt. Gleichzeitig werden bei der Umsetzung der rechtlichen Grundlagen zu inklusiver Bildung auf der Mikroebene der Schule deutliche Exklusionsprozesse sichtbar. International vergleichend zu den regionalen Strukturen inklusiver Beschulung in Andalusien steht als „Extremfall“ - im Sinne eines kontrastierenden Falls hinsichtlich struktureller Eigenschaften - das Land Berlin, konkret: Maßnahmen im Rahmen des „Gesamtkonzepts Inklusive Schule in Berlin“. Dabei soll folgende Fragestellung beantwortet werden: Welche Gemeinsamkeiten und Unterschiede bzgl. organisationaler Maßnahmen zur Umsetzung inklusiver Schulbildung lassen sich für diese beiden Länder föderaler Staaten (die Autonome Region Andalusien und das Land Berlin) im Kontext der politischen Vorgaben feststellen?
\end{abstract}

\section{Einleitung}

Im Zuge des Demokratisierungsprozesses in Spanien nach der Diktatur Francos führte das Land die Autonomie der einzelnen Regionen bzgl. Bildung im Jahre 1981 in Andalusien ein (BOE 11/01/1982; Nohlen/Hildenbrand 2005). Die Bundesrepublik Deutschland wurde hingegen bereits nach Ende des 2. Weltkriegs in damals 12 Bundesländer aufgeteilt. Hierin kam Berlin aufgrund der Teilung in Ost- und West-Berlin bis 1990 eine Sonderrolle zu (Wolff 2019). Diese Gemeinsamkeit einer föderalen Bildungsverwaltung, ebenso wie eine Vorreiterrolle bzgl. inklusiver Bildung im jeweiligen Land bieten die Basis für einen tiefergehenden und vergleichenden Blick auf Andalusien als Autonome Region Spaniens und Berlin als ein Stadtstaat in Deutschland ${ }^{1}$ (zum Vergleich Madrid und Baden-Württemberg, siehe Powell et al. 2021 i.d.B.). Sowohl Andalusien als auch (West-)Berlin führten zwar in den 1980er Jahren die gemeinsame Beschulung aller Schüler*innen ein, dies jedoch vor dem Hintergrund

1 Der Blick auf die historische Entwicklung fokussiert Deutschland und Berlin ohne die Entwicklung in den Landes- bzw. Stadtteilen der DDR miteinzubeziehen. 
divergenter historischer Entwicklungen und mit voneinander abweichenden Resultaten. Dies wird u.a. bei einem Vergleich der Segregationsquoten beider Länder deutlich (European Agency 2018), welche zwar nur marginal Aussagen über die Qualität der inklusiven Praxis zulassen, aber dennoch als ein Tertium Comparationis in Form eines Hinweises auf den Stand der Etablierung inklusiver Bildung an dieser Stelle herangezogen werden.

Die seit Jahren im internationalen Vergleich sehr niedrige Integrations- und Inklusionsquote Spaniens, die das spanische Bildungsministerium für das Schuljahr 2017/18 mit 0,45\% (Ministerio de Educación y Formación Profesional (MEFP) 2019) angibt und worunter sowohl die Beschulung in separaten Klassen innerhalb der Regelschulen als auch die Beschulung in sog. Schulen für Sonderpädagogik, wie sie in Spanien bezeichnet werden, fallen, sprechen insgesamt und auf den ersten Blick für eine konsequente Umsetzung inklusiver Bildung. Die European Agency (2018) differenziert diese Zahl in ihren Statistiken zu inklusiver Bildung noch einmal und unterscheidet zwischen der Beschulung in Sonderklassen und jener in Sonderschulen. Diese Zahlen sind für Spanien insgesamt etwas höher, jedoch im Vergleich immer noch weit unter dem Durchschnitt der an der Erhebung beteiligten europäischen Länder. So werden hier 0,55\% der Gesamtpopulation der Schüler*innen in Schulen für Sonderpädagogik unterrichtet, in Deutschland sind es 3,47\% aller Schüler*innen, die an Förderschulen unterrichtet werden (Durchschnitt aller beteiligten Länder: 1,54\%) (ebd.). Im Gegensatz zu diesen niedrigen Separations- und Segregationsraten in Spanien kritisierte das UN-Komitee im Jahr 2017, dass das Land aufgrund seines segregierenden Schulsystems für Menschen mit Behinderungen gegen Artikel 24 der UN-Konvention über die Rechte von Menschen mit Behinderungen (UN-Convention on the Rights of Persons with Disabilities; i.F. UN-CRPD) verstößt (UN-Ausschuss zu Rechten von Menschen mit Behinderungen 2017; UN 2019). Die Untersuchung des spanischen Bildungssystems nach Verstößen gegen die UN-CRPD durch die UN war die Folge einer Klage eines großen Verbandes von Menschen mit Behinderungen $^{2}$, der sich an die unabhängige Anlaufstelle gem. Par. 33 der UN-CRPD zur Umsetzung ebendieser in Spanien ${ }^{3}$ gewandt hatte (ebd.: 3). Dies erklärt auch, wieso es diese Untersuchung in einem Land mit einer so niedrigen Quote gibt, in Deutschland, das eine sehr viel höhere Zahl separierter und segregierter Beschulung aufweist, jedoch (noch) nicht.

Der international vergleichende Blick auf Aspekte inklusiver Bildung in Andalusien (Spanien) und Berlin (Deutschland) bietet sich u.a. aufgrund der Gemeinsamkeit des föderalen Systems im Bereich Bildung und der unterschiedlichen schulstrukturellen Umsetzung an. Die niedrige Segregationsquote in Spanien, die lange Tradition integrativer und inklusiver Beschulung und die Tatsache, dass inklusive Bildung in Spanien im Kontext der internationalen Inklusionsforschung insgesamt bislang noch ein Desiderat darstellt (s. u.a. Dyson 2004; Johnson 2013; Köpfer 2013; Kiuppis/Peters 2014; Powell 2014; Brüggemann et al. 2016; Schäfer et al. 2016; Köpfer et al. 2018; Powell 2018; Schäfer et al. 2018), macht Spanien für einen Vergleich interessant. Konkret wird in diesem Artikel der Frage nachgegangen, welche Unterschiede und Gemeinsamkeiten sich im Vergleich der bildungspolitisch definierten Maßnahmen zur Umsetzung inklusiver Bildung in Andalusien und Berlin als Resultat jahrelanger Entwicklungen feststellen lassen Dabei wird im Kontext dieses Artikels - anschließend an die punktuelle Komparation der historischen Entwicklung inklusiver Schulbildung auf rechtlicher Ebene in beiden Ländern - das Konzept zur „Berücksichtigung der Diversität“ (Consejería de Educación 2008) in Andalusien analysiert und dem „Gesamtkonzepts Inklu-

2 Asociación SOLCOM para la solidaridad comunitaria de las personas con diversidad funcional y la inclusión social.

3 Comité Español de Representantes de Personas con Discapacidad (CERMI) 
sive Schule in Berlin" (Abgeordnetenhaus von Berlin 2011) gegenübergestellt. Andalusien befindet sich im Süden Spaniens und ist die bevölkerungsreichste Autonome Region des Landes mit der höchsten Anzahl an Schüler*innen, doch gleichzeitig ist die Bevölkerungsdichte relativ gering (Instituto Nacional de Estadísticas 2019). Laut Bildungsbericht von 2019 gingen im Schuljahr 2017/18 ca. 1,8 Mio. Kinder und Jugendliche in Andalusien in die Schule, davon 7949 in separaten und segregierten Beschulungsformen $(0,44 \%)$ (Consejería de Educación y Deporte 2019: 2). Mit einer Integrationsquote von 84,2\% liegt Andalusien über der spanischen Durchschnittsquote von 83,6\% und im Mittelfeld aller Autonomen Regionen (MEFP 2019: 7).

Der Schritt des Vergleichs beider Vorgaben zu inklusiver schulischer Bildung entspricht im Kontext international vergleichender Forschung jenem der Juxtaposition oder Komparation nach Hilker (1962), welchem die Exploration und Selektion bereits vorausgingen. Im Kontext dieser Komparation werden beide Konzepte hinsichtlich des Gegenstands der Maßnahmen inklusiver Bildung im Schulsystem beider Länder analysiert. Der Fokus liegt hierbei auf der rechtlichen Verortung, den möglichen Beschulungsformen sowie den konkreten Maßnahmen inklusiver Schulbildung. Die abschließende Diskussion dient dazu, die gewonnenen Ergebnisse hinsichtlich Gemeinsamkeiten und Unterschiede einzuordnen und daran anschließende Forschungsdesiderate zu formulieren.

Der Artikel enthält bzgl. Spanien und Andalusien Ergebnisse aus meiner Dissertation (erfolgreiche Disputation 09/2019, Kultur-, Sozial- und Bildungswissenschaftliche Fakultät der Humboldt-Universität zu Berlin). Im Rahmen dieser Studie wurde der institutionelle Wandel von Diversität im spanischen und andalusischen Schulsystem mittels einer qualitativen Mehrebenenanalyse sowohl historisch als auch durch Expert*inneninterviews rekonstruiert und analysiert. Ebenso wie die genannte Studie wird der vorliegende Artikel aus der Perspektive einer Wissenschaftlerin aus Berlin in Deutschland geschrieben, was in Bezug auf die Analyse in Andalusien bedeutet, dass mit dieser eine Fremdbetrachtung durchgeführt wird, die einerseits das Verständnis kultureller Dimensionen einschränkt und gleichzeitig die Möglichkeit eröffnet, von außerhalb auf ein Land und eine Region zu schauen, ohne Teil dessen zu sein. Alle spanischen Quellen wurden von mir übersetzt.

\section{Historische Meilensteine inklusiver Schulbildung in Spanien zwischen 1960 und 1999}

Die nationale und die regionale Ebene, die im Fokus der Analyse stehen, werden hier durch Andalusien (Spanien) und Berlin (Deutschland) repräsentiert. Dieses komplexe Anliegen kann an dieser Stelle die historische Entwicklung auf nationaler Ebene nur skizzieren. Die historischen Entwicklungsschritte auf nationaler Ebene sind in beiden Ländern die Voraussetzung für die Entwicklungen auf regionaler Ebene, da sowohl für Andalusien das spanische Bildungsgesetz als auch für Berlin die Empfehlungen der Kultusministerkonferenz (KMK) bindend sind. Außerdem wurden zwei Zeitabschnitte festgelegt: die Phase von 1960 bis 1999 und jene von 2000 bis 2013. Damit ist die Komparation des aktuellen Standes in Andalusien und Berlin bzgl. der Maßnahmen zur Berücksichtigung der Diversität von Schüler*innen insofern vorbereitet, als dass auch die historischen Meilensteine zum Verständnis der aktuellen Situation in Andalusien und Berlin dienen können. 
Das Bildungsministerium der Zentralregierung Spaniens gibt den einzelnen Autonomen Regionen die Bildungsziele wie u.a. die Chancengleichheit vor, die insbesondere durch inklusive Bildung und Maßnahmen zur Berücksichtigung von Diversität gewährleistet werden sollen (Ecoem 2007).

Die adäquate schulische Antwort für alle Schüler*innen wird nur durch das Prinzip der Inklusion gewährleistet. Nur auf diese Weise wird die Entwicklung aller garantiert, wird die Gerechtigkeit fokussiert und ein größerer sozialer Zusammenhalt erreicht. Die Berücksichtigung der Diversität ist eine Notwendigkeit, die alle Schulstufen und alle Schülerinnen und Schüler umfasst. D.h. es geht darum, die Diversität der Schülerinnen und Schüler als ein Prinzip zu betrachten und nicht als Maßnahme für die Bedürfnisse einiger weniger (Ecoem 2007: 66).

Bereits auf nationaler Ebene wird mit dem Bildungsgesetz LOE (2006) (Ecoem 2007) so die Relevanz inklusiver Bildung für Bildungsgerechtigkeit festgelegt und für alle Autonomen Regionen rechtlich vorgegeben. Dies bedeutet, dass Andalusien die gesetzliche Vorgabe der nationalen Regierung auf regionaler Ebene in seiner Gesetzgebung konkretisieren muss, die dann wiederum bindend für weitere Verordnungen des Bildungssystems und schließlich auch für die Schulen ist. Dieser klaren rechtlichen Positionierung ging eine jahrzehntelange Entwicklung hin zur Berücksichtigung von Diversität im Kontext inklusiver Bildung voraus, die nun in Komparation mit der Entwicklung in Deutschland und Berlin skizziert werden soll.

Die erste Phase von 1960 bis 1999 ist in Spanien von großen Veränderungen geprägt. Der wirtschaftliche Aufschwung des Landes seit dem „Stabilisierungspakt“ im Jahr 1959 erforderte von der Regierung Francos eine internationale Öffnung sowie die Annäherung an Inhalte und Ziele von Organisationen wie den United Nations (UN) und der UNESCO (Organisation der Vereinten Nationen für Bildung, Wissenschaft und Kultur). Zudem machte die Bildungsexpansion zwischen 1961 und 1967 eine Neustrukturierung des seit 1857 nahezu unveränderten Bildungssystems notwendig (Capitán 2002; Nohlen/Hildenbrand 2005). So reagierte noch während der Diktatur Francos im Jahre 1970 der damalige Bildungsminister Spaniens Villar Palasí mit dem Allgemeinen Bildungsgesetz (LGE) auf die vielfältigen Veränderungen innerhalb der spanischen Gesellschaft und der damit einhergehenden massiven Kritik am spanischen Bildungssystem (Capitán 2002). Dieser erste Versuch der Demokratisierung der Bildung - in Zeiten der diktatorischen Herrschaft - legte den Grundstein für alle anschließend vollzogenen rechtlichen Reformen und bildete damit die Grundlage eines auf rechtlicher Ebene auf Chancengleichheit ausgerichteten Bildungssystems. Gleichzeitig wurde im Jahr 1975 die Gründung des Nationalen Instituts der Sonderpädagogik beschlossen, das zum Ziel hatte, „Personen, die unter Defiziten oder mangelndem Anpassungsvermögen leiden, durch die adäquate schulische Förderung auf ihre Eingliederung in das soziale Leben und - im Rahmen des Möglichen - in das Arbeitsleben vorzubereiten“ (Ministerio de Educación y Ciencia (MEC) 1975: 11769). Der Ausbau des Sondersystems für bestimmte Schüler*innen mit dem expliziten Ziel der Integration wurde damit rechtlich festgelegt und legitimiert (MEC 1975). Im Kontext der Formulierung grundlegender Prinzipien für die schulische Integration wie Normalisierung und Individualisierung des Schulsystems fand im Nationalen Plan für sonderpädagogische Schulen die Differenzierung zwischen „,vollständiger“, „,kombinierter“, „partieller“ Integration sowie der Beschulung in speziellen Einrichtungen der Sonderpädagogik in Form von vier Stufen der Integration statt (Instituto Nacional de Educación Especial 1978: 7).

Für Spanien waren die Schritte der Demokratisierung des Landes und damit auch des Bildungssystems sowie die internationale Öffnung in dieser Phase entscheidend für die Entwicklung der inklusiven Schulbildung. Andalusien wurde im Zuge dessen im Jahr 1981 zur 
Autonomen Region und verpflichtete sich als eine der ersten vier Autonomen Regionen dazu, die Verantwortlichkeit für das Bildungssystem zu übernehmen (BOE 11/01/1982; Nohlen/Hildenbrand 2005) - eine Entwicklung, die sich für Andalusien als wegweisend herausstellte. Mit dem „Recht über die Planung der Sonderpädagogik und der Erweiterung der Erprobungsphase der Integration in der Autonomen Region Andalusien für das Schuljahr 1986/87“"vom 25.03.1986 verankerte die Regierung als eine der ersten Autonomen Regionen die Ausweitung der Integration. Das Ziel sollte die „,vollständige Integration und eine systematische und schrittweise Überführung“ von Schüler*innen mit ,physischen, psychischen und sensorischen Behinderungen oder Unangepasstheiten“ sein (Consejería de Educación 1986: 1095).

Die Ziele, an denen sich das Bildungssystem von da an orientieren sollte, sind gesellschaftlicher Art, wofür das Bildungsgesetz LOGSE im Jahr 1990 ein entscheidendes Element sein sollte, „um die sozialen Stereotype, verbunden mit der Differenzierung aufgrund von Geschlechtern, angefangen bei derselben Konstruktion und durch den Gebrauch von Sprache“ zu verhindern (LOGSE 1990: 28928). Im Rahmen dieses Gesetzes wurde - in Anlehnung an den Warnock-Report in England - die Zielgruppe schulischer Integration auf Schüler*innen mit besonderen Bedürfnissen erweitert (Sánchez/Torres 2009). Dieses Gesetz gilt als die entscheidende Bildungsreform, auf die alle weiteren Veränderungen bzgl. inklusiver Schulbildung in der Bildungsgesetzgebung aufbauen.

\section{Historische Meilensteine inklusiver Schulbildung in Deutschland zwischen 1960 und 1999}

Im Rückblick auf die Entwicklung inklusiver Bildung in Deutschland - mit Fokus auf die Entwicklung der Regulative - ist der Ausbau des Sonderschulwesens im Anschluss an das Gutachten der Ständigen Konferenz der Kultusminister (KMK) im Jahr 1960 als ein Resultat vielfältiger gesellschaftlicher Entwicklungen zu sehen, das in der Folge weitreichenden Einfluss auf ebendiese Entwicklung hatte. In diesem „Gutachten zur Ordnung des Sonderschulwesens“ wurden zwölf eigenständige Sonderschulen legitimiert, empfohlen und damit im Bildungssystem etabliert, was eine Verdopplung der Sonderschulen von 1960 bis 1973 und eine Verdreifachung der Schüler*innen in diesen Schulen zur Folge hatte (Muth 1986: 10). Die Empfehlungen ,Zur pädagogischen Förderung behinderter und von Behinderung bedrohter Kinder und Jugendlicher" des Deutschen Bildungsrates von 1973 formulieren als erstes bildungspolitisches Dokument schulische Integration als gemeinsame Unterrichtung von Behinderten und Nichtbehinderten (Muth 1986).

Insgesamt waren dann die 1980er und 1990er Jahre in Deutschland geprägt von integrativen Schulversuchen und Integrationsklassen mit wissenschaftlicher Begleitforschung in Berlin, Hamburg und Bonn (Preuss-Lausitz 2001; Müller 2018). Das Berliner Schulgesetz von 1990 legt schließlich die Integration von Schüler*innen mit Behinderung in die Regelschule fest, womit eine „Gleichrangigkeit“ von gemeinsamer Erziehung und Unterricht in der Sonderschule hergestellt wurde.

Das Jahr 1994 beinhaltete für Deutschland zwei relevante Meilensteine, die die Entwicklung inklusiver Bildung mitgeprägt haben. Auf der einen Seite die Ergänzung in Artikel 3 des Grundgesetzes „Niemand darf wegen seiner Behinderung benachteiligt werden“ 
(Bundesregierung 2014) und auf der anderen Seite die „Empfehlung zur sonderpädagogischen Förderung in den Schulen in der Bundesrepublik Deutschland" der Kultusministerkonferenz (KMK). Ersterer stellt die Grundlage für die explizite rechtliche Berücksichtigung von Menschen mit Behinderung auf Bundesebene dar. Die KMK-Empfehlungen von 1994 formulieren dann für die einzelnen Bundesländer Integration als mögliche Form der Beschulung für ,Kinder und Jugendliche mit Sonderpädagogischem Förderbedarf (...) wenn (...) die notwenige sonderpädagogische und auch sächliche Unterstützung sowie die räumlichen Voraussetzungen gewährleistet sind“" (Sekretariat der Ständigen Konferenz der Kulturminister 1994: 14). Dieser Haushaltsvorbehalt findet sich auch 10 Jahre danach im, bis heute gültigen, Berliner Schulgesetz von 2004 wieder (ebd.).

\section{Der Vergleich: historische Meilensteine inklusiver Schulbildung zwischen 1960 und 1999 in Spanien und Deutschland}

Die Entwicklung in dieser Phase zeigt, dass durch die Einführung der zwölf Förderschwerpunkte auf nationaler Ebene in Deutschland die Zahl der Sonderschulen bis 1973 verdoppelt wurde und erst die Empfehlungen des Bildungsrates im selben Jahr Integration auf formaler Ebene ermöglichten. Damit wurde das segregierende Schulsystem fortgeführt und massiv ausgebaut. Spanien war politisch während dieser Zeit in der letzten Phase der Diktatur angekommen und strebte u.a. aufgrund der Bildungsexpansion nach der Demokratisierung von Bildung und Chancengleichheit, für welche das nationale Bildungsgesetz LGE als wegweisend gelten kann. Gleichzeitig stellt die Gründung des Nationalen Instituts für Sonderpädagogik mit dem Ziel der Förderung des Ausbaus von Sonderschulen und der parallelen Etablierung von speziellen Übergangsklassen in Regelschulen, um Integration zu gewährleisten, für Spanien keinen Widerspruch dar, sondern die Methode um Chancengleichheit zu ermöglichen. Auch die Sonderpädagogik hatte einen klaren regulativen Auftrag: die Gewährleistung von Chancengleichheit durch unterschiedliche Formen von Integration, die auch innerhalb der Schulen separierende und außerhalb des Regelschulsystems auch segregierende Formen enthielt.

Diese beiden grundverschiedenen Ausgangslagen für die Einführung von Integration in Spanien und Deutschland führten dennoch in beiden Ländern zu Schulversuchen bzgl. Integration in den 1980er Jahren. In Spanien ausgehend von der nationalen und regionalen Gesetzgebung und in Deutschland zumindest in einzelnen Städten ausgehend von Einzelschulen und Elterninitiativen und mit der Empfehlung von Integration als Beschulungsform. Deutschland definiert Integration weiterhin lediglich als eine Empfehlung der Beschulungsform. Dies zeigt eine Fortsetzung der rechtlichen Unverbindlichkeit in Deutschland, wie bereits in der vorherigen Phase herausgestellt. Gleichzeitig stellt die Salamanca-Erklärung 1994 (UNESCO 1994) nun einen internationalen Referenzrahmen inklusiver Bildung dar, der für beide Länder ein Orientierungspunkt für die Umsetzung sein könnte. Die nächste Phase ab 2000 wird zeigen, inwiefern dieser Rahmen genutzt wurde. 


\section{Die Entwicklung inklusiver Schulbildung in Spanien zwischen 2000 und 2013}

In der Analyse der Entwicklungen in dieser Phase wird an dieser Stelle der Fokus auf die nationale Ebene gelegt, da die Maßnahmen in Andalusien und Berlin Gegenstand der daran anschließenden differenzierten Darstellung sind.

Diese Phase beginnt mit einem für beide Länder einschneidenden Ereignis: die PISAStudie 2000. Die - im europäischen Vergleich - schlechten Ergebnisse Spaniens wurden von der konservativen PP-Regierung (Partido Popular) als Anlass genommen, das Bildungssystem durch ein neues Bildungsgesetz zu verändern: vom Fokus auf Chancengleichheit und Dezentralisierung der Bildungsverantwortung auf die Autonomen Regionen in der vorherigen Bildungsgesetzgebung der PSOE, hin zu einer Zentralisierung der Ziele des Bildungssystems auf europäische und internationale neoliberale Entwicklungen (Beltrán et al. 2008). Eine Schule für alle, wie sie noch im LOGSE als eines der Hauptziele formuliert wurde, stand während der konservativen PP-Regierung bis Anfang der 2000er Jahre nicht mehr im Mittelpunkt - die (Bildungs-)Gesetzgebung war nun insgesamt auf Separation und Selektion ausgelegt (Pohl 2005). Hier wird offensichtlich, wie die PISA-Ergebnisse Einfluss auf die Bildungspolitik und letztlich auf die Bildungsgesetzgebung in Spanien nahmen. Die schlechten Ergebnisse wurden politisch unter der Regierung der PP verwendet, um in Form staatlicher Kontrolle und externer Evaluation auf unterschiedlichen Ebenen eine Standardisierung einzuführen. Die Anpassung an die Entwicklung zentraleuropäischer Bildungssysteme sowie die Europäisierung im Allgemeinen, wird als Schlüssel zu wirtschaftlichem und sozialem Aufstieg angesehen, als Notwendigkeit, damit Spanien ,in der Mitte“ Europas ankommt (Bonal/Tarabini 2013). In Deutschland hingegen war die erste PISA-Studie 2000 insbesondere aufgrund der Schüler*innenleistungen, die sich tendenziell eher im Mittelfeld befanden sowie aufgrund des deutlichen Zusammenhangs zwischen sozialer Herkunft und Bildungserfolg, dem sog. Pisa-Schock, relevant (Baumert 2003).

Der erneute Regierungswechsel in Spanien im Jahr 2004 hatte zur Folge, dass die sozialistische Partei PSOE die Reformen im Bildungssystem weiter fortsetzen konnte und 2006 das Allgemeine Bildungsgesetz LOE verabschiedete (Ecoem 2007). Bereits im Rahmen der Beschreibung der Gesetzesteile in der Präambel wird als übergeordnetes Ziel des II. Titels über die Bildungsgerechtigkeit die ,,vollständige Inklusion und Integration“ von Schüler*innen als Ziel genannt, die aufgrund eines ,spezifischen Bedarfs an schulischer Unterstützung“ eine andere schulische Aufmerksamkeit benötigen (Ecoem 2007: 66). Dieses Inklusionsverständnis unterscheidet sich deutlich vom bisherigen Ziel der Integration durch unterschiedliche Stufen, die das Bildungssystem strukturell ermöglichte.

Gleichzeitig wird die Zielgruppe der Schüler*innen, die eine ,andere Unterstützung als die reguläre schulische Unterstützung“ (ebd.) benötigen, in diesem Bildungsgesetz erweitert und ein weiterer Begriff dafür eingeführt. Das bereits genannte Kapitel 1 mit dem Titel „Schüler*innen mit einem spezifischen Bedarf der schulischen Unterstützung“ (NEAE) ist in drei Abschnitte eingeteilt, die jeweils eine bestimmte Gruppe dieser Schüler*innen mit besonderen Bedarfen umfassen. Grundsätzlich sind darunter Schüler*innen zu verstehen, die aufgrund folgender Merkmale diesen Anspruch haben: Spezielle schulische Bedürfnisse (NEE), spezielle Lernschwierigkeiten, Hochbegabung, späte Einschulung, persönliche Bedingungen oder aufgrund der Schullaufbahn (Ecoem 2007: 111f.). 
Mit diesem Gesetz wurden spezielle Lernschwierigkeiten und die späte Einschulung als Gründe für inklusive Beschulung aufgenommen. NEE wird als vorübergehende oder dauerhafte Ursache für besondere Förderung aufgrund von Behinderung oder gravierenden Verhaltensstörungen konkretisiert (ebd.). Jedoch obliegt es nach wie vor den einzelnen Autonomen Regionen, für sich selbst zu definieren, welche Gruppen von Schüler*innen darunter zu verstehen sein sollen. Neu ist an diesem Gesetz, dass besondere schulische Bedarfe definiert werden, um entsprechende Maßnahmen zur Sicherstellung der Bildungsgerechtigkeit daraus abzuleiten.

Im Jahr 2006 verabschiedeten die UN die UN-CRPD, welche von Spanien 2007 und von Deutschland 2009 ratifiziert wurde (UN 2019). In der Folge waren beide Länder dazu aufgerufen, alle vier Jahre einen Staatenbericht über die aktuelle Situation ihres Landes - entsprechend Artikel 24 auch bzgl. Bildung - zu formulieren. Der Nationale Aktionsplan des spanischen Bildungsministeriums, der die Bildungsziele 2010-2020 auf Grundlage der UN-CRPD festlegt, fasst unter Punkt 12 ,Inklusive Bildung, Diversität und Interkulturalität: das Recht auf Differenz ohne rechtliche Differenzierungen“ (Ministerio de Educación, Cultura y Deporte 2010: 139) zusammen und sichert inklusive Bildung zu ebenso wie die Anerkennung von Diversität und Interkulturalität. Die vier formulierten Maßnahmen bleiben dabei jedoch äußerst ungenau und umfassen u.a. die Zusicherung der Ressourcen für Schüler*innen mit Behinderung und die Erleichterung der Chancen auf dem Ausbildungs- und Arbeitsmarkt für Schüler*innen mit NEAE - gleichzeitig soll die Finanzierung dieser Maßnahmen durch die Setzung anderer Schwerpunkte im Bildungssystem abgesichert sein. Außerdem wurde die Umsetzung der UN-CRPD als Bildungsziel in die nationale Gesetzgebung aufgenommen (ebd.).

Eine Konsequenz der Finanzkrise in Spanien ab 2008 war, dass der Ministerpräsident der PP, Mariano Rajoy, im Jahr 2012 verkündete, dass Spanien im Gesundheits- und Bildungssystem 10 Billionen Euro einsparen müsse - 3 Billionen allein in der Grund- und Sekundarstufe (Wandler 2012). Im selben Jahr veröffentlichte der Minister für Bildung, Kultur und Sport, José Ignacio Wert, die erste Version des neuen Bildungsgesetzes für die Verbesserung der Bildungsqualität, LOMCE, was vielfältige Proteste und Generalstreiks nach sich zog (Planelles 2013; Aunión/Álvarez 2013). Bereits in der Präambel wurde die Notwendigkeit für ein neues Bildungsgesetz erläutert: erforderliche Maßnahmen zur Reduzierung der Schulabbruchquote sowie notwendige Abschlussexamen am Ende der Pflichtschulzeit (Ministerio de Educación, Cultura y Deporte (MECD) 2013: 7). Große Verbände, wie der Verband für Angestellte im Bildungswesen, benannten ansteigende Klassengrößen, die Zusammensetzung von Klassen nach Kompetenzniveau bereits ab dem zwölften Lebensjahr (bislang erst ab 14 Jahren möglich) sowie die Reduzierung der Maßnahmen zur Berücksichtigung von Diversität im Schulsystem als Folgen dieses Gesetzes, welche weder zu mehr Qualität noch Gleichheit oder Inklusion im Sinne der UN-CRPD führen würden (Federcación de Trabajadores de la Enseñanza (FETE-UGT) 2013: 11). Insgesamt spiegelte sich in diesem Gesetz im Jahr 2013 die Entwicklung hin zu mehr staatlicher Kontrolle, Kompetenzfokussierung und insgesamt einer Standardisierung zu Ungunsten der Berücksichtigung der Diversität der Schüler*innenschaft, durch die Reduktion der Ausgaben für die Chancengleichheit und des Rechts auf Bildung für alle, wieder. Für die Berücksichtigung von Diversität hieß dieses Gesetz das Ersetzen der Maßnahme der Diversifizierung in den letzten beiden Schulstufen durch die Einführung des „Programm[s] zur Lern- und Leistungsverbesserung“ (MECD 2014: 5) bereits im 2. Jahr der Sekundarstufe. Diese beinhaltet „spezifische Methoden, die veränderte Struktur der Inhalte, der praktischen Anteile sowie der Fächer“ (ebd.). 
Zusammenfassend kann diese Umbenennung der Maßnahme der Diversifizierung ab der 2. Sekundarschulklasse als Ausweitung der Separation interpretiert werden. Eine weitere regulative Neuerung der PP-Regierung war im Jahr 2012 die Verabschiedung eines Gesetzes, das sowohl die Erhöhung der Unterrichtsstunden der Lehrkräfte erlaubte wie auch die Erhöhung der Klassengröße um bis zu 20\% über die maximale Anzahl der Schüler*innen, das im Frühjahr 2019 vom Bildungsministerium wieder aufgehoben wurde (MEFP 2019). Dieses Gesetz auf nationaler Ebene ist die Grundlage der Umsetzung inklusiver Bildung in Andalusien, auf die im weiteren Verlauf des Artikels differenziert eingegangen wird.

\section{Die Entwicklung inklusiver Schulbildung in Deutschland zwischen 2000 und 2013}

Der UN-Berichterstatter Vernor Muñoz bezeichnete 2007 das deutsche Schulsystem noch als „menschenrechtsfeindlich“ und kritisierte vor allem die starke Segregation und die dadurch verursachte Benachteiligung bestimmter Personengruppen, wie z.B. der Kinder sozial benachteiligter Schichten und der Kinder mit Behinderungen (Platte 2009: o.S.). 2011 wurde dann der erste Staatenbericht und der Nationale Aktionsplan der Bundesregierung veröffentlicht (BMAS 2011) und in der Folge die Aktionspläne der einzelnen Bundesländer, so auch das „Berliner Inklusionskonzept“ (Abgeordnetenhaus von Berlin 2011) als Aktions- und Maßnahmenplan, auf welches im Zuge des Vergleichs der Maßnahmen in Abschnitt 4 konkret eingegangen wird. Hierin heißt es: „Das Bildungssystem auf allen Ebenen steht Menschen mit Behinderung chancengleich und ohne Diskriminierung unter grundsätzlich unentgeltlicher Zurverfügungstellung der behinderungsbedingt notwendigen Nachteilsausgleiche offen“" (ebd.: 3).

Die UN-CRPD hatte auf das Berliner Schulgesetz bislang keine Auswirkung, weshalb auch der Befund und die Empfehlungen des UN-Ausschusses für die Rechte von Menschen mit Behinderungen 2015 für Deutschland nicht überraschen. In seinen „Abschließenden Bemerkungen über den ersten Staatenbericht Deutschlands" hatte dieser bzgl. Artikel 24 der UN-CRPD folgende Anmerkungen:

- Empfehlung einer Strategie in Form eines Aktionsplans mit konkretem Zeitplan und Zielen, die für alle Bundesländer gültig sind.

- Empfehlung des Rückbaus des segregierenden Förderschulwesens, „um Inklusion zu ermöglichen“ (Ausschuss für die Rechte von Menschen mit Behinderungen 2015: 9).

- Sicherstellung der Bereitstellung angemessener Vorkehrungen auf allen Ebenen des Bildungssystems.

- Schulung aller Lehrkräfte auf dem Gebiet der inklusiven Bildung (ebd.)

Die Empfehlung zum „Rückbau des segregierenden Förderschulwesens“ deckt sich mit der Einschätzung des UN-Ausschusses zum spanischen Bildungssystem, wobei letztere auf einer großen Studie im Anschluss an eine Klage von Betroffenenverbänden basiert (UN-Ausschuss zu Rechten von Menschen mit Behinderungen 2017). 


\section{Die Entwicklung inklusiver Schulbildung in Spanien und Deutschland zwischen 2000 und 2013 im Vergleich}

Diese dritte Phase - beginnend mit der PISA-Studie - ist bzgl. inklusiver Schulbildung in beiden Ländern von vielen Ereignissen geprägt, die Einfluss auf das Schulsystem hatten. In Spanien gab es einen Regierungswechsel und das nationale Bildungsgesetz LOE wurde im Jahr 2006 verabschiedet, dessen Umsetzung durch die Finanzkrise ab 2008 ins Stocken geriet. Gleichzeitig weitet dieses Gesetz, als Grundlage für alle regionalen Gesetze und Verordnungen, das Verständnis von Diversität als Zielgruppe für inklusive Schulbildung über Behinderung und Lernschwierigkeiten hinaus aus. Darüber hinaus ist die Ratifizierung der UN-CRPD für Spanien nur ein logischer Schritt in der Gesetzgebung, die bereits mit dem LOE im Sinne des Art. 24 der UN-CRPD an die Diversität der Schüler*innen angepasst wurde. Dennoch werden die Inhalte in die nationale und regionale Gesetzgebung mit aufgenommen, im Gegensatz zu Deutschland und Berlin, wo zwar der Nationale Aktionsplan und der Staatenbericht auf nationaler Ebene existieren, das Berliner Inklusionskonzept jedoch keinen rechtlich verbindenden Charakter hat. Vor diesem Hintergrund stellt sich die Frage, welche konkreten Maßnahmen inklusiver Bildung auf regionaler Ebene in Andalusien und Berlin aus den rechtlichen Vorgaben bzw. Empfehlungen resultieren.

\section{Maßnahmen inklusiver Beschulung im andalusischen Bildungsgesetz}

Für das andalusische Schulsystem ist das Bildungsgesetz LEA rechtlich bindend. In Abschnitt II wird betont, dass die Bildung in Andalusien dem durchschnittlichen Niveau der am höchsten entwickelten europäischen Länder angepasst werden soll, insbesondere durch eine steigende Zahl von Abiturient*innen und die Reduzierung der Schulabbruchzahlen (Junta de Andalucía 2007: 5). Ziele des Gesetzes sind u.a. die Verbesserung der Qualität der Bildung, die Gewährleistung von Chancengleichheit aller Schüler*innen, das Erreichen schulischer Inklusion und die Bereitstellung angemessener Bildungsmaßnahmen, um den Verbleib von Schüler*innen, die Schwierigkeiten in ihrer Schullaufbahn aufweisen, zu gewährleisten (ebd.).

Im Abschnitt III, Bildungsgerechtigkeit, wird auf die Definition von Ursachen für besondere Förderung und den Umgang mit Schüler*innen mit NEAE eingegangen. Die Kategorien des nationalen Bildungsgesetzes LOE werden dabei weitgehend beibehalten, wenngleich die Begrifflichkeiten differenzierter ausformuliert werden. Die Kategorie NEE, die „,besonderen schulischen Bedürfnisse“, beinhaltet dabei die Schüler*innen, die in Berlin unter Schüler*innen mit sonderpädagogischem Förderbedarf gefasst sind: NEE umfasst auf nationaler Ebene „Behinderung und schwerwiegende Verhaltensstörung“ (Ecoem 2007) und auf Ebene der Autonomen Region Andalusien konkretisiert „Beeinträchtigungen der Mobilität, schwerwiegende Verhaltensstörungen, allgemeine Entwicklungsstörungen, Trisomie 21, auditive Behinderung, visuelle Behinderung und Taubblindheit, chronische Erkrankungen und geistige Behinderung" (Consejería de Educación o.J.: 1). 
Durch dieses Bildungsgesetz LEA von 2007 sowie durch die Verordnung zur Berücksichtigung von Diversität von 2008 (Consejerìa de Educación 2008) und die aktuellen Vorgaben der andalusischen Bildungsverwaltung von 2017 (ebd. 2017) wird auf Ebene der Region bereits sehr konkret rechtlich vorgegeben, wie die Diversität der Schüler*innen im bereits genannten Sinne auf Ebene der Organisation Schule sowie auf Unterrichtsebene umgesetzt werden soll. Alle Maßnahmen sind dabei vorzugsweise im Regelunterricht durchzuführen. Wenn zeitliche oder räumliche Abweichungen notwendig sind, sind diese ohne Diskriminierung oder Exklusion zu gewährleisten (Junta de Andalucía 2007, Art. 4.4). In Artikel 48 werden Strategien und Maßnahmen für die Berücksichtigung von Diversität der Schüler*innen aufgelistet, die durch die Autonome Verwaltung Andalusiens reguliert und finanziert werden sollen. Grundsätzlich sind die Schulen frei bzgl. der Auswahl notwendiger Maßnahmen, z.B. der Klassenorganisation und des Unterrichtsmaterials, um sich an die Diversität ihrer Schüler*innen und die Förderung der Geschlechtergleichheit flexibel anzupassen - jedoch ohne bestimmte Gruppen zu diskriminieren (Art. 48.1). Artikel 113.5 legt fest, dass die Beschulung von Schüler*innen mit NEAE nach den „Prinzipien der Normalisierung, der schulischen und sozialen Inklusion, der Flexibilisierung, der Personalisierung des Unterrichts und der interadministrativen Koordination" (ebd.) zu erfolgen hat. Auf die Gruppe der gehörlosen/taubstummen Schüler*innen geht Artikel 113.8 ein: Diese sollen bevorzugt in Schulen beschult werden, die Gebärdensprachdolmetscher*innen oder eine andere spezielle Art der technischen Ausstattung für diese Schüler*innen zur Verfügung stellen. Außerdem weist Artikel 113.9 darauf hin, dass für Schulbezirke, die wirtschaftlich, sozial oder kulturell benachteiligt sind, spezielle Programme der Kompensation dieser Benachteiligungen vorgehalten werden (ebd.).

Castaño (2009) stellt für das LEA bzgl. der Berücksichtigung von Diversität der Schüler*innen im Schulsystem zwei Aspekte besonders heraus:

1. Es legt die Weiterbildung der Lehrkräfte bzgl. der Handlungskompetenzen für den Umgang mit Diversität fest (Artikel 115) und

2. formuliert deutlich, dass die Beschulung der Schüler*innen mit NEAE Aufgabe aller Lehrkräfte ist. Diese soll in Kooperation mit Unterstützungslehrkräften, dem Beratungsfachbereich innerhalb der Sekundarschulen und weiteren spezialisierten Fachkräften der Abteilung für Bildungsberatung in Grundschulen im Schulbezirk stattfinden (Junta de Andalucía 2007: Artikel 116, Absatz 1-3, Art. 138.3, 27).

Diese multiprofessionell angelegten Teams, die seit dem Bildungsgesetz LODE 1985 bereits regulativ verankert sind, fungieren auf organisationaler Ebene der Schule als die Unterstützungsstruktur auf Mikroebene. Ihnen kommen diverse Aufgaben zu, um auf die Diversität der Schüler*innen eingehen zu können, wie die Beratung der Klassenlehrkräfte, die Prävention und Früherkennung von Schüler*innen mit NEAE, die Unterstützung der curricularen Anpassungen der Lehrpläne für einzelne Schüler*innen sowie Koordination der Unterstützungsmaßnahmen auf Schul- und Unterrichtsebene. Zudem sind die Schulen dazu verpflichtet, ihre Maßnahmen zur Berücksichtigung von Diversität der Schüler*innen im Schulkonzept festzulegen (ebd.: Artikel 127).

Die Programme und Maßnahmen, die auf struktureller Ebene des Unterrichts laut LEA möglich sind, sind vorgegeben und werden in den Instruktionen zur Berücksichtigung von Diversität der Bildungsverwaltung konkret formuliert (ebd.: Artikel 48.1). Als didaktische Methoden, die für inklusive Beschulung bevorzugt eingesetzt werden sollen, werden insbesondere Projektarbeit und kooperatives Lernen genannt und im Detail ausgeführt. Darüber hinaus werden organisatorische Möglichkeiten bzgl. räumlicher und zeitlicher Anpassung an 
die Schüler*innen sowie diverse Formen der Evaluation von Schüler*innenleistungen genannt, um individuelle Bedürfnisse der Schüler*innen, insbesondere auf Ebene des Unterrichts,-berücksichtigen zu können (ebd.).

Neben diesen allgemeinen und spezifischen Möglichkeiten zur Berücksichtigung von Diversität der Schüler*innen, um Chancengleichheit zu gewährleisten, existieren gemäß Kapitel III des Gesetzes zur Berücksichtigung der Diversität in Andalusien von 2008 diverse Programme, um darüberhinausgehend als Organisation auf die Diversität eingehen zu können. Diese drei Programme können jeweils bei unterschiedlichen Graden besonderer Bedürfnisse umgesetzt werden (Consejería de Educación 2008). So gibt es Unterstützungsprogramme für Schüler*innen in der Grundschule oder im ersten Jahr der Sekundarschule, die Schwierigkeiten in den Kernfächern Spanisch und Literatur, erste Fremdsprache und Mathematik aufweisen und deren Versetzung in die nächste Klasse ggf. gefährdet ist. Mit dem LOGSE wurde außerdem das Basiscurriculum für alle sowie geringe und signifikante Lehrplananpassungen eingeführt (Muntaner/Rosselló 1999; Consejería de Educación 2008).

Die Diversifizierung des Lehrplans, als maximale Form der Lehrplananpassung stellt eine Maßnahme ab dem dritten, bzw. seit dem LOMCE (2013) ab dem zweiten Schuljahr, der Sekundarschule dar. Sie beinhaltet eine ,adäquate Organisation der Inhalte, praktische Aktivitäten und andere Fächer des Lehrplans sowie spezifische Methoden, um die Basisziele und -kompetenzen der Schulstufe und den Abschluss der Sekundarschule zu erreichen“ (ebd.). Die Dauer der Maßnahme beträgt in der Regel ein Jahr. Wenn jedoch nach dem Schuljahr deutlich ist, dass der oder die Schüler*in den Sekundarschulabschluss nicht erlangen wird, kann die Maßnahme für ein weiteres Jahr verlängert werden (ebd.).

Dieser Abschnitt zu Andalusien zeigt sehr deutlich, wie differenziert die Unterstützungsprogramme für unterschiedliche Bedarfe auf Seiten der Schüler*innen auf rechtlicher Ebene bereits festgelegt sind. Die Autonomen Regionen haben bei der Interpretation der nationalen Vorgaben $\mathrm{zu}$ inklusiver Bildung viel Spielraum und gleichzeitig eröffnen diese Vorgaben auch jeder Einzelschule Möglichkeiten, zwischen den Maßnahmen - je nach Bedarf vor Ort einzelne auszuwählen und ihre Ressourcen hierfür einzusetzen. Welche Maßnahmen das Berliner Gesamtkonzept „Inklusive Schule“ vorsieht, das rechtlich nicht denselben Grad der Verbindlichkeit aufweist wie das andalusische Bildungsgesetz, ist Gegenstand der Analyse im nächsten Abschnitt.

\section{Maßnahmen inklusiver Beschulung im Berliner Gesamtkonzept „Inklusive Schule“}

Die Verordnung über die sonderpädagogische Förderung (Sonderpädagogikverordnung; SopädVO) in Berlin von 2005 (letzte Änderung vom 20.09.2019) legt die Rahmenbedingungen für inklusive Bildung fest. Rahmenbedingungen in der Grundschule sind hier:

- $\quad$ angemessene „räumliche, sächliche und personelle Ausstattung muss gewährleistet sein“ (SopädVO 2005: §19, Abs.1),

- die sonderpädagogische Förderung soll von Lehrkräften mit sonderpädagogischer Qualifikation durchgeführt werden,

- $\quad$ max. 3-5 Kinder mit Förderbedarf pro Klasse (ebd.: §19, Abs. 3\&6). 
Für die Sekundarstufe I und die gymnasiale Oberstufe liegt die max. Anzahl von Schüler*innen mit sonderpädagogischem Förderbedarf bei vier, wobei $§ 20$ Absatz 2 die Möglichkeit geringfügiger Abweichungen nach oben oder unten festlegt (ebd.: §19, Abs. 3\&6).

Diese Vorgaben der Sonderpädagogikverordnung bilden den Rahmen inklusiver Bildung in Berlin, da der gemeinsame Unterricht rechtlich ausschließlich an Schüler*innen mit sonderpädagogischem Förderbedarf adressiert ist, welcher in Berlin die Förderschwerpunkte Sehen, Hören und Kommunikation, Körperliche und motorische Entwicklung, Sprache, Lernen, Geistige Entwicklung, Emotionale und soziale Entwicklung sowie Autismus umfasst (ebd.: $\S 7-\S 14)$. Im Berliner Schulgesetz von 2004 finden sich darüber hinaus noch „Kranke Schülerinnen und Schüler“ unter den „Grundsätzen Sonderpädagogischer Förderung“ (Berliner Schulgesetz 2004: Abschnitt V, §36). In Absatz 2 wird dem gemeinsamen Unterricht als Ort sonderpädagogischer Förderung Vorrang vor Unterricht an Schulen mit sonderpädagogischem Förderschwerpunkt gewährt (ebd.). Gleichzeitig werden gesonderte Schüler*innengruppen legitimiert, wenn dies ,pädagogisch“ notwendig erscheint und zieldifferenter Unterricht für Schüler*innen mit dem Förderschwerpunkt „Geistige Entwicklung“ umgesetzt. Schüler*innen mit Förderschwerpunkt dürfen von allgemeinen Schulen nur dann abgewiesen werden, ,wenn für eine angemessene Förderung die personellen, sächlichen und organisatorischen Möglichkeiten nicht vorhanden sind“ (ebd.: Abschnitt V, §37, Absatz 3\&4).

Das Berliner Gesamtkonzept „Inklusive Schule“ aus dem Jahr 2011 verfolgt das Ziel der Erhöhung des Anteils der inklusiven Beschulung von Schüler*innen mit sonderpädagogischem Förderbedarf in den Grund- und weiterführenden Schulen, neben der Reduzierung der Anzahl an Förderzentren und dem Absenken der Förderquote auf 6,5\% (Abgeordnetenhaus Berlin 2011).

Folgende drei Maßnahmenbündel sollen inklusive Bildung gewährleisten:

1. Die Einrichtung „Inklusiver Schwerpunktschulen“ in den einzelnen Bezirken Berlins. Diese sind für die Förderschwerpunkte „körperliche und motorische Entwicklung, Sehen, Hören und Kommunikation, Geistige Entwicklung (und) Autismus"vorgesehen, um laut Senatsverwaltung die inklusive Beschulung von Schüler*innen mit schweren Behinderungen zu gewährleisten.

2. Die Einführung einer zentralen Diagnostik. Diese soll zur Erhöhung von Vergleichbarkeit und Transparenz bzgl. des Diagnostikprozesses führen und damit zu einer Vereinheitlichung und zentralen Steuerung der Diagnose- und Feststellungsverfahren. Hintergrund sei die große Differenz der Förderquoten in den einzelnen Berliner Bezirken, die durch eine zentrale Koordinierungsstelle und die personelle Trennung der Aufgaben von Feststellung des sonderpädagogischen Förderbedarfs und die der Förderung verringert werden soll (ebd.).

3. Die Einrichtung von regionsübergreifenden „Beratungs- und Unterstützungszentren für die Bereiche Hören/Gehörlose, Sehen/Blinde" (BUZ) (ebd.: 71). Diese sollen an bereits bestehende Förderzentren angegliedert werden und sind verantwortlich für „die fachspezifische Diagnostik, Beratung und Unterstützung der Eltern“ (ebd.) sowie für die Gewährleistung der sonderpädagogischen Förderung und spezifischen Fortbildungen.

Darüber hinaus formuliert das Berliner Gesamtkonzept weitere Maßnahmen wie die Veränderung der Lehrkräftebildung, die seit dem Jahr 2015 an den Berliner Universitäten umgesetzt wird. Als weiteren Punkt zur Implementierung inklusiver Bildung in Berlin wird die Einführung ,integrierter Sekundarschulen“ genannt, die seit dem Schuljahr 2010/11 neben den Gymnasien die Sekundarstufe II im Berliner Schulsystem bilden. Damit soll eine „Verbesserung der individuellen Förderung“ sowie „der Abbau des starken Zusammenhangs 
zwischen sozialer Herkunft sowie Migrationshintergrund und schulischem Erfolg" (Abgeordnetenhaus Berlin 2011: 67) erreicht werden. Diverse Schulversuche in Hinblick auf die drei genannten Schwerpunkte inklusiver Bildung in Berlin sollten dann ab Schuljahr 2009/10 zu der „Erprobung der Umsetzung des Inklusionskonzepts“ (ebd.: 76) dienen.

Das Berliner Gesamtkonzept „Inklusive Schule“ stieß nach seiner Veröffentlichung im Jahre 2011 auf scharfe Kritik von diversen Gremienvertretungen und Verbänden. Im Anschluss daran wurde 2012 der Beirat „Inklusive Schule Berlin“ gegründet, welcher auf Grundlage des Gesamtkonzepts und unter Hinzuziehung der Kritikpunkte Empfehlungen für die Überarbeitung formulierte (Lange 2017). Diesem Beirat gehörten 20 Mitglieder aus Bezirksvertretungen, Vertreter*innen aus Wissenschaft, Gremien und Schulen an. Die Empfehlungen beinhalteten u.a. die Streichung des Haushaltsvorbehalts im Schulgesetz, die Implementierung eines Zentrums für Inklusion in jeder Schule mit der Aufgabe der Koordination der Schulentwicklung sowie eine ,verlässliche Grundausstattung an sonderpädagogischen Ressourcen für die Förderschwerpunkte Lernen und Emotionale und soziale Entwicklung“ (ebd.: 11), die Abschaffung der Feststellungsdiagnostik der Förderschwerpunkte Lernen, Emotionale und soziale Entwicklung sowie Sprache (LES) sowie die Bereitstellung zusätzlicher Personalressourcen (Senatsverwaltung für Bildung, Jugend und Familie 2020a). Diese Empfehlungen wurden dann im Jahr 2013 der Senatsverwaltung zur Überprüfung der Umsetzbarkeit vorgelegt. Das Ergebnis der Überarbeitung des Gesamtkonzepts auf Grundlage der Empfehlungen des Beirats waren die Eckpunkte „Auf dem Weg zur Inklusiven Schule“4, die von einer Projektgruppe Inklusion in der Senatsverwaltung Ende 2014 formuliert wurden. Um den Umsetzungsprozess weiter zu begleiten, wurde das Nachfolgeorgan des 2012 eingesetzten Beirats, der „Fachbeirat Inklusion“, eingesetzt, der die Eckpunkte hinsichtlich der Umsetzbarkeit überarbeitet (ebd.; Lange 2017).

Obgleich bis heute keine offiziell überarbeitete Version des Berliner Gesamtkonzepts „Inklusive Schule“ existiert, wurden bereits einige Inhalte der drei formulierten Eckpfeiler inklusiver Bildung - 1. Schwerpunktschulen, 2. Zentrale Diagnostik und 3. Beratungs- und Unterstützungszentren - sowie einige Punkte der Empfehlungen des Beirats in der Schulpraxis umgesetzt. So wurde ein „Rahmenkonzept Inklusive Schwerpunktschule“ und ein „Rahmenkonzept für Beratungs- und Unterstützungszentren“ erarbeitet (Senatsverwaltung für Bildung, Jugend und Wissenschaft 2014; 2015). Nach einer Pilotphase im Schuljahr 2013/14 und einem weiteren Ausbau der letztgenannten Zentren erfolgte die Umbenennung der aktuell 13 Zentren in „Schulpsychologische und inklusionspädagogische Beratungs- und Unterstützungszentren“" (SIBUZ) (Senatsverwaltung für Bildung, Jugend und Familie 2019).

Die „Inklusiven Schwerpunktschulen“ konnten nicht in der geplanten Geschwindigkeit ausgebaut werden, sodass das Ziel von jährlich sechs hinzukommenden Schwerpunktschulen nicht erreicht werden konnte. Bis zum Schuljahr 2019/20 gibt es 19 dieser Schulen, 36 sollen es insgesamt bis zum Schuljahr 2023/24 werden (ebd.: 6). Die Förderschwerpunkte Lernen, Emotionale und soziale Entwicklung und Sprache (LES) werden in Berlin an allen Schulen und potentiell auch an Schwerpunktschulen unterrichtet (ebd.).

Für die Unterstützung der Schüler*innen mit den Förderschwerpunkten LES wurde im Schuljahr 2017/18 auf Empfehlung des Beirats die „Verlässliche Grundausstattung“ eingeführt und seitdem schrittweise innerhalb von sieben Schuljahren ausgebaut (Senatsverwaltung für Bildung, Jugend und Familie 2020b). Aktuell im Schuljahr 2019/20 ist sie bis Jahrgangsstufe 5 an allen Grund- und Gemeinschaftsschulen angekommen (Senatsverwaltung für

4 https://www.berlin.de/sen/bildung/schule/inklusion/fachinfo/eckpunkte-inklusion-stand-10-11-14.pdf

[Zugriff: 08.01.2020] 
Bildung, Jugend und Familie 2019). Diese Grundausstattung der Schulen wird anhand der „,von Zuzahlung zu den Lernmitteln befreiten Schülerinnen und Schülern (Imb-Quote)“ berechnet, welche als ,ein relativ zuverlässiger Indikator für die sozio-ökonomischen Bedingungen, unter denen die Schule arbeitet" gilt (Dobe et al. 2017: 1). Hinzugezogen werden neben dieser Quote die durchschnittliche Förderquote für die sonderpädagogischen Förderschwerpunkte LES sowie der Umgang an sonderpädagogischer Förderung der Einzelschule. Hierdurch soll eine größere Verteilungsgerechtigkeit erreicht werden (ebd.).

Die ,,verlässliche Grundausstattung“ zielt laut Senatsverwaltung zudem auf die sonderpädagogische Förderung innerhalb des Unterrichts sowie in temporären Lerngruppen, auf sonderpädagogische Diagnostik, Präventionsmaßnahmen und ggf. weitere Maßnahmen (ebd.: 3). Die Grundausstattung ist damit ein Instrument für die Gewährleistung sonderpädagogischer Unterstützung für Schüler*innen mit den Förderschwerpunkten LES durch unterschiedliche Maßnahmen.

Seit dem Schuljahr 2017/18 gibt es für Berlin und Brandenburg einen gemeinsamen Rahmenlehrplan, der die Inhalte und Ziele in den einzelnen Fächern festlegt und laut Senatsverwaltung besser auf unterschiedliche Lerngeschwindigkeiten eingeht. So gibt es für die Jahrgangsstufe 1-10 einen Rahmenlehrplan, welcher die „Grundsätze der Bildung und Erziehung“ festlegt, auf ,überfachliche Kompetenzentwicklung mit den Schwerpunkten auf der Sprach- und Medienbildung“ eingeht und schließlich die ,angestrebten Kompetenzen sowie Themen und Inhalte" der einzelnen Fächer definiert (Senatsverwaltung für Bildung, Jugend und Familie 2020c: o.S.). Zusätzlich werden Schüler*innen mit dem sonderpädagogischen Förderschwerpunkt „Geistige Entwicklung“ zieldifferent nach dem „Rahmenlehrplan sonderpädagogischer Förderbedarf" unterrichtet (ebd.).

Die Inklusiven Schwerpunktschulen, die zentrale Diagnostik, die Beratungs- und Unterstützungszentren als strukturelle Unterstützung aller beteiligten Akteur*innen und letztlich auch die verlässliche Grundausstattung sowie der gemeinsame Rahmenlehrplan haben das Ziel einer flächendeckenden inklusiven Schulbildung in Berlin. Die Analyse zeigte jedoch auch, dass für den Förderschwerpunkt „Geistige Entwicklung“weiterhin Ausnahmen gelten, der Haushaltsvorbehalt auch weiter besteht und die Vorgaben der UN-CRPD noch nicht in die Berliner Schulgesetzgebung eingegangen sind. Außerdem bleiben die Gymnasien neben den Integrierten Sekundarschulen bestehen, was einer Schule für alle entgegensteht, ebenso wie die erneuten Schulversuche zur Überprüfung der Umsetzung des Berliner Inklusionskonzepts ab 2010, anstatt einer rechtlichen Verpflichtung für alle Schulen, Inklusion umzusetzen.

\section{Komparative Analyse: Inklusive Bildung in Andalusien und Berlin}

Die Darstellung der Beschulungsformen und Maßnahmen zur Berücksichtigung von Diversität in Andalusien und jene der inklusiven Schulbildung in Berlin rücken nun im Schritt der komparativen Analyse die Ausgangsfrage dieses Artikels in den Fokus: Welche Gemeinsamkeiten und Unterschiede der politischen Vorgaben hinsichtlich organisationaler Maßnahmen zur Umsetzung inklusiver Schulbildung lassen sich für diese beiden Länder föderaler Staaten (die Autonome Region Andalusien und das Land Berlin) feststellen? 
Tabelle 1: Zusammenfassende komparative Darstellung: Rechtliche Verortung und Beschulungsformen inklusiver Bildung in Andalusien und Berlin

\begin{tabular}{|c|c|c|}
\hline & Andalusien & Berlin \\
\hline \multirow{4}{*}{$\begin{array}{l}\text { Rechtliche } \\
\text { Verortung }\end{array}$} & International: UN-CRPD & International: UN-CRPD \\
\hline & $\begin{array}{l}\text { National: Spanisches Bildungsgesetz } \\
\text { LOMCE (2013) }\end{array}$ & Empfehlungen der KMK \\
\hline & Regionales Bildungsgesetz (Andalusien) & $\begin{array}{l}\text { Berliner Schulgesetz (2004) } \\
\text { Sonderpädagogikverordnung (2012) }\end{array}$ \\
\hline & $\begin{array}{l}\text { Verordnung auf regionaler Ebene } \\
\text { (Andalusien) }\end{array}$ & $\begin{array}{l}\text { Berliner Inklusionskonzept/ } \\
\text { Inklusionsbeirat }\end{array}$ \\
\hline \multirow{6}{*}{$\begin{array}{l}\text { Beschulungs- } \\
\text { formen }\end{array}$} & Regelschulen & Gemeinsamer Unterricht \\
\hline & $\begin{array}{l}\text { Diversifizierung: Unterricht hauptsächlich } \\
\text { Kleingruppenunterricht ab dem 2. Schuljahr } \\
\text { der Sekundarschule }\end{array}$ & $\begin{array}{l}\text { Temporäre Lerngruppen in } \\
\text { Regelschulen }\end{array}$ \\
\hline & Schwerpunktschulen in Städten & Inklusive Schwerpunktschulen \\
\hline & $\begin{array}{l}\text { Bevorzugte Beschulung von } \\
\text { gehörlosen/taubstummen Schüler*innen } \\
\text { in Schulen mit Gebärdensprach- } \\
\text { dolmetscher*innen oder spezieller } \\
\text { technischer Ausstattung }\end{array}$ & \\
\hline & Förderschulen & Förderschulen \\
\hline & $\begin{array}{l}\text { Eine Sekundarschule für alle, Trennung } \\
\text { erst nach } 10 . \text { Klasse }\end{array}$ & $\begin{array}{l}\text { Integrierte Sekundarschulen, neben } \\
\text { anderen Schulformen }\end{array}$ \\
\hline Einschränkung & $\begin{array}{l}\text { Zeitliche oder räumliche Abweichungen zu } \\
\text { inklusiver Beschulung - segregierte } \\
\text { Beschulung ohne Diskriminierung oder } \\
\text { Exklusion }\end{array}$ & $\begin{array}{l}\text { Fehlende Ressourcen, um } \\
\text { Schüler*innen angemessen zu } \\
\text { beschulen }\end{array}$ \\
\hline
\end{tabular}

Quellen: Junta de Andalucía 2007; Consejería de Educación 2008; Abgeordnetenhaus Berlin 2011

In Bezug auf die Beschulungsformen hat Andalusien - ausgehend von den Stufen der Integration von 1978 auf nationaler Ebene - ab 1986 bereits vier verschiedene Formen der Integration festgelegt und 1983 multiprofessionelle Teams etabliert.

Die Beschulungsformen in Berlin (s. Tabelle 1) sehen gemeinsamen Unterricht, temporäre Lerngruppen in Regelschulen, die Beschulung in inklusiven Schwerpunktschulen, genauso wie in Förderschulen und integrierten Sekundarschulen vor. In Andalusien sind diese Formen differenziert rechtlich und durch vorgegebene Möglichkeiten der Beschulung festgehalten: Diversifizierung als Möglichkeit der Beschulung in separaten Lerngruppen der Sekundarschule oder auch die Tatsache, dass es in Andalusien nur eine Sekundarschule für alle bis zur 10 Klasse gibt, sind die hauptsächlichen Unterschiede. Gemeinsam sind die für Berlin beschriebenen Beschulungsformen, wobei die genannte Segregations- und Separationsrate in Förderschulen sehr unterschiedlich ist, wie bereits in der Einleitung ausgeführt.

Die Einschränkung inklusiver Beschulung liegt in Andalusien in der Tatsache begründet, dass es insbesondere für Schüler*innen mit Schwerst-Mehrfachbehinderung weiterhin segregierte Schulen gibt. Im Vergleich hält in Berlin der Haushaltsvorbehalt bzgl. fehlender personeller und sächlicher Ressourcen die Legitimation segregierter Beschulung aufrecht. In Spanien führte dies bereits zu einer ersten Klage und intensiver Forschung zur Verletzung 
der UN-CRPD. In Deutschland, mit einer sehr viel höheren Segregationsquote, merkte der UN-Ausschuss zumindest die Aufrechterhaltung der Förderschulen kritisch an.

Die Unterstützungsstrukturen für die Berücksichtigung von Diversität bzw. inklusive Bildung sehen in Andalusien pauschale Mittel für die Schulen vor, die sie entsprechend ihrer Bedarfe einsetzen können, in Berlin ist die verlässliche Grundausstattung an die sonderpädagogischen Förderschwerpunkt LES gebunden (siehe Tabelle 2). In beiden Regionen gibt es finanzielle Unterstützung zur Kompensation sozialer Ungleichheiten, die organisationalen Strukturen sind jedoch in Andalusien auf den verschiedenen Ebenen sehr viel komplexer und differenzierter aufgebaut. Die SIBUZ in Berlin sind vergleichbar mit der Abteilung für (spezialisierte) Bildungsberatung auf Ebene der Provinzen - ein relevanter Unterschied sind jedoch die Strukturen auf Ebene der Schulen, auf der in Andalusien für jede Sekundarschule ein multiprofessionelles Team eingesetzt wird, welchem mindestens ein*e Koordinator*in für die Berücksichtigung von Diversität und die Koordination alle personellen, sachlichen und finanziellen Ressourcen in diesem Kontext obliegt. Diese Person, die fester Bestandteil des Schulteams ist, übernimmt die sonderpädagogische Diagnostik gemeinsam mit dem multiprofessionellen Team und der Klassenlehrkraft.

Die dargestellten Maßnahmen (Tabelle 2) verdeutlichen, dass zentrale Gemeinsamkeiten die Notwendigkeit der Weiterbildung der Lehrkräfte sowie die Möglichkeit zieldifferenter Unterrichtung sind - in Berlin für Schüler*innen mit dem Förderschwerpunkt „Geistige Entwicklung“ und in Andalusien, bzw. Spanien insgesamt, für Schüler*innen, die ,signifikante Lehrplananpassungen“" benötigen. Diese beinhalten eine Veränderung der Bildungs- und Lernziele und sind für Schüler*innen gedacht, die eine Klasse zum zweiten Mal wiederholen müssen und bei denen klar ist, dass sie die geforderten Mindeststandards dieser Stufe nicht erreichen werden (Consejería de Educación 2008). Die differenzierte rechtliche Verankerung stellt sich also auch hier als entscheidender Unterschied dar - so sind Nachhilfeprogramme für Schüler*innen mit Schwierigkeiten in einem Fach vorgeschrieben, ebenso wie Projektarbeit und kooperatives Lernen als förderliche Maßnahmen zur Gewährleitung inklusiver Beschulung (ebd.).

Tabelle 2: Zusammenfassende komparative Darstellung: Unterstützungsstrukturen und Maßnahmen inklusiver Schulbildung in Andalusien und Berlin

\begin{tabular}{lll}
\hline & Andalusien & Berlin \\
\hline $\begin{array}{l}\text { Unterstützungs- } \\
\text { strukturen }\end{array}$ & $\begin{array}{l}\text { Pauschale Mittel zur Berücksichtigung } \\
\text { von Diversität in Schulen }\end{array}$ & Verlässliche Grundausstattung \\
\cline { 2 - 3 } & $\begin{array}{l}\text { Programme der Kompensation der Schu- } \\
\text { len in Schulbezirken, die wirtschaftlich, } \\
\text { sozial oder kulturell benachteiligt sind }\end{array}$ & Lernmittelbefreiung \\
\cline { 2 - 3 } & $\begin{array}{l}\text { Abteilung für (spezialisierte) Bildungs- } \\
\text { beratung auf Provinzebene }\end{array}$ & $\begin{array}{l}\text { Schulpsychologische und inklusions- } \\
\text { pädagogische Beratungs- und } \\
\text { Unterstützungszentren (SIBUZ) }\end{array}$ \\
\cline { 2 - 3 } & $\begin{array}{l}\text { Pro Bezirk: Team von Fachkräften für } \\
\text { Bildungsberatung an Grundschulen }\end{array}$ \\
\cline { 2 - 3 } & $\begin{array}{l}\text { Multidisziplinärer Beratungsfachbereich } \\
\text { in allen Sekundarschulen }\end{array}$ & \\
\cline { 2 - 2 } & $\begin{array}{l}\text { Koordinator*innen für die Berücksichti- } \\
\text { gung von Diversität an Sekundarschulen }\end{array}$ & Zentrale Diagnostik \\
\hline
\end{tabular}




\begin{tabular}{|c|c|c|}
\hline & Andalusien & Berlin \\
\hline \multirow[t]{14}{*}{ Maßnahmen } & Fachübergreifender Unterricht & \\
\hline & $\begin{array}{l}\text { Flexible Gruppenzusammensetzung der } \\
\text { Klassen, Bildung von Kleingruppen }\end{array}$ & \\
\hline & $\begin{array}{l}\text { Flexibler Umgang mit Unterrichts- } \\
\text { materialien }\end{array}$ & \\
\hline & $\begin{array}{l}\text { Reduzierung Schüler*innenanzahl pro } \\
\text { Klasse }\end{array}$ & \\
\hline & $\begin{array}{l}\text { Programme zur Förderung und } \\
\text { Unterstützung im Bereich Sprache und } \\
\text { Mathematik }\end{array}$ & \\
\hline & $\begin{array}{l}\text { Verpflichtende Weiterbildung für } \\
\text { Lehrkräfte }\end{array}$ & Lehrkräftebildung \\
\hline & & $\begin{array}{l}\text { Schulversuche zur } \\
\text { Erprobung des Inklusionskonzeptes }\end{array}$ \\
\hline & $\begin{array}{l}\text { Nachhilfeprogramme zum Übergang } \\
\text { Grundschule-Sekundarschule }\end{array}$ & \\
\hline & $\begin{array}{l}\text { Angebot von Wahlfächern, um an } \\
\text { Interessen der Schüler*innen ansetzen } \\
\text { zu können }\end{array}$ & \\
\hline & $\begin{array}{l}\text { Für inklusive Beschulung: v.a. } \\
\text { Projektarbeit und kooperatives Lernen }\end{array}$ & \\
\hline & Unterschiedliche Evaluationsformen & \\
\hline & Formen von Unterstützungsprogrammen: & \\
\hline & $\begin{array}{l}\text { 1. Schwierigkeiten in den Kernfächern } \\
\text { Spanisch und Literatur, erste Fremd- } \\
\text { sprache und Mathematik } \\
\text { Wiederholung der Inhalte }\end{array}$ & \\
\hline & $\begin{array}{l}\text { 2. Lehrplananpassungen, d.h. personali- } \\
\text { sierte Lehrpläne: } \\
\text { - geringe Lehrplananpassungen } \\
\text { - } \text { signifikante Lehrplananpassungen } \\
\text { - Lehrplananpassungen für Schü- } \\
\text { ler*innen mit Hochbegabung }\end{array}$ & $\begin{array}{l}\text { Zieldifferente Beschulung von } \\
\text { Schüler*innen mit Förderschwerpunkt } \\
\text { „Geistige Entwicklung“ }\end{array}$ \\
\hline
\end{tabular}

Quellen: Junta de Andalucía 2007; Consejería de Educación 2008; Abgeordnetenhaus Berlin 2011

\section{Fazit und Ausblick}

Die Beantwortung der Ausgangsfrage dieses Artikels nach den Gemeinsamkeiten und Unterschieden im Kontext der Maßnahmen für inklusive Schulbildung in Andalusien und Berlin beinhaltete die Betrachtung der historischen Entwicklung auf nationaler und regionaler Ebene, die rechtliche Verortung inklusiver Schulbildung, mögliche Beschulungsformen sowie die Unterstützungsstrukturen für alle beteiligten Akteur*innen.

Die konkreten Maßnahmen zur Berücksichtigung von Diversität und der Gewährleistung von Bildungsgerechtigkeit durch inklusive Bildung knüpfen in Andalusien an die nationalen 
Vorgaben dazu an. Diese haben sich historisch seit der aufkommenden Kritik an der Diktatur durch die Bildungsexpansion, die wachsende Bedeutung von Bildung, die internationale Öffnung und schließlich durch die Demokratisierung des Landes immer weiter ausdifferenziert ebenso wie das Verständnis von Beeinträchtigung und der damit verbundenen Förderung durch multiprofessionelle Teams in jeder Schule. Die Ausdifferenzierung und der Ausbau des sonderpädagogischen Fördersystems in Deutschland seit den 1960er Jahren, die auch von Powell (2009) im Vergleich zu den USA festgestellt wurden, und gleichzeitig das Thema Inclusive Education auf der internationalen Agenda global agierender Organisationen wie z.B. der EU, der UN oder der UNESCO, tragen in Deutschland dazu bei, dass beide Systeme parallel existieren - sowohl ein segregierendes System für bestimmte Gruppen von Schüler*innen als auch integrative und inklusive Beschulung, dies jedoch in Berlin ohne konsequent rechtliche Verankerung inklusiver Schulbildung. Im Vergleich hierzu Spanien, das mit sehr viel weniger separierter und segregierter Beschulung auskommt. Gleichzeitig sind jedoch seit dem nationalen Bildungsgesetz LOMCE von 2013 als Reaktion auf die Finanzkrise steigende Zahlen zu beobachten, sowohl was den prozentualen Anteil der Schüler*innen mit NEAE und NEE angeht, als auch hinsichtlich der Separations- und Segregationsquote, die in Spanien zusammengefasst erhoben werden (MEFP 2019; Schäfer 2019). Die Tatsache, dass jedoch weiterhin ein segregierter Beschulungsort aufrechterhalten wird, stellt eine Gemeinsamkeit zwischen beiden Ländern dar, wenn auch die Zahlen sehr unterschiedlich sind.

Die Unterschiede zwischen den Ländern geben darüber hinaus Hinweise für Entwicklungsrichtungen für beteiligte Akteur*innen der Bildungspolitik und -praxis in Deutschland und Berlin. Spanien ermöglicht an dieser Stelle mit der rechtlichen Verankerung inklusiver Schulbildung auch auf nationaler Ebene, als bindende Vorgabe für die Regionen, mit ausgebauter struktureller Unterstützung auf Ebene der Region, der Provinzen und der Schulbezirken ebenso wie mit den multiprofessionellen Teams in jeder Sekundarschule mit eine*m Koordinator*in für die Berücksichtigung der Diversität der Schüler*innen potenzielle Anregungen. Insbesondere die Rolle der Koordinator*innen in den Schulen ist positiv hervorzuheben, die auch in den Empfehlungen Nr. 5 des Inklusionsbeirats von 2013 deutlich formuliert und für Berlin empfohlen wird (Senatsverwaltung für Bildung, Jugend und Wissenschaft 2013). Zudem gibt es Initiativen in den einzelnen Berliner Bezirken, die Aktionspläne zur Umsetzung der UN-CRPD erstellt haben, wie z.B. in Tempelhof-Schöneberg (Bezirksamt Tempelhof-Schöneberg 2020).

Weitere Anregungen aus den Erfahrungen des spanischen und andalusischen Schulsystems könnten eine breitere Auffassung von Schüler*innen, die Anspruch auf spezifische Unterstützung haben, sein, bis hin zur Abkehr von der Abhängigkeit finanzieller Ressourcen von bestimmten Diagnosen sowie eine selbstverständliche Auffassung inklusiver Schulbildung auf allen Ebenen und ein einheitliches Regelschulsystem für alle Schüler*innen. Vor dem Hintergrund der Ergebnisse wird deutlich, dass die Fragen nach der Rolle von Verbänden, nach der Sonderpädagogik als Profession, der Einstellung und Bedeutung von Eltern im Kontext der Umsetzung inklusiver Bildung und eben jene in den einzelnen Schulen offen bleiben. Darüber hinaus könnte der Einbezug weiterer föderaler Staaten in die international vergleichende Analyse Aufschluss über weitere Zusammenhänge geben und in einem größeren Kontext insbesondere die Umsetzung vor Ort in den Blick nehmen. 


\section{Literatur}

Abgeordnetenhaus von Berlin (2011): Gesamtkonzept „Inklusive Schule“. Umsetzung der UNKonvention über die Rechte von Menschen mit Behinderungen. https://www.berlin.de/sen/bildung/schule/inklusion/gesamtkonzept inklusion.pdf [Zugriff: 20.02.2020].

Aunión, J. A./Álvarez, Pilar (2013): La escuela, en el disparadero. https://elpais.com/sociedad/ 2013/09/01/actualidad/1378052615_066096.html [Zugriff: 20.02.2020].

Ausschuss für die Rechte von Menschen mit Behinderungen (2015): Abschließende Bemerkungen über den ersten Staatenbericht Deutschlands. https://www.gemeinsam-einfach-machen.de/ GEM/DE/AS/UN_BRK/Staatenpruefung/Erste_Staatenpruefung/staatenpruefung_node.html [Zugriff: 20.02 .2020 ].

Baumert, Jürgen (Hrsg.) (2003): PISA 2000. Ein differenzierter Blick auf die Länder der Bundesrepublik Deutschland. Deutsches PISA-Konsortium (OECD). Opladen: Leske und Budrich.

Berliner Schulgesetz (2004): Schulgesetz für das Land Berlin. http://gesetze.berlin.de/jportal/?quelle $=$ jlink\&query $=$ SchulG $+\mathrm{BE} \& \mathrm{psml}=$ bsbeprod.psml\&max $=$ true\&aiz $=$ true $\quad$ [Zugriff: 20.02.2020].

Bezirksamt Tempelhof-Schöneberg (2020): Bezirkliches Inklusionskonzept gemäß UN-Behindertenrechtskonvention. https://www.berlin.de/ba-tempelhof-schoeneberg/politik-und-verwaltung/beauftragte/menschen-mit-behinderung/aktuelles/artikel.870996.php [Zugriff: 22.07. 2020].

Biermann, Julia/Powell, Justin J.W. (2014): Institutionelle Dimensionen inklusiver Schulbildung - Herausforderungen der UN-Behindertenrechtskonvention für Deutschland, Island und Schweden im Vergleich. In: Zeitschrift für Erziehungswissenschaft, 17, 4, S. 679-700.

BMAS (2011): Nationaler Aktionsplan der Bundesregierung. https://www.bmas.de/DE/Service/ Medien/Publikationen/a740-aktionsplan-bundesregierung.html [Zugriff: 20.02.2020].

BOE 11/01/1982: Ley Organica 6/81, de 30 de diciembre, de Estatuto de Autonomía para Andalucía. http://www.juntadeandalucia.es/educacion/portals/abaco-portlet/content/cf3281d9-96 68-4a33-8c7f-efcb8e15d1c1 [Zugriff: 20.02.2020].

Bonal, Xavier/Tarabini, Aina (2013): The Role of PISA in Shaping Hegemonic Educational Discourses, Policies and Practices. The case of Spain. In: Research in Comparative and International Education 8, 3, S. 335-341.

Brüggemann, Christian/Köpfer, Andreas/Bergmüller-Hauptmann, Claudia (Hrsg.) (2016): The Global Paradigm of Inclusive Education. Global Diffusion and Local Translations. In: ZEP Zeitschrift für international Bildungsforschung und Entwicklungspädagogik, 19, 3, S. 4-35.

Bundesregierung (2014): 20 Jahre Gleichheitsgebot im Grundgesetz. Menschen mit Behinderung nicht benachteiligen. https://www.bundesregierung.de/breg-de/aktuelles/menschen-mit-behinderung-nicht-benachteiligen-409764 [Zugriff: 20.02.2020].

Capitán Díaz, Alfonso (2002): Breve historia de la educación en España. Madrid: Alianza Editorial.

Castaño Gómez, Ana (2009): La atención a la diversidad en el marco de una escuela inclusiva. Evolución histórica. In: Berruezo Albéniz, R./ Conejero López, S. (Hrsg.): El largo camino hacia una educación inclusiva: la educación especial y sovial del siglo XIX a nuestros días: XV Coloquio de Historia de la Educación. Universidad de Navarra, S. 405-416.

Comité sobre los Derechos de las Personas con Discapacidad (2017): Informe de la investigación relacionada con España bajo el artículo 6 del Protocolo Facultativo. http://www.convenciondiscapacidad.es/2018/05/30/informe-de-la-investigacion-relacionada-con-espana-bajo-el-articulo-6-del-protocolo-facultativo/ [Zugriff: 20.02.2020]. 
Comunidad Autónoma de Andalucía (1999): Ley 9/1999 de 18 de noviembre, de Solidaridad en la Educación. https://www.boe.es/buscar/pdf/1999/BOE-A-1999-24195-consolidado.pdf [Zugriff: 22.02 .2020$]$.

Consejería de Educación (o.J.): Manual de servicios, prestaciones y recursos educativos para el alumnado von necesidades específicas de apoyo educativo, Junta de Andalucía. https://es.slideshare.net/fundacioncadah/manual-de-servicios-prestaciones-y-recursos-educativos-para-elalumnado-con [Zugriff: 20.02.2020].

Consejería de Educación (1983): DECRETO 238/1983, de 23 de noviembre, por el que se crean los Equipos de Promoción y Orientación Educativa. https://www.juntadeandalucia.es/boja/ 1983/98/2 [Zugriff: 20.02.2020].

Consejería de Educación (1986): Orden de 25 de marzo de 1986, sobre planificación de la Educación Especial y ampliación de la experimentación de la integración en la Comunidad Autónoma de Andalucía para el curso 1986/87. https://www.juntadeandalucia.es/boja/1986/32/2 [Zugriff: 20.02.2020].

Consejería de Educación (2008): ORDEN de 25 de julio de 2008, por la que se regula la atención a la diversidad del alumnado que cursa la educación básica en los centros docentes públicos de Andalucía. https://www.juntadeandalucia.es/boja/2008/167/2 [Zugriff: 20.02.2020].

Consejería de Educación y Deporte (2019): La Educación en Andalucía. Datos y cifras del curso 2017-2018. http://www.iseandalucia.es/c/document_library/get_file?uuid=3e4fa120-a56c-46 74-8544-1f5eb0ff926a\&groupId=10137 [Zugriff: 20.02.2020].

Dobe, Mario/Heuel, K.-Jürgen/Winter-Witschurke, Christiane/Dietmann, Thurid (2017): Fragen und Antworten über die verlässliche Grundausstattung im Unterricht für die sonderpädagogischen Förderschwerpunkte Lernen, Emotionale und soziale Entwicklung sowie Sprache (L-ES). https://www.berlin.de/sen/bildung/schule/foerderung/sonderpaedagogische-foerderung/ fachinfo/\#grundausstattung [Zugriff: 03.02.2020].

Dyson, Alan (2004): Inclusive Education. A Global Agenda? In: The Japanese Journal of Special Education 41, 6, S. 613-625.

Ecoem (2007): LODE (Ley Orgánica de Derecho a la Educación) y LOE (Ley Orgánica de Educación), Sevilla: Fundación Ecoem.

European Agency for Special Needs and Inclusive Education (2018): European Agency Statistics on Inclusive Education. 2016 Dataset Cross-Country Report. https://www.european-agency. org/resources/publications/european-agency-statistics-inclusive-education-2016-datasetcross-country [Zugriff: 03.02.2020].

Federcación de Trabajadores de la Enseñanza (FETE-UGT) (2013): Análisis del Proyecto de Ley Orgánica para la Mejora de la Calidad Educativa. Cabinete Técninco de FETE-UGT.

Flick, Uwe (2011): Qualitative Sozialforschung. Eine Einführung. Reinbek: Rowolht.

Hilker, Frank (1962): Vergleichende Pädagogik. Eine Einführung in ihre Geschichte, Theorie und Praxis. München: Max Hübner Verlag.

Instituto Nacional de Educación Especial (1978) Plan Nacional de Educación Especial. https://studylib.es/doc/7763355/plan-nacional-de-educación-especial.-1978 [Zugriff: 20.02.2020].

Johnson, Magdalena (2013): Schulische Inklusion in den USA - ein Lehrbeispiel für Deutschland? Eine Analyse der Vermittlung von Ansätzen der Inklusion durch die Zusammenarbeit mit einem outside change agent. Bad Heilbrunn: Klinkhardt.

Junta de Andalucía (2007): Ley 17/2007, de 10 de diciembre, de Educación de Andalucía. In: Boletín Oficial de la Junta de Andalucía (BOJA) núm. 252, Sevilla: 26.12.2007, S. 5-36.

Kiuppis, Florian/Peters, Susan (2014): Inclusive Education for All as a Special Interest within the Comparative and International Education Research Community. In: Annual Review of Comparative and International Education. Emerald Group Publishing Limited, S. 53-63. 
Köpfer, Andreas (2013): Inclusion in Canada. Analyse inklusiver Unterrichtsprozesse, Unterstützungsstrukturen und Rollen am Beispiel kanadischer Schulen in den Provinzen New Brunswick, Prince Edward Island und Québec. Bad Heilbrunn: Klinkhardt.

Köpfer, Andreas/Kotthoff, Hans-Georg/Rakhkochkine, Anatoli (Hrsg.) (2018): Inklusive Bildung/Inclusive Education. Bildung und Erziehung, 71, 4.

Lange, Valerie (2017): Inklusion in der Schule und der Beruflichen Bildung in Berlin. In: Lange, Valerie/Dobe, Mario (Hrsg.): Inklusive Bildung in Berlin. Friedrich-Ebert-Stiftung, S. 9-26.

Ley Orgánica 1/1990 de Ordenación General del Sistema Educativo (LOGSE). In: BOE núm. 238, jueves 4 octubre 1990. https://www.boe.es/eli/es/lo/1990/10/03/1/dof/spa/pdf [Zugriff: 02.02. 2020].

Ministerio de Educación, Cultura y Deporte (MECD) (2010): Objetivos de la Educación para la década 2010-2020. Plan de acción 2010-2011. http://www.mecd.gob.es/dctm/ministerio/horizontales/prensa/documentos/2010/plan-de-accion-2010-2011vdefinitivafinal.pdf?documentId=0901e72b801b3cad [Zugriff: 20.02.2020].

Ministerio de Educación, Cultura y Deporte (MECD) (2013): Proyecto de Ley Orgáncia para la Mejora de la Calidad Educativa. http:/www.mecd.gob.es/servicios-al-ciudadano-mecd/dms/ $\mathrm{mecd} /$ servicios-al-ciudadano-mecd/participacion-publica/lomce/20130517-aprobacionproyecto-de-ley.pdf [Zugriff: 20.02.2020].

Ministerio de Educación, Cultura y Deporte (MECD) (2014): LOMCE. Paso a paso. Atención a la diversidad en Educación Secundaria y Bachillerato. https://www.educacionyfp.gob.es/dam/ jcr:09a49003-ec3d-4093-af30-d715ea35c9fb/lomced-pasoapaso-secundariaybac-diversidadv51.pdf [Zugriff: 20.02.2020].

Ministerio de Educación y Formación Profesional (MEFP) (2019): Estadística de las Enseñanzas no Universitarias. Alumnado con Necesidad específica de apoyo educativo. Curso 2016-2017. http://www.educacionyfp.gob.es/dms/mecd/servicios-al-ciudadano-mecd/estadisticas/educacion/no-universitaria/alumnado/necesidades-apoyo/2016-17/Nota-resumen.pdf [Zugriff: 20.02.2020].

Ministerio de Educación y Ciencia (MEC) (1975). DECRETO 1151/1975, de 23 de mayo, por el que se crea el Instituto Nacional de Educación Especial. https://www.boe.es/boe/dias/1975/06/ 03/pdfs/A11769-11771.pdf [Zugriff: 20.02.2020].

Müller, Frank J. (2018): Interview mit Ulf Preuss-Lausitz. In: ders (Hrsg.): Blick zurück nach vorn - WegbereiterInnen der Inklusion, Band 1. Gießen: PsychosozialVerlag.

Muntaner Guasp, Joan J./Rosselló Ramón, Maria R. (1999): Las adaptaciones curriculares individualizadas. In: Sánchez Palomino, Antonio/Torres González, José A.: Educación Especial. Una perspectiva curricular, organizativa y profesional. Madrid: Ediciones Pirámide, S. 263-273.

Muth, Jakob (1986): Integration von Behinderten. Essen: DSV.

Nohlen, Dieter/Hildenbrand, Andreas (2005): Spanien: Wirtschaft - Gesellschaft - Politik. Wiesbaden: VS Verlag für Sozialwissenschaften.

Platte, Andrea (2009): Das Recht auf Bildung und das besondere Recht auf Bildung. In: Zeitschrift für Inklusion, 2. http://www.inklusion-online.net/index.php/inklusion/article/view/36/43 [Zugriff: 20.02.2020].

Planelles, Manuel (2013): En busca de alternativas a la ley Wert. https://elpais.com/ccaa/ 2013/07/04/andalucia/1372962691_875987.html [Zugriff: 20.02.2020].

Pohl, Ursula (2005): Ökosystemische Integrationspädagogik in schulischen Kontexten. Ressourcen und Perspektiven in Deutschland und Spanien. Dissertation. http://publikationen.ub.unifrankfurt.de/opus4/frontdoor/index/index/year/2006/docId/2680 [Zugriff: 20.02.2020].

Powell, Justin J.W. (2014): Comparative and International Perspectives, on Special Education. In: Florian, Lani (Hrsg.): The SAGE Handbook of Special Education. London: SAGE, S. 335-349. 
Powell, Justin J.W. (2018): Inclusive Education: Entwicklungen im internationalen Vergleich. In: Sturm, Tanja/Wagner-Willi, Monika (Hrsg.): Handbuch schulische Inklusion. Opladen: Verlag Barbara Budrich, S. 127-142.

Powell, Justin, J.W./Merz-Atalik, Kerstin et al. (2021 i.d.B.): Heterogene Lerngruppen inklusionsorientiert unterrichten. In: Köpfer, Andreas/Powell, Justin J.W./Zahnd, Raphael (Hrsg.): Handbuch Inklusion international. Opladen: Budrich, S. 357-379.

Preuss-Lausitz, Ulf (2001): Gemeinsamer Unterricht Behinderter und Nichtbehinderter. In Zeitschrift für Erziehungswissenschaft 4, 2, S. 209-224.

Sánchez Palomino, Antonio/Torres Gonzalés, José A. (2009): Educación Especial. Centros educativos y profesores ante la adversidad. Madrid: Ediciones Pirámide.

Schäfer, Lea (2019): Institutioneller Wandel und Steuerung von „Förderbedürftigkeit“ im Zuge inklusiver Bildung. Ein qualitativer Mehrebenenvergleich am Fallbeispiel von Sekundarschulen in Andalusien. Diss. Berlin: Kultur-, Sozial-, und Bildungswissenschaftlichen Fakultät der Humboldt-Universität zu Berlin.

Schäfer, Lea/Köpfer, Andreas/Aust, Robert/Powell Justin J.W./Brendel, Michelle (2018): Human Rights - Inclusion - Education. Interdisciplinary and Comparative Perspectives. $4^{\text {th }}$ Annual I.INT Network Meeting at the University of Luxembourg. In: Bildung und Erziehung 71, 4, S. 481-482.

Schäfer, Lea/Köpfer, Andreas/Aust, Robert (2016): Workshoptage Inklusion International. In: ZEP - Zeitschrift für international Bildungsforschung und Entwicklungspädagogik 19, 3, S. 37.

Sekretariat der Ständigen Konferenz der Kulturminister der Länder in der Bundesrepublik Deutschland (1994): Empfehlungen zur sonderpädagogischen Förderung in den Schule in der Bundesrepublik Deutschland. https://www.kmk.org/fileadmin/veroeffentlichungen_beschluesse/1994/1994_05_06-Empfehlung-sonderpaed-Foerderung.pdf [Zugriff: 20.02.2020].

Senatsverwaltung für Bildung, Jugend und Familie (2019): Haushaltsgesetz 2020/12 Inklusion/Inklusive Schule. 42. Sitzung des Ausschusses für Bildung, Jugend und Familie vom 08.08.2019. https://www.parlament-berlin.de/ados/18/Haupt/vorgang/h18-2485-v.pdf [Zugriff: 08.01 . 2020].

Senatsverwaltung für Bildung, Jugend und Familie (2020a): Inklusion - Fachinformation. https://www.berlin.de/sen/bildung/schule/inklusion/fachinfo/ [Zugriff: 08.01.2020].

Senatsverwaltung für Bildung, Jugend und Familie (2020b): Sonderpädagogische Förderung Fachinformation. https://www.berlin.de/sen/bildung/schule/foerderung/sonderpaedagogischefoerderung/fachinfo/\#grundausstattung [Zugriff: 08.01.2020].

Senatsverwaltung für Bildung, Jugend und Familie (2020c): Rahmenlehrpläne. https://www.berlin.de/sen/bildung/unterricht/faecher-rahmenlehrplaene/rahmenlehrplaene/ [Zugriff: 08.01. 2020].

Senatsverwaltung für Bildung, Jugend und Wissenschaft (2013): Inklusive Schule in Berlin. Empfehlungen des Beirats. https://www.berlin.de/sen/bildung/schule/inklusion/fachinfo/ [Zugriff: 03.01.2020].

Senatsverwaltung für Bildung, Jugend und Wissenschaft (2014): Rahmenkonzept für Beratungsund Unterstützungszentren im Land Berlin. https://www.berlin.de/sen/bildung/schule/inklusion/fachinfo/ [Zugriff: 08.01.2020].

Senatsverwaltung für Bildung, Jugend und Wissenschaft (2015): Rahmenkonzept für Schwerpunktschulen im inklusiven Schulsystem. https://www.berlin.de/sen/bildung/schule/inklusion/fachinfo/ [Zugriff: 08.01.2020].

UNESCO (1994): Die Salamanca Erklärung und der Aktionsrahmen zur Pädagogik für besondere Bedürfnisse. https://www.unesco.de/sites/default/files/2018-03/1994_salamanca-erklaerung.pdf [Zugriff: 20.02.2020]. 
United Nations (UN) (2019): Convention on the Rights of Persons with Disabilities (CRPD). https://www.un.org/development/desa/disabilities/convention-on-the-rights-of-persons-withdisabilities.html [Zugriff: 20.02.2020].

UN-Ausschuss zu Rechten von Menschen mit Behinderungen (2017): Informe de la investigación relacionada con España bajo el artículo 6 del Protocolo Facultativo. http://www.convenciondiscapacidad.es/2018/05/30/informe-de-la-investigacion-relacionada-con-espana-bajo-el-articulo-6-del-protocolo-facultativo/ [Zugriff: 20.01.2020].

Verordnung über die sonderpädagogische Förderung in Berlin (Sonderpädagogikverordnung SopädVO) (2005). http://gesetze.berlin.de/jportal/?quelle=jlink\&query $=$ SondP $\% \mathrm{C} 3 \% \mathrm{~A} 4 \mathrm{dV}+$ BE\&psml=bsbeprod.psml\&max=true [Zugriff: 20.02.2020].

Wolff, Frank (2019): Vom Mauerbau zum Mauerfall. 28 Jahre deutsch-deutscher Migrationsgeschichte. https://www.bpb.de/gesellschaft/migration/laenderprofile/299944/vom-mauerbauzum-mauerfall [Zugriff: 23.7.2020]. 


\title{
12 Inklusive Bildung als Institution am Beispiel Schweiz
}

\author{
Mathias Mejeh
}

\begin{abstract}
Im folgenden Beitrag wird der Versuch unternommen, den Institutionalisierungsprozess Inklusiver Bildung in der Schweiz aufzuzeigen. Im Fokus stehen governancetheoretische Überlegungen zum schweizerischen Bildungssystem, die mit verschiedenen Kernelementen des Neo-Institutionalismus verbunden werden. In einem ersten Schritt wird daher über einen kurzen historischen Abriss der heutige Stand Schulischer Integration skizziert. Im zweiten Teil werden das Säulenmodell, die Entkopplung, die Isomorphie und die Pfadabhängigkeit beschrieben und deren Relevanz für den Institutionenbegriff im Neo-Institutionalismus ausgewiesen. Im dritten Teil wird der Institutionalisierungsprozess Inklusiver Bildung in der Schweiz beschrieben, bevor im vierten Teil weitergehende Gedanken zukünftiger Analysemöglichkeiten Inklusiver Bildung in der Schweiz angestellt werden.
\end{abstract}

\section{Entwicklung der Inklusiven Bildung in der Schweiz}

Die Diskussion zur Umsetzung Schulischer Inklusion und Inklusiver Bildung sowie deren Implikationen wird seit einigen Jahren, auch in der Schweiz (s. z.B. Haeberlin et al. 2003; Kronig 2007; Mejeh 2016; Labhart 2019), vielfältig geführt. Die Komplexität und Vielschichtigkeit dieser Thematik, die allein schon durch die fundamentale Unschärfe der Begriffsbestimmung Inklusion (damit auch: Inklusive Bildung) bedingt ist, stellt in Wissenschaft und Praxis nach wie vor eine große Herausforderung dar (Werning 2014). Eine Definitionsmöglichkeit besteht darin, die Realisierung Inklusiver Bildung über schulbezogene messbare Daten festzulegen, die sich beispielsweise an den Merkmalen des Zugangs und der Anwesenheit, der Beteiligung und Teilhabe sowie der Lernleistung beziehungsweise dementsprechender Zertifizierung von Schülerinnen und Schülern beobachten lassen (Powell 2018). Die entsprechenden menschenrechtlichen Forderungen erstrecken sich freilich weit über die Schulbildung hinaus, inzwischen auf alle Bildungsstufen bis hin zum lebenslangen Lernen, darüber hinaus aber auch auf gesellschaftliche Barrierefreiheit und Teilhabe im Allgemeinen (Degener 2017). In diesem Zusammenhang wird Inklusive Bildung in diesem Text als Teil der Inklusion im Sinne einer menschenrechtlichen Forderung verstanden, die sich über die Schule hinaus auf alle Lebensbereiche von Menschen bezieht und somit als gesellschaftlicher Institutionalisierungsprozess verstanden werden kann. Um den aktuellen Stand der Umsetzung Inklusiver Bildung in der Schweiz zu verstehen, ist daher ein Blick in die Vergangenheit unerlässlich. 


\subsection{Institutionalisierung der Heilpädagogik in der Schweiz}

Zu Beginn des 19. Jahrhunderts setzt in der Schweiz, durch verschiedene gesellschaftliche, politische und wissenschaftliche Entwicklungen, ein Prozess ein, der sich heute noch auf die Ermöglichung des Bildungsangebotes für alle Menschen gemeinsam und gleichzeitig auswirkt. Erste Institutionalisierungsprozesse der Heilpädagogik lassen sich für die Schweiz im ausgehenden 18. Jahrhundert, unter anderem durch eine einsetzende Klassifikation verschiedener Menschengruppen, ausmachen. Die Verhandlung der Bildungsfähigkeit sogenannter „taubstummer“ Menschen spielt so beispielsweise in einem ideell-normativen Sinn während des gesamten 19. Jahrhunderts eine essentielle Rolle für den Institutionalisierungsprozess der schweizerischen Heilpädagogik, hat sich hier doch gezeigt, wie sich die Diffusion der Idee einer speziellen oder besonderen Pädagogik in verschiedenen Kantonen vollzogen hat (Wolfisberg 2002). Die Notwendigkeit systematischen heilpädagogischen Wissens zeigt sich in dieser Zeit darüber hinaus beispielsweise auch an der Einführung kantonaler Statistiken oder der Etablierung von Hilfsschulen sowie -klassen. Im letzten Viertel des 19. Jahrhunderts erhielt die Heilpädagogik insbesondere durch die Systematisierung heilpädagogischen Fachwissens sowie, und dies ist vor allem für die rechtliche Entwicklung der Umsetzung Inklusiver Bildung elementar, der Einführung der allgemeinen Schulpflicht im Jahre 1874 einen weiteren „Institutionalisierungsschub“. Während sich das schweizerische Bildungssystem in dieser Phase weiter stratifiziert, findet gleichzeitig ein differenzierter Ausbau heilpädagogischer Organisationen, der in der Regel privat finanziert wird, statt. Dabei ist auch zu erkennen, dass bereits im 19. Jahrhundert oftmals schulorganisatorische Gründe angebracht werden, um Schüler*innen mit besonderem Förderbedarf zu segregieren. Während sich die Heilpädagogik als Fach anfänglich noch aus den Überlegungen anderer Fachgebiete bestimmen lässt (bspw. Medizin, Recht, Psychologie, Pädagogik), wird ihre Etablierung (und damit Institutionalisierung) als eigenständiger Wissenschaftsbereich spätestens zu Beginn des 20. Jahrhunderts mit der Ernennung Heinrich Hanselmanns zum ersten Extraordinarius für Heilpädagogik an der Universität Zürich deutlich, denn nicht nur wurde damit der erste universitäre Lehrstuhl für Heilpädagogik in der Schweiz gegründet, gleichzeitig hat sich ein quantitativer Ausbau von Hilfs- und Spezialklassen vollzogen, der in anderen gesellschaftlichen Bereichen zu einer verstärkten rechtlichen und finanziellen Verankerung der Heilpädagogik insgesamt geführt hat (Wolfisberg 2002). Mit einer verwaltungstechnischen Institutionalisierung und weitergehenden Professionalisierung hat zu Beginn des 20. Jahrhunderts ein Verwissenschaftlichungs- und Modernisierungsprozess der Heilpädagogik eingesetzt, der der Situation in anderen Ländern Europas ähnlich ist.

\subsection{Beginn der Schulischen Integration}

In der zweiten Hälfte des 20. Jahrhunderts lassen sich in der Schweiz erstmalig Forderungen zur Umsetzung Inklusiver Bildung in Form der Schulischen Integration feststellen, die ab den 1970er Jahren wesentlich durch Eltern formuliert wurden. Diese Forderungen und damit verbundene erste Integrationsversuche, die auch gegen das stratifizierte Bildungssystem gerichtet waren, besaßen allerdings in erster Linie pragmatischen Charakter und sind weniger einer pädagogischen Leitidee gefolgt. So ist bei Bless (2007: 11) zu lesen: 
Gegenüber dieser Entwicklung der Ausdifferenzierung des Schulwesens und der unvermeidbar damit verbundenen Ausgrenzung von behinderten Menschen setzte sich seit Ende der 70er Jahre vermehrt eine kritische Haltung durch, welche eine bis heute andauernde fachliche Kontroverse über die Beschulung von behinderten Kindern, die sogenannte „Integrationsdiskussion“, auslöste. Die entstandene Gegenbewegung fordert die gemeinsame Schulung von behinderten und nichtbehinderten Kindern in der Regelschule [...] An dieser Stelle muss am Rande jedoch erwähnt werden, dass die in der Schweiz praktizierte Integration insbesondere aus pragmatischen Gründen entstehen konnte und demnach nicht als Folge einer ernsthaften Auseinandersetzung konzeptioneller Art mit dem Integrationsgedanken betrachtet werden kann.

In dieser Zeit ist auch ein deutlicher Rückgang von Schüler*innen in Hilfsklassen zu erkennen. So nehmen beispielsweise im Zeitraum von 1977 bis 1982 die Schüler*innenzahlen in Regelklassen um 9\% ab, während die Schüler*innenzahlen in Hilfsklassen im gleichen Zeitraum um 28\% zurückgehen (Sturny 1984; zit. n. Haeberlin et al. 2003: 31). Die Erklärungsversuche für diese Entwicklung sind nach Haeberlin et al. (2003: 31) vielfältig. So sehen sie zunehmende Legitimationsprobleme von Schüler*innen in Hilfsklassen, Eigeninteresse der entsprechenden Lehrpersonen Schüler*innen mit besonderem Förderbedarf aufgrund der Klassengröße weiterhin zu beschulen, einen Rückgang von Kindern und Jugendlichen in Gastarbeiterfamilien, der Klassengröße grundsätzlich und der Tatsache, dass es in dieser Zeit in diversen Kantonen sogenannte Versuchsklassen gab, in denen Kinder und Jugendliche mit besonderem Förderbedarf beschult wurden, als mögliche Gründe. In dieser Argumentationskette wird deutlich, dass die Integrationsbemühungen der 1970er Jahre, die die Anfänge in der Schweiz darstellen, vorwiegend auch aus schulorganisatorischen Gründen vollzogen wurden: ,[...] weil die erforderliche Schülerzahl für eine Sonderklasse nicht mehr erreicht wurde, suchte man eine neue schulorganisatorische Lösung für Lernbehinderte" (ebd.).

Wesentliche rechtliche Entwicklungen hinsichtlich integrativer Beschulung haben in der Schweiz erst später eingesetzt. Mit der Ratifizierung des Übereinkommens über die Rechte von Menschen mit Behinderungen (UN-BRK), hat sich die Schweiz im Jahre 2014 rechtlich dazu verpflichtet, allen Schüler*innen ausnahmslos die Beteiligung an Bildungsangeboten im Regelschulbereich zu ermöglichen (für einen Vergleich zu anderen deutschsprachigen Ländern siehe Biermann 2021 i.d.B. sowie Biewer 2021 i.d.B.). Einige Jahre zuvor wurde bereits auf nationalstaatlicher Ebene das Behindertengleichstellungsgesetz (BehiG, 2002) verabschiedet, durch das die Kantone rechtlich dazu verpflichtet wurden, im Rahmen der Neugestaltung des Finanzausgleichs und der Aufgabenteilung zwischen Bund und Kantonen (NFA), die 2008 in Kraft getreten ist, die Beschulung aller Schüler*innen gleichermaßen zu gewährleisten. Somit wurde die pädagogische und finanzielle Verantwortung der Beschulung von Schüler*innen mit besonderem Förderbedarf in den letzten Jahren sukzessive vom Bund auf die Kantone übertragen. Diese Änderungen haben unter anderem dazu geführt, dass in der jüngeren Vergangenheit kantonale Konzepte zur Beschulung von Kindern und Jugendlichen mit besonderem Förderbedarf entwickelt wurden, die sich grundsätzlich an einem Kaskadenmodell orientieren (beispielsweise Erziehungs-, Kultur- und Umweltschutzdepartement Graubünden 2007 [Graubünden]; Dienststelle Volksschulbildung Kanton Luzern 2012 [Luzern]; Direktion für Bildung und Kultur Zug 2008 [Zug] oder Department für Bildung und Kultur Solothurn 2013 [Solothurn]). Gleichzeitig sind auch Änderungen auf interkantonaler Ebene zu beobachten (Sonderpädagogikkonkordat), die nachhaltige Auswirkungen auf die Gemeindeebene und damit auf die Schulen haben. Es ist also zu erkennen, dass die Institutionalisierung Inklusiver Bildung in der Schweiz in eine zeitliche, organisationale, rechtliche und ideell-normative Perspektive einzuordnen ist, die sich auf verschiedenen nationalstaatlichen Ebenen vollzieht. Dabei stellt sich unter anderem die Frage, wie sich Inklusive 
Bildung als Institution bzw. in ihrem Institutionalisierungsprozess verstehen lässt? Um auf diese Frage eine Antwort zu finden, soll folgend ein analytisches Modell beschrieben werden, das verschiedene Elemente des Neo-Institutionalismus verbindet.

\section{Institutionalisierung und Neo-Institutionalismus}

Nachfolgend wird der Veränderungsprozess des schweizerischen Bildungssystems im Kontext der Umsetzung Inklusiver Bildung betrachtet. Dazu sollen governance-theoretische Überlegungen mit verschiedenen Elementen des Neo-Institutionalismus wie dem Drei-Säulen Modell (Scott 2014), den Entkopplungsmechanismen (Meyer/Rowan 1977), der Isomorphie (DiMaggio/Powell 1983) und der Pfadabhängigkeit von Organisationen (Bulmer 1998; Pierson 2000; Sydow et al. 2009) verbunden werden.

\subsection{Die Begriffe Institution und Organisation im Neo-Institutionalismus}

Um Institutionalisierungsprozesse aus neo-institutionalistischer Perspektive zu beschreiben, ist in einem ersten Schritt eine diesbezügliche Klärung des Begriffes „Institution“ notwendig. Demnach werden Organisationen nicht als losgelöste Systeme in einem größeren Gefüge betrachtet, sondern es sind gerade die interdependenten Beziehungen zwischen Organisationen und der damit verbundenen Legitimationsfrage, die in den Fokus des Interesses rücken. Traditionelle Ansätze des Institutionalismus lassen den Kontext von Organisationen weitestgehend unberücksichtigt. Im Neo-Institutionalismus wird durch „Institution“ eine Auffassung ausgedrückt, die die Analyse einer Organisation mit der Analyse gesellschaftlicher Organisationsweisen verschmelzen lässt (Bonazzi 2014), was Institutionen zur entscheidenden Einflussgröße auf die Geschehnisse einer Organisation macht (Senge 2011). Die formale Struktur von Organisationen, so das Argument, wird nun als wichtiger Teil des Institutionalisierungsprozesses gesehen, auch oder gerade weil Teile dieser formalen Struktur durch die verschiedenen Akteur*innen nicht mehr hinterfragt werden (DiMaggio/Powell 1983; 1991). Gleichzeitig wird eine Institution allerdings nicht nur durch einzelne Elemente einer Organisation konstituiert, sondern auch durch die mit der Organisation befassten Akteur*innen und deren Verhaltensweisen selber (Zucker 1977). Institutionen werden demnach durch „social patterns" (Jepperson 1991: 145) begründet und aufrechterhalten. Hierbei wird die Institution allerdings nicht durch die Handlung der Akteur*innen selber begründet, sondern vielmehr durch die Reproduktion bestimmter, in diesem Sinne dann institutionalisierter, Verhaltensund Handlungsweisen. Eine institutionalisierte Verhaltensweise ändert sich daher auch langsamer als eine nicht institutionalisierte Verhaltensweise (Zucker 1977).

Es lässt sich daraus ableiten, dass Institutionalisierung grundsätzlich sowohl als Zustand wie auch als Prozess verstanden werden kann: letzteres beschreibt Institutionalisierung als eine Form der Wirklichkeitsvermittlung, einen Ablauf also, durch den ganz bestimmte Deutungen, Handlungen, Beziehungen und Denkweisen etabliert und danach nicht mehr hinterfragt werden. Damit sind jene gesellschaftlichen Handlungen gemeint, die durch ihre Ak- 
teur*innen produziert und reproduziert werden. Institutionalisierung als Zustand bezeichnet hingegen die Tatsache, bestimmte Deutungssysteme innerhalb einer Gesellschaft als objektive und außerhalb des Individuums liegende Entitäten zu verstehen, die historisch gewachsen sind und längeren Bestand haben (Walgenbach 2019). Es wird also deutlich, dass der Begriff der Institution im Neo-Institutionalismus als Schablone verstanden werden kann, die hinter jeglicher gesellschaftlichen Organisation liegt und sie in ihrer Wesenheit (formalisierte Vorgaben bis hin zu empirischer Handlung der Akteur*innen) ausmacht. Darüber hinaus haben sich gerade, wie weiter oben angedeutet, im Neo-Institutionalismus verschiedene Blickweisen auf Gesellschaft entwickelt, die grob in makro-institutionalistische und mikro-institutionalistische Ansätze unterteilt werden können. Damit wird deutlich, dass der Begriff der Institution im Neo-Institutionalismus eine elementare Rolle spielt. Der umfassendste Versuch, den Begriff definitorisch festzulegen, stammt von Richard Scott (2014) und soll in der Folge daher kurz erläutert werden.

\subsection{Die drei Säulen einer Institution}

Richard Scott (2014) differenziert in seinem Drei-Säulen Modell Institutionen nach ihrer Funktion und schreibt ihnen eine ,handlungsgenerierende Kraft“ (Senge 2011: 84) zu. Eine Institution wird hierbei durch verschiedene Elemente bestimmt, die er in eine regulative, normative und kulturell-kognitive Säule unterteilt. Regelhafte Handlungsmuster und unbesehene Vorstellungen von Akteur*innen in Organisationen, werden somit durch den Begriff der Institution beschreibbar. Diese Mechanismen sind es, die die Beständigkeit einer Institution sichern, denn die drei Säulen werden auf einem Kontinuum zwischen bewussten und unbewussten Praktiken sowie gesetzlichen Regelungen und Strukturen, die als selbstverständlich erachtet werden (,taken for granted“), konzipiert (s. Abbildung 1).

Die erste Säule ist durch Gesetze, Vorgaben und Verordnungen (regulativ), die zweite Säule durch Normen und Werte (normativ) und die dritte Säule durch Handlungen, Deutungen, Vorstellungen und Interpretationen eines bestimmten, kulturell geprägten, Kontextes (kulturell-kognitiv) gekennzeichnet.

Die regulative Säule bezeichnet Prozesse, die das Handeln von Akteur*innen steuern sollen, beispielsweise indem durch Gesetze und Regeln bestimmte Kontrollmechanismen vollzogen werden, die an ganz konkrete Sanktionen oder Belohnungen gekoppelt sind und durch die die betroffenen Akteur*innen basierend auf einem Kosten-Nutzen-Kalkül die Möglichkeit haben, der Einhaltung der formalen Vorgaben zu entsprechen (Walgenbach 2019). Der Legitimitätsanspruch einer Organisation führt auf regulativer Ebene also zur Einhaltung formaler Vorgaben oder zumindest zu einem Verhalten, bei dem so getan wird als halte man die Vorgaben ein. 
Abbildung 1: Kontinuum der Handlungs- und Denkpraktiken in Scotts Säulenmodell

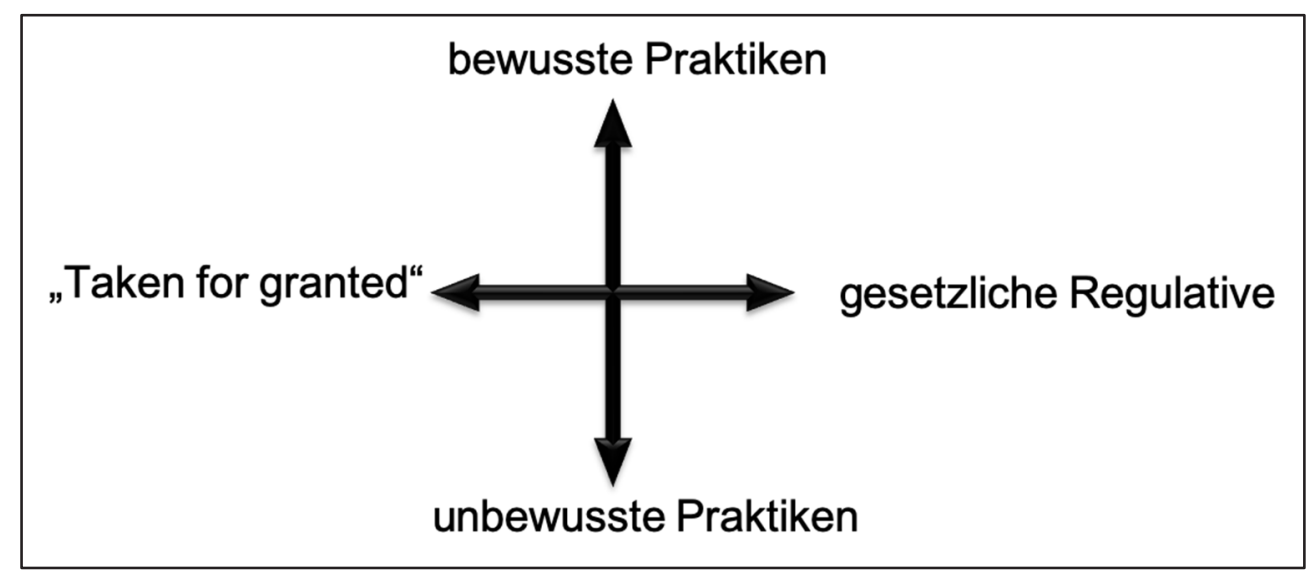

Quelle: eigenen Darstellung.

Die normative Säule beinhaltet eher vorschreibende, bewertende und verpflichtende Aspekte einer Institution (Walgenbach 2019) und trägt gleichermaßen zu ihrer Stabilisierung bei. Sie konstituiert sich über herrschende Werte sowie Normen und findet ihren konkreten Niederschlag in feststehenden Handlungsmaximen, also darüber, wie etwas sein soll. Im Gegensatz zur regulativen Säule, in der Akteur*innen einer direktiven Autorität folgen, ist hier vielmehr eine moralische Autorität handlungsleitend. Durch Normsetzungen wird dabei festgelegt, ob ein bestimmtes Verhalten oder ein Zustand als gut oder schlecht verstanden wird, sodass die Erfüllung einer Norm verfolgt wird, weil es von den betroffenen Akteur*innen erwartet wird und sie sich dazu verpflichtet fühlen. In diesem Fall ist der Legitimitätsanspruch der Organisation im Bereich der Einhaltung akzeptierter Normen angesiedelt und unterliegt weniger einem Kost-Nutzen-Kalkül (Walgenbach 2019).

Durch die kognitiv-kulturelle Säule wird beschrieben, wie Akteur*innen die Wirklichkeit erfahren. Es wird hier also ausgedrückt, wie der kulturelle Rahmen einer Organisation die kognitiven Prozesse ihrer Mitglieder (mit-)bestimmt (Walgenbach 2019). In diesem Zusammenhang wird kulturell-kognitiv allerdings nicht nur - im Sinne eines klassischen topdown Verfahrens - deterministisch verstanden, sondern beschreibt auch wie die Wirklichkeit, in Form einer Sinngebung, aktiv durch die Akteur*innen erschlossen wird. Damit rückt nicht nur das, was als objektive Voraussetzung des Handelns bezeichnet werden kann in den Fokus, sondern darüber hinaus auch kognitive Repräsentationen der Umwelt (Walgenbach 2019). Dieser Aushandlungsprozess, der an anderer Stelle als „Skript“ bezeichnet wird (Berger/Luckmann, 1966), wird in Scotts Konzeption für eine Organisation übernommen, ohne dass damit zusammenhängende Rollen und Vorgaben hinterfragt werden. 


\subsection{Erzwungene, normative und mimetische Isomorphie}

Das organisationale Feld und der damit verbundene Umweltbezug von Organisationen ist ein weiteres Kernkonzept des Neo-Institutionalismus (DiMaggio/Powell 1983). Aufgrund seines nachhaltigen Einflusses auf die Kultur und Werte einer Organisation, spielt deren gesellschaftliches Umfeld, auch in analytischer Hinsicht, eine wichtige Rolle. Diesem Gedanken folgend ist die formale Organisationsstruktur einer Organisation wesentlich durch die gesellschaftliche Umwelt bestimmt, was in der Konsequenz bedeutet, dass alle Organisationen, mehr oder weniger, in ein gemeinsames Sinnsystem eingebunden sind und Institutionalisierung somit als Prozess zu verstehen ist, der sich interorganisational vollzieht (DiMaggio 1986; DiMaggio/Powell 1983; Powell 1991; Scott 1994; Scott/Meyer 1991). In Folge der Legitimitätsbestrebungen und interdependenten Orientierungsprozesse kommt es in organisationalen Feldern dabei zu Strukturangleichungsprozessen, die auch als Isomorphie bezeichnet werden. Organisationen eines Feldes gleichen sich demnach durch drei Prozesse immer stärker aneinander an: durch Zwang, durch Imitation und durch normativen Druck.

Isomorphie durch Zwang beruht auf autoritativen Zwängen zwischen Organisationen, so beispielsweise in Form eines neuen Bildungsgesetzes, das durch einen Staat erlassen wird und in der Folge durch eine Schule umgesetzt werden muss. Zwänge können dabei formell, informell oder kulturell ausgeübt werden, d.h. dass eine Angleichung durch Zwang lediglich dann stattfindet, wenn erstens eine Abhängigkeit von Organisation A zu Organisation B besteht und zweitens implizite oder explizite Erwartungen durch die Gesellschaft an diese Organisation gestellt werden (Walgenbach 2019). Demnach ist auch hier von einem KostenNutzen-Kalkül auszugehen, nämlich insofern, dass Organisationen im Normalfall versuchen dem Zwang nachzukommen, um entweder positive Sanktionen (z.B. einen Förderpreis) zu erhalten oder negative Sanktionen (z.B. eine Strafe) zu vermeiden.

Isomorphie durch Imitation kann als organisationale „Reaktion“ auf Unsicherheit innerhalb des organisationalen Feldes verstanden werden, die Nachahmungstendenzen zur Folge hat. Dazu findet eine Orientierung an anderen (legitimierten) Organisationen statt, wobei die Grundlage für isomorphe Imitationsprozesse immer ein wahrgenommenes Problem ist, das, um das Überleben der Organisation zu sichern, gelöst werden muss. Dies führt zu einer Orientierung an ausgemacht erfolgreichen Organisationen und konsequenterweise zu einer Angleichung des organisationalen Feldes insofern, dass alternative Organisationsmodelle mit der Zeit abnehmen, da als legitim erachtete Modelle eine Vorbildfunktion einnehmen.

Normativer Druck hängt hingegen sehr stark mit dem Prozess der Professionalisierung zusammen. Dabei steht die Definition dessen was als „richtig“ erachtet wird im Zentrum und nimmt einen vorschreibenden, verpflichtenden Charakter ein, der direkt an normative Sanktionen gekoppelt ist (Becker-Ritterspach/Becker-Ritterspach 2006). Vergleicht man in diesem Zusammenhang Organisationen mit Professionen, stehen diese gleichermaßen unter permanentem Adaptions- und Wandlungsdruck. Es zeigt sich dabei einerseits, dass sich normativer Druck auch auf die Legitimierung von Professionen, beispielsweise in Form von Zertifikaten durch Ausbildungsorganisationen, auswirkt. Andererseits führt normativer Druck gleichzeitig zu grundsätzlich ähnlichen Denk- und Handlungsweisen, die dann als professionelles Handeln bezeichnet werden. In diesem Kontext entstehen auch professionelle Netzwerke, die nach DiMaggio und Powell (1991) mehr und mehr zu homogenen Akteur*innengruppen mit ähnlichen Überzeugungen, Normen und Werten führen. 


\subsection{Entkopplungsmechanismen}

Wie im vorhergehenden Kapitel beschrieben, unterliegt jede Organisation einem gewissen Legitimationsdruck, da ihr Überleben von externen Faktoren, in diesem Sinne Ressourcen, abhängig ist (Meyer/Rowan 1977). Demnach werden Organisationen in einer Gesellschaft dadurch legitimiert, dass gesetzte Anforderungen morphologisch angepasst werden. Vielfältige (An-)Forderungen werden in diesem Sinne von den entsprechenden Akteur*innen interpretiert und umgesetzt, was zur Konstruktion von Rationalitätsfassaden führt, die sich als „Mythen“ etablieren und langfristig als unreflektierte Tatsachen in der Gesellschaft institutionalisieren. Neben dem Prinzip der Legitimation stellt sich hierbei auch die Frage der Effizienz, nämlich dann, wenn innerhalb von Organisationen tatsächliche Arbeitsaktivitäten von formalen Strukturen entkoppelt werden (Weick 1976; Meyer/Rowan 1977; Hasse/Krücken 2005). Die skizzierten Prozesse können allerdings zu Konflikten führen, weshalb Organisationen zu zwei grundlegenden Strategien tendieren (Meyer/Rowan 1977; Bromley/Powell 2012).

Durch Entkopplung werden tatsächliche Aktivitäten einer Organisation von den formalen Vorgaben, die die durch die Umwelt festgelegten Ziele und Anforderungen zur Erreichung dieser Ziele beinhalten, losgelöst. Diese Strategie verfolgen Organisationen aus Effizienzgründen, da so beispielsweise auch widersprüchliche gesellschaftliche Anforderungen, die in Form institutionalisierter Regeln an eine Organisation gestellt werden, entsprochen werden kann, wenn etwa

$[\ldots]$ the relational networks involved in economic exchange and political management become extremely complex, bureaucratic structures are thought to be the most effective and rational means to standardize and control sub-units (Meyer/Rowan, 1977: 342).

Die dafür etablierten Rationalitätsfassaden verhindern die Überprüfung der Effizienz der formalen Struktur und ermöglichen es gleichzeitig, dass Probleme durch die betroffenen Akteur*innen der Organisation gelöst werden können, obwohl die dafür gewählte Vorgehensweise nichts mit der formalen Struktur selber zu tun haben muss.

Gleichzeitig besteht die Aufgabe von Organisationen darin, nach innen und außen ein vertrauensvolles Klima zu schaffen. Gesellschaftliche Zuversicht und Vertrauen (extern) in die formale Struktur einer Organisation, bei gleichzeitiger Stärkung des Verpflichtungsgrades und der Identifikation betroffener Akteur*innen (intern) in deren Aktivitätsstruktur, bilden dabei erst die Grundlage für erfolgreiche Entkopplungsprozesse.

\subsection{Organisationale Pfadabhängigkeit}

Das Konzept der Pfadabhängigkeit, das sich ursprünglich auf Technologiekonkurrenz und damit verbundener Marktselektion (Arthur 1989; David 1985) bezogen hat, wird heute vielseitig und in unterschiedlichen Disziplinen verwendet. Pfadabhängigkeit fokussiert institutionelle Persistenz in historischer Perspektive (Ebbinghaus 2009; Mahoney 2000; Pierson 2000), wobei sich eine neuere Konzeption von Sydow et al. (2009) explizit auf die Pfadabhängigkeit einer Organisation bezieht. Demnach besteht die organisationale Pfadabhängigkeit aus drei Phasen: 
Die erste Phase, die Vorformationsphase (preformation phase), ist wesentlich durch einen freien Handlungsspielraum der Organisationen gekennzeichnet, wobei die Unvorhersehbarkeit bestimmter, bewusst gewählter Optionen diese Phase genauso kennzeichnet, wie die Möglichkeit, unabsichtlich einen selbstverstärkenden Prozess auszulösen. Verfestigt sich in einer Organisation, bedingt durch diese selbstverstärkenden Prozesse, ein dominantes Handlungsmuster, wird dieser Zeitpunkt auch als „critical juncture“ bezeichnet und bedeutet gleichzeitig das Ende der Vorformationsphase.

In der zweiten Phase, auch als Formationsphase bezeichnet, hat sich ein bestimmtes Handlungsmuster in der Organisation etabliert, das zunehmend nicht mehr umkehrbar ist. Der Pfad unterschiedlicher Handlungsmöglichkeiten der Organisation wird dadurch enger, ohne jedoch ausschließlich auf eine Handlung reduziert zu sein.

Die dritte Phase, auch als Lock-In Phase bezeichnet, manifestiert sich ab dem Zeitpunkt, ab dem für die Organisation nur noch eine Handlungsmöglichkeit besteht (Lock-In). Das ausgewählte Handlungsmuster der Organisation hat sich nun etabliert und alle Handlungen sind nun auf einen Pfad fixiert und reduziert. Damit geht der Organisation nicht nur die Handlungsflexibilität verloren, sondern sie wird darüber hinaus auch ineffizient, so beispielsweise, wenn sie sich mit effizienteren Handlungsalternativen konfrontiert sieht, die dann nicht mehr adaptiert werden können.

Diese Perspektive ermöglicht eine Analyse gesellschaftlicher Gegebenheiten, die insbesondere historische Aspekte der Institutionalisierung (Mahoney 2000) fokussiert, die langanhaltende Stabilität von Institutionen erklärt und ,[...] sie als historische Hinterlassenschaften von Richtungsentscheidungen an kritischen Weggabelungen“ (Hartong/Nikolai 2016: 107) versteht.

\section{Die Institutionalisierung Inklusiver Bildung in der Schweiz}

Geht man also der Frage nach, wie sich Inklusive Bildung als Institution in der Schweiz konstituiert, lässt sich eine Verbindung verschiedener Elemente des Neo-Institutionalismus zur Klärung heranziehen. Ausgangspunkt sollen hierfür die auf globaler Ebene entwickelten überstaatlichen Agenden sein, die im Sinne einer Weltkultur (Meyer 2005) für die Forderung und Umsetzung Inklusiver Bildung längere Zeit zurückzuverfolgen sind (beispielsweise Salamanca-Erklärung 1994) und als top-down Prozesse durch verschiedene staatliche Ebenen diffundieren. Damit lässt sich hinsichtlich der Diffusion der UN-BRK eine Vorgehensweise nachzeichnen, wie sie in Abbildung 2 abgebildet ist.

Über die überstaatliche Festlegung Inklusiver Bildung in Form der Behindertenrechtskonvention (konkret für den hiesigen Kontext: Artikel 24 der UN-BRK) stellt sich die nächste Ebene (national) beispielsweise in Form der Bundesverfassung dar, vollzieht sich über die interkantonalen (Beispiel: Sonderpädagogikkonkordat) sowie kantonalen Festlegungen (Beispiel: Volksschulgesetz) und manifestiert sich in der Organisation Schule, nämlich dann, wenn es um die gemeinsame Beschulung aller Schülerinnen und Schüler in einer Klasse geht. Der Wandel eines nationalen Bildungssystems besitzt demnach nicht ausschließlich endogene Triebfedern, sondern steht auch unter exogenem Einfluss (Meyer 2005). Die Vereinten Nationen sind so als Organisation Teil einer globalen Ordnungsstruktur, die durch ihre in den Konventionen festgelegten Zielsetzungen nationale Reformprozesse auslösen können (Blanck et al. 2013: 267). Gleichzeitig ist aber auch davon auszugehen, dass sich top-down 
Prozesse nicht nur einseitig vollziehen, denn regulative Vorgaben sind immer auch ein Ausdruck kultureller und normativer Aushandlungsprozesse, die ihren Niederschlag zwischen Makroebene (Weltgesellschaft) und Mikroebene (Individuum) in Form bestimmter ,Skripte“ (Berger/Luckmann 1966) finden. Es lässt sich also festhalten, dass bildungspolitische Reformagenden, wie die der Inklusiven Bildung, somit durch verschiedene innerstaatliche Faktoren genauso konstituiert sind, wie durch internationale Entwicklungen (Blanck et al. 2013). Mit dem von DiMaggio und Powell (1983) beschriebenen Prozess der Isomorphie zieht sich hier ein Spannungsfeld auf, auf dessen einer Seite sich Veränderungsdruck und auf der anderen Seite Persistenz institutioneller Strukturen, im Mehrebenensystem eines Staates, zeigen (Powell 2016). In dieser Hinsicht kann eine Entwicklung auf überstaatlicher Ebene, wie die UN-BRK inklusive ihrer Ratifizierung, für einen Nationalstaat (und seinen entsprechenden Suborganisationen) als ,exogener Schock“ (Blanck et al. 2013: 268) verstanden werden, wobei dabei zu bedenken ist, dass ,sich die genannten Beharrungskräfte keineswegs allein aufgrund veränderter Rechtslage verflüchtigen“ (ebd.). Um diese Differenzen und die entsprechenden Konsequenzen offensichtlich zu machen, eignet sich der Vergleich verschiedener Bildungssysteme, insbesondere, so das Argument, föderaler Systeme, in denen Aushandlungsprozesse stärker auf niedriger Steuerungsebene stattfinden, da globale Normen immer auch national, regional und lokal implementiert werden (müssen).

Abbildung 2: Diffusion einer überstaatlichen Festlegung durch verschiedene staatliche Ebenen der Schweiz

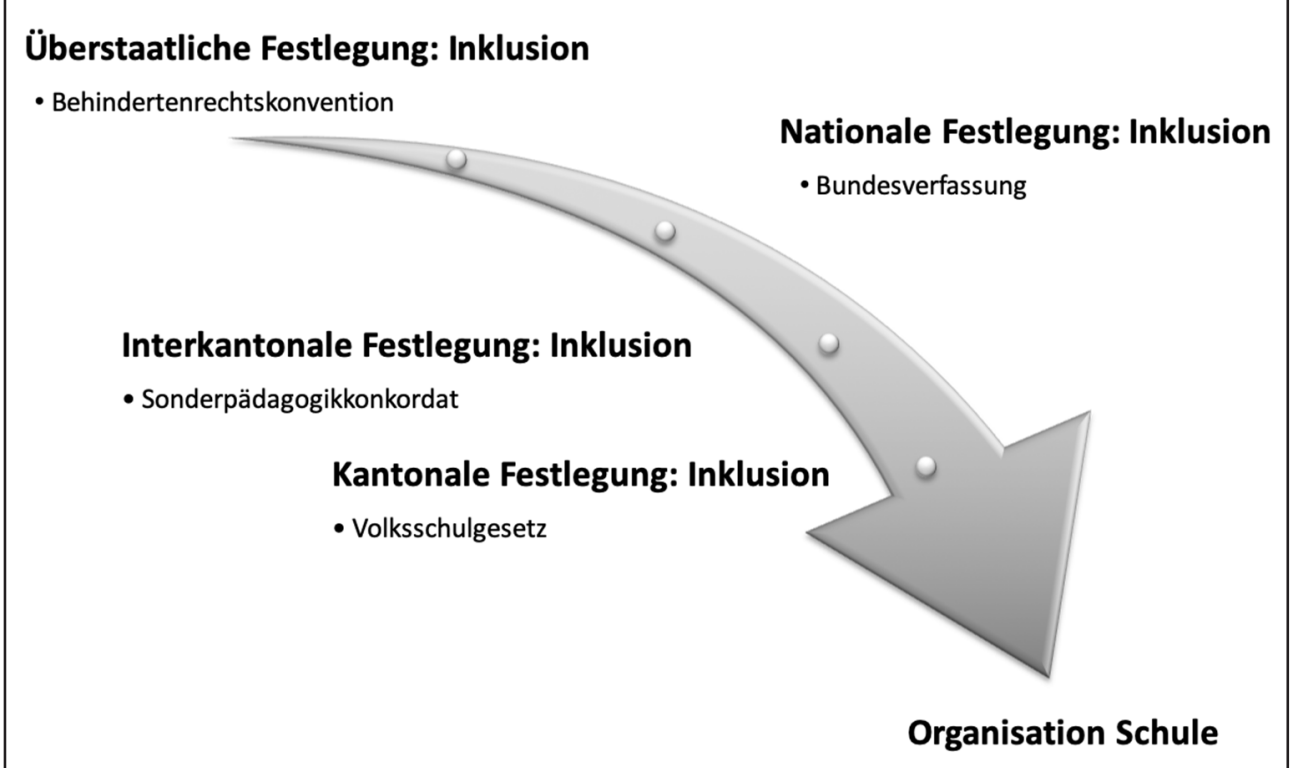

Quelle: eigene Darstellung.

In diesem Kontext stellt der kulturell stark verankerte (Bildungs-)Föderalismus in der Schweiz einen tragenden Grundwert der Gesellschaft dar. In den 26 Kantonen haben sich je eigene bildungsverwalterische Traditionen entwickelt, die sich nicht nur durch diverse bildungspolitische Strömungen und Einflüsse auszeichnen, sondern zunehmend auch durch die 
beschriebenen internationalen Entwicklungen beeinflusst werden. Es wird also eine Steuerungslogik des schweizerischen Bildungswesens erkennbar, die sich erstens durch ihre Vielfältigkeit auszeichnet und zweitens top-down genauso wie bottom-up bestimmt wird. Konsequenterweise beeinflussen (inter-)nationale Monitoringverfahren wie beispielsweise PISA, TIMMS, IGLU, CHECK P3/CHECK P6 oder Stellwerk die Entwicklung auf ähnliche Weise wie rechtliche Setzungen, beispielsweise die UN-BRK oder die UN-Kinderrechtskonvention. Daher kann von einem Mehrebenensystem ausgegangen werden, bei dem die verschiedenen Ebenen als unterschiedliche, vertikal angeordnete Akteur*innen bezeichnet werden (Maag Merki 2016). Hier spielen insbesondere intermediär agierende Akteur*innen eine wichtige Rolle, d.h. Organisationen die zwischen den Ebenen vermittelnd tätig sind (für die Schweiz in diesem Kontext beispielsweise: Schweizerische Konferenz der kantonalen Erziehungsdirektoren, Kantonale Bildungsdirektionen, Inclusion Handicap oder Schweizerisches Zentrum für Heil- und Sonderpädagogik). Variationen bestehen zwischen Bund und Kantonen, den Kantonen selbst und verschiedenen Bildungsbereichen (Volkschulbereich, Sekundarschulbereich, Tertiärer Bildungssektor), wobei die vielfältigen Einflussfaktoren berücksichtigt werden müssen, die die Governance im Bildungsbereich gerade in den Kantonen ausmachen, wie beispielsweise Größe des Kantons oder soziodemographische, politische und administrative Struktur (Maag Merki 2016). Darüber hinaus ist der schweizerische Bildungsföderalismus von vielfältigen Spannungsverhältnissen durchdrungen (Künzli/Weber 2016: 136f.). So stehen sich politische Legitimation und wissenschaftliche Begründung genauso gegenüber, wie die zunehmend zu erkennende Tatsache, dass ausgewählte Gremien und Einrichtungen legislative Funktionen übernehmen und somit ohne öffentliche Meinungsbildung oder parlamentarische Kontrolle Zuständigkeiten verwischt werden können. Gleichzeitig sind immer wiederkehrende Umsetzungsvorschläge zur Governance des Bildungssystems auszumachen, obwohl starke Disparitäten zwischen den Kantonen und Regionen liegen, während nationale und lokale Orientierungen und Bedingungen - trotz Harmonisierungsversuchen voneinander entkoppelt werden. Vor diesem Hintergrund stellt sich also die Frage, in welchem Sinne der erzeugte Zwang zu verstehen ist und was sich insbesondere für die Interpretation der Diffusion der UN-BRK als grundlegend erweist. Demnach ist zwar in den letzten 20 Jahren ein sukzessiver Abbau segregierter Beschulung im schweizerischen Bildungssystem erkennbar, aber dennoch bleibt die Segregationsquote einerseits im internationalen Vergleich vergleichsweise hoch, während andererseits nach wie vor große interkantonale Unterschiede bestehen (Mejeh/Powell 2018). Im Rahmen der Harmonisierung der obligatorischen Schule (Harmos-Konkordat) sind daher, insbesondere im Hinblick auf die zunehmende Steuerungskompetenz der Kantone (Walther-Müller/Häfeli 2005), diese interkantonalen Unterschiede erklärungsbedürftig. Denn auch in der Schweiz bildet die hierarchische Struktur regulativer Setzungen, die sich von überstaatlichen Verpflichtungen wie beispielsweise der UN-Behindertenrechtskonvention bis auf schulpraktische Ebene in Form von Schulkonzepten zieht, keine zusammenhängende Einheit. So wird beispielsweise durch die Analyse rechtlicher Setzungen klar, dass regulative Prozesse überstaatlicher Natur offensichtlich von nationalstaatlichen Entwicklungen überlagert oder gar konterkariert werden, denn

[...] insbesondere das schweizerische Behindertengleichstellungsgesetz und die Neugestaltung des Finanzausgleichs und der Aufgabenteilung zwischen Bund und Kantonen, haben offenbar einen weitaus stärkeren Einfluss auf das schweizerische Bildungssystem, als die Ratifikation der UNBRK bis dato entwickelt hat. Die Konsequenz einer Entkopplung von Vorgaben der UN-BRK erachten wir daher als insofern problematisch, dass das schweizerische Behindertengleich- 
stellungsgesetz keine klaren Ableitungen zulässt, wann und wie Schüler*innen mit besonderem Förderbedarf segregiert, separiert oder inklusiv beschult werden sollen (Mejeh/Powell 2018: 16).

Es zeigt sich also, dass divergente Entwicklungen im Bildungsbereich durchaus genauso ein Ergebnis der Governance sein können, wie die Tatsache, dass konvergente Entwicklungen nicht notwendigerweise ein Ergebnis bildungspolitischer Verflechtungen sein müssen (Gonon et al. 2016). Die globale Diffusion des Menschenrechts Inklusive Bildung fordert etablierte Bildungsorganisationen, die Sonderschule genauso wie die Regelschule, sowie kulturell verankerte Paradigmen von Bildung und Behinderung zwar heraus (Biermann/Powell 2014), der Institutionalisierungsprozess, so das Argument hier, vollzieht sich allerdings erst durch die Entkopplungsmechanismen die auf den verschiedenen nationalstaatlichen Ebenen zum Tragen kommen. Daraus ergibt sich für die Analyse der Umsetzung der UN-BRK eine wichtige Differenzierung, die ihren Unterschied in der Einhaltung rechtlicher Vorgaben und konkreter Umsetzung in Form normativer und kulturell-kognitiver Setzungen findet.

So lässt sich aufgrund des Drei-Säulen Modells am Beispiel der Schweiz aufzeigen, wie die Umsetzung Inklusiver Bildung vertikalen und horizontalen Wirkmechanismen unterliegt, die für eine genaue Analyse gemeinsam gedacht werden müssen. Demzufolge ist hier von einer mehrdimensionalen Diffusion eines top-down Prozesses auszugehen, der sich in drei Bereichen vollzieht: in einer kulturell-kognitiven, einer normativen und einer regulativen Dimension. Dadurch wird durch die Beschreibung von Praktiken, Handlungen und Strukturen, die bewusst oder unbewusst durchgeführt werden und sich im Rahmen gesetzlicher Vorgaben bewegen bzw. als selbstverständlich erachtet werden, die Analyse der Persistenz von Institutionen möglich (Scott 2014). Dieser analytische Rahmen wird durch das Konzept der organisationalen Pfadabhängigkeit ergänzt, zeigt sich doch hier welche Entscheidungen entsprechender Organisationen in der Vergangenheit getroffen wurden und welche Auswirkungen dies auf den Institutionalisierungsprozess der ,,besonderen Pädagogik“ (Heilpädagogik) und ihrer dementsprechenden professionellen sowie organisationalen Ausgestaltung hat. Unter Berücksichtigung von Entkopplungsmechanismen und isomorphen Prozessen können so, im hier zu diskutierenden Kontext mit der kulturell-kognitiven Dimension, die aktuell herrschenden Behinderungsparadigmen (etwa medizinische, sozialpolitische oder menschrechtsbasierte Modelle von Behinderung), mit der normativen Dimension die sonderpädagogische aber auch allgemeinpädagogische Profession sowie deren Organisation, und mit der regulativen Dimension die aktuelle Bildungspolitik und Rechtsprechung erfasst und analysiert werden (Powell 2009; 2016; Biermann/Powell 2014).

Ordnet man Scotts Säulenmodell in die dargestellte Diffusionslogik ein, ergibt sich also eine Vielzahl an Anschlussmöglichkeiten an weitere neo-institutionalistische Konzepte (s. Abbildung 3), denn obwohl die neo-institutionalistischen Ansätze eigentlich keine geschlossene Theorie darstellen (Schaefers 2002), zeigt sich, dass insbesondere die Einführung des Legitimitäts- und Effizienzprinzips hohe Erklärungskraft besitzt, da sich hiermit vor allem die Relevanz der Entkopplungsmechanismen und der Isomorphie herausstellt. Durch Entkopplung wird offensichtlich, wie Vorgaben und Orientierungen durch die unterschiedlichen Verhandlungsebenen voneinander losgelöst werden (Effizienz), während sich durch den Prozess der Isomorphie auf horizontaler und vertikaler Ebene eine Angleichung der verschiedenen Organisationen ergibt (Legitimität). 
Abbildung 3: Institutionenmodell Inklusiver Bildung

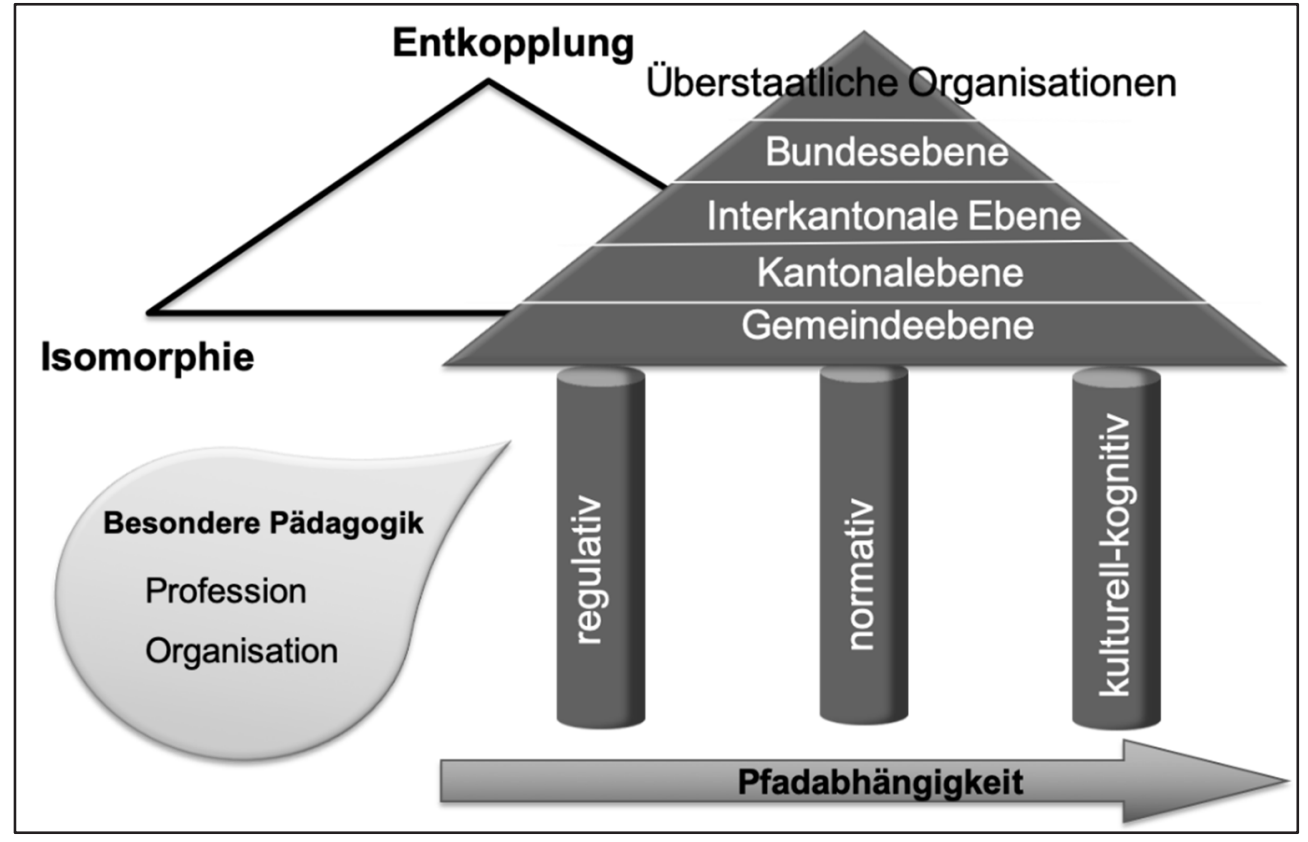

Quelle: eigene Darstellung.

Demnach ist Inklusion (hier: Inklusive Bildung) in ihrer institutionalisierten Form das, was in unterschiedlichen Organisationen der Gesellschaft interdependent verhandelt, gedacht und gemacht wird. Inklusive Prozesse sind dabei sowohl über verschiedene organisationale Hierarchiestufen (strukturell), wie auch konkrete Handlungen (akteur*innenspezifisch) erkennund analysierbar. Durch die Festlegung dessen, was als Inklusion zu verstehen ist, wird in einem distinktiven Sinne immer auch festgelegt, was nicht als Inklusion zu verstehen ist.

\section{Fazit}

Im vorliegenden Beitrag wurde versucht, den Institutionalisierungsprozess Inklusiver Bildung in der Schweiz mit Hilfe neo-institutionalistischer Kernkonzepte darzustellen. Um die Institutionalisierung Inklusiver Bildung in der Schweiz analysierbar zu machen, ist ein Blick auf die unterschiedlichen Voraussetzungen ihrer Umsetzung notwendig. Dabei ist, wie weiter oben bereits angedeutet, von einer Diffusionslogik auszugehen, die sich in vielfacher Hinsicht durch mehrere Staatsebenen zieht, allerdings nicht nur in rechtlicher (regulativ) Sichtweise, sondern auch hinsichtlich ihrer Normen und Werte (normativ) sowie tatsächlichen Praxis (kulturell-kognitiv). Darüber hinaus hat sich auch gezeigt, dass die Historizität der Organisationen selber, aber auch die Entwicklung verschiedener Professionen genauso eine elementare Bedeutung besitzen, wie die Tatsache, dass im beschriebenen Mehrebenensystem 
Entkopplungsmechanismen und isomorphische Wandlungsprozesse den inkrementellen Wandel des Bildungssystems bestimmen.

International mag sich so durch einen zunehmend normativen Druck die Ratifikation der UN-BRK in der Schweiz zwar vollzogen haben, grundsätzlich bleibt das Recht der Inklusiven Bildung aber nicht einklagbar, da das entsprechende Fakultativprotokoll nicht unterzeichnet wurde. Im Sinne eines globalen Wertesystems wie es in der world-polity-theory (Meyer 1980; Meyer et al. 1987; Meyer 2005) formuliert wird, hat sich die Schweiz damit weltweit zwar anderen Staaten angeglichen, allerdings können bereits die rechtlichen Entkopplungen auf nationalstaatlicher Ebene als problematisch erachtet werden. Daneben ist der Legitimationsdruck, den Organisationen erfahren, von grundlegender Bedeutung, denn gerade durch die Inkorporation institutionalisierter Elemente, die als Ziel und Zielerreichung durch die Gesellschaft festgelegt werden, wird die Umsetzung Inklusiver Bildung erst analysierbar. Bedingt durch die Tatsache, dass die Anforderungen, die an eine Organisation gestellt werden, nicht immer konsistent und zuweilen sogar paradox sind, ist es hierbei entscheidend, dass der Austausch von Ressourcen jeglicher Art in erster Linie der Herstellung von Legitimation, nicht aber einem Optimierungsgedanken unterliegt. Man möge sich hier an das weiter oben skizzierte Beispiel erinnern, durch das klar wird, wie variabel und vielfältig alleine schon die unterschiedlichen gesellschaftspolitischen Ausgestaltungen der Schule auf Gemeindeebene in der Schweiz sind. Dabei sind es gerade die gesellschaftlichen Rationalitätsfassaden, bzw. „Mythen“, die in den Blick zu nehmen sind. So hält sich beispielsweise nach wie vor hartnäckig die allgemeine Überzeugung des meritokratischen Leistungsprinzips im schweizerischen Bildungssystem, obwohl dies schon vielfach empirisch widerlegt worden ist (Eberle/Brüggenbrock 2013; Glauser/Becker 2016; Hofstetter 2017; Kronig 2007). Im Hinblick auf die Persistenz institutioneller Strukturen zeigt sich, wie zu Beginn des Artikels aufgeworfen, eine mittlerweile zwei Jahrhundert andauernde etablierte Praxis: nach wie vor zählen kantonale Statistiken mit zu einem der wichtigsten Instrumente der Bildungssteuerung, Kleinklassen (vorher: Hilfsklassen) und Sonderschulen (vorher: Hilfsschulen) wurden in den letzten Jahren zwar sukzessive abgebaut, sind aber nach wie vor noch ein wichtiger Bestandteil des Bildungssystems. Ähnlich wie im 19. Jahrhundert sind es während der zweiten Hälfte des 20. Jahrhunderts bis heute in erster Linie schulorganisatorische Gründe, die für eine segregative, separative oder inklusive Beschulung verantwortlich sind. All dies sind Indikatoren dafür, wie Inklusive Bildung heute in der schweizerischen Gesellschaft verhandelt und umgesetzt wird.

Aufgrund der vorhergehenden Darlegungen ist es nun angezeigt, die Institutionalisierung Inklusiver Bildung in der Schweiz weitergehend zu untersuchen. Das bedeutet, auch die Grundlagen des föderalen Bildungssystems zu fokussieren, um die institutionellen Reproduktionsmechanismen im Mehrebenensystem zu untersuchen. Beispielsweise ist nicht deutlich, wie stark die beschriebenen Entkopplungsmechanismen in der Praxis tatsächlich eine Rolle spielen und ob sie sich auf den verschiedenen staatlichen Ebenen unterscheiden. Darüber hinaus wurde hier eine radikale Konzeption der Entkopplung dargestellt, der eine binäre Idee, im Sinne von vorhanden/nicht vorhanden, zugrunde liegt. Es ist jedoch auch denkbar, dass Entkopplungen teilweise oder temporär bedingt stattfinden. Gleichzeitig wäre herauszufinden, ob beispielsweise Entkopplungsprozesse der regulativen und normativen Dimension den gleichen Mechanismen unterliegen. Hierfür könnten soziale Praktiken untersucht werden, um die Bedeutung, die einzelne, zentrale Akteur*innen bestimmten Maßnahmen, Gesetzen oder Programmen zuschreiben, zu erfahren. Nach wie vor sind aber auch Untersuchungen auf Aggregatebene notwendig, so beispielsweise die landesweite Segregation/ 
Separation von Schüler*innen mit besonderem Förderbedarf. Denn aufgrund der aktuellen Datenlage lässt sich davon ausgehen, dass mit dem Rückgang segregierter Beschulung eine Verlagerung separativer Beschulung in das Regelschulsystem stattgefunden hat (Pfister et al. 2012). So lässt sich auch im hier aufgezeigten Fall davon ausgehen, dass es durch die sukzessive Auflösung separativer und segregativer Systeme zu Substitutionseffekten hinsichtlich Inklusiver Bildung im schweizerischen Bildungssystem gekommen ist, die genauer zu analysieren wären. Aktuell lassen sich hier keine genaueren Aussagen treffen, da mit den entsprechenden Daten (etwa zu den Kleinklassen), die durch das schweizerische Bundesamt für Statistik erhoben werden, erst noch zu rechnen ist.

\section{Literatur}

Arthur, Brian (1989): Competing Technologies, Increasing Returns, and Lock-In by Historical Events. In: Economic Journal 99, 394, S. 116-131.

Becker-Ritterspach, Florian A. A./Becker-Ritterspach, Jutta C. E. (2006b): Isomorphie und Entkopplung im Neo-Institutionalismus. In: Senge, Konstanze/Hellmann, Kai-Uwe (Hrsg.): Einführung in den Neo-Institutionalismus. Wiesbaden: VS Verlag, S. 102-117.

Berger, Peter L./Luckmann, Thomas (1966): The Social Construction of Reality. New York: Anchor Books.

Biermann, Julia (2021 i.d.B.): Der Einfluss der UN-BRK auf inklusive Bildung in Nigeria und Deutschland. In: Köpfer, Andreas/Powell, Justin J.W./Zahnd, Raphael (Hrsg.): Handbuch Inklusion international. Opladen: Budrich, S. 167-178.

Biermann, Julia/Powell, Justin J.W. (2014): Institutionelle Dimensionen inklusiver Schulbildung. Herausforderungen der UN-Behindertenrechtskonvention für Deutschland, Island und Schweden im Vergleich. In: Zeitschrift für Erziehungswissenschaften 17, 4, S. 679-700.

Biewer, Gottfried (2021 i.d.B.): Inklusive Bildung in Österreich. In: Köpfer, Andreas/Powell, Justin J.W./Zahnd, Raphael (Hrsg.): Handbuch Inklusion international. Opladen: Budrich, S. 239246.

Blanck, Jonna M./Edelstein, Benjamin/Powell, Justin J.W. (2013): Persistente schulische Segregation oder Wandel zur inklusiven Bildung? Die Bedeutung der UN-Behindertenrechtskonvention für Reformprozesse in den deutschen Bundesländern. In: Schweizerische Zeitschrift für Soziologie, 39, 2, S. 267-292.

Bless, Gérard (2007): Zur Wirksamkeit der Integration. Bern: Haupt.

Bonazzi, Giuseppe (2014): Geschichte des organisatorischen Denkens. Wiesbaden: Springer VS.

Bromley, Patricia/Powell, Walter (2012): From smoke and mirrors to walking the talk. Decoupling in the contemporary world. In: The Academy of Management Annals 6, 1, S. 483-530.

Bulmer, Simon (1998): New institutionalism and the governance of the Single European Market. In: Journal of European Public Policy 5, 3, S. 365-386.

David, Paul (1985): Clio and the Economics of QWERTY. In: American Economic Review 75, 2, S. 332-337.

Degener, Theresia (2017): A New Human Rights Model of Disability. In: Della Fina, Valentina/Cera, Rachele/Palmisano, Giuseppe (Hrsg.): The United Nations Convention on the Rights of Persons with Disabilities. New York: Springer, S. 41-59.

Department für Bildung und Kultur Solothurn (2013): Leitfaden Sonderpädagogik. https://www. so.ch/fileadmin/internet/dbk/dbk-vsa/Foerdern/Unterstuetzung_Behinderung/Leitfaden_Sonderpaedagogik.pdf [Zugriff: 13.06.2020]. 
DiMaggio, Paul (1986): Structural analysis of organizational fields: A blockmodel approach. In: Research in Organizational Behaviour 8, S. 335-370.

DiMaggio, Paul/Powell, Walter (1983): The Iron Cage Revisited. Institutional Isomorphism and Collective Rationality in Organizational Fields. In: American Sociological Review 48, 2, S. 147-160.

DiMaggio, Paul/Powell, Walter (1991): Introduction. In: Powell, Walter/DiMaggio, Paul (Hrsg.): The New Institutionalism in Organizational Analysis. Chicago: University of Chicago Press, S. 1-38.

Dienststelle Volksschulbildung Kanton Luzern (2012): Kantonales Konzept für die Sonderschulung 2012.https://volksschulbildung.lu.ch/media/Volksschulbildung/Dokumente/syst_schulen/ss_hps_luzern_emmen/kantonales_konzept_der_sonderschulung.pdf?la $=$ de-CH [Zugriff: 13.06.2020].

Direktion für Bildung und Kultur Zug (2008). Konzept Sonderpädagogik KOSO. edudoc.ch/record/39117/files/ZG_Konzept_Sonderpaedagogik_KOSO.pdf [Zugriff: 13.06.2020].

Ebbinghaus, Bernhard (2009): Can path dependence explain institutional change? In: Magnusson, Lars/Ottosson, Jan (Hrsg.): The Evolution of Path Dependence. Cheltenham: Edward Elgar, S. 191-212.

Eberle, Franz/Brüggenbrock, Christel (2013). Bildung am Gymnasium. Bern: Schweizerische Konferenz der kantonalen Erziehungsdirektoren.

Erziehungs-, Kultur- und Umweltschutzdepartment (2007): Sonderpädagogisches Konzept (Sonderschulkonzept) Graubünden. edudoc.ch/record/4108/files/sonderschulkonzept_maerz_2007 -1.pdf [Zugriff: 13.06.2020].

Glauser, David/Becker, Rolf (2016): VET or general education? Effects of regional opportunity structures on educational attainment in German-speaking Switzerland. Empirical Research in Vocational Education and Training 8, 1, S. 1-25.

Gonon, Philipp/Hügli, Anton/Künzli, Rudolf/Maag Merki, Katharina/Rosenmund, Moritz/ Weber, Karl (2016): Einleitung. In: Gonon, Phillip/Hügli, Anton/Künzli, Rudolf/Maag Merki, Katharina/Rosenmund, Moritz/Weber, Karl (Hrsg): Governance im Spannungsfeld des schweizerischen Bildungsföderalismus. Bern: hep Verlag, S. 11-19.

Haeberlin, Urs/Bless, Gérard/Moser, Urs/Klaghofer, Richard (2003): Die Integration von Lernbehinderten. Versuche, Theorien, Forschungen, Enttäuschungen, Hoffnungen. Haupt: Bern.

Hartong, Sigrid/Nikolai, Rita (2016): Schulstrukturreform in Bremen: Promotoren und Hindernisse auf dem Wege zu einem inklusiveren Schulsystem. In: Moser, Vera/Lütje-Klose, Birgit (Hrsg.): Schulische Inklusion. 62. Beiheft zur Zeitschrift für Pädagogik, S. 105-123.

Hasse, Raimund/Krücken, Georg (2005): Neo-Institutionalismus. Bielefeld: transcript.

Hofstetter, Daniel (2017): Die schulische Selektion als soziale Praxis: Aushandlungen von Bildungsentscheidungen beim Übergang von der Primarschule in die Sekundarstufe I. Weinheim: Beltz Juventa.

Jepperson, Ronald L. (1991): Institutions, Institutional Effects, and Institutionalism. In: DiMaggio, Paul/Powell, Walter W. (Hrsg.): The New Institutionalism in Organizational Analysis. Chicago: The University of Chicago Press: S. 143-163.

Kronig, Winfried (2007): Die systematische Zufälligkeit des Bildungserfolges. Bern: Haupt.

Künzli, Rudolf/Weber, Karl (2016): Kontexte der Bildungsgovernance in der Schweiz. In: Gonon, Phillip/Hügli, Anton/Künzli, Rudolf/Maag Merki, Katharina/Rosenmund, Moritz/Weber, Karl (Hrsg): Governance im Spannungsfeld des schweizerischen Bildungsföderalismus. Bern: hep Verlag, 127-139.

Labhart, David (2019): Interdisziplinäre Teams in inklusiven Schulen: Eine ethnografische Studie zu Fallbesprechungen in multiprofessionellen Gruppen. Bielefeld: transcript. 
Maag Merki, Katharina (2016): Selektion und Übertritte. In: Gonon, Phillip/Hügli, Anton/Künzli, Rudolf/Maag Merki, Katharina/Rosenmund, Moritz/Weber, Karl (Hrsg): Governance im Spannungsfeld des schweizerischen Bildungsföderalismus. Bern: hep, S. 107-126.

Mahoney, James (2000): Path Dependence in Historical Sociology. In: Theory and Society 29, 4, S. 507-548.

Mejeh, Mathias (2016): Absicht und Wirklichkeit integrativer Bildung - Ein netzwerkanalytischer Beitrag zum Neo-Institutionalismus. Wiesbaden: Springer VS.

Mejeh, Mathias/Powell, Justin J.W. (2018): Inklusive Bildung in der Schweiz. Zwischen globalen Normen und kantonalen Besonderheiten. In: Bildung und Erziehung 71, 4, S. 412-431.

Meyer, John W. (1980): The World Polity and the Authority of the Nation-State. In: Bergesen, Albert J. (Hrsg.): Studies of the Modern World-System. New York: Academic Press, S. 109137.

Meyer, John W. (2005): Weltkultur. Wie die westlichen Prinzipien die Welt durchdringen. Frankfurt am Main: Suhrkamp.

Meyer, John W./Rowan, Brian (1977): Institutionalized Organizations. Formal Structure as Myth and Ceremony. In: American Journal of Sociology, 83, 2, S. 340-363.

Meyer, John W./Boli, John/ Thomas, Georg M. (1987): Ontology and Rationalization in the Western Cultural Account. In: Thomas, Georg M./Meyer, John W./Ramirez, Francisco O./Boli, John (Hrsg.): Institutional Structure Constituting State, Society, and the Individual. Newbury Park: Sage, S. 12-37.

Pfister, Mirjam/Eckhart, Michael/Bärtschi, Simon (2012): Integrierte Sonderklassenschülerinnen und -schüler. Analyse bildungsstatistischer Daten. In: Schweizerische Zeitschrift für Heilpädagogik 9, S. 22-30.

Pierson, Paul (2000): Increasing Returns, Path Dependence, and the Study of Politics. In: American Political Science Review 94, 2, S. 251-267.

Powell, Justin J.W. (2009): Sonderpädagogische Fördersysteme im Vergleich. In: Koch, Sascha/Schemmann, Michael (Hrsg.): Neo-Institutionalismus in der Erziehungswissenschaft. Wiesbaden: VS Verlag, S. 212-232.

Powell, Justin J.W. (2016): Barriers to Inclusion. Special Education in the United States and Germany. Abingdon: Routledge.

Powell, Justin J.W. (2018): Inclusive Education. Entwicklungen im internationalen Vergleich. In: Sturm, Tanja/Wagner-Willi, Monika (Hrsg.): Handbuch schulische Inklusion. Opladen: Budrich UniPress, S. 127-142.

Powell, Walter (1991): Expanding the Scope of Institutional Analysis. In: Powell, Walter/DiMaggio, Paul (Hrsg.): The New Institutionalism in Organizational Analysis. Chicago: University of Chicago Press, S. 183-203.

Schaefers, Christine (2002): Der soziologische Neo-Institutionalismus. Eine organisationstheoretische Analyse- und Forschungsperspektive auf schulische Organisationen. In: Zeitschrift für Pädagogik 48, 2, S. 835-855.

Scott, W. Richard (1994): Conceptualizing Organizational Fields. In: Derlien, Hans-Ulrich/ Gerhardt, Uta/Scharpf, Fritz W. (Hrsg.): Systemrationalität und Partialinteresse. Baden-Baden: Nomos, S. 203-221.

Scott, W. Richard (2014): Institutions and Organizations. Thousand Oaks: SAGE.

Scott, W. Richard/ Meyer John W. (1991): The Organization of Societal Sectors: Propositions and Early Evidence. In: Powell, Walter/DiMaggio, Paul (Hrsg.): The New Institutionalism in Organizational Analysis. Chicago: University of Chicago Press, S. 108-140.

Senge, Konstanze (2011): Das Neue am Neo-Institutionalismus. Wiesbaden: Springer VS.

Sturny, Gabriel (1984): Die Schulung Lernbehinderter in der Schweiz. Luzern: SZH.

Sydow, Jörg/Schreyögg, Georg/Koch, Jochen (2009): Organizational Path Dependence. Opening the Black Box. In: Academy of Management Review 34, 4, S. 689-709. 
UNESCO [United Nations Educational, Scientific and Cultural Organization] (1994). The Salamanca Statement and Framework for Action on Special Needs Education. Paris.

Walgenbach, Peter (2019): Neoinstitutionalistische Ansätze in der Organisationstheorie. In: Kieser, Alfred/Ebers, Mark (Hrsg.): Organisationstheorien. Stuttgart, Berlin, Köln: Kohlhammer, S. 300-350.

Walther-Müller, Peter/Häfeli, Kurt (2005): Das Wachstum des sonderpädagogischen Angebots im interkantonalen Vergleich. In: Schweizerische Zeitschrift für Heilpädagogik 7-8, 5, S. 3644.

Weick, Karl E. (1976): Educational Organizations as Loosely Coupled Systems. In: Administrative Science Quarterly, 21, 1, S. 1-19.

Werning, Rolf (2014): Stichwort. Schulische Inklusion. In: Zeitschrift für Erziehungswissenschaft 17, 4, S. 601-623.

Wolfisberg, Carlo (2002): Heilpädagogik und Eugenik. Zur Geschichte der Heilpädagogik in der deutschsprachigen Schweiz (1800-1950). Zürich: Chronos Verlag.

Zucker, Lynne (1977): The Role of Institutionalization in Cultural Persistence. In: American Sociological Review, 42, 5, S. 726-743. 


\title{
13 Inklusive Bildung in Österreich - Von den Anfängen der Entwicklung eines rechtlich-normativen Auftrags bis zu den Perspektiven für die Zukunft
}

\author{
Gottfried Biewer
}

\begin{abstract}
Inklusive Bildung ist im elementarpädagogischen Bereich eine Aufgabe der Bundesländer, die recht unterschiedliche Angebote bereithalten. Sowohl bei der Berufsausbildung wie auch im tertiären Bildungssektor sind Konzepte für inklusive Bildung nur begrenzt vorhanden. Für den schulischen Bereich gibt es eine einheitliche Bundesgesetzgebung, die bereits seit Beginn der 1990er Jahre eine Ausweitung integrativer Beschulungsformen ermöglichte. Mit der Einführung des Elternwahlrechts zwischen integrativen und sonderschulischen Settings wurde ab 1993 für rund 10 Jahre ein kontinuierlicher Anstieg der Zahl integrativ beschulter Kinder ermöglicht. Seit 2004 ist die Quote zur Beschulung in den beiden Formen ungefähr gleich hoch. In der Sekundarstufe wurden integrative Systeme fast nur an den Hauptschulen bzw. Neuen Mittelschulen etabliert, nicht jedoch an den Allgemeinbildenden Höheren Schulen. Die seit 2014 etablierte neue Lehrer*innenbildung, die Stufenlehrämter für Primar- und Sekundarschulen schuf und gleichzeitig die vorherige Sonderschullerer*innenausbildung durch eine Spezialisierung in Inklusiver Pädagogik ersetzte, kann als langfristig nachhaltige Maßnahme zur Etablierung inklusiver Strukturen im Schulbereich betrachtet werden.
\end{abstract}

\section{Institutionelle Rahmenbedingungen für inklusive Bildung}

Die Darstellung inklusiver Bildung in Österreich kann nicht ohne Bezugspunkte des Vergleichs erfolgen, auch wenn diese nicht fortlaufend explizit benannt werden. Dies können Normen sein, die in einem internationalen Rahmen Geltung beanspruchen. Es kann aber auch die kolportierte Situation anderer Länder sein, denen die bessere Lösung institutioneller und pädagogischer Problemlagen unterstellt wird und die somit als Modelle dienen können (Biewer/Luciak 2010). Der nachfolgende Beitrag nimmt sowohl international geltende Normen wie auch die Situation anderer, insbesondere deutschsprachiger Länder als Maßstab für die Darstellung.

Österreich gehört zu denjenigen europäischen Ländern, die bereits sehr früh eine Unterrichtspflicht für alle Kinder gesetzlich verankerten (Engelbrecht 2015). Maria Theresia legte mit ihrer Schulordnung bereits 1774 eine Schulpflicht zwischen dem sechsten und zwölften Lebensjahr fest. Viele strukturelle Elemente des heutigen Bildungssystems haben ihre Grundlage noch in administrativen Festlegungen in den Zeiten der Donaumonarchie, an die mit der Gründung der ersten Republik im Jahre 1918 angeknüpft wurde. 
Administrative Strukturen des Bildungswesens haben eine lange Geschichte, die in die Zeit vor der Gründung der Republik zurückreichen. Eine föderale Verwaltungsstruktur legt Aufgaben für Bund, Länder und Gemeinden fest mit sehr unterschiedlichen Verantwortlichkeiten. Während für die Gesetzgebung zu elementarpädagogischen Einrichtungen wie Kindergärten und Krippen die 9 Bundesländer zuständig sind, gibt es eine einheitliche staatliche Gesetzgebung für die Schulen durch den Bund, die alle Bereiche schulischer Organisation und des Unterrichts für Lehrer*innen abdeckt. Dies ist ein Unterschied, beispielsweise zu Deutschland, wo die Bundesländer über Gesetzgebungskompetenz in schulischen Belangen verfügen.

Österreich bietet Primarschulbildung von der 1. bis zur 4. Klasse für alle Kinder in der Volksschule an, die als Einheitsschule gestaltet ist. Ab dem 5. Schulbesuchsjahr werden die Schüler*innen zwischen Neuer Mittelschule (NMS, ehemals Hauptschule) und Allgemeinbildender Höherer Schule (AHS) aufgeteilt. Es wird damit eine Gabelung in einen eher berufsorientierten und einen akademischen weiteren Bildungsweg eröffnet. Die Allgemeinbildende Höhere Schule (AHS) beginnt im Alter von 10 Jahren, umfasst 8 Jahre mit einer Unterund Oberstufe und soll im Alter von 18 Jahren mit einer Studienberechtigung abschließen. Auch wenn diese Unterteilung einschneidend ist, so hat Österreich insbesondere im Bereich der Sekundarstufe II mit einer Vielzahl berufsbildenden Höheren Schulen höhere Durchlässigkeit zwischen diesen Zweigen, als dies etwa in Deutschland der Fall ist.

Die frühe Selektion bezüglich der weiteren Bildungslaufbahn im Alter von 10 Jahren ist seit den 1920er Jahren Gegenstand politischer Debatten zwischen konservativen und sozialdemokratischen Politiker*innen. Kritisiert wird an ihr, dass Karrierechancen begabter Kinder aufgrund ökonomischer, sprachlicher und kultureller Barrieren eingeschränkt werden. Die Übergangsrate zur AHS nach der 4. Klasse der Grundschule beträgt 35\%, d.h. 2/3 der Schüler*innen verbleiben in Neuen Mittelschulen (ehem. Hauptschulen). Da grundlegende strukturelle Veränderungen im österreichischen Bildungssystem eine Zweidrittelmehrheit im Parlament erfordern, sind notwendige Strukturreformen in wichtigen und umstrittenen Bereichen des Bildungswesens nur schwer durchsetzbar (Biewer/Proyer 2017).

Auf die Neue Mittelschule der Schulbesuchsjahre 5 bis 8 (für Schüler*innen von 10 bis 14 Jahren) folgt eine einjährige Berufsvorbereitung über die Polytechnische Schule. Das duale System der daran anschließenden, meist dreijährigen Lehrlingsausbildung in Unternehmen in Kombination mit dem Besuch der Berufsschule führt zu einer im europäischen Vergleich niedrigen Jugendarbeitslosenquote.

\section{Strukturen inklusiver Bildung und ihre Genese}

Elementarpädagogische Einrichtungen sind Angelegenheiten der Bundesländer, welche die Zielsetzungen von Integration und Inklusion sehr unterschiedlich berücksichtigen. Das Bundesland Niederösterreich hat bereits in den 1980er Jahren damit begonnen vorschulische Integration für Kinder mit Behinderungen für die Altersgruppe von 2 1/2 bis 6 Jahren zu etablieren und flächendeckend umzusetzen. Niederösterreich, für das diese Entwicklung gut dokumentiert ist (Kerschbaumer 1999) begann mit der Erneuerung des niederösterreichischen Kindergartengesetzes 1987 die Möglichkeit für integrative Kindergartengruppen zu schaffen. Nach einigen Jahren der Erprobung der Integrationsmodelle werden diese schließlich 1996 als Regelform eingeführt. 2010 wird dann im Bildungsplan für Kindergärten in NÖ Inklusion 
als allgemeines Bildungsprinzip und pädagogische Leitlinie definiert und sie löst damit den Begriff Integration weitgehend ab.

Die Rate der Schüler*innen mit sonderpädagogischem Förderbedarf (SPF) liegt bei fast 4\% der Schüler*innen im Pflichtschulbereich. Diese Zahl umfasst sowohl die Schüler*innen an Sonderschulen wie auch in integrativen und inklusiven Settings. Die Durchschnittsquote der Schüler*innen mit SPF liegt an Volksschulen bei 1,5\% und an weiterführenden Schulen (5. bis 8 . Klasse) bei 2,2\%. In der 5. Klasse besuchen rund 1,9\% Sonderschulen. Diese Quote steigt bis zur 9. Klasse auf 2,3\% (Biewer/Proyer 2017).

Schüler*innen mit schwerer intellektueller Behinderung besuchen häufiger Sonderschulen als andere Gruppen, obwohl die rechtliche Situation für alle Gruppen gleich ist. Zusätzliche, von den Ländern oder Gemeinden finanzierte Serviceangebote wie die Tagesbetreuung am Nachmittag sind in Sonderschulen häufiger zugänglich als in integrativen Einrichtungen, was die Tendenz der Eltern, Sonderschulumgebungen für ihr Kind zu wählen, zusätzlich verstärkt.

Sonderschulen sind für Schüler*innen im unterrichtspflichtigen Alter der 1. bis 9. Klasse (6 bis 15 Jahre) verfügbar. Diese Zeitspanne kann für integrative Formen der Lehrlingsausbildung und der Unterstützung an Schulen der Sekundarstufe II (beide zwischen 15 und 18 Jahren) verlängert werden.

Gruppen, denen ein SPF zugesprochen wird, umfassen Schüler*innen mit Lernbehinderungen, erhöhtem Förderbedarf, motorischen Störungen, Seh- und Hörbehinderungen, emotionalen und Verhaltensproblemen sowie Schüler*innen an Krankenhausschulen. Infolge der Wahl der Eltern zwischen sonderschulischem und integrativem Setting stieg die Zahl der Schüler*innen mit SPF in integrativen Klassen zwischen 1994 und 2004 kontinuierlich an. Seitdem sind beide Zahlen mit 50\% nahezu gleich hoch. In den letzten Jahren ist die Quote der Schüler*innen mit SPF leicht gestiegen auf fast 4\%, allerdings als Folge einer etwas höheren Bereitschaft in Regelschulen Kindern einen SPF zuzuschreiben.

Es können mehrere Phasen der Entwicklung inklusiver Strukturen in der Schule betrachtet werden. Österreich verfügt seit den 1970er Jahren über ein ausgereiftes Sonderschulsystem mit Sonderschultypen für Schüler*innen mit Lernbehinderungen (,Allgemeine Sonderschule“), geistigen Behinderungen (,Schule für Schwerstbehinderte“), körperlichen Beeinträchtigungen, Hör- und Sehstörungen, emotionalen und Verhaltensstörungen sowie an Krankenhäusern (,Heilstättenschulen“).

Erste Versuche Kinder integrativ zu beschulen, gab es ab der Mitte der 1980er Jahre. Nach der Gründung der ersten Integrationsklasse in Oberwart im Burgenland, brachten die nachfolgenden Jahren eine Ausweitung über Modellversuchsklassen insbesondere für Kinder mit körperlichen und Sinnesbehinderungen (Anlanger 1993).

Ein großer Einschnitt kam mit einer Änderung des Schulorganisationsgesetzes im Jahre 1993, als Eltern von Volksschüler*innen (Klassen 1 bis 4) mit sonderpädagogischem Förderbedarf das Recht eingeräumt wurde zu wählen, ob ihr Kind eine Sonderschule oder eine integrative Klasse besuchen sollte. 1996 wurde dieses Recht ebenfalls durch Bundesgesetzgebung auf die Sekundarstufe mit den Schulbesuchsjahren 5 bis 8 ausgeweitet.

Der gesetzliche Wechsel war auch Folge des Wirkens einer außerordentlich aktiven Elternbewegung für Integration in Österreich, die im Zusammenwirken mit reformorientierten Lehrkräften nicht nur diese gesetzlichen Veränderungen ab 1993 durchsetzte, sondern danach auch noch die Umsetzung genau verfolgte und als Vertretungsorganisation mit eigenen Vorschlägen in Erscheinung trat. 
Angesichts einer einheitlichen Bundesgesetzgebung für das Schulwesen könnte unterstellt werden, dass der Level der Umsetzung integrativer und inklusiver Strukturen in allen Teilen Österreichs vergleichbar wäre. Dies ist nicht der Fall, sondern es gibt sogar erhebliche Unterschiede zwischen den Bundesländern, die von einer Sonderbeschulungsquote von rund $4 \%$ im Bundesland Vorarlberg bis zu 0,6\% in der Steiermark reichen. Die Gründe für diese auffälligen Unterschiede wurden nie systematisch untersucht, so dass es zwar Vermutungen gibt, aber keine über empirische Daten belegte Aussagen. Als relevante Faktoren werden die Angebote gut ausgebauter Sonderschulen gesehen, mit denen eine Nachfrage erzeugt wird. Ein relevanter Faktor ist auch die Bereitstellung einer Ganztagesbetreuung in sonderschulischen Settings im Vergleich zu integrativen Settings, in denen das Nachmittagsangebot nicht vorhanden oder nicht gesichert ist. Auch Eltern, die den integrativen oder inklusiven Weg priorisieren, nehmen angesichts der sehr unterschiedlichen Versorgungsgrade dann doch lieber segregative schulische Settings in Kauf. Eltern haben vom Gesetz her ein Wahlrecht zwischen beiden Formen, nehmen aber im Vorfeld ihrer Entscheidung schulische Beratungsangebote über die bestehenden Strukturen in Anspruch. So kann auch vermutet werden, dass die institutionellen Beratungsprozesse hier noch eine weitere Steuerungsfunktion einnehmen (Klicpera 2007; Biewer 2018).

Österreich hat unterschiedliche Lehrpläne für Schüler*innen an Sonderschulen. Daher gibt es spezielle Lehrpläne für Schüler*innen mit Hörstörungen, Sehstörungen, motorischen Störungen, geistigen Behinderungen, Lernstörungen und emotionalen bzw. Verhaltens-Störungen. Schüler*innen mit SPF werden gemäß den Zielen des Sonderschullehrplans unterrichtet, auch wenn sie integrative Klassen besuchen. Die Verwendung eines gemischten Lehrplans, der die speziellen Lehrpläne und die allgemeinen Lehrpläne umfasst, ist für Schüler*innen mit SPF in integrativen Klassenräumen möglich. So könnten beispielsweise für Deutsch und Mathematik spezielle Sonderschullehrpläne für Schüler*innen mit Lernproblemen relevant sein, während für alle anderen Fächer der reguläre Regelschullehrplan Verwendung findet.

\section{Zielsetzungen im Nationalen Aktionsplan Behinderung}

Die Umsetzung Inklusiver Bildung ist eine Aufgabe für die Zukunft, die bereits mit dem Nationalen Aktionsplan Behinderung 2012-2020 propagiert wurde (BMASK 2016), aber immer noch vor großen Hürden steht, insbesondere seit veränderte bildungspolitische Schwerpunktsetzungen auf Bundesebene dem Ausbau inklusiver Strukturen keine Priorität mehr einräumen. Mit dem bildungspolitischen Wechsel zu einer Koalition aus einer konservativen und einer rechtspopulistischen Partei auf Bundesebene wurde ab Ende des Jahres 2017 sogar vorübergehend die Stärkung der Sonderschulen im Regierungsprogramm propagiert. Ansonsten hat Österreich im Unterschied zu den beiden anderen deutschsprachigen Ländern Inklusion als gesellschaftspolitische und bildungspolitische Zielsetzung deutlicher proklamiert. So verabschiedete sich Österreich im Juni 2016 von der gemeinsamen Übersetzung der UN-Behindertenrechtskonvention durch die deutschsprachigen Länder und legte eine eigene Übersetzung vor. Diese, im österreichischen Bundesgesetzblatt veröffentlichte Fassung, übertrug ,,inclusion“ mit „Inklusion“, ,,accessibility“ mit „Barrierefreiheit“, independence“ mit „Selbstbestimmung“ und ,assistance“ mit „Assistenz“. Diese Begriffswahlen entsprachen den langjährigen Forderungen der Vertretungsorganisationen behinderter Menschen 
und zogen gleichzeitig auch die Konsequenz aus den Länderüberprüfungen durch den Ausschuss in Genf.

Eine integrative Ausbildung ist ein Mittel, um Jugendlichen mit Behinderungen nach Abschluss der Schulpflicht die Berufsausbildung zu erleichtern. Unterstützungssysteme für den Tertiärbereich sind unterentwickelt, was zum Teil auf die fehlende Gesetzgebung des Bundes zu diesem Thema zurückzuführen ist. Die Höhe der Unterstützung hängt also von den Leistungen ab, die von Universitäten, Ländern und Gemeinden erbracht werden und über die vorgeschriebenen Zuwendungen hinausgehen. Persönliche Assistenz ist ein wichtiger Faktor, um Studierende dabei zu unterstützen, positivere Ergebnisse im tertiären Bildungsbereich zu erzielen. Es besteht jedoch kein Anspruch auf solche Dienste, und ihre Verfügbarkeit hängt von der freiwilligen Bereitstellung durch Universitäten, Länder und Gemeinden $\mathrm{ab}$.

Österreich plante im Nationalen Aktionsplan Behinderung, das Sonderschulsystem durch die Entwicklung inklusiver Regionen zu ersetzen. Einige Regionen mit ohnehin bereits hohen Integrationsraten, wie die Steiermark im Südosten Österreichs, haben weitere Fortschritte in diese Richtung erzielt, während andere mit stärkeren Sonderschulstrukturen, wie Vorarlberg oder Tirol im Westen, stagnierten. Der Prozess der Einrichtung inklusiver Modellregionen im Land erhöht daher leider die bestehenden Ungleichheiten bei den Raten der Sonderschulung und der integrativen oder inklusiven Beschulung.

Ein problematisches Thema ist die Beschäftigungssituation von Menschen mit geistigen Behinderungen. Ein Projekt des Wissenschaftsfonds FWF untersuchte in einer bundesweiten Erhebung die Eingliederung von Menschen mit einer intellektuellen Beeinträchtigung, die ihre Schulbildung zuvor zum Teil in integrativen Strukturen erhalten hatten, in den Arbeitsmarkt in Österreich. Hierzu gab es eine bundesweite Befragung der Bezirksschulinspektor*innen und der Eltern für einen kompletten Jahrgang von Schulabsolvent*innen mit einer intellektuellen Beeinträchtigung (Fasching/Mursec 2010), eine Befragung der Träger arbeitsmarktpolitischer Unterstützungsmaßnahmen (Fasching/Koenig 2010) und aller Werkstattstandorte (Koenig 2010) für diese Gruppe. Als Resultat dieser umfänglichen Erhebungen zeigte sich, dass der integrative Weg der Beschulung, dort wo er eingeschlagen wurde, nach der Schulzeit abrupt endete und dass der Ersatzarbeitsmarkt aus Arbeits- und Beschäftigungstherapie über die Jahre hinweg weiter ausgebaut wurde und expandierte, statt zu inklusiven Arbeitsmarktstrukturen hinzuführen. Die Verfügbarkeit von Unterstützung für Arbeitsplätze auf dem allgemeinen Arbeitsmarkt ist nach wie vor sehr begrenzt.

\section{Inklusive Pädagogik in der Lehrer*innenbildung}

Einen ähnlich wichtigen Schritt wie die Einführung des Elternwahlrechts zwischen Integration und Sonderbeschulung in den 1990er Jahren, stellen die gegenwärtigen Umgestaltungen im Bereich der Lehrer*innenbildung dar. Es handelt sich um einen Prozess, der bereits 2009 mit einem Beschluss der Bundesregierung eingeleitet wurde, die einer Expert*innenkommission den Auftrag erteilte, die Strukturen der Ausbildung von pädagogischen Fachkräften für alle Altersgruppen von 0 bis 18 Jahren auf den Prüfstand zu stellen. Ein Expert*innenpapier aus dem Jahre 2011 machte eine Anzahl sehr weitgehender Vorschläge für strukturelle Veränderungen, die auch das System der bisherigen Ausbildung sonderpädagogischen Personals grundlegend reformierten (Braunsteiner/Spiel 2019). 
Passend zur Gliederung eines selektiven Schulsystems gab es bis 2014 zwei unterschiedliche Gruppen von Lehrkräften, die unterschiedlich lang an verschiedenen Institutionen ausgebildet wurden. Die Lehrkräfte an Pflichtschulen (Volksschule, Hauptschule bzw. Neue Mittelschule, Sonderschule) erhielten ihre Ausbildung an den Pädagogischen Hochschulen in einem dreijährigen, einphasigen Bachelorstudium. Lehrkräfte an Allgemeinbildenden und an Berufsbildenden Höheren Schulen studierten ca. 4 1/2 bis 5 Jahre an Universitäten und schlossen mit einem Magister-Grad ab. Diese Struktur endete mit dem neuen Lehrer*innenbildungsgesetz 2013, das ab dem Wintersemester 2014 bundesweit zu neuen Studienplänen führte. Mit einem vierjährigen Bachelorstudium und einem anschließenden $1 \frac{1}{2}$ bis 2 -jährigen Masterstudium ist die Studiendauer für angehende Pflichtschullehrkräfte etwa doppelt so lang wie zuvor. Statt der bisherigen Festlegung auf den Beruf Sonderschullehrer*in gibt es zukünftig ein einheitliches Primarstufenlehramt, innerhalb dessen Inklusive Pädagogik als Spezialisierung gewählt werden kann. Für das Sekundarstufenlehramt ist nunmehr eine ähnliche Struktur etabliert. Jede Sekundarstufenlehrkraft muss zwei Unterrichtsfächer im jeweils gleichen Ausmaß studieren. Im neuen System ist es möglich, eines der beiden Fächer durch eine Spezialisierung in Inklusiver Pädagogik zu ersetzen (Biewer/Proyer 2018).

Im neuen Lehrer*innenbildungssystem muss jede angehende Lehrkraft Grundkenntnisse in inklusiver Pädagogik erwerben. Die Möglichkeit, dass einige Sekundarschullehrer*innen ein Unterrichtsfach durch eine Spezialisierung in Inklusiver Pädagogik ersetzen können (z.B. Mathematik/Inklusive Pädagogik statt Mathematik/Chemie) trägt dazu bei, das vorhandene Wissen zu erweitern und neues Fachwissen für die künftige Entwicklung inklusiver Schulen zu generieren. Die Etablierung dieser neuen Struktur der Lehrer*innenausbildung ist eine vielversprechende Perspektive für die Zukunft, um einerseits alle Lehrkräfte in die Verantwortung für Schüler*innen mit Behinderungen zu nehmen, andererseits aber auch um notwendiges, vertieftes professionelles Wissen zur Spezialisierung bereit zu stellen.

Sowohl Neue Mittelschulen wie auch Allgemeinbildende Höhere Schulen haben seit 1996 das Recht Integrationsklassen zu bilden. Diese Möglichkeit wird von den AHSen aber selten genutzt. Eine wichtige Ursache für die ungleiche Verteilung stellt der unterschiedliche Habitus der Lehrkräfte an den Höheren Schulen dar, die sich vorwiegend als Wissensvermittler*innen verstehen. Dieser ist bedingt durch die überkommene Trennung der Ausbildungen an Universitäten und Pädagogischen Hochschulen. Mit dem einheitlichen Stufenlehramt für die Sekundarstufe besteht erstmals die Chance, diese Trennung der Lehrer*innenschaft nachhaltig aufzubrechen.

\section{Ausblick}

Auch wenn die Strukturen des Schulsystems sich in den deutschsprachigen Ländern sehr ähneln, so ist die unterschiedliche Entwicklung integrativer Systeme augenfällig, insbesondere im Vergleich zwischen Österreich und Deutschland (Biewer 2006), aber auch im Vergleich zur Schweiz. Unter den deutschsprachigen Ländern ist Österreich das einzige, das die rechtlichen Rahmenbedingungen für integrative Bildung bereits Mitte der 1990er Jahre geschaffen hat. Es ist zudem das einzige der drei Länder, das über eine einheitliche Bundesgesetzgebung einheitliche Strukturen in den Regionen generieren konnte.

Eine sehr engagierte Elternbewegung erreichte ab der Mitte der 1990er Jahre einen strukturellen Wechsel, der bis $2004 \mathrm{zu}$ einer konstanten Ausweitung der Quote integrativ 
beschulter Kinder führte. Die Versuche Inklusion flächendeckend umzusetzen und dabei Sonderschulen weitgehend zurückzubauen, waren regional unterschiedlich erfolgreich und stellen weiterhin eine wichtige Aufgabe der Bildungspolitik dar. Die Etablierung der neuen Lehrer*innenbildung mit der Ersetzung des Sonderschullehramtsstudiums durch die Spezialisierung in Inklusiver Pädagogik erscheint als langfristig nachhaltigste Maßnahme zur Etablierung inklusiver Bildung im Schulsystem.

\section{Literatur}

Anlanger, Otto (1993): Behinderten Integration. Geschichte eines Erfolges. Wien: Volk und Wissen.

Biewer, Gottfried (2006): Schulische Integration in Deutschland und Österreich im Vergleich. Erziehung und Unterricht - Österreichische Pädagogische Zeitschrift 156, 1-2, S. 21-28.

Biewer, Gottfried (2010): Inklusive Schule - Folgerungen für die Aus-, Fort- und Weiterbildung von allgemeinen Pädagogen und Pädagoginnen sowie Sonderpädagogen und Sonderpädagoginnen mit Blick auf die Konzeptionen der Bachelor- und Master-Studiengänge. In: EllgerRüttgardt, Sieglind/Wachtel, Grit (Hrsg.): Pädagogische Professionalität und Behinderung. Herausforderungen aus historischer, nationaler und internationaler Perspektive. Stuttgart: Kohlhammer, S. 159-165.

Biewer, Gottfried (2018): Schulische Inklusion in Österreich. In: Leonhardt, Annette/Pospischil, Melanie (Hrsg.): Internationale Ansätze zur schulischen Inklusion. Bad Heilbrunn: Klinkhardt, S. 80-95.

Biewer, Gottfried/Luciak, Michael (2010): Der internationale Vergleich in der Sonderpädagogik. In: Enzyklopädie Erziehungswissenschaft online, S. 1-19.

Biewer, Gottfried/Proyer, Michelle (2017): The Republic of Austria. In: Wehmeyer, Michael/Patton, James (Hrsg.): The Praeger International Handbook of Special Education. Band 2. Santa Barbara, Denver: Praeger, S. 239-248.

Biewer, Gottfried/Proyer, Michelle (2018): Lehrer/innenbildung ohne Sonderschullehramt. Chancen und Herausforderungen des Systemwechsels in Österreich. In: Sonderpädagogische Förderung heute 63,3 , S. 307-315.

BMASK (Hrsg.) (2016): Nationaler Aktionsplan Behinderung 2012 - 2020. Strategie der österreichischen Bundesregierung zur Umsetzung der UN-Behindertenrechtskonvention. Inklusion als Menschenrecht und Auftrag. Wien: Bundesministerium für Arbeit, Soziales und Konsumentenschutz.

Braunsteiner, Maria-Luise/Spiel, Christiane (Hrsg.) (2019): PädagogInnenbildung. Festschrift für Andreas Schnider. Heiligenkreuz: BeBe Verlag.

Engelbrecht, Helmut (2015): Schule in Österreich. Die Entwicklung ihrer Organisation von den Anfängen bis zur Gegenwart. Wien: New Academic Press.

Fasching, Helga/Koenig, Oliver (2010): Arbeitsmarktpolitische Unterstützungsmaßnahmen in Österreich. Dokumentation der bundesweiten Trägerbefragung arbeitsmarktpolitischer Unterstützungsmaßnahmen. Datenband II der dreibändigen Reihe "Die Übergangs-, Unterstützungsund Beschäftigungssituation von Menschen mit einer intellektuellen Beeinträchtigung in Österreich". Wien, Universität Wien. Online: https://vocational-participation.univie.ac.at/de/publikationen/2010/ [Zugriff: 20.09.2020].

Fasching, Helga/Mursec, Diana (2010): Schulische Ausgangssituation und Übergang in Ausbildung und Beruf in Österreich. Dokumentation der bundesweiten Befragung der Bezirksschul- 
inspektor/innen und Eltern. Datenband I der dreibändigen Reihe „Die Übergangs-, Unterstützungs- und Beschäftigungssituation von Menschen mit einer intellektuellen Beeinträchtigung in Österreich“. Wien: Universität Wien. Online: https://vocational-participation.univie.ac.at/ de/publikationen/2010/ [Zugriff: 20.09.2020].

Kerschbaumer, Franz Xaver (1999): Gemeinsame Erziehung von behinderten und nichtbehinderten Kindern im Kindergarten. Konzeptentwicklung, theoriegeleitete Diskussion und pädagogische Umsetzung in NÖ Kindergärten. St. Pölten: Amt der NÖ Landesregierung.

Klicpera, Christian (2007): Erfahrungen von Eltern und Schulaufsicht mit dem Elternwahlrecht in der Entscheidung über den Unterrichtsort. Wien, Berlin: List.

Koenig, Oliver (2010): Werkstätten und Ersatzarbeitsmarkt in Österreich. Dokumentation der Befragung der österreichischen Sozialabteilungen zu Stand und Umsetzung der Werkstättenstandorte in Österreich sowie der Befragung aller österreichischen Werkstättenträger und Standorte im Zeitraum 2008 - 2009. Datenband III der dreibändigen Reihe „Die Übergangs-, Unterstützungs- und Beschäftigungssituation von Menschen mit einer intellektuellen Beeinträchtigung in Österreich“. Wien: Universität Wien. Online: https://vocational-participation.univie.ac.at/ de/publikationen/2010/ [Zugriff: 20.09.2020]. 


\title{
14 Einflüsse von Bildungspolitiken auf biographische Verläufe in international vergleichender Perspektive erforschen: Erkenntnisse aus dem Quali-TYDES- Projekt
}

\author{
Tobias Buchner
}

\begin{abstract}
Das vorliegende Kapitel setzt sich anhand des Quali-TYDES-Projekts mit den Möglichkeiten zur Erforschung des Einflusses von Bildungspolitiken auf Personen mit Behinderungen in international vergleichender Perspektive auseinander. Dabei wird nach einer Einleitung der heuristische Rahmen des Projekts, der sich aus lebenslauftheoretischen Überlegungen und dem sozialen Modell von Behinderung zusammensetzt, entfaltet. Anschließend erfolgt eine methodologische Erörterung des im Projekt vertretenen Verständnisses biographischer Texte. Zudem wird dargelegt, was die damit verbundenen Annahmen für die Erforschung der Wirkungen von Implementierungsversuchen inklusiver Bildung auf Personen mit Behinderungen bedeuten. Im nächsten Abschnitt wird erörtert, wie spezifische Konzepte aus der Lebenslaufforschung für den internationalen Vergleich der Daten genutzt wurden. Zur Illustration der mit einem solchen method(olog)ischen Ansatz produzierbaren Ergebnisse wird das im Rahmen von Quali-TYDES entwickelte Konzept des ,mainstream pathway“ vorgestellt. Zum Abschluss erfolgt eine Diskussion der Potenziale, aber auch Limitationen des gewählten Designs.
\end{abstract}

\section{Einleitung}

In den letzten Jahrzehnten haben die meisten europäischen Staaten versucht, ihre Bildungssysteme inklusiver zu gestalten (Smyth et al. 2014). Diese Prozesse wurden einerseits durch entsprechende Forderungen der Behindertenbewegungen und Elterninitiativen vorangetrieben. Andererseits, und mit dem vorigen Punkt in Beziehung stehend, erfolgten die Bemühungen zum Aufbau inklusiver Bildungsstrukturen auch aufgrund eines sich etablierenden internationalen ,policy environment", das durch Dokumente wie das mittlerweile berühmt gewordene Salamanca Statement (UNICEF 1994), die Aktionspläne der Europäischen Union (z.B. Council of Europe 2006) sowie die Vorgaben von Menschenrechtskonventionen (hier vor allem die Konvention über die Rechte des Kindes (UN 1989) und die Konvention über die Rechte von Menschen mit Behinderungen (UN-BRK) (UN 2006)) geprägt wurde. Den Dokumenten zufolge sollen Kinder bzw. Jugendliche eine wohnortnahe (schulische) Bildung erhalten, in deren Rahmen sie an einer Regelschule ihren individuellen Bedürfnissen entsprechend unterstützt werden und lernen können - bzw. haben sie ein Recht auf eine solche 
Qualität von Bildung. Der sich in diesen Dokumenten manifestierende diskursive Wandel vom Paradigma der sonderschulischen Förderung zu einer inklusiven Bildung hat dazu geführt, dass inklusive Bildung auf den Agenden der Bildungspolitiken der meisten europäischen Länder steht. Neben den darin zum Ausdruck kommenden, scheinbar einheitlichen Zielsetzungen in puncto inklusiver Bildung zeigen sich allerdings starke Unterschiede hinsichtlich der Umsetzungsversuche und der dabei verfolgten Strategien. Diese Divergenzen hängen einerseits mit den jeweiligen historischen Entwicklungspfaden und damit auch Strukturen der Bildungssysteme zusammen (Richardson/Powell 2011). Andererseits zeigen sich in den Implementierungsstrategien zwischen den Ländern auch Differenzen hinsichtlich der Vorstellungen von dem, was schulische Inklusion bedeuten soll (Hardy/Woodcock 2015). Die genannten Aspekte stellen für sich bereits erhebliche Herausforderungen für einen wissenschaftlichen, internationalen Vergleich dar - verweisen aber gleichzeitig auf die potenziellen Vorzüge einer solchen Unternehmung. Schließlich kann der Aufbau inklusiver Bildungssysteme mit Verweis auf die obigen policy papers und Menschenrechtserklärungen (Heyer 2021 i.d.B., Biermann 2021 i.d.B.) als globales Projekt erachtet werden - und europäische, aber auch nationale Regierungen sowie DPOs, NGOs und weitere Akteur*innen können anhand eines wissenschaftlichen Vergleichs nicht nur eine Standortbestimmung vornehmen, sondern auch aus den Umsetzungsversuchen anderer lernen.

Betrachtet man das Feld der komparativen Forschung von Policies zu inklusiver Bildung, so zeigt sich, dass - analog zum Mainstream der vergleichenden Bildungsforschung - zur Bearbeitung der zuvor genannten Herausforderungen häufig auf quantitative Designs zurückgegriffen wird, wobei anhand von Indikatoren eine gewisse Kamparabilität angestrebt wird. Konstruktionen von Kategorien, die sich aus schulischem Setting und sonderpädagogischem Förderbedarf zusammensetzen, wie etwa die sogenannten Inklusions- und Exklusionsquoten, stellen ein prominentes Beispiel für einen solchen Ansatz dar (Biermann/Powell 2014; Powell 2017; Buchner et al. i.E.) - und in der Tat hat sich der besagte Zugang, zumindest auf der Ebene der Platzierungen, als ertragreich erwiesen. Schließlich können so gewisse Trends und Bewegungen in Relation zu Label und (Bildungs-)Raum über Zeit nachgezeichnet werden. Die über derartige Verfahren erzeugten Daten sagen jedoch lediglich etwas über die ,Oberfläche“ der Umsetzung inklusiver Bildung in den unterschiedlichen Staaten aus. Denn welche Qualität ,darunter', also welche Tiefenstruktur im Unterricht zu finden ist und wie die Bemühungen aus Sicht der involvierten Akteur*innen wahrgenommen werden, kann anhand solcher Daten freilich nicht bestimmt werden. ${ }^{1}$ Letzterer Aspekt - und hier insbesondere die Perspektive von Schüler*innen mit Behinderungen - hat allerdings in den letzten Jahren immer mehr an Bedeutung gewonnen. Dieser Anspruch generiert sich aus Diskursen zu ,voices' in den Disability Studies, wonach die ,Stimmen“ von Personen mit Behinderungen für die Bewertung der Qualität von Implementierungsversuchen von Policies zentral gesetzt werden sollten (Shah 2005; Gibson 2006); Perspektiven, die tatsächlich in der bildungsund sozialwissenschaftlichen Forschung häufig nicht berücksichtigt wurden (vgl. für den deutschsprachigen Raum Buchner/Koenig 2008) - was nicht nur angesichts der Normen inklusiver Bildung überrascht. Der Anspruch, die Perspektive von Personen mit Behinderungen bezüglich der Umsetzung inklusiver Bildung zu erforschen, lässt sich zudem aus den Anforderungen der Konvention der Rechte von Menschen mit Behinderungen ableiten. Schließlich heißt es hier in Artikel 31, dass die vertragsunterzeichnenden Staaten Daten produzieren müssen, mit Hilfe derer die Implementierung der Konvention überwacht werden

1 Siehe hierzu auch die Differenzierung zwischen Sicht- und Tiefenstrukturen in der empirischen Unterrichtsforschung (Thieme 2006). 
kann. Diese Daten sollten eine Beschaffenheit aufweisen, die dazu dienen kann, „Hindernisse, denen sich Menschen mit Behinderungen bei der Ausübung ihrer Rechte gegenübersehen, zu ermitteln und anzugehen“(UN-BRK, Art. 31 (2)). Somit stellt sich die Frage, wie ein solches Vorhaben - alternativ oder in Ergänzung zu quantitativen Verfahren - methodisch realisiert werden kann und welche Herausforderungen, aber auch Möglichkeiten damit verbunden sind (siehe hierzu auch die Debatte zur Entwicklung von menschenrechtsbasierten Indikatoren, vgl. Hirschberg 2012; BRK-Allianz 2013).

Qualitative Zugänge bieten Möglichkeiten, die Blickwinkel von Menschen mit Behinderungen als Adressat*innengruppe von Policies (zu inklusiver Bildung) zu erheben und ihre Erfahrungen sichtbar zu machen (Waldschmidt 2003). In den Disability Studies wurde insbesondere auf das Potenzial von Biographieforschung für die Untersuchung der Auswirkungen von Policies auf Menschen mit Behinderungen verwiesen (z.B. Priestley 2001, 2003; Shah/Priestley 2011; für den deutschsprachigen Raum: Pfahl 2011; Buchner 2018). Demnach würde ein biographieforscherisches Vorgehen Räume eröffnen, in denen Menschen mit Behinderungen ausführlich ihre Erfahrungen in lebensgeschichtlicher Perspektive artikulieren könnten. Hinsichtlich der damit verwobenen Diskurse um ,voice' wurde jedoch von Vertreter*innen der Disability Studies zurecht angemerkt, dass ein simples Erheben der ,Stimmen“ Gefahren birgt. So hat unter anderem Colin Barnes kritisiert, dass der oben erwähnte Anspruch in einigen Studien lediglich zu einer Romantisierung, vor allem aber Individualisierung der Erfahrungen von Menschen mit Behinderungen führe (Barnes 2003; Barnes/Sheldon 2007) - ohne die sozialen Kontexte, in welche diese eingebettet sind, zu hinterfragen. Daher sei es von Nöten, ,Stimmen“ nicht einfach unhinterfragt deskriptiv abzubilden, sondern Erlebnisse auf der Mikro- zu den Strukturen auf der Makro-Ebene in Relation zu setzen und eine entsprechende kritische theoretische Rahmung vorzunehmen (vgl. Freitag 2005) wozu Barnes das soziale Modell von Behinderung vorschlägt (Barnes 2003). So können im Hinblick auf Policies zu inklusiver Bildung die schulbiographischen Elemente von Erzählungen daraufhin befragt werden, ob diese auf Lernumwelten verweisen, die den Zielen bzw. Normen der Policies entsprechen, oder ob sich hierin Barrieren des Lernens eingelagert finden.

Wie Shah und Priestley betonen, kann Biographieforschung mit einer solchen kritischen Heuristik dazu beitragen, „the micro-level impacts of macro-level policies over time“ (Shah/Priestley 2010: 156) zu erforschen - womit ein weiteres Potenzial von Biographieforschung angesprochen ist: der Einfluss von Policies auf Subjekte über Zeit. Ein Aspekt, der in herkömmlichen Designs zur Erforschung der Wirkungen von Policies häufig nicht oder zu wenig berücksichtigt wird, wie Aspitzsch und Kolleginnen ausführen (Aspitzsch et al. 2008). Biographieforscherische Ansätze verfügen jedoch über die Möglichkeit, die Prozesshaftigkeit von Erfahrungen, Veränderungen über Zeit und damit auch die ,Nachhaltigkeit' von Policies im Sinne von deren Wirkung auf die Selbst-Bildung über verschiedene Lebensphasen hinweg zu rekonstruieren (ebd.). Demzufolge kann mit Hilfe eines biographischen Ansatzes potenziell auch in den Blick genommen werden, wie sich Übergänge zwischen Schulabschnitten gestalteten oder wie sich Erfahrungen inklusiver Bildung auf nachschulische Lebenswelten auswirken - womit Aspekte der, Qualität' von Policies in Zielperspektive inklusiver Bildung sowie deren Implementierung rekonstruiert werden können, welche in anderen Verfahren wenig oder auch schlicht nicht erhoben werden können.

Bis hierhin sollte bereits deutlich geworden sein, dass Biographieforschung spezifische Möglichkeiten bietet, den Einfluss von Policies im Allgemeinen und Implementierungsversuche inklusiver Bildung im Besonderen aus der Perspektive von Personen mit Behinde- 
rungen zu untersuchen. Unter anderem die Studie von Shah und Priestley (Shah/Priestley 2010, 2011), in welcher mit einem solchen Zugang der Einfluss von Bildungs- sowie Sozialpolitiken auf drei Generationen von Personen mit körperlicher Beeinträchtigung nachgezeichnet wurde, zeigt dieses Potenzial exemplarisch für Großbritannien auf. Trotz dieser Vorteile wurde innerhalb der sozial- und bildungswissenschaftlichen Biographieforschung als auch der Disability Studies kaum erprobt, wie ein solcher Ansatz für den internationalen Vergleich nutzbar gemacht werden könnte. An dieser Leerstelle setzte das Projekt QualiTYDES an. Im Projekt wurden mit einer Kombination aus Policy-Analyse und Biographieforschung die Einflüsse von Politiken auf Menschen mit Behinderungen, die in den 1980er Jahren geboren wurden, international vergleichend untersucht. Die dabei untersuchte Kohorte wuchs demgemäß in genau jenem Zeitraum auf, in dem die meisten europäischen Staaten eine Veränderung ihrer Bildungssysteme mit der Zielperspektive Inklusion anstrebten. Anliegen des vorliegenden Artikels ist es, das dafür entworfene Design detailliert darzulegen und anhand ausgewählter empirischer Ergebnisse die Auswirkungen des politischen Wandels im Bildungsbereich auf die Leben von jungen Menschen mit Behinderungen aus vier europäischen Ländern vergleichend nachzuzeichnen.

Dazu wird die folgende Gliederung vorgenommen. Zunächst wird ein Überblick über die Anliegen sowie die Struktur von Quali-TYDES gegeben. Anschließend wird der heuristische Rahmen, der sich aus lebenslauftheoretischen Überlegungen und dem sozialen Modell von Behinderung zusammensetzt, entfaltet. Das darauffolgende Kapitel ist einer methodologischen Erörterung des im Projekt vertretenen Verständnisses biographischer Texte gewidmet. Zudem wird dargelegt, was die damit verbundenen Annahmen für die Erforschung der Wirkungen von Implementierungsversuchen inklusiver Bildung auf Personen mit Behinderungen bedeuten. Im nächsten Schritt wird vorgestellt, wie spezifische Konzepte aus der Lebenslaufforschung für den internationalen Vergleich der Daten genutzt wurden. Zur Illustration der mit einem solchen method(olog)ischen Ansatz produzierbaren Ergebnisse wird das im Rahmen der Analyse von Quali-TYDES entwickelte Konzept des ,mainstream pathway 'vorgestellt. Zum Abschluss erfolgt eine Diskussion der Potenziale, aber auch der Limitationen des gewählten Designs.

\section{Das Quali-TYDES Projekt: Anliegen und Struktur}

Das Projekt Quali-TYDES (Qualitative Tracking with Young Disabled People in European States) setzte es sich zum Ziel, die Einflüsse von Wohlfahrtsstaatsregimen, Bildungsinstitutionen, Policies und Gesetzen auf die Lebensverläufe junger Menschen mit Behinderungen in unterschiedlichen europäischen Ländern zu untersuchen. ${ }^{2}$ Die internationale Projektpartnerschaft, bestehend aus Wissenschaftler*innen aus Irland, Spanien, Tschechischer Republik und Österreich, war darauf ausgerichtet, eine länderübergreifende komparative Betrachtung dieser Phänomene zu ermöglichen (Buchner/Biewer 2013). Die genannten Länder wurden für den Vergleich ausgewählt, da sie eine gewisse Vielfalt bezüglich nationaler Policy-Kulturen aufweisen als auch eine Bandbreite wohlfahrtsstaatlicher Regime repräsentieren. So

2 Das Projekt wurde aus Mitteln der European Science Foundation, des Fonds zur Förderung der wissenschaftlichen Forschung (FWF), des Irish Research Council for the Humanities and Social Sciences, des Ministerio de Ciencia e Innovación (MICINN) und der Czech Science Foundation finanziert. 
können in Referenz auf die Typologie von Esping-Anderson $(1990,1996)$ Irland als liberaler, Spanien und Österreich als konservativer und die Tschechische Republik als Wohlfahrtsstaat in Transition kategorisiert werden - Wohlfahrtsstaaten, die sich in der Verschränkung von sozialen Dienstleistungen sowie Versicherungs- und Arbeitsmarktsystemen unterscheiden und damit auch, so die These, im Kontext Behinderung unterschiedlich auf Lebensverläufe wirken. Zudem weisen alle vier Länder unterschiedlich strukturierte Bildungssysteme auf und wählten, was besonders für den Kontext dieses Artikels von Interesse ist, unterschiedliche Strategien der Implementierung von Bildungsangeboten für Menschen mit Behinderungen. Folgt man den Kategorien der European Agency for Special Needs and Inclusive Education (2003), so verfolgt(e) Spanien diesbezüglich einen so genannten one-track approach (Fokussierung auf den Aufbau eines umfassenden inklusiven Schulsystems), Irland und Österreich einen multi-track approach (Beschulung von Kindern und Jugendlichen mit Behinderungen in einem Spektrum aus Sonder- und inklusiven Schulen) sowie die Tschechische Republik einen two-track approach (Schüler*innen mit Behinderungen werden grundsätzlich in Sonderschulen unterrichtet), der allerdings in den späten 1990er Jahren in einen multitrack approach umgewandelt wurde. Bereits auf der Ebene der ausgewählten Staaten wird deutlich, dass für das Projekt ein diversifiziertes Sample angestrebt wurde, das letztlich aus insgesamt 107 Personen mit Behinderungen aus den vier Partnerländern bestand (Irland $n=20$, Österreich $n=34$, Spanien $n=19$, Tschechische Republik n=34), die in den 1980ern geboren waren. Diese Kohorte wurde hinsichtlich der Ziele des Projekts gewählt, da ihre Mitglieder in einer historischen Phase aufwuchsen, in der in den meisten europäischen Staaten politische Bestrebungen erfolgten, die Lebenssituation von Menschen mit Behinderungen zu verbessern - wie z.B. durch die ab den frühen 1980er Jahren einsetzenden Policies zu inklusiver Bildung, zu Deinstitutionalisierung oder der Arbeitsmarkintegration. Die Teilnehmer*innen unterschieden sich allerdings nicht nur bezüglich der Länder, in denen sie aktuell wohnen, sondern auf der Ebene der nationalen Samples hinsichtlich des Geschlechts, Alters (Bandbreite von 1980-1989 geborenen Personen), Herkunft (in allen nationalen Samples wurden Teilnehmer*innen mit einem so genannten ,Migrationshintergrund' rekrutiert), Region (rural vs. urban, unterschiedliche Bundesländer) sowie der Beeinträchtigungsform (visuell, auditiv, physisch, kognitiv).

Im Projekt wurde Biographieforschung mit kritischer Policy-Analyse kombiniert. Dazu wurden mit den Teilnehmer*innen im Frühjahr und Sommer 2011 biographische Interviews sowie mit ausgewählten Fällen in den beiden folgenden Jahren Follow-up-Interviews durchgeführt. Mit diesem Design sollte einerseits der Einfluss von Policies und den darüber verbundenen institutionellen Umwelten, Unterstützungsleistungen, etc. auf die bisherige biographische Entwicklung nachgezeichnet werden. Andererseits sollte über das Längsschnittdesign ermöglicht werden, die Auswirkungen von rezenten Policies bzw. Implementierungsbemühungen der UN-Konvention auf die Leben der Teilnehmer*innen zu erforschen. Zur Analyse wurden schließlich für alle Länder Dokumentenanalysen von Policies und Gesetzen erstellt, die sich auf unterschiedliche Kontexte von Behinderung bezogen (z.B. für Österreich die Novellierungen des Schul-Organisationsgesetzes in Richtung integrative Bildung oder das so genannte ,Behindertenkonzept ${ }^{6}$ des Sozialministeriums 1992). ${ }^{3}$ Im Rahmen der Dokumentenanalysen wurden Ziele, relevante Definitionen (z.B. von Behinderung oder integrativer Bildung), Rechtsansprüche, Design von Unterstützungsleistungen, etc. herausgearbei-

3 Für Österreich wurden aufgrund der föderalen Struktur des Staates sowohl Policies und Gesetze auf der Ebene des Bundes, aber auch jener Bundesländer durchgeführt, aus denen die Teilnehmer*innen kamen (Oberösterreich, Steiermark, Tirol, Wien). 
tet. So wurde ein Kontextwissen für die Interpretation der biographischen Texte erarbeitet. Denn diese konnten nun danach befragt werden, welche Möglichkeiten die Policies auf der Ebene des individuell Erlebten kreierten - und ob die hier rekonstruierbaren Implementierungen auf der Mikroebene den Zielsetzungen von Policies entsprachen.

\section{Heuristischer Rahmen des Projekts}

Der heuristische Rahmen von Quali-TYDES setzte sich aus dem sozialen Modell von Behinderung sowie lebenslauftheoretischen Überlegungen zusammen. Beide Komponenten werden zunächst vorgestellt und anschließend aufeinander bezogen.

Quali-TYDES verortete sich in den Disability Studies, einer interdisziplinären Forschungsrichtung, die nicht zuletzt auch aufgrund ihrer Herkunft als ,akademischer Arm der Behindertenbewegung' überwiegend darauf ausgerichtet ist, Behinderung als soziales Phänomen zu erforschen - im Sinne eines ,behindert Werdens' (Cloerkes 2003) bzw. der sozialen Konstruktion von Behinderung (UPIAS 1975; Oliver 1990). Zufolge des sozialen Modells von Behinderung ist nicht die im medizinischen Sinne vorliegende Beeinträchtigung (impairment) als Behinderung zu denken, sondern die von der Gesellschaft in Replik auf die Beeinträchtigung hervorgebrachten, im eigentlichen Sinne behindernden Strukturen:

It is not individual limitations, of whatever kind, which are the cause of the problem but society's failure to provide appropriate services and adequately ensure the needs of disabled people are fully taken into account in its social organisation. Further, the consequences of this failure does not simply and randomly fall on individuals but systematically upon disabled people as a group who experience this failure as discrimination institutionalised throughout society (Oliver 1990, o.S.).

Demnach handelt es sich bei Menschen mit Behinderungen um eine Personengruppe, die aufgrund gesellschaftlicher Barrieren, wie physische Hindernisse, aber auch stereotype Denkmuster und institutionelle Diskriminierungsformen, systematisch benachteiligt wird. Dementsprechend gilt es für die Forschung, diesen theoretischen Prämissen folgend, Barrieren und Unterdrückungsmechanismen, welche häufig verdeckt auf die Leben von Menschen mit Behinderungen wirken, empirisch freizulegen und zu thematisieren. Dadurch soll eine wissenschaftlich fundierte Kritik an diskriminierenden Praktiken und Strukturen entstehen, die letztlich nicht als akademischer Selbstzweck, sondern als Mittel zum Abbau von Barrieren dienen soll (Finkelstein 2001). Im Rahmen von Quali-TYDES wurde das soziale Modell von Behinderung wie erwähnt mit lebenslauftheoretischen Überlegungen verbunden.

Lebenslauftheorie setzt sich mit der Korrespondenz zwischen Individuum und Struktur über Zeit auseinander. So wird unter einer Doppelperspektive danach gefragt, wie Individuen im Laufe der Zeit durch soziale Strukturen geformt werden - und umgekehrt, wie Individuen ihre Lebenswege in sozialen Strukturen gestalten (Elder 1995). Dies bedeutet für Lebenslaufforschung, dass es darum geht, Leben(swege) in Relation zu ihren (sozialen) Kontexten $\mathrm{zu}$ untersuchen (Moen et al. 1995) - und hierbei interessieren vor allem die Umwelten der Institutionen des Lebenslaufs, welche von Individuen in einer bestimmten Abfolge durchlaufen werden müssen - wie es im Konzept des institutionalisierten Lebenslaufs zum Ausdruck kommt. Der institutionalisierte Lebenslauf, der von Kohli für das fordistisch geprägte Deutschland der 1950-1970er Jahre nachgezeichnet wurde, stellt eine Anordnung dar, die um das Erwerbssystem herum organisiert und in drei Phasen gegliedert ist, die durch ver- 
schiedene Institutionen strukturiert werden (Kohli 1985). Die Lebensphasen der Kindheit und Jugend werden von den Institutionen des Bildungssystems, insbesondere des Kindergartens und der Schule, bestimmt. In dieser, Vorbereitungsphase“ erfolgt eine ,Ertüchtigung für den Arbeitsmarkt', die wiederum die zentrale Institution der zweiten, ,Aktivitätsphase ' darstellt, die mit dem Erwachsenenleben gleichgesetzt ist. Die abschließende dritte, als ,Ruhephase bezeichnete Sequenz, wird durch das Rentensystem bestimmt. Die skizzierten Lebenslaufphasen erweisen sich als durch institutionalisierte Übergänge miteinander verwoben und bestehen gleichzeitig selbst aus verschiedenen Übergängen und Subphasen (z.B. für die Vorbereitungsphase der Übergang zwischen Elementarbildung und Grundschule oder der Übergang zwischen der Primar- und der Sekundarstufe; Powell/Pfahl 2019). Sie stellen dementsprechend eine ,integrierte' Abfolge dar bzw. sollen das Prozessieren von Individuen durch eine solche Abfolge gewährleisten.

Kohlis Modell der Dreiteilung des institutionalisierten Lebenslaufs wurde zurecht bezüglich der zeitlichen Gültigkeit und eines geschlechtlichen Bias hinterfragt (diese Aspekte können aus Platzgründen nicht ausgeführt werden, siehe hierzu u.a. Dausien 1996; Sackmann 2013). Das Modell stellt aber trotz alledem einen Gewinn für die theoretische Rahmung des Quali-TYDES-Projekts dar, schließlich zeigt es die Strukturierung von Lebensverläufen durch miteinander verzahnte Institutionen und die Wirkmächtigkeit dieses Regimes in Hinblick auf Lebensverläufe sehr deutlich auf - gerade in Bezug auf die Funktion von Schule. So ist das Durchlaufen schulischer Sequenzen für Individuen (nach wie vor) obligatorisch (Schulpflicht) und stellt somit einen unvermeidbaren Abschnitt des Lebenslaufs eines Individuums dar (zumindest in Hinblick auf die Staaten, die im Quali-TYDES-Sample repräsentiert waren). Zudem wird der Einfluss von Schule auf das Postschulische deutlich: Die Bewertung schulischer Performanzen und Bildungszertifikate regeln demzufolge, welche Teilhabemöglichkeiten in nachschulischen Lebensphasen bestehen. Gerade im letzten Hinweis deutet sich ein Aspekt an, der selbstverständlich erscheint, für die Analyse von Lebensverläufen aber von wesentlicher Bedeutung ist: Das Modell des institutionalisierten Lebenslaufs zeichnet ein Schema, das einerseits von den Individuen europäischer Nationalstaaten durchschritten werden muss, andererseits aber unterschiedlich durchschritten wird. Mit anderen Worten: Das Lebenslaufregime führt zu einer Ausdifferenzierung von Lebensverläufen, die mit der Einweisung in unterschiedliche Statuspositionen und damit auch unterschiedlichen Möglichkeiten ökonomischer und auch sozialer Partizipation einhergeht. So werden in Österreich z.B. die Weichen für die Ausdifferenzierung von Bildungsverläufen durch den institutionell angelegten schulischen Übergang zwischen Primar- und Sekundarschule nach der vierten Schulstufe, in dessen Rahmen Schüler*innen auf unterschiedlich prestigeträchtige Tracks verteilt werden, bereits sehr früh gestellt. Im weiteren Durchschreiten der Statuspassage Schulzeit entscheidet sich schließlich, ob ein*e Jugendliche*r die Schule nach der obligatorischen Pflichtzeit mit einem niedrigen Bildungszertifikat oder nach der Oberstufe mit der Qualifikation für ein universitäres Studium verlässt. Schulverläufe unterscheiden sich demzufolge hinsichtlich der institutionellen Orte, ihrer zeitlichen Länge und des Levels von Befähigung für postschulische Lebensbereiche. Über die Vergabe unterschiedlicher Zertifikate wird letztlich geregelt, wer in welchen beruflichen Segmenten eine Lehre beginnen kann, wer welche Chancen zur Teilhabe am ersten Arbeitsmarkt hat, wer sich in welchen Studiengang einschreiben kann, etc. Neben diesen institutionell geprägten Strukturierungen hängt die Gestalt von Lebens- und insbesondere von Bildungsverläufen aber auch von den Ressourcen der Individuen ab, welche das Lebenslaufregime durchschreiten. Zahlreiche Studien haben gezeigt, dass der familiale Hintergrund von wesentlicher Relevanz für die 
Partizipation in Bildungseinrichtungen, vor allem der Schule, ist. Darauf wurde nicht zuletzt auch mit der Kapitaltheorie Pierre Bourdieus aufmerksam gemacht, wonach Bildungserfolg und, damit zusammenhängend, die Positionierung in der Gesellschaft, stark durch das kulturelle, soziale und ökonomische Kapital einer Person beeinflusst wird (Bourdieu 1986). Individuen nutzen (nicht immer bewusst) solche und andere Ressourcen, um ihre Lebensverläufe innerhalb der gegebenen institutionellen Strukturen zu beeinflussen. Zum Beispiel, wenn die Ausbildung zur Pilotin durch das Vermögen der Eltern finanziert oder wenn der Zugang zum prestigeträchtigeren Gymnasium ermöglicht werden kann, weil die Direktorin der Mutter noch einen Gefallen schuldet. Individuen verfügen also auf ihrer Route durch das Lebenslaufregime über Handlungsfähigkeit und beeinflussen ihre Lebensverläufe ganz konkret - im Falle fehlender oder geringer Ressourcen sind diese Gestaltungsmöglichkeiten jedoch reduziert.

Wie können die angeführten theoretischen Annahmen zu Lebenslauf und Lebensverläufen nun auf das weiter oben behandelte soziale Modell von Behinderung bezogen werden? Nun, eine solche Verzahnung bedeutet zunächst einmal, grob formuliert, Behinderungen in Lebensverläufen in den Blick zu nehmen - also danach zu fragen, durch welche Barrieren Individuen im Kontext des Lebenslaufregimes und seiner institutionellen Umwelten behindert werden und wie sich dies auf den weiteren Lebensweg auswirkt. Zum Beispiel durch fehlende Unterstützungsleistungen (Ressourcen) oder diskriminierende Praktiken von Professionellen der Bildungsadministration. So ist empirisch bereits belegt worden, dass die Strukturen des institutionalisierten Lebenslaufs sowie die damit verflochtenen Umwelten, insbesondere jene der Schule, die Form von Lebensverläufen sowie Möglichkeitsräume für Menschen mit Behinderungen entscheidend beeinflussen:

The evidence illustrates how policies, institutions, environments and relationships framed the choices for young disabled people and their families as they negotiated their lives. Public policies and institutions have a powerful influence in shaping life chances and choices (Shah/Priestley 2011: 2).

Konkreter kann mit Bezugnahme auf die Arbeiten von Priestley $(2001,2003)$ angenommen werden, dass in jeder Phase des Lebenslaufs über Institutionen, Wissensstrukturen und Normen Benachteiligungen für Personen mit Behinderungen produziert werden. So generieren sich sozial konstruierte, scheinbar ,vorgefertigte` Lebensentwürfe und -verläufe, die als ,typisch“ in gesellschaftlichen Erwartungshaltungen und Vorstellungshorizonten von Individuen sedimentieren. Zum Beispiel so genannte „Exklusionskarrieren“ (Wansing 2006: 99), die aufgrund der Prozessierung von Menschen mit kognitiven Beeinträchtigungen durch Sonderinstitutionen entstehen (von der Sonderschule in die Werkstatt und das Wohnheim) und häufig zu eingeschränkten bzw. lediglich spezifischen Teilhabemöglichkeiten führen.

Gegen diese institutionell angelegte Benachteiligung begehrten, wie bereits zu Beginn des Artikels erwähnt, Behindertenbewegungen und Elterninitiativen seit den 1980er Jahren in vielen europäischen Staaten auf, was zur Verabschiedung zahlreicher Policies führte, die das jeweilige Bildungssystem inklusiver machen sollten. Die Öffnung von Regelschulen für Kinder und Jugendliche mit Behinderungen sollte nicht nur Partizipation an den dortigen Lernprozessen und das Schließen von Freundschaften mit nichtbehinderten Peers, sondern auch den Erwerb der (im Vergleich zu Sonderschulabschlüssen) höherwertigen Bildungszertifikate und eine postschulische Fortsetzung der Lebensverläufe am ersten Arbeitsmarkt oder an Hochschulen ermöglichen. Unter der hier vorgeschlagenen Perspektive bleibt diesbezüglich allerdings danach zu fragen, inwiefern diese Policies tatsächlich Umwelten in den Regeleinrichtungen des Bildungssystems generierten, die ermöglichend wirkten, oder ob hierin 
nicht institutionelle Behinderungen eingelagert wurden, die zu einer Akkumulation von Benachteiligungen über Zeit führen - wie zum Beispiel jene Marginalisierungsprozesse, die mit der Etikettierung als Schüler*in mit sonderpädagogischen Förderbedarf einhergehen (Powell 2003). Eine solche Perspektive bedeutet schließlich, die Erfahrungen von Personen mit Behinderungen im Kontext der Institutionen des Bildungssystems in den Blick zu nehmen bezüglich der Qualität der institutionellen Umwelten, aber auch der Verläufe, der verfügbaren Ressourcen und der so bedeutenden Übergänge zwischen den Segmenten, insbesondere der ,großen “ Übergange Elementarbildung zur Primarstufe oder Schule zu Arbeitsmarkt/Studium. Bezüglich der Policies können diese Erfahrungen dann befragt werden, ob die Ziele der Politiken sich in den Erlebnissen auf der biographischen Mikroebene spiegeln, ob schulische Umwelten als ermöglichend oder behindernd wahrgenommen wurden, ob Schüler*innen mit Behinderungen von schulischer Seite die notwendigen Ressourcen erhielten (wie z.B. ein Tafellesegerät oder Assistenz) oder andere Ressourcen aktiviert werden mussten.

Im nachfolgenden Kapitel wird diese analytische Perspektive mit methodologischen Überlegungen verbunden und dargelegt, wie die vorangestellte Heuristik für den internationalen Vergleich nutzbar gemacht wurde. Dabei wird auf die Analyse des Datenmaterials fokussiert, das in der ersten Erhebungswelle von Quali-TYDES über biographische Interviews generiert wurde. Die Vorgehensweise zur Verzahnung dieser Daten mit den im Rahmen des Längsschnittdesigns (Follow Up Interviews) generierten Daten aus der zweiten sowie dritten Welle wird in diesem Artikel nicht behandelt.

\section{Biographische Erzählungen als Produkt von Text-Kontext- Relationen}

Als Mittel zur Datenerhebung wurde das narrative biographische Interview gewählt, um ein Datenmaterial zu produzieren, auf welches die zuvor entfaltete Heuristik ertragreich bezogen werden sollte. Dabei gilt es allerdings, die über narrative Interviews produzierten biographischen Texte als komplexe Gebilde zu verstehen; als Erzählungen, in denen Zeit auf verschiedenen Ebenen relevant und soziale Realität nicht einfach ,abgebildet' wird - wie es Vorstellungen von ,voice“ als ,authentische“ Lebensgeschichte mitunter suggerieren. Daher ist zunächst einmal zu klären, welche Auskunft ein solches Datenmaterial über das Leben einer Person überhaupt geben kann. Im Rahmen von Quali-TYDES wurde einem Verständnis gefolgt, wonach es sich bei den über narrative Interviews hervorgebrachten Daten um Texte handelt, die als Produkte spezifischer Kontexte zu denken sind (Text-Kontext-Relationen, Dausien 2006). Dieses Verständnis biographischer Texte soll im Folgenden umrissen werden.

Bei der Methode des narrativen biographischen Interviews handelt es sich um ein Verfahren, ,das die Erfahrungs- und Orientierungsbestände des Informanten bei weitgehender Zurücknahme des Forschereinflusses unter den Relevanzgesichtspunkten des Informanten möglichst immanent zu rekonstruieren versucht" (Schütze 1987: 254). Die Interviewer*in versucht dabei, durch eine offene Eingangsfrage eine möglichst umfassende lebensgeschichtliche (Stegreif-)Erzählung auf Seiten der interviewten Person zu stimulieren und sich während des Erzählens auf die Rolle der Zuhörerin zurück zu ziehen bzw. höchstens zu versuchen, die Erzählung durch Aufmunterungen weiter in Gang zu halten. Erst wenn die so 
genannte Haupterzählung (in der die Interviewpartner*in ihre Lebensgeschichte erzählt) beendet ist, sollten Nachfragen gestellt werden. Derart soll ein Setting geschaffen werden, in dem die interviewte Person idealer Weise ,ad hoc' versucht, eine Erzählung zu ihrem Leben zu produzieren, in der sie für sie relevante Erfahrungen und Erlebnisse zu einer biographischen Storyline zusammenfügt.

Erzähler*innen adressieren in diesen Vorgängen ihren umfangreichen ,Erfahrungsschatz' retrospektiv und konstruieren aus verschiedenen Elementen daraus eine Lebensgeschichte. Allerdings ist es von methodologischer Bedeutung, dass es sich bei diesen Erfahrungen nicht um so etwas wie eine ,authentische oder faktische Wiedergabe' vergangener, sozialer Wirklichkeit handelt, sondern - da Menschen ihre Erlebnisse fortwährend deuten um Interpretationen, die auf soziale Realitäten verweisen (Alheit/Dausien 1996). Biographische Erzählungen sind also keine ,eins zu eins'- Abbildungen sozialer Wirklichkeit (von Felden 2008: 14). Das ,erzählte Leben“ kommt aber ohne das ,gelebte Leben“ auch nicht aus, handelt es sich hierbei doch um den ,Rohstoff‘ für biographische Erzählungen (Alheit/Dausien 1996). Schließlich verweisen Erzählungen auf soziale Kontexte, die erlebt wurden, zu denen sich das Subjekt in Relation setzen muss(te) - und die das Leben sowie die Subjektivität der Person maßgeblich geprägt haben bzw. prägen. Was in einem biographischen Interview erzählt wird bzw. erzählt werden kann, welche Erfahrungen in einer Erzählung relevant gemacht werden, hängt also von den gelebten Erfahrungen ab, die einen wesentlichen Kontext des Textes bilden. Allerdings nicht nur, wie nachfolgend erläutert wird.

Die Rede von der Zusammensetzung spezifischer Elemente von Erfahrungen zu einer Geschichte verdeutlicht den Konstruktionscharakter von biographischen Erzählungen und auch, dass Erzähler*innen rhetorische Mittel und Erzählweisen einsetzen, wie z.B. Dramatisierungen, um ihrer Geschichte bestimmte Effekte zu verleihen und Wirkungen sozialer Kontexte auf das Selbst zu verdeutlichen. Schließlich sagt die Gesamtgestalt einer Lebensgeschichte, also die biographisierende Zusammensetzung von Erfahrungen, etwas über den aktuellen Blickwinkel auf die Vergangenheit, bzw. den biographischen Standpunkt, von dem aus über das bisherige Leben resümiert wird, aus (Schütze 1984). Erzählungen enthalten dementsprechend Bilanzierungen von Erlebnissen, die Hinweise auf so etwas wie die ,Moral der Geschichte' geben und zudem erklären sollen, wie die erzählende Person ,so wurde, wie sie heute ist ${ }^{\star}$. Der biographische Standpunkt bildet demzufolge einen weiteren Kontext, der auf den biographischen Text wirkt.

Wie und was erzählt wird, hängt allerdings nicht nur vom ,gelebten Leben', dem biographischen Standpunkt und den verfügbaren erzählerischen Mitteln ab, sondern auch von der sozialen Konstellation der Interviewsituation, die einen weiteren wichtigen Kontext der Textproduktion darstellt. So ist davon auszugehen, dass z.B. dis/ability, Geschlecht, sozialer Herkunft, Nationalität, Religion, sowie Migrationserfahrungen von Forscher*in und Interviewpartner*in die biographische Erzählung beeinflussen (können). Die damit verbundenen Diskurse und möglichen Positionierungen ,stehen` demgemäß während des Interviews ,im Raum' und beeinflussen das Sagbare bzw. auch das Fragbare. Ebenso wirkt aber auch die Vorgeschichte zum Interview auf die Erzählsituation; vor allem die Rekrutierung der Teilnehmer*in sowie die dabei kommunizierten Hintergründe und Ziele der Studie, in deren Rahmen das Interview stattfindet. Teilnehmer*innen überlegen aufgrund dieser Vorabinformationen vor und während dem Interview, welche Aspekte ihrer Erfahrungen interessant bzw. relevant sein könnten und was sie aus ihrem Erfahrungsschatz diesbezüglich berichten wollen. 
Zusammenfassend kann zu den vorigen Ausführungen nun gesagt werden, dass biographische Erzählungen keine bloßen Abbildungen von sozialen Realitäten darstellen, wie es etwa im Rahmen verkürzter Konzeptualisierungen von ,voice' suggeriert wird. Biographische Erzählungen enthalten viel mehr Hinweise auf soziale Kontexte und deren Auswirkungen auf das Subjekt - bei deren Rekonstruktion gilt es allerdings die oben genannten TextKontext-Relationen, die letztlich im Zuge der Textproduktion ineinander spielen, zu beachten. Im Rahmen von Quali-TYDES flossen Rekonstruktionen der sozialen Konstellation der Interviewsituation sowie der Rekrutierungsprozess in die Analyse ein (siehe ausführlich hierzu Buchner 2018). Für die Erforschung der Wirkungen von Policies auf schulische Umwelten bedeuten die obigen Ausführungen, dass die Erzählungen zur Schulzeit fokussiert werden - und ihre Verzahnung mit den daran angegliederten Institutionen des Lebenslaufs, wie z.B. Einrichtungen der Elementar-, der tertiären oder der beruflichen Bildung. Dabei wurde ein besonderes Aufmerksamkeitsinteresse auf jene Textelemente gelegt, die Schütze als soziale Rahmungen (Schütze 1981, 1984) bezeichnet. Soziale Rahmungen skizzieren „heteronome Systembedingungen lebensgeschichtlichen Handelns und Erleidens" (Schütze 1981: 99). Sie müssen nicht Gegenstand expliziter Elaborationen sein, sondern können sich auch dem Bewusstsein der Erzähler*in entziehen - von der Forscher*in jedoch rekonstruiert werden. So wurden bei der Analyse von schulbiographischen Sequenzen relevante Beschreibungen routinemäßiger professioneller Praktiken und pädagogischer Settings eingehender untersucht - vor allem Darstellungen integrations- bzw. inklusionspädagogischer Arrangements und spezieller Unterstützungsmaßnahmen. In vielen Erzählungen wurden systemstrukturelle Aspekte thematisiert, wie etwa die Schulwahl, Transitionen zwischen Schulstufen bzw. Schulen (sowie Übergänge zwischen Elementarbildung und Primarstufe, bzw. zwischen Schule und nachschulischem Leben) als auch die Wirkungen der institutionellen Kategorisierung als Schüler*in mit sonderpädagogischem Förderbedarf. Dabei galt es in den Blick zu nehmen, mit welchen Erfahrungsqualitäten diese Aspekte verbunden waren, also ob pädagogische Arrangements als den Bedürfnissen entsprechend, als ermächtigend oder eher besondernd wahrgenommen wurden; ob Interaktionen mit Peers als unterstützend beschrieben oder als Belegepisode für einen Marginalisierungsprozess angeführt wurden. Wie hier deutlich wird, wurde nicht nur das was des Gesagten (z.B. Akteur*innenkonstellationen, räumliche Aspekte, etc.) rekonstruiert, sondern auch das wie (z.B. aktive oder passive Formulierungen). Zudem wurden der biographische Standpunkt sowie die Gesamtgestalt der Lebensgeschichte in Relation zu den Institutionen des Lebenslaufs rekonstruiert. Derart wurden verschiedene zeitliche Ebenen bzw. Text-Kontext-Relationen bedacht und hinsichtlich ihrer Bedeutungen für das Erkenntnisinteresse des Projekts reflektiert. Für den internationalen Vergleich wurde, darauf aufbauend, eine spezifische Vorgehensweise mit einem besonderen Analyseraster entwickelt, wofür Konzepte der empirischen Lebenslaufforschung adaptiert wurden.

\section{Analysen biographischer Texte in international vergleichender Perspektive}

Auf nationaler Ebene wurden die biographischen Texte zunächst einer Grobanalyse unterzogen. Im Rahmen dieser wurden formale Übersichten zum Interviewverlauf, der sequenziellen Abfolge der thematisierten Inhalte, dominante Sprechweisen, Rekonstruktionen der sozialen 
Rahmung des Interviews sowie Skizzen zum biographischen Standpunkt erstellt. Die im Zuge der Grobanalysen gewonnenen Befunde stellten die Basis für biographische Kurzportraits dar, die eine chronologische Darstellung des biographischen Verlaufs, Hinweise auf biographisch besonders bedeutsame Ereignisse sowie Vermerke zur erzählerischen Darstellung der Lebensgeschichten enthielten. Die Portraits erfüllten eine orientierende Funktion in dieser Phase der Auswertung; konnte anhand von ihnen doch eine erste Übersicht zu ähnlichen Verläufen bzw. auch Kontrasten zwischen den Biographien generiert werden. Portraits ausgewählter Fälle, die sich nach der ersten Durchsicht des Materials als besonders markant hinsichtlich spezifischer Verlaufsformen erwiesen hatten, wurden schließlich für eine erste gemeinsame Sichtung durch das Quali-TYDES Konsortium auf Englisch übersetzt, in der erste Thesen zu länderübergreifenden Aspekten, aber auch zu Differenzen zwischen der Beschaffenheit der nationalen Datensätze, erstellt wurden.

Im nächsten Schritt wurden fünf sensibilisierende Konzepte, die aus der Lebenslauftheorie entnommen wurden, an die Fälle herangetragen: Trajekte, Transitionen, Wendepunkte (Turning Points), Ressourcen und Pathways. Diese Konzepte wurden in der Lebenslaufforschung überwiegend zur Analyse quantitativer Daten angewendet, auch wenn sie im Laufe der letzten Jahre zunehmend auch Anwendung in qualitativen Studien erfuhren (z.B. Shah/Priestley 2011). In der Literatur zeigen sich jedoch mitunter erhebliche definitorische Unterschiede, weshalb an dieser Stelle die im Rahmen von Quali-TYDES bestimmten Verständnisse der Begrifflichkeiten kurz umrissen werden sollen.

Trajekte stehen für Verläufe durch unterschiedliche institutionelle Sphären und Lebensbereiche - wobei vor allem die Richtung als auch die Geschwindigkeit eines Trajekts von Relevanz sind. Trajekte können zu analytischen Zwecken isoliert voneinander rekonstruiert werden (z.B. Gesundheitstrajekt, Familientrajekt oder Erwerbstätigkeitstrajekt), sie erweisen sich allerdings als miteinander verknüpft und ergeben das, was Elder als, life course trajectory' bezeichnet (Elder 1985). Trajekte bestehen ihrerseits wiederum aus Subtrajekten. So setzt sich das Bildungstrajekt eines Individuums aus den Trajekten der Elementarbildung, Primar- und Sekundarstufe(n) sowie möglicherweise Studientrajekten und Trajekten der Erwachsenenbildung zusammen. Aufgrund des Erkenntnisfokus von Quali-TYDES wurden in der Analyse besonders jene Trajekte in den Blick genommen, die durch die Institutionen des Lebenslaufs maßgeblich geprägt werden - insbesondere das Bildungstrajekt. Ziel der Auswertung war es, im Unterschied zu quantitativen Operationalisierungen des Konzepts, allerdings nicht eine bloße deskriptive Darstellung der Abfolge von institutionellen Stationen, Statuspassagen und den entsprechenden Stratifizierungseffekten zu leisten, sondern Erlebnisqualitäten, die Beschaffenheit sozialer Kontexte, in die Trajekte eingelagerte Barrieren aber auch ermöglichende Aspekte, nachzuzeichnen. So wurde in Bezug auf den schulischen Verlauf unter anderem die Beschaffenheit unterrichtlicher Settings, Lernerfahrungen, die mit den schulischen Umwelten verknüpfte Sozialität und deren Wirkung auf die Subjektivität der Biograph*in rekonstruiert.

Trajekte sind durch Transitionen miteinander verzahnt: „Trajectories [...] are themselves made up of transitions, or changes in state and role“ (Elder et al. 2006: 8). Das Konzept der Transition/des Übergangs ${ }^{4}$ wird in der soziologischen Forschung für Wechselphasen zwischen Rollen, Lebensaltern (z.B. Jugend - Erwachsenenalter), Beschäftigungsverhältnissen, Erwerbstätigkeit und Arbeitslosigkeit, etc. verwendet. Im Kontext des thematischen Schwerpunktes des vorliegenden Artikels interessieren besonders institutionell angelegte Übergänge

In diesem Artikel werden beide Begriffe synonym verwendet 
im Rahmen von Bildungstrajekten. Transitionen weisen das Potenzial für Disruptionen, Verzögerungen und Veränderungen (im Sinne einer Modifizierung der Richtung des Verlaufs) auf: Zum Beispiel das Wiederholen einer Jahrgangsstufe, den Wechsel vom Gymnasium auf die Realschule, etc. Übergänge unterscheiden sich bezüglich ihrer Bedeutungen und damit auch biographischen Tragweite. Als Beispiele für ,große' Übergänge gelten der Übergang von der Primarstufe in die Sekundarstufe sowie die Transition von der Schule in das Arbeitsleben. Mit dem sozialen Modell von Behinderung kann in Hinblick auf Übergänge danach gefragt werden, ob und wie Personen in diesen Prozessen behindert werden.

Transitionen können letztlich auch die Bedeutung eines turning points annehmen. Turning Points stellen einschneidende Erlebnisse in einer Biographie dar, die ein Trajekt unterbrechen bzw. das Leben einer Person in eine gänzlich neue Richtung lenken, wie zum Beispiel Flucht, die Trennung von einer Lebensgefährt*in, eine Kündigung, ein neues Arbeitsverhältnis, etc. Turning Points stehen also für einen bedeutenden Richtungswechsel von Trajekten: „Turning points involve a substantial change in the direction of one's life“ (ebd.). Als ein Exempel für einen Turning Point aus dem Bereich Schule kann auf die Erfahrungen eines Teilnehmers von Quali-TYDES verwiesen werden, der wegen des Umzugs seiner Familie in ein anderes österreichisches Bundesland aus einer als harmonisch erlebten Integrationsklasse in eine neues schulisches Setting kam, das von gewaltvollen Praktiken zwischen den Schüler*innen geprägt war (Buchner 2018).

Unter dem Konzept Ressourcen wurden Mittel und Dinge subsumiert, die eine Person für die Gestaltung eines Trajekts nutzt oder die für sie in unterstützender Absicht genutzt werden. Ressourcen verweisen in besonderer Form auf das lebenslauftheoretische Axiom der Interdependenz von Lebensverläufen (Hutchison 2011), besonders in Hinblick auf soziale Ressourcen, aber auch darüber hinaus. Die Unterstützung durch eine wohlmeinende Lehrerin kann dementsprechend als Ressource gelesen werden, aber auch eine soziale Dienstleistung (z.B. Persönliche Assistenz) oder das finanzielle Vermögen einer Familie. Wie weiter oben bereits erwähnt, stellt Bourdieus Kapitalientheorie einen brauchbaren Rahmen für das Verständnis von Ressourcen dar, insbesondere in Bezug auf Bildungsprozesse. Im Laufe des Auswertungsprozesses erwiesen sich zudem Ausdifferenzierungen des Konzepts ,soziales Kapital' als ertragreich, die von Allan und Kolleginnen in Hinblick auf Bildungsprozesse vorgenommen wurden (bonding, bridging and linking social capital, Allan et al. 2009). Wesentlich für die Analyse bezüglich der Wirkungen von Politiken ist auch die Identifizierung von nicht vorhandenen Ressourcen in Bildungstrajekten - obwohl sie der Person zugestanden hätten bzw. im System verankert hätten sein sollen.

Der Pathway wurde im Projekt als sensibilisierendes Konzept für den Vergleich von Trajekten verwendet. Pathways ,illuminate structure“ (Pallas 2006: 168) und zwar im Hinblick auf Muster von Lebensverläufen die als Effekte von Lebenslaufregimen verstanden werden können. Pathways stehen diesbezüglich für ähnlich verlaufende Trajekte, die durch ähnliche institutionelle Umwelten und strukturelle Aspekte geprägt werden, wie zum Beispiel die Einspurung in den weiter oben bereits erwähnten Sonderlebenslauf. Bildungs- Pathways sind häufig durch Stratifizierungsmechanismen gekennzeichnet, wie ,ability ${ }^{`}$ oder ,age grouping' (Natriello 1994). Schulische Pathways im Kontext von Behinderung beinhalten oft Schemata institutioneller Diskriminierung - wie die Einsortierung in die Kategorie Sonderpädagogischer Förderbedarf als Voraussetzung zur Teilhabe an Bildung.

Die Analyse des empirischen Materials unter dem zuvor erörterten Aufmerksamkeitsschwerpunkt erscheint angesichts der im vorigen Kapitel thematisierten Komplexität biographischer Erzählungen zunächst als relativ enge Fokussierung auf spezifische Aspekte von 
Leben(sverläufen). Die Konzeptionalisierung des beschriebenen Analyserasters erwies sich aber letztlich als theoretisch fundierter Blickwinkel, mit dem genau jene Aspekte von Biographien gezielt betrachtet werden konnten, die stark durch institutionelle Strukturen beeinflusst sind - ohne die Handlungsfähigkeit von Subjekten zu unterschlagen. Zudem wurden, wie oben bereits deutlich geworden ist, die Konzepte nicht bloß auf ihre ,faktische ' Ebene reduziert (z.B. eine Transition von einer Hauptschule auf ein Gymnasium), sondern die Vielschichtigkeit biographischer Erzählungen wie Erlebnisqualitäten, Sprechweisen, Deutungen aus dem Heute und Wirkungen auf das Selbst analytisch berücksichtigt. Diese Form von Schwerpunktsetzungen ermöglichten es darüber hinaus, Biographien aus den unterschiedlichen Ländern zu vergleichen, wie noch eingehender gezeigt werden wird.

Präzisiert werden soll an dieser Stelle noch, wie die Auswirkungen von Policies auf Lebensverläufe im Hinblick auf das empirische Material erforscht wurden. Hierfür wurde eine kritische Lesart der Texte entwickelt, für die das durch die Policy-Analyse produzierte, breite Hintergrundwissen zu Zeitpunkten, Anliegen, administrativen Aspekten sowie Umsetzungsweisen von Policies unabdingbar war. Zum Beispiel für Österreich die Modalitäten des für die Primarstufe 1993 sowie die Sekundarstufe I 1996 eingeführten ,Elternwahlrechts` (Eltern dürfen formal wählen, ob ihr Kind in einer Regel- oder einer Sonderschule unterrichtet werden soll, müssen allerdings vor ihrer Entscheidung einen von der lokalen Bildungsadministration gesteuerten Beratungsprozess durchlaufen), Schemata der Umsetzung integrativer Bildung (Integrationsklasse, Einzelintegration, Kleinklassen) oder Regelungen zu Persönlicher Assistenz (PA) (Antragsberechtigung und verschiedene Modelle (Arbeitgeber*innen-Modell vs. PA als Sachleistungen) sowie die diesbezüglich existierenden Differenzen zwischen den Bundesländern. So konnten zum Beispiel in Bezugnahme auf das Konzept der Transition Erzählungen zum Übergang zwischen Kindergarten und Schulzeit danach befragt werden, ob diese auf eine tatsächliche Realisierung des Elternwahlrechtes hindeuteten, oder ob der mit dem Modell der Integrationsklasse verbundene kooperative Unterricht von zwei Lehrer*innen als gute Unterstützung im Sinne einer Ressource erfahren wurde oder eher nicht, ob der Erwerb einer Beeinträchtigung in der Schulzeit als Turning Point des Bildungstrajekts erlebt wurde oder ob die betreffende Person die entsprechende Unterstützung erhielt, um ihren Bildungsweg im gleichen schulischen Setting fortzuführen. Das Policy-informierte Wissen stellt somit eine Voraussetzung dafür dar, die rekonstruierbaren Umwelten, biographischen Verläufe und die darin eingelagerten Praktiken und Akteur*innen in Bezug zu den Zielsetzungen von Policies zu lesen, sprich in den Blick zu nehmen, welche ,Abdrücke'dieser Policies im Sinne von deren lokalen Umsetzungen sich in den Erzählsegmenten finden, wie diese erlebt wurden, ob sie ermächtigend angeeignet werden konnten, etc. Diese Aufmerksamkeitsrichtung geht allerdings über die Analyse des Gesagten, also der in Erzählungen enthaltenen Beschreibungen von Settings hinaus - denn ebenso ist es von Bedeutung, biographische Texte daraufhin zu beleuchten, was nicht gesagt wird oder werden kann - z.B. wenn eine Teilnehmerin erklärte, sie sei nach ihrem Unfall von der Sozialabteilung ihres Krankenhauses gleich an einen traditionellen Anbieter der Behindertenhilfe verwiesen worden (und die Person nicht über die Option der PA informiert wurde). Es gilt also in der Auswertung danach zu fragen, was wäre (aufgrund der Rechtslage, Policies, usw.) möglich gewesen, wurde dieses Potenzial umgesetzt oder nicht und was waren die Gründe hierfür? Wie wird über spezifische Elemente, die in Relation zu Policies stehen, berichtet, werden sie in einer aktiven Sprache, die auf Aneignung und Handlungsfähigkeit hindeutet, erzählt? Werden Barrieren als stetig präsent im Bildungstrajekt gerahmt, etc. 
Die hier umschriebene, Policy-informierte, kritische Lesart förderte in international vergleichender Perspektive spezifische Gemeinsamkeiten zwischen den so diversen biographischen Erzählungen der Teilnehmer*innen von Quali-TYDES zu Tage - trotz unterschiedlicher Implementierungsstrategien, die nachfolgend skizziert werden.

\section{Policies in Richtung inklusiver Bildung in Irland, Österreich, Spanien und der Tschechischen Republik}

Im Folgenden werden zunächst die politischen Entwicklungen in Richtung inklusiver Bildung in den vier am Quali-TYDES beteiligten Länder nachgezeichnet, wobei aus Platzgründen lediglich eine relativ grobe Darstellung der Abläufe und Implementierungsstrategien geleistet werden kann. Wie anschließend anhand des empirischen Konzepts des ,mainstream pathway ' erläutert wird, führten die recht unterschiedlichen Bemühungen zu relativ ähnlichen Wirkungen auf der Mikro-Ebene - zumindest angesichts der rekonstruierbaren Lebensverläufe von jenen jungen Menschen mit Behinderungen aus den vier Ländern, die eine Regelschulkarriere verfolgten.

Wie an anderer Stelle ausführlich dargestellt (Smyth et al. 2014), unternahmen Irland, Österreich, Spanien und die Tschechische Republik in den letzten dreißig Jahren zahlreiche politische Bemühungen, ihre Bildungssysteme, insbesondere Schulen, inklusiver zu gestalten. Diese Politiken firmierten zunächst noch unter dem Schlagwort ,Integration', seit den 1990er Jahren lassen sich in den betreffenden Ländern allerdings zunehmend Verwendungen des Konzepts der inklusiven Bildung in politischen Leitpapieren finden. Vergleicht man die Entwicklungspfade dieser Policies miteinander, so zeigen sich in allen Ländern politische Bestrebungen in Richtung inklusiver Bildung, die auf nationaler Ebene aber relativ unterschiedlich gestaltet wurden.

Spanien kündigte bereits 1978 in Artikel 49 der Konstitution eine Strategie an, alle Menschen mit Behinderungen in das Regelschulsystem zu integrieren und dabei spezialisierte Unterstützung zur Verfügung zu stellen. Diese Strategie führte letztlich dazu, dass 1982 ein entsprechendes Gesetz verabschiedet wurde (vgl. ebd.). Der in Spanien verfolgte ,one track approach' führte zu einer stetig steigenden Anzahl von Schüler*innen mit Behinderungen in Regelschulen im Laufe der Jahre. In Österreich wurden ab Mitte der 1980er Jahre Schulversuche zur Integration von Schüler*innen mit Behinderungen durchgeführt. Aufgrund von deren positiven Evaluationen wurde in den 1990er Jahren das bereits erwähnte Elternwahlrecht in das Schulorganisationsgesetz aufgenommen. Anders als in Spanien wurden Sonderschulen allerdings nicht systematisch reduziert, sondern als Alternative beibehalten (multi-track approach). So stieg bis Ende der 1990er Jahre die Zahl der Kinder und Jugendlichen mit Behinderungen in Regelschulen bis auf rund 50\% an, stagnierte auf diesem Level aber bis Ende der 2000er Jahre (vgl. Buchner/Gebhardt 2011). Erst ab 2010 lässt sich wieder ein Aufwärtstrend ausmachen (61\% im Schuljahr 2016/2017, vgl. Buchner et al. i.E.). Eine relativ ähnliche Entwicklung lässt sich auch für Irland nachzeichnen, wo zunächst für die 1980er Jahre eine relativ zögerliche Entwicklung festgestellt werden kann, die seit den 1990er Jahren allerdings an Fahrt aufnahm und zur gesetzlichen Verankerung des gemeinsamen Unterrichts im Jahr 1998 führte. Seitdem wurden weitere Policies in Richtung inklusiver Bildung implementiert, allerdings stets im Rahmen eines ,multi-track approach', wobei die klare Mehrheit 
an Schüler*innen mit Behinderungen Regelschulen besuchten (73\%, EADSNE 2018). In der Tschechischen Republik führte der Systemwandel zu Beginn der 1990er Jahre auch zu einer langsamen Transformation des bis dahin verfolgten ,two-track ${ }^{6}$ in einen ,multi-track approach'. Nachdem 1991 ein Gesetz zur Ermöglichung des gemeinsamen Unterrichts für Schüler*innen mit körperlichen, visuellen und sprachlichen Beeinträchtigungen für die Primarstufe erlassen wurde, strebte das 2001 verabschiedete nationale Programm zur Transformation des tschechischen Bildungswesens die bestmögliche Förderung aller Schüler*innen an. 2005 wurde mit dem neuen Bildungsgesetz die individuelle Integration aller Schüler*innen mit Behinderungen in Regelschulen legistisch etabliert (bis dahin wurden Schüler*innen mit intellektueller Beeinträchtigung lediglich in Form einer ,Gruppenintegration ' (Sonderklassen) unterrichtet). Durch weitere Policies in Richtung inklusiver Bildung stieg die Zahl von Schüler*innen mit Behinderungen in Regelschulen in diesem Land bis heute stetig an (60\% im Jahr 2018, EADSNE 2018).

Auf der Ebene der Verankerung von Ressourcen im System zeigen sich schließlich Differenzen und Gemeinsamkeiten zwischen den Staaten. In allen vier Ländern wurde das in der Sonderschulära entwickelte Etikettierungs-Ressourcen-Schema auch auf den Kontext der Regelbeschulung übertragen: Kinder und Jugendliche mit Behinderungen werden als sonderpädagogisch förderbedürftig diagnostiziert, um spezialisierte Unterstützung in Regelschulsettings zu erhalten. Unterschiedlich sind diesbezüglich jedoch die Definitionen des sonderpädagogischen Förderbedarfs als auch deren beeinträchtigungsspezifischen Subsparten. In allen Staaten werden zusätzliche Bedarfe durch zusätzliches Personal abzudecken versucht, wobei sich wiederum verschiedene Unterstützungsmodelle abzeichnen, die von Schulassistenz einzelner Schüler*innen bis hin zu kooperativen Lehrsettings von mindestens zwei Lehrer*innen reichen.

\section{Regelschulverläufe zwischen Ermächtigung und Zumutung: Der ,mainstream pathway' als Korrelat politischer Bemühungen in Richtung inklusive Bildung}

Wie erwähnt, wird in der folgenden Ergebnisdarstellung auf ein Konzept referiert, das ein zentrales Resultat des im Rahmen von Quali-TYDES geleisteten länderübergreifenden Vergleichs darstellt: der , mainstream pathway'. So förderte der Vergleich von Bildungstrajekten, die in Regelschulsettings durchlaufen wurden, zwar einige Differenzen zu Tage, wie zum Beispiel unterschiedliche schulische Unterstützungsstrukturen. Bemerkenswerterweise zeigten sich jedoch relativ viele Gemeinsamkeiten - trotz der zuvor herausgearbeiteten unterschiedlichen Strategien der Transformation in Richtung inklusive Bildung in den Ländern. Der ,mainstream pathway‘ junger Menschen mit Behinderungen ist dementsprechend länderübergreifend durch ähnliche Möglichkeiten, aber auch ähnliche Formen von Barrieren gekennzeichnet, die zu spezifischen Verläufen und Subjektivitäten der betreffenden Individuen führen. ${ }^{5}$

5 Der Vollständigkeit halber sei darauf hingewiesen, dass die vergleichende Analyse der Bildungstrajekte von Quali-TYDES-Teilnehmer*innen neben dem ,mainstream pathway' auch starke Ähnlichkeiten von Verläufen durch segregierte schulische Settings zeigte. Dieser , segregated pathway` zeichnete sich unter 
Die Präsentation der Ergebnisse zum ,mainstream pathway ' erfolgt in einer komprimierten Form. So werden jeweils die charakteristischen Elemente des ,pathway verallgemeinernd beschrieben und anschließend anhand von Fallbeispielen aus dem Sample illustriert. Anschließend werden die Ergebnisse in Relation zu den politischen Bemühungen gesetzt.

Betrachtet man die Bildungsverläufe in Regelinstitutionen im Hinblick auf die Transition Schule zum postschulischen Leben, so ist zunächst einmal festzustellen, dass diese Trajekte relativ häufig Transitionen in tertiäre Bildungseinrichtungen oder Beschäftigungsverhältnisse am ersten Arbeitsmarkt ermöglichten. Wie viele der Interviewpartner*innen bilanzierend im Hinblick auf ihren Bildungsweg anmerkten, konnten sie sich in Regelschulen Wissen und die notwendigen Fähigkeiten aneignen, um in den besagten nachschulischen Lebensbereichen zu reüssieren. Insofern ermöglichte diese Form von Verläufen eine Ermächtigung und gesellschaftliche Teilhabe in Regelinstitutionen. Diese Verläufe sind jedoch neben den genannten ermöglichenden Aspekten auch durch den unsicheren Zugang zu den Räumen der Regelschule gekennzeichnet, insbesondere im Kontext von Transitionen. Solche Unsicherheiten zeigen sich bereits beim Übergang von der Elementarbildung auf die Primarstufe. So berichten einige Erzähler*innen von den enormen Bemühungen ihrer Eltern (zumeist ihrer Mütter), eine Regelschule zu finden, die sich bereit erklärte, ein Kind mit Behinderung aufzunehmen. Eltern sahen sich dabei in als ,Beratung' deklarierten Gesprächen mal implizit, mal explizit artikulierten Widerständen ausgesetzt - wie im Fallbeispiel Jane McCarthy, das dem irischen Sample entnommen ist.

In ihren Erzählungen führt Jane McCarthy ${ }^{6}$ aus, wie der Wunsch ihrer Mutter, ihre schwerhörige Tochter in einem Regelschulsetting unterrichten zu lassen, von Professio-

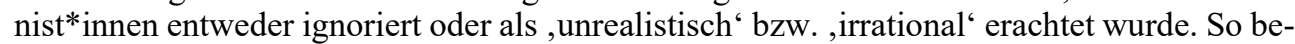
kam die Mutter laut der Biographin im Prozess der Schulsuche häufig nahegelegt, ihre Tochter an einer Sonderschule anzumelden - da dies von Professionist*innen als das geeignetere Setting zu deren Beschulung erachtet wurde. Dabei wurde der Wunsch zur Unterrichtung in der Regelschule von einem Lehrer gar als Ausdruck von Verleugnung (,denial') der Beeinträchtigung der Tochter erachtet. Nach einer Reihe von Absagen schaffte es die Mutter schließlich aber doch, einen Platz für ihre Tochter an einer Regelschule zu erhalten. An dieser Schule sah sich Jane McCarthy allerdings sehr häufig negativen Fähigkeitsbeurteilungen von Lehrer*innen ausgesetzt, die in Relation zur Beeinträchtigung gesetzt wurden. Implizit wurde dadurch von den Lehrkräften die Regelschule als ,passendes Setting' stets in Frage gestellt. Die sich in dieser Phase abzeichnende, negativ konnotierte Verlaufskurve und die darin eingelagerte Anbahnung einer Transition in ein segregatives schulisches Setting konnte letztlich durch den Wechsel an eine andere Regelschule durchbrochen bzw. verhindert werden. Die Lehrer*innen im neuen schulischen Setting wurden als unterstützend wahrgenommen und Jane McCarthy zeigte überwiegend gute Leistungen hier. Wie exemplarisch an diesem Fallbeispiel erläutert, wurde die Transition von der Elementarbildung in die Primarstufe für viele Teilnehmer*innen als mit wesentlichen Barrieren versehen erinnert. Der mühsam erstrittene Zugang konnte zudem, wie auch am obigen Beispiel deutlich wird, nie gänzlich als gesichert erachtet werden. Die betreffenden Sequenzen der biographischen Texte sind dementsprechend häufig von einer dramatisierenden Erzählweise gekennzeichnet, wobei insbesondere die Schulsuche zum familialen ,Kampf um Zugang zu Bildung in der Regelschule“

anderem durch einen starken Fokus auf Therapie und ein geringes Ausmaß an akademischem Lernen aus und führte häufig zu Übergängen in Werkstätten für Menschen mit Behinderungen oder in Schleifen von Qualifizierungsprogrammen (dazu ausführlicher Buchner 2019).

6 Bei allen in diesem Text verwendeten Namen handelt es sich um Pseudonyme. 
stilisiert wird. Wie auch im dargelegten Beispiel deutlich wird, wird dieser Konflikt als Kampf mit machtvollen Gatekeeper*innen inszeniert. Hierbei wird insbesondere auf Direktor*innen referiert, die trotz der Policies und Gesetzgebungen zum Recht auf gemeinsamen Unterricht (wie z.B. das Elternwahlrecht in Österreich) in der Lage sind, Kinder mit Behinderungen mit Verweis auf die mangelnde Barrierefreiheit von Schulen oder nicht entsprechend geschultem Personal abzulehnen.

Ähnliche Dynamiken zeigen sich letztlich auch in weiteren Transitionen, wie jener von der Primar- zur Sekundarstufe. Die biographische Wirkung derartiger Kämpfe um Zugang lässt sich anhand der nachfolgenden, aus dem Fallbeispiel Markus Oberndorfer entnommenen, Sequenz aufzeigen. In dieser thematisiert der Biograph seine Erfahrungen im Zuge des genannten Übergangs. Markus Oberndorfer und seine Mutter versuchen in dieser so bedeutenden Transitionsphase zunächst ein Gymnasium in der Nähe des Wohnorts zu finden, erhalten allerdings durchgehend Absagen - obwohl der Schüler über ein Zeugnis mit der Benotung ,sehr gut' in allen Hauptfächern verfügt (was an diesem Übergang als das gängige, relevante Kriterium zur Indizierung der so genannten ,Gymnasiumsreife‘ zu erachten ist). Im Zeugnis sind allerdings einige Nebenfächer mit dem Zusatz ,nach dem Lehrplan für körperbehinderte Kinder unterrichtet' versehen. Die Markierung als Schüler mit sonderpädagogischem Förderbedarf wird von den Direktor*innen schließlich mal implizit, mal explizit als Beleg für die Nicht-Eignung des Schülers für einen Gymnasiumbesuch herangezogen. Infolgedessen versuchen Markus Oberndorfer und seine Mutter Alternativen zur eigentlich präferierten Schulform zu finden.

MO: ja, dann ha dann hat sie's in, dann ham mas bei den Hauptschulen probiert in C-Stadt, die wollten auch nicht und die ham das, ich mein, Sportschule war sowie, Sportschule war sowieso, $\mathrm{ja}$, is nicht drin, is eh klar, ja, aber mir hat der Musikschuldirektor dezidiert gesagt, er nimmt mich nicht und [...] dann hat meine Mutter gesagt, so, jetzt meldet sie mich in die, in der Sonderschule an (MO: 849-872)

Der Übergang wird in der Passage als eine Art Absagemarathon geschildert. Die Weigerungen der Direktor*innen führen zu einer spürbaren Frustration auf Seiten des Schülers als auch seiner Mutter, die schließlich die Sonderschule als letzte Option auf Bildung in Erwägung zieht. Die damit verbundenen Erzählungen lassen die Schulsuche als eine Art ,downgrading'-Prozess erscheinen, in dessen Rahmen der Schüler implizit mitgeteilt bekommt, wo sein Platz innerhalb des Bildungssystems ist. Nach langer Suche findet Markus Oberndorfer allerdings doch noch ein relativ weit entfernt gelegenes Gymnasium, dessen Direktorin Willens ist, ihn aufzunehmen. Der hier exemplarisch aufgezeigte, für den ,mainstream pathway charakteristische ,Kampf um Zugang ' wirkt, verallgemeinernd betrachtet, nachhaltig auf die Subjektivitäten der Betroffenen. Schließlich lernen sie anhand der beschriebenen Erfahrungen, dass die Fortführung ihres Bildungstrajekts an einer Regelschule potenziell mit Verweis auf ihre Beeinträchtigung behindert werden kann bzw. eine Änderung der Richtung des Trajekts im Sinne eines erzwungenen Wechsels an eine weniger prestigeträchtige Schulform droht.

Die Darstellung von Direktor*innen als Ressourcen, über die Zugang zu Regelschulbildung ermöglicht bzw. aufrechterhalten werden konnte, findet sich in zahlreichen weiteren Fällen aus den anderen Ländern des Quali-TYDES-Samples und stellt ein weiteres Charakteristikum des ,mainstream pathway“ dar. Hier zeigt sich ein Spektrum von Direktor*innen, die selbst unkonventionelle Wege gingen, um ihre Schule barrierefrei zu gestalten, bis hin zu Direktor*innen, die zwar eine Aufnahme akzeptierten, aber ansonsten keinerlei besondere Unterstützung leisteten. Direktor*innen, die Zugang zur Regelschule gewährten, werden 
trotz der aufgezeigten Varianten in den Erzählungen rückblickend als ,Türöffner*innen ' erachtet, für deren Unterstützung die Biograph*innen auch heute noch dankbar sind. Denn die Erzähler*innen verfügen aufgrund der erlebten Bedrohungen des Verbleibs im Regelschultrajekt über ein Wissen um die Kontingenz dieses Verlaufs. Angesichts der Ergebnisse der Policy-Analyse kann jedoch gesagt werden, dass die meisten dieser Direktor*innen lediglich das realisierten, was gesetzlich vorgesehen war bzw. im Rahmen der Policies umgesetzt werden sollte (oder sogar noch weniger).

Zudem erweisen sich die Unsicherheit bezüglich passender Unterstützung und das Erleben von besondernden pädagogischen Settings als charakteristisch für den ,mainstream pathway'. Dies bedeutet nicht, dass die betreffenden Personen im Kontext von Regelschule durchgehend schlechte Unterstützung erfuhren. Schließlich berichten einige der im Zuge von Quali-TYDES interviewten Personen über didaktische Praktiken von Lehrer*innen, die ein gutes Lernen ermöglichten bzw. Teilhabe am Unterricht generierten. Hierbei handelt es sich um Erinnerungen an schulische Umwelten, die als temporäre inklusive Abschnitte des Schultrajekts gelesen werden können und häufig in der Primarstufe verortet werden - inklusive Umwelten, die sich aber nicht als durchgängiger Bestandteil des Regelschultrajekts erwiesen. Denn Teilnehmer*innen aus allen vier Ländern erlebten zumindest phasenweise schulische Settings, in denen sie nicht adäquat unterstützt wurden. Kritisiert wurde diesbezüglich mitunter ein zu starker medizinischer Fokus der Unterstützung, der auf eine Normalisierung von Beeinträchtigungen abzielte und besondernd wirkte, da die betreffenden Praktiken in einem räumlich separierten Setting parallel zum Unterricht stattfanden. Viele Biograph*innen berichten zudem von lediglich begrenzter oder manchmal auch gar nicht vorhandener Unterstützung, wobei sich ein gewisses Spektrum dieser Kategorie ausmachen lässt.

So erzählt beispielsweise Eduardo Herreira, ein Teilnehmer aus dem spanischen Sample, von mangelnder Unterstützung im Kontext seiner Sehbeeinträchtigung. Auch wenn Herreira auf eine überaus erfolgreiche schulische Karriere zurückblicken kann, so stellte die von einigen Lehrer*innen nicht erfolgte Anerkennung spezifischer Unterstützungsbedürfnisse des Schülers ein permanentes Spannungsfeld in seiner Schulzeit dar:

it has been a constant struggle with school and at university as well ... the needs that I had. I need more time on exams, I need the exam paper with large print, etc. Some teachers understood, but others didn't (Eduardo Herreira, in Buchner et al. 2015: 420).

Pavla Svoboda, ein Fall aus dem tschechischen Sample, berichtet ebenfalls von nicht passender Unterstützung, die sich im Unterschied zum vorigen Beispiel allerdings in starker Besonderung und der Zwangsverpflichtung von Peers als Assistent*innen äußerte. So erinnert die Biographin nach guten Unterstützungserfahrungen auf der Primarstufe ihren Klassenlehrer in der Sekundarstufe als stark paternalisierend. Seine besondernden Adressierungen evozierten bei der Schülerin über Zeit eine Selbstwahrnehmung als „disabled and poor“ (Biewer et al. 2015: 286). Aufgrund dieser Positionierung als hilfsbedürftig teilte die Lehrkraft letztlich vier Mitschülerinnen als Assistent*innen ein - gegen deren Willen, was letztlich zu Spannungen auf der Peer-Ebene führte.

Marianne Kaltenbrunner (österreichisches Sample) erlebte ihre professionellen Schulassistent*innen als unzuverlässig, da sie häufig fehlten. Ihre Lehrer*innen sahen sich laut der Biographin jedoch für das Sicherstellen einer adäquaten, durchgehenden Unterstützung nicht verantwortlich. 
MK: Das Problem war halt das ich wieder niemanden gehabt habe der mir hilft und ja, dann weiß ich noch einmal da war ich dann wirklich so fertig dass ich keine Ahnung wie lange auf der Toilette war und immer wieder probiert habe, dass ich aufstehe / und mir ist aber immer wieder schwindlig geworden

\section{TB: Mhm}

MK: Und dann habe ich einfach ja die Mama angerufen und gesagt: „Mama ich kann einfach nicht mehr aufstehen, weil mir so schwindlig ist.“"Und sie ist dann wirklich, es ist niemandem aufgefallen, dass ich nicht in der Klasse bin (MK: 324-339)

Mit der Anekdote illustriert die Biographin eine schulische Umwelt, die durch einen klaren Mangel an personalen Ressourcen gekennzeichnet ist. Die Schülerin Marianne Kaltenbrunner ist hier auf sich alleine gestellt und versucht den Toilettengang auch ohne ihre Assistentin, die an diesem Tag erneut fehlt, zu bewältigen. Aufgrund eines Schwindelanfalls ist sie gezwungen, auf der Toilette zu verbleiben - die Absenz der Schülerin fällt allerdings niemandem auf. In dieser Szenerie sieht sich die Schülerin letztlich gezwungen, ihre Mutter als Ressource zu aktivieren, womit ein weiteres zentrales Merkmal des ,mainstream pathway' angesprochen ist.

Denn in vielen Erzählungen zu den Erfahrungen in Regelschulen wird der besondere Einsatz von Eltern, insbesondere Müttern, hervorgehoben, worüber Partizipation ermöglicht werden konnte. Gerade Mütter unterbrachen nach Angaben der Biograph*innen ihre eigenen Arbeits- oder Bildungstrajekte, um sich ganz der Unterstützung ihrer Kinder widmen zu können. Bezüglich dieser Formen von care work zeigt sich in den biographischen Texten eine gewisse Skala. Diese reicht von der Unterstützung durch Mütter bei extracurricularen Aktivitäten, Exkursionen oder Klassenfahrten (da Lehrer*innen erklärt hatten, dass eine Teilnahme der Schüler*in ohne diese ,zusätzliche 'Unterstützung nicht in Frage käme), über die Assistenz für Toilettengänge (in Pausen) und das Überwinden baulicher Barrieren (beim Wechsel von einem Stockwerk in das andere), bis hin zu Fahrdiensten, da die Schulen, welche sich zu einer Aufnahme der Schüler*innen bereit erklärt hatten, häufig vom Wohnort relativ weit entfernt lagen. Mütter stellten demzufolge eine entscheidende Ressource für schulische Teilhabe dar.

Simon Harrauer, ein Teilnehmer aus dem österreichischen Sample, hebt in seiner Erzählung die Bedeutung seiner Mutter an verschiedenen Stellen hervor, wobei er überwiegend auf die Teilhabe ermöglichender Unterstützungsleistungen in verschiedenen schulischen Kontexten referiert, wie den Transport zur Schule, aber auch die Begleitung bei Exkursionen: „Volksschule/ auch da begleitete meine Mutter uns eben bei, ah Ausflügen“" (SH, 110-111). So äußert sich der Biograph, retrospektiv auf seine Schulzeit blickend, als äußerst dankbar gegenüber seiner Mutter, welche ihre berufliche Tätigkeit beendete, um sich besser der Unterstützung ihres Sohnes widmen zu können.

Jane McCarthy macht in ihrer Erzählung eine different gelagerte Form von care work relevant, nämlich Praktiken emotionaler Unterstützung. Neben der bereits weiter oben erwähnten Durchsetzung der Unterrichtung an einer Regelschule werden von der Biographin besonders die kontinuierlich erfolgende, von der Mutter geleistete Ermutigung zur Fortsetzung des Regelschultrajekts und die Zurückweisung ableistischer Deutungsmuster von Professionellen angeführt. Dieses care work ermöglichte letztlich den Aufbau von Resilienz, welche Jane McCarthy dazu befähigte, trotz der zahlreichen institutionellen Barrieren die Räume der Regelschule erfolgreich zu durchschreiten (vgl. Buchner et al. 2015). 
Ein weiteres Kennzeichen des ,mainstream pathway' stellen soziale Marginalisierungserfahrungen im Kontext von Peers dar. Auch diese sind allerdings keine das gesamte Trajekt durchziehende Erfahrung und werden nicht von allen Teilnehmer*innen erzählt, die ihre Schulzeit in Regelsettings verbrachten - aber von vielen. Einige Biograph*innen verknüpfen erlebte, ableistische Anfeindungen von Mitschüler*innen mit der Subjektposition ,Schüler*in mit sonderpädagogischem Förderbedarf* und den damit verbundenen, als besondernd erlebten pädagogischen Praktiken. Viele berichten zudem von Lehrer*innen, welche der Aggressionen durch Peers gewahr wurden, aber nichts dagegen unternahmen. Die betreffenden Schüler*innen konnten demzufolge auf keine Ressourcen des Schulsystems zurückgreifen. Marginalisierungserfahrungen, die überwiegend im Trajekt der Sekundarstufe stattfanden, werden von den Erzähler*innen oft als Erleidensprozesse dargestellt, die wesentliche Auswirkungen auf das Schultrajekt hatten.

KH: das war aber nicht nur irgendwie blödes Gerede oder so, sondern das ging über jeglichste Grenzen, die ein Mensch hat, also das war (langgezogen) // ja u' unter der Gürtellinie einfach, das ging in Bereiche, die mein Leben dermaßen irgendwie erschüttert haben [...] und das war auch so, dass ich auch wirklich in, nach der dritten Klasse, also wo die vierte begonnen hatte, nicht mehr in die Schule gehen konnte.

TB: $\mathrm{mhm}$

KH: Ich war am ersten Tag dort und habe gesagt, ich gehe da keinen Schritt mehr nie wieder rein (KH: 86-105)

In der ausgewählten Sequenz werden die tiefgehenden Verletzungen deutlich, welche die Schülerin Kathrin Horvath in der umrissenen institutionellen Umwelt erlitt (,ihr Leben wird erschüttert'). Auf sich alleine gestellt (auch Kathrin Horvath verweist in einer anderen Passage auf die mangelnde Unterstützung von Lehrer*innen gegen die Anfeindungen) erscheint der Schüler*in nur noch die Transition an eine neue Schule als Ausweg. Wie die Erzählerin an anderer Stelle ausführt, hinterließen die Erlebnisse an der Schule ,eine riesen-klaffende Wunde“ (KH: 875).

Steven O'Hara, Teilnehmer aus dem irischen Sample, berichtet ebenfalls von verletzenden Konfrontationen mit Peers, in deren Rahmen diese den Schüler in Verweis auf seine körperliche Beeinträchtigung verandernd positionierten. Als Basis für diese Praktiken werden in den schulbiographischen Erzählungen des Teilnehmers ableistische Diskurse deutlich, welche das Denken der Mitschüler*innen stark prägten:

and that was kind of oh there's a guy with some form of bloody disability in the school and, you know, the attitude back then was probably 'God I hope it's not contagious', you know (laughs) (SO: 452-454)

Den hier angeführten Beispielen ist allerdings hinzuzufügen, dass die Anfeindungen durch Peers und die Reaktionen der biographischen Subjekte darauf innerhalb der Fälle eine gewisse Bandbreite aufweisen. So berichtet Simon Harrauer von seinem erfolgreichen, für seine Opponenten durchaus schmerzhaften Widerstand und rahmt aus seinem heutigen biographischen Standpunkt heraus diese Erlebnisse als wichtige Erfahrung, da er anhand dieser lernen konnte, sich gegen ableistische Aggressionen zur Wehr zu setzen.

Was kann angesichts der zuvor beschriebenen Charakteristika des ,mainstream pathway bezüglich des Einflusses von Politiken im skizzierten Transformationsprozess in Richtung inklusiver Bildung gesagt werden? 
Nun, zunächst einmal verweisen die biographischen Erzählungen darauf, dass diese Politiken Spielräume für fortgesetzte Partizipation in Regelbildungseinrichtungen eröffneten. Insofern ermöglichten es die Policies in Richtung inklusiver Bildung der Generation der in den 1980er Jahren geborenen Menschen mit Behinderungen, im Unterschied zur, bis dorthin dominanten Beschulung in Sondereinrichtungen, sich für Regelschulkarrieren zu entscheiden. Gleichzeitig stellt der ,mainstream pathway 'ein spezifisches Verlaufsmuster durch das Regelschulwesen im Kontext von Behinderung dar, das von großen Herausforderungen und Barrieren geprägt ist. Wie ersichtlich wurde, führten die politischen Bemühungen auf der Makro-Ebene häufig nicht zu einem problemlosen Zugang zum Regelschulwesen und guter Unterstützung auf der Mikro-Ebene. Stattdessen zeigen die Lebensgeschichten, dass der Eintritt und der Verbleib in der Regelschule in allen Ländern trotz entsprechender gesetzlicher Grundlagen häufig mühsam erkämpft werden musste und gerade im Kontext von Übergängen bedroht wurde. Diese empirischen Befunde verweisen auf Verharrungstendenzen von Institutionen, die sich auf der Mikroebene der schulischen Umwelten dekuvrieren - und zwar in Form von gegenüber Menschen mit Behinderungen und ihren Familien relativ offen vorgetragener, aber auch kaschierter, Ablehnung von Seiten wirkmächtiger schulischer Gatekeeper. Gegen diesen lokalen Widerstand des Systems musste der Zugang oft erst erkämpft werden und dies, wie die Erzählungen zu den Unterstützungsleistungen der Mütter zeigen, unter Mobilisierung der zur Verfügung stehenden familialen Ressourcen. Die biographischen Texte liefern aber auch Hinweise darauf, dass die Policies Möglichkeiten auf Ebene der Schulen generierten, zumindest temporär inklusive Räume zu schaffen und einige Direktor*innen dazu veranlasste, Zugang zu gewähren. Die Erzählungen dieser Generation als biographische Zeug*innen des zähen schulischen Wandels verweisen jedoch auch darauf, dass es sich in allen vier Ländern nicht um eine tiefgreifende, systematische Transformation in Richtung inklusiver Bildung handelte; besonders nicht in jenen Ländern, die auf einen ,multi-track approach setzten. Denn über den Trajekten der Regelschule schwebte stets die Gefahr der Disruption bzw. der Stratifizierung , nach unten ' in einen weniger prestigeträchtigeren Track des Schulsystems, bis hin zur Sonderschule. Zudem erwiesen sich die schulischen Umwelten häufig als von ableistischen Feindseligkeiten von Peers geprägt, was in Kombination mit den durch schulische Strukturen und Praktiken hervorgerufenen Besonderungseffekten auf einen wesentlichen Problembereich der Umsetzungsversuche inklusiver Bildung innerhalb eines unverändert meritokratisch strukturierten Bildungssystems verweist. Durch die derart konstituierten schulbiographischen Umwelten wurde schließlich eine spezifische Erfahrungsqualität generiert, durch die Kinder und Jugendliche mit Behinderungen lernen, dass ihre ,Aufenthaltserlaubnis‘ in den Territorien der Regelschule stets bedroht ist und ihre Beeinträchtigung jederzeit zu schulischer Behinderung (Powell 2017) führen kann.

\section{Abschließende Betrachtungen: Der Beitrag einer lebenslauforientierten Biographieforschung zur Untersuchung von Bildungspolitiken}

Welche Vorteile, aber auch Limitierungen sind nun mit dem in diesem Artikel vorgestellten Ansatz zur Erforschung des Einflusses von Bildungspolitiken auf die Lebensverläufe von jungen Menschen mit Behinderungen verbunden? 
Allgemein betrachtet ermöglicht ein solcher Zugang Einblicke in die biographischen Erfahrungen zu institutionellen Umwelten, die im Kontext von bildungspolitischen Implementierungsbemühungen in Richtung Inklusion stehen. Die rekonstruierbaren schulbiographischen Räume verweisen auf die ,Abdrücke' solcher Policies, aber auch auf Mängel in der Umsetzung - und wie die darüber entstehenden Umwelten auf die darin platzierten Individuen wirkten. Durch die Erhebung des Blickwinkels der involvierten Subjekte können letztlich auch unter der Oberflächenstruktur, Regelschule 'verborgene, jedoch wie gezeigt wurde, äußerst wirkmächtige Mechanismen und Dynamiken freigelegt werden. Ein wesentlicher Mehrwert des biographieforscherischen Zugangs liegt schließlich in der Rekonstruierbarkeit der Wirkungen schulischer Kontexte auf das Innenleben der Subjekte über Zeit - also das Potenzial, die zu den biographischen Räumen der Regelschule in Relation stehenden Subjektivitäten nachzuzeichnen; in Betracht nehmen zu können, inwiefern die betreffenden Umwelten ermächtigend bzw. befähigend wirkten.

Anhand der biographischen Texte lassen sich allerdings die Wirkungen von spezifischen Policy-Elementen bzw. von Implementierungsstrategien nur bedingt nachweisen. Wie die Illustration empirischer Befunde gezeigt hat, konnten im Rahmen der Analyse zwar einige solcher Aspekte, wie die Lücken und Problematiken der Umsetzung des so genannten Elternwahlrechts, rekonstruiert werden. Die über Policies anvisierte didaktische Anlage von Lernprozessen oder die Wirkung von kooperativem Unterricht konnte allerdings in relativ wenigen Fällen nachgezeichnet werden - auch aufgrund des zeitlichen Abstands zu diesen biographischen Zeiträumen.

Die im Rahmen der Auswertung des Quali-TYDES-Projekts vorgenommene Interpretation des Materials unter lebenslauftheoretisch fundierten ,sensitizing concepts ${ }^{\text {} ~}$ erwies sich für die Analyse institutioneller Aspekte der Biographien als äußerst ertragreich. Auch wenn es sich hierbei um eine relativ starke Fokussierung handelte, zeigte sich dieser Analyserahmen als flexibel genug, um das internationale Datenmaterial in komparativer Perspektive auszuwerten:

The broad analytical framework demonstrated particular relevance in educational comparison, as concepts proved to be wide enough for analysing biographical experiences in varying education systems, and at the same time to be precise enough for describing developments in specific educational segments over time in a shared language (Buchner et al. 2015: 414).

Wie das Konzept des ,mainstream pathway“ verdeutlicht, können bei aller Unterschiedlichkeit zwischen den Teilnehmer*innen, ihrer sozialen Herkunft, Geschlechtern, Beeinträchtigungstypen sowie den unterschiedlichen Bildungssystemen und Policies in Richtung inklusiver Bildung wesentliche Gemeinsamkeiten identifiziert werden, welche die schulbiographischen Verläufe dieser Generation von Menschen mit Behinderungen in verschiedenen europäischen Ländern prägten und wesentliche Rückschlüsse auf Mängel in der Umsetzung der Politiken zulassen. Die Schwerpunktsetzung auf die lebenslauftheoretischen Konzepte erwies sich letztlich auch für die vertiefende international vergleichende Beleuchtung von spezifischen Aspekten des ,mainstream pathway' als vorteilhaft, wie zum Beispiel der Transitionen von der Regelschule in den tertiären Bildungsbereich (Biewer et al. 2015) oder eine Beleuchtung des Zusammenspiels von sozialem Kapital und Konstruktionen von Resilienz in ableistischen schulischen Umwelten (Buchner et al. 2015).

Die relativ ,groben' analytischen Matrizen ermöglichen, wie an den angeführten Fallbeispielen klargeworden sein sollte, einerseits die Subsumierung, aber auch differenzierte Betrachtung spezifischer Phänomene unter einer verzeitlichten Perspektive. So wird z.B. an den im vorigen Kapitel thematisierten, phasenweise nicht vorhandenen oder als unpassend 
empfundenen Unterstützungsressourcen deutlich, dass dieses Phänomen in unterschiedlichen institutionellen Passagen auftritt und Subjekte behindert. Trotz der entsprechenden temporalen und örtlichen Differenzen können diese anhand der theoretischen Matrix von QualiTYDES jedoch als übergreifendes, gewissermaßen , internationales ' Phänomen erachtet werden. Ähnlich verhält es sich mit der Gemeinsamkeit der Fallbeispiele hinsichtlich der identifizierten familialen Ressourcen, insbesondere in Person von Müttern. Hier konnten verschiedene Varianten von Care-Praktiken an verschiedenen Zeitpunkten von Schultrajekten festgestellt werden, die von den Biograph*innen als Voraussetzung für Zugang und fortgesetzte Teilhabe an Regelschule gerahmt werden. Diese Aspekte auf der Mikro-Ebene verweisen letztlich auf soziale Ordnungen, welche die unterschiedlichen Implementierungsversuche und Politiken inklusiver Bildung in differenten Settings, Lokalitäten und sozialen Konstellationen durchdringen, wie z.B. ein patriarchales Geschlechterverhältnis oder fähigkeitsbezogene Hierarchien.

Der im Rahmen von Quali-TYDES verfolgte, international vergleichende Ansatz ermöglicht zudem die Erforschung der Auswirkungen der besagten Policy-Regime auf die Subjektivitäten von Menschen mit Behinderungen in verschiedenen europäischen Ländern, also zu welchen Selbstbildungen die Zumutungen mangelnder Unterstützung, Verletzungen auf der Peer-Ebene, aber auch ,erlebter Inklusion' über Zeit führten. Auch hier zeigen sich, wie ersichtlich geworden sein sollte, bemerkenswerte Übereinstimmungen bezüglich der biographischen Haftung von als inklusiv intendierten Settings und Policies.

\section{Literatur}

Alheit, Peter/Dausien, Bettina (1996): Bildung als „,biographische Konstruktion“? Nichtintendierte Lernprozesse in der organisierten Erwachsenenbildung. In: Report. Literatur- und Forschungsreport Weiterbildung 37, 1, S. 33-45.

Allan, Julie/Ozga, Jenni/Smith, Geri (2009): Social Capital, Professionalism and Diversity. Rotterdam: Sense.

Aspitzsch, Ursula/Inowloki, Lena/Kontos, Maria (2008): The method of biographical policy evaluation. In: Aspitzsch, Ursula/Kontos, Maria (Hrsg.): Self-Employment-Activities of Women and Minorities. Wiesbaden: VS-Verlag, S. 12-18.

Barnes, Colin (2003): Disability Studies. What's the point? https://disability-studies.leeds.ac.uk/ wp-content/uploads/sites/40/library/Barnes-Whats-the-point.pdf [Zugriff: 20.06.2020]

Barnes, Colin/Sheldon, Alison (2007): ,Emancipatory“ Disability Research and Special Educational Needs. In: Florian, Lani (Hrsg.): The Sage Handbook of Special Education. London: Sage, S. 233-246.

Biermann, Julia (2021 i.d.B.): Der Einfluss der UN-BRK auf inklusive Bildung in Nigeria und Deutschland. In: Köpfer, Andreas/Powell, Justin J.W./Zahnd, Raphael (Hrsg.): Handbuch Inklusion international. Opladen: Budrich, S. 167-178.

Biermann, Julia/Powell, Justin J.W. (2014): Institutionelle Dimensionen inklusiver Schulbildung. Herausforderungen der UN-Behindertenrechtskonvention für Deutschland, Island und Schweden im Vergleich. In: Zeitschrift für Erziehungswissenschaft 17, 4, S. 679-700.

Biewer, Gottfried/Buchner, Tobias/Shevlin, Michael/Smyth, Fiona/Šiška, Jan/Kánová Sarka/Ferreira, Miguel/Toboso-Martín, Mario/Rodriguez Diaz, Susana (2015): Pathways to inclusion in European higher education systems. In: Alter - European Journal of Disability Research/Revue Européenne de Recherche sur le Handicap 9, 4, S. 278-289. 
Bourdieu, Pierre (1986): The Forms of Capital. In: Richardson, John G. (Hrsg.): Handbook of Theory and Research for the Sociology of Education. New York: Greenwood Press, S. 241258.

BRK-Allianz (Hrsg.) (2013): Für Selbstbestimmung, gleiche Rechte, Barrierefreiheit, Inklusion! Erster Bericht der Zivilgesellschaft zur Umsetzung der UN-Behindertenrechtskonvention in Deutschland. Berlin: BRK-Allianz. http://www.brk-allianz.de/attachments/article/93/parallelbericht_barrierefrei_layoutfassung.pdf [Zugriff: 21.04.2020]

Buchner, Tobias (2018): Die Subjekte der Integration. Biographie, Schule und Behinderung. Bad Heilbrunn: Klinkhardt.

Buchner, Tobias (2019): Die Scharniere der Segregation. Zur biographischen Wirkmächtigkeit von Fähigkeitskonstruktionen an Übergängen in (Sonder-)Bildungsverläufen. In: Sonderpädagogische Förderung, 2. Beiheft, S. 298-307.

Buchner, Tobias/Biewer, Gottfried (2013): Forschung zum Einfluss von Policies und Gesetzen auf die Leben von Menschen mit Behinderung: Das Quali-TYDES Projekt. In: Sehrbrock, Peter/Erdélyi, Andrea/Gand, Sina (Hrsg.): Internationale und Vergleichende Heil- und Sonderpädagogik und Inklusion. Bad Heilbrunn: Klinkhardt, S. 30-38.

Buchner, Tobias/Gebhardt, Markus (2011): Zur schulischen Integration in Österreich. Historische Entwicklung, Forschung und Status Quo. In: Zeitschrift für Heilpädagogik 62, 8, S. 289-304.

Buchner, Tobias/Koenig, Oliver (2008): Methoden und eingenommene Blickwinkel in der sonder- und heilpädagogischen Forschung von 1996-2006 - eine Zeitschriftenanalyse. In: Heilpädagogische Forschung 34, 1, S. 15-34.

Buchner, Tobias/Shevlin, Michael/Donovan, Mary-Ann/Gercke, Magdalena/Goll, Harald /Siska, Jan/Janyskova, Kristyna/Smogorzewska, Joanna/Szumski, Gregorsz/Vlachou, Anastasia/ Demo, Heidrun/Feyerer, Ewald/Deirdre Corby (i.E.): Same progress for all? Inclusive Education, the United Nations Convention on the Rights of Persons with Disabilities and Persons with Intellectual Disabilities in European Countries. In: Journal of Policy and Practice in Intellectual Disabilities.

Buchner, Tobias/Smyth, Fiona/Biewer, Gottfried/Shevlin, Michael/Ferreira, Miguel/TobosoMartín, Mario/Rodriguez Diaz, Susana/Latimier, Camille/Šiška, Jan/Kánová Sarka (2015): Paving the way through mainstream education. The interplay of families, schools and disabled students. In: Research Papers in Education 30, 4, S. 433-445.

Cloerkes, Günther (Hrsg.) (2003): Wie man behindert wird. Texte zur Konstruktion einer sozialen Rolle und zur Lebenssituation betroffener Menschen. Heidelberg: Winter.

Council of Europe (2006): Action Plan to Promote the Rights and Full Participation of People with Disabilities in Society. Improving the Quality of Life of People with Disabilities in Europe 2006-2015. Brussels: CE.

Dausien, Bettina (1996): Biographie und Geschlecht. Zur biographischen Konstruktion sozialer Wirklichkeit in Frauenlebensgeschichten. Bremen: Donat.

Dausien, Bettina (2006): Repräsentation und Konstruktion. In: Brombach, Sabine/Wahrig, Bettina (Hrsg.): LebensBilder. Leben und Subjektivität in neueren Ansätzen der Gender Studies. Bielefeld: transcript, S. 179-211.

Elder, Glenn H. Jr. (1985). Perspectives on the life course. In: Glenn H. Elder Jr. (Hrsg.): Life course dynamics. Ithaka: Cornell University Press, S. 23-49.

Elder, Glenn H., Jr. (1995). The life course paradigm: Social change and individual development. In: Phyllis Moen, Glenn H./Elder, Jr./Kurt Luscher (Hrsg.): Examining lives in context: Perspectives on the ecology of human development. Washington, DC: American Psychological Association, S. 101-140.

Elder, Glenn H., Jr./Kirkpatrick Johnson, Monica/Crosnoe, Robert (2006): The Emergence and development of Life Course Theory. In: Mortimer, Jeylan T./Shanahan, Michael J. (Hrsg.), Handbook of the Life Course. New York: Springer, S. 3-19. 
Esping-Andersen, Gøsta (1990): The three worlds of welfare capitalism. Princeton, New Jersey: Princeton University Press.

Esping-Andersen, Gøsta (Hrsg.) (1996): Welfare states in Transition. New York: Sage.

European Agency for Special Needs and Inclusive Education (EADSNE) (2018): European Agency Statistics on Inclusive Education. 2016 Dataset Cross. Odense, Denmark: European Agency for Development in Special Needs Education.

Finkelstein, Vic (2001): A Personal Journey Into Disability Politics. First presented at Leeds University Centre for Disability Studies. http://www.independentliving.org/docs3/finkelstein 01a.pdf [Zugriff: 03.09.2020].

Freitag, Walburga (2005): Contergan. Eine genealogische Studie des Zusammenhangs wissenschaftlicher Diskurse und biographischer Erfahrungen. Münster: Waxmann.

Gibson, Susanne (2006): Beyond a ,culture of silence'. Inclusive education and the liberation of ,voice'. In: Disability \& Society 21,4 , S. 315-329.

Hardy, Ian/Woodcock, Stuart (2015): Inclusive education policies. Discourses of difference, diversity and deficit. In: International Journal of Inclusive Education 19, 2, S. 141-164.

Heyer, Katharina (2021 i.d.B.): What is a Human Right to Inclusive Education? In: Köpfer, Andreas/Powell, Justin J.W./Zahnd, Raphael (Hrsg.): Handbuch Inklusion international. Opladen: Budrich, S. 45-57.

Hirschberg, Marianne (2012): Menschenrechtsbasierte Datenerhebung - Schlüssel für gute Behindertenpolitik. Berlin: Deutsches Institut für Menschenrechte. https://www.institut-fuermenschenrechte.de/fileadmin/_migrated/tx_commerce/Policy_Paper_Menschenrechtsbasierte _Datenerhebung_Schluessel_füer_gute_Behindertenpolitik_01.pdf [Z̄ugriff: 28.04.2020].

Hutchison, Elizabeth D. (2011): Life Course Theory. In: Levesque, Roger J.R. (Hrsg.) Encyclopedia of Adolescence. Springer, New York, NY.

Klieme, Eckhard (2006): Empirische Unterrichtsforschung: aktuelle Entwicklungen, theoretische Grundlagen und fachspezifische Befunde. In: Zeitschrift für Pädagogik 52, 6, S. 765-773.

Kohli, Martin (1985): Die Institutionalisierung des Lebenslaufs. Historische Befunde und theoretische Argumente. In: Kölner Zeitschrift für Soziologie und Sozialpsychologie 37, 1, S. 1-29.

Moen, Phyllis/Elder, Glen H. Jr./Luscher, Kurt (Hrsg.) (1995): Examining Lives in Context: Perspectives on the Ecology of Human Development. Washington, DC: American Psychological Association.

Natriello, Gary (1994): Coming together and breaking apart: Unifying and differentiating processes in schools and classrooms. In: Research in Sociology of Education and Socialization 10, 2, S. 111-145.

Oliver, Michael (1990): The Individual and Social Models of Disability. https://disability-studies.leeds.ac.uk/wp-content/uploads/sites/40/library/Oliver-in-soc-dis.pdf [Zugriff: 4.9.2019].

Pallas, Aaron M. (2006): A subjective approach to schooling and the transition to adulthood. In: Advances in Life Course Research 11, 2, 173-197.

Pfahl, Lisa (2011): Die Techniken der Behinderung. Der deutsche Lernbehinderungsdiskurs, die Sonderschule und ihre Auswirkungen auf Bildungsbiografien. Bielefeld: transcript.

Powell, Justin J. W. (2017): Räumliche Vielfalt der Inklusiven Bildung und sonderpädagogische Fördersysteme im Vergleich. In: Gercke, Magdalena/Opalinski, Saskia/Thonagl, Tim (Hrsg.): Inklusive Bildung und gesellschaftliche Exklusion. Wiesbaden: Springer VS, S. 25-38.

Powell, Justin J. W. (2007): Behinderung in der Schule, behindert durch Schule? Die Institutionalisierung der ,schulischen Behinderung“. In: Waldschmidt, Anne/Schneider, Werner (Hrsg.): Disability Studies, Kultursoziologie und Soziologie der Behinderung. Bielefeld: transcript, S. 321-343.

Powell, Justin J. W. (2003): Constructing Disability and Social Inequity Early in the Life Course. In: Disability Studies Quarterly 23, 2, S. 57-75. http://dsq-sds.org/article/view/414/575 [Zugriff: 20.08.2020]. 
Powell, Justin J. W./Pfahl, Lisa 2019. Disability and Inequality in Educational Opportunities in Life Course Perspective. In: Becker, Rolf (Hrsg.): Research Handbook on Sociology of Education. Cheltenham: Edward Elgar, S. 383-406.

Priestley, Mark (2003): Disability. A life course approach. Cambridge: Polity Press.

Priestley, Mark (Hrsg.) (2001): Disability and the Life Course. Global perspectives. Cambridge, Cambridge University Press.

Richardson, John G./Powell, Justin J. W. (2011): Comparing Special Education. Origins to Contemporary Paradoxes. Stanford/CA: Stanford University Press.

Sackmann, Reinhold (2013): Lebenslaufanalyse und Biographieforschung. Wiesbaden: VS-Verlag.

Schütze, Fritz (1987): Das narrative Interview in Interaktionsfeldstudien. Lehrbrief der Fernuniversität Hagen, Kurseinheit 1. Hagen: Fernuniversität Hagen.

Schütze, Fritz (1984): Kognitive Figuren des autobiographischen Stehgreiferzählens. In: Kohli, Martin/Robert, Günther (Hrsg.): Biographie und soziale Wirklichkeit. Neue Beiträge und Forschungsperspektiven. Stuttgart: Metzler, S. 78-117.

Schütze, Fritz (1981): Prozessstrukturen des Lebensablaufs. In: Matthes, Joachim (Hrsg.): Biographie in handlungswissenschaftlicher Perspektive. Nürnberg, S. 67-156.

Shah, Sonali/Priestley, Mark (2011): Disability and social change. Bristol: Policy Press.

Shah, Sonali/Priestley, Mark (2010): Home and away: the changing impact of educational policies on disabled children's experiences of family and friendship. In: Research Papers in Education 25, 2, S. 155-175.

Shah, Sonali (2005): Voices and choices. How education influences the career choices of young disabled people. In: Journal of Research in Special Educational Needs 5, 3, S. 112-117.

Smyth, Fiona/Shevlin, Michael/Buchner, Tobias/Biewer, Gottfried/Flynn, Paula/Latimier, Camille/Šiška, Jan/Kánová Sarka/Ferreira, Miguel/Toboso-Martín, M./Rodriguez Diaz, S. (2014): Inclusive education in progress. Policy evolution in four European countries. In: European Journal of Special Needs Education, 29, 4, S. 433-445.

Union of the Physically Impaired Against Segregation (UPIAS) (1975): Fundamental Principles of Disability. https://disability-studies.leeds.ac.uk/wp-content/uploads/sites/40/library/UPIAS-fundamental-principles.pdf [Zugriff: 21.07.2019].

UNICEF (1994): The Salamanca Statement and Framework for Action on Special Needs Education. New York: United Nations.

United Nations (1989): Convention on the Rights of the Child. New York: United Nations.

United Nations (2006): Convention on the Rights of Persons with Disabilities. New York: United Nations.

Vereinte Nationen (2006): Konvention über die Rechte von Menschen mit Behinderungen. https://broschuerenservice.sozialministerium.at/Home/Download?publicationId=19 [Zugriff: 18.06.2020].

von Felden, Heide (2008): Einleitung. Traditionslinien, Konzepte und Stand der theoretischen und methodischen Diskussion in der erziehungswissenschaftlichen Biographieforschung. In: von Felden, Heide (Hrsg.): Perspektiven der erziehungswissenschaftlichen Biographieforschung. Wiesbaden: VS-Verlag, S. 7-26.

Waldschmidt, Anne (2003): Wozu ein weiteres (Ethik)institut? Anmerkungen zur Eröffnung des Instituts Mensch, Ethik und Wissenschaft. In: Behindertenpädagogik 42, 3/4, S. 418-423.

Wansing, Gudrun (2006): Teilhabe an der Gesellschaft Menschen mit Behinderung zwischen Inklusion und Exklusion. Wiesbaden: VS Verlag. 



\section{B \\ Schulstrukturen, -kulturen und -praktiken}





\title{
15 Inklusive Unterstützungsstrukturen in Schulen in Island und Kanada - Eine kulturvergleichende Analyse mit Blick auf schulrechtliche Entwicklungen und In-School-Support
}

\author{
Edda Óskarsdóttir, Andreas Köpfer
}

\begin{abstract}
Dieser Beitrag vergleicht die Unterstützungssysteme im Kontext Inklusiver Bildung an isländischen und kanadischen Schulen. Diese beiden Unterstützungssysteme vergleichend in den Blick zu nehmen, liegt darin begründet, dass beide nordischen Länder bzw. Regionen auf eine relativ lange Tradition inklusiver Schulentwicklung zurückblicken können und sich daher Unterstützungsstrukturen und -praktiken Inklusiver Bildung längerfristig entwickelt und schulkulturell manifestiert haben. Beiden Ländern bzw. Regionen ist gemeinsam, dass sie die Notwendigkeit für Unterstützung in die Verantwortung der allgemeinen Schule gegeben haben - wie dies die Salamanca Erklärung von 1994 (UNESCO 1994) als Leitidee ausgewiesen hat. Für den durchgeführten Vergleich wurde Island sowie die kanadische Provinz New Brunswick ausgewählt. Die Fokussierung auf eine kanadische Provinz liegt darin begründet, dass in Kanada Provinzen die Hoheit über Bildungsfragen besitzen. Der Vergleich der beiden Regionen stützt sich inhaltlich auf zwei ethnographische Studien (Óskarsdóttir 2017 zu Island; Köpfer 2013 zu Kanada) und fokussiert dabei insbesondere die Rolle von Unterstützung als integraler Bestandteil inklusiver Praktiken an Schulen. Unterstützungssysteme - so das Ergebnis des interkulturellen Vergleichs - sind als notwendige und zugleich ambivalente Bestandteile inklusiver Schulen anzusehen, um gerechte Bildung für alle Schüler*innen zu gewährleisten. Wenngleich die Organisation und Implementation von konkreten Unterstützungsstrukturen von den kulturellen, politischen und sozialen Rahmenbedingungen des jeweiligen Landes abhängt, zeigt der Vergleich, dass Island und Kanada (New Brunswick) die Unterstützung für Inklusive Bildung - mit all ihren Ambivalenzen - mit einem menschenrechtlichen Zugang zu Bildung und einem schulkulturellen Entwicklungsprozess verbinden.
\end{abstract}

\section{Einleitung}

Inklusive Bildung kann als ein internationales Phänomen beobachtet werden (Biermann/Powell 2014), das insbesondere durch die UN-Behindertenrechtskonvention (UNBRK) (UN 2006) internationale Bedeutung und Bekanntheit erlangt hat und im Bereich Bildung als kritischer Hinweis darauf verstanden werden kann, dass bildungsgerechte und menschenrechtlich adäquate Bildungsorganisationen in den ratifizierenden Staaten bereitzu- 
stellen sind. In den letzten Dekaden hat sich Inklusive Bildung - unterstützt durch die genannten internationalen Entwicklungslinien der Salamanca-Erklärung (UNESCO 1994) und der UN-Behindertenrechtskonvention - sukzessive als schulisch-pädagogisches Konzept (weiter-)entwickelt, das Behinderungen bzw. Barrieren in Bildungsorganisationen hinterfragt und diversitätsorientiertes Lernen sowie die Teilhabe aller Schüler*innen zu ermöglichen sucht (Florian 2005; Booth/Ainscow 2011). Es zeigt sich jedoch, dass unterschiedliche Staaten, (Bundes-)Länder, Kommunen und Schulen die rechtlichen Vorgaben höchst divergent umsetzen. Diese Rekontextualisierung ist vor dem Hintergrund der Unterschiedlichkeit von Ausgangslagen und Ressourcen erklärbar (Allan/Catts 2014). Dennoch sind, grob skizziert, zwei Stränge erkennbar: Nach Slee und Weiner (2001) gibt es im Rahmen inklusiver Schulentwicklung entweder die Tendenz, den Reformauftrag ,Inklusion“ als technisches Problem (technical problem) bzw. Herausforderung anzusehen und Maßnahmen in Schule und Unterricht anzustoßen, um den Inklusionsauftrag möglichst reibungslos in deren bestehende Strukturen und Kulturen zu integrieren (auch kritisch Feuser 2016) - z.B. durch die Aufnahme von Schüler*innen mit sonderpädagogischem Förderbedarf in eine bestehende, tendenziell homogenisierende Unterrichts- und Schulpraxis. Eine zweite Tendenz besteht darin, Inklusion als einen weitreichenden Transformationsprozess (cultural politics) von Schule und Unterricht $\mathrm{zu}$ erachten und vor diesem Hintergrund ganzheitliche, systemische und schulkulturelle Veränderungen zu initiieren, die das soziale Lernen und die Leistungsentwicklung aller Schüler*innen in den Blick nehmen (Slee/Weiner 2001). In letztgenannter Tendenz - die in diesem Handbuchbeitrag im Zentrum steht - wird Inklusive Bildung als Ansatz verstanden, Bildungsorganisationen so weiterzuentwickeln, dass sie den Bedürfnissen aller Schüler*innen gerecht werden können, insbesondere denjenigen, die von Marginalisierung, Diskriminierung und Aussonderung bedroht sind (Ainscow/Sandill 2010; European Agency 2016). Dies wurde bereits in der Salamanca-Erklärung festgehalten:

Inclusive schools must recognize and respond to the diverse needs of pupils, accommodation of both different styles and rates of learning and ensuring quality education to all through appropriate curricula, organisational arrangements, teaching strategies, resource use and partnerships with their communities (UNESCO 1994: 11).

Die Verlagerung des Inklusionsverständnisses von den individuellen Bedürfnissen von Schüler*innen mit sonderpädagogischem Förderbedarf hin zu einem rechtlich gesicherten Anspruch auf hohe Bildungsqualität für alle Schüler*innen erfordert gleichzeitig einen Wandel hinsichtlich des Verständnisses von Unterstützung und Förderung in Schulen. Der Grundgedanke der Bereitstellung von Unterstützung liegt dann in einer sozialen Perspektive auf Behinderung begründet, dem sog. ,sozialen Modell‘ von Behinderung, das die Konstruktion von Behinderung durch gesellschaftliche Behinderungen/Barrieren betont (Rieser 2011; Köbsell/Hirschberg 2021 i.d.B.). Vor diesem Hintergrund zielt inklusionsorientierte Unterstützung in systemischem Sinne darauf ab, Behinderungen/Barrieren abzubauen, die die Partizipation und das Lernen von Schüler*innen auf ontologische und naturalisierende Personenmerkmale reduzieren, und stattdessen spezifische und subsidiäre Unterstützungsmaßnahmen und -ziele entlang von fordernden und qualitätsvollen Bildungsinhalten zu entwerfen und bereitzustellen. Somit treten Fragen von Platzierung zugunsten von Fragen von Unterstützung in den Hintergrund (Burns 2004; Ekins 2013). Die Unterstützungsziele sind dann z.B. entsprechend des individuellen Lernfortschritts der Schüler*innen angelegt und bestimmen nicht in summativer Hinsicht über Erfolg oder Versagen, sondern erlauben es der Lehrperson, individuell und mit anderen (u.a. auch unter Einbezug der Eltern), ein Verständnis 
dahingehend zu entwickeln, wie Schüler*innen zum Lernerfolg geführt werden können (European Agency 2014).

Laut Hart, Drummond und McIntyre (2007) ist eines der zentralen Prinzipien Inklusiver Bildung die Verantwortungsübernahme der Lehrer*innen für alle Schüler*innen, nicht nur für eine Teilgruppe bestehend aus Schüler*innen mit/ohne sonderpädagogischen Förderbedarf. Die Verantwortung und die Zuständigkeit können dabei in Kollaboration mit weiterem pädagogischem Personal (u.a. Sonderpädagog*innen, Schulassistenz) gestaltet sein. Dieser holistische und multiprofessionelle Ansatz wird im internationalen Kontext als menschenrechtlich adäquat angesehen - und wird vonseiten der Studienlage gestützt. So zeigen sich multiprofessionelle Teams aus unterschiedlichen Disziplinen (z.B. auch Schulsozialarbeit und externe Dienstleistende, u.a. Frühförderung) laut rezenten Studien (Mulholland/O'Connor 2016; Finkelstein et al. 2019) als geeignet, um der Heterogenität der Schüler*innen gerecht zu werden. Kollaboration erhöht hierbei die Möglichkeiten, die Lern- und Entwicklungsstände der Schüler*innen adäquat zu erfassen, so früh wie möglich zu erkennen und entsprechende Unterstützungsmaßnahmen anzubieten.

\section{Unterstützungsstrukturen in Island und Kanada (New Brunswick) im Vergleich}

In dem vorliegenden Handbuchbeitrag werden nun in einem interkulturellen Vergleich multiprofessionelle Teams und Unterstützungsstrukturen an inklusiven Schulen untersucht. Dabei werden die Entwicklungslinien für inklusionsorientierte Schulentwicklung in Kanada und Island skizziert und herausgearbeitet, welche entscheidenden Wegmarken die beiden Länder hinsichtlich der Implementierung von Unterstützung in inklusiven Schulen getroffen haben. Kanada und Island scheinen auf den ersten Blick, aufgrund ihrer geographischen Lage und Größe, ihrer Kultur, Geschichte und Sprache, sehr unterschiedlich. Die Auswahl liegt darin begründet, dass diese beiden Länder - neben weiteren wie z.B. USA, Schweden oder Finnland - als Pioniere im Bereich Inklusiver Bildung gelten (Kozleski et al. 2011: 3) und bereits in den 1980er Jahren Reformprozesse im Bildungssystem angestoßen haben, die inklusive Schulentwicklung begünstigen und im Einklang mit dem zuvor erläuterten Perspektivenwechsel der Salamanca-Erklärung von 1994 stehen, der die Regelschule als ersten Bildungsund Förderort für alle Schüler*innen erachtet und den Abbau von Sondereinrichtungen unterstützt.

Aufgrund des vergleichenden Forschungsansatzes musste die Vergleichbarkeit der Regionen gewährleistet werden, wozu wir den regionalen Kontext des zu untersuchenden Feldes eingegrenzt haben. Da in Kanada die Hoheit über Bildungsfragen den Provinzen obliegt, haben wir uns für die Provinz New Brunswick entschieden, der bereits von mehreren Seiten (z.B. Timmons 2006; Arbeitsgruppe Internationale Vergleichsstudie 2007; Hinz 2007; Kruschel/Jahr 2019) eine progressive und inklusionsorientierte Schulentwicklung attestiert wurde. In Island hingegen werden - sicherlich auch aus demographischen und geographischen Gründen - Bildungsfragen auf nationaler Ebene entschieden. Beide Länder haben Entwicklungen im Kontext inklusiver Schulentwicklung bereits in den 1980er Jahren - also noch vor dem internationalen Meilenstein der Salamanca-Erklärung von 1994 - angestoßen und können demnach auf vier Dekaden von ,Inclusive Education“ auf Policy- und Praxisebene 
blicken. In unserem Vergleich geht es uns dabei nicht darum, so genannte ,Best Practice'Beispiele für Inklusion herauszuarbeiten, sondern aufzuzeigen, wie sich Unterstützungsstrukturen in diesen beiden ausgewählten Ländern entwickelt haben.

Inklusive Bildung kann als eine Infragestellung sozialer Ungleichheit im Kontext von Bildungssystemen und -organisationen angesehen werden (Artiles/Dyson 2005). In international vergleichender Forschung wird häufig auf Fragen von Bildungsstruktur und Steuerung fokussiert und es werden darin enthaltene Benachteiligungen und Barrieren analysiert (Jahnukainen 2015; Powell 2011). Unterstützungsstrukturen in ihrer Funktion der ganzheitlichen Etablierung und Aufrechterhaltung inklusiver Bildungs- und Schulsysteme und der Unterstützung der Partizipation aller Schüler*innen hinsichtlich ihrer Lernentwicklung und ihrer sozialen Partizipation rücken selten in den Blickpunkt. Daher werden im Folgenden die Charakteristika für Unterstützung, z.B. deren Zugänglichkeit und Flexibilität für alle Schüler*innen (European Agency 2016), und deren Implementation im Rahmen inklusiver Schulen in Kanada und Island vergleichend in den Blick genommen.

Der in diesem Beitrag vorgenommene Vergleich fokussiert die Unterstützungssysteme als integralen Teil inklusiver Schul- und Unterrichtsentwicklung. Unterstützung wird daher als theoretische wie empirische Analysefolie für den Vergleich herangezogen. Die empirische Basis bilden die zwei umfassenden ethnographischen Studien „Constructing support as inclusive practice“ (Óskarsdóttir 2017) und „Inclusion in Canada“ (Köpfer 2013), die in Island respektive Kanada (New Brunswick, Prince Edward Island, Québec) durchgeführt wurden. Erstere Studie untersucht, wie die Koordination von Unterstützungsleistungen in isländischen Regelschulen während der Einführungsphase des Reformauftrags ,Inklusion“ in der Praxis ablief. Es handelt sich dabei um eine autoethnographische Studie, d.h. die Autorin ist - als Mitarbeitende an der Universität Reykjavik und als Lehrerin an isländischen Schulen - aktiv in die inklusive Schulentwicklung und -steuerung in Island eingebunden. Die zweite Studie ist eine ethnographische Feldstudie, die in den drei kanadischen Provinzen New Brunswick, Prince Edward Island und Québec durchgeführt wurde und inklusive Unterrichtsprozesse, Unterstützungsstrukturen und (para-)professionelle Rollen an Primar- und Sekundarschulen fokussiert. Ungleich zur autoethnographischen Studie in Island ist der Autor der kanadischen Untersuchung im Schul- und Forschungskontext eines anderen Landes verortet. Er blickte als im deutschen Schulsystem sozialisierter Forschender und Wissenschaftler einer deutschen Hochschule in den kanadischen Bildungs- und Schulkontext und führte eine auf den Prinzipien der Grounded Theory Methodology (Glaser/Strauss 2008; Mey/Mruck 2011) beruhende Feldforschung mittels eines triangulierenden Methodensets von teilnehmender Beobachtung, leitfadengestützter (Experten-)Interviews und Forschungstagebuch durch (Köpfer 2013).

Ohne an dieser Stelle vertieft die methodologischen Grundlagen international vergleichender Bildungsforschung (u.a. Phillips/Schweisfurth 2008) auszuführen, werden im Folgenden einige Vorzüge hervorgehoben. Internationale Vergleiche bieten sich an, um Gemeinsamkeiten und Unterschiede unterschiedlicher Bildungssysteme herauszuarbeiten und durch Kontrastierungen eine Reflexionsfläche für die Bildungsvorstellungen des bzw. der Forschenden zu erhalten (Crossley/Watson 2003). Indem wir den oben skizzierten Idealtypus ,Unterstützung ' als tertium comparationis, d.h. als vergleichenden Gegenstand, heranziehen, zielt der Vergleich auf eine detailreiche Erkundung der beiden Bildungssysteme entlang der Charakteristika für Unterstützung im Kontext Inklusiver Bildung innerhalb von Schulen und auf Policy-Ebene. 
Ein Vergleich zweier Bildungssysteme bietet die Möglichkeit, einen vom Standpunkt des/der Forschenden (und deren/dessen länderspezifischer Vorerfahrung) aus transzendentalen und reflexiven Blick auf die Kulturen, Strukturen und Praktiken bezogen auf Inklusion und Exklusion in Bildungssystemen unterschiedlicher Länder zu richten (Powell 2018). In der international und interkulturell vergleichenden Fachliteratur werden zahlreiche Dimensionen vorgeschlagen, um Inklusive Bildung im Sinne eines ganzheitlichen und schulkulturellen Transformationsprozesses im Bildungssystem zu vergleichen. So werden zum Beispiel durch Artiles und Kollegen (2006) die weit verbreiteten Dimensionen ,access', ,acceptance', ,participation' und ,learning outcomes‘ genannt. Darüber hinaus schlägt Peters (2007) vier konzeptionelle Bausteine Inklusiver Bildung vor, die auf den Grundgedanken der SalamancaErklärung fußen. Letztere werden als Vergleichsebenen herangezogen, um Unterstützung als tertium comparationis zu konturieren bzw. operationalisieren:

All learners come to school with diverse needs and abilities, so no learner is fundamentally different. It is the responsibility of the general education system to be responsive to all learners. A responsive general education system provides high expectations and standards, quality academic curriculum and instruction that are flexible and relevant, an accessible environment, and teachers who are well prepared to address the educational needs of all learners. Progress in general education is a process evidenced by schools and communities working together to create citizens for an inclusive society who are educated to enjoy the full benefits, rights, and experiences of societal life (Peters 2007: 99).

Diese Bausteine stellen einen qualitativen konzeptionellen Rahmen für unsere vergleichende Analyse zu Unterstützungsstrukturen an inklusiven Schulen in Kanada und Island dar.

Verständlicherweise können durch den Vergleich keine direkten Maßnahmen für das jeweils andere Land vorgeschlagen werden, da direkte Übertragungen aufgrund der unterschiedlichen kulturellen, sozialen und politischen Ausgangslagen der Länder zu Verzerrungen führen können. Allerdings können Impulse für den internationalen Diskurs um Unterstützung im Kontext Inklusiver Bildung formuliert werden (Singal/Muthukrishna 2014), was abschließend vorgenommen wird (siehe Kap. 5).

\section{Inklusive Schulentwicklung in Island}

Der Grundgedanke Inklusiver Bildung ist im isländischen Gesetz bereits seit 1995 enthalten, wenngleich die Verankerung inklusiver Beschulung aller Schüler*innen erst in der Schulgesetzänderung von 2008 (Lög um grunnskóla nr. 91/2008) rechtlich bindend festgesetzt wurde. Frühere Schulgesetze hatten - in der Tendenz mit integrativem Grundgedanken - ausgewiesen, dass Schulen alle Schüler*innen aus dem wohnortnahen Einzugsbereich aufnehmen und sie entsprechend ihrer Bedürfnisse unterrichten sollten. Dies wurde jedoch nicht mit spezifischem Blick auf Inklusion und nicht mit besonderem Augenmerk auf den Abbau von Segregation ausgewiesen, was zur Folge hatte, dass Schüler*innen mit sonderpädagogischem Förderbedarf zwar in den gleichen Schulgebäuden unterrichtet wurden und sozusagen den gleichen Bildungs-, Raum` zur Verfügung hatten, aber ein gleichberechtigter Zugang zu Bildung dennoch nicht gewährleistet war (z.B. bezogen auf Curriculum, Abschlüsse, Unterstützungsmaßnahmen etc.) (Jóhannesson 2006; Marinósson 2011). 


\subsection{Die Grundlegung Inklusiver Bildung}

Rückblickend kann konstatiert werden, dass das Fundament für Inklusive Bildung in Island im Jahr 1974 gelegt wurde, als ein neues Schulgesetz für die öffentlichen Schulen verabschiedet wurde, das einige zentrale Entwicklungen aufnahm, die das Schulsystem in der vorangegangenen Dekade tangierten (Jónasson 1996). Dieses Gesetz brachte grundlegende Veränderungen in Bezug auf soziale Ungleichheit im Schulsystem mit sich. So sicherte es gleichberechtigen Zugang zu Bildung hinsichtlich des Wohnorts (städtisch vs. ländlich) und nahm spezifisch die Heterogenitätsdimensionen Gender und Behinderung in den Blick. Hier zeichnete sich nun ein Wandel im Rollenverständnis und in der Verantwortung und Aufgabe von Schulen ab - von einem gegenstands- und fachbezogenen Fokus auf Lerninhalte (die den Schüler*innen curricular vorgegeben werden) hin zu einem Verständnis von Lernen, das sich an den individuellen Lern- und Entwicklungsständen der Schüler*innen orientiert (Jónasson 1996). Dieses Schulgesetz formulierte zudem zum ersten Mal in der isländischen Schulgeschichte explizit, dass Schule ein Lern- und Bildungsort für alle Schüler*innen ist und dass alle Schüler*innen - wenn möglich - in einer Regelschule zu beschulen sind. Es kategorisierte dabei sonderpädagogischen Förderbedarf (,Special Educational Needs' (SEN)) in fünf Kategorien und sah vor, dass Schüler*innen einzelner Förderbedarfe in speziellen Einrichtungen beschult werden sollten (Jónasson 2008a). Die Schulgesetzänderung von 1974 wurde später durch die erste Verordnung für sonderpädagogische Förderung im Jahr 1977 ergänzt, die die Einteilung und Diagnose von Schüler*innen in Behinderungskategorien weiter konkretisierte und die Institutionalisierung von Sonderschulen und Sonderklassen durch die Vergabe spezifischer finanzieller Ressourcen unterstützte (Jónasson 1996, 2008a).

Im Jahr 1990 trat eine neue Verordnung für sonderpädagogische Förderung in Kraft, die auf der Schulgesetzänderung von 1974 beruhte und zu einer spezialisierten Unterstützungspraxis führte. Diese Verordnung betonte explizit das Recht aller Kinder und Jugendlichen, eine wohnortnahe Schule zu besuchen (Reglugerð um sérkennslu 98/1990), wenngleich Eltern nach wie vor auch eine Sonderschule als Förderort für ihr Kind auswählen konnten. Dennoch ist hier ein Perspektivenwechsel hin zur Diagnose von Unterstützungsbedürftigkeit von Schüler*innen innerhalb ihrer bestehenden Lernumgebung zu erkennen, die zwar in der Tendenz das medizinische Modell der ontologisierenden Zuschreibung von Förderbedürftigkeit entlang defizitärer Behinderungskategorien aufrechterhält - so jedoch Ressourcen für die Schüler*innen sichert. Es wurde ein Modell der Ressourcenzuweisung für sonderpädagogischen Förderbedarf entwickelt, das 20\% der Schüler*innenschaft als pauschale Richtlinie zur Ressourcenzuteilung vorsah (Óskarsdóttir 1993; Jónasson 1996, 2008a).

In der Schulgesetzänderung für allgemeine Schulen von 1995 wurde dann die Zuständigkeit und rechtliche Verantwortung für Schulen - inklusive der Sonderschulen - von der Staats- auf die Kommunalebene verlagert. Diese Reform schuf eine Dezentralisierung des Bildungssystems (Lög um grunnskóla nr. 66/1995). Zugleich kamen Bedenken darüber auf, wie der steigende Bedarf an sonderpädagogischem Förderbedarf finanziell gedeckt werden könne. Hierfür richteten die Kommunen einen sogenannten Ausgleichs-Fonds ein, um die Unterschiede in der finanziellen Situation der Kommunen zu egalisieren (Jónasson 2008b). Um einen Beitrag aus diesem Fonds für eine Schülerin bzw. einen Schüler zu gewähren, bedarf es jedoch einer medizinischen Diagnose. Das heißt, dass ein medizinisches Verständnis von Behinderung und deren Feststellung nach wie vor Teil der Ressourcenvergabe ist (Jónasson 2008b). In der Schulgesetzänderung von 1995 wurden neue Vorgaben für sonderpädagogischen Förderbedarf präsentiert, insbesondere bezogen auf die Finanzierung. So 
wurde ein quotenbasiertes Ressourcierungsmodell für den sonderpädagogischen Förderbedarf eingeführt, das für Schulen mit über 200 Schüler*innen eine zusätzliche Lehrperson vorsieht, um die spezifischen Förder- und Unterstützungsbedarfe zu koordinieren. Mit dem Nationalen Lehrplan von 2010, vor dem Hintergrund des Bildungsgesetzes von 2008 ins Leben gerufen wurde, wird die Tendenz der Flexibilisierung der Lernwege und der Fokussierung auf Lernergebnisse im Sinne der Kompetenzorientierung weiter unterstützt (vgl. Powell et al. 2021 i.d.B.).

\subsection{Der Wandel von sonderpädagogischer Förderung zu Unterstützungsstrukturen}

Bezogen auf das letzte Schulgesetz für allgemeine Schulen von 2008 kann konstatiert werden, dass inklusive Schulpraxis und Unterstützung sich in erster Linie auf die Erreichung eines adäquaten Bildungsabschlusses, auf die Förderung individueller Bedarfe, des Wohlbefindens und von allgemeiner Bildung bezieht. Artikel 17 des Gesetzes, der von Schüler*innen mit sonderpädagogischem Förderbedarf handelt, sieht vor:

Pupils are entitled to be treated equally, their educational needs are to be met in a common compulsory school without exclusion, without regard to their physical or mental abilities (Lög um grunnskóla nr. 91/2008).

In diesem Gesetz gibt es einige Innovationen, zum Beispiel, dass (1) die Begriffe Unterstützungssystem und Unterstützungsmaßnahmen anstelle von sonderpädagogischer Förderung verwendet werden; (2) dass der Aufbau von Kompetenzen bei Schüler*innen statt Fachbereichen angestrebt wird; und (3) dass Schulen ab der ersten Klasse regelmäßige Screenings durchführen, um den Lern- und Entwicklungsständen entsprechende Bildungsangebote für die Schüler*innen bereitzustellen (Lög um grunnskóla nr. 91/2008). Gemäß dem Gesetz steht allen Kindern und Jugendlichen eine angemessene Bildung in allgemeinen Schulen zu, und die Schulaufsicht ist verpflichtet, angemessene Bildungsmöglichkeiten und -angebote für alle zu schaffen. Dies bezieht sich auf alle Kinder: Kinder mit und ohne Behinderungen, mit chronischen Krankheiten, hochbegabte Kinder, Kinder mit geistigen Behinderungen, Kinder aus sozioökonomisch schwächeren Wohngebieten und Kinder von ethnischen, sprachlichen oder kulturellen Minoritäten (Ministry of Education, Science and Culture 2011).

Fundamentale Veränderungsprozesse sind zwischen dem Schulgesetz von 2008 über Schüler*innen mit sonderpädagogischem Förderbedarf (Reglugerð um nemendur með sérparfir 585/2010) und der Verordnung über sonderpädagogischen Förderbedarf von 1996 zu erkennen. Der zentrale Unterschied besteht darin, dass nicht mehr die Förderbedürftigkeit der Schüler*innen entlang ihrer Defizite im Vordergrund steht, sondern - im Sinne einer ressourcenorientierten Pädagogik - die Stärken der Schüler*innen und die Rahmenbedingungen, die diese zur Entfaltung bringen können. Demnach werden die Schulen adressiert, auf die Diversität der Schüler*innen gleichberechtigt einzugehen und die Partizipation aller sicherzustellen. Die Verordnung geht über das Schulgesetz von 2008 hinaus - insbesondere hinsichtlich des Fokusses. Sie sieht vor, dass Unterstützung, wie sie an Schulen organisiert ist, inklusiv ausgerichtet sein muss. Erstaunlicherweise ist die Definition von Inklusion dabei in der Verordnung expliziert, nicht jedoch im Schulgesetz selbst, was insofern zu der Ambivalenz führt, dass Inklusion noch immer durch einen sonderpädagogischen Kontext gerahmt 
wird. In der Verordnung wird Inklusion als allgemeine, wohnortnahe Schule für alle Schüler*innen definiert, die sich durch Werteorientierung, Demokratie und soziale Gerechtigkeit auszeichnet und den sozialen wie Lern- und Bildungsbedürfnissen aller Schüler*innen gerecht wird (Reglugerð um nemendur með sérparfir 585/2010). Darüber hinaus wird explizit festgelegt, dass die Schulleitung für die Implementierung der entsprechenden Voraussetzungen für die Umsetzung der Verordnung verantwortlich ist - ohne dabei eine Delegation dieses Tätigkeitsbereichs an eine spezielle Lehrkraft vorzusehen.

Sonderpädagogische Fachkräfte haben noch immer eine unterstützende Funktion in jeder Regelschule, wenngleich ihr professioneller Status nicht mehr durch ein Gesetz oder eine Verordnung geregelt ist - und in einigen Schulen hat sich ihre Rolle tendenziell in die einer lehrer*innenberatenden Rolle entwickelt. Die Diskussionen darüber, wie das allgemeine Schulsystem auf die Diversität von Schüler*innen adäquat eingehen sollte und kann, hat sich also von einer Fokussierung auf die Bereitstellung sonderpädagogischer Förderung für Schüler*innen, denen ein sonderpädagogischer Förderbedarf zugeschrieben wird, hin zu einem inklusiven und menschenrechtsbasierten Unterstützungssystem für alle Schüler*innen gewandelt, was durch den Fachdiskurs und die dortige terminologische Auseinandersetzung und Abgrenzung von ,Integration“ und ,Inklusion` unterstützt wurde (Marinósson/Bjarnason 2014).

Diese Entwicklung kann jedoch nicht als zeitlich linearer und chronologischer Prozess angesehen werden, da sich bisweilen unterschiedliche theoretische Perspektiven auf Behinderung und Inklusion überlagern und parallel zu neu entwickelten Perspektiven fortbestehen. Vor dem Hintergrund einer unlängst durchgeführten Evaluation des inklusiven Bildungssystems in Island (European Agency 2017), bilden die Gesetzgebung und Policy-Ebene die Ziele und Perspektiven Inklusiver Bildung adäquat ab. Eine durch die Evaluation identifizierte Herausforderung ist die unterschiedliche Auslegung und Interpretation der gesetzlichen Richtlinien - und dementsprechend die Pluralität ihrer Umsetzungsformen. Eine weitere Hürde zur Umsetzung Inklusiver Bildung ist die Ressourcenfrage. Die relevanten Bildungsakteur*innen fordern hier noch stärkere Flexibilität, die es den Schulen erlaubt, auf die von den Schüler*innen oder Eltern artikulierten Bedarfe zu reagieren, ohne diagnostisch auf vorbereitete Kategorien von sonderpädagogischem Förderbedarf zurückzugreifen. Eine weitere zentrale Herausforderung in der Umsetzung der Richtlinien für Inklusive Bildung sind die fehlenden interdisziplinären Kooperationen mit Akteur*innen externer Träger*innen wie z.B. aus dem Gesundheits-, Wohlfahrts- oder Bildungsbereich. Auch stellt die Rolle der Schulleitungen in inklusiven Schulen ein zentrales Echolot für Gelingen/Nicht-Gelingen inklusiver Schul- und Unterrichtsentwicklung dar. Zudem gibt es auf ministerieller Ebene erste, aber noch unzureichende, kollaborativ angelegte Tätigkeitsbereiche und Strukturen, die die bildungsgerechte Unterstützung aller Schüler*innen fokussieren und nicht - aufgrund ihrer tätigkeitsspezifischen Parzellierung - verhindern (European Agency 2017; Óskarsdóttir 2017; Powell et al. 2021 i.d.B.).

\section{Inklusive Schulentwicklung in Kanada (New Brunswick)}

Kanada kann auf eine nahezu vier Dekaden umfassende inklusive Schulentwicklung zurückblicken, die auf rechtlicher Ebene in erster Linie durch die so genannte „Canadian Charter of Rights and Freedoms“ (Department of Justice 1982) aus dem Jahr 1982 angestoßen wurde. 
Vor dem Hintergrund dieser Zeitspanne kann Kanada ein weitreichender Erfahrungsschatz zugeschrieben werden, was die Einführung, Implementierung und Etablierung von (Unterstützungs-)Maßnahmen im Kontext von Inklusion anbelangt und es erscheint daher gewinnbringend, die Mechanismen und Strukturen näher zu betrachten, die zu einem inklusionsorientierten Gesamtschulsystem geführt haben bzw. dieses aufrechterhalten. Zudem wird Kanada häufig als Land dargestellt, das als Einwanderungsland mit der Multikulturalität und Heterogenität der Menschen auf positive und wertschätzende Art und Weise umgeht (Timmons 2007; Kelley/Trebilcock 2010; Berry 2013; Löser 2019), sei es im Bereich Migration oder in Bezug auf andere Heterogenitätsdimensionen wie zum Beispiel Geschlecht, Religion, Behinderung etc. Der Begriff und insbesondere der spezifische Gedanke von ,Inklusion ‘ fand vergleichsweise früh Eingang in den kanadischen Bildungs- und Erziehungsdiskurs, noch bevor er durch die Salamanca-Erklärung zu einem internationalen anerkannten Begriff avancierte (Porter/Richler 1991; UNESCO 1994):

The vision of inclusion is that all children would be served in their neighbourhood schools in regular classrooms with children their own age. The idea is that these schools would be restructured so that they are supportive, nurturing communities that really meet the needs of all the children within them [...] (O’Neil 1994, zit. nach Perner/Porter 1998).

Wenngleich an dieser Stelle kein ausführlicher Einblick in das kanadische Bildungssystem möglich ist (vgl. hierzu Arbeitsgruppe Internationale Vergleichsstudie 2007), sollen einige zentrale und für Inklusion relevante Charakteristika der Schulentwicklung skizziert und insbesondere der Aspekt von Unterstützung (support) berücksichtigt werden (Mittler 2000; Köpfer 2013). Wir beziehen uns dabei hauptsächlich auf eine ausgewählte Provinz, New Brunswick, die, wie bereits genannt, im Zentrum mehrerer internationaler Studien stand und häufig als beispielhaft für Inklusive Bildung genannt wird. Im Rahmen der ethnographischen Studie ,Inclusion in Canada' (Köpfer 2013) wurde eine empirische Analyse zur inklusiven Schulorganisation und -entwicklung in New Brunswick durchgeführt, die die Unterrichtsprozesse, Unterstützungsstrukturen und (para-)professionellen Rollen an Grund- und weiterführenden Schulen in den Blick nahm. Ein zentraler Erkenntnisgewinn der Feldstudie war, dass Unterstützung als conditio sine qua non für die Entwicklung und Praxis Inklusiver Bildung gelten kann und dass diese die Dimensionen Koordination und Reflexion beinhalten muss. In chronologischer Hinsicht wurde aufgezeigt, dass nach einer Phase der De-Segregation, in der Förderschulen zugunsten von integrativen Bildungsangeboten umstrukturiert wurden, eine Phase inklusiver Schulentwicklung folgte, die die schulinterne Umstrukturierung von Unterstützung und Förderung - u.a. auch die Umgestaltung von Rollen und Zuständigkeiten - vorsah. Im Folgenden wird, auf Basis der ethnographischen Untersuchungsergebnisse, das Unterstützungssystem unter Berücksichtigung unterschiedlicher Ebenen von Unterstützung erläutert.

\subsection{Menschenrechtsbasierter Entwicklungsprozess}

Die „Canadian Charter of Rights and Freedoms“ (Department of Justice 1982) kann als Ausgangspunkt für inklusive Schulentwicklung in Kanada angesehen werden und demnach ebenso für New Brunswick. Artikel 15 der Charter, die 1982 in Kraft trat, verankert gesetzlich und explizit 
[...] that every individual is equal before and under the law and has the right to the equal protection and equal benefit of the law without discrimination and, in particular, without discrimination based on race, national or ethnic origin, colour, sex, age or mental or physical disability (ebd.: 15(1)).

Diese gesetzliche Regelung war entscheidend für die Initiierung eines De-Segregationsprozesses in unterschiedlichen kanadischen Provinzen, der sich aufgrund der Bildungshoheit der Provinzen und der unterschiedlichen bildungspolitischen Prioritäten höchst divergent ausgestaltete (Thompson et al. 2015). In einigen Provinzen, wie zum Beispiel New Brunswick oder Prince Edward Island, führte dies durch Schulgesetzänderungen zur nahezu vollständigen Abschaffung von Sonderschulen (vgl. kritisch Schroeder 2016). Während Schüler*innen mit sonderpädagogischem Förderbedarf bis in die 1980er Jahre noch in Sonderschulen unterrichtet und gefördert wurden, ergaben die Schulrechtsgesetzänderungen mit der Verpflichtung zur Aufnahme, der sog. ,duty to accommodate“ eine vollkommen neue Situation: „This new Act [Bill 85, A.K/E.O.] was the only one that required justification of the exclusion of an exceptional pupil from a regular class, not his/her inclusion“ (MacKay 2005: 4f.). Diese gesetzliche Veränderung des Schulrechts - die in anderen Provinzen auf Basis der Canadian Charter of Rights and Freedoms unterschiedlich ausfiel - markierte in New Brunswick einen schulorganisatorischen Bruch, da von nun an nicht die Integration von Schüler*innen mit sonderpädagogischem Förderbedarf argumentiert werden musste, sondern deren Aussonderung. In institutioneller Hinsicht führte dies zu einem Rückbau von Sonderschulen und zu einem Aufbau von innerschulischen Unterstützungs- und Förderangeboten.

Die zeitliche Dauer für diesen Transformations- bzw. De-Segregationsprozess in der Provinz New Brunswick wird von Gordon Porter - ehemaliger Governor und Superintendant in New Brunswick - auf zwei bis drei Jahre geschätzt, ähnlich zu Ländern wie z.B. Italien. Dieser vergleichsmäßig kurze institutionelle Wandlungsprozess muss jedoch einerseits vor dem Hintergrund der relativ geringen Bevölkerungsdichte und der hohen Anzahl ländlicher Gegenden in New Brunswick betrachtet werden. Darüber hinaus war die Zahl von Sonderschulen, die überdies cross-kategorial ausgerichtet waren, relativ gering und die Schüler*innen wurden in wohnortnahe Schulen umgeschult.

Die ethnographische Studie und die darin geleistete Rekonstruktion der historischen Entwicklungsprozesse im Bildungssystem New Brunswicks zeigt also auf, dass die inklusiven Schulentwicklungsprozesse in zwei Phasen eingeteilt werden können. Zunächst wurden sogenannte „special classes“ für Schüler*innen mit ,Special Educational Needs“ (SEN) in den Regelschulen eingerichtet, um die Kluft zwischen den Sonderschulen und Regelschulen zu verringern und um die Schüler*innen mit SEN ihren wohnortnahen Schulen zuzuweisen. Diese Binarität von Allgemeinem und Besonderem (Regelklasse und Sonderklasse) bildete sich zunächst auch noch auf der Ebene der Lehrkräfte ab. Lehrpersonen, die zuvor in Sonderschulen tätig waren, waren nun verantwortlich für die Sonderklassen in den Regelschulen. Die nun freistehenden Sonderschulen wurden entweder in Ressourcenzentren oder - in wenigen Fällen - auch in Regelschulen umgestaltet. Dieser pragmatisch und schulorganisatorisch angelegte Reformprozess ,Inclusive Education“ sah also eine nahezu vollständige Verlagerung von Schüler*innen und Lehrpersonen aus den Sonderschulen in die Grund- und weiterführenden Schulen vor. Um die weiteren Entwicklungen im Sinne einer Auflösung von Sonderklassen voranzubringen, wurden erste Unterstützungsmaßnahmen für alle Schüler*innen eingeführt. Dabei war es in erster Linie die (Klassen-)Lehrperson, die Unterstützung erhielt - entweder durch Fort- und Weiterbildungsmaßnahmen (mit Fokus auf Differenzieren, Adaptieren und Classroom Management) oder durch direkte und indirekte personelle Unterstützungsmaßnahmen. So wurden zum Beispiel Schulbegleitungen (Teacher Assistants) 
eingestellt und eine Koordinationsstelle für innerschulische Beratung in Form eines „Methods and Resource Teams“" (M\&RT) eingerichtet, die den Lehrpersonen didaktisch-methodische Beratung und Unterstützung bezogen auf den Unterricht in heterogenen Lerngruppen anbietet. Dies erfolgte mit dem Ziel, dass sonderpädagogische Maßnahmen nicht mehr mit Allokations- und Platzierungsfragen verbunden sind, sondern dass sie als systemische und subsidiäre Unterstützungs- und Beratungsangebote innerhalb einer wohnortnahen Schule fungieren. Dennoch bleiben Klassifizierungen in Form von curricularen Anpassungsstufen (s.u.) weiter bestehen und auch das professionelle Handeln der M\&RT ist in die grundsätzliche Ambivalenz von spezifischer Förderung und Selektion eingebunden.

\subsection{Der Aufbau von schulinternen und kommunalen Unterstützungsstrukturen}

Durch die systematische Entwicklung von Kernkategorien im Forschungsprozess der ethnographischen Feldstudie, trat sukzessive ein Aspekt des Unterstützungssystems in den Vordergrund, der als „flexible Unterstützung auf unterschiedlichen Bildungsebenen“ bezeichnet werden kann. Das menschenrechtliche Verständnis und Ziel, dass allen Schüler*innen Bildung in einer wohnortnahen Schule innerhalb der Gemeinschaft (,Community') zusteht, führte zum Ausbau eines Unterstützungssystems innerhalb und außerhalb der Schulen. Die grundlegende Transformation des Mechanismusses von Delegation und Abstufung entlang von Schüler*innenleistung hin zu einer subsidiären Unterstützungsstruktur für Vielfalt an einer Schule kann als Unterstützungspyramide gedacht werden (s. Visualisierung in Köpfer 2013: 148). Aufbauend auf dem rechtlichen Rahmen werden sowohl außerhalb der Schule (Student Services Teams in den sog. School Boards) als auch innerhalb der Schule (Guidance Team, Native Support Workers, Methods \& Resource Team, Therapists, Teacher Assistants) Unterstützungssysteme und -rollen kreiert, die den Unterricht und insbesondere die Lehrperson unterstützen. Beispielhaft kann hier die Rolle der so genannten Methods \& Resource Teachers hervorgehoben werden. Diese Rolle, die das Kernelement des so genannten „Collaborative Consultant Model“ (Porter/Richler 1991) bildet, stellt den Versuch dar, eine professionelle Rolle zu definieren, die die artifizielle Dichotomie von Sonder- und Regellehrperson überwindet. Sie ist demnach nicht als sonderpädagogische Rolle anzusehen, sondern als systemische Beratungsrolle mit einer Bandbreite von Zuständigkeiten - bezogen auf alle Schüler*innen der Schule bzw. Klasse. Sie bildet sozusagen eine Scharnierstelle zwischen Unterrichtspraxis und schulischem Unterstützungsangebot, indem sie direkte und indirekte Unterstützungsmaßnahmen miteinander verbindet. So ist das Methods and Resource Team zum Beispiel zuständig für die Koordination und fachliche Vorbereitung von adaptierten und individualisierten Entwicklungsplänen (Adapted/Individualized Education Plans) und für die Anleitung von Teacher Assistants für den Unterricht. Gleichsam ist das Team in der Lage, koordinative Aufgaben zur Organisation der Elternarbeit oder der Freiwilligenbeteiligung am Schulgeschehen zu übernehmen. Im Bühnenbild von Goffman (1959/2003) gesprochen: Um zwischen der Vorderbühne der unterrichtlichen Praxis und der Hinterbühne der Koordination von Unterstützung adäquat und für alle Schüler*innen zu vermitteln, wurde die Rolle als didaktische ausgerichtet (Köpfer/Óskarsdottir 2019) und mit entsprechender Qualifizierung (verpflichtender Masterabschluss in Inclusive Education), Zeit sowie Raum (Resource Room) ausgestattet. Neben den ressourcen- und qualifikationsbezogenen Aspekten, die 
zweifelsohne eine bedeutsame Ebene inklusiver Schulentwicklung darstellen, sind auch Dimensionen von Haltung und Reflexion an die Rolle von Methods \& Resource Teacher geknüpft. So soll sie die Lehrpersonen darin unterstützen, das eigene pädagogisch-professionelle Handeln fallbasiert zu reflektieren und Handlungsschritte systemisch und kooperativ zu planen. Dies erhält für den Kontext inklusiver Schulentwicklung insofern besondere Bedeutung, da unterrichtliche Situationen (z.B. Differenzierung, Umgang mit Störungen etc.) nicht vor dem Hintergrund unzulänglicher Leistungen bzw. störenden Verhaltens einzelner Schüler*innen betrachtet werden, sondern als komplexe didaktische Aufgabe mit zum Teil ambivalenten Handlungsanforderungen für Lehrpersonen (Valle/Connor 2011).

Abschließend zeigte die ethnographische Analyse, dass neben den soeben diskutierten direkten Unterstützungsrollen auch eine Dimension von Unterstützung relevant ist, die als schulkulturelle und kommunikative Unterstützungsebene bezeichnet werden kann. Die Bedeutung von Kommunikation in inklusionsorientierten Schulen wurde konzeptuell im internationalen Fachdiskurs insbesondere als „community of practice“ (Laluvein 2010: 35) oder "professional learning community" (DuFour/Eaker 2008: 10) ausgearbeitet. Im Vordergrund steht dabei die gemeinschaftsbildende Idee, dass Lehrpersonen innerhalb ihrer Schule eine kommunikative Struktur entwickeln, um Expertise, Fachwissen und Erfahrungen auszutauschen und - datenbasiert und in Rückkoppelung mit Wissenschaftler*innen - Unterrichtsentwicklung voranzutreiben (Carrington/Robinson 2006; Köpfer 2019).

Um den Bedürfnissen aller Schüler*innen in inklusiven Schulen in New Brunswick gerecht zu werden, wurde eine Kommunikationsstruktur entwickelt, die niederschwellige und fallbasierte Absprachen zu unterrichtlichen Herausforderungen in einem so genannten „Student-based Student Services Team Meeting“ vorsieht. Unter Beteiligung auch paraprofessioneller Akteur*innen (z.B. School Intervention Worker, Therapists, Guidance Counsellor, etc.) sowie Eltern werden kindbezogene Anliegen besprochen und mit dem auf Ebene der School Boards angesiedelten „Student Services Teams“ rückgekoppelt (Löser 2019).

Zusammenfassend kann konstatiert werden, dass in der kanadischen Provinz New Brunswick ein schulischer De-Segregationsprozess bezogen auf Sonderinstitutionen durchlaufen wurde, der durch schulgesetzliche Veränderungen in Gang gesetzt wurde. Dieser wurde infolgedessen durch einen längerfristigen innerschulischen Entwicklungsprozess abgelöst, in dem sich sukzessive direkte Unterstützungsstrukturen und -rollen (u.a. das Methods \& Resource Team) sowie indirekte Unterstützungs- und Kommunikationsstrukturen herausgebildet haben. Die Hürden bzw. die fehlenden Möglichkeiten, Schüler*innen mit sonderpädagogischem Förderbedarf auf Sonderschulen zu überweisen, führte zu einem paradigmatischen Wechsel von Selektions- zu Unterstützungsstrukturen innerhalb der Schule, die subsidiär und entlang der Bedürfnisse der Schüler*innen Unterstützung im Sinne von ,situated agency“ (Danforth/Naraian 2015: 80) anbieten sollen.

\section{Die Unterstützungssysteme an isländischen und kanadischen Schulen im Vergleich}

Aus den vorangegangenen Entwicklungslinien inklusiver Schulentwicklung in Island und Kanada (New Brunswick) seit den 1980er Jahren lassen sich - mit Bezug auf Aspekte von Unterstützung als tertium comparationis (vgl. Kap. 2; Peters 2007) - eine Reihe von Gemein- 
samkeiten und Unterschieden ableiten, wie sich inklusive Schulen und der Aufbau von Unterstützungsstrukturen auf gesetzlicher und schulischer Ebene entwickelt haben. Eine weitreichende Gemeinsamkeit findet sich im Transformationsprozess auf gesetzlicher Ebene. Hier ist sowohl in Island als auch in Kanada eine paradigmatische Veränderung des Fokusses von einer binären Bereitstellung von Unterstützung und Förderung (bezogen auf Schüler*innen mit und ohne sonderpädagogischem Förderbedarf) hin zu einer Ausrichtung auf Unterstützung, die - im generalistischen Sinne - die Diversität der Schüler*innen fokussiert und demnach alle Schüler*innen innerhalb einer wohnortnahen Schule in den Blick nimmt. So wurde die Verantwortung für alle Schüler*innen in die Hände der Regelschule gelegt. Während dieser rechtliche Transformationsprozess in Island jedoch durch die Dezentralisierung der Bildungshoheit bzw. einer Verantwortungsübertragung auf Ebene der Schulen (insbesondere in die Hände der Schulleitungen) begleitet wurde, blieb die Zuständigkeit für Bildungsfragen in Kanada bei den Provinzen und es wurden z.B. in der Provinz New Brunswick sog. Student Services Teams auf der Ebene der School Boards eingerichtet, die die Schulen mit bedarfsorientierter Unterstützung begleiten wollen.

Sowohl in Island wie auch in Kanada können innerhalb des inklusiven Schulentwicklungsprozesses zwei Phasen unterschieden werden: Zunächst wurden im Rahmen einer kürzeren Phase die Schüler*innen in die Regelschule integriert. Dann wurden die rechtlichen Rahmenbedingungen der Regelschule so ausgerichtet, dass diese für alle Schüler*innen zuständig ist. Entsprechend wurde nun der Fokus von Unterstützung und Förderung auf alle Schüler*innen gelegt und eine Phase des längerfristigen und fortwährenden Aufbaus und der Etablierung von Unterstützungsstrukturen begann. In der zweiten Phase gibt es eine Reihe von Gemeinsamkeiten zwischen Island und Kanada (New Brunswick). Sie ist charakterisierbar als „,community-based approach“. Das bedeutet, dass im Rahmen inklusiver Schulentwicklung Wohnortnähe und die Einbettung von Schulen in Gemeinde- und Quartiersstrukturen als relevante Kriterien betrachtet wurden (Porter/AuCoin 2012: 154). Dies umfasste neben lokalen Schulkooperationen auch die Einbindung von Freiwilligen (Volunteers) (ebd.).

Die innerschulischen Entwicklungen zeigten in beiden Ländern eine Transformation der Ausrichtung sonderpädagogischer Unterstützung. So wurden Maßnahmen getroffen, ein nonkategoriales Unterstützungssystem für alle Schüler*innen zu implementieren. In Kanada (New Brunswick) zeigte sich dies in der Einrichtung der Rolle von „Methods \& Resource Teachers“ - verbunden mit dem Versuch, den Fokus der Unterstützung sukzessive vom Defizit des Kindes hin zur Unterstützung von Lehrer*innen zu verlagern, damit diese der Heterogenität der Schüler*innen gerecht werden können (Crawford/Porter 2004). In Island wurde Inklusive Bildung auf rechtlicher Ebene sukzessive als Bildungsauftrag für alle Schüler*innen begriffen - ohne Beschränkung auf Schüler*innen mit sonderpädagogischem Förderbedarf. Jedoch bleibt die Herausforderung noch immer bestehen, die schulischen Unterstützungsstrukturen so zu verändern, dass die Klassifizierung von sonderpädagogischem Förderbedarf keine Voraussetzung für den Erhalt finanzieller Ressourcen für die Schulen darstellt. Darüber hinaus forcierten beide Länder Veränderungsprozesse auf Steuerungsebene, um alle Schüler*innen auf ihrem Lern- und Entwicklungsniveau zu unterstützen. So wurden zum Beispiel in Kanada (New Brunswick) Adaptionsstufen im Curriculum (adapted, modified and individualized) verankert - die jedoch die Ambivalenz von Klassifizierung und Unterstützung nicht aufzulösen vermögen. In Island zeigte sich diese Transformation von Unterstützung in der Emphase des Aufbaus von Schüler*innenkompetenzen entlang von flexiblen Curricula, die fächerbezogene Anforderungen abgelöst haben. 


\section{Zusammenfassung}

Unser interkultureller Vergleich zeigt auf, dass Island und die kanadische Provinz New Brunswick seit den 1980er Jahren einen vergleichbaren inklusiven Schulentwicklungsprozess durchlaufen haben, der sich in erster Linie durch den sukzessiven Aufbau und die Etablierung von Unterstützung für alle Schüler*innen an Regelschulen auszeichnet. Allerdings bleiben weitreichende Ambivalenzen bestehen, die ebenso in anderen Ländern und im internationalen Fachdiskurs um Inklusive Bildung verhandelt werden. So bleibt nach wie vor die Frage nach einer umfassenden systemischen - und zugleich schulbasierten - Klassifikation von Unterstützungsbedarf ungelöst. Während die historischen Entwicklungen zeigten, dass Island und Kanada Bemühungen hin zu non-kategorial und flexiblen Unterstützungssystemen (z.B. bezogen auf Rollen und Zuständigkeiten, Curriculum, etc.) anstießen, bleibt die Ambivalenz des ,Besonderen“ und ,Allgemeinen“ bestehen. So wurden in Island und Kanada einige Schritte unternommen, insbesondere die Rolle von Sonderpädagog*innen in eine didaktische Unterstützungsrolle für alle Schüler*innen zu transferieren. Auf der Hinterbühne bleiben medizinische/psychologische Klassifikationen so genannter ,Special Needs', die als personenbezogene Kategorien an das Individuum geheftet sind, bestehen und bedingen hierdurch ressourcenorientierte Versuche der Unterstützungsvergabe. In Kanada erfolgt diese, wie beschrieben, u.a. entlang der Modifikation und Individualisierung des Curriculums. Dies zeigt letztlich, dass inklusive Schulentwicklung ein komplexer und auf unterschiedlichen Ebenen des Bildungssystems angesiedelter Reformprozess mit Fort- und Rückschritten ist (Kruschel/Jahr 2019).

Zusammenfassend kann konstatiert werden, dass die Bildungssysteme in Island und Kanada (New Brunswick) für die menschenrechtsbasierte und gemeinschaftsorientierte Bildung von Unterstützungssystemen stehen, die das Lernen und die Entwicklung aller Schüler*innen in der Schule unterstützen sollen. Der Vergleich der Schulentwicklungsprozesse zeigte, dass der Versuch unternommen wurde, ein ganzheitliches und prozessorientiertes Verständnis von Unterstützung in den Schulen zu etablieren, das sich anschlussfähig zeigt an Diskurse um Beratung, Individualisierung und Unterstützung für Lehrer*innen in der Schule.

Die nachhaltige Verankerung von Unterstützungsrollen deutet dabei auf einen Prozess hin, in dem Inklusive Bildung nicht als personenbezogenes oder ,technisches ' Problem, sondern als schulkulturelle Entwicklungsaufgabe angesehen wird (,cultural politics') (Slee/Weiner 2001). Um Halls (1985, vgl. auch Köpfer 2020) Konzept der articulation zu bemühen: Inklusive Bildung wird mit der Idee von Unterstützung für alle Schüler*innen entsprechend ihrer unterschiedlichen Bedürfnisse artikuliert. Dies steht in Kontrast zu inklusiven Schulentwicklungsprozessen in anderen Ländern. So zeigen international vergleichende Studien (z.B. Biermann 2021 i.d.B.), dass in manchen Ländern der Diskurs um Inklusive Bildung mit Diskursen von sonderpädagogischer Förderung (zum Beispiel Deutschland, Schweiz) oder Bildung für alle (z.B. Nigeria) verbunden wird und hierdurch zu einer latenten Sonderpädagogisierung von Inklusion führt. Dies zeigt sich unter anderem in steigenden Diagnostizierungsquoten und dem Fokus auf die Ausweitung sonderpädagogischer Klassifizierungssysteme (Biermann/Pfahl 2018). Hierbei ist kritisch anzumerken, dass Praktiken von Kompensation und Intervention Gefahr laufen, systemstabilisierend zu wirken und Marginalisierungen bzw. Segregation zu reproduzieren.

Vor dem Hintergrund der geleisteten Analyse des Aufbaus von inklusionsorientierten Unterstützungssystemen in Kanada und Island erscheint es daher im international ver- 
gleichenden Forschungsdiskurs notwendig, Unterstützung/support als Analysekategorie für Forschung mit Blick auf Inklusive Bildung/Inclusive Education weiterzuentwickeln. Dies kann z.B. durch weitere komparative Analysen mit Ländern/Regionen erfolgen, die sich durch ein hohes Maß an Spezialisierung und Separation bezogen auf die Unterrichtung von Schüler*innen mit und ohne sonderpädagogischen Förderbedarf auszeichnen. Dabei sind insbesondere Fragen von professionellen Rollenkonstellationen und dem Umgang mit Klassifikationen in den Blick zu nehmen (Biermann/Pfahl 2018). Weiter zeigt sich als Forschungsdesiderat die empirische Verknüpfung von Diskursen Inklusiver Bildung mit Demokratieentwicklung und sozialem Lernen. Hier hat die vorangegangene Analyse einen engen Zusammenhang von inklusiver und schulkultureller (und auch community- bzw. quartiersorientierter) Schulentwicklung gezeigt, den es empirisch weiter zu bearbeiten gilt.

Für die entsprechenden Reformprozesse in Deutschland und in anderen Ländern, die unter „Inklusion“" gefasst werden, lässt sich aus dem Vergleich von Island und Kanada ableiten, dass Inklusive Bildung als ganzheitlicher Schulentwicklungsprozess (Ainscow 2021 i.d.B.) anzusehen ist, der sich nicht in Fragen von Platzierung und Förderung von Schüler*innen mit sonderpädagogischem Förderbedarf erschöpfen kann, sondern den Aufbau ganzheitlicher und sozialer Unterstützungsstrukturen für alle Schüler*innen forcieren sollte, z.B. entlang von schulinternen Beratungs- und Unterstützungslehrpersonen - um hierdurch auch das Prinzip der inhärenten Delegation von Fragen von Inklusion an die sonderpädagogischen Fachkräfte zu vermeiden (vgl. kritisch Merz-Atalik 2018).

\section{Literatur}

Ainscow, Mel (2021 i.d.B.): Inclusion and Eequity in Education. In: Köpfer, Andreas/Powell, Justin J.W./Zahnd, Raphael (Hrsg.): Handbuch Inklusion international. Opladen: Budrich, S. $75-87$.

Ainscow, Mel/Sandill, Abha (2010): Developing Inclusive Education Systems. The Role of Organizational Cultures and Leadership. In: International Journal of Inclusive Education 14, 4, S. 401-416.

Allan, Julie/Catts, Ralph (2014): Schools, social capital and space. In: Cambridge Journal of Education 44, 2, S. 217-228.

Arbeitsgruppe Internationale Vergleichsstudie (Hrsg.) (2007): Schulleistungen und Steuerung des Schulsystems im Bundesstaat. Kanada und Deutschland im Vergleich. Studien zur International und Interkulturell Vergleichenden Erziehungswissenschaft, Band 9. Münster: Waxmann Verlag.

Artiles, Alfredo/Kozleski, Elisabeth/Dorn, Sherman/Christensen, Carol (2006): Learning in Inclusive Education Research. Re-mediating Theory and Methods With a Transformative Agenda. In: Review of Research in Education 30, 1, S. 65-108.

Artiles, Alfredo/Dyson, Alan (2005): Inclusive education in the globalization age: the promise of comparative cultural historical analysis. In: Mitchell, David (Hrsg.): Contextualizing Inclusive Education. London: Routledge, S. 37-62.

Berry, John (2013): Research on multiculturalism in Canada. In: International Journal of Intercultural Relations 37, 6, S. 663-67.

Biermann, Julia (2021 i.d.B.): Der Einfluss der UN-BRK auf inklusive Bildung in Nigeria und Deutschland. In: Köpfer, Andreas/Powell, Justin J.W./Zahnd, Raphael (Hrsg.): Handbuch Inklusion international. Opladen: Budrich, S. 167-178. 
Biermann, Julia/Pfahl, Lisa (2018): Wissen um Behinderung und das Recht auf Bildung - ein nigerianisch-deutscher Vergleich sonderpädagogischer Klassifikationssysteme und schulischer Inklusion. In: Bildung und Erziehung 71, 4, S. 432-448.

Biermann, Julia/Powell, Justin J.W. (2014): Institutionelle Dimensionen inklusiver Schulbildung - Herausforderungen der UN-Behindertenrechtskonvention für Deutschland, Island und Schweden im Vergleich. In: Zeitschrift für Erziehungswissenschaft 17, 4, S. 679-700.

Booth, Tony/Ainscow, Mel (2011): Index for Inclusion. Developing Learning and Participation in Schools. Bristol: CSIE.

Burns, Edward (2004): The special education consultant teacher. Enabling children with disabilities to be educated with nondisabled children to the maximum extent appropriate. Springfield, IL: C.C. Thomas.

Carrington, Suzanne/Robinson, Robyn (2006): Inclusive school community. Why is it so complex? In: International Journal of Inclusive Education 10, 4-5, S. 323-334.

Crawford, Cameron/Porter, Gordon (2004): Supporting teachers. A foundation for advancing inclusive education. Toronto: Roeher Institute.

Crossley, Michael/Watson, Keith (2003): Comparative and International Research in Education. London: Routledge Falmer.

Danforth, Scot/Naraian, Srikala (2015): This New Field of Inclusive Education. In: Intellectual and Developmental Disabilities 53, 1, S. 70-85.

Department of Justice (1982): Canadian Charter of Rights and Freedoms. Ottawa: Government of Canada.

DuFour, Rebecca/Eaker, Robert (2008): Revisiting Professional Learning Communities at Work. New Insights for Improving Schools. Toronto: Solution Tree.

Ekins, Alison (2013): Special education within the context of an inclusive school. In: Mac Ruairc, Gerry/Ottesen, Eli/Percey, Robin (Hrsg.): Leadership for inclusive education. Rotterdam: Sense Publishers, S. 19-33.

European Agency for Special Needs and Inclusive Education (2014): Organisation of Provision to Support Inclusive Education - Summary Report. Odense, Denmark: EASNIE.

European Agency for Special Needs and Inclusive Education (2016): Agency position on inclusive education systems. Odense, Denmark: EASNIE.

European Agency for Special Needs and Inclusive Education (2017): Education for all in Iceland. External audit of the Icelandic system for inclusive education. Odense, Denmark: EASNIE.

Feuser, Georg (2016): Die Integration der Inklusion in die Segregation. In: Böing, Ursula/Köpfer, Andreas (Hrsg.): Be-Hinderung der Teilhabe. Bad Heilbrunn: Klinkhardt, S. 26-43.

Finkelstein, Simon/Sharma, Umesh/Furlonger, Brett (2019): The inclusive practices of classroom teachers. A scoping review and thematic analysis. In: International Journal of Inclusive Education, S. 1-28.

Florian, Lani (2005): Inclusive Practice. What, Why and How. In: Maloney, Sheelagh/Topping, Keith (Hrsg.): The Routledge Falmer Reader in Inclusive Education. London: Routledge, S. 29-30.

Glaser, Barney/Strauss, Anselm (2008): Grounded Theory. Strategien qualitativer Forschung. Bern: Verlag Hans Huber.

Goffman, Erving (1959/2003): Wir spielen alle Theater. München: Pieper.

Hall, Stuart (1985): Signification, representation, ideology: Althusser and the post-structuralist debates. In: Critical Studies in Mass Communication 2, 2, S. 91-114.

Hart, Susan/Drummond, Mary Jane/McIntyre, Donald (2007): Learning without limits. Constructing a pedagogy free from determinist beliefs about ability. In: Florian, Lani (Hrsg.): The Sage handbook of special education. London: Sage, S. 499-514.

Hinz, Andreas (2007): Inklusion - Vision und Realität! Herausforderungen in Deutschland und Praxis in Kanada. In: Katzenbach, D. (Hrsg.): Vielfalt braucht Struktur. Heterogenität als 
Herausforderung für die Unterrichts- und Schulentwicklung. Frankfurt am Main: Goethe-Universität, S. 81-98.

Jahnukainen, Markku (2015): Inclusion, integration, or what? A comparative study of the school principals' perceptions of inclusive and special education in Finland and in Alberta, Canada. In: Disability \& Society 30, 1, S. 59-72.

Jóhannesson, Ingólfur Ásgeir (2006): 'Strong, independent, able to learn more... ' Inclusion and the construction of school students in Iceland as diagnosable subjects. In: Discourse: Studies in the Cultural Politics of Education 27, 1, S. 103-119.

Jónasson, Helgi (1996): Um sérkennslu og sérfræðipjónustu skóla. Ráðstefna Menntamálaráðuneytisins um ýmis málefni barna og unglinga með sérparfir.

Jónasson, Jón Torfi (2008a): Skóli fyrir alla? [School for all?]. In: Guttormsson, Loftur (Hrsg.): Almenningsfræðsla á Íslandi 1880-2007. Reykjavík: Háskólaútgáfan, S. 272-291.

Jónasson, Jón Torfi (2008b): Innlend stefnumið og alpjóðleg viðmið. [Local policies and international standards]. In: Guttormsson, Loftur (Hrsg.): Almenningsfræðsla á Íslandi 1880-2007. Reykjavík: Háskólaútgáfan, S. 254-269.

Kelley, Ninette/Trebilcock, Michael J. (2010): The making of the mosaic. A history of Canadian immigration policy. Toronto: University of Toronto Press.

Köbsell, Swantje/Hirschberg, Marianne (2021 i.d.B.): Disability Studies in Education. In: Köpfer, Andreas/Powell, Justin J.W./Zahnd, Raphael (Hrsg.): Handbuch Inklusion international. Opladen: Budrich, S. 127-146.

Köpfer, Andreas (2013): Inclusion in Canada. Bad Heilbrunn: Klinkhardt.

Köpfer, Andreas (2019): Creation of supportive inclusive learning communities. In: Peters, Michael (Hrsg.): Encyclopedia of Teacher Education. Heidelberg: Springer Meteor.

Köpfer, Andreas/Óskarsdóttir, Edda (2019): Analysing Support in Inclusive Education Systems a Comparison of Inclusive School Development in Iceland and Canada since the 1980ies focusing on Policy and In-School Support. In: Ainscow, Mel/Slee, Roger (Hrsg.): International Journal of Inclusive Education 23, 7-8, S. 876-890.

Köpfer, Andreas (2020): Artikulationen Inklusiver Bildung zwischen Strukturkritik und funktionaler Integration - Erkundungen mit Stuart Hall. In: Vierteljahresschrift für wissenschaftliche Pädagogik 93, 2, S. 296-310.

Kozleski, Elisabeth/Artiles, Alfredo/Waitoller, Federico (2011): Introduction: Equity in Inclusive Education. Historical Trajectories and Theoretical Commitments. In: Artiles, Alfredo/Kozleski, Elisabeth/Waitoller, Frederico (Hrsg.): Inclusive Education. Examining Equity on Five Continents. Cambridge, MA: Harvard Education Press, S. 1-14.

Kruschel, Robert/Jahr, David (Hrsg.) (2019): Inklusion in Kanada. Weinheim: Beltz Verlag.

Laluvein, Jackie (2010): School inclusion and the 'community of practice'. In: International Journal of Inclusive Education 14, 1, S. 35-48.

Lög um grunnskóla, 66/1995 §. 1995. Act on compulsory schools. Reykjavik: Government of Iceland.

Lög um grunnskóla, 91/2008 §. 2008. Act on compulsory schools. Reykjavik: Government of Iceland.

Löser, Jessica (2019): Inklusion systematisch im schulischen Kontext verankern. Ein Blick auf die kanadischen Provinzen Ontario, New Brunswick und Alberta. In: Kruschel, R./Jahr, D. (Hrsg.): Inklusion in Kanada. Weinheim: Beltz Verlag, S. 240-251.

MacKay, Wayne (2005): Inclusive Education: A Review of Programming and Services in New Brunswick. Fredericton: New Brunswick Department of Education and Early Childhood Development. https://ssrn.com/abstract=2126723 [Zugriff: 13.02.2020]

Marinósson, Gretar (2011): Responding to diversity at school. An ethnographic study. Berlin: Lambert Academic Publishing. 
Marinósson, Gretar/Bjarnason, Dóra (2014): Special education today in Iceland. In: Rotatori, Anthony/Bakken, Jeffrey/Burkhardt, Sandra/Obiakor, Festus/Sharma, Umesh: Special education international perspectives: Practices across the globe. Bingley, UK: Emerald Group Publishing Limited, S. 271-309.

Merz-Atalik, Kerstin (2018): Von einem Versuch „der Integration der Inklusion in die Segregation“?! In: Zeitschrift für Inklusion-Online 12, 4. https://www.inklusion-online.net/index.php/inklusion-online/article/view/508/371 [Zugriff: 10.02.2020]

Mey, Günter/Mruck, Katja (2011): Grounded-Theory-Methodologie. Entwicklung, Stand, Perspektiven. In: Mey, Günter/Mruck, Katja (Hrsg.): Grounded Theory Reader. Wiesbaden: VS Verlag, S. 11-48.

Ministry of Education, Science and Culture (2011): The Icelandic national curriculum guide for compulsory schools. General section. Reykjavik: Government of Iceland.

Mittler, Peter (2000): Towards Inclusive Education. London: Falmer.

Mulholland, Monica/O'Connor, Una (2016): Collaborative classroom practice for inclusion. In: International Journal of Inclusive Education 20, 10, S. 1070-1083.

Óskarsdóttir, Edda (1993): Developing a school in Iceland. Masters project, University of Oregon, Eugene.

Óskarsdóttir, Edda (2017): Constructing support as inclusive practice. A self-study. Doctoral dissertation, University of Iceland, Reykjavík.

Perner, Darlene/Porter, Gordon (1998): Creating inclusive schools: Changing roles and strategies. In: Hilton, Alan/Ringlaben, Ravic (Hrsg.): Best and Promising Practices in Developmental Disabilities. Austin, Texas: Pro-Ed, S. 521-541.

Peters, Susan (2007): 'Education for all?' A historical analysis of international inclusive education policy and individuals with disabilities. In: Journal of Disability Policy Studies 18, 2, S. 98108.

Phillips, David/Schweisfurth, Michele (2008): Comparative and international education. London: Continuum.

Porter, Gordon/Richler, Diane (1991): Changing Canadian Schools. Perspectives on Disability and Inclusion. North York, Ontario: Roeher Institute.

Porter, Gordon/AuCoin, Angela (2012): Strengthening Inclusion, Strengthening Schools. Report of the Review of Inclusive Education Programs and Practices in New Brunswick Schools. Fredericton: Government of New Brunswick.

Powell, Justin J.W. (2011): Barriers to Inclusion. Special Education in the United States and Germany. Abingdon: Routledge.

Powell, Justin J.W. (2018): Inclusive Education. Entwicklungen im internationalen Vergleich. In: Sturm, Tanja/Wagner-Willi, Monika (Hrsg.): Handbuch schulische Inklusion. Opladen, Toronto: Barbara Budrich, S. 127-142.

Powell, Justin J.W./Merz-Atalik, Kerstin et al. (2021 i.d.B.): Heterogene Lerngruppen inklusionsorientiert unterrichten. In: Köpfer, Andreas/Powell J.W./Zahnd, Raphael (Hrsg.): Handbuch Inklusion international. Opladen: Budrich, S. 357-379.

Reglugerð um sérkennslu 98/1990. [Regulation on special needs education].

Reglugerð um nemendur með sérparfir 585/2010. [Regulation on pupils with special needs].

Rieser, Richard (2011): Disability, human rights and inclusive education, and why inclusive education is the only educational philosophy and practice that makes sense in today's world. In: Richards, Gill/Armstrong, Felicity (Hrsg.): Teaching and learning in diverse and inclusive classrooms. New York: Routledge, S. 156-169.

Schroeder, Joachim (2016): Kanada kann auch anders. In: Zeitschrift für Inklusion-Online 10, 4. www.inklusion-online.net/index.php/inklusion-online/article/view/397/313 [Zugriff: 03.10 . 2019] 
Singal, Nidhi/Muthukrishna, Nihiti (2014): Introduction. Education, childhood and disability in countries of the South - re-positioning the debates. In: Childhood 21, 3, S. 293-307.

Slee, Roger/Weiner, Gaby (2011): Education Reform and Reconstruction as a Challenge to Research Genres. Reconsidering School Effectiveness Research and Inclusive Schooling. In: School Effectiveness and School Improvement. An International Journal of Research, Policy and Practice 12, 1, S. 83-98.

Thompson, Scott/Lyons, Wanda/Timmons, Vianne (2015): Inclusive education policy. What the leadership of Canadian teacher associations has to say about it. In: International Journal of Inclusive Education 19, 2, S. 121-140.

Timmons, Vianne (2006): Impact of a multipronged approach to inclusion. Having all the partners on side. In: International Journal of Inclusive Education 10, 4-5, S. 469-480.

Timmons, Vianne (2007): Towards Inclusive Education in Canada. In: Barton, Len/Armstrong, Felicity (Hrsg.): Policy, Experience and Change. Cross-Cultural Reflections on Inclusive Education. Dordrecht: Springer, S. 133-146.

United Nations (2006): Convention on the Rights of Persons with Disabilities and Optional Protocol.

UNESCO (1994): The Salamanca Statement and Framework for action on special needs education. World Conference on Special Needs Education; Access and Quality. Salamanca, Spain, 7-10 June 1994. UNESCO.

Valle, Jan/Connor, David (2011): Rethinking disability. A disability studies approach to inclusive practices. New York: McGraw Hill. 



\title{
16 Vergleichende Perspektiven auf die Kultur(-en) der (schulischen) Inklusion in Addis Abeba und Bangkok
}

\author{
Michelle Proyer, Margarita Bilgeri
}

\begin{abstract}
Das internationale Forschungsprojekt CLASDISA (Classifications of Disabilities, 20102015) untersuchte soziale und kulturelle Aspekte, die schulische Inklusion in drei Hauptstädten beeinflussen. Narrative Interviews mit Kindern mit Behinderung, deren Eltern und Lehrer*innen und Expert*innen ermöglichten Einblicke in den schulischen Alltag. Die Komplexität der Faktoren in den einzelnen Städten führte dazu, dass der vorgesehene Vergleich bisher stark unterbelichtet blieb. Ausgehend davon werden hier ausgewählte Aspekte zwischen Addis Abeba und Bangkok miteinander verglichen. So sollen unterschiedliche Perspektiven und kulturell bedingte Einflussfaktoren auf Inklusion illustriert, die Herausforderung der inter- bzw. transkulturellen Ggegenüberstellung problematisiert und kritisch im Sinne einer globalen Perspektive auf inklusive Bildung kontrastiert werden.
\end{abstract}

\section{Zum Geleit}

Wir betreten einen Schulcampus - ein Hof umgeben von einem dreistöckigen Gebäude, das, neben einem Internat für jene Schüler*innen, die von weither kommen, auch Platz bietet für eigene Werkstätten, eine Kantine und Klassen- und Therapieräume für mehrere hundert Kinder und Jugendliche. In der Mitte prangt eine sich kaum bewegende blau-weiß-rote Flagge. Es ist kurz vor 9 Uhr bei etwa 30 Grad. Auf dem zentralen Gebäude in der Mitte des Schulhofes findet sich ein überdimensionales Bild des damals noch regierenden Königs Bumiphol Adulyadej gerahmt von einem breiten goldenen Rahmen. Es ist kurz vor dem Vatertag, einem der höchsten Feiertage Thailands. Überall hängen von Schüler*innen gemalte Portraits des Königs. Sie sind nach künstlerischer Qualität geordnet. Die Maler*innen der schönsten haben in den letzten Tagen Preise verliehen bekommen und seien sehr stolz gewesen erklärt man uns - auch die Schulleitung sei sehr stolz auf die Besten der Besten. Die Schule ist mit zahllosen wunderschön-kitschigen aus buntem und goldenem Stoff gebundenen Maschen verziert. Alle scheinen in erwartender Vorfreude. Aufführungen werden geprobt, Kostüme genäht und probiert, Kekse in der hauseigenen Bäckerei vorbereitet. Alles läuft geordnet und mit engelsgleicher Beherrschtheit ab. Der betörende Duft lässt den Smog der zahllosen Autos, die am Schulgebäude vorbei preschen, fast vergessen. Der Schulhof wird zu einer Oase. Wir dürfen einen Blick in eine Klasse werfen, nachdem uns die Schuladministration mit Kaffee, Kuchen und tropischen Früchten herzlich willkommen geheißen hat. In der Klasse befinden sich etwa acht Schüler*innen im Alter von zwölf Jahren. Sie lernen mittels einer von 
einem Lehrer selbst, für uns ungewöhnlichen, aber wunderschön anmutenden, hergestellten DVD, Bewegungen mit ihren Händen und Fingern zu machen. Es handelt sich um Unterricht in traditionellem thailändischem Tanz. Diese Gruppe soll in den nächsten Tagen in komplex arrangierten Kostümen auftreten, zur Feier des Geburtstags des Königs.

Wie bei jedem Schulbesuch in Addis Abeba sehen wir uns nach der Begrüßung durch die Schulleiter*innen als erstes das gesamte Schulgelände genauer an. Die Schule hat mehrere Gebäude, einige älteren, einige neueren Datums. Der Schulhof ist hier riesig, was nicht bei jeder Schule so ist. Auf dem Weg zu den Toiletten finden wir ein einen Meter auf einen Meter großes und ebenso tiefes Loch mitten auf dem Schulhof. Es gibt keine Absperrung rund herum. Die Toiletten sind, wie meistens, einfache kleine Häuschen mit Löchern im Boden. Es wurde allerdings extra eine Sitztoilette für Schüler*innen mit Behinderung errichtet. Dies ist vermutlich auch eine Folge davon, dass schon einige Kinder darauf hingewiesen hatten, dass sie beim Benutzen der Toiletten schmutzig werden, da sie ihre Kleidung aufgrund von unterschiedlichen Behinderungen nicht vom schmutzigen Boden fernhalten können. Wir gehen hinüber zur speziell errichteten Toilette. Sie ist abgesperrt und nicht benutzbar, weil sie kaputt ist.

An dieser Schule gibt es Sonderklassen für Kinder mit Behinderung. Sobald die Kinder ein bestimmtes Level erreicht haben, können sie in die regulären Klassen wechseln. Nicht jede*r Schüler*in kann dieses Level erreichen. Die Sonderklassen befinden sich in einem eigenen Gebäude, gleich nach dem Eingang zum Schulgelände, abgesondert und weit weg vom regulären Schulgebäude. Beim täglichen Antreten zum Singen der Nationalhymne sind die Kinder der Sonderklasse nicht dabei. Sie sitzen draußen in einem Stuhlkreis vor ihrem Klassenzimmer. Als wir hinzu kommen springt einer der Schüler auf, und bringt mir einen Sessel, den er zuvor mit Hingabe mit einem Tuch sauber gemacht hat. Mit einem übers ganze Gesicht strahlenden Lächeln fordert er mich auf, mich hinzusetzen.

\section{Einleitung}

So oder ähnlich, würde man eine Abhandlung zu Kulturen der Inklusion in verschiedenen Ländern wohl erwarten - dichte Beschreibungen der exotischen und von einer westlich gedachten Norm von Inklusion abweichenden Zugänge. Beschreibungen von Situationen, in denen man sich denken könnte: „So geht Inklusion ja wohl gar nicht, aber klar, wie sollen die das besser wissen." Wir müssen die hoffnungsvollen Erwartungen der Leser*innen an exotische Darstellungen dahingehend allerdings enttäuschen. Vielmehr wollen wir auf den folgenden Seiten kritisch reflektieren, welche Aspekte in Bezug auf eine globale Kultur der Inklusion sich im Verlauf eines mehrjährigen Forschungsprojektes in unterschiedlichen Kulturen als relevant ergeben haben und wo die Vergleichbarkeit Grenzen aufweist. Kein Land weltweit kann bisher mit einer umfassend realisierten inklusiven schulischen Praxis aufwarten. Einblicke in inklusive Zugänge unterschiedlicher Länder (vgl. Powell et al. 2021 i.d.B.) können unserer Meinung nach einen breiter gedachten Zugang zu inklusiven Praktiken ermöglichen, wenn von essentialistischen, auf kulturelle Gegebenheiten bezogenen und in der Darstellung reduzierten Praktiken Abstand genommen wird. Wir plädieren so also für eine globale Kultur der Behinderungsforschung (vgl. Biermann 2021 i.d.B.) bzw. Inklusionsforschung. Inklusion darf keine territorialen Grabenkämpfe hervorrufen oder gar ein kolonial geprägter Diskurs werden. 
Vorab stellen wir die Hintergründe des Projektes dar und beziehen uns dann auf zwei ausgewählte Ebenen, die wir vergleichend-diskursiv einführen, um dann in einem kritischen finalen Statement, die Notwendigkeit einer globalen Vision auf Inklusion zu diskutieren, welche innterkulturelle bzw. transkulturelle Momente berücksichtigt und respektiert. Transkulturell verstehen wir dabei als über einen interkulturellen Vergleich zwischen Ländern hinausgehenden Ansatz, der darauf abzielt auch individuelle und national unterschiedliche Facetten aufzuzeigen und zu verstehen.

\section{CLASDISA - International, interkulturell oder transnational vergleichend?}

Im Projekt CLASDISA ${ }^{1}$ (Proyer et al. 2011), auf dessen Ergebnissen dieser Beitrag aufbaut, wurden zwischen 2010 und 2015 in den Hauptstädten Österreichs, Thailands und Äthiopiens mit einem, auf einem mixed-methods Ansatz basierenden, Forschungsdesign mit Schwerpunkt auf qualitativem Material, Daten gesammelt, um in Schulen Barrieren und Förderfaktoren für Kinder mit Behinderung im schulpflichtigen Alter der jeweiligen Länder in den unterschiedlichen Kulturen zu analysieren. Zu diesem Zweck wurden Kinder mit Behinderungen, deren Eltern, Lehrer*innen und andere Expert*innen interviewt. Es fanden zudem Unterrichtsbeobachtungen und Erkundungen des Schulgeländes statt. Ziel der Untersuchung war es, die kulturellen und sozialen Grundannahmen und einflussreiche Aspekte zu erforschen, die Barrieren und Förderfaktoren. Mit Bezugnahme auf die Logik der Umweltfaktoren der ICF (WHO 2001) wurden Barrieren und Förderfaktoren für die Beschulung von Kindern mit unterschiedlichen Behinderungen im Schulalter erhoben und beschrieben. Dabei stand weniger im Vordergrund, ob diese inklusiv oder besondert beschult wurden, sondern inwieweit soziale und kulturelle Gegebenheiten die jeweils vorhandenen Systeme beeinflussen. $\mathrm{Zu}$ jeder der drei Städte entstand je eine Dissertation (Proyer 2014; Schiemer [Bilgeri] 2014; Kramann 2017), die über eine Annäherung durch Ansätze aus dem Methodenkomplex der Grounded Theory darauf abzielten, für bildungsbezogene Kontexte, die von Kindern mit Behinderungen frequentiert werden oder von diesen ausgespart werden, relevante und kulturspezifische Aspekte herauszuarbeiten. Zum Beispiel interessierte es das Projektteam welchen Einfluss religiöser Glaube oder gesellschaftliche Praktiken auf das Bild von Behinderung haben bzw., ob diese sich überhaupt auswirken. Der konstante Austausch zwischen den Forschenden, die in den drei Kontexten agierten, führte zu einem breiten Spektrum an Themen, die in den jeweiligen urbanen Räumen erforscht wurden. Die zentralen Ergebnisse der jeweiligen Studien sind in den Dissertationen bzw. daraus entstandenen Publikationen nachzulesen.

Nachfolgend sollen überblicksmäßig relevante Aspekte der Arbeiten der beiden Autorinnen angeführt werden, um vergleichende Perspektiven einzuleiten.

Trotz der langen Laufzeit des Projektes blieb aufgrund des umfangreichen Datenkorpusses und der Komplexität der regionalen Gegebenheiten und innerhalb des Landes auftretender inter- (z.B. im Sinne unterschiedlicher kultureller Hintergründe der involvierten Forschungsteilnehmenden v.a. in Österreich) und transkultureller Unterschiede (Bsp. Unter-

1 Projektnummer: P22178, finanziert vom österreichischen Wissenschaftsfonds (FWF). https://classifications-of-disabilities.univie.ac.at/ 
schiedliche Relevanz von Religion, Spiritualität, Ansichten über Bildung etc.), die erwartet aber im Umfang unterschätzt wurden, wenig Zeit, um die unterschiedlichen Standorte zu vergleichen. Die vergleichende Ebene zwischen den drei Forschungsfeldern ist - abgesehen von einer komparativen Dissertation (Ozik-Scharf 2015), die sich auf die quantitativen Daten fokussierte - abseits von wenigen Publikationen (Schiemer [Bilgeri]/Proyer 2013; Schiemer [Bilgeri]/Proyer 2015; Biewer et al. 2016) - noch wenig elaboriert.

\subsection{Addis Abeba (Margarita Bilgeri ehem. Schiemer):}

Mit Blick auf die Forschung in Äthiopien können spezifische Aspekte identifiziert werden, die im Kontext Behinderung und Kultur als wichtig erscheinen. So wurde als wichtigstes Moment in den gesammelten Daten die Dimension „feeling like a family“ identifiziert. Diese Hauptkategorie stand im Zusammenhang mit einem Zugehörigkeitsgefühl zwischen den Interviewten untereinander und im Kontext der jeweiligen Schule. Am deutlichsten konnte das Gefühl der Familienzugehörigkeit zwischen Lehrer*innen und Eltern beobachtet werden. Es führte bei den Schüler*innen mit Behinderung zu mehr Selbstbewusstsein und sie erfuhren vermehrt Unterstützung. Andererseits waren Familie und Zugehörigkeit auf Seiten der Kinder wichtige Elemente auf zwei Ebenen. Einerseits ging es darum, als Kind die Eltern später versorgen zu können und andererseits ging es darum, durch diese Leistung auch ein geschätztes Mitglied der Gesellschaft zu werden. Für die Kinder war also eines der wichtigsten Ziele, ein Zugehörigkeitsgefühl zur eigenen Familie aber auch zur Gesellschaft bzw. Gemeinschaft allgemein zu entwickeln (Schiemer [Bilgeri] 2017). Es wird hier deutlich, dass es spezifische kulturell geprägte und traditionell tief verwurzelte Wertesysteme gibt, die generell im Alltag der Kinder, aber auch spezifisch im Bezug auf ihre Bildung eine extrem wichtige Rolle einnehmen. Dies zeigt sich auch weiter unten in den von uns gewählten zwei Bereichen zum Vergleich.

\subsection{Bangkok (Michelle Proyer):}

Zentrales Ergebnis der Forschung in Bangkok ist die enge Verwobenheit des Themas Behinderung mit der soziokulturellen Komponente des Glaubens an das Karma. Die meisten der Befragten titulierten sich selbst als Buddhist*innen. Aus den Ergebnissen konnte eine Abstufung, die Empathie (Toleranz und Akzeptanz) gegenüber Menschen mit Behinderungen und mögliche Auswirkungen auf Schulentscheide (sonderschulische, inklusive oder integrative Settings werden meist alle als inklusiv bezeichnet) generiert werden. Das abgeleitete breitere Modell fokussiert auf die Interrelation zwischen Medizin, Kultur und Behinderung: KarmaMedical-Model of Disability (Proyer 2014). Dies deutet auf die Verwobenheit von religiös motivierten Praktiken und dem Glauben an medizinische Erkenntnisse ab. Eine zentrale soziokulturelle Kompenoente nahm bei den untersuchten Fällen das Bestreben nach dem NichtAuffallen bzw. sich in die Gesellschaft einfügen an. Eltern scheinen großen Wert auf die Aufrechterhaltung genormter gesellschaftlicher Realitäten zu legen (z.B. besonderes Augen- 
merk auf die gutsitzende Schuluniform, unauffälliges Verhlaten in der Öffentlichkeit, wenn das Kind eine sichtbare Behinderung hat etc.).

Mit Blick auf die Forschungsvorhaben in beiden Ländern gilt anzumerken, dass es keinerlei Probleme im Zugang zum Forschungsfeld gab und sich bis auf eine Ausnahme im Verlauf der Sammlung von ca. 140 (Äthiopien) bzw. mehr als 200 (Thailand) qualitativen Datensätzen keine Herausforderungen ergaben.

Im Folgenden haben wir zwei Themen gewählt, die unterschiedliche Ebenen schulischer Inklusion, Integration und sonderschulischer Bildungssettings für Kinder mit Behinderungen in den jeweiligen Forschungsregionen abbilden. Wir werden diese beispielhaft gegenüberstellen, um aufzuzeigen, wie Unterschiede zwischen den Städten identifiziert und in weiterer Folge verglichen werden können. Zusätzlich werden aber auch Grenzen der Vergleichbarkeit aufgezeigt, die unter anderem etwas damit zu tun haben, dass die Unterschiede innerhalb eines Forschungskontexts für sich genommen schon der Vergleichbarkeit bedürfen. Vorab wird nun in gesetzliche Rahmenbedingungen und den Stand der Umsetzungsbemühungen bzw. der Implementierung inklusiver Bildung in den beiden Ländern eingeführt, um eine gute Basis des Nachvollziehens von Unterschieden zu entwickeln.

\section{Rahmenbedingungen schulischer Inklusion in Äthiopien und Thailand}

All children will have access to schooling and remaining barriers to learners' participation in schooling will be removed. There will be fair treatment for each child and no child will be discriminated against because of low income, gender, creed, race, location or disability. The policy intention is that no child should be out-of-school at any stage during the primary school years (Ethiopian Ministry of Education 2015: 35).

Dieses politische Statement bildet prinzipiell eine solide Basis, um inklusive Bildung in die Realität umzusetzen. Herausforderungen finden sich v.a. an zwei Orten: den Schulen und der Gesellschaft generell, wobei es teilweise natürlich Überschneidungen zwischen beiden gibt. Betrachtet man die Schulen, so wird schnell deutlich, dass Lehrer*innen sich mehr Ausbildung hinsichtlich der Organisation von heterogenen Klassenräumen wünschen. Ihnen mangelt es an Wissen, Information und passenden Methoden für den Unterricht. Ebenso finden sich oft Ängste und Ablehnung unter den Kolleg*innen oder auch bei der Schulleitung. Diese grundlegenden Barrieren spiegeln sich auch in der Gesellschaft wider. Negative Einstellungen gegenüber Behinderung sind meist auf religiöse Hintergründe zurückzuführen. So wird die Behinderung eines Kindes meist als eine Strafe Gottes wahrgenommen (Schiemer [Bilgeri] 2017). Scham, kein Zugang zu Informationen und andere fehlende Unterstützung führen bei der Familie zu Schwierigkeiten im Umgang mit der Behinderung eines Kindes. Auf der anderen Seite werden traditionelle Settings, bei denen viele Mitglieder einer Gemeinschaft anwesend sind, oft dazu genutzt, um Aufklärung, Information oder Hilfe anzubieten.

Blickt man auf die gesetzlichen Grundlagen hinsichtlich inklusiver Bildung scheint in Äthiopien, wie in so vielen anderen Ländern, alles auf einem guten Weg zu sein. Die UNKonvention über die Rechte von Menschen mit Behinderung wurde unterzeichnet und ratifiziert, im Gesetz findet man Schulpflicht für alle Kinder, die Verpflichtung für Schulen, Kinder mit Behinderung aufzunehmen und an den Ausbildungsstätten für zukünftige Leh- 
rer*innen findet man Sonderpädagogik bzw. special needs education, wenn auch nicht sehr prominent, im Lehrplan.

Der aktuelle „Education Sector Development Plan“ der äthiopischen Regierung ist bereits der 5. und für die Periode 2015/16-2019/20 gültig. Es wird darin festgehalten, dass 2013/14 von 5 Millionen Kindern mit besonderen Bedürfnissen lediglich 77.850 in den Stufen 1-12 registriert waren. Aktuelle Zahlen waren nicht verfügbar, sind aber in Planung, erhoben zu werden (Ethiopian Ministry for Education 2015: 26). Dies verdeutlicht den Handlungsbedarf auf der Ebene inklusiver Bestrebungen vor allem mit Blick auf die Zielsetzungen der Agenda 2030 (UN online).

Informationsmangel und die Angst davor, aufgrund eines Kindes mit Behinderung gesellschaftliche Erwartungen nicht erfüllen zu können oder unnötig aufzufallen, waren - ähnlich wie in Äthiopien - Themen, die von thailändischen Forschungsteilnehmer*innen als relevant für den gesellschaftlichen Diskurs rund um Behinderung angeführt wurden (Vorapanya/Dunlap 2014). Auf der einen Seite sind gesellschaftlich und institutionell verankerte Meinungsbilder, sowie Ressourcenmangel zwei Aspekte, die die Implementierung von inklusiver Bildung eher hemmen. Ein auf Mitleid beruhender Zugang bestimmt dabei weitestgehend die Beschulung von Kindern und Jugendlichen mit Behinderung und lässt akademische Orientierung in den Hintergrund treten. Auf der anderen Seite sind Bestrebungen, internationale Verpflichtungen (UN-BRK und Sustainable Development Goals-SDGs-2030, UN online) einzuhalten, ausgeprägt und treiben die inklusive Agenda voran. Seit 2002 ist die verpflichtende Beschulung für Kinder und Jugendliche zwischen 7 und 16 Jahren, unabhängig von der Art der Behinderung, verankert. Im thailändischen Jahr 2550 (2007) wurde der ,Persons with Disabilities Empowerment Act' eingeführt, der explizit darauf verweist, dass Bildung für Kinder und Jugendliche mit Behinderung auch non-formal erfolgen kann. Ein Jahr später folgte der ,Education for Persons with Disabilities Act', in welchem erstmals explizit festgehalten wird, dass Bildungsangebote für diese Gruppe qualitative hochwertig sein müssen und, dass freie Wahl hinsichtlich der Art der Schulform bestehen muss (Vibulpatanavong 2017). Im Jahr 2017 wurde schließlich der gesetzlich nicht bindende ,National Education Plan 2017-2036“ verabschiedet, welcher vor allem auf die Steigerung des allgemeinen Schulzugangs fokussiert, aber auch explizit auf ,Education for All` und ,Inclusive Education' verweist. Laut Vibulpatavongs Übersetzung ist in jener Passage, welche auch Behinderung betrifft, zwar explizit auf die Relevanz der Offenheit jeglicher Schulart verwiesen, allerdings auch ein klar medizinischer Fokus gelegt, indem darauf verwiesen wird, dass der/die jeweilige Schüler*in auch den akademischen Inhalten folgen können muss (ebd.). Laut der Autorin unterscheidet sich der Zugang zu inklusiver Bildung in diesem Dokument maßgeblich von jenem in internationalen Dokumenten. Das Primat des medizinischen Modells fand sich wie bereits beschrieben auch sehr klar im Datenmaterial der Dissertation zu Thailand wieder (Proyer 2014). Explizit wurde auch auf die mangelnde Qualität der Bildungsprovision verwiesen. Dies lässt einerseits auf Mängel in der Ausbildung schließen, welche nach wie vor stark in der Vermittlung eines medizinisch geprägten Zugangs auf Behinderung verharrt. Viele Standorte weisen daher Therapieräume auf, in denen beispielsweise audiometrische Messungen vorgenommen werden. Des Weiteren lässt sich die niedrige Qualität der Bildung für Personen mit Behinderung auch hinsichtlich niedriger Erfolgsquoten von Studierenden mit Behinderungen an Hochschulen ablesen (Vibulpatanavong 2018).

Die vorangegangenen Zeilen mögen dazu animieren eine westliche Betrachtungsweise einzunehmen und dementsprechende Erwartungshorizonte bezüglich schulischer Inklusion zu erzeugen. Gerade der Verweis darauf, dass die theoretische Orientierung an interna- 
tionalen Dokumenten, wie der UN-BRK (UN 2006), zu Recht zu unterschiedlichen Umsetzungsstrategien geführt hat und dadurch auch den Umsetzungsgrad bestimmt, scheint hinsichtlich einer kultursensiblen Perspektive auf Inklusion besonders relevant. Es besteht die Notwendigkeit, diese Dokumente auf der Basis unterschiedlicher Zugangsweisen neu zu interpretieren und zu nutzen.

\section{Ausgewählte Vergleichsebenen schulischer Inklusion in Addis Abeba und Bangkok}

Als Ausgangspunkt einer vergleichenden Perspektive beziehen wir uns auf zwei Dimensionen: Die Schul- und Unterrichtsgestaltung als Faktor auf einer Mesoebene, also auf der Ebene der Institution Schule, und die Motivation der Lehrer*innen auf einer Mikroebene, also des individuellen Levels.

Wir haben diese Themen auch deshalb gewählt, da sie aktuelle Fragen betreffen, die rund um die Implementierung von inklusiver Schule stark diskutiert werden: Schul- und Unterrichtsgestaltung sind hinsichtlich der Grenzen der Umsetzbarkeit von Inklusion relevant. Unter anderem sei hier auch auf den sich langsam auf den deutschsprachigen Raum ausweitenden Ansatzes des Universal Designs (for Learning) verwiesen. Dieser war im Verlauf der Forschung zwar noch kein Thema, retrospektiv gesehen bietet dieser Ansatz aber ein geeignetes ,framing“. Das zweite Thema - die Motivationslage von Lehrpersonen bzw. verwandte Aspekte der Lehrer*innenbildung (beispielsweise Grenzen der Vermittelbarkeit von Inklusion als gesamtgesellschaftliche Vision) - spielt aktuell eine relevante Rolle. Wichtig gilt es hier anzumerken, dass einige der dargestellten Zusammenhänge, Ergebnisse weiterer Auseinandersetzung mit dem Forschungsfeld und der kritischen Reflexion unserer Abschlussarbeiten sind.

\subsection{Schul- und Unterrichtsgestaltung}

Der einzige für mich äußerlich sichtbare Hinweis auf eine inklusive Gestaltung des Schulgeländes waren in Addis Abeba Rampen. Allerdings waren auch diese nicht überall vorhanden, ganz zu schweigen von den neuen fünfstöckigen Schulgebäuden. Mit dem Ziel vor Augen, den Zugang zu Bildung für Kinder zu verbessern, wurden landesweit zahlreiche solche identischen Schulen gebaut. Obwohl die Idee der Inklusion in den Dokumenten des Bildungsministeriums tief verwurzelt ist (Ethiopian Ministry of Education 2015), wurde weder bei den neuen fünfstöckigen Schulen noch bei neu errichteten Universitäten auf die Vermeidung von baulichen Barrieren Rücksicht genommen. In den im Projekt besuchten Schulen waren vereinzelt Kinder in Rollstühlen zu sehen - viel häufiger jedoch Kinder mit Krücken oder anderen Mobilitätshilfen. Aus den Interviews ging hervor, dass Rollstühle oft zu teuer oder nicht verfügbar waren. Kann daraus geschlossen werden, dass vorhandene Barrieren aus dieser Perspektive beurteilt werden? Also vor dem Hintergrund der eingesetzten und vorhandenen Hilfsmittel? Schließlich wurden Kinder in Rollstühlen meist kurzerhand in den Klassenzimmern im Erdgeschoss untergebracht. Ein nicht zu unterschätzender Aspekt war die Lage der 
Schulen. Die meisten von uns besuchten Schulen lagen an Hängen, teilweise schon nahe am Wald. Dies hatte zur Folge, dass das Erreichen der Schule einerseits schon für einige Kinder problematisch war und andererseits die Klassenräume oft sehr kalt waren. Während es auf der Straße meist um die $25^{\circ} \mathrm{C}$ hatte, trugen die Kinder (und wir dann auch) in den Klassenzimmern Pullover und Jacken. Nach dem Singen der Hymne (an der wie erwähnt nicht immer alle Kinder teilnahmen) während der auch die äthiopische Fahne gehisst wurde, marschierten die Klassen meist in Reih und Glied in ihre Klassenzimmer. Die Unterrichtsgestaltung selbst war fast ausschließlich durch Frontalunterricht in sehr großen Klassen geprägt.

Betrachten wir die Gestaltung des Unterrichts (aus der Perspektive des Universal Design for Learning, UDL) so kann angemerkt werden, dass die Lehrer*innen in einigen Schulen äußerst motiviert waren, unterschiedliche Materialen selbst herzustellen. In einzelnen Schulen fanden wir teilweise spannende Materialien, die jedoch immer von Spender*innen oder Organisationen kamen. Der generelle Mangel an Materialien war an allen Schulen ein Thema. Aus diesem Grund ist die Kreativität, die einige Lehrer*innen an den Tag legten, besonders hervorzuheben. Dies spielt vor allem dann eine Rolle, wenn nicht vorgegebene (meist westliche) Konzepte die Grundlage für die Entwicklung solcher Materialien bildet, sondern die Erfahrung des Unterrichts heterogener Klassen im eigenen Kulturraum. Es geht in anderen Worten darum, dass nicht davon ausgegangen werden kann, dass im Klassenzimmer alle Schüler*innen einen einheitlichen Hintergrund haben (Rose et al. 2006). UDL setzt an diesem Punkt an und setzt den Fokus auf diverse Lernwege für unterschiedliche Schüler*innen.

With UDL, there is a focus on learning relevance, value, and authenticity in terms of learners' needs and desires through the inclusion of real-life tasks and an understanding of the importance of flexibility (Rogers-Shaw et al. 2017: 21).

Der Vorteil einer solchen Perspektive liegt hier z.B. in den „real-life tasks“, die sich in unterschiedlichen Kulturen essentiell hinsichtlich des Inhalts, der Bedeutung und des Werts unterscheiden. Das heißt, es kann auf diese Unterschiede entsprechend eingegangen werden.

Im Kontext des Unterrichts spielen kulturelle Hintergründe, Werte und Traditionen eine große Rolle. Rogers-Shaw et al. (2017) erwähnen im Kontext von UDL auch die Rolle der Gemeinschaft. In der Publikation wird dies zwar nur als Nebenaspekt behandelt, die Ergebnisse der Studie in Äthiopien gab jedoch klare Hinweise auf die große Bedeutung von „,community“ und „family“. Eine solche Erkenntnis kann dazu genutzt werden, entsprechende Schwerpunkte bei der Anwendung von Lehr-Lernkonzepten zu setzen. Dabei muss auch darauf hingewiesen werden, dass manche (westliche) Konzepte wichtige (kulturelle) Aspekte womöglich völlig außer Acht lassen.

Die in Bangkok besuchten Schulen waren bezüglich ihrer Ausstattung sehr unterschiedlich. Barrierefreie Gestaltung hat dabei unter anderem auch etwas mit dem Alter der jeweiligen Schule zu tun: Einige der Standorte sind an benachbarte Tempel (Wats) angegliedert, was auf die Anfänge schulischer Bildung in Thailand zurückzuführen ist, als ausschließlich männliche Angehörige höherer Gesellschaftsschichten von Mönchen unterrichtet wurden (McDaniel 2010). Diese als klassisch zu bezeichnenden Schulgebäude weisen wenig Berücksichtigung universeller Designansätze auf, je nach Größe sind diese eher verwinkelt und wirken dunkel und kahl. Meist sind die Gebäude mehrstöckig, ohne Aufzüge. Auffallend war, dass durchwegs alle Standorte extrem sauber wirkten und viel Personal zur Instandhaltung ständig vor Ort war. Die eingangs beschriebene Dekoration durfte an keinem Standort an den zahllosen Feiertagen fehlen. Zeremoniell und Ordnung bestimmen den Schulalltag und damit auch die Gestaltung und Funktionalität des Schulraumes. Neben der freiwilligen Morgen- 
meditation, die an einigen Standorten angeboten und von allen Schüler*innen und Lehrer*innen besucht wird, findet ähnlich wie in Äthiopien allmorgendlich ein Appell statt, bei dem die Fahne gehisst und die Hymne gesungen wird. Bei dieser Gelegenheit werden auch aktuelle Informationen, Verhaltensregeln etc. an die Schüler*innen weitergegeben und ausgewählte, wechselnde Lehrpersonen sind zur Kontrolle der Schüler*innen eingeteilt. Sie achten auf Pünktlichkeit, kontrollieren die Sauberkeit und Passform der Uniformen, sowie mitunter die Haarlänge und das Verhalten der Schüler*innen.

Schulen, die auf bestimmte Gruppen von Schüler*innen spezialisiert sind, unterscheiden sich architektonisch nicht unbedingt von anderen Standorten. Einzig die Größe und Nähe zu Stiftungen, Krankenhäuser oder anderen sozialen oder therapeutischen Einrichtungen, die an der Finanzierung oder Pflege der Schüler*innen beteiligt sind, können relevante Marker sein. Häufig finden sich Statuen oder Bildnisse von Vertreter*innen der Königsfamilie an einem zentralen Ort der Schule. Spannenderweise findet sich in einem Außenbezirk von Bangkok eine Ballung an Einrichtungen. Neben Schulen finden sich dort auch Waisenhäuser, Einrichtungen für Kinder, wie auch für sozial benachteiligte Erwachsene. Dieser Standort ist umgangssprachlich auch als soi pikarn (spezielle Straße) bekannt.

Integrative oder gar inklusive Standorte weisen sogenannte ressource rooms auf, in denen speziell zusammengesetzte Gruppen unterrichtet werden, ihre Freizeit miteinander verbringen oder meist selbst erstelltes Material für den Unterricht gelagert wird. Die Ausstattung der Schulräume kann entlang der Kriterien der Umweltfaktoren der ICF (WHO 2001; Proyer 2014) als ausreichend beschrieben werden. Meist sind eigene Räumlichkeiten für spezifische Diagnostik oder Therapieformen mit funktionierender technischer - wenn auch nicht aktuellsten technischen Standards entsprechend - Ausstattung vorhanden. Besonders sich aus klimatischen Verhältnissen ergebende Herausforderungen (durchgehende Hitzephasen mit Temperaturen über 35 Grad und starke Monsoon-Regenschauer, die zu Überschwemmungen führen), sowie umfeldbezogene Einflussfaktoren, wie Lärm- und Abgasbelastung durch die hohe Dichte an Fahrzeugen in Thailands Hauptstadt werden als beeinträchtigend angeführt. So wäre der Einsatz von Aircondition in allen Klassenräumen für fast alle Schulen unbezahlbar. Deckenventilatoren sind meist vorhanden, aber nicht immer einsatzbereit (Stromausfälle). Interessanterweise sind neben den Administrationsräumen, die Räume für Therapien diejenigen, die Klimaanlagen aufweisen.

In den regulären Klassenräumen findet sich meist eine höhere Anzahl an Schüler*innen als man diese aus dem europäischen Kontext kennt. Meist finden sich in den Räumen Einzeltische, die mitunter sehr eng zusammengeschoben werden, damit die Kinder und Jugendlichen zusammenarbeiten können bzw. ihre Aufmerksamkeit auf die Lehrperson fokussieren können (vgl. Proyer/Sriwanyong 2016).

Die Unterrichtsgestaltung kann grob als ,traditionell‘ beschrieben werden. Ähnlich wie in Äthiopien ist diese von Frontalunterricht geprägt, welcher sehr eng an das Curriculum und die jeweiligen zum Einsatz kommenden Bücher gestaltet ist. Eines der zentralen Themen sind die Lehren des Buddha, die auch Regeln für das Zusammenleben und gesellschaftliche Orientierung vermitteln sollen. Nachsagen, Auswendiglernen von einfachen Dialogen und Abschreiben von Tafelbildern stehen didaktisch gesehen an der Tagesordnung. Meist wird zusätzliches Material, so es eingesetzt wird, selbst gestaltet. Mitunter scheint es einen informellen Wettbewerb unter den Lehrpersonen zu geben, wer das liebevollere und detailreicher gestaltete Material zum Einsatz bringt. Häufig handelt es sich bei diesem Material um Zeigetafeln, welche den Schüler*innen anzeigen, wie etwas geschrieben, ausgesprochen oder ge- 
zeichnet wird. Diese werden dann als Vorlagen verwendet oder es gilt Bilder oder Begriffe zuzuordnen.

Entsprechend der Unterrichtsgestaltung stehen für die Lehrpersonen meist die Vermittlung von Alltagsfertigkeiten im Zentrum. Der Fokus liegt je nach Behinderung weniger oder mehr auch auf der Vermittlung von akademischen Kompetenzen. Vor allem im Bereich der Sinnesbehinderung ist der Zugang zu weiterführender Bildung in Thailand ausgeprägt. Leider bleibt aber häufig wenig Zeit sich tiefergehend in besondere Kompetenzen, wie Braille oder Gebärdensprache einzuarbeiten, was mitunter zu Herausforderungen im Unterricht führt und Auswirkungen auf die Qualität der Bildungsabschlüsse hat.

In diesem Kapitel zur Schul- und Unterrichtsgestaltung wird deutlich, dass - wie zu erwarten war - Unterschiede in den vorhandenen Ressourcen auffallen. Die genannten Bereiche der ICF (Umweltfaktoren) sind in den beiden Ländern unterschiedlich zu bewerten. Bezüglich technischer Ausstattung liegen die Schulen in Addis Abeba eindeutig auf einem niedrigeren Niveau als die beforschten Schulen in Bangkok.

Die Lösungsansätze, die bei den jeweiligen Lehrer*innen für ressourcenbedingte Problemlagen herangezogen werden, gleichen sich in Bezug auf ihren hohen kreativen Anteil, sind jedoch auch spezifisch auf kulturelle Gegebenheiten bezogen. So sind Tätigkeiten wie Kaffeekochen für die traditionelle Kaffeezeremonie in Äthiopien mit wichtigen traditionellen Werten verbunden. Das Erlernen dieser Tätigkeit hat in diesem kulturellen Kontext einen höheren gesellschaftlichen Wert als in anderen Kulturen. Die Lehrer*innen können sich auch in der Unterrichtsgestaltung auf solche Aspekte beziehen und Alltagskultur mit dem Lernen verknüpfen. Dieses Beispiel bezieht sich v.a. auf den Unterricht von Kindern mit Lernschwierigkeiten. Im Kontext von Thailand konnte in Bezug auf sogenannte Moralstunden (Dharma-Teaching) beobachtet werden, dass die Vermittlung dieser, für das gesellschaftliche Zusammenleben wichtiger Normen allen Schüler*innen vermittelt wurden. Anhand von Geschichten zu den Lehren Buddhas werden in diesen Stunden gute und schlechte Taten definiert, besprochen, künstlerisch interpretiert und als relevante Leitlinien vermittelt.

Bezüglich der Gestaltung von Lehrmaterialien scheinen Lehrpersonen stets bereit und motiviert. Wie es darüber hinaus bezüglich der Motivation von Lehrpersonen in den beiden Hauptstädten aussieht, wird im Folgenden erläutert. Wie eingangs erwähnt scheint dies relevant, weil die Umsetzung von Inklusion tief mit der Bereitschaft von Lehrpersonen verbunden ist sich auf Neues einzulassen (Leo/Barton 2006).

\subsection{Motivation der Lehrpersonen}

Ein besonderer Umstand, der die Motivation der Lehrer*innen grundlegend beeinflusste, war in Äthiopien derjenige, dass manche Lehrer*innen diesen Beruf ursprünglich gar nicht ausüben wollten. Dies ist einem System ähnlich dem Numerus Clausus zu verdanken, welches dazu führte, dass Schulabsolvent*innen aufgrund ihrer unzureichenden Ergebnisse, lediglich für Pädagogik oder dergleichen auf der Universität zugelassen wurden. Studien der Rechtswissenschaften oder der Medizin lagen dagegen im oberen Bereich des Leistungsmaßstabs.

Weitere Aspekte in Bezug auf Motivation konnten in Äthiopien vor allem im Vergleich von Lehrer*innen von Sonderschulen/-klassen und denen in integrativen Settings an öffentlichen Schulen beobachtet werden (Schiemer [Bilgeri] 2015): 
In öffentlichen Schulen kann ein konstanter LehrerInnenwechsel beobachtet werden, was an den Schulen mit sonder-Settings nicht der Fall ist. Man könnte deswegen daraus schließen, dass LehrerInnen in sonder-Settings mehr Solidarität, Engagement und Einsatz gegenüber der Schule an der sie unterrichten zeigen - was wiederum zu mehr Motivation im Beruf führt (Schiemer [Bilgeri] 2015: 77).

Betrachtet man nun die Hintergründe dieses Phänomens, fällt auf, dass Lehrer*innen in integrativen Settings oft auf Unverständnis bei Kolleg*innen stoßen und wenig Unterstützung im Allgemeinen erhalten. Dies wurde auch von Lehrer*innen von Sonderklassen angeführt, die an öffentlichen Schulen angesiedelt waren. Die Hintergründe wurden von einem Lehrer dadurch erklärt, dass ihnen vorgeworfen wurde, sie würden sich nur mit Kindern mit Behinderung beschäftigen um vor Gott ,gut dazustehen“. Es finden sich also mehrere Widersprüche, mit denen sich die Lehrer*innen auseinandersetzen müssen.

Im Kontext der Motivation der Lehrer*innen spielt auf jeden Fall auch eines der bereits erwähnten Hauptergebnisse der Studie in Äthiopien eine wichtige Rolle: „developing a sense of belonging“" (Schiemer [Bilgeri] 2017). Das Gefühl der Zugehörigkeit, oder eben auch das Gegenteil, spielte eine entscheidende Rolle für die Motivation der Lehrer*innen. Die Herausforderung der Übernahme von Verantwortung im Beruf konnte durch ein Gefühl der Zugehörigkeit und eine damit verbundene Wertschätzung v.a. durch Eltern von Kindern mit Behinderung gemeistert werden. Als weitere Folge konnte ein höheres „,commitment" und eine Identifikation mit dem Lehrer*innenberuf beobachtet werden. Als kulturelles Moment auf dieser Ebene kann auf jeden Fall die hohe Bedeutung von Gemeinschaft und Familie herangezogen werden. Familie aus dem Grund, dass sich motivierte Lehrer*innen oftmals als Teil einer größeren Familie sahen. Ebenso fühlten sich Eltern den Lehrer*innen in einer solchen Beziehung verbunden (vgl. Schiemer [Bilgeri] 2017). Auf diesen Aspekt wurde bereits im ersten Teil dieses Beitrags hingewiesen.

Die Situation von Lehrpersonen, die für den thailändischen Anteil am Projekt befragt wurden, ist ähnlich wie in Äthiopien von einigen Dilemmata durchzogen. Wie bereits eingangs beschrieben, war Bildung anfangs wohlhabenden Personen vorbehalten. Buddhistische Inhalte (Dharma) standen im Zentrum der Vermittlung und werden auch heute noch von Mönchen an Schulen unterrichtet. Mittlerweile agieren auch religiöse Lai*innen als Lehrpersonen, das gesellschaftliche Ansehen diesen gegenüber steht früheren Zeiten aber um nichts nach. So wird beim jährlich stattfindenden teacher day zuerst dem König, dann der Religion und gleich danach der Lehrpersonen gedacht. An diesem Tag erhalten die Lehrpersonen Geschenke, auch ehemalige Schüler*innen kommen zu Besuch um sich bei früheren Lehrpersonen für den gelungenen Unterricht zu bedanken (eine Praxis, die übrigens auch an der Universität weitergeführt wird und darin mündet, dass sich in manchen Sprechstunden zahllose Geschenke als Dank für die Konsultationen anhäufen).

Als Lehrperson zu arbeiten, ist also erstrebenswert, man übt dann einen hochangesehenen Beruf aus, der junge Menschen auf den richtigen Weg bringen kann. Dieser Sachverhalt wirkt sich aber nicht auf das Gehalt aus, das von den meisten Befragten als verhältnismäßig niedrig eingestuft wird. Hier lassen sich also gewisse Parallelen zu Äthiopien ziehen. Dies wird besonders eklatant rechnet man die vielen Zeitressourcen ein, die die Lehrpersonen in die Entwicklung von Lehrmaterialien investieren, vor allem, wenn es um die Arbeit mit Kindern und Jugendlichen mit Behinderung geht.

Der Unterricht der vorab genannten Gruppe scheint für viele besonders erstrebenswert und Geld scheint zwar ein Thema, aber kein ausschlaggebender Motivationsgrund zu sein. Ein zentrales Moment, warum sich die meist weiblichen Lehrpersonen für die Arbeit mit 
Kindern mit Behinderungen entscheiden, ist Mitleid für diese Personengruppe. Mitleid für das von ihnen erlittene Karma. Der Wunsch diesen Menschen mit Behinderungen zu helfen ist mitunter stark ausgeprägt, nicht zuletzt, weil man hofft damit auch sein eigenes Karma nachhaltig positiv zu beeinflussen. Eine Befragte ging sogar so weit zu sagen, dass sie mit Kindern mit Lernschwierigkeiten arbeite, weil diese es am schwersten haben. Sie hätten, ihrer Meinung nach, diese Behinderung, weil sie in einem ihrer früheren Leben Alkohol missbraucht hätten. Auch der ungehorsame Umgang meist mit Eltern wird als Grund für Behinderungen ins Treffen geführt. Die Gründe sind mannigfaltig.

Ein kleinerer Teil der Lehrpersonen hat sich aufgrund der Nähe bestimmter Schulstandorte für dieses spezielle Arbeitsfeld entschieden oder gar nicht genau gewusst, auf was er oder sie sich einlässt.

Um eine weitere Vergleichsebene hervorzuheben: es ist interessant, dass Religion bzw. mit Glauben assoziierte und in der Gesellschaft verankerte moralische Grundhaltungen (z.B. Mitleid in Thailand und die Grundhaltung, Menschen mit Behinderung zu helfen in Äthiopien) in beiden Ländern ein Aspekt zu sein scheint, der Lehrer*innen die Motivation gibt, mit Kindern mit Behinderung arbeiten zu wollen. An dieser Stelle wäre selbstverständlich eine tiefere Analyse notwendig, die deutlich herausstellen könnte, welche kulturellen und traditionellen Werte etc. tatsächlich dahinterstehen. Dies könnte dazu dienen, wichtige Unterschiede in einer scheinbaren Überschneidung herauszuarbeiten.

Abschließend wollen wir uns über die Gegenüberstellungs- und Vergleichsebene hinaus mit einer globaleren Perspektive auf Kultur und Inklusion beschäftigen und weiterführende Forschungsinteressen aufzeigen.

\section{Kritische Perspektiven auf Kultur(en) der Inklusion und Ausblick}

Anhand der Erläuterung von nur zwei Aspekten wird bereits ersichtlich, wie komplex sich vergleichende Ansätze in groß angelegten kulturvergleichenden Studien zum Themenkomplex Bildung von Kindern und Jugendlichen mit Behinderungen gestalten können. Einige Ebenen sind gut miteinander vergleichbar oder es ergeben sich zumindest vergleichbare Tendenzen. Hierbei ist es immer wichtig genauer hinzusehen und kulturelle, traditionelle etc. Hintergründe mitzuberücksichtigen, die oft die eigentliche Unterschiedlichkeit im vermeintlich Gleichen herausstellen. Andere Bereiche sind aber auch klar voneinander abgrenzbar und damit sind Unterschiede offensichtlich.

Es geht uns hierbei nicht um eine Bewertung des Grades und der Güte der Umsetzung von Inklusion in einer Art imaginärem Länder- oder Kulturkontest, sehr wohl wollen wir aber aufzeigen, dass die Umsetzung der inklusiven Idee mitunter auch völlig falsch interpretiert werden kann und die Namensgebung schlichtweg nicht mit der Grundidee korrespondiert (bspw. sonderschulische Einrichtungen, die einzelne Klassen 'inclusive classrooms' nennen). Dies scheint eine der zentralen Vorteile transkulturell vergleichender Forschungspraxis zu sein: Einblicke in falsch interpretierte Umsetzungstendenzen von Inklusion können in die klarere Beschreibung von inklusiven Kontexten münden. Der Vergleich dieser Interpretationen vor dem Hintergrund unterschiedlicher sozikultureller Kontexte ermöglicht ein klareres Verständnis inklusiver Tendenzen und Güte, die über einen Vergleich zwischen den Ländern 
hinausgeht. Zudem ist es von Bedeutung, die unterschiedlichen Problemlagen und Chancen ins Zentrum zu rücken, die die alltägliche Gestaltung von Bildungswegen von Kindern und Jugendlichen mit Behinderung beeinflussen. Schnell wird auch klar, wie eng verwoben Aspekte der Entwicklung und unsere diesbezüglichen Einschätzungen mit den beschriebenen Phänomenen sind. Globale Bemühungen zur Gleichschaltung von Bildungsagenden haben dabei einen Einfluss, wie wir im Kapitel zu den Rahmenbedingungen erläutert haben. Nicht nur der Vergleich und die Abgrenzung zwischen den beiden erläuterten Länderkontexten ist relevant, sondern auch weitere Ebenen, die über nationalstaatliche Grenzen hinweg wirken. Eine dieser Ebenen ist z.B. der Einfluss, der sich über die westliche Prägung internationaler Dokumente und Sichtweisen ergibt. Eine Beobachtung scheint aus dieser transkulturellen Perspektive interessant und soll beispielhaft erläutern, welche Ausmaße eine gleichgeschaltete Perspektive auf Inklusion bewirken kann: Einer der Schulstandorte im Großraum Bangkok, welcher auf Kinder und Jugendliche mit einer bestimmten Sinnesbehinderung spezialisiert ist, ist eines der ersten Beispiele für eine Sonderschule in Thailand. Spannenderweise erinnert die Gestaltung vielfach eher an einen US-amerikanischen Standort und einige der architektonischen Besonderheiten wurden zweckentfremdet oder werden einfach nicht im eigentlichen Sinne verwendet. Ein Beispiel dafür sind auch PCs, die mittlerweile veraltet oder aufgrund US-amerikanischer Tastaturen und Software nicht gut einsetzbar sind.

Es gilt auf der anderen Seite nicht zu unterschätzen, dass es beispielsweise in puncto Technologie noch einiges voneinander zu lernen gibt. Thailand kämpft seit langer Zeit mit einem Lehrer*innenmangel, aktuell vor allem in kleinen ruralen Orten, weit abseits von Ballungszentren. Diesem Umstand begegnet man zurzeit mit computergestützter Fernlehre, die entweder real-time von einer Lehrperson für mehrere Schulklassen durchgeführt wird oder in Form von fertig aufgezeichneten interaktiven Modulen angeboten wird. Auch in Äthiopien wurde vor allem „Plasma TV“ dazu eingesetzt, Bildung durch die Nutzung von im Fernsehen übertragenen Lehreinheiten (durch den Einsatz von sogenannten Plasma Fernsehern) auch im ländlichen Gebiet auszubauen (Assefa 2017).

Generell kann also festgehalten werden, dass der Einfluss westlicher Konzepte auf Bildung in anderen Kulturen weitreichende Folgen haben kann:

Despite the fact that Ethiopia has a long and rich tradition of indigenous philosophy and education that could have been a solid foundation for its development and modern education like for example, the case in East Asia, however, it had simply assimilated the Western system of education instead of integrating the important cultural and philosophical values with the new education system. At least to me, therefore, there seem [to be] cultural metamorphoses caused by Western-oriented modern education and a consequent emerging confusion of the Ethiopian youth as far as identity formation is concerned (Demeke 2011: 18).

Eine ebensolche Gefahr sehen wir in Bezug auf globale Inklusionsbestrebungen: Das Verhüllen kultureller Aspekte durch ein Überstülpen westlich geprägter Ideen und damit eine Verwaschung inklusiver Qualitätskriterien unter dem Deckmantel einer standardisierten Interpretation von Inklusion. Dies führt im Weiteren zur Gefahr der Verunmöglichung der Umsetzung in bestimmten kulturellen Kontexten. Es bleibt zu hoffen, dass aktuelle Agenden, wie die SDGs 2030, weiterhin kultursensible Ansätze über eine einheitlich standardisierte und nach westlichen Maßstäben festgesetzte Vorstellung von inklusiver Bildung von allen Kindern und Jugendlichen stellen.

Kulturen der Inklusion zu vergleichen, erfordert einen besonderen Fokus auf die Kulturen selbst und deren vielfältige Ausprägungen. Die Erforschung des Bedeutungsgehalts bestimmter kultureller Aspekte liefert oft Erklärungen für Phänomene, die aus einer etischen 
Perspektive nicht nachvollziehbar sind. Dies beschreibt wohl auch eine der größten Herausforderungen, der wir uns als Forscherinnen aus einer fremden Kultur stellen mussten. Das Bedürfnis zu verstehen, einzuordnen, hinzusehen und zuzuhören war Motor und Benzin zugleich. Aber auch die Fähigkeit, sich zurück zu nehmen und sich bewusst zu machen, dass unsere Einsichten immer durch unseren (westlichen) Hintergrund beeinflusst sind und sein werden, ist ein Lehrstück, das wir mit Respekt und Achtung vor einer fremden Kultur aus dieser Forschung mitgenommen haben.

Aktuelle weiterführende Forschungsfelder beziehen sich auf eine Hochschulkooperation, die drei Universitäten in Äthiopien umfasst. Die zentralen Bemühungen innerhalb des Projekts ,Inclusion in Education for Persons with Disabilities“ (INEDIS, APPEAR 2017) fokussieren auf die Verbesserung des Hochschulzugangs für Studierende mit Behinderungen und die Entwicklung der Bildungsangebote für Fachkräfte, die im Bereich der communitybased rehabilitation agieren.

Mit den Kolleg*innen aus Thailand laufen derzeit Erhebungen für das Projekt ,Professional Development of Teachers to Foster Inclusive Teaching Practices in the Context of Education, Disability and Migration“ (ProITEM, OEAD 2018). Darin werden die Hintergründe der Lehrpersonen erforscht, welche mit Personen mit Behinderungen und Kindern und Jugendlichen mit Migrationshintergrund arbeiten. Mängel in diesem Bereich sollen aus mehreren Ebenen erläutert und bearbeitet werden. Im Zentrum steht ein partizipativer Forschungsansatz eines kulturell durchmischten und erfahrenen Forscher*innenteams, das auf Augenhöhe agiert.

Ein weiteres Feld, mit dem wir uns aktuell in der Phase der Projektantragstellung auseinandersetzen ist die Verschränkung zwischen Grenzen, Bildung, Behinderung und Flucht. Gerade an dieser Schnittstelle zeigen sich weitere sehr spannende Ebenen der Unterscheidung und unterschiedlicher Zugänge auf bildungspolitischer, gesellschaftlicher, wie auch individuumsbezogener Ebene, welche Auswirkungen auf eine Gruppe von Menschen haben, die von mehrfacher Diskriminierung betroffen sind.

Als Abschluss dieses Handbuchbeitrages gilt es anzumerken, dass es bezüglich der Forschung noch einen enormen Handlungsbedarf gibt. Die Problemlagen, die dabei zu berücksichtigen sind, sind nicht nur die im Artikel angesprochenen kulturellen Unterschiede, die einerseits durch die vermehrte und intensivere Rezeption und Förderung kulturell originärer bzw. nicht-westlich orientierter Forschung oder kulturell gemischte Forscher*innenteams aufgebrochen werden könnten, oder andererseits auch durch partizipative Forschungsmethoden beforscht werden können. Ebenso wichtig ist die Frage in welchen kulturellen Kreisen wir uns in unserer Wissenschaftskultur bewegen. Auch hier wäre ein Vergleich fruchtbar, um festzustellen, wo innerhalb der Forschungsumgebung, in der wir kultiviert wurden, unsere „blind spots“ liegen.

\section{Literatur}

APPEAR (2017): Inclusion in Education for People with Disabilities (INEDIS). https://appear. at/en/projects/current-projects/project-websites/project167-inedis/ [Zugriff: 04.08.2020].

Assefa, Temtim (2017): Educational Technology Implementation in Ethiopian High Schools. Benefits and Challenges of the Instructional Plasma TV. In: Marcus-Quinn, Ann/Hourigan, Tríona (Hrsg.): Handbook on Digital Learning for K-12 Schools. Cham: Springer, S. 413-427. 
Biermann, Julia (2021 i.d.B.): Der Einfluss der UN-BRK auf inklusive Bildung in Nigeria und Deutschland. In: Köpfer, Andreas/Powell, Justin J.W./Zahnd, Raphael (Hrsg.): Handbuch Inklusion international. Opladen: Budrich, S. 167-178.

Biewer, Gottfried/Proyer, Michelle/Schiemer [Bilgeri], Margarita (2016): Klassifikationsfragen im Kulturvergleich. In: Hedderich, Ingeborg/Biewer, Gottfried/Hollenweger, Judith/Markowetz, Rainhard (Hrsg.): Handbuch Inklusion und Sonderpädagogik. Bad Heilbrunn: Klinkhardt, S. 151-155.

Demeke, Yeneayhu (2011): Ethiopian Education Policy Analysis. Using Foucault's Genealogy. https://www.academia.edu/11891083/Ethiopian_Education_Policy_Analysis_Using_Fou cault_s_Genealogy [Zugriff: 04.08.2020].

Dingle, Mary P./Brownell, Mary T./Leko, Melinda M./Boardman, Alison G./Haager, Diana (2011): Developing Effective Special Education Reading Teachers. The Influence of Professional Development, Context, and Individual Qualities. In: Learning Disability Quarterly 34, 1, S. 87-103.

Ethiopian Ministry of Education (2015): Education sector development program V (ESDP V). 2015/2016-2019/2020. Addis Ababa: Ethiopian Ministry of Education.

Hodge, Samuel R./Haegele, Justin/Gutierres Filho, Paulo/Rizzi Lopes, Gleides (2018): Brazilian Physical Education Teachers' Beliefs About Teaching Students With Disabilities. In: International Journal of Disability, Development and Education 65, 4, S. 408-427.

Kramann, Michaela (2017): Soziale Partizipation von Kindern mit Beeinträchtigung an schulischen Lebensbereichen in Wien. Diss. Wien: Universität Wien.

Leo, Elizabeth/Barton, Leon (2006): Inclusion, Diversity and Leadership. Perspectives, Possibilities and Contradictions. In: Educational Management Administration \& Leadership 34, 2, S. 167-180.

McDaniel, Justin T. (2010): Gathering leaves and lifting words. Histories of Buddhist monastic education in Laos and Thailand. Seattle, WA: University of Washington Press.

OEAD (2018): Professional Development of Teachers to Foster Inclusive Teaching Practices in the Context of Education, Disability and Migration (proITEM). https://oead.at/en/projects/development-research/current-projects/geospatial-methodology-geom/ [Zugriff: 04.08.2020].

Ozik-Scharf, Anja (2015): Empirische Studie im Rahmen eines internationalen Forschungsprojekts mit Mixed Methods Ansatz über kooperative Beziehungen zwischen Elternhaus und Schule, deren Unterschiede und Gemeinsamkeiten im Bildungskontext von Kindern mit Behinderungen in Äthiopien, Thailand und Österreich. Diss. Wien: Universität Wien.

Powell, Justin J.W./Merz-Atalik, Kerstin et al. (2021 i.d.B.): Heterogene Lerngruppen inklusionsorientiert unterrichten. In: Köpfer, Andreas/Powell J.W./Zahnd, Raphael (Hrsg.): Handbuch Inklusion international. Opladen: Budrich, S. 357-379.

Proyer, Michelle/Sriwanyong, Siriparn (2016): Exploring Cultures of Exclusion of Children with Disabilities in Greater Bangkok. In: Zeitschrift für Inklusion, 10, 4. https://www.inklusion-online.net/index.php/inklusion-online/article/view/322/274 [Zugriff: 10.08.2020].

Proyer, Michelle (2014): Educational environments of children with disabilities in Greater Bangkok. Accepting disability and leading a happy life in a transiting urban area. Diss. Wien: Universität Wien.

Proyer, Michelle/Schiemer [Bilgeri], Margarita/Luciak, Mikael (2011): CLASDISA - Classifications of Disabilities in the Field of Education in Different Societal and Cultural Contexts. Insights Into the Current State of Research. In: Austrian Journal of South-East Asian Studies/Österreichische Zeitschrift für Südostasienwissenschaften ASEAS 4, 1, S. 158-165.

Rogers-Shaw, Carol/Carr-Chellman, Davin J./Choi, Jinhee (2017): Universal Design for Learning. Guidelines for Accessible Online Instruction. In: Adult Learning 29, 1, S. 20-31.

Rose David H./Harbour Wendy S./Johnston Catherine S./Daley Samantha G./Abarbanell Linda (2006): Universal design for learning in postsecondary education. Reflections on principles 
and their application. In: Journal of Postsecondary Education and Disability 19, 2, S. 135-151. http://files.eric.ed.gov/fulltext/EJ844630.pdf [Zugriff: 18.07.2020].

Schiemer [Bilgeri], Margarita/Proyer, Michelle (2015): Bildung für Kinder mit Behinderungen. Kritik an einem universalen Konzept der Inklusion am Beispiel Thailands und Äthiopiens. In: Faschingeder, Gerald/Kolland, Franz (Hrsg.): Bildung und ungleiche Entwicklung. Globale Konvergenzen und Divergenzen in der Bildungswelt. Wien, New Academic Press, S. 160-174.

Schiemer [Bilgeri], Margarita (2015): Die „Kultur“ inklusiver Bildung ab Beispiel der Situation von LehrerInnen von Kindern mit Behinderung in unterschiedlichen Schulsettings in Addis Abeba. In: Graf, Erich Otto (Hrsg.): Behinderung im globalen Kontext. Über die Grenzen der Sonderpädagogik hinaus. Berlin: epubli, S. 74-83.

Schiemer [Bilgeri], Margarita/Proyer, Michelle (2013): Teaching children with disabilities. ICTs in Bangkok and Addis Ababa. In: Multicultural Education \& Technology Journal 7, 2/3, S. 99112.

Schiemer [Bilgeri], Margarita (2014): Developing a sense of belonging. A study on the impact of environmental factors regarding education for children with disabilities in Addis Ababa, Ethiopia. Diss. Wien: Universität Wien.

Schiemer [Bilgeri], Margarita (2017): Education for Children with Disabilities in Addis Ababa, Ethiopia - Developing a Sense of Belonging. Cham: Springer.

UN (online): About the Sustainable Development Goals. https://www.un.org/sustainabledevelopment/sustainable-development-goals/ [Zugriff: 18.07.2019].

Vibulpatanavong, Kanokporn (2015): Inclusive Education in Thailand. In: Advances in Social Science, Education and Humanities Research (ASSEHR), Proceeding of the 2017 International Conference of Early Childhood Education (ICECE) 169, S. 67-70.

Vorapanya, Sermsap/Dunlap, Diane (2014): Inclusive education in Thailand. Practices and challenges. In: International Journal of Inclusive Education 18, 10, S. 1014-1028.

WHO (2001): International Classification of Functioning, Disability and Health. Geneva: World Health Organization. 


\title{
17 Exploring Successful Inclusive Practice in China: An Inclusive Public Kindergarten in Shanghai
}

Run Tan

\begin{abstract}
As international influences increase, the Chinese government has joined the worldwide endeavour to implement inclusion on the preschool level. One kindergarten was selected for its high quality and longest history of inclusive practices in Shanghai. A qualitative case study was conducted to explore the potential factors that contribute to this success. Findings indicated that the foremost factor is the substantial community-based support identified on three different levels: the community level, the kindergarten level, and the classroom level. Among the many community factors, the principal's support to guarantee the successful implementation of inclusive practices stands out. Based on these findings, the author intends to provide insights of relevance for key community stakeholders in German preschools as they strengthen inclusive practices.
\end{abstract}

\section{Introduction}

\subsection{Inclusive Education in China}

Together with the increasing interest to develop early childhood inclusion on the international level, Asian countries like Singapore (Yeo et al. 2011) and cities like Hong Kong (Cheuk/Hatch 2007) have gradually started to explore the implementation of inclusion on the preschool level. The Chinese government has joined the worldwide endeavour to improve the implementation of inclusion on the preschool level, aiming at improving the quality of current inclusive services by granting more children with disabilities equal educational opportunities for quality early childhood education (ECE) programs (Hu/Szente 2010).

\section{Policies for Inclusive Early Childhood Education and Their Shortcomings}

The development of preschool inclusion in China was strongly influenced by UNESCO. While UNESCO was advocating the implementation of inclusive education, China responded by initiating two national laws: (1) 'the People's Republic of China on Protection of Disabled Persons Act' (National People's Congress 1990); (2) the 'Educational Guidelines for People with Disabilities' ('National Education Committee of the People's Republic of China' 1994). Both laws acknowledged the needs for early intervention and early special education service (Gargiulo/Piao 1995) and argued that early childhood inclusion should be the main format to 
offer children with disabilities the access to general preschools (Chen 1996). They serve as the fundamental ones for the advocacy and preliminary implementation of early inclusive education, calling for national endeavours to establish quality kindergarten (from birth to six) programs (Chen 1996).

The Chinese government furthered its support of the rights of children with 'Special Educational Needs (SEN)'1 by carrying out: (1) the 'Tenth Five-Year Plan (2001-2005)', explicitly stating that (a) the education of infants and toddlers with disabilities in China is of great importance and (b) to place those children in inclusive preschools (National People's Congress 2001); (2) the 'Eleventh Five-Year Plan 2006-2010'(National People's Congress 2006) fulfilling the promise of including the majority of children with disabilities in regular preschools; (3) 'fourth Plenary Session of the 16th Central Committee' (Meng 2005) dedicating to establish a harmonious society, which can't be accomplished when the educational needs of children with SEN are not met (Zhu 2005). Under those policies, more and more national planning concerning equity in education have taken place. Specifically, the Chinese government called upon professionals to explore the concept of quality preschool inclusion in the Chinese context and methods for assessment (Hu/Li 2012). Following these government initiatives, a few megacities such as Shanghai and Beijing initiated pilot preschools to include children with disabilities, but tens of millions of other children in the majority of the country still await appropriate services.

While examining the policies initiated by the Chinese government, I cannot deny their fundamental role to emphasize the value of early childhood inclusion. Nevertheless, they failed to provide specific guidelines for kindergartens and teachers to implement inclusion. A closer examination on the guidelines reveals a lack of: (1) the evaluation and assessment of the performance of children with SEN; (2) teachers' professional knowledge of children with SEN and of inclusion; (3) an inclusive curriculum and activity designs; (4) clarifications of governmental roles in the implementation process, and (5) sustainable funding resources. Therefore, I argue that despite the strong advocacy from the laws and legislation, the situation of early childhood inclusion shares the same unfulfilled status as inclusive education on the primary and secondary school levels: most children with recognized disabilities lack access to general schools. Most regular preschools in China have neither been familiarized with the principles of inclusion nor have they considered providing this type of service (Wang/Shen 2009). A survey conducted in Hebei province revealed that none of the regular kindergartens had enrolled any children with disabilities (Jiao et al. 2004).

\subsection{Preschool Inclusive Education in Shanghai}

Lewin, Wang and Qu (1994) explained that China's vast regional variations makes it challenging for researchers to conduct research that is thoroughly representative of the whole country. Thus, the current study intends only to explore the factors that contribute to the successful implementation of inclusive practices within one inclusive preschool, instead of making generalizable or transferable claims. I chose Shanghai to focus on in the current study since I am quite familiar with its inclusive education development. What's more, since

1 Children with special educational needs (SEN) and children with disabilities are used interchangeably in this article. 
Shanghai is the forefront of educational reform in China, this study will likely to generate interesting and promising findings.

Shanghai is the largest city in China, having a residential population of over 24 million of whom 9.9 million are migrants. As the economic centre of Mainland China, Shanghai has a GDP that is well above the national average (Wang 2016). Since 1949, Shanghai has served as the exemplary city for experimenting educational reforms and those that prove effective will be potentially further implemented in other parts of China (Arens 1952). It was the first city in China to implement the 9-year compulsory education (6-year primary education and 3 -year junior secondary education), a policy which plays a fundamental role in developing inclusive education. Starting from 2009, this city attracted more attention for its extraordinary performance of Shanghai students in the Programme for International Student Assessment (PISA), vividly described as to have provoked a 'PISA-shock' (Takayama 2008).

In the past years, while many cities are still striving for a higher enrolment rate of students in general school settings, Shanghai's education guiding philosophy shifts from quantity to quality (ShCoE, 2010). Specifically, four perspectives were proposed to reach the goal of a quality education for all: advocating equity, pursuing excellence, promoting innovation and supporting development. Specifically, on the preschool level, Shanghai Municipal Commission of Education (SMCE) has started to persistently pursue the goal of providing children with SEN with three years of high-quality early childhood education and has dedicated itself to reaching this goal since then $(\mathrm{Hu} /$ Szente 2010). Meanwhile, the Shanghai government has carried out the 'Three-Year Special Education Action Plan (2014-2016)' to develop its special and inclusive education programs. Taking the district where the researched kindergarten is located as an example: since 2013, within Chang Ning district, six kindergartens accepted children with disabilities and by 2016 , there were eleven kindergartens that engaged themselves in early childhood inclusion. In order to guarantee its current progress, Chang Ning district further implemented its 'Chang Ning District Long-Term Education Reform and Development Plan (2010-2020)' (Chang Ning District 2010) that aims to improve overall preschool education quality.

\section{Theoretical Framework}

\subsection{The Importance of Community Involvement in the School Inclusion Process}

According to Sharma, Forlin, Loreman, and Earle (2006) and Donohue and Bornman (2014), the direction of discussions about inclusive education is slowly shifting from the justification of why schools should adopt this approach to how they can successfully implement inclusion. Bronfenbrenner's (1979) 'ecological theory' strongly emphasizes the importance of school and community partnerships. Specifically, this theory states that children's learning and development are influenced significantly by the contexts of home, community, and school and that when continuity between these is improved they exert more positive influence on children's development. Empirical studies also show the essential role of the community involvement in schools in the successful implementation of inclusive education (e.g., Loreman 2007; Rouse/Florian 1996): (1) the participation of the community as a critical element fostering 
students' better achievement (Apple/Bean 2007; McAlister 2013); (2) the involvement of the community as one of the main factors contributing to the creation of a climate conducive to learning (Stoll 1991). In some countries where inclusion is at its preliminary stage, such as South Africa, the participation of the community is perceived as key to tackle the challenges of developing inclusion (e.g., Lomofsky/Lazarus 2001). Meanwhile, this connection between schools and communities is more strongly called for, since schools have, to a strong degree, become disconnected from our fractured modernist society (Elkins 2005).

Traditional perceptions regard schools as organizations where members involved are motivated by self-interests and therefore tend to seek to maximize gains and cut losses (Sergiovanni 1994). Nevertheless, community involvement in schools emphasizes the need for intersectional collaboration of all vital actors in the school and the community members to promote inclusive education together (Gómez-Zepeda et al. 2017; Rouse/Florian 1996; Turnbull et al. 2007). It emphasizes that different community members are socially organized around relationships and the strongly felt interdependencies that nurture them. It thus contributes to the construction of schools themselves as communities where the connections of people are involved with purposes and commitment, which is essential for democratic collaboration and thus more inclusive practice (Sergiovanni 1991; 1994).

\section{Methodology}

\subsection{Methodological Considerations}

While taking a thorough look at the research methodologies from the previous research studies conducted to examine inclusion at Chinese preschools, I identified that many of them are exclusively quantitative, applying questionnaires and generating quantitative observations (e.g., Hu et al. 2016). Only very few follow a non-positivist paradigm that recognizes the value of social constructivism where the explorations of the researched phenomenon take place in its most naturalistic settings (e.g., Hu et al. 2011). Thus, a single case-study design was applied in the current inquiry for its strong explorative orientation and relatively flexible design. Moreover, the core characteristic of a case study is its outward-looking nature (Cohen 2003), so the factors identified from the current study that contribute to the success of the researched kindergarten could thus provide indications for inclusive practice in Germany and other countries.

\subsection{The Researched Kindergarten}

A detailed description of the researched kindergarten is an essential component in a case study report and will direct others in deciding for themselves the appropriateness of generalizing case study findings to other contexts (Stake 2010). Specifically, the researched kindergarten was founded in 1991, being then the only inclusive kindergarten in Chang Ning district. Located within a middle-class living community, the kindergarten has been the largest 
public one, taking up around 2283 square meters. By the time I collected the data in 2017, there were 175 children aged from three to six and 21 people working as teaching staff. In total, there are seven classes: two classes for children aging from 3 to 4; two classes for children aging from 4 to 5; two classes for children aging from 5 to 6 ; one special class for children with mixed ages from 3 to 6 .. Each classroom, according to the 'Shanghai Guideline for Kindergarten Education', has two main teachers and one teacher's aide who helps out with the daily routine and clean-up (called bao yu yuan (BYY) in Chinese), leading to an average student-teacher ratio of 15:1 to 20:1. The kindergarten has good basic infrastructure including some technological equipment (e.g., PC, Beamers, LED screen) to make teaching more diversified and fun.

\subsection{Research Methods}

In total, seven in-depth, face-to-face interviews were conducted: one with the principal, one with the teaching director, two with special educators, and three with general teachers. Those interviews were designed to explore the potential factors that contribute to the successful implementation of inclusive practices within the kindergarten. Participatory observation was also applied in the current study. In total, the researcher conducted ten intensive sessions (15 minutes for each) of participatory observations during 'free play' time. In addition, all the kindergarten website news and updates regarding inclusive practices (from September 2017 until November 2017) were systematically examined to analyse the potential factors that contribute to the inclusive practices of the researched kindergarten.

\subsection{Data Analysis}

Since most of the data were in Chinese, the researcher needed to first translate them into English. During the translation process, several challenges appeared: 1) there were some words that appeared specifically only in the researched kindergarten context, which were quite challenging to translate into English words that could express their full meanings (e.g., the translation of the 'qi zhi class' due to its transitional role differing from a special class). 2) there were many words that only existed in the general Chinese kindergarten context, e.g., bao yu yuan (BYY) with their varied responsibilities and roles differing from aide teachers; 3) some teachers sometimes spoke in Shanghai dialect during interviews and observations, which made the translation process more difficult.

Overall, qualitative content analysis was applied to analyse the observational, interview and document data in the current research project (Kohlbacher 2005). Mayring (2004) proposed the process of an inductive approach to qualitative content analysis consisting of the following main aspects: 1) selecting the units of analysis, 2) open coding, 3) preliminary code formulation, 4) creating categories, and 5) creating levels of the categories. Open coding was conducted with Altas.ti and all data were clustered and coded, then analysed to confirm and build on the findings from the previous study. Specifically, data from the interviews with the principal were analysed by noting patterns and themes and clustered according to those 
themes. Data from the participatory observations and document review were utilized to support or refute developing patterns and themes.

\section{Results}

From the current study, I have identified the active involvement of different members from the community that serves as the main factor contributing to the successful implementation of inclusive education in this Chinese kindergarten. In total, I have categorized those community members into three levels: (1) the first level talks about the involvement of members from the community, such as parents, the shadow teacher, ${ }^{2}$ and the 'Chang Ning Special Education Centre'; (2) the second level focuses on the involvement of people within the kindergarten level: the principal and the (special) educators; (3) the third level targets the involvement of members on the classroom level: other main teachers (including intern teachers) and bao yu yuan (BYY).

\subsection{Involvement of the Members on the Community Level}

\subsubsection{Involvement of the Special Education Centre}

'Chang Ning Special Education Centre' serves as the key source of support for the researched kindergarten since its first day of inclusive practice: (1) it provides regular in-service teacher training on special and inclusive education to all staff, in the hope of informing everyone with different theories and effective practices; (2) it puts much effort into constructing a 'central expert team' consisting of a select group of different stakeholders (e.g., teachers, professors, principals, parents) that organizes regular meetings to visit the researched kindergarten for systematic assessment of inclusion practice and supports official diagnosis of children with SEN by working with the researched kindergarten.

\subsubsection{Involvement of the Professor}

Professor $\mathrm{Zhou}^{3}$ is an expert in inclusive preschool education from East China Normal University and has established a long-term cooperation with the researched kindergarten. Her involvement includes the following aspects, improving the perceptions teachers have towards inclusion and empowering them with concrete strategies to implement inclusive education. She shows teachers how to do action research projects to improve their daily inclusive practice: "Our teachers really love to work with Professor Zhou; she always brings us her

2 The shadow teachers: they are mainly college students still majoring in special education and are employed by parents of children with SEN to accompany the learning and participation of the children in the general schools (usually starting from primary schools). When placed in the international context, they can be most nearly compared to special educators.

3 Professor Zhou: a pseudonym for the professor. 
experiences of how inclusion works in other cultures as well as her in-depth understanding of what challenges our teachers face" said the principal in the interview. "She knows what we are most afraid of," said teacher Chen in her interview, "she spent so much time in the classroom with us, so she knows how we are doing daily and what we find challenging". To cooperate with the main teachers is her another important role, for example, when Professor Zhou went to the classroom two when the "small group life-skill activity" session was on and the children were busy peeling the beans for the kitchen. Child E was not interacting with his peers but observing them, showing interest. Professor Zhou saw him and bent down on her knees. "This looks fun!" she said and then waited until E responded to her. "Yes," said E slowly, "Look, child $\mathrm{Li}^{4}$ seems to have difficulty in putting the bean skins in the bowl because her hands are too full. Would you like to help her by passing her the other bowl?" "Okay," said E but did not push the bowl. "See, like this, just push it like this," encouraged teacher Liu. ${ }^{5}$ Professor Zhou then pushed the bowl gently lying in front of E a bit and E also started to imitate her by pushing the bowl further. "Exactly, you are doing great!" commented Professor Zhou after E did that. "Thank you," said child Li, smiling at E. Then child Li invited E to join her in peeling the beans together. During the whole process, teacher Liu supported Professor Zhou whenever it was needed and she also did not interrupt them even though the session was already over. In this type of cooperation, teachers play a more cooperative role and Professor Zhou plays a more leading role.

\subsubsection{Involvement of the Parents}

Parents play a crucial role in supporting more inclusive practices from the researched kindergarten. Specifically, some are actively involved in teachers' activity design that targets promoting the participation and social interactions of children with SEN. Teacher Liu from the inclusive class three asked child E's parents to continue to play the 'pretend play' that was carried out in her classroom during the 'free play' session, seeking to enhance child E's interest to play as a 'doctor'. This continuation of efforts would, in the end, contribute to E's interest in the 'role-play' session, increasing his participation in the classroom activities and potentially promote his interactions with his peers. Teacher Chen, on the other hand, talked to child F's parents to stick to the 'social rules (strictly implemented in the classroom)' at home: which was to have the same requirement for child F's certain behaviours as for his peers, during, for example, a conflict situation. Through emphasizing that child F should try to ask his peers nicely first before grabbing the toys from others, teachers' cooperation with parents directly contributed to the decrease of F's challenging behaviours and the increase of socially appropriate behaviours.

\subsubsection{Involvement of the "Shadow Teacher"}

Shadow teachers are teachers that some parents of children with SEN have employed to support their children to learn and participate in a general school. Within the researched kindergarten, shadow teachers are highly regarded and teachers try to get them involved as much as possible. Firstly, main teachers seek opportunities to exchange with them regarding how

4 Child Li: a pseudonym for this child.

5 All teachers' names are pseudonyms. 
to improve the social inclusion and participation of children with disabilities whenever the shadow teachers are present in the classroom. They were often observed to consult the shadow teachers for certain strategies they needed to deal with children's problems: teacher Chen was observed to consult the shadow teacher about the method of 'social story' and to learn more to better deal with child F's disruptive behaviour against his peers. Secondly, they also try to involve shadow teachers in certain classroom activities to encourage children to interact with their peers: "E, I saw you were playing the ball with the others during the mixedage activity time and that looks really fun. Would you like to share it with us?" suggested teacher Liu, "Yes, E, I also saw that and that you played with this girl, right? Would you like to tell us something about it?" encouraged the shadow teacher. Then E stood up and went to the centre of the classroom, starting to tell his peers what he played.

\subsection{Involvement of the Members on the Kindergarten Level}

This level focuses intensively on the involvement of professionals within the kindergarten, those who are officially employed by the kindergarten compared to the level of stakeholders from the community level. Specifically, it mainly addresses the active involvement of the principal and special educators.

\subsubsection{The Active Involvement of the Principal}

A supportive administrator serves as one of the fundamental sources for the successful implementation of inclusive education (Shogren et al. 2015). The principal in this researched kindergarten serves as the core individual who tries to establish an inclusive culture by involving everyone actively in the inclusion process. From the macro-level, she serves as the critical role to advocate and promote different policies regarding (1) how to sustainably develop inclusive education and (2) how to create an inclusive culture within the kindergarten. All teachers spoke very highly of her and emphasized her key role in establishing inclusion within the kindergarten: "Our principal is very determined and she has committed almost 20 years to inclusive education" said the teaching director from the interview; "we would never continue this inclusion journey if we do not have our principal. She is like the light guiding us for inclusion" mentioned the special educator Gao in her interview. From the micro-level, she was observed to take a very active part in participating in the inclusion process from codesigning a more inclusive curriculum and activities with all teachers and supporting their daily inclusion practice to having regular one-to-one talks with teachers whenever they faced challenges or problems in the inclusion process.

\subsubsection{The Active Involvement of the Special Educators}

There are four special educators for the special class ('qi zhi class' in Chinese), which serves as the transitional class for children with diagnosed disabilities who are first enrolled in the kindergarten and waiting to be transferred to the general class based on their progress. There are, in total, ten children with a wide range of disabilities in the class. The kindergarten values 
the professional knowledge and skills special educators have and regard their involvement as key in promoting inclusion. They have created various opportunities to involve the special educators. Firstly, regular and official exchanges between special educators and main teachers occur every month. Those exchanges mainly focus on how to improve the participation and learning of children with SEN in the general classroom as well as how to deal with different challenges in the inclusion process and it is observed that there is constant communication between special educators and general teachers in daily practice. Secondly, both special educators and general teachers are invited to co-design and evaluate the inclusive activities, for example, the 'mixed-age group activity' is one of the cooperation. Thirdly, establishing mentoring relationships between special educators and general teachers, such as that each of the four special educators act as a mentor for two to three general teachers and meet monthly for exchange.

\subsection{Involvement of the Members on the Classroom Level}

\subsubsection{The Involvement of Other Main Teachers (Including Intern Teacher)}

Cooperation among teachers is crucial for successful inclusive teaching and it has been an unspoken but well-shared value. When one main teacher is occupied by some unforeseen event, another teacher will take the main responsibility to continue the current teaching or the ongoing activity without being asked to. Specifically, teachers cooperate to enable more participation and learning of children with SEN to take place. They were always observed together to encourage children with SEN to participate in a certain activity: "Come on, you can do that" said teacher Liu, "yes, you can, you can first try it out," said teacher Chen immediately after teacher Liu. In another situation, main teachers from inclusive classroom one were observed discussing how to promote children's peer interactions while designing the "outdoor play session". In inclusive classroom three, teacher Li was on medical leave and an intern teacher served as her substitute. Teacher Yao allocated tasks to the intern teacher to support the learning and participation of children with SEN and had regular communications and exchanges with her. It was often observed that the intern teacher participated in different seminars focusing on learning as well as special and inclusive education.

\subsubsection{Cooperation with the BYY}

Bao yu yuan (BYY)'s responsibilities mainly contain the following aspects: maintain the classroom's cleanliness, arrange for lunch and snack time, and support the organization of classroom activities. In this kindergarten, their ages range from 40 to 65 and they work for the same class from the moment the children enter the kindergarten. Apart from the basic tasks they all share, some BYYs also tend to have more responsibilities compared to the others: for example, supporting main teachers to implement different learning tasks and activities or supporting the child with SEN to learn and participate. Specifically, BYY three from inclusive class three sometimes acts as the main teacher and seems to be given more credit by the teachers compared to the intern teacher. 
The kindergarten tries to involve every BYY in the inclusion process since they serve as valuable sources for cooperation to promote more inclusive practices. Teachers actively involve them in some activity designs: (1) communicating with the BYYs regularly on how to work together to encourage more participation of children with SEN; (2) inviting BYYs to reflect together on how to improve their cooperation: "after the whole day, we also like to invite our BYY to join us to talk about the whole day together and see how we can improve for the future" said Cai in her interview. Meanwhile, it was also observed that the two sides cooperate to engage children with SEN to participate. For example, during the outdoor freeplay session, teacher Cai and BYY one tried to invite child $\mathrm{C}$ to join in the ball-throwing: "hey, C, do you also want to play the ball with us" suggested teacher Cai after seeing child $\mathrm{C}$ was left alone. "This looks really fun, C, and look how Gui Gui is doing it," BYY one encouraged $\mathrm{C}$ further. Child $\mathrm{C}$ started to observe child Gui Gui and how he interacted with teacher Cai, then she also joined them playing with the ball. Teacher Cai and BBY one first both played with the two children, but they then slowly withdrew themselves.

\section{Conclusions}

The current study focuses on one of the pilot inclusive preschools in Shanghai and examines the potential factors that contribute to the successful implementation of inclusive education by applying a constructivist qualitative case-study approach. Open-ended interviews, participatory observations, and documents were collected during fieldwork over three months. To start with, very active involvement of community members from three levels is defined as the core factor. The three levels of community members are from the classroom, the kindergarten and the community - stakeholders from within and outside of the researched kindergarten. Secondly, new community members to support more inclusion are also identified: the shadow teacher and the bao yu yuan (BYY). Thirdly, among the many factors, the key role of the principal stands out since she serves as the instructional leader who is critical to the implementation of inclusion for the kindergarten. Those successful experiences can provide valuable guidelines for kindergartens in Germany and other countries to implement inclusive practices.

\subsection{The Active Involvement of the Community from the Three Levels}

Consistent with what Loreman (2007) and Rouse and Florian (1996) emphasized, I present from the current study further evidence indicating the crucial role of the involvement of the community in preschools for the successful implementation of inclusive education. Specifically, I identified the active involvement of various community members from three different levels who contribute to the inclusive practices of the researched kindergarten, which echoes with previous studies indicating that multiple agencies from the community, including general teachers, peers, support teachers, families, volunteers, and other social agents are key to the successful inclusive practices (e.g., Gómez-Zepeda et al. 2017). Meanwhile, the three levels of cooperation also contribute to a more thorough and systematic understanding of the 
different community agencies compared to previous literature (e.g., Turnbull et al. 2007). German researchers (e.g., Prengel 2017) discussed the value of establishing a "caring community" to promote better inclusive practice in which children's emotional, social, and cognitive learning support and enhance each other. Nevertheless, few talked about how specifically to establish such community. Thus, based on the findings of the current study and my own experience of working as a support teacher for children with disabilities in German inclusive kindergartens, I suggest that German kindergartens start conversations with a wide range of different community members such as support teachers to discuss concrete strategies to involve them in the inclusion process.

As Loreman et al. (2005) argued about parents' involvement as the most important element for successful inclusion, teachers in the current researched kindergarten actively try to involve parents in certain activities targeting to increase participation and social interactions of children with SEN. Though some German researchers (Peters 2015; Platte 2014) emphasized the key role parents play in the kindergarten inclusion process, discussions on specific ways of how to engage them into the kindergarten have not been comprehensively researched. The current study thus provides some specific examples for kindergartens and teachers in Germany and other countries to include parents in the inclusion process, aiming to create more sustainable inclusive practice.

\subsection{New Community Factors Identified from the Current Study}

The current study makes vital contributions to the literature on the potential factors that influence the successful implementation of inclusive education by identifying new community members in the kindergarten's inclusion process, such as the involvement of "shadow teachers" from the community level and the involvement of "bao yuan yuan (BYY)"from the kindergarten level.

To start with, shadow teachers' active involvement in classroom life and their cooperation with the main teachers contribute to more inclusive practices. The concept of 'shadow teachers' first appeared in some Chinese NGOs (non-governmental organizations) specifically for children with autism. Their main responsibilities include accompanying children to learn and participate in general school settings; carrying out regular assessment of children's development; and giving children extra "one-to-one" intervention programs after school (Wang 2016). Since there are very few special educators in most Chinese kindergartens, some parents would have to employ shadow teachers to fulfil the special educators' role. Most of the shadow teachers are still students majoring in special education in universities, having a good knowledge of special education and some experiences with children with disabilities (Wang 2016). They are potentially very valuable to the sustainable development of inclusive practices for every Chinese inclusive kindergarten. In the researched kindergarten, main teachers hold very positive attitudes towards the presence of the shadow teachers and involve them in certain activity designs (e.g., European Commission 2005). Furthermore, main teachers initiate conversations with the shadow teachers when facing certain challenges and shadow teachers also try to talk with the main teachers for some updates of certain children with SEN.

That the general teachers and the shadow teachers continuously work together to design some curricula and activities demonstrates the very characteristics of an interactive co- 
teaching relationship, which is considered as the key to implement successful inclusive practice (Mastropieri/Scruggs 2013). This interactive and interdependent relationship also directly contributes to more social interactions between children with and without SEN. Team teaching between general teachers and special educators has been widely recognized as one of the biggest challenges facing many inclusive preschools, for example, in Germany (see Schelle/Friederich 2015; Tures/Neuß 2017). In fact, while working in two German inclusive kindergartens, there were almost no cooperation between the main teachers and me. For most of the time, I was the support teacher who only needed to take care of the children with disabilities and there were rare communications taking place regarding curriculum or activity plans. Thus, the current study offers some valuable guidance to kindergartens regarding (1) how to define the role of special educators in the general classroom; (2) how to develop the relationship between the general teachers and special educators; (3) how general teachers could engage special educators in their lesson planning and activity designs. Nevertheless, it should also be noted the exact roles of the shadow teachers in this researched kindergarten still differ from the ones of the special educators in Germany and other countries and thus certain adaptations should be made to fit in the concrete inclusive preschool context (Stangvik 2010).

Secondly, though a clear definition of their roles is lacking, the involvement of the BYYs serves as one key element for making more inclusive practices possible, either through their support directly to the children with SEN or collaboration with the main teachers. This positive experience with the BYYs from the researched kindergarten offers a good example to illustrate the key value of involving every staff within the kindergarten in the inclusion process, one of the key features of an inclusive school culture (e.g., Strathern 2012; Zollers et al. 1999). During the one and a half years working as the support teacher in German inclusive kindergartens, I seldom was offered any training opportunity on special/inclusive education compared to the main teachers. Therefore, German kindergartens that aim for successful implementation of inclusive education could learn from the researched kindergarten about the specific ways they involve the BYYs and every other staff in the whole inclusion process to establish an inclusive culture. Specifically, they should learn from the researched kindergarten to involve every staff member in inclusive and special education training, equipping everyone with necessary theoretical knowledge and practical strategies to promote inclusive education. Only when everyone in the kindergarten holds similar goals and acquires the necessary knowledge and skills will sustainable inclusion be possible.

\section{The Key Role of the Principal}

The key role of the principal is very much emphasized from the researched kindergarten to successfully implement inclusive education, which resonates with previous research studies showing that school principals are key instructional leaders to both the attitudinal shifts and behavioural practices associated with success in inclusive education (Shogren et al. 2015; McLeskey et al. 2014; Robinson et al. 2008). Consistent with previous research studies (DeMatthews 2014; Hoppey/McLeskey 2013; Villa/Thousand 2005), the principal's expectations, support, modelling, and guidance are the major influencing factors for the successful implementation of inclusion. Moreover, previous research failed to show a comprehensive understanding of a principal's concrete involvement in the inclusion process (Praisner 2003). The current study, based on the "comprehensive approach to school administration and diversity" proposed by Riehl (2000), identified concrete strategies the principal has applied to 
promote more inclusive practice from the following three perspectives: (1) fostering new meanings about diversity; (2) promoting inclusive practices within schools; (3) building connections between schools and communities. Thus, the current study contributes to a clearer understanding of the principal's involvement in the kindergarten's inclusion process and thus provides useful guidelines for kindergartens in Germany and other countries to better develop inclusion.

(1) Fostering new meanings about diversity: the principal helps establish a democratic system of communication that allows every teacher to disagree with certain policies and practices and to make recommendations for changes; involves every staff to collaboratively build the inclusion philosophy together, which has been effective to create educational practices that respond actively to the needs of diverse students (Booth 2011; Corson 1995; Riehl 2000).

(2) Promoting inclusive practices within schools: the principal organizes training for every staff member to master knowledge and skills to realize inclusion philosophy through practice; values the "social relationships" with teachers by involving them in activity design and evaluations of inclusive practices (Hopkins 2001).

(3) Building connections between kindergartens and communities (see, e.g., Rouse/Florian 1996): the principal leads the "central expert team" from the "Chang Ning Special Education Centre"; initiates and participates in a teacher training program and acts as a strong advocate to develop inclusive education within the researched kindergarten and within kindergartens from other districts in Shanghai; and includes shadow teachers and parents into activity designs and establishes close cooperation with parents of children with SEN.

Johnston and Hayes (2007) indicated the necessity of conceptualizing professional learning as the pedagogical practice of educational leaders, explaining that every practitioner, including the principals, need to "learn new things" not just "do new things." The current study identifies the principal's key roles and clusters concrete strategies, initiatives and behaviours into three perspectives focusing specifically on how such leaders may foster new meanings of diversity, promote inclusive practices as well as build connections between kindergartens and communities. These lessons from Shanghai provide valuable insight into the conceptualization of potential leadership development and professional learning curricula for inclusive preschools in Germany and other countries.

\section{References}

Apple, Michael/Bean, James (2007): Democratic schools: Lessons in powerful education. Portsmouth, NH: Heinemann.

Arens, Richard (1952): The impact of communism on education in China, 1949-1950. Chicago: University of Chicago.

Booth, Tony (2011): The name of the rose: Inclusive values into action in teacher education. In: Prospects 41, 3, pp. 303-318.

Bronfenbrenner, Urie (1979): The ecology of human development: Experiments by nature and design. Cambridge, MA: Harvard University Press.

Chang Ning District (2010): Long-Term Education Reform and Development Plan (2010-2020). https://max.book118.com/html/2017/0911/133437976.shtm. 
Chen, Yun Ying (1996): Making special education compulsory and inclusive in China. In: Cambridge Journal of Education 26, 1, pp. 47-58.

Cheuk, Jenny/Hatch, Amos (2007): Teachers' perceptions of integrated kindergarten programs in Hong Kong. In: Early Child Development and Care 177, 4, pp. 417-432.

Cohen, Arie (2003): Ethnography and case study. In: Academic Exchange Quarterly 7, 3, pp. $283-$ 288.

Corson, David (1995): Discourse and power in educational organizations. Cresskill, NJ: Hampton Press.

DeMatthews, David (2014): Principal and teacher collaboration. In: International Journal of Educational Leadership and Management 2, 2, pp.176-206.

Donohue, Dana/Bornman, Juan (2014): The challenges of realising inclusive education in South Africa. In: South African Journal of Education 34, 2, pp. 1-14.

Elkins, John (2005): Inclusive education in Queensland: Where are we going and how will we get there? In: Social Alternatives 24, 4, pp. 45-49.

European Commission (2005): Report on social inclusion 2005: An analysis of the National Action Plans on Social Inclusion (2004-2006). Brussels.

Gargiulo, Richard/Piao, Yong Xin (1995): Early childhood special education in the People's Republic of China. In: Early Child Development and Care 118, 1, pp. 35-43.

Gómez-Zepeda, Gabriela/Petreñas, Cristina/Sabando, Dorys/Puigdellívol, Ignasi (2017): The role of the Support and Attention to Diversity Teacher (SADT) from a community-based perspective: Promoting educational success and educational inclusion for all. In: Teaching and Teacher Education 64, pp. 127-138.

Hoppey, David/McLeskey, James (2013): A case study of principal leadership in an effective inclusive school. In: The Journal of Special Education 46, 4, pp. 245-256.

$\mathrm{Hu}, \mathrm{Bi}$ Ying/Lim, Chih-Ing/Boyd, Brian (2016): Examining engagement and interaction of children with disabilities in inclusive kindergartens in China. In: Infants \& Young Children 29, 2, pp. 148-163.

Hu, Bi Ying/Roberts, Sherron/Wang, Yan Hua/Zhao, Hong Mei (2011): The initiation of early childhood inclusion in China: A case study from Beijing. In: International Journal of Early Years Education 19, 2, pp. 111-131.

$\mathrm{Hu}, \mathrm{Bi}$ Ying/Li, Ke Jian (2012): The quality rating system of Chinese preschool education: Prospects and challenges. In: Childhood Education 88, 1, pp. 14-22.

$\mathrm{Hu}, \mathrm{Bi}$ Ying/ Szente, Judit (2010): Education of young Chinese migrant children: Challenges and prospects. In: Early Childhood Education Journal 37, 6, pp. 477-482.

Hopkins, David (2001): School improvement for real. Lewes: Falmer.

Jiao, Yun Hong/Tang, Jian/He, Hong Ying/Wu, li Xin/An, Li Ge (2004): 河北省城市普通幼儿 园学前特殊教育调查与分析 [Investigation and analysis on the status of preschool special education in regular kindergartens]. In Chinese Journal of Special Education 44, 2, pp. 91-94.

Johnston, Ken/Hayes, Debra (2007): Supporting student success at school through teacher professional learning: The pedagogy of disrupting the default modes of schooling. In: International Journal of Inclusive Education 11, 3, pp. 371-381.

Kohlbacher, Florian (2005): The Use of Qualitative Content Analysis in Case Study Research [89 paragraphs]. In: Forum Qualitative Sozialforschung / Forum: Qualitative Social Research 7, 1, Art. $21 \mathrm{http} / / \mathrm{nbn}$-resolving.de/urn:nbn:de:0114-fqs0601211

Lewin, Keith/Wang, Ying Ji/Qu, Heng Chang (1994): Implementing basic education in China: Progress and prospects in rich, poor and national minority areas. Paris: UNESCO, International Institute for Educational Planning.

Lomofsky, Lilian/Lazarus, Sandy (2001): South Africa: First steps in the development of an inclusive education system. In: Cambridge Journal of Education 31, 3, pp. 303-317. 
Loreman, Tim (2007): Seven Pillars of Support for Inclusive Education. In: International Journal of Whole Schooling 3, 2, pp. 22-38.

Loreman Tim/Deppeler Joanne/Harvey, David (2005): Inclusive education: A practical guide tosupporting diversity in the classroom. Sydney: Allen \& Unwin.

Mastropieri, Margo A./Scruggs, Thomas E. (2013): The Inclusive Classroom: Strategies for Effective Differentiated Instruction. New York: Pearson.

Meng, Qiu Ming (2005): Thoughts on Strengthening Administrative Capacity Construction of Party in Colleges - Reflections on Thoroughgoing Study of the Fourth Plenary Session of Party's Sixteenth Central Committee. Duyun: Journal of Qiannan Normal College of Nationalities.

Mayring, Philipp (2004): Qualitative content analysis. In: Flick, Uwe/von Kardoff, Ernst/Steinke, Ines (eds.) (2000): A companion to qualitative research. London: Sage, pp.266-269.

McAlister, Sara (2013): Why community engagement matters in school turnaround. In: Voices in Urban Education 36, pp. 35-41.

McLeskey, James/Waldron, Nancy/Redd, Lacy (2014): A case study of a highly effective, inclusive elementary school. In: The Journal of Special Education 48, 1, pp. 59-70.

National People's Congress (1990): 中华人民共和国残疾人保障法 [The People's Republic of China on Protection of Disabled Person's Law]. http://www.spe-edu.net/Html/jiaoyufagui/200901/ 2633.html.

National Education Committee of the People's Republic of China (1994): 残疾人教育条例 [Educational Guidelines for Persons with Disabilities Bill]. http://www.moe.gov.cn/s78/A06/jcys left/moe_709/s3330/201001/t20100128_82037.html.

National People's Congress (2001): 关于“十五”期间进一步推进特殊教育改革和发展的意见 [The Tenth Five-Year Plan]. http://www.gov.cn/gongbao/content/2002/content_61549.htm.

National People's Congress (2006): 中国残疾人事业十一五发展纲 [The eleventh five-year plan]. http://www.gov.cn/jrzg/2006-06/08/content_304096.htm.

Peters, Susanne (2015): Inklusive Bildung für Kinder mit komplexen Beeinträchtigungen? Elternperspektiven auf Schule. Koblenz-Landau: Universität Koblenz-Landau. https://dnb.info/1074470605/34.

Platte, Andrea (2014): Inklusion-Implikationen eines Leitbegriffes für die Pädagogik der frühen Kindheit. In: Braches-Chyrek, Rita/Sünker, Heinz/Röhner, Charlotte/Hopf, Michaela (Hg.): Handbuch Frühe Kindheit. Opladen, Berlin, Toronto: Budrich, pp. 85-96.

Praisner, Cindy (2003): Attitudes of elementary school principals toward the inclusion of students with disabilities. In: Exceptional Children 69, 2, pp. 135-145.

Prengel, Annedore (2017): Individualisierung in der „Caring Community“-Zur inklusiven Verbesserung von Lernleistungen. In: Leistung inklusive, pp. 13-27.

Riehl, Carolyn (2000): The principal's role in creating inclusive schools for diverse students: A review of normative, empirical, and critical literature on the practice of educational administration. In: Review of Educational Research 70, 1, pp. 55-81.

Robinson, Viviane/Lloyd, Claire/Rowe, Kenneth (2008): The impact of leadership on student outcomes: An analysis of the differential effects of leadership types. In: Educational administration quarterly 44, 5, pp. 635-674.

Rouse, Martyn/Florian, Lani (1996): Effective inclusive schools: a study in two countries. In: Cambridge Journal of Education 26, 1, pp. 71-85.

Schelle, Regine/Friederich, Tina (2015): Weiterentwicklung pädagogischer Qualität durch inklusive Frühpädagogik. eine Analyse der Schlüsselprozesse in Kitas. In: Diskurs Kindheits- und Jugendforschung. In: Journal of Childhood and Adolescence Research 10, 1, pp. 67-80.

Sergiovanni, Thomas (1991): Constructing and changing theories of practice: The key to preparing school administrators. In: The Urban Review 23, 1, pp. 39-49. 
Sergiovanni, Thomas (1994): Building community in schools. San Francisco: Jossey-Bass Publishers.

Sharma, Umesh/Forlin, Chris/Loreman, Tim/Earle, Chris (2006): Pre-Service Teachers' Attitudes, Concerns and Sentiments about Inclusive Education: An International Comparison of Novice Pre-Service Teachers. In: International Journal of Special Education 21, 2, pp. 80-93.

Shogren, Karrie/McCart, Amy/Lyon, Kristin/Sailor, Wayne (2015): All means all: Building knowledge for inclusive school wide transformation. In: Research and Practice for Persons with Severe Disabilities 40, 3, pp. 173-191.

ShCoE (2010): Mid-term and Long-term Plan of Educational Reform. Shanghai: Shanghai Committee of Education.

Stake, Robert (2010): Qualitative Research: Studying How Things Work. New York: Guilford Press.

Stangvik, Gunnar (2010): Special education in society and culture: Comparative and developmental perspectives. In: European Journal of Special Needs Education 25, 4, pp. 349-358.

Stoll, Louise (1991): School effectiveness in action: supporting growth in schools and classrooms. In Ainscow, Mel (1991) (ed.): Effective Schools for All. London: David Fulton, pp. 68-91.

Strathern, Marilyn (2012): The nice thing about culture is that everyone has it. In: Strathern, Marilyn (ed.): Shifting Contexts: Transformations in Anthropological Knowledge. New York: Routledge, pp. 165-188.

Takayama, Keita (2008): The politics of international league tables: PISA in Japan's achievement crisis debate. In: Comparative Education 44, 4, pp. 387-407.

Tures, Andrea/Neuß, Norbert (2017): Multiprofessionelle Perspektiven auf Inklusion. Zur Notwendigkeit eines interdisziplinären und multiperspektivischen Diskurses. In: Tures, Andrea/Neuß, Nobert (2017) (eds.): Multiprofessionelle Perspektiven auf Inklusion. Opladen: Verlag Barbara Budrich, pp. 7-20.

Turnbull, Anna/Zuna, Nina/Turnbull III, Rutherford/Poston, Denise/Summers, Jean Ann (2007): Families as Partners in Educational Decision Making: Current implementation and future directions. In: Odom, Samuel/Horner, Robert/Snell, Martha/Blacher, Jan (eds.): Handbook of developmental disabilities. New York: Guilford Press, pp. 570-590.

Villa, Richard/Thousand, Jaqueline (eds.) (2005): Creating an Inclusive School. Alexandria, VA: Association for Supervision and Curriculum Development (ASCD).

Wang, Yu Chen (2016): Imagining inclusive schooling: An ethnographic inquiry into disabled children's learning and participation in regular schools in Shanghai. Edinburgh: University of Edinburgh.

Wang, Zhi Qiang/Shen, Ren Hong (2009): 学前随班就读的困境与思考-基于某幼儿园个案的描述与分 析 [The challenge and discussion of including children with disabilities in regular classroomsA case study]. In: Journal of Chongqing Normal University Edition of Social Science 2, pp. 141-147.

Yeo, Lay See/Neihart, Maureen/Tang, Hui Nee/Chong, Wan Har/Huan, Vivien (2011): An inclusion initiative in Singapore for preschool children with special needs. In: Asia Pacific Journal of Education 31, 2, pp. 143-158.

Zhu, Xiao Jin (2005): Semi-supervised learning literature survey. Wisconsin-Madison: University of Wisconsin-Madison.

Zollers, Nancy/Ramanathan, Arun/Yu, Moonset (1999): The relationship between school culture and inclusion: How an inclusive culture supports inclusive education. In: International Journal of Qualitative Studies in Education 12, 2, pp. 157-174. 


\title{
18 Inclusive School Development: The First Years of an English Free School
}

\author{
Julie Allan, Clara Rübner Jørgensen
}

\begin{abstract}
This chapter discusses inclusion in the context of a newly opened free school in England. The school has since its inception sought to be inclusive though its admissions policies, physical design and mixed ability teaching, and the chapter analyses and discusses the impact of these three elements on the experiences of students at the school. Data are drawn from the initial application to the UK government to open a free school, the school website, photoelicitation interviews with students and an interview with the first principal of the school. Based on an understanding of inclusion as involving the participation of students in the culture and curricula of mainstream schools, and the removal of barriers to their participation, the chapter investigates both how the school has tried to be inclusive and the extent to which young people at the school have felt the school is inclusive. The findings highlight the importance of social capital and trust in students' sense of belonging and inclusion, and furthermore illustrate the significance of school space in the development of relationships. The lessons learned from the start up and realisation of the inclusive vision described in the chapter, and the links between inclusion and social capital, trust and space provides insights that are relevant across different types of schools and international contexts.
\end{abstract}

\section{Introduction}

This chapter reports on the first years of a new school in England and its efforts to be inclusive. The school, which is the only secondary University Training School in the UK, is also a "free school," established under relatively recent English legislation that takes schools out of local authority control and gives them greater freedoms, including the freedom to set their own admissions policies. They are not formally allowed to be selective in their admissions, but because anyone can apply to set up a school (including charities, universities, community and faith groups, parents, teachers and businesses), in effect they may be developed to appeal to select groups or communities.

The school which forms the basis of this chapter sought to be inclusive from the outset through an admissions policy that brought children from across the city, through its architecture and design and by practising mixed ability class teaching rather than setting or streaming, as is the common practice in English secondary schools. Here, we draw on documentary evidence, including the original application to the UK government to open a free school, to report on how the school was initially conceived and has developed as an inclusive school. We report on the findings from research undertaken in the school within a project entitled 
Our space, our school, particularly focusing on data from the students, obtained through photo-elicitation methods and group interviews. These reveal how the students mostly experienced the school as inclusive, but with some exclusionary "moments," and we suggest that their inclusive experiences come through the acquisition of social capital. Finally, we include the perspective of the first principal of the school, drawing on an interview conducted three years after the school opened its doors in 2015. We argue for greater attention to the documentation and evidencing of inclusion "processes" and "outcomes" to ensure inclusion is recognized and valued and to acknowledge the explicitly inclusive efforts of schools such as the one discussed here. As such we hope that the lessons learned from the start up and realisation of the vision of a free school and the explicit articulation of the school as inclusive provide insights that are relevant across international contexts.

\section{Free Schools and Inclusion}

The Academies Act 2010, passed by a UK Conservative-Liberal Democrat Government, established a number of new types of schools, among them "free schools". These schools, funded by central government, are effectively taken out of local authority control and are then at liberty to make their own decisions about the curriculum, admissions (with the above mentioned limitations), the length of the school day, who to employ as teachers and the salaries and conditions of service of teachers. The idea behind free schools was that they would create competition, choice and innovation (Wiborg et al. 2018). Free schools were also intended to improve attainment, especially among socially disadvantaged young people (Doward 2011), as they were expected to respond to a demand for better schools in deprived neighbourhoods. However, evidence of improved educational outcomes of academies in England (of which free schools are a subset), Charter Schools in the US and Swedish free schools is limited (OECD 2010; Hatcher 2011) and their social impact has been mixed and widely debated. Furthermore, Wiborg et al. (2018) note a lack of innovation, arguing that free schools have remained conservative, constrained by parents and by the Ofsted ${ }^{1}$ Inspection regime and have become relatively isolated from other schools. Free schools in England have experienced tensions between the decentralisation and reduction in local authority control and heightened accountability regimes (Wilkins, 2020; Done, 2020) and provide an important point of comparison with other contexts through their selective adoption of free school policy from Sweden and the US. It is also potentially a unique point of reference, marked by its substantial adoption of the neoliberal agenda and strong engagement with market ideologies.

In addition to being a free school, the school discussed in this chapter is the only secondary university training school in the UK. As such, it is mandated to promote the learning of adults and of student teachers through formal and non-formal teacher education and benefits students and teachers at the school, student teachers and lecturers at the university and the wider university community. Students from the school make visits to the University for

1 Ofsted is the Office for Standards in Education, Children's Services and Skills. It is a non-ministerial UK government department that inspects and regulates services that care for children and young people, and services providing education and skills for learners of all ages. Ofsted reports directly to Parliament and is independent and impartial. 
a range of educational, sporting and cultural activities; student teachers observe and interact with classes in state-of-the art laboratories and classrooms; teachers at the school engage in knowledge exchange and continuing professional development activities together with university staff; and the school provides a location for both the sharing and generation of research. University staff deliver lectures at the school and contribute to the school's enrichment programme and regular Rush Hour Research events ensure a lively exchange of ideas. The staff of the University, including these authors, have an inside track and are able to undertake primary research in the school, however measures are in place to control the amount of research undertaken in order to protect the staff and students from excessive exposure.

From the outset, the school was conceived of as an inclusive school for the city. Inclusion of children with special educational needs in mainstream schools is generally encouraged in the UK, but $44.2 \%$ of children with SEN statements of Education and Health Care Plans (EHC) attend state sponsored special schools rather than mainstream schools and this number has been increasing since 2010 (Department for Education 2018). Furthermore, a significant degree of school segregation along ethnic lines has been identified in England, particularly in primary schools, although it has been noted that patterns are not static, nor uniform across the country (Burgess 2016; DEMOS 2015). As we will describe below, the school's admissions system seeks to bring students from across the city and from different backgrounds. Since its opening, it has had a gradual intake of students, admitting Year 7 and Year 12 only in its first year, and then gradually "filling up" with two new cohorts each year. The academic year 2019/2020 was the first year the school reached full capacity, with 750 students.

\section{The Research}

There have been few attempts to measure a school's inclusiveness that go beyond head counts of children with categorised special educational needs. This absence is strongly connected to the vagueness surrounding the concept of inclusion itself and the tendency to view it as a technical matter, concerned only with placing children with special needs in mainstream schools. Our understanding of inclusion, and that of the school's, is aligned with Booth's (1996: 36) definition as both increasing the participation of pupils in the cultures and curricula in mainstream schools and decreasing the schools' exclusionary pressures - "to attempt the first without the second is self-defeating". Inclusive school development takes account of the school's efforts to build an environment that welcomes a diverse student body and ensures that staff have the competence to ensure inclusive pedagogy. It should be said that free schools, because they can be set up by parents, groups or communities, could have the potential not to be inclusive, for example by catering for specific faith groups or serving a particular area. However, the leaders of this school sought to use the affordances of the free school legislation to create a school that is inclusive and followed a model of inclusive school development. In this research, we have investigated how the school has tried to be inclusive and have sought to gauge the extent to which the young people have felt the school is inclusive.

In order to examine how the school set about being inclusive, the original application to the government (Department for Education 2012) to open a free school, school policies and the school's website were scrutinised. Additional data were derived from an interview with the first principal of the school, carried out in January 2019, which was published as a 
conversation between the principal and a governor (Roden/Allan 2020). Finally, student perspectives on their social life within the school were obtained using photo-elicitation methods which are increasingly used in research with children and young people as it gives them more control over the research and acknowledges them as active participants in their own lives (Torre/Murphy 2015; Cooper 2017). The photo-elicitation exercise was undertaken in the first year of the school's operation. Each student was given a Kindle and asked to take at least five pictures within the school that made them think of friendships, relationships and wellbeing. To ensure the privacy of other students, they were instructed to avoid taking clear photos of people's faces. The photographs were used as the basis for the subsequent groupinterviews, in which the students were invited to show their photographs and describe why they had taken them before talking about their school and the extent to which they experienced it as inclusive.

In order to gauge how inclusive the school felt to the pupils, we utilised a framework of social capital. This allowed us to attend to the relationships among the students and between students and staff and involved an inference on our part that positive and strong connections would be conducive to inclusion. Social capital is the actual or potential resources that are linked to being part of a network (Bourdieu 1986), and these have been described as consisting of three types: bonding social capital, generated in homogenous groups, bridging social capital, generated in heterogenous networks and linking social capital, generated in relations between people of different status. Bridging and linking social capitals are generally considered more beneficial or productive than bonding social capital, although it is recognised that different types of capital may supplement or support each other (Holland et al. 2007; Terrion 2006). Trust is a key element that is built and maintained through these capitals and which, for Fukuyama $(1995 ; 2001)$ is the extent to which people believe that others act responsibly and for the public good. The link between trust and feelings is both strong and tenuous. Fukuyama (1995: 1) likens trust to 'chicken soup of social life,' which brings good things that are reciprocated but which, like chicken soup, works in mysterious ways. We can infer that high levels of trust among people within a given context can strengthen their feelings of belonging. Fukuyama (1995: 5) argues that strong organisations and structures cannot be legislated into existence but have to be "nourished through an increased awareness and respect for culture" and for cultural difference. This is similarly a key element of inclusion, involving sustained strategies by schools to facilitate relations and trust between students of diverse backgrounds. There is also increasing awareness of the importance of the relationship between the built environment and social capital (Mazumdar et al. 2017), and the importance of different types of spaces for young people producing social capital (Allison/Catts 2012; Holt et al. 2013). Young people's own networks have been identified as a source of social capital (Choudry et al. 2017; Holland et al. 2007; Ryabov 2009; Weller 2010) leading to positive outcomes, such as social well-being (Jørgensen 2017) and emotional support (Stanton-Salazar/Spina 2005).

\section{Making a School Inclusive}

The application to open a free school made explicit the ambition that the school should be inclusive. The word inclusive was not used in the stated vision of the school in the application; rather the intention to have a "diverse and comprehensive student body" was declared. Inclusion became much more explicit in the school's own website, stating on its landing page: 
"Together we're pioneering a school for a truly diverse and inclusive school community that creates great citizens". The school's inclusive ambition was realised through the explicit effort to create a school for the whole city, in the design and building of the school and in the deliberate rejection of setting and streaming in favour of mixed ability teaching.

\subsection{Creating a School for the City}

An ambitious school, meeting a need for more secondary places in [the city], accessible without examination and without reference to religious creed, seeking to bring together children from all over [the city] and beyond into a single learning community and to create an environment that raises aspirations and in which all have the opportunity to excel (DfE 2012: 13).

The creation of a school for the city - a comprehensive school - "serving a diverse and geographically dispersed student body, reaching across both deprived and more affluent regions of the city" (DfE 2012: 16) - was fundamental to the vision of the school, as stated in the application, to "transform lives" (DfE 2012: 13). As the first secondary training school application coming from a university, the applicants declared the school's:

unapologetic focus on aspiration, academic achievement, and ultimately success in higher education [...] a key and proven route to social mobility, and this School will seek to help less advantaged students raise their aspirations and achievement to promote social mobility (DfE 2012: 19).

It was acknowledged that the diversity of the city would bring with it some challenges to this aspiration:

If trends continue [the city] is set to become Britain's first city with a majority non-white population by 2024. It is also a city that suffers higher than average levels of deprivation, with $34 \%$ of children living in poverty overall compared to $20.9 \%$ nationally. This creates challenges for schools. Different ethnic and religious groups have different attitudes to education and aspiration. Children growing up in poverty face documented challenges accessing education, from lack of resources for transportation to unstable home lives to poor nutrition (DfE, 2012: 20).

The admissions policies of schools in England generally draw children from their local catchment area, based on distance from the school. This school, in its application and its stated endeavour to create an inclusive school for the whole city, considered all approaches to admissions, including distance from the school; fair banding tests, that seek to ensure a range of abilities through testing; random allocation of places across sub-regions; and working with feeder schools. Each of these was ruled out on the basis of the disadvantages that they would bring. Instead, an admissions policy that would create a highly diverse pupil profile in terms of ethnicity and social mix in its year 7 (eleven year old) students was selected. Four "nodal points" of the city were identified, with postcodes, and with the following distribution based on the train station at the heart of three of the nodes:

- $1.50 \%$ (up to 75 pupils) from around the school site: an area with a mixture of private and council housing and a predominantly white population but with some ethnic diversity.

- $\quad 2.16 .7 \%$ (up to 25 pupils) from around Rail Station A, an area with a large amount of 19th Century housing and high numbers of people from Irish, West Indian, East African, South Asian and Pakistani communities. 
- $3.16 .7 \%$ (up to 25 pupils) from around Rail Station B, a working class, inner city area, with a culturally diverse mix of people.

- $\quad$ 4. $16.7 \%$ (up to 25 pupils) from around Rail Station C, an inner city area that is subject to urban regeneration, with a large business area, a mixture of housing and an ethnically diverse population.

As part of the admissions process, the distance between the applicants' home and the main entrance of the node (the school in the case of node 1 or the railway station entrance of nodes 2, 3 and 4 as the crow flies) is calculated in order to apply the distance criteria. The idea of an inclusive school for the city, based on this particular system for admissions, was one of the key motivations of the first principal to want to lead the school, as he revealed in an interview:

The big attraction was the proposed admissions, the fact it was being done with the local authority and [...] [including] the African Caribbean group, which were probably the ones with the lowest numbers getting into the grammar schools. When they did get into the grammar schools, invariably, they were the ones with the most trouble because of their difficult home life. So, it was a chance to have something that really did reflect the city.

In addition to the nodal system, the admissions policy of the school gives priority to pupils with an Education and Health Care Plan (EHC) - a legal document issued for children with significant special educational needs, to 'Looked after Pupils' (pupils who are in the care of or who are provided with accommodation by the local municipality) and to siblings of pupils attending the school. Year 7, 8, 9 and 10 at the school has a diverse school population in terms of socio-economic background and ethnicity, and a significantly higher number of children on EHC plans than city average.

The school has a separate admissions policy for its year 12 (sixth form) students. This is based on academic achievement as evidenced through exam results, with a supporting reference from the school; this admissions policy is not intended to create the same level of diversity as among the year $7 \mathrm{~s}$. School staff hope that as many students as possible who have been admitted through the year 7 admission criteria will remain in the school for their entire school career but recognize that some students will not meet the academic criteria and will be forced to go elsewhere.

\subsubsection{Designing and Building the School}

The school was built from scratch, designed to be inclusive and realised through its physical openness, an absence of boundaries and walls and a maximization of visibility. It was also constructed with, as its central feature, a huge social space (the atrium) intended for shared dining by teachers and students and social interaction. The atrium bears some resemblance to Jeremy Bentham's panoptic model of surveillance that Foucault (1977) saw as the ultimate model for the exercise of power; but it was intended to also deliver a reciprocity of surveillance, with students being able to look in on teachers and to improve student safety by providing no enclosed spaces where bullying could take place.

Upon entering the school building, there is a reception and a sofa area, where visitors wait to be collected and students sometimes sit, if they have an issue that needs attending to, such as sickness. In the middle of the building is the large atrium with 12 long rows of tables and a canteen (pictured by one of the students in Figure 2 below). On both sides of the atrium 
there is a large open staircase leading to the second and third floor. The staircase has a railing in the middle and students are instructed to walk up on the left and down on the right. A favourite position of the first principal was at the top of the stairs, looking down to the door through which students arrived in the mornings. This, he said, enabled him to both see and be seen by the students each day, something that was important to him. The main school building has three floors with wide corridors connecting classrooms, staff rooms, break out space (small clusters of 2-3 tables and chairs), toilets and some staff offices. All classrooms, staff rooms and offices have large windows, which can be overlooked from the corridors. The corridor walls are white and there are large windows on all sides of the building and natural light from above. In the arts corridor, posters and arts creations are displayed on the walls, but in the remaining areas, the walls are relatively bare. On each floor there is a set of TV screens displaying pictures from school events, quotes from former students, inspirational quotes, and sometimes the news. On all floors there are regular and disabled toilets and there is a lift connecting the floors. Outside, there is a large green area and a tarmacked playground, including a football field. Other spaces of relevance are the assembly hall - a large room with theatre style seating, and the library, containing fiction and non-fiction books, magazines, newspapers and other resources and with one area with tables and chairs and another with sofa arrangements and bean bags. Not all students found the same areas inclusive. For example, the atrium could be a stressful place for some students when it was full. But there were alternative areas, such as the library, where students could go for some quiet time and/or to meet up with friends.

\subsubsection{Rejecting Setting and Streaming}

Secondary schools in England generally group students by attainment, at least in core subjects such as Maths, English and Science. Successive research studies have shown that streaming and ability groupings have little or no impact on overall school attainment (Boaler/William 2001; Francis et al. 2017) and may have a negative impact for lower sets and streams. In a review of the international literature on streaming, Johnston and Wildy (2016) found that while the practice may have some benefits for high-achieving students, it also has a significant cost for low-achieving students as it increases inequality between higher and lower achieving groups. Because students of lower income and minority ethnic backgrounds tend to be overrepresented in lower sets, ability groupings may furthermore exacerbate the effect of socio-economic background on educational achievement and result in increased segregation of students along the lines of race and class. Hallam (2012) argues that countries that practise early educational selection, including streaming, tend to have higher levels of social segregation, and that streaming may also have a negative effect on intergenerational mobility. This is supported by the OECD report Equity and Quality in Education (OECD 2012) which notes that "Early student selection has a negative impact on students assigned to lower tracks and exacerbates inequities, without raising average performance" (p. 10). Finally, Johnston and Wildy's review identifies the psychological impact of streaming, particularly on students in lower sets, but also shows that the extent of these depends on the context and that teachers play a key role in mediating the adverse effects of streaming upon students.

Francis et al. (2017), in their review of the literature, identified seven key problems with ability groupings which relate both to the process and the outcomes of the practice: 1) certain 
groups of children (from lower socio-economic groups and particular minority ethnic groups) are overrepresented in low sets and the allocation doesn't always match their ability; 2) there is a lack of fluidity between groups and pupils thus tend to stay in their ability group even if their attainment improves; 3 ) higher and lower sets receive a different quality of teaching and teachers; 4) teacher expectations for pupils vary depending on sets; 5) different pedagogy, curriculum and assessment are applied to different groups; 6) pupil perception and experiences of 'ability' groupings can serve to label students in low streams and 7) these factors together may provoke self-fulfilling prophecies about low achievement. Francis et al. (2017: 6) therefore conclude that " $[t]$ he evidence concerning the impact of placement in low 'ability' groups on (poorer) educational progress and outcomes, coupled with the findings about the disproportionate representation of economically disadvantaged pupils in these low sets, provides a clear hypothesis that these practices may contribute to the socio-economic attainment gap in the UK education system".

The evidence against setting and streaming and its effect on inclusion influenced the decision by the applicants for the school and its first principal to adopt mixed ability class organization. This was seen as a natural extension of the "aim to create a mixed ability, coeducational, state school" (DfE 2012: 112) a message which was communicated to parents of potential students of the school and explained on the school website:

We believe in helping everybody to do the best that they can. Evidence suggested that setting or streaming students does not have a positive impact on learner achievement. We teach in mixed attainment groups and work hard to ensure that all students are stretched and challenged to meet our high expectations.

Staff have admitted to finding the practice of mixed ability teaching challenging but have continued to see its value and its role in inclusion. They have asked for and have received support in the form of Continuing Professional Development through, for example sessions on stretching and challenging all learners. In addition, the School has looked to its full and extensive enrichment programme "to provide a varied approach to teaching the curriculum which will maximise its ability to 'hook' students who learn in different ways" (DfE 2012: $67)$.

\section{Our School, Our (Inclusive) Space}

The students clearly identified their school as inclusive. For them, this was marked by two key elements, both interlinked in various ways with the admissions policy, the design of the school building and the mixed ability teaching. The elements that they focused on were the positive regard for the diversity of the students attending the school and their sense of belonging together. Both of these seemed to have come about through the social capital they had acquired since coming to the school. The students' understanding of their inclusive school space as inherently social, but also determined by physical factors, is evidenced by their photographs and associated narratives. It accords with the spatial theories of Lefebvre (1991) and Soja (1996) and with spatial analyses of schools by Leander et al (2010) and Burnett (2011). 


\subsection{Relishing Diversity}

Figure 1: Students interacting in a school corridor

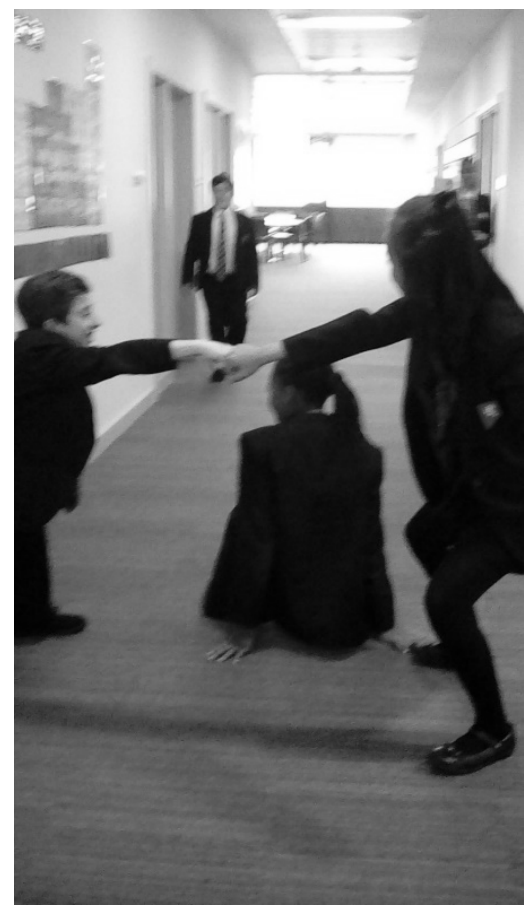

Source: own research.

The image above was taken by a group of students, who saw it as representing the high degree of diversity within the school. This was something that the students liked a great deal because it created more interest for them:

Everybody has different taste[s]. Like, I have a Korean friend and he literally hates sports ... Just because you like something else it doesn't mean that you are out of the ordinary, because some people like this and some people like that.

You can't just have the same thing, because that would just be boring. You want to find people with different personalities, different things they like.

The diverse ability range was also remarked upon positively by the students, especially since it was accompanied by good teacher support:

There are a lot of people with problems in this school, and special needs. And other people who don't have those problems, they are not used to having to talk with those people ... In the past, people with learning disabilities wouldn't be treated or cured as they are now.

There is really good support. ... if they get carried away or don't know how to handle themselves, there is someone who can support them almost all the time. 
Other students compared the management of special needs favourably with their experience in primary school:

They are dealing with [special needs] well. They don't want children to feel bad in the school ... In my primary school they would shout at you and they thought that was the way to make you not do it ... the thing is, people who have Special needs, they can't help it.

Yeah and especially when you start your education in primary you can't quite tell your special needs. You need to study for a while to be able to see.

Although students considered the school's inclusivity of diverse students to be one of its most important features, there were, however, a few negative comments about the impact of making adjustments for students with special educational needs on the learning environment. One student commented that disabled students seemed to get away with behaviours that, coming from themselves, would have been admonished, but on balance thought the support available was good:

This girl, she does stand up and get out of class, whereas if we did that we would get amber stamps and she never gets told off. But I do think they treat disabled people quite well ... but sometimes people do distract our learning but they don't get told off for it or anything.

A student with special educational needs indicated that his difference was treated only with "surprise," but nothing more than that:

Student: well, sometimes I feel a little weird. Everyone in my class has never seen a dyslexic person in their life. So when I first came along and had a laptop they were like surprised. I was surprised to get a laptop as well.

Interviewer; so does it help you?

Student: it is extremely helpful

Interviewer; and do your friends understand?

Student; my friends understand and some of them don't.

\subsection{Belonging Together}

Students described the relationships in the school as:

generally good, there are a lot of people laughing, talking to each other, talking about new things. In primary school it was just a game of tag, game of hide and seek.

Many of the students described their favourite spaces in the school as places, including the atrium, where new relationships were forged and maintained or others, such as the library, where they could have quiet conversations with friends:

I also like the atrium. That is where I go when I don't have lessons. In the beginning, we met friends there.

The stairs were where I met my first friend.

The green area was where you would go when school first started, hang out and do most of the work - basically, it is just our favourite area to hang out with friends. 
Figure 2: The central atrium of the school

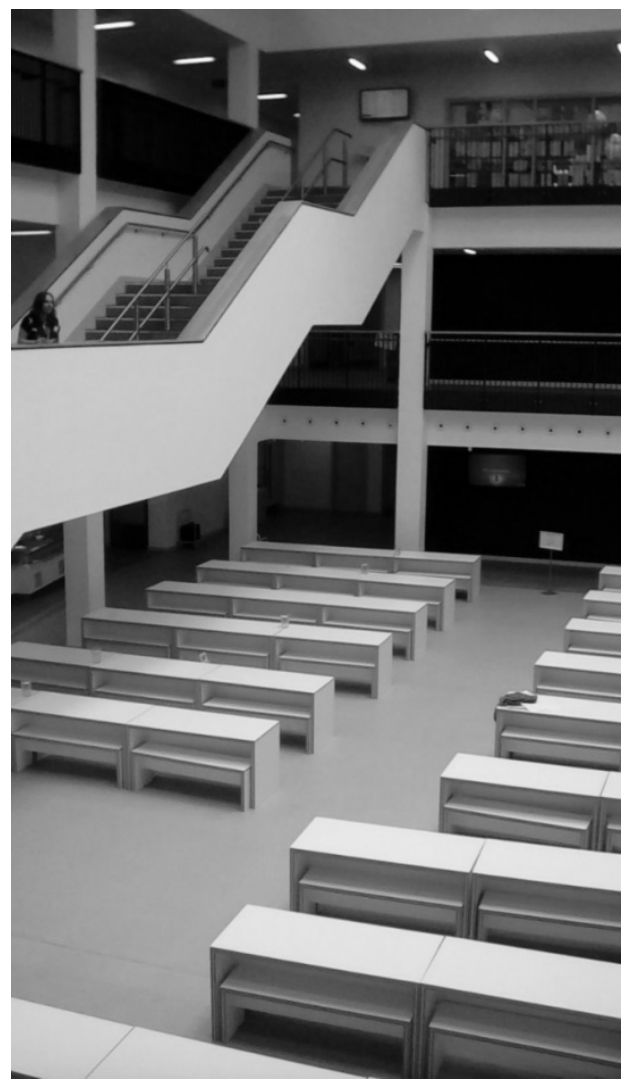

Source: own research.

The students relished the newness of the school and considered that it both belonged to them and that they belonged to it. The school was "a happy place to be" and one which was "welcoming". This image of the lockers was a symbol of belonging because it was a place where they could physically mark their presence; it also marked a moment in the school day where they belonged together:

I took the picture of people opening their locker because it is like the end of the day or before the school day and people go to their lockers to get their stuff or hang with their friends, have conversations, everyone doing their thing ... Lots of us hang out around the lockers a lot. So then there is a lot more memories there.

Other students marked out specific spaces (for example the library or the art room) where they felt they particularly belonged. As suggested earlier, the precipitating factor in their belonging was the relationships that they enjoyed within these particular spaces, and in the school in general. 
Figure 3: Students in the locker area

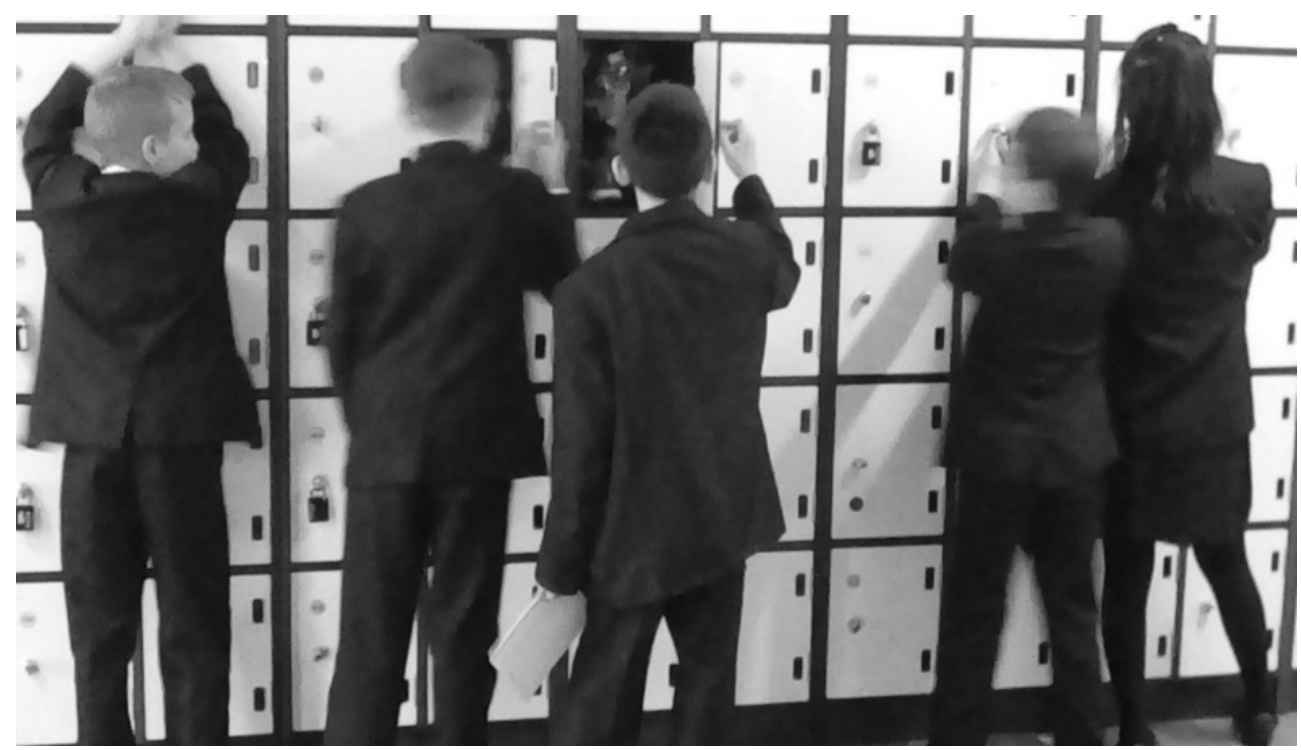

Source: own research.

\section{Discussion: Including Social Capital}

Mazumdar et al. (2017: 149), in their systematic review on the relationship between the built environment and social capital, found that "chance and formal encounters facilitated by the built environment can enhance social capital." The creation and construction of this school, with its deliberate attempts to bring together and foster interaction among students from different backgrounds and teachers, appears to have actively generated social capital. The admissions policy, by bringing students to the school from different parts of the city, established a student population that was richly diverse in terms of ethnicity, background and ability. This diversity was converted to (bridging) social capital through the insistence on mixed ability teaching, whereby students with 'different tastes', different attributes and different abilities were required to learn and to live together. The refusal of the separatist practices of setting and streaming avoided the creation of 'dis-belonging' (Erevelles 2011: 2157) for particular students, an all too frequent phenomenon within the "quintessentially [...] ableist" (Goodley 2014: 100) mainstream school environment.

The students appeared to welcome the diversity in the school, at the very least not as boring as other, more homogeneous, arrangements. Converted to bridging social capital, diversity became positive, interesting and a resource, an argument also made by Goodley (2014: 104) in describing disability as offering "provocation" because it "disturbs our image of the typical student". Garland Thomson (2011: 598-599) further elaborates on the strategic value of difference: 
So whereas the benefit of fitting is material and visual anonymity, the cost of fitting is perhaps complacency about social justice and a desensitizing to material experience. Misfitting, I would argue, ignites a vivid recognition of our fleshliness and the contingencies of human embodiment.

The support for some disabled students, and the possibility that they might take advantage of it, troubled a few individuals but even they, on balance, recognised the overall benefits, thereby suggesting a further increment of bridging social capital. The students' strong sense of belonging to the school, and the school belonging to them, seemed to suggest that they had acquired some bonding social capital, in a familial sense of being connected to the school and to each other, and seemed also to contribute to their strong sense that their school actively included and welcomed them. Relationships, or networks in the language of social capital, were of great importance and value to the students, as suggested by one student who, asked to describe the school to an outsider, said "If you are worried about making friends, there are loads of people to make friends with. You'll make a lot of friends".

Trust, as Fukuyama (2001) argues, is acquired through habituation to the common norms of a group or community and is always social rather than individual. The trust within the school was developed through the strategic cultivation of bridging social capital and had a moralistic quality that Uslaner (2010: 3 ) describes as bringing people who are different together and making them want more for each other:

If we believe we are connected to people who are different from ourselves and have a moral responsibility for their fate, we see that trust is a fundamentally egalitarian ideal ... A culture of trust depends upon the idea that things will get better for those who have less and it is in our power to make the world better.

This type of trust differs from the more strategic kinds that are based on prior knowledge and experience (Yamagishi/Yamagishi 1994). Although apparently less rational, moralistic trust can be considered to be less risky because it makes the normative assumption that people should - and usually do - behave.

\section{Conclusion}

In many ways this school is no different from others in their aspiration to be inclusive. What has marked this school out as distinctive is its efforts to create a more diverse student population than it would have if it only took students from its own catchment area; designing a school to maximise opportunities for interaction and relationship building and continuing to expose the students to difference within mixed ability classes.

The students apprehended the school's inclusive intentions. They recognised the richness of the diversity in their school lives and considered themselves enriched by it. And, given the space - literally - and the opportunity to develop relationships, they articulated a strong sense of belonging - to each other and to the school. The school was not without its fractious relationships among students, misbehaviour and the occasional exclusion. Yet there existed among them a level of respect and the kind of moralistic trust that Uslaner (2010) has identified. Social capital provided a useful framework for exploring the impact of trust on students' sense of belonging and inclusion in the school. It also highlighted the significance of the space - and its highly social nature - in the development of relationships and trust within the school and there is a clear need for further investigation of school space and inclusion. 
These inclusion 'outcomes' are derived from what the students feel about the school, their and others' presence in it and the relationships they had developed. We sought to gain some traction on inclusion by using the concept of social capital, starting from the premise that "relationships matter" (Field 2005: 2) and inferring an association between the acquisition of social capital and inclusion. This may not be the most propitious way of identifying inclusion outcomes, but it is at least a start and we urge policymakers and researchers to give further and more detailed attention to both the processes and the outcomes of inclusion. This is all the more important at a time when, as Hamre et al. (2018: 258) argue, inclusion is itself increasingly succumbing to "accountability inclusion", which is, once again, about individual students' performances but is extended to incorporate - and judge - teachers' capabilities in delivering these performances.

\section{References}

Allison, Marion/Catts, Ralph (2012): Youth Club Connections. In: Allan, Julie/Catts, Ralph (eds.): Social Capital, Children and Young People. Bristol: Policy Press, pp. 77-98.

Boaler, Jo/William, Dylan (2001): Setting, streaming and mixed-ability teaching. In: Dillon, Justin/Maguire, Meg (eds.): Becoming a teacher. Issues in secondary teaching. Buckingham: Open University Press.

Booth, Tony (1996): Stories of exclusion. Natural and unnatural selection. In: Blyth, Eric/Milner, Judith (eds.): Exclusion from School. Inter-professional Issues for Policy and Practice. London: Routledge.

Bourdieu, Pierre (1986): The Forms of Capital. In: Richardson, John G. (ed.): Handbook of Theory and Research for the Sociology of Education. London: Greenwood Press.

Burgess, Simon (2016): Are we headed to integration or segregation for England's pupils? http:// www.integrationhub.net/are-we-headed-to-integration-or-segregation-for-englands-pupils/ [Accessed: 06.01.20].

Burnett, Cathy (2011): The (Im)Materiality of Educational Space: Interactions between Material, Connected and Textual Dimensions of Networked Technology Use in Schools. E-Learning and Digital Media 8, 214-227. https://doi.org/10.2304/elea.2011.8.3.214

Choudry, Sophina/Williams, Julian/Black, Laura (2017): Peer Relations and Access to Capital in the Mathematics Classroom. A Bourdieusian Social Network Analysis. In: British Journal of Sociology of Education 38, 7, pp. 1037-1053.

Cooper, Victoria (2017): Lost in Translation. Exploring Childhood Identity Using Photo-elicition. In: Children's Geographies 15, 6, pp. 625-637.

DEMOS (2015): 61\% of ethnic minority kids in England - and 90\% in London - begin Year 1 in schools where ethnic minorities are the majority of the student body. https://demos.co.uk/pressrelease/61-of-ethnic-minority-kids-in-england-and-90-in-london-begin-year-1-in-schoolswhere-ethnic-minorities-are-the-majority-of-the-student-body/ [Accessed: 04.07.2019]

Department for Education (DfE) (2012): Free schools in 2013. Application form. Mainstream and 16-19 Free Schools. London: DfE.

Department for Education (2018): Special educational needs in England. London: DfE.

Department for Education and Science (DfES) (2004): Governing the School of the Future. London: DfES. 
Done, Elizabeth J. (2020): Education governance and the responsibility to include: Teachers as a site of discursive tension. In: Allan, Julie/Harwood, Valerie/Joergensen, Clara Rübner (eds.): Schooling, governance and inequalities. World Yearbook in Education. London: Routledge.

Doward, Jamie (2011): Government gives go-ahead to first eight "free schools". In: The Guardian, January 29.

Erevelles, Nirmala (2011): 'Coming out crip' in inclusive schools. In: Teachers College Review 113,10 , pp. 2155-2185.

Field, John (2005): Social capital. London: Routledge.

Foucault, Michel. (1977): Discipline and punish. New York: Random House.

Francis, Becky/Archer, Louise/Hodgen, Jeremy/Pepper, David/Taylor, Becky/ Travers, MaryClaire (2017): Exploring the relative lack of impact of research on 'ability grouping' in England. A discourse analytic account. In: Cambridge Journal of Education 47, 1, pp. 1-17.

Fukuyama, Francis (1995): Trust. The social virtues and the creation of prosperity. London: Hamish Hamilton.

Fukuyama, Francis (2001): Social capital, civil society and development. In: Third World Quarterly 22, 1, pp. 7-20.

Garland Thomson, Rosemarie (2011): Misfits: A feminist materialist disability concept. In: Hypatia 26, 3, pp. 591-609.

Goodley, Dan (2014): Disability studies. Theorizing disablism and ablesim. London: Routledge.

Hallam, Susan (2012): Streaming and Setting in UK Primary Schools. Evidence from the Millennium Cohort Study. In: FORUM, 54, 1, pp. 57-64.

Hamre, Bjørn/Morin, Anne/Ydesen, Christian (2018) Optimizing the educational subject between testing and inclusion in an era of neoliberalism: Musings on a research agenda and its future perspectives. In: Hamre, Bjørn/Morin, Anne/Ydesen, Christian (eds.): Testing and inclusive schooling: International challenges and opportunities. London: Routledge.

Hatcher, Richard (2011): The Conservative-Liberal Democratic Coalition Government's 'free schools' in England. In: Educational Review 63, 4, pp. 485-503.

Holland, Janet/Reynolds, Tracey/Weller, Susie (2007): Transitions, Networks and Communities. The Significance of Social Capital in the Lives of Children and Young People. In: Journal of Youth Studies 10, 1, pp. 97-116.

Holt, Louise/Bowlby, Sophia/Lea, Jennifer (2013): Emotions and the habitus. Young people with socio-emotional differences (re)producing social, emotional and cultural capital in family and leisure space-times. In: Emotion, Space and Society 9, pp. 33-41.

Jørgensen, Clara Rübner (2017): 'Peer Social Capital' and Networks of Migrants and Minority Ethnic Youth in England and Spain. In: British Journal of Sociology of Education 38, 4, pp. 566-580.

Leander, Kevin/Phillips, Nathan/Taylor, Katherine (2010): The Changing Social Spaces of Learning: Mapping New Mobilities. In: Review of Research in Education 34, pp. 329-394. https:// doi.org/10.3102/0091732X09358129

Lefebvre, Henri (1991): The production of space. Oxford: Blackwell.

Mazumder, Soumya/Learnihan, Vincent/Cochrane, Thomas/Davey, Rachel (2017): The built environment and social capital. A systematic review. In: Environment and behaviour 50, 2, p. 119-158.

Johnston, Olivia/Wildy, Helen (2016): The effects of streaming in the secondary school on learning outcomes for Australian students - A review of the international literature. In: Australian Journal of Education 60, 1, pp. 42-59.

OECD (2010): PISA 2009 results. Executive Summary. Paris: Organisation for Economic and Co-operative Development.

OECD (2012): Equity and Quality in Education - Supporting disadvantaged students and schools, Paris: Organisation for Economic and Co-operative Development. 
Roden, Michael/Allan, Julie (2020): Governing inclusion. A school principal and a governor in conversation. In: Allan, Julie/Harwood, Valerie/Joergensen, Clara Rübner (eds.): Schooling, governance and inequalities. World Yearbook in Education. London: Routledge.

Ryabov, Igor (2009): The Role of Peer Social Capital in Educational Assimilation of Immigrant Youths. In: Sociological Inquiry 79, 4, pp. 453-480.

Soja, Edward W. (1996): Thirdspace: Journeys to Los Angeles and other real-and-imagined places. Cambridge, MA: Blackwell.

Stanton-Salazar, Ricardo D./Spina, Stephanie Urso (2005): Adolescent Peer Networks as a Context for Social and Emotional Support. In: Youth Society 36, 4, pp. 379-417.

Terrion, Jenepher Lennox (2006): Building Social Capital in Vulnerable Families. Success Markers of a School-based Intervention Program. In: Youth and Society 38, 2, pp. 155-176.

Torre, Daniel/Murphy, Joseph (2015): A Different Lens. Changing Perspectives Using Photo-elicitation Interviews. In: Education Policy Analysis Archives 23, 1, p. 111.

Uslaner, Eric (2010): Segregation, mistrust and minorities. In: Ethnicities 10, 4, pp. 415-434.

Yamagishi, Toshio/Yamagishi, Midori (1994): Trust and commitment in the United States and Japan. In: Motivation and Emotion 18, 2, pp. 129-166.

Weller, Susie (2010): Young People's Social Capital. Complex Identities, Dynamic Networks. In: Ethnic and Racial Studies 33, 5, pp. 872-888.

Wiborg, Susane/Green, Francis/Taylor-Gooby, Peter/Wilde, Rachel (2018): Free schools in England. 'Not unlike other schools?' In: Journal of Social Policy 47, 1, pp. 117-137.

Wilkins, Andrew (2020): Technologies in rational self-management: Interventions in the 'responsibilisation' of school governors. In: Allan, Julie/Harwood, Valerie/Jørgensen, Clara Rübner (eds.): Schooling, governance and inequalities. World Yearbook in Education. London: Routledge. 


\title{
19 Steps to Inclusion? The Role of Tiered Intervention in Finland and in the United States
}

\author{
Markku Jahnukainen, Tiina Itkonen
}

\begin{abstract}
This article contrasts the Finnish policy context of a tiered support model with that of the United States, and discusses subsequent approaches in identifying and supporting students with learning disabilities in inclusive general education settings. The Finnish public school is well-known for being highly equitable. One key ingredient of equity is the broad variety of additional supports delivered inclusively in general education under the mandatory Learning and Schooling Support system. The U.S. Department of Education does not subscribe to a particular framework of RtI but acknowledges that the core characteristics include research-based instruction in general education settings. In both school systems the role of tiered systems in teacher education needs to be implemented more systematically.
\end{abstract}

\section{Introduction}

There has been an enormous interest in comparative education research since early 2000 . The international school attainment tests have offered a platform for comparing the results between countries and jurisdictions. Many governments and educational actors all over the world have turned their eyes to the top performing countries to find explanations for their success (e.g. Lien Foundation 2018). Since 2000 Finland has been one of those countries under siege of both academics and practitioners in education. Educational researchers (e.g. Chong 2016; Tomlinson 2012) as well as journalists (e.g. The Editorial Board, 2013; see Takayama et al. 2013) have pointed out some ingredients of the "Finnish miracle" like high level teacher education, trust and teacher admiration. In some cases also the ways of meeting the needs of diverse learners have been suggested as possible sources of Finnish success (Itkonen/Jahnukainen 2007; 2010; Lien Foundation 2018; Sahlberg 2015).

In this chapter, we utilize our previous research work comparing inclusive and special education policies in Finland and in the United States (Itkonen/Jahnukainen 2007; 2010; Jahnukainen/Itkonen 2016). For making the contrasting of two different school systems possible and meaningful, our perspective focuses mainly on system-level developments. Therefore, our angle on inclusive education is also restricted to define the education policy and system level actions to promote the access to support for every student in general education settings.

We first present the tiered intervention/support model in Finland in its broader educational policy context. We further provide a state-by-state review of policies as they relate to identifying students with learning disabilities in the U.S. We then compare the tiered support 
models in Finland and the United States; the mandatory Learning and Schooling Support in Finland and the most common U.S. model consisting of three tiers (e.g. Fuchs et al. 2008). This chapter concludes with some thoughts on implications of tiered models of support to education policy, including teacher education.

\section{From Special Education to Learning and Schooling Support in Finland}

The Finnish comprehensive education system is historically based on the idea of "education for all" since the first Compulsory Education Act 1921 (Jahnukainen 2011). However, it took decades to fundamentally change the system considering the long tradition of parallel school systems and exclusion of students with severe special educational needs. Finally, since 1997 children of every school age - including students with severe disabilities - are being educated in the same comprehensive school system (Graham/Jahnukainen 2011). Even though special schools still exist, the number of students placed in them have decreased steadily since the late 1990s (Figure 1). Also, the funding reform in 2010 affected the use of full-time special classes (small group) located in regular schools (see Pulkkinen 2019). The increase of the use of partial placement in general education (Figure 1) since 2010 was partly affected by the implementation of more specific statistical units in data gathering but also partly by the service model reform to a tiered system starting 2011. It could be said that on the system level the Finnish comprehensive school has had a clear shift towards a more inclusive direction by decreasing the number of students placed in segregated settings, however, in partly unsystematic ways (see Honkasilta et al. 2019).

Figure 1: Number of Tier 3 (special support) students in Finland by placement options 1998-2017

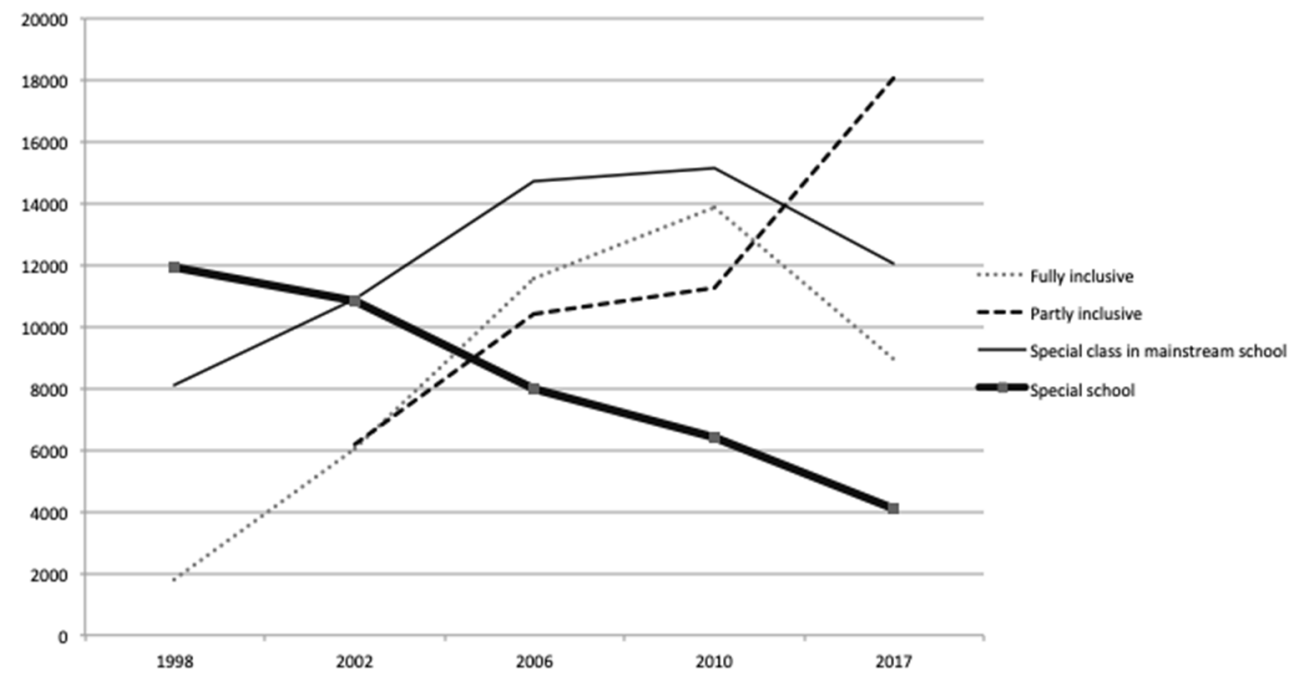

Source: Jahnukainen 2011; OSF 2018. 
The earlier service system with full-time and part-time special education was modified to a three-tiered system defined under the title of Learning and Schooling Support effective from 2011 (The Amendment of the Basic Education Act 642/2010) (see Pulkkinen/Jahnukainen 2016). The reform started from the committee work of Strategy of Special Education and finalised during 2011, when the Amendment of Basic Education Law was enacted. Inbetween, following the Strategy of Special Education, there was an intensive period of inservice teacher training in every region of Finland organized jointly by the special teacher education units of different universities and by the Finnish National Board of Education (see Thuneberg et al. 2014).

The Finnish tiers are functionally equivalent to US Tiers 1, 2 and 3 (see Table 2), but in Finland there is only one fixed national model mandatory for all school boards. Tier 1 (general support) consists of every action made by the regular classroom teacher in terms of differentiation as well as in terms of school-wide efforts to meet the diversity of students. This is basically transferring more responsibility to classroom teachers as well as the school community. Tier 2 (intensified support) consists of remedial support by the class teacher, coteaching with the special educator and temporal individual or small group learning with the part-time special educator. In practice, this is highly equivalent with the former definition of "part-time special education". Tier 3 (special support) consists of the whole continuum of special education services from fulltime general education to a special school placement and is mostly equivalent with the previous "full-time special education" (see Jahnukainen/Itkonen 2016).

\section{United States: Learning Disability Diagnosis and RtI}

The Individuals with Disabilities Education Act (IDEA), initially passed in 1975, is a federal law that guides special education policymaking in the United States. The main provisions of the statute include "free appropriate public education" for every eligible child regardless of the type or severity of disability, education in the least restrictive environment, due process rights for parents, and a non-discriminatory, multi-disciplinary evaluation to determine if a child qualifies to receive services. Thus, the provision of special education is diagnosis-based. Today there are thirteen categories under which a child's diagnosis must fall in order to receive services. This section discusses the evaluation of students for suspected learning disabilities (LD), and the use of RtI in that process.

It is necessary to explain the U.S. education policy context before proceeding to review the variety with which states have designed their RtI and LD diagnostic processes. The U.S. are grounded on the concept of federalism, or the vertical independence between the federal government and the states. Thus, the federal government cannot mandate states to adopt educational policies (although state policies must align with the Constitution), but the federal government can provide incentives or carrots such as funding for the adoption of federal legislation. For example, special education law, initially passed in 1975 by Congress, was last adopted by New Mexico in 1989, over ten years after the law's passage. Similarly, there is variation in how states have approached the use of RtI and discrepancy models in diagnosing LD.

RtI is an effort to "un-do" the medical model set forth in the law. Traditional "LD" eligibility required discrepancy between academic potential and actual achievement. The un- 
intended consequence was that once eligible, the student would receive special education, often in a "resource room" where the student went for part of the day (Fuchs/Fuchs 2005). In the U.S. regulations of the part-time special education placements are called "the continuum of services" and "least restrictive envrironment". RtI's underlining assumption is the opposite - the place for instruction is the general education classroom. Whereas the burden of learning was placed on the child in the traditional LD evaluation (discrepancy), the burden under RtI rests on the "educational system" to provide interventions to keep the child in the general classroom without a special education label (Itkonen/Jahnukainen 2010; Jahnukainen/Itkonen 2016).

RtI is a concept that emerged empirically (e.g. Fuchs/Fuchs 2005), but is not used as a term in special or general educational legislation in the U.S. However, the 2004 reauthorized IDEA permits a local education agency to use up to $15 \%$ of federal funds to develop and implement early intervention services to students "who are not currently identified as needing special education or related services, but who need additional academic and behavioural support to succeed in a general education environment" (20 U.S.C. $\$ 1413[\mathrm{f}][\mathrm{p} 1])$.

The U.S. Department of Education does not subscribe to a particular framework of RtI but acknowledges that the core characteristics include research-based instruction in the general education setting, continuous monitoring of student progress, early screening for academic and behavioural problems, and multiple tiers of intervention that provide progressively more intensive support.

An indication that the U.S. as a whole is moving away from a discrepancy model (which compares a student's potential with actual performance) in diagnosing a specific learning disability was articulated in IDEA 2004, where it stated that states could use science-based interventions as part of the evaluation procedure to determine if the student responded to interventions or needed special education services. The guidelines given to the states stated that the criterion for a learning disability (a) must not require the use of discrepancy between intellectual ability and performance; (b) must permit the use of response to science-based interventions; and (c) may allow the use of alternative research-based procedures to determine if a child has LD (IDEA, 20 U.S.C. $\$ 1414$ (b)(6)(A)).

However, the Office of Special Education Programs of the U.S. Department of Education has since issued a memo (Musgrove 2011) to the states to remind them that tiered models cannot be used to delay or deny special education evaluations under IDEA. Specifically, parents can continue to request a special education evaluation at any time, and local schools cannot deny or delay providing it using RtI strategies as a reason. Similarly, all due process protections for students and parents under IDEA (e.g. consent for evaluation, eligibility, and individualized education program decisions) supersede local school district or state RtI policies.

All fifty states have adopted an RtI method for identifying LD in accordance with the federal regulations described earlier (Coomer 2015; Schultz/Stephens 2019). Out of these, eleven states (22\%) allow only RtI as a method to diagnose LD. Twenty-four states (48\%) allow school-based evaluation teams to use RtI or the severe discrepancy model. Twelve states (24\%) allow RtI or a pattern of strengths and weaknesses model for identifying LD. Three states (Alabama, Arkansas, and California) allow school-based evaluation teams to use any of the three LD identification models (discrepancy, RtI, or strengths and weaknesses assessment). Table 1 summarizes these data. 
Table 1: Diagnostic models by state

\begin{tabular}{lll}
\hline LD Diagnosis Model as Adopted by the State & Number of States & \% of States \\
\hline Only Rtl & 11 & 22 \\
\hline School-level choice: Rtl or Discrepancy & 24 & 48 \\
\hline $\begin{array}{l}\text { School-level choice: Rtl or Ts strength/weakness } \\
\text { pattern }\end{array}$ & 12 & 24 \\
\hline $\begin{array}{l}\text { School-level choice: Any of the three (discrepancy, } \\
\text { Rtl, or strengths and weaknesses assessment) }\end{array}$ & $\begin{array}{l}3 \text { (Alabama, Arkansas, } \\
\text { and California) }\end{array}$ & \\
\hline
\end{tabular}

Source: Adapted from Jahnukainen/Itkonen 2016.

\section{Comparing the Tiered Models}

Students with learning disabilities have been the main groups receiving special educational services in both countries. In the U.S., this student group comprises $34 \%$ of all students in special education (National Center for Education Statistics 2019). In Finland, statistics based on grounds of special education decisions have not been gathered since the law reform of 2011. However, it can be assumed that the main reasons have remained the same (OSF 2010).

The definitions are slightly different. In the U.S., the IDEA defines a learning disability as "a disorder in one or more of the basic psychological processes involved in understanding or in using language, spoken or written, that may manifest itself in the imperfect ability to listen, think, speak, read, write, spell, or to do mathematical calculations." In Finland, learning disabilities were split (2001-2010) into different categories based on their origin, e.g. learning difficulties caused by impaired linguistic development (dysphasia) or learning difficulties related to autism or the Asperger's syndrome or specific learning difficulties based on the subject area e.g. learning difficulty in mathematics, learning difficulty in foreign languages or reading or writing disorder (OSF 2019). However, this kind of data is currently not gathered anymore.

In the Finnish system, all students might have received support without a diagnosis by decisions made on pedagogical groundings in student welfare groups at school level even before the 2011 reform. We have characterised the Finnish system as a "difficulty model" and the U.S as "disability model" (see Itkonen/Jahnukainen 2010). However, if the RtI model is used in the U.S., it allows similarly low threshold services without "wait to fail" as in Finland, at the Tier 2 level. Tier 2 is also the typical level of additional support in terms of learning difficulties in the area of reading, writing and mathematics in both countries. As we have shown earlier (Itkonen/Jahnukainen 2007), the services in the Finnish system concentrate on early intervention and there is a striking difference compared to the share of students served for LD in secondary education.

The special feature of the Finnish special support system is still the extensive use of Tier 2-level part-time special education (Table 2; see also Jahnukainen/Itkonen 2016). The key player in this low threshold system of meeting the diverse population is a special teacher, whose workload is not dedicated to a small group of students only. Instead, s/he is dealing with the whole school population, doing assessment, co-teaching, teaching small groups and consulting the classroom teachers (e.g. Rytivaara et al. 2012). Basically, there is at least one 
of this kind of teacher available in every school. During the school year 2016-2017, about 120,000 (or 21.8 per cent) comprehensive school students (age group 6 to 16) were taking advantage of this service (OSF 2018). This service simultaneously delivers preventive as well as rehabilitative functions. In practice, this means that as soon as the classroom teacher or the parents notice that some additional support is needed, the possible intervention can be started. This form of special education is basically open for any student who struggles with learning for whatever reason. In particular, there is considerable pressure to rehabilitate early reading, writing and arithmetic problems using part-time special education.

Table 2: Overview of tiered intervention models in the United States and in Finland

\begin{tabular}{lll}
\hline & US model & FIN model \\
\hline Program & Response to Intervention & Learning and schooling support \\
\hline Implementation status & Several models & One national model \\
\hline Legal status & $\begin{array}{l}\text { Must be permitted in states' identification } \\
\text { methods of LD since 2004 }\end{array}$ & Mandatory since 2011 \\
\hline Tier 1 & Universal interventions & General support \\
\hline Tier 2 & Targeted interventions & Intensified support \\
\hline Tier 3 & Intensive interventions & Special support \\
\hline
\end{tabular}

Source: Adapted from Jahnukainen/Itkonen 2016.

The first tier in both models focuses on offering quality instruction for everybody and meeting the so-called "normal diversities" (see Table 3). The U.S. model focuses more heavily on evidence-based practices and scientifically validated curriculum. This difference might be explained by the different basic educational standards in these countries. In Finland, the variation between schools in terms of quality of instruction and the curriculum expectations is very narrow (Sahlberg 2015). Also, teacher education is rather similar and based on the same standards in all Finnish universities.

Table 3: Features of the Tier 1 intervention in the United States and in Finland

\begin{tabular}{lll}
\hline Tier $\mathbf{1}$ & US & FIN \\
\hline Target group & All students & All students \\
\hline Interventions & Evidence-based practices to all students & Quality instruction \\
& Scientifically validated core curricula & Differentiation, additional support \\
\hline Key professional & General education teachers & $\begin{array}{l}\text { General education teachers/Special } \\
\text { education teacher }\end{array}$ \\
\hline
\end{tabular}

Source: Jahnukainen/Itkonen 2016.

In both models, Tier 2 focuses on "at-risk students," for whom the general classroom or Tier 1 primary instruction is not sufficient. These students need "occasional and perhaps relatively short-term additional instruction or curriculum adaptations to meet their needs" (Vaughn/Denton 2008: 52) (see Table 4). Students with SLD (reading, writing, mathematics) receive services mainly at this Tier 2 level in the Finnish system (Thuneberg/Vainikainen 2015). Only students with severe language delay or combined with other disabilities will be guided to Tier 3 . The Tier 2 target group is estimated to be more than 20 per cent of all students. If taking into account all students served yearly by the part-time special education, 
this seems to be a quite correct estimation based on the past Finnish experiences (e.g. OFS 2018). At Tier 2 level the specialist also plays an important role in the implementation of the more targeted interventions in more traditional small groups (resource rooms) or in coteaching. The special educator on this level needs to be a kind of jack-of all trades who is able to work with different kinds of students with a variety of special needs.

Table 4. Features of the Tier 2 intervention in the United States and in Finland

\begin{tabular}{lll}
\hline Tier 2 & US & FIN \\
\hline $\begin{array}{l}\text { Target group } \\
\text { (Estimated) size }\end{array}$ & $\begin{array}{l}\text { At-risk student, not successful in Tier 1 } \\
\text { Also, students who have been evaluated and } \\
\text { qualify for special education under IDEA, but } \\
\text { whose individualized education program has } \\
\text { minimal support needs regarding goals, } \\
\text { services, accommodations. }\end{array}$ & $\begin{array}{l}\text { At-risk students with mild learning } \\
\text { and behavioral special needs }\end{array}$ \\
& $\begin{array}{l}\text { 20-30\% of the age group } \\
\text { 25-40\% of the age group }\end{array}$ & \\
\hline Interventions & $\begin{array}{l}\text { Individualised and/or small group support, in } \\
\text { general classrooms or pull-out service }\end{array}$ & $\begin{array}{l}\text { Targeted short term individualised } \\
\text { and/or small group instruction, } \\
\text { co-teaching or pull-out service }\end{array}$ \\
\hline Key professional & $\begin{array}{l}\text { Specialist (e.g. reading specialist) and } \\
\text { other designated personnel as decided } \\
\text { locally } \\
\text { Special education teacher will be a case } \\
\text { manager (working with classroom } \\
\text { teachers) if the student is part of special } \\
\text { education }\end{array}$ & $\begin{array}{l}\text { Special teacher in co-operation with } \\
\text { classroom teacher }\end{array}$ \\
& \begin{tabular}{l} 
\\
\hline
\end{tabular} & \\
\hline
\end{tabular}

Source: Jahnukainen/Itkonen 2016.

Finally, Tier 3 in both countries consists of students who require more individualised, more intensive support. Table 5 depicts the two countries' systems.

Table 5: Features of the Tier 3 intervention in the United States and in Finland

\begin{tabular}{lll}
\hline Tier $\mathbf{3}$ & US & FIN \\
\hline $\begin{array}{l}\text { Target group } \\
\text { (Estimated) size }\end{array}$ & $\begin{array}{l}\text { Students who require extensive amount } \\
\text { of time and intensity to reach desired } \\
\text { levels of proficiency; Students who } \\
\text { qualify under IDEA for special education } \\
\text { 4-6\% of the age group }\end{array}$ & $\begin{array}{l}\text { Students with significant continuing } \\
\text { need(s) for special support } \\
6-7 \% \text { of the age group }\end{array}$ \\
\hline Interventions & Special education, as well as other long- & Inclusive or special education based \\
& term services for students who are not & on IEP. \\
& eligible for special education under IDEA & General curricula can be \\
& & individualised \\
\hline Key professional & Highly qualified teacher & Special teacher \\
\hline Additional & EA, classroom teacher & EA, classroom teacher \\
professional & Problem solving teams & Student welfare groups \\
\hline
\end{tabular}

Source: Adapted from Jahnukainen/Itkonen 2016. 


\section{Policy Implications for Teacher Education}

The Finnish "Education for All"-approach has developed during the decades and currently every student is served in the same comprehensive school system. Finnish public school is well known for its high equity and narrow standard deviation in terms of student performance. One key ingredient of equity is the broad variety of additional supports delivered under the Learning and Schooling Support system. Special teachers play an important role in this system as consultants, co-teachers and educators specialized in learning and behaviour support and student welfare. Every school in Finland has at least one special teacher working with the whole student population.

In Finland, special educators with this kind of profile have been trained since 1977 (Hautamäki et al. 1996), instead of traditional special teachers focusing on more specific disability groups. They are mostly responsible for organizing the Tier 2 level but participate also in organizing and consulting the actions in other levels. In teacher education, these special teachers do have their own route; however, the collaboration with other teacher students starts during undergraduate studies sharing the same basic studies in educational science. The inservice training is an important part of the professional development of these teachers. Also, the successful implementation of large-scale reforms calls for extensive in-service training offered to every teacher.

As U.S. states have similarly adopted RtI policies, the use of a tiered model obviously places new demands for teachers, and institutions that train them. The remainder of the section examines California as a U.S. case study for a state-wide teacher education innovation. States often function as "policy laboratories" for federal policies, as federal policies often grow from state initiatives (Manna 2007). In special education policy, California was the first state (1992) to require functional behavioural assessments and positive behavioural interventions for students who exhibited challenging behaviours. IDEA included such language in its 1997 reauthorization.

According to a policy memo from 2018, the California State Commission for Teacher Credentialing will be restructuring its elementary and special education teacher licensing. This means that universities, which provide such credentials, must rethink their teacher education programs. All teacher candidates in California will soon receive the same "trunk" of methods and foundations, after which specialization will occur. According to the new requirements, everyone receives a shared training on core curriculum, classroom management, and foundations. The idea is also that co-teaching and collaboration are infused in the training. After a shared core training, specialization on "mild to moderate support needs" will then occur. Other special education teacher licenses will include extensive support needs, deaf and hard of hearing, visual impairments, and early childhood special education. It is beyond the scope of this chapter to discuss whether these teacher education reforms are a response to the tiered models already being implemented in school districts across California, or are a result of another event (e.g. an expected teacher retirement wave in the state). 


\section{Conclusion}

This chapter has shown that both countries have adopted a tiered model for serving and/or diagnosing students who require more academic or behavioural support than what the general curriculum and classroom teaching provide.

In the United States, there is state-by-state variation in how a tiered model of support is implemented. This chapter focused on the use of RtI as a mechanism to diagnose students with LD. Hence, the tiered intervention concept did not override the medical model -children still need a diagnosis in order to receive special education. The ability for school-level teams to consider an intervention-based diagnosis in all 50 states however, is a move away from the "wait to fail" approach, which was a criticism of the discrepancy model (Itkonen/Jahnukainen 2010). It was beyond the scope of this chapter to discuss the implementation of RtI at the school level, for example, the fidelity of the intervention models; or their effect in states' LD prevalences. Also, beyond the scope here were the interactions between diagnoses using tiered models of intervention and the due process rights that IDEA guarantees for families (e.g., due process protections to disagree with a child's evaluation or individualized education program). Thus, what appears to be the case in the U.S. is that it may, as a nation, implement a parallel system. IDEA has its eligibility evaluation requirements on one hand, and entitlements on the other (families' right to request an evaluation or challenge the school district in instances of disagreement), both of which trump intervention-based diagnostic and service efforts (Musgrove 2011). IDEA is headed toward a regular reauthorization process sooner than later. It remains to be seen whether (and how) tiered supports will be further reflected in legislative special education language.

In the Finnish context, the implementation of the learning and schooling support has succeeded well, at least at the system level, and the latest statistics show a development towards more inclusive settings. However, the recent national evaluation investigating the actualized educational support system (Vainikainen et al. 2018) found regional differences in operational models of the tiered system. This is related to the fact that the Finnish legislation is rather loose, as it gives a lot of flexibility to municipalities to choose the actual ways to organise the support at the school level. Because part-time special education, in particular at the Tier 2 level, plays an important role, the reported lack of resources and professionals is an alarming finding, which needs to be solved by offering more funding for this purpose from both state and municipality sources.

In light of the international discourse on inclusion (see Richardson/Powell 2011) and our findings on the challenges in implementing Tier 2, a tiered system presents both possibilities and challenges. A tiered model works insofar as the people implementing it have the strategies, willingness, and both individual and systemic capacities to provide high-quality instruction and to determine when students' movement between tiers is required (Lipsky 2010; Wilson 1989). By systemic capacity we refer to, for example, a school-wide approach where there are enough resources (e.g., staff, knowledge) that are utilized creatively to meet the needs of all students.

Finally, time will tell the relative success of tiered systems. The two countries compared here represent very different special education structures and disability constructions. A comparative, historical-institutional perspective can provide a deeper understanding of the efficacy and fidelity of tiered systems. U.S. special education originated in courts to remedy the lack of access to public schooling, and to provide individualized supports for those with 
invisible disabilities who had access to schools but were left to their own devices. Thus, the development of special and inclusive education in the U.S. continues to move between civil rights, educational and medical frameworks. Eligibility determination continues to be required in order to receive services. In contrast, Finnish special and inclusive education originated from educator initiatives (Pesonen et al. 2015; Thuneberg et al. 2014). Tiered supports in general education context were provided inclusively as "best practice" even before legislation formalized them (Sahlberg 2015). Therefore, implementing a tiered model of supports in Finland does not require the un-doing of previously institutionalized structures. More research is needed on institutional and organizational structures and their effects on the outcomes of tiered systems. For example: Is the formalization in the Finnish context helping or hindering local support provisions by establishing implementation procedures and requirements? Such research would help school districts across countries and regions in planning and delivering education that reaches all learners.

\section{References}

Amendment of the Basic Education Act 642/2010 (2010): Helsinki: Government Printing Office.

California Commission on Teacher Credentialing (2018): Commission adoption of preliminary Education Specialist program standards and teaching performance expectations. https://www. ctc.ca.gov/docs/default-source/educator-prep/ps-alerts/2018/psa-18-03.pdf?sfvrsn=b69450b1 6 [Accessed: 02.08.2018].

Chong, Pei Wen (2016): The Finnish "Recipe" Towards Inclusion. Concocting Educational Equity, Policy Rigour, and Proactive Support Structures. In: Scandinavian Journal of Educational Research 62, 11, pp. 1-18.

Coomer, Lauren Faith (2015): Definitions and Criteria Used by State Education Departments for Identifying Specific Learning Disabilities. MA Thesis Western Kentucky: Western Kentucky University/Department of Psychology. https://digitalcommons.wku.edu/theses/1515 [Accessed:15.02.2019].

Fuchs, Douglas/Fuchs, Lynn. S. (2005): Responsiveness-to-Intervention: A blueprint for practitioners, policymakers and parens. In: Teaching Exceptional Children 38, 1, pp. 57-61.

Fuchs, Douglas/Fuchs, Lynn S./Vaughn, Sharon. (Eds.) (2008): Response to intervention: A framework for reading educators. Newark, DE: International Reading Association.

Graham, Linda/Jahnukainen, Markku (2011): Wherefore Art Thou, Inclusion? Analysing the Development of Inclusive Education in New South Wales, Alberta and Finland. In: Journal of Education Policy 26, 2, pp. 263-288.

Hautamäki, Jarkko/ Kuusela, Jorma/Mänty, Tarja (1996): Erityisopettajien Suomessa 1960-1994 (Special Education Teacher Graduation in Finland 1960-1994). In: Blom, Heikki/Laukkanen, Reijo/Lindström, Aslak/Saresma, Ulla/Virtanen, Pirkko (eds.): Erityisopetuksen Tila (The Current State of Special Education). Helsinki: National Board of Education, pp. 75-80.

Honkasilta, Juho/Ahtiainen, Raisa/Hienonen, Ninja/Jahnukainen, Markku (2019): Inclusive and Special Education and the Question of Equity in Education. The Case of Finland. In: Schuelka, Matthew J./Johnstone, Christopher J./ Thomas, Gary/Artiles, Alfredo J. (eds.): The SAGE Handbook of Inclusion and Diversity in Education. Thousand Oaks, CA: Sage, pp. 481- 495.

Individuals with Disabilities Education Improvement Act of 2004 (IDEA): Pub.L.No.108-446, 118 Stat. 2647 (2004). 
Itkonen, Tiina/Jahnukainen, Markku (2007): An Analysis of Accountability Policies in Finland and the United States. In: International Journal of Disability, Development and Education 54, 1, pp. 5-23.

Itkonen, Tiina/Jahnukainen, Markku (2010): Disability or Learning Difficulty? Politicians or Educators? Constructing Special Education in Finland and the United States. In: Comparative Sociology 25, pp. 182-201.

Jahnukainen, Markku (2011): Different Strategies, Different Outcomes? The History and Trends of the Inclusive and Special Education in Alberta (Canada) and in Finland. In: Scandinavian Journal of Educational Research 55, 5, pp. 489-502.

Jahnukainen, Markku/Itkonen, Tiina (2016): Tiered Intervention. History and Trends in Finland and the United States. In: European Journal of Special Needs Education 31, 1, pp. 140-150.

Lien Foundation (2018): Inclusion matters database. http://inclusionmatters.sg [Accessed: 21.05. 2019].

Lipsky, Michael (2010): Street-Level Bureaucracy: Dilemmas of the Individual in Public Services. New York: The Russell Sage Foundation.

Manna, Paul (2007): School's In: Federalism and the National Education Agenda. Washington, DC: Georgetown University Press.

Musgrove, Melody (2011): A Response-to-Intervention (RTI) Process Cannot be Used to DelayDeny an Evaluation for Eligibility under the Individuals with Disabilities Education Act (IDEA). United States Department of Education, Office of Special Education Programs (Memo 11-07). https://www2.ed.gov/policy/speced/guid/idea/memosdcltrs/osep11-07rtimemo.pdf [Accessed: 10.12.2018].

National Center for Education Statistics (2019): Children and Youth with Disabilities. https://nces. ed.gov/programs/coe/indicator_cgg.asp [Accessed: 23.052019].

Official Statistics of Finland (OSF) (2018): Special Education. http://www.stat.fi/til/erop/index en.html [Accessed: 29.05.2019].

Official Statistics of Finland (OSF) (2019): Concepts and definitions. Helsinki: Statistics Finland http://www.stat.fi/til/erop/kas_en.html [Accessed: 26.11.2019]

Pesonen, Henri/ Itkonen, Tiina/Jahnukainen, Markku/Kontu, Elina/Kokko, Tiina/Ojala, Terhi/ Pirttimaa, Raija (2015). The Implementation of New Special Education Legislation in Finland. In: Educational Policy 29, 1, pp. 162-178.

Pulkkinen, Jonna (2019): Reforming policy, changing practices? Special education in Finland after educational reforms. Jyväskylä: Jyväskylä University Press.

Pulkkinen Jonna/Jahnukainen, Markku (2016): Finnish Reform of the Funding and Provision of Special Education. The Views of Principals and Municipal Education Administrators. In: Educational Review 68, 2, pp. 171-188.

Richardson, John, G./Powell, Justin J.W. (2011): Comparing Special Education: Origins to Contemporary Paradoxes. Stanford, CA: Stanford University Press.

Rytivaara, Anna/Pulkkinen, Jonna/Takala, Marjatta (2012): Erityisopettajan Työ. Opettamista Yksin ja Yhdessä (The Work of a Special Teacher. Teaching Alone and Together). In: Jahnukainen, Markku (ed.) (2012): Lasten Erityishuolto ja - Opetus Suomessa (The special care and education in Finland). Tampere: Vastapaino, pp. 333-352.

Sahlberg, Pasi (2015): Finnish Lessons 2.0. What Can the World Learn from Educational Change in Finland. New York: Teachers College Press.

Schultz, Edward K./Stephens, Tammy L. (2019): SLD Identification. An Analysis of State Policies. https://www.advocacyinstitute.org/resources/SLD.Identification.State.Policy.shtml [Accessed: 15.12 .2018$]$.

Takayama, Keita/Waldow, Florian/Sung, Youl-Kwan (2013): Finland has it All? Examining the Media Accentuation of ,Finnish Education“ in Australia, Germany and South Korea. In: Journal in Comparative and International Education 8, 3, pp. 307-325. 
The Editorial Board (2013): Why Other Countries Teach Better? New York: The New York Times. https://www.nytimes.com/2013/12/18/opinion/why-students-do-better-overseas.html [Accessed: 21.05.2019].

Thuneberg, Helena/Hautamäki, Jarkko/Ahtiainen, Raisa/Lintuvuori, Meri/Vainikainen, MariPauliina/Hilasvuori, Touko (2014): Conceptual Change in Adopting the Nationwide Special Education Strategy in Finland. In: Journal of Educational Change 15, 1, pp. 37-56.

Thuneberg, Helena/Vainikainen, Mari-Pauliina (2015): Uuden Lain Toteutuminen Pedagogisten Dokumenttien Perusteella. In: Jahnukainen, Markku/Kontu, Elina/Thuneberg, Helena/Vainikainen Mari-Pauliina (eds.) (2015): Erityisopetuksesta Oppimisen ja Koulunkäynnin Tukeen. Helsinki: Suomen Kasvatustieteellinen Seura Fera, pp. 135-162.

Tomlinson, Sally (2012): Ignorant Yobs? Low Attainers in a Global Knowledge Economy. London: Routledge.

Vainikainen, Mari-Pauliina/Lintuvuori, Meri/Paananen, Maiju/Eskelinen, Mervi/Kirjavainen, Tanja/Hienonen, Ninja/Jahnukainen, Markku/Thuneberg, Helena/Asikainen, Mikko/Suhonen, Eira/Alijoki, Alisa/Sajaniemi, Nina/Reunamo, Jyrki/Hotulainen, Risto (2018): Oppimisen tuki varhaislapsuudesta toisen asteen siirtymään: tasa-arvon toteutuminen ja kehittämistarpeet. (Educational support from early childhood to the transition to upper secondary education: actualisation of equity and needs for development). Publication of the Government's analysis, assessment and research activities 55/2018. Helsinki.

Vaughn, Sharon/Denton, Carolyn A. (2008): The Role of Intervention. In: Fuchs, Douglas/Fuchs, Lynn/Vaughn, Sharon (eds.) (2008): Response to Intervention. A Framework for Reading Educators. Newark, DE: International Reading Association, pp. 51-70.

Wilson, James Q. (1989): Bureaucracy: What government organizations do and why they do it. New York: Basic Books. 


\title{
20 Heterogene Lerngruppen in Europa inklusionsorientiert unterrichten: Inspirierende Praktiken und Erkenntnisse aus Deutschland, Island, Litauen, Luxemburg, Schweden und Spanien
}

\author{
Justin J.W. Powell, Kerstin Merz-Atalik, Stefania Ališauskienè, Michelle Brendel, \\ Geraldo Echeita, Hafdis Guðjónsdóttir, Jóhanna Karlsdóttir, Lina Miltenienè, \\ Rita Melienè, Edda Óskarsdóttir, Bengt Persson, Elisabeth Persson, Cecilia Simón, \\ Marta Sandoval, Anneli Schwartz, Heike Tiemann, Katja Weber ${ }^{1}$
}

\begin{abstract}
Inklusive Bildung ist zu einem globalen Ziel geworden. Dieses Ziel wird durch die Anerkennung des Menschenrechts auf Bildung für alle und die Vision einer demokratischen Gesellschaft unterstützt, die Vielfalt in all ihren Facetten wertschätzt. Die Förderung angesichts der Vielfalt der Schüler*innen mit dem Ziel der Verbesserung des Lernens aller, bleibt für Lehrer*innen in allen nationalen Kontexten eine herausfordernde Aufgabe, da sowohl die gemeinsame Bildung für alle ausgeweitet als auch inklusive Bildung universell werden soll. Die erfolgreiche Unterstützung verschiedener Schüler*innen in ihren Lernprozessen gilt seit Jahrzehnten als das Herzstück einer ausgezeichneten Pädagogik. Dennoch finden wir in ganz Europa signifikante, persistente Unterschiede, sowohl hinsichtlich des Umfangs als auch der Qualität der inklusiven Schulbildung, die auf einer Reihe von institutionalisierten Strukturen und Kulturen beruhen und sich unter anderem in organisatorischen Rahmenbedingungen und Lehrmethoden heterogener Bildungssysteme niederschlagen. Aufbauend auf einer dreijährigen Zusammenarbeit im Rahmen des von der Europäischen Kommission finanzierten Comenius-Netzwerkprojekts „Teaching Diverse Learners in School Subjects“ (TdiverS), werden in diesem Beitrag Erkenntnisse über ,inspirierende Praktiken“ der inklusiven Bildung, die in den sechs teilnehmenden Ländern - Deutschland, Island, Litauen, Luxemburg, Schweden und Spanien - gefunden wurden, zusammengefasst. Trotz erheblicher Unterschiede in den Bildungssystemen in Europa sind überall inklusive Bildungspraktiken zu finden. Bei den Schulhospitationen fanden wir inspirierende Inklusionspraktiken in allen Ländern, auch wenn diese durchaus sehr differente Niveaus der (inklusiven) Bildungssysteme aufweisen.
\end{abstract}

1 Wir danken Simone Tiegs (Studierende und Tutorin an der PH Ludwigsburg) für die aus dem Englischen übersetzte Vorlage. Eine englischsprachige Version des Beitrags erschien 2019 (Powell et al. 2019). 


\section{Einleitung: Inklusive Bildungswerte von einer globalen Norm zu lokalen Realitäten}

Inklusive Bildung ist zu einem globalen Ziel geworden. Dieses Ziel wird durch die Anerkennung des Menschenrechts auf Bildung für alle und die Vision einer demokratischen Gesellschaft unterstützt, die Vielfalt in all ihren Facetten wertschätzt. Die Förderung angesichts der Vielfalt der Schüler*innen mit dem Ziel der Verbesserung des Lernens aller, bleibt für Lehrer*innen in allen nationalen Kontexten eine herausfordernde Aufgabe, da sowohl die gemeinsame Bildung für alle ausgeweitet, als auch inklusive Bildung universell werden soll. Die erfolgreiche Unterstützung verschiedener Schüler*innen in ihren Lernprozessen gilt seit Jahrzehnten als das Herzstück einer ausgezeichneten Pädagogik. Dennoch finden wir in ganz Europa signifikante, persistente Unterschiede sowohl hinsichtlich des Umfangs als auch der Qualität der inklusiven Schulbildung, die auf einer Reihe von institutionalisierten Strukturen und Kulturen beruhen und sich unter anderem in organisatorischen Rahmenbedingungen und Lehrmethoden heterogener Bildungssysteme niederschlagen (Europäische Kommission 2017). Die Förderung der Zusammenarbeit von Wissenschaftler*innen, politischen Entscheidungsträger*innen und Praktiker*innen an Orten mit unterschiedlichen Traditionen in (mehr oder weniger) inklusiven Bildungssystemen ist sowohl eine Schlüsselaufgabe für vergleichende Forscher*innen als auch ein Kernziel für europäische Entscheidungsträger*innen in der Bildung.

Aufbauend auf einer dreijährigen Zusammenarbeit im Rahmen des von der Europäischen Kommission finanzierten Comenius-Netzwerkprojekts „Teaching Diverse Learners in School Subjects“ (TdiverS) werden in diesem Beitrag Erkenntnisse über ,inspirierende Praktiken“ der inklusiven Bildung, die in den sechs teilnehmenden Ländern - Deutschland, Island, Litauen, Luxemburg, Schweden und Spanien - gefunden wurden, zusammengefasst.

Das Projekt konzentrierte sich auf aktuelle Praktiken der Organisation und des Unterrichts von heterogenen Lernenden und war bemüht, dabei alle in den Reformprozess an den Schulen involvierten Akteur*innen einzubeziehen. Dem Projekt wurden drei Säulen der Vielfalt zugrunde gelegt (Projektjahresberichte TdiverS, 2015, 2016, 2017). Die erste Säule umfasst die Vielfalt an theoretischem und praktischem Wissen, die unter anderem aus der Zusammenarbeit von Praktiker*innen und Wissenschaftler*innen resultiert. Ziel war es, sich über das vorhandene Wissen und die Erfahrungen bezüglich eines Fachunterrichts in inklusiven Settings aus verschiedenen Perspektiven auszutauschen. Mit der zweiten Säule stärkte das Projekt das Bewusstsein für die internationale und nationale Vielfalt in Bezug auf grundlegende Strukturen, Rahmenbedingungen und bestimmende Faktoren des inklusiven Unterrichtens in den sechs beteiligten Ländern in Nord-, Ost-, Süd- und Westeuropa. Die dritte Säule beruhte auf der Erforschung diverser Konzepte der inklusiven Bildung, um von den multikulturellen und multidisziplinären Perspektiven auf verschiedenen Ebenen zu profitieren. Der ursprünglich von den deutschen Antragstellerinnen avisierte Fokus auf die Fachdidaktiken der Unterrichtsfächer, musste nach zahlreichen Diskussionen im Konsortium erweitert werden: so schien dies eher eine deutsche Perspektive und in den stärker am Klassenlehrerprinzip und Gemeinschaftsschulsystem orientierten Partnerländern, hat man von den traditionellen Fachstrukturen der Unterrichtsorganisation im Schulalltag eher Abstand genommen (z.B. durch fächerübergreifendes Lernen oder durch eine Orientierung an den zu den Fächern quer liegenden Schlüsselkompetenzen; z.B. in Anlehnung an den Index für Inklu- 
sion, Booth/Ainscow 2017, Ainscow 2016). In diesem Beitrag sollen die Werte der inklusiven Bildung hervorgehoben, die aktuelle Situation der inklusiven Einstellungen und Praktiken in den verschiedenen Regionen Europas dokumentiert und die gegenwärtigen Tendenzen und Trends in den Bildungsreformen und bei der Implementierung inklusiver Praktiken zusammengefasst sowie die Ergebnisse des TdiverS-Projekts diskutiert werden. Diese bestehen hauptsächlich aus einer Sammlung von Videos mit inspirierenden Praktiken und Erkenntnissen sowie englischsprachigen Übersetzungen zugehöriger Originaltexte.

Dem Projekt wurde durch die weltweite Ratifizierung der UN-Konvention über die Rechte von Menschen mit Behinderungen (UN-BRK) Relevanz verliehen. In dieser Menschenrechtscharta (in Artikel 24) wird das Recht auf lebenslange, inklusive Bildung eindeutig definiert. Somit stehen alle Schulen und Bildungssysteme in Europa vor der Herausforderung inklusive Kulturen, inklusive Strukturen und inklusive Praktiken zu entwickeln (Ainscow et al. 2006). Darüber hinaus definiert die Agenda 2030 für nachhaltige Entwicklung als Ziel 4 die „Gewährleistung einer inklusiven und qualitativ hochwertigen Bildung für alle und die Förderung des lebenslangen Lernens" (UN 2015). Am 16. Oktober 2015 fand im Rahmen des luxemburgischen Ratsvorsitzes der EU eine europäische Konferenz mit dem Titel „Inclusive Education - Take Action!" vom luxemburgischen Ministerium für Bildung, Kinder und Jugend (MENJE) zusammen mit der Europäischen Agentur für sonderpädagogische Förderung und inklusive Bildung (EASNIE) statt. 72 junge Menschen mit unterschiedlichen Fähigkeiten aus 28 Ländern nahmen an dieser Veranstaltung teil und erarbeiteten übereinstimmend fünf Kernbotschaften: (1) „Alles über uns, mit uns“: Dies bedeutet, dass junge Menschen direkt in alle Entscheidungen eingebunden werden sollten, die sie betreffen; (2) barrierefreie Schulen - physische und technische Barrieren sollten beseitigt werden; (3) Klischees müssen abgebaut werden, und das Konzept der „Normalität“ muss kritisch reflektiert werden; (4) „Vielfalt ist die Mischung, Inklusion macht die Mischung möglich“; und 5) vollwertige Bürger*innen zu werden bezieht sich auf die Wirkung von inklusiver Bildung im Hinblick auf die vollständige Eingliederung in die Gesellschaft (EASNIE 2015).

Eingebettet in institutionalisierte Bildungssysteme hängen solche grundlegenden Reformprozesse zur Weiterentwicklung inklusiver Bildungssysteme von den Strategien und Praktiken ab, auf die sich Lehrkräfte in regulären Klassenzimmern einlassen und die sie im Hinblick auf das Eröffnen von Lernmöglichkeiten innerhalb dieser Klassen, die aus einer Vielfalt von Lernenden bestehen, anwenden (Prengel 2016). Die drei Hauptelemente, die diese Forschung und unsere interkulturelle Zusammenarbeit vorangetrieben haben, waren:

(1) Verknüpfung von Theorie und Praxis, mit der expliziten Absicht, theoretisch begründete Reflexionen von „inspirierenden Praktiken“ der Inklusion im (u.a. im Fachunterricht) bereitzustellen. Dazu sollten Erfahrungen (z.B. bezüglich eines Fachunterrichts) in inklusiven Klassenzimmern gesammelt werden und ein Dialog von Lehrpersonen und anderen im Bildungsbereich tätigen, oder damit in Verbindung stehenden Personen auf nationaler und internationaler Ebene ermöglicht werden (um dazu beizutragen, die Kluft zwischen Theorie und Praxis zu überbrücken);

(2) Neue Perspektiven zu eröffnen, indem Forschungsergebnisse und konkrete Erfahrungen mit inklusiver Bildung (im Fachunterricht) sowie Reformen der Lehrer*innenbildung im Zusammenhang mit inklusiver Bildung unter den verschiedenen Ländern ausgetauscht werden; und

(3) sowohl gemeinsame Konzepte für die Lehrer*innenbildung (für inklusive Bildung), als auch Wissen über spezifische Themen und die Ermöglichung von inklusiver Lehrer*innenbildung zu reflektieren und $\mathrm{zu}$ etablieren. Die Materialien finden sich in einer 
Sammlung von Videos auf einem weltweit kostenlos vertriebenem USB-Flash-Laufwerk (siehe http://www.tdivers.eu/).

In Bezug auf die Werte lag der Schwerpunkt auf der Wertschätzung der Vielfalt der Lernenden - wobei diese als eine Ressource und ein Vorteil für Lernprozesse betrachtet wird - und auf Unterstützungsmaßnahmen, die sicherstellen, dass alle Schüler*innen gemeinsam lernen können und dass die Lehrer*innen hohe Erwartungen an alle Lernenden haben (Hart et al. 2004; European Agency 2012). Auf der Basis der länderübergreifenden Forschung wurden Einstellungen und Erfahrungen von Schüler*innen, Lehrer*innen und Schulleiter*innen, sowie Beispiele für effektive Lehransätze in heterogenen Klassenzimmern in verschiedenen Schulfächern gesammelt, die als Inspiration für Lehrkräfte an anderen Schulen, gemäß dem ein Schlüsselmotiv für länderübergreifende vergleichende Studien zur (inklusiven) Bildung (z.B. Powell 2014), auch in anderen Ländern dienen können.

\section{Zur Geographie der Inklusion in Europa}

Das TdiverS-Projekt führte zur Erkenntnis, dass trotz erheblicher Unterschiede in den Bildungssystemen in Europa überall inklusive Bildungspraktiken zu finden sind. Bei den Schulhospitationen im Rahmen der Projektmeetings fanden wir inspirierende Inklusionspraktiken in Deutschland, Island, Litauen, Luxemburg, Schweden und Spanien, auch wenn diese Länder durchaus sehr differente Niveaus der (inklusiven) Bildungssysteme aufweisen. Obwohl Europa über viele verschiedene Kulturen und Sprachen verfügt, war der Wunsch nach grenzübergreifender Zusammenarbeit, sei es kulturell, sprachlich, disziplinär oder professionsbezogen, ebenso offensichtlich wie die Notwendigkeit zu kooperieren, um inklusive Praktiken erfolgreich zu verstehen und sie in so unterschiedlichen Kontexten zu ermöglichen, eine wichtige Voraussetzung für Policy- und Praxistransfer. Drei Jahre intensiven Austauschs durch Länderstudien, Schulhospitationen, das Teilen der videographischen Praxisbeispiele sowie durch internationale Konferenzen und Konsortialtreffen, die mindestens zweimal pro Jahr in Europa und einmalig in Nordamerika (Projektmeetings und öffentliche Präsentationen des Projektes) stattfanden - ermöglichten uns Konvergenz und Divergenz in den laufenden Prozessen der Entwicklung einer inklusiveren Schulbildung in ganz Europa besser zu begreifen. Das Verstehen dieses gemeinsamen und unterschiedlichen Verständnisses erforderte Zeit für einen vertieften Dialog und profitierte enorm von der gemeinsamen Erforschung unterschiedlicher Schulen, Systemen und kultureller Kontexte (Artiles et al. 2011; Richardson/Powell 2011). Die Beachtung von verschiedenen Ebenen war von entscheidender Bedeutung, da die in vielen länderübergreifenden Analysen verwendeten, kumulierten Daten auf Länderebene (Makroebene) nicht mit regionalen oder lokalen Erfahrungen und Praktiken sowie der Praxis in Schulen - die sich während der Schulbesuche offenbarten - übereinstimmten, diese jedoch für unsere kulturübergreifende Analyse und Videografie (auf der Meso- und Mikroebene einzelner Schulen und einzelner Klassen) bedeutsam waren.

Die Reflexion der verschiedenen Ebenen der Bildungspolitik und -reformen, deren Analyse, Bewertung und die Aufschlüsselung nationaler Daten, insbesondere in föderalen Systemen, war notwendig (vgl. Schäfer 2021 i.d.B., zu den Maßnahmen inklusiver Bildung in den föderalen Ländern Deutschland und Spanien). Tatsächlich sind einige der Einzelschulstandorte, mit denen wir zusammengearbeitet haben, unter den wenigen Schulen, die sich der 
Inklusion verpflichtet haben, selbst wenn dies der Systemlogik des nationalen oder regionalen Bildungssystems widerspricht, welches überwiegend dauerhaft getrennte oder abgesonderte Bildungsangebote für Schüler*innen (mit festgestelltem besonderem Bildungsbedarf ${ }^{2}$ ) vorsieht (Powell [2011] 2016). Das Erkennen von und Reagieren auf bildungsbedingte und soziale Benachteiligungen - und von Schüler*innen, die in der Schule und/oder beim Übergang ins Erwachsenenalter „,besondere Bildungsbedürfnisse haben“ und „,behindert werden“ - hängt maßgeblich von der Institutionalisierung des Bildungssystems und von den vorhandenen (sonder-)pädagogischen Unterstützungssystemen ab (vgl. Óskarsdóttir/Köpfer 2021 i.d.B.). Obwohl ein generelles Ausschließen von Schüler*innen mit Behinderungen durch die Entwicklung von sonderpädagogischen Bildungsangeboten überwunden wurde, weisen diese dennoch häufig eine Überrepräsentation von Kindern und Jugendlichen auf, die in Armut oder in Familien mit niedrigem sozioökonomischem Status leben; Jungen und ethnische Minderheiten (und Kinder aus Migrantenfamilien aus bestimmten Ländern) sind oft erheblich überrepräsentiert (Tomlinson 2017, 2021 i.d.B.).

Im Vergleich der TdiverS-Partnerländer fanden wir 2014 eine bemerkenswerte Variationsbreite bei den Klassifikationsquoten von Schüler*innen mit SEN (,special educational needs") (gemessen als Prozentsatz der Schüler*innen mit SEN an der Gesamtschüler*innenpopulation), von $0,5 \%$ in Schweden bis zu $16 \%$ in Island (EASNIE 2017a). Die bedeutsame Differenz, selbst in den nordischen Ländern, die wohl zu den inklusivsten Bildungssystemen der Welt zählen, ist bemerkenswert, da sie die sehr unterschiedlichen Ideen in Bezug auf Klassifikations- und Lernendenkategorien aufzeigt (Biermann/Powell 2014). Zusätzlich unterscheiden sich die erhobenen Statistiken der einzelnen TdiverS Länder auch nach Klassifizierungskategorien, -verfahren und Berichterstattungen an die European Agency for Special Needs and Inclusive Education (EASNIE), eine von der EU finanzierte Organisation, die erheblich zur Entwicklung vergleichender Studien und Entwicklungsprojekte für die Zusammenarbeit bei SEN/besonderen Bedürfnissen und inklusiver Bildung beigetragen hat (und die hier dargestellten Daten zur Verfügung gestellt hat).

In den sechs Partnerländern unterscheidet sich die Notwendigkeit der Diagnose, die häufig eine Bedingung für zusätzliche Unterstützung ist, sehr stark. Dies erfordert die Beachtung des Dilemmas der Balance zwischen der positiven Bereitstellung von Ressourcen und den negativen Folgen der Etikettierung, das in den Bildungssystemen vorherrscht. In den meisten Bildungssystemen ist Klassifizierung als Voraussetzung für den Erhalt individualisierter Unterstützung und Leistungen erforderlich. Die Klassifizierungsbegriffe (Kategorien; Labels) hängen eng mit den Sonderschultypen (z.B. in Deutschland), oder dem System der sonderpädagogischen Unterstützung zusammen (z.B. abhängig von der Verfügbarkeit interner professioneller (sonder-)pädagogischer Ressourcen in inklusiven Schulen oder der Notwendigkeit einer ambulanten Unterstützung für Schüler*innen mit SEN). In vielen Ländern - sowohl größeren als auch kleineren, reicheren und ärmeren - finden wir anhaltende schulische und soziale Benachteiligungen, unter denen insbesondere Kinder und Jugendliche mit Beeinträchtigungen und Behinderungen, aber auch Menschen, die Unterstützung zur Erreichung festgelegter Lernziele benötigen, leiden: diese Gruppen sind nicht immer gleich (Richardson/Powell 2011).

$\mathrm{Zu}$ den größten Hindernissen für das inklusive Schulwesen zählen hoch differenzierte Systeme aus getrennten Sonderschulen und separaten Klassenzimmern. Vergleicht man den Anteil der Schüler*innen in gesonderten Settings an der Gesamtschüler*innenschaft in

2 Im Sinne des englischsprachigen Begriffs ,special educational needs“. 
ausgewählten europäischen Ländern und den TdiverS-Partnerländern, zeigt sich die beträchtliche Spanne: von nahezu keiner schulischen Segregation in Schweden und Spanien mit weniger als 1\% (der Gesamtschüler*innenschaft) über Island, Litauen und Luxemburg, die im unteren mittleren Bereich zwischen 1\% und 2\% liegen und bis zu Deutschland am oberen Ende mit fast 4\% in Sonderschulen. Die Quoten für Kinder in gesonderten Schulen und Klassen variieren europaweit zwischen fast $0 \%$ in Italien oder Malta und über $7 \%$ in Belgien (Daten von 2014, EASNIE 2017a). In ganz Europa haben die Länder unterschiedliche Konstellationen von Unterstützungsdiensten und Organisationsformen entwickelt, um Kinder mit Behinderungen oder Lernschwierigkeiten sowie solche, die von unzähligen Benachteiligungen und Diskriminierungen betroffen sind, zu versorgen. Trotz des weltweiten, normativen, und mit der UN-BRK-Ratifizierung auch rechtlichen, Drucks gab es in den letzten Jahrzehnten nur eine begrenzte globale Konvergenz in der sonderpädagogischen und inklusiven Bildung (Richardson/Powell 2011). Umfassende inklusive Bildungsreformen müssen sich daher explizit mit der langfristigen, unterschiedlichen Institutionalisierung der sonderpädagogischen Angebote und Strukturen und ihrer Persistenz befassen, wenn diese Veränderungsprozesse erfolgreich sein sollen.

\section{Inklusive Bildungsreformen: Von politischen Initiativen und Forschung zur Nachhaltigkeit in Schulen}

Bei der Kartierung der internationalen Entwicklung einer inklusiven Bildung ist die wissenschaftliche Aufmerksamkeit auf die Kluft zwischen politischer Rhetorik, die sich oft den Menschenrechten und internationalen Normen verschrieben hat (gemäß der UN-BRK, Art. 24, oder den Sustainable Development Goals, SDG 4 Quality Education), und den schulischen Realitäten von entscheidender Bedeutung. Die normative Dimension von Menschenrechtsvorgaben durch die UN-BRK hat erfolgreich zu einer breiten Sensibilisierung für inklusive Bildung geführt, aber gleichzeitig Gegendruck hervorgerufen, der mithin Rückwärtsentwicklungen hervorruft (Blanck/Edelstein/Powell 2013). In den teilnehmenden Ländern, darunter auch die erfolgreichsten im Hinblick auf Inklusion, benötigen die Schulen, ihre Leitungen und das Lehrpersonal als change agents in der Reform fortwährend breite Unterstützung, Ressourcen und Vernetzungsmöglichkeiten über die Grenzen hinweg, wenn sie zu kompetenten Akteur*innen der Organisationsentwicklung werden und bleiben sollen. Ausdrücklich müssen die vorhandene Bereitschaft zur und die Praktiken der inklusiven Bildung an den Schulen angesichts kontrastierender Systemlogiken wertgeschätzt werden: Inklusive Beschulung ist nicht mit segregierten Strukturen und Rahmenbedingungen vereinbar, die jedoch in vielen Ländern allgegenwärtig bleiben. Inklusive Bildung muss daher inhärent als ein fortlaufender (politischer) Prozess und als ein dauerhaftes Ziel, nicht als ein einmal erreichter, stabiler Status verstanden werden. Die Herausforderungen für die Inklusion variieren und verändern sich historisch und regional, sind auf Kontexte zu beziehen und unterscheiden sich z.B. je nach Ausgangsniveau im Bildungssystem. Als Prozess ist die inklusive Reform von Natur aus herausfordernd und hat politische Implikationen. Das Projekt TdiverS bot Lernmöglichkeiten, um zu verstehen, dass viele dieser Herausforderungen in ganz Europa ähnlich sind: Keine Einzelschule oder kein Land steht diesen alleine gegenüber. 
Wenn solche Reformen vorangekommen sind, sollten sie nicht als selbstverständlich betrachtet werden, da die sie untermauernden Reformprozesse und die inklusiven Werte immer Gefahr laufen, gestoppt oder angefochten zu werden. Sowohl Forscher*innen als auch Praktiker*innen müssen daher um die Nachhaltigkeit der Umsetzung von Reformen bemüht sein, die darauf abzielen, die Bildung inklusiver zu gestalten. Ein individuelles Modellieren der Inklusion in den einzelnen Schulen wurde so bspw. in TdiverS als wichtig erachtet, und zwar für alle Beteiligten, um „Inklusion zu leben“, unter der Einbeziehung von außerschulischen Programmen in den Gemeinschaften sowie der aktiven Beteiligung von Schüler*innen und Eltern an der Schulentwicklung. Ferner kann eine von allen Schulmitgliedern unterzeichnete Schul-Charta (Harris et al. 2017) sowie team-teaching wichtig sein, um Rollenmodelle auf der Basis der Erwachsenen für die Zusammenarbeit und die inklusiven Praktiken innerhalb der Schule zu entwickeln. Festzuhalten bleibt: Eine Schlüsselmethode zur Förderung der Nachhaltigkeit inklusiver Schulbildung besteht darin, auf das Prinzip des Dialogs zu setzen, d.h. die Kommunikation zwischen allen Akteur*innen und allen am Schulleben und an der Bildung der nächsten Generation beteiligten Personen und Institutionen sicherzustellen.

Forschung und Studien zur Implementation sind entscheidend, um solche Prozesse zu unterstützen. Als ein von der Europäischen Union finanziertes Projekt ist TdiverS ein Beispiel für grenzübergreifenden, multikulturellen Austausch und Vernetzung, mit dem Potential die Verbreitung und Weitergabe von Ideen im Zusammenhang mit inklusiver Bildung zu erleichtern und deren Nachhaltigkeit zu erhöhen. Zu den fast zwanzig einzelnen Projektpartner*innen gehörten Vertreter*innen von Universitäten, Lehrer*innenausbildungsorganisationen, Bildungsverwaltungen und Schulen und weitere assoziierte Partner*innen aus der Bildungspolitik, die über 36 Monate lang (2013-2016) an der Erstellung und Erarbeitung verschiedener Materialien mitgearbeitet haben, die auf multidisziplinärer Forschung in einem Methodenmix basierten und in mehreren Sprachen aufbereitet wurden. Wesentliche Ressource waren die Länderberichte, die eingangs von Projektmitglieder*innen verfasst wurden, Schulbesuche und Unterrichtshospitationen in allen Ländern, Diskussionen mit Referenzgruppen der Schulgemeinschaften (z. B. junge Menschen mit Behinderungen), Präsentationen im Rahmen internationaler Konferenzen (AERA \& EERA) sowie viele Diskussionen zwischen den Mitglieder*innen des Konsortiums und mit den beteiligten Lehrer*innen (im Rahmen der Videoproduktion) sowie mit Projektgästen, insbesondere Lehrer*innen und Wissenschaftler*innen aus den sechs Ländern oder aus verwandten EU-Forschungsprojekten. Dies ermöglichte das Projektziel: Grundsätze für inklusives Lehren und Lernen zu identifizieren, die von den Partner*innen gemeinsam genutzt und überprüft werden können, um bei der Auswahl der ,inspirierenden Praktiken“, die in den Partnerschulen gefunden werden, für die Verbreitung in ganz Europa und darüber hinaus, zu helfen. Wir wenden uns im Folgenden kurz jeweils den Debatten über die Implementation von inklusiver Bildung sowie den aus dem Projekt TdiverS gezogenen Lehren für die beteiligten Länder in vergleichender Perspektive zu. 


\section{Vielfalt, Unterschiede, Verschiedenheit: Die Umsetzung von inklusiver Bildung in sechs Ländern in Europa}

Die Studie und das Projekt betonten die generelle Vielfalt der Lernenden, die Unterschiede zwischen den einzelnen Ländern und auch innerhalb der Länder sowie Differenzen zwischen den Lern-/Bildungsangeboten, die alle mit den Reformen der inklusiven Bildung in verschiedenen Stadien der Umsetzung in den sechs europäischen Ländern zusammenhängen. Inklusive Bildung wurde systematisch als übergreifendes, umfassendes Veränderungsprojekt verstanden. Für inklusivere Systeme bedeutet das Vorantreiben der Inklusion das Beenden der Segregation der Lernenden in auf schulische Leistungen basierende Gruppen innerhalb der allgemeinen Schule oder im Klassenzimmer. In weniger inklusiven Systemen ermöglichen Entwicklungen, wie z.B. ambulante Dienstleistungen, eine inklusivere Schulbildung auf kurze Sicht, indem die Notwendigkeit einer Selektion oder Segregation von Schüler*innen zur Bereitstellung von Unterstützung verringert wird, obwohl Sonderschulen in diesen Ländern noch in gewissem Umfang vorhanden sind. In einigen von ihnen - wie in Deutschland wird die Mehrheit der Schüler*innen mit special educational needs (SEN) in Sonderschulen unterrichtet, während in anderen - wie in Island, Litauen, Luxemburg, Schweden und Spanien - nur (noch) eine Minderheit von Schüler*innen mit SEN in Sondersystemen betreut wird, obwohl die Klassifizierungsraten ebenfalls deutlich variieren (EASNIE 2017a). Die räumliche Komponente des Schulwesens ist entscheidend, da Schulen auch „Lebensräume“ sein sollten, die das Wohlbefinden fördern und verschiedene Lernmöglichkeiten bieten. Änderungen des Lehrplans und des Schullebens mit dem Ziel inklusive Prinzipien zu reflektieren, wirken sich auf die gesamte Schule und die gesamte Schulkultur aus und erfordern, dass die Schule offen ist und sich als Teil der Gesellschaft versteht. Lehrer*innen in Ländern mit Gesamtschulen für alle Schüler*innen haben mehr Erfahrung darin, tatsächlich heterogene Lerngruppen zu unterrichten, als solche in horizontal gegliederten Schulsystemen mit mehreren weiterführenden Schultypen (Merz-Atalik et al. 2016).

In Bezug auf Lernprozesse erwiesen sich Lernstandserhebungen und Diagnostik (MerzAtalik 2013a; Prengel 2016) abhängig von der gewählten Form als Hemmnisse oder Unterstützer von Inklusion. Standardisierte Leistungsbewertungen (defizitorientiert) führen zu Etikettierung und wirken damit den Grundsätzen der inklusiven Bildung entgegen. Im Projektkonsortium wurde betont, wie wichtig es ist, dass jede*r Schüler*in einen individuellen Bildungsplan hat. Einige Schulen haben Wege gefunden, um die übermäßige Betonung von standardisierten Leistungsbewertungen zu verringern und alternative Bewertungsmöglichkeiten zu entwickeln, wie etwa Portfolios, die alle Lernenden erstellen und mit ihren Klassenkamerad*innen teilen, um Peer-Interaktion und horizontales Lernen zu stärken (Brendel/Noesen 2014). Vor allem an Übergängen führen die vorherrschenden Systemorientierungen und -normen in selektiven Bildungssystemen zu Problemen für einzelne Lehrer*innen und Schüler*innen, weswegen inklusive Beschulung eine systemweite Reform sein muss.

Ein traditioneller Fachunterricht wurde als mögliche Einschränkung der Inklusion - im Gegensatz zu multidisziplinärer, projektbezogener Arbeit und offenen Strukturen, die die Inklusion erleichtern - diskutiert. Nicht alle (Fach-)Lehrer*innen sind gleichermaßen offen für inklusives Lernen, weil ihr Fachwissen möglicherweise ,gefährdet" wird, oder die Vielfalt der Lernenden eine komplexere Vorbereitung von Lernmaterialien erfordert. Die fachspezifischen Lernstandards sollten den „Lernprofilen“ der einzelnen Schüler*innen entsprechend 
angepasst werden, wobei die Fächer häufig noch traditionell lehrer*innenzentriert unterrichtet werden.

Das Projekt TdiverS setzte sich beispielhaft mit den schulpolitischen Herausforderungen auseinander, welche die Unterstützung inklusiver Praktiken, Programme und Richtlinien zum Ziel haben. Drei Jahre sind in Bezug auf das Projektmanagement eine willkürliche Dauer und es stellte sich die Frage nach der optimalen Zeitspanne für die Entwicklung von Ideen im Kontext einer Zusammenarbeit mit einem derart großen Umfang und solch ehrgeizigen Zielen. Zufällig wurde TdiverS weitgehend auf bestehenden Netzwerken (Projekt TE4i ,Teacher Education for Inclusion“ der Europäischen Agentur) und früheren Forschungskooperationen zwischen einzelnen Partner*innen aufgebaut. Grundlegende Diskussionen waren dennoch notwendig, um eine gemeinsame Sprache zu entwickeln und Prioritäten und die Aufgabenverteilung zu bestimmen. Es blieben Unterschiede in den Ansichten und Perspektiven bestehen und diese sind unter den gegebenen Bedingungen kaum lösbar. Beispielsweise wurden im deutschsprachigen Raum die Didaktiken in Fachgebieten (so genannte Fachdidaktiken) als eigenständige Bereiche und Abteilungen innerhalb von Universitäten betrachtet, oft getrennt von der allgemeinen Lehrer*innenausbildung und der Sonderpädagogik. Während in Deutschland aktuell sehr stark eine Verknüpfung von Sonderpädagogik und Fachdidaktik thematisiert wird, ging es in den meisten anderen europäischen Ländern bereits bei der Umsetzung eines Gemeinschaftsschulsystems und des längeren gemeinsamen Lernens um die Entwicklung einer diversitätsbewussten, differenzierten Fachdidaktik (Merz-Atalik 2018a), d.h. die in Deutschland vorhandene Kluft ist in anderen Ländern weniger ausgeprägt oder gar nicht vorhanden. Im Sinne der Inklusion akzeptierten die Projektpartner*innen diese Unterschiede und erkannten deren Wert (und die Kontexte, aus denen sie entstanden sind).

\section{Inspirierende Praktiken und Lessons Learned in Deutschland, Island, Litauen, Luxemburg, Schweden und Spanien}

Mit dem Bestreben „inspirierende Praktiken“ zu katalogisieren (anstatt einer einzigen für ganz Europa gültigen ,best practice“) entdeckte das TdiverS Konsortium, wie komplex und intensiv Videografie in Schulen ist: insbesondere in sehr differenten schulischen und kulturellen Kontexten, in sechs verschiedenen Sprachen und mit unterschiedlicher technischer Expertise sowie technologischem Equipment. Das Team erkannte, dass die Videos einen Rahmen sowie Struktur benötigen, um den Kontext zu verstehen sowie um die Botschaft hinter den ausgewählten Situationen und ihre jeweiligen Determinanten (Barrieren und unterstützende Faktoren) zu verstehen, die das Bildungssystem im Allgemeinen sowie das jeweilige spezifische Schulumfeld aufwerfen. Die Videos, Reviews und Übersetzungen wichtiger wissenschaftlicher Texte konnten als widersprüchliche Ziele und Aspekte verstanden werden, die reflektiert werden mussten (so wurden die Videos von und für die Praxis und die Lehrer*innenbildung nach anderen Kriterien ausgewählt als die wissenschaftliche Literatur). Das Projektergebnis enthält Literaturlisten und -texte sowie Videos, um vielfältige Einsatzmöglichkeiten für diverse Nutzer*innen in der Lehrer*innenbildung bereitzustellen. So wurde die wissenschaftliche Literatur durch die Länderteams überwiegend in Anlehnung an die Erweiterung des Verständnisses für die Videosequenzen ausgewählt, hat daher vielfältige thematische Schwerpunkte und kann nicht als Basisliteratur bezeichnet werden, da die Videos 
diverse Aspekte inklusiver Schul- und Unterrichtsentwicklung herausarbeiten. So wurden am Ende nur wenige grundlegende Texte mit einer übergreifenden Perspektive eingebunden (z.B. ein Text von Annedore Prengel (2016), der dafür ins Englische übersetzt wurde). Diese grundlegenden Texte hatten zum Teil bereits zu Beginn des Projektes einen ähnlich hohen Stellenwert für alle beteiligten Wissenschaftler*innen und Praktiker*innen (z.B. der Index für Inklusion). Eine wichtige lesson learned war es dennoch, dass es in solchen Projekten die Notwendigkeit gibt, explizit die Definitionen und Werte von inklusiver Bildung zu diskutieren sowie den Beitrag vergleichender Methoden zu solchen Forschungsprojekten umfassender zu reflektieren.

Gemeinsam diskutierten wir Fragen zum Zweck von Bildung und Unterricht in einer umfassenderen Perspektive. In ganz Europa ist die Bedeutung der evidenzbasierten Politikgestaltung und der Begriffe Qualität, Exzellenz, Relevanz und Impact gestiegen, denn die EU erweitert ihre Rolle beim Aufbau grenzüberschreitender Netzwerke und bei der Gestaltung von Forschungsprogrammen durch Anreize zur Konvergenz zu allumfassenden Prioritäten und Themen - sowie komparative Perspektiven oder interkulturelle Zusammenarbeit - in EU-Projekten (Zapp et al. 2018) wie die von TdiverS. Heutzutage wird die Wettbewerbsfähigkeit kontinuierlich anhand vergleichender Indikatoren überwacht, die von politischen Entscheidungsträger*innen, Wissenschaftler*innen und Administrator*innen verwendet werden, um Reformziele zu generieren, Standards und bewährte Verfahren zu identifizieren sowie politische Maßnahmen und Programme empirisch zu überprüfen. Zunehmend haben Länder Richtlinien erlassen, die erfolgreiche ausländische Modelle nachahmen oder die sich durch Bezugnahmen auf diese legitimieren. Mechanismen eines solchen interkulturellen Transfers umfassen „kontinuierliche konkurrierende Vergleiche“ in Form von Rankings, Leistungsvergleichen und ,, best practices, “ „policy learning “ und Netzwerken, sowie zwischenstaatliche Verhandlungen und Koordinierung. Supranationale Koordination erleichtert das Definieren von Standards, erweitert die grenzüberschreitende Mobilität und ermöglicht Zusammenarbeit. Eine solche Zusammenarbeit und Kooperation, der Austausch inspirierender Praktiken aus inklusiven Lernumgebungen und das Lernen aus Reformprozessen und Erfahrungen andernorts, ist von entscheidender Bedeutung. In der Tat ist es für alle Lehrer*innen eine Herausforderung, die Vielfalt aller Lernenden im standardisierten fachbezogenen Unterricht zu unterrichten. Seit der TdiverS-Abschlusskonferenz in Ludwigsburg (Deutschland) im Jahr 2016 wurde die Videosammlung (USB-Flash-Laufwerk) sehr breit international präsentiert und wahrgenommen, tausendfach verteilt und wird zunehmend in den Partnerländern und darüber hinaus in der Lehrer*innenausbildung eingesetzt. Solche Projekte, sowie die Sammlung und Verbreitung inspirierender Praktiken in der inklusiven Bildung, sind von entscheidender Bedeutung für Länder, die noch eine wissensbasierte Professionalisierung benötigen, um eine inklusivere Bildung und „Pädagogik der Vielfalt“ (Prengel 2016) zu ermöglichen. Im Folgenden werden Skizzen der jeweiligen Länderkontexte und der Erkenntnisse vorgelegt. Die Verfasser*innen haben dabei unterschiedliche Gewichtungen verschiedener Aspekte vorgenommen: 


\subsection{Deutschland: Das Paradoxon der gleichzeitigen Entwicklung inklusiver Bildung und Co-Teaching (kooperatives Unterrichten) ohne einen Rückgang der Sonderbeschulung}

Deutschlands relativ kurze Geschichte der inklusiven Bildung beginnt in den 1970er Jahren mit Pilotprojekten in mehreren Bundesländern. Die Ständige Konferenz der Kultusminister (KMK) veröffentlichte 1994 erstmals die Empfehlung, dass „,Die Bildung behinderter junger Menschen [...] verstärkt als gemeinsame Aufgabe für grundsätzlich alle Schulen anzustreben [ist]. Die Sonderpädagogik versteht sich dabei immer mehr als eine notwendige Ergänzung und Schwerpunktsetzung der allgemeinen Pädagogik". Trotz des terminologischen Wandels von Sonderschulbedürftigkeit zum sonderpädagogischen Förderbedarf in politischen Dokumenten besucht immer noch die Mehrheit dieser klassifizierten Schüler*innen räumlich-getrennte Sonderschulen (EASNIE 2017a). Seit 2009 ist die UN-BRK in Deutschland rechtlich bindend, aber die 16 Bundesländer behalten die Autorität über die Bildung (Bildungshoheit) und interpretieren das Inklusionsideal in ihren bestehenden Systemen sehr unterschiedlich: Während einige die bestehenden Sonderschulen schützen, streben andere nach einer Implementierung umfassender inklusiver Bildungsreformen (Blanck et al. 2013). Sonderpädagogische Unterstützungssysteme haben je nach regionalen Bedingungen unterschiedliche Profile. Die Politik verlangt die professionelle organisatorische Entwicklung von Sonderpädagogik, Beratung und Unterstützung, einschließlich präventiver, inklusiver und kooperativer Formen (KMK 2011). Die meisten Bundesländer garantieren ein Elternwahlrecht, die Schule für ihre Kinder mit einem sonderpädagogischen Förderbedarf selbst zu wählen. Dies führte jedoch zu Debatten um den Erhalt von Sonderschulen, was ein großes Hindernis für die inklusive Bildungsreform darstellt.

Obwohl sich die inklusive Bildung in Deutschland allmählich entwickelt, geschieht dies ohne den Rückgang der Sonderbeschulung in den meisten Bundesländern. Ein Paradoxon, das erhöhte Normen und Erwartungen, festgelegte Standards sowie das Bewusstsein für und die Diagnose von sonderpädagogischem Förderbedarf widerspiegelt (Powell [2011] 2016). Die Inklusionsrate (Anteil der Schüler*innen mit diagnostiziertem sonderpädagogischen Förderbedarf in inklusiven Settings an allen klassifizierten Schüler*innen) variiert beträchtlich zwischen den 16 Bundesländern: von $83 \%$ in inklusiven Settings in Bremen bis zu $25 \%$ in Hessen. Gleichsam variiert die Sonderbeschulungsquote unter der Gesamtschüler*innenschaft zwischen 6,5\% in Mecklenburg-Vorpommern und 1,2\% in Bremen (Autorengruppe Bildungsbericht 2016). Trotz der UN-BRK-Ratifizierung stieg die Segregationsrate in einigen Bundesländern von 2012 bis 2016 sogar an (ebd.).

Inklusive Bildung und Didaktik sind aufgrund verschiedener Faktoren im Entwicklungsrückstand. Als mögliche Gründe können beispielsweise folgende aufgeführt werden: die Trennung zwischen den Disziplinen Allgemeine Erziehungswissenschaft, Schulpädagogik und Sonderpädagogik sowie Fachwissenschaft und Fachdidaktik an Universitäten und Pädagogischen Hochschulen und den niveausegregierenden Schultypen im segregativen Bildungssystem; die Herausforderung für Inklusionsforscher inklusive Settings für Untersuchungen zu finden, selbst in jenen Bundesländern wo diese Forschung zugelassen oder sogar finanziert wird (Merz-Atalik 2013b); sowie die erst kürzlich gestiegene Anerkennung des Bedarfs an Ausbildung und Schulung aller Lehrkräfte für die Ausarbeitung individualisierter Bildungspläne und bei der Bewältigung inklusiver Unterrichts- und Lehrkonzepte (MerzAtalik 2014). Im Jahr 2015 haben die KMK und die Deutsche Hochschulrektorenkonferenz 
(HRK) Empfehlungen für die Lehrer*innenbildung für ein Bildungssystem herausgegeben, das sich der Lerner*innenvielfalt annimmt. Hier wird ausgeführt, dass die Entwicklung einer Beschulung, die die Vielfalt als Stärke und als „Normalität“ anerkennt und schätzt, ein Ziel von Lehrer*innen an allen Schulen darstellt.

Bei den beiden TdiverS-Partnerschulen handelt es sich noch um eher seltenere Fälle inklusiver Bildung im Land Baden-Württemberg, das erst 2013 mit der Entwicklung von Gesamtschulen begonnen hat, in dem - trotz der Schulgesetzänderung im Jahr 2015 und vielfältiger Modellprojekte zur inklusiven Bildung - nach wie vor die Quote der Schüler*innen an Sonderschulen steigend ist (Merz-Atalik 2018b) und das eine lange Geschichte selektiver und gegliederter Sekundarschulen (Trennung in niveaudifferenzierte Schultypen nach der Primarstufe, ab Klasse 4) aufweist. Die Akteur*innen, welche uns im Rahmen der Videosequenzen aus Deutschland Einblicke in ihre Schul- und Unterrichtspraxis ermöglicht haben, haben in vielfacher Hinsicht aufzeigen können, dass die Entwicklung einer qualitativ hochwertigen, inklusiven Praxis auch unter ungünstigen Rahmenbedingungen möglich sein kann. Zum Beispiel durch eine ausgewiesene Expertise im Co-teaching, durch differenzierten Unterricht (Sabine Meixner, Grundschullehrerin; Kerstin Bohl, Sonderpädagogin an einer Grundschule in Ludwigsburg) oder durch die besondere Kompetenz, den Unterricht im Hinblick auf die Diversität der Schüler*innen zu personalisieren (Markus Plath, Sportlehrer an einer Gemeinschaftsschule in Tübingen). Dies bestätigt, dass positive Einstellungen und die selbstmotivierte Professionalisierung der einzelnen Akteur*innen als bedeutende Ressourcen für die Reform zur inklusiven Bildung wirken. Es bleibt die Frage: Wie kann dieses Engagement in laufenden Reformprozessen erhalten und gestärkt werden? Und wie kann es gelingen inklusionsfreundliche kulturelle und strukturelle Veränderungen auf den Ebenen der Bildungspolitik und -verwaltung einzufordern und aufzubauen, welche für die nachhaltige Sicherung dieser hochwertigen Praxis erforderlich sind (Powell/Merz-Atalik 2019).

Wir wenden uns nun von dem bevölkerungsmäßig größten Land, welches am geringsten einem inklusiven Bildungssystem entspricht, zum flächenmäßig kleinsten, aber jenem mit dem am stärksten inklusionsorientierten Bildungssystem unter den Projektpartnerländern.

\subsection{Island: Chancengleichheit und Verbesserung des Wohlbefindens der Schüler*innen in inklusiven, auf Schüler*innen und Zusammenarbeit ausgerichteten Schulen}

Nach dem Bildungsgesetz von 2008, wonach alle Schulen inklusiv sein sollten, trat 2011 in Island ein neuer nationaler Lehrplan für die frühkindliche, verpflichtende und weiterführende Sekundarstufe in Kraft (Sigurðardóttir et al. 2014). Der Leitfaden für Lehrpläne bewegte sich von detaillierten Zielen hin zu einem Fokus auf Lernergebnisse und fundamentale Bildungsideen. Dieses nationale Curriculum basiert auf sechs Grundpfeilern: Bildung im weitesten Sinne; Bildung für Nachhaltigkeit; Demokratie und Bürgerschaft; Erziehung zur Gleichberechtigung; Kreativität; und Gesundheit (Icelandic Ministry of Education, Science and Culture 2011). Kürzlich hat die Europäische Agentur für sonderpädagogische Förderung und inklusive Bildung das isländische Bildungssystem einer externen Prüfung unterzogen und dabei auf die zu verbessernden Bereiche abgezielt (EASNIE 2017b; siehe auch Köpfer/ Óskarsdóttir 2021 i.d.B.). 
Wie auch anderenorts, suchen Lehrer*innen in Island kontinuierlich nach pädagogischen Ansätzen, um sowohl den Anforderungen des Lehrplans als auch der Vielfalt der Schüler*innen in inklusiven Schulen gerecht zu werden. Lehrer*innen, die am TdiverS-Projekt teilgenommen haben, organisieren ihren Fachunterricht in diversen und inklusiven Klassenräumen. In ihren Reaktionen auf verschiedene Schüler*innen tauchten drei Hauptthemen auf: (1) Wie Lehrkräfte inklusiven Unterricht verstehen und wie sich dies auf ihren Unterricht auswirkt; (2) Wie Lehrer*innen ihren Unterricht in verschiedenen Fächern für unterschiedliche Schüler*innen organisieren und vorbereiten; und (3) die Wichtigkeit der Zusammenarbeit mit anderen Lehrer*innen und dem Unterstützungspersonal (pädagogisches Personal) bei der Schaffung inklusiver (Lern-)Umgebungen. Das konzeptionelle Verständnis von Inklusion, wie es von den Lehrer*innen erklärt wird, ist durch ihre Haltung charakterisiert, dass das Wohlergehen der Schüler*innen das wichtigste Ziel inklusiver Schulen sei: d.h. dafür zu sorgen, dass alle Menschen dabei unterstützt werden, ihre Ziele zu erreichen, indem den Schüler*innen gleiche Chancen geboten werden, ihre unterschiedlichen Stärken zu zeigen, zu erweitern und zu genießen. Die Beachtung der Unterschiede zwischen den Schüler*innen sowie ihrer Gemeinsamkeiten ist von entscheidender Bedeutung, da inklusives Lernen von Lehrer*innen unterschiedliche Lehrmethoden erfordert.

Bei der Vorbereitung und Organisation des Lehrens und Lernens betonen die Lehrer*innen, wie wichtig es sei, über die gesamte Gruppe nachzudenken und die Planung auf jede individuelle Schüler*innengruppe abzustimmen. Bei ihrer Vorbereitung berücksichtigen diese Lehrer*innen die Interessen und Motivation der Schüler*innen beim Wunsch zum Erledigen von Aufgaben. Sie haben festgestellt, dass Gruppenarbeit, Spiele und Spaß beim Lernen positive Auswirkungen auf den Schulerfolg haben.

Die Zusammenarbeit mit anderen wurde als wichtig für das Erreichen aller Schüler*innen hervorgehoben. Mit der Unterstützung von Sonderpädagog*innen, Lehrer*innenassistent*innen und Sozialpädagog*innen war es laut den Lehrer*innen möglich, dass sie in der Lage waren, Lernräume zu schaffen, die alle Schüler*innen einbeziehen können, die erforderliche Differenzierung bereitzustellen und die individuellen Bedürfnisse der Schüler*innen zu erfüllen. Einer der Lehrer erklärte: „Wenn ich mit den Sonderpädagogen arbeite, entscheide ich mich zuerst für eine Aufgabe und dann lasse ich diese für mich draufschauen und Möglichkeiten finden, die Aufgaben für manche Schüler anzupassen. Diese Zusammenarbeit war sehr erfolgreich. Sie macht mein Leben und meine Arbeit etwas einfacher." Die Praktiken um diverse Schüler*innen zu unterrichten basieren auf einer Pädagogik, die professionelles Wissen über Lehren, Lernen und die kindliche Entwicklung integriert und eine ethische und soziale Verpflichtung gegenüber den Kindern beinhaltet (Guðjónsdóttir 2000).

Inspirierende Unterrichtspraktiken fördern die Inklusion, würdigen die Vielfalt in und von Kulturen und ethnischem Erleben und erstellen die Lernumgebung basierend auf der differenten Beteiligung und den Identitäten jedes*r Schülers*in. Diese Lehrer*innen entwickeln eine inklusionsorientierte Pädagogik, die auf die Schüler*innen ausgerichtet ist und sich durch Offenheit gegenüber der Vielfalt der Schüler*innen, den Einsatz innovativer Strategien und den Aufbau von Kooperationen innerhalb und außerhalb von Schulen auszeichnet. 


\subsection{Litauen: Lehrer*innen unterstützen Lernende als Kooperationspartner*innen in stärker personalisierten und barrierefreien Klassenräumen}

Die wichtigsten Leitideen zu inklusiver Bildung und zum Inklusionskonzept sind in nationalen strategischen Dokumenten wie dem Bildungsgesetz (2011) und der nationalen Bildungsstrategie 2013-2022 ausgeführt. Chancengleichheit ist einer der Grundsätze, auf denen das Bildungssystem beruht. Sie zielt darauf $a b$, die Gleichstellung von Individuen mit Unterschieden bezüglich Fähigkeiten, Geschlecht, Ethnie, Nationalität, Sprache, Herkunft, sozialem Kontext, Religion, Glauben oder Überzeugung sicherzustellen. In der nationalen Bildungsstrategie ist die Priorität festgelegt, Möglichkeiten für die Bildung von Kindern mit unterschiedlichen Fähigkeiten und Bedürfnissen zu schaffen und sicherzustellen, dass jede*r Einzelne Zugang zu Bildung und Gelegenheiten zur Erlangung von Allgemeinbildung erhält. In der nationalen Bildungsstrategie sind Ziele für die Entwicklung der Bildung und die Mittel zur Erreichung dieser Ziele festgelegt. Zusätzlich wurden quantitative und qualitative Ergebnisindikatoren festgelegt, die als Grundlage für die Weiterentwicklung des litauischen Bildungssystems und seiner Evaluation dienen. Es sollen Lernmöglichkeiten für Kinder aus ethnischen Minderheiten und Migrantenfamilien sichergestellt und das Unterstützungssystem für Kinder und Jugendliche mit außergewöhnlichen Fähigkeiten entwickelt und ausgebaut werden.

Seit der postsowjetischen Transformation wurden viele Studien mit dem Schwerpunkt auf inklusive Bildung von Kindern mit sonderpädagogischem Förderbedarf durchgeführt. In den meisten dieser Studien wurden die allgemeinen Grundsätze der inklusiven Bildung, die Qualität der pädagogischen Assistenz, die Unterstützung verschiedener Gruppen von Kindern mit besonderen Bildungsbedürfnissen, die Zusammenarbeit und die Rolle von Lehrer*innen und Spezialist*innen sowie die Einstellung der Lehrkräfte analysiert (Ališauskas et al. 2009; Milteniene/Venclovaite 2012; Ališauskas et al. 2013). Die Konzeptualisierung der inklusiven Bildung in der litauischen Forschung beinhaltet die explizite Beteiligung der Sonderpädagogik.

Der Erfolg von inklusiver Bildung hängt im Wesentlichen mit der Verfügbarkeit von Ressourcen und der Fähigkeit von Lehrer*innen zusammen, diese zu erkennen und gemeinsam zu nutzen, um Lernprozesse zu erleichtern. Lehrer*innenkompetenz und -bereitschaft gehören zu den wichtigsten Faktoren für die Entwicklung eines günstigen Umfelds und einer qualitativ hochwertigen Bildung für jede*n Lernende*n (Ališauskas et al. 2013). TdiverS bot Lernmöglichkeiten, um zu verstehen, dass viele Herausforderungen an Schulen ähnlich sind, wobei Schulvertreter, insbesondere Renata Geleziniene, Kestutis Saltis und Saulius Vilutis, zu den Projektergebnissen beitrugen. Die jüngste Forschung stützte sich auf neue Paradigmen und Methoden, wie beispielsweise kooperatives Lehren und Lernen und personalisiertes Lehren und Lernen. Dieses neue Paradigma leitet das gesamte Bildungssystem an, um die sich ändernden Bedürfnisse und Rollen der Lernenden zu erkennen. Es findet graduell ein Wechsel von der Ansicht der Lernenden als „Nutzer*innen“ zu „Kooperationspartner*innen“ statt. In diesem Zusammenhang wird die Unterstützung des einzigartigen Lernweges jedes*r einzelnen Schülers*in als Schwerpunkt einer inklusiven Bildung betrachtet. 


\subsection{Luxemburg: Intersektionale Inklusion und dialogischer Unterricht in kulturell vielfältigen, mehrsprachigen Schulen}

Luxemburg liegt an der Grenze zwischen den germanischen und frankophonen Sprachgemeinschaften Europas. Mit einer der kulturell und sprachlich vielfältigsten Bevölkerungsgruppen in Europa müssen luxemburgische Pilotprojekte im Bereich der inklusiven Bildung unter Berücksichtigung zahlreicher Merkmale aus einer intersektoralen Perspektive evaluiert werden (Powell et al. 2017). Wie in ganz Europa waren vor einem Jahrhundert ausgrenzende Praktiken sowie die Orientierung an durchschnittlichen Lernfortschritten im allgemeinen Schulunterricht weit verbreitet. Vor allem nach der Universalisierung der Schulpflicht wurde die Verantwortung auf immer unterschiedlichere Schüler*innengruppen ausgedehnt, aber spezialisierte Organisationen, die Menschen mit erkannten sensorischen oder intellektuellen Beeinträchtigungen betreuen, führten häufig zu Stigmatisierung und Segregation. Nach dem Zweiten Weltkrieg verbreitete sich die Idee der Bildung als Menschenrecht global, was letztendlich dazu führte, dass „Bildung für alle“ und inklusive Bildung weltweit gefordert werden. In Luxemburg sind solche Forderungen nach mehr Gerechtigkeit in der Schule schon lange vorhanden, beispielhaft durch die luxemburgische Regierungskampagne „A School for Everyone“ („Eine Schule für alle“) in den 1970er Jahren.

Trotz vieler Bemühungen in den letzten Jahrzehnten, wie beispielsweise einer umfassenden Schulreform 2009, ist es nicht gelungen, grundlegende Veränderungen im Schulwesen zur Verbesserung der Gleichstellung herbeizuführen. Tatsächlich finden Forscher*innen eine beträchtliche Beharrlichkeit in Bezug auf soziale und schulische Segregation, auf unterschiedliche Schulleistung und in Bezug auf die hohen Zahlen von Klassenwiederholungen und Schulabbrecher*innen vor (Ministère de l'Éducation nationale, de l'Enfance et de la Jeunesse 2017) - dies ist jedoch kein ungewöhnliches Bild, es zeigt sich auch in den Nachbarländern Luxemburgs Belgien, Frankreich und Deutschland. Neue Gesetze und Verordnungen, viele Projekte und Initiativen sowie die Ausweitung der Bildungsangebote, ein dramatisches demografisches Wachstum und soziale Veränderungen in der Gesellschaft haben den Rahmen für das Schulwesen verändert. Luxemburg hat internationale Menschenrechtsabkommen unterzeichnet, die globale Normen widerspiegeln. Die Strukturen des Schulsystems haben sich aber nicht grundlegend verändert.

Es wurden jedoch mehrere Schulprojekte entwickelt, um ein integratives Lernumfeld zu fördern, einschließlich der Vorschul- und Grundschulbildung in Esch/Alzette (1995-2000), der Grundschule Weiswampach (2000) und der Eis Schoul auf Kirchberg (seit 2008), des Instituts für Sehbehinderte sowie der TdiverS-Partnerschule Ecole Jean Jaurès in Esch/Alzette (seit 2006). In dieser letztgenannten Ganztagsschule haben sich inklusive Praktiken entwickelt, die auf langjähriger gemeinschaftlicher Lehrforschung basieren, um eine Gemeinschaft von Lernenden zu schaffen, vielfältige Konzepte für das Sprachenlernen im Vorschulalter anzubieten und einen inklusiven Ansatz für alle Schüler*innen in allen Schulfächern zu entwickeln. Eine offene architektonische Struktur, ein offener Stundenplan, eine einvernehmliche Schulcharta, alternative Beurteilungen, Portfolioarbeit und ein narrativer Ansatz für das Lehren und Lernen gehören zu den Schlüsselaspekten, um die Schulbildung inklusiver zu gestalten. Beispielsweise können narrative Bewertungen zeigen, was ein Kind weiß und wie bestimmte Lernaktivitäten am besten in das „Wissensuniversum“ eines Kindes passen, sowie die Ausdrucksfähigkeit und Kommunikation von Kindern fördern und sie als Experten für ihr eigenes Lernen positionieren. Das TdiverS-Video, das auch von Schüler*innen 
konzipiert wurde, sammelte inspirierende Praktiken, indem es die dialogische, dialektische Art und Weise demonstrierte, in der Lehrer*innen und Schüler*innen miteinander im Raum interagieren. Das Video bietet einen Leitfaden für ihre Visionen und ihr Verständnis von „Inklusion“, die in ihrer Schulgemeinschaft gelebt werden.

\subsection{Schweden: Respekt für Vielfalt, Toleranz und Solidarität fördern die Inklusion, um Gleichstellung und Leistung zu ermöglichen}

Im Jahr 1960 verabschiedete das schwedische Parlament ein Gesetz zur Einrichtung eines neunjährigen Schulpflichtsystems für alle Kinder. Eine einjährige Vorschule, die Kinder ab sechs Jahren besuchen, stimuliert die Entwicklung und das Lernen der Schüler*innen und bereitet sie auf ihren weiteren Bildungsweg vor. Das Parlament hat dieses Einführungsjahr ab dem Schuljahr 2018/2019 zur Pflicht gemacht: die Schulpflicht umfasst nun zehn Jahre. Während der Begriff „Inklusion“ in politischen Texten wie dem Schulgesetz, der Schulverordnung und den nationalen Lehrplänen nur sparsam verwendet wird, wurde er in politischen Kontexten häufig missbraucht. Gleichzeitig ist die Inklusion Gegenstand lebhafter und intensiver Diskussionen in Kommunen und in Schulen, die bei der Anpassung des Konzepts an die örtlichen Gegebenheiten oft kreativ sind.

In der im Projekt TdiverS profilierten Gemeinde heißt das Leitbild „Eine Lernumgebung für alle - eine moderne Schule auf wissenschaftlicher Basis“. Die teilnehmende Schule baut darauf auf, dass alle Mitarbeiter*innen zusammenarbeiten, um ein gutes Lernumfeld zu schaffen, in dem sich jedes Kind so weit wie möglich entsprechend seiner eigenen Fähigkeiten entwickelt - im Rahmen eines inklusiven Ansatzes. Dies bedeutet curriculare kognitive Lernziele mit demokratischer Bildung zu verbinden, mit Konzepten und Prinzipien wie Gerechtigkeit, Vertrauen und Teilhabe im Kern, die als entscheidend angesehen werden, um die Problemlösefähigkeit der Schüler*innen zu entwickeln. Schüler*innen, die besondere Unterstützung benötigen, erhalten ihre Hauptunterstützung im Rahmen der allgemeinen Bildung an der Regelschule.

Die Lehrer*innen nehmen zusätzliche Anpassungen vor, um den unterschiedlichen Bedürfnissen der Schüler*innen gerecht zu werden. Ein Beispiel sind alternative Kommunikationstechniken, die parallel zur gesprochenen Sprache eingesetzt werden, wobei auch die Klassenkamerad*innen die Nutzung von unterstützter Kommunikation erlernen, was wiederum die sozialen Aspekte der Schultage erleichtert. Weitere Beispiele sind die Änderung der Unterrichtsmethoden und die Verwendung von IuK (Informations- und Kommunikationstechnik) in verschiedenen Formen, wobei der Fokus stets auf der Wissensentwicklung der Schüler*innen in den verschiedenen Fächern liegt. Schüler*innen mit unterschiedlichen Diagnosen oder Lernschwierigkeiten können zusammen mit ihren Klassenkamerad*innen am allgemeinen Unterricht teilnehmen. Spezialisierte Lehrer*innen können bei Bedarf auch stets mit Fachlehrer*innen im Klassenzimmer zusammenarbeiten.

Eine weitere schwedische Studie zeigt eine Gemeinde, die sich gewandelt hat. Innerhalb von drei Jahren stieg sie von einer Position am Ende des nationalen Bildungsrankings nach oben auf - durch die Einführung einer inklusiven Bildungsreform. Inklusion ist nicht nur aus der Perspektive der Gleichstellung gerechtfertigt, sondern kann auch allen Schüler*innen zum Erfolg und zur Verbesserung ihrer Leistung verhelfen (Persson 2013). Die Aktivitäten der Schule gingen von der Allgemeinen Erklärung der Menschenrechte der Vereinten Natio- 
nen und unserer gemeinsamen Verantwortung für Nachhaltigkeit in einer globalen Perspektive aus. Ein Modell des sozialen Kapitals wurde verwendet, um die Perspektiven und Erfahrungen der Schüler*innen zu interpretieren und es zeigten sich die starken Normen und Werte, denen die Schüler*innen ausgesetzt waren, und die Verbindungen, Brücken und Verknüpfungspraktiken, die sie bedeutungsvoll miteinander verbanden (Schuller et al. 2000).

Eine Stichprobe von 20 Schüler*innen wurde zu ihren Erfahrungen in dieser inklusiven Lernumgebung befragt. Die Schüler*innen formulierten ein starkes Engagement, anderen zum Erfolg verhelfen zu wollen, und ein klares Verständnis und Selbstvertrauen bezüglich der eigenen Lernprozesse. Sie schätzten den Respekt für Vielfalt, Toleranz und Solidarität mit den verletzlichsten Personen und entwickelten Reife sowie Ansätze für ihre eigene Rolle in einer herausfordernden Zukunft. Diese Ergebnisse haben Konsequenzen für die Praxis, einschließlich des Verständnisses von inklusiver Bildung als notwendiger Weg, um jungen Menschen zu helfen, in einer unsicheren Zukunft für eine nachhaltige Welt zu arbeiten.

\subsection{Spanien: Werte der Inklusion und ethische Anleitung zur Förderung der Zusammenarbeit aller Beteiligten}

Spanien verfügt über eine territoriale und politische Struktur, die sich aus 17 autonomen Gemeinden und zwei autonomen Städten (Ceuta und Melilla) zusammensetzt, mit einer großen Vielfalt an Steuerung und Ausrichtung ihrer eigenen Bildungspolitik im Rahmen eines gemeinsamen staatlichen Gesetzes (LOMCE 2013) - das ist Deutschlands Bundesländern sehr ähnlich (vgl. Blanck/Edelstein/Powell 2013; Schäfer 2021 i.d.B.). Daher sind auch in diesem Land die Unterschiede zwischen den autonomen Gemeinden in Bezug auf die Verpflichtung zu inklusiver Bildung beachtlich. Das geltende Rahmengesetz hat jedoch einen eindeutig inklusiven Ansatz: So fordert Art. 1 Gerechtigkeit, die Chancengleichheit, inklusive Bildung und Nichtdiskriminierung garantiert, sodass Bildung als kompensatorisches Element für persönliche, kulturelle, wirtschaftliche und soziale Ungleichheiten fungieren kann, wobei behinderungsbedingten Ungleichheiten besondere Aufmerksamkeit gewidmet wird. Diese rechtliche Anerkennung bezieht sich auf das bereits seit Mitte der 1990er Jahre bestehende Engagement Spaniens für die „Inklusion“, gemäß der Weltkonferenz zur Sonderpädagogik: Zugang und Qualität 1994 (UNESCO 1994) - abgehalten in Salamanca (Spanien) (Saleh 2004).

Dennoch, wie im Vergleich zu anderen Ländern hervorgehoben wurde (Ainscow 2016) und unter Anerkennung der wichtigen Fortschritte bei der Integration von Schüler*innen, die am stärksten von Ausgrenzung betroffen sind (diejenigen, die sonderpädagogischen Förderbedarf haben), ist die große Kluft zwischen formal festgelegten Verpflichtungen und der Realität hartnäckig (Echeita 2017). Gleichzeitig gibt es im ganzen Land und ähnlich wie in anderen an TdiverS teilnehmenden Ländern viele inspirierende Beispiele für die Möglichkeit einer inklusiven Bildung, die zur Entwicklung einer Gesellschaft mit größerer sozialer Gerechtigkeit beiträgt.

Wir haben eng mit drei Schulen in Madrid zusammengearbeitet, um die zentralen Elemente zu ermitteln, die in ihrer/ihren Schulkulturen, -richtlinien und -pädagogiken verändert wurden (Booth/Ainscow 2011; Florian/Linklater 2010), um die Vielfalt der Schüler*innen $\mathrm{zu}$ begrüßen und anzuerkennen und um mit Maßnahmen zur Förderung von Gerechtigkeit und zur Wertschätzung der Vielfalt der Schüler*innen, die diese Schulen besuchen (Simón et al. 2016), zu reagieren. Im Rahmen eines kollaborativen Ansatzes zwischen Schulpersonal 
und Forscher*innen von Universitäten (Messiou 2017) wurden Fokusgruppen durchgeführt, die einer qualitativen Inhaltsanalyse unterzogen wurden.

Gemäß der Analyse von Tyack und Cuban (1995) passt die Entwicklung einer inklusiveren Bildung nicht in den Rahmen der „Grammatik der Schule“, der unsere formalen Bildungssysteme definiert hat. Die Ergebnisse zeigen die Haupthindernisse und Herausforderungen auf, die sich den Schulen stell(t)en, um diese Veränderungsprozesse in Richtung ihrer Ziele voranzutreiben: eine ,dialogische Kultur“ unter den Stakeholdern, die auf starken inklusiven Prinzipien und Werten beruht; mehr kooperative Arbeit unter den Lehrer*innen (einschließlich derjenigen mit Unterstützungsfunktionen), nicht nur bei der Vorbereitung des Unterrichts, sondern auch bei der Realisierung im Klassenzimmer; Legen des Schwerpunktes auf die kooperative Arbeit unter Schüler*innen; multidisziplinäre, projektbezogene Arbeit in Schulfächern sowie flexible Strukturen zur Anpassung an Lernbedürfnisse und -erwartungen.

Als wichtigste Erkenntnis aus der Auseinandersetzung mit den kooperierenden Schulen kann festgehalten werden, dass ethische Leitung, Zusammenarbeit zwischen den Interessengruppen auf allen Ebenen und angemessene Schulbedingungen erforderlich sind, um bestehende Barrieren, Schulstrukturen, Lehrpläne und Bewertungspraktiken zu durchbrechen und zu ersetzen. Wenn dies nicht der Fall ist, bleibt die inklusive Bildung ein Ideal außerhalb der Reichweite der Schulen.

\section{Implikationen aus TdiverS für Forschung und Politikgestaltung in Bezug auf Inklusive Bildung}

Zusammenfassend stellten wir fest, dass deskriptive, kumulierte Statistiken Paradoxien überdecken: zum Beispiel können das berufliche Engagement und die Entwicklung von inklusiver Bildung und Co-Teaching unterstützt werden, ohne dass zeitgleich die Quote der Sonderbeschulung insgesamt sinkt (Deutschland). Selbst in inklusiven, schüler*innenzentrierten und auf Kooperation ausgerichteten Schulen bleibt das Egalisieren der Möglichkeiten und das Wohlbefinden der Schüler*innen ein zentrales Ziel der Schulentwicklung (Island). Wenn Lehrer*innen Lernende als Kooperationspartner unterstützen, insbesondere in gut ausgestatteten, individualisierten und in mehrfacher Hinsicht barrierefreien Klassenräumen, verbessert sich die schulische Bildung (Litauen). Inklusion aus intersektionaler Perspektive und dialogisches unterrichten in kulturell diversen, mehrsprachigen Schulen wird durch alternative Bewertung, Portfolio-Arbeiten und schülerzentrierte Pädagogik erleichtert (Luxemburg). Die grundlegenden Werte der Inklusion sowie eine ethische Leitung zur Förderung der Zusammenarbeit zwischen allen Stakeholdern sind für die langfristige Entwicklung einer inklusiven Bildung von entscheidender Bedeutung (Spanien). Die Schüler*innen sind bereit, Vielfalt zu respektieren, Toleranz zu entwickeln und Solidarität zu fördern, was inklusive Bildung unterstützt, die im Gegenzug Gleichheit und Leistung ermöglicht (Schweden). Die wissenschaftliche Bedeutung dieser Ergebnisse aus Forschung und Praxis wurde mit dem Material für die Lehrer*innenbildung in Englisch und dem halben Dutzend Sprachen, die von den Partnerländern in TdiverS vertreten werden, veröffentlicht: als Sammlung mehrsprachiger, multidisziplinärer Ressourcen zum Unterrichten von diversen Lernenden im (Fach-) unterricht in inklusiven Settings (TdiverS USB-Flash-Laufwerk). 
In diesem Beitrag wurden kurz unsere Erfahrungen und Erkenntnisse zusammengefasst, die aus der dreijährigen Zusammenarbeit mit ausgewählten Schulen und Lehrer*innenteams in Bezug auf wirksame Konzepte, Didaktik und Unterrichtsmethoden gewonnen wurden und die den Unterricht von Lehrer*innen und die Entwicklung von Lernenden in inklusiven Klassenräumen unterstützen sollen. Interessenvertreter, politische Entscheidungsträger und die Öffentlichkeit waren durch öffentliche Konferenzen und die Publikation von (vorläufigen) Erkenntnissen im Rahmen des Projekts beteiligt.

In den teilnehmenden Ländern arbeiteten wir gemeinschaftlich mit Universitäten, Organisationen der Lehrer*innenausbildung und ausgewählten Schulen, Lehrer*innen, Schulleiter*innen und Eltern. Während des kooperativen Prozesses und des Dialogs mit reflektierenden Praktiker*innen, Eltern und Schüler*innen der inklusiven Schulen haben wir nützliche Kenntnisse über inklusives Lernen gewonnen. In gewisser Weise sind sich Lehrkräfte oft nicht bewusst, welche Kompetenzen und inklusiven Praktiken sie in ihrer alltäglichen Praxis entwickelt haben. Bei der Rekonstruktion im Rahmen des dialogischen Ansatzes wurden diese Kompetenzen und Adaptationen herausgearbeitet und konnten bei der Diskussion der Videosequenzen der eigenen Unterrichtspraxis häufig zum ersten Mal öffentlich reflektiert werden. Beispielsweise fasste ein Team, bestehend aus einer Grundschullehrerin und einer Sonderschullehrerin, die in einem inklusiven Klassenzimmer (in Deutschland) arbeiten, seine Erfahrungen nach einem einstündigen Interview über den gemeinsamen Unterricht und die Teamarbeit folgendermaßen zusammen:

Dies war das erste Mal, dass wir intensiv und explizit über ,,das, was wir tun “ und „,wie wir es tun“ sowie „das, was wir gelernt haben“ gesprochen haben, und dies hat zu einem größeren Bewusstsein für die bereits professionell geleisteten Dinge, aber auch über die Dinge, über die wir in Zukunft noch viel lernen müssen, geführt.

Da die Videosequenzen und mit ihnen die Lehrkonzepte und Lernsituationen in den Situationen von den Lehrer*innen selbst authentisch beschrieben wurden, waren die dahinterliegenden (teilweise impliziten) Theorien klar zugänglich. Die Präsentation persönlicher Praktiken als Lehrer*in, Schulleiter*in oder als Vertreter*in einer Organisation (manchmal unterstützt durch, oder in Verbindung mit, theoretischen Ressourcen) gehört zu den Projekterfolgen, die in der Lehrer*innenbildung einen Unterschied bewirken können: reflektiertes Erfahrungswissen wird damit für die Ausbildung anderer Lehrer*innen zur Verfügung gestellt, dies ist in Materialien für die Lehrer*innenbildung nicht oft der Fall.

Wir sind der Auffassung, die an der Lehrer*innenbildung beteiligten Personen müssen die Ideale und Prinzipien der Inklusion - inklusive deren Umsetzung in spezifischen Kontexten - reflektieren, damit es möglich wird die konkreten Effekte zwischen Lehrer*innenbildungscurricula, eigener Seminargestaltung und dem Aufbau von Kompetenzen bei den Studierenden einzuschätzen, mit dem Ziel die Selbstwirksamkeitserwartung der angehenden Lehrer*innen im Hinblick auf das Unterrichten von Schüler*innen mit unterschiedlichen Lernniveaus und -stufen in inklusiven Umgebungen zu erhöhen (Merz-Atalik 2014). Neben der Verwendung von Videomaterial mit inspirierenden Praktiken des inklusiven Unterrichts in den Bildungsbereichen ist es erforderlich, zahlreiche und aktive professionelle Erfahrungen in differenten inklusiven Situationen und Strategien für alle angehenden und bereits praktizierenden Lehrer*innen zu ermöglichen, um sich über ihre bedeutsame Rolle bei der Förderung der Inklusion bewusst zu werden (z.B. durch Schulhospitationen, Exkursionen, Gastreferent*innen aus der Praxis).

Länderübergreifende Kooperationsprojekte wie TdiverS fördern den vertieften Vergleich, die Zusammenarbeit und das Verständnis zwischen Vertreter*innen von Ländern mit 
unterschiedlichen Bildungssystemen und sehr unterschiedlichen Institutionalisierungspfaden inklusiver Schulbildung (Merz-Atalik 2017; Merz-Atalik/Tiemann/Weber 2017; MerzAtalik/Weber 2017). Ein solcher Austausch erweist sich als bedeutsam für die aktuelle Inklusionsforschung und inklusive Bildungsreformen. In diesen sechs Ländern betonten Professionelle des Bildungssystems sowie Familien die Notwendigkeit, zusammenzuarbeiten und Perspektiven auszutauschen, wobei selbst die bereits inklusivsten Schulen und Gemeinschaften die Inklusion eher als einen laufenden Prozess denn als einen erreichten Status betrachteten. Ressourcen verschiedener Quellen und Vernetzungsmöglichkeiten über organisatorische und institutionelle Grenzen hinweg unterstützen den Fortschritt bei der Verwirklichung einer inklusiveren Schulbildung. Dies ist besonders wichtig in solchen Kontexten, in denen die Systemlogik auf die Platzierung in speziellen Einrichtungen ausgerichtet bleibt, was mit einem inklusiven Schulwesen nicht vereinbar ist. Dies schließt ebenso die (bildungs)politischen Sphären ein, welche die inklusive Bildung umgeben, zumal sich die Herausforderungen zeitlich und im regionalen Umfeld dauerhaft verändern. Vergleiche bieten Lernmöglichkeiten: so können Gemeinsamkeiten in Bezug auf die Herausforderungen erkannt werden, die auf allen Ebenen gegenwärtig sind, während gleichzeitig erfolgreichere Reformansätze andernorts und ,inspirierende Praktiken“ von anderswo als Modelle und Unterstützung für Innovation dienen.

Auch wenn insgesamt inklusive Reformen in ganz Europa vorangekommen sind, obschon in unterschiedlichem Tempo und in unterschiedlichem Ausmaß, sollten sie nicht als selbstverständlich betrachtet, sondern wertgeschätzt werden. Politische Entscheidungsträger*innen, Forscher*innen, Praktiker*innen und Schulverwaltungsmitarbeiter*innen sind gemeinsam dafür verantwortlich, die Nachhaltigkeit der durchgeführten Reformen zu einer inklusiveren Gestaltung von Schulbildung sicherzustellen. Eine solche Reform steht im Einklang mit der globalen Norm der inklusiven Bildung als Menschenrecht und bekennt sich zu den Chancen von Vielfalt in der Bildung.

\section{Literatur}

Ainscow, Mel (2016): Diversity and equity. A global challenge. In: New Zealand Journal of Educational Studies 51, 2, S. 143-155.

Ainscow, Mel/Booth, Tony/Dyson, Alan (2006): Improving schools, developing inclusion. London: Routledge.

Ališauskas, Algirdas/Ališauskienė, Stefanija/Gerulaitis, Darius/Meliene, Rita/Milteniene, Lina/ Šapelytė, Odeta (2009): Special educational support. In: Specialusis ugdymas/Special Education 1, 20, S. 131-143.

Ališauskas, Algirdas/Ališauskienė, Stefanija/Miltenienė, Lina (2013): Meeting children's special educational needs in school. A Lithuanian case. In: Society, integration, education. Proceedings of the international scientifical conference 24-25, Vol. II, Rēzekne: Rēzeknes Augstskola, S. 113-125.

Artiles, Alfredo J./Kozleski, Elizabeth/Waitoller, Federico R. (Hrsg.) (2011): Inclusive education. Examining equity on five continents. Cambridge, MA: Harvard Education Press.

Autorengruppe Bildungsberichterstattung (2016): Bildung in Deutschland 2016. Bielefeld: Bertelsmann. 
Biermann, Julia/Powell, Justin J.W. (2014): Institutionelle Dimensionen inklusiver Schulbildung. Herausforderungen der UN-Behindertenrechtskonvention für Deutschland, Island und Schweden im Vergleich. In: Zeitschrift für Erziehungswissenschaften 17, 4, S. 679-700.

Blanck, Jonna M./Edelstein, Benjamin/Powell, Justin J.W. (2013): Persistente schulische Segregation oder Wandel zur inklusiven Bildung? Die Bedeutung der UN-Behindertenrechtskonvention für Reformprozesse in den deutschen Bundesländern. In: Schweizerische Zeitschrift für Soziologie 39, 2, S. 267-292.

Booth, Tony/Ainscow, Mel (2011/2017): Index for inclusion. Developing learning and participation in schools. Bristol: CSIE.

Brendel, Michelle/Noesen, Melanie (2014): Portfolioarbeit und Entwicklung inklusiven Unterrichts. In: Schuppener, Saskia/Hauser, Mandy/Bernhardt, Nora/Poppe, Frederik (Hrsg.): Inklusion und Chancengleichheit. Bad Heilbrunn: Klinkhardt, S. 244-255.

Echeita, Gerardo (2017): Educación inclusiva. Sonrisas y lgrimas. In: Aula Abierta 46, S. 17-24.

Edwards, Carolyn/Gandini, Lella/Forman, George (2012): The hundred languages of children. The Reggio Emilia experience in transition. Santa Barbara, CA: Praeger.

EADSNE - European Agency for Development in Special Needs Education (2012): Profile of inclusive teachers. Odense, DK: EADSNE.

EASNIE - European Agency for Special Needs and Inclusive Education (2015): European hearing. Luxembourg recommendations - inclusive education take action! Odense, DK. https:// www.european-agency.org/sites/default/files/Luxembourg_Recommendations_Flyer_EN.pdf [Zugriff: 02.08.2020]

EASNIE - European Agency for Special Needs and Inclusive Education (2017a): European Agency statistics on inclusive education: 2014 dataset cross-country report. (Ramberg, J./Lénárt A./Watkins, A. (Hrsg.)). Odense, DK: EASNIE.

EASNIE - European Agency for Special Needs and Inclusive Education (2017b): Education for all in Iceland. External audit of the Icelandic system for inclusive education. Odense, DK: EASNIE.

Florian, Lani/Linklater, Holly (2010): Preparing teachers for inclusive education. Using inclusive pedagogy to enhance teaching and learning for all. In: Cambridge Journal of Education 40, 4, S. 369-386.

Guðjónsdóttir, Hafdis (2000): Responsive professional practice. Teachers analyze the theoretical and ethical dimensions of their work in diverse classrooms. Dissertation, University of Oregon.

Harris, Jess/Carrington, Suzanne/Ainscow, Mel (2017): Promoting equity in schools. Collaboration, inquiry and ethical leadership. Abingdon: Routledge.

Hart, Susan/Dixon, Annabelle/Drummond, Mary/McIntyre, Donald (2004): Learning without limits. Maidenhead: Open University Press.

Icelandic Ministry of Education, Science and Culture (2011): Aðalnmskr grunnskóla. Reykjavík, Iceland: Mennta- og Menningamlarðuneytið. http://www.menntamalaraduneyti.is/utgefidefni/namskrar/nr/3953 [Zugriff: 02.08.2020]

KMK (2011): Inklusive Bildung von Kindern und Jugendlichen mit Behinderungen in Schulen. Beschluss der Kultusministerkonferenz vom 20.10.2011. Bonn: Kultusministerkonferenz.

Lithuanian Law on Education [2011-03-17 No XI-1281]. http://www3.lrs.lt/pls/inter3/dokpaieska.showdoc_1?p_id=407836 [Zugriff: 02.09.2019].

Lithuanian National Education Strategy 2013-2022 [2013-12-23 No2 XII-745]. http://www.smm. lt/web/lt/teisesaktai/tei/valstybine-svietimo-strategija_1 [Zugriff: 02.09.2020].

LOMCE (2013): Ley orgnica para la mejora de la calidad educativa. https://www.boe.es/buscar/ pdf/2013/BOE-A-2013-12886-consolidado.pdf [Zugriff: 02.09.2020]

Ministère de l'Éducation nationale, de l'Enfance et de la Jeunesse (2017): Les chiffres-clé de l'education nationale. Statistiques et indicateurs 2015/2016. Luxembourg: MENJE. 
Merz-Atalik, Kerstin (2013a): Inklusion/Inklusiver Unterricht an der Gemeinschaftsschule. In: Bohl Thorsten/Meissner, Sibylle (Hrsg.): Expertise Gemeinschaftsschule: Forschungsergebnisse und Handlungsempfehlungen für Baden-Württemberg. Weinheim: Beltz Juventa, S. 6176.

Merz-Atalik, Kerstin (2013b): Der Forschungsauftrag aus der UN-Behindertenrechtskonvention Probleme, Erkenntnisse und Perspektiven einer Inklusionsforschung im schulischen Feld. In: Trumpa, Silke/Seifried, Stefanie/Franz, Eva-Kristina/Klauß, Theo (Hrsg.): Inklusive Bildung. Erkenntnisse und Konzepte aus Fachdidaktik und Sonderpädagogik. Weinheim, Germany: Beltz Juventa, S. 24-46.

Merz-Atalik, Kerstin (2014): Lehrer*innen_innenbildung für Inklusion - ,Ein Thesenanschlag“. In: Schuppener, Saskia/Hauser, Many/Bernhardt, Nora/Poppe, Frederik (Hrsg.): Inklusion und Chancengleichheit. Diversity im Spiegel von Bildung und Didaktik. Bad Heilbrunn, Germany: Klinkhardt, S. 266-277.

Merz-Atalik, Kerstin (2017): „WOW“ in Madrid. Interview zum Projekt TdiverS Teaching diverse learners in (School-)Subjects. In: E\&W, Zeitschrift der Gewerkschaft Erziehung und Wissenschaft, 7-8, S. 37.

Merz-Atalik, Kerstin (2018a): Inklusive Fachdidaktik - eine Kernaufgabe nicht nur für „inklusive“ Schulen. In: Olsen, Ralph/Hochstadt, Christiane: Handbuch Deutschunterricht und Inklusion. Weinheim: Beltz, S. 16-32.

Merz-Atalik, Kerstin (2018b): Von einem Versuch „der Integration der Inklusion in die Segregation“?!. Zeitschrift Für Inklusion 4, https://www.inklusion-online.net/index.php/inklusion-online/article/view/508 [Zugriff: 02.09.2020]

Merz-Atalik, Kerstin/Beuse, Kira/O'Brien, Chris (2016): Sentiments, concerns and attitudes towards inclusive education. In: Zeitschrift für Inklusion 1, https://www.inklusion-online.net/index.php/inklusion-online/article/view/313 [Zugriff: 02.09.2020].

Merz-Atalik, Kerstin/Tiemann, Heike/Weber, Katja (2017): Abschlussbericht zum Projekt TdiverS - Comenius Netzwerk „Teaching diverse learners in (School-)Subjects“. Unveröffentlichtes Manuskript.

Merz-Atalik, Kerstin/Weber, Katja (2017): Das Comenius Projekt „TdiverS“. In: Grundschule aktuell 137, 2, S. 48-49.

Messiou, Kyriaki (2017): Research on the field of inclusive education. Time for a rethink? In: International Journal of Inclusive Education, 21, 4, S. 146-159.

Miltenienè, Lina/Venclovaitè, Indrè (2012): Teacher collaboration in the context of inclusive education. In: Specialusis ugdymas/Special Education 27, 2, S. 111-123.

Óskarsdóttir, Edda/Köpfer, Andreas (2021 i.d.B.): Inklusive Unterstützungsstrukturen in Schulen in Island und Kanada. In: Köpfer, Andreas/Powell, Justin J.W./Zahnd, Raphael (Hrsg.): Handbuch Inklusion International. Opladen: Budrich, S. 277-295.

Persson, Elisabeth (2013): Raising achievement through inclusion. In: International Journal of Inclusive Education, 17, 11, S. 1205-1220.

Powell, Justin J.W. ([2011] 2016): Barriers to inclusion. Special education in the United States and Germany. Abingdon: Routledge.

Powell, Justin J.W. (2014): Comparative and international perspectives on special education. In: Florian, Lani (Hrsg.): The SAGE handbook of special education. London: Sage, S. 335-349. http://hdl.handle.net/10993/13854 [Zugriff: 02.09.2019]..

Powell, Justin J.W./Limbach-Reich, Arthur/Brendel, Michelle (2017): Grand Duchy of Luxembourg. In: Wehmeyer, Michael L./Patton, James R. (Hrsg.): The Praeger handbook of special education. Santa Barbara, CA: Praeger, S. 296-309.

Powell, Justin J.W./Merz-Atalik, Kerstin (2019): Die Notwendigkeit inklusiver Bildung für die Erneuerung der Governancekonzepte: Deutschland und Luxemburg im Vergleich. In: Budde, Jürgen/Dlugosch, Andrea/Herzmann, Petra/Rosen, Lisa/Panagiotopoulou, Julie A./Sturm, 
Tanja/Wagner-Willi, Monika (Hrsg.): Inklusionsforschung im Spannungsfeld von Erziehungswissenschaft und Bildungspolitik. Opladen: Barbara Budrich, S. 71-96.

Prengel, Annedore (2016): Humane structures in inclusive education foster development and achievement. In: Teaching Diverse Learners in (School) Subjects. USB flash drive. Ludwigsburg: TdiverS.

Richardson, John G./Powell, Justin J.W. (2011): Comparing special education. Origins to contemporary paradoxes. Stanford, CA: Stanford University Press.

Saleh, Lena (2004): Desde Torremolimos a Salamanca y ms all. Un tributo a España. In: Echeita, Gerardo/Verdugo, Miguel A. (Hrsg.): La Declaración de Salamanca sobre NEE 10 años después. Valoración y prospectiva. Salamanca: INICO, S. 23-34.

Schäfer, Lea (2021 i.d.B.): Maßnahmen inklusiver Bildung. In: Köpfer, Andreas/Powell, Justin J.W./Zahnd, Raphael (Hrsg.): Handbuch Inklusion International. Opladen: Budrich, S. 197220.

Schuller, Tom/Baron, Stephen/Field, John (2000): Social capital. A review and critique. In: Baron, Stephen/Field, John/Schuller, Tom (Hrsg.): Social capital. Critical perspectives. Oxford: Oxford University Press, S. 1-38.

Sigurðardóttir Anna K./Guðjónsdóttir, Hafdis/Karlsdóttir, Johanna (2014): The development of a school for all in Iceland. Equality threats and political conditions. In: Blossing, Ulf/Imsen, Gunn/Moos, Leif (Hrsg.): The Nordic education model. Heidelberg: Springer, S. 95-113.

Simón, Cecilia/Sandoval, Marta/Echeita, Gerardo/Calero, Cristóbal/Núñez, Beatriz/de Sotto, Pilar/Pérez, Monserrat M./García de la Torre, Ana Belén (2016): Transformando la ,gramatica escolar' para ser más inclusivos: La experiencia de tres centros educativos. In: Contextos Educativos. Revista de Educación 19, S. 7-24.

TdiverS (2015, 2016, 2017): Annual reports. http://www.tdivers.eu/downloads/ [Zugriff: 02.09. 2020].

Tomlinson, Sally (2017): A sociology of special and inclusive education. Abingdon: Routledge.

Tomlinson, Sally (2021 i.d.B.): Sociology of special and inclusive education: Insights from the UK, US, Germany, and Finland. In: Köpfer, Andreas/Powell, Justin J.W./Zahnd, Raphael (Hrsg.): Handbuch Inklusion International. Opladen: Budrich, S. 59-73.

Tyack, David/Cuban, Larry (1995): Tinkering toward utopia. Cambridge, MA: Harvard University Press.

UNESCO (1994): The Salamanca statement and framework for action on special needs education. Paris: UNESCO.

United Nations (2015): 2030 Agenda for sustainable development. http://www.un.org/sustainabledevelopment/development-agenda/ [Zugriff: 02.09.2020].

Zapp, Mike/Marques, Marcelo/Powell, Justin J.W. (2018): European Educational Research (Re)constructed: Institutional Change in Germany the United Kingdom Norway and the European Union. Oxford: Symposium Books. 

C Schule, Unterricht und Professionalisierung 



\title{
21 Schulleitungshandeln in integrations-/inklusionsorientierten Schulentwicklungsprozessen - Empirische Befunde aus der Schweiz und Deutschland
}

Benjamin Badstieber, Bettina Amrhein

\begin{abstract}
In einer differenztheoretischen und international vergleichenden Perspektive auf Inklusion untersucht der Beitrag sowohl erste bedeutsame Gemeinsamkeiten als auch Unterschiede des Schulleitungshandelns in Deutschland und der Schweiz. Davon ausgehend werden Perspektiven für eine international und transnational vergleichende Inklusionsforschung unter besonderer Berücksichtigung von Schulleitenden erarbeitet.
\end{abstract}

\section{Erkenntnisinteresse und einleitende Positionierungen}

Die Ausgestaltung eines inklusiven Bildungssystems ist eine zentrale bildungspolitische Aufgabe im internationalen Raum (Ainscow 2021 i.d.B.). Mit dem Aufkommen der Debatte um Integration/Inklusion in den 1980 Jahren stellen sich vermehrt Fragen nach einer heterogenitätssensiblen Ausgestaltung schulischer Strukturen, Praktiken und Kulturen (Booth/Ainscow 2011). Durch die Ratifizierung der UN-BRK (UN 2006) werden die seither erarbeiteten Forderungen in der schulischen Praxis und auch in der wissenschaftlichen Debatte aus einer menschenrechtlichen Perspektive verstärkt vorgetragen (Biermann 2021 i.d.B.; Heyer 2021 i.d.B.) und inklusionsorientierte Veränderungen insbesondere im schulischen Kontext verpflichtend eingefordert (Deutsches Institut für Menschenrechte 2015). Die vorrangig bottomup entstandenen Prozesse und formulierten Forderungen werden in Deutschland und der Schweiz zunehmend durch bildungspolitische und administrative Top-down-Strategien ergänzt. Waren es zunächst insbesondere die Eltern und einzelne Schulen, die sich für eine inklusionsorientierte Ausgestaltung engagierten, wird nun auch von Seiten der Bildungspolitik und der Bildungsadministrationen etwa durch Schulgesetzänderungen versucht, inklusionsorientierte Schulentwicklungsprozesse zu initiieren.

Es erfolgt eine auch für Menschenrechtsabkommen schnelle und starke Verbreitung der UN-BRK und der Begrifflichkeit ,inclusion“ als Menschenrecht im internationalen Raum (Degener 2009: 200). Obwohl damit eine weitergehende Formalisierung und rechtliche Verankerung inklusionsorientierter Bildung im internationalen Kontext geleistet wurden, bleiben die menschenrechtlichen Forderungen im nationalen Kontext interpretationsbedürftig und weitestgehend an das dortige (bildungspolitische) Engagement der Unterzeichnerstaaten gebunden. Trotz unterschiedlicher Strategien zur Erhöhung der internationalen Verbindlichkeit 
der UN-BRK (z.B. einer schriftlichen Berichterstattung, formale Ratifizierung, etc.) „stehen den Vereinten Nationen wohl keine mächtigen Mittel zur Verfügung, die Umsetzung dieser Normen in den Beitrittsstaaten durchzusetzen" (Altrichter/Feyerer 2011).

Demzufolge obliegt es den unterschiedlichen Akteur*innen in den nationalen Schul- und Bildungssystemen das Recht auf einen integrativen/inklusiven Unterricht zu interpretieren und zu realisieren. Die Frage, inwieweit und in welcher Ausrichtung die Forderungen nach Inklusion eine Transformation der bestehenden Schul- und Bildungssysteme verlangen, wird entsprechend im internationalen Vergleich aber auch auf nationaler Ebene höchst unterschiedlich und mitunter überaus kontrovers diskutiert (Amrhein 2011: 15ff; Boban/Hinz 2009; Böing/Köpfer 2016; Feuser 2016; Feuser 2017; Hinz 2013).

Die Positionen in Wissenschaft und schulischer Praxis reichen hier seit Beginn der Debatten von der systemkonformen Einpassung von sonderpädagogischen Unterstützungsleistungen in die bestehenden Strukturen und Praktiken der allgemeinbildenden Schulen (kritisch Boban/Hinz 2009; Hinz 2008: 37ff.; Hinz 2013), ohne diese grundlegend verändern zu wollen, bis hin zur Forderung nach einer grundlegenden Revision derselbigen und ihrer Transformation mit Blick auf alle Schüler*innen bzw. unter Berücksichtigung unterschiedlicher Differenzlinien (Badstieber 2020; Feuser 2016; Feuser 2017).

In dem vorliegenden Beitrag soll auf die Entwicklungen zweier deutschsprachiger Länder, der Schweiz (s. Mejeh 2021 i.d.B.) und Deutschlands (s. Gasterstädt 2021 i.d.B.), näher eingegangen werden (Powell/Hadjar 2019). In einer Mehr-Ebenen-theoretischen-Perspektive (Fend 2008a; 2008b) soll nach einem kurzen Überblick über die bildungspolitischen Entscheidungen und verwaltungsorganisatorischen Maßnahmen dabei insbesondere die zentrale Akteur*innengruppe der Schulleitenden näher betrachtet werden. Wie internationale Befunde zeigen, übernehmen diese in den nationalen Schul- und Bildungssystemen eine zentrale Schnittstellenfunktion zwischen inner- und außerschulischer Gestaltungsbemühung und damit insbesondere auch in der Übersetzung bildungspolitisch und administrativ gesteuerter Veränderungsbemühungen auf Ebene der Einzelschule (Badstieber/Moldenhauer 2016).

So soll anhand exemplarischer Analysen herausgestellt werden, wie die Schulleitenden unter den jeweiligen nationalen bildungspolitischen und administrativen Vorgaben den Auftrag integrations- bzw. inklusionsorientierter Schulentwicklungsbemühungen interpretieren und bearbeiten. Die rekonstruktiven Analysen (vgl. Sturm 2013) erfolgen dabei unter Rückgriff auf ausgewählte Daten eines umfangreichen Forschungsprojektes in Deutschland mit dem Titel „Mit Schulleitung inklusive Schule gestalten“ (Amrhein/Badstieber 2019; Badstieber 2020) und durch einen explorativen Blick auf die Erkenntnisse zum Schulleitungshandeln im Kontext integrations- bzw. inklusionsorientierter Schulentwicklung in der Schweiz (Köpfer 2015; Köpfer/Mejeh 2017). In diesem Sinne positioniert sich der Beitrag innerhalb einer differenztheoretischen Perspektive auf Inklusion, ,,in der Behinderungen/Benachteiligungen, wie auch Ermöglichungen als Produkte der Praktiken, Strukturen und Kulturen eines Feldes gefasst" (Köpfer 2019: 146) werden und sich der Blick auf die behindernden/benachteiligenden Barrieren in den institutionellen Strukturen und Praktiken der Akteur*innen in Schule richtet.

Durch eine international vergleichende Perspektive werden mit Blick auf die Schulleitenden sowohl erste bedeutsame Gemeinsamkeiten als auch Unterschiede zwischen Deutschland und der Schweiz exploriert und damit Anforderungen an ein inklusionsorientiertes Schulleitungshandeln identifiziert (Kugelmass/Ainscow 2004). Durch einen explorativen, international vergleichenden Blick lässt sich hier in einer ersten Andeutung herausarbeiten, ob die Schulleitenden unter den jeweiligen bildungspolitischen und administrativen Vorgaben 
in der Schweiz und in Deutschland die inklusionsorientierten Schulentwicklungsprozesse unterschiedlich ausgestalten oder ob sich übergreifend ähnliche Anforderungen erkennen lassen.

Auf eine darüberhinausgehende, explizite zusammenfassende Darstellung der internationalen Forschungsergebnisse im Kontext Schulleitung und Inklusion bzw. Integration wird in diesem Artikel hingegen verzichtet. Hier liegen bereits ausführliche Beiträge vor (Badstieber et al. 2018; Badstieber/Moldenhauer 2016; Scheer et al. 2014; Sturm et al. 2015).

\section{Entwicklungen auf bildungspolitischer und administrativer Ebene und der Anspruch schulischer Integration/Inklusion}

Es kann davon ausgegangen werden, dass die bildungspolitischen und administrativen Vorgaben in der Schweiz und in Deutschland für das jeweilige Schulleitungshandeln von zentraler Bedeutung sind (Fend 2008b). Entsprechend werden in diesem Kapitel zunächst die bildungspolitischen und administrativen Vorgaben in der Schweiz und Deutschland als relevante Rahmenbedingungen für die Schulleitenden skizziert, bevor die empirischen Ergebnisse zum Schulleitungshandeln dargestellt werden.

\subsection{In der Schweiz}

In der Schweiz wird vorrangig der Begriff der „Integration“ verwendet und die Forderungen nach Integration werden vor allem unter der Berücksichtigung der Differenzlinie des sogenannten besonderen Bildungsbedarfs seit geraumer Zeit diskutiert (Bless 2007; Bless/Kronig 1999; Mejeh 2021 i.d.B.). Auf bildungspolitischer Ebene gewinnt die integrative Beschulung von Schüler*innen mit einem besonderen Bildungsbedarf an allgemeinbildenden Schulen zunehmend an Bedeutung. Wichtige Meilensteine bilden hier das Behindertengleichstellungsgesetz (BeHiG 2002) und das Sonderpädagogik-Konkordat (EDK 2007), die einer integrativen Beschulung gegenüber separierten Sonderbeschulung den Vorzug geben und eine sukzessive Ausweitung sonderpädagogischer bzw. heilpädagogischer Unterstützungen an allgemeinbildenden Schulen proklamieren.

Folgt man Achermann et al. (2017), erweisen sich ausgehend von diesen Entwicklungen die aktuellen bildungspolitischen Bemühungen integrationsorientierter Veränderungen im Schul- und Bildungssystem der Schweiz als überaus unübersichtlich. Die Schweiz hat die UN-BRK im April 2014 ratifiziert (Schweizerischer Bundesrat 2014) und im Jahr 2016 einen „Ersten Bericht der Schweizer Regierung über die Umsetzung der BRK“ vorgelegt (Schweizerischer Bundesrat 2016). Darin werden weitreichende bildungspolitische und administrative Umstrukturierungen auf nationaler Ebene (eher) nicht adressiert. Mit Blick auf die Beschulung von Schüler*innen mit besonderem Bildungsbedarf wird festgehalten, dass das „Recht auf einen angemessenen und unentgeltlichen Grundschulunterricht und die Gewährleistung eines qualitativ hochstehenden Aus- und Weiterbildungsangebots für alle Menschen, auch für Menschen mit Behinderungen, [...] im Zentrum des schweizerischen Bildungsrechts“ stehen und „eine ausreichende Sonderschulung für Kinder und Jugendliche mit 
Behinderung bis zum vollendeten 20. Altersjahr entweder in Sonderschulen, in besonderen Kleinklassen oder integrativ in Regelklassen unter Beizug heilpädagogischer Dienste ermöglicht wird" (Schweizerischer Bundesrat 2016). Eine Priorisierung der Beschulungsform wird hier nicht ersichtlich. Passend dazu stellt Hollenweger (2016) mit Blick auf die Frage nach der Bedeutung der Behindertenrechtskonvention (BRK) für die Schweiz zwei Jahre nach ihrem Inkrafttreten fest, dass die UN-BRK bis heute politisch, medial und gesellschaftlich eine eher untergeordnet Rolle spielt (vgl. auch Acherman et al. 2017). Auch die Behindertenverbände verweisen darauf, dass eine ,umfassende, kohärente Strategie zur Umsetzung der BRK“ (Inclusion Handicap 2017) in der Schweiz bisher fehlt.

Der Begriff Inklusion im Sinne der Behindertenrechtskonvention (BRK) spielt tatsächlich in der bildungspolitischen Debatte in der Schweiz keine bzw. eine deutlich untergeordnete Rolle (ebd.). Handlungsleitend für den Schweizer Raum ist auch nach der UN-BRK der Begriff der Integration (Acherman et al. 2017). Im Zentrum der, vergleichsweise verhaltenen, gesellschaftlichen und bildungspolitischen Debatten stehen entsprechend Fragen der sonderpädagogischen Unterstützung von Schüler*innen mit einer Behinderung. Eine darüberhinausgehende Perspektive auf andere Differenzlinien bzw. eine intersektionale Ausrichtung werden auch unter dem Begriff Integration nicht ersichtlich.

So stellen Acherman et al. (2017) fest, dass die Schweizer Kantone nach wie vor ein sehr selektives Schulsystem mit stark ausdifferenzierten, personell, finanziell und räumlich gut ausgestatteten Sonderschulen vorzuweisen haben und verweisen insbesondere auf die Studien von Kronig (2007) und Hofstetter (2017). Auch Mejeh und Powell (2018) kommen in ihrem Beitrag zur Institutionalisierung Inklusiver bzw. Integrativer Bildung in verschiedenen Regionen der Schweiz zu dem Schluss, dass zwar ein inkrementeller Rückgang segregierter Beschulung im schweizerischen Bildungssystem abbildbar ist, jedoch die Segregationsquote insgesamt vergleichsweise hoch bleibt (ebd.: 426) und in den Kantonen ,,persistente Bildungsungleichheiten" (ebd.: 412) festzustellen sind.

Besonders zu berücksichtigen ist dabei sicherlich die föderalistische Struktur, in der die staatliche Hoheit für die Sicherstellung und Ausgestaltung des schweizerischen Schul- und Bildungssystems auf die Ebene der 26 Kantone delegiert wurde. Die Souveränität der Kantone im Bereich der Bildung ist in der Schweiz entsprechend auch im Kontext integrationsbzw. inklusionsorientierter Schulentwicklungsprozesse - anders als in Deutschland - nahezu unbestritten. Auch mit Blick auf die ,gesamte fachliche, rechtliche und finanzielle Verantwortung für die Schulung von Kindern und Jugendlichen mit besonderem Bildungsbedarf" (EDK 2007) tragen die Kantone ausdrücklich die alleinige Verantwortung.

Zwar wurde durch die EDK (2007) eine Harmonisierung der sonderpädagogischen Grundangebote angestrebt, allerdings zeigt sich für die Deutschschweiz eine beachtliche Varianz in den Integrationskonzepten, v.a. in den Ressourcen- und Pensenmodellen (Kummer-Wyss 2012). Eine schweizweite Systematisierung fehlt bislang (Huber et al. 2017: 43).

Als besonders erklärungsbedürftig bezeichnen Mejeh und Powell (2018) entsprechend die teilweise extremen kantonalen Unterschiede und verweisen u.a. auf historische, pfadabhängige Entwicklungen in den Kantonen und Unterschiede zwischen städtischen und ländlichen Gebieten. Achermann et al. (2017) kommen in ihrem Beitrag zu dem Schluss, dass obwohl es kaum möglich ist, nationale inklusive Entwicklungen zu erkennen und darzustellen, eine große Vielfalt beispielhafter, singulärer Entwicklungen in verschiedenen Kantonen sichtbar werden, die innerhalb der eher verhaltenen Entwicklungen bemerkenswerte integrationsbzw. inklusionsorientierte Potentiale in der Schweiz erkennen lassen. 


\subsection{In Deutschland}

Im Vergleich zu den Entwicklungen in der Schweiz erfährt die Debatte um Integration/Inklusion im Voraus und insbesondere auch im Nachgang der Ratifizierung der UN-BRK im Jahr 2009 eine enorme gesellschaftliche und bildungspolitische Resonanz in Deutschland. Aus Platzgründen kann an dieser Stelle kein Überblick über die Entwicklungen in Deutschland gegeben werden. Die bundes- und bundeslandspezifischen Entwicklungen innerhalb des auch in Deutschland föderal organisierten Schul- und Bildungssystems wurden an unterschiedlichen Stellen bereits ausführlich zusammengefasst (Gasterstädt 2021 i.d.B.; Lange 2017) und kritisch reflektiert (Feuser 2017; Hinz 2013). Dabei erweisen sich nicht nur die bildungspolitischen Entwicklungen in den Bundesländern als überaus different, sondern auch die methodologischen Überlegungen für eine mögliche Standortortbestimmung und Prozessbegleitung (Preuss-Lausitz 2019).

Bedeutsam erscheint, dass im Kontrast zu den bildungspolitischen Entwicklungen in der Schweiz der Begriff Inklusion spätestens mit der Ratifizierung der UN-BRK und der von NETZWERK ARTIKEL 3 e.V. im deutschsprachigen Raum erstellten ,Schattenübersetzung“ (Deutsches Institut für Menschenrechte 2019) umfassend Einzug in die bildungspolitische Debatte in Deutschland erhält.

Unter dem Leitbegriff Inklusion werden in allen Bundesländern, wenn auch in unterschiedlicher Intensität und Ausführlichkeit Schulgesetzänderungen geplant und sogenannte allgemeinbildende Schulen zur Aufnahme von (ausgewählten) Schüler*innen mit attestierten sonderpädagogischen Förderbedarfen verpflichtet (Blanck 2014; BRK-ALLIANZ 2013; Mißling/Ückert 2014). Der Begriff Integration wird mit Blick auf die Differenzlinie Behinderung nicht zuletzt auf Kosten einer Vernachlässigung bereits bestehender Konzeptionen (Feuser 2012: 6) dabei nahezu vollständig aus dem Diskurs verdrängt, während er als Begriff etwa mit Blick auf Fragen der Migration und Flucht nach wie vor Verwendung findet.

Gleichzeitig verstärken sich Tendenzen einer uneinheitlichen Begriffsverwendung um Inklusion. Insbesondere Wissenschaftler*innen, die sich bereits vor der Ratifizierung der UN-BRK mit der Begrifflichkeit Inklusion auseinandergesetzt haben, verweisen auf den zunehmend inflationären Gebrauch (Amrhein 2011: 15ff; Boban/Hinz 2009; Böing/Köpfer 2016; Feuser 2016; Feuser 2017; Hinz 2009; Hinz 2013; Wocken 2011). So steht die starke inklusive Rhetorik in der schulischen, bildungspolitischen und gesellschaftlichen Programmatik in einem erheblichen Spannungsfeld zu den letztlich geplanten und umgesetzten (bildungspolitischen) Aktivitäten. Diese setzen die Forderungen schulischer Inklusion vorrangig im Sinne einer teilweisen „De-Segregation“ (Hinz 2009) und im Sinne eines Gemeinsamen Lernens von ausgewählten Schüler*innen mit sonderpädagogischen Förderbedarf an allgemeinbildenden Schulen um, ohne letztlich eine darüberhinausgehende Transformation der bestehenden institutionellen Strukturen und Praktiken des segregierenden und homogenisierenden Schul- und Bildungssystems zu adressieren.

Ähnlich zu den bildungspolitischen und administrativen Entwicklungen in der Schweiz bleiben damit die bildungspolitischen und administrativen Maßnahmen hinter den mit dem Begriff Inklusion assoziierten Auseinandersetzungen in der Wissenschaft (Feuser 2016) und internationalen Policies (UNESCO 2009; 2017) sowie bestehenden einzelschulischen Entwicklungsbemühungen (Lütje-Klose et al. 2017) in Deutschland zurück. Diese Entwicklungen wurden unter anderem unter dem Begriff der ,Sonderpädagogisierung“ kritisch reflektiert (Schumann 2016). 


\section{Die Programmatik bildungspolitischer und verwaltungsorganisatorischer Anforderungen an Schulleitende im Kontext schulischer Integration/Inklusion}

\subsection{In der Schweiz}

Aus internationaler Perspektive ist die Institutionalisierung einer Schulleitung in den schweizerischen Kantonen ein vergleichsweise junges Unternehmen. So beginnen den bildungsföderalistischen Strukturen in der Schweiz folgend erste Kantone erst in den 1990er Jahren an einzelnen Volksschulen Schulleitungen einzurichten. Wurde die Einzelschule zuvor durch eine Rektoratsperson ohne Weisungsbefugnisse verwaltet, wurden seither einzelne Schulen zu sogenannten „Geleiteten Schulen“ und die Funktion einer operativ tätigen Schulleitung auf Ebene der Einzelschule eingerichtet, wobei die Entwicklung von Kanton zu Kanton sehr unterschiedlich verläuft (Stemmer Obrist 2012: 1f.).

Demnach hat sich auch in der Schweiz neben der sogenannten Schulpflege eine Schulleitung als funktionale Akteur*innengruppe an Einzelschulen in allen Kantonen fest etabliert (Huber et al. 2013; Krause et al. 2013; Pekruhl et al. 2011). Während die Schulpflege zumeist die Gesamtverantwortung für die strategische Führung der Schule und das Schulpersonal trägt, ist die Schulleitung insbesondere für operative Tätigkeiten in der Leitung der Schule zuständig. Benannt werden hier pädagogische Führungsaufgaben, die Personalführung, die Organisation und Administration sowie Information und Kommunikation (Stemmer Obrist 2012: 50f.). Zudem wird der Schulleitung eine tragende Rolle in der Qualitätssicherung und -entwicklung in den meisten Kantonen zugesprochen (ebd.). So wird entsprechend den internationalen Debatten um veränderte Steuerungsmodelle im Bildungssystem (Bonsen 2016) auch in der Schweiz die Etablierung einer Schulleitung insbesondere mit der Forderung nach einer Steigerung der Qualität schulischer Angebote durch eine zunehmende Delegation der Verantwortlichkeiten auf die Ebene der Einzelschule in Verbindung gebracht. Ergänzt werden Maßnahmen eines schulinternen Qualitätsmanagements und die Einführung einer externen Schulevaluation durch die Kantone (Stemmer Obrist 2012: 46ff.).

In dieser Funktion kann der Schulleitung etwa mit Blick auf eine mögliche Leitbildentwicklung, Fort- und Weiterbildungsplanung oder Evaluation im Kontext inklusions- bzw. integrationsorientierter Schulentwicklung und auch im Hinblick auf verwaltungs-organisatorische Aspekte eine bedeutsame Rolle zukommen, obwohl die Schulleitung in den nationalen bildungspolitischen Dokumenten als Akteur*innengruppe nicht direkt adressiert wird. Auch auf kantonaler Ebene ist eine explizite und differenzierte Beschreibung sowie Professionalisierung der Funktion der Schulleitung im Kontext Inklusion bzw. Integration für uns nicht unmittelbar erkennbar (Huber et al. 2017).

\subsection{In Deutschland}

Auch in den deutschen Bundesländern spielen bisher Fragen der Professionalisierung der Schulleitenden im Kontext Inklusion eher eine untergeordnete Rolle (Amrhein/Badstieber 
2013). Obwohl die Funktion der Schulleitenden bereits in der zweiten Hälfte des 19. Jahrhunderts etabliert und das bildungspolitische wie wissenschaftliche Interesse an ihrer Funktion insbesondere in den vergangenen Jahrzenten deutlich gestiegen ist (Wissinger 2014), erscheinen die Schulleitenden in der starken Verknüpfung von Fragen schulischer Inklusion mit Fragen der Sonderpädagogik eher als „Randfiguren“ in der politischen Reformagenda und wissenschaftliche Auseinandersetzung (Badstieber/Moldenhauer 2016). Spezifische Unterstützungs- und Qualifizierungsprogramme für Schulleitende werden im Zuge der Debatte um Inklusion und im Nachgang der Ratifizierung nach unserem Kenntnisstand nicht umfassend reformiert oder adressiert.

Nichtsdestotrotz kommt den Schulleitenden in den bildungspolitischen Entwicklungen der deutschen Bundesländer eine bedeutsame Rolle zu. So umfasst das Aufgabenspektrum der Schulleitenden neben der Lehrtätigkeit aus rechtlicher Sicht die „unmittelbare Führung im Unterrichts- und Erziehungs-, Verwaltungs- und Ordnungsbereich sowie die Vertretung der Schule nach außen“ (Avenarius 2010: 151) und wird in dieser Ausrichtung auch von den Schulrechtsänderungsgesetzen im Kontext Inklusion unmittelbar tangiert. Von Fragen der Personalplanung (insbesondere mit Blick auf die sonderpädagogische Expertise) über die Aufnahme von Schüler*innen mit sonderpädagogischem Förderbedarf, etc. werden unmittelbar veränderte Anforderungen sichtbar, die in den Verantwortungsbereich der Schulleitenden fallen. Dieser verpflichtet die Schulleitenden in administrativer Hinsicht nachdrücklich zur Verantwortungsübernahme. Inwieweit den Schulleitenden darüber hinaus im Kontext des auch in Deutschland erweiterten Verantwortungsbereiches für eine inklusionsorientierte Schul- und Unterrichtsentwicklung eine bedeutsame Funktion in Fragen der Schulentwicklung zukommen soll, bleibt in den offiziellen Dokumenten der Bildungspolitik weitestgehend offen. Hier sind der Auftrag und Anforderungen in den Bundesländern weitestgehend vieldeutig und indirekt formuliert und damit vermehrt an die Selbstbindung der Einzelschulen und ihrer Schulleitenden gebunden.

\section{Ein Blick in die Praxis der Schulleitenden im Kontext integrations-/inklusionsorientierter Schulentwicklung}

Vor dem Hintergrund der hier skizzierten Programmatiken auf bildungspolitischer und verwaltungsorganisatorischer Ebene und damit verbundener indirekt bzw. direkt formulierter Anforderungen integrations- bzw. inklusionsorientierter Schulentwicklung an die Akteur*innengruppe der Schulleitenden stellt sich die Frage, wie Schulleitende diese auf Ebene der Einzelschule in der Schweiz und in Deutschland praktisch erleben und umsetzen.

\subsection{In der Schweiz}

Insgesamt ist zunächst festzustellen, dass die empirische Datenlage zur Rolle der Schulleitenden im Allgemeinen und mit Blick auf ihre Funktion im Kontext schulischer Integration/Inklusion in der Schweiz übersichtlich ist. Es existieren nur wenige, empirische Studien 
(Sturm et al. 2015), die einen ersten Blick auf die Bewältigung integrations-/inklusionsorientierter Schulentwicklungsprozesse in der Schweiz durch die Schulleitenden ermöglichen.

Einen empirischen Blick auf die Situation und Handlungspraxis von Schulleitenden im Kontext schulischer Integration/Inklusion liefert Köpfer (2015) in einer gemeinsamen Studie mit Sturm (et al. 2015). Sie befragen explorativ mittels problemzentrierten (Expert*innen-) Interviews (Nohl 2012; Witzel 2000) Schulleitende an drei Schulen unterschiedlicher Schulstufen und Schulformen aus verschiedenen Kantonen der Nordwestschweiz (Köpfer 2015). Auf der Grundlage der dokumentarischen Methode der Textinterpretation und unter Rückgriff auf einzelne zentrale Interviewsequenzen untersucht Köpfer (2015) die handlungspraktische Bearbeitung der Schulentwicklungsanforderungen durch Integration an die Schulleitenden. Dabei wurde den Schulleitenden ,derselbe erzählgenerierende Einstiegsimpuls gestellt, indem gefragt wurde, wie es an ihrer Schule zur Integration gekommen sei und was sich bis heute wie entwickelt und möglicherweise in diesem Prozess auch verändert habe“ (ebd., o.S.).

Die Analysen der beiden Sequenzen zeigen beispielhaft die Ausgestaltung des Auftrages schulischer Integration in der Schweiz und liefern einen ersten, detaillierten Blick in ihre Praxis auf Ebene der Einzelschule. Obwohl in den beiden hier dargestellten Fällen „die Entscheidungspraxis für die Einführung von Integration auf Basis eines äußeren Impulses stattfindet“ und diese damit als Beispiele für die „Reaktion“ auf einen bildungspolitisch gesteuerten Prozess der Veränderung von Schule im Kontext von Integration gelesen werden können, verweisen die Untersuchungen insbesondere auf ,eine differente handlungspraktische Bearbeitung des bildungspolitischen Auftrags schulischer Integration“ (ebd., o.S.) durch die Schulleitenden.

Ein interviewter Schulleitender einer Sekundarschule aus der Nordwestschweiz bearbeitet den Auftrag vorrangig im Sinne einer ,pragmatischen und zeitlich unaufwändigen Einführung“ (3, Abs. 8). In dieser wird „,keine grundlegende Umstrukturierung der schulischen Tätigkeiten bzw. Organisation“ (3, Abs. 10) adressiert und vorhandene Strukturen werden mit einer vorrangig ökonomischen Orientierung beibehalten. Im Kontrast dazu zeigt sich im anderen Falle eines Schulleitenden einer Primarschule aus der Nordwestschweiz, die Bearbeitung schulischer Integration im Sinne eines ,gewünscht inhaltlichen, abgestimmten, wertegeleiteten und mit Ressourcierung und Professionalisierung verknüpften Prozesses der Nichtaussonderung bzw. Integration“" (3, Abs. 16). So wird im zweiten Fall die Forderung nach Integration im Sinne eines ,umsetzungsrealistisch-strategischen Vorhaben[s] kodiert, das mit Professionalisierungs- und Strukturierungsversuchen verbunden wird und erste Anzeichen eines kulturellen Schulentwicklungsprozesses aufzeigt“" (3, Abs. 20).

Kontrastierend gesprochen lassen sich damit zwei latente Schulleitungstypen und Schulentwicklungsstrategien im Kontext schulischer Integration/Inklusion durch die Schulleitenden nachzeichnen:

Ersterer verbindet den Auftrag inklusiver Schulentwicklung mit einem Professionalisierungs- und Ressourcierungsanspruch, der als notwendig für die Erfüllung des anspruchsvollen Auftrags der Inklusion von Schülerinnen und Schülern mit besonderem Bildungsbedarf in der Volksschule angesehen wird. Zweiterer verfolgt tendenziell die Orientierung, dass der Anspruch von Integration in die bereits bestehenden Team- und Unterrichtsstrukturen einzupassen ist und hierfür keine Anpassungs- respektive Modifikationsleistungen innerhalb der bestehenden Schul- und Unterrichtspraxis vorzunehmen sind (Köpfer/Mejeh 2017: 173f.).

Neben diesen beiden kontrastiven Fällen konnten in der Studie jedoch auch übergreifende, gemeinsam geteilte Orientierungen rekonstruiert werden. So wird in beiden Fällen Unter- 
richtsentwicklung und -gestaltung von Schulleitenden in erster Linie als Auftrag an die Schulische Heilpädagog*innen gesehen, und Integration insbesondere ,entlang bildungspolitischer Vorgaben - als Auftrag der Inklusion von Kindern mit besonderem Bildungsbedarf bearbeitet“ (Köpfer/Mejeh 2017: 175). Zudem wird der Auftrag der Integration ,als topdown gesteuerter Prozess bearbeitet, im Zuge dessen Schulleitungen sich selbst als Zuständige für Ressourcensicherung und Steuerung erachten. Inklusion wird von den Schulleitungen dabei als ein Reformauftrag unter vielen wahrgenommen, der in keinem ambivalenten Verhältnis zu bestehenden Strukturen und Praktiken steht“"(ebd.).

Im Rückgriff auf die bereits von Köpfer (2015) präsentierten Daten, heben Köpfer und Mejeh (2017) die besondere Bedeutung der Schulleitenden im Kontext schulischer Integration/Inklusion noch einmal heraus. So können sie neben den bereits dargestellten Befunden auf der Basis einer quantitativ ausgerichteten „Netzwerkstudie im Kanton Solothurn (Nordwestschweiz) [...] (vgl. Mejeh 2015), bei der insgesamt 136 Akteur*innen (Teilstichproben: Eltern, Lehrpersonen, Heilpädagog*innen, Logopäd*innen, Psycholog*innen, Schulleitungen) mittels Onlinefragebogen (Limesurvey) zu ihrem alltäglichen Handeln in schulintegrativen Kontexten befragt wurden“ (Köpfer/Mejeh 2017: 170), herausarbeiten, dass die Schulleitenden mit Blick auf Fragen der Diagnostik, des Schulrechts und der Finanzierung eine wichtige Vermittlungsfunktion einnehmen und maßgeblich zur Steuerung der Netzwerke zwischen den befragten Akteur*innen im Kontext Integration beitragen.

\subsection{In Deutschland}

Die hier von Köpfer (2015) und von Mejeh (2015) erarbeiteten Analysen für die Schweiz fordern dazu auf, die erarbeiteten Gemeinsamkeiten aber insbesondere sichtbarwerdende Differenzen in der handlungspraktischen Bearbeitung der bildungspolitischen top-down gesteuerten Prozesse im Kontext Integration/Inklusion zum Anlass zu nehmen, mögliche analoge Typisierungen auch im deutschen Kontext zu ergründen.

Ähnlich wie in der Schweiz, muss auch mit Blick auf Deutschland zunächst konstatiert werden, dass empirische Befunde zur Funktion der Schulleitenden im Kontext schulischer Integration/Inklusion weitestgehend fehlen (Badstieber/Moldenhauer 2016). Eine Ausnahme bilden hier die Analysen von Scheer, Laubenstein und Lindemeier (2014), welche die Rolle der Schulleitungen sowie ihre jeweilige Ausgestaltung in der inklusiven Schulentwicklung in Schwerpunktschulen in Rheinland-Pfalz anhand von Experteninterviews und strukturierenden qualitativen Inhaltsanalysen an 18 Schulen untersuchen. Sie arbeiten die Notwendigkeit heraus, inklusionsorientierte Schulentwicklungsprozesse aus unterschiedlichen Perspektiven (structural, human resource, symbolic und political frames) zu betrachten und kommen zu dem Ergebnis, dass Schulleitende, die entsprechend unterschiedliche Perspektiven in ihrem Führungsverständnis artikulieren, Inklusion eher im Sinne eines umfassenden Schulentwicklungsprozesses adressieren (Scheer 2020).

Um weitergehende Perspektiven einer international vergleichenden Forschungsperspektive herauszuarbeiten, wird im Folgenden auf Daten des Projektes „Mit Schulleitung gesunde, inklusive Schule gestalten“ (Amrhein/Badstieber 2019) zurückgegriffen. Hier wurden im Nachgang an eine explorative, quantitativ ausgerichtete Fragebogenerhebung in 10 Bundesländern in Deutschland zu dem expliziten Selbstverständnis der Schulleitenden im Kontext schulischer Integration/Inklusion an 20 ausgewählten Fallschulen problemzentrierte 
Experteninterviews mit den Schulleitenden geführt. In diesen hatten die Schulleitenden, ähnlich der Studie von Köpfer und Sturm (2015), die Möglichkeit, die Ereignisse und Aktivitäten ihrer Schule und ihrer eigenen Person im Kontext inklusionsorientierter Veränderungsprozesse zu beschreiben. Auch wurden zur Rekonstruktion der Handlungspraxis der Schulleitenden die Interviews auf der Grundlage der Dokumentarische Methode der Textinterpretation (Bohnsack 2014) analysiert und ausgewertet. Um den Schulleitenden eine Möglichkeit zu geben, innerhalb ihrer Relevanzsetzungen von den Ereignissen inklusionsorientierter Veränderungsprozesse im Nachgang der UN-BRK und damit verbundener Schulrechtsänderungsgesetze zu berichten, wurde den Schulleitenden nach kurzen Fragen zu ihrer Person auch hier allen die ähnliche, offene, erzählgenerierende Einstiegsfrage gestellt, seit wann Inklusion an ihrer Schule eine Rolle spielt und wie es dazu gekommen ist. Nach den ausführlichen, anfänglichen Erzählungen der Schulleitenden und erzählimmanenten Rückfragen der interviewenden Person, wurden die Schulleitenden im weiteren Verlauf zu unterschiedlichen Aspekten inklusionsorientierter Schulentwicklungsprozesse befragt, die sich im Zuge der explorativen, quantitativ ausgerichteten Fragebogenerhebung für die Schulleitenden als offensichtlich besonders bedeutsam herausstellten. Hierzu zählten unter anderem Fragen nach der Aufnahme von Schüler*innen mit unterschiedlichen sonderpädagogischen Förderbedarfen, der Zusammenarbeit mit dem Kollegium und der Schulaufsicht sowie dem Einsatz und der Akquise personeller und sachlicher Ressourcen.

Entlang der von Nohl (2012) vorgeschlagenen Auswertungsschritte der Dokumentarischen Methode wurden ausgewählte Interviewsequenzen kontrastierend analysiert und unterschiedliche Orientierungen der Schulleitenden herausgearbeitet. Auf diese Weise konnten in einer umfangreichen Studie Unterschiede und Gemeinsamkeiten in der handlungspraktischen Bearbeitung der Ausgestaltung schulischer Inklusion sichtbar gemacht und systematisiert werden, die sich für eine international vergleichende Perspektive mit den Ergebnissen von Köpfer (2015) und mit Einschränkung von Mejeh (2015) anbieten.

Im folgenden Kapitel werden exemplarisch zwei Sequenzen aus den Interviews unserer Studie dargestellt und die Orientierungen der dort interviewten Schulleitenden sichtbar gemacht. Sie stehen stellvertretend für eine differenziert ausgearbeitete Typologie, die die von Köpfer (2015) für die Schweiz erarbeiteten Andeutungen für den deutschen Kontext erhärten, differenzieren und durch weitere Typenbildungen ergänzen können (siehe dazu Badstieber 2020). Die hier vorgelegten Analysen stellen nur einen kleinen Teil der umfangreichen Daten und Forschungsergebnisse des Forschungsprojektes dar, eignen sich jedoch in besonderen Maße für einen internationalen Vergleich mit den für die Schweiz erarbeiteten Ergebnissen und ermöglichen auf diese Weise eine zielführende Erarbeitung möglicher international vergleichender Perspektiven zur Rolle der Schulleitenden im Kontext Inklusion. Um eine verständliche Darstellung im Rahmen dieses Beitrages zu ermöglichen, wurden die Sequenzen teilweise gekürzt (gekennzeichnet mit [...]).

\subsubsection{Interviewsequenz Schulleitende S1: Inklusion als Schulentwicklungsprozess}

Wie in den gesamten Fällen unserer Studie führt auch in dem Interview mit der Schulleitenden S1 die Einstiegsfrage nach den Anfängen der Auseinandersetzung mit der Thematik Inklusion zu einer ausführlichen Auseinandersetzung mit der Entwicklung des Lernens von Schüler*innen mit einem sonderpädagogischen Förderbedarf an der eigenen Schule durch 
die Schulleitenden. Insgesamt zeigt sich in allen in unserer Studie untersuchten Interviews, entsprechend den Ausführungen von Köpfer (2015) und Köpfer und Mejeh (2017) zur Situation in der Schweiz, eine Orientierung seitens der Schulleitenden entlang der bildungspolitischen Forderungen zur Unterrichtung von Schüler*innen mit sonderpädagogischem Förderbedarf und eine Fokussierung auf die Differenzlinie der Behinderung. Zu Beginn legt die Schulleitende S1 ausführlich die einzelnen Schritte des Entscheidungsprozesses zur Einführung des Gemeinsamen Lernens an ihrem Gymnasium unter der Einflussnahme der Schulaufsicht dar, wie folgende Interviewpassage exemplarisch zeigt.

\section{Oberthema: Die Einführung des gemeinsamen Lernens von Schüler*innen mit und ohne sopäd. Förderbedarf}

\begin{tabular}{lll}
\hline Zeile & Sprecher & Transkription \\
\hline 1 & S1 & So, der Impuls dazu ist eigentlich von außen, von der Schulaufsicht gekommen, die \\
2 & uns - den Schulleitungen der Gymnasien - gesagt haben: Ihr müsst euch damit ausei- \\
3 & nandersetzen. Es wird kommen, dass auch ihr Kinder mit sonderpädagogischem För- \\
4 & derbedarf auch zieldifferent beschulen müsst. Und ich habe das dann in die Schulge- \\
5 & meinschaft reingetragen und habe gesagt: Dann lasst uns doch gucken, dass wir so früh \\
6 & wie möglich damit anfangen, um Erfahrungen zu sammeln, und dann auch noch zu \\
7 & einer Zeit, wo die Ressourcenlage auch noch eine andere war. [...] Und das hat dann, \\
8 & wie gesagt, [...] Jahre gedauert. Wir haben dann eine Expertengruppe gebildet aus - \\
9 & oder eine offene Expertengruppe - aus Mitgliedern aller Gruppen der Schulgemein- \\
10 & schaft. Da waren auch externe Leute dabei. Eine Gruppe, die - an der grundsätzlich \\
11 & auch jeder teilnehmen konnte - die anfangs ziemlich engmaschig getagt hat und die \\
12 & Frage vor allem beantworten musste: Was brauchen wir? Was müssen wir tun? Was \\
13 & müssen wir in der Schulentwicklung anbahnen, um kompetent genug - ich sage es mal \\
14 & ganz vorsichtig - um kompetent genug in die Inklusion einsteigen zu können? \\
15 & \#00:04:27-3\# \\
\hline
\end{tabular}

Die Beschreibung des Prozesses zur Aufnahme von Schüler*innen mit sonderpädagogischem Förderbedarf wird durch die Erzählung mit der Ankündigung bzw. dem Appell der Schulaufsicht - bzgl. der offiziellen Verpflichtung zur Aufnahme zieldifferent beschulter Schüler*innen mit sonderpädagogischem Förderbedarf auch an Gymnasien - verbunden (S1, Z. 1-4). Implizit sichtbar wird hier zunächst eine administrative Orientierung, in der die Initiative zur Einführung schulischer Inklusion in der Schulaufsicht verortet und eine Differenzierung zwischen sogenannten zielgleich und zieldifferent unterrichteten Kindern vorgenommen wird. Es zeigt sich eine Orientierung an den bildungspolitischen und administrativen Vorgaben des sogenannten Gemeinsamen Lernens, in denen die verpflichtende Aufnahme von zieldifferent unterrichteten Schüler*innen mit Blick auf das Gymnasium, als Schulform für den auf das Abitur zielenden Bildungsgang, offensichtlich die wesentliche Veränderung darstellt.

Die Validierung als „Impuls eigentlich von außen“ (S1, Z. 1) dient dann weiterführend der Vorbereitung der Erzählung der eigenen Handlung und der Übernahme des äußeren Drucks als Startpunkt für den langandauernden, ,eigentlich' doch dann ,innenliegenden ' Prozess der Auseinandersetzung in der Schulgemeinschaft, im weiteren Verlauf der Passage (S1, Z. 6-10). Durch das ,eigentlich“ wird hier der positive Horizont von auch innerhalb der Schule erwünschter Prozesse sichtbar. So dokumentiert sich in der folgenden Darstellung, 
wie auch in anderen Passagen des Interviews, eine unterstützende Orientierung gegenüber dem Auftrag Inklusion bzw. Gemeinsamen Lernen und gegenüber den Forderungen der vorgesetzten Behörde seitens der Schulleitenden. In dieser Orientierung wird implizit ein prozessuales Verständnis der Einführung schulischer Inklusion sichtbar, das mit Fragen der Professionalisierung (,um Erfahrungen zu sammeln“ S1, Z. 6) und Ressourcenakquise (,wo die Ressourcenlage auch noch eine andere war" S1, Z. 7) verbunden wird. Dabei wird an dieser Stelle in der differenzierenden Beschreibung der Ereignisse in die erste Person Singular gewechselt (,Und ich habe das dann in die Schulgemeinschaft reingetragen“ S1, Z. 6) und die Geschehnisse als individuell verantwortete Handlung der eigenen Person proklamiert. Sichtbar wird hier implizit die Inanspruchnahme einer Führungsfunktion durch die Schulleitende und eine aktive Handlungspraxis in der Bearbeitung des Auftrages schulischer Inklusion.

In der wörtlich zitierten Rede formuliert die Schulleitende S1, im Kontrast zu der offensichtlich verpflichtend bzw. mahnend erinnerten Ansprache der Schulaufsicht („müsst“", „müsst“ S1, Z. 2f.) an sie, dann gegenüber dem Lehrer*innenkollegium keine harte Direktive, sondern eher einen gemeinschaftsstiftenden, sich selber einschließenden Appell („,Dann lasst uns doch gucken, dass" S1, Z. 6) mit zusätzlicher argumentativer Unterstützung des eigenen Vorgehens (,um Erfahrungen zu sammeln, und dann auch noch zu einer Zeit, wo die Ressourcenlage auch noch eine andere war" S1, Z. 7f.). So zeigt sich hier eine Orientierung an einem eher horizontalen, gemeinschaftsstiftenden Führungsstil der Schulleitenden gegenüber dem Kollegium im Kontext der Schulentwicklungsprozesse, in der ihr offensichtlich die Aufgabe zukommt, zwischen den externen Anforderungen und den internen Interessen zu vermitteln. Die Verwendung des Verbs „reintragen“ (Z. 5) emphasiert diese Orientierung an einer zielgerichteten, aber vermittelnden, behutsam vorgehenden Handlungspraxis. Gleichzeitig wird erneut ein Führungsanspruch der Schulleitenden im Versuch deutlich, die anstehenden Erwartungen an die Schule frühzeitig zu antizipieren und dann auch in der eigenen Schule bedienen zu können. Die Orientierung liegt hier, ganz ähnlich den Ergebnissen von Köpfer (2015), weiterführend auf Fragen der Ressourcenakquise (insbesondere auch Zeit) und der Professionalisierung des Kollegiums, ,um kompetent genug in die Inklusion einsteigen zu können“ (S1, Z. 14-15). Dabei zeichnet sich latent auch die Sorge um eine zukünftige Überforderung der eigenen Schule bzw. des Kollegiums vor der bevorstehenden Verpflichtung ab. So zeigt sich eine Orientierung des Schulleitungshandelns, in der die Schulleitende nicht voraussetzungslos den neuen Auftrag in das eigene System überführt, sondern auf eine prozessuale Veränderung der bestehenden Systemvoraussetzungen mit Blick auf den neuen Auftrag Inklusion bzw. gemeinsames Lernen hinzuarbeiten versucht.

Die Schulleitende S1 schließt ihre Darstellung dann mit einer weiterführenden Beschreibung des andauernden, als ,engmaschig“ (S1, Z. 13) bezeichneten Prozesses der Entscheidungsfindung und der Beschreibung der Gründung einer Expertenrunde. Der vorgenommene Einschub ,an der grundsätzlich jeder teilnehmen konnte“ (S1, Z. 11f.) sowie die Aufzählung der Fragestellungen nach den eigenen bzw. kollegialen Verpflichtungen (,Was brauchen wir? Was müssen wir tun? Was müssen wir..."S1, Z. 13-15) dokumentiert nicht nur erneut die Orientierung an einem eher horizontalen, gemeinschaftsstiftenden Führungsstil, sondern auch die unterstützende Orientierung an dem Auftrag Inklusion bzw. Gemeinsamen Lernen, in der die Verantwortungsübernahme und Professionalisierung der eigenen Schule in den Vordergrund gerückt wird. Der abschließend vorgenommene validierende Einschub ,ich sage es mal ganz vorsichtig“ (S1, Z. 15) markiert die Orientierung an einer eher zurückhaltend optimistischen Einschätzung einer einfachen und pragmatischen Umsetzbarkeit schulischer Inklusion im eigenen System. So deutet sich hier stellvertretend für das Interview von 
S1 und für weitere Interviews in unserer Studie insgesamt eine Orientierung von Schulleitenden als aktiv, gestaltende Akteur*innen ab, die entsprechend den Ergebnissen von Köpfer (2015) in der Schweiz, Inklusion vorrangig im Sinne des gemeinsamen Lernens adressieren und gleichzeitig als ,umsetzungsrealistisch-strategischen Vorhaben kodier[en], das mit Professionalisierungs- und Strukturierungsversuchen verbunden wird und erste Anzeichen eines kulturellen Schulentwicklungsprozesses aufzeigt" (3, Abs. 20) unter der aktiven Beteiligung der eigenen Person und Führung.

\subsubsection{Interviewsequenz Schulleitende S2: Inklusion als administrativer Akt}

Auch im Falle der Schulleitenden S2 evoziert die Einstiegsfrage eine Beschreibung der Prozesse zur Aufnahme von Schüler*innen mit sonderpädagogischem Förderbedarf an ihrer Schule, dieses Mal einer Schule mit mehreren Bildungsgängen. Dabei zeigen sich die Orientierungen seitens der Schulleitenden S2.

\section{Oberthema: Die Einführung des Gemeinsamen Lernens}

\begin{tabular}{|c|c|c|}
\hline Zeile & Sprecher & Transkription \\
\hline $\begin{array}{l}1 \\
2 \\
3 \\
4 \\
5 \\
6 \\
7\end{array}$ & $\mathrm{~S} 2$ & $\begin{array}{l}\text { Und wir sind dann vor zwei Jahren, also wir sind jetzt im zweiten Jahre, also vor knapp } \\
\text { zweieinhalb Jahren angeschrieben worden vom Schulträger, dass wir mit dem nächs- } \\
\text { ten fünften Schuljahr dann auch inklusive Schule werden. Für uns etwas überraschend, } \\
\text { weil da war eigentlich die Anmeldung für das fünfte Schuljahr schon durch, also quasi } \\
\text { im Nachhinein für das nächste Schuljahr verpflichtet worden. Und sind jetzt eben im } \\
\text { zweiten Jahr Schule des inklusiven Lernens. }\end{array}$ \\
\hline 8 & $\mathrm{~S} 2$ & {$[\ldots]$} \\
\hline $\begin{array}{l}9 \\
10 \\
11 \\
12 \\
13 \\
14 \\
15\end{array}$ & $\mathrm{~S} 2$ & $\begin{array}{l}\text { Und haben dann tatsächlich eine Klasse gebildet, die wir auch- Kollegen und Kolle- } \\
\text { ginnen eingesetzt hat, die dann auch ganz klar erklärt haben "wir möchten am Projekt } \\
\text { Inklusion teilnehmen". Aber das ist natürlich ein Verfahren, was auf Dauer nicht } \\
\text { durchführbar ist, weil die Zahlen gehen momentan in eine Richtung, der neue fünfte } \\
\text { Jahrgang hat zum Beispiel elf Anmeldungen mit Förderbedarf bei } 4 \text { Klassen, dass wir } \\
\text { das auf Dauer eben doch breit aufstellen werden und letzten Endes natürlich alle Kol- } \\
\text { leginnen und Kollegen an Inklusion beteiligt sind. }\end{array}$ \\
\hline
\end{tabular}

Ähnlich wie im Interview von S1 wird die Initiative zur Einführung schulischer Inklusion bzw. der Aufnahme von Schüler*innen mit sonderpädagogischem Förderbedarf auf der administrativen, hier der kommunalen, Ebene in dem Akteur Schulträger verortet. Dabei wird die Schule in den Erzählungen von S2 per Anschreiben durch den Schulträger schließlich zur „,inklusiven Schule“ (S2, Z. 4). Es wird eine Orientierung sichtbar, in der Inklusion als ein schriftlich-administrativer, pragmatisch zu vollziehender Akt durch den Schulträger dargestellt wird und latent ein prozessuales Verständnis schulischer Inklusion und eine aktiv befürwortende Bearbeitung zurückgewiesen werden.

Lediglich kurz wird die Erzählung durch eine bewertende Passage unterbrochen und die Entscheidung des Schulträgers zurückhaltend als „etwas überraschend“ (S2, Z. 4) bewertet. Auch hier bleibt eine darüberhinausgehende negative Validierung oder Opposition aus, 
jedoch wird im Kontrast zu S1 hier nicht mit einer Beschreibung der eigenen Aktivitäten angeschlossen. Die sich anschließende Begründung wird vielmehr entlang pragmatisch-organisatorischer Ereignisse, hier der bereits abgeschlossenen Anmeldung der fünften Klasse, geführt und damit Inklusion dezent als störender Eingriff in die berechenbaren, administrativen Abläufe auf Ebene der Einzelschule adressiert.

So wird weiterführend, wie auch in anderen Passagen des Interviews, eine administrative, pragmatische Orientierung sichtbar, in der eine möglichst einfache und nach organisatorischen Gesichtspunkten zu vollziehende Umsetzung schulischer Inklusion bzw. des Gemeinsamen Lernen als positiver Horizont deutlich wird. Auch das „Und sind jetzt eben im zweiten Jahr Schule des inklusiven Lernens" (S2, Z. 6f.) verweist hier implizit eher auf eine sachlich, nüchterne aber letztlich akzeptierende Haltung im Hinblick auf die Entwicklungen um Inklusion bzw. das Gemeinsame Lernen an der eigenen Schule. Die sich an anderer Stelle wiederholende Verwendung des Adverbiums „eben“ validiert diese Orientierung. Eher hinnehmend, nüchtern wird hier das administrative Vorgehen des Schulträgers nachvollzogen.

Eine Darstellung eines, wie im ersten Fall, umfassenden Schulentwicklungsprozesses bleibt im weiteren Verlauf der Interviewpassage aus. Vielmehr wird hier die bekannte Strategie der Delegation an Freiwillige aufgerufen (vgl. Amrhein 2011). Wie auch in anderen Interviews wird hier offensichtlich die Möglichkeit gesehen, den Auftrag Inklusion bzw. Gemeinsames Lernen über die Auswahl von Freiwilligen pragmatisch in das eigene System zu überführen. Die abschließende Evaluation dieses Vorgehens ,,als auf Dauer nicht durchführbar" (S2, Z. 12) wird argumentativ erneut entlang der Anmeldezahlen und der damit verbundenen Notwendigkeit, die Bündelung aus dem „Pilotjahrgang“" „eben doch breit aufstellen“ (S2, Z. 15) zu müssen, elaboriert. In einem eher administrativ-organisatorischen Sprachgebrauch (,durchführbar“ S2, Z. 12, „breit aufstellen“ S2, Z. 15) wird wiederum die Notwendigkeit zur Beteiligung aller Kolleginnen und Kollegen an Inklusion rein administrativ-organisatorisch begründet und das eigene Handeln im Sinne einer Reaktion auf die administrativen Vorgaben der Bildungsadministration markiert. Dabei liegt die Orientierung auf der Suche nach einem, den Prozess der Veränderung abschließenden, dauerhaft „durchführbaren“ und damit letztlich berechenbaren Vorgehen. So deutet sich hier stellvertretend für das Interview von S2 und für weitere Interviews in unserer Studie insgesamt eine Orientierung von Schulleitenden als eher reaktiv, administrativ tätige Akteur*innen ab, die entsprechend den Ergebnisse von Köpfer (2015) in der Schweiz, Inklusion im Sinne des gemeinsamen Lernens eher pragmatisch in die bestehenden Routinen und Strukturen zu überführen versuchen.

\section{Vergleichende Analysen und Interpretation der Ergebnisse}

Die hier vorliegenden Ergebnisse eröffnen einen explorativen, empirischen Blick in die Praxis der Schulleitenden im Nachgang der Ratifizierung der UN-BRK im Kontext schulischer Integration/Inklusion in der Schweiz und in Deutschland. Trotz der unterschiedlichen Traditionen und unterschiedlichen bildungspolitischen Entwicklungen in den beiden Nachbarländern einschließlich der im politischen und gesellschaftlichen Diskurs verwendeten Begrifflichkeiten (siehe oben) lassen sich anhand der Daten länderübergreifend gemeinsame Tendenzen im Schulleitungshandeln erkennen.

Leitend für die Entwicklungen beider Länder ist zunächst offensichtlich die Verknüpfung der Forderung schulischer Integration/Inklusion mit der Aufnahme von Schüler*innen 
mit sonderpädagogischem Förderbedarf und damit verbundener Fragen nach der Einbindung sonderpädagogischer Unterstützungsleistung in die allgemeinbildenden Schulen (kritisch Schumann 2016). Die Fokussierung auf die Differenzlinien der administrativ konstruierten sonderpädagogischen Förderbedarfe deutet sich in den Analysen von Köpfer (2015) als bedeutsame Orientierung für die Schulleitenden an und zeigt sich nachdrücklich auch in unseren Daten. Die Aufnahme von Schüler*innen, die anhand sonderpädagogischer Differenzsetzungen vormals nicht zum Kreis der Schüler*innenschaft der Einzelschulen gehörten und nun an der jeweiligen Einzelschule unterrichtet werden sollen, bilden den Anlass der Auseinandersetzung mit Fragen schulischer Integration/Inklusion und strukturieren grundlegend die Handlungspraxis der Schulleitenden auf Ebene der Einzelschule. Die auch im internationalen Kontext sichtbarwerdende und (kritisch) diskutierte Kategorisierung (Ziemen 2016) spiegelt sich hier offensichtlich nicht nur auf Ebene der Bildungspolitik und Bildungsadministration wieder, sondern evoziert in den zunehmend top-down gesteuerten Prozessen letztlich auch eine dementsprechende Orientierung in der Handlungspraxis der Schulleitenden. In dieser bleibt Integration/Inklusion ein Reformauftrag neben anderen, mitunter auch neben solchen, die andere Differenzlinien bildungspolitisch adressieren.

Davon ausgehend lassen sich im internationalen Vergleich der Studien zwei voneinander unterscheidbare Strategien auf Ebene der Einzelschule in beiden Ländern identifizieren. Diese ermöglichen es offensichtlich den Schulleitenden die aus integrations- bzw. inklusionspädagogischer Perspektive widersprüchlichen und spannungsreichen Forderungen (Amrhein 2014) innerhalb bzw. trotz der exkludierenden und klassifizierenden Strukturen, Kulturen und Praktiken sinnstiftend zu bearbeiten.

Die Strategie einer pragmatisch vollzogenen Aufnahme von Schüler*innen mit sonderpädagogischem Förderbedarf und Eingliederung sonderpädagogischer Unterstützungsleistungen rückt die Forderung nach Integration/Inklusion, wie Köpfer (2015) in Anlehnung an Slee und Weiner (2001) bereits bemerkt, im Sinne eines ,technical problems“ (Slee/Weiner 2001: 94) insbesondere in den Bereich vorrangig organisatorischer Fragestellungen. Im Zentrum stehen die Bemühungen den sich verändernden administrativen Rahmungen durch die Bildungsadministration durch eine eigene entsprechende organisatorische Planung reaktiv und verlässlich zu begegnen. Die von Seiten der Bildungspolitik und Bildungsadministration aufgeworfene Fokussierung auf sonderpädagogische Fragestellungen und die aufrechterhaltene Logik der Zuweisung sonderpädagogischer Förderbedarfe und sonderpädagogischer Unterstützungsleistungen, so die Interpretation, eröffnet hier durchaus für die Schulleitenden die Gelegenheit, Integration/Inklusion mehr oder weniger als zusätzlichen „Baustein“ administrativ in die vorhandenen Systembedingungen einzugliedern. Hier geht es also vor allem um Fragen nach der Verteilung der Schüler*innen mit sonderpädagogischem Förderbedarf, der Lehrkräfte etc. Eine weitreichende Veränderung der Strukturen, Kulturen und Praktiken der Schule müssen sie nicht unmittelbar evozieren.

Ihr Gegenüber bzw. zur Seite steht die Strategie einer transformierenden Bearbeitung, in der Integration/Inklusion (auch) als „,cultural problem“ (ebd.) in der die Wahrnehmung der Schulleitenden eine Veränderung der bestehenden Ressourcen und Kompetenzen und einen gemeinsamen Diskurs über die Auslegung des Reformauftrages initiieren. Dabei muss und kann, das zeigen die Beispiele, die Fokussierung sonderpädagogischer Fragestellungen nicht gänzlich aufgegeben, aber doch insoweit flexibilisiert werden, als das die an Schule Beteiligten in einen Prozess einer gemeinsamen Professionalisierung geführt werden, in denen Fragen nach Verantwortlichkeiten, Differenzsetzungen etc. möglich und wahrscheinlich 
werden. Entgegen der ersten Strategie ist die Bearbeitung hier (zunächst) nicht reaktiv und (ab)schließend, sondern eher aktiv und öffnend.

Die Wirkungen und Bedeutung der jeweiligen Strategie durch die Schulleitenden für die Lehrer*innen, Schüler*innen, Eltern etc. wäre weitergehend zu untersuchen und zu diskutieren. Die empirischen und theoretischen Auseinandersetzungen mit der Funktion von Schulleitenden verweisen durchaus auf die Notwendigkeit einer ausgewogenen Balance einer systemerhaltenden und transformierenden Handlungspraxis in den Führungspositionen, obwohl das verbreitete Narrativ des „Motoren für Schulentwicklung“ „transformational leadership“ und ,change agent" insbesondere auch im internationalen Diskurs um Integration/Inklusion bzw. inclusion mit Blick auf die Akteur*innengruppe der Schulleitenden vorherrschend ist (zsfd. Badstieber/Moldenhauer 2016). So werden unter diesen Begrifflichkeiten sowohl von Seiten der Bildungspolitik als auch von Seiten der Wissenschaft eine herausgehobene, innovative Führungsfunktion der Schulleitenden im Kontext von Schulentwicklungsprozessen propagiert und Konstitutionen eines systemverändernden Führungsstils thematisiert.

Die insbesondere durch den angloamerikanischen Sprachraum bestimmte empirische Auseinandersetzung fokussiert hier wiederholt die Frage, wie Schulleitende weitreichende Veränderungsprozesse auf Ebene der Einzelschule unterstützen können (Angelides 2012; Hoppey/McLeskey 2012; Keyes et al. 1999), wohingegen Fragen nach der systemerhaltenden Bearbeitung der Schulleitenden und ihrer möglichen bzw. notwendigen (Re-)Produktion der exkludierenden und etikettierenden Logiken des Schul- und Bildungssystem unter den gegeben Bedingungen im Zusammenspiel mit den anderen an Schule beteiligten Akteur*innen eher unberücksichtigt bleiben (Engelbrecht et al. 2006; Ryan 2012).

Sichtbar wird in den vorliegenden Ergebnissen diesbezüglich eine auch in anderen Beiträgen (Amrhein 2011; Hinz 2013; Trumpa/Janz 2014; Wocken 2011) aufgezeigte und empirisch weiter zu untersuchende Diskrepanz zwischen dem normativen Anspruch schulischer Inklusion und ihrer handlungspraktischen Bearbeitung. Dabei sollten weitere Untersuchungen die jeweiligen nationalen Bedingungen innerhalb derer Schulleitende als „Motoren für Schulentwicklung“ „transformational leader" oder „,change agent" taktieren können und müssen, vermehrt berücksichtigen.

\section{Vorläufiges Fazit: Herausforderungen der Rolle der Schulleitung im internationalen Kontext}

Unter Berücksichtigung des aktuellen Forschungsstandes zu inklusionsorientierten Schulentwicklungsprozessen und zur Rolle der Schulleitenden auf Ebene der Einzelschule werden länderübergreifend komplexe, vielfältige und mitunter diffuse Anforderungen an Schulleitende sichtbar (Kugelmass/Ainscow 2004). Diese gehen, wie auch die beispielhaften Analysen andeuten, über rein administrative Tätigkeiten hinaus und umfassen unter anderem die Professionalisierung des Kollegiums, eine Reflexion des eigenen Verantwortungsbereiches, eine Veränderung der Schulkultur im Hinblick auf die Heterogenität der Schüler*innen etc. Dabei geht es insbesondere für die Schulleitenden in ihrer zentralen Schnittstellenfunktion zwischen der Bildungspolitik und -administration auf der einen und ihrem Kollegium und den Schüler*innen auf der anderen Seite darum, eine jeweils angemessene Balance zwischen einer innovierenden und pragmatischen bzw. systemstabilisierenden Auslegung ihrer Rolle 
zu finden. Hier deuten sowohl die Ergebnisse aus der Schweiz als auch aus Deutschland auf einen bedeutsamen Handlungs- und Deutungsspielraum seitens der Schulleitenden hin. Möglichkeiten einer vertiefenden und differenzierten Systematisierung unterschiedlicher Umsetzungsszenarien auf Ebene der Einzelschule lassen sich hier anschließen und wurden im nationalen Kontext auf der Grundlage der Methodologie rekonstruktiver Auswertungsverfahren bereits weiterführend erarbeitet (Badstieber 2020). So konnten am Beispiel des Bundesland Nordrhein-Westfalen typische Umsetzungsszenarien schulischer Inklusion identifiziert werden, innerhalb derer Schulleitende die Forderungen schulischer Inklusion in einer transformatorischen Artikulation auf Ebene der Einzelschule aufgreifen und subventionieren oder aber auch strategisch im Sinne des Erhalts bestehender Kulturen, Strukturen und Praktiken systematisch unterminieren können (ebd.).

Eine über den hier dargestellten explorativen Blick in Deutschland und der Schweiz hinausgehende Bearbeitung des Themenfeldes in einer international vergleichenden Ausrichtung würde es insbesondere ermöglichen, diesen Handlungs- und Deutungsspielraum der Schulleitenden unter der Berücksichtigung der jeweiligen nationalen institutionellen Strukturen, Praktiken und Kulturen respektive der bildungspolitischen und administrativen Vorgaben weiterführend untersuchen zu können.

Dies berührt insbesondere auch die Frage, inwieweit die hier aufgezeigten Strategien tatsächlich Ausdruck individueller Dispositionen der Schulleitenden und/oder Ausdruck systemischer Zusammenhänge und Akteur*innenkonstellationen innerhalb der auch in der Schweiz und in Deutschland international propagierten neuen Steuerungsmodelle sind (Altrichter/Feyerer 2011; Rürup 2011). So ließen sich im internationalen Vergleich aus einer Mehr-Ebenen-theoretischen-Betrachtung mit Blick nach „oben“ mögliche Handlungs- und Deutungsspielräume der Schulleitenden differenziert sichtbarmachen und davon ausgehend auch Möglichkeiten eines die Schulleitenden unterstützenden bildungspolitischen und administrativen Akteur*innenhandelns weitergehend explorieren.

Gleichzeitig wäre mit Blick nach ,unten“ hier die Frage nach der Zusammenarbeit mit den Akteur*innen auf Ebene der Einzelschule und insbesondere auf der Ebene des Lehrens und Lernens näher in den Blick zu nehmen. Die Frage nach einem inklusionsorientierten Schulleitungshandeln ist hier schließlich vor den möglich werdenden Praktiken einer inklusionsorientierten Ausgestaltung der Lehr- und Lernprozesse und des durch die UN-BRK international formulierten Rechtsanspruchs der Schüler*innen zu reflektieren.

\section{Abschließender Ausblick: Der Weg zu einem inter- und/oder transnationalen Verständnis von Inklusion?}

Auch etwas selbstkritisch soll abschließend mit Blick auf den hier rezipierten und (re-)produzierten internationalen Forschungsstand auf die starke „Northernness“ dieser vergleichenden Ergebnisse aufmerksam gemacht werden. So bleibt für zukünftige Forschungsbemühungen eine deutliche Erweiterung der international vergleichenden Perspektive zu den Inklusionsentwicklungen im sogenannten „Global North“ durch die Berücksichtigung des „Global South" bisher offen (Biermann 2021; Hummel 2021; Schäfer 2021; Schreiber-Barsch/Rule 2021; jeweils i.d.B.). Insbesondere jüngere Untersuchungen zur integrativen/inklusiven Bildung im internationalen Vergleich haben die hegemoniale Bedeutung dieses Konzepts in 
Frage gestellt (Grech 2011; Rambla et al. 2008; Urwick/Eliiot 2010). So machen Naraian und Amrhein (2020) darauf aufmerksam, dass die Aufrufe zur Überprüfung des Verständnisses von Inklusion und ihrer theoretischen wie praktischen Implikationen innerhalb der Wissenschaft im Globalen Norden noch wenig Beachtung gefunden haben (siehe Naraian 2016; Messiou 2017). Auch der internationale Diskurs um ein inklusionsorientiertes Schulleitungshandeln ist insbesondere durch den angloamerikanischen Sprachraum geprägt (hierzu Badstieber et al. 2018).

An dieser Stelle sei sehr knapp festgestellt, dass gerade die Inklusionsentwicklungen im „Global South“ der Erde für die Schulleitenden und andere Akteur*innen unwiderruflich mit regionalen Zwängen verbunden sind - kulturell, wirtschaftlich und politisch -, in denen etwa mit Aspekten hoher Armut, niedriger Alphabetisierungsrate, hoher Bevölkerungsdichte und begrenzten Ressourcen umgegangen werden muss. Auch das häufig verborgene Verschweigen der kolonialen Grundlage für die Wissensbildung verewigt nicht nur Ungleichheit, sondern privilegiert auch weiterhin ein Inklusionsverständnis, das für die Erfahrungen der Menschen im globalen Süden mitunter wenig relevant ist (Naraian 2016). Für die Analyse im vorliegenden Text kann jedoch vermutet werden, dass auch im Globalen Süden die Rollen der Schulleitungen als "tranformational leader" oder „,change agent" sehr relevant sein könnten. So wären die Erfahrungen der südlichen Nationen innerhalb der international vergleichenden Inklusionsforschung verstärkt zu fokussieren und unter der Berücksichtigung der nationalen „Artikulationen“ (Stein et al. 2016) international vergleichende Studien als methodisches Mittel zur Entwicklung eines transnationalen Verständnisses von und Bemühens um integrative/inklusive Bildung denkbar. Damit verbunden sind für uns Wissenschaftler*innen insbesondere zwei Fragen: Welche Erkenntnisse über die Artikulationen integrativer/inklusiver Bildung (Köpfer 2020) werden durch unsere Arbeit als Forscher*innen, die in sehr verschiedenen nationalen Kontexten entstehen, vermittelt? Wie können auch wir durch unsere eigenen, kooperativen, internationalen Partnerschaften mehr Nord-Süd-Gerechtigkeit bei der Konzeption integrativer/inklusiver Forschung und Bildung erreichen?

In Bezug auf das hier zur Anwendung gekommene Inklusionsverständnis würden wir gerne mit Weisser enden, der im aktuellen Inklusionsdiskurs die Chance sieht, in der Schule und im Bildungssystem ,historische und lokale Beschränkungen des Angebots zu reflektieren und das Angebot mit Blick auf die Rechte aller Kinder und Jugendlichen auf Bildung und die Entfaltung ihrer Fähigkeiten weiterzuentwickeln“ (Weisser 2017: 148).

\section{Literatur}

Achermann, Bruno/Buholzer, Alois/Däppen, Sandra/Hubmann, Fabienne/Sahli-Lozano, Caroline (2017): Länderbericht Schweiz. In: Zeitschrift für Inklusion 11, 2. https://www.inklusion-online.net/index.php/inklusion-online/article/view/421 [Zugriff: 10.10.2019].

Ainscow, Mel (2021 i.d.B.): Inclusion and Equity in Education. In: Köpfer, Andreas/Powell, Justin J.W./Zahnd, Raphael (Hrsg.): Handbuch Inklusion international. Opladen: Budrich, S. 7587.

Altrichter, Herbert/Feyerer, Ewald (2011): Auf dem Weg zu einem inklusiven Schulsystem? Die Umsetzung der UN-Konventionen in Österreich aus der Sicht der Governance-Perspektive. In: Zeitschrift für Inklusion 5, 4. https://www.inklusion-online.net/index.php/inklusion-online/article/view/73/73 [Zugriff: 09.10.2019]. 
Amrhein, Bettina (2011): Inklusion in der Sekundarstufe. Eine empirische Analyse. Bad Heilbrunn: Verlag Julius Klinkhardt.

Amrhein, Bettina (2014): Am und im Widerspruch arbeiten - Wege aus dem professionellen Unbehagen in inklusiven Bildungsreformen. In: Friedrich Jahresheft, S. 17-20.

Amrhein, Bettina/Badstieber, Benjamin (2013): Lehrerfortbildungen zu Inklusion. Eine Trendanalyse. Im Auftrag der Bertelsmann Stiftung. http://www.bertelsmann-stiftung.de/fileadmin/files/BSt/Publikationen/GrauePublikationen/Studie_IB_Lehrerfortbildungen_zu_Inklusion_ 2013.pdf [Zugriff 03.02.2019].

Amrhein, Bettina/Badstieber, Benjamin (2019): Handreichung. Gesunde, inklusive Schule gestalten - Handlungsempfehlungen und Reflexionsimpulse für Schulleitende. Ms. (unveröff.).

Angelides, Panayiotis (2012): Forms of Leadership that Promote Inclusive Education in Cypriot Schools. In: Educational Management Administration \& Leadership 40, 1, S. 21-36.

Avenarius, Hermann (2010): Schulverfassung II (Schulleitung, Lehrerkonferenz, Schulkonferenz). In: Avenarius, Hermann/Füssel Hans-Peter (Hrsg.): Schulrecht. Ein Handbuch für Praxis, Rechtsprechung und Wissenschaft. Köln: Carl Link, S. 147-160.

Badstieber, Benjamin (2020, i.V.): Zwischen systemkonformer Anpassung und transformierender Innovation - Eine empirische Analyse der Rekontextualisierungsstrategien von Schulleitenden an Schulen der Sekundarstufe I in Nordrhein-Westfalen im Kontext schulischer Inklusion. Inauguraldissertation an der Humanwissenschaftlichen Fakultät der Universität zu Köln.

Badstieber, Benjamin/Köpfer, Andreas/Amrhein, Bettina (2018): Schulleitungen im Kontext inklusiver Bildungsreformen. In: Sturm, Tanja/Wagner-Willi, Monika (Hrsg.): Handbuch schulische Inklusion. Opladen/Toronto: Verlag Barbara Budrich, S. 235-250.

Badstieber, Benjamin/Moldenhauer, Anna (2016): Schulleitungshandeln in inklusionsorientierten Schulentwicklungsprozessen. In: Böing, Ursula/Köpfer, Andreas (Hrsg.): Be-Hinderung der Teilhabe. Soziale, politische und institutionelle Herausforderungen inklusiver Bildungsräume. Bad Heilbrunn: Verlag Julius Klinkhardt, S. 209-219.

BehiG (2002): Behindertengleichstellunggesetz. Bundesgesetz über die Beseitigung von Benachteiligungen von Menschen mit Behinderungen. https://www.edi.admin.ch/edi/de/home/fachstellen/ebgb/recht/schweiz/behindertengleichstellungsgesetz-behig.html [Zugriff: 16.10. 2019].

Biermann, Julia (2021 i.d.B.): Der Einfluss der UN-BRK auf inklusive Bildung in Nigeria und Deutschland. In: Köpfer, Andreas/Powell, Justin J.W./Zahnd, Raphael (Hrsg.): Handbuch Inklusion international. Opladen: Budrich, S. 167-178.

Blanck, Jonna M. (2014): Organisationsformen schulischer Integration und Inklusion. Eine vergleichende Betrachtung der 16 Bundesländer. In: WZB Discussion Papers, SP I 2014-501. Berlin: Wissenschaftszentrum Berlin für Sozialforschung.

Bless, Gerard (2007): Zur Wirksamkeit der Integration. Forschungsüberblick, praktische Umsetzung einer integrativen Schulform, Untersuchungen zum Lernfortschritt. Beiträge zur Heilund Sonderpädagogik, 18. Bern: Haupt.

Bless, Gerard/Kronig, Winfried (1999): Wie integrationsfähig ist die Schweizer Schule geworden? - Eine bildungsstatistische Analyse über schulorganisatorische Maßnahmen bei „Normabweichungen“. In: Vierteljahresschrift für Heilpädagogik und ihre Nachbargebiete 68, 4, S. 414-426.

Boban, Ines/Hinz, Andreas (2009): Inklusive Pädagogik zwischen allgemeinpädagogischer Verortung und sonderpädagogischer Vereinnahmung - Anmerkungen zur internationalen und zur deutschen Debatte. In: Börner, Simone/Glink, Andrea/Jäpelt, Birgit/Sanders, Dietke/Sasse, Ada (Hrsg.): Integration im vierten Jahrzehnt. Bilanz und Perspektiven. Bad Heilbrunn: Verlag Julius Klinkhardt, S. 220-228.

Böing, Ursula/Köpfer, Andreas (2016): Einleitung - Inklusion zwischen Bewahrung und Transformation von Bildungsräumen. In: Böing, Ursula/Köpfer, Andreas (Hrsg.): Be-Hinderung der 
Teilhabe. Soziale, politische und institutionelle Herausforderungen inklusiver Bildungsräume. Bad Heilbrunn: Verlag Julius Klinkhardt, S. 7-14.

Bohnsack, Ralf (2014): Rekonstruktive Sozialforschung: Einführung in qualitative Methoden. Opladen: Budrich.

Bonsen, Martin (2016): Schulleitung und Führung in der Schule. In: Altrichter, Herbert/Maag Merki, Katharina (Hrsg.): Handbuch Neue Steuerung im Schulsystem. Wiesbaden: VS Verlag für Sozialwissenschaften, S. 301-323.

Booth, Tony/Ainscow, Mel (2011): Index for Inclusion. Developing Learning and Participation in Schools. London: Centre for Studies on Inclusive Education.

BRK-ALLIANZ (2013): Erster Bericht der Zivilgesellschaft zur Umsetzung der UN Behindertenrechtskonvention in Deutschland. https://www.institut-fuer-menschenrechte.de/fileadmin/ user_upload/Word/Erster_Bericht_der_Zivilgesellschaft_zur_Umsetzung_der_UN_Behindertenrechtskonvention_in_Deutschland_2013.doc [Zugriff: 16.10.2019].

Degener, Theresia (2009): Die UN-Behindertenrechtskonvention als Inklusionsmotor. In: Recht der Jugend und des Bildungswesens 57, 2, S. 200-219. https:/www.studentenwerke.de/sites/ default/files/un_behindertenrechtskonvention_degener2.pdf [Zugriff: 16.10.2019].

Deutsches Institut für Menschenrechte (2015): Parallelbericht an den UN-Fachausschuss für die Rechte von Menschen mit Behinderungen anlässlich der Prüfung des ersten Staatenberichts Deutschlands gemäß Artikel 35 der UN- Behindertenrechtskonvention durch die MonitoringStelle zur UN-Behindertenrech. Berlin. http://www.institut-fuer-menschenrechte.de/fileadmin/ user_upload/PDF-Dateien/Parallelberichte/Parallelbericht_an_den_UN-Fachausschuss_fuer_ die_Rechte_von_Menschen_mit_Behinderungen_150311.pdf [Zugriff 16.10.2019]

Deutsches Institut für Menschenrechte (2019): Behindertenrechtskonvention (CRPD). https:// www.institut-fuer-menschenrechte.de/menschenrechtsinstrumente/vereinte-nationen/menschenrechtsabkommen/behindertenrechtskonvention-crpd [Zugriff: 10.10.2019].

EDK (2007): Interkantonale Vereinbarung über die Zusammenarbeit im Bereich Sonderpädagogik. https://edudoc.educa.ch/static/web/arbeiten/sonderpaed/konkordat_d.pdf [Zugriff: 10.10. 2019].

Engelbrecht, Petra/Oswald, Marietje/Forlin, Chris (2006): Promoting the implementation of inclusive education in primary schools in South Africa. In: British Journal of Special Education 33, 3, S. 121-129.

Fend, Helmut (2008a): Neue Theorie der Schule. Einführung in das Verstehen von Bildungssystemen. Wiesbaden: VS Verlag für Sozialwissenschaften.

Fend, Helmut (2008b): Schule gestalten. Systemsteuerung, Schulentwicklung und Unterrichtsqualität. Wiesbaden: VS Verlag für Sozialwissenschaften.

Feuser, Georg (2012): Eine zukunftsfähige „Inklusive Bildung“ - keine Sache der Beliebigkeit! Vortrag im Rahmen eines durch den Landesverband Bremen von Bündnis90/DIEGRÜNEN zur Frage der Inklusion und der damit verbundenen Entwicklungen in Bremen im KonsulHackfeld-Haus veranstalteten Forums vom 06. Juni 2012. https://www.georg-feuser.com/wpcontent/themes/pdf/Feuser_G._Zukunftsf_hige_Inklusive_Bildung_HB_06_06_2012.pdf [Zugriff: 16.10.2019].

Feuser, Georg (2016): Die Integration der Inklusion in die Segregation. In: Böing, Ursula/Köpfer, Andreas (Hrsg.): Be-Hinderung der Teilhabe Soziale, politische und institutionelle Herausforderungen inklusiver Bildungsräume. Bad Heilbrunn: Verlag Julius Klinkhardt, S. 26-43.

Feuser, Georg (Hrsg.) (2017): Inklusion - ein leeres Versprechen? Zum Verkommen eines Gesellschaftsprojekts. Gießen: Psychosozial-Verlag.

Gasterstädt, Julia (2021 i.d.B.): Same same but different. In: Köpfer, Andreas/Powell, Justin J.W./Zahnd, Raphael (Hrsg.): Handbuch Inklusion international. Opladen: Budrich, S. 179196. 
Grech, Shaun (2011): Recolonising Debates or Perpetuated Coloniality? Decentring the Spaces of Disability, Development and Community in the Global South. In: International Journal of Inclusive Education 15, 1, S. 87-100.

Heyer, Katharina (2021, i.d.B.): What is a Human Right to Inclusive Education? In: Köpfer, Andreas/Powell, Justin J.W./Zahnd, Raphael (Hrsg.): Handbuch Inklusion international. Opladen: Budrich, S. 45-57.

Hinz, Andreas (2008): Inklusion - historische Entwicklungslinien und internationale Kontexte. In: Hinz, Andreas/Körner, Ingrid/Niehoff, Ulrich (Hrsg.): Von der Integration zur Inklusion: Grundlagen - Perspektiven - Praxis. Marburg: Lebenshilfe-Verlag, S. 33-52.

Hinz, Andreas (2009): Inklusive Pädagogik in der Schule - veränderter Orientierungsrahmen für die schulische Sonderpädagogik!? Oder doch deren Ende?? In: Zeitschrift für Heilpädagogik, 5, S. 171-179.

Hinz, Andreas (2013): Inklusion - von der Unkenntnis zur Unkenntlichkeit!? - Kritische Anmerkungen zu einem Jahrzehnt Diskurs über schulische Inklusion in Deutschland. In: Zeitschrift für Inklusion 1. https://www.inklusion-online.net/index.php/inklusion-online/article/view/26 [Zugriff: 16.10.2019].

Hofstetter, Daniel (2017): Die schulische Selektion als soziale Praxis. Aushandlungen von Bildungsentscheidungen beim Übergang von der Primarschule in die Sekundarstufe I. Weinheim/Basel: Beltz Juventa.

Hollenweger, Judith (2016): Inklusion. Kein Handlungsbedarf? In: Bildungspolitik, 196, S. 1112.

Hoppey, David/McLeskey, James (2012): A Case Study of Principal Leadership in an Effective Inclusive School. In: The Journal of Special Education 46, 4, S. 245-256.

Huber, Stephan G./Sturm, Tanja/Köpfer, Andreas (2017): Inklusion und Schulleitung - Schulleitende als Gestaltende inklusiver Schulen (auch) in der Schweiz. In: Lütje-Klose, Birgit/Miller, Susanne/Schwab, Susanne/Streese, Bettina (Hrsg.): Inklusion: Profile für die Schul- und Unterrichtsentwicklung in Deutschland, Österreich und der Schweiz. Theoretische Grundlagen Empirische Befunde - Praxisbeispiele. Münster/New York: Waxmann, S. 43-57.

Huber, Stephan G./Wolfgramm, Christine/Kilic, Selin (2013): Vorlieben und Belastungen im Schulleitungshandeln: Ausgewählte Ergebnisse aus der Schulleitungsstudie 2011/2012 in Deutschland, Österreich, Lichtenstein und der Schweiz. In: Huber, Stephan G. (Hrsg.): Jahrbuch Schulleitung 2013. Befunde und Impulse zu den Handlungsfeldern des Schulmanagements. Schwerpunkt: gesunde Schule. Köln: Carl Link, S. 259-271.

Hummel, Myriam (2021 i.d.B.): Die diskursive Konstruktion inklusiver Bildung im südlichen Afrika. In: Köpfer, Andreas/Powell, Justin J.W./Zahnd, Raphael (Hrsg.): Handbuch Inklusion international. Opladen: Budrich, S. 147-163.

Inclusion Handicap (2017): Schattenbericht. Bericht der Zivilgesellschaft anlässlich des ersten Staatenberichtsverfahrens von dem UN-Ausschuss für die Rechte von Menschen mit Behinderungen. Bern. https://www.promentesana.ch/fileadmin/user_upload/News_Events/News/1708 29 schattenbericht_unobrk_inclusion_handicap_barrierefrei.pdf [Zugriff: 16.10 .2019$]$.

Keyes, Maureen W./Hanley-Maxwell, Cheryl/Capper, Colleen A. (1999): „Spirituality? It's the Core of my Leadership“. Empowering Leadership in an Inclusive Elementary School. In: Educational Administration Quarterly 35, 2, S. 203-237.

Köpfer, Andreas (2015): Rekonstruktion von Handlungspraktiken professioneller Akteur/-innen im Kontext inklusiver Schulentwicklung am Beispiel Schulleitender. In: Zeitschrift für Inklusion, 4. https://www.inklusion-online.net/index.php/inklusion-online/article/view/326/278 [Zugriff: 10.10.2019].

Köpfer, Andreas (2019): Rekonstruktion behinderungsbedingter Differenzproduktion in inklusionsorientierten Schulen. In: Budde, Jürgen/Dlugosch, Andrea/Herzmann, Petra/Rosen, Lisa/ Panagiotopoulou, Argyro/Sturm, Tanja/Wagner-Willi, Monika (Hrsg.): Inklusionsforschung 
im Spannungsfeld von Erziehungswissenschaft und Bildungspolitik. Opladen/Toronto: Verlag Barbara Budrich, S. 143-164.

Köpfer, Andreas (2020): Artikulationen Inklusiver Bildung zwischen Strukturkritik und funktionaler Integration - Erkundungen mit Stuart Hall. In: Vierteljahresschrift für wissenschaftliche Pädagogik 93, 2, S. 296-310.

Köpfer, Andreas/Mejeh, Mathias (2017): Inklusive Schulentwicklung in der Schweiz im Spannungsfeld proaktiver und reaktiver Steuerung: Ein Beitrag zur Handlungspraxis von Schulleitungen unter Berücksichtigung ihrer formalen Rolle. In: Sonderpädagogische Förderung heute 62, 2, S. 168-179.

Krause, Andreas/Baeriswyl, Sophie/Dorsemagen, Cosima (2013): Die Arbeits- und Gesundheitssituation von Schulleitungen. In: Huber, Stephan G. (Hrsg.): Jahrbuch Schulleitung 2013. Befunde und Impulse zu den Handlungsfeldern des Schulmanagements: Schwerpunkt: gesunde Schule. Köln: Carl Link, S. 241-258.

Kronig, Winfried (2007): Die systematische Zufälligkeit des Bildungserfolgs. Theoretische Erklärungen und empirische Untersuchungen zur Lernentwicklung und zur Leistungsbewertung in unterschiedlichen Schulklassen. Bern: Haupt.

Kugelmass, Judy W./Ainscow, Mel (2004): Leadership for inclusion. A comparison of international practices. In: Journal of Research in Special Educational Needs 4, 3, S. 133-141.

Kummer-Wyss, Annemarie (2012): Das sonderpädagogische Grundangebot in der Deutschschweiz. https://www.lch.ch/fileadmin/files/documents/Positionspapiere/130301_Studie_Umsetzung_Sonderpaedagogik.pdf [Zugriff: 10.10.2019].

Lange, Valerie (2017): Inklusive Bildung in Deutschland. Ländervergleich. Bericht im Auftrag der Friedrich-Ebert-Stiftung. http://library.fes.de/pdf-files/studienfoerderung/13493.pdf [Zugriff: 16.10.2019].

Lütje-Klose, Birgit/Neumann, Phillip/Streese, Bettina (2017): Schulische Inklusion in NordrheinWestfalen (NRW) - sieben Jahre nach Ratifizierung der UN-BRK. In: Zeitschrift für Inklusion, 2. https://www.inklusion-online.net/index.php/inklusion-online/article/view/416 [Zugriff 16. 10.2019].

Mejeh, Mathias (2015): Absicht und Wirklichkeit Integrativer Bildung - Ein netzwerkanalytischer Beitrag zum Neo-Institutionalismus. Wiesbaden: Springer VS.

Mejeh, Mathias (2021 i.d.B.): Inklusive Bildung als Institution am Beispiel Schweiz. In: Köpfer, Andreas/Powell, Justin J.W./Zahnd, Raphael (Hrsg.): Handbuch Inklusion international. Opladen: Budrich, S. 221-238.

Mejeh, Mathias/Powell, Justin J.W. (2018): Inklusive Bildung in der Schweiz. Zwischen globalen Normen und kantonalen Besonderheiten. In: Bildung und Erziehung 71, 4, S. 412-431.

Messiou, Kyriaki (2017): Research in the Field of Inclusive Education. Time for a Rethink? In: International Journal of Inclusive Education, S. 1-14

Mißling, Sven/Ückert, Oliver (2014): Inklusive Bildung. Schulgesetze auf dem Prüfstand. Im Auftrag des Deutschen Instituts für Menschenrechte. Berlin. http://www.institut-fuer-menschenrechte.de/fileadmin/_migrated/tx_commerce/Studie_Inklusive_Bildung_Schulgesetze_auf_dem_Pruefstand.pdf [Z̄ugriff: 16.10.2019].

Naraian, Srikala (2016): Inclusive Education Complexly Defined for Teacher Preparation: The Significance and Uses of „Error“. In: International Journal of Inclusive Education 20, 9, S. 946961.

Naraian Srikala/Amrhein Bettina (2020): Learning to read 'inclusion' divergently: Enacting a transnational approach to inclusive education. In: International Journal of Inclusive Education, online first: doi: 10.1080/13603116.2020.1807624

Nohl, Arnd-Michael (2012): Interview und dokumentarische Methode. Anleitungen für die Forschungspraxis. Wiesbaden: Springer VS. 
Pekruhl, Ulrich/Dorsemagen, Cosima/Kölliker, Alexandra/Krause, Andreas (2011): Arbeitszeit und Arbeitsorganisation der Schulleitungen an den Volksschulen des Kantons BaselLandschaft. Projektbericht für Bildungs-, Kultur- und Sportdirektion. Fachhochschule Nordwestschweiz, Hochschule für Wirtschaft und Hochschule für angewandte Psychologie, Olten. https://www.researchgate.net/publication/274138474_Arbeitszeit_und_Arbeitsorganisa tion_der_Schulleitungen_an_den_Volksschulen_des_Kantons_Basel-Landschaft [Zugriff: 09. $01.2020]$.

Powell, Justin J.W./Hadjar, Andreas (2018): Schulische Inklusion in Deutschland, Luxemburg und der Schweiz: Aktuelle Bedingungen und Herausforderungen. In: Rathmann, Katharina/ Hurrelmann, Klaus (Hrsg.): Leistung und Wohlbefinden in der Schule: Herausforderung Inklusion. Weinheim: Beltz Juventa, S. 46-64.

Preuss-Lausitz, Ulf (2019): Ergebnisse der Inklusions- und Separationsforschung nach zehn Jahren UN-Konvention über die Rechte von Menschen mit Behinderungen. Bilanz und Perspektiven. In: Zeitschrift für Heilpädagogik 10, S. 468-483.

Rambla, Xavier/Ferrer, Ferran/Rarabini, Aina/Verger, Antoni (2008): Inclusive Education and Social Inequality. An Update of the Question and Some Geographical Considerations. In: Prospects: Quarterly Review of Comparative Education 38, 1, S. 65-76.

Rürup, Matthias (2011): Inklusive Bildung als Reformherausforderung. Zur Perspektive der Educational Governance Forschung. In: Zeitschrift für Inklusion 4. http://www.inklusion-online. net/index.php/inklusion/article/view/132/128 [Zugriff: 16.10.2019].

Ryan, James (2012): Struggling for Inclusion. Educational Leadership in a Neo-Liberal World. Information Age Publishing Inc.

Schäfer, Lea (2021 i.d.B.): Maßnahmen inklusiver Bildung. In: Köpfer, Andreas/Powell, Justin J.W./Zahnd, Raphael (Hrsg.): Handbuch Inklusion international. Opladen: Budrich, S. 197220.

Scheer, David (2020, i. E.): Schulleitung und Inklusion. Empirische Untersuchung zur Schulleitungsrolle im Kontext schulischer Inklusion. Wiesbaden: Springer VS.

Scheer, David/Laubenstein, Désirée/Lindmeier, Christian (2014): Die Rolle von Schulleitung in der Entwicklung des inklusiven Unterrichts in Rheinland-Pfals. In: Zeitschrift für Heilpädagogik, 4, S. 147-155. https://www.researchgate.net/publication/261229770_Die_Rolle_von_ Schulleitung_in_der_Entwicklung_des_inklusiven_Unterrichts_in_Rheinland-Pfalz_Vorstellung_eines_Forschungsdesigns_im_Rah̄men_der_Schulbegleitforschung [Zugriff: 16.10 . 2019].

Schreiber-Barsch, Silke/Rule, Peter (2021 i.d.B.): Shifting Lenses to a Participatory Ethos in Research. In: Köpfer, Andreas/Powell, Justin J.W./Zahnd, Raphael (Hrsg.): Handbuch Inklusion international. Opladen: Budrich, S. 547-572.

Schumann, Brigitte (2016): „Auf dem Weg zur Inklusion“ oder zur „Sonderpädagogisierung“ der allgemeinen Schule? In: Amrhein, Bettina (Hrsg.): Diagnostik im Kontext inklusiver Bildung Theorien, Ambivalenzen, Akteure, Konzepte. Bad Heilbrunn: Verlag Julius Klinkhardt, S. 168177.

Schweizerischer Bundesrat (2014): Beitritt der Schweiz zur UNO-Behindertenrechtskonvention. https://www.admin.ch/gov/de/start/dokumentation/medienmitteilungen.msg-id-52666.html [Zugriff: 10.10.2019].

Schweizerischer Bundesrat (2016): Erster Bericht der Schweizer Regierung über die Umsetzung des Übereinkommens über die Rechte der Menschen mit Behinderungen. https://www.edi.admin.ch/dam/edi/de/dokumente/gleichstellung/bericht/Initialstaatenbericht\%20BRK.pdf.download.pdf/Initialstaatenbericht_BRK_v1.0.pdf [Zugriff: 10.10.2019].

Slee, Roger/Weiner, Gaby (2001): Education Reform and Reconstructions as a Challenge to Research Genres. Reconsidering School Effectiveness Research and Inclusive Schooling. In: School Effectiveness and School Improvement 12, 1, S. 83-98. 
Stein, Sharon/Andreotti, Vanessa/Bruce, Judy/Susa, Rene (2016): Towards Different Conversations About the Internationalization of Higher Education. In: Comparative and International Education 45, 1, S. 1-18.

Stemmer Obrist, Gabriele (2012): Schulleiterinnen und Schulleiter in der Aargauer Volksschule. Eine qualitative Studie zum beruflichen Selbst- und Führungsverständnis von Schulleitenden und zu den Gelingensbedingungen operativer Führung im Paradigmenwechsel. Baden: UZH Dissertationen. https://www.zora.uzh.ch/id/eprint/63920/1/Stemmer_Dissertation_vollstText_ SL.pdf [Zugriff: 10.10.2019].

Sturm, Tanja (2013): Lehrbuch. Heterogenität in der Schule. München/Basel: Reinhardt.

Sturm, Tanja/Köpfer, Andreas/Huber Stephan G. (2015): Schulleitungen. Gestaltende einer inklusionsfähigen Schule - Begriffserklärung und Stand der Forschung. In: Huber, Stephan G. (Hrsg.): Jahrbuch Schulleitung 2015. Kronach: Karl Link, S. 193-210.

Trumpa, Silke/Janz, Frauke (2014): „Ich mach mir die Welt, wie sie mir gefällt!“ Rekontextualisierungen und Verantwortungsübernahme im Implementierungsprozess der Inklusion. In: Trumpa, Silke/Seifried, Stefanie/Franz, Eva/Klauß Theo (Hrsg.): Inklusive Bildung. Erkenntnisse und Konzepte aus Fachdidaktik und Sonderpädagogik. Weinheim/Basel: Beltz Juventa, S. 61-78.

UN (2006): The UN-Convention on the Rights of Persons with Disabilities. https://www.un. org/disabilities/documents/convention/convoptprot-e.pdf [Zugriff: 10.10.2019].

UNESCO (2009): Policy Guidelines on Inclusion in Education. Paris. https://unesdoc.unesco. org/ark:/48223/pf0000177849 [Zugriff: 16.10.2019].

UNESCO. (2017): A guide for ensuring inclusion and equity in education. Paris. http://unesdoc.unesco.org/images/0024/002482/248254e.pdf [Zugriff 16.10.2019].

Urwick, James/Elliott, Julian C. (2010): International orthodoxy versus national realities. Inclusive schooling and the education of children with disabilities in Lesotho. In: Comparative Education 46, 2, S. 137-150.

Weisser, Jan (2017): Konfliktfelder schulischer Integration/Inklusion im 20. Jahrhundert. Weinheim: Beltz.

Wissinger, Jochen (2014): Schulleitung und Schulleitungshandeln. In: Terhart, Ewald/Bennewitz, Hedda/Rothland, Martin (Hrsg.): Handbuch der Forschung zum Lehrerberuf. Münster: Waxmann, S. 144-176.

Witzel, Andreas (2000): Das problemzentrierte Interview. In: Forum Qualitative Sozialforschung/Forum: Qualitative Social Research 1, 1, S. 1-13. https://www.researchgate.net/publication/228581012_Das_problemzentrierte_Interview [Zugriff: 09.01.2020].

Wocken, Hans (2011): Über die Entkernung der Behindertenrechtskonvention. Ein deutsches Trauerspiel in 14 Akten, mit einem Vorspiel und einem Abgesang. In: Zeitschrift für Inklusion. https://www.inklusion-online.net/index.php/inklusion-online/article/view/80 [Zugriff 16.10 . 2019].

Ziemen, Kerstin (2016). De-Kategorisierung. Vierteljahresschrift für Heilpädagogik und ihre Nachbargebiete, 2, S. 93-97. 


\title{
22 Konstruktion von (Leistungs-)Differenzen in der Schule - Ein transnationaler Fallvergleich unterrichtlicher Praxen
}

Tanja Sturm

\begin{abstract}
Der Beitrag widmet sich der Bedeutung schulstruktureller Rahmenbedingungen für die Genese unterrichtlicher Praxen der Konstruktion von Leistungsdifferenzen und damit verbundener Behinderungen sozialer und akademischer Teilhabe und der jeweiligen sozialisierenden Implikationen für die Schüler*innen. Anhand von Ergebnissen aus dem Projekt „Differenzkonstruktionen in unterrichtlichen Praxen - internationale Vergleiche“ wird aufgezeigt, dass sich die Konstruktionsprozesse von Lehrpersonen, die in ein- und mehrgliedrigen Schulsystemen tätig sind, unterscheiden.
\end{abstract}

\section{Einleitung}

Dass die Gestaltung inklusiver Bildung und Erziehung sich nicht darin erschöpft, alle Schüler*innen eines Einzugsgebiets und/oder eines Jahrgangs in einem Klassenraum zu platzieren, sondern über diese räumliche Situation hinaus v.a. Fragen der Gestaltung des schulischunterrichtlichen Zusammenlebens umfasst, kann als geteiltes Wissen erziehungswissenschaftlicher Diskurse zum Themenfeld angesehen werden. Eine vergleichbare Perspektive findet sich in internationalen, bildungspolitischen Dokumenten der UNESCO (2009). Inklusive Bildung und Erziehung werden dort mit der Zielperspektive verbunden, Teilhabe an Lernen, Kultur und Gemeinschaft zu eröffnen, bei gleichzeitiger Überwindung und Reduktion bestehender Exklusionen, Benachteiligungen und Marginalisierungen. Die Verantwortung hierfür wird in der gesellschaftlichen Organisation Schule und bei allen, dort tägigen professionellen Akteur*innen gleichermaßen verortet (vgl. ebd.). Damit greift die UNESCO ein kulturelles Verständnis der Ermöglichung sowie der Behinderung von Teilhabe an Erziehungs- und Bildungsprozessen auf (Waldschmidt 2005) und verortet die Verantwortung für den Abbau erkannter Barrieren entsprechend als gesellschaftliche und schulische Herausforderung. Hieran anknüpfend lässt sich mit Blick auf Schule und Unterricht u.a. fragen, welche Formen von Inklusion und Exklusion aktuell wie hervorgebracht werden und wie diese Praxen in Relation zu den jeweiligen schulischen Rahmenbedingungen steht, in denen sie hervorgebracht wwerden.

Die Bearbeitung dieser Fragen bedarf einer genauen Betrachtung der Komplexität des Gegenstands, dem Unterricht, der einen Interaktionsraum innerhalb der gesellschaftlichen Organisation Schule darstellt. Dieser zeichnet sich u.a. durch Erwartungen aus, die gesellschaftlich an die Schule und ihre professionellen Akteur*innen gestellt werden, die einen 
Rahmen ihrer Praxis darstellen. Die Aufgaben, die an Schule gerichtet werden, sind vielfältig und stehen z.T. im Widerspruch zueinander. Ein zentraler Widerspruch besteht zwischen den Erwartungen sowohl auf ein demokratisches als auch ein kapitalistisch-meritokratisches gesellschaftliches Miteinander vorzubereiten bzw. in dieses einzuführen. Bezogen auf das schulische Ziel der Qualifikation durch Erziehung und Bildung verweist dies gleichermaßen auf den Erwerb von Wissen und Kompetenzen für berufliche Tätigkeiten, im Sinne der Teilnahme an der Arbeitswelt, und für die Partizipation am politischen Leben in einer demokratischen Gesellschaft. Die Vorstellungen von Gleichheit/Ungleichheit der beiden unterscheiden sich. In schulisch-unterrichtlichen Settings findet sich dieser Unterschied ebenfalls als bessere/schlechtere Leistungen (vgl. Luhmann 2002: 62ff.) einerseits und, zugespitzt im Begriff der Inklusion als gleichberechtigte soziale und akademische Teilhabe oder, mit Annedore Prengel (2001), als „egalitäre Differenz“ andererseits. Diese Zielsetzungen, die in formalen Dokumenten, wie z.B. in Form von Schulgesetzen und Lehrplänen formuliert sind, stellen einen Rahmen dar, mit dem sich Lehrpersonen auseinandersetzen. Dies erfolgt u.a. mit erziehungswissenschaftlichen Theorien und/oder eigenen pädagogischen Idealen und Überzeugungen. Letztgenannte sind zu einem großen Teil implizite Wissensbestände, die in Erfahrungen generiert werden, nicht unbedingt explizit gemacht und reflektiert werden können, aber die Handlungspraxen der Lehrpersonen wesentlich leiten (vgl. Bohnsack 2017: 128ff.). Pädagogisches Handeln in Schule und Unterricht ist durch diese Grundambivalenz gerahmt, die sich im deutschen Schulsystem mit ihrer mehrgliedrigen Struktur insofern zuspitzt, als allokative und selektive Vorgänge vorgesehen sind und so eine stetige „Selektionsbedrohung“ (Schuck 2014: 163) besteht. Innerhalb dieses Rahmens entfaltet sich die Alltagspraxis der Akteur*innen (vgl. Amling 2017). Pädagogische Praxen, die sich in diesem Kontext entwickeln und vollzogen werden, stellen für die Schüler*innen nicht allein Qualifikationsanlässe dar, sondern sie sind bzw. wirken zugleich sozialisierend (vgl. Biesta 2011). Die unterrichtliche Praxis, das Wie des Miteinanders, lässt Schüler*innen nicht allein inhaltsbezogenes Wissen erfahren, wie es z.B. im Lehrplan als Ziel formuliert ist, sondern sie erfahren in der alltäglichen Praxis, mit den Lehrpersonen und ihren Mitschüler*innen, welche Formen von Teilhabe und Marginalisierung, oder Inklusion und Exklusion, welche Kultur des Miteinanders, der sozialen (Un)Gleichheit legitim und anerkannt sind und welche nicht.

Während alle Schüler*innen diese Erfahrungen in konkreten unterrichtlichen Settings machen, unterscheiden sie sich je nach ihrer Positionierung innerhalb des Spannungsfeldes von Teilhabe und Marginalisierung. So machen als ,erfolgreich' geltende Schüler*innen vermutlich andere Erfahrungen als jene, deren Leistung als ,nicht den Erwartungen entsprechend' eingeschätzt wird. Bezogen auf das ausgeführte Praxisverständnis wäre dafür zunächst zu fragen, was in einer konkreten unterrichtlichen Praxis als Leistung verstanden wird, wie diese zu einer Bewertung als Miss-/Erfolg und damit verbunder Teilhabe und/oder Marginalisierung wird. Leistung als schuleigene und -spezifische Differenzdimension und ihre unterrichtliche Hervorbringung ist bis dato deutlich weniger untersucht, als z.B. die (Re)Produktion von Gender (vgl. z.B. Budde/Scholand et al. 2008; Willems 2007) und familiärer, sozial-ökonomischer Herkunft in Schule und Unterricht (vgl. z.B. Jünger 2008; Wellgraf 2011). Die wenigen Studien, die das Thema im deutschsprachigen Raum bisher bearbeitet haben, zeigen, dass Leistungsunterschiede die zentrale Differenzdimension unterrichtlicher Praxis von Lehrpersonen darstellt, die hierarchisiert (besser/schlechter) und individualisiert den Schüler*innen zugeschrieben wird (vgl. Sturm 2010; Rabenstein et al. 2013; WagnerWilli et al. 2018). Diese weitgehend homogene Praxis findet sich über unterschiedliche Schulformen und Sozialräume des deutschsprachigen Raums hinweg und geht mit 
unterschiedlichen Formen der De/Gradierungen, der Besser-/Schlechterstellung der Schüler*innen einher sowie mit entsprechenden sozialisierenden Erfahrungen (vgl. z.B. Sturm et al. 2020).

Hieran schließt sich die Frage nach möglichen Erklärungen für diesen hohen Grad der Gemeinsamkeit der rekonstruierten unterrichtlichen Praxen mehrgliedriger deutschsprachiger Schulsysteme an. In einer explorativen Studie, in der Gruppendiskussionen zur unterrichtlichen Praxis mit Lehrpersonen aus Norwegen und den USA geführt werden, deutet sich an, dass die Delegationsmöglichkeiten bzw. Selektionsnotwendigkeiten, die durch unterschiedlich konzipierte Angebote, denen die Schüler*innen zugeordnet werden können, eine Erklärung für die Individualisierung von schulischem Miss-/Erfolg darstellen könnte. Diese Resultate legen nahe, dass in der schulischen Struktur, die sich im deutschen Sprachraum mit der Ausnahme Südtirols - durch unterschiedliche Formen der Mehrgliedrigkeit auszeichnet, eine Erklärung für die Genese der unterrichtlichen Praxis der Konstruktion und Bearbeitung von Leistungsdifferenzen individualisierender und hierarchisierender Form und der damit verbundenen sozialisierenden Erfahrungen wiederholter Degradierungen und Marginalisierungen sogenannter leistungsschwacher Schüler*innen liegen könnte. Ergebnisse aus der explorativen Studie - also auf der Grundlage erster, zufällig ausgewählter Fälle -, die Praxen in eingliedrigen Schulsystemen rekonstruiert, sollen hier dargelegt werden. Dies erfolgt mit dem inhaltlichen Ziel, mehr über die Relevanz sozialer Rahmenbedingungen für die Genese, also die Entwicklung von schulisch-unterrichtlichen Praxen der Differenzkonstruktion zu erfahren. Hervorzuheben ist, dass in dem gewählten Vorgehen - bedingt durch die Samplestruktur - methodisch eher von einem Fall- als von einem transnationalen Vergleich zu sprechen ist. Das explorative Interesse liegt mithin v.a. darin, zu erkennen, ob die Praxen sich unterscheiden und in der Folge eine systematisch angelegte Studie lohnenswert wäre. Um dem skizzierten Anliegen nachzukommen, wird zunächst der Projektkontext, in dem die Fälle generiert wurden, entlang seiner theoretischen und methodologischen Verankerung dargelegt (Abschnitt 2). Anschließend werden anhand ausgewählter Ausschnitte aus Gruppendiskussionen und ihrer sozialen Kontextualisierung die Ergebnisse der unterrichtlichen Praxis mit Fokus auf Leistung eines norwegischen und eines deutschen Falls vorgenommen und verglichen (Abschnitt 3). Abschließend werden im vierten Abschnitt inhaltliche und methodische Perspektiven mit Blick auf transnational vergleichende Forschung im Kontext schulisch-unterrichtlicher Inklusion formuliert.

\section{Differenzkonstruktionen in unterrichtlichen Praxen}

Das Projekt Differenzkonstruktionen in unterrichtlichen Praxen (Sturm 2011) folgt dem leitenden Erkenntnisinteresse, die Konstruktion und Bearbeitung von Differenzen durch Lehrpersonen in unterrichtlichen Praktiken zu rekonstruieren. Mit der systemtheoretischen Perspektive Niklas Luhmanns (2015: 86) lässt sich dies als „Beobachten von Beobachtungen“ oder als „Beobachtungen zweiter Ordnung“ beschreiben. Das Projekt selbst ist in der Praxeologischen Wissenssoziologie und der Dokumentarischen Methode verortet. Beide Ansätze gehen auf Arbeiten von Karl Mannheim zurück, der die Wissenssoziologie als „Lehre von der ,Seinsverbundenheit“ menschlichen Wissens“ (Mannheim 1995: 227, Herv. im Orig.) bezeichnet und sie von Erkenntnisformen abgrenzt, die auf den Prinzipien der Fremdheit und Entpersönlichung gegenüber den untersuchten sozialen und materiellen (Forschungs-) 
Gegenständen fußen. So ist es das Primat der Wissenssoziologie, das je spezifische Wissen und die jeweilige Sicht auf die Welt der Akteur*innen konstitutiv einzubeziehen. Die „existenziellen Beziehung[en]“ (Mannheim 1980: 210) zur sozialen und materialen Welt der Akteur*innen werden also nicht ausgeblendet, sondern ins Zentrum des Erkenntnisinteresses gestellt. Am Beispiel von Geschlecht lässt sich der Unterschied beispielhaft illustrieren: interessiert im ersten Ansatz die Zugehörigkeit zu einem der drei anerkannten Geschlechter, sind im zweiten die Erfahrungen, die konkreten Erlebnisse und die Bedeutung von Geschlecht und (zugeschriebener) Geschlechtlichkeit im Alltag und ihre Genese der beforschten Praxis von Interesse.

Ein weiteres konstitutives Element des Ansatzes, der maßgeblich von Ralf Bohnsack (2017) weiterentwickelt wurde, ist die „Doppelstruktur“ (Bohnsack 2007: 183) menschlichen Wissens: das kommunikative, das v.a. alltagssprachlich vorliegt und sich anknüpfend an Bourdieu (1996) als common sense-Wissen verstehen lässt, wird vom konjunktiven oder handlungspraktischen Wissen unterschieden. Das letztgenannte stellt eine Wissensform dar, die in existenziellen Erfahrungen und Beziehungen, die Mannheim (ebd.: 208) als „Kontagion“ oder als „konjunktiven Erfahrungsraum“ bezeichnet, erworben wird. Die Erfahrungen umfassen v.a. körperbasierte, sinnesphysiologische Formen und markieren und beschreiben die Einheit zwischen dem erkennenden Subjekt und dem sozialen und/oder materialen Gegenstand. Bohnsacks (2017) begriffliche Differenzierung der Wissenssoziologie als praxeologische betont die „handlungspraktische Herstellung der Realität“, also die „habitualisierten Praktiken, die auf dem handlungsleitenden und zum Teil inkorporierten Erfahrungswissen der Akteure basieren“ (Bohnsack 2007: 182f., Herv. im Orig.). Das hierin aufgerufene Wie der Herstellung von Realität lässt sich vom Was abgrenzen, das sich als explizites Wissen in Vorstellungen des common sense findet. Es basiert auf unhinterfragten Regeln bzw. einer illusio sowie der damit verknüpften Unterstellung (zweck-)rationaler Handlungsmuster (vgl. Bohnsack 2014: 38). Beide Wissensformen sind in ihrem Zusammenspiel konstitutiv und relevant für alltägliche Handlungen und zwischenmenschliche Kooperationsformen und lassen sich nur analytisch trennen.

Konjunktives Wissen ist weder allgemeingültig, da es sozial, kulturell und historisch verankert ist, noch individuell. Als kollektives Wissen, das Akteur*innen in den gleichen oder strukturell vergleichbaren Zusammenhängen erwerben, findet es seinen Ausdruck in einer geteilten Alltagspraxis. Die Akteur*innen verfügen hierüber, ohne dass sie dies (immer) reflexiv benennen können. Kollektive, geteilte Handlungspraxen werden als Milieus bezeichnet. Milieuspezifisches Handeln findet sich z.B. in Bezug auf Geschlecht sowie in professionellen Kontexten (vgl. Bohnsack 2014). Soziale Alltagspraxen, die im Kontext gesellschaftlicher Organisationen angesiedelt sind, stellen einen spezifischen Typ von Milieu dar. Sie zeichnen sich nicht allein durch die skizzierte Doppelstruktur expliziten und impliziten Wissens aus, sondern durch weitere Bezüge, zu denen u.a. formale Regeln der Organisation, die u.a. Rollenerwartungen, wie z.B. die Bewertung der Leistung von Schüler*innen durch die Lehrpersonen, zählen. Bohnsack spricht vor diesem Hintergrund von einer ,,doppelten Doppelstruktur“" (vgl. Bohnsack 2017: 129f.). Für Lehrpersonen bedeutet dies, dass sie nicht nur über Wissen über etwas, sondern auch und v.a. über handlungsleitendes Wissen des Unterrichtens verfügen. Dieses unterrichtspraktische Wissen zeichnet sich durch die Fähigkeit aus, in der Alltagspraxis Entscheidungen zu treffen und ist analytisch von theoretischem, explizitem Wissen über Unterricht abzugrenzen. Professionelle Unterrichtspraxen speisen sich entsprechend wesentlich aus der Sozialisation in konkreten organisationalen und pädagogischen Erfahrungsräumen. Schulisch-unterrichtliche Erfahrungsräume erfordern mithin auch die 
Integration widersprüchlicher Erwartungen und/oder Normen. Dies erfolgt - vergleichbar anderen Milieus - sowohl in expliziter als auch in handlungspraktischer Hinsicht (vgl. Bohnsack 2017: 103ff.).

Die Dokumentarische Methode und ihre Analyseschritte der formulierenden und reflektierenden Interpretation sowie der Komparativen Analyse eröffnen eine Rekonstruktion des konjunktiven Wissens, indem entweder die Praxis selbst in den Blick genommen wird, wie dies z.B. Unterrichtsvideografien ermöglichen (vgl. z.B. Bohnsack 2009), oder indem Erzählungen und Beschreibungen der alltäglichen Unterrichtspraxis, die in Interviews und/oder Gruppendiskussionen formuliert werden, herangezogen werden. Die Rekonstruktion der, der beschriebenen und erzählten Praxis zugrunde liegenden, handlungsleitenden Orientierungen entsprechen denen, die auch in der Hervorbringung der Praxis selbst leitend sind (vgl. Bohnsack 2014: 105ff.).

Vor dem Hintergrund dieser Bezüge konkretisiert sich der inhaltliche Fokus des Beitrags: die Frage nach der Erklärungskraft des schulstrukturellen Rahmens - mehr-/eingliedrig - für unterrichtlichen Praxen in Bezug auf die Konstruktion von Leistungsdifferenzen und damit verbundene sozialisierende Erfahrungen der (Behinderung von) Teilhabe.

\section{Konstruktion von Leistungsdifferenzen durch Lehrpersonen - transnationaler Vergleich}

Der bildungspolitisch formulierte Anspruch, Schule und Unterricht inklusiv zu gestalten, der in der Konvention über die Rechte von Menschen mit Behinderungen der Vereinten Nationen, kurz UN-BRK, (UN 2006; 2008) formuliert ist, und von zahlreichen Regierungen ratifiziert wurde, trifft in den Ländern und Schulsystemen auf je spezifische Rahmenbedingungen. Die nachfolgend darzustellenden Ausschnitte aus den geführten Gruppendiskussionen werden durch Kontextinformationen zu den Schulsystemen, die hier von besonderem Interesse sind, mit dem Fokus auf Fragen schulisch-unterrichtlicher Inklusion ergänzt.

\section{1 „Kinder aus dem Niemandsland“ - unterrichtliche Praxis der Gruppe Kontinent}

\subsubsection{Formaler schulischer Rahmen mit Fokus auf Inklusion in der Bundesrepublik Deutschland}

Anders als viele andere (west)europäische Länder verfügt Deutschland ebenso wie andere deutschsprachige Länder über mehrgliedrige Schulsysteme. Das heißt, es werden Bildungsgänge unterschieden, denen die Schüler*innen zugeordnet werden. Neben einem differenzierten Sonderschulwesen, das seinerseits neun Schulformen unterscheidet, werden die sogenannten Regelschüler*innen in den meisten Bundesländern nach der vierten Klasse auf Bildungsgänge, die je eigene Curricula bzw. Bildungspläne haben, aufgeteilt (vgl. Europäische Kommission 2016). Die Bildungsgänge gehen historisch auf die Stände- 
gesellschaft und die für die Stände offerierten Bildungsangebote zurück. Sie unterscheiden sich hierarchisch entlang von Leistungserwartungen und eröffnen eine unterschiedliche Breite an Möglichkeiten der an Schule anschließend zu besuchenden Bildungsangebote. Während in der DDR ein eingliedriges - bzw. mit den sonderschulischen Einrichtungen ein zweigliedriges (vgl. Becker/Große 1990) - Schulsystem bestand, blieb die Struktur der Schule der Weimarer Republik in der Bundesrepublik weitgehend erhalten. Im Wesentlichen - bei zahlreichen länderspezifischen Varianten - lassen sich die Bildungsgänge des Gymnasiums, der Haupt- und Real- sowie der Sonderschulen unterscheiden. Letztgenannte differenzieren sich insofern, als von den sieben Förderschwerpunkten fünf (Hören und Kommunikation, Sehen, Sprache und Kommunikation, Körperlich-Motorische Entwicklung sowie emotional-soziale Entwicklung) mit den sogenannten regelschulischen Bildungsgängen korrespondieren, während die Förderschwerpunkte Lernen und Geistige Entwicklung diese Möglichkeit nicht eröffnen (KMK 2011).

Die Themen Behinderung und Inklusion, verstanden als Überwindung und/oder Reduktion von Exklusion und Benachteiligungen, waren im erziehungswissenschaftlichen Diskurs lange selbst marginalisiert und wurden (fast) ausschließlich innerhalb sonderpädagogischer Diskussionen thematisiert (vgl. Tervooren 2001). Mit der Ratifizierung der UN-BRK durch Bund und Länder sowie den bildungspolitisch angestoßenen Reformen der Schulgesetze wie auch der Lehrer*innenbildung an den Universitäten (vgl. HRK/KMK 2015), hat sich dies zugunsten eines breiteren Diskurses verändert.

\subsubsection{Gruppe Kontinent}

Die fünf an der Gruppendiskussion beteiligten Lehrerinnen sind an einer Grundschule in einer deutschen Großstadt tätig. Die Schule arbeitet seit dem Schuljahr, in dem das Gespräch stattfindet, dem formalen Anspruch nach inklusiv. Neben Schüler*innen, die dem Bildungsgang der Grundschule angehören, wird die Schule von Schüler*innen besucht, die sonderpädagogischen Förderbedarf in den Förderschwerpunkten Lernen, Sprache und sozial-emotionale Entwicklung attestiert haben.

Die Gruppendiskussion hat mit dem Eingangsimpuls begonnen, der die Lehrpersonen auffordert, zu erzählen und zu beschreiben, was ihnen am Herzen liegt, wenn sie an ihre Schüler*innen denken. Die nachfolgend dargestellte Sequenz ist am Anfang des Gesprächs entstanden. Unmittelbar vorher unterhalten sich die Lehrerinnen über die Besonderheit der Bewertung von Schüler*innen mit zugeschriebenem sonderpädagogischen Förderbedarf. Diese Thematisierung von Differenzen erfolgte, wie in den meisten weiteren Gesprächen ebenfalls, meist zeitnah nach dem Gesprächseinstieg.

\section{Ausschnitt: „Kinder aus dem Niemandsland“}

${ }_{\text {„Cf }}{ }^{1}$ : die haben ja son Sonderstatus die sind ja ausgewiesene Sonderschüler und alles gilt irgendwie etwas anders aber bei den restlichen Kindern haben wir ja auch Kinder dabei die ganz sch-viele

1 Die Namen der beteiligten Lehrer*innen sind anonymisiert: Die Großbuchstaben stehen jeweils für eine Person, das f verweist auf das weibliche Geschlecht der Personen. Männliche Lehrer sind an dem Gespräch nicht beteiligt. Namen von Personen, z.B. Schüler*innen, die im Gespräch genannt werden, sind alle durch andere Namen ersetzt. Die Transkription ist entlang der Regeln des „TiQ: Talk in 
Schwierigkeiten mit m Lernen haben und dann lassen wir sie manchmal überprüfen weil ich denke das Kind kann ja gar nichts und dann wird eben festgestellt hat nicht diesen Sonderschul eh Bedarf sondern es ist ein Regelkind nicht hochbegabt aber $n$ Regelkind und die die Schwierigkeiten liegen in ganz vielen Bereichen die ehm in der gesamten Entwicklung dazu geführt haben bis hin zu stark auffälliges Verhalten dass das Kind gar nicht in der Lage ist zu lernen weil es nicht zur Ruhe kommt weil es sich nicht konzentrieren kann und und und und die eh find sind also für mich diejenigen die mir Schwierigkeiten machen in den Ansprüchen und in der Bewertung denn auch wenn ich Lernstand nehme wo eh wo Ef eben von gesprochen hat diese Lernstandserhebungen dann nehmen die I-Kinder nicht teil

Bf: $\quad$ Und wenn sie Noten bekämen bekämen sie sowieso andere

Df: $\quad$ Also die ärmsten Würmer sind eigentlich die finde ich die so gerade an der Grenze

Cf:

$\mathrm{Ja}$

Df: vorbeischrammen die dann irgendwie n IQ von hundert haben sag ich jetzt mal oder neunzig

Cf: Ja drunter meistens

Df: Meistens noch drunter irgendwie bei neunzig wo eben definitiv keine sonderpädagogische Förderung irgendwie

Bf: $\quad$ Förderbedarf schon aber kein sonderpädagogischer

Cf: ja genau so $[\ldots]$

Thematisch geht es in dieser Passage um unterschiedliche Gruppen von Schüler*innen und ihre Möglichkeiten, in der Schule zu lernen sowie die Verantwortung für die Gestaltung adäquater Lehr-Lernangebote durch die Lehrpersonen. Die Lehrerinnen unterscheiden zunächst zwei Gruppen von Schüler*innen: die sogenannten I-Kinder und die der „hochbegabten Regelkinder". Zwischen beiden Gruppen verorten sie die ,scheiternden Regelkinder'. Wenngleich die letztgenannte Gruppe formal zu den Regelkindern gezählt wird, unterscheidet sie sich von dieser. Die Zwischenposition zwischen „hochbegabten Regelkindern“ und denen mit Förderbedarf führen die Lehrerinnen auf deren niedrigen „Intelligenzquotienten“ zurück. Dieser ist aber nicht niedrig genug, um formal Anspruch auf ,sonderpädagogische Förderung" und den damit verbundenen Sonderstatus, wie z.B. nicht an Lernstandsvergleichen teilzunehmen, zu legitimieren. Im Verlauf des Gesprächsausschnitts wird diese Gruppe von Schüler*innen mit den Metaphern „Kinder aus dem Niemandsland“ und ,ärmste Würmer“ weiter charakterisiert. Mit Victor Turner (1966) kann ihre Position als "betwixt and between the positions assigned and arrayed by law, custom, convention, and ceremonial" (Turner 1966: 95) beschrieben werden. Das heißt, sie stehen zwischen zwei anerkannten und legitimen Positionen. Dieser inhaltliche Faden wird - nach einer kurzen Klärung eines anderen Themas - wie folgt von der Gruppe weiterbearbeitet:

Qualitative Social Research“ (Bohnsack 2010: 236) erstellt. @ steht für Lachen, (.) für eine kurze Pause, L verweist auf gleichzeitiges Sprechen. 
Df: $\quad$ und da muss ich dann auch so schwach sein damit ich irgendwie die Unterstützung bekomme das ist also ich find es immer so Kinder aus dem Niemandsland

Cf:

Df: $\quad$ irgendwie so kommen nicht gut mit und haben auch von zuhause aus

Cf: $\quad$ Und es steht ihnen noch keine Förderung

Aus der skizzierten Zwischenposition, das dokumentiert sich in dem zweiten Ausschnitt, kommen die Kinder im Unterricht nicht heraus. Mögliche kompensatorische Maßnahmen, die dies eröffnen könnten, verorten die Lehrpersonen durchgängig außerhalb des Unterrichts: im Elternhaus und in der Option, sonderpädagogischen Förderbedarf attestiert zu bekommen, um dann - dies bleibt implizit - in den Verantwortungsbereich der Sonderpädagogik zu fallen. Sonderpädagogische Förderung wäre ihrer Ansicht nach notwendig, sie wird im Bundesland jedoch unter den Bedingungen nicht gewährt. Die Unterscheidung der Schüler*innen entlang der formalen schulischen Kategorie des Förderbedarfs und des angeführten IQ-Werts hat für die Lehrerinnen scheinbar objektive Gültigkeit und bezieht sich auf die Schüler*innen in ihrer Position insgesamt und auch dauerhaft. Zugleich wird eine statische Objektivität insofern in Frage gestellt, als kompensatorische Maßnahmen das Ziel hätten, die Schüler*innen zu unterstützen, die an sie gerichteten Erwartungen zu erfüllen, wenngleich dies von den lehrerinnen außerhalb ihres Unterrichts verortet wird. In dem Ausschnitt werden die Probleme und Schwierigkeiten der Lehrerinnen in der Arbeit mit der Gruppe der „Kinder aus dem Niemandsland" deutlich. Während sie einerseits Empathie für die Kinder zeigen, wird handlungspraktisch deutlich, dass sie ihnen keine unterrichtlichen Angebote bereitstellen; vielmehr suchen sie die notwendige Unterstützung außerhalb des Unterrichts und sehen sich selbst nicht als zuständig.

In der Passage dokumentiert sich nicht nur eine habituelle Unterrichtspraxis, die sich dadurch auszeichnet, Schüler*innen in drei Gruppen zu kategorisieren und unterschiedliche unterrichtliche Anforderungen an sie zu stellen, sondern auch, dass für sie verschiedene Professionen zuständig sind: für die „hochbegabten Regelkinder“ sind dies die Regellehrpersonen, also die Grundschullehrpersonen, und für die Schüler*innen mit attestiertem Förderbedarf die Sonderpädagog*innen. Schüler*innen, die keiner der zwei Gruppen zugeordnet werden, haben keine schulisch-unterrichtliche Profession, die für sie und ihre Lernprozesse verantwortlich ist. Vergleichbare Ergebnisse einer professionsspezifischen Zuständigkeit für unterschiedliche Schülergruppen konnten auch in anderen Projekten zu inklusivem Unterricht rekonstruiert werden (vgl. z.B. Heinrich et al. 2014; Sturm/Wagner-Willi 2016). Es zeigt sich eine professionelle Praxis, in der Schüler*innen unterrichtliche Lehr-Lernangebote eröffnet werden, verbunden mit der Erwartung, dass diese zu ihnen passen bzw. sie die Erwartungen erfüllen können. Es liegt in der Verantwortung der Schüler*innen bzw. ihrer Eltern und Familien, dass sie passen(d sind). Hier wird ein Verständnis von unterrichtlichem Mitkommen und Folgen erkennbar, das an schulrechtlichen Kategorien sowie den damit verbundenen Überprüfungsformen (z.B. sonderpädagogischer Förderbedarf, Intelligenztest) ausgerichtet ist. Diese Kategorien sind zugleich mit der Struktur des Sortierens und der Zuordnung im deutschen Schulsystem konform, die ihrerseits eine Vergleichbarkeit der Schüler*innen voraussetzen. Hiermit gehen Normen und Erwartungen an die Rolle von Lehrpersonen einher. Die Differenz, die für die Lehrkräfte leitend ist, liegt in der Relation zu dieser gesetzten Norm und der je individuellen Möglichkeit der Schüler*innen, sie selbstständig zu erreichen.

In dem kurzen Gesprächsausschnitt dokumentiert sich entsprechend das Dilemma, dass zwischen dem formal-expliziten Anspruch, als Schule inklusiv zu arbeiten und alle Schü- 
ler*innen in ihrem Lernstand anzuerkennen und entsprechend zu unterstützen, und der schulstrukturellen und professionellen Situation, die für spezifische Gruppen von Schüler*innen unterschiedliche schulische Angebote vorbehält und verschiedene, auf diese Strukturen bezogene Lehrpersonen (Sonder-/Grundschullehrkräfte) einbezieht. Beide basieren auf der Annahme, dass Schüler*innen nach besser/schlechter unterschieden werden und entsprechend Erfolg und Scheitern vorgesehen sind. In dem dargelegten Beispiel scheitern nicht die Schüler*innen mit attestiertem ,sonderpädagogischem Förderbedarf', sondern eine Gruppe von Schüler*innen, die dem Bildungsgang der Grundschule zugeordnet ist. Wenngleich diese Gruppe besonders benachteiligt wird, so machen in einem derartigen unterrichtlichen Setting alle Schüler*innen die Erfahrung, dass schulischer Leistungserfolg - ebenso wie Misserfolg - individualisiert wird. Die Ergebnisse verweisen auf einen modus operandi, also ein Muster, entlang dessen Differenzen im Unterricht von den Lehrpersonen hergestellt und bearbeitet werden; in diesem irritieren Unterschiede die unterrichtliche Praxis der Lehrpersonen. Anhand weiterer Ausschnitte aus der Gruppendiskussion der Gruppe Kontinent konnte gezeigt werden, dass dieses Muster sich homolog durch das gesamte Gespräch zieht. Es beschreibt zugleich die rekonstruierte Basistypik, also die spezifische Typik, die sich über alle Fälle des Samples hinweg zeigt (vgl. Sturm 2014).

\section{2 „Vertrauen“ - unterrichtliche Praxis der Gruppe Planet}

\subsubsection{Formaler schulischer Rahmen mit Fokus auf Inklusion im Königreich Norwegen}

Während die Schulsysteme der deutschsprachigen Länder im Kern und über die verschiedenen Länder und Bundesländer hinweg mehrgliedrig organisiert sind, zeichnet sich das norwegische durch einen integrativen bzw. inklusiven Grundgedanken aus. Es kennt seit der Wende vom 19. zum 20. Jahrhundert keine den deutschsprachigen Schulsystemen vergleichbare Aufteilung der Schüler*innen in unterschiedliche, hierarchisch gegliederte Bildungsgänge. 1974 wurde zudem ein nationales Curriculum entwickelt, das integrative gegenüber separativen schulischen Settings vorzieht und für alle Schüler*innen gilt. Die stärkste Selektion, die das norwegische Schulsystem kennt - und die in den letzten Jahren zugenommen hat - ist die zwischen staatlichen und privaten Schulen (vgl. Nilsen 2010). Der Anteil der Privatschüler*innen liegt mit unter 4\% (vgl. Norway 2016: 9) deutlich niedriger als die 10\% in Deutschland (vgl. Bildungsbericht 2018: 44).

Das bildungspolitisch leitende Verständnis von Inklusion beschreiben Bjørnsrud und Nilsen (2019) in Übereinstimmung mit den Empfehlungen der UNESCO: Es fokussiert die Prämisse, allen Schüler*innen in Schule und Unterricht die Erfahrung sozialer und akademischer Gemeinschaft in einer Gesamtschule zu eröffnen. Diese Perspektive weist deutlich über Fragen der Platzierung einzelner Schüler*innen in Schulen und/oder Sonderklassen innerhalb von Schulen resp. Schultypen und Bildungsgängen hinaus. Seit den 1970er Jahren verfolgt Norwegen - parallel zu den Entwicklungen in anderen europäischen Ländern - einen inklusiven Ansatz. Bereits 1990 lag der Anteil derjenigen Schüler*innen, die separativ beschult wurden, bei 0,7 Prozent; sechs Jahre später waren es nur noch 0,5\% (vgl. Vislie 2003). Das Absinken der Quote erklärt Vislie (2003) mit den schulstrukturellen Reformen. 


\subsubsection{Gruppe Planet}

An der Gruppendiskussion haben zwei Lehrerinnen teilgenommen, die an einer sogenannten Jugendschule (umfasst die Klassenstufen 8 bis 10) tätig sind. Die Schule ist in einer urbanen Gegend Norwegens angesiedelt. Sie arbeitet inklusiv, ohne dass dies ein besonderes Merkmal der Schule im Vergleich zu anderen darstellt, das als solches ausgewiesen würde. Die Schüler*innen dieser Schule werden nach Aussagen der Lehrpersonen erst ab Klasse 10 im Notensystem bewertet. Die Kollegien entscheiden in Norwegen darüber, ob Leistungsbewertung an ihrer Schule ab Klasse 9 oder 10 erfolgt.

Das Gespräch wurde auf Englisch geführt, einer Sprache, die sowohl für die Interviewten als auch für die Interviewerin nicht die Erst- und Alltagssprache darstellt. Es wurde durch den gleichen Eingangsimpuls gestartet, wie die Diskussion der beschriebenen Gruppe Kontinent. Der folgende Gesprächsausschnitt entstammt dem Anfang des Gesprächs, also kurz nachdem der Impuls vonseiten der Gesprächsleitung gesetzt wurde.

\section{Ausschnitt: „Vertrauen“}

Af: $\quad$ So (.) the relationship to them is really important, and it's important too that they feel included, (.) in all the material, and I think we sort of always think about explaining words. (.) Er so when they go to the classroom yeah I want to be there (.) I want them to feel safe with me (.) in the classroom; I don't want them to feel like they're pushed into something they don't want to do. I want them that they should feel um (.) that I back them up; if they gonna try something hard I want them to feel that I'm backing them up; not that I'm pushing them into it. That they really want to do it themselves in the end. You know they might not want to do it in the beginning @(.)@

Yf: $\quad$ Do you have an example of that?

In diesem Gesprächsausschnitt geht es inhaltlich um Vertrauen zwischen Lehrpersonen und Schüler*innen, insbesondere das Vertrauen der Schüler*innen in die und zu den Lehrpersonen. Zudem um das Gefühl der Zugehörigkeit zu der Gemeinschaft und um die lernende Auseinandersetzung mit den unterrichtlichen Gegenständen. Die Lehrerin betont, dass es ihr wichtig ist, dass die Schüler*innen sich in ihrem Unterricht sicher fühlen. Sie distanziert sich davon, dass die Schüler*innen sich im Unterricht zu etwas gedrängt fühlen, das sie nicht wollen und hebt - in anderer Formulierung - hervor, den Schüler*innen Sicherheit vermitteln zu wollen. Der Abgrenzung vom Drängen zu etwas stellt sie die Idee gegenüber, dass die Schüler*innen selbst etwas lernen wollen und dabei von ihr unterstützt werden. Nach diesen eher theoretisierenden Beschreibungen fragt die Interviewerin die Lehrerinnen, ob sie dies an einem Beispiel erläutern können. Daraufhin entwickelt sich folgende Erzählung:

Af: $\quad$ Er we're doing er (.) some presentations in Norwegian now, where (.) I'm pushing them into using less slides and talk more about one picture, and I feel a bit sort of at least one girl I (Yf: ahem) took yesterday I said I don't want you to have that many slides they are to many, I want you to at least at least take away four (Yf: ahem) and she was um (.) a bit annoyed with me. it was Nicole she thought mhh and she was a bit annoyed and she felt sort of (.) like I was pushing her into something she was not used to. First I was thinking if "caus-cause she is a really (.) good student and I felt if she tries to just talk out to the poem and show everything with one text and one slide, she will be able to sort of show her knowledge better than sort of racing through all the slides. (Yf: ahem) And she did it today and I asked her afterwards how did it feel, and she said it felt really good. So she did listen to me but I said you (Yf: ahem) weren't really really pleased with me yesterday when I asked you to take them away ah n-no she felt I was sort of a mad woman asking her to do that. 
Bf:@@(.)@

Af: $\quad$ So ahem she trusted me, and she sort of (.) she did it, but she didn't feel really secure yesterday when I told her to.

Die Lehrerin elaboriert die Frage nach einem Beispiel performativ, indem sie von einer Begebenheit des gestrigen und heutigen Tags im Norwegischunterricht erzählt. Die Schüler*innen sollten in dem Unterricht Präsentationen erstellen. Die Lehrerin ,drängt' (push them) die Schüler*innen dazu, weniger Folien zu nutzen, bzw. durch sie ,zu rauschen', mit dem Ziel, mehr zu einem Bild erzählen zu können. Sie verwendet hier die Worte push them to, einer Tätigkeit, von der sie sich zuvor begrifflich dezidiert abgegrenzt hat. Gleichwohl wird darin die Enaktierung deutlich, dass sie im Unterricht die Schüler*innen immer wieder an ihre Grenzen und darüber hinaus zu bringen versucht. Damit diese sich lernend auseinandersetzen, müssen sie ihr in diesem Prozess vertrauen. Die Lehrerin berichtet von der Schülerin Nicole, die ihr gegenüber am gestrigen Tag mit Ablehnung reagiert hat, als Af sie aufgefordert hat, ihre Präsentation auf eine Folie zu reduzieren. Trotz ihrer Kritik, die sich v.a. gegen die Lehrerin gerichtet hat, hat sie sich darauf eingelassen, ihre Folienanzahl zu reduzieren. Auf Nachfrage der Lehrerin am heutigen Tag hat die Schülerin berichtet, dass es gut war, diesen Schritt zu gehen. Af elaboriert bzw. konkludiert anschließend, dass die Schülerin ihr vertraut hat, obwohl sie sich zunächst nicht sicher gefühlt hat.

Das Beispiel veranschaulicht die Brüchigkeit und Ambivalenz des Vertrauens vonseiten der Schüler*innen in die Lehrperson im Unterricht. Die Schülerin gewährt der Lehrerin eine Art Vertrauensvorschuss, sich auf die Veränderung ihres Arbeitsprodukts einzulassen. Dies erfolgt zunächst mit Widerstand, der nicht allein auf der Sachebene, sondern v.a. auf der Beziehungsebene zwischen Schülerin und Lehrerin erfolgt bzw. von den Schüler*innen problematisiert wird. Das Vertrauen von Schüler*innen in die Lehrperson stellt dabei die Voraussetzung (lernender) schulisch-unterrichtlicher Auseinandersetzungen aufseiten der Schüler*innen dar. Dass dieses nicht immer vorhanden ist, sondern kontinuierlich herzustellen ist, zeigt der weitere Verlauf der Sequenz:

Af: $\quad$ So ahem she trusted me, and she sort of (.) she did it, but she didn't feel really secure yesterday when I told her to. I tried the other (.) one he's down from nineteen to thirteen I think.

Bf:

ᄂ@(.)@

Af:

But he's not@getting there yet@ (.) so telling them to make them sort of do things in a way that they haven't done things before, (.) is is the one thing that we need to make them trust you. And I'm a new teacher to this this group this year; so um (Yf: $\mathrm{mmh})($.$) it takes a bit for them also to sort of trust that I know what I'm doing, I think.$

Bf: I think that's common for most of our students. um (.) the lack of uh (.) trust in grown-ups, and in teachers $($.

Af: $\quad$ Yeah

Bf: $\quad$ you have to work with them for quite a long time until you get kind of an interest that you would get from a lot of other teenagers just because being, and because you are an adult; (Yf: Mhm) but here, different kinds of reasons that makes it hard for them to develop relationships to to grownups, and to trust them, and to trust our um opinions, our views, and our feedback to them and that's uh that's a challenge.@(.)@

Af differenziert ihre Ausführungen an dem Beispiel eines weiteren Schülers, der die Anzahl seiner Folien ebenfalls reduziert hat, allerdings von 19 auf 13. Das kurze Lachen ihrer Kollegin deutet auf deren Verständnis und/oder Kenntnis der Situation hin. Af führt in ihrer 
Äußerung weiter aus, dass der Schüler noch nicht dort sei, wo er hinsolle. Hier dokumentiert sich ein Vertrauen der Lehrerin in den Lernprozess des Schülers: sie hat das Vertrauen, dass ihm dies zu einem späteren Zeitpunkt gelingen wird. Sie konkludiert dann proponierend, d.h. hier explizit, dass es ihre Aufgabe ist, die Schüler*innen auf eine ihnen unbekannte Art und Weise herauszufordern und dass es hierfür notwendig ist, dass die Schüler*innen ihr vertrauen. Ihre Kollegin Bf differenziert die Aussage, indem sie heraushebt, dass die meisten Schüler*innen ihrer Schule kein bzw. kaum Vertrauen in Erwachsene generell und in Lehrpersonen im Besonderen haben. Sie führt weiter aus, dass es eine Herausforderung für sie darstellt, dass die Schüler*innen ihnen vertrauen. Dabei führt sie implizit an, dass es sich um eine Besonderheit der Schüler*innenschaft an dieser Schule handelt, die sich von anderen Schulstandorten in dem gewährten und erfahrenen Vertrauen gegenüber Erwachsenen unterscheidet und das seinerseits Voraussetzung für eine lernende Auseinandersetzung in Schule und Unterricht darstellt.

\subsection{Vergleich}

Die Kontrastierung zeigt zunächst, dass Leistung in beiden Fällen relevant ist und dass sie inhaltliche Erwartungen umfasst, die vonseiten der Schule und der Lehrpersonen an die Schüler*innen gestellt werden. In der Gruppe Kontinent wird dabei nicht nur gemäß einer ZweiGruppen-Theorie (behindert/nicht-behindert) (Hinz 2004) unterschieden, welche Anforderungen an einzelne Schüler*innen gestellt werden, sondern es wird auch, mit Bezug auf den IQ-Wert, von einer scheinbar objektiven und natürlichen Erklärung davon ausgegangen, welcher Gruppe Schüler*innen zugeordnet werden. Die Schüler*innen werden entlang der Norm der Erwartung verglichen. In der Gruppe Planet hingegen wird Leistung zwar auch den Schüler*innen zugeschrieben, sie ist aber zugleich das Ergebnis oder Produkt der Beziehung zwischen Lehrperson und Schüler*innen und wird nicht in einen unmittelbaren Vergleich mit den Leistungen anderer gestellt. Erfolgreich, so die Lehrpersonen, sind Lehr-Lernprozesse nur, wenn eine vertrauensvolle, pädagogische Beziehung aufgebaut ist bzw. besteht. Die Schüler*innen werden im norwegischen Fall mit sich selbst verglichen und die Lehrpersonen blicken auf einen Schüler, der etwas noch nicht kann, als jemanden, der es potenziell können wird und kann. Eine vergleichbare Sicht finden wir bei den Lehrpersonen der Gruppe Kontinent nicht.

Vor diesem Hintergrund stellt sich nun die Frage, welche sozialisierenden Erfahrungen Schüler*innen in dem jeweiligen Unterricht machen; was lernen sie als legitimen Umgang miteinander kennen? Diese Frage bezieht sich auf alle Schüler*innen gleichermaßen; wenngleich sich ihre individuellen Erfahrungen innerhalb der Unterscheidung von Miss-/Erfolgs unterscheiden können. Im Fall der Gruppe Kontinent machen alle Schüler*innen die Erfahrung, dass schulischer Miss/Erfolg ein individuelles, persönliches Schicksal ist, da unterrichtliche Angebote derart gestaltet sind, dass Schüler*innen an ihnen Scheitern können. Vor allem die Schüler*innen, die nicht ,erfolgreich“ sind, erhalten unterrichtlich keine Unterstützung und in der Folge - dies gilt v.a. für die „Kinder aus dem Niemandsland“ - wird ihnen der Förderbedarf vermutlich attestiert. Die Schüler*innen, so lässt sich zusammenfassen, müssen zur Schule passen, zu dem jeweiligen unterrichtlichen Angebot. Ihnen ist es kaum möglich, unterrichtlich in sozialer und/oder akademischer Hinsicht Zugehörigkeit und Anerkennung zu erfahren. Dies verstärkt sich umso mehr, als der Miss/-Erfolg individualisiert und 
nicht, wie im Fall der Gruppe Planet, als in der interaktiven Beziehung zwischen Lehrpersonen und Schüler*innen liegend, verstanden wird. Mit Annedore Prengel (2013: 13, Herv. im Orig.) lässt sich vermuten, dass hier die Möglichkeit und die Gefahr besteht, die Schüler*innen in diskriminierende Praxen einzusozialisieren, da eine „leistungshierarchisch legitimierte Entwertung so genannter ,schlechter' Schüler" stattfindet. Zugleich berührt diese Praxis die pädagogische Beziehung, die ihrerseits zentrale Voraussetzung für Lehr-Lernprozesse ist und sich vermutlich nur schwer als vertrauensvoll erweisen kann.

Die Sozialisation der Schüler*innen im Unterricht der norwegischen Lehrpersonen zeichnet sich durch keinen vergleichbaren Bruch der pädagogischen Beziehung aus, die Schüler*innen können die Lehrpersonen als Unterstützende ihres Lernprozesses kennenlernen und werden zudem in der Art und Weise ihres Lernens akzeptiert.

\section{Resümee und Perspektiven}

Vor dem Hintergrund der exemplarischen und illustrierenden Ausführungen soll das Erkenntnisinteresse der sozialisierenden Erfahrungen der Schüler*innen in den skizzierten schulisch-unterrichtlichen Kontexten mit Blick auf die Bedeutung für Inklusion und Exklusion bzw. Teilhabe und Marginalisierung betrachtet werden. Entlang der Ergebnisse deutet sich an, wenngleich dies für Norwegen lediglich entlang eines Einzelfalls erfolgt, dass die Schulstruktur und die mit ihr einhergehende Notwendigkeit der kontinuierlichen Bewertung der Schüler*innen erklären können, wie sich pädagogische Praxen der Differenzkonstruktion entwickeln. Diese Ergebnisse differenzieren die der australischen Studie von Anne Petriwskyj (2010) aus dem vorschulischen Bereich insofern, als dass der strukturelle Kontext unterschiedliche pädagogische Praxen hervorbringt bzw. konstituiert, die sich in der Verantwortlichkeit für alle bzw. für ausgewählte Schüler*innengruppen zeigt. Der strukturelle Rahmen legitimiert nicht nur eine hierarchische Unterscheidung der Schüler*innen nach Leistung, sondern scheint sie notwendig zu machen. Dies steht zugleich im Widerspruch zur Idee des Aufbaus sozialer und akademischer Anerkennung aller Kinder und Jugendlichen im schulisch-unterrichtlichen Rahmen.

Die Ergebnisse des explorativen Vorgehens zeigen, dass es von Interesse ist, diese in einem systematisch angelegten transnationalen Vergleich zu bearbeiten (vgl. Nohl 2013). Ein solcher eröffnet die Möglichkeit, unterrichtliche Praxen unterschiedlicher Schulsysteme methodisch kontrolliert zu vergleichen (Bohnsack 2014: 143). Entsprechend wären Lehrpersonen, die in Schulen in unterschiedlichen Regionen und Sozialräumen (z.B. urban/ländlich; privilegiert/benachteiligt) tätig sind, und/oder Lehrpersonen mit unterschiedlichem Umfang an Berufserfahrung (vgl. Hericks et al. 2019) einzubeziehen. Sollten sich die Ergebnisse, die hier mit Blick auf Norwegen auf einem Einzelfall basieren, generalisieren lassen, so wäre dies ein Beleg für die Notwendigkeit die Frage schulisch-unterrichtlicher Inklusion im deutschsprachigen Raum deutlich stärker als bisher als schulstrukturelle Entwicklungsaufgabe - die wesentlich im Verantwortungsbereich der Bildungspolitik liegt - zu bearbeiten. Dies verweist unmittelbar auf die bildungspolitische Notwendigkeit der Gestaltung entsprechender Rahmenbedingungen, in denen sich pädagogische Praxen entwickeln. 


\section{Literatur}

Amling, Steffen (2017): Formen von und Umgang mit Diskrepanzerfahrungen von LehrerInnen Reflexionen auf die dokumentarische Analyse der Organisation Schule. In: Vogd, Werner/Amling, Steffen (Hrsg.): Dokumentarische Organisationsforschung. Perspektiven der praxeologischen Wissenssoziologie. Opladen, Berlin, Toronto: Barbara Budrich. S. 103-121.

Becker, Klaus-Peter/Große, Klaus-Dietrich (1990): Educational Rehabilitation in the German Democrativ Republic. In: International Journal of Disabilty Development and Education 37, 2, S. 99-108.

Biesta, Gert (2011): Evidenz, Erziehung und die Politik der Forschung. In: Bellmann, Johannes/Müller, Thomas (Hrsg.): Wissen, was wirkt. Kritik evidenzbasierter Pädagogik. Wiesbaden: VS Verlag für Sozialwissenschaften, S. 269-278.

Bildungsbericht, Autorengruppe (2018): Bildung in Deutschland 2018. Ein indikatorengestützter Bericht mit einer Analyse zu Wirkungen und Erträgen von Bildung. https://www.bildungsbericht.de/de/bildungsberichte-seit-2006/bildungsbericht-2018/pdf-bildungsbericht-2018/bildungsbericht-2018.pdf [Zugriff: 23.06.2018].

Bjørnsrud, Halvor/Nilsen, Sven (2019): Joint reflection on action - a prerequisite for inclusive education? A qualitative study in one local primary/lower secondary school in Norway. In: International Journal of Inclusive Education 23, 2, S. 158-173.

Bohnsack, Ralf (2007): Dokumentarische Methode und praxeologische Wissenssoziologie. In: Schützeichel, Rainer (Hrsg.): Handbuch Wissenssoziologie und Wissensforschung. Konstanz: UVK Verlagsgesellschaft, S. 180-190.

Bohnsack, Ralf (2009): Qualitative Bild- und Videointerpretation. Die dokumentarische Methode. Opladen/Framington Hills: Verlag Barbara Budrich.

Bohnsack, Ralf (2014): Rekonstruktive Sozialforschung. Einführung in qualitative Methoden. Opladen/Farmington Hills: Verlag Barbara Budrich.

Bohnsack, Ralf (2017): Praxeologische Wissenssoziologie. Opladen, Toronto: Verlag Barbara Budrich.

Bourdieu, Pierre (1996): Die Praxis der reflexiven Anthropologie. In: Bourdieu, Pierre/Wacquant, Loïc (Hrsg.): Reflexive Anthropologie. Frankfurt/Main: Suhrkamp, S. 251-294.

Budde, Jürgen/Scholand, Barbara/Faulstich-Wieland, Hannelore (2008): Geschlechtergerechtigkeit in der Schule. Eine Studie zu Chancen, Blockaden und Perspektiven einer gender-sensiblen Schulkultur. Weinheim/München: Juventa Verlag.

Europäische Kommission (2016): Struktur der europäischen Bildungssysteme 2016/17 (Publication no. 10.2797/072740). https://publications.europa.eu/de/publication-detail/-/publication/ becafa9c-9a85-11e6-9bca-01aa75ed71a1 [Zugriff: 11.09.2016]

Heinrich, Martin/Arndt, Ann-Kathrin/Werning, Rolf (2014): Von „Fördertanten“ und „Gymnasialempfehlungskindern“. Professionelle Identitätsbehauptung von Sonderpädagog/innen in der inklusiven Schule. In: ZISU, 3, S. 48-71.

Hericks, Uwe/Keller-Schneider, Manuela/Bonnet, Andreas (2019): Lehrerprofessionalität in berufsbiographischer Perspektive. In: Gläser-Zikuda, Michaela/Harring, Marius/Rohlfs, Carsten (Hrsg.): Handbuch Schulpädagogik. Münster, New York: Waxmann, S. 597-607.

Hinz, Andreas (2004): Vom sonderpädagogischen Verständnis der Integration zum integrationspädagogischen Verständnis der Inklusion!? In: Schnell, Irmtraud/ Sander, Alfred (Hrsg.), Inklusive Pädagogik. Bad Heilbrunn: Verlag Julius Klinkhardt. S. 41-74.

HRK/KMK (2015): Lehrerbildung für eine Schule der Vielfalt. Gemeinsame Empfehlung von Hochschulrektorenkonferenz und Kultusministerkonferenz. https://www.kmk.org/fileadmin/ 
Dateien/veroeffentlichungen_beschluesse/2015/2015_03_12-Schule-der-Vielfalt.pdf [Zugriff: 31.07.2018].

Jünger, Rahel (2008): Bildung für alle? Die schulischen Logiken von ressourcenprivilegierten und -nichtprivilegierten Kindern als Ursache der bestehenden Bildungsungleichheit. Wiesbaden: VS Verlag für Sozialwissenschaften.

KMK (2011): Inklusive Bildung von Kindern und Jugendlichen mit Behinderungen in Schulen. http://www.kmk.org/fileadmin/Dateien/veroeffentlichungen_beschluesse/2011/2011_10_20Inklusive-Bildung.pdf [Zugriff: 16.09.2016].

Luhmann, Niklas (2002): Das Erziehungssystem der Gesellschaft. Frankfurt/Main: Suhrkamp.

Luhmann, Niklas (2015): Die Wissenschaft der Gesellschaft. Frankfurt/Main: Suhrkamp.

Mannheim, Karl (1980): Strukturen des Denkens. Frankfurt/Main: Suhrkamp.

Mannheim, Karl (1995): Ideologie und Utopie. Frankfurt/Main: Vittorio Klostermann.

Nilsen, Sven (2010): Moving towards an education policy for inclusion? Main reform stages in the development of the Norwegian unitary school system. In: International Journal of Inclusive Education 14, 5, S. 479-497.

Nohl, Arnd-Michael (2013): Relationale Typenbildung und Mehrebenenvergleich. Neue Wege der dokumentarischen Methode. Wiesbaden: Springer VS.

Norway, Statistics (2016): Facts about education in Norway 2017 - key figures 2015. Statistics Norway, Division for Education Statistics: https://www.ssb.no/en/utdanning/artikler-og-publikasjoner/_attachment/287176?_ts=158d834b638 [Zugriff: 22.07.2018].

Petriwskyj, Anne (2010): Diversity and inclusion in the early years. In: International Journal of Inclusive Education, 14 (2). S. 195-212.

Prengel, Annedore. (2001): Egalitäre Differenz in der Bildung. In: Lutz, Helma/Wenning, Norbert (Hrsg.): Unterschiedlich verschieden. Differenz in der Erziehungswissenschaft (1. Aufl.). Opladen: Leske + Budrich, S. 93-107.

Prengel, Annedore (2013): Geleitwort: Diversität und Bildung. In: Hauenschild, Katrin/Robak, Steffi/Sievers, Isabel (Hrsg.): Diversity Education. Zugänge - Perspektiven - Beispiele. Frankfurt am Main: Brandes \& Apsel Verlag. S. 11-14.

Rabenstein, Kerstin/Reh, Sabine/Ricken, Norbert/Idel, Till-Sebastian (2013): Ethnographie pädagogischer Differenzordnungen. In: Zeitschrift für Pädagogik, 59, 5, S. 668-690.

Sturm, Tanja (2010): Heterogenitätskonstruktionen durch Lehrende. Zur Bedeutung des Habituskonzepts für die Lehrerbildung. In: Müller, Florian H./Eichenberger, Astrid/Lüders, Manfred/Mayr, Johannes (Hrsg.): Lehrerinnen und Lehrer lernen - Konzepte und Befunde zur Lehrerfortbildung. Münster: Waxmann, S. 89-105.

Sturm, Tanja (2011): Differenzkonstruktionen in unterrichtlichen Praktiken. In: Flieger, Petra/ Schönwiese, Volker (Hrsg.): Menschenrechte, Integration, Inklusion. Aktuelle Perspektiven aus der Forschung. Bad Heilbrunn: Verlag Julius Klinkhardt, S. 99-104.

Sturm, Tanja (2014): Unterrichtliche Praktiken der (Re-)Produktion von Differenz in der Freiarbeit. In: Lichtblau, Michael/Blömer, Daniel/Jüttner, Ann-Kathrin/Koch, Katja/Krüger, Michaela/Werning, Rolf (Hrsg.): Gemeinsamen Jahrestagung der DGfE Kommission Grundschulforschung und Didaktik der Primarstufe und der DGfE Sektion Sonderpädagogik. Forschungsorientierter Band. Bad Heilbrunn: Klinkhardt, S. 275-288.

Sturm, Tanja/Wagner-Willi, Monika (2016): Kooperation pädagogischer Professionen. Bearbeitung und Herstellung von Differenz in der integrativen Sekundarstufe. In: Kreis, Annelies/ Wick, Jeannette/Kosorok Labhart, Carmen (Hrsg.): Kooperation im Kontext schulischer Heterogenität. Münster, New York, München, Berlin: Waxmann, S. 207-221.

Sturm, Tanja/Wagener, Benjamin/Wagner-Willi, Monika (2020): Inklusion und Exklusion im Fachunterricht. Dynamische Relationen in Schulformen der Sekundarstufe 1. In: Pfaff, Nicolle/ Kessl, Fabian (Hrsg.): Bewegungen. DGfE-Kongressband. Opladen: Barbara Budrich Verlag. 
Schuck, Karl Dieter (2014): Individualisierung und Standardisierung in der inklusiven Schule ein unauflösbarer Widerspruch? DDS - Die Deutsche Schule 106, 2, S. 162-174.

Tervooren, Anja (2001): Pädagogik der Differenz oder differenzierte Pädagogik? Die Kategorie Behinderung als integraler Bestandteil von Bildung. In: Tervooren, Anja/Hartmann, Jutta/ Fritzsche, Bettina/Schmidt, Andreas (Hrsg.): Dekonstruktive Pädagogik. Erziehungswissenschaftliche Debatten unter poststrukturalistischen Perspektiven. Wiesbaden: Springer Fachmedien, S. 201-216.

Turner, Victor (1966): The Ritual Process. Structure and Anti-Structure. Ithaca, New York: Cornell Paperbacks.

UN, United Nations (2006; 2008): Übereinkommen über die Rechte von Menschen mit Behinderungen. (dreisprachige Fassung im Bundesgesetzblatt Teil II Nr. 35 vom 31.12.2008). http:// www.un.org/depts/german/uebereinkommen/ar61106-dbgbl.pdf [Zugriff: 16.09.2016].

UNESCO (2009): Policy Guidelines on Inclusion in Education. https://unesdoc.unesco.org/ark:/ 48223/pf0000177849 [Zugriff: 23.03.2019].

Vislie, Lise (2003): From integration to inclusion. focusing global trends and changes in the western European societies. In: European Journal of Special Needs Education 18,1, S. 17-35.

Wagner-Willi, Monika/Sturm, Tanja/Wagener, Benjamin/Elseberg, Anika (2018): Herstellung und Bearbeitung von Differenz im Fachunterricht der Sekundarstufe I. Eine Vergleichsstudie zu Unterrichtsmilieus in inklusiven und exklusiven Schulformen. Abschlussbericht zum SNFProjekt 100019_152751. https://irf.fhnw.ch/bitstream/handle/11654/27462/Abschlussbericht snf_06-18_geku \%cc\%88rzt.pdf?sequence=1 [Zugriff: 13.03.2019].

Waldschmidt, Anne (2005): Disability Studies: Individuelles, soziales und/oder kulturelles Modell von Behinderung? In: Psychologie und Gesellschaftskritik 29, 1, S. 9-31.

Wellgraf, Stefan (2011): Hauptschule. Formationen von Klasse, Ethnizität und Geschlecht. In: Hess, Sabine/Langreiter, Nikola/Timm, Elisabeth (Hrsg.): Intersektionalität revisited. Empirische, theoretische und methodische Erkundungen. Bielefeld: transcript, S. 119-147.

Willems, Katharina (2007): Schulische Fachkulturen und Geschlecht. Physik und Deutsch - natürliche Gegenpole? Bielefeld: transcript. 


\title{
23 Teachers' Learning in Schools Moving Towards Inclusion: Experiences from England and Germany
}

\author{
Elisabeth Plate, Alison Peacock
}

\begin{abstract}
The chapter emphasises the importance of recognising teachers as learners and their continuing learning as crucial elements of inclusion in schools. It complements other perspectives that consider teacher professional development and learning first and foremost as preparation for inclusive practices. Following theoretical considerations to rationalise and define teachers as learners and teacher learning, the article explores current implementation and practices on different levels: on national policy level in England and Germany and on school level. The exemple of Wroxham Primary School in England serves as a basis for further reflections and questioning of educational developments in schools in general and in Germany in particular.
\end{abstract}

\section{Introduction}

Learning is like breathing - we do it all the time, indeed we cannot live without breathing. [...] Learning, like breathing (and indeed ingesting), is a natural process of engaging with our everchanging environment and taking from it what we need in order to function and to grow (Rogers, A. 2014: 35).

At first sight this quote appears to be unquestionable. However, in some contexts learning is predominantly ascribed to some people and less to others. So, for instance in schools, the institution we are going to focus on here, some people are assigned the role of "learners", others the role of "teachers". Does this division imply that teachers do not learn in schools? Do they not need to grow anymore? Is learning in school considered as unprofessional or inadequate for teachers? The scientific discourse on teacher education and professional development shows that quite the opposite is the case: The interdependence of continuing teacher learning and professional development on one side and school development on the other has been emphasised for a long time (see e.g. Plate 2017; Schimank 2014; Rolff 2010; Heise 2009; Bastian et al. 2002): "[...] there is little significant teacher development without school development $[\ldots]$. This challenges the more simple view that improved schools result from improved teachers" (Hargreaves 1994: 436). Over the last three decades there has been a growing call for further professional development in education systems internationally following the introduction of the so-called "inclusion agenda" and "standard agenda" (see Ackeren/Brauckmann 2010). This led to an increasing number of measures and programmes for continuing professional development (CPD), yet mostly outside school, disconnected from school development focussing on particular professional competencies and knowledge. It indicates an understanding of teacher development as a prerequisite for school developments - 
according to Hargreaves (1994: 436) "the more simple view" - but not conversely, also as a result of and supported through school developments. The findings of a small student research project carried out at the Humboldt-Universität zu Berlin (Plate 2018, unpublished), in which 17 teachers from different primary and secondary schools in Berlin were interviewed about opportunities for their own learning inside school, confirm this understanding of teacher development. The majority of participating teachers referred to school external CPD courses and only a few recognised opportunities for their learning inside school, yet mostly outside their classrooms, such as staff meetings and conversations with colleagues. Learning from students as part of and supported through educational practice was only mentioned by one teacher.

For these reasons, in this chapter we intend to emphasise the importance of recognising teachers ${ }^{1}$ as learners and their continuing learning as crucial elements of school development and educational practice in general and, more specificalls, in the context of inclusion guided by the following questions:

- What do we mean by "teachers as learners" and "teacher learning"? (section 1)

- What are rationales for teacher learning and teachers as learners as elements of daily educational practice and (inclusive) school developments? (section 1)

- What are current resources and barriers to implement continuing teacher learning and support teachers as learners in schools in England and Germany:

- on national policy level? (section 2)

- $\quad$ on school level? (section 3)

- How can developments in schools equally support teachers' and students' learning and participation? (section 3)

Our explorations are based on our experiences in educational practice and research for inclusion in England and Germany: Alison was a school teacher and a headteacher for many years. A few years ago, she founded the Chartered College for Teaching in England where she holds the post of chief executive officer. The college is a charitable membership organisation for teachers and educationalists to support their professional developments and teaching as a global profession. Elisabeth qualified as a teacher in Germany. Afterwards she did her PhD in England carrying out an international research study on inclusive and excluding responses to diversity in schools in England and Germany, focusing on supporting staff for inclusion (see Plate 2012). She stayed in academia and is now employed at the Humboldt-Universität $\mathrm{zu}$ Berlin qualifying teachers and teacher students for inclusive education. Alison and Elisabeth started their dialogue on inclusive school developments a few years ago, especially focusing on the importance of supporting not only students but also teachers and other staff in their learning. The reason for their first meeting at Alison's former school, Wroxham Primary in England, was two research studies in which Alison participated, conducted by colleagues from the University of Cambridge (see Swann et al., 2012; Hart et al. 2004): the first study was about the educational practices of nine teachers, from a variety of primary and secondary schools in England, each of whom held strong views that all children in class should be valued for their capacities to learn - rather than being categorised, ranked or labelled. The second research project was a follow-up study at Wroxham Primary on developments towards inclusion and therefore towards an educational practice in which ideas of fixed ability had

1 Although this article focusses on teachers, the remarks on teachers as learners and teacher learning may well apply to other professionals in schools, too. 
been rejected. Both studies may be considered as most impressive, thought-provoking accounts on inclusive developments.

In this chapter, we apply an international perspective. By this means we neither presume nor want to pretend that there is such thing as one coherent "national" perspective. And in the specific cases of Germany and England, this would be even more questionable because Germany is a nation consisting of sixteen Länder with different education systems and England has not always been considered a nation. For instance, the United Nations defines England as a country within the nation of the United Kingdom. Therefore, in the following, references in the text to "international developments" address differences between England and Germany but at the same time do not exclude existing differences within each country.

Correspondingly, using a comparative approach does not necessarily mean to look at the tertium comparationis, i.e. teachers' learning, evenly in the selected countries and to identify successful practices in one context in order to adopt them in the other following "the notion that practice can be generalized across countries without attention to local contexts and meanings" (Booth/Ainscow 1998: 4). Instead, we intend to evoke increased reflection and questioning on cultures, structures and practices by taking a stance outside. We acknowledge each context's uniqueness, as opposed to supporting uniformity, and, in this way, emphasise diversity as a potential to inform developments and to initiate further dialogue.

\section{Understanding "Teachers as Learners": A Resource for Inclusive School Developments}

Being considered a "learner" is often interpreted as an indication of lacking competence or being incompetent; mostly without specifying the area of learning but making a general judgement. Additionally, it can be accompanied by a sense of powerlessness (see Agostini et al. 2018; Rancière 2007). In contrast, being considered a "teacher" is widely perceived as being knowledgeable, skilled and also powerful. This ascription of the roles of learners and teachers to young people and adults, seemingly self-evident and without questioning its discriminatory potential, is detrimental for inclusive processes: In schools it establishes hierarchies and inequality between teachers and students (and quite often also the students' families) that prove to be a barrier to processes of learning, teaching and participation for everyone (see Peschel 2009; Rancière 2007; Hart et al. 2004).

In this context, we have chosen to focus on teacher learning and to use the phrase "teachers as learners" to challenge the persisting view on teachers as "all-knowing experts", delivering knowledge to the "unknowing" students, which - from our perspective - even gained strength in the context of New Public Management (NPM) and market-oriented developments in education systems. This also suggests to question the implications of established roles of learners and teachers and so-called "teacher-learner-relationships". We want to acknowledge the growing requests for teachers' professional development ${ }^{2}$ and support since schools have been required to develop inclusively (see e.g. Waitoller/Artiles 2013) and emphasise the importance of continuing learning. Last but not least, we argue that being a

2 The term “professional development" here relates to Hoyle's definition (1991, in Dlugosch 2005: 28; transl. EP): "a process in which a practitioner acquires or improves the necessary knowledge and skills for the effective professional practice". 
"learner" implies competence and participation and infact is a crucial element of inclusive school developments as well as professional action.

In support of our argument, in this chapter we firstly define what we mean by "teachers as learners" and "teacher learning" and give rationales for their importance for educational developments. Secondly, we describe the particular potential of inclusive developments as processes to support teacher learning as much as student learning.

\subsection{Constructing "Teachers as Learners"}

The notion of "teachers as learners" as an element of daily practice in school - as opposed to a role outside educational practice, for instance in further education courses - will be described and reasoned in the following by drawing on different theoretical as well as evidencebased rationales allocated to the discourses on learning and teaching, teacher professionalism and (inclusive) school developments.

\subsubsection{Teachers as Learners - More than Formalised Professional Learning}

"We cannot not learn" (Lemke 2013: 66). Learning can be defined as an ongoing, life-long process, "an aspect of living, an aspect of development" (ibid.) for everyone. Learning brings about change not only in knowledge, but also in understanding, skills and attitudes (see Rogers, A. 2014). It is a contextualised, social as well as individual, self-directed and self-reflective process: "While it is the individual who learns, learning always happens in a social context and is socially constructed within normative demands and values of different cultures" (Livingston 2010: 73). It is a self-regulating process, when facing a challenging situation, or "when the subject matter is perceived [...] as having relevance" (Bélanger 2011: 36). By considering learning as "relearning" Agostini et al. (2018: 19; transl. EP) emphasise the process of restructuring a learner's existing knowledge following a new experience. Whilst this is accompanied by a feeling of helplessness - since one's own knowledge is put into question - at the same time the learner's competence is acknowledged. In other words, the learner experiences herself as the "(knowing) unknowing" (Agostini et al. 2018: 19; transl. EP).

Not all learning happens consciously. Informal learning - in contrast to formal and nonformal learning - can also be unconscious. According to empirical evidence, informal learning is "both larger and more important than formal learning" (Rogers, A. 2014: 34), because it is "ubiquitous, universal and continuous" (Rogers, A. 2014: 35). Built on experiential social learning and greater learner control, even when unconscious, it is very deep learning, affecting attitudes and values, perceptions of one's self and the content of learning, one's confidence or lack of it (see Rogers, A. 2014: 43). Yet, internationally formal learning still dominates in educational research and practice and therefore also in teacher education (see Harring et al. 2015). Especially in Germany, informal learning has hardly been noticed thus far as a resource to support teachers' continuing professional development and there have been even fewer attempts for its systematic implementation (see Heise 2009).

On the basis of these understandings of learning, the necessity to recognise teachers as learners in school can be reasoned with regards to different aspects: Teachers, like all other 
persons, learn all the time. Learning happens automatically when "people engage socially in conversation and action on shared projects and problems" (Bélanger 2011: 29). When this is not acknowledged, it is a lost resource for developments in schools and for teaching and learning and may well be counterproductive: Learning is a way to cope with challenges. And feeling helpless, which teachers have voiced for instance in regard to inclusion (see Plate $2017 ; 2012$ ), has to be acknowledged as a natural element of this process whilst still valuing one's competencies (see above, Agostini et al. 2018). It is important to recognise schools and educational practices as much more significant learning contexts for teachers than any external further education course (see Calvert 2016; Terhart 2011). Particular attention should be paid to informal learning not only as a resource for teachers' professional development but also - especially when ignored - as potentially counteracting intended developments, such as inclusion, and subconsciously reifying existing, i.e. excluding cultures, structures and practices:

The basic norms and assumptions on which we build our social interactions are the result of unconscious informal learning. [...] And because informal learning reflects the power structures of the society from which we are learning, it has an inherent tendency to confirm the existing rather than encourage change (Rogers, A. 2014: 37f.).

In sum, constructing "teachers as learners" refers to the process of teacher learning not only in the sense of acquiring specific professional skills and knowledge, which we therefore call "professional learning", but also in every other sense: It includes any learning content relevant to the individual in daily educational practice and implies an attitude of learning which will be specified in the following part on theoretical approaches to teacher professionalism.

\subsubsection{Teachers as Learners - A Core Element of Teacher Professionalism}

"Ideally, teachers are learners themselves" (Agostini et al. 2018: 82; transl. EP). In this quote, teachers being learners is valued as a core element of teacher professionalism. This can be defined as a continuous, individual and contextualised process of developing professional competencies and responding to the indeterminacy of pedagogical relationships and the professional dilemmas and paradoxes in educational practice (see e.g. Helsper 2004). It requires ongoing (self-)reflection and non-standardised actions instead of predefined measures. It is based on what some authors call a "professional consciousness" (Agostini et al. 2018: 34ff.; see also Hin-Wai Yung 2002; transl. EP); what others may refer to as a "researching attitude" (Abels 2011: 143; see also Peschel 2009; transl. EP) and we call a "learning attitude". It can be characterised more specifically as being open to the unexpected and welcome uncertainties and perceived differences, being aware of the limits of one's own knowledge about the learning contents and about the other learners (i.e. students) instead of believing to "know what is right" (see Terhart 2011; Seitz 2010). In other words, it implies questioning apparent certainties and the familiar, such as preconceived images of students and work-related routines, and paying attention to irritations and surprise, taking risks and being creative in responding to challenges (see Swann et al. 2012: 88).

Consequently teachers as learners are professionals. In contrast to the prevalent connotation of "learners" as deficient, this perspective places very high aspirations on teachers" professionalism: It does not only emphasise a continuous development of professional competencies but also to mediate between them and the individual context of educational practice. 
This requires acknowledgement that teachers' professional actions will always remain precarious (see Terhart 2011: 206; Oevermann 2008: 62).

\subsubsection{Teachers as Learners - A Resource for School Developments and Innovation}

Acknowledging teachers as learners in their daily practice and thereby their learning as an element of and not just a prerequisite for school developments can have positive, i.e. unburdening and motivating effects for teachers, especially when they face external pressures to implement school reforms and innovation. In this way, school developments can become processes in which teachers' uncertainties, needs of support and individual developments are valued as indispensable elements of educational practice and change (see Plate 2017). If teachers experience school developments as beneficial to their own individual learning and professional development, it can enhance their motivation to contribute and increase their courage and feelings of security to take risks. This can reduce deviations between the originally intended reform and the actual outcomes of the implementation process (see Fend 2008; Rogers, E. 1995). Such deviations were often observed to derive from teachers' non-participation and therefore little or non-acceptance of the reform, which appeared to be rather disconnected from teachers' realities and educational practices (see e.g. Calvert 2016; Plate 2012; Zeitler et al. 2012: 23ff.; Heinrich/Altrichter 2008: 210; Bastian et al. 2002: 421): 'Changes, if implemented bureaucratically 'top down', are far too often non-accepted, consequently delayed, completely ignored or, if there is no alternative, implemented without selfmotivation and engagement. [...] a circle of disappointments and misunderstandings with demotivating effects for all people involved and tieing up resources that are crucially needed elsewhere" (Kruip 2011: 13; transl. EP).

Consequently, recognising reform implementations in schools as processes of organisational and teacher learning increases participation and, in turn, supports teachers to develop a professional, i.e. "reflective, researching and casuistic" (Bastian et al 2002: 420; transl. EP) attitude that "the heuristic character of school development" (ibid.; transl. EP) as well as learning organisations (see Holtappels 2010) in general require.

\subsubsection{Teachers as Learners - A Prerequisite for Student Learning}

The importance of teachers seeing themselves as learners to support the learning of students has already been pointed out above in the context of teacher professionalism. What we call a "learning attitude" is necessary to deal with the indeterminacy of teacher-student-relationships. In addition, there are further aspects regarding student learning and in support of our argument to be mentioned in the following.

On one side my teaching doesn't lead to learning. On the other side, teaching and learning depend on each other. [...] By being a learner I teach and by being teachers the others learn (Schratz et al. 2012: 26; transl. EP).

What this quote highlights is the interdependence of processes of learning and teaching and moreover, the equality between teachers' and students' roles that has to be created by sharing, instead of dividing, powers and responsibilities to develop conditions for everyone's learning and teaching and to engage together in the learning content: "Teachers cannot exercise their 
power, and fulfil their responsibility and commitment to making a difference to young people's future lives, except by working in partnership with young people" (Hart et al. 2004: 200; see also Seitz 2006). Correspondingly, Rosenholtz (1991) found out that so-called "learning-enriched schools" (Rosenholtz 1991: 87ff.), i.e. schools in which teachers and students learn alongside and in support of each other, lead to higher learning achievements than "learning impoverished schools" (Rosenholtz 1991: 87ff.).

Consequently, teachers have to recognise not only teaching but also learning opportunities for themselves (see Agostini et al. 2018: 10). Additionally, it is necessary to actively replace predominant and persistently assumed hierarchies of knowledge and skills between teachers as "professional know-it-all" (Holzkamp 1991: 20; transl. EP) and students by trust in everyone's individual competencies and unlimited learning capacities (see Hart et al. 2004). Young people, having been engaged in learning and teaching long before entering school, must be valued as skilled and experienced, too: "There is no human on earth, who wouldn't have learnt something through herself and without an explaining teacher" (Rancière 2007: 26; transl. EP). Teachers who aim to support students' self-directed learning, their growing autonomy and emancipation - and not just the students' knowledge gains - focus their actions on adapting external conditions for learning to the students and consult the students' perspectives in order "to be taught and not to teach them" (Rancière 2007: 42; see also Hart et al. 2004; transl. EP). It is a participatory process, i.e. a "joint enterprise" (Hart et al. 2004: 200) between students and teachers, in which teachers repeatedly become learners and, vice versa, students become teachers. By "teaching in the mode of learning", as Agostini et al. (2018: 22; transl. EP) call it, "the interweaving of teaching and learning can be taken into account. [...] not the student is in the centre of the process but the learning experiences of students on one side and of the teacher on the other" (Agostini et al. 2018: 22; transl. EP).

\subsection{Summary: Teachers as Learners for and through Inclusive School Developments}

"We need [...] to stop conceptualising learning as preparation of social life but rather as part of social life" (Hager/Halliday 2009: 100). Similarly, following the explanations above, we need to stop seeing teacher learning mainly as preparation of school developments and educational practices but rather as part of them, permeating all areas: organisational developments as well as developments of personnel and of learning and teaching (see Rolff 2010). It is an ongoing, unconscious as well as conscious process, happening in dialogue and through self-reflection, which - if taken into account - can benefit the learning of others (young people and adults) as well as the whole organisation.

"Teachers as learners" is more than a synonym for teachers as "reflective practitioners" (Schoen 1983). It implies what we call a "learning attitude", acknowledging teaching and learning as interdependent, requiring teachers and students to engage together in these processes, sharing their responsibilities and powers: Students also teach and teachers also learn. It emphasises professional and organisational developments as mutually supportive: If teachers perceive organisational developments as beneficial for themselves, for their own professional development and educational practices, they are more motivated to engage in instead of neglect institutional reforms and innovation. 
In this context, for teachers to become learners, to experience school developments as supportive and, as a result, feel enabled to support others, participation has proven to be key (see Plate 2017, 2016, 2012; Calvert 2016; Amrhein/Badstieber 2013). Participation can be defined as a two-sided process: On one hand it includes one's own participation, i.e. "the participating self" (Booth/Ainscow 2016: 25), namely having the opportunity to contribute and finding one's own interests, needs and strengths recognised. On the other hand, it means to support others' participation, i.e. "participatory action or activity" (Booth/Ainscow 2016: 25), removing barriers they experience and valuing their individual perspectives and contributions (see also Prengel 2016; Black-Hawkins et al. 2010: 46ff.). Following this definition, participation carries the potential to liaise organisational and professional developments (see Plate 2017), two areas that have repeatedly been described as conflicting (see e.g. Schimank 2014; Oevermann 2008).

Participation in school has especially been emphasised in the context of inclusion in education, yet mainly in regards to students, whilst teacher participation - ironically - has internationally been decreasing, especially following NPM. Implemented in education systems in the 1990s, the NPM, which has also been called the "standard agenda", initiated massive changes in educational governance. Based on economic principles, such as output orientation, standardisation, competition and accountability, which are executed in practice through comparative exams, school inspections, etc., the NPM counteracts developments of inclusion (see e.g. Plate 2012; Ainscow et al. 2006; Ball 2003).

Based on the following definition of inclusion, inclusive school developments can be considered as a particular resource to acknowledge teachers as learners, supporting their participation and learning and thereby enhance their capacities to develop schools inclusively: teacher learning for inclusion through inclusion.

[Inclusion is] a never ending process of increasing participation for everyone, children, young people and adults. It involves challenging and reducing all forms of exclusion. Increasing participation for all implies not only that everyone is entitled to participate in their local educational settings, but that education systems and settings are developed to be responsive to diversity in ways that value equally all children, young people and their families and the adults who work with them. A principle of equality of value [...] is central to inclusion... It is not sufficient that our systems and settings are simply responsive to diversity since they frequently respond in ways that create a hierarchy of value amongst children [and adults; EP \& AP] within and between schools [...] (Booth 2011: 1).

Following previous theoretical considerations, outlining the importance and implications of teachers as learners and teacher learning as a condition, a continuous and inevitable element and an aim of inclusive school developments, the question of putting them into practice remains and will be looked at in subsequent sections, focussing on different levels: national policies in England and Germany and individual school developments.

\section{Recognising "Teachers as Learners": Policy Developments in England and Germany}

To what extent have the ideas of teachers as learners and continuing teacher learning and participation in daily practice in schools been considered in national policy developments for 
teacher education in England and Germany? Due to the width of this question, we chose to focus on selected policy documents we have recognised as most influential for teachers' roles and developing professional identities and for the support of teacher learning and participation in schools.

\subsection{Policies for Teacher Education and Continuing Professional Development in Germany}

Teacher education and continuing professional development (CPD) in Germany are in the responsibility of each federal state resulting in a great variety of arrangements. Yet, there are a few national policy documents which inform the arrangements and developments on federal state level for the purpose of national quality assurance and consistency (see Terhart 2002). Amongst them the "Standards for teacher education" (KMK 2004/2014; transl. EP) - published by the Standing Conference of the Ministers of Education and Cultural Affairs of Germany's federal states (KMK) - and the "Recommendations for teacher education for a school welcoming diversity" (KMK/HRK 2015; transl. EP) - which is a joint publication by the KMK and the German Rector's Conference (HRK) - have been two of the most influential documents. They will be explored subsequently regarding their indications of teachers' continuing learning as well as teachers as learners in schools following the understanding introduced before (see section 2).

Both documents predominantly locate teachers' CPD in Germany outside educational practice (see KMK/HRK 2015; KMK 2004/2014; Waitoller/Artiles 2013; Hascher 2011): the first phase of teacher education, pre-service, primarily takes place in universities; the second phase is to a greater extent located in school, yet also in seminars outside school. The third phase, i.e. further education for qualified teachers, is generally recognised to a much smaller degree in educational policy papers than the first and second phases (see e.g. KMK 2017a, 2004/2014; KMK/HRK 2015). It is mostly referred to by external, formalised courses. In fact, in some federal states, for instance in Berlin, teachers have even been told to obtain formalised further education outside educational practice: "Teachers are required to take part in further education courses on a regular basis and especially outside lesson time" (Berlin school law 2004, §67 (7); transl. EP). This is surprising, because internationally, external arrangements for further teacher education have repeatedly been criticised: They would be "isolated events" (Terhart 1990: 164; transl. EP), disconnected from individual practice and settings and therefore with little or no lasting effects (see Calvert 2016; Heise 2009). Vice versa it has been recognised, when professional teacher development is included in developments and processes of reflection in the individual school, it proves to be particularly effective (see Allemann-Ghionda 2013; Amrhein/Badstieber 2013: 20; Bastian et al. 2002: 424). Yet, despite these findings, instruments and materials for (inclusive) school developments, such as the Aargauer evaluation tool (Fachhochschule Nordwestschweiz Pädagogische Hochschule 2012) or the Quick Guides for Inclusion (LISUM 2011a \& b), very rarely consider professional development. Neither have there been many references to informal learning opportunities for teachers on the job, namely as part of educational practice (see Heise 2009).

"There is probably no area of teachers' responsibilities like continuing professional development, which on one hand is so clearly defined as a duty [see KMK 2017b; EP] and on the other hand so unclear in its specification when the duty is fulfilled" (GEW Berlin 2012, 1; 
transl. EP). Policy papers predominantly emphasise teachers as "experts" (KMK 2004/2014: 7) in educational practice, characterised through responsibilities such as "supporting students" (ibid.: 8), "plan and implement lessons correctly" (ibid.: 7), "convey values and norms" (ibid.: 10), "make judgements" (ibid.: 11), "find solutions" (ibid.: 10) etc. But this leaves hardly any space for teachers to be also acknowledged as continuous learners alongside their students, although shown to be a condition for students' participation and learning, teachers' professionalism and school developments (see section 2).

However, there are a few indications of elements of teachers as learners in the "Standards for teacher education" (KMK 2004/2014; transl. EP) and in the "Recommendations for teacher education for a school welcoming diversity" (KMK/HRK 2015; transl. EP): the Standards emphasise teachers' continuous reflection of processes of teaching and learning as a core responsibility. And the recognition of the teaching job "as a continuous learning task" (KMK 2004/2014: 13; transl. EP) implying to "continuously advance their competencies" (KMK 2004/2014: 13; transl. EP) is considered as part of teacher professionalism. Similarly, the Recommendations (KMK/HRK 2015; transl. EP) stress the importance of teachers' "ongoing evaluation of their lessons" (KMK/HRK 2015, 4; transl. EP) and "a professional attitude towards the limits of one's own competencies" (KMK/HRK 2015: 3; transl. EP).

The apparent limited emphasis on teachers as learners and continuing teacher learning inside as opposed to outside school in German education policies was reflected in the results of the student research project mentioned before (see section 1): most of the interviewed teachers from Berlin referred to further education opportunities outside school when they talked about themselves as learners. Only a few identified informal learning opportunities, such as conversations with colleagues and teacher meetings, to support their own learning and professional development. Just one teacher described herself as learning also in the lesson from students when engaging with them in the learning content. Yet, she was a lateral entry employee in her first years of teaching and considered her learning from students as temporary state: "I am only three and a half years in this job. So, of course I am yet learning from the students every day - if you allow it to happen [...] that you build a learning community together with the students" (Plate 2018, unpublished; transl. EP).

\subsection{Policies for Teacher Education and Continuing Professional Development in England}

In England a growing majority of teachers qualifies in schools. This is the result of the White Paper "The importance of Teaching" (DfE 2010) in which the Department of Education (DfE) from the English government published its vision for teacher education outlining a socalled "self-improving school-led system". Since then, schools have been given the opportunity to apply for the status of "Teaching School", namely schools, judged by Office for Standards in Education, Children's Services and Skills (Ofsted) ${ }^{3}$ as "outstanding”, i.e. with high standards of student attainment. Such schools "will take a leading responsibility for providing and quality assuring initial teacher training in their area and receive funding to offer professional development for teachers and leaders" (DfE 2010: 23). At the same time, higher 
education institutions still play a crucial role in the provision of initial teacher education resulting in a confusing array of entry routes into teacher education (see Sorenson 2019).

The majority of professional development and learning for student teachers in England is now taking place within schools, but the opportunities lack coherence: The quality of experience, of mentoring and day-to-day support may be hugely variable depending on the leadership priorities of the school. Moreover, the encouragement for schools to contract directly with the government as charitable trusts, i.e. academies, has led in many cases to a diminishing local offer of regional CPD. There are currently over 800 Teaching Schools many of which are also part of multi-academy trusts. The vision of the government's White Paper (DfE 2010) was that Teaching Schools would not only begin engaging with recruitment and initial teacher training but would also trade their services as agents of school improvement and providers of CPD. Previously, Local Education Authorities had taken responsibility for teachers' continuing professional development. But this has almost disappeared in many areas of the country, now been replaced with local clusters of schools trading professional development opportunities of variable quality alongside unregulated commercial CPD companies (see Greany/Higham 2018).

As in Germany, another core policy paper for teacher education and professional development in England to support coherence between professional learning opportunities are the "Teachers' Standards" (DfE 2011/2013: 5). They "apply to the vast majority of teachers regardless of their career stage" (DfE 2011/2013: 5). Underlying the eight Standards there is the expectation of teachers' continuing professional development, reflection and self-evaluation of their educational practice (see ibid.: 7). The Standards define the "level of practice" (ibid.: 6) that is expected from teachers and against which their performance is assessed throughout their career by teachers themselves, by headteachers (or appraisers), school inspectors and through feedback from colleagues. Beside reflection and self-evaluation, the only reference made to the notions of teachers as learners and teacher learning as continuous elements of their professionalism and educational practice can be identified in the preamble of the Standards which points to the necessity for teachers to be "self-critical" (ibid.: 10). Predominantly, the Standards define the teachers' role as "performers" in educational practice: "teachers are expected to perform" (ibid.: 6). This for instance implies to "demonstrate", "guide", "be accountable", "have a secure understanding", "assess" and "manage" (ibid.: 10ff.). It offers teachers hardly any possibilities to perceive themselves also as learners and to engage in learning processes together with students. The $8^{\text {th }}$ standard, "fulfil wider professional responsibilities" (DfE 2011/2013: 13), directly addresses teachers' own professional development and learning ("take responsibility for improving teaching through appropriate professional development, responding to advice and feedback from colleagues" (ibid.: 11)), but places it amongst other responsibilities all located outside lesson practice and not as part of it.

Another English policy document that is supposed to support teacher professional development - especially in the first two years of teaching - is a new "Early Career Framework" (DfE 2019) the government has recently consulted upon. The framework builds on the Teachers' Standards (DfE 2011/2013) and specifies their implications for teacher professional development in educational practice. Moreover, it indicates a move towards a perspective of teachers as learners as part of their professionalism by emphasising the indeterminacy of pedagogical relationships and students' developments. So for instance, originally within the Teachers' Standards (DfE 2011/2013) students were described in terms of their "abilities and dispositions". This is no longer referenced in the new Early Career Framework (DfE 2019). 
Instead, new teachers are helped to learn to "communicate a belief in the academic potential of all pupils" (DfE 2019: 8) and to create "a culture of respect and trust in the classroom that supports all pupils to succeed" (ibid.: 8). This represents a welcome shift in attitude, against a perception that has dominated education systems internationally up to now, namely that, according to the notion of so-called "ability", a student's capacity to learn is fixed. In contrast, the new Early Career Framework (DfE 2019) requires teachers to explore a young person's learning process and believe in every student's unlimited capacities to learn (see Hart et al. 2004), rather than judging on and pretending to be able to predict students' learning.

Furthermore, in contrast to the Teachers' Standards (DfE 2011/2013), the term "differentiation" is no longer used in the Early Career Framework and it is recognised that, whenever ability based grouping is applied in educational practice, "avoiding the perception that groups are fixed" (DfE 2019: 18) is very important. This can be interpreted as a call for novice teachers to maintain a learning attitude, i.e. to recognize the discriminatory potential of groupings based on ascribed fixed ability and instead to constantly explore ways to increase every student's learning capacities, holding high expectations of attainment and achievement for all students and their contributions to processes of teaching and learning (see Hart et al. 2004). On the other hand, despite indications of its discriminatory effects, attainmentbased streaming is still accepted practice in the Early Career Framework (DfE 2019: 18): "ensuring that any groups based on attainment are subject specific". Equally contradictory is the descriptor "adaptive teaching" (ibid.: 17), linking to the fifth Teachers' Standard (DfE 2011/2013: 11): "Adapt teaching to the strengths and needs of all pupils". The descriptor elaborates on students' potential needs of support, but, surprisingly, does not expand on their strengths at all. This raises the question to what extent students can actually participate in educational practice, meaning to be recognised as contributors to teaching and learning, supporting adults and young people as much as feeling supported by them, i.e. together forming a "joint enterprise" (Hart et al. 2004: 200; also see section 2).

\subsection{Policy Effects for Teacher and Student Participation in England and Germany}

In both countries policy papers recognise teachers' professional development as a continuous process throughout a teacher's work life. Since in Germany this process is mainly organised to take place outside school, usually in time-limited, formalised seminars at universities or in other further education institutions, in England it is primarily located in school - yet, not necessarily integrated in daily educational practice, but as initial teacher qualification in a Teaching School, in in-service trainings and in the context of teachers' annual appraisals (see HM Government 2012). A set of competence-based standards in both countries is supposed to guide teachers' professional development, to identify their "professional development needs" (TDA 2007: 3) and overall to raise educational quality. This approach has also been called teachers' "New Professionalism" (Hargreaves 1994). It is based on economic values such as accountability, competition, standardisation and output-orientation. This indicates a cultural change which has gained strength and permeated all areas of education systems internationally following the introduction of NPM. In this context teacher education has turned into teacher training: "The training model sidesteps thinking, questioning and inventiveness in favour of 'right' answers, 'what works' and 'best practice'. Pre-digested ideas about 'best 
practice' are 'delivered' [...]" (Swann et al. 2011: 107f.) promoting a teacher's role as "a producer increasingly focused on compliance" (Ballard 2004: 99).

These developments oppose other approaches to teachers' professionalism, professional development and teacher agency: Those place teachers' capacities to respond to the indeterminacies of pedagogical relationships at the core of professional action (see section 1). They rely upon a combination of intuitive and theoretical knowledge that builds confidence to speak up about values and a seemingly innate sense of "what is right". In other words, they acknowledge the development of teachers as learners and teacher learning as continuous, participatory, self-directed and -reflective processes which have proven to be crucial to support students' learning and participation. Vice versa, approaches to teachers' professional development as learning the craft of a trade have been criticised as a barrier towards teacher professionalism (see Plate 2017, 2016; Schimank 2014; Tacke 2005; Ball 2003) leading to further excluding processes for all people in educational practice and for students in particular (see Plate 2012):

One example of excluding effects is the rise in scripted lessons in England to be "delivered" by teachers. It could be argued that shared planning and lesson scripts are a way to support teachers in their subject knowledge whilst writing individual lesson plans is unreasonable considering the increasing teacher workload. However, this allows little room for planning with the individuality of all young people in mind within such classrooms and the indeterminableness of pedagogic situations. ${ }^{4}$ Instead, it assumes willing compliance from all students as well as teachers' "willingness necessary for instructing students" (Stephens et al. 2004: 109) rather than supporting students' participation and "intellectual emancipation" (Rancière 2007).

Another example, showing fairly drastically the excluding effects for teachers and students in educational practices, are high-stakes school inspections taking place on a regular basis in the English and German school systems. The education inspection framework in England (Ofsted 2019) that has been in place emphasised evidence of progress made by individual students. This has led to an expectation by some leaders and inspectors that progress should be evident within every lesson. Lessons have been observed and judged by inspectors to be "good" if progress of all students was clear to see. This may provide a powerful disincentive for school leaders and teachers to welcome all students into their setting - not only in England but also in Germany (see Plate 2012). There have been reports about recent challenges in England to a tendency amongst some school leaders to remove students from their school if their attainment progress was likely to negatively impact upon the overall assessment data profile of the institution (see e.g. Weale 2018). Correspondingly, in particular in regards to students' behaviour management, some schools in England have begun to take what they describe as a "zero tolerance approach" to any infringements of school rules, whilst others have argued such approach, that refuses to allow for individual differences, is discriminating (see Roberts 2019).

4 The newly established Chartered College of Teaching (https://chartered.college/) aims to offer a more nuanced debate about issues such as these. 


\subsection{Summary: From Teacher Participation to Teacher Compliance and Exclusion}

Internationally, teachers are required to engage in CPD (see OECD 2006; KMK 2004/2014; Terhart 2000, 2004). In scientific discourses teacher professionalism has primarily been defined as a (self-)reflective, participatory process. This understanding appears to be increasingly suppressed in policies in England and Germany in the context of NPM to the benefit of an approach which defines processes of teaching first and foremost as performance management based on economic principles. Correspondingly, teacher professionalism and professional development in both countries are emphasised as continuous processes orientated on predefined professional standards setting out professional competencies to gain expertise. They are primarily to be acquired in formalised pre- and in-service trainings. This understanding is far off the implications of continuing teacher learning and teachers as learners we have outlined before, referring to a learning attitude, to any learning content relevant to the individual teacher, to teachers' roles as structures for learning and to learning as a participatory process alongside students and as part of educational practice. Here, students and teachers can both perceive themselves as learners and teachers, as supporters and supported.

It can be concluded that teachers are affected by the same standards agenda as students. This originally followed the logic that "high standards" for teachers will lead to "high standards [of] pupils' performance" (TDA 2002: 1). In this context, resulting from pressures of standardisation, output orientation and (hyper-)accountability (see Schimank 2014; Ball 2003), teachers' participation has been continuously reduced causing further detrimental effects on educational practice and school developments and on the learning and participation of students (see Plate 2012). Looking at it the other way round, it suggests once more to acknowledge teacher participation as a condition not only for teacher professionalism and CPD but for teaching and learning as well as for (inclusive) school developments and the connection of both (see section 1 ).

From previous outlines the question arises how the notions of continuing teacher learning and teachers as learners can be put into practice in schools in a climate of standardisation, accountability and output orientation. Therefore, the following section provides an example of a school development process in England led by a headteacher, who saw the necessity that increasing learning and participation for young people requires the same for all adults.

\section{Supporting "Teachers as Learners": A School in England Moving towards Inclusion}

As outlined above, governmental requirements limit teachers' as well as students' learning and participation. Yet, there are schools that still manage to provide space for participatory and individualised developments to support teaching and learning. One example, a school in England, will be introduced in the following. According to the chapter's main focus, particular attention will be paid to the support of teachers' learning and their recognition as learners as an element of, and not just a prerequisite for, inclusive school developments. 
One central question regarding the learning and professional development of teachers in their daily educational practice in schools is: By whom and what is it supported, if not by formalised further education settings and a teacher educator? Ideally, processes for teacher learning and professional development in school are supported on different levels and through various people (see Haeffner 2012; Swann et al. 2012; Heise 2009): on the individual level through the teachers themselves, their willingness to learn and to reflect; on classroom level through dialogue with others, for instance colleagues, students and their significant other; on institutional level, e.g. through school leaders and headteachers as well as other people who are not present in the lessons. Furthermore, other institutionalised structures supporting teacher learning are necessary, such as forms of collegiate learning circles (Bonsen/Rolff 2006), and facilities to access different sources of information, such as the internet, a school library or a resource room. However, structures are not enough. To ensure that teachers use them, as Heise (2009) found out in her study on informal teacher learning in schools, they have to rely on school cultures based on values such as participation, openness, trust and diversity (see Booth/Ainscow 2016: 24f.). Moreover, it presupposes an understanding that teachers can only support students when both engage in processes of learning and teaching together.

\subsection{Wroxham Primary}

How might a more inclusive approach that values every teacher as an individual learner and celebrates the opportunity for them to diversify throughout their career, work within an individual school community and in an education system predominantly led by counteracting economic principles? How can barriers to teacher learning and professional development be removed and their participation be supported? Alison Peacock was headteacher of Wroxham Primary School (3-11 years) where the story of an alternative approach to school development, called Learning without Limits (LwL) (Hart et al. 2004), was researched over a period of six years (see Swann et al. 2012). How did change come about so quickly and with such transformative success throughout the school? How can the example from one small school begin "a quiet revolution"? There is much to be said about this empirical case study (see Swann et al. 2012), but the story of what happened to the teachers as the school moved from being judged as "failure" to "success" is of most interest here.

\subsubsection{Background and Initiation}

In January 2003 Alison took up the post of headteacher at The Wroxham School in Hertfordshire, England. In 2001 the school had been judged by the inspectorate to require "Special Measures" due to inadequate leadership, poor attainment, underachievement and behaviour that led inspectors to describe some students as "un-teachable". Termly inspections since that date stubbornly showed that "no progress" had been achieved. Arriving as a novice headteacher, Alison was charged with improving the school or facing its closure. As challenges go, this was a huge one. Alison had studied for a professional headship qualification and read many books on leadership. However, the greatest influence on her approach to headship was her Masters study on inclusion and the early years, a project on pupil voice at the 
University of Sussex (see Fielding/Bragg 2003) and then, whilst working as a deputy headteacher, her participation in the first LwL-study (see Hart et al. 2004). The latter played a particular role for the developments at Wroxham.

When Alison took up the headship, she received a phone call from a researcher from the University of Cambridge suggesting that maybe she would be prepared to continue the LwLproject in a second study, now from the perspective of school leadership, to explore and reflect the impact of an alternative school approach applying "a fundamentally different view of learners and learning, of curriculum and pedagogy, from that promoted by the standards agenda, and a radically different approach to the distribution of leadership, power, monitoring and accountability" (Swann et al. 2012: Preface). In response to the researchers request to participate in a second LwL-study Alison recalls thinking: "Don't agree to this... They may find you out. They will realize that you don't know what you are doing and that you have no idea how to improve this school." But, instead of declining, she found herself agreeing that the university should proceed with the study and that she would be willing to take on a dual role as both a member of the research team and a member of the school staff. This dual role she found challenging and confusing at times but ultimately provided a wonderful opportunity for richly contextual professional mentoring, questioning and thereby - of course - learning.

\section{Excursus: Learning without Limits}

Initially, Learning without Limits (LwL) was a research study based at the University of Cambridge (see Hart et al. 2004) aiming "to bring together a group of teachers who had rejected ideas of fixed ability and to study their practice, in order to explore and try to identify what was distinctive about teaching free from ability labelling" (Hart et al. 2004: 14). From this study resulted what can be considered a philosophy of learning and teaching and - explored more in-depth in the second (see Swann et al. 2012) - an inclusive approach to school developments. And both have been called Learning without Limits, too.

The core of this philosophy is the "idea of transformability" (Hart et al. 2004: 163), emphasising every person's unlimited capacities to learn. It is based on "a fundamentally different sense of the relation between present and future: a firm and unswerving conviction that there is potential for change in current patterns of achievement and response, that things can change and be changed for the better, sometimes even dramatically, as a result of what happens and what people do in the present" (Hart et al. 2004: 166). This strongly opposes the concept of a person's fixed ability, which is based on "the assumption that, in the future, things will remain much as they are at present, and therefore present patterns of attainment and response are reliable predictors of young people's future development" (ibid.). Fixedability thinking has dominated education systems internationally up until now and even gained strength in the context of NPM, requiring increased accountability and output-orientation, resulting in excluding practices such as ability labelling. However, despite these systemic constraints, there are teachers who have found ways to work inclusively, setting aside the concept of ability aiming to continuously enhance all students' learning capacities. Underlying their educational practice, i.e. supporting Learning without Limits, are three interdependent "practical pedagogical principles" (Hart et al. 2004: 172):

"The principle of everybody" (Hart et al. 2004: 187f.) emphasises the importance to provide learning arrangements always for everybody and not only for selected groups, because teachers' actions will inevitably affect every student - positively or negatively (Hart et al. 
2004: 261). Students shall be given the opportunity to participate in, and thus contribute to, processes of learning and teaching. In contrast, ability labelling (e.g. labelled as having "special educational needs") can support the presumption that some students can only contribute very little to their own and to others' learning (Hart et al. 2004: 189).

"The principle of co-agency" (Hart et al. 2004: 180f.) reflects the idea of processes of teaching and learning as a "joint enterprise" (Hart et al. 2004: 180f.) of teachers and students. This means that responsibilities for learning and teaching have to be shared - instead of split - between both, based on the understanding that teachers are powerless if students do not engage (see Florian/Linklater 2010: 372). Consequently, the teachers' actions aim to provide a learning environment for students and to change external conditions in a way that students are enabled to "join the enterprise".

"The principle of trusting the learner" (Hart et al. 2004: 191), on one hand, points out that trust "sustains the teachers' belief that students will choose to engage if the conditions are right [...]" (Hart et al. 2004: 191). On the other hand, it stresses that there is always potential for development and change as a result of our actions in the present, independent of what has happened in the past (see Hart et al. 2004: 192). Trust is a precondition for students to take up the invitation to join processes of learning and teaching.

\subsubsection{How to Start}

During her first week of headship Alison put up a display in the main corridor stating that this was a "Listening School", listening to everyone, young people and adults. Looking back she realises that this was a very clear message that the team, staff as well as students and parents were all going to work together to move beyond the Ofsted label of "Special measures". Later, this was described as learning as a "collective endeavour" (Swann et al. 2012: 112).

The school had spent the last two years monitoring learning. The deficit notion of monitoring implies that everything needs to be checked upon and improved. Many advisors had visited the school since it was judged as "inadequate". The effects for teachers were clearly noticeable: they felt completely disempowered. Thus, Alison decided to take control of the programme of relentless advisory visits. She insisted that every advisor meet with her before and after any classroom work and ensured that also teachers had the opportunity to met with advisors after each visit. This shifted the emphasis from external advisors "telling" teachers top-down what to improve to having an equal conversation in which teachers are valued as competent professionals engaging in their own learning and continuing professional development. This quickly resulted in the co-ordination of both purpose and action.

\subsubsection{Establishing Structures for Staff Learning}

As headteacher Alison regarded it as one of her primary responsibilities to support the learning and professional development of the staff. "Seven key dispositions that increase the capacity for professional learning" (Swann et al. 2012: 88; see Table 1) guided the development of structures for staff learning. Additionally, they were modelled by the headteacher in her interactions with staff and in her own learning. In contrast, current developments in education systems internationally following NPM rather support "States of mind that inhibit learning" (Swann et al. 2012: 88; see Table 1). 
Table 1: Positions for and against learning

\begin{tabular}{llll}
\hline $\begin{array}{l}\text { Seven key dispositions that increase the } \\
\text { capacity for professional learning }\end{array}$ & States of mind that inhibit learning \\
\hline Openness - to ideas, to possibilities, to surprise & not & $\begin{array}{l}\text { belief that there is one right way, that } \\
\text { outcomes are predictable }\end{array}$ \\
\hline Questioning - restlessness, humility & not & $\begin{array}{l}\text { reliance on certainties and ready-made } \\
\text { solutions }\end{array}$ \\
\hline Inventiveness - creative responses to challenges & not & $\begin{array}{l}\text { compliance with imposed models and } \\
\text { materials }\end{array}$ \\
\hline Persistence - courage, humility & not & $\begin{array}{l}\text { settling for easy answers, rejecting } \\
\text { complexity }\end{array}$ \\
\hline Emotional stability - taking risks and resistance & not & fear of failure, fear of trying new things \\
\hline Generosity - welcoming difference & not & deficit thinking, desire for uniformity \\
\hline Empathy - mutual supportiveness & not & fear, defensiveness, blame \\
\hline
\end{tabular}

Source: Swann et al. 2012: 88

\section{Mentoring and Coaching Arrangements for All Staff}

A new deputy headteacher was appointed quite soon. Together, she and Alison were able to begin to support the staff team to regain confidence through informal coaching and mentoring conversations. Crucially, these conversations were almost always about the teachers' worries or confusion about students, rather than about their professional "performance" (HM Government 2012: 3). The teachers were incredibly responsive in such conversations, which they perceived as supportive and enabling for themselves. Every member of staff in the school had a mentor from elsewhere in the team. They met individually each term to reflect on their developments. Sometimes, mentors and mentees carried out small lesson-based studies and the findings were shared amongst the whole staff (see Swann et al. 2012: 21). This was much more than officially required for the statutory appraisal, which exclusively addressed teachers and only has to take place approximately once a year (see HM Government 2012). The arrangements at Wroxham supported all staff to develop trust in their own unlimited learning capacities and to follow their aspirations: for instance, Alison had agreed to support and mentor four teaching assistants, who wanted to apply for a foundation degree at the University of Hertfordshire. Wroxham funded their study and provided them with one day a week release for four years. Additionally, three of the four went onto attain a teaching degree and one was subsequently employed as a teacher at Wroxham. Later, in an Ofsted inspection, she was recognised as "a magical teacher".

A shift from a culture of fear and compliance to one of optimism and inquisitiveness was quickly noticeable amongst staff. Uninformed optimism, however, is not enough. Teacher or, rather, staff learning and professional development needs to be placed at the heart of school developments as much as students' learning and development. Therefore, at Wroxham every opportunity was taken to build on it. 


\section{More Collaborative Structures for Learning}

Different collaborative structures for adults and young people were established to learn with and from each other and to support the development of their school:

"Faculty Teams" (Swann et al. 2012: 19f.), in contrast to usual teachers' conferences, consist of teachers from different subjects, teaching assistants and members of school committees, such as parent governors. Everybody can choose the team by themselves. The faculty teams meet once a month for two hours and have the responsibility to develop the school's curriculum. The results of such meetings are displayed for everyone at the school's noticeboard and put into practice in lessons. Colleagues are invited to observe the lessons and afterwards reflect on them together.

"Circle Group Meetings" (Swann et al. 2012: 16f.) are another structure established to support collaborative learning and participation of staff and students. They take place every Tuesday morning for fifteen minutes in mixed-year groups, led and protocolled by students from year six. The idea of these groups has been to give students and staff the opportunity to voice their ideas and perspectives and thereby contribute to school developments and learn from each other.

\subsubsection{Challenging Fixed-ability Thinking}

As the school rapidly began to improve, it was important to connect with individual members of staff to begin to challenge notions of "fixed ability" and to call out comments that belied inherent bias towards thinking of students in terms of past performance, instead of being open to the "idea of transformability" (Hart et al. 2004: 163). Findings of the research project based at the school showed that teacher attitudes and behaviours were influenced much more by individual conversations and dialogue related to young people within their class, than they were by staff meetings and policies (see Swann et al. 2012). In fact, as time went on teachers spent less time in formal meetings and more time in practices such as shared lesson planning, observations and reflections. This approach, as opposed to monitoring, combined with external study and educational reading began to move teachers from talking about Learning without Limits and transformability to living it. Working as a "collective endeavour" (Swann et al. 2012: 112) at Wroxham helped teachers to respond to students who experienced increased barriers to their learning. Through enhanced empathy and knowledge about the child, relationships noticeably had a greater and more supportive impact on the students' learning. And not only young people, but also adults were more and more recognised to exemplify transformability. These examples served to reinforce and embed this alternative way of school development that everyone who heard about or visited the school could begin to see was working.

\subsubsection{Becoming a Teaching School - Engaging in Learning with External Professionals}

In 2012, the Wroxham School was in the first cohort of teaching schools within England. This meant that the school was expected to establish a local alliance of schools for school improvement purposes. The reality was that they began to build a "Transformative Learning 
Alliance" of schools keen to learn more about their inclusive approaches to teaching and learning. Therefore, Sally, a recently retired headteacher, was appointed to support building a community of schools that became a local partnership. She used her coaching skills in conversations with various school leaders, such as headteachers, deputy and assistant headteachers and CEOs, to build their confidence and expertise.

A small group of teachers, funded by the Local Authority and led by a colleague from Wroxham, began to support teachers' learning about approaches that have been proven to enhance learning for all students. The teachers in this group worked together to develop the support for schools and the quality of local offers for students who experience barriers to their learning.

Wroxham School began to run courses in the school assembly hall with students joining a session to talk to the whole group of visiting teachers about learning, about challenges and successes they experienced and to share their exercise books. Classrooms became places that regularly included large numbers of visiting teachers working and learning alongside the students. In some lessons, this meant that visiting teachers would sit in an adjacent viewing area to observe the class teacher and the students. On other occasions, teachers would simply mingle within the classroom and engage with the students, learning from them about barriers and resources for learning and teaching. The teachers were able to meet with the class teacher prior to the lesson to talk about the aims and plans of the lesson and seek to understand the underlying pedagogical theory. When the lesson was complete, the group would re-convene to engage in shared reflections on what took place.

This approach to teacher learning was almost overwhelming for the school but the impact across the Transformative Learning Alliance was huge. Teachers visiting Wroxham were able to benefit from "seeing", not just "being told about" or "listening" to, inclusive practices. The learning and professional development of teachers at Wroxham became quite extraordinary. The opportunities, afforded by ensuring that growth of talent was predominant, meant that the school rapidly went from strength to strength. Young people and adults were supported to increase their learning capacities and, vice versa, were recognised as supporters for and amongst each other. Learning without Limits principles (see Hart et al. 2004) applied to everybody in the school:

This support across the school was based on trust, on the belief that people could be empowered to think for themselves, to find their own way without being told what to do. [...] To be continually learning is everybody's responsibility; all members of staff should have opportunities to learn, to develop new practices and to share their growing expertise (Swann et al. 2012: 20f.).

\subsection{Summary: Reinterpreting Systemic Conditions and Developing Shared Responsibility}

Introducing Wroxham Primary was to exemplify how the notion of "teachers as learners" and teacher learning (see section 2) can be put into practice and how this affects inclusive developments at the level of the individual school.

It was noticeable that inclusive school developments are possible when opposing systemic requirements (see section 3) are reinterpreted in the individual school context. As a result, practices, which formerly were shown to impede inclusive developments, can be coun- 
teracted: For example, at Wroxham inspections have been developed as occasions on which teachers and inspectors engage on an equal level to reflect on educational practice. The concept of "fixed ability" has been replaced by the "idea of transformability" (Hart et al. 2004: 163 ) and ability-based labelling has been rejected. Statutory annual teacher appraisals have been turned into termly mentoring and coaching conversations for every member of staff. Formal staff meetings and conferences have been opened to students, parents and significant others. In this way, the seven key dispositions for so-called "professional" learning at Wroxham (see Table 1) have also been reinterpreted as equally applicable to students' and everybody else's individual and collective learning.

Furthermore, exemplified at Wroxham, valuing teachers as learners has positive effects for students' participation and learning and generally for inclusive developments: Engaging in dialogue with students, acknowledging them as experts for their own learning, enables teachers to reflect on and inform their pedagogical actions, i.e. a central element of teacher professionalism (see 2.1.2). In this way, as a "joint enterprise" (see 2.1.4), teachers and students feel supported by and to be learning from each other. Together with all other people at Wroxham, professionals, parents and significant others, they formed what has been called a "collective endeavour" (Swann et al. 2012: 112). This has been supported by various structures across the school promoting a sense of shared responsibility: for instance, the establishment of circle groups and faculty teams or the display in the school entrance stating "this is a listening school", which immediately indicates an approach that is opposite to instruction and order but in valuation of and seeking everyone's perspectives. In addition, a sense of shared responsibility across the whole school is supported by the structure of so-called "coordinators", which has generally been established in schools in England. These teachers coordinate one aspect of educational practice for the entire school: there are, for example, "assessment coordinators", "literacy coordinators" and "numeracy coordinators".

Following the previous summary of school developments at Wroxham, one question is of particular interest to us with regard to our international perspective: What do we learn from the descriptions of cultures, structures and practices at Wroxham for developing inclusion in education internationally and specifically in Germany? Finding ways to work inclusively whilst facing opposing requirements appears to be equally relevant for developments in schools in Germany. Practices, such as external monitoring, comparative national exams and other assessments of standardised attainment targets have continued to pressure teachers and other professionals with ongoing detrimental effects on student learning and participation (see Plate 2012). This has been reflected in the steady increase of students' labelling in schools in Germany over the last years (see KMK 2018: XIV): Between 2007 and 2016, the percentage of students labelled as "sonderpädagogisch förderbedürftig", went from 5,2\% up to $7,0 \%$ (KMK 2018: XIV). In the academic year 2017/18, the number again rose, to $7,2 \%$. And ironically, various national statistics define inclusion as educating students labelled as "sonderpädagogisch förderbedürftig" in mainstream schools (see e.g. Friedrich-Ebert-Stiftung 2017): the higher the percentage of students with this label in a mainstream school depicted in the so-called "inclusion rate" (Friedrich-Ebert-Stiftung 2017; transl. EP) - the more a school is judged as "inclusive".

5 The German term "sonderpädagogisch förderbedürftig” refers to the concept of ,sonderpädagogischer Förderbedarf" in Germany's education system. In English publications it has often been translated with the concept of "special educational needs". Yet, it must not be ignored that both concepts differ, following differences between the education systems in which they have been constructed (see Plate 2008). 
Furthermore, perspectives to develop a sense of shared responsibility in schools, such as teachers' "coordinator" roles in England (see above), can be of particular interest for schools in Germany, where many teachers still see their primary responsibility to be their own teaching practice in class, despite the increased official emphasis on teachers' engagement in whole-school developments (see KMK 2004, 2014: 14). Additionally, structures for collaboration in schools in Germany rather support role-homogeneous groupings: for example steering groups, subject conferences and the whole teachers' conference, parent governing bodies, students' committee, etc. Committees like at Wroxham, with members from all groups (students, parents, significant others, etc.), are much less established. Their development maybe worth considering as another way to support a growing sense of shared responsibility and a whole school community, emphasising the perspective "we and our school" instead of "me and my class".

In sum, Wroxham Primary School has exemplified that innovation is possible if it is not imposed but allows space for everyone's participation and learning. The described developments would not have been possible without the great efforts of the headteacher. This confirms once again the powerful role of school leaders not only in England but also in Germany: whether teacher agency is stifled or enabled often strongly depends on the school leader. Where school leaders can embrace individualism and learning as a strength within their schools there is space for teachers to be valued as learning professionals. Conversely, there are also other leaders who view teacher education as an apprenticeship towards learning the craft of a trade providing false certainties and one-sided solutions with proven detrimental effects for students' learning and participation (see Plate 2012). At Wroxham, the headteacher's understandings of learning and teaching, of school development and of her role as supporter for the learning and participation of all young people as well as adults in the school were key to the developments that took place. She recognised the school's resources and potential, which had not been noticed before when the school was considered "failing". Staff and students had always been the same, but now they were given the opportunity to contribute.

It can be concluded that teachers as well as everybody else must be valued and supported as competent, continuous learners whilst implementing change. In this way, they can become excited and motivated about new, inclusive ways of developing schools and educational practice.

\section{Conclusion: Making Space for Students and Teachers to Surprise}

The intention of this chapter has been to emphasise the importance of valuing teachers as learners and their continuing learning as crucial for inclusion in schools. Using an international perspective, we have aimed to support critical reflection and questioning regarding our surrounding systems - cultures, structures and practices - and how we may promote them ourselves, sometimes even without knowing. We have wanted to motivate change and open up new perspectives for inclusive developments.

Therefore, at first (see section 1) we highlighted that in the education systems of England and Germany teacher learning and teachers as learners have yet primarily been recognised in 
the context of so-called "continuing professional development" (CPD) mostly taking place outside school and educational practice, i.e. teacher pre-service trainings and further education courses. CPD mainly aims at the acquisition of specific professional competencies and skills in preparation for educational practice and school developments. Thus, based on broader definitions of "teachers as learners" and "teacher learning", for instance including learning attitudes and learning as an ongoing process, we argued that teachers being learners and engaging in their own continuing learning are also inevitable elements of inclusion and supported through inclusive processes in schools. Accordingly, by applying the terms "learning" and "learners" to teachers, we did not mean to indicate a deficiency or lack of competence but very much the opposite: Such terms place very high aspirations on teachers' professionalism, emphasising its quality as a continuous, participatory, individual and contextualised process to respond to the indeterminacies of pedagogical relationships and the limits of one's own knowledge. At the same time, they can be unburdening for teachers as they make it professionally reasonable for them to admit if and when they need support.

Following such theoretical considerations, we looked at current implementations in practice, at existing barriers and resources to support teachers as learners and teacher learning in schools, firstly in national policies for teacher education in England and Germany (see section 3) and later at school level exemplified by Wroxham Primary School (see section 4). It has been noticeable that policy requirements in England and Germany, driven by New Public Management (NPM), counteract the notions of teachers as learners and teacher learning. They reduce teacher participation and agency, leading to a diminishing of teacher professionalism and inhibit inclusive developments in schools (see Plate 2017). However, Wroxham Primary illustrated that it is possible to move towards a school in which learning and participation for teachers is acknowledged as a core condition to raise students' learning achievements and participation. In order to be sustainable, such developments, supporting everyone in a school as life-long and competent learners, have to be rooted in a school's cultures based on shared values like trust, equality, diversity and, of course, participation - in other words "inclusive values" (Booth/Ainscow 2016). This cultural basis offers the opportunity for systematic change: the establishment of new structures for learning as well as the adjustment and reinterpretation of already existing, yet counteracting structures, such as regular inspections and other monitoring procedures, according to the school's values. At Wroxham, Alison, the headteacher, played a key role in this process which - instead of being perceived as burdensome and work-overload - became a resource supporting teachers in various respects, such as in developing positive attitudes towards change, supporting their well-being and their motivation to engage in school developments and learning and teaching.

Beyond the introduced possibilities to support teachers as learners and their continuing learning and professional development as part of schools' educational practice, there are of course many more, exemplified in the following:

- Materials for inclusive school developments should widen their focus from supporting learning and participation for students to supporting teachers and everyone else in the school. One source that applies such a widened perspective is the Index for Inclusion (Booth/Ainscow 2016).

- According to the clear emphasis in national policies on teachers' continuing professional development as part of their roles, current opportunities should be diversified. Particular attention ought to be paid to teachers' informal learning since it has rarely been considered in teacher education (see Heise 2009). 
- As indicated above, teacher professional development for inclusion has to be inclusive itself (see Plate 2016), i.e. not only considering inclusion as a curricular content but as underlying the development of cultures, structures and practices in programmes and institutions for teacher education.

- Output-oriented standards for teachers (DfE 2011, 2013) in England and for teacher education (KMK 2004, 2014) in Germany may be replaced by standards focusing on the process of teacher learning and professional development. The "Standards for Professional learning" (Learning forward 2011), published in the US, may serve as orientation since they "call for professional learning that is ongoing, embedded, connected to practice, aligned to school and district goals, and collaborative" (Calvert 2016: 3).

- The different phases of teacher professional development and learning in England and Germany need to be more connected to increase coherence and thereby the understanding of teacher professionalism as a continuous process. This may also be supported through the establishment of professional bodies, such as the Chartered College of Teaching in England: it supports teachers and educationalists to become inclusive professional communities of learning, celebrating, supporting and connecting their professionalism through lifelong professional learning opportunities available regardless of one's educational setting or current experience. ${ }^{6}$

Furthermore, there is hardly any research on teachers as learners and opportunities for teacher learning as part of inclusive school developments and educational practice. And there are even fewer international studies. Examples of research questions deriving from previous descriptions and to be empirically addressed are: Which opportunities for teacher learning are provided in schools internationally and as part of educational practice and what are the rationales? What relevance do teachers ascribe to their own learning and the notion of teachers as learners in their classrooms and schools and how does it affect inclusive developments?

To conclude, we must ensure that in seeking to support the development of young people we also pay close attention to the growth, continuing learning and agency of the professionals, outside and especially inside schools. The principles that guide inclusive educational work with young people have to apply to staff and communities, too. Inclusion is not just the aim of teachers' actions but the process that enables them to act inclusively. If you don't feel included, how can you include others? In schools and classrooms, where values such as trust, shared ownership, listening, voice and participation form the basis of educational practice, where teachers and students are free from practices that seek to categorise and rank them, there is space for them (and everyone else) to surprise.

\section{References}

Abels, Simone (2011): LehrerInnen als “Reflective Practitioner". Reflexionskompetenz für einen demokratieförderlichen Naturwissenschaftsunterricht. Wiesbaden: VS Verlag für Sozialwissenschaften.

Ackeren, Isabelle van/Brauckmann, Stefan (2010): Internationale Diskussions-, Forschungs- und Theorieansätze zur Governance im Schulwesen. In: Altrichter, Herbert/Maag Merki, Katharina

6 For further information on the Chartered College for Teaching, see https://chartered.college. 
(eds.): Handbuch Neue Steuerungen im Schulsystem. Wiesbaden: VS Verlag für Sozialwissenschaften, pp. 41-61.

Agostini, Evi/Schratz, Michael/Risse, Erika (2018): Lernseits denken - erfolgreich unterrichten. Personalisiertes Lernen in der Schule. Hamburg: AOL Verlag.

Ainscow, Mel/Booth, Tony/Dyson, Alan (2006): Inclusion and the standards agenda. Negotiating policy pressures in England. In: International Journal of Inclusive Education, 10, 4-5, pp. 295308.

Allemann-Ghionda, Cristina (2013): Bildung für alle, Diversität und Inklusion. Internationale Perspektiven. Paderborn: Ferdinand Schöningh.

Amrhein, Bettina/Badstieber, Benjamin (2013): Lehrerfortbildungen zu Inklusion - eine Trendanalyse. Gütersloh: Bertelsmann. http://www.jakobmuthpreis.de/uploads/tx_itao_download/ Lehrerfortbildung_Inklusion.pdf [Accessed: 28.08.2015].

Ball, Stephen J. (2003): The teacher's soul and the terrors of performativity. In: Journal of Education Policy 18, 2, pp. 215-228.

Ballard, Keith (2004): Learners and outcomes. Where did all the children go? In: New Zealand Journal of Teachers' Work 1, 2, pp. 95-103.

Bastian, Johannes/Combe, Arno/Reh, Sabine (2002): Professionalisierung und Schulentwicklung. In: Zeitschrift für Erziehungswissenschaften 5, pp. 417-435.

Bélanger, Paul (2011): Theories in Adult Learning and Education. Opladen \& Farmington Hills: Barbara Budrich Publishers.

Berlin (2004): School law. http://gesetze.berlin.de/jportal/;jsessionid=DD1ABC52C9B06FB4B8 C976430B9D1F5E.jp21?quelle=jlink\&query=SchulG+BE\&psml=bsbeprod.psml\&max=true \&aiz=true\#jlr-SchulGBErahmen [Accessed 11.06.2019].

Black-Hawkins, Kristine/Florian, Lani/Rouse, Martyn (2010): Achievement and inclusion in school. London: Routledge.

Bonsen, Martin/Rolff, Hans-Günter (2006): Professionelle Lerngemeinschaften von Lehrerinnen und Lehrern. In: Zeitschrift für Pädagogik 52, 2, pp. 167-184.

Booth, Tony/Ainscow, Mel (1998) (eds.): From them to us. London: Routledge.

Booth, Tony (2011): The name of the rose. Inclusive values into teacher education. In: Prospects 41, 3, pp. 303-318.

Booth, Tony/Ainscow, Mel (2016): Index for inclusion. A guide to school development led by inclusive values. Cambridge: Index for Inclusion Network.

Calvert, Laurie (2016): Moving from compliance to agency. What teachers need to make professional learning work. Oxford, OH: Learning Forward and NCTAF.

DfE (Department for Education) (2010): The importance of Teaching. The Schools White Paper. https://www.gov.uk/government/publications/the-importance-of-teaching-the-schools-whitepaper-2010 [Accessed: 01.06.2019].

DfE (Department for Education) (2011/2013): Teachers' Standards. Guidance for school leaders, school staff and governing bodies. https://assets.publishing.service.gov.uk/government/uploads/system/uploads/attachment_data/file/665520/Teachers__Standards.pdf [Accessed: 01.06. 2019].

DfE (Department for Education) (2019): Early Career Framework. https://assets.publishing.service.gov.uk/government/uploads/system/uploads/attachment_data/file/773705/EarlyCareer_Framework.pdf [Accessed: 01.06.2019].

Dlugosch, Andrea (2005): Professionelle Entwicklung in sonderpädagogischen Kontexten. In: Horster, Detlef/Hoyningen-Suess, Ursula/Liesen, Christian (eds.): Sonderpädagogische Professionalität. Beiträge zur Entwicklung der Sonderpädagogik als Disziplin und Profession. Wiesbaden: Springer VS, pp. 27-52.

Fachhochschule Nordwestschweiz Pädagogische Hochschule (2012): Bewertungsraster zu den schulischen Integrationsprozessen an der Aargauer und Solothurner Volksschule. https:// 
www.schulen-aargau.ch/media/schulen-aargau/schulorganisation/qualitaet/schulinternes-qm/ bksvs-bewertungsraster-schulintegration.pdf [Accessed 30.11.2019].

Fend, Helmut (2008): Neue Theorie der Schule (second edition). Wiesbaden: VS Verlag für Sozialwissenschaften.

Fielding, Michael/Bragg, Sara (2003): Students as Researchers. Making a Difference. Cambridge: Pearson Publishing.

Florian, Lani/Linklater, Holly (2010): Preparing teachers for inclusive education. Using inclusive pedagogy to enhance teaching and learning for all. In: Cambridge Journal of Education 40, 4, pp. 369-386.

Friedrich-Ebert-Stiftung (2017): Ländervergleich. Inklusive Bildung in Deutschland. http:// library.fes.de/pdf-files/studienfoerderung/13493.pdf [Accessed 06.12.2019].

GEW Berlin (2012): Fortbildungspflicht für LehrerInnen. https://www.gew-berlin.de/public/media/SG20.pdf [Accessed 11.6.2019].

Greany, Toby/Higham, Rob (2018): Hierarchy, Markets \& Networks. Analysing the "self-improving school-led system' agenda in England and the implications for schools. London: UCL IOE Press.

Haeffner, Johannes (2012): Professionalisierung durch Schulentwicklung. Eine subjektwissenschaftliche Studie zu Lernprozessen von Lehrkräften an evangelischen Schulen. Münster: Waxmann.

Hager, Paul/Halliday, John (2009): Recovering Informal Learning: Wisdom, judgement and community. Dordrecht: Springer.

Hargreaves, David H. (1994): The new professionalism: The synthesis of professional and institutional development. In: Teaching \& Teacher Education 10, 4, pp. 423-438.

Harring, Matthias/Witte, Matthias D./Burger, Timo (eds.) (2015): Handbuch informelles Lernen. Interdisziplinäre und internationale Perspektiven. Weinheim: Beltz Juventa.

Hart, Susan/Dixon, Annabelle/Drummond, Mary Jane/McIntyre, Donald (2004): Learning without limits. Maidenhead: Open University Press.

Hascher, Tina (2011): Forschung zur Wirksamkeit der Lehrerbildung. In: Terhart, Ewald/Bennewitz, Hedda/Rothland Martin (Eds.): Handbuch der Forschung zum Lehrerberuf. Münster: Waxmann, pp. 418-440.

Heinrich, Martin/Altrichter, Herbert (2008): Schulentwicklung und Profession. Der Einfluss von Initiativen zur Modernisierung der Schule auf die Lehrerprofession. In: Helsper, Werner/Busse, Susann/Hummrich, Merle/Kramer, Rolf-Torsten (eds.): Pädagogische Professionalität in Organisationen. Neue Verhältnisbestimmungen am Beispiel Schule. Wiesbaden: VS Verlag für Sozialwissenschaften, pp. 205-224.

Heise, Maren (2009): Informelles Lernen von Lehrkräften. Ein Angebots-Nutzungs-Ansatz. Münster: Waxmann.

Helsper, Werner (2004): Antinomien, Widersprüche, Paradoxien. Lehrerarbeit - ein unmögliches Geschäft? Eine strukturtheoretisch-rekonstruktive Perspektive auf das Lehrerhandeln. In: Koch-Priewe, Barbara/Kolbe, Fritz-U./Wildt, Johannes (eds.): Grundlagenforschung und mikrodidaktische Reformansätze zur Lehrerbildung. Bad Heilbrunn: Klinkhardt, pp. 49-98.

Hin-Wai Yung, Benny (2002): Same Assessment, Different Practice. Professional consciousness as a determinant of teachers' practice in a school-based assessment scheme. In: Assessment in Education. Principles, Policy \& Practice 9, 1, pp. 97-117.

HM Government (2012): The Education (School Teachers Appraisal) (England) Regulations. http://www.legislation.gov.uk/uksi/2012/115/pdfs/uksi_20120115_en.pdf [Accessed: 16.6. 2019].

Holtappels, Hans G. (2010): Schule als lernende Organisation. In: Bohl, Thorsten/Helsper, Werner/Holtappels, Heinz G./Schelle, Carla (eds.): Handbuch Schulentwicklung. Bad Heilbrunn: Klinkhardt, pp. 99-105. 
Holzkamp, Klaus (1991): Lehren als Lernbehinderung? In: Forum Kritische Psychologie 27, pp. $5-22$.

KMK (Kultusministerkonferenz) $(2004,2014)$ : Standards für die Lehrerbildung. Bildungswissenschaften. (Beschluss der Kultusministerkonferenz vom 16.12.2014) http://www.kmk.org/ fileadmin/Dateien/veroeffentlichungen_beschluesse/2004/2004_12_16-Standards-Lehrerbildung.pdf [Accessed: 08.12.2016].

KMK (Kultusministerkonferenz) (2017a): Sachstand in der Lehrerbildung in Deutschland. https:// www.kmk.org/fileadmin/Dateien/pdf/Bildung/AllgBildung/2017-03-07_Sachstand_LB_o_ EW.pdf [Accessed: 14.05.2019].

KMK (Kultusministerkonferenz) (2017b): Lehrkräftefortbildung in den Ländern (Stand 19.12.2017) https://www.kmk.org/fileadmin/Dateien/pdf/Bildung/AllgBildung/2017-12-19 Lehrerfortbildung_in_den_Laendern_003_.pdf [20.05.2019].

KMK (Kultusministerkonferenz) (2018): Statistische Veröffentlichungen der Kultusministerkonferenz. Dokumentation Nr. 214. Sonderpädagogische Förderung in Schule 2007-2016.

https://www.kmk.org/fileadmin/Dateien/pdf/Statistik/Dokumentationen/Dok_214_SoPaeFoe_2016.pdf [Accessed: 02.12.2019].

KMK \& HRK (Kultusminister- \& Hochschulrektorenkonferenz) (2015): Lehrerbildung für eine Schule der Vielfalt. Gemeinsame Empfehlung von Hochschulrektorenkonferenz und Kultusministerkonferenz. (Beschluss der Kultusministerkonferenz vom 12.03.2015, Beschluss der Hochschulrektorenkonferenz vom 18.03.2015) https://www.kmk.org/fileadmin/Dateien/veroeffentlichungen_beschluesse/2015/2015_03_12-Schule-der-Vielfalt.pdf [Accessed: 30.11 . 2019].

Kruip, Gerhard (2011): Educational Governance und kooperative Bildungsverantwortung. In: Steinbach-Heims, Marianne/Kruip, Gerhard (Eds.): Kooperative Bildungsverantwortung. Bielefeld: Bertelsmann, pp. 11-33.

Learning forward (2011): Standards for Professional learning. Oxford, OH: Learning forward.

Lemke, Jay L. (2013): Thinking about feeling. Affect across literacies and lives. In: Erstad, Ola/Sefton-Green, Julian (eds.): Identity, Community, and Learning Lives in the Digital Age. Cambridge: Cambridge University Press, pp. 57-69.

LISUM (Landesinstitut für Schule und Medien Berlin-Brandenburg) (2011a): Quick-Guides für Inklusion. Teil 1: Zusammen leben. https://bildungsserver.berlin-brandenburg.de/fileadmin/ bbb/schule/inklusion/2012/Quick-Guides_Teil_1.pdf [Accessed: 30.11.2019].

LISUM (Landesinstitut für Schule und Medien Berlin-Brandenburg) (2011b): Quick-Guides für Inklusion. Teil 2: Lehren und Lernen. https://bildungsserver.berlin-brandenburg.de/fileadmin/ bbb/schule/inklusion/2013/Quick-Guides_Teil_2.pdf [Accessed: 30.11.2019].

OECD (Organisation for Economic Co-operation and Development) (2006): Stärkere Professionalisierung des Lehrerberufs. Wie gute Lehre gewonnen, gefördert und gehalten werden können. Paris: OECD Publishing.

Ofsted (2019): The education inspection framework. Manchester: Office for Standards in Education, Children's Services and Skills.

Oevermann, Ulrich (2008): Profession contra Organisation? Strukturtheoretische Perspektiven zum Verhältnis von Organisation und Profession in der Schule. In: Helsper, Werner/Busse, Susann/Hummrich, Merke/Kramer, Rolf-Thorsten (eds.): Pädagogische Professionalität in Organisationen. Neue Verhältnisbestimmungen am Beispiel Schule. Wiesbaden: VS Verlag für Sozialwissenschaften, pp. 55-77.

Peacock, Alison (2016): Assessment for Learning without Limits. London: Open University Press.

Peschel, Falko (2009). Offener Unterricht - Idee, Realität, Perspektive und ein praxiserprobtes Konzept zur Diskussion. Teil I: Allgemeindidaktische Überlegungen. Baltmannsweiler: Schneider Verlag Hohengehren. 
Plate, Elisabeth (2008): Betrachtungen “inklusiver" Schulentwicklungen in England aus einer internationalen Perspektive. In: Sonderpädagogische Förderung heute 53, 4, pp. 399-426.

Plate, Elisabeth (2012): Staff support for inclusion. An international study. Canterbury: Canterbury Christ Church University. http://create.canterbury.ac.uk/12105/ [Accessed: 06.06.2019].

Plate, Elisabeth (2016): Lehrer_innenbildung für Inklusion braucht Lehrer_innenbildung durch Inklusion. In: Dannenbeck, Clemens/Dorrance, Carmen/Moldenhauer, Anna/Oehme, Andreas/ Platte, Andrea (eds.): Inklusive Hochschule. Ansätze - Perspektiven - Kritik. Bad Heilbrunn: Klinkhardt, pp. 194-214.

Plate, Elisabeth (2017): Professionalisierung durch inklusive Schulentwicklung. In: Moser, Vera/Egger, Marina (eds.): Schulentwicklung und Inklusion. Konzepte, Instrumente und Befunde. Stuttgart: Kohlhammer, pp. 193-211.

Plate, Elisabeth (2018): Student research project on opportunities for teacher learning in schools in Berlin: Humboldt Universtät zu Berlin (unpublished).

Prengel, Annedore (2016): Bildungsteilhabe und Partizipation in Kindertageseinrichtungen. München: Deutsches Jugendinstitut e.V. \& Weiterbildungsinitiative Frühpädagogische Fachkräfte (WiFF). http://www.weiterbildungsinitiative.de/uploads/media/WiFF_Exp_47_Prengel_web. pdf [Accessed: 08.12.2016].

Rancière, Jacques (2007): Der unwissende Lehrmeister. Fünf Lektionen über die intellektuelle Emanzipation. Wien: Passagen Verlag.

Roberts, Carolyn (2019): Ethical Leadership for a Better Education System. London: Routledge.

Rogers, Alan (2014): The Base of the Iceberg. Informal Learning and Its Impact on Formal and Non-formal Learning. Toronto: Barbara Budrich Publishers.

Rogers, Everett M. (1995): Diffusion of Innovations. New York: The Free Press.

Rolff, Hans-Günter (2010). Schulentwicklung als Trias. In: Bohl, Thorsten/Helsper, Werner/Holtappels, Heinz Günther/Schelle, Carla (eds.): Handbuch Schulentwicklung. Bad Heilbrunn: Verlag Julius Klinkhardt. pp. 29-36.

Rosenholtz, Susan J. (1991): Teachers' Workplace. The Social Organization of Schools. New York: Teachers College Press.

Schimank, Uwe (2014): Governance und Professionalisierung. Notizen zu einem Desiderat. In: Maag Merki, Katharina/Langer, Roman/Altrichter, Herbert (eds.): Educational Governance als Forschungsperspektive. Strategien. Methoden. Ansätze. (2. edition). Wiesbaden: Springer Fachmedien, pp. 127-150.

Schoen, Donald A. (1983): The reflective practitioner. How professionals think in action. New York: Perseus Books.

Schratz, Michael/Schwarz, Johanna F./Westfall-Greiter, Tanja (2012): Lernen als (bildende) Erfahrung. Vignetten in der Praxisforschung. Innsbruck: Studienverlag.

Seitz, Simone (2006): Inklusive Didaktik. Die Frage nach dem "Kern der Sache". http://www.inklusion-online.net/index.php/inklusion-online/article/view/184/184 [Accessed: 25.05.2019].

Seitz, Simone (2010): Erziehung und Bildung. In: Kaiser, Astrid/Schmetz, Dieter/Wachtel, Peter/Werner, Birgit (eds.): Bildung und Erziehung. Enzyklopädisches Handbuch der Behindertenpädagogik vol. 3, pp. 43-58.

Sorenson, Nick (ed.) (2019): Diversity in Teacher Education: Perspectives on a school-led system. London: UCL Institute of Education Press.

Stephens, Paul/Tønnessen, Finn. E./Kyriacou, Chris (2004): Teacher training and teacher education in England and Norway: A comparative study of policy goals. In: Comparative Education 40, 1, pp. 109-130.

Swann, Mandy/Peacock, Alison/Hart, Susan/Drummond, Mary Jane (2012): Creating Learning without Limits. Maidenhead: Open University Press. 
Tacke, Veronika (2005): Schulreform als aktive Deprofessionalisierung? Zur Semantik der lernenden Organisation im Kontext Erziehung. In: Klatetzki, Thomas/Tacke, Veronika (eds.): Organisation und Profession. Wiesbaden: VS-Verlag, pp. 165-198.

TDA (2002): Qualifying to Teach - Professional Standards for Qualified Teacher Status and Requirements for Initial Teacher Training. London: Training and Development Agency for Schools.

TDA (Training and Development Agency for Schools) (2007): Professional Standards for Teachers - Why sit still in your career? London: TDA.

Terhart, Ewald (1990): Professionen in Organisationen. Institutionelle Bedingungen der Entwicklung von Professionswissen. In: Alisch, Lutz M./Baumert, Jürgen/Beck, Klaus (eds.): Professionswissen und Professionalisierung. Braunschweig: Technische Universität, pp. 151-170.

Terhart, Ewald (2000): Perspektiven der Lehrerbildung in Deutschland. Weinheim: Beltz Juventa.

Terhart, Ewald (2002): Standards für die Lehrerbildung - Eine Expertise für die Kultusministerkonferenz. Münster: Zentrale Koordination Lehrerausbildung.

Terhart, Ewald (2004). Lehrerkompetenzen für die Grundschule. Kontext, Entwicklung und Bedeutung. In: Grundschule 36, 6, pp. 10-12.

Terhart, Ewald (2011): Lehrerberuf und Professionalität. Gewandeltes Begriffsverständnis - neue Herausforderungen. In: Helsper, Werner/Tippelt, Rudolf (eds.): Pädagogische Professionalität, 57. Beiheft, Zeitschrift für Pädagogik. Weinheim: Beltz Juventa, pp. 202-224.

Waitoller, Frederico R./Artiles, Alfredo J. (2013): A Decade of Professional Development Research for Inclusive Education. A Critical Review and Notes for a Research Program. In: Review of Educational Research, 83, 3, pp. 319-356.

Weale, Sally (2018): 300 Schools picked out in GCSE “off-rolling” investigation. In: The Guardian, 26 June 2018. https://www.theguardian.com/education/2018/jun/26/300-schools-pickedout-in-gcse-off-rolling-investigation [Accessed: 15.07.2019].

Zeitler, Sigrid/Heller, Nina/Asbrand, Barbara (2012): Bildungsstandards in der Schule. Eine rekonstruktive Studie zur Implementation der Bildungsstandards. Münster: Waxmann. 



\title{
24 Multilingual Teaching Assistants in the UK: Translators in the Field of Inclusive Education
}

\author{
Bettina Fritzsche, Michalis Kakos
}

\begin{abstract}
In our contribution we introduce and discuss the practice of employing multilingual teaching assistants to support students who have English as a second language in English schools. Whereas in many European countries, newly arrived migrants are received in the school system by being assembled in reception classes, in England they are directly included in regular classes, yet are often provided additional support by (multilingual) teaching assistants and by attending EAL (English as an Additional Language) classes. Typically, multilingual teaching assistants are themselves first-generation or second-generation migrants. We explore their practices in supporting students with EAL, focusing on the task of translating on a lingual, but also on a cultural, level.

Based on literature review and the results from empirical studies, we explore the chances and challenges connected with the (para-)profession of multilingual teaching assistants as translators in inclusive education. In particular, we focus on multilingual teaching assistants' own perception of their tasks and analyse them with reference to theories on translation. Our objective is to understand the strengths of this practice and the ways that these could be transferred and inform the inclusive educational practices concerning migrant students in Germany and other countries. For this reason, we will consider the situation in Germany with regard to the support that is available to students who are not native speakers in German schools and the potentials of our findings to address its shortcomings.
\end{abstract}

\section{Introduction}

Following Ainscow's (2008: 241) concept of inclusion as overcoming discrimination and disablement, the ideal of an inclusive school is associated with a modification of those structures and practices that contribute to exclusion or marginalisation of certain students or groups of students or disable their learning processes (Sturm 2016: 13ff.). In many countries, one recognised cause of marginalisation within schools is the expectation towards students to speak and understand the respective dominant language. As Knappik and Thoma (2015: 9) argue, despite the fact that multilingualism is a characteristic of many modern societies, often their educational institutions are organised on the assumption that their members were monolingual. In relation to German schools, in 1994, Ingrid Gogolin (1994) identified the "monolingual Habitus".

Especially students who recently immigrated with their families are confronted with exclusive effects on the basis of language in educational institutions but also on their un- 
familiarity with the education system (Koehler et al. 2018). Niedrig (2015) points out that the ability to speak English or French of students coming from postcolonial African countries has been institutionally degraded in German schools. Since the intensified movements of flight and migration, European schools are more than ever confronted with the task to include students who are not able to speak the respective country's first language. Simultaneously, education is regarded as one of the cornerstones of the integration of migrants in the EU (see, e.g., European Commission 2011).

As in many other European countries - Austria, Germany, Finland, France, Italy, Sweden, Denmark, Greece, Hungary, the Netherlands and Poland (see Koehler et al. 2018: 12) in Germany the educational inclusion of students who have recently migrated with their families and do not speak German is organised via reception classes, which are usually called "Vorbereitungsklassen" (preparatory classes) or "Willkommensklassen" (welcome classes). Those reception classes have been restored since 2015 and students usually attend them for 1-2 years (Autorengruppe Bildungsberichterstattung 2018: 93). Those reception classes can be offered as classes, which exist in parallel to the regular classes but also as a (partially) integrative model, which offers students the possibility to at least partially attend regular classes during their time in reception classes (Decker-Ernst 2017: 2). In a report from 2016, Karakayalı and colleagues emphasize the marginalising effects of those classes, strengthening the critique about the potentially exclusive effects of this model of educational provision.

England, following the post-1990s tradition set by inclusive education policies and the arguments against most forms of separate educational provision, gave up on the concept of reception classes as a means of including new students who do not speak English. Those students are supported by attending special parallel programmes of study offered in "English as a second language (ESL)" or "English as an additional language (EAL)" departments that are organised by and hosted in the schools that the students attend. Another means of support to students in some English schools is the employment of multilingual or bilingual teaching assistants (MTAs) who offer in-class, one-to-one or group support. Until 2011 their employment was funded directly by the Minority Achievement Grant (Cable 2003) but since then the relevant funding has been absorbed into general school funding, arguably with mixed results (Hutchinson 2018: 16).

Here, we explore the chances and challenges connected with this model of support as identified by four MTAs whom we interviewed. From an international perspective on inclusion, the role of (para-)professionals ${ }^{1}$ is of special interest, as an international trend in favour of subsidiary support services in order to enable an education oriented towards inclusion (Fritzsche/Köpfer 2019, 2020). The rise of popularity of such roles in the field of education has led to an increase of research in this field (Chambers 2015; Sharma/Salend 2016). Whereas in England, as in some other European countries and in North America, (para-)professional assistance has been an integral element of school for a long time, in Germany the increased claim of subsidiary support services within schools is a relatively new phenomenon.

1 Pedagogical assistance roles are often characterized as "para-professional". As we argue in above, their pedagogical practice in certain aspects can be considered as professional practice and labels like "semiprofessional" and "para-professional" are rather problematic. For this reason, we use the notation " (para-)professional" here. 
As we will argue, the (para-)professional role of multilingual teaching assistants very much consists in providing translations, both lingual and cultural. ${ }^{2}$ For this reason, the theoretical framework we will relate to in our analysis consists of theories on professionalism and theories on translation. Emanating from those theories, we qualitatively analyse interviews with multilingual teaching assistants based on a sample of schools in North England.

We begin with a short overview on international research findings on teaching assistants' and especially multilingual teaching assistants' roles in inclusive education. Afterwards, we present our theoretical approach, our sample and the applied methods. After this, based on interview interpretations, we elaborate our assumptions on the chances and challenges connected with the employment of multilingual teaching assistants for the educational inclusion of non-native speakers in education. In our conclusions, we summarise main findings and relate them to the discussion in Germany on ways to include students who do not speak German as a first language.

\section{2. (Multilingual) Teaching Assistants and Their Roles in Inclusive Education: The International Research Discourse}

Generally, it can be stated, that the assignment of (para-)professionals has enormously increased with growing expectations towards schools to work inclusively (Sharma/Salend 2016). Terms for such roles include "teacher assistants", "teaching assistants", "educational assistants", "classroom assistants", "learning support assistants", "specialist teaching assistants", "learning supporters", or "integration aids". Looking at their working conditions in different states, strong variances can be stated in relation to their formal qualification. For example, in Germany no formal qualifications are necessary, whereas in the US a two-year training programme must be completed (see Butt/Lowe 2012; Butt 2016). In England, such training is available, but the employment of such personnel is not conditional on it. The lack of training and professional development opportunities of (para-)professionals in schools has been criticized in the international discussion (Ashbaker/Morgan 2012; Webster et al. 2013; Butt 2018) and especially in relation to the situation in Germany (Heinrich 2016). A shared characteristic of (para-)professionals in schools are their precarious working conditions and the phenomenon that far more women than men take on assistant roles (Mansaray 2012: 20; Henn et al. 2014).

Another important topic in international research is the widespread problem concerning the lack of clarity with regard to the roles and responsibilities that "(para-)professionals" have, as well as sometimes the fact that formal roles and expectations they meet during work diverge (Giangreco 2010; Egilson/Traustadottir 2009; Tucker 2009). In relation to the demands of inclusive education, the one-to-one support of certain students by (para-)professionals can isolate them from teachers as well as from their peers and thus have a negative impact on their learning and their inclusion in the classroom (Blatchford et al. 2009; Sharma/Salend 2016). This is despite the fact that TAs often occupy a central role in the implementation plans of schools' inclusion policies, especially in English schools (Tucker 2009).

2 We are relating to an understanding of translation as practice of transition between different languages and cultural contexts (Renn et al. 2002: 8; see section 2). 
A comparative study on (para-)professionals employed in schools in Canada, England and Germany notes their general lack of autonomy due to their role as assistants but also to formal regulations and instructions. This produced a permanent struggle for autonomy for the (para-)professionals involved in the study and it was shown that their relationship with the assigned students as well as their incremental development of professional skills was used as a means to increase autonomy and professional agency (Fritzsche/Köpfer 2019, 2020).

In Germany, (para-)professionals have been increasingly employed in schools over the last 20 years. Due to the federalist education system, the organisation of school assistance differs a lot in relation to state-specific rules. In German research, the fundamental lack of clarity in relation to teaching assistances' job profiles has been criticised. In most federal states, their job descriptions refer to practical, physical and psychosocial care. Simultaneously, it is well known that they often take on pedagogical and instructional tasks, even if they are not officially authorised to do so (Dworschak 2012; Kremer 2012). An ethnographic study stated that teacher assistants take on variable positions and functions in classroom instruction and often serve as a link between different groups (Blasse 2017).

In comparison to Germany, in England the assignment of teaching assistants in schools has a much longer tradition. It goes back to the 1980s and has steadily increased ever since, so that nowadays they constitute about one quarter of all professionals employed in schools (Webster et al. 2013). Their main task is to support teachers and they are not subject to comparably restrictive job descriptions as are their colleagues in Germany. Some teaching assistants carry out more general roles, while others assume specific responsibilities for a child, subject area or age group(s) (Tyrer et al. 2009: 9).

Since 2011 EMA was integrated into the general schools funding (Education Services Grant). There is a long tradition of employment of bilingual/multilingual teaching assistants in English schools. Since mid-1980s and the introduction of the "bilingual support" policy, which aimed to support bilingual learners from minority ethnic groups, bilingual teaching assistants were routinely employed by schools in multilingual urban areas of England and they were often "appointed to low-status posts, with ill-defined job descriptions" (MartinJones/Saxena 1996: 106). Currently, the terms of their employment are usually not different to other teaching or classroom assistants and the model of their work follows similar patterns: they usually offer classroom based support while in some schools (usually secondary schools) they support groups of students with EAL away from the classroom (withdrawal from classroom support) (Slater/Gazeley 2019). Such support is usually coordinated and delivered in the EAL departments. However, as discussed below, these changes in their employment seem not to have brought significant changes in their status.

\section{Theoretical Framework: Profession Theory and Theories on Translation}

As it remains rather contested whether teaching itself is a profession or only a "semi-profession" (Etzioni 1969) or if it is in need of being professionalised (Dewey 1988), at first glance it seems somewhat far-fetched to think about professionalism in relation to teaching assistants. Evidently (Tucker 2009; Dávila 2018), many of the most commonly cited criteria of a profession, such as theoretical and practical expertise and especially a high degree of 
individual autonomy (Carr 2000), do not seem to apply to their occupation. But in some approaches in professionalism theory, other criteria are discussed that very well apply to teaching assistants, for example, the assumption that professionals deal with the unique problems of individual clients and or take on responsibility for existential risks (Evetts 2003; Vanderstaeten 2007: 623f.).

Therefore, in our reflections on teaching assistants' professionalism, we take this type of close relationship to potentially vulnerable clients as a starting point to discuss their professionalism. In professionalism theory, Ulrich Oevermann (1996: 146) in particular emphasises explicitly the therapeutic dimension of pedagogical work and argues that pedagogical actors are inescapably entangled in diffuse interactional practices critical for the further development of students' personal integrity (Oevermann 1996: 110). From Oevermann's point of view, the lack of professionalisation of teachers' pedagogical practices is made particularly clear by the fact that they are unable to resolve the contradictions between diffusion and specificity in their relationships to students and either lose all distance and infantilise students or take on the administrative role of an expert. On the contrary, in the field of special education, a responsibility for a wide range of case-specific disorders makes it necessary to enter into case-specific working alliances. Therefore, in this field Oevermann sees clear trends towards the professionalisation of pedagogical practice. From this point of view, school assistants can be seen as professionalised, notwithstanding their frequent lack of formal qualifications.

Similar to Oevermann, Ursula Rabe-Kleberg (1996) criticises theories that describe professionalism on the basis of the above-mentioned characteristics. Rabe-Kleberg makes clear that the labelling of certain occupations as semi-professional (Etzioni 1969) discriminates especially precarious service occupations, often carried out by women. In her own understanding of professionalism, it is rather "the ability and willingness to not only withstand but also actively balance the contradictory structures of professional work" (Rabe-Kleberg 1996: 298, own translation).

Thus, following Oevermann and Rabe-Kleberg, we are especially interested in looking at teaching assistants' own strategies in dealing with the uncertainties they are exposed to through their (para-)professional occupation and in their negotiations of the working-alliances they build with students.

As shortly mentioned above, an important characteristic of teaching assistants' professional practice is the fact that they often serve as a link between different actors in school (see also Blasse 2017): They negotiate not only between the students, they have been assigned to and the teachers, but also between the students and other students and sometimes between the students' parents and the schools (Tucker 2009: 294). From our point of view, those negotiations can be regarded as translations. In the case of multilingual teaching assistants, their translations include and simultaneously go beyond the pure transfer of a certain language's meanings into another's. Next to the mentioned theories on professionalism, theories on cultural translation are also an important for our analysis.

Since the 1980s the notion of translation has become increasingly freed from its limitation on a textual dimension and employed as a means to analyse cultural life worlds (Bachmann-Medick 2006: 239). The term "translation" in this discussion is not any more reduced to a transfer of meanings on a lingual (written or oral) level, but rather as technique of dealing with cultural differences. Our analyses will be based on an understanding of translation developed in cultural theory that describes translation as a "practice of transition between different languages and cultural contexts, in the course of which 'both sides' get into motion" 
(Renn et al. 2002: 8). This means that translation is understood as a daily practice performed in a social context, which involves any sort of negotiating differences in situations of confrontation with other, potentially foreign, languages and cultures.

In this sense, translation also means to put oneself into another, formerly external, maybe foreign position, which may lead to a transformation of one's own perspective (Mannheim 1931/1995: 258f.).

Those reflections by Mannheim hint at the close connection between the operations of translating and comparing, a topic also discussed by Ilja Srubar (2002). Srubar argues, that acts of translation always are also acts of comparison. Translating always means to try to connect to a different potentially strange meaning system and it goes along with the comparing selection of elements of meanings. Following Srubar, a translation is a negotiation between different meaning systems and can never be at home in one of those systems (Srubar 2002: 327). This also means, translations necessarily go along with an uncertainty, which can never be completely dissolved (Srubar 202: 329).

Relating to those ideas on translation, our analysis is based on the following assumptions:

1. MTAs often serve as a link between different actors in school (Blasse 2017), which means that acts of cultural translation are part of their professionalism.

2. As we understand translation as a "practice of transition between different languages and cultural contexts, in the course of which 'both sides' get into motion" (Renn et al. 2002: 8 ), we are especially interested in the range of practices of transition characterising MTAs daily work and in their results: do they have effects on their respective cultural contexts?

3. Understanding translation also as an act of comparison between different meaning systems we are also interested in the potential of such ongoing comparisons for MTAs professionalism.

\section{Sampling, Data and Methods}

As a follow-up study of our research in the field of para-professional assistants in schools (Fritzsche 2014; Fritzsche/Köpfer 2019, 2020) in 2018, we conducted five semi-structured interviews with multilingual teaching assistants, working at four different inner-city schools in North England. Two of these schools are primary schools, and the rest comprehensive secondary schools. All schools have a higher than average percentage of students with EAL. The secondary schools have special EAL provision, delivered by a small number of staff (12 teachers and the MTAs), coordinated by an assigned member of staff. The two primary schools do not have specialised provision for students with EAL and any matters related to support for these students is overseen by senior members of staff: The Head Teacher in one school and the Inclusion Coordinator in the other.

The interviews were interpreted inspired by the documentary method (Bohnsack 2003; Nohl 2006). This qualitative method of interpretation is based on Karl Mannheim's sociology of knowledge and is focused on a reconstruction of patterns of orientation and implicit knowledge. ${ }^{3}$ Thus, it was especially useful in order to interpret recurring patterns of teaching

3 In relation to this methodological approach, see also Sturm's chapter in this volume (2021). 
assistants' perceptions of their own professionalism as well as an implicit professional knowledge.

\section{Multilingual Teaching Assistant as a Translating Profession: Interpretations}

In the following, we characterise the central aspects of multilingual teaching assistants' professionalism that we were able to analyse on the basis of our interpretations. Our main arguments are that (1) multilingual teaching assistants' professionalism was very much based on the utilisation of personal resources for professional requirements, and (2) that their professional practice is characterised by translations, firstly of school topics towards the students they are responsible for and secondly of an expertise attained through the role as assistant towards the school.

\subsection{Personal Experience as Educational Resources: Empathy, Care and Trust}

Not only multilingual teaching assistants, but also other teaching assistants, who were interviewed in earlier studies, emphasised the usefulness of personal experiences and resources for the development of a professional attitude. For example, an assistant who works in a school in Berlin made clear in an interview conducted in another study (Fritzsche 2017), that in her work she also relies on her personal experiences as a person with a disability.

When interacting and working with students, due to the lack of professional training, the MTAs personal experiences and the skills they have acquired in other settings are the only available source to tap into. Similar to the German TA above, for some MTAs it is often the similarity of their personal experiences to those of the students that allows them to fulfil their professional role as supporters of students' inclusion, acting at the same time as role model (Dávila 2018: 965). Dora, the bilingual teaching assistant in Lucero's study, was able to use her (marginal) position constructively, partially because of her ability to draw "on her social and professional networks as well as her personal experience as an immigrant and a learner of English as a second language to meet the goals of the biliteracy program" (Lucero 2010: 137) that she has developed.

The significance of personal experiences when supporting students and in order to relate to them was highlighted by all MTAs whom we interviewed.

Rabia, an MTA from Iran, who works in secondary school, is able to guide newly arrived students in England in choosing subjects for their General Certificate of Secondary Education (GCSE) exams, the English qualification that marks the completion of compulsory education. In doing so, Rabia draws from her experience supporting her own children who had to go through the same system: 
Rabia: I know from my kids and [I explain to the students that] if you want to [go to] university you should choose as a minimum four or five [subjects] and [..] if you want [to study] medicine you should get good marks in chemistry and in biology and in maths as well, and English.

Eleni, the Czech and Greek native speaker who was born in Czech Republic, moved to Greece in the early years of her primary education. Currently residing in England, she explains how her personal experiences allow her to help students overcome language barriers:

Eleni: In terms of progression and learning I definitely relate to them because when I was a child and I arrived to Greece my Greek was minimum .... and actually it [...] wasn't proper Greek, it was what I had learned from my grandparents $[. .$.$] . So I [\ldots]$ know that it is hard and especially when you see children with potential and initiative and you can see that language barrier there it's just heart-breaking, you just you think we need we need to do something about this, because they can, they are clever, you know if you give them a mathematical thing or something they just do it straight away, or if you give them a problem solving [exercise], they will just finish it. It's the literacy, the language, the writing, the reading that they can't do so definitely [...] I definitely relate to them and I just want to help them. [...]. So [I] indirectly check on some things that we think they do know [...]. I casually play with them and find out how far they are [in language learning] $[\ldots]$. And then work on strategies with the teachers on how [to] support them.

Eleni's ability to support her students is evidently not based on any particular professional skill but in her ability to empathise with them and to comprehend the scale of the task that they have to fulfil. Most importantly, she knows also that the difficulty of the task lies also in its complexity and that the language barrier is only one of its dimensions:

Eleni: [When] we arrived to Greece we were the Czech people. And [it] was [...] so obvious and there was a lot of ... there was a lot of bullying at school. Yeah ... Because you know even from the outfit and the different habits.

On the basis of her experience, she describes the objectives and methods of the support that she aspires to offer from her position:

Eleni: We can actually make that child feel comfortable ... [...] I will make sure that all the peers will know the child's name when they arrive. I will make sure that I have a buddy ready for that child so when they arrive, this is going to be your buddy for the first few days ... and you can do things together, they will show you around ... and if you don't understand anything you can ask. So things like that, $[\ldots]$ or they can be asked to bring their favourite thing to school or go through a ... process when they are part of a small group, just to see how we do lunchtimes ... or just having a walk in the school.... So those first weeks to be kind of ... like a welcoming environment and attitude towards that child.

\subsection{Expertise, Autonomy and MTAs Professional Status}

The MTAs' job, as it has been described by our interviewees, requires the utilisation of a set of skills and the handling of resources developed outside any special training that the MTAs might have received. The performance of their role, on the other hand, aims to offer support to students with EAL in their inclusion in the new educational setting, which is described by the MTAs as a process of transition. In order to do so and as it appears from their descriptions, they themselves participate in it, not merely conducting (written or oral) linguistic translations whenever this is necessary. They also engage in a process of understanding the students' 
backgrounds, evaluate and map their understandings, empathise and oversee their difficulties, and, using their own experiences, consider the process of settling in the new environment more holistically than what would normally be expected from a linguistic translator.

Therefore, their job profile and their position in the school are rather unique. Rooted in their life experiences, the set of skills that are put into motion for the performance of their roles are such that their incorporation into the objectives of professional training programmes would be a challenge, especially for courses that are comfortable with the performative culture of modern schooling. Such culture which operates within a logic of dissociation of the personal from the professional that leads to a values schizophrenia (Ball 2003: 223) seems to be inherently incompatible to personalised interaction and to the mobilisation of personal experiences as teaching resources (Kakos 2017). It is exactly the ability to bring these personal experiences into the classroom that place MTAs in an exceptional position and allows them to perform their roles. As Lucero points out, "many [para-educators] (although by no means all) [...] have experienced immigration and second-language learning themselves, and, thus, may have the ability to connect with families and children in ways that teachers do not" (Lucero 2010: 134; see also Rueda et al. 2004).

However, and despite our interviewees stating in one way or another that their contribution to the inclusion of the students with EAL is recognised by colleagues, this recognition does not always reflect their status in the school. Similarly to the participants in Dávila's study who "described their work as simultaneously valued and marginalized by lead teachers within the classroom" (Dávila 2018: 961), the five MTAs in this study seem to share feelings of exclusion from what is being considered (by some of their colleagues) as the central function of the school:

Eleni: Now there are individuals so I am not generalizing here, there are individuals that think ... that at the end of the day you are teaching assistant ... and that's fine, that's in every job.

Svetlana, a trained biologist who works in the primary school that her son attends, states that she enjoys the recognition by her colleagues of the value of her work. She offers one-to-one classroom support to Key Stage 1 students (4-6 years old) with EAL, but she works also with small groups from the Nursery and Key Stage one outside the classroom. She has the confidence to develop rather complex programmes of study for these groups and she has a very good understanding of individuals' needs. However, she is hesitant in approaching teachers directly and advising them on the strategies to teach these students and she is always waiting for them to instigate such discussions:

Svetlana: I think we've got really high qualified teachers and they know ... well they've got lots of strategies how to teach our EAL children, how to write, how to read [...]. What I do is it's very basic and it's ... it's just help for children who are completely new to English [...] and then my role stops actually to be honest.

Rabia, explaining the good working relationships that she has with teachers in the school, takes on most of the responsibility for the quality of this collaboration. She offers a description that echoes the "turn-taking" processes described by Martin-Jones and Saxena (1996):

Rabia: [I have a good relationship] Because I obey the rule and I respect the teacher [...]. I should not interrupt her. Because I obey the rule, I think the teacher don't have any problem with me.

Evidently, the picture of their position that the MTAs draw is somewhat in contrast to the significance and complexity of their role. In their interviews, Eleni and Rabia described initiatives and future plans for the development of resources for teachers and of small-scale, 
school-based staff training. However, and despite the understanding of the uniqueness of their role that these plans signify, they seem to feel that their role is being considered (and they consider themselves it as being) peripheral to the main educational activities. Their role is a supportive one to the actual teaching taking place in the classroom and conducted by the teacher. In that respect, the picture that they draw is not different to that described by ErnstSlavit and Wenger as a (significant) bridge between the students and the school (2006: 77).

As Lucero (2010) has shown, operating in this marginal space has its advantages: MTAs seem to enjoy an autonomy perhaps not purposefully built into their roles at the design stage but often getting relevant later, as the schools recognise the significance of their contribution and the uniqueness of the MTAs skills set.

However, this is not to say that the MTAs enjoy an advantageous position. Three out of five of the MTAs that we interviewed have completed university degrees in their countries of origin. They do not see themselves remaining in the MTA posts for much longer, much as they enjoy working with children. As they report, the main reasons for this are the limited opportunities for progression and the low pay, while the main advantages that keep them in their posts have little to do with the status of their job and are mostly related to the working hours and the flexibility of their employment. If the schools value the contribution of the MTAs and if their skills are so unique, we think that efforts should be made for the MTAs' roles to become more attractive to those who are in position to perform them - efforts not only on the level of single schools, but also in the realm of policy and in relation to the way their role is politically designed.

\subsection{Translating Languages, Curricula and Systems}

The knowledge of an additional language has been the first (and for some it appears to remain the main) reason for the employment of all the MTAs whom we interviewed. Three of the five MTAs have responded to Teaching Assistant's job advertisements that required the knowledge of particular languages: Punjabi and Urdu in one case, Arabic in the second and third. The fourth MTA, a Polish native speaker who immigrated in the UK in 2002, was offered a temporary post in her son's primary school when her son's teacher recognised her skills in supporting him in building his confidence in English. The fifth MTA whom we interviewed was initially employed as a TA working part-time but her knowledge of additional languages earned her a permanent position, which was largely created by herself as she was looking for full-time employment:

Eleni: Then last year it was just that we had that discussion [with the Head Teacher] that if I can't have my $[\mathrm{f} / \mathrm{t}]$ hours, then, although I love this school and I love our head ... and I didn't want to go because I feel- it ticks a lot of boxes for me ... I thought ... if I need to work full time ... I need to find something that pays better. So we had that discussion with the head and she says: I don't want to lose you, how can we make it work? So I said, well I've had these thoughts about I would like to run interventions to support the children [with EAL] with their speech and language. And she was like: Let's do it!

Eleni is a trilingual speaker. English is an additional language for Eleni who is a native speaker of Czech and Greek. Although there is only one student in the school who speaks one of these two languages (Czech), her language skills seem to be of value for more children, 
especially the large number of Polish students in the school, since she is able to understand the process of translation and the peculiarities of particular languages:

Eleni: You know, when I work with the Czech child or Polish child (.) it makes sense that they don't use determiners, because they don't use them in their own language so I'm just thinking that in the sentence when I teach them the sentence, I need to tell them that we use the determiners in English language. [...] And that's why I'm thinking that ... we would need to have like a plan for the teachers, just for few languages to know: Oh, I have a Czech child. That child uses that grammar, so I should actually train that child to use a different grammar.

Many teaching assistants, in Germany as well as in England, told us about the special didactic material they collected or produced for the students they were responsible for, in order to enable them to follow the curriculum. In the case of multilingual teaching assistants such material is interconnected with translations on the level of language. But, in the interviews conducted with them, it became very clear that their work cannot be characterised as a simple transfer of meaning from the English language into another language. Instead, a new language is created, which sometimes takes on meanings from both languages, but also produces new meanings. Rabia explains:

Rabia: [Then they ask:] What's the mean of condensation? What [does this mean? [...]And I find a pictures ... pictures for this [...]. And I make a lot of vocabulary as an imagines. [...] And I prepared this for the new-to-English students because the image express a lot of meaning. And I make a lot of vocabulary as images. Yes and I prepared this for the New-to-English students because the image express a lot of meaning. And for the maths also I made a maths model which means 'What's the meaning of?' Sometimes you can use lot of terms in maths but you don't know what they mean.

Similar to Rabia, Eleni is using colours to help newly arrived in England primary school students to formulate sentences in English:

Eleni: Trying to help them formulate sentences [...] we use many visuals and we use colourful semantics. [...] So basically, we link words to colours and so if a sentence needs to have a 'who', is orange, a "doing what", is yellow, a "what" is green, blue is "where".

The translation that MTAs are producing is evidently not of language but goes well beyond it. It is a process of interpretation of situations and, most importantly, a kind of support of students' transition process from one language to another, from one curriculum to another, even from one educational system to another. Sabi, who came from Iraq more than 10 years ago and is currently working as MTA in a secondary school, has described how she is able to combine her language skills, her knowledge of the English educational system as well as that of the system in Iraq in order to offer guidance to her students:

Sabi: You see, I know [what] they do not understand [about the system] and when they say 'I don't understand', I can ask them about their systems [the systems in the countries that they come from] and you know, many are similar to Iraq, I mean in countries in Middle East at least, and then I can explain to them the differences [from the systems in their own country] and the English name of everything and then it becomes much easier for them. For a while we discuss like this [...] explaining the equivalent of everything to system at [their] home [country].

Sabi is not alone in taking up the role of the translator of educational systems. In Dávila's study, a teacher is able to compare schooling in Somalia with that in Sweden to help students understand the system in their new country (Dávila 2018: 962). 
Evidently, such translations are not instant: Sabi continues to translate each element of the educational system that students encounter for the first time until they build their confidence in their knowledge of it. During this process, students may need to explain the system in their home countries to Sabi who assesses the compatibility of the two systems before explaining to them this new element. Importantly, Sabi will need to compare the system of the students' home country to the Iraqi system, conducting a translation within the translation. Her cultural translations are based on a comparison of three meaning systems and probably go along with many uncertainties. But it allows students to take an active part in the cultural translation and to act as experts for their own experiences. And it may also mean, that schools' routines (not only in the field of inclusion) are irritated and confronted with new meanings.

Cultural translation for MTAs includes simultaneous interpretation, written translation, the comparison of different meaning systems, but also the creation of new symbols and didactic ideas and it may also go along with transforming schools' former (implicit) rules (see also: Lucero 2010). Moreover, the resources and skills that MTAs refer to in their interviews are not those acquired in any professional training for their role (which is often organically developed and partially for this reason often unclear) but those that relate to their own experiences and the cultural capital that they had access to prior to their employment in the schools. From the position that this capital allows them to construct they are able not only to perform but also to define their role and to offer guidance to teachers, as experts in a classroom process that teachers do not (and possibly cannot) master or own. This is perhaps a space for MTAs to exercise professional autonomy emerging organically and interactively.

\section{Conclusions}

The situation with regard to the role of MTAs as described above seems to echo some of the findings from other studies, especially with regard to the position of para-professionals and the in-between space within which they operate. Discussing the findings from their threeyear ethnographic study in the US, Ernst-Slavit and Wenger report that "in spite of their indepth knowledge of the students' languages and cultures and their pivotal role in educating language minority students, these educators held marginal positions that were complex, multifaceted, and fragmented" (2006: 62). The five MTAs whom we interviewed seem to verify this. They also describe the processes and tasks that they undertake from within these marginal positions. Central in these tasks is that of comparison and cultural translation, which, however, is not only linguistic and it is not only an interpretation from one language to another. Their narratives seem to describe a constant transition from one language and culture to another, which, as it takes place in this marginal space, becomes a new language and the culture of this space. As Lucero (2010) has pointed out, if the conditions are right, then the marginality of these spaces can become their strength. The translation as a culture of transition that they create goes along with uncertainties, but does not seem to be one of constant and unsettling motion but one of a safe passage the pace of which is to a large extent controlled by the MTAs and the students themselves.

We think that the skills to facilitate and participate in the construction of this transition culture and the control of its pace is key in explaining the MTAs' advantaged position as EAL educators and a demonstration of their "day in and day out - a deep understanding of 
what it takes to be a language and culture learner" (Ernst-Slavit/Wenger 2006: 78). Also, it can be stated that, in relation to Oevermann's theory on professionalism, teaching assistants' professionalism consists in the ability to translate general requirements in ways that they can be case-specifically understood. This can also have as a consequence that it is not only the positions of MTAs and students that get into move, but also in some aspects, schools' normal processes (e.g. in the grammar lesson) are challenged and undergo changes. Moreover, the cultural capital to which they have access and their skills to utilise it secures them an exclusive position among their colleagues. From this position they can define their role while offering guidance to teachers, as experts in a classroom process that teachers do not (and possibly cannot) master or own. This is perhaps a space for MTAs to exercise professional autonomy emerging organically and interactively.

Our results from England, we argue, enable the following conclusions to be drawn on the support that is available to students, who are not native speakers, in schools in Germany: The support of migrant students by personal responsible MTAs in England compared to the reception class system bares the advantage that the targeted students are not excluded within schools as organisations and enabled to participate in regular lessons. Also, the fact that their support is organised by the EAL departments means that it is constructed as support on the basis of lingual, sometimes cultural translation, which helps to avoid a perspective on migrant students as being generally in deficit. As our interpretation shows, the processes of translations initiated by MTAs go along with a valorisation of their own and the students' native language(s), e.g., when the MTA explains to the teacher, how she/he should explain English grammar to students, who are used to another language. This sort of translation, from our point of view, may challenge the mentioned "monolingual Habitus" diagnosed in relation to the German school system by Gogolin (1994) - and replace it by a culture of translation.

Of course, by looking at the work of (para-)professionals in schools, the above-mentioned problems stated in research have to be considered: Also, the close work with TA's can lead to an isolation of the targeted students from other students and from teachers. It seems to be a general characteristic of inclusion, that the (para-)professionals who are employed to support students with the precarious status are assigned a precarious status themselves. When the working alliance between those two groups leads to a marginalisation within schools, the aim of inclusion is contradicted. A central aim of policies in this sense should be the upgrading of (para-)professionals employed in inclusive settings on the basis of increased salaries and a higher and more profiled status within schools. Also, it is important, that this working alliance is continuously connected with alliances to other students and professionals within schools. Such a connection may also be helpful in avoiding, that the translation provided by MTAs risks to be patronising in the way that they consist in a "speaking about" instead of a "speaking for" students. Our results also show that the lack of a formal qualification and clear instructions in the case of MTAs can lead to a rather creative use of personal resources that can have very productive results. But this is only possible in cases in which the responsible managers in the schools are able to appreciate their unique position, skills and expertise. MTAs' existing creativity in using personal resources and in processing translations should be used as a starting point for professional development in this field. 


\section{References}

Ainscow, Mel (2008): Teaching for Diversity. The Next Big Challange. In: Connelly, Michael F./ He, Ming Fang/Phillion, JoAnn (eds.): The Sage Handbook of Curriculum and Instruction. Los Angeles: SAGE Publications, pp. 240-258.

Ashbaker, Betty J./Morgan, Jill (2012): Team Players and Team Managers. Special Educators Working with Paraeducators to Support Inclusive Classrooms. In: Creative Education 3, 3, pp. 322-327.

Autorengruppe Bildungsberichterstattung (2018): Bildung in Deutschland 2018. Ein indikatorengestützter Bericht mit einer Analyse zu Wirkungen und Erträgen von Bildung. https://www.bildungsbericht.de/de/bildungsberichte-seit-2006/bildungsbericht-2018/pdf-bildungsbericht2018/bildungsbericht-2018.pdf [Accessed: 24.01.2020].

Bachmann-Medick, Doris (2006): Cultural Turns. Neuorientierungen in den Kulturwissenschaften. Reinbek bei Hamburg: Rohwolt.

Ball, Stephen J. (2003): The teacher's soul and the terrors of performativity. In: Journal of Education Policy 18, 2, pp. 215-228.

Blasse, Nina (2017): Vielfältige Positionen von Schulbegleitung im Unterricht. In: Laubner, Marian/Lindmeier, Bettina/Lübeck, Anika (eds.): Schulbegleitung in der inklusiven Schule. Weinheim: Beltz Verlag, pp. 107-117.

Blatchford, Peter/Bassett, Paul/Brown, Penelope/Koutsoubou, Maria/Martin, Clare/Russell, Anthony/Webster, Rob/Rubie Davies, C. (2009): The Impact of Support Staff in Schools. Results from the Deployment and Impact of Support Staff (DISS) project. London: DCSF.

Bohnsack, Ralf (2003): Rekonstruktive Sozialforschung. Opladen: Budrich.

Butt, Rosemary (2016): Teacher assistant support and deployment in mainstreaming schools. In: International Journal of Inclusive Education 20, 9, pp. 995-1007.

Butt, Rosemary (2018): "Pulled in off the street" and available. What qualifications and training do Teacher Assistants really need? In: International Journal of Inclusive Education 22, 3, pp. 217-234.

Butt, Rosemary/Lowe, Kaye (2012): Teaching assistants and class teachers. Differing perceptions, role confusion and the benefits of skills-based training. In: International Journal of Inclusive Education 16, 2, pp. 207-219.

Cable, Carrie (2003): Bilingual teaching assistants. Their contribution to learning Paper presented at the British Educational Research Association Annual Conference, Heriot-Watt University, Edinburgh, 11-13 September 2003. http://www.leeds.ac.uk/educol/documents/00003209.htm [Accessed: 22.3.2019].

Carr, David (2000): Professionalism and Ethics in Teaching. London: Taylor \& Francis Books Ltd.

Chambers, Dianne (2015): The Changing Nature of the Roles of Support Staff. In: Chambers, Dianne (ed.): Working with Teaching Assistants and Other Support Staff for Inclusive Education. International Perspectives on Inclusive Education, Volume (4). UK: Emerald Group Publishing Limited, pp. 3-25.

Dávila, Liv Thorstensson (2018): The pivotal and peripheral roles of bilingual classroom assistants at a Swedish elementary school. In: International Journal of Bilingual Education and Bilingualism 21, 8, pp. 956-967.

Decker-Ernst, Yvonne (2017): Vorbereitungsklassen. Wortschatzarbeit mit neu eingewanderten Schüler/innen. In: unterrichtspraxis. Beilage zu bildung \& wissenschaft 50, 7, pp. 1-8.

Dewey, John (1988): Culture and professionalism in education. In: Dewey, John (ed.): The middle works, 1899-1924. Carbondale/Edwardsville, IL: Southern Illinois University Press. 
Dworschak, Wolfgang (2012): Assistenz in der Schule. Pädagogische Reflexionen zur Schulbegleitung im Spannungsfeld von Schulrecht und Eingliederungshilfe. In: Lernen konkret 31, 4, pp. 2-7.

Egilson, Snaefridur/Traustadottir, Rannveig (2009): Assistance to students with physical disabilities in regular schools. Providing inclusion or creating dependency. In: European Journal of Special Needs Education 24, 1, pp. 21-36.

Ernst-Slavit, Gisella/Wenger, Kerri J. (2006): Teaching in the Margins. The Multifaceted Work and Struggles of Bilingual Paraeducators. In: Anthropology \& Education Quarterly 37, 1, pp. 62-81.

Etzioni, Amitai (ed.) (1969): The Semi-Professions and their Organisation. New York: Free Press.

European Commission (2011): European agenda for the integration of third-country nationals. Communication from the Commission to the Council, the European Parliament, the European Economic and Social Committee and the Committee of the Regions. http://aei.pitt.edu/38019/ [Accessed: 22.3.2019].

Evetts, Julia (2003): The sociological analysis of professionalism. Occupational change in the modern world. In: International Sociology 18, 2, pp. 395-415.

Fritzsche, Bettina (2014): Inklusion als Exklusion. Differenzproduktionen im Rahmen des schulischen Anerkennungsgeschehens. In: Tervooren, Anja/Engel, Nicolas/Göhlich, Michael/Miethe, Ingrid/Reh, Sabine (eds.): Ethnographie und Differenz in pädagogischen Feldern. Internationale Entwicklungen erziehungswissenschaftlicher Forschung. Bielefeld: transcript, pp. 329-345.

Fritzsche, Bettina (2017): Anerkennungsverhältnisse an deutschen und englischen Grundschulen. Bericht zu einem binational-vergleichenden ethnographischen Projekt. In: Burger, Timo/Micelli, Nicole (eds.): Empirische Forschung im Kontext Schule. Einführung in theoretische Aspekte und methodische Zugänge. Wiesbaden: Springer VS, pp. 343-355.

Fritzsche, Bettina/Köpfer, Andreas (2019): (Para-)Professionalität im Umgang mit Ungewissheitsstrukturen. Eine kulturvergleichende Rekonstruktion von Interviews mit Assistenzkräften im inklusionsorientierten Unterricht. In: Donlic, Jasmin/Jaksche-Hoffman, Elisabeth/Peterlini, Hans Karl (eds.): Ist inklusive Schule möglich? Nationale und internationale Perspektiven, pp. 133-160.

Fritzsche, Bettina/Köpfer, Andreas (2020): (Para-)professionalism in dealing with structures of uncertainty. A culture comparative reconstruction of interviews with teaching assistants in inclusion-oriented classrooms. Disability \& Society: in press.

Giangreco, Michael F. (2010): One-to-One Paraprofessionals for Students with Disabilities in Inclusive Classrooms. Is Conventional Wisdom Wrong? In: Intellectual and Developmental Disabilities 48, 1, pp. 1-13.

Gogolin, Ingrid (1994): Der monolinguale Habitus der multilingualen Schule. Münster: Waxmann.

Heinrich, Martin (2016): Professionalisierbarkeit von Schulbegleitung? In: Heinrich, Martin/Lübeck, Anika (eds.): Schulbegleitung im Professionalisierungsdilemma. Rekonstruktionen zur inklusiven Beschulung. Münster: MV-Wissenschaft, pp. 5-31.

Henn, Katharina/Thurn, Leonore/Besier, Tanja/Künster, Anne K./Fegert, Jörg M./Ziegenhain, Ute (2014): Schulbegleiter als Unterstützung von Inklusion im Schulwesen. In: Zeitschrift für Kinder- und Jugendpsychiatrie und Psychotherapie 42, 6, pp. 397-403.

Hutchinson, Jo (2018): Educational Outcomes of Children with English as an Additonal Language. London: Education Policy Institute and Bell Foundation.

Kakos, Michalis (2017): The interaction between students and teachers in times of performativity. In: Borgnakke, Karen/Dovemark, Marianne/Silva, Sofia Marques da (eds.): The construction of the post-modern professional. Stoke-on-Trent: Trentham, pp. 53-72. 
Karakayalı, Juliane/zur Nieden, Birgit/Kahveci, Çağrı/Groß, Sophie/Heller, Mareike/Güleryüz, Tutku (2016): Mit Segregation zur Inklusion? Eine Expertise für den Mediendienst Integration. https://mediendienst-integration.de/fileadmin/Dateien/Expertise_Willkommensklassen.pdf [Accessed: 22.3.2019].

Knappik, Magdalena/Thoma, Nadja (eds.) (2015): Sprache und Bildung in Migrationsgesellschaften. Machtkritische Perspektiven auf ein prekarisiertes Verhältnis. Bielefeld: transcript.

Koehler, Claudia/Kakos, Michalis/Sharma-Brymer, Vinathe/Schneider, Jens/Tudjman, Tomislav/Van den Heerik, Alexandra/Ravn, Stiene/Lippens, Michiel/Nouwen, Ward/Belloni, Milena/Clycq, Noel/Timmerman, Christiane/Denkelaar, Monique/Palaiologou, Nektaria/Toumpoulidis, Giorgos (2018): Multi-country partnership to enhance the education of refugee and asylum-seeking youth in Europe. PERAE (Comparative Report). https://www.researchgate.net/publication/ 326113676_SIRIUS-Policy_Network_on_Migrant_Education_MULTICOUNTRY_PARTNERSHIP_TO_ENHANCE_THE_EDUCATTION_OF_REFUGEE_AND ASYLUM-SEEKING_YOUTH_IN____EUROPEPERAE_Comparative_Report. [Accessed: 04.09.2019].

Kremer, Gabriele (2012): Wer passt denn heute auf mich auf? Chancen und Probleme des Einsatzes von Integrationshelfern in der Schule. In: Systhema 26, 2, pp. 152-161.

Lucero, Audrey (2010): Dora's Program. The Role of a Constructively Marginalized Paraprofessional and her Developmental Biliteracy Program. In: Anthropology and Education Quarterly 41, 2, pp. 126-143.

Mannheim, Karl (1931/1995): Ideologie und Utopie. Frankfurt am Main: Vittorio Klostermann.

Mansaray, Ayodele Abdul (2012): The roles and positions of teaching assistants in two urban primary schools. An ethnographic study of educational work and urban social change. Doctoral thesis, Institute of Education, University of London. http://discovery.ucl.ac.uk/10020720/ 1/554335.pdf [Accessed: 22.02.2018].

Martin-Jones, Marilyn/Saxena, Mukul (1996): Turn-Taking, Power Asymmetries, and the Positioning of Bilingual Participants in Classroom Discourse. In: Linguistics and Education 8, 1, pp. 105-123.

Niedrig, Heike (2015): Postkoloniale Mehrsprachigkeit und "Deutsch als Zweitsprache". In: Thoma, Nadja/Knappik, Magdalena (eds.): Sprache und Bildung in Migrationsgesellschaften. Machtkritische Perspektiven auf ein prekarisiertes Verhältnis. Bielefeld: transcript, pp. 69-86.

Nohl, Arnd-Michael (2006): Interview und dokumentarische Methode. Wiesbaden: Springer VS.

Oevermann, Ulrich (1996): Theoretische Skizze einer revidierten Theorie professionalisierten Handelns. In: Combe, Arno/Helsper, Werner (eds.): Pädagogische Professionalität. Untersuchungen zum Typus pädagogischen Handelns. Frankfurt am Main: Suhrkamp, pp. 70-182.

Rabe-Kleberg, Ursula (1996): Professionalität und Geschlechterverhältnis. Oder: Was ist "semi" an traditionellen Frauenberufen? In: Combe, Arno/Helsper, Werner (eds.): Pädagogische Professionalität. Untersuchungen zum Typus pädagogischen Handelns. Frankfurt am Main: Suhrkamp, pp. 276-302.

Renn, Joachim/Straub, Jürgen/Shimada, Shingo (2002): Vorwort. In: Renn, Joachim/Straub, Jürgen/Shimada, Shingo (eds.): Übersetzung als Medium des Kulturverstehens und sozialer Integration. Frankfurt am Main: Campus, pp. 7-12.

Rueda, Robert/Monzo, Lilia D./Higareda, Ignacio (2004): Appropriating the Sociocultural Resources of Latino Paraeducators for Effective Instruction with Latino students. Promise and Problems. Urban Education 39, 1, pp. 52-90.

Sharma, Umesh/Salend, Spencer J. (2016): Teaching Assistants in Inclusive Classrooms. A Systematic Analysis of the International Research. In: Australian Journal of Teacher Education 41, 8, pp. 118-134.

Slater, Edwina/Gazeley, Louise (2019): Deploying teaching assistants to support learning. From models to typologies. In: Educational Review 71, 5, pp. 547-563. 
Srubar, Ilja (2002): Strukturen des Übersetzens und interkultureller Vergleich. In: Renn, Joachim/ Straub, Jürgen/Shimada, Shingo (eds.), Übersetzen als Medium des Kulturverstehens und sozialer Integration. Frankfurt am Main: Campus, pp. 323-345.

Sturm, Tanja (2016): Heterogenität in der Schule. München: Reinhardt.

Tucker, Stanley (2009): Perceptions and reflections on the role of the teaching assistant in the classroom environment. In: Pastoral Care in Education 27, 4, pp. 291-300.

Tyrer, Richard/Gunn, Stuart/Lee, Chris/Parker, Maureen/Pittman, Mary/Townsend, Mark (2009): A Toolkit for the Effective Teaching Assistant. London: Sage.

Vanderstraeten, Raf (2007): Professions in organizations, professional work in education. In: British Journal of Sociology of Education 28, 5, pp. 621-635.

Webster, Rob/Blatchford, Peter/Russell, Anthony (2013): Challenging and changing how schools use teaching assistants. Findings from the Effective Deployment of Teaching Assistants project. In: School Leadership \& Management. Formerly School Organisation 33, 1, pp. 78-96. 



\section{III}

Inklusion, Hochschule und Lebenslanges Lernen 



\title{
25 Diversität, Inklusion und Internationalisierung in der Hochschule: Eine komparative Perspektive
}

\author{
Cristina Allemann-Ghionda
}

\begin{abstract}
In diesem Beitrag werden Diskurse und Forschungsergebnisse referiert und zusammengeführt, die sich sonst eher in getrennten Veröffentlichungen finden. Leitend ist dabei die Idee, dass Diversität mit der Internationalisierung des Hochschulstudiums einhergeht. Studierende (und Lehrende) sind hinsichtlich ihrer Herkunft zunehmend diversifiziert, werden mit einem zuvor nicht bekannten Hochschulsystem und einer neuen Gesellschaft konfrontiert und müssen sich mit neuen Aspekten von Diversität auch in den Lehr-/Lerninhalten und in der Lehrpraxis auseinandersetzen. Nach geschichtlichen Umrissen der Begriffe Diversität (und komplementär dazu Inklusion) sowie Internationalisierung werden konkrete Beispiele vorgestellt und schließlich Desiderate für Forschung und Entwicklung skizziert.
\end{abstract}

\section{Einleitung}

Seit den 1960er Jahren ist in vielen Ländern der Welt das Thema der Diversität allmählich zu einer Leitidee in Teilen der Gesellschaft und Politik, in der Erziehungswissenschaft, im Bildungsdiskurs und in der Gestaltung von Bildungspolitik geworden. Dabei wurde die Begrifflichkeit weiterentwickelt und inhaltlich ausgeweitet. Je nach Land und gesellschaftlicher sowie politischer Lage standen ursprünglich, oder stehen vorwiegend, soziale und ethnische Diskriminierung von historischen oder zugewanderten Minderheiten sowie Rassismus im Vordergrund, oder interkulturelle Beziehungen, oder aber die ungleiche Behandlung bzw. Gleichstellung der Geschlechter, oder das Verhältnis zwischen Menschen ohne und mit Behinderungen bzw. deren Wahrnehmung und Stellung in Institutionen.

Anders als im anfänglichen Stadium der Diskussion über kulturelle Unterschiede und Multikulturalismus (seit den 1960er Jahren) wird im heutigen wissenschaftlichen Verständnis - zumindest in westlichen Diskursen - der Begriff „Diversität“ breit aufgefasst. Im deskriptiven Sinne ist es die Feststellung und im normativen Sinne die moralisch begründete, individuelle und politische Anerkennung aller wahrgenommenen Formen soziokultureller Vielfalt. In jeder Person und in jeder Gruppe überlagern und kreuzen sich zahlreiche „Differenzlinien“", so dass von Intersektionalität gesprochen wird (Dietz 2007). Die Anerkennung der Vielfalt und die gerechte Behandlung aller Individuen und Gruppen - auch unterstützt durch Richtlinien und Gesetze - ist der zugrunde liegende Gedanke.

Parallel und komplementär dazu wird etwa im gleichen Zeitraum der Begriff „Inklusion“ im Gegensatz zur „Exklusion“ der jeweils designierten „Anderen“ thematisiert. Inklusion wird insbesondere in der deutschsprachigen Literatur oft als Gegenbegriff zur Integration 
dargestellt, wobei Integration eher auf Migrant*innen, Inklusion eher auf Personen mit körperlichen oder anderen Behinderungen oder Beeinträchtigungen (in der Literatur oft als disability bezeichnet) bezogen wird. In den Texten supranationaler Organisationen besteht diese Unterscheidung nicht. Inklusion bedeutet die akzeptierende, konstruktive, nicht trennende Behandlung von Menschen mit Behinderungen in Bildungseinrichtungen. Inklusion wurde zunehmend - sowohl deskriptiv als auch normativ - auf alle Formen der Diversität im Einklang mit der Idee der Intersektionalität ausgedehnt (zur Geschichte der Begriffe Diversität und Inklusion siehe Allemann-Ghionda 2013: 17ff. und 125ff.).

Somit können die Begriffe Diversität und Inklusion als komplementär gesehen werden. Diversität (bzw. Aspekte davon) kann in Bildungsinstitutionen, etwa an Hochschulen, auf verschiedene Weise thematisiert und berücksichtigt werden: eher exklusiv (ignorierend, negierend oder die Unterschiede penetrant hervorhebend) oder eher inklusiv, also im Sinne der anerkennenden Teilhabe und der akzeptierenden Einbettung (vgl. Heyer 2021 i.d.B.).

Im Einklang mit und als Ergebnis von Empfehlungen supranationaler Körperschaften (Europäische Kommission, Vereinigte Nationen, UNESCO, OECD, Weltbank) bekennt sich gegenwärtig jede Hochschule zu einer Politik der Diversität sowie zu einer Politik der Inklusion. Zumindest und mit Nachdruck gilt dies in den Mitgliedstaaten der OECD, in Mitgliedstaaten der Europäischen Union und in den Ländern, die im Hochschulbereich etwa im Rahmen von Mobilitätsprogrammen mit Letzteren kooperieren.

Seit den 1980er Jahren ist die Internationalisierung der Hochschulbildung ein die Hochschulpolitik prägendes, lebhaft diskutiertes, aber kaum in Frage gestelltes Desiderat. Hierbei geht es um inner- und interkontinentale Mobilität der Studierenden, Dozierenden und Forschenden, um Veränderung der Lehrinhalte (der Curricula), sowie um Kompatibilität und Vergleichbarkeit der Studiengänge im Hinblick auf die Mobilität der Studierenden und Absolvent*innen.

Inwiefern Internationalisierung einerseits und Diversität und Inklusion andererseits miteinander verflochtene empirisch erfahrbare Tatsachen und Prozesse sind und als hochschulpolitische Diskurse formuliert werden, beleuchtet dieser Beitrag. Aufgrund der Datenlage und der kulturellen Verortung der Verfasserin geht es hierbei vorwiegend um Hochschulsysteme in dem Teil der Welt, der von Programmen der Europäischen Union und der OECD abgedeckt wird.

Diskurse über Diversität und Internationalisierung im Sinne eines offenen, akzeptierenden (aber nicht unkritischen) Umgangs damit sind charakteristisch für supranationale Organisationen. Diese sind wiederum stark von europäischen und westlichen gedanklichen Traditionen und Wertesystemen (Aufklärung, Demokratie, Pluralismus, Menschenrechte, Gleichheit der Geschlechter) geprägt. Ob und wie in anderen Teilen der Welt und politischen Systemen im jeweiligen wissenschaftlichen Diskurs und in Bildungseinrichtungen über Diversität und Internationalisierung gedacht, geschrieben und gehandelt wird, ist in der (westlichen) wissenschaftlichen Literatur weniger bekannt und kann in diesem Rahmen daher nicht berücksichtigt werden. So gesehen, ist die Sichtweise in diesem Beitrag bewusst und reflektiert eurozentrisch und westlich. Eine interkulturelle Auseinandersetzung mit anderen Sichtweisen ist in diesem Rahmen nicht möglich.

Die komparative Perspektive bedeutet hier: Zur Beantwortung der Frage, welchen Stellenwert Diversität und Internationalisierung in der Hochschule haben, werden Beispiele aus verschiedenen Ländern herangezogen. Die Auswahl kann nur exemplarisch sein. Der Diskurs wurde und wird maßgeblich von angloamerikanischen Universitäten geprägt. Daher wird Literatur und werden konkrete Beispiele aus jenen Hochschulen aufgeführt. Punktuell werden 
Studien und Beispiele aktueller Programme aus deutschsprachigen Hochschulen aufgeführt. Ziel ist es, Ähnlichkeiten und Unterschiede aufzuzeigen, die auf unterschiedliche gesellschaftliche Lagen zurückzuführen sind. Systematische Vergleiche im Sinne der Methoden der Vergleichenden Erziehungswissenschaft (Allemann-Ghionda 2004) würden jedoch den Rahmen sprengen; in einigen Fällen wird exemplarisch auf komparative (vergleichende) Untersuchungen verwiesen.

Für die nachfolgenden Ausführungen ist es hilfreich, zunächst einige Begriffe zu klären. Der Begriff, ,global“ bedeutet „,weltumspannend“ und ist in diesem Zusammenhang relevant, weil die hier angesprochenen Prozesse so gut wie die ganze Welt betreffen. „International“ betont die Inter-Aktion, also die Wechselbeziehung, zwischen Personen, Institutionen und Diskursen der einzelnen Nationen. „Supranational“" bezeichnet Organisationen, die alle Nationen oder eine große Anzahl von Nationen umfassen mit dem Ziel, das gegenseitige Verständnis zu fördern und nach Möglichkeit zu gemeinsamen Stellungnahmen zu finden. „Transnational“ bedeutet „quer durch die Nationen“. „Multikulturell“ bezeichnet das Nebeneinander von Kulturen, während ,,interkulturell“ die Interaktionen zwischen Kulturen (oder Personen oder Gruppen oder Institutionen, die Kulturen zugeordnet werden oder sich selbst zuordnen) meint. „Transkulturell“ bedeutet sinngemäß „quer durch die Kulturen“. Ableitungen von den oben genannten Begriffen, zum Beispiel „Globalisierung“ und „Internationalisierung“, werden in den entsprechenden Teilen dieses Beitrags erläutert. „Diversität“ kann mit „Heterogenität“ gleichgesetzt werden.

Der Beitrag befasst sich zunächst mit der Transformation vom nationalen zum zwischenstaatlichen zum transnationalen Raum und beleuchtet anschließend die Entwicklung der Begriffe Diversität und Inklusion sowie die Baustellen der Internationalisierungs- und Diversitätspolitik an Hochschulen. Aus der Gesamtperspektive werden Kommentare und Desiderate für Forschung und Entwicklung abgeleitet und vorgeschlagen. Durch die verknüpfende Betrachtung der Diskurse und Forschungsfelder Internationalisierung, Diversität und Inklusion wird beabsichtigt, die zahlreichen Verschränkungen dieser empirischen Realitäten und Diskurse in der gegenwärtigen Hochschulpolitik hervorzuheben und auch die darin enthaltenen Chancen erkennbar zu machen. Nicht die bedingungslose Hervorhebung von Unterschieden um der Zelebrierung willen, sondern das Ideal der Überwindung von Ungleichbehandlung und das Streben nach Chancengerechtigkeit im Rahmen der Regeln der Meritokratie im aufklärerischen Sinne sind hierbei leitend.

\section{Vom nationalen zum zwischenstaatlichen zum transnationalen Raum}

\subsection{Ursprung der Internationalisierung}

Die Institution „Universität“ ist seit der Gründung der ersten Universitäten (Bologna 1088; Paris 1200) per definitionem international. Für die magistri, licentiati und doctores der Universitäten im Mittelalter, in der Renaissance und darüber hinaus war es selbstverständlich zu reisen. Lange vor der Erfindung des Buchdruckes, der modernen Transport- und Kommunikationsmittel bis hin zum Flugzeug und zum Internet gehörte es zum Beruf der Forschenden 
und Lehrenden, sich international physisch zu bewegen und wissenschaftlich auszutauschen. Auch im Zeitalter der Nationalstaaten und im postnationalen Zeitalter ist die internationale Offenheit der Wissenschaftler*innen ein kaum wegzudenkendes Merkmal - mit Ausnahme der Restriktionen und Repressionen unter faschistischen, nationalsozialistischen und anderen Diktaturen (Koch 2008: 200ff.). Die Hochschule ist die kosmopolitischste unter allen Bildungsinstitutionen (Teichler 2007: 24), d.h.: nationale Zugehörigkeit und Grenzen waren und sind im Selbstverständnis der Hochschulen unbedeutend; was zählt, sind (nicht nur formale) Qualifikationen sowie die Generierung und das Weitergeben von Wissen ohne Rücksicht auf geografische Grenzen. Frauen erkämpften sich und erhielten erst ab dem neunzehnten Jahrhundert (abgesehen von seltenen Ausnahmen in früheren Jahrhunderten) schrittweise die offizielle Möglichkeit zu studieren und dann auch zu lehren und zu forschen. Dies erfolgte zuerst in den Vereinigten Staaten und Frankreich (1833), in der Schweiz (1864), in den Niederlanden (1869) und im Deutschen Reich nach und nach ab 1899 (Koch 2008: 192ff.). Die Öffnung von Universitäten für Frauen führte in beschränktem Rahmen zu individueller internationaler Mobilität derselben. Frauen trugen also zusätzlich zur Internationalisierung der Hochschulen bei. Bis zur Bildungsexpansion (in Deutschland ab den 1960er Jahren), mit der schrittweisen Erweiterung der Bildungsbeteiligung bis hin zur Massenuniversität, gingen Dozierende und Studierende fast ausschließlich aus dem Adel und aus gebildeten und wohlhabenden Familien hervor. Diese hatten dank ihrer soziokulturellen und wirtschaftlichen Lage die Mittel und die Beziehungen, die Ihnen das Reisen ermöglichten. Viele Jahrhunderte lang waren Hochschulen also ohne spezifische Programmatiken international, bevor der Begriff „Internationalisierung“ zur Anwendung kam. Zugleich waren sie stillschweigend oder explizit (was zum Beispiel Frauen, Farbige, ethnische und andere Minderheiten betrifft) von Formen der sozialen Ungleichheit und der Exklusion bestimmter Personen und Gruppen gekennzeichnet.

Die Internationalisierung der Hochschulbildung ist seit Ende der 1980er Jahre - ebenso wie der Trend hin zur Massenuniversität - gemäß der OECD eines der Schlüsselmerkmale des Wandels der tertiären Bildung in hochindustrialisierten Ländern (Santiago et al. 2008: 235). Letztere bezeichnen die Internationalisierung als third mission. Internationalisierung der Hochschulpolitik prägt substanziell den „Funktionswandel der Universitäten“ (Kehm et al. 2012). Bis zu den 1990er Jahren wurde vor allem in englischsprachigen Ländern der Begriff international education gebraucht (de Wit 2013: 6). In den Anfängen wurden unter Internationalisierung lose miteinander verbundene Aktivitäten subsumiert: Auslandsstudium, Beratung von ausländischen Studierenden, akademischer Austausch von Dozierenden und Studierenden, einzelne Kooperationsprojekte mit Schwellenländern, Studiengänge in Regionalwissenschaften (area studies).

Seit den 1990er Jahren lässt sich weltweit ein wachsendes Interesse an der Internationalisierung von Hochschulen feststellen, das mit einer Systematisierung von zuvor isolierten, als international bezeichneten Maßnahmen einhergeht. Nach Knight (2001) kann Globalisierung als der Katalysator, Internationalisierung als die proaktive Antwort darauf angesehen werden. Über die Weiterentwicklung der Internationalisierung von Hochschulen unter den Bedingungen der Globalisierung wird permanent reflektiert (Lanzendorf/Teichler 2003). Am Beispiel der deutschen Hochschullandschaft lässt sich verfolgen, wie nach und nach eine explizite, organisierte Strategie der Internationalisierung entsteht. Dabei sind sowohl Hochschulleitungen als auch Ministerien und beratende Instanzen aktiv (Deutscher Wissenschaftsrat 2017). Entscheidend ist dabei die Rolle der world polity oder world culture (Lechner/Boli 2005) als Steuerungsinstanz. Selbst ohne über gesetzliche Instrumente der Politikbildung zu 
verfügen erweisen sich global players wie die OECD und die Europäische Kommission bei der Gestaltung von internationaler bzw. transnationaler Hochschulpolitik als zunehmend einflussreich (Adick 2012). Dabei bleibt es nicht nur bei Absichtserklärungen und dem Entwerfen erwünschter Szenarien, sondern empirische komparative Studien dokumentieren die Europäisierung und Globalisierung der Hochschulbildung beispielsweise in Mittel- und Osteuropa (Kwiek 2017).

In Europa hat das Programm ERASMUS (European Community Action Scheme for the Mobility of University Students), 1987 auf Initiative der italienischen Erziehungswissenschaftlerin Sofia Corradi gegründet, die Internationalisierung der tertiären Bildung eingeleitet und spürbar vorangetrieben. Die Nachfolgeprogramme ERASMUS plus und ERASMUS Mundus haben die Reichweite nicht nur in geografischer Hinsicht erweitert, geht es doch bei Letzterem um Kooperation zwischen Hochschulen in der Europäischen Union (einschließlich der Partnerländer) und solchen in anderen Erdteilen. Es folgte und entfaltete sich gleichzeitig der nicht unumstrittene Bologna-Prozess, der stufenweise zur Erschaffung eines gemeinsamen europäischen Hochschulraumes führen sollte. Die Umsetzung des Bologna-Prozesses war und ist nicht einfach, da historisch gewachsene, nationale Traditionen, kulturelle Denkmuster und Strukturen schwer zu ändern sind (Schriewer 2007). Ungeachtet der oft berechtigten Kritik ist kaum zu übersehen, dass die allgemeine (nicht nur europaweite) Umstellung der alten Studiengänge in das in anglo-amerikanischen Ländern und deren ehemaligen Kolonien übliche Bachelor-Master-Modell die unabdingbare strukturelle Grundlage für Mobilität, Anerkennung der Studienleistungen und Internationalisierung in ihren verschiedenen Formen (vgl. 2.2.) darstellt.

Weitere Handlungsfelder eröffnen sich mit der fortschreitenden Transnationalisierung der Hochschulpolitik. Zu den Kooperationsverträgen zwischen Ländern der Europäischen Union kommen zunehmend Kooperationsverträge mit Hochschulen in Asien, Afrika, Nordamerika, Lateinamerika und Australien hinzu. An westeuropäischen Universitäten gelten Studierende aus wirtschaftlich expandierenden, technologisch starken Ländern als besonders attraktiv, da sie die Zukunft wirtschaftlicher Kooperation verkörpern. Vor allem die stärkeren Universitäten der asiatischen und lateinamerikanischen Länder, deren Wirtschaften sich durch technologische Innovation auszeichnen, treten immer mehr als Akteure der Hochschulpolitik und -praxis im Rahmen globaler Prozesse in Erscheinung (Ng et al. 2016).

\subsection{Formen der Internationalisierung}

Nach der Klassifizierung der OECD äußert sich die Internationalisierung der tertiären Bildung in vier Formen: 1. Konvergenz der Systeme tertiärer Bildung und Vereinbarungen der internationalen Anerkennung von Studiengängen und Abschlüssen; 2. Internationalisierung der curricularen Inhalte und der Vermittlung derselben; 3. Mobilität der Menschen (der Studierenden, der Dozierenden und Forschenden); 4. Mobilität der Institutionen und Programme (Santiago et al. 2008: 238ff.). Nach Knight (2012) hat Internationalisierung zwei Pfeiler, denen zahlreiche, zunehmend diversifizierende Aktivitäten zugeordnet sind: Internationalisierung ,zu Hause“ und ,im Ausland“. Transnationale Hochschulbildung wird als dritte Generation von cross-border higher education bezeichnet. Länderberichte und empirische Untersuchungen über spezifische Fragestellungen und Prozesse unter veränderten Umständen 
beleuchten die Wechselwirkung von Internationalisierung und Globalisierung (Streitwieser 2014).

Internationalisierung setzt voraus, dass die beteiligten Akteure - Studierende, Lehrende, Forschende, Verwaltungspersonal - miteinander reden und sich möglichst verstehen können. In den frühen Phasen der europäischen Universität und bis weit in die Neuzeit hinein war Latein die lingua franca, die den internationalen wissenschaftlichen Austausch ermöglichte. Im gegenwärtigen Zeitalter der Internationalisierung und der Globalisierung übernimmt die englische Sprache diese Aufgabe. Tagungen und Kongresse werden in englischer Sprache abgehalten, Veröffentlichungen in englischer Sprache sind in der Regel unabdingbar, um eine akademische Laufbahn einzuschlagen und mit Erfolg fortzusetzen, nicht zuletzt um außerhalb des eigenen Herkunftslandes und des eigenen Sprachraums rezipiert und zitiert zu werden. Dies ist wiederum nicht zuletzt unumgänglich, um Zugang zu Forschungsgeldern und zu internationalen Kooperationsprojekten zu bekommen. Die Dominanz der englischen Sprache hat ihre Berechtigung, da die englische Sprache in den meisten Fällen die einzige Sprache darstellt, der die Beteiligten auf der globalen Arena mehr oder weniger mächtig sind. Durch ihre Geschichte und Beschaffenheit, durch den Status als Weltsprache, aber auch aufgrund der Tatsache, dass das Internet und alle damit verbundenen Medien die englische Sprache benutzen, ist diese am ehesten geeignet, früher oder später von sehr vielen Menschen erlernt und - nicht nur im wissenschaftlichen Betrieb - aktiv genutzt zu werden (Fischer 2001: 234 ff.). Daraus ergibt sich die Paradoxie, dass einerseits Internationalität und die damit einhergehende Diversität als gesellschaftlich normal und deren Wertschätzung als wünschbar deklariert werden, andererseits aber die Vielfalt der Sprachen und Soziokulturen verflacht und wie von manchen nicht ganz zu Unrecht befürchtet wird - zugunsten einer oft holprigen, vereinfachten englischen Sprache und eines transnationalen, transkulturellen Einheitsdenkens verdrängt werden könnte.

\subsection{Von der geografischen zur virtuellen Internationalisierung}

Die in Gebäuden untergebrachte Hochschullehre mit physischer Anwesenheit von Studierenden und Lehrenden (abgesehen von der grundsätzlichen Opportunität, den jeweils geltenden gesetzlichen Grundlagen und der oft faktischen Unmöglichkeit, die Teilnahme der Studierenden zu kontrollieren) ist gegenwärtig die vorherrschende Variante. Für alle Typen von Hochschulen zeichnet sich jedoch weltweit eine folgenreiche Tendenz ab. Die zunehmende Virtualisierung und Entmaterialisierung des Studiums geschieht auf globaler Skala. Im Zeitalter der Digitalisierung wird die Wolke (cloud) zum unsichtbaren, nicht geografischen Ort, an dem nicht nur Dateien jeglicher Art gespeichert, sondern an welchem Ideen ausgetauscht und Inhalte konstruiert werden, an welchem kommuniziert, gelehrt und gelernt werden kann (Bergmann/Grané 2013). Fragte die OECD vor Jahren noch rhetorisch, ob virtuelle Hochschulen als Instrument der Internationalisierung zu betrachten seien (Santiago et al. 2008: 261), so kann die Frage heute bejaht werden.

Zwar ist der Übergang zum elektronischen Lernen (e-learning) keine plötzlich auftretende Veränderung, sondern eine zusätzliche Form von Entlokalisierung, eine Weiterentwicklung des traditionellen Fernstudiums, die mit den Mitteln der digitalen Kommunikation virtuelle Mobilität ermöglicht. Allerdings haben Sichtbarkeit und quantitative Bedeutung des virtuellen Studierens seit 2012 exponentiell zugenommen und eine neue Dimension und 
Qualität erhalten, seitdem die Idee der Massive Open Online Courses (MOOCs) geboren und umgesetzt wurde. Die Reihe der an Kataklysmen angelehnten Metaphern im Zusammenhang mit den Zuständen im Bildungswesen vor der Bildungsexpansion (,Bildungskatastrophe“, Picht 1964) und bezogen auf den enormen Zuwachs bis hin zur Massenuniversität (,, Studentenberg "), wird um das furchterregende, weil das Unausweichliche suggerierende Bild des „,campus tsunami “ reicher (Brooks 2012). Unaufhaltsame, revolutionäre Veränderungen des Hochschulstudiums werden als ,avalanche“ (Lawine) beschworen (Barber et al. 2013).

Bemerkenswert ist bei der Bestrebung, Online-Hochschulbildung in großem Stil zu veranstalten, die Tatsache, dass Elitehochschulen sie ins Leben gerufen haben, und dass bis dahin unerreichte Massen von Studierwilligen angesprochen werden sollten und auch konnten (Brooks 2012). Die Elite-Hochschule Wesley stellte sich als ,premium “-Produkt auf dem Gebiet kostenfreier Kurse dar und begann Kurse mit 370’000 Studierenden (Salido 2013). Anfänglich fast ausschließlich in den Vereinigten Staaten basiert, etablieren sich MOOCs seither - wenngleich oft mit Skepsis bedacht - auch in Europa.

Neben den MOOCs werden zunehmend auch andere Formen virtueller oder hybrider, semi-virtueller Lehre veranstaltet. So ist es möglich, eine Vorlesung in einer Universität zu halten und diese dank digitalen Technologien in mehreren anderen Hörsälen im selben Bundesland, Land oder international zu hören. Es wird mit Mischformaten (blended learning) experimentiert, bei denen Studierende und Lehrende zeitweise im Hörsaal anwesend sind gleichzeitig oder nicht - und teils digital kommunizieren.

Auch in diesem Falle geht es um Kosteneinsparung, aber auch um die Entwicklung neuer hochschuldidaktischer Formen, die möglicherweise der Affinität junger Menschen zur digitalen Kommunikation entgegenkommen.

Virtuelle Lehre in den vielen verfügbaren Formen erlaubt es Studierenden, die sonst diese Möglichkeit nicht hätten, in einem Land ihrer Wahl zu studieren, andere akademische Kulturen kennenzulernen und ihre Kompetenzen im Umgang mit Interkulturalität und Diversität zu entwickeln. Zum Beispiel wählen Tausende von Studierenden aus Schweden, Russland, Deutschland und China an norwegischen Universitäten nicht nur Kurse im Rahmen von ERASMUS-Semestern, sondern auch Studiengänge, die teilweise oder ganz auf dem Weg der Online-Lehre absolviert werden können. Dieses Phänomen kann als Erleichterung und Demokratisierung der Internationalisierung des Studiums aus Sicht der Studierenden gelesen werden. Da diese aber den geografischen Ort nicht wechseln, könnte der wissenschaftliche Gewinn fraglich sein. Auch aus der Perspektive der Qualität der Hochschullehre wird diese Form von virtueller Internationalisierung hinterfragt (Vabø/Wiers-Jenssen 2014: 714ff.).

Weitere potentielle Vorteile virtueller hochschuldidaktischer Formate sind: Barrierefreiheit, potentieller Abbau sozialer Ungleichheiten (auch zwischen den Regionen der Welt), sofern die Kurse kostenfrei oder kostengünstig sind sowie flexibles Zeitmanagement ermöglichen. Es können im Hinblick auf die berufliche Zukunft nützliche internationale Kontakte geknüpft werden.

Digitalisierung bedeutet auch: Bibliotheken und gedruckte Bücher und Zeitschriften besitzen nicht mehr das Monopol der Archivierung von Information und Wissen. Wikipedia und andere digitale Datenbanken sorgen für globale Ubiquität und fast uneingeschränkte Abrufbarkeit - ein Vorteil für Studierende und Lehrende/Forschende, besonders für solche, die eingeschränkt mobil sind; allerdings gilt dies nur für Menschen, die über einen Internetzugang verfügen. 
Die Entmaterialisierung und Virtualisierung des Studiums und des Zugriffs zu Informationen und Forschungsergebnissen bergen aber auch potentielle Nachteile und nicht eingehaltene und wohl kaum einzuhaltende Versprechungen in sich.

Virtuelle Lehre ist stark angelehnt an Prozesse der Digitalisierung, wie auch der omnipräsente Buchstabe ,i“ - zum Beispiel im Firmennamen „Iversity“ (Iversity 2019). signalisiert. Damit geht eindeutig ein Trend der Kommerzialisierung von Hochschullehre einher, die sich auf dem globalen Markt präsentieren kann - unter Umständen als teures, exklusives „,premium "Produkt, was an Mechanismen beim kommerziellen Wettbewerb erinnert, wie er bei Angeboten von Online-Sprachkursen zu beobachten ist.

Die Erfinder*innen und Betreiber*innen von MOOCs warben mit dem Argument der Kostenfreiheit für die Studierenden. In Wirklichkeit können MOOCs nur zu einem Bruchteil ohne Entgelt genutzt werden. Eher ist es so, dass Hochschulen große Einsparungen vor allem an Kosten für Lehr- und Verwaltungspersonal und Räumlichkeiten sowie zugehörigen Infrastrukturen, Apparaten usw. tätigen können, wenn eine einzige Person (eventuell in Form einer reproduzierbaren Videoaufzeichnung, also ohne Diskussionsmöglichkeit) gleichzeitig Tausende von Studierenden mit Lehre beliefern kann. Mit anderen Worten: akademische und nicht akademische Arbeitsplätze werden vernichtet.

Virtuelle Lehre in einem solchen Ausmaß verunmöglicht interaktives Lernen nahezu. Reproduktives, oberflächlich rezipiertes Wissen droht Bildung zu ersetzen und somit zu verhindern, weil Möglichkeiten der Reflexion, der Vertiefung und des direkten Austauschs fehlen und implizit als unnötig dargestellt werden. Ein solches Risiko ist für geistes- und sozialwissenschaftliche Fächer offensichtlich. Die Beurteilung der Lernergebnisse von Studierenden im Rahmen von MOOCs muss zwingend standardisiert und automatisiert werden. Neben dem Einsatz von multiple-choice Tests besteht die Möglichkeit, sogar individuelle Essays mit software zu beurteilen. Als noch ökonomischer (weil effizienter) wird die Einstellung von Gruppen von Studierenden für die Korrektur gewertet. Die so entlasteten Lehrenden könnten sich der Forschung oder anderen Tätigkeiten widmen. Was als Vorteil gepriesen wird, kann sich leicht als Entwertung wissenschaftlicher Qualifikation und Kompetenz und somit als dramatischer Qualitätsverlust erweisen.

Ein weiteres Argument rückt die Demokratisierung von Hochschulbildung in den Mittelpunkt. MOOCs sollen weltweit für alle frei verfügbar sein, die Internetzugang haben. In absoluten Zahlen mag die potentiell globale Teilnahme an MOOCs beeindruckend erscheinen und als Erfolg gewertet werden, doch nach ersten Auswertungen war die Abbruchquote extrem hoch (Salido 2013: 268). Insgesamt ist nach einer neueren Studie die Bilanz über den Nutzen von MOOCs ernüchternd. Die überwältigende Mehrheit der Studierenden kommt nach dem ersten Studienjahr nicht zurück, also bestätigt sich das anfänglich festgestellte Phänomen der hohen Abbruchquoten. Die Zunahme der Beteiligung an MOOCs betrifft fast nur die reicheren Länder, also ändert sich an der Beteiligung von sozioökonomisch Benachteiligten am Studium nichts (Hüther et al. 2020; Reich/Ruipérez-Valiente 2019).

Insgesamt verändern sich durch die internationale Digitalisierung des Zugangs zu Informationen und zu Wissen und durch die digitalisierte Internationalisierung der Lehre Methoden, Dynamiken und Ergebnisse der Konstruktion von Wissen und Bildung. Ob und wie die weitgehende Digitalisierung auf vielen Ebenen der Lehre und Forschung dazu beiträgt, Wissen zu generieren (anstatt bloß Wissen unkritisch zu reproduzieren und Informationen anzuhäufen), ist eine offene Frage. Die sinnvolle Nutzung von online verfügbaren Daten und Informationen steht und fällt mit der durch eigenständiges Denken und Studieren zu erwerbenden Fähigkeit, Informationen zu suchen, zu verstehen, einzuordnen und deren Zuverlässig- 
keit und wissenschaftliche Relevanz zu prüfen. Vieles deutet darauf hin, dass durch die zunehmende Vermischung von digitaler Lehre und Kommunikation über soziale Medien die Art der verbalen und nichtverbalen Kommunikation zwischen Studierenden und Lehrenden, möglicherweise auch die Qualität des schriftlichen Ausdrucks beim Verfassen von Qualifikationsarbeiten, sich in beträchtlichem Ausmaß ungünstig verändert.

\section{Zur Entwicklung der Begriffe Diversität und Inklusion}

\subsection{Komponenten und Verflechtungen der „Superdiversität"}

Dass Internationalisierung und Diversität im tertiären Sektor der Bildung miteinander verbundene und verflochtene, wahrnehmbare und empirisch erfassbare Tatsachen und Dynamiken sind, ist eine Feststellung, die sich in der einschlägigen Literatur eher selten findet. Bezeichnenderweise behandelt ein Artikel diese Frage ausgehend von der japanischen Hochschullandschaft (Rivers 2010). Bemerkenswert ist dies deshalb, weil Japan hinsichtlich der sprachlichen und kulturellen Diversität ein ausnehmend homogenes Land ist. Durch die erhöhte, teilweise organisierte und institutionell gewollte Mobilität der Studierenden (und der Dozierenden) kommt es jedoch zu einer verstärkten Präsenz von Menschen, die nicht nur unterschiedliche Staatsangehörigkeiten haben und deshalb oft „,international“ genannt werden, sondern auch unterschiedliche soziokulturelle Hintergründe, religiöse Erfahrungen, Sprachen und akademische Kulturen - mit anderen Worten: alle Facetten der Diversität und der Internationalität - verkörpern und mit sich bringen. Daher erscheint es berechtigt, Diversität unter den Bedingungen der Globalisierung und der Internationalisierung unter die Lupe zu nehmen.

Heute wird in den Sozialwissenschaften und in der Bildungsforschung ein Begriff der Diversität angewandt, der viel umfassender ist als der Begriff der Multi- und der Interkulturalität, der seit den 1960er und 1970er Jahren in den Vereinigten Staaten und Kanada, einige Jahre später auch in westeuropäischen und anderen Ländern aller Erdteile zur Anwendung kam (Länderstudien in Banks 2009). Die vom kanadischen Politikwissenschaftler und Sozialphilosophen Charles Taylor begrifflich gefasste und breit rezipierte Konzeption einer „Politik der Anerkennung“, allerdings damals fokussiert auf Multikulturalismus und Kommunitarismus (Taylor 1992), entstand als Antwort auf seit den 1960er Jahren zunehmende soziale Kämpfe und Forderungen vieler Personen und Gruppen, angefangen bei den Farbigen, bis hin zu ethnischen und anders definierten Minderheiten sowie Frauen, gegen Diskriminierung, für die Idee der Gleichheit und für die gerechte Behandlung aller.

Im gegenwärtigen Diskurs geht es um mehr und auch um Anderes als um die Hervorhebung und Anerkennung von ,kulturellen“ Unterschieden im ethnonationalen oder -lokalen Sinne. Es geht auch nicht um Abgrenzung oder Gegenüberstellung oder um Sonderbehandlung in jedem Falle. Die Kritik am essentialistischen und statischen Kulturbegriff der frühen Diskurse über Multikulturalismus und der multikulturellen und interkulturellen Erziehung bzw. Pädagogik, wie sie in den 1980er Jahren diskutiert wurde, hat ebenso wie gesellschaftliche Transformationen, die seit dem Ende des Zweiten Weltkrieges und vor allem seit den 1960er Jahren zu beobachten sind, zu einem erweiterten und dynamischen Verständnis von 
Diversität beigetragen. Der Mensch (oder eine bestimmte Gruppe) ist - laut dem gegenwärtigen sozialwissenschaftlichen Diskurs - nicht entweder schwarz oder weiß. Frauen und Männer, LGBTQI, weitere, differenzierende Orientierungen und Bezeichnungen der sexuellen und Genderzugehörigkeit, religiös oder ideologisch definierte Gruppen, Menschen mit körperlichen oder anderen Beeinträchtigungen, Zugewanderte und viele andere, gehören zum heterogenen Ganzen der Menschheit. Die Liste der möglichen Identitätskennzeichen, Zugehörigkeiten und Zuschreibungen ist lang und wird ständig erweitert.

Unterschiede aufgrund von körperlichen oder anderen wahrnehmbaren Merkmalen oder sozialen Zugehörigkeiten gelten deshalb als „Differenzen“, weil sie gesellschaftlich als solche konstruiert, in vielen Fällen problematisiert und zu Faktoren der Exklusion gemacht werden. Sie sind nicht hierarchisch zu verstehen und für Aus- oder Abgrenzungen zu nutzen. Vielmehr sind Zeichen der Identität und Zugehörigkeit einer Person oder einer Gruppe immer multipel und miteinander verschränkt, wie der Begriff Intersektionalität (Dietz 2007) besagt. Gleichzeitig sind Zeichen der Identität auch dynamisch und flüssig (liquid) - oder flüchtig, wie es in den deutschen Übersetzungen der zeitdiagnostischen Werke des polnisch-britischen Soziologen Zygmunt Bauman (2000) heißt. Gerade auch das dynamische Verständnis von Diversität unterstreicht die Nichtigkeit einer Hierarchisierung von Individuen und Gruppen aufgrund von als solchen designierten Unterschieden und Identitäten.

Eine Steigerung des Begriffs Diversität hin zur „Superdiversität“ beschreibt der britische Soziologe Vertovec (2007) ausgehend vom Phänomen der Migration und dessen Veränderungen um die Wende zum einundzwanzigsten Jahrhundert. Seine Grundthese lautet: Gesellschaften verändern sich auch - aber nicht nur - durch vielfältige Migrationsprozesse in alle Richtungen, sie werden zunehmend divers (also heterogen) und umfassen die ganze Bandbreite möglicher Unterschiede (aus der Sicht einer hypothetischen, von der Mehrheit einer jeweiligen Gesellschaft angenommenen und als ,richtig“ empfundenen Normalität) - daher super-diversity.

Seit einigen Jahren (aus deutscher und europäischer Sicht war 2015 wegen der zum Politikum verdichteten „Flüchtlingskrise“ ein Wendejahr) ist das Motiv der Flucht und der Asylsuche stärker als zuvor in den Mittelpunkt gerückt. Durch die zahlenmäßige und aufgrund der politischen Brisanz außerordentliche Zuwanderung von Menschen aus Kriegsgebieten und/oder von Menschen, die aus europäischen, asiatischen und afrikanischen Regionen fliehen, weil sie aus nachvollziehbaren Gründen ein besseres Leben suchen, nimmt die Diversität zusätzlich zu und verdichtet sich zum erstrangigen, zunehmend kontroversen politischen Thema.

Wenn von aktueller Diversität die Rede ist, darf nicht außer Acht gelassen werden, dass zunehmend hochqualifizierte Migrant*innen (Expats) aus vielen Ländern der Welt nach Europa kommen und innerhalb Europas sowie interkontinental zirkulieren und für kürzere oder längere Zeit in ein neues Land übersiedeln. Gleichzeitig gibt es auch eine neue Migration von Menschen aus zahlreichen (auch europäischen) Ländern, die nicht oder wenig qualifiziert sind, oder die eine nur geringe Schulbildung aufweisen. Sie haben Kinder oder werden Kinder bekommen. Dieser Aspekt der Diversität tritt früher oder später in Schulen und Hochschulen in Erscheinung. Was für die Gesamtpopulation gilt, gilt auch für Studierende. Zur nationalen, soziokulturellen und sozioökonomischen Herkunft der Studierenden kann verallgemeinernd festgestellt werden:

Erstens: Infolge gesteigerter Zuwanderung, einschließlich der Aufnahme anerkannter Flüchtlinge, und dank der Mobilitätsprogramme, sind Studierende zunehmend heterogen hinsichtlich ihrer nationalen, soziokulturellen und sonstigen Herkunft, ihrer Religion, ihres 
sozioökonomischen Status, ihrer Vorbildung. In Deutschland wird zwischen „Bildungsausländern“ und „Bildungsinländern“ unterschieden; Letztere werden in neueren amtlichen Publikationen mit dem Attribut ,mit Migrationshintergrund“ versehen (Autorengruppe Bildungsberichterstattung 2016). In Ländern, die sich als Einwanderungsländer bezeichnen oder diese Bezeichnung ablehnen, aber de facto Einwanderungsländer sind, kann der prozentuale Anteil besonders hoch sein, vor allem wenn der Erwerb der Staatsangehörigkeit nicht nach dem ius soli, (wörtlich: Recht des Bodens) sondern nach dem ius sanguinis (wörtlich: Recht des Blutes) oder abgeschwächten bzw. Mischformen der beiden Modelle erfolgt.

Zweitens: Auch die Bildungsexpansion und die ihr zugrundeliegende, relative Demokratisierung der Bildungsbeteiligung haben - schon seit den 1960er Jahren - zu einer Vielfalt in sozioökonomischer Hinsicht beigetragen, indem immer mehr Studierende (darunter immer mehr Frauen) aus verschiedenen sozialen Milieus Hochschulen besuchen.

Drittens: Mobilität betraf bis in die 1980er Jahre fast nur eine Elite und vorwiegend Männer, die aus eigenem Antrieb und dank vorhandener persönlicher Ressourcen reisen konnten. Im Rahmen der Internationalisierung handelt es sich um gelenkte und von vielen Studierenden auch gewollte und gesuchte Mobilität.

Viertens: Die Altersgruppe der millennials (der zwischen 1980 und 2000 Geborenen) trägt in der Europäischen Union und in den Partnerländern zu einer neuen Kategorie von Migrant*innen bei. Neben den nicht gut Ausgebildeten gibt es viele, die eine gute berufliche Ausbildung absolviert oder einen Hochschulabschluss haben, aber im eigenen Land (zum Beispiel Italien und Spanien) keine für sie angemessene Arbeit finden. Andere wollen im Ausland ein Studium beginnen oder sich weiter akademisch qualifizieren, oder sie interessieren sich für neue kulturelle Erfahrungen. Diese Generation der ,,nativi mobili “ (in Anlehnung an digital natives) (Pasqualini/Rosina 2016) ist dank den ERASMUS-Programmen, der Einheitswährung Euro, den Billigflügen und der globalen digitalen Kommunikation durch das Internet und soziale Medien mit der Idee der selbstverständlichen Mobilität groß geworden.

Studierende, die sich ins Ausland begeben, erleben und erzeugen Unterschiede und manchmal Reibungen etwa beim Verständnis von akademischer Kultur. Die akademische Sprache kann ihnen Schwierigkeiten bereiten, selbst wenn Kurse in englischer Sprache angeboten werden. Auch im persönlichen Umgang mit Kommiliton*innen und Lehrenden kann es zu interkulturellen Missverständnissen kommen. Möglicherweise benötigen einige von ihnen Unterstützung, was ebenso für Studierende ohne Migrations- und Mobilitätsgeschichte gelten kann. Die Anwesenheit und Erfahrung der „Mobilen“ kann aber auch die Lehre bereichern und neue Forschungsfragen anregen, wenn Lehrende die Chance erkennen und die Studierenden - ungeachtet ihrer Herkunft - produktiv einbinden.

\subsection{Verlagerung der Schwerpunkte im Diskurs über Diversität}

Das Wissen um sozioökonomische Ungleichheit als ernstzunehmende Kategorie, die sich auf die Bildungsbeteiligung nachweislich auswirkt, war in den 1960er Jahren in vielen Ländern der Motor für Reformen zugunsten der Bildungsbeteiligung. Das Thema der sozialen Ungleichheit sowie der geschlechts- und genderbezogenen Ungleichheit der Bildungschancen ist eine Konstante in Untersuchungen der OECD, insbesondere der Berichte über international vergleichende Untersuchungen über die Lesekompetenz und die mathematische Kom- 
petenz (OECD 2018). Auch in den nationalen Berichterstattungen einzelner Länder wird das Problem der ungleichen Chancen - auch im Zusammenhang mit Migration - wieder vermehrt in den Mittelpunkt gerückt (Autorengruppe Bildungsberichterstattung 2016). Als kulturell wahrgenommene und bezeichnete Spezifika und Unterschiede müssen nicht zwingend $\mathrm{zu}$ ungleicher und ungerechter Behandlung und zu verhinderter oder erschwerter Bildungsbeteiligung namentlich auf Hochschulebene führen. Geschlecht und Gender müssen ebenso wenig wie Behinderung nicht zwingend der Anlass für Diskriminierung sein. In Kombination mit dem sozioökonomischen Status können jedoch die genannten Aspekte von Diversität verstärkt zu mangelnder Beteiligung oder im Verlauf des Studiums zu Diskriminierung beitragen, wenn Eingriffe oder Missachtungen durch einzelne Personen, oder die Logik von Institutionen den Zugang zu Bildung erschweren.

Beim Versuch, gesellschaftliche Konflikte und ungleiche Bildungsbeteiligung zu erklären, sind weitere Erklärungsfaktoren hinzugekommen und zeitweise als herausragend postuliert worden.

Das Thema der kulturellen Zugehörigkeiten und Unterschiede nahm seit den späten 1960er bis in die 1990er Jahre hinein einen wichtigen Platz ein, wobei die Dimension der ökonomisch bedingten sozialen Ungleichheit oft in den Hintergrund geriet oder ausgeklammert wurde.

Im Gegensatz zu analytischen Modellen, welche die Frage nach der Beteiligung und dem Erfolg von Menschen unterschiedlicher Herkunft mit Gesellschaft und Kultur erklären, stehen biologische bzw. biologistische Modelle und Argumente. Diese gerieten in der genannten Zeitspanne (späte 1960er bis 1990er Jahre) in Vergessenheit, ja sie wurden im sozialwissenschaftlichen Diskurs kategorisch abgelehnt, während sie in einem Teil der Politik periodisch herangeführt wurden, um Ungleichheit, mangelnde Bildungsbeteiligung und ungleiche Behandlung zu rechtfertigen. Im zwanzigsten Jahrhundert ist es dank der Untersuchungen in Anthropologie und Genetik gelungen nachzuweisen, dass es in biologischer Hinsicht keine verschiedenen menschlichen Rassen gibt (Cavalli-Sforza/Cavalli-Sforza 1995). Gemäß dem Soziologen Rogers Brubaker (2015) hat aber die bahnbrechende Aufschlüsselung des menschlichen Genoms im Jahr 2000 Informationen zu Tage gefördert, die für genetische Unterschiede zwischen Bevölkerungsgruppen sprechen. Dadurch sei der Diskurs über Diversität in Medien und Politik über Gebühr beeinflusst und für bestimmte Zwecke benutzt worden, wobei Brubaker in erster Linie Entwicklungen in den Vereinigten Staaten meint. Allerdings seien genetische Unterschiede zwischen zwei zufällig ausgewählten Individuen prozentual so geringfügig (0,1 Prozent), dass sie keinerlei Bedeutung hätten (ebd.: 52). In Bezug auf den Stellenwert von Diversität in Gesellschaft und Bildung ist dies ein wichtiger Befund, hilft er doch, diejenigen Positionen zu entkräften, die Ungleichheit und Diskriminierung mit biologischen „Fakten“ zu rechtfertigen trachten.

Religion (oder besser: das Religiöse, das Geistliche) wurde in den ersten Jahrzehnten der Diskussion über interkulturelle Bildung und über Diversität so gut wie ignoriert. Im gegenwärtigen, post-säkularen Zeitalter (Habermas 2001) ist jedoch die Bedeutung von Religion(en) für zwischenmenschliche Beziehungen und für das Verständnis und die Gestaltung von Politik - auch für die Politik und Praxis der Bildungsinstitutionen - weniger denn je zu leugnen, ganz unabhängig vom persönlichen Verhältnis zu Glaubensfragen oder von der Art und Weise, in welcher in einem Land das Verhältnis von Staat und Kirche(n) definiert wird.

Doch weder soziale noch kulturelle noch religiöse Differenzen oder biologistische Sichtweisen scheinen für sich allein die gegenwärtige gesellschaftliche Diversität ausreichend beschreiben und erklären zu können. 
In seinem Buch Grounds for Diversity analysiert Rogers Brubaker (2015) die Verschränkungen der vielen Aspekte von Diversität und stellt fest: Die kulturelle Wende (cultural turn) im sozialwissenschaftlichen Diskurs über Diversität scheint sich erschöpft zu haben. Der gegenwärtige gesellschaftliche Kontext (die Rede ist im Buch vor allem von den Vereinigten Staaten) lässt drei Tendenzen erkennen, die Brubaker im Hinblick auf die Gestaltung einer „Politik der Differenzen“ für wegweisend hält: Rückkehr der Ungleichheit: ,Inequality as a public concern “ (Ungleichheit als öffentliche Sorge); Rückkehr der Biologie: „,Biology as an asserted basis for ethnic and racial differences " (Biologie als behauptete Grundlage für ethnische und rassenbedingte Unterschiede); Rückkehr des Geistlichen: „,Religion as a key terrain of public contestation " (Religion als Schlüsselgebiet der öffentlichen Auseinandersetzung) (ebd.: 2ff.).

Diese Tendenzen sind nicht nur für Nordamerika charakteristisch. In Europa haben vorwiegend die Schwerpunkte soziale Ungleichheit, Gleichberechtigung der Geschlechter und inklusive Behandlung von Menschen mit Behinderungen oder disabilities die Diversitätspolitik der Bildungsinstitutionen, insbesondere der Hochschulen, geprägt. Doch etwa seit der Jahrhundertwende (um 2000) haben auch religiös definierte Unterschiede und Konflikte in der Öffentlichkeit und in Bildungsinstitutionen von der vorschulischen bis zur Hochschulbildung an Sichtbarkeit gewonnen. In europäischen (ebenso wie nordamerikanischen) Hochschulen, die entweder laizistisch oder mehrheitlich christlich geprägt sind, ist es keineswegs ungewöhnlich, Studierende mit äußeren Zeichen unterschiedlicher religiöser Zugehörigkeiten zu sehen, außer wenn solche Zeichen verboten sind. Folgerichtig hält die religiöse Dimension auch in die Diversitätspolitik der Hochschulen - wenngleich unterschiedlich ausgeprägt - Einzug (vgl. 3.3.).

Vor dem oben skizzierten Hintergrund der sich wandelnden gesellschaftlichen Diversität, ihrer kollektiven Wahrnehmung und des Wandels der Schwerpunkte im sozialwissenschaftlichen Diskurs (und in der Politik) erscheint es angebracht, Diversität an der Hochschule nicht als Summe getrennter, autonomer Problemlagen zu betrachten, sondern als komplexe, vielschichtige, sich ständig wandelnde Wirklichkeit. Diversität ist unter anderem mit Migration und anderen Faktoren der Internationalisierung verwoben. Internationalisierung unterstreicht und intensiviert die Dimension der Diversität. Daraus ergeben sich komplexe Aufgaben, was Strukturen und Inhalte der Hochschulbildung, Herausforderungen an die Studierenden, Kompetenzen der Lehrenden und neue Forschungsfelder betrifft.

\subsection{Zur Berücksichtigung von Diversität an Hochschulen}

Da der Begriff „Inklusion“ Hauptgegenstand des vorliegenden Handbuches ist, sei an dieser Stelle ein nur kurzer Hinweis erlaubt. In deutschsprachigen Schriften bezieht sich Inklusion sehr oft hauptsächlich auf den Aspekt von Diversität, der gegenwärtig häufig als ability bzw. disability bezeichnet wird, um den belasteten und undifferenzierten Begriff der Behinderung zu umgehen. Im Diskurs der supranationalen Organisationen und in zahlreichen Bildungssystemen der Welt wird Inklusion jedoch auf alle, oder zumindest auf für Bildung besonders relevante Aspekte der Diversität angewandt. Das liegt daran, dass zahlreiche Bildungssysteme der Welt im weitesten Sinne inklusiv sind, zumindest was ihre formalen Strukturen angeht, oft aber auch darüber hinaus. Das heißt: sie praktizieren nicht nur inklusive oder integrierte Heil- bzw. Sonderpädagogik (Bürli et al. 2009), sondern sie kennen außerdem 
keine frühe Selektion nach einer kurzen Grundstufe. Sie orientieren sich an einer umfassenderen Agenda der Inklusion mit Fokus auf die bestmögliche Förderung sowie auf höhere Bildung für möglichst Viele (vergleichende Fallstudien in Allemann-Ghionda 2015).

Die Beteiligung von Personen mit körperlichen oder anderen Beeinträchtigungen an der Hochschulbildung wurde bis zum späten zwanzigsten Jahrhundert in der einschlägigen Literatur kaum erwähnt. Die gegenwärtige Diskussion über Inklusion im Hochschulbereich erschöpft sich nicht darin, Forderungen zur Beseitigung von Beteiligungsbarrieren durchzusetzen, sondern Exklusionsmechanismen theoretisch und empirisch zu untersuchen, sei es bezogen auf die Hochschulbildung (Heitzmann/Klein 2012), sei es auf politische Rahmenbedingungen im europäischen Vergleich (Waldschmidt 2019).

Die explizite Inklusion (im Gegensatz zu Ausblendung, willentlicher Missachtung oder Diskriminierung) von Diversität an Hochschulen ist zuerst prominent in den Vereinigten Staaten und in Kanada als programmatisches Thema in Erscheinung getreten. Nach der Devise der positiven Diskriminierung haben nordamerikanische Hochschulen Programme der Verbesserung von Chancen für historisch diskriminierte Schwarze, ethnische Minderheiten unterschiedlicher Herkunft, andere benachteiligte Personen und Gruppen, sowie allgemein im Sinne der geschlechtlich und nach der Kategorie Gender definierten Gleichstellung zu verwirklichen versucht (Nieto 2009). Zahlreiche Zentren für Studien über Diversität wurden in den Vereinigten Staaten und in Kanada gegründet, denen ein breites Verständnis von Diversität und Inklusion zugrunde liegt. Die Bezeichnungen der Zentren erhalten spezifische Akzentuierungen, so zum Beispiel beim Center for Diversity, Inclusion, and Belonging an der New York University, School of Law (NYU School of Law 2019). Ähnliches gilt für andere Weltteile. Exemplarisch sei das Wits Centre for Diversity Studies der University of the Witwatersrand in Johannesburg erwähnt. Dort wird ein Masterprogramm angeboten mit dem Ziel, ,critical diversity literacy “ zu fördern (University of the Witwatersrand 2019). In Kontinenten und Ländern (Vereinigte Staaten, Südafrika), die historisch scharfe Gegensätze zwischen der weißen und der farbigen Bevölkerung gekannt haben und unter veränderten politischen und gesetzlichen Bedingungen immer noch kennen, steht das Thema der rassistisch motivierten Konflikte weiterhin an prominenter Stelle.

Auch in Europa kam es - zu einem späteren Zeitpunkt - zu zahlreichen Gründungen von Zentren für Studien über Diversität. Ein Beispiel ist das Kompetenzfeld SINTER (Soziale Ungleichheiten und Interkulturelle Bildung) der Universität zu Köln, das 2013 im Rahmen der Exzellenzinitiative aus dem 2006 gegründeten CEDIS (Center for Diversity Studies) hervorgegangen ist. Im Programm stehen Studien über ungleiche Bildungschancen und über die Grenzen und Möglichkeiten, Chancengerechtigkeit zu begünstigen:

Soziale Ungleichheiten hängen mit gesellschaftlichen (Macht-)Verhältnissen, Strukturen und Praktiken zusammen und werden auch im Kontext von Bildungsinstitutionen (re-)produziert. Zugleich gilt der Abbau sozialer Ungleichheiten und ungleicher Bildungschancen als zentrales gesellschaftliches und bildungspolitisches Ziel. Wie aber hängt die Entstehung und Reproduktion sozialer Ungleichheiten - entlang der Differenzlinien sozioökonomischer Hintergrund, ethnische Zugehörigkeit, Sprache(n), Religionen, Geschlecht/Gender, ability/disability, Alter etc. - mit Strukturen, Diskursen und Praktiken der schulischen und außerschulischen Bildung zusammen? Und wie begegnen Akteur*innen der Bildung den relevanten Herausforderungen im Kontext von Diversität und dem Anspruch von Chancen- und Bildungsgerechtigkeit (SINTER 2019)?

Des Weiteren existiert an der Universität zu Köln das Studienzentrum GESTIK (Gender und queer studies), dessen Wirkungsfeld sich auf alle Bereiche und Problemfelder der Gesellschaft erstreckt (GeStiK 2019). Schließlich bearbeitet das IDIS (Internationale Forschungs- 
stelle Disability Studies) die Forschungsfelder Disability Studies, Soziologie der Behinderung und politische Partizipation behinderter Menschen (iDiS 2019). Die drei zuletzt genannten Beispiele signalisieren zugleich eine spezialisierte Profilbildung der Hochschule, die Dotierung des Forschungsschwerpunktes Diversität mit erheblichen Ressourcen, und die Einbindung in internationale Netzwerke.

Die Tätigkeit der fakultätseigenen oder fakultätsübergreifenden Studienzentren und der dort betriebenen Forschungsaktivitäten ist das Kerngeschäft der systematischen Etablierung von Diversität als relevantes Studienfeld, in das möglichst viele, idealerweise alle Fakultäten und Fächer einbezogen werden sollen. Ebenso tragend ist das strukturelle Gerüst, nämlich die offizielle Politik der Internationalisierung und der Diversität auf Rektoratsebene. Am Beispiel des Portals der Universität zu Köln wird deutlich, dass eine Aufgabentrennung zwischen Internationalisierung und Diversität als nötig erachtet wird - und sei es nur aus organisatorischen Gründen. Eine Rubrik heißt Gleichstellung und Diversität, eine andere Internationalisierung. Sie sind jeweils einer Prorektorin beziehungsweise einem Prorektor zugeteilt. Die Rubrik Gleichstellung und Diversität führt eine lange Liste von Anlaufstellen auf und widerspiegelt die Bemühung, alle Aspekte von Inklusion der Diversität zu berücksichtigen. Die Gleichstellung der Geschlechter erscheint als der Ausgangspunkt. Zusätzlich werden unter Gleichstellung und Diversität zahlreiche Stichworte und konkrete Angebote aufgeführt: Barrierefreiheit, Beratung ausländischer Studierender, Einrichtung von WCs für alle Geschlechter (sic: die besonderen WCs richten sich kumulativ an alle Geschlechter, die sich nicht in den Bezeichnungen Frau und Mann wiedererkennen), bis hin zum überkonfessionellen Gebetsraum - analog zu ähnlichen Einrichtungen in großen Flughäfen und Bahnhöfen. Im Sinne der Vernetzung werden thematisch affine außeruniversitäre Beratungsstellen aufgelistet (Universität zu Köln 2019).

An einem Beispiel aus Nordamerika wird exemplarisch ersichtlich, wie die Mission der Inklusion von Diversität gestaltet und öffentlich bekannt gemacht werden kann. Die Universität Kaliforniens in Berkeley, das Flaggschiff der zehn Standorte des University of California Systems (mit einer Viertelmillion Studierenden) gehörte zu den prominenten Stätten der Veränderungsbewegungen in den turbulenten 1960er Jahren und darüber hinaus. Die Revolte begann als Protest gegen den Vietnamkrieg und gegen das establishment und dessen Werte und Normen und mündete in eine Programmatik der gleichen Rechte und der Anerkennung der „Anderen“ (der Vernachlässigten, der Ausgeschlossenen) in der Hochschulbildung. Auf dem Portal der School of Information der Universität Kaliforniens in Berkeley werden alle Ressourcen bekanntgegeben, die insbesondere auf dem Gebiet engineering and sciences verfügbar sind, um ,nicht traditionellen Studierende“ (will sagen: nicht weiße, nicht bildungsbürgerliche oder wohlhabende oder männliche oder heterosexuelle usw.) Chancengleichheit zu ermöglichen. Das Angebot ist auf zwei Ebenen verteilt: Campus Resources und I School Resources. Es genügt, die in alphabetischer Reihenfolge angeführten Campus Resources anzusehen, um etwas von dem Geist und der Logik zu erahnen, die das Programm in einem wohl längeren Prozess hervorgebracht hat.

Einige der Anlaufstellen wenden sich an einzelne ethnisch oder nach besonderen Fähigkeiten oder Problemlagen definierte Gruppen, während andere transversalen Charakter haben. Einiges spricht dafür, dass es sich etwa bei den ethnisch definierten Angeboten um ältere Gründungen im Verlauf einer bottom-up Bewegung handelt. Einige Sammelangebote benennen ein Problemfeld (zum Beispiel Harassment and Discrimination oder Student Parent Center) und richten sich an alle Interessierten. Ein drittes Muster ist die Benennung eines spezifischen Problemfeldes (zum Beispiel STEM = Science, Technology, Engineering, Mathe- 
matics), jedoch gerichtet an die heterogene Gruppe der ,,diverse NERDS“. Hierbei ist wohl die Annahme leitend, dass solche Personen, unter einem Sammelbegriff gruppiert (diverse $N E R D S$, auch non-traditional students), in der Regel systematisch ausgeschlossen werden oder geringere Chancen hatten bzw. haben und daher besonderer Aufmerksamkeit und Unterstützung bedürfen. Ebenso wie beim Internetauftritt der Universität zu Köln zum Thema Diversität ergänzt auch hier eine Liste außeruniversitärer Anlaufstellen das helfende Angebot (Berkeley School of Information 2019).

Am Konzept der Universität Kaliforniens in Berkeley ist exemplarisch die Absicht erkennbar, alle potentiell oder nachweislich benachteiligten Gruppen und Einzelpersonen zu nennen und einzubeziehen. Bemerkenswert ist, dass Inklusion von Diversität als relevantes Thema für eine naturwissenschaftliche Fakultät (school) erklärt wird, während an europäischen Hochschulen in der Regel vor allem Geistes- und sozialwissenschaftliche Fakultäten und Fächer Interesse am Schwerpunkt „Diversität und Inklusion“ bekunden. Dabei fällt in Berkeley die sehr prägnante, fast stereotypisch anmutende Verbindung „diverser“ Gruppen auf, denen Unterstützung und Schutz gewährt werden muss, als ob sie prinzipiell nicht in der Lage wären, ohne besondere Hilfe den Weg zum Hochschulstudium zu finden und ihr Studium erfolgreich zu beenden. In gewisser Weise widerspricht dieser Ansatz dem heutigen wissenschaftlichen Diskurs, wonach Diversität nicht eine Summe spezifischer, als "anders" etikettierte Gruppen, sondern die ganze Gesellschaft (und somit die ganze Population der Universität) kennzeichnet. Das Beispiel Kaliforniens regt zum Nachdenken an: Das Bestreben, Diversität anzuerkennen, inklusiv wertzuschätzen und gleichzeitig „diverse“ Studierende zu fördern, kann zu einem Boomerangeffekt führen und unter Umständen Exklusion und Stigmatisierung durch übermäßige Exposition hervorrufen.

Vergleicht man die exemplarisch genannten Studien- und Forschungsangebote miteinander, wird ein gemeinsames Anliegen erkennbar: das Bestreben, allen Studierenden ungeachtet ihrer möglichen Unterschiede und objektiven Startbedingungen die bestmöglichen Chancen zu eröffnen und ihnen gleichzeitig das Forschungspotential der Diversität aufzuzeigen.

\section{Baustellen der Internationalisierungs- und Diversitätspolitik an Hochschulen}

\subsection{Kompetenzbildung der Studierenden und Lehrenden}

Mit der enormen Diversifizierung der Studierenden, die in ganz besonderem Ausmaß seit der zweiten Hälfte des zwanzigsten Jahrhunderts kontinuierlich erfolgt ist, haben sich auch die Ziele und Erwartungen ihnen gegenüber, zugleich aber auch die Voraussetzungen der Studierenden diversifiziert. Dies gilt für die alte und die neue Welt, unter anderen Vorzeichen aber auch für die anderen Erdteile, da Globalisierung Grenzen relativiert und sprengt. Von einer verhältnismäßig homogenen sozialen und kulturellen Struktur der Studierenden kann heute kaum die Rede sein, sei es in sozioökonomischer kultureller, religiöser oder anderer Hinsicht; und keine Hochschule würde in der heutigen Zeit Homogenität oder eine nationale, geschlossene Ausrichtung des Studiums offen als wünschenswert angeben. Daraus ergeben 
sich Aufgaben für die Hochschulbildung, welche die Philosophin Martha Nussbaum so zusammenfasst:

It is relatively easy to construct a gentleman's education for a homogeneous elite. It is far more difficult to prepare people of highly diverse backgrounds for complex world citizenship (Nussbaum 1998: 295).

Auf diese Herausforderung wird durch verschiedene Strategien und Reformen reagiert. Eine erste Strategie besteht darin, dass die einstige, eher Eliten vorbehaltene, Universität sich selbst vermehrt und diversifiziert. Nach den 1968er Unruhen wird die ursprüngliche Pariser Universität Sorbonne auf Druck der Studierendenbewegung auf dreizehn Nachfolgeuniversitäten in Paris und Umgebung aufgeteilt. In vielen Ländern entstehen in größeren und kleineren Städten im Zuge der Bildungsexpansion neue Universitäten traditionellen Zuschnitts, die jedoch eine weit größere Anzahl von Studierenden aufnehmen als es in der Gründungszeit vorstellbar war. Es entstehen colleges, praxisorientierte Fachhochschulen, und fast überall wird die Lehrer*innenbildung tertiarisiert. In jeder Variante von Hochschule ist ungeachtet der im Hinblick auf Zielgruppen und Bildungsbedürfnisse gewollten Aufgabentrennung und Spezialisierung weiterhin und zunehmend jeweils ein heterogenes Publikum zugegen, das komplexe Anforderungen an die Lehre stellt, die sich zum Teil von denen unterscheiden, die sozial homogene Gruppen von Studierenden stellen.

Eine zweite Strategie ist die Diversifizierung des Lehrpersonals, denn konsequenterweise müsste der Diversität der Studierenden eine Diversität der Lehrenden gegenüberstehen. Ein gewisses $\mathrm{Maß}$ an Internationalität des Lehrkörpers und die freie Zirkulation von Gelehrten sind normale Phänomene im Zeichen der Tradition der abendländischen Universität. Aus demselben Grund (Qualifikation ist das, was zählt) galt die Diversität des Lehrkörpers im Sinne der sozialen Herkunft und aller Aspekte von Diversität nach heutigem Verständnis bis zum späten zwanzigsten Jahrhundert als nicht besonders hervorzuhebendes Thema - mit Ausnahme der Variablen Geschlecht und Gender. Es war selbstverständlich, dass Dozierende und Forschende mehrheitlich eine bildungsbürgerliche Herkunft hatten, was aber offiziell keine soziale Barriere im Hinblick auf die Laufbahn darstellen sollte.

In den anglo-amerikanischen Ländern, allen voran den Vereinigten Staaten und Kanada, wurde im Rahmen der Diskurse und Bemühungen um multicultural education und diversity education auch die Rekrutierung von Hochschullehrenden nach den Kriterien Ethnizität (in der anglo-amerikanischen Literatur und Bildungspolitik wird auch der Begriff „,race“ verwendet, der im Englischen eine andere Konnotation als im Deutschen hat), Geschlecht und Gender, sexuelle Orientierung, disability sowie sozioökonomische Herkunft seit den 1960er Jahren mit Hilfe von Programmen der positiven Diskriminierung thematisiert und forciert. In Europa sind die meisten Hochschuleinrichtungen eher vorsichtiger hinsichtlich der absichtlichen Rekrutierung von ,diversem“ Lehrpersonal, nicht zuletzt auch weil der soziale Aufstieg von Migrant*innen zweiter und dritter Generation sowie die innereuropäische Mobilität mit der damit verbundenen Zirkulation von Sprachen und Kulturen auch ungeplant für Diversität sorgen. Dabei bestehen nationale Unterschiede, indem etwa in den ehemaligen Kolonialmächten (Großbritannien, Niederlande, Frankreich) Lehrende aus ehemaligen Kolonien keine Seltenheit sind. In deutschsprachigen Ländern wird erst in jüngster Zeit die Diversität des Lehrkörpers unter dem Stichwort „Management von Diversität“ diskutiert und empirisch untersucht. Das Hochschulpersonal wird als „Black-Box“ bezeichnet, über welches kaum etwas bekannt sei (Richter 2012).

Eine dritte Strategie ist die Kompetenzbildung der Lehrenden. Die Internationalität und im weitesten Sinne die Diversität der Lehrenden sind von Bedeutung, weil die Gestaltung 
und die Vermittlung curricularer Inhalte maßgeblich von den Lehrenden beeinflusst werden. Vertiefende Untersuchungen über die Einstellungen und Praxen von Lehrenden - neben Studierenden die Hauptakteure der Hochschulbildung - zum Verständnis von Internationalisierung und zu den Aufgaben, die sich aus der „Superdiversität“ der Gesellschaft und der Studierenden ergeben, erscheinen daher notwendig. Ein Beispiel dafür, wie Leitlinien der ERASMUS-Programme in Forschungspraxis umgesetzt werden können, ist eine empirische Untersuchung über die Einstellungen von Lehrenden zum Thema Diversität, verbunden mit der Weiterentwicklung von Diversitätskompetenz der Lehrenden (Resch/Raschauer 2019). Das Projekt wurde im Rahmen von ERASMUS plus durchgeführt, in einem von 2016 bis 2018 dauernden Teilprojekt, dessen Ziel so lautete:

The HE4u2 project has developed an approach to the intercultural dimension of existing curricula, a generic CPD course for staff to understand how cultural diversity can enhance their work, and a set of policy recommendations focusing on the curricula and the teachers of adults in higher education, who often provide access for disadvantaged groups (European Commission 2019).

Diese Agenda fasst effektiv die Synergien zusammen, die notwendig sind, um dem Thema Diversität und Inklusion im Rahmen von Internationalisierung gerecht werden zu können.

\subsection{Internationalisierung, Diversität und Bildungsinhalte}

Für Akteure der Hochschulpolitik auf Rektorats- und Fakultätsebene, die sich auf der internationalen Arena positionieren wollen und müssen, bilden Internationalisierung und Diversität als Leitideen gegenwärtig und perspektivisch den unverzichtbaren Rahmen. Zudem spricht Einiges dafür, dass die Mobilität von Studierenden (und von Lehrenden) die Erweiterung kultureller Horizonte und den Aufbau von interkultureller und Diversitätskompetenz fördert. Allerdings ist fraglich, ob die Programmatik der Institution und die Mobilität der Individuen alleine ausreichen. Neben der Kompetenzbildung der Lehrenden stellt sich die Frage nach den Inhalten der Hochschullehre, wenn das Ziel darin besteht, eine Lehre zu gestalten, die Wissen über und Verständnis von Diversität unter den Bedingungen der Internationalisierung zum relevanten Inhalt macht (Kreft/Leichsenring 2012). Die bisherige Forschung über interkulturelle Beziehungen und Kommunikation geht oft noch von einem ethnokulturellen oder ethnonationalen Verständnis von Interkulturalität aus. Auf die Hochschulbildung bezogen gilt es jedoch die Ziele der interkulturellen Kompetenz so festzulegen, dass eine Diversitätskompetenz anvisiert wird. Dazu gehören Sensibilität gegenüber Diversität, natürlicher Umgang mit Personen mit Migrationshintergrund, anderer Staatsangehörigkeit oder Religion, mit Personen mit Beeinträchtigungen oder die auf andere Weise in den Augen mancher von einer hypothetischen „Normalität“ abweichen, Offenheit gegenüber und Respekt vor ,anderen“, unbekannten Lebensweisen, Bildungssystemen u.a. (Allemann-Ghionda 2017:73ff.).

Ein erster Ansatz ist das Angebot von Lehrveranstaltungen über Fragestellungen, die explizit im Zusammenhang mit Diversität und Inklusion stehen. Hierbei sind Disziplinen wie Psychologie, Soziologie, Anthropologie, Erziehungswissenschaften (Knobloch 2014), aber auch Philologie besonders geeignet. Potentiell können alle Disziplinen und Fachkulturen die Dimensionen der Diversität und der Internationalität integrieren. Nicht nur für zukünftige Lehrpersonen (Allemann-Ghionda 2017) ist der Sinn einer solchen Kompetenzerweiterung 
unmittelbar ersichtlich, sondern auch in der sozialen und Jugendarbeit (Wolfsgruber 2015) und in der Humanmedizin (Hallal 2015). Es existieren spezifisch auf Jurist*innen zugeschnittene Programme der Diversitätsstudien (NYU School of Law 2019), andere richten sich an alle Studierenden. Das Interesse in Forschung und Lehre an interkultureller oder Diversitätskompetenz, auf Hochschulbildung bezogen, beinhaltet empirische Forschung im internationalen Vergleich, Lehre und Evaluation gegebenenfalls erworbener Kompetenzen (Deardorff/Aarasaratnam 2017).

Ein zweiter Ansatz ist die Vision der Bildung zum Weltbürgertum (englisch education for world citizenship). Die Politikwissenschaftlerin und Sozialphilosophin Amy Gutmann entwirft eine Kombination von demokratischer Erziehung, Kosmopolitanismus (Weltbürgertum) und egalitärem Multikulturalismus, womit die Nähe zur Konzeption von Charles Taylor (1992) dokumentiert wird:

Egalitarian cosmopolitanism flows from the same moral source as a deliberative democratic education: both are fed by a commitment to equal respect for persons (Gutmann 1987/1999: 311).

Frühere Konzepte des Multikulturalismus bergen das Risiko der Fragmentierung und der Abgrenzung von Gruppen gegeneinander in sich. Dagegen postuliert „Bildung zum Weltbürgertum" die Positionierung des Individuums und der Gruppen als Mitglieder des gemeinsamen Planeten - im Gegensatz zur Beschränkung auf den lokalen und nationalen Horizont, die leicht in Kirchturmmentalität und Fremdenfeindlichkeit kippen kann.

In der Konzeption der Philosophin Martha Nussbaum (1998) verbindet sich die klassische, humanistische Bildung mit der liberalen Bildung und mit dem Ideal, dass alle sozialen Milieus und alle Aspekte der menschlichen Diversität bewusst wahrzunehmen und zu inkludieren sind. Aus einer anderen Weltregion (dem Südpazifik) kommt der Vorschlag, die Curricula der Hochschulbildung in der Hinsicht zu internationalisieren, dass sie nicht dem Diktat der Wirtschaft folgen, sondern zum friedlichen Zusammenleben aller Menschen beitragen mögen (Ng 2012).

Die beiden oben erwähnten Entwürfe haben gemeinsam, dass sie um eine theoretische Konturierung bemüht sind. Aufgrund der strukturellen Voraussetzungen des jeweiligen Hochschulsystems und der jeweiligen akademischen Kulturen ergibt sich, ob diese Ideen in den curricularen Inhalten einzelner Disziplinen, oder in kompletten Kursen zu verwirklichen sind.

Ein dritter Ansatz ist das in Europa bisher wenig beachtete Thema der religiösen Bildung im Rahmen des Hochschulstudiums und knüpft an die erweiterte Konzeptualisierung von Diversität an (Brubaker 2015: 2ff.; zur Religion siehe 85ff. und 102ff.; vgl. 3.1):

Professional exposure to ,religious literacy“ is another priority, starting with higher education [...] and extending to professional organizations such as diplomatic services and United Nation institutions (Marshall 2018: 37).

„Religiöse Bildung“ kann als Variante oder Bestandteil des expliziten Studiums der Diversität verstanden werden, oder als Element eines umfassenden Programms einer Bildung zum Weltbürgertum. Ziel ist nicht religiöse Bildung und Erziehung im konfessionellen Sinne, sondern vergleichendes, reflektierendes, historisches, soziologisches, philosophisches Wissen (nicht nur einführende, elementare Informationen und Begriffe) über Religionen, das dazu befähigen soll, in den Professionen zu handeln, die besonders offensichtlich mit Internationalität und Diversität, also auch mit religiöser Diversität, zu tun haben. Dieses Plädoyer findet an anglo-amerikanischen Hochschulen Resonanz in Form institutioneller Angebote, so etwa beispielsweise am The-Woolf-Institute in Cambridge (Vereinigtes Königreich) 
(Woolf Institute, The (2019) und an der Harvard Divinity School (Vereinigte Staaten). Letztere stellt sich vor als

a nonsectarian school of religious and theological studies that educates students both in the pursuit of the academic study of religion and in preparation for leadership in religious, governmental, and a wide range of service organizations (Harvard Divinity School 2019).

Dem kann hinzugefügt werden, dass Wissen über Religionen für alle Professionen und nichtberuflichen Tätigkeiten (nicht zuletzt für Elternschaft) von Bedeutung ist, da ohne solches Wissen kaum etwas vom gesellschaftlichen und (welt-)politischen Geschehen, vielleicht auch von zwischenmenschlichen interkulturellen Beziehungen in Familie und Schule zu verstehen ist.

Selbstverständnis und Struktur der anglo-amerikanischen, der von Letzteren inspirierten, und der europäischen Hochschulen unterscheiden sich insofern, als allgemeine Bildung in europäischen Bildungssystemen Sache der höheren Bildung (Sekundarstufe II) ist, während in der Hochschulbildung anglo-amerikanischer Prägung eine Phase der allgemeinen Bildung dem Fachstudium vorausgeht. Daher können einige der oben angeführten Beispiele schwer zu verwirklichen erscheinen. Dennoch ist es denkbar, auch außerhalb der dazu prädestinierten Disziplinen (unter anderen: Philologien, Geschichte, Philosophie, Anthropologie, Religionswissenschaft) im Rahmen des studium generale Raum für Bausteine einer Bildung zum Weltbürgertum bzw. Bildung zur Diversität zu schaffen.

\subsection{Internationalisierung, Diversität und Qualitätssicherung}

In programmatischen Dokumenten der supranationalen sowie der nationalen Institutionen wird die Wünschbarkeit der Internationalisierung der Hochschule mit verschiedenen Argumenten bekräftigt. Für einige Länder (vor allem für die Mitgliedstaaten der OECD) stelle die Anwerbung von auswärtigen Studierenden aufgrund des Geburtenrückgangs eine ökonomische Notwendigkeit dar. Im Vergleich zu einem ausschließlich nationalen Bildungsangebot sei die Öffnung der Grenzen und der Hochschulen die kostengünstigere Alternative (Santiago et al. 2008: 237). Internationalisierung wird mit dem Argument der Qualitätssteigerung von Forschung und Lehre verknüpft. Es wird als plausibel dargestellt, dass Qualitätssteigerung und Marktorientierung nicht gegensätzliche, sondern sich ergänzende Ziele sind. Durch die OECD werden Leitlinien für die Qualitätssicherung von cross-border education vorgeschlagen (Vincent-Lancrin/Pfotenhauer 2012). Wie wichtig die Internationalisierung der Hochschulen im Hinblick auf deren materielles Überleben und Anschlussfähigkeit (also: das Gegenteil von nationaler Isolation) ist, wird in folgender Feststellung aus der Sicht der finnischen Hochschulen ersichtlich:

As for higher education policy, a new funding model of universities rewards them on the basis of international staff members, students and degrees. In addition, international publishing is one of the key criteria for the funding of universities. Simultaneously, however, HEIs (Higher Education Institutions) are struggling to become more international in their teaching and administrative practices in addition to the increased emphasis put on international cooperation in research (Välimaa/Weimer 2014: 708). 
Aus der Sicht norwegischer Hochschulen beleuchten Vabø/Wiers-Jenssen (2014) die Beziehungen zwischen Internationalisierung, Diversifizierung und Qualität in der Hochschulbildung, insbesondere nach der Einführung der Bologna-Reform. Sie warnen vor dem drohenden Verlust an Qualität, falls Aktionen zur Beteiligung von ausländischen Studierenden (geografisch mobil oder virtuell oder in Mischformen) sporadisch, zufällig anmutend und oberflächlich bleiben. Auch in Bezug auf Inklusion von Diversität stellt sich die Frage nach der Verbesserung von Forschung und Lehre und somit nach der Qualitätssicherung zugunsten der Studierenden.

\section{Kommentar und Desiderate für Forschung und Entwicklung}

Die internationale Mobilität, globale Prozesse und die allgemein erhöhte Heterogenität (,Superdiversität") der Studierenden, in Teilen auch der Lehrenden, wirkt sich automatisch, aber bei weitem nicht systematisch, bis zu einem gewissen Grad auf die Inhalte der Lehre und auf die akademischen Kulturen in Theorie und Praxis aus. Die Aufmerksamkeit für Internationalisierung und Diversität scheint zu wachsen, ist in den Fächern und Disziplinen aber ungleich verteilt, wie Fallstudien über Finnland (Välimaa/Weimer 2014) und Norwegen (Vabø/WiersJenssen 2014) dokumentieren.

Was allerdings noch nicht das Selbstverständnis der Hochschulen im Ganzen durchdringt, ist die Idee, dass Curricula im umfassenden Sinne der Diversität und Inklusion gegenüber zu öffnen sind. Dazu reichen offiziell proklamierte Programme auf Rektoratsebene nicht aus, sondern es bedarf des Einverständnisses der Lehrenden an den einzelnen Fakultäten und Instituten.

Die wachsende Diversifizierung der Population stellt vor allem die Massenuniversitäten vor gewaltige Aufgaben. Diesen ist wohl kaum mit dem quantitativ orientierten Mittel der Massenveranstaltungen gegebenenfalls im virtuellen Format von MOOCs angemessen, im Sinne eines respektablen Studiums, zu begegnen. Eher bedarf es einer ausreichenden Anzahl qualifizierter Lehrender, die nicht in prekären Arbeitsverhältnissen arbeiten und die bereit und fähig sind, sich mit Internationalisierung, Diversität und Inklusion konstruktiv zu befassen.

Die von supranationalen Organisationen propagierten Strategien der Internationalisierung und der wertschätzenden, nicht nur emotional befürworteten, sondern auch professionell gestalteten Einbindung von Diversität sind kaum zu umgehen. Ein Teil der akademischen Gemeinschaft kritisiert die Marktorientierung der global aufgestellten tertiären Bildung scharf (Portnoi/Bagley 2014). Unter anderem wird auf die Paradoxie hingewiesen, dass gerade die Internationalisierung, die untrennbar mit der Inklusion von jeder Manifestation von Diversität verknüpft ist, soziale Ungleichheiten nicht immer neutralisiert oder ihnen aktiv entgegenwirkt, sondern sogar zu mehr Ungleichheit beitragen kann.

Das Credo der Internationalisierung und der Akzeptanz gesellschaftlicher Diversität sowie der Wünschbarkeit von Inklusion wird in Bildungssystemen und Gesellschaften (sogar innerhalb eines Landes) nicht universell geteilt und kann niemandem verordnet werden. Es kann sein, dass in einer bestimmten Gesellschaft und akademischen Kultur die Förderung von Diversität, Inklusion und Internationalisierung nicht als erstrebenswert gilt. Nicht zuletzt muss sich die Durchsetzung einer derartigen Ausrichtung mit dem für die Universität typischen Grundsatz der Freiheit von Forschung und Lehre auseinandersetzen. Daher muss 
dieses Ideal bzw. dessen Vernachlässigung oder Verwirklichung in den Fakultäten und Instituten diskutiert werden.

Vor dem Hintergrund des bisher Erreichten zeichnen sich einige Forschungsdesiderate $\mathrm{ab}$ :

- Von den vier Formen der Internationalisierung (vgl. 2.2) ist laut OECD (Santiago et al. 2008: 237) die Internationalisierung auf der curricularen Ebene die am wenigsten erforschte. Ähnliches gilt für die Berücksichtigung der verschiedenen Aspekte von Diversität. Daher sind dies zwei längerfristig zu bearbeitende Forschungsfelder.

- Das Problem der sozialen Ungleichheiten und der ungleichen Beteiligung an Hochschulbildung wird als nach wie vor signifikant beschrieben (Teranishi et al. 2015), so dass auch hier sowohl Forschung als auch Entwicklung von Strategien erwünscht sind.

- Empirische Forschung über die Hintergründe und die Auswirkungen der auf Dauer stattfindenden internationalen Mobilität der Studierenden unter Berücksichtigung des relativ neuen Phänomens der Geflüchteten, die ein Studium aufnehmen, ist langfristig zu leisten (Streitwieser/Light 2018).

- Ebenso gilt es, die Einstellungen und den Beitrag der Dozierenden sowie des Verwaltungspersonals weiter zu erforschen.

Vergleichende, empirische Untersuchungen in internationaler Kooperation zu den genannten Fragestellungen ermöglichen es am ehesten, neue und weiterführende Erkenntnisse zu gewinnen.

Wie Ng (2012) aus der Warte der asiatisch-pazifischen Region bemerkt, dominiert in der Hochschulpolitik das anglo-amerikanische Paradigma, in welchem Marktorientierung und wirtschaftlicher Pragmatismus leitend sind. Das müsse nicht so sein, sondern jedes Hochschulsystem müsse aus der Sicht der Bedürfnisse der eigenen Region eigene Paradigmen und Programme entwickeln. Das Ziel solle Bildung zum Weltbürgertum sein. In diesem Punkt besteht Einigkeit unter kritisch eingestellten und liberal (im Gegensatz zu neoliberal) orientierten Wissenschaftler*innen in verschiedenen Weltregionen - auch in anglo-amerikanischen Ländern. Internationalisierung kann ihr ganzes interkulturelles, verbindendes Potential wohl besser entfalten, wenn Mobilität vor allem real und nicht vor allem virtuell - digitalisiert - stattfindet.

Apokalyptische Prophezeiungen (avalanche, tsunami) haben sich nicht bewahrheitet, aber die Globalisierung ist nicht aufzuhalten; deren Folgen sollen daher Gegenstand von rationaler Hochschulpolitik für eine bestmögliche Berücksichtigung von Diversität und Internationalität im Einklang mit wissenschaftlichen Forschungsergebnissen und Diskursen werden. Gelingende Konkretisierungen in anderen Ländern und Bildungssystemen können als Inspiration dienen. Die philosophische Arbeit zu einer Politik der Anerkennung von Charles Taylor (1992) hat entscheidend und nachhaltig dazu beigetragen, dass vor allem in den geistes- und sozialwissenschaftlichen Diskursen eine nicht propagandistische, sondern philosophisch basierte Agenda in die Hochschulinstitutionen, zumindest in Teilen, Einzug gehalten hat. Aus zum Teil gescheiterten Programmen, die das Hervorheben von Diversität so sehr zelebriert haben, dass dadurch othering (exzessives, essentialistisches Hervorheben des Andersseins) und Feindschaft zwischen Gruppen entstanden, kann gelernt werden. Unterschiede und besondere Bedürfnisse sollen so wenig wie möglich, aber so viel wie nötig Gegenstand besonderer Aufmerksamkeit und spezifischer Hilfe sein (Sen 1995: xii); dies immer in einem Klima der gegenseitigen Rücksichtnahme. 


\section{Literatur}

Adick, Christel (2012): Transnationale Bildungsorganisationen. In: Tertium Comparationis, 18, 1, S. 82-107.

Allemann-Ghionda, Cristina (2004): Einführung in die vergleichende Erziehungswissenschaft. Weinheim /Basel: Beltz.

Allemann-Ghionda, Cristina (2013): Bildung für alle, Diversität und Inklusion. Internationale Perspektiven. Paderborn: Schöningh.

Allemann-Ghionda, Cristina (2014): Internationalisierung und Diversität in der Hochschule. Zum Wandel von Diskurs und Praxis. In: Zeitschrift für Pädagogik, 60, 5, Thementeil „Internationalisierung, Diversität, Hochschule“, S. 668-680.

Allemann-Ghionda, Cristina (2015): Inklusive Politik und Praxis im internationalen Vergleich. In: Hensen, Gregor/Beck, Anneka (Hrsg.): Inclusive Education. Internationale Strategien und Entwicklungen Inklusiver Bildung. Weinheim/Basel: Beltz Juventa, S. 29-48.

Allemann-Ghionda, Cristina (2017): Interkulturalität, Internationalität, Diversität. Was kann Lehrer_innenbildung anbieten? In: Allemann-Ghionda, Cristina/Kula, Gülbeyaz/ Mignon, Laurent (Hrsg.): Diversität in europäischen Bildungssystemen und in der Lehrer_innenbildung. Frankfurt am Main: Peter Lang, S. 67-88.

Autorengruppe Bildungsberichterstattung (2016): Bildung in Deutschland 2018. Ein indikatorengestützter Bericht mit einer Analyse zu Bildung und Migration. Bundesministerium für Bildung und Forschung und Ständige Konferenz der Kultusminister der Länder der Bundesrepublik Deutschland. Bielefeld: W. Bertelsmann Verlag.

Banks, James A. (Hrsg.) (2009): The Routledge International Companion to Multicultural Education. New York: Routledge.

Barber, Michael/Donnelly, Katelyn/Rizvi, Saad (2013): An Avalanche is Coming. Higher education and the revolution ahead. London: Institute for Public Policy Research.

Bauman, Zygmunt (2000): Liquid Modernity. Cambridge: Polity Press.

Bergmann, Juliana/Grané, Mariona (Hrsg.): La Universidad en la nube - A universidade na nuvem. Laboratori de Mitjans Interactius, Barcelona: Universitat de Barcelona,

Berkeley School of Information (2019): Diversity and Inclusion Resources. https://ischool.berkeley.edu [Zugriff: 24.04.2019].

Brooks, David (2012): The Campus Tsunami. New York Times 03.05.2012. https://www.nytimes.com/2012/05/04/opinion/brooks-the-campus-tsunami.html [Zugriff: 24.04.2019].

Brubaker, Rogers (2015): Grounds for Difference. Cambridge, MA: Harvard University Press.

Bürli, Alois/Strasser, Urs/Stein, Anne-Dore (Hrsg.) (2009): Integration/Inklusion aus internationaler Sicht. Bad Heilbrunn: Klinkhardt.

Cavalli-Sforza, Luigi Luca/Cavalli-Sforza, Francesco (1995): The Great Human Diasporas. The History of Diversity and Evolution. Cambridge, MA: Perseus.

Deardorff, Darla K./Arasaratnam-Smith, Lily (2017): Intercultural Competence in International Higher Education. International Approaches, Assessment, Application. New York \& London: Routledge.

Deutscher Wissenschaftsrat (2017): Empfehlungen zur Internationalisierung von Hochschulen. Berlin: Bundesministerium für Bildung und Forschung.

De Wit, Hans (2013): Reconsidering the Concept of Internationalization. In: International Higher Education, 70, Winter, S. 6-7.

Dietz, Gunther (2007): Keyword: Cultural Diversity. A Guide Through the Debate. In: Zeitschrift für Erziehungswissenschaft 10, 1, S. 7-30. 
European Commission (2019): Erasmus+ Project Integrating Cultural Diversity in Higher Education HE4U2 (Key Action 3). Lifelong Learning Platform. European Civil Society for Education. www.lllplatform.eu [Zugriff: 24.04.2019].

Fischer, Steven Roger (2001). Eine kleine Geschichte der Sprache. Aus dem Englischen von Andreas Simon. (Originalausgabe A History of Language 1999). Frankfurt am Main: Campus.

GeStiK (2019): Gender Studies in Köln. https://gestik.uni/koeln.de [Zugriff: 24.04.2019].

Gutmann, Amy (1997): Democratic Education (first published in 1987). With a New Preface and Epilogue. Princeton (NJ): Princeton University Press.

Habermas, Jürgen (2001): Glauben und Wissen. Friedendensspreis des Deutschen Buchhandels 2001. Frankfurt am Main: Sonderdruck Edition Suhrkamp.

Hallal, Houda (2015): Diversität in der humanmedizinischen Ausbildung. Eine empirische Rekonstruktion kollektiver Orientierungen in den institutionellen Erfahrungsräumen des Medizinstudiums. Marburg: Tectum Verlag.

Harvard Divinity School (2019). Startseite. http://hds.harvard.edu [Zugriff: 11.12.2019].

Heitzmann, Daniela/Klein, Uta (2012): Zugangsbarrieren und Exklusionsmechanismen. In: Klein, Uta/Heitzmann, Daniela (Hrsg.): Hochschule und Diversity. Theoretische Zugänge und empirische Bestandsaufnahme. Weinheim/Basel: Beltz, S. 11-45.

Heyer, Katharina (2021 i.d.B.): What is a Human Right to Inclusive Education? In: Köpfer, Andreas/Powell, Justin J.W./Zahnd, Raphael (Hrsg.): Handbuch Inklusion international. Opladen: Budrich, S. 45-57.

Hüther, Otto/Kosmützky, Anna/Asanov, Igor/Bünstorf, Guido/Krücken, Georg (2020): Massive Open Online Courses after the Gold Rush: Internationale und nationale Entwicklungen und Zukunftsperspektiven. Hannover: Institutionelles Repositorium der Leibniz Universität Hannover, 2020 (LCSS Working Papers ; 4), 107 S. DOI: https://doi.org/10.15488/9775 [Zugriff: 14.07.2020].

iDiS (2019): Internationale Forschungsstelle Disability Studies. https://idis.uni.koeln.de [Zugriff: 24.04.2019].

Iversity (2019): http://iversity.org [Zugriff: 24.04.2019].

Kehm, Barbara M./Schomburg, Harald/Teichler, Ulrich (2012): Funktionswandel der Universitäten: Differenzierung, Relevanzsteigerung, Internationalisierung. Frankfurt am Main: Campus.

Klein, Uta/Heitzmann, Daniela (Hrsg.) (2012): Hochschule und Diversity. Theoretische Zugänge und empirische Bestandsaufnahme. Weinheim/Basel: Beltz Juventa.

Knight, Jane (2001): Issues and Trends in Internationalization. A Comparative Perspective. In: Bond, Sheryl/Lemasson, Jean Pierre (Hrsg.): A New World of Knowledge. Canadian Universities and Globalization. Ottawa: International Development Research Center.

Knight, Jane (2012): Internationalization. Three Generations of Cross-Border Higher Education. Occasional Publications, 38, New Delhi: India International Centre, S. 1-23.

Knobloch, Phillip D. Th. (2014): Internationalisierung als Herausforderung für die Pädagogik. Entwurf eines meta-theoretischen Vermittlungsansatzes zwischen Vergleichender Erziehungswissenschaft und Allgemeiner Pädagogik. In: Zeitschrift für Pädagogik, 60, 5, S. 724-735.

Koch, Hans-Albrecht (2008): Die Universität. Geschichte einer europäischen Institution. Darmstadt: Wissenschaftliche Buchgesellschaft.

Kreft, Anne-Kathrin/Leichsenring, Hannah (2012): Studienrelevante Diversität in der Lehre. In: Klein, Uta/Heitzmann, Daniela (Hrsg): Hochschule und Diversity. Theoretische Zugänge und empirische Bestandsaufnahme. Weinheim/Basel: Beltz Juventa, S. 145-163.

Kwiek, Marek (2017): Europeanisation and Globalization of Higher Education in Central and Eastern Europe: 25 Years of Changes Revisited (1990-2015). Introduction to a Special Issue (with Michael Dobbins). In: European Educational Research Journal, 16, 5, S. 519-528. 
Lanzendorf, Ute/Teichler, Ulrich (2003): Globalisierung im Hochschulwesen - ein Abschied von etablierten Werten der Internationalisierung? In: Zeitschrift für Erziehungswissenschaft, 6, 2, S. 219-238.

Lechner, Frank J./Boli, John (Hrsg.) (2005): World Culture. Origins and Consequences. Oxford: Blackwell.

Marshall, Katherine (2018): Global Education Challenges. Exploring religious dimensions. In: Sivasubramaniam, Malini/Hayhoe, Ruth (Hrsg.) (2018): Religion and Education. Comparative and international perspectives. Oxford: Symposium Books, S. 29-50.

$\mathrm{Ng}$, Shun Wing (2012): Rethinking the mission of internationalization of higher education in the Asia-Pacific region. In: Compare. A Journal of Comparative and International Education, 42, 3, S. 439-459.

Ng, Chi-hung Clarence/Fox, Robert/Nakano, Michiko (Hrsg.) (2016): Reforming Learning and Teaching in Asia-Pacific Universities. Influences of Globalised Processes in Japan, HongKong and Australia. Singapore: Springer.

Nieto, Sonia (2009): Multicultural education in the United States. Historical realities, ongoing challenges, and transformative possibilities. In: Banks, James A. (Hrsg.): The Routledge International Companion to Multicultural Education. New York: Routledge, S. 79-95.

Nussbaum, Martha C. (1998): Cultivating Humanity. A Classical Defense of Reform in Liberal Education. Cambridge, MA: Harvard University Press.

NYU School of Law (2019): Center for Diversity, Inclusion, and Belonging. https://www.law. nyu.edu/centers/belonging [Zugriff: 24.04.2019].

OECD (2018): Programme for International Student Assessment. PISA 2018 Results. http:// oecd.org [Zugriff: 10.12.2019].

Pasqualini, Cristina/Rosina, Alessandro (2016): IN or OUT? Inclusione, innovazione, internazionalizzazione dei millennials italiani. In: Rapporto italiani nel mondo, 2016, Roma: Fondazione Migrantes, S. 139-148.

Picht, Georg (1964): Die deutsche Bildungskatastrophe. Analyse und Dokumentation. Olten/Freiburg: Walter.

Portnoi, Laura M./Bagley, Sylvia S. (Hrsg.) (2014): Critical Perspectives on Global Competition in Higher Education. San Francisco: Jossey-Bass.

Reich, Justin/Ruipérez-Valiente, José A. (2019): The MOOC Pivot: From Teaching the World to Online Professional Degrees. Pots-print Manuscript. Original in Science 363, 6423, S. 130131. http://science.sciencemag.org/content/363/6423/130. [Zugriff: 29.11.2019].

Resch, Katharina/Raschauer, Agnes (2019): Kritische Ereignisse in der Hochschullehre. Ein Beitrag zur Weiterentwicklung von Diversitätskompetenz. In: Die Hochschullehre 5, S. 115-134.

Richter, Caroline (2012): „Black-Box“ Hochschulpersonal. Plädoyer für die Einbindung einer kaum beachteten Zielgruppe. In: Klein, Uta/Heitzmann, Daniela (Hrsg.): Hochschule und Diversity. Theoretische Zugänge und empirische Bestandsaufnahme. Weinheim/Basel: Beltz Juventa, S. 126-144.

Rivers, Damian J. (2010): Ideologies of internationalisation and the treatment of diversity within Japanese higher education. In: Journal of Higher Education Policy and Management 32, 5, S. 441-454.

Salido, Jordi Sancho (2013): Muerte y resurrección de la universidad (again) en manos de la colaboración masiva: avanzar los MOOC. In : Bergmann, Juliana /Grané, Mariona (Hrsg.) : La Universidad en la nube - A universidade na nuvem. Laboratori de Mitjans Interactius, Barcelona: Universitat de Barcelona, S. 263-278.

Santiago, Paulo/Tremblay, Karine/Basri, Ester/Arnal, Elena (2008): Tertiary Education for the Knowledge Society. Volume 2 - Special Features: Equity, Innovation, Labour Market, Internationalisation. Paris: OECD. 
Schriewer, Jürgen (2007): „Bologna“ - ein neu-europäischer „Mythos“? In: Zeitschrift für Pädagogik 53(2), S.182-199.

Sen, Amartya (1995): Inequality Reexamined. New York/Cambridge (MA): Russell Sage Foundation/ Harvard University Press.

SINTER (2019): Soziale Ungleichheiten und Interkulturelle Bildung. https://sinter.uni/koeln.de [Zugriff: 24.04.2019].

Streitwieser, Bernhard Thomas (Hrsg.) (2014): Internationalisation of Higher Education and Global Mobility. Oxford: Symposium Books.

Streitwieser, Bernhard Thomas/Light, Gregory J. (2018): Student Conceptions of International Experience in the Study Abroad Context. In: Higher Education 75, 3, S. 471-487.

Taylor, Charles (1992): Multiculturalism and the „Politics of Recognition“. An Essay. With commentary by Amy Gutmann (Editor), Steven C. Rockefeller, Michael Walzer, Susan Wolf. Princeton: Princeton University Press.

Teichler, Ulrich (2007): Die Internationalisierung der Hochschulen. Neue Herausforderungen und Strategien. Frankfurt am Main: Campus.

Teranishi, Robert T./Bordoloi Pazich, Loni/Knobel, Marcelo/Allen, Walter (Hrsg.) (2015): Mitigating Inequality: Higher Education, Research, Policy, and Practice in an Era of Massification and Stratification (Advances in Education in Diverse Communities: Research, Policies, and Praxis, Vol. 11. Emerald.

University of the Witwatersrand (2019): WICDS (Wits Centre for Diversity Studies). https://wits. ac.za [Zugriff: 24.04.2019].

Universität zu Köln (2019): Internationalisierung / Gender Equality \& Diversity. https://www.portal.uni/koeln.de [Zugriff: 24.04.2019].

Vabø, Agnete/Wiers-Jenssen, Janneke (2014): Internationalization, Diversification and Quality in Higher Education. In: Zeitschrift für Pädagogik 60, 5, S. 710-723.

Välimaa, Jussi/Weimer, Leasa (2014): The Trends of Internationalization in Finnish Higher Education. In: Zeitschrift für Pädagogik 60, 5, S. 696-709.

Vertovec, Steven (2007): Super-diversity and its implications. Ethnic and Racial Studies 29, S. 1024-1054.

Vincent-Lancrin, Stéphan/Pfotenhauer, Sebastian (2012): Guidelines for Quality Provision in Cross-Border Higher Education: Where do we Stand? Paris: OECD, Directorate for Education.

Waldschmidt, Anne (2019): Behindertenpolitik (in) der Europäischen Union: Geschichte und aktueller Stand. In: Degener, Theresia/Miquel, Marc von (Hrsg.): Aufbrüche und Barrieren, Behindertenpolitik und Behindertenrecht in Deutschland und Europa seit den 1970er Jahren. Bielefeld: Transcript, S. 79-105.

Wolfsgruber, Gabi (2015): Soziale Arbeit und soziokulturelle Diversität. Handlungskompetenzen für Fachkräfte der Kinder- und Jugendarbeit. Opladen u.a.: Budrich UniPress Ltd.

Woolf Institute, The (2019): Improving relations between religion \& society through education. http://woolf.cam.ac.uk. [Zugriff: 11.12.2019]. 


\title{
26 Studieren mit Behinderungen im Spannungsfeld zwischen Behindertenrechtskonvention und Neoliberalismus: Entwicklungen im europäischen Hochschulkontext
}

Arthur Limbach-Reich

\begin{abstract}
Inklusion bei Studierenden mit Behinderungen oder mit chronischen Krankheiten erobert zumindest terminologisch im Zuge der UN-Behindertenrechtskonvention nach primärer und sekundärer Bildung nun auch den tertiären Bildungsbereich in Europa. Global lässt sich im europäischen Vergleich hier eine eher zögerliche Umsetzung beobachten. Das Vorliegen unterschiedlicher Datengrundlagen macht jedoch eine differenzierte Interpretation der empirischen Befunde schwierig. Unterschiedliche Programme und bildungspolitische Maßnahmen spiegeln sich in unterschiedlichen Inklusionsquoten wider. Dennoch verharren die Teilhaberaten Studierender mit Behinderungen ubiquitär unterhalb der Gesamtpopulation. Eine inklusive Öffnung der Universitäten steht in Konflikt mit neoliberalen und an Exzellenz ausgerichteten Hochschulpolitiken.
\end{abstract}

\section{Inklusion: Unschärfen im universitären Diskurs}

Eine der Komplexität gerecht werdende Darstellung gegenwärtiger Perspektiven Studierender mit Behinderungen im Hochschulbereich steht vor mehreren Herausforderungen: Dies wurzelt einerseits im Inklusionsbegriff, der sich zwar als allgegenwärtiges Synonym moderner (Bildungs-)Politik im internationalen wie nationalen Diskurs eine Vormachtstellung erobert hat, ohne dass aber gleichzeitig ein ausreichend einheitliches Inklusionsverständnis entwickelt wurde (Ahrbeck 2014; Limbach-Reich 2016; Wansing 2015). Andererseits wächst erst allmählich und zögerlich die Bereitschaft inklusive Perspektiven auf den tertiären Bildungssektor auszudehnen (Knauf 2015). Darüber hinaus konturiert sich die Dreigestalt der Inklusion (Inklusion als gesellschaftspolitisches Ziel, Inklusion als menschenrechtliches Prinzip und Inklusion als zu präferierende Methode um eine inklusive Gesellschaft zu erreichen) wie diese in der UN-BRK (2006) aufscheint, bisher noch recht unscharf im universitären Kontext. Weder als theoretischer Ansatz inklusiver Hochschulbildung und Didaktik, noch als ethisches Grundprinzip einer ,Hochschule für Alle' und schließlich auch kaum im Kontext empirischer Untersuchungen zur Umsetzung der Inklusion an Hochschulen liegen allgemein anerkannte Ausarbeitungen vor (s. Allemann-Ghionda 2021 i.d.B.). Koinzident erwächst aus neoliberaler Perspektive ein gänzlich anderes Universitätsverständnis, das auf 
individuelle Leistungsfähigkeit, Humankapital, Marktorientierung, Employability und Kompetitivität der Student*innen ${ }^{1}$ und Hochschulen zielt.

Hochschulen als Orte der Inklusion zu konzipieren erscheint einerseits erleichtert gegenüber vorgeordneten Schulen, da bis auf sehr wenige Ausnahmen keine spezialisierten Institutionen existieren ${ }^{2}$, die überwunden werden müssten, aber andererseits auch erschwert, da akademische Bildung traditionell hoch selektiv organisiert wurde und wird. So lässt sich trotz rhetorischen Formulierungen wie „Hochschule für Alle“ (Hochschulrektorenkonferenz 2009) mit Katzenbach (2013) vermuten, dass bei genauerem Hinsehen klar wird, dass hier gar nicht „alle“ gemeint sind, insbesondere nicht Personen mit kognitiven Beeinträchtigungen.

Universitäten zeichnen sich im Wettringen um Exzellenz und Rankingplätze eher durch Publikationsindizes, Drittmittelquoten und Auftragsforschung aus, als durch Inklusionsquoten, Barrierefreiheit und Teilhabegerechtigkeit. In den international führenden Vergleichslisten, deren Bekannteste die seit 2003 publizierte, sogenannte Shanghai Liste (Academic Ranking of World Universities, ARWU 2018) sein dürfte, findet sich kein Indikator, der den Einbezug von Studierenden mit Behinderungen abbildet. Gleiches gilt für das seit 2004 erscheinende und seit 2010 in Kollaboration mit der Unternehmensberatungsagentur PwC konzipierte Times Higher Education World University Ranking aus Großbritannien (PwC/Times 2019). Auch die im deutschsprachigen Raum von der Bertelsmann Stiftung 1994 ins Leben gerufene Bewertungsagentur CHE Ranking (Centrum für Hochschulentwicklung, 2014) wartet nicht mit Indikatoren auf, die sich mit der Frage der Inklusion und dem Umgang mit Heterogenität oder Behinderung beschäftigen.

Nach der Lissaboner Erklärung des Europäischen Rats (2000), erfordert die ökonomische Situation und der demographische Wandel, Menschen mit Behinderung in den Arbeitsmarkt zu integrieren, was unter anderem mit der Erhöhung der erzielten Bildungsabschlüsse erleichtert werden solle. Tertiäre Bildung und Inklusion werden hier prioritär unter einer ökonomischen Prämisse betrachtet, die sich auch die Frage von Studieren mit Behinderung zu stellen habe. Nicht zuletzt in diesem Sinne hebt die Europäische Kommission mit der EUStrategie 2020 für Menschen mit Behinderungen die Notwendigkeit der Beseitigung von Barrieren und Hindernissen hervor.

Auf den ersten Blick mag einer ökonomischen Sichtweise durchaus ein positiver Impuls der Inklusion entspringen, auf den zweiten Blick aber wird klar, dass Inklusion und Bildung nicht als Werte an sich, als Ausschöpfung des individuellen Entwicklungspotentials betrachtet werden, die auch ohne eine entsprechende berufliche Einmündung als Menschenrecht anzusehen seien, sondern als ökonomisch notwendige Beiträge zum Wirtschaftswachstum. So wird beispielsweise in Frankreich mit der Charte Université/handicap (2007) eine Studienwahl vorausgesetzt, die auch eine Beschäftigungsmöglichkeit erwarten lässt. Diese Charta definiert und postuliert Maßnahmen zur Inklusion von Student*innen mit Behinderungen, die befähigt und bestrebt sind, ein Universitätsstudium erfolgreich abzuschließen und befähigt sind, sich beruflich zu integrieren. Die Prämisse der Befähigung zur Einbindung in den Arbeitsmarkt macht deutlich, dass keine bedingungslose Öffnung der Universitäten für alle Personen unabhängig der Art und Schwere ihrer Behinderungen angestrebt wird, sondern

1 Studierende und Student*innen wird hier meist synonym gebrauch. Bisweilen verweist das nominalisierte Partizip Präsens stärker auf die Tätigkeit des Studierens (gerade bemüht sein), während „Student“ den Status (z.B. beim Nutzen kultureller Einrichtungen, Campusleben, Sport, usw.) stärker mitschwingen lässt und bei entsprechenden Übertragungen aus der Literatur der Vorzug gegeben wurde.

2 Die Gehörlosenuniversität Gallaudet University in Washington, D.C. kann hier als Beispiel genannt werden. 
dass einer späteren beruflichen Tätigkeit dezisive Entscheidungsmacht eingeräumt wird. Ein Studium als berufsunabhängiges Bildungsangebot wahrzunehmen, scheint nicht angedacht zu sein (Charte Université/handicap 2007: 2). Ähnlich formuliert das Universitätsgesetz in Luxemburg bezüglich Student*innen mit Behinderungen:

Nutzer*in mit besonderen pädagogischen Bedürfnissen ist jede*r Nutzer*in mit einer Behinderung oder besonderen Beeinträchtigung, deren Auswirkungen den normalen Studienfortschritt behindern oder ihn*sie daran hindern, in Prüfungssituationen die erworbenen Kenntnisse und Fähigkeiten zu nutzen, wobei die Behinderungen und Einschränkungen durch die in Abschnitt 39 vorgesehenen angemessenen Vorkehrungen behoben werden können (Loi du 27 juin 2018 ayant pour objet l'organisation de l'Université du Luxembourg, Art. 1, 12; Übersetzung durch den Autor). ${ }^{3}$

Auch hier wird demnach Inklusion verkürzt auf Studierende, deren eingeschränkte Anpassungsfähigkeit sich mit den vorgegebenen Maßnahmen kompensieren lässt. In Deutschland gibt es keinen einheitlichen durch das Hochschulrecht definierten Behindertenbegriff, sodass der Begriff der jeweiligen Landesbehindertengleichstellungsgesetze maßgebend ist (Gaentzsch 2020).

Zwischen der hier kursorisch dargestellten neoliberalen Marktorientierung und menschenrechtlich begründeter Inklusion im Hochschulbereich spannt sich europaweit ein dynamisches Feld unterschiedlicher nationaler Hochschulpolitiken und Bildungsrealitäten. Für alle Staaten, die sich der Umsetzung der UN-BRK (2006) verpflichtet haben, bietet deren menschenrechtliche Perspektive eine gemeinsame Orientierung bezüglich der Inklusion von Menschen mit Behinderungen an. Problematisch erscheint jedoch, dass weder Behinderung noch Inklusion in der UN-BRK explizit definiert werden, was unterschiedlichen nationalen Interpretationen Vorschub leistet und somit einen internationalen Vergleich erschwert. Nicht zuletzt ist es wohl dieser Unschärfe geschuldet, dass sich Universitäten meist am medizinischen Paradigma orientieren und ärztliche Gutachten (Diagnosen) als Voraussetzung für angemessene Vorkehrungen (reasonable accommodation) und Nachteilsausgleiche verlangen. Nach den Recherchen der EADSNE (2009) ${ }^{4}$ gehören hierzu Universitäten in Deutschland, Griechenland, Ungarn, Island, Malta, den Niederlanden, Slowenien und Spanien, die strikt vom unterschiedlichen Grad an funktionaler oder struktureller Beeinträchtigung ausgehen. In Ländern wie Belgien, Estland, Finnland, Irland, Lettland, Litauen, Norwegen, Portugal, der Slowakischen Republik, der Tschechischen Republik, und dem Vereinigten Königreich wird diese einseitige Ausrichtung am medizinischen Paradigma teilweise überwunden und Student*innen mit Migrationshintergrund, sozialbenachteiligte Student*innen, Angehörige ethnischer Minderheiten, oder besonders Begabte oder Hochleistungssport betreibende Student*innen werden ebenfalls in besondere Förderprogramme, Nachteilsausgleiche und Vorkehrungen miteinbezogen (EADSNE 2009; Ebersold et al. 2011).

„usager à besoins éducatifs particuliers » : tout usager présentant une déficience ou une incapacité particulière dont les répercussions entravent une progression normale dans les études ou l'empêchent de faire valoir, lors des épreuves d'évaluation, les connaissances et compétences acquises et qui est telle que ces entraves et empêchements puissent être palliés par les aménagements raisonnables prévus à l'article 39“" (Loi du 27 juin 2018 ayant pour objet l'organisation de l'Université du Luxembourg, Art. $1,12)$.

4 Datenbank „Barrierefreie Hochschule“ (HEAG) der Europäischen Agentur für Entwicklungen in der sonderpädagogischen Förderung (EADSNE), zur Zeit nicht abrufbar. 


\section{Historische Markieritems universitärer Inklusion}

Inklusion im Kontext von Universitäten kann einerseits unter dem Prinzip eines allgemeinen Wachstumsprozesses verstanden werden, der sich aus der Hereinnahme von zuvor ausgeschlossenen Gruppen speist, was sich in historischer Hinsicht exemplarisch an der Aufnahme afro-amerikanischer Student*innen an US-Universitäten aufzeigen lässt (Parsons 1966). Andererseits widerspricht eine umfassende Inklusion tertiären Bildungseinrichtungen, die ihr Renommee nicht zuletzt daraus beziehen, dass nur die vorgeblich Begabtesten (und somit nicht jeder oder alle) aufgenommen werden. Während in Kontinentaleuropa Eliteuniversitäten $^{5}$ durchaus auch kritisch gesehen werden, verweisen angelsächsische Universitäten offensiver auf ihre eingeschränkte Zugänglichkeit, wodurch Fragen der Inklusion stärker juristisch als im Sinne einer allgemeinen Bildungszugänglichkeit betrachtet werden (Equality Act 2010) ${ }^{6}$.

Stodden und Roberts (2014) stellen in einem historischen Überblick ausgewählte Meilensteine universitärer Inklusion dar, beginnend mit der Zulassung von Frauen zum Studium bis zu aktuellen Entwicklungen. Das Privileg, sich an einer Universität einschreiben zu dürfen war, ausgenommen weniger Einrichtungen in Italien, wo bereits früh Universitäten für Frauen offenstanden, weitgehend bis ins 20. Jahrhundert lediglich Männern vorbehalten. Als ein erster Wendpunkt auf dem Weg einer inklusiven Öffnung der Universitäten wird meist die Schweiz genannt, wo bereits um 1860 bis 1870 Frauen sukzessive an den meisten Universitäten zu Studium und Examen zugelassen wurden (Wecker 2007) ${ }^{7}$. Nach England, Russland und Skandinavien und weiteren europäischen Staaten ermöglichte das Großherzogtum Baden erstmals um 1900 Frauen den vollen Zugang zum Universitätsstudium (Dreier 2000). Auch aktuell lässt sich noch immer eine vergleichsweise geringe Präsenz von Frauen unter der Professorenschaft feststellen. So betrug der Anteil an Professorinnen 2018 deutschlandweit lediglich $24,7 \%$ mit einem starken Nord-Süd-Gefälle: $20,5 \%$ in Bayern und $32,3 \%$ in Berlin (Statistisches Bundesamt 2019). Nach dem ersten und stärker noch nach dem zweiten Weltkrieg rückten vereinzelt Personen mit körperlichen (kriegsbedingten) Einschränkungen und etwas später auch mit sensoriellen Einschränkungen in den Blickpunkt einer Öffnung der universitären Bildungsprogramme. Mit dem Einsetzen der sogenannten Bildungsexpansion zu Beginn der 1960er Jahre und verstärkt unter dem Leitbegriff der Chancengleichheit setzen schließlich umfassende Integrationsbemühungen ein, die auch Student*innen mit Behinderungen einschlossen. Rechtliche und administrative Regelungen zu Integration und Nicht-Diskriminierung, angestoßen durch die Studentenbewegungen, setzten diesen Trend weiter fort. Standen zu dieser Zeit sichtbare Behinderungen im Mittelpunkt der Diskussionen, so verlagerte sich insbesondere im angelsächsischen Raum ab den 1980er Jahren der Schwerpunkt zunehmend auf weniger sichtbare Behinderungen wie psychische Beeinträchtigungen und Teilleistungsstörungen (,,hidden disabilities “). In Europa etablierte sich mit den Bolog-

5 Hierzu passt die Meldung, dass der französische Präsident Emmanuel Macron konkrete Schritte eingeleitet hat, die nationale Elite Hochschule l'École nationale d'administration (ENA) zu schließen (Floc'h 2019).

6 Gleichstellungsgesetz der Regierung des Vereinigten Königreichs (Equality Act 2010) http://www.legislation.gov.uk/ukpga/2010/15/contents [Zugriff: 25.01.2020].

7 Einschränkend sei darauf hingewiesen, dass bspw. Marie Vögtlin als erste Medizinstudentin der Universität Zürich nur unter Miteinbezug ihres Vaters akzeptiert wurde (Köpfer, Andreas 2019, mündliche Mitteilung). 
na-Reformen (ab 1999) ein gemeinsamer Hochschulraum, in dessen Folge auch der Situation Studierender mit Behinderungen ein besonderes Augenmerk entgegengebracht werden sollte. Das sogenannte Brügge-Memorandum der Europäischen Kommission (2008) hob unter anderem als Kernpunkte hervor:

- die Gewährleistung internationaler Mobilität der Studierenden und Absolvent*innen mit Behinderungen;

- die Chancengleichheit von Menschen mit Behinderungen an den Hochschulen;

- die Partizipationsmöglichkeiten behinderter Student*innen an den Angeboten der Hochschule und an den sie betreffenden Entscheidungen auf allen Ebenen;

- die Verankerung des Aspekts der Barrierefreiheit in der Qualitätssicherung und schließlich die Verbesserung des Erkenntnisstandes über die Situation Studierender mit Behinderungen.

Ausdrücklich bezieht sich das Memorandum auf die aus der UN-BRK (2006) erwachsenden Verpflichtungen der Schaffung eines inklusiven Bildungssystems auf allen Ebenen. In Fortführung der Erklärungen von Jomtien (UNESCO 1990) und Salamanca (UNESCO 1994) wird in der Incheon Erklärung (UNESCO 2015) die Gewährleistung inklusiver, gleichberechtigter und hochwertiger Bildung und Möglichkeiten lebenslangen Lernens für alle als nachhaltiges Entwicklungsziel (sog. SDG-4) ${ }^{8}$ formuliert. Powell (2016) weist jedoch mit Recht darauf hin, dass im Zuge des Bologna-Prozesses vielfach die soziale Dimension wie der Zugang zu Hochschulbildung im Kontext sozialer Benachteiligung oder Behinderung trotz entsprechender Verlautbarungen selten mitgedacht wurde - und noch immer nicht wird, wie man wohl hinzufügen muss.

Eine weitere bisher kaum thematisierte Herausforderung besteht in der Frage des Einbezugs von Personen mit kognitiven Beeinträchtigungen im Rahmen universitärer Inklusion. $\mathrm{Zu}$ den wenigen Universitäten, die hier Maßnahmen anbieten, gehört das Trinity College Dublin (Irland), das in Zusammenarbeit mit dem University College of Cork ein Zertifikat für Student*innen mit einer kognitiven Beeinträchtigung entwickelt hat. In Island hat die Universität Reykjavik ein ähnliches Programm entwickelt. In Malta bietet das Malta College of Arts, Science \& Technology (MCAST) ein Programm an, das teilweise aus dem Europäischen Sozialfonds finanziert wird und darauf abzielt, die unabhängigen Lebenskompetenzen von Menschen mit einer intellektuellen Beeinträchtigung zu entwickeln. Ein ähnliches Projekt gibt es an der Universität Elche in Spanien. An der pädagogischen Hochschule in Salzburg (o.J.) bietet das BLuE-Hochschulprogramm inklusive tertiäre Bildungsmaßnahmen für Menschen mit kognitiver und oder psychischer Beeinträchtigung an.

\section{Universitäre Inklusion im internationalen Vergleich}

Im Gefolge der europäischen Politik zur Inklusion von Menschen mit Behinderungen entstanden mehrere umfassende Studien mit dem Ziel, belastbare Daten zur Umsetzung der Inklusionsziele zu liefern. Einschlägige Studien wurden durch die Organisation für wirt-

8 SDG (Sustainable Development Goal) von der UNESCO (2015) verabschiedete Entwicklungsziele: Ziel 4: Inklusive, gleichberechtigte und hochwertige Bildung gewährleisten und Möglichkeiten lebenslangen Lernens für alle fördern. 
schaftliche Zusammenarbeit und Entwicklung (OECD 2003), die Weltbank (World Bank 2011) und die Weltgesundheitsorganisation (WHO 2011) beauftragt. Der von der Weltgesundheitsorganisation und der Weltbank gemeinsam herausgegebene Weltbericht Behinderung (WHO/World Bank 2011) greift den Themenbereich tertiärer Bildung jedoch nur am Rande auf und widmet sich an nur wenigen Stellen der Frage des Universitätszuganges für Menschen mit Behinderungen. So wird festgehalten, dass von den postsekundär weiterlernenden Student*innen mit Behinderungen sich $8 \%$ an einer Hochschule oder Universität mit vierjährigem Studium eingeschrieben hatten, während für die Gesamtpopulation ein Anteil von $29 \%$ angegeben wird (ebd.: 208).

Der nationale wie internationale Vergleich empirischer Daten ist hier jedoch dadurch eingeschränkt, wie dies Ramsden (2005) bereits bezogen auf das Vereinigte Königreich kritisch anmerkte, dass keine allgemein anerkannte Definition von Behinderungen vorliegt, Selbsteinschätzungen der Befragten zu Behinderung und deren Ausmaß dominieren, Kategorisierungen und Verkodungen in den Erhebungen voneinander abweichen und keine einheitliche Identifikation bezüglich universitärer Ausbildung, Hochschule oder tertiärer Bildung vorliegt. So weist die Organisation der nationalen tertiären Bildungssysteme spezifische Unterschiede auf. „University“ und „College“ im angelsächsischen Bereich sind nicht ohne Weiteres mit deutschen Universitäten und Hochschulen gleichzusetzen. Auch das französische oder belgische System des „Enseignement Supérieur“, das italienische „Sistema di istruzione superiore“, die spanische „Educación Superior“ und das polnische „system szkolnictwa wyższego" sind nicht deckungsgleich. Während beispielsweise die meisten paramedizinischen Berufe in Deutschland bisher keine akademische Ausbildung darstellen, sind z.B. Ergotherapeut*in, Hebamme, Krankenpfleger*in, Logopäd*in und Physiotherapeut*in international meist akademische Ausbildungsgänge.

Zum Vergleich der Bildungsabschlüsse wird auf das ISCED ${ }^{9}$-Referenzsystem der UNESCO (1997; 2011) zurückgegriffen. Hier ist jedoch im zeitlichen Verlauf ein Vergleich erschwert, da 2013 eine Umstellung des Referenzsystems erfolgte (von ISCED 1997 mit den Stufen 5-6, bis zum Jahre 2013, auf ISCED 2011 mit den Stufen 5-8 für Daten ab dem Jahr 2014; in Deutschland ab 2015 in Gebrauch). Während ISCED Stufe 5 (Kurzes tertiäres Bildungsprogramm) im OECD-Durchschnitt bei 10\% eines Jahrganges liegt, spielt diese Kategorie so gut wie keine Rolle in Deutschland (Statistische Ämter des Bundes und der Länder 2018).

\subsection{OECD-Studien}

Es ist bezeichnend für die Dominanz einer (neoliberal ausgerichteten) ökonomischen Sichtweise auf Bildung, dass die Organisation für wirtschaftliche Zusammenarbeit und Entwicklung als Herausgeber für internationale Vergleichsstudien universitärer Bildungssysteme in vorderster Reihe steht. Eine erste Studie der OECD (2003) beschäftigte sich mit der Inklusion von Menschen mit Behinderungen im tertiären Bildungsbereich auf der Grundlage von Daten und Berichten aus Ontario (der bevölkerungsreichsten Provinz Kanadas), Frankreich, dem Vereinigten Königreich, der Schweiz und Deutschland. Zusammenfassend konstatiert die

9 Die Internationale Standardklassifikation des Bildungswesens wurde bereits in den 1970er Jahren entwickelt und mehrfach modifiziert, um Bildungsabschlüsse klassifizieren zu können. 
von Serge Ebersold geleitete Arbeit sichtbare Erfolge bei der Einbeziehung von Student*innen mit Behinderungen auf universitärer Ebene, da vorangegangene Maßnahmen zur Bekämpfung von Diskriminierung wirksam würden, Unterstützungsprogramme verbessert worden seien und institutionelle Strategien sich nun zunehmend etablierten. Gleichzeitig hebt die Studie aber auch hervor, dass ein grundlegender Mangel an zuverlässigen Statistiken bestehe und es generell Schwierigkeiten bei Finanzierungsmöglichkeiten von Maßnahmen, die Student*innen mit Behinderungen zugute kämen, gebe. Schließlich wird festgehalten, dass das Verständnis bezüglich Student*innen mit Behinderungen noch nicht ausreichend entwickelt sei und Fragen des Zuganges zum Arbeitsmarkt wesentlich seien, insbesondere dort, wo hohe Studiengebühren erhoben werden (OECD 2003).

Nachfolgende Veröffentlichungen der OECD (2011), die auf einer Datenkompilation von Ebersold (2008) basieren, bestätigen, dass behinderte Menschen häufiger als nicht behinderte während ihres Hochschulstudiums mit Diskontinuitäten des Studienverlaufs konfrontiert sind, häufiger als Teilzeitstudent*innen eingeschrieben sind, ihr Studium häufiger bereits nach dem ersten Jahr abbrechen und insgesamt seltener als nichtbehinderte Student*innen einen Abschluss erzielen. Wie im OECD-Bericht festgehalten wird, weisen in Deutschland behinderte Student*innen während ihres Studiums tendenziell mehr Um- und Abmeldungen auf, benötigen mehr Zeit für ihr Studium, wechseln häufiger als nicht behinderte Student*innen ihre Kurse (23\% vs. $19 \%)$ oder ihre Universität (18\% vs. $16 \%)$ und erreichen seltener einen Hochschulabschluss. In Ermangelung geeigneter Unterstützungssysteme wirken sich die bestehenden Schwierigkeiten stärker auf Student*innen mit schwereren oder komplexeren Beeinträchtigungen aus, wie insbesondere Berichte aus Österreich und Litauen aufzeigen (Ebersold 2008: 13). Auffallend häufig sind nach Angaben aus Frankreich Studierende mit Behinderungen in Studiengängen mit ungewissen Berufswegen und einem erwartbar höheren Arbeitslosigkeitsrisiko oder geringerem Lohnniveau vertreten (Ebersold 2008).

\subsection{Erhebungen der Europäischen Agentur zur Zugänglichkeit des Hochschulstudiums}

Im Rahmen des europäischen Projektes „Führer zur Zugänglichkeit des Hochschulstudiums“ (Higher Education Accessibility Guide, HEAG) der Europäischen Agentur für sonderpädagogische Förderung und inklusive Bildung (ehemals EADSN aktuell EADSNIE (European Agency for Development in Special Needs Education and Inclusive Education)) wurden Barrierefreiheit, Zugänglichkeit und Studiermöglichkeiten jeweils national erhoben, um so Studieninteressierten und Student*innen mit Behinderungen eine Orientierungshilfe zu geben. Der Führer zur Zugänglichkeit des Hochschulstudiums erfasste erstmals 2005 bis 2006 die Barrierefreiheit von Universitäten und Hochschulen und wurde 2009 aktualisiert. Eine ursprünglich im zweijährigen Turnus vorgesehene Wiederauflage wurde jedoch nicht realisiert. Auf den Landesseiten der eigens eingerichteten Internetplattform wurden Informationen zum landesspezifischen Ansatz in Bezug auf das Thema Studieren mit Behinderung bereitgestellt. Zusätzlich wurde über Angebote spezifischer, unterstützender Dienste für Studierende mit Behinderung an Hochschulen informiert. Der HEAG lieferte Studierenden mit Behinderung insbesondere auch relevante Informationen zu einem geplanten Auslands-Semester/Studium. Alle Informationen wurden auf Englisch und in den jeweiligen Landessprachen verfasst. $\mathrm{Zu}$ 
den Teilnehmerstaaten zählten: Belgien (Flandern und Wallonien), Dänemark, Deutschland, Estland, Finnland, Frankreich, Griechenland, Island, Irland, Kroatien, Lettland, Litauen, Luxemburg, Malta, Niederlande, Norwegen, Österreich, Polen, Portugal, Slowakei, Slowenien, Spanien, Schweden, Schweiz, Tschechische Republik, Ungarn, Vereinigtes Königreich (England, Nordirland, Schottland und Wales) und Zypern. ${ }^{10}$

\subsection{Berichte des Akademischen Netzwerks europäischer Experten im Behindertenbereich (ANED)}

Einen stärkeren Fokus auf die europäische Sozial- und Bildungspolitik für Menschen mit Behinderungen hat die 2007 gegründete ANED-Expertengruppe ${ }^{11}$, die jährliche Berichte zur Situation von Menschen mit Behinderungen sowie einen verdichteten gesamteuropäischen Synthesereport publiziert. Ebersold, Schmitt und Priestley (2011) fassen die verschiedenen nationalen ANED Berichte in einer Synthese über inklusive Bildung im tertiären Sektor für junge Menschen mit Behinderungen in Europa, zusammen. Die nachfolgenden Informationen und Daten beziehen sich insbesondere auf die Publikationen des ANED-Netzwerks, wobei es hier die besondere Art der Datengenerierung zu berücksichtigen gilt. Partizipationsraten im tertiären Bildungsbereich beruhen auf Antworten aus Haushaltsbefragungen des EUSILC $^{12}$ und LFS ${ }^{13}$ wodurch Personen, die in Institutionen leben, nicht erfasst werden. Daher werden die Partizipationsraten tendenziell überschätzt. Aufgrund des relativ geringen Bevölkerungsanteils institutionalisierter Personen, ist hier jedoch von einer geringen Größenordnung der Überschätzung im Bereich weniger als ein Prozent auszugehen.

Auf der Grundlage der Haushaltsbefragungen (EU-SILC und LFS) für das Referenzjahr 2014 wird die Quote der Personen im Alter von 30-34 Jahren, die einen Hochschul- oder einen gleichwertigen Abschluss haben mit 41,2\% (EU-SILC), respektive mit 37,9\% (LFS) angegeben (Grammenos 2017). Die Zahl der Menschen mit Behinderungen im gleichen Altersbereich (30-34), die eine tertiäre oder gleichwertige Ausbildung abgeschlossen haben, beträgt 29,7\%. Dies entspricht einer Differenz von 12,9 Punkten gegenüber der Referenzgruppe ohne Behinderungen (42,6\%), was eine Vergrößerung um 0,1 Punkte im Vergleich zu 2013 (12,8\%) darstellt. Hierbei muss jedoch die bereits angesprochene Modifikation der ISCED Skalen berücksichtigt werden: 2013 ISCED Stufen 5-6 versus 2014 ISCED Stufen 58. Im weiteren zeitlichen Verlauf lässt sich noch keine kontinuierliche Verringerung der Differenz beobachten, wenngleich sowohl die Partizipationsraten allgemein als auch die

10 Zurzeit ist die Internet-Plattform nicht erreichbar, jedoch ist eine Wiederauflage des Programms geplant.

11 Academic Network of European Disability Experts ist ein Akademisches Netzwerk europäischer Experten im Behindertenbereich (ANED), das von der Europäischen Kommission bis 2019 finanziert wurde und von thematischen Berichterstattern und externen nationalen Beratern unterstützt wird.

12 Die EU-Statistik über Einkommen und Lebensbedingungen (EU Statistics on Income and Living Conditions - EU-SILC) stützt sich auf Haushaltsbefragungen primär zu Einkommen und sozialen Lebensbedingungen innerhalb der Mitgliedstaaten der Europäischen Union sowie Norwegen und Island (Eurostat 2016).

13 Der europäische Labour Force Survey (Arbeitskräfteerhebung der Europäischen Union) basiert auf Haushaltsbefragungen in den 28 Mitgliedstaaten der Europäischen Union, Beitrittskandidaten und Ländern der Europäischen Freihandelszone, die seit 1983 durchgeführt werden. https://ec.europa.eu/eurostat/de/web/microdata/european-union-labour-force-survey [Zugriff: 28.05.2019] 
Teilhabe der Menschen mit Behinderungen im tertiären Bildungsbereich sich erhöht haben. Für 2017 liegt mit 39,0\% bisher nur eine zusammenfassende Statistik der Teilhabe an tertiärer Bildung auf einer etwas anderen Datenbasis vor ${ }^{14}$ (s. Abb. 6).

Im empirischen Vergleich der Teilhabe von Menschen mit Behinderungen an tertiären Bildungsangeboten auf europäischer Ebene zeigen sich deutlich unterschiedliche Partizipationsraten, die teilweise quer zum Stand der Inklusion/Integration im schulischen Bildungsbereich stehen. So nimmt beispielsweise Italien trotz seiner Vorreiterrolle und bereits Jahrzehnte früher einsetzenden ,integrazione scolastica“ mit hohen Inklusionsquoten im Schulbereich einen nur unterdurchschnittlichen Platz im tertiären Bildungsbereich ein. Daten aus einzelnen Ländern machen deutlich, dass junge Menschen mit Behinderungen generell weniger deutlich von der Bildungsexpansion profitieren als Menschen ohne Behinderungen: Während in Irland die Gesamtzahl der Student*innen zwischen 2000 und 2006 um 80\% stieg, ist die Zahl der Studierenden mit Behinderungen nur um 2,6\% gestiegen. In Norwegen haben 9\% der jungen Menschen mit Behinderungen eine Hochschulausbildung absolviert, gegenüber $21 \%$ der Gesamtbevölkerung dieses Alters. Im Vereinigten Königreich absolvierten 28\% der behinderten jungen Menschen im Alter von 19 Jahren eine Hochschulausbildung, verglichen mit 41\% der nicht behinderten jungen Menschen (Grammenos 2017).

Abbildung 1: Anteil der Menschen mit Behinderungen im Alter 30-34 mit einem Abschluss im tertiären Bildungsbereich im europäischen Ländervergleich (2015).

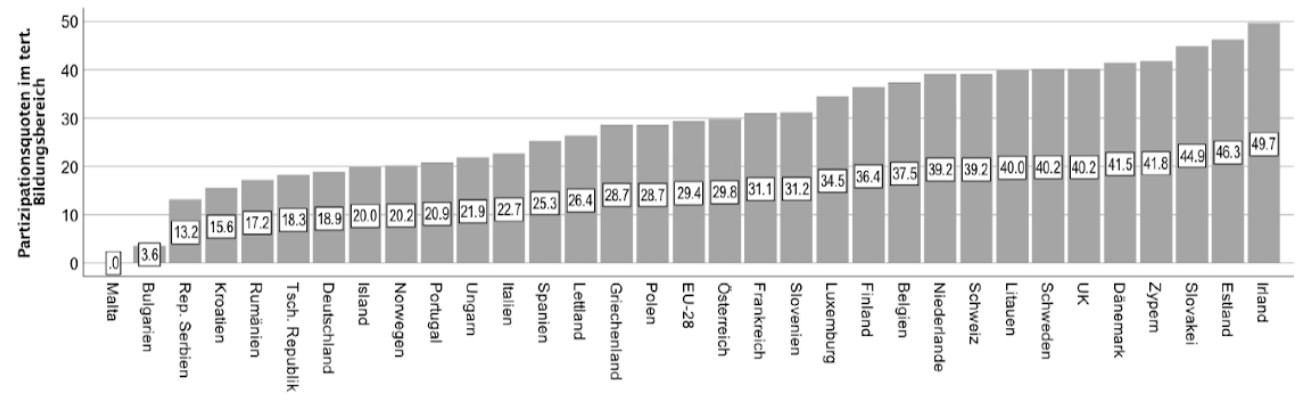

Quelle: EU-SILC 2015, eigene Darstellung

Wie die Abbildung 1 zeigt, beläuft sich die Spanne der Teilhaberaten von unter einem Prozent in Malta bis hinauf zu annähernd $50 \%$ in Irland. Da bis auf Bulgarien und Malta eine sehr dichte Datenfolge vorliegt, lässt sich über eine grobe Kategorisierung in unteres Drittel (über 10\% bis unter 25\% mit Serbien, Kroatien, Rumänien, Tschechische Republik, Deutschland, Irland, Norwegen Portugal Ungarn und Italien) mittleres Drittel (ab 25\% bis unter 40\% mit Spanien, Lettland, Griechenland, Polen, Österreich, Frankreich, Slowenien, Luxemburg, Finnland, Belgien, Niederlande und der Schweiz) und oberes Drittel (ab 40\% mit Litauen, Schweden Vereinigtes Königreich Dänemark, Zypern, Slowakei, Estland und Irland) kaum eine bedeutungstragende Strukturierung der Daten vornehmen.

In einem weiteren Analyseschritt wurde daher der spezifische Anteil der Menschen mit Behinderungen zum Anteil der Menschen ohne Behinderungen mit einem tertiären

14 Das Europäische Statistikamt EUROSTAT (2019) ermittelt auf der Basis aller EU-28 Bürger im Altersbereich 25-34 Jahre, mit und ohne Behinderungen einen Prozentsatz von 39,0\%. http://appsso.eurostat.ec.europa.eu/nui/submitViewTableAction.do [Zugriff: 28.05.2019] 
Bildungsabschluss ins Verhältnis gesetzt und eine sogenannte „Behindertenlücke“ (disability gap) errechnet.

Abbildung 2: Behinderungsspezifische Differenz der Teilhabe an tertiären Bildungsabschlüssen im europäischen Ländervergleich (2015).

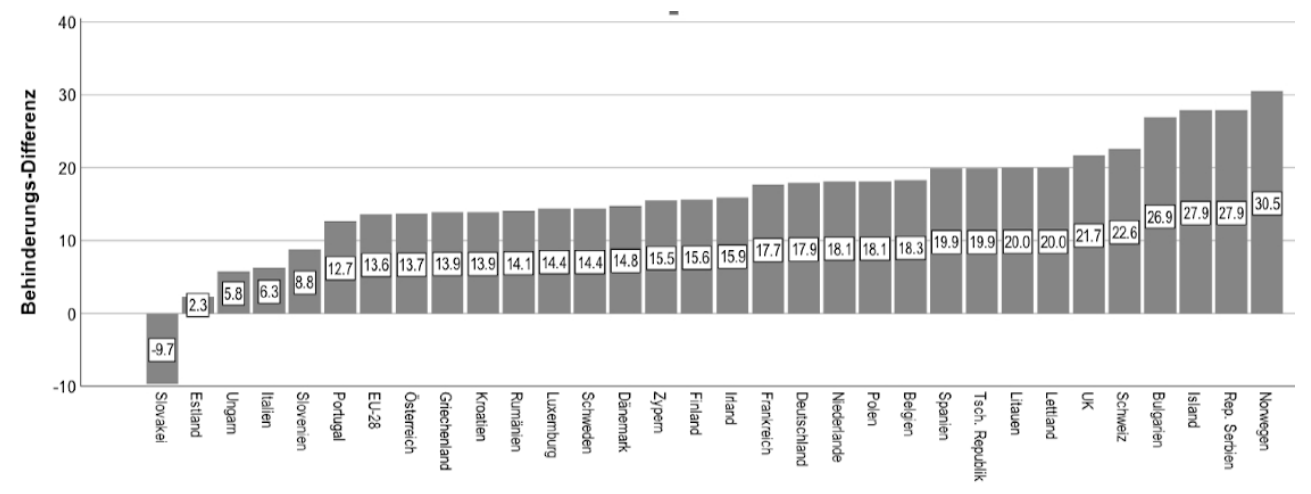

Quelle: EU-SILC-UDB, 2015, eigene Darstellung

Mit Ausnahme der Slowakei zeigen alle Länder eine Lücke in der Teilhabe von Menschen mit Behinderungen im tertiären Bildungssektor auf. Die Varianz der differentiellen Partizipation speist sich aus den unterschiedlichen nationalen Bemühungen, diese Lücke zu verringern, wobei jeweils verschiedene nationale Maßnahmen und Programme in Gang gesetzt wurden: Wie die nationalen ANED-Berichte ${ }^{15}$ zeigen, bestehen in den meisten Ländern zwar nationale Rahmengesetze und rechtliche Vorgaben allgemeiner Natur - beziehungsweise speziell für das Hochschulwesen bestimmte Antidiskriminierungsvorschriften. Einen Automatismus der Beibehaltung bereits im Sekundarbereich gewährter Assistenzmaßnahmen und Nachteilsausgleiche gibt es insbesondere in Ländern mit föderalen Bildungsstrukturen wie in Deutschland aber auch in Luxemburg nicht. In einigen Ländern, in denen eine Verpflichtung zur Unterstützung analog zur Sekundarstufe gegeben ist, werden darüber hinaus zusätzliche Ressourcen sowohl für Studierende als auch für Hochschulen bereitgestellt, um den Zugang behinderter Menschen zur Hochschulbildung zu fördern. So sind beispielsweise im Vereinigten Königreich die Hochschulen gesetzlich verpflichtet, ein Gleichstellungssystem für Menschen mit Behinderungen einzurichten und jährlich über die ergriffenen Initiativen und die erzielten Fortschritte zu berichten. Meist liegt es jedoch in der Verantwortung der Universitäten und nicht der Behörden, behinderten Studierenden Unterstützung und Vorkehrungen anzubieten, die ihnen Zugang zu den Kursen und deren erfolgreiche Teilnahme ermöglichen. In Malta können beispielsweise die Universitäten motorisierte Rollstühle oder Sprachsynthesizer während des Studiums für Studierende bereitstellen, die nicht über eine solche Ausrüstung verfügen. Aus Spanien wird berichtet, dass Universitäten Gebärdendolmetscher*innen, Bücher in Blindenschrift, Notiznehmer*innen und andere Hilfsmittel zur Verfügung stellen können. In Finnland besteht keine staatliche Verpflichtung zu behinderungsrelevanten Unterstützungsleistungen, die Universitäten können aber Sonderregelungen für Aufnahmeprüfungen, Campuszugänglichkeit und Lernförderung treffen. Im Falle der Slowakei wird die Einrichtung eines Zentrums zur Unterstützung der Hochschulbildung im 
Rahmen eines Sonderprojekts erwähnt, das sich an Schüler*innen, Lehrkräfte und Eltern richtet, um Studierende unterstützen zu können.

Wie aus den nationalen ANED-Berichten weiterhin hervorgeht, haben sich einige Länder im Hinblick auf die Unterstützung der Zulassung zur Universität für ein präferenzielles Einschreibungsverfahren entschieden. So gibt es beispielsweise in Portugal eine spezielle Zulassungsquote von 2\% für körperlich oder sensoriell eingeschränkte Studierende und in Griechenland besteht eine Quote von 5\% auf Universitäts- respektive Fachbereichsebene. In Deutschland wird behinderten Bewerber*innen ein bevorzugter Zugang bei der Vergabe von Studienplätzen im Falle gleicher Eignung zugesichert. In Irland und Ungarn gibt es ein Bonussystem bei Aufnahmeprüfungen. In Norwegen können behinderte Studienbewerber*innen, die noch keinen Sekundarschulabschluss haben, einen vorläufigen Zugang zum Hochschulbereich erhalten, vorausgesetzt, sie erhalten ihren Schulabschluss noch während des ersten Semesters ihres Universitätsstudiums. Im Vereinigten Königreich bieten Colleges und Universitäten Vorbereitungskurse für Personen an, die keinen Hochschulzugang besitzen, wobei sie Menschen mit Behinderungen explizit einschließen. In Ländern, in denen Zulassungsprüfungen und Eingangstests vorgeschrieben sind, können bei diesen Eingangstests den Studierenden besondere Unterstützungen und Nachteilsausgleiche gewährt werden. In der Tschechischen Republik ist gegebenenfalls eine Adaptation der Aufnahmeprüfungsverfahren vorgeschrieben, wenn Studienbewerber*innen dies wünschen. In Dänemark haben Schulabsolvent*innen mit sonderpädagogischem Unterstützungsbedarf auch als Student*innen Anspruch auf Unterstützung und Beratung auf der Universität. In Irland finanziert ein Fonds für behinderte Student*innen angepasste Lernmittel (z.B. Computer, Drucker, Scanner, Diktiergeräte), personelle Unterstützung (z.B. persönliche Assistenz, Notiznehmer*in ${ }^{16}$, pädagogische Unterstützung, spezifische Kurse) und Transportkosten.

Die finanzielle Unterstützung für die Aufnahme Studierender mit Behinderungen an Hochschulen variiert je nach nationaler Organisation der Finanzierung der Universitäten. ANED-Berichte aus Ländern, in denen Studiengebühren zu zahlen sind, heben hervor, dass hier Studierende mit Behinderungen in der Regel Zugang zur gleichen finanziellen Unterstützung wie nichtbehinderte Studierende haben. Norwegen gewährt einen staatlich finanzierten Studienkredit der Staatsbank, der bei erfolgreichem Bestehen der Prüfungen teilweise in ein Stipendium umgewandelt wird (ähnlich auch z.B. Dänemark und Schweden), was auch beantragt werden kann, wenn Studierende mit Behinderungen aus gesundheitlichen Gründen gezwungen sind, ihr Studium abzubrechen. Dies gilt jedoch nicht für Urlaubs- oder Freisemester im Zusammenhang mit einer Behinderung. In Dänemark gleicht die „Behindertenzulage“ einen Einkommensverlust aufgrund eines erschwerten Zugangs zur Arbeitstätigkeit für Studierende mit Behinderungen aus. In einigen Ländern kann die Einschreibegebühr bei Studierenden mit Behinderungen reduziert oder ganz gestrichen werden (z.B. in Deutschland, Island und Spanien). In manchen Ländern beantragt die Universität nach der Einschreibung eines behinderten Studierenden eine spezielle, direkte staatliche Förderung, die dem ermittelten Bedarf der Studierenden entspricht. Die Universität wird dann die notwendigen Unterstützungen leisten (z.B. in Spanien und Lettland). Andere Länder gewähren individuell Studenten bzw. Studierenden mit Behinderungen eine Unterstützungsleistung, die dann mit Hilfe einer Behindertenabteilung oder eines Behindertenbeauftragten organisiert wird oder individuell zu bezahlen ist. Einschränkungsspezifische finanzielle Unterstützung für das Studium gibt es zum Beispiel für Student*innen mit Hörbehinderungen, die damit eine*n 
Gebärdendolmetscher*in engagieren können. In Island sind es Nichtregierungsorganisationen von Menschen mit Hörbehinderungen oder Sehbehinderungen, die Student*innen Zuschüsse für ihre besonderen Bedürfnisse gewähren. In Litauen können sehbehinderte Studierende Sonderzuschüsse für technische Geräte erhalten, die entweder von der Universität, von Sponsoren oder von einer Behindertenorganisation bereitgestellt werden. Dem slowakischen Bericht zufolge wird die Unterstützung für behinderte Studierende durch einen von Universitäten eingerichteten Sonderfonds (Fonds zur Unterstützung von Studierenden mit Behinderungen) gewährt, der aus verschiedenen Töpfen gespeist wird. In einigen Ländern (Tschechien, Dänemark, Deutschland, Griechenland, Ungarn, Island und Slowenien) haben sich Sponsorensysteme für Studierende mit Behinderungen etabliert.

Über einen längeren Beobachtungszeitraum verfolgt die ANED-Gruppe die Partizipation von Studierenden mit Behinderungen im tertiären Bildungsbereich.

Abbildung 3: Personen mit Behinderungen mit einem tertiären Bildungsabschluss (EU28), 2006-2016.

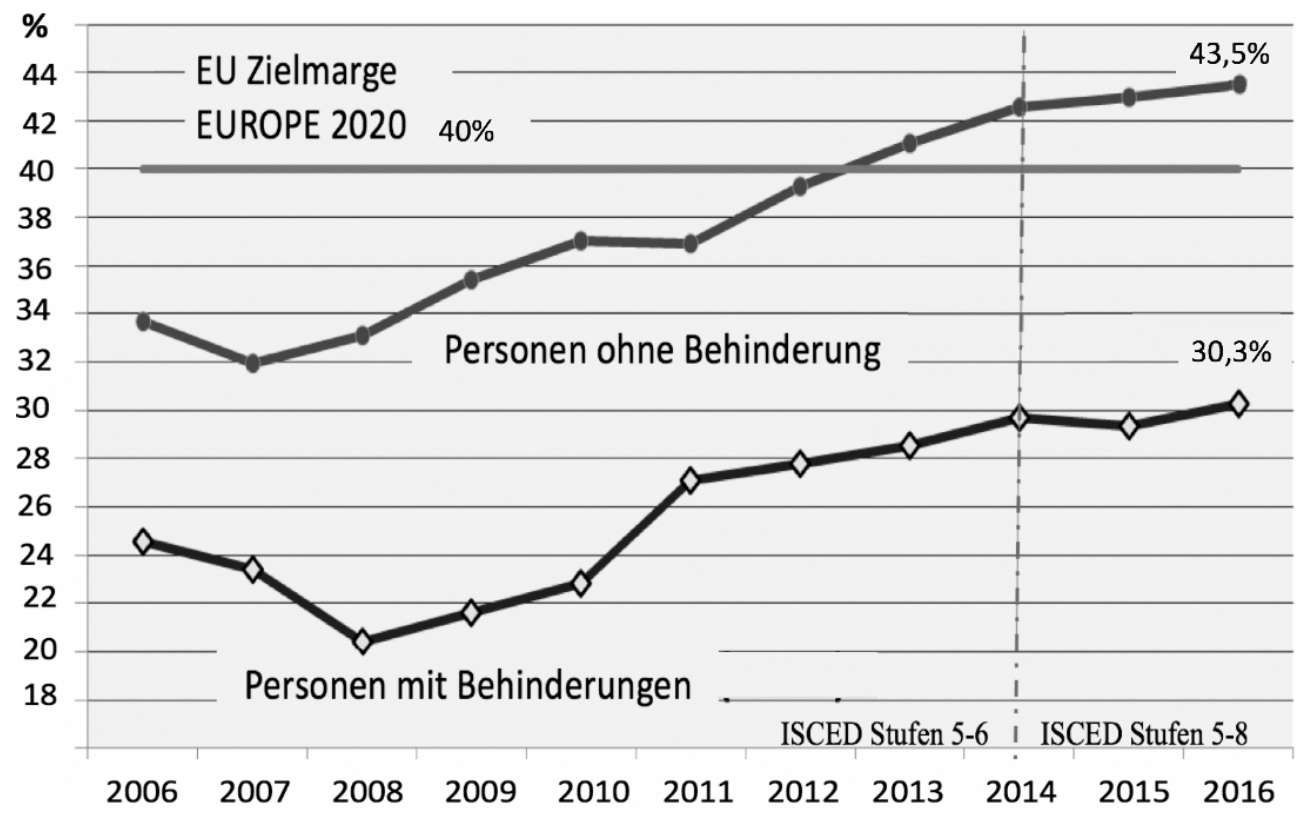

Quelle: Grammenos 2019, 15 EU-SILC-UDB Daten, Modifikationen durch den Autor

Wie die Darstellung zeigt, lässt sich langfristig eine deutliche Zunahme des Anteils von Personen mit einem universitären Abschluss beobachten. Dennoch liegt dieser Anteil stets und noch immer deutlich unterhalb der Rate von Personen ohne Behinderungen, was auf noch immer bestehende Herausforderungen hinweist. Auch der deutliche Rückgang des Anteils behinderter Personen gegenüber nicht Behinderten im Zuge der globalen Finanzkrise (20072010) zeigt an, dass Personen mit Behinderungen einem höheren Risiko unterliegen, in Krisenzeiten aus Bildungsprozessen ausgeschlossen zu werden. 
Abbildung 4: Behinderung und akademischer Abschluss für ausgewählte Länder (2010- 2015)

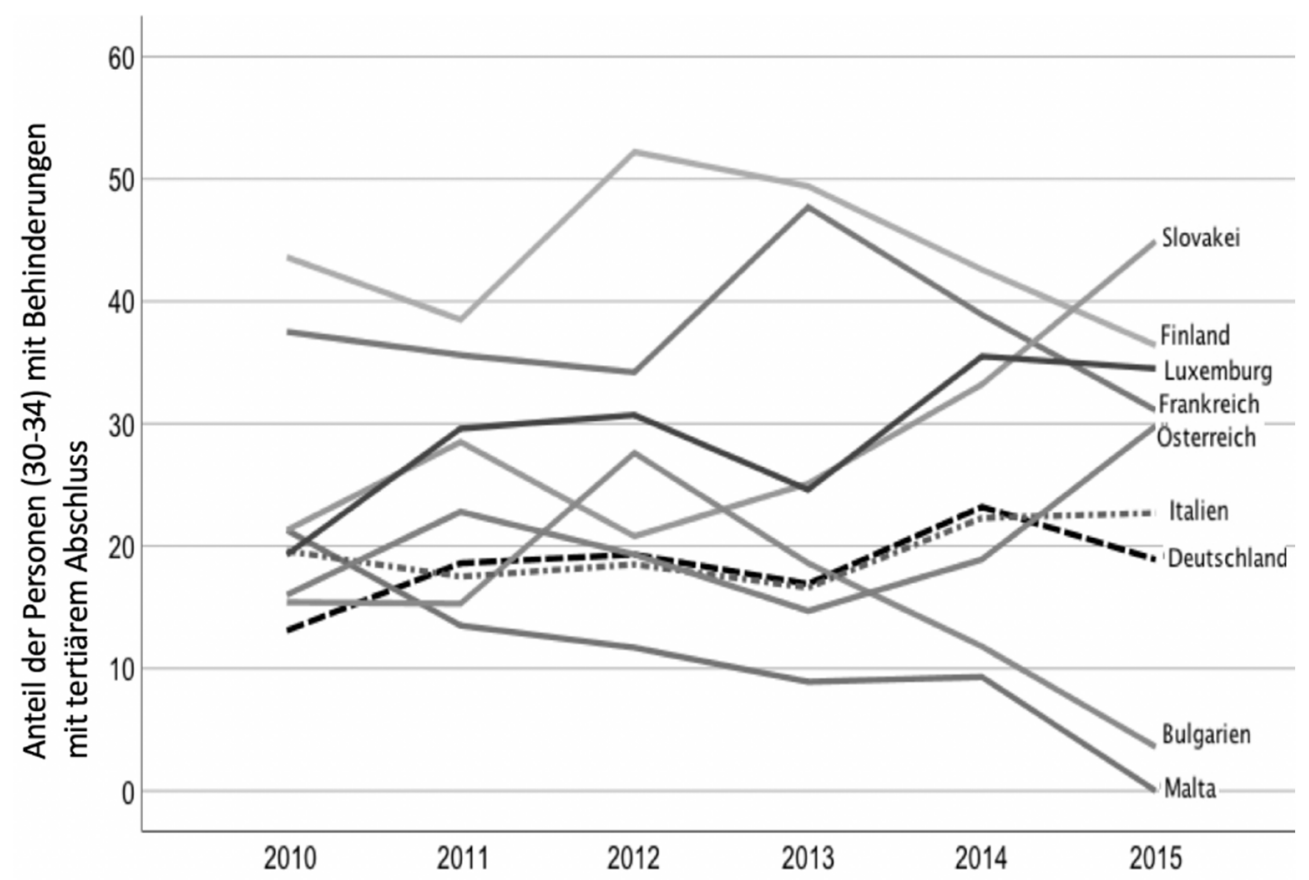

Quelle: ANED 2010-2015, eigene Darstellung

Anhand der ausgewählten neun Länder ${ }^{17}$ lassen sich insbesondere ab 2012 vier verschiedene Muster der Entwicklung aufzeigen:

- $\quad$ kontinuierlich steigende Anteile bei ursprünglich eher niedrigem Ausgangsniveau (Österreich und Slowakei),

- in etwa auf gleichem Niveau verharrend bei eher unterdurchschnittlichem Niveau (weitgehend paralleler Verlauf von Italien und Deutschland),

- $\quad$ fallende Werte bei bereits niedriger Ausgangslage (Bulgarien und Malta)

- zurückgehende Partizipationswerte bei ursprünglich hohem Ausgangsniveau (Finnland und Frankreich)

Diese kurz skizzierte Gesamtschau der von den ANED-Expert*innen zusammengetragenen Daten der Teilhabe von Student*innen mit Behinderungen macht deutlich, dass hinter den recht unterschiedlichen empirischen Teilhaberaten entsprechend unterschiedliche Ansätze und Angebote stehen.

17 Auf den Einbezug aller Länder wurde hier aus Gründen der Übersichtlichkeit verzichtet. 


\section{Befragung Studierender mit Behinderungen}

Eine Reihe nationaler wie internationaler Untersuchungen gehen von der direkten Befragung Studierender aus, so der EUROSTUDENT VI Survey ${ }^{18}$, der im Wesentlichen auf OnlineBefragungen Studierender aus 29 europäischen Ländern basiert (DZHW 2018). Insgesamt schwankt nach diesem Datensatz der Anteil der Studierenden mit Behinderungen zwischen $7 \%$ bis 39\%. Einen Anteil von weniger als 15\% wird in Italien, Österreich, Slowakei, Malta, Frankreich, Serbien und Rumänien angegeben, während mehr als 25\% für Island, die Niederlande und Schweden vermeldet werden. ${ }^{19}$

Abbildung 5: Studierende mit Behinderungen/chron. Krankheiten im Befragungszeitraum, ausgewählte europäische Staaten, 2016-2018

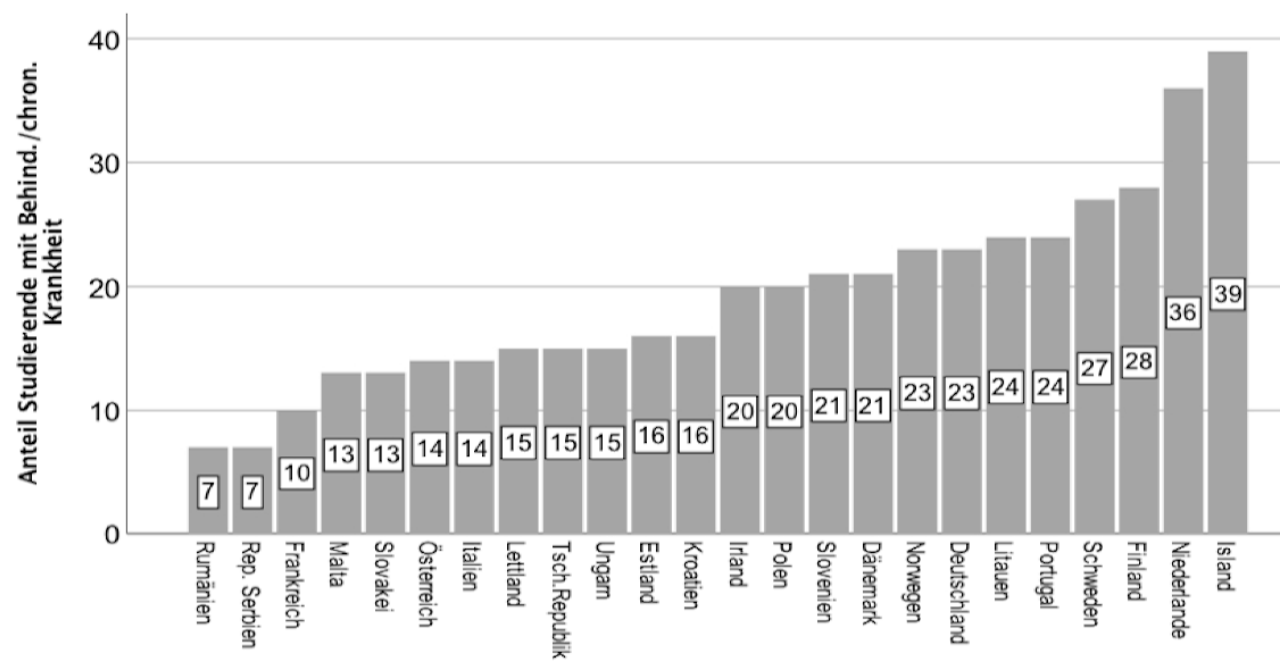

Quelle: EUROSTUDENT VI (2016-2018), eigene Darstellung

Nicht alle Studierende mit Behinderungen sehen durch ihre Behinderung/Krankheit auch ihre Studienmöglichkeiten eingeschränkt oder behindert. Anhand einer Einstufungsskala wurden der Prozentsatz der Studierenden errechnet, die sich eingeschränkt beziehungsweise stark eingeschränkt sehen (,limited or severely limited“/,,big or quite big obstacle“).

18 DZHW (2018) Social and Economic Conditions of Student Life in Europe EUROSTUDENT VI 20162018. Synopsis of Indicators.

19 Die entsprechende Frage (5.8) lautet: „Bitte geben Sie an, ob Sie eine Behinderung, Beeinträchtigung, ein langanhaltendes Gesundheitsproblem oder eine Funktionseinschränkung haben.“ 
Abbildung 6: Studierende deren Behinderung das Studium erschwert (2016-2018)

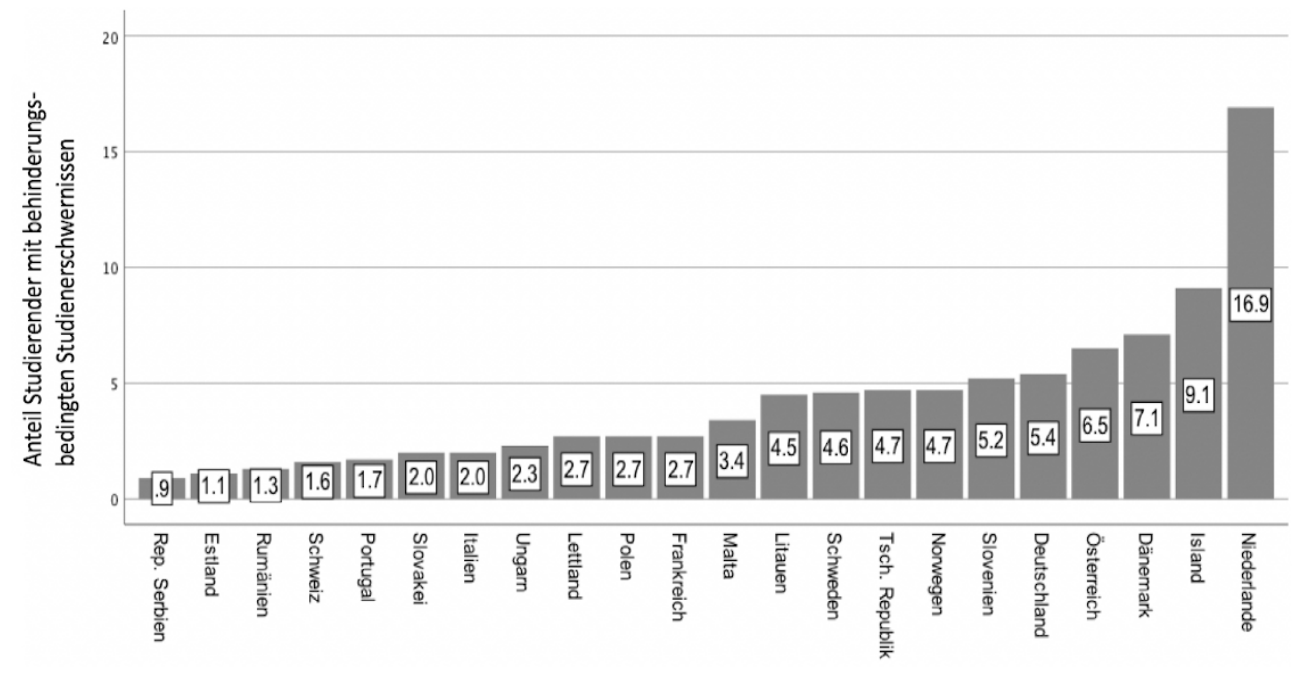

Quelle: EUROSTUDENT VI, eigene Darstellung

Die Interpretation der Daten ist jedoch durch die hohe Schwankungsbreite der Rücklaufquoten $(1,5 \%$ bis $66 \%)$, beziehungsweise aufgrund fehlender Angaben zum Rücklauf in einigen Teilnehmerstaaten, eingeschränkt. Einige Länder wie Belgien, Griechenland, Spanien und das Vereinigte Königreich, die im ANED-Verbund vertreten sind, haben an den Erhebungen der EUROSTUDENT-Surveys zudem nicht teilgenommen. Luxemburg nahm ab 2019 teil. Schließlich zeigt sich, dass die Zufriedenheit der Studierenden mit der Betreuung von Land $\mathrm{zu}$ Land variiert und sich insbesondere Studierende mit nicht sichtbaren Behinderungen am wenigsten zufrieden äußern.

Eine zusammenfassende Interpretation der Befunde ist schwierig, da die nationalen Kennzahlen hoch variieren und ebenso die verschiedenen Indizes auf nationaler Ebene unterschiedlich ausfallen. In Deutschland beispielsweise werden Teilleistungsstörungen (ADHS, Dyslexie und Dyskalkulie) kaum erfasst, während diese Beeinträchtigungsgruppe in angelsächsischen Ländern zu den häufigsten Störungen respektive Behinderungen zählt. Auch eine Betrachtung über den zeitlichen Verlauf liefert kein einheitliches Bild. Für 18 Länder lassen sich die Daten aus früheren Erhebungen (EUROSTUDENT Survey V 20122015) mit den aktuellen Werten (Survey VI 2016-2018) vergleichen. In fast der Hälfte der Länder (45\%) hat sich der Anteil der Studierenden mit Behinderungen, die auf Hindernisse in ihrem Studium stoßen, nicht verändert (Irland, Norwegen, Lettland, Kroatien, Österreich, Polen, Slowakei, Slowenien). Eine leichte Zunahme wird aus Dänemark, Malta, Rumänien, Schweden, der Tschechischen Republik und Ungarn berichtet (1-2\%). Eine leichte Abnahme der Teilhabe vermelden Litauen, Estland und Serbien (ebenfalls im 1-2 Prozentbereich). Aus den fast 40\% der Länder, in denen höhere Anteile von Studierenden mit Behinderungen zu verzeichnen sind, ragen die Niederlande mit einem Anstieg um 4\% hervor. Hier scheint eine Neuregelung der Unterstützung verantwortlich zu sein. 


\section{Beratungsstellen für Studierende mit Behinderungen}

Die im Positionspapier der Universitäten der europäischen Hauptstädte (UNICA) ${ }^{20}$ festgehaltenen Mindeststandards haben viele Universitäten mittlerweile von sich aus oder aufgrund gesetzlicher Verpflichtungen erfüllt und entsprechend eine Kontaktperson, eine*n Behindertenbeauftragte*n, eine*n Koordinator*in benannt oder eine Abteilung für behinderte Studierende eingerichtet. Die Aufgabenbereiche dieser Personen sind von Land zu Land und auch innerhalb der Länder von einer Universität zur anderen sehr unterschiedlich ausgestaltet. Über die Beratungsaufgabe hinaus sind sie auch in einigen Ländern für die Zugänglichkeit zuständig und sorgen dafür, dass die notwendigen Änderungen an den Gebäuden vorgenommen werden. In manchen Ländern, wie z.B. in Griechenland und Spanien, können sie einen Behindertendienst anbieten und direkt andere spezialisierte Dienste, wie Gebärdenübersetzung, persönliche Assistenz, oder Notiznehmer organisieren. Bisweilen sind sie auch Ansprechpartner*innen für Anbieter (und für die Installation) von technischen Geräten für Menschen mit Behinderungen. Behindertenbeauftragte können auch als Ansprechpartner*innen für das wissenschaftliche Personal fungieren, wenn ein*e Student*in einer Anpassung in den Prüfungsverfahren oder im Curriculum bedarf. Sie können auch Leitlinien erstellen und über bewährte Verfahren berichten. An den Universitäten, an denen Behindertenkoordinator*innen oder -büros gut etabliert und mit Ressourcen ausgestattet sind, stellen sie die wichtigste Ressource dar, von der die Studierenden für Information, Beratung, Fürsprache und Koordination der praktischen Unterstützung abhängig sind. Seale (2014: 26) fasst eine Reihe von Untersuchungen mit der Feststellung zusammen: „The most common prais is for support provided by central disability services and professionals who work with them." Die Existenz solcher Unterstützungsmechanismen bleibt jedoch sehr lückenhaft und wird nicht ausreichend wahrgenommen. Die meisten Länder bieten einen rechtlichen Rahmen um Hochschulen und Universitäten bei der Verbesserung ihrer Zugänglichkeit (Barrierefreiheit) zu unterstützen. Irland gewährt 1\% des jährlichen Budgets für den Hochschulbereich für die Unterbringung benachteiligter Gruppen (einschließlich behinderter Studierender). In Norwegen müssen 5\% der staatlichen Unterhaltsbeihilfen für Universitäten für den Bau von barrierefreien Gebäuden verwendet werden. Finanzielle Anreize können auch darauf abzielen, pädagogische Innovationen, die Qualifizierung des Personals oder die Forschung im Bereich der Hochschulbildung und Ausbildung behinderter junger Erwachsener zu unterstützen. So finanziert beispielsweise der 2006 gegründete irische New Strategic Innovation Fund Projekte zur Unterstützung einer Bildungspolitik, um die Qualität der Ausbildung und das akademische Niveau der Student*innen zu verbessern und das lebenslange Lernen zu fördern. In einigen Ländern haben sich auch nationale wie internationale Netzwerke herausgebildet, die Universitäten und Hochschulen in Inklusionsanliegen unterstützen, wie z.B. das Nationale Büro für behinderte Student*innen (SKILL) im Vereinigten Königreich oder das Disability Advisers Working Network (DAWN) in Irland. In der Großregion Saar-Lor-Lux und Wallonien haben die Behindertenbeauftragen eine gemeinsame Charta zu Studierenden mit Behinderungen entwickelt, nach der Universitäten die an einer Universität der Großregion

20 UNICA: Network of Universities from the Capitals of Europe. Der recht lose Zusammenschluss der Universitäten der Hauptstädte Europas entwickelte in Warschau 2007 anlässlich der Konferenz Bildung für Alle (Education for all, 10.-12. September 2009) ein Positionspapier zu Mindeststandards der Inklusion Studierender mit Behinderungen (UNICA 2007). 
gewährten Unterstützungsmaßnahmen ohne weitere Prüfung auch in der jeweiligen nationalen Hochschule beibehalten sollen (Charta Universität der Großregion 2013) ${ }^{21}$.

\section{Szenarien universitärer Inklusion}

Eine mögliche Erklärung für die beschriebenen unterschiedlichen Entwicklungen mag in der Verfolgung unterschiedlicher Strategien der Umsetzung der UN-BRK verwurzelt sein. Eine Sammlung diesbezüglicher Ansätze, Konzepte und Organisationsentwicklungen finden sich z.B. im Sammelband „Inklusionssensible Hochschule“ (Dannenbeck et al. 2016). Mit Fokus auf die deutschsprachige Hochschullandschaft werden sowohl theoretische Grundlagen als auch bereits bestehende praktische Umsetzungen inklusiver Strukturen diskutiert.

Einen Blick in die Zukunft wagt Helen Knauf (2015) in ihren Darstellungen zu drei ineinander verschränkten, möglichen Szenarien der Umsetzung der Inklusion im Bereich Hochschule: (a) die Universität als barrierefreies Lernumfeld, (b) die Universität als eine von Diversity-Management getriebene Organisation und (c) das Konzept einer inklusiven Universität.

a) Staaten, die Inklusion im Hochschulbereich primär als Einführung und Gewährleistung von Nachteilsausgleichen, Barrierefreiheit und via gesetzlicher Verankerung von vernünftigen Vorkehrungen betrachten, beziehen sich hierbei häufig auf entsprechende Maßnahmen, die bereits im sekundären Bildungsbereich umgesetzt wurden. Als Beispiele wären hier Luxemburg und länderspezifisch deutsche Universitäten zu nennen. Dieses Vorgehen stellt insofern einen Fortschritt dar, als dass Studierende mit Behinderungen sich nun auf ein Recht auf Anpassungen berufen können und weniger als Bittsteller erscheinen müssen. Insbesondere in Prüfungsangelegenheiten haben sich hier Maßnahmenbündel etabliert, die von globaler Prüfungszeitverlängerung bis hin zu differenzierten, störungsspezifischen Adaptationen reichen (Gattermann-Kasper 2014). Aber auch im Bereich der Forschung und Lehre werden zunehmend barrierefreie Ansätze diskutiert (Orr/Bachman Hammig 2009). Nationale Gesetzgebungen zur Barrierefreiheit machen es in der Regel erforderlich, dass auch Universitäten und deren Infrastrukturen eine möglichst barrierefreie Nutzung erlauben. Die Beschränkung des Inklusionskonzeptes auf Nachteilsausgleiche und Modifikationen der Lehr-Lern-Angebote, erlaubt die grundsätzliche Ausrichtung der Universitäten als Elitenausbildungsstätte zu erhalten und erfordert keine radikale Umstrukturierung universitärer Aufgabenstellung. Andererseits fehlen häufig soziale Begleitmaßnahmen und Stigmatisierungsprozesse können folgen, so dass Studierende auf die Wahrnehmung ihres Rechts auf vernünftige Vorkehrungen verzichten. Schließlich besteht die Gefahr einer mangelnden Bereithaltung adaptiver Vorkehrungen. Werden aus Kostengründen Angebote und technische Hilfsmittel erst dann realisiert, wenn eine Nachfrage gestellt wird (bedarfsorientierte Anschaffung), so antizipieren Studieninteressierte mit Behinderungen möglicherweise einen lokalen Mangel an Barrierefreiheit und entscheiden sich für eine andere Universität, wodurch keine Nachfrage entsteht und Barrieren bestehen bleiben.

b) Ausgehend von Modellen an angelsächsischen und skandinavischen Universitäten werden Aspekte der Inklusion Studierender mit Behinderungen unter dem Konzept der

21 Universität der Groß-Region http://www.uni-gr.eu/de/warum-unigr/nuetzliche-infos/studium-und-behinderung [Zugriff: 02.06.2019] 
Vielfalt (Diversity-Management) behandelt. Ausgehend von Verschiedenheitsdimensionen wie ethnische Zugehörigkeit, religiöse Orientierung und Geschlecht wird angestrebt, möglichst vielen Verschiedenheitsaspekten (vgl. Allemann-Ghionda 2021 i.d.B.) z.B. über Präferenz- und Quotensysteme gleichzeitig gerecht zu werden. Stabsstellen für Gleichstellungsbeauftragte und Behindertenbeauftragte haben sich an den meisten Universitäten fest etabliert. Quotenregelungen für Studierende mit speziellem pädagogischen Förderungsbedarf existieren jedoch nur in wenigen Ländern (Griechenland, Italien, Portugal). Vielfalt umfasst in Belgien auch Hochleistungssportler, die einen besonderen Förderbedarf anmelden und Studienmodifikationen beantragen können. Aktuell werden auch Asylbewerber*innen und Geflüchtete in universitäre Programme der Vielfalt miteinbezogen. Die Inklusion Studierender mit Behinderungen im Rahmen eines generellen Ansatzes der Vielfalt zu betrachten, birgt die Gefahr, dass hier insbesondere in Zeiten knapper finanzieller Ressourcen interne Verteilungskonflikte entstehen. Studierende mit Behinderungen als relativ kleine Gruppe geraten hier möglicherweise schnell aus dem Blickfeld, wenn ökonomische Gesichtspunkte wie Kosteneffizienz und ,return on investment" in den Vordergrund gestellt werden, wie dies in neoliberal ausgerichteten Universitäten zu beobachten ist.

c) Inklusionssensible Universitäten stehen im engen Bezug zur UN-BRK, indem sie die Schaffung inklusiver Strukturen für alle Personen mit Behinderung unabhängig der Art und des Ausmaßes der Behinderung als Menschenrecht betrachten und anstreben. Dies bezieht sich damit auch auf Personen mit Lernleistungsstörungen und mit kognitiven Beeinträchtigungen. Nur in einigen Ländern wie in Irland, Österreich und Spanien finden sich hierzu spezielle Angebote. Inklusion wird darüber hinaus nicht auf Nachteilsausgleiche beschränkt, sondern auch auf soziale Aspekte und Freizeiteinrichtungen im Umfeld der Universitäten erweitert. Die inklusive Öffnung der Universitäten für Menschen mit Behinderungen, unabhängig der Art und des Ausmaßes der Behinderung, erfordert eine grundlegende Umgestaltung und Umdeutung des gesellschaftlichen Auftrags an Universitäten. Hier erwächst die Herausforderung, dass inklusive Universitäten sich ihrer ausschließlichen Elitebildungsfunktion entledigen und ihre Fixierung auf Employability abbauen sollten. Der latente Widerspruch zu einer neoliberal organisierten Universität ist evident. Zunehmend bieten Universitäten neben den akkreditierten Programmen auf BA, MA und PhD-Ebene Zertifikatskurse an, die auch Personen offenstehen, die nicht den gängigen Zulassungskriterien entsprechen und somit eine Öffnung im Sinne zunehmender Inklusion erlauben.

Platte und Schultz (2011: 246) präzisieren als Zielsetzungen inklusiver Strukturen an Hochschulen - als Orte der Zukunftsgestaltung - die Verfügbarkeit von angemessener personeller und materieller Ausstattung (Availability), die Zugänglichkeit auch unter erschwerten Bedingungen - wirtschaftliche, bauliche und informationstechnische Barrierefreiheit (Accessibility), die Qualität von Bildung in Bezug auf Inhalt und Form - Hochschuldidaktik (Acceptability), die Adaptierbarkeit im Blick auf gesellschaftliche, bildungspolitische und internationale Veränderungsprozesse (Adaptability).

Diese konzeptionellen Überlegungen gehen über quantitative Erhebungen nationaler Inklusionsraten hinaus und lenken den Blick auf die individuelle Erfahrung als Student*in mit Behinderungen an einer Universität willkommen zu sein. Weitgehend von Studierenden mit Behinderungen geteilte Erfahrungen beziehen sich auf den Mangel an Verständnis und Akzeptanz sowohl durch Kommiliton*innen als auch durch Lehrkräfte, sowie auf das Gefühl mehr leisten zu müssen als ,nicht behinderte Student*innen“ (having to work harder) (Seale 2014: 27). 


\section{Fazit}

Inklusion an europäischen Hochschulen vollzieht sich trotz gemeinsamer Orientierungsrichtlinien wie der UN-BRK auf unterschiedlichen Ebenen und erfasst in unterschiedlichem Ausmaß die Teilhabe von Studierenden mit Behinderungen im tertiären Bildungssektor. Die komplexe Datenlage (unterschiedliche Konzeptionalisierung Studierender mit Behinderungen, uneinheitliches Inklusionsverständnis, differierende Hochschulsysteme, unterschiedliche Methoden der Datenerhebungen, etc.) gestattet nur bedingt vergleichende Analysen zum Einbezug Studierender mit Behinderungen an Hochschulen und Universitäten im internationalen Vergleich. Dennoch kann festgehalten werden, dass insgesamt in Betrachtung des europäischen Kontextes immer noch eine bedeutsame Lücke zwischen den Anteilen nichtbehinderter Personen und Personen mit Behinderungen in Bezug auf das Erreichen eines akademischen Abschlusses klafft. Inklusionsquoten im Hochschulbereich sind noch weit von denen im schulischen Sektor entfernt. Eine Diskussion über inklusionsorientierte Neubestimmungen der Universitäten etabliert sich in Folge der UN-BRK (2006) erst allmählich.

Das vorliegende Zahlenmaterial bezüglich nationaler Bildungsmaßnahmen ermöglicht keine kausale Zuordnung und Bewertung einzelner Staaten oder deren Ansätze zur erfolgreichen Inklusion von Menschen mit Behinderungen. Der internationale Vergleich zeigt aber, wie sich Weichenstellungen entwickeln können und bietet somit Anregungen, wie auf dem Weg hin zu einer inklusiven Hochschulbildung weitergegangen werden kann. Dies ist um so wichtiger, da neoliberal ausgerichtete Hochschulen und der Konkurrenzkampf um Exzellenz die Gefahr birgt, dass in internationalen Vergleichsstudien erhobene Indikatoren korrumpiert werden und unter dem Primat ökonomischer Interessen Menschen mit Behinderungen aus dem tertiären Bildungssektor ausgeschlossen bleiben. Ein neoliberal ausgerichteter, europäischer Hochschulraum lässt sich kaum mit den Forderungen einer menschenrechtlich fundierten Inklusion vereinbaren, wenn auf Grund geringerer Arbeitsmarktattraktivität Studierende mit Behinderungen geringeren wirtschaftlichen Gewinn versprechen, wenn es aufgrund nicht vorhandener Kriterien keine Anerkennung der Inklusion in Rankinglisten gibt. Studierende mit Behinderungen stehen in Gefahr, im Wettrennen der Universitäten um Leuchtturminitiativen, internationale Anerkennung und Exzellenz auf der Strecke zu bleiben. Hier ist eine parteigreifende Forschung und entsprechende Neubestimmung der Kennzeichnung einer Exzellenzuniversität angezeigt.

\section{Literatur}

Academic Network of European Disability experts (ANED) (2011): Inclusive Education for Young Disabled People in Europe. Trends, Issues and Challenges. A synthesis of evidence from ANED country reports and additional sources. https:/www.humanconsultancy.com/projects/ academic-network-of-european-disability-experts-aned [Zugriff: 06.06. 2020].

Ahrbeck, Bernd (2014): Inklusion. Eine Kritik. Stuttgart: Kohlhammer.

Allemann-Ghionda, Cristina (2021 i.d.B.): Diversität, Inklusion und Internationalisierung in der Hochschule. In: Köpfer, Andreas/Powell, Justin J.W./Zahnd, Raphael (Hrsg.): Handbuch Inklusion international. Opladen: Budrich, S. 473-498. 
ARWU (Academic Ranking of World Universities) (2018): Ranking Criteria and Weights. http://www.shanghairanking.com/ARWU-Methodology-2018.html [Zugriff: 06.06.2020].

Berhanu, Girma (2019): Inclusive Education and the "Balkanization" Professionalization of the Specialized Field of Studies in Special Education Postgraduate Programs: The Case of Sweden. In: International Journal of Special Education 33, 4, S. 869-876.

Charta Universität der Großregion (2013): Bildung, Forschung und Mobilität für alle/ Université de la Grande Région Formation, recherche et mobilité pour tous. Gemeinsames Positionspapier der Behindertenbeauftragten der Universitäten der Großregion. http:/www.uni-gr.eu/sites/tstuni-gr.univ-lorraine.fr/files/users/documents/offre_unigr/unigr_charta_studium-behinderung_20131122_de.pdf [Zugriff: 06.06. 2020].

CHE Centrum für Hochschulentwicklung (2014): CHE Ranking (2014). http://www.che-ranking.de/cms/?getObject=64\&getLang=de [Zugriff: 29.04 .2019$]$.

Dannenbeck, Clemens/Dorrance, Carmen/Moldenhauer, Anna/Oehme, Andreas/Platte, Andrea (Hrsg.) (2016): Inklusionssensible Hochschule. Grundlagen, Ansätze und Konzepte für Hochschuldidaktik und Organisationsentwicklung. Bad Heilbrunn: Klinkhardt.

Deutsches Zentrum für Hochschul- und Wissenschaftsforschung / German Centre for Higher Education Research and Science Studies (DZHW) (Ed.) Eurostudent VI (2018): Social and Economic Conditions of Student Life in Europe Synopsis of Indicators (2016-2018). https://www. eurostudent.eu/download_files/documents/EUROSTUDENT_VI_Synopsis_of_Indicators.pdf [Zugriff: 06.06. 2020].

Dotcom the Disability Online Tool of the Commission (Update date: Mon, 2019-05-20): www.disability-europe.net/dotcom [Zugriff: 25.01.2020].

Dreier, Rudolf-Werner (2000): Vor einhundert Jahren Beginn des Frauenstudiums an der Universität Freiburg. https://idw-online.de/de/news18330 [Zugriff: 29.04.2019].

Ebersold, Serge (2008): Pathways for People with Disabilities Towards Tertiary Education and Employment. Preliminary Findings from a Literature Review Covering Selected OECD Countries. OECD $2011 \mathrm{http}$ //www.oecd.org/education/innovation-education/inclusionofstudentswithdisabilitiesintertiaryeducationandemployment.htm [Zugriff: 06.06. 2020].

Ebersold, Serge/Schmitt, Marie José/Priestley, Mark (2011): Inclusive Education for Young Disabled People in Europe. Trends, Issues and Challenges. A synthesis of evidence from ANED country reports and additional sources. http://www.youthmetro.org/uploads/4/7/6/5/ 47654969/aned_2010_task_5_education_final_report.pdf [Zugriff: 06.06. 2020].

EADSNE, Europäische ${ }^{-}$Agentur für sonderpädagogische Förderung und inklusive Bildung (2003): Grundprinzipien für bildungspolitische Maßnahmen zur Förderung der integrativen/inklusiven Bildung (European Agency for Special Needs and Inclusive Education) https://www.european-agency.org/sites/default/files/key-principles-in-special-needs-education_keyp-de.pdf [Zugriff: 06.06. 2020].

EADSNE, Europäische Agentur für Entwicklungen in der sonderpädagogischen Förderung (2006): https://www.european-agency.org/sites/default/files/special-needs-education-in-europe-volume-2-provision-in-post-primary-education_Thematic-DE.pdf_Zugriff: 25.01.2020].

Europäische Kommission (2007): Situation von Menschen mit Behinderungen in der Europäischen Union. Europäischer Aktionsplan 2008-2009. Brüssel, 26.11.2007 KOM (2007) 738. https://eur-lex.europa.eu/LexUriServ/LexUriServ.do?uri=COM:2007:0738:FIN:DE:PDF [Zugriff: 06.06. 2020].

Europäische Kommission (2008): Brügge-Memorandum. Von Bologna nach Brügge und weit darüber hinaus. Chancengleichheit für Menschen mit Behinderungen ("From Bologna to Bruges and far beyond. Equal opportunities for people with disabilities.”). Brügge-Konferenz Brügge (Belgien) 1.-4.12.2008. http://www.esok.fi/esok-hanke/kv/bruges.pdf [Zugriff: 06.06. 2020]. 
European Commission (2016): EU Labor Force Survey database. https://ec.europa.eu/eurostat/ statistics-explained/index.php/EU_labour_force_survey___data_and_publication [Zugriff: 25.01.2020].

Europäische Kommission (2010): Mitteilung der Kommission an den Rat, das europäische Parlament, den europäischen Wirtschafts- und Sozialausschuss und den Auschuss der Regionen. Europäische Strategie zugunsten von Menschen mit Behinderungen 2010-2020. Erneuertes Engagement für ein barrierefreies Europa. https://eur-lex.europa.eu/LexUriServ/LexUriServ.do? uri=COM:2010:0636:FIN:de:PDF [Zugriff: 06.06. 2020].

Europäischer Rat (2000): Die Lissabon-Strategie. Schlussfolgerungen des Vorsitzes. Lissabon, 23.-24. März 2000. http://www.europarl.europa.eu/summits/lis1_de.htm [Zugriff: 06.06. 2020].

EUROSTAT (2016): Glossar: EU-Statistik über Einkommen und Lebensbedingungen (EU-SILC) https://ec.europa.eu/eurostat/statistics-explained/index.php?title=Glossary:EU_statistics_on_ income_and_living_conditions_(EU-SILC)/de [Zugriff: 09.09.2020].

EUROSTĀT (2019): Europäisches Statistikamt. Behinderung und Tertiärer Bildungsabschluss. https://ec.europa.eu/eurostat/de/web/products-datasets/product?code=hlth_de020 [Zugriff: 06. 06. 2020].

EU-SILC (2018): EU-Statistik über Einkommen und Lebensbedingungen (EU Statistics on Income and Living Conditions - EU-SILC). https://ec.europa.eu/eurostat/statistics-explained/index.php?title=Living_conditions_in_Europe_-_poverty_and_social_exclusion [Zugriff: 06 . 06.2020].

Floc'h, Benoît (2019): L'ENA, dernière réforme avant liquidation. Le Monde, 26. Oktober 2019. https://www.lemonde.fr/politique/article/2019/10/26/1-ena-derniere-reforme-avant-liquidation _6017003_823448.html [Zugriff: 09.09.2020].

Gaentzsch, Annette (2020). Das Recht auf Inklusion an Hochschulen. Forschung \& Lehre, Heft 4/20, S. 314 - 320. https://www.forschung-und-lehre.de/recht/das-recht-auf-inklusion-an-hoch schulen-2661/ [Zugriff: 06.06. 2020].

Gattermann-Kasper, Maike (2014): Nachteilsausgleiche für Studierende mit Beeinträchtigungen bei Lehrveranstaltungen, Prüfungen und Fristen - Ein Überblick. In: Zeitschrift für Inklusion 2014, 1-2, o.A. https://www.inklusion-online.net/index.php/inklusion-online/article/view/213 [Zugriff: 06.06. 2020].

Global Education Monitoring Report Team (2015). Education for All 2000-2015: achievements and challenges; EFA global monitoring report, 2015. https://unesdoc.unesco.org/ark:/48223/ pf0000232205 [Zugriff: 06.06. 2020].

Grammenos, Stefanos (2017): European comparative data on Europe 2020 and People with disabilities. (Published 9 August 2017 in cooperation with ANED Academic Network on European Disability Research). https://www.disability-europe.net/theme/statistical-indicators [Zugriff: 06.06. 2020].

Grammenos, Stefanos (2019): Quantitative Data Europe 2020, SDGS 2030, Social scoreboard and EU trends (European Network of Academic Experts in the Field of Disability meeting, 29 March 2019). https://www.disability-europe.net/theme/statistical-indicators [Zugriff: 06.06. 2020].

Hausotter, Anette (2008): Integration und Inklusion in Europa. In: Eberwein, Hans/Mand, Johannes (Hrsg.): Integration konkret. Begründung, didaktische Konzepte, inklusive Praxis. Bad Heilbrunn: Klinkhardt, S. 75-91.

HEAG: Higher Education Accessibility Guide (o. J.): www.heagnet.org [Zugriff zur Zeit nicht möglich].

Hochschulrektorenkonferenz (HRK) (2009): Empfehlung „Eine Hochschule für Alle“ - Arbeitshilfe zur Umsetzung. Informations- und Beratungsstelle Studium und Behinderung. Berlin: 
IBS. https://www.hrk.de/positionen/beschluss/detail/eine-hochschule-fuer-alle/ [Zugriff: 06. $06.2020]$.

ISCED (2011): International Standard Classification of Education - Internationale Standardklassifikation des Bildungswesens. http://uis.unesco.org/en/topic/international-standard-classification-education-isced [Zugriff: 06.06. 2020].

Katzenbach, Dieter (2013): Inklusion - Begründungsfiguren, Organisationsformen, Antinomien. In: Burtscher, Reinhard/Ditschek, Eduard J./Ackermann, Karl-Ernst/Kil, Monika/Kronauer, Martin (Hrsg.): Zugänge zu Inklusion. Erwachsenenbildung, Behindertenpädagogik und Soziologie im Dialog. Bielefeld: Bertelsmann, S. 27-38.

Kaufmann, Sabine (2017): Frauenstudium. https://www.planet-wissen.de/gesellschaft/lernen/universitaet/pwiefrauenstudiumamanfangstanddieschulefuerhoeheretoechter100.html [Zugriff: 06.06. 2020].

Knauf, Helen (2015): Paths to Inclusion. Implementing the CRPD in German Higher Education. In: HSW, 63, 1, S. 21-28.

Kyriazopoulou, Mary/Weber, Harald (2009): Entwicklung eines Satzes von Indikatoren - für die inklusive Bildung in Europa. Odense, Dänemark: European Agency for Development in Special Needs Education, S.13.

LFS (siehe European Commission) (2016): EU Labor Force Survey database. Labour Force Survey- Arbeitskräfteerhebung der Europäischen Union). https://ec.europa.eu/eurostat/de/web/ microdata/european-union-labour-force-survey [Zugriff: 06.06. 2020].

Limbach-Reich, Arthur (2016): Inklusion. Analyse eines aktuellen Leitbegriffs sozial- und bildungspolitischer Auseinandersetzungen. Konzeptionelle Ankerpunkte und empirische Antworten. In: Bliemetsrieder, Sandro/Bäuml-Rossnagl, Maria-Anna (Hrsg.): Inklusion im interdisziplinären Diskurs. Band 1: Eine Herausforderung für Schule und Hochschule. München: Utz, S. 191-233.

Luhmann, Niklas (1984): Soziale Systeme. Grundriss einer allgemeinen Theorie. Frankfurt am Main: Suhrkamp.

Pädagogische Hochschule Salzburg (o.J.): BLuE Hochschulprogramm. https://www.phsalzburg. at/studium/studienangebot/blue-hochschulprogramm/ [Zugriff: 09.09.2020].

OECD (2003): Disability in Higher Education. Paris: OECD Publications Service. http://leshem. telhai.ac.il/articles/Disability\%20in\%20Higher\%20Education.pdf [Zugriff: 06.06. 2020].

OECD (2010): Sickness, Disability and Work: Breaking the Barriers. A Synthesis of findings across OECD countries. Paris: OECD Publications Service. https://read.oecd-ilibrary.org/ social-issues-migration-health/sickness-disability-and-work-breaking-the-barriers_97892640 88856-en\#page1 [Zugriff: 06.06. 2020].

OECD (2011): Pathways for People with Disabilities Towards Tertiary Education and Employment. Preliminary Findings from a Literature Review Covering Selected OECD Countries by Serge Ebersold. Paris: OECD Publications Service. http://www.oecd.org/education/innovation-education/inclusionofstudentswithdisabilitiesintertiaryeducationandemployment.htm [Zugriff: 06.06. 2020].

Orr, Ann C./Bachman Hammig, Sara (2009): Inclusive Postsecondary Strategies for Teaching Students with Learning Disabilities. A Review of the Literature. In: Learning Disability Quarterly 32, 3, S. 181-196.

Parsons, Talcott (1966): Societies. Evolutionary and Comparative Perspectives. Englewood Cliffs, NJ: Prentice Hall.

Petz, Andrea/Miesenberger, Klaus (2010): Success through Exchange - The Higher Education Accessibility Guide (HEAG). In: Miesenberger, Klaus/Klaus, Joachim/Zagler, Wolfgang/Karshmer, Arthur (Hrsg.): Computers Helping People with Special Needs. ICCHP 2010. Lecture Notes in Computer Science vol 6179. Berlin:S Springer, 53-58. Heidelberg. DOI: 10.1007/9783-642-14097-6_85. 
Platte, Andrea/Schultz, Christian-Peter (2011): Inklusive Bildung an der Hochschule - Impulse für LehrerInnenbildung und Soziale Arbeit. In: Flieger, Petra/Schönwiese, Völker (Hrsg.): Menschenrechte Integration Inklusion. Aktuelle Perspektiven aus der Forschung. Bad Heilbrun: Klinkhardt, S. 245-251.

Powell, Justin J.W. (2016): Von Ableismus zur universal design Universität. In: Dannenbeck, Clemens/Dorrance, Carmen/Moldenhauer, Anna/Oehme, Andreas/Platte, Andrea (Hrsg.): Inklusionssensible Hochschule. Grundlagen, Ansätze und Konzepte für Hochschuldidaktik und Organisationsentwicklung. Bad Heilbrunn: Klinkhardt, S. 34-51.

PwC/Times (2019): Higher Education World University Rankings 2019 report. https://www.timeshighereducation.com/world-university-rankings/methodology-world-university-rankings2019\#survey-answer [Zugriff: 25.01.2020].

Ramsden, Brian (2005): Participation in Higher Education: A Study to Determine Whether the Higher Education Initial Participation Rate Should be Disaggregated, Department for Education and Skills Research Report RR676. http://letr.org.uk/references/storage/CDXEEMIW/RR 676.pdf

Seale, Jean K. (2014): E-Learning and Disability in Higher Education. Accessibility Research and Practice. New York: Routledge.

Sidiropoulou-Dimakakou, Despina (2009): Greek home-page of the "Higher Education Accessibility Guide" [zurzeit nicht abrufbar].

Statistische Ämter des Bundes und der Länder (2018): Internationale Bildungsindikatoren im Ländervergleich. https://www.statistikportal.de/sites/default/files/2018-10/Internationale_Bildungsindikatoren_Ausgabe_2018.pdf [Zugriff: 06.06. 2020].

Statistisches Bundesamt (2019): Frauenanteil in der Professorenschaft in Deutschland im Jahr 2018 nach Bundesländern. https://de.statista.com/statistik/daten/studie/197898/umfrage/frauenanteil-in-der-professorenschaft-nach-bundeslaendern/\#professional [Zugriff: 06.06. 2020].

Stodden, Robert/Roberts, Kelly (2014): Students with Disabilities in Postsecondary Education. In: Florian, Lani (Ed.): The SAGE Handbook of Special Education. Thousand Oaks, CA: Sage, pp. 675-688.

UNESCO (1990): Final Report. World Conference on Education for All: Meeting Basic Learning Needs. https://unesdoc.unesco.org/ark:/48223/pf0000097551 [Zugriff: 09.09.2020].

UNESCO (1994): Die Salamanca Erklärung und der Aktionsrahmen zur Pädagogik für besondere Bedürfnisse (Original: The Salamanca Statement and Framework for Action on Special Needs Education). In deutscher Übertragung von Petra Flieger. UNESCO World Conference on Special Needs Education (1994). http://bidok.uibk.ac.at/library/unesco-salamanca.html [Zugriff: 06.06. 2020].

UNESCO (1997): International Standard Classification of education ISCED 1997. http://uis.unesco.org/sites/default/files/documents/international-standard-classification-of-education-1997en_0.pdf [Zugriff: 25.01.2020].

UNESCO (2011): ISCED (International Standard Classification of Education - Internationale Standardklassifikation des Bildungswesens). http://uis.unesco.org/en/topic/international-standard-classification-education-isced [Zugriff: 06.06. 2020].

UNESCO (2015): Education 2030. Incheon Declaration and Framework for Action for the implementation of Sustainable Development Goal 4. Ensure inclusive and equitable quality education and promote lifelong learning opportunities for all. https://unesdoc.unesco.org/ark:/48223/ pf0000245656 [Zugriff: 06.06. 2020].

UNICA (2007): Recommendations for UNICA Universities: Minimum standards for disabled persons for UNICA Universities. Paper of Unica-participants, established at the Education for all Conference, 10-12 September 2009, Warschau. http://www.unica-network.eu/sites/default/files/ga_recommendations-for-unica-universities-1.pdf [Zugriff: 06.06. 2020]. 
UN-BRK (2006): Übereinkommen der Vereinten Nationen über die Rechte von Menschen mit Behinderungen (Convention of the United Nations on the rights of persons with disabilities). Bonn: Bundesministerium für Arbeit und Soziales. http://www.bmas.de/SharedDocs/Down loads/DE/PDF-Publikationen/a729-un-konvention.pdf?_blob=publicationFile [Zugriff: 06. 06.2020].

Wansing, Gudrun (2015): Was bedeutet Inklusion? Annäherung an einen vielschichtigen Begriff. In: Degener, Theresia/Diehl, Elke (Hrsg.): Handbuch der Behindertenrechtskonvention. Teilhabe als Menschenrecht - Inklusion als Aufgabe. Bonn: bpb, S. 43-54.

Wecker, Regina (2007): Die Schweiz, das europäische Land des Frauenstudiums. In: Nagelschmidt, Ilse (Hrsg.): 100 Jahre Frauenstudium an der Alma Mater Lipsiensis. Leipzig: Leipziger Universitäts Verlag, S. 235-252.

WHO/World Bank (2011): World Report on Disability. New York: World Health Organization. https://www.who.int/disabilities/world_report/2011/en/ [Zugriff: 06.06. 2020]. 


\title{
27 Vulnerabilität als (neues) europäisches bildungspolitisches Deutungsmuster? Ein Beispiel aus dem Bereich des Lebenslangen Lernens
}

\author{
Marcelo Parreira do Amaral, Jozef Zelinka
}

\begin{abstract}
Das Konzept der Vulnerabilität hat seit kurzem in bildungspolitischen Diskussionen auf europäischer Ebene an Popularität gewonnen. Insbesondere wird das Konzept im Rahmen zahlreicher politischer Maßnahmen genutzt, die sich um die Bekämpfung von sozialer Exklusion mithilfe von Lebenslangem Lernen (LLL) bemühen. Unter Bezugnahme auf den Forschungsstand fokussiert der Beitrag die gesellschaftliche Konstruiertheit sowie Kontextabhängigkeit des Konzepts der Vulnerabilität, um der Frage nachzugehen, ob die verbreitete Bezugnahme auf das Konzept in bildungspolitischen Debatten um LLL möglicherweise ein (neues) bildungspolitisches Deutungsmuster konstituiert und um zu erörtern, welche Implikationen daraus erwachsen. In diesem Beitrag erörtern wir erstens einige Merkmale des Konzepts Vulnerabilität. Im zweiten Schritt wenden wir uns vor dem Hintergrund der Forschungsliteratur den pluralen Verständnissen und unterschiedlichen Verwendungsweisen des Konzepts zu und erörtern Vulnerabilität als anthropologische Konstante, als sozialwissenschaftliches Forschungskonzept sowie als politischen Mechanismus. Als drittes wird dann mit Bezug auf Ergebnisse des kürzlich abgeschlossenen europäischen Forschungsprojekts YOUNG ADULLLT Vulnerabilität in ihrem kontextuellen und relationalen Charakter thematisiert, womit gleichzeitig die stigmatisierenden Effekte ihres essenzialisierenden Gebrauchs - d.h. als Attribut Einzelner oder Gruppen - aufgezeigt werden. Hierbei wird die dynamische sowie relative und multidimensionale Natur des Konzepts Vulnerabilität betont, mit dem Verweis auf eine wichtige Veränderung in der Verwendungsweise dieses Begriffs auf der Ebene europäischer Regionen. Der Beitrag schließt, viertens, mit einer Diskussion der Ergebnisse sowie einigen kritischen Überlegungen zur Nutzung des Konzepts Vulnerabilität.
\end{abstract}

\section{Einleitung}

Das Konzept Vulnerabilität hat seit einigen Jahren in bildungspolitischen Diskussionen an Popularität gewonnen. Insbesondere wird das Konzept im Rahmen zahlreicher politischer Maßnahmen genutzt, die sich um die Bekämpfung sozialer Exklusion mithilfe Lebenslangen Lernens (LLL) bemühen. Vor allem im Rahmen von zielgruppenspezifischen Jugendpolitiken der europäischen Kommission erfährt der Begriff Konjunktur und Bezeichnungen wie vulnerable youth bzw. young people in vulnerable situations, die die soziale und strukturelle Benachteiligung junger Erwachsener umschreiben, wurden zu bestehenden Ausdrücken für die Adressierung von Zielgruppen. Diese Zielgruppen sollen mittels Arbeitsmarktpolitiken 
,aktiviert' und (wieder) integriert werden. Anders als Konzepte wie beispielsweise (diagnostizierter) ,sonderpädagogischer Förderbedarf" wird Vulnerabilität in der Regel aufgrund der (angenommenen) Zugehörigkeit zu einer statistisch erfassten Gruppe zugeschrieben. Daher scheint es relevant zu fragen, welche Implikationen sich aus dieser Labeling-Praxis insbesondere für junge Erwachsene ergeben. Aus einer übergreifenden, international vergleichenden Perspektive lässt sich insbesondere nach der Rolle und Wirkung von Konzepten fragen, welche sich in bildungspolitischen Entwicklungen auf europäischer Ebene etablieren.

Im europäischen Kontext ist vor dem Hintergrund der zunehmenden Aufmerksamkeit für die Vulnerabilität der jüngeren Generationen soziale Inklusion zu einem der zentralen politischen Ziele in Bezug auf Beschäftigung, Lebenslanges Lernen und berufliche Orientierung geworden. Sowohl der Europarat als auch die Europäische Kommission haben sich bei der Gestaltung von sozialer Inklusion vorrangig für die Förderung des sozialen Zusammenhalts eingesetzt. Zu Beginn des neuen Jahrtausends wurde sozialer Zusammenhalt dabei definiert als:

the capacity of a society to ensure the welfare of all its members, minimising disparities and avoiding polarisation. A cohesive society is a mutually supportive community of free individuals pursuing these common goals by democratic means (European Committee for Social Cohesion 2004: 2).

Jedoch hat die Europäische Kommission diese Zielsetzung zunehmend mit den Zielen zur wirtschaftlichen Wettbewerbsfähigkeit im globalen Kontext verknüpft. Sie betonte zunächst die Notwendigkeit, das Armutsrisiko zu verringern und sicherzustellen, dass Frauen, Jugendliche und Zuwanderer*innen im Visier von Inklusionspolitiken stehen. Mit der LissabonStrategie (und den anschließenden Initiativen) wurde jedoch größere Aufmerksamkeit auf Wirtschaftswachstum und Arbeitsplätze gelegt. Dies wurde im Entschluss des Europäischen Rates von 2000 in Lissabon auf den Punkt gebracht. Darin wurden Wirtschaftswachstum und soziale Inklusion als gleichrangige Ziele festgelegt, um Europa ,the most dynamic and competitive, sustainable knowledge-based economy in the world capable of sustaining economic growth with more and better jobs and greater social cohesion“ (CEU 2000: 2) zu machen.

Relevant in diesem Kontext ist das spezifische Verständnis, wie soziale Inklusion erreicht werden soll, nämlich durch die Förderung von active inclusion (Europäische Kommission 2008). Dies wurde als ein Mittel angesehen, um allen europäischen Bürger*innen, insbesondere den vulnerabelsten, d.h. denen, die am meisten von den strukturellen, wirtschaftlichen, sozialen oder kulturspezifischen Ungleichheiten betroffen sind, die Möglichkeit zu geben, sich voll und ganz im Rahmen ihrer Möglichkeiten an der Gesellschaft zu beteiligen. Diese Beteiligung soll insbesondere durch Beschäftigung im Arbeitsmarkt und (Re-)Integration in das (Aus-)Bildungssystem gewährleistet werden, womit ein Bezug zu inklusiver Bildung hergestellt wurde (ebd.: L 307/14). In Frage steht, welche Verknüpfung zwischen den strukturellen Problemen (Wirtschaft, Arbeitsmarkt etc.) und den individuellen Lösungen in den bildungspolitischen Initiativen und Programmen besteht; und vor allem, welche Rolle das Konzept Vulnerabilität darin spielt.

Vulnerabilität hat sich inzwischen sowohl als alltäglicher Begriff (vgl. Digitales Wörterbuch der deutschen Sprache 2019) als auch als fachlicher Terminus etabliert (vgl. Deutsche Forschungsgemeinschaft 2019). ${ }^{1}$ Die zunehmende Thematisierung von Vulnerabilität geht mit einem größeren Bewusstsein für Risiko, Unsicherheit und Kontingenz einher (vgl. Beck

1 Bis zum Jahr 2019 unterstützte die Deutsche Forschungsgemeinschaft 132 Forschungsprojekte, die das Thema Vulnerabilität in unterschiedlichen Kontexten auffassten. 
1992; Greven 2007; Han 2015). Gleichzeitig entsteht mit dieser größeren Aufmerksamkeit für individuelle und gesellschaftliche Verletzbarkeit bzw. Vulnerabilität jedoch oft auch ein inflationärer und undifferenzierter Gebrauch solcher Begrifflichkeiten. Dies zieht nicht nur die Notwendigkeit eines reflektierten Umgangs mit dem Begriff selbst und seinen Verwendungsweisen nach sich, sondern erfordert auch ein kritisches Nachdenken über die (potenziellen) Implikationen der allgegenwärtigen Bezugnahme auf das Konzept - sowohl für die Formulierung von öffentlicher Politik als auch für die Implementation inklusiver Bildungspraktiken. Die Thematisierung und Feststellung von Vulnerabilität führt zugleich - zumindest implizit - die Aufforderung mit, die Faktoren und Dispositionen zu identifizieren, auf die eingewirkt werden kann, um diesen Zustand aufzuheben. Dabei können in dieser Interaktion selbst vulnerable Umgangsweisen erzeugt, reproduziert oder weiter verschärft werden. Daher ist zu fragen, welche Stigmatisierungs-, Exklusions- oder Diskriminierungseffekte durch Interaktionen in der Bildungspraxis ausgelöst werden (können) und welche Konsequenzen dies für die Entwicklung einer genuin inklusiven Bildung hat.

Ausgangspunkt für den Beitrag ist die Frage, ob sich im öffentlich-politischen Diskurs gegenwärtig Vulnerabilität als neues Interpretations- und Deutungsmuster gesellschaftlicher Phänomene durchsetzt und ob mithilfe dessen bestimmte Risiken oder problematische gesellschaftliche Lebensumstände erfasst, thematisiert und bearbeitet werden sollen. Vor diesem Hintergrund betont der Beitrag die gesellschaftliche Konstruiertheit sowie Kontextabhängigkeit des Konzepts Vulnerabilität. Dafür werden erstens einige Merkmale des Konzepts erörtert, zweitens seine pluralen Verständnisse und unterschiedlichen Verwendungsweisen herauspräpariert, drittens, mit Bezug auf die Ergebnisse des kürzlich abgeschlossenen europäischen Forschungsprojekts $Y O U N G_{-} A D U L L L T^{2}$, der kontextuelle und relationale Charakter von Vulnerabilität thematisiert und schließlich werden viertens einige kritische Überlegungen zur Nutzung des Konzepts Vulnerabilität angestellt.

\section{Vulnerabilität - ein Konzept mit pluralen Verständnissen und widersprüchlichen Verwendungsweisen}

Der Begriff Vulnerabilität wird in verschiedenen Kontexten verwendet, um ein (höheres) Risiko bestimmter Personen oder Gruppen zu markieren oder um auf die Gefahr einer Verschlechterung von (Lebens-)Bedingungen zu verweisen. Mehrere Faktoren können als Ursache von Vulnerabilität (oder als diese beeinflussend) angesehen werden. Dazu gehören physische (z.B. Krankheit, Behinderung, körperliche Verfassung), emotionale/psychische (z.B. psychische Erkrankungen, Unreife, Abhängigkeit), strukturelle (z.B. Armut, Obdachlosigkeit, Gesundheitsfürsorge, Bildungsniveau) und soziale (mangelnde Unterstützung durch Familie oder Peer-Gruppe, Fehlen von Beratung in schwierigen Situationen und unmittelbare

2 Als Antwort auf die Ausschreibung der Europäischen Kommission YOUNG-3-2015 und im Rahmen der Zuwendungsvereinbarung Nr. 693167 hat das Forschungsprojekt YOUNG_ADULLLT zwischen den Jahren 2016 und 2019 eine vergleichende Analyse von über 180 Politiken des Lebenslangen Lernens vorgenommen. Die Analysen haben in 18 Funktionalen Regionen (Functional Regions), in neun EULändern stattgefunden, inklusive Bulgarien, Deutschland, Finnland, Italien, Kroatien, Österreich, Portugal, Spanien und dem Vereinigten Königreich. Für mehr Informationen zu den Ergebnissen des Projekts siehe http://www.young-adulllt.eu/. 
Risiken aus der Umwelt) Faktoren (vgl. Palumbo et al. 2018; Wustmann 2004). Als solche kann Vulnerabilität aus unterschiedlichen Blickwinkeln thematisiert werden, zum Beispiel aus der Perspektive individueller Bedingungen und Verhaltensweisen, struktureller und systemisch-materieller Bedingungen oder auch sozial-relationaler Beziehungen und Unsicherheiten, und stellt daher ein multidimensionales, relationales Konzept dar.

In der Forschungsliteratur hat die Konjunktur des Begriffs Vulnerabilität zu verschiedenen Versuchen seiner konzeptuellen Schärfung und Bestimmung geführt (vgl. Brown et al. 2017; Burghardt et al. 2017; Mackenzie et al. 2014). Vulnerabilität - aus dem lateinischen vulnus $=$ Wunde, Verwundung, Verletzung - stellt für einige Forscher*innen einerseits eine anthropologische Grundbedingtheit dar, die mit der Leidensfähigkeit des Menschen verbunden ist. Andererseits wird sie als Anfälligkeit bzw. Verwundbarkeit bestimmter Personen und Personengruppen gegenüber gewissen Bedrohungen und Schädigungen verstanden (vgl. Mackenzie et al. 2014: 5ff.). Sie kann zudem auf mannigfachen Ebenen materialisiert, erlebt und gespürt werden, etwa bei den einfachen leiblichen Zuständen und Prozessen, die bei einer Krankheit oder Behinderung deutlich anstrengender zu vollziehen sind (Trinken, Essen, Bewegen, Spüren, Wahrnehmen) oder bei den täglichen sozialen und situativen Erfahrungen in der Interaktion mit den Mitmenschen (Ausgrenzung, Einsamkeit, Belästigung, Diskriminierung, Xenophobie) (vgl. Bieler 2017: 27), die in dem schulischen Kontext intensiviert werden. Vulnerabilität kann allerdings auch symbolisch zum Ausdruck kommen (Respektlosigkeit, Unterschätzung, Paternalismus, Vertrauensverlust, Lästern) oder subtilere Formen annehmen (Kontaktvermeidung, Kommunikationsblockade, Lieblosigkeit, Verkennen), und damit ein inneres Unbehagen und kollektives Unwohlsein erzeugen.

Die verschiedenen Erlebniswelten der Lernenden - wie etwa Unterricht, Freizeitaktivitäten, Mini-Job, Familie, Clique etc. - , in denen vulnerable Situationen auftreten können, deuten auf die Ambiguität dieses Begriffs hin und betonen die wesentliche Rolle diverser Faktoren, die eine vulnerable Erfahrung begünstigen oder mildern. Wann und wie eine Erfahrung vulnerabel erscheint und verletzend wirkt, hängt außerdem davon ab, unter welchen Umständen sie vorkommt und wie sie dabei empfunden wird. Deswegen ist es wichtig, soziale Konstruktionen von Vulnerabilität zu analysieren,

in denen Vorstellungen von Verletzlichkeit sprachlich-diskursiv und institutionell verfestigt und in Rückwendung auf das leibeigene Spüren sukzessive materialisiert werden (Bieler 2017: 47).

Daher scheint es zentral, nicht von der Vulnerabilität konkreter Individuen auszugehen, sondern Situationen zu untersuchen, die eine mehr oder weniger intensive vulnerable Erfahrung begünstigen oder wahrscheinlicher machen (vgl. Luna 2009: 128). Zentrale Frage bleibt dabei, wie Vulnerabilität identifiziert und behandelt wird, ohne dass dadurch negative (Neben-) Folgen entstehen; darauf gehen wir unten ein.

Im Bereich inklusiver Bildung wird die Debatte um Vulnerabilität aus mindestens zwei Perspektiven geführt. Als erstes wird der essenzialisierende Gebrauch dieser Kategorie diskutiert. Mit der Annahme, dass in den westlichen (Risiko-)Gesellschaften das Sensibilisieren für Gefahr und Bedrohung eine Kultur der Angst installiert hat (vgl. Eisch-Angus 2019), die zur Suche nach Resilienz und Vorsorge drängt (vgl. Conze 2018: 114ff.; auch Burghardt et al. 2017: 9), wird Vulnerabilität zunehmend als ontologische Bedingung profiliert, mit deren Hilfe soziale Gerechtigkeit und Menschenrechte gefördert werden sollen (vgl. Brunila et al. 2016: 70). Dies betrifft auch die Integration sozial oder gesundheitlich benachteiligter Schüler*innen. Allerdings wird mit dem Einbeziehen solcher Kategorien wie Vulnerabilität auch 
die Diskussion eröffnet, wem das Recht zugeteilt wird, über vulnerable Existenzweisen oder prekäre Lebenslagen und -bedingungen zu entscheiden. Mit anderen Worten,

Aussagen darüber, wer wie, wieweit und wodurch als vulnerabel zu gelten hat und insofern verletzbar ist, ist einer kontingenten Ordnungslogik von diskursiven und nicht-diskursiven Elementen, von Interessen und Machtkonstellationen anheim gegeben (Burghardt et al. 2017: 14).

Wenn Vulnerabilität von vorneherein eine ontologische Dimension bestimmter Individuen darstellt, so klassifiziert sie diese nach ausgewählten Merkmalen oder Unterscheidungskriterien und erzeugt damit eine Zwangslage, für welche bestimmte Personengruppen von schwachen, immigrierten oder sonst marginalisierten jungen Erwachsenen anfällig sind - sie sind sozusagen vulnerabel, um als vulnerabel bestimmt zu werden (vgl. McLeod 2012: 21).

Ein zweiter Aspekt der Debatte um Vulnerabilität in der inklusiven Bildung bezieht sich auf die individualisierende und pathologisierende Wirkung der Zuschreibung vulnerabler Dispositionen. Individualisierend wirken vor allem die Hinweise darauf, dass sich junge Erwachsene aus eigener Schuld oder Unfähigkeit zu lernen in prekären Lebenssituationen wiederfinden und die Rolle sozialer Verhältnisse bei der Herstellung prekärer Umstände dabei manchmal zum Teil, manchmal auch völlig ausgeblendet werden (vgl. ebd.). Pathologisierend wirken dagegen die Versuche, jungen Erwachsene, die nicht im Arbeitsmarkt integriert sind und damit, so die Logik, von Exklusion bedroht sind, einem standardisierten bzw. normalen Lebenslauf (wieder) zuzuführen. Dem widmen sich zahlreiche Initiativen, etwa die europäische Initiative Jugendgarantie (Youth Guarantee) (vgl. Brunila 2016: 70; Europäische Kommission 2019). Allerdings wird damit der komplexen Natur der Vulnerabilität nicht gerecht. Vulnerabilität umfasst nämlich nicht nur negative Attribute, sondern auch positive Dimensionen zwischenmenschlicher Interaktion, wie beispielsweise Zärtlichkeit, Barmherzigkeit, Offenheit gegenüber anderen, Milde und Zerbrechlichkeit (vgl. McLeod 2012: 22). Mehr noch, die Kondition der Vulnerabilität wird ziemlich oft mit der Präposition gegen (vulnerability of) (ich bin vulnerabel gegenüber Kollektivdruck, Autoritäten, Unbekanntem etc.) statt mit der Präposition für (vulnerability for) (ich bin vulnerabel bzw. sensibel für Unterschiede, Gerechtigkeit, Schutz der Schwachen etc.) konnotiert. Während erstere eine Abgeschlossenheit und Grenze suggeriert, impliziert letztere eine Offenheit, ein zu erreichendes Ziel bzw. einen zu aktivierenden Prozess (vgl. Batchelor 2006: 789f). Eine inklusive Bildung, die um Wiedereingliederung bemüht ist, fokussiert die offene Kondition der Vulnerabilität, die zur (Selbst-)Kritik, Akzeptanz der eigenen Fehlbarkeit und zum persönlichen Voranschreiten ermutigt (vgl. ebd.) und welche die differenzierenden Praktiken meidet (vgl. McLeod 2012: 22). Was in diesem Vulnerabilitätsdiskurs jedoch oft verloren geht, sind kritische Fragen danach, ob nicht dadurch auch hilfebedürftige Subjekte mitkonstruiert werden (vgl. Langemeyer 2007), denn oftmals werden Perspektiven auf die Handlungsmächtigkeit bzw. -fähigkeit der Individuen (Stichwort agency) hierbei nicht berücksichtigt.

In den wissenschaftlichen Diskussionen wird das Konzept der Vulnerabilität aus unterschiedlichen Perspektiven gedacht. Dabei lassen sich zunächst zwei Bereiche voneinander abgrenzen, die die Vulnerabilität systemischer oder subjektiver Art pointieren. Im zweiten Schritt lassen sich drei Verwendungsweisen unterscheiden, die die Anwendung der Vulnerabilität als ein bestimmendes Deutungsmuster präzisieren.

Auf der einen Seite könnte mit Blick auf die Forschungsliteratur über eine Vulnerabilität der Systeme gesprochen werden. Darunter werden Schwachstellen und Verletzungsmöglichkeiten systemischer Art verstanden, die die Fragilität und Kontingenz jedweder Systeme offenbaren. Das Konzept der systemischen Vulnerabilität findet in verschiedenen Bereichen Anwendung, wie etwa (Cyber-)Sicherheit (vgl. Friedrichsen/Bisa 2016; Subrahmanian et al. 
2015), Wirtschaft (vgl. Ritchie 2010), Finanzen (vgl. Johnston et al. 2000), Umwelt (vgl. Vatsa 2004; Weichselgartner 2001), Politik (vgl. Ferrarese 2018; Oliviero 2018), Soziales (vgl. Bütow/Gómez Jiménez 2015), Gesundheit (vgl. Petersen/Wilkinson 2008) oder Infrastruktur (vgl. Birkmann et al. 2010). Die Vulnerabilität der Systeme betrifft dabei nicht nur ein konkretes, geschlossenes System, sondern zeigt vielmehr das Zusammenhängen und Ineinandergreifen komplexer Verhältnisse, die an einer vulnerablen Stelle sichtbar werden. So zeigen beispielsweise die bedrohenden Umweltveränderungen nicht nur die Zerbrechlichkeit der Ökosysteme, sondern auch die Grenzen globaler politischer Aktionsfähigkeit.

Auf der anderen Seite ließe sich im gleichen Zuge eine Vulnerabilität des Subjekts festhalten. In den Blick kommen dann die Verletzungsmöglichkeiten des Subjekts, welche seine Existenz und Entwicklung eingrenzen. Eine subjektive Vulnerabilität umfasst Bereiche sowohl sozialer Interaktion als auch innerer Erlebniswelt, wie etwa Körperlichkeit (vgl. Knudsen/Stage 2015; Springhart 2016), psychische Gesundheit (vgl. De Masi 2009; Möller/ Deister 1996), soziale Lebensbedingungen und -verhältnisse (vgl. Bishawjit 2014; Ranci et al. 2014), Spiritualität (vgl. Kohl 2017), Identitätsbestimmung (vgl. Cover 2016; McLaughlin 2012), Lebenszyklen (vgl. Andresen et al. 2015; Kruse 2017) oder Professionalisierung (vgl. Beicht/Granato 2011; Burghardt et al. 2017). Die Vulnerabilität des Subjekts ließe sich durch die Verschränkung bzw. Intersektion verschiedener Bereiche intensivieren, beispielsweise die Kombination von Erfahrungen mit Rassismus, Sexismus, religiösem Hass oder weiteren Störfaktoren. Dadurch werden eine soziale Inklusion und gesellschaftliche Integration vieler Individuen beinahe verunmöglicht.

Zwischen den zwei genannten Bereichen existieren zahlreiche Überschneidungen und eine gegenseitige Bedingtheit. So zeigt beispielsweise die Verbreitung des sexuellen Missbrauchs in sozialen Netzwerken, auch als Cybergrooming bekannt, wie die Vulnerabilität virtueller Systeme (Internet), mit der Vulnerabilität der Kinder und Jugendlichen in ihren Lebensübergängen koinzidieren (vgl. Martellozzo 2013). Diese grundlegende Differenzierung zweier unterschiedlicher Bereiche reduziert die Komplexität der Verhältnisse und Themen, in denen Vulnerabilität als relevantes Deutungsmuster auftritt. Im Rahmen einer inklusiven Bildungspraxis zeigt sich indessen, wie die Verschränkung systemischer und subjektiver Vulnerabilität synergetisch Effekte produziert, welche im schulischen Alltag ko-präsent und permanenten, situativen Wandlungen unterworfen sind.

Der folgende Abschnitt geht auf die unterschiedlichen Verwendungsweisen des Konzepts in der Forschungsliteratur ein.

\subsection{Die Verwendung des Vulnerabilitätskonzepts in der Forschungsliteratur}

Die allgemeine Konjunktur und beinahe Beliebtheit des Begriffs Vulnerabilität hat sich schrittweise in verschiedenste Bereiche sozialwissenschaftlicher Disziplinen integriert und zu analytischen Neubestimmungen geführt. Beim Versuch, die gängigen Verwendungsweisen der Vulnerabilität zu dechiffrieren, lassen sich dann zahlreiche Konzeptualisierungen festhalten (vgl. Alwang et al. 2002; Bankoff et al. 2004; Hammer et al. 2019; Hogan/Marandola 2005). Diese Versuche zeugen zugleich von der Ambiguität des Konzepts sowie von der Notwendigkeit seiner weiteren Präzision und Bestimmung in der Forschungsliteratur. Umfassend und doch anwendungsbereit haben Kate Brown, Kathryn Ecclestone und Nick 
Emmel das Konzept untersucht und dabei seine drei Facetten herauspräpariert. Sie verstehen Vulnerabilität

first, as an anthropological condition of humans and as a cultural trope about the problems of life in increasingly fragmented and unequal societies; second, as a policy and practice mechanism, which plays out in interventions, sometimes overtly and explicitly, sometimes subtly or unnoticed; and third as a more robust concept to facilitate social and political research and analysis (Brown et al. 2017: 498).

Die Unterscheidung dieser drei Perspektiven auf Vulnerabilität - als eine anthropologische und kulturelle Konstante, als praktischer und politischer Mechanismus und als soziales und politisches Forschungskonzept - trägt zum besseren Verständnis der gesellschaftstheoretischen Etablierung der Vulnerabilität als eines (möglicherweise neuen) Deutungsmusters insbesondere im Bereich inklusiver Bildung - bei und wird im Folgenden näher in den Blick genommen.

\subsubsection{Vulnerabilität als anthropologische Konstante}

Vulnerabilität wird als eine anthropologische Konstante insbesondere in der Theologie (vgl. Bieler 2017; Leidinger 2018), Philosophie (vgl. Achtenberg 2017; Batchelor 2006; Mackenzie et al. 2014) oder Bioethik (vgl. Bergemann/Frewer 2018; Levine et al. 2004; Luna 2019) aufgeworfen. In diesen Disziplinen wird sie auf grundsätzliche menschliche Erfahrungen wie Schmerz, Leid, Trauer, Liebe oder Tod - bezogen. Damit eröffnet sich mit ihrer Hilfe die Frage, ob sie „Unterscheidungskriterium für die Differenzierung vulnerabler Gruppen im Unterschied zu nicht-vulnerablen Gruppen“" ist (Springhart 2018: 33) oder ob sie eher eine „allgemein menschlich geteilte conditio humana“ (ebd., Herv.i.O.) darstellt. Dies wird je nachdem unterschieden, mit welchem Menschenbild, mit welcher Vorstellung von Würde und Stellenwert des Menschen in der Gesellschaft die eine oder andere Kultur operiert (vgl. Burghardt et al. 2017: 13). Die kulturelle und soziale Tradition entscheidet indessen auch darüber, wo die Grenzen vulnerabler Erfahrungen liegen und mit welchen Mitteln ihre Überschreitung sanktioniert wird. Das betrifft insbesondere den Umgang mit Schwachen, Kranken, Benachteiligten, Verletzten, mit schwangeren Frauen, mit Kindern sowie mit Menschen im höheren Alter.

Vulnerable Erfahrungen begrenzen sich allerdings nicht nur auf bestimmte Personengruppen, sondern finden sich auch in den Übergangsphasen, die im Leben eines jeden Menschen vorkommen, wieder. Gerade der Übergang von einer bereits etablierten Lebensphase in eine andere, noch flüchtige Lebensphase - sei es die Änderung des sozialen Status von ledig auf verheiratet, von verheiratet auf geschieden, von geschieden auf liiert etc., oder die Änderung der Berufsrolle von ausgebildet auf angestellt, von angestellt auf arbeitslos, von arbeitslos auf requalifiziert etc., wird von vulnerablen Zuständen begleitet. Bei der Produktion solch vulnerabler Verhältnisse und Umstände spielen außer dem sozialen Status auch das Alter und vor allem die Zukunftsvorstellung und -planung des Menschen eine besondere Rolle (vgl. Delor/Hubert 2000). Gerade die letzteren Aspekte erweisen sich als besonders anspruchsvolle Aufgaben für junge Erwachsene gleichwie für die Praxis inklusiver Bildung. Die wachsenden Ansprüche an Leistungsfähigkeit, Flexibilität und ständige Erreichbarkeit erzeugen eine Atmosphäre, in der feste Grenzen (etwa zwischen Arbeitszeit und Freizeit) und klare Verantwortungsbereiche (etwa zwischen institutionalisierter Bildung und lebenslanger Selbstbildung) sukzessive verwischen und instabil werden (vgl. Bauman 2000; Bührmann 
2012). In einer solchen gesellschaftlichen Atmosphäre gedeihen Risikokalkulierung, Gefährdungsbeurteilung und Versuche, die entstehenden Vulnerabilitäten - etwa Armut, Arbeitslosigkeit oder Exklusion - zu bekämpfen bzw. zu reduzieren. Diese Versuche bleiben allerdings auch stets limitiert:

Der moderne Versuch, Vulnerabilität zu minimieren bzw. aufzuheben, verweist auf die Grenzen dieses Versuchs, offenbart weitere Vulnerabilitäten und bringt - paradoxerweise - selbst neue Vulnerabilitäten hervor. Bisher haben sich faktisch alle historischen Versuche, Vulnerabilität aufzuheben oder den Menschen invulnerabel zu machen, als vergeblich erwiesen (Burghardt et al. 2017: $10)$.

Eine Invulnerabilität erzeugen und den Menschen in seinen Lebensübergängen schützend begleiten zu wollen, würde zugleich heißen, die negativ erscheinenden Erfahrungen aus dem Lernrepertoire herauszunehmen und damit die Fähigkeit, sich neuen Herausforderungen zu stellen, zu schwächen (vgl. United Nations Development Programme 2014: 56). Dies kann die Vulnerabilität bzw. die Veranlagung zur Vulnerabilität paradoxerweise vertiefen. Vulnerabilität als anthropologische Konstante ließe sich demnach vor dem Hintergrund einer inklusiven Bildung nicht nur als eine begleitende Lebenserscheinung thematisieren, sondern auch als Lernmöglichkeit begreifen und in den Lernprozess schrittweise integrieren.

\subsubsection{Vulnerabilität als (sozial)wissenschaftliches Forschungskonzept}

Vulnerabilität scheint in bestimmten Kontexten ,geradezu eine Chiffre für zu kritisierende gesellschaftliche Verhältnisse oder Krisenerfahrungen zu sein“ (Burghardt et al. 2017: 11). Vor dem Hintergrund kommt Vulnerabilität in den Sozialwissenschaften ein besonderer Platz in der Thematisierung gesellschaftlich relevanter Fragen zu. Zwar gab es in der Geschichte immer wieder vulnerable Erfahrungen und Existenzweisen, doch heutzutage wird dies viel deutlicher in den Blick genommen. Diese Sensibilisierung kollidiert mit der Annahme, dass Vulnerabilität nicht zu denken, sondern lediglich zu fühlen ist. Dieser Verweis auf die emotionale bzw. affektive Seite des Erlebens und Wahrnehmens geht mit der Beobachtung einer affektiven Wende bzw. eines affective turn einher, insbesondere in den Gender Studien (vgl. Clough/Halley 2007; Illouz 2006; Mouffe 2014; Penz/Sauer 2016).

Die affektive Wende signalisiert ein Umdenken im Zugang mit sozialen Problemen. Diese werden vermehrt durch das Zusammenspiel zwischen Risiken und Vulnerabilitäten (vgl. Brown et al. 2017: 506) bzw. durch die „Faktoren, die als Ursachen und Gründe für die Aktualisierung der vulnerablen Disposition sorgen können“ (Burghardt et al. 2017: 12), untersucht. Burghardt et al. schlagen deshalb vor, den Begriff der Vulnerantialität als eine analytische Kategorie einzuführen:

Dieser Begriff bezeichnet komplementär zum Begriff der Vulnerabilität, der die Möglichkeiten des Verletzt-Werdens umfasst, die Möglichkeiten des Verletzens, Beschädigens, Diskriminierens usw. Vulnerabilität erscheint mit Vulnerantialität verschränkt: Man kann sich nur als verletzbar wahrnehmen oder auf diese Weise gesehen werden, wenn gleichzeitig unterstellt wird, dass es auch vulnerante, d.h. verletzende Faktoren gibt, die die Vulnerabilität hervorbringen (können). Vulnerabilität als potentielle oder aktuelle Disposition ist immer von ,vulneranten Momenten` und ,Bedingungen', die sie ,auslösen', abhängig (ebd.).

Sodann wird Vulnerabilität nicht nur als eine Möglichkeit der Erkenntnis verstanden, sondern auch als „eine Möglichkeit der Erfahrung, die erst unter bestimmten, sich realisierenden 
Bedingungen wirklich werden kann“ (ebd.: 13). Für die inklusive Bildungsforschung heißt das, dass Vulnerabilität und Vulnerantialität nicht nur als diskursive Kategorien erscheinen, welche die systemischen und strukturellen Fehlpassungen analytisch zugänglich machen und somit auf Inklusionsmöglichkeiten hinweisen. Vielmehr müssen diese auch als Steuerungsund Subjektivierungstechnologien problematisiert werden, mit deren Hilfe soziale Probleme und Bildungssubjekte geschaffen werden (Sattler 2009; Schneider 1985). Wer und nach welchen Prinzipien, auf welche Dauer und mit welchen Konsequenzen als vulnerabel oder als gefährdet identifiziert wird, entscheidet darüber, welche Bildungswege ihm*ihr zuteilwerden und welche dagegen ausgeschlossen bleiben bzw. erschwert werden. Im Umkehrschluss bedeutet dies auch Organisationen und Praktiken im Bildungsbereich ebenfalls als, zumindest potenziell, vulnerant zu betrachten.

Auch politische Konsequenzen der affektiven Wende haben weitreichenden Einfluss auf alltägliche Bildungspraktiken. Insbesondere wird Vulnerabilität als eine prekäre Lebenssituation dekodiert, die des Schutzes bedarf. Allerdings können nicht alle Regierungen und Organisationen allen Personengruppen den gleichen Schutz gewährleisten, weshalb sich die Vulnerabilität bestimmter Schichten maximiert (Butler 2009: ii). Dazu kommt, dass je nach Gesellschaft Vulnerabilität unterschiedlich stark medial repräsentiert und daher an Gewicht und Bedeutung gewinnt. Man denke hier vor allem an den Terroranschlag an Charlie Hebdo am 7. Januar 2015 (11 Opfer), der vergleichsweise viel präsenter in der medialen Öffentlichkeit war, als zum Beispiel der Anschlag auf das Garissa University College in Kenya am 2. April desselben Jahres (148 Opfer) (vgl. Digitales Wörterbuch der deutschen Sprache 2019). Abgesehen von den multiplen Faktoren, welche die Vulnerabilität eines Individuums bzw. einer Gruppe potenzieren und ihr Auftreten mehr oder weniger wahrscheinlich machen, besteht eine besondere Herausforderung für die inklusive Bildung darin, die Vulnerabilität nicht als Attribut der modernen Subjekte, die problematisch und zum Teil gefährlich sind (vgl. Chandler 2016: 121) zu stilisieren, sondern sie vielmehr ,als eine relationale Kategorie zu entwerfen" (Burghardt et al. 2017: 10; Herv. i. Orig.).

Vulnerabilität als (sozial)wissenschaftiches Forschungskonzept rückt das komplementäre Verhältnis des Konzeptes mit den es aktualisierenden bzw. hervorbringenden Faktoren in den Blick, womit Vulnerabilität nicht als Attribut von Individuen und Gruppen erscheint, sondern als relationale Kategorie angesehen werden muss.

\subsubsection{Vulnerabilität als praktischer und politischer Mechanismus}

Vor allem in den westlichen Industriegesellschaften hat spätestens seit der kulturellen Wende des Jahres 1968 eine Sensibilisierung für die subtilen und immanenten Formen der Unterdrückung und Herrschaft stattgefunden. Dies bezeugen vor allem die neuen Wellen des Feminismus (vgl. Millett 2018; Rivers 2017), der Kampf gegen Rassismus und Xenophobie (vgl. Oeser 2015; Weiß 2013) oder die Ermächtigung sexueller Minderheiten (vgl. Ayoub 2017; Pierceson 2016). Somit hat auch Vulnerabilität als Deutungsmuster für die Legitimierung prekärer gesellschaftlicher Verhältnisse in politische Steuerungsmechanismen Eingang gefunden (vgl. Brown 2017: 423).

Durch den Verweis auf die prekäre Lebenslage vulnerabler Gruppen werden auch zahlreiche bildungspolitische Programme und Politiken auf verschiedenen Ebenen eingeleitet und legitimiert. Dabei wird das Augenmerk zuerst oft auf sichtbare Vulnerabilitäten gerichtet, wie etwa das Fehlen von Grundkompetenzen, und weniger auf die scheinbar unauf- 
fälligen oder verschleierten Vulnerabilitäten, wie etwa psychische Schwierigkeiten, soziale Deprivation oder Armut (vgl. Toiviainen et al. 2019: 18; auch Dutta et al. 2011). Solche bildungspolitischen Maßnahmen können allerdings die Situation junger Erwachsene weiter verschärfen. Indem beispielsweise der Trend zur De-Standardisierung des Lebenslaufs (vgl. Kohli 1978) nicht berücksichtigt und stattdessen normative Erwartungen eines standardisierten Lebenslaufs als alleinige Option an die Schüler*innen und Student*innen gesetzt werden (vgl. Parreira do Amaral/Jornitz 2019), entsteht bei ihnen ein Anpassungs- und Konformitätsdruck (vgl. Brown et al. 2017: 501; auch Priestley 2001). In einer solchen Situation werden vulnerable Erfahrungen vorprogrammiert, was nicht zuletzt jedwede Inklusionsbemühungen scheitern lässt. Ähnlich verhält es sich mit sozialen und strukturellen Barrieren, die erst Beeinträchtigungen in Behinderungen verwandeln. Wenn Desinteresse, Exklusion und Entfremdung junger Erwachsener ihrer psycho-emotionalen Vulnerabilität zugeschrieben wird, erscheint die Einführung individuell und therapeutisch orientierter Maßnahmen zur Verbesserung ihres emotionalen Wohlseins als ein Akt sozialer Gerechtigkeit. Die Vorstellung, dass junge Erwachsene therapeutisch begleitet werden müssen, da sie psycho-emotionale Mängel aufweisen, wandelt soziale Probleme in individuelle Defizite um (vgl. Brunila et al. 2016: 71). In diesem Zusammenhang sind europaweit Bildungspolitiken entstanden, welche in ihren Bemühungen um Inklusion eher individuelle Maßnahmen entwickeln, statt strukturelle und sozial-bedingte Zwänge als Ursache mangelnder Beteiligung anzusehen, auch wenn sie dabei unterschiedlichen Interventionslogiken folgen (vgl. Parreira do Ama$\mathrm{ral} /$ Zelinka 2019).

Vulnerabilität wird in politische Programme auch im Hinblick auf die Entstehung neuer sozialer Risiken bzw. New Social Risks (vgl. Taylor-Gooby 2004) eingeschrieben, die insbesondere mit den Nebeneffekten des modernen Vorsorgestaats sichtbar werden (vgl. Castel 2005; Ewald 1993). Die neoliberalen Gesellschaften erleben heutzutage eine Veränderung der Arbeitsweise, die nicht mehr auf direkten Vorgaben beruht, sondern vielmehr Eigeninitiative und Selbstverantwortung fordert. Dieser Übergang von klar abgegrenzten Verantwortungsgebieten zur Vertrauensarbeit und flexiblen Arbeitszeiten hat auch eine konkrete Auswirkung auf die Sorge um die soziale Sicherheit und betrifft nicht nur ausgewählte Personengruppen, sondern mehr und mehr jede*n Einzelne*n. Die Emergenz neuer sozialer Risiken wird besonders anschaulich an der internen Kohäsion in den Wohngebieten industrieller Länder, in denen die neuen Haushaltspolitiken zu weiten Marginalisierungs- und Exklusionseffekten ganzer sozialer Schichten geführt haben (vgl. Harvey 1989). Auch weitere Faktoren, wie die Flexibilisierung des Arbeitsmarkts, Schwierigkeiten mit der Vereinbarkeit von Familie und Beruf, ethnische Pluralisierung oder die schwächer werdende familiäre Solidarität führen zum erhöhten Ungleichheitsniveau und spornen die Konflikthaftigkeit in den Städten und Wohngebieten an (vgl. Ranci et al. 2014: 277). Nicht selten wird dann der Preis für die genannten sozialen Spannungen in Form psychopathologischer Erscheinungen wie Depression oder Burnout gezahlt (vgl. Bianchi et al. 2017; Krüger 1997). Diese wiederum ergänzen die äußeren Störfaktoren um innere Spannungen und transformieren die Vulnerabilität in eine Normalerscheinung und Gewöhnungssache. Die Alltäglichkeit, Verallgemeinerung und Umdeutung der Vulnerabilität als ein zu behandelndes Problem, auch in der inklusiven Bildungspraxis, erzeugen eine essenzialisierende Vorstellung von vulnerablen Erfahrungen, die hinterfragt werden muss (Brown et al. 2017: 506).

Zusammenfassend lassen sich nach dieser ersten Sichtung einige Merkmale des Konzepts identifizieren, welche für eine inklusive Bildungspraxis relevant sind. Diese berücksichtigen insbesondere das Ineinandergreifen und Wirken systemischer und subjektiver 
Vulnerabilität beim Eingliederungsprozess. Das Konzept Vulnerabilität geht also einerseits von Verwundbarkeit als Grundbedingtheit des Menschseins aus und wird andererseits vor allem dazu genutzt, die Anfälligkeit oder das Risiko bestimmter Gruppen zu thematisieren. Dabei ist Deskription oftmals nur schwer von Präskription zu trennen, denn im selben Zuge wie Vulnerabilität benannt wird, tauchen (normative) Forderungen zu ihrer Aufhebung auf. Zugleich zeichnet sich das Konzept durch eine ausgeprägte Relationalität zwischen Individuen/Gruppen, ihren Lebensbedingungen, aber auch zwischen den unterschiedlichen Lebensbereichen aus, was die Relevanz eines intersektionalen Blicks für die Phänomene deutlich macht.

Der intersektionale Blick auf Vulnerabilität lässt sich als eines der zentralen Gewinne dieses Diskurses erkennen, denn hier wird eine produktive Verbindung teilweise disparater wissenschaftlicher Diskurse zu verschiedenen Problemlagen hergestellt. Eine eigentümliche Dialektik lässt sich ebenfalls ausmachen, indem Vulnerabilität der Individuen/Gruppen mit der Vulnerantialität der sozialen, ökonomischen usw. Kontexte und Bedingungen, die Letztere hervorrufen (können), verknüpft wird. Schließlich lassen sich ebenfalls Aspekte der Zuschreibung von Zuständen und Dispositionen festmachen, welche oftmals mit Fragen sozialer Kontrolle einhergehen.

Im nächsten Abschnitt gehen wir auf die Ergebnisse eines kürzlich abgeschlossenen Projektes ein, um ein relationales und kontextsensibles Verständnis von Vulnerabilität zu erörtern.

\section{Das Europäische Forschungsprojekt YOUNG_ADULLLT}

Im Rahmen des EU-Programms für Forschung und Innovation Horizont 2020 hat das europäische Forschungsprojekt YOUNG_ADULLLT untersucht, wie die Politiken des Lebenslangen Lernens (lifelong learning policies) zur sozialen Inklusion und zum wirtschaftlichen Wachstum der EU-Länder beitragen. Die Garantie einer sozialen Inklusion und eines nachhaltigen Wachstums ist zu einer der wichtigsten Prioritäten für die Mitgliedsländer der Europäischen Union geworden, insbesondere seit der Finanzkrise im Jahr 2008/2009 und der darauffolgenden andauernden Arbeitsmarktrezession (vgl. Europäische Kommission 2010). In vielen dieser Länder wurde die Politik des Lebenslangen Lernens als eine Möglichkeit angesehen, die Folgen der Krise zu entschärfen, allgemeine Mobilität zu fördern und regionale Kohäsion sowie eine stärkere soziale Inklusion zu unterstützen (vgl. Europäische Kommission 2013).

Gerade die Politiken des Lebenslangen Lernens standen im Fokus vergleichender empirischer Analysen des YOUNG_ADULLLT Projekts, die anhand eines Methodenmix-Verfahrens quantitative mit qualitativen Daten kombiniert und ausgewertet haben (vgl. Parreira do Amaral et al. 2020). Das Projekt hat die Wechselwirkungen zwischen drei Elementen Lebenslangen Lernens analysiert, und zwar zwischen der regionalen und lokalen Ebene der Politikgestaltung, der Politikformulierung und -implementierung im Bereich des Lebenslangen Lernens und den Lebensläufen junger Erwachsener. Die Ergebnisse des Projekts haben gezeigt, dass im Rahmen der untersuchten EU-Mitgliedsländer (Bulgarien, Deutschland, Finnland, Italien, Kroatien Österreich, Portugal, Spanien und Schottland) eine große Diversität angesichts der Ziele, Orientierungen und Adressat*innengruppen herrscht. Allerdings trat ebenfalls zu Tage, dass die Beschäftigungsfähigkeit (employability) der Adressat*innen eines 
der wichtigsten Ziele vieler Politiken ist, die vornehmlich durch präventive, kompensierende oder aktivierende Strategien erreicht werden soll (vgl. Parreira do Amaral/Zelinka 2019). Für das Erreichen dieses Hauptziels spielt die Definition der Zielgruppe einer Politik eine zentrale Rolle; in der Regel beziehen sich diese Definitionen vornehmlich auf Zustände und Dispositionen junger Erwachsener, weshalb das Konzept der Vulnerabilität als ein zentrales Unterscheidungskriterium herangezogen wird. Insbesondere betrifft dies die Definition von Adressat*innen, für die die Politiken des Lebenslangen Lernens maßgeschneidert werden. Diese werden als vulnerabel, gefährdet, exkludiert, prekär oder diskriminiert bezeichnet und sollen daher durch fachliche Requalifizierung bzw. durch psychosoziale und emotionale Unterstützung befähigt werden, sich wieder vollständig in den Arbeitsmarkt und in die Gesellschaft einzugliedern. Einem solchen Blick auf Vulnerabilität als ein essenzielles Attribut bzw. als eine natürliche Charakteristik junger Erwachsener oder ganzer sozialer Gruppen hat das $Y$ OUNG_ADULLLT Projekt eine Perspektive gegenübergestellt, welche die Vulnerabilität als eine relationale und kontextuelle Kategorie auffasst. Sie kann je nach kultureller und sozialer Deutung variieren und verschiedene Implikationen für die Gestaltung der Politik des Lebenslangen Lernens haben (Scandurra et al. 2017: 10f). Wie Vulnerabilität als ein bildungspolitisches Deutungsmuster aus dieser Perspektive betrachtet werden kann, zeigen die folgenden Beispiele aus den untersuchten Funktionalen Regionen. ${ }^{3}$

\section{Vulnerabilität als relationales und kontextsensibles Konzept: Beispiele aus den Funktionalen Regionen Europas}

In manchen Regionen erscheinen Individuen als vulnerabel, weil sie zu einer ethnischen, religiösen oder sexuellen Minderheit gehören und daher beispielsweise schlechteren Wohnund Lebensverhältnissen ausgesetzt sind, wie etwa die Roma in der Funktionalen Region (FR) Plovdiv, Bulgarien. Basierend auf der Vorstellung, dass diese Zielgruppe besonders vulnerabel ist, wurden in der FR Plovdiv bildungspolitische Maßnahmen eingeleitet, die die kritische Situation vieler Roma verbessert und diese Minderheit in die Gesellschaft konsistenter integrieren sollte. Ein Beispiel dafür bietet das Programm des Lebenslangen Lernens Land Source of Income, das eine Personalqualifizierung im Agrarbereich anbietet. ${ }^{4}$ Die Weiterbildung dieser ethnischen Minderheit wird als eine Möglichkeit angesehen, Wirtschaftswachstum mit sozialer Inklusion zu verbinden. Mit der Einleitung solcher Maßnahmen wird die Gruppe der Roma a priori als förderungsbedürftig deklariert, was die Tatsache ausblendet, dass ihre Lebensverhältnisse strukturell und historisch bedingt sind.

Wenn zu den genannten vulnerablen Zuständen weitere Faktoren hinzukommen, wie zum Beispiel gesundheitliche Beeinträchtigungen oder soziale Exklusion, kann dies die relative Vulnerabilität eines Individuums bzw. einer ganzen Gruppe multiplizieren. Die dadurch

3 Eine funktionale Region wird durch die Intensität der Infrastruktur (Verkehr, Handel, Kommunikation) bzw. durch die Funktionalität der Beziehungen (wirtschaftlichen, technologischen, sozialen) bestimmt. Sie entspricht nicht immer einer administrativen Einheit und ihre Beziehungen sind weder zentrifugal noch zentripetal, sondern entsprechen einer Maximierung von Innenbeziehungen und Minimierung von Außenbeziehungen (siehe auch Parreira do Amaral et al. 2020).

4 Weiterführende Informationen zu dieser und weiteren untersuchten Maßnahmen befinden sich auf der Webseite des Projekts: http://www.young-adulllt.eu/policy-mapping/index.php [Zugriff: 31.07.2020] 
entstehenden Vulnerabilitätsschichten (vgl. Luna 2009: 128) zeigen, dass Vulnerabilität je nach materiellen und zeitlichen Umständen unterschiedlich stark ausgeprägt und mehr oder weniger augenfällig sein kann. Um diese Komplexität und Verflechtung von Vulnerabilitäten $\mathrm{zu}$ berücksichtigen und den jungen Erwachsenen einen personalisierten Service anbieten zu können, werden in manchen Funktionalen Regionen Maßnahmen entwickelt, die sich um eine aktive Wiedereingliederung und Begleitung junger Erwachsener bemühen, so beispielsweise auch das Programm NUPPA No-Threshold Guidance Centre in der FR Kainuu, Finnland (vgl. Rinne et al. 2016). Im Rahmen dieses Programms werden den jungen Erwachsenen zu ihren konkreten Belangen Hilfsinstrumente wie Beratung, psychosoziale Unterstützung oder Rehabilitation angeboten, damit sie sich sowohl in akuten wie auch in dauerhaften Lebenssituationen orientieren können. Durch diese ermächtigenden Maßnahmen lernen die jungen Erwachsenen, wie sie mit den entstehenden Inkonsistenzen umgehen und wo sie eine passende Hilfe finden können. Ihre Vulnerabilität wird dadurch behandelbar und ihre Lebensprojekte realisierbar gemacht, was die positive Seite des Vulnerabilitätsdiskurses offenbart, und zwar die Ermächtigung, die durch das Gestehen und Handhaben vulnerabler Erfahrungen stattfinden kann (vgl. Brown 2013).

Schließlich wird die Übergangsphase in das Erwachsenenleben nicht selten auf die Fähigkeit junger Erwachsenen zurückgeführt, sich während bzw. nach der Ausbildung in den Arbeitsmarkt integrieren und selbstständig unterhalten zu können. In dieser Phase des Lebenszyklus besteht für die jungen Erwachsenen eine Gefahr darin, wegen einer unabgeschlossenen Ausbildung, mangelnder Arbeitserfahrung, prekärer sozialer Lage oder Unter- bzw. Überqualifizierung den Vollzeit-Arbeitsmarkt nicht zu erreichen und somit nicht ausreichend für ihre sozialen Sicherheiten sorgen zu können. Die Erfahrung ihrer sozialen Vulnerabilität versetzt sie in einen Zustand, in dem sie ihre Gegenwart nicht kontrollieren und ihre Zukunft nicht planen können (vgl. Castel 2005). In vielen Funktionalen Regionen Europas, beispielsweise in den FR Bremen (Deutschland), Plovdiv (Bulgarien), Istria (Kroatien) und Girona (Spanien) werden die jungen Erwachsenen als passive Empfänger*innen von Politiken des Lebenslanges Lernen betrachtet, wobei diese nicht im ganzen Maße auf deren Bedürfnisse, Wünsche und Pläne eingehen (Rambla et al. 2018: 66). Stattdessen wird von den jungen Erwachsenen mehr oder weniger implizit erwartet, dass sie einem linearen Lebenslauf folgen, das heißt in ihren Lebensphasen graduell - Schule, Beruf, Familie, Rente - vorankommen werden. Unter diesem Blickwinkel werden mögliche Missverhältnisse als rückfällig und die soziale Vulnerabilität als problematisch gedeutet, was ihre Behandlung in Form aktivierender Programme rechtfertigt. Außerdem kann eine solche Perspektive zu beträchtlichen negativen Folgen führen, indem sie die jungen Erwachsenen als passiv, unwillig oder vulnerabel ansieht und sie damit weiteren Stigmatisierungseffekten aussetzt.

Die vergleichenden Analysen des YOUNG_ADULLLT Projekts haben Vulnerabilität als ein sozio-politisches Deutungsmuster beobachtet und ihre Einschreibung in die Politiken des Lebenslangen Lernens auf struktureller und individueller Ebene festgehalten.

\subsection{Strukturelle und individuelle Vulnerabilitäten relational verstehen und erfassen}

Vulnerabilität als eine relationale und kontextuelle Kategorie zu deuten, heißt, die Bedingungen und Umstände, unter welchen vulnerable Lebensverhältnisse entstehen, zu untersuchen. 
In Europas Funktionalen Regionen herrschen zahlreiche Disparitäten, sowohl innerhalb auch als außerhalb der Regionen. Dem liegt die Tatsache zugrunde, dass die Regionen unterschiedlich stark wirtschaftlich, infrastrukturell oder institutionell aufgebaut sind und daher unterschiedliche Dynamiken erzeugen. Zu der Heterogenität regionaler und lokaler Verhältnisse tragen außerdem weitere Faktoren bei, wie beispielsweise demographische, soziale oder politische Trends, Bildungs- und Arbeitsmarktstruktur, Gesundheits- und Sozialsysteme usw., die miteinander interagieren und die Lebensbedingungen von jungen Erwachsenen damit bedeutend prägen. Dies ist gleichzeitig ein Plädoyer für einen kontext-spezifischen Blick auf die Lebensbedingungen junger Erwachsener, welche einen direkten Einfluss auf ihre Chancen haben, kontinuierliche und gehaltvolle Lebensprojekte zu entwickeln und sich damit aktiv an der Gesellschaft zu beteiligen. Deshalb werden im Folgenden die strukturellen Formen der Vulnerabilität sowie ihre individuellen Faktoren in der Gestaltung von den Politiken des Lebenslangen Lernens, wie sie aus dem Methodenmix-Verfahren des Projekts resultieren, diskutiert.

YOUNG_ADULLLT hat den kontextuellen Lebensbedingungen junger Erwachsener eine besondere Aufmerksamkeit geschenkt (vgl. Scandurra et al. 2017), um die Wechselwirkungen zwischen den verschiedenen Sphären des sozialen Lebens zu analysieren. Diese Wechselwirkungen entscheiden nämlich darüber, welche Opportunitätsstrukturen für die jungen Erwachsenen an unterschiedlichen Orten und Regionen Europas entstehen und mithin auch darüber, welchen strukturellen Vulnerabilitäten die jungen Erwachsenen ausgesetzt sind (vgl. Cloward/Ohlin 2013; Kerckhoff 1995, 2001; Lehmann 2005). Bisher wurden vor allem Nationalstaaten bzw. Individuen als Analyseeinheiten gewählt (vgl. Ranci 2010), selten aber die lokalen und regionalen Ebenen, wo die jungen Erwachsenen die Exklusionseffekte empfindsamer erleben. Die vergleichende Analyse hat nahegelegt, dass immer mehr junger Erwachsene eine tertiäre Bildung anstreben, dass aber gleichzeitig in vielen Funktionalen Regionen Europas, insbesondere in Süd- und Südosteuropa, ein Anstieg von Jugendarbeitslosigkeit zu beobachten ist, da diese sehr sensibel auf die Wirtschaftszyklen reagiert. Außerdem wurde deutlich, dass die wirtschaftliche Stärke eines Landes bessere Berufsoptionen schafft, wobei die formellen Bildungsstrukturen ziemlich gleich aufgebaut sind. Die jungen Erwachsenen haben demnach in gewissen Funktionalen Regionen, wie etwa Bremen (Deutschland) oder Oberösterreich (Österreich) vergleichbar bessere Opportunitätsstrukturen für den Übergang von Schule zum Beruf als in anderen Funktionalen Regionen, wie etwa Ligurien (Italien) oder Plovdiv (Bulgarien) (vgl. Scandurra et al. 2017: 52). Des Weiteren erzeugen die unterschiedlichen strukturellen Bedingungen Missverhältnisse zwischen dem Fachkraftangebot und der Fachkraftnachfrage, von denen die schon ohnehin benachteiligten jungen Erwachsenen am meisten betroffen sind. Bei begrenzter Nachfrage in schwach wirtschaftlich entwickelten Regionen bleibt die Frage offen, ob eine höhere Bildung oder Investition in Requalifizierung in Form vom Lebenslangen Lernen die Chancen auf dem Arbeitsmarkt tatsächlich garantieren oder umgekehrt die vulnerablen Zustände eher weiter verschärfen (vgl. CapsadaMunsech et al. 2018: 39).

Neben den strukturellen Bedingungen, unter denen die Politiken des Lebenslangen Lernens gestaltet werden, hat das YOUNG_ADULLLT Projekt auch die individuellen Faktoren der Vulnerabilität junger Erwachsener in den Blick genommen. Diese spielen insbesondere dann eine bedeutende Rolle, wenn sie bei der Definition der Zielgruppe als Marker oder Kriterien ausgewählt werden. Die Politiken des Lebenslangen Lernens können somit je nach der Definition der Zielgruppe intervenieren und die notwendigen Eingriffe rechtfertigen. Anhand der Analyse von Zielgruppendefinitionen konnten im Rahmen des Projekts vier unter- 
schiedliche Kategorien von Vulnerabilität junger Erwachsener identifiziert werden. Diese unterscheiden sich in Bezug:

- Zur Bildung oder zum Training: junge Erwachsene mit niedrigem Bildungsniveau, niedriger Qualifikation, Schulabbrecher*innen, Schulabgänger*innen;

- Zum aktuellen Beschäftigungsverhältnis: vor allem arbeitslose junge Erwachsene und diejenigen, die sich nicht in Beschäftigung, Unterricht, Aus- oder Fortbildung befinden (engl. NEET: Not in Employment, Education, or Training);

- $\quad Z u$ strukturellen Verhältnissen: junge Erwachsene, die wegen materieller Lage (Armut, Obdachlosigkeit, mangelnde gesundheitliche Sorge), sozialer Verhältnisse (mangelnde Unterstützung von der Familie, Peer-Gruppen oder Mentor*innen, insbesondere in Krisensituationen), unmittelbarer Naturrisiken (Segregation), oder ihres Hintergrunds (ethnische oder sexuelle Minderheit) verstärkt verwundbar sind;

- Zur physischen oder kognitiven Beeinträchtigung: junge Erwachsene, die unter Krankheit, mentaler Störung, Behinderung oder mentalen Folgen der Unreife, Drogenabhängigkeit oder Sucht beeinträchtiget sind (vgl. Palumbo et al. 2018: 217).

Hierbei ist es wichtig anzumerken, dass die genannten Kategorisierungen dazu tendieren, die jungen Erwachsenen als defizitär, passiv oder unwillig zu beschreiben und damit riskieren, weitere Stigmatisierungseffekte zu erzeugen (vgl. auch Hirschberg/Köbsell 2016). Die hier genannten Kategorien repräsentieren nicht lediglich junge Erwachsene, sondern konstruieren und konstituieren diese als vulnerable youths. Viele Politiken des Lebenslangen Lernens neigen allerdings dazu, gerade die kurativen und restaurativen Maßnahmen einzuführen, mit denen die individuellen Defizite durch präventive, aktivierende, kompensierende oder ermächtigende Interventionen beseitigt oder mindestens reduziert werden könnten (vgl. Parreira do Amaral/Zelinka 2019). Gleichzeitig wird durch die normativen Erwartungen eines standardisierten Lebenslaufs ein enormer Druck auf die jungen Erwachsenen ausgeübt, der jedwedes Scheitern als Unfähigkeit deutet, sich im Leben erfolgreich zu etablieren. Die hier beobachtete selbsterfüllende Prophezeiung zeugt von der Übernahme dieser Norm, die, wenn sie als einzige Option erscheint, an Vulnerabilität als eine individuelle Eigenschaft glauben lässt. Deshalb ist es wichtig hervorzuheben, dass junge Erwachsene nicht für die Umstände, auf die sie keinen Einfluss nehmen können, verantwortlich gemacht werden dürfen und dass verschiedene politische Maßnahmen des Lebenslangen Lernens verschiedene Effekte in verschiedenen Kontexten haben können (vgl. Schweisfurth et al. 2018: 251). Schließlich müssen auch weitere Behauptungen hinterfragt werden; und zwar die Vorstellung, dass die Risiken, mit denen die jungen Erwachsenen konfrontiert sind, wie zum Beispiel die Balance zwischen Arbeit und Familie, schwache Fachkompetenzen, Unreife usw. auf deren Unfähigkeit, einem standardisierten Lebenslauf zu folgen, zurückzuführen sind. Die jungen Erwachsenen sehnen sich stattdessen nach aktiver Unterstützung, Mitbeteiligung und Mobilisierung ihrer Wünsche, Bedürfnisse und Sehnsüchte, für die sie die nötigen Instrumente, auch in Form von Politiken des Lebenslangen Lernens, benötigen (vgl. Rambla et al. 2018: 63).

Um die strukturellen und individuellen Vulnerabilitäten methodologisch erfassen und kontextspezifisch abbilden zu können, wurden einerseits Aussagen von jungen Erwachsenen und Expert*innen aus Bildungs- und Sozialberufen (164 und 128 Interviews) erhoben, die Auskunft geben sollten mit welchen Schwierigkeiten und Beeinträchtigungen, sich die jungen Erwachsenen konfrontiert fühlen und welchen Einfluss diese auf ihre Lebensläufe nehmen. Andererseits wurden Indikatoren auf der NUTS-2-Ebene erfasst, anhand derer die strukturelle und soziale Lage junger Erwachsener auf lokaler und regionaler Ebene klarer erkennbar wurde. Wie Abbildung 1 zeigt, zeichnet sich jede Funktionale Region durch die 
Kombination bestimmter Merkmale aus, die über den Wahrscheinlichkeitsgrad für die Entwicklung vulnerabler und vulneranter, die Vulnerabilität begünstigender, Lebensbedingungen entscheidet. Diese Merkmale lassen sich für sechs grundlegenden Lebenssphären aufweisen und liefern ein Instrumentarium zur Beschreibung der lokalen und regionalen Kontexte, in denen sich die jungen Erwachsenen während ihrer Übergangs- und Entscheidungsphasen befinden. Damit die Analysen möglichst nah an die Lebensverhältnisse dieser Untersuchungsgruppe herankommen, wurden solche Indikatoren ausgewählt, die statistisch aufgefasst und auf der regionalen Ebene zugänglich sind. Daraus entstand im Weiteren eine Plattform für vergleichbare Analysen, die die Struktur der Funktionalen Region und die damit einhergehenden unterschiedlichen Lebensbedingungen junger Erwachsener problematisiert haben.

Abbildung 1: Kontextuelle Lebensbedingungen von jungen Erwachsenen

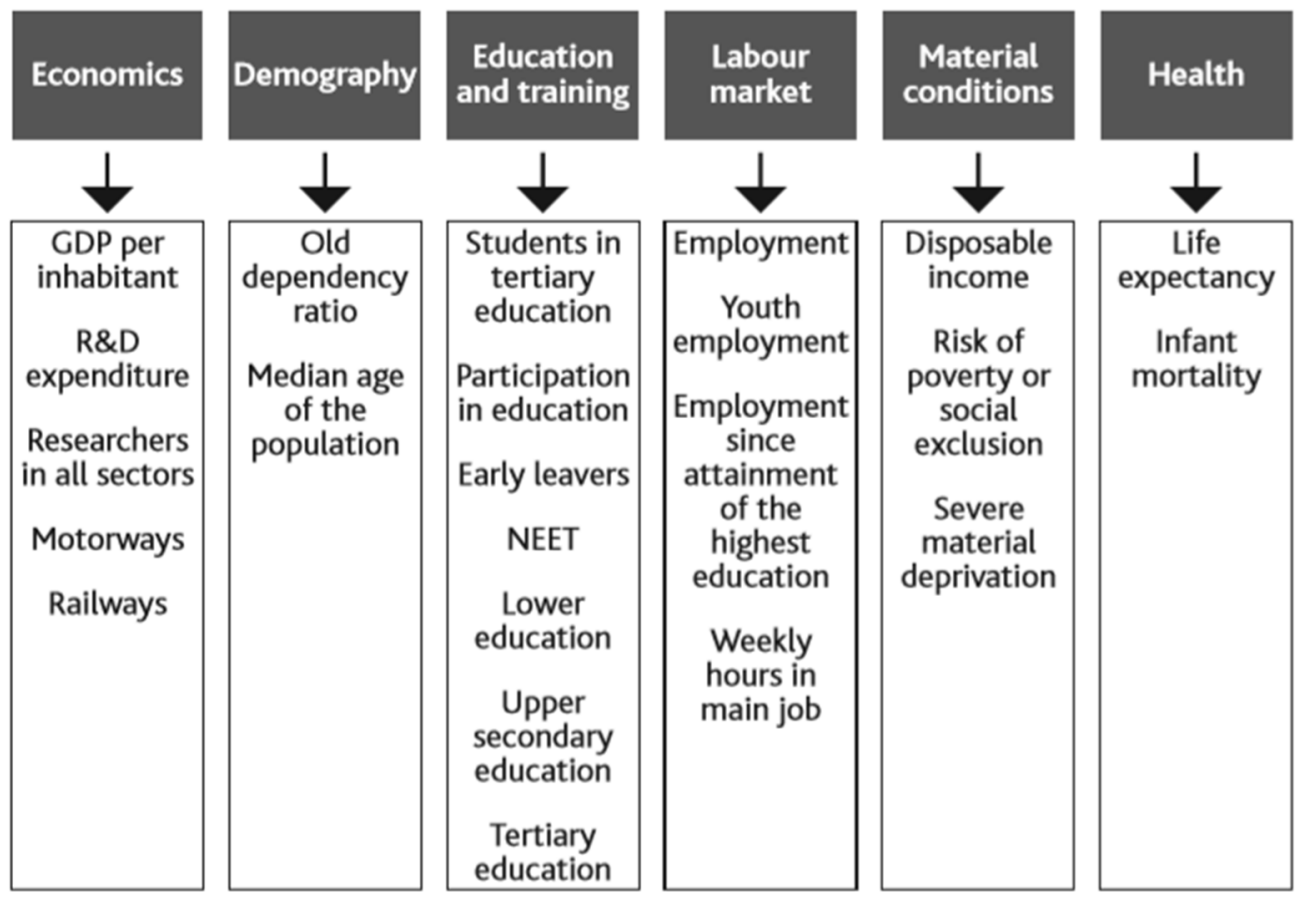

Quelle: Scandurra et al. 2020: 178

Die Abbildungen 2 und 3 veranschaulichen zum Beispiel, unter welchen Bedingungen die jungen Erwachsenen in Österreich und Spanien ihre Übergangsphase erleben und die Programme des Lebenslangen Lernens wahrnehmen. So sind die jungen Erwachsenen in den Funktionalen Regionen Spaniens im Vergleich zu den jungen Erwachsenen in Österreich durch ein relativ schwächeres Wirtschaftswachstum, einen weniger flexiblen Arbeitsmarkt sowie durch einen niedrigeres Bildungsniveau konditioniert. Zudem verändern sich die Lebensverhältnisse beider Gruppen unterschiedlich stark je nach dem, wie die Regionen auf die äußeren Einflussfaktoren reagieren. So weisen die Funktionalen Regionen Österreichs im 
Vergleich zu den Funktionalen Regionen Spaniens eine relativ gleiche Entwicklung auf sowohl vor (2007) als auch nach (2014) der globalen Wirtschaftskrise.

Abbildung 2: Kontextuelle Lebensverhältnisse junger Erwachsener am Beispiel Österreichs [Der Indikator „Material Conditions“ für das Jahr 2007 in der Funktionalen Region Wien konnte nicht ermittelt werden]

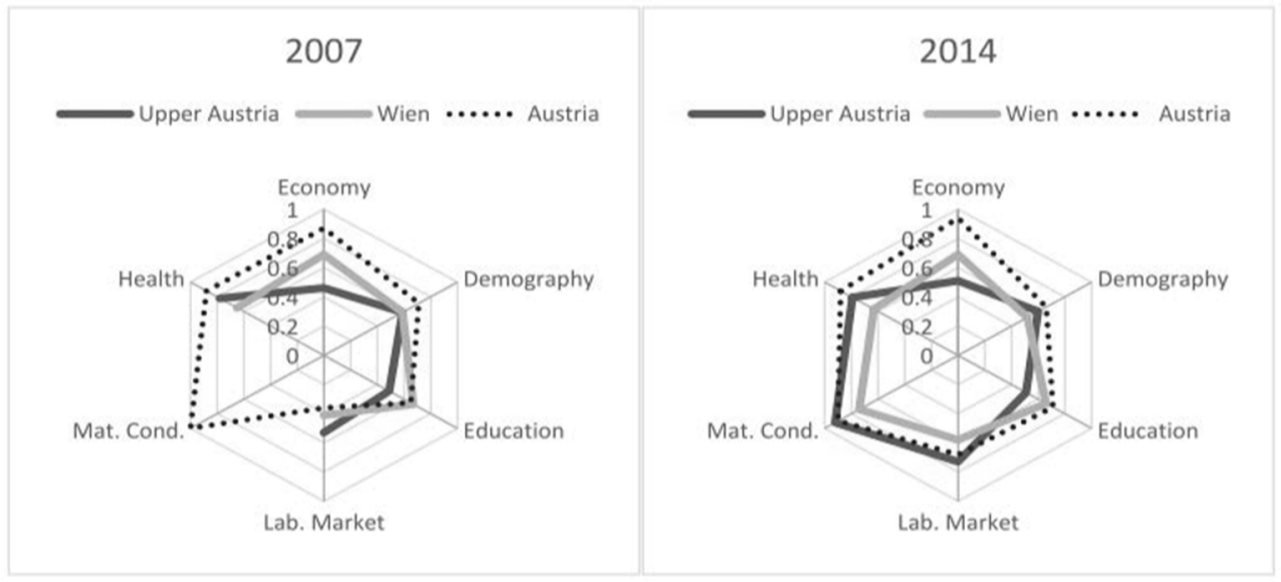

Quelle: Alexander et al. 2018: 79

Abbildung 3: Kontextuelle Lebensverhältnisse junger Erwachsenen am Beispiel Spaniens

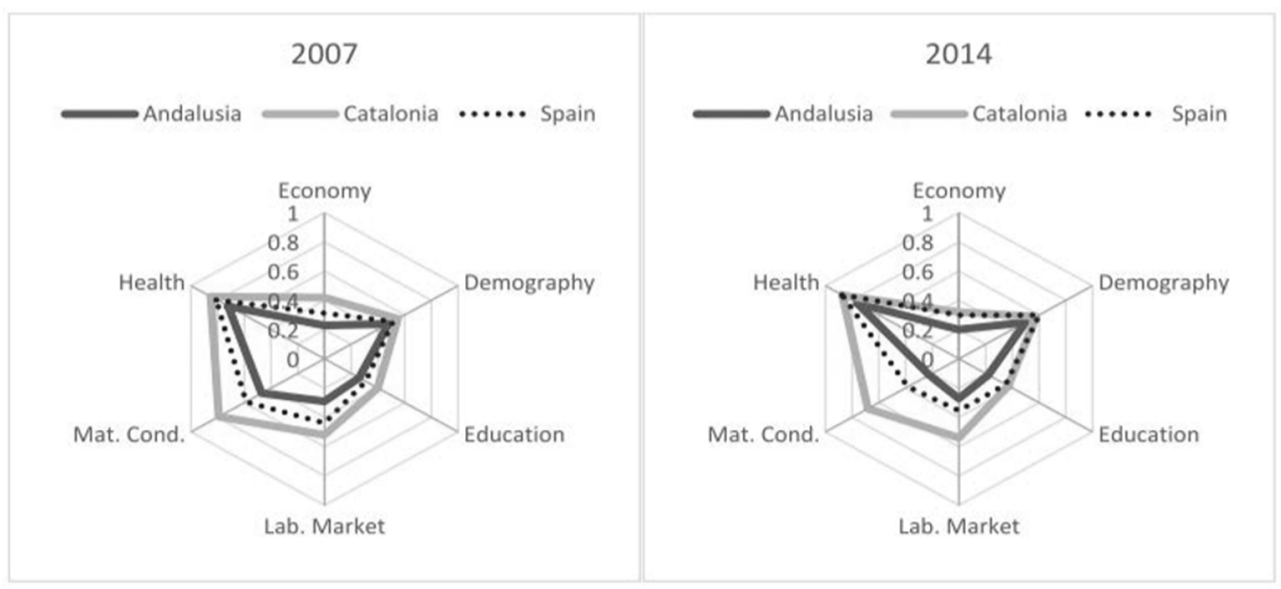

Quelle: Alexander et al. 2018: 88

Mit dem Fokus auf kontextsensible und relationale Indikatoren für die Erfassung regionaler Vulnerabilitäts- und Vulnerantialitätsfaktoren hat YOUNG_ADULLLT konkrete Hinweise zu strukturellen Unstimmigkeiten geliefert, die bei der Planung und Durchführung von Politiken des Lebenslangen Lernens berücksichtigt werden können. Vulnerabilität kann auf diese 
Weise empirisch erfasst und auf der regionalen Ebene untersucht werden. Deutlich wurde jedoch auch, dass die Datenlage auf der regionalen Ebene (NUTS-2) sehr unterschiedlich ist und hier weitere Anstrengungen notwendig sind, damit bildungspolitische Maßnahmen gut informiert werden.

\section{Diskussion und Schluss}

Vulnerabilität, so lässt sich resümierend festhalten, ist ein in der Gegenwart vielseitig verwendetes Konzept. Der Begriff fand sowohl auf europäischer als auch auf nationaler Ebene Eingang und dient maßgeblich der Charakterisierung bestimmter Gruppen in der Bearbeitung gesellschaftlicher Probleme, wie die Bekämpfung von sozialer Exklusion. Es ist ein Konzept, das in seinem Bedeutungsumfang sehr breit und in seiner Verwendung stark uneinheitlich ist. Der inflationäre Gebrauch zieht die Notwendigkeit nach sich, konzeptuelle Anstrengungen zu unternehmen, um Vulnerabilität mit Gewinn analytisch und reflektiert anwenden zu können. Eine ähnliche wissenschaftliche und politische Rezeption erfährt auch der Begriff der Inklusion bzw. der inklusiven Bildung. Mit der Aufforderung zur Nichtaussonderung der Individuen, gleichgültig welche individuellen oder strukturellen Hindernisse sie ausgesetzt sind, aus den institutionellen (Aus-)Bildungsprogrammen, wird einerseits das Bemühen erkennbar, die Chancengleichheit für jede*n zumindest realisierbar zu machen. Andererseits wird mit inklusiver Bildung auch dafür gesorgt, dass eine differenzierende Praxis in die Programmatik der (Aus-)Bildung eingeführt wird, denn Inkludieren setzt ein Exkludieren voraus und damit eine kontinuierliche Auseinandersetzung mit Unterscheidungsmerkmalen, die zur endlosen Selbstreproduktion der Inklusion führen kann.

Vulnerabilität, so wurde hier argumentiert, stellt gegenwärtig ein neues bildungspolitisches Deutungsmuster dar, mit dem gesellschaftliche (strukturelle) Probleme bearbeitet werden sollen, vornehmlich durch eine individualisierende Deutung. Mit Blick auf Politiken des Lebenslangen Lernens wird Vulnerabilität genutzt, um auf die relative Position junger Erwachsener im Bildungssystem oder bei ihrem Übergang von der Bildung in den Arbeitsmarkt hinzuweisen. Dies mit dem Ziel, diese Gruppe bei der Bekämpfung der sozialen Ausgrenzung zwischen verschiedenen Bevölkerungsgruppen zu priorisieren. Trotzdem wird in politischen Diskursen Vulnerabilität sehr oft mit Umständen oder Dispositionen von Individuen/Gruppen in Verbindung gebracht. Als solches wird der Begriff vulnerabel zu einem Verweis auf intrinsische Merkmale oder Attribute von Zielgruppen, anstatt Vulnerabilität als sozial-relationalen Begriff zu betrachten (vgl. Scandurra et al. 2017: 10f.).

Eine Beobachtung ist bemerkenswert, wenn es um die Prozesse geht, junge Erwachsene hinsichtlich ihrer Position auf dem Arbeitsmarkt, ihrer Bildungsergebnisse, ihres sozioökonomischen Status als vulnerabel zu rahmen: Da lineare Lebensläufe immer noch als die Norm angesehen werden, übernehmen sie die Funktion eines gesellschaftspolitischen Interpretationsrahmens, ambivalent und in ihren Implikationen sehr potent. Soziopolitische Interpretationsrahmen, so Axel Pohl (2015: 57), leiten ihre Wirksamkeit sowohl aus ihrem normativen Gehalt als auch aus ihrer tatsächlichen Verbreitung ab.

Vulnerabilität als gesellschaftspolitischer Interpretationsrahmen, so wie er in LLL-Politiken sichtbar wird, fördert das Verständnis eines normalen Lebenslaufs und einer Biografie entlang institutionalisierter, mehr oder weniger linearer Wege von der Schule zum Arbeitsmarkt. Von jungen Erwachsenen wird erwartet, dass sie Lebensprojekte entwickeln, die 
solchen legitimen Erzählungen entsprechen. Diejenigen, die als unfähig oder nicht willens angesehen werden, lineare Lebensläufe zu verfolgen, werden dann als benachteiligt und/oder vulnerabel (mit negativen Konnotationen) wahrgenommen.

Dieser gesellschaftspolitische Interpretationsrahmen ist ambivalenter Natur, da er zwar der Politik ermöglicht, integrative Interventionen zu rechtfertigen, aber auch junge Menschen als passiv und durch Defizite oder Mängel gekennzeichnet darstellt, was die Gefahr birgt, dass sie stigmatisierende und schädliche Auswirkungen auf ihr Leben erfahren. Ähnliche Ambivalenzen wurden bereits hinsichtlich des Themas sonderpädagogischer Förderung „schulische Behinderung“ - diskutiert (vgl. Powell 2006). Es ist wichtig zu beachten, dass diese Repräsentationen junger Erwachsener sie nicht einfach neutral widerspiegeln, sondern sie konstituieren und konstruieren. Letzteres ist nicht nur ein Potenzial, sondern ein tatsächlicher Effekt, da sich, wie bereits erwähnt, viele LLL-Politiken mit strukturellen Problemen befassen, indem sie hauptsächlich/primär individuelle Defizite angehen.

Der vorliegende Beitrag fokussierte das Konzept Vulnerabilität aus einer übergreifenden, international vergleichenden Perspektive und thematisierte dessen Verwendung auf europäischer Ebene. Dadurch ließ sich die Etablierung des Konzepts als ein bildungspolitisches Deutungsmuster ausweisen, das gegenwärtig europäische Bildungsinitiativen im Bereich des Lebenslangen Lernens maßgeblich prägt. Zukünftige Forschung wird sich jedoch stärker mit den nationalen Ausprägungen des Phänomens auseinandersetzen müssen, um die unterschiedlichen Wirkweisen von vulnerablen und vulneranten Faktoren in den verschiedenen europäischen Kontexten verstehen zu können. Für die Politik und Praxis bleibt vorerst der Hinweis auf die Notwendigkeit, so reflexiv wie möglich mit den verwendeten Konzepten und Begriffen umzugehen.

\section{Literatur}

Achtenberg, Deborah (2017): Essential Vulnerabilities. Plato and Levinas on Relations to the Other. Evanston: Northwestern University Press.

Alexander, Lukas/Lipp, Mathias/Görgen, Nina/Scandurra, Rosario/Cefalo, Rugero/Kazepov, Yuri (2018): Cross-regional measures of contextual living conditions of young adults in Europe. In: Parreira do Amaral, Marcelo/Zelinka, Jozef/Schweisfurth, Michele (Hrsg.): Comparative Analysis Report. YOUNG_ADULLLT Working Paper. Münster: University of Münster, S. 70-92.

Alwang, Jeffrey/Siegel, Paul B./Jorgenson, Steen L. (2002): Vulnerability as Viewed from Different Disciplines. Presented at International Symposium Sustaining Food Security and Managing Natural Resources in Southeast Asia: Challenges for the 21st Century. Chiang Mai, Thailand, 8-11 January 2002.

Andresen, Sabine/Koch, Claus/König, Julia (Hrsg.) (2015): Vulnerable Kinder. Interdisziplinäre Annäherungen. Wiesbaden: Springer Fachmedien.

Ayoub, Phillip M. (2017): Das Coming-out der Staaten. Europas sexuelle Minderheiten und die Politik der Sichtbarkeit. Bielefeld: transcript Verlag.

Bankoff, Greg/Frerks, Georg/Hilhorst, Dorothea (Hrsg.) (2004): Mapping Vulnerability. Disasters, Development, and People. London, Sterling, VA: Earthscan.

Batchelor, Denise Claire (2006): Vulnerable Voices. An examination of the concept of vulnerability in relation to student voice. In: Educational Philosophy and Theory 38, 6, S. 787-800.

Bauman, Zygmunt (2000): Flüchtige Moderne. Frankfurt am Main: Suhrkamp Verlag. 
Beck, Ulrich (1992): Risikogesellschaft. Frankfurt am Main: Suhrkamp.

Beicht, Ursula/Granato, Mona (2011): Prekäre Übergänge vermeiden - Potenziale nutzen. Junge Frauen und Männer mit Migrationshintergrund an der Schwelle von der Schule zur Ausbildung. Expertise im Auftrag des Gesprächskreises Migration und Integration der FriedrichEbert-Stiftung. Bonn: Friedrich-Ebert-Stiftung.

Bergemann, Lutz/Frewer, Andreas (2018): Autonomie und Vulnerabilität in der Medizin. Menschenrechte - Ethik - Empowerment. Bielefeld: transcript Verlag.

Bianchi, Renzo/Schonfeld, Irvin Sam/Laurent, Eric (2017): Burnout or depression. Both individual and social issue. In: The Lancet 390, 10091, S. 230.

Bieler, Andrea (2017): Verletzliches Leben. Horizonte einer Theologie der Seelsorge. Göttingen: Vandenhoeck \& Ruprecht.

Birkmann, Jörn/Bach, Claudia/Guhl, Silvie/Witting, Maximilian/Welle, Torsten/Schmude, Miron (2010): State of the Art der Forschung zur Verwundbarkeit kritischer Infrastrukturen am Beispiel Strom/Stromausfall. Forschungsforum Öffentliche Sicherheit. Schriftenreihe Sicherheit Nr. 2 Berlin: Freie Universität Berlin.

Bishawjit, Mallick (2014): Der gesellschaftliche Umgang mit zunehmender Verwundbarkeit. eine Analyse der sozialen Bedingungen für vulnerabilitätsorientierte räumliche Planung in den Küstenzonen von Bangladesch. Karlsruhe: Scientific Publishing.

Brown, Brené (2013): Verletzlichkeit macht stark. Wie wir unsere Schutzmechanismen aufgeben und innerlich reich werden. München: Kailash Verlag (Verlagsgruppe Random House GmbH).

Brown, Kate (2017): Introduction. Vulnerability and Social Justice. In: Social Policy \& Society 16, 3, S. 423-427.

Brown, Kate/Ecclestone, Kathryn/Emmel, Nick (2017): Review Article. The Many Faces of Vulnerability. In: Social Policy \& Society 16, 3, S. 497-510.

Brunila, Kristiina/Ikävalko, Elina/Kurki, Tuuli/Mertanen, Katariina/Mikkola, Anna (2016): Revisiting the vulnerability ethos in cross-sectoral transition policies and practices for young people in the era of marketisation of education. In: Research in Comparative \& International Education 11, 1, S. 69-79.

Bührmann, Andrea D. (2012): Das unternehmerische Selbst. Subjektivierungsform oder Subjektivierungsweise? In: Keller, Reiner/Schneider, Werner/Viehöver, Willy (Hrsg.): Diskurs, Macht und Subjekt: Theorie und Empirie von Subjektivierung in der Diskursforschung. Wiesbaden: VS Verlag für Sozialwissenschaften, S. 145-164.

Bütow, Birgit/Gómez Jiménez, María Luisa (Hrsg.) (2015): Social Policy and Social Dimensions on Vulnerability and Resilience in Europe. Opladen, Berlin, Toronto: Barbara Budrich Publishers.

Burghardt, Daniel/Dederich, Markus/Dziabel, Nadine/Höhne, Thomas/Lohwasser, Diana/Stöhr, Robert/Zirfas, Jörg (2017): Vulnerabilität. Pädagogische Herausforderungen. Stuttgart: Verlag W. Kohlhammer GmbH.

Butler, Judith (2009): Performativity, precarity and sexual politics. In: AIBR Revista de Anthropologia Iberomericana 4, 3, S. i-xiii.

Capsada-Munsech, Queralt/Valiente, Oscar/Palumbo, Mauro/Cossetta, Anna/Pandolfini, Valeria/Bouillet, Dejana/Ganter de Otero, Jan Peter (2018): Work Package 6. Comparative Analysis of Skills Supply and Demand. International Report. YOUNG_ADULLLT Working Paper. Glasgow: University of Glasgow.

Castel, Robert (2005): Die Stärkung des Sozialen. Leben im neuen Wohlfahrtsstaat. Hamburg: Hamburger Edition.

Clough, Patricia Ticineto/Halley, Jean (Hrsg.) (2007): The Affective Turn. Theorizing the Social. Durham, London: Duke University Press. 
Chandler, David (2016): The Self-Construction of Vulnerability. In: Chandler, David/Reid, Julian: The Neoliberal Subject. Resilience, Adaptation and Vulnerability. London, New York: Rowman \& Littlefield, S. 121-143.

Cloward, Richard A./Ohlin, Lloyd E. (2013): Delinquency and opportunity. A study of delinquent gangs. Abingdon: Routledge.

Conze, Eckart (2018): Die Geschichte der Sicherheit. Entwicklung - Themen - Perspektiven. Göttingen: Vandenhoeck \& Ruprecht GmbH \& Co. KG.

Cover, Rob (2016): Queer Youth Suicide, Culture and Identity: Unliveable Lives? Abingdon, New York. Routledge.

Delor, François/Hubert, Michel (2000): Revisiting the concept of 'vulnerability'. In: Social Science \& Medicine 50, 11, S. 1557-1570.

De Masi, Franco (2009): Vulnerability to Psychosis. A Psychoanalytic Study of the Nature and Therapy of the Psychotic State. Abingdon, New York: Routledge.

Deutsche Forschungsgemeinschaft (2019): Ergebnisliste der Suchanfrage im Bereich „Projekte“ mit dem Stichwort „Vulnerabilität“. https://gepris.dfg.de/gepris/OCTOPUS?keywords_criterion $=$ Vulnerabilit $\% \mathrm{C} 3 \% \mathrm{~A} 4 \mathrm{t} \&$ findButton $=$ Finden\&task $=$ doSearchSimple\&context $=$ projekt [Zugriff: 10.05.2019].

Digitales Wörterbuch der deutschen Sprache (2019): DWDS-Wortverlaufskurve für „Vulnerabilität". https://www.dwds.de/r/plot?view=1\&corpus=zeitungen\&norm=date $\% 2$ Bclass\&smooth $=$ spline $\&$ genres $=0 \&$ grand $=1 \&$ slice $=1 \&$ prune $=0 \&$ window $=3 \&$ wbase $=0 \&$ logavg $=0 \&$ logscale $=0 \&$ xrange $=1945 \% 3 \mathrm{~A} 2017 \& \mathrm{q} 1=$ Vulnerabilit\%C3\%A4t_[Zugriff: 10.05 .2019$]$.

Dutta, Indranil/Foster, James/Mishra, Ajit (2011): On Measuring Vulnerability to Poverty. In: Social Choice and Welfare 37, 4, S. 743-76.

Eisch-Angus, Katharina (2019): Absurde Angst - Narrationen der Sicherheitsgesellschaft. Wiesbaden: Springer Fachmedien $\mathrm{GmbH}$.

Europäische Kommission (2008): Commission Recommendation of 3 October 2008 on the active inclusion of people excluded from the labour market. https://eur-lex.europa.eu/legal-content/ EN/TXT/PDF/?uri=CELEX:32008H0867\&from=EN\%22 [Zugriff: 20.05.2019].

Europäische Kommission (2010): Europe 2020. A European strategy for smart sustainable and inclusive growth. http://ec.europa.eu/eu2020/pdf/COMPLET\%20EN\%20BARROSO $\% 20 \%$ 20\%20007\%20-\%20Europe\%202020\%20-\%20EN\%20version.pdf [Zugriff: 20.05.2019].

Europäische Kommission (2013): Lifelong learning for young adults: better policies for growth and inclusion in Europe. RIA Research and Innovation action - YOUNG-3-2015: http://ec.europa.eu/research/participants/portal/desktop/en/opportunities/h2020/topics/young-3-2015. html [Zugriff: 20.05.2019].

Europäische Kommission (2019): Die Jugendgarantie. https:/ec.europa.eu/social/main.jsp? catId=1079\&langId= de [Zugriff: 09.05.2019].

Ewald, François (1993): Der Vorsorgestaat. Frankfurt am Main: Suhrkamp Verlag.

Ferrarese, Estelle (Ed.) (2018): The Politics of Vulnerability. Abingdon, New York: Routledge.

Friedrichsen, Mike/Bisa, Peter-J. (2016): Digitale Souveränität. Vertrauen in der Netzwerkgesellschaft. Wiesbaden: Springer Fachmedien.

Greven, Michael T. (2007): Politisches Denken in Deutschland nach 1945: Erfahrung und Umgang mit der Kontingenz in der unmittelbaren Nachkriegszeit. Opladen: Barbara Budrich Verlag.

Hammer, Charlotte Christiane/Brainard, Julii/Innes, Alexandria/Hunter, Paul R. (2019): (Re-) conceptualising vulnerability as a part of risk in global health emergency response: updating the pressure and release model for global health emergencies. In: Emerging Themes in Epidemiology 16, 2, S. 1-8.

Han, Byung-Chul (2015): The Burnout Society. Stanford, CA: Stanford Press.

Harvey, David (1989): The Condition of Postmodernity. Oxford: Blackwell. 
Hirschberg, Marianne/Köbsell, Swantje (2016): Behindernde gesellschaftliche Realität heute, Studienbrief I 07, Fernstudiengang „Inklusion und Schule“, zfuw, Universität Koblenz-Landau.

Hogan, Daniel Joseph/Marandola, Eduardo (2005): Towards an Interdisciplinary Conceptualisation of Vulnerability. In: Population, Space and Place 11, S. 455-471. https://doi.org/10. $1002 / \mathrm{psp} .401$

Illouz, Eva (2006): Gefühle in Zeiten des Kapitalismus. Adorno-Vorlesungen 2004. Frankfurt am Main: Suhrkamp Verlag.

Johnston, Barry, R./Chai, Jingqing/Schumacher, Liliana (2000): Assessing Financial System Vulnerabilities. International Monetary Fund. Working Paper 00/76.

Kerckhoff, Alan C. (1995): Institutional arrangements and stratification processes in industrial societies. In: Annual review of sociology 21, 1, S. 323-347.

Kerckhoff, Alan C. (2001): Education and Social Stratification Processes in Comparative Perspective. In: Sociology of Education 74, (Extra Issue), S. 3-18.

Knudsen, Britta Timm/Stage, Carsten (2015): Global Media, Biopolitics, and Affect: Politicizing Bodily Vulnerability. New York, London: Routledge.

Kohl, Bernhard (2017): Die Anerkennung des Verletzbaren. Eine Rekonstruktion der negativen Hermeneutik der Gottebenbildlichkeit aus den Anerkennungstheorien Judith Butlers und Axel Honneths und der Theologie Edward Schillebeeckx'. Würzburg: Echter Verlag.

Kohli, Martin (1978) (Hrsg.): Soziologie des Lebenslaufs. Darmstadt, Neuwied: Luchterhand.

Krüger, Fred (1997): Urbanisierung und Verwundbarkeit in Botswana. Existenzsicherung und Anfälligkeit städtischer Bevölkerungsgruppen in Gaborone. Pfaffenweiler: Centaurus-Verlagsgesellschaft.

Kruse, Andreas (2017): Lebensphase hohes Alter. Verletzlichkeit und Reife. Berlin: Springer Verlag.

Langemeyer, Ines (2007): Wo Handlungsfähigkeit ist, ist nicht immer schon Unterwerfung. Fünf Probleme des Gouvernementalitätsansatzes. In: Anhorn, Roland/Bettinger, Frank/Stehr, Johannes (Hrsg.): Foucaults Machtanalytik und Soziale Arbeit. Eine kritische Einführung und Bestandsaufnahme. Wiesbaden: VS Verlag für Sozialwissenschaften. S. 227-243.

Lehmann, Wolfgang (2005): Choosing to Labour. Structure and Agency in School-Work Transitions. In: The Canadian Journal of Sociology 30, 3, S. 325-350.

Levine, Carol/Faden, Ruth/Grady, Christine/Hammerschmidt, Dale/Eckenwiler, Lisa/Sugarman, Jeremy (2004): The Limitations of "Vulnerability" as a Protection for Human Research Participants. In: The American Journal of Bioethics 4, 3, S. 44-49.

Luna, Florencia (2009): Elucidating the Concept of Vulnerability: Layers not Labels. In: The International Journal of Feminist Approaches to Bioethics 2, 1, S. 121-139.

Luna, Florencia (2019): Identifying and evaluating layers of vulnerability - a way forward. In: Developing World Bioethics 19, S. 86-95. https://doi.org/10.1111/dewb.12206.

Mackenzie, Catriona/Rogers, Wendy/Dodds, Susann (2014): Vulnerability: New Essays in Ethics and Feminist Philosophy. New York: Oxford University Press.

Martellozzo, Elena (2013): Online Child Sexual Abuse. Grooming, Policing and Child Protection in a Multi-Media World. Abingdon, New York: Routledge.

McLaughlin, Kenneth (2012): Surviving Identity: Vulnerability and the Psychology of Recognition. Hove, New York: Routledge.

McLeod, Julie (2012): Vulnerability and the neoliberal youth citizen. A view from Australia. In: Comparative Education 48, 1, S. 11-26.

Millett, Kate (2018): Sexus und Herrschaft. Die Tyrannei des Mannes in unserer Gesellschaft. Köln: Kiepenheuer \& Witsch.

Möller, Hans-Jürgen/Deister, Arno (1996): Vulnerabilität für affektive und schizophrene Erkrankungen. Wien, New York: Springer Verlag.

Mouffe, Chantal (2014): Agonistik. Die Welt politisch denken. Berlin: Suhrkamp Verlag. 
Oeser, Erhard (2015): Die Angst vor dem Fremden: Die Wurzeln der Xenophobie. Darmstadt: Wissenschaftliche Buchgesellschaft.

Oliviero, Katie (2018): Vulnerability Politics. The Uses and Abuses of Precarity in Political Debate. New York: New York University Press.

Palumbo, Mauro/Benasso, Sebastiano/Parreira do Amaral, Marcelo (2018): Patterns of LLL policy-making: Parameters for LLL Coordinated Policy-Making. In: Parreira do Amaral, Marcelo/Zelinka, Jozef/Schweisfurth, Michele (Hrsg.): Work Package 8. Comparative Analysis and Reporting. Comparative Analysis Report. YOUNG_ADULLLT Working Paper. Münster: University of Münster, S. 212-247.

Parreira do Amaral, Marcelo/Jornitz, Sieglinde (2019): Die Konzeptualisierung von Bildungsverläufen Jugendlicher: eine Governance-Perspektive auf Lebenslauf. In: Langer, Roman/Brüsemeister, Thomas (Hrsg.): Handbuch Educational Governance Theorien. Wiesbaden: Springer Fachmedien, S. 417-440.

Parreira do Amaral, Marcelo/Kovacheva, Siyka/Rambla, Xavier (Hrsg.) (2020): Lifelong Learning Policies for Young Adults in Europe. Navigating between Knowledge and Economy. Bristol: Policy Press.

Parreira do Amaral, Marcelo/Lowden, Kevin/Pandolfini, Valeria/Schöneck Nikolas (2020): Coordinated policy-making in Lifelong Learning. Functional Regions as dynamic Units." In: Parreira do Amaral, Marcelo/Kovacheva, Siyka/Rambla, Xavier (Hrsg.), Lifelong Learning Policies for Young Adults in Europe. Navigating between Knowledge and Economy. Bristol: Policy Press, S. 21-42.

Parreira do Amaral, Marcelo/Zelinka, Jozef (2019): Lifelong learning policies shaping the life courses of young adults. An interpretative analysis of orientations, objectives and solutions. In: Comparative Education. DOI: 10.1080/03050068.2019.1619333

Penz, Otto/Sauer, Birgit (2006): Affektives Kapital. Die Ökonomisierung der Gefühle im Arbeitsleben. Frankfurt am Main, New York: Campus Verlag GmbH.

Petersen, Alan/Wilkinson Iain (Hrsg.) (2008): Health, Risk and Vulnerability. London, New York: Routledge.

Pierceson, Jason (2016): Sexual Minorities and Politics: An Introduction. London: Rowman \& Littlefield.

Pohl, Axel (2015): Konstruktionen von „Ethnizität“ und „Benachteiligung“. Eine international vergleichende Untersuchung von Unterstützungssystemen im Übergang Schule - Beruf. Weinheim \& Basel: Beltz Juventa.

Powell, Justin J.W. (2006): Special Education and the Risk of Becoming less Educated. In European Societies 8, 4, S. 577-599.

Priestley, Mark (2001): Disability and the Life Course: Global Perspectives. New York: Cambridge University Press.

Rambla, Xavier/Bouillet, Dejana/Petkova, Borislava/Boutiuc-Kaiser, Alina (2018): Do Lifelong Learning Policies Contribute to Tackling the Vulnerability of The Target Groups in EU Regions? In: Parreira do Amaral, Marcelo/Zelinka, Jozef/Schweisfurth, Michele (Hrsg.): Work Package 8. Comparative Analysis and Reporting. Comparative Analysis Report. YOUNG_ADULLLT Working Paper. Münster: University of Münster, S. 43-69.

Ranci, Constanzo (2010): Bringing territory back in social comparative research. In: Ranci, Constanzo (Hrsg.): Social vulnerability in Europe. The new configuration of social risks. Basingstoke, New York: Palgrave Macmillan, S. 25-34.

Ranci, Constanzo/Brandsen, Taco/Sabatinelli, Stefania (2014): Local Welfare Systems in Europe in the Age of Aucterity. In: Ranci, Constanzo /Brandsen, Taco/Sabatinelli, Stefania (Hrsg.): Social Vulnerability in European Cities. The Role of Local Welfare in Times of Crisis. New York, Basingstoke: Palgrave Macmillan, S. 279-298. 
Rinne, Risto/Järvinen, Tero/Kinnari, Heikki/Plamper, Raakel/Silvennoinen, Heikki/Tikkanen, Jenni/Vanttaja, Markku (2016): Work Package 3. Policy Mapping, Review and Analysis. National Report: Finland. YOUNG_ADULLLT Working Paper. Turku: University of Turku.

Ritchie, Bryan K. (2010): Systemic Vulnerability and Sustainable Economic Growth. Skills and Upgrading in Southeast Asia. Cheltenham, Northampton: Edward Elgar.

Rivers, Nicola (2017): Postfeminism(s) and the Arrival of the Fourth Wave: Turning Tides. Basingstoke, New York: Palgrave Macmillan.

Sattler, Elisabeth (2009): Die riskierte Souveränität: Erziehungswissenschaftliche Studien zur modernen Subjektivität. Bielefeld: transcript Verlag.

Scandurra, Rosario/Cefalo, Ruggero/Hermannson, Kristinn/Kazepov, Yuri (2017): Work Package 4. Quantitative Analysis Young Adults' Data. International Report. YOUNG_ADULLLT Working Paper. Vienna: University of Vienna.

Scandurra, Rosario/Hermannsson, Kristinn/Cefalo, Rugero (2020): Assessing young adults' living conditions across Europe using harmonised quantitative indicators: Opportunities and risks for policy makers. In: Parreira do Amaral, Marcelo/Kovacheva, Siyka/Rambla, Xavier (Hrsg.): Lifelong Learning Policies for Young Adults in Europe. Navigating Between Knowledge and Economy. Bristol: Policy Press. S. 171-197.

Schneider, Joseph W. (1985): Social Problems Theory. The Constructionist View. In: Annual Review of Sociology 11, S. 209-229.

Springhart, Heike (2016): Der verwundbare Mensch. Sterben, Tod und Endlichkeit im Horizont einer realistischen Anthropologie. Tübingen: Mohr Siebeck.

Springhart, Heike (2018): Inklusion und Vulnerabilität - systematisch-theologische Überlegungen. In: Geiger, Michaela/Stracke-Batholmai, Matthias (Hrsg.): Inklusion denken. Theologisch, biblisch, ökumenisch, praktisch. Stuttgart: Verlag W. Kohlhammer GmbH, S. 33-42.

Subrahmanian, V.S./Ovelgönne, Michael/Dumitras, Tudor/Prakash, B. Aditya (2015): The Global Cyber-Vulnerability Report. Switzerland: Springer International Publishing.

Taylor-Gooby, Peter (2004): New social risks in postindustrial society. Some evidence on responses to active labour market policies from Eurobarometer. In: International Social Security Review 57, 3, S. 45-64.

Toiviainen, Hanna/Kersh, Natasha/Hyytiä, Jaakko (2019): Understanding vulnerability and encouraging young adults to become active citizens through education. The role of adult education professionals. In: Journal of Adult and Continuing Education 25, 1, S. 45-64.

United Nations Development Programme (2014): Human Development Report 2014. Sustaining Human Progress. Reducing Vulnerabilities and Building Resilience. New York: United Nations Development Programme.

Vatsa, Krishna S. (2004): Risk, Vulnerability, and Asset-based Approach to Disaster Risk Management. In: International Journal of Sociology and Social Policy 24, 10/11, S. 1-48.

Weichselgartner, Jürgen (2001): Disaster mitigation. The concept of vulnerability revisited. In: Disaster Prevention and Management: An International Journal 10, 2, S. 85-95.

Weiß, Anja (2013): Rassismus wider Willen. Ein anderer Blick auf eine Struktur sozialer Ungleichheit. 2. Auflage. Wiesbaden: Springer Fachmedien.

Wustmann, Corina (2004): Resilienz. Widerstandsfähigkeit von Kindern in Tageseinrichtungen fördern. Weinheim, Basel: Beltz Verlag. 


\title{
28 Shifting Lenses to a Participatory Ethos in Research: Adult Learners with Disabilities in Germany and South Africa
}

\author{
Silke Schreiber-Barsch, Peter Rule
}

\begin{abstract}
This chapter compares the systems of inclusive adult education in Germany and South Africa. It uses disability as the meta-criterion in adopting a comparative education approach. The conceptual framework is guided by a relational approach to disability, as well as the notion of intersectionality, and features of inclusive adult education. After providing a comparative overview of the relevant history and policy, the chapter presents, analyses, and compares qualitative data that are relevant to disability from adult education contexts in the two countries. It finds that in both Germany and South Africa two policy discourses tend to predominate: rights and ethics; and the political - with the pragmatic emerging to some extent. This chapter contends that the efficacy discourse needs to be further developed and applied. It also argues for the epistemological, methodological, ethical, and political importance of a participatory ethos in research with regard to persons with disabilities.
\end{abstract}

\section{Introduction}

Disability, seen from an anthropological perspective, is part of the conditio humana; "all of us either are or will become disabled to one degree or another during the course of our lives" (United Nations 2015). Beyond the essentials of human existence, however, what disability means, how it is framed in a nation-state architecture, and how it is experienced by individuals in a certain geographical place and at a given time are likely to vary. Sayed and Soudien (2003) argue accordingly that the living conditions and environments encountered by persons "as gendered, raced or classed subjects are neither equivalent nor interchangeable and the solutions that are generated in response to these individually are not transposable one to the other" (Sayed/Soudien 2003: 11).

An extensive body of research has contributed to the investigation of these nuances. For example, with regard to intersecting dimensions of inequalities across the life course, the multiple fields of daily life, or the regions of the world (ISSC/IDS/UNESCO 2016), the frameworks of inclusive education as an aspect of public education (Artiles et al. 2015) are relevant, as is the academic landscape of dominant discourses, classification systems, and research paradigms (Watson et al. 2014; WHO 2007). In order to extend this extant body of knowledge, the chapter draws on an intersectional lens to explore disability in relation to the categories of age and race. In addition, it shifts the focus to inclusive adult education. We found that too little attention has been paid so far to adult learners - and thus to learning 
beyond the formal setting of schools and the schooling system - in scholarly work on disability and learning. Overall, the chapter utilizes a comparative education research approach. Following comparative education's methodological principles, we identify disability as our tertium comparationis, the meta-criterion upon which our comparative inquiry is established (Bereday 1964). To investigate this meta-criterion, we define as our unit of analysis the field of inclusive adult education in two nation-states: Germany and South Africa.

To this end, we adhere to qualitative approaches in comparative education research that seek to understand educational phenomena within the framework of an interpretive analysis (Fairbrother 2016; Manzon 2016). Thus, particular emphasis will be put on unravelling and explaining individuals' perspectives on disability (which might be those of adult learners with disabilities, or of adult education practitioners) and the meanings they attach to inclusive adult education, as well as the surrounding cultural, geographical, political, and social frameworks and contexts of relevance. This approach ties in with Mason's (2016) argument that "comparative education is best conceptualized as a critical social science, incorporating an emancipatory interest focused on the distribution of power and its associated attributes" (Mason 2016: 253). The purpose of comparative education research is, thus, in Mason's opinion, to "identify the axes along which educational and other goods are differentially distributed, and to disaggregate their object of study along those axes" (Mason 2016: 253). Yet, to not only describe, but capture, understand, and explain commonalities, similarities, or differences, fundamentally requires the prior identification of a tertium comparationis (disability). This meta-point of reference determines the comparative basis of our unit of analysis.

For a country-to-country comparison of disability, we have chosen the field of inclusive adult education in the two nation-states, Germany and South Africa. However, we will also argue beyond the context of these two nation-state contexts, aiming to provide insights that might prove explanatory and useful for research on inclusive adult education in general. The choice of the two nation-states has been guided by a variety of aspects.

First, there is a substantial gap in research when it comes to comparing disability with a focus on inclusive adult education. There is a body of research on cross-national studies that compare educational systems of inclusive education, but these focus on schooling for children and youth (for example, Armstrong 2003; Richardson/Powell 2011; Biermann/Powell 2014; Smyth et al. 2014; Artiles et al. 2015). This gap might be due to the much more pluralistic, far less regulated, structure of nation states' adult education systems compared to school systems - a quality that makes cross-country comparative inquiries in adult education exceedingly challenging. Desjardins (2017) provides one of the few multi-country comparative studies in his analysis of the political economy of adult learning systems. However, he only touches upon western countries of the Global North, and the comparative analysis is primarily based on large-scale quantitative studies. For example, those made available by the Programme for the International Assessment of Adult Competencies (PIAAC), led by the Organisation for Economic Co-operation and Development (OECD). No African country, including South Africa, has yet been part of one of the three PIAAC cycles. Overall, also beyond a comparative lens, little attention has been paid so far to issues of inclusion and disability in adult education studies (Ross-Gordon 2018). Therefore, we see a need to bring not only the field of inclusive adult education to the fore, but also to expand territorial patterns in research to include a global North-South dimension, as advocated by Grech and Soldatic in their edited volume, Disability in the Global South (2016). Within this handbook, our chapter links with Julia Biermann's comparative focus on Germany and Nigeria (Biermann 
2021 in this volume), as well as Raphael Zahnd's discussion of the conceptualization of disability in his study of The World Bank (Zahnd 2021 in this volume).

Second, the comparability of our units of analysis is appropriate because the category (disability) and the unit of analysis (adult education) exist in both countries. Furthermore, both countries have adopted and ratified the United Nation's Convention on the Rights of Persons with Disabilities (United Nations 2006). This is significant because in both countries, the same policy document has been launched in the sense of a "travelling policy", introduced via intergovernmental organizations and woven into "embedded policy" (Ozga/Jones 2006), like the existing nation-state policy architectures. To what extent and within what contextualization this has occurred will be systematized by a brief look at educational discourses and the impact of policy agendas on institutional settings in adult education.

Third, both countries show a common heritage of totalitarian political regimes. In Germany, this took the form of the Nazi regime of terror (1933-1945) (Poore 2009); while in South Africa, the Apartheid regime (1948-1990), was built upon antecedents of colonial oppression and segregation dating from the seventeenth century (Davenport/Saunders 2000). In exploring this common heritage, different layers of consequences for the category of disability and adult education in both countries will appear. An intersectional approach will be used in order to show the interconnectedness and far-reaching consequences of the interplay between different and pivotal markers of identity. This will be done by discussing disability and age and disability and race.

Finally, we shift the attention in the remaining part of this handbook chapter to research in the field of inclusive adult education. In particular, we seek to address projects following participatory research approaches, favouring qualitative bottom-up-approaches in data generation. In doing so, the chapter argues for the epistemological, methodological, ethical and political importance of a participatory ethos in research with regard to persons usually relegated to society's and academia's periphery and aims to scrutinise the wealth of existing theoretical approaches in their explanatory potential.

Summing up, in this handbook chapter, we begin by drawing on relevant theoretical strands and conceptual viewpoints from the scholarly educational debate on disability and adult learners in order to explain our approach to both our investigation and our understanding of what constitutes an inclusive adult education (Section 2). This section, in turn, frames a comparative inquiry into the two nation-states: Germany and South Africa. In the first step of our inquiry, we adhere to the meta level of both general strands in the educational discourse and dominant drivers in the policy agendas of each country (Section 3). To complement this, we present two more in-depth analyses, one linking disability with age and race (Section 4), and one using principles of participatory research in small-scale qualitative empirical studies to investigate the issues under scrutiny (Section 5). This leads to a conclusion addressing the benefits and limitations of our comparative inquiry and further desiderata in this scholarly field (Section 6). 


\section{Disability: Nuancing the tertium comparationis and the Unit of Analysis under Scrutiny}

Here, we advocate for the notation disability, following Campbell's (2009) arguments on the generic impact of societies' deeply entrenched able/not-able divide. Ableism, a specific strand of disability studies (Goodley 2017; Waldschmidt 2020), calls for shifting the focus from exploring deviation of whatever kind - such as disability or impairment - to fundamentally questioning the premise of impairment: the logic of ability (see also Buchner et al. 2015). This shift questions societal technologies of classification and attribution systems with respect to inter alia education (specific kinds of ratings; types of schools; places of learning; curricula; learning outcomes and the like), as well as its normative justifications through political agenda-setting (knowledge-based society; neoliberal meritocracy), including its consequences for individual human beings. The latter are, accordingly, individually labelled as abled, less abled, or non-abled; as normal or deviant; and as gifted or un-gifted. And thus, these individuals are conventionally classified, wherein their abilities are (mis)matched with respective educational standards, "rather than the educational sector being seen as problematic for excluding them in the first instance", as Sayed and Soudien (2003: 12) criticize. This is what Weisser (2018) calls a generic "ability-clash," which, furthermore, positions the non/less-abled as "being naturally the subject of marginalization" due to their problems (Armstrong 2003: 2; emphasis in original). Within this logic, disability, then, is "cast as a diminished state of being human" (Campbell 2009: 5). Summing up, the notation disability does not indicate the idea of a binary dividing line between the respective abilities of human beings, but rather points to the understanding of disablement as being relational (Corbett/Slee 2000: 143) and to the power-driven terrain of contestation in societies - to be investigated and elicited by research.

Following this understanding, we shall argue that disability is not an ontological category, that is, the defining feature or essence of a person. In this regard, we view the medical model of disability as reductive, because it locates disability as an essence within the person (an illness, medical condition to be cured, or personal tragedy), to be "fixed" by medical or other treatments (Barnes 2014). We acknowledge both the value and the limitations of the social model, which sees disability as a set of constraints imposed on the person by society. The social perspective is crucial but should not neglect the individual's embodied experience of disability. A holistic view recognizes that disability can be congenital, acquired (by accident, or through disease or age, etc.), and/or socially constructed, which reminds us of the wide range of possible impairments or difficulties (physical, intellectual, mental and so on). Moving away from viewing disability as an essence, we adopt a relational approach that views disability as "a function of the relationship between the individual and the environment, including biological, cultural, psychological, and sociopolitical aspects" (Rule/Modipa 2012:141).

Thus, disability needs to be contextualized in terms of the interrelatedness of being - in whatever sense - impaired, as well as living in disabling societal conditions. This is mirrored in the UN Convention on the Rights of Persons with Disabilities (United Nations 2006). The convention has been ratified in both countries under scrutiny here and, thus, represents a common policy framework; or, more precisely, an international human rights treaty launched by the parties ratifying the convention (such as the South African and the German govern- 
ments). The convention pursues the so-called social model of disability. This is clarified in Article 1: instead of defining "disability" as such, the convention clarifies that "persons with disabilities include those who have long-term physical, mental, intellectual or sensory impairments which in interaction with various barriers may hinder their full and effective participation in society on an equal basis with others" (United Nations 2006: Art. 1). This definition reflects the useful distinction between "disabilities" and "impairments." The World Health Organization's (WHO) International Classification of Functioning (ICF) views disability as an "umbrella term for impairments, activity limitations and participation restrictions", whereas an impairment specifically is understood as a problem with body function or structure (WHO 2002: 10).

Though the social model of disability is widely used at the policy level, for our comparative approach we consider a relational model of disability - as elaborated in scholarly works within the field of disability studies - as adequate and enriching. This approach advocates for a multi-factorial account of disability; it emphasizes the interwoven linkages between biological, social, cultural, and psychological aspects, as well as attitudes, norms, and personal perceptions, and explicitly pays attention to power structures (see, for example, Snyder/Mitchell 2006; Campbell 2009; Shakespeare 2014; Goodley 2017).

\subsection{Focusing on Adult Learners with Disabilities}

Against this backdrop, this chapter seeks to put adults into the limelight of educational endeavours. Here we enter - compared to, for example, the juvenile school system - a very heterogeneous and fuzzy terrain, starting with the question of under what conditions someone is defined as an adult. There are legal criteria tied to a distinct chronological age, allowing, for example, a contractual capability or the right to vote from a certain minimum age; defining the age cohorts for compulsory education is also a consideration. However, the point at which adult learning and education are to start - as the (usually) longest temporal period of lifelong learning - seems to be more of a moving target.

The relational nature of defining adult learning is mirrored by the shifting definitions of the sample's age limits in international representative surveys on adult learning and education. Whereas the Adult Education Survey (AES) (EUROSTAT 2019), to be implemented by all member states of the European Union (EU), sets its age limit as between 25 and 64 years old, in Germany, AES's data collection includes a wider age range - 18 to 64 year-olds (Bilger et al. 2017) - in order to incorporate professional training and education. Beyond the European context, the OECD survey Education at a Glance uses data from the PIAAC survey, whose sample draws on subjects aged 25 to 64 years (OECD 2018). However, as stated above, this research does not yet involve any African country. Apart from age indicators, the adult learning and education landscape is likewise diverse when it comes to drawing a clear line between formal, non-formal, and informal learning activities; of standardizing curricula and learning outcomes; and of systematizing the patterns and settings of adult education practice.

This general heterogeneity might explain to a certain degree the substantial research gaps with regard to adults with disabilities and, in particular, with regard to adults with learning difficulties (the latter is a notation proposed by People First, a self-advocacy group of individuals with so-called intellectual disabilities). This hierarchy of impairments persists even, 
as Stalker (2014: 125) has elaborated, within the field of disability studies; this leaves people with learning difficulties and mental distress at the bottom of the ladder of research endeavours. This holds true for both of the countries in question.

Germany is one of those countries with a traditionally highly segregated education system according to the aforementioned "able/not-able divide" (Campbell 2009; Poore 2009; Richardson/Powell 2011). This has resulted in a territorial segregation, which has persisted for centuries, between the abled and the non-abled in "normal" and "special" learning institutions, showing ultrastable effects until today (see Biermann 2021 in this volume). In contrast, in the field of adult learning and education, there is, in general, very little empirical evidence when it comes to disability and adult learning. However, what little evidence there is clearly shows the very low participation rate of adults with disabilities and, in particular, learning difficulties in learning opportunities meant for the abled (Autorengruppe Bildungsberichterstattung 2014; BMAS 2016; Koscheck et al. 2013). Learning opportunities for adults with impairments and/or learning difficulties continue to be provided almost exclusively in sheltered workshops or in care institutions without any primary adult education mandate hence, not in public places such as a public adult education centres (Ackermann/Ditschek 2015; Heimlich/Behr 2018).

In South Africa, a substantial body of research on inclusive education has developed since the emergence of White Paper 6 in 2001 (Walton 2011; Donohue/Bornman 2014; Dreyer 2017), including some comparative literature (Engelbrecht et al. 2017). The predominant focus of this scholarship has been on inclusive education in schooling, particularly on the relation between policy and practice, and the problems of implementation. This scholarship acknowledges the progress made in inclusive education but identifies problems regarding a lack of clarity in policy and shortcomings in implementation. Furthermore, while there has been some attention paid to inclusive education in higher education, especially at universities, little attention has been afforded to inclusive education in adult education. Exceptions include work on the inclusion of a range of marginalized groups in Kha Ri Gude, the mass literacy campaign, including adults with disabilities (McKay/Romm 2015; McKay 2018), and on the attitudes and experiences regarding education of adults with disabilities (Rule/Modipa 2012). McKay (2018) also usefully frames adult literacy in relation to lifelong learning and sustainable development.

\subsection{Outlining Core Features of an Inclusive Adult Education}

In consequence of these contextualizing factors, we agree with Allan (2013) that, for the most part, the concept of inclusion seems to "remain a political concept tied more closely to special education than to democratic education." However, our understanding of inclusive adult education follows approaches that reject individualizing disability (in the sense of a person-related root cause or medical model) or devaluing persons as solely passive victims of social conditions. Rather, we recognize, by calling on Rancière's (1999: 33) work in political philosophy, individuals' conditio humana as based on the "equality of speaking beings" (see also Schreiber-Barsch 2018).

Thus, we fundamentally acknowledge the human ability to act and to influence one's life course and living environment. Autonomy, in this sense, means acting - as far as possible self-determinately (not necessarily independently), according to the matrix of societal values 
and common understandings of a "good" life and tenable living standards. This premise inevitably requires a triad: the authorization and legitimacy (given by collective bodies and societal frameworks) to render a decision, the individual willingness to render a decision, and the ability to carry out a decision. Taking this into account, we clarify our understanding of inclusive adult education by referring to a table in a recent World Bank report on children with disabilities (The World Bank 2019: 12). This table had been developed with respect to Article 24 of the UN Convention and its core features of inclusive education (United Nations 2006; see also Zahnd's (2021) discussion of the The World Bank's conceptions of disability). This we adapt to adult education (see Table 1):

Table 1: Core Features of Inclusive Adult Education

\begin{tabular}{|c|c|}
\hline Whole systems approach & Strengthen the capacity of lifelong learning systems to reach out to all learners. \\
\hline $\begin{array}{l}\text { Whole educational } \\
\text { environment }\end{array}$ & $\begin{array}{l}\text { Transform adult education culture, policy, and practice in all formal, non-formal, } \\
\text { and informal educational environments to achieve lifelong learning at all levels. }\end{array}$ \\
\hline Whole person approach & $\begin{array}{l}\text { Recognize the capacity of every individual to learn and set high expectations } \\
\text { for all learners. Accommodate diverse requirements and identities of individual } \\
\text { learners, together with a commitment to remove the physical, social-structural, } \\
\text { and mental barriers that impede that possibility, aiming for flexible learning inf- } \\
\text { rastructures. }\end{array}$ \\
\hline $\begin{array}{l}\text { Supported } A E \\
\text { professionals }\end{array}$ & $\begin{array}{l}\text { Focus on all staff within the adult learning and education eco-system receiving } \\
\text { education and training on values and competencies to accommodate diverse } \\
\text { learners and build inclusive learning environments. }\end{array}$ \\
\hline $\begin{array}{l}\text { Learning-friendly } \\
\text { environment }\end{array}$ & $\begin{array}{l}\text { Transform learning places into welcoming spaces for all. Focus on creating ac- } \\
\text { cessible formal, non-formal, and informal environments where everyone feels } \\
\text { safe, supported, stimulated, and able to express themselves, with a strong em- } \\
\text { phasis on involving learners themselves in building a positive learning commu- } \\
\text { nity. }\end{array}$ \\
\hline $\begin{array}{l}\text { Respect for value } \\
\text { and diversity }\end{array}$ & $\begin{array}{l}\text { Focus on the full and active participation, accessibility, attendance and achie- } \\
\text { vement of all learners, especially those who, for different reasons, are excluded } \\
\text { or vulnerable (in the sense of being at risk of being marginalized). }\end{array}$ \\
\hline
\end{tabular}

Source: Adapted from The World Bank 2019, 12; United Nations 2006.

This conceptual approach illustrates the high interdependency between society's framework and conditions, adult education's normative values, theoretical discourses and practice, and, furthermore, the realities of individual learners. We agree with Lindmeier and Lütje-Klose (2015: 8) that inclusive adult education is thus not meant to be restricted to being exclusively responsible for persons with disabilities, which represents a narrow definition of inclusion. Rather, we see inclusive adult education as meant to address all (potential) learners on an equal level, and - as part of this - in particular, those individuals and/or groups with a high risk of vulnerability, in the sense of being marginalized from lifelong learning opportunities.

Within this broader view of inclusion, the lens of intersectionality, which acknowledges the relations among vulnerability factors (for example, disability, gender, income, race, and age), is of vital importance in developing strategies for access and inclusion. Such an understanding is based on fundamental values of maximizing opportunities to participate in learning and minimizing mechanisms of exclusion or devaluation (Lindmeier/Lütje-Klose 2015: 8) for all lifelong learners. It neither seeks to shift responsibility for realizing inclusive adult education exclusively to one of the parties involved, nor to problematize one of them as being the burden to overcome or the barrier to remove, nor to promote the idea that moving toward inclusive adult education might happen beyond the legacies of power structures. For to "be 
welcomed" is a consequence of being "positioned as the one who is not at home" (Ahmed 2012: 156).

\section{Comparing Educational Discourses and the Impact of Policy Agendas}

Comparing the phenomenon of inclusive adult education in Germany and South Africa, we touch upon the question of which strands of educational discourses and, more precisely, conceptual approaches, can be identified as having been influential and characteristic for the issue under scrutiny in the overall debate since the mid-twentieth century (specifically since the mid-1990s). Since then, both countries have been subject to profound changes: in 1994, South Africa, on its way to transforming into a post-apartheid democratic republic, held its first universal elections, won by the African National Congress (ANC). Germany, on the other hand, saw the non-violent reunification of its Western and Eastern halves in 1990. It embarked on a long-term route of profound societal transformation toward a unified country - a unified country which nevertheless continues to grapple with inequalities due to the stratified schooling system, which had been then implemented in both parts of the nation. Yet, these transformations are deeply rooted in the decades that came before.

We aim to link the discursive level with the level of political agenda setting, acknowledging the latter's primary relevance for the nation-state framework of inclusive adult education. To this end, two systematizations seem particularly rewarding. One is provided by Sayed and Soudien (2003: 15-17). Following work by Dyson (1999), Sayed and Soudien differentiate between four dominant public policy discourses on the issue of inclusion since the mid-twentieth century. These are guided by one of the following rationales: First, rights and ethics (human rights treaties; redressing global inequities); second, efficacy (an inclusive educational system as "more cost-efficient, socially beneficial and educationally effective" (2003: 16); third, political (political struggle of marginalized individuals and groups); and four, pragmatic (the transfer into educational practice; manuals on how to achieve inclusive education). We aim to link these overall trends in the debate over public policy discourses on inclusion with another systematization, elaborated by Biermann and Powell (2014). The latter focus on the aforementioned rights and ethics discourse by exploring in their comparative work the implementation of the UN Convention into the realities of schooling in Germany, Iceland, and Sweden. Within this, Biermann and Powell distinguish three forms of institutional dimensions that strongly influence the path toward realizing inclusive education in the aftermath of the UN Convention: a cultural-cognitive dimension (educational leitmotif and disability paradigm); a normative dimension (types of organizing education and places of learning); and a regulative one (educational rights). We would argue that both systematizations provide relevant guiding frameworks for our comparative inquiry between Germany and South Africa, encompassing both overall educational discourses as well as the much narrower focus of inclusive adult education. 


\subsection{A Deficit-oriented Medical Model as the Historical Starting Point}

Similar to the German context, in South Africa during the pre-democratic era (since the midtwentieth century until the mid-1990s), the field of disability was dominated by a number of discourses, which had in common the understanding of disability as a deficit (physical, intellectual, social, moral, and/or spiritual). This understanding represented the prevalent educational leitmotif and disability paradigm and manifested in strongly segregated places of learning. According to this medical model, which was dominant in contexts of institutional health and education, disability was viewed as a condition internal to the person that needed to be "fixed" by medical and/or educational intervention. For example, in South Africa, deaf children were prohibited from using Sign Language at school and were forced to conform to the hearing world by learning to lip read; in a related practice, they were also medicalized with cochlear implants to "cure" their deafness. This was accompanied by a paternalistic charity discourse, often prominent in non-governmental organizations, which positioned persons with disabilities as helpless victims and recipients of aid to be pitied and assisted. A traditional discourse that viewed disability as a "curse" which might reflect "witchcraft" or the displeasure of the ancestors was also prevalent in indigenous communities. This was similar to a religious discourse, which viewed disability as an indication of God's punishment for "sin," requiring repentance by and forgiveness of the disabled person and/or their parents and family. Persons with disability who were not healed by prayer were blamed and ostracized for their lack of faith. Disability was thus associated with deficits (inability, lack, shame, sin, curse), which was reflected in naming practices in various South African languages ("handicapped," and "cripple" in English; gestremde in Afrikaans).

With regard to Germany, the prevalence of the deficit-oriented medical model in the decades after the Second World War shows very similar features. Whereas Germany had, in the nineteenth century, once been a pioneer in establishing (segregated) learning opportunities for children and young adults with disabilities (Powell/Pfahl 2012), and, thus, recognizing the educational rights of these individuals, this system had also paved the way for a highly differentiated system of segregation according to impairment. This system showed utmost persistence. Its logic furthered the idea of disburdening the "normal" schools and the "normal" pupils from less-abled ones, while, at the same time, disburdening the less-abled pupils by offering them special care and paternalistic welfare in territorially segregated and more-or-less closed sanctuaries (Powell/Pfahl 2012). In the 1980s, this framework provided support to the idea that special care providers were responsible for providing learning opportunities for adult learners with disabilities - but not, for example, public adult education centres (Schreiber-Barsch/Fawcett 2019). However, from the 1960s onward in Germany, people with disabilities also formed a social movement; they argued for their political rights and, by seeking empowerment via their own voices ("Nothing about us without us"), they slowly started to gain strength and, in time, contributed to amending the deficit-oriented discourse on disability in academia, policy and the public sphere. In consequence, in the adult education arena, and in some public adult education centres, adults with impairments started to work as course leaders and, thus, challenged the dominant disability paradigm (SchreiberBarsch/Fawcett 2019). 


\subsection{The Emergence of the Social Model (1990s)}

These discourses persist in the present era, even while alternative discourses based on human rights, social justice, and the social model of disability have come to the fore (with regard to South Africa, see Schneider 2006; Elphick et al. 2015; Nuwagaba/Rule 2015a). In South Africa during the democratic era (meaning from the mid-1990s onward), the notion of disability has been strongly informed by the social model of disability. This model, in turn, has been informed by what Sayed and Soudien (2003) term the "political rationale," arising from the struggles of the disability movement, as well as a human rights rationale. This is also true of the German context in the wake of UNESCO's Salamanca process (which dates from the early 1990s) and its attempt to situate inclusive schooling as a global agenda. And, ultimately, the launch of the UN Convention in 2006, which has given voice to the social model of disability (Schreiber-Barsch/Fawcett 2017). In South Africa, for example, the Integrated National Disability Strategy (Office of the Deputy President 1997), which strongly influenced subsequent social and educational policy, rejected medical and welfare models of disability in favour of a human rights approach and a social model. It argues that "if society cannot cater for people with disabilities, it is society that must change" (Office of the Deputy President 1997: 4). It retains the distinction between "impairment" (organic/medical condition) and "disability" (socially constructed barrier that makes an impairment disabling). Similarly, the understanding of "inclusive education" in South Africa is informed by human rights and social justice agendas. The South African Constitution (Republic of South Africa 1996) provides the basis of this approach by enshrining the fundamental right of all South Africans to basic education, including adult basic education (Article 29). It also proscribes unfair discrimination on the grounds of "race, gender, sex, pregnancy, marital status, ethnic or social origin, colour, sexual orientation, age, disability, religion, conscience, belief, culture, language and birth" (Article 9, 3). Inclusive education in South Africa thus adopts a broad stance toward inclusion as "overcoming the barriers in the system that prevent it from meeting the full range of learning needs," including the needs of learners with disabilities (Department of Education 2001: 17). The focus of the White Paper is on schooling, for which it has specific structural recommendations, but it does not mention inclusive education on other levels, including vocational, higher, and adult education.

\subsection{Moving toward a Pragmatic Discourse - With Adult Learners still at the Periphery?}

More recently, in South Africa, the White Paper for Post-School Education and Training (Department of Higher Education and Training 2013) includes an entire chapter on "Addressing disability", strongly informed by the rights and ethics discourse. However, the document acknowledges that there is no national policy to guide disability inclusion in the post-school sector, that policy and practices are uneven across institutions, and that adult learners with disabilities are poorly represented at post-school institutions. Adult inclusive education is thus still at a fledgling stage within South African adult education, although there are pockets of excellence within literacy campaigns (McKay 2015) and adult learning centres (Rule/Modipa 2012). In Germany, the post-school sector as an explicit part of a policy agenda likewise 
refers to an almost empty box. The UN Convention had the merit of finally initiating a profound transformation of Germany's education system; however, this transformation almost exclusively affected the school sector, rather than adult education. Two key policy documents were launched as a result of the UN Convention. The first one, from 2011 (BMAS 2011; "Our way to an inclusive society"), is grounded in the rights and ethics discourse. The second one, from 2016, shifted to a pragmatic discourse, as it outlines a National Action Plan (BMAS 2016); however, it too had only very limited reference to adult learners. Besides, the traditionally strong legal autonomy of the sixteen German federal states (Bundesländer) with regard to the spheres of education and culture serve as an additional barrier for navigating through the lifelong learning system. In consequence, sixteen more or less different educational systems exist, greatly affecting the learner's mobility and range of choices.

All in all, this comparison illuminates a high degree of similarity between Germany and South Africa with regard to the respective dominance of public policy discourses and corresponding forms of institutional settings. However, the policy discourse that appears to be missing is the one regarding efficacy, which advocates for an inclusive educational system as "more cost-efficient, socially beneficial and educationally effective" (Sayed/Soudien 2003: 16). Though current policy papers emphasize this point (UNESCO/Leonard Cheshire Disability/2019: 18), it seems that the policy level still tends to ignore the economic "price of exclusion" (Buckup 2009) and the empirically proven "disability-poverty cycle" (Banks/Polack 2014).

\section{Nation-state Legacies: Interlinking Disability with Age and Race}

As stated in the introduction, the everyday experience of disability of whatever kind and its socio-structural surroundings are very likely to vary, not only in relation to individuals, but also within different countries, due to the historicity of each given place at a given time. Sayed and Soudien (2003) argue in this sense that it is not about merely adding one factor upon another. Rather, "the concept of an interlocking framework recognizes the highly complex ways in which race, class, gender and other categories intersect and inter-relate to produce unique individual and group experiences" (Sayed/Soudien 2003: 14). In this sense, an inclusive adult education approach does not seek to iron out the category and realities of difference by claiming a supposed meta-norm (as in, everybody is different, everybody is equal); instead, an inclusive approach to adult education acknowledges the ineluctability of difference. By doing so, it avoids the much higher risk of disguising individual or collective discrimination (Dederich 2014: 48; Katzenbach 2015: 25). It shifts the focus to analysing and reconstructing the conflicts, contradictions, and everyday realities related to inclusion and, thus, counters mechanisms of individualizing disability. This shall be exemplarily illustrated by the intersecting categories of disability with age (Germany), on the one hand, and with race (South Africa), on the other hand. Whereas Germany and South Africa might appear to provide very different contexts, a country-to-country-comparison illuminates similarities in the axes of inequality and injustice within the context of disability due to a common heritage of totalitarian political regimes. 
In South Africa, there was and remains a particular intersection between race and disability. Under apartheid, education was differentially funded on the basis of race, with white children receiving, for instance, up to fifteen times more resources than black African children in 1975/1976 (Christie et al. 1991). While there were special remedial classes and special schools for white children with disabilities, there were very few such facilities for black children. Many black adults with disabilities thus never had the opportunity to attend school as children or dropped out of school at an early stage because of discrimination and/or lack of accommodations (Rule/Modipa 2012). While in the democratic era, the middle class in South Africa has grown to include persons of all races, the working-class majority remains largely black and poor, and includes most persons with disabilities. Although the democratic government has instituted a system of social grants (including a disability grant), the poor have unequal access to services such as health and education, particularly those living in rural areas and informal settlements. There is therefore an intersection of race, class, location, and disability that accentuates oppression and marginalization. In addition, there is evidence that women and girls with disabilities experience oppression and abuse; this adds gender to other intersecting identities, particularly in the context of the HIV/AIDS pandemic (Chappell 2013; Hanass-Hancock 2009).

In Germany, on the other hand, research on the intersection of disability and migration (in the sense of ethnic origin) - raised, for example, by Wansing and Westphal (2014) - has been slowly coming to the fore. However, race is not a category usually elaborated on, not least because of Germany's Nazi regime of terror (1933-1945) that installed the category of race at the very heart of its inhuman political ideology (Poore 2009). Within this ideology, disability was been labelled as a "diminished state of being human" (Campbell 2009: 5). Put into force by the euthanasia program (called "Aktion T4"), the systematic killing of more than 70,000 people with all kinds of disabilities between 1939 and 1945 was legitimized as a government program (Poore 2009). The long-term effects of the euthanasia program are still present, because the gaps between people with and without disabilities of similar age has only recently been narrowing in Germany (Dieckmann/Giovis 2012: 15). As Dieckmann and Giovis (2012) clarify, in consequence, the number of individuals aged sixty and older with learning difficulties will almost quadruple within the next 15 years - requiring a profound transformation of the educational and social systems' premises, and the demands to be met with regard to providing learning opportunities across the lifespan.

\section{Doing Research in Disability Studies and Adult Education: Insights from the Field}

This section shifts the focus to empirical studies of disability and adult education in the two countries, using participatory research. By referring to this approach, we aim to use this handbook chapter to emphasize and showcase the value of qualitative, small-scale, and participatory-oriented research. It allows researchers - and society at large - to derive insights that are not, or cannot yet be, satisfactorily achieved by large-scale assessment surveys of, for example, adults' literacy and numeracy skills (see, e.g., Evans 2019: 68).

Participatory research is a generic term for approaches that aim to explore, reconstruct and, ultimately, influence social reality in participatory ways of doing research. Implemen- 
ting such research in the context of disability has noticeably increased during the last decades; different strands of participatory research have emerged (see, for example, Kiernan 1999; Chappell 2000; Buchner et al. 2011; Stalker 2014; von Unger 2014; Walmsley/Johnson 2016). Overall, participatory research aims to develop research questions from the convergence between social reality and science, thus encouraging alliances and dividing power between scientists, practitioners, and the people under scrutiny. Participatory research is also defined by its objectives of initiating change (its political dimension); questioning the legitimacy and appropriateness of norms and values; and using the richness of methodological pathways in order to adapt methods to the sample's needs and demands, not vice versa.

Hence, by participatory methods, we refer to research methodologies and methods that, to varying degrees, actively engage participants as agents in planning and conceptualizing the research, collecting and analysing the data, writing up the findings, and/or disseminating the research. This approach falls broadly within the tradition of participatory research following the concepts of von Unger (2014) and, more explicitly, emancipatory disability research (Barnes 2003). Both approaches target "the empowerment of disabled people through the transformation of the material and social relations of research production" (Barnes 2003: 6). The tradition of participatory research is thus a reaction against positivist research, which excludes the subject and the subjective from research. It also reacts against professional social science research, which excludes the agency of the research participants in favour of "professionals" and "experts". Participatory research recognizes that research involves questions of power: the power to conceive, plan, conduct, analyse, interpret, disseminate, and benefit from research; it also recognizes that participants, in this case persons with disabilities, are experts in their own lives (Rule 2018). And it leads to empirical findings that would not be possible to obtain with the usual social science methods and methodological approaches. As Corbett and Slee (2000: 136) state, it allows "listening to voices from the outside" by "bringing sub groups in from the margins and initiates a reordering of the politics of knowledge."

Drawing on examples of participatory research implemented in Germany and South Africa, we aim to provide insight into not only the richness of methods used, but also the commonalities, similarities, and differences between such approaches.

\subsection{Insights from Participatory Research}

With the aim of providing a range of insights, we draw on a small body of South African scholarship. It uses participatory methods to engage adults and youths with disabilities in relation to a range of adult learning domains: adult basic education (Rule/Modipa 2012), HIV/AIDS and disability (Rule 2011; Chappell et al. 2014), vocational education and training (Siwela 2017) and mature adults with disabilities in higher education (De Lange 2016). Moreover, we refer to research currently conducted in Germany that examines numeracy as part of adult basic education and explores the everyday numeracy practices of adults with learning difficulties $^{1}$ (also termed intellectual disabilities) (Schreiber-Barsch et al. 2020). The relevance of numeracy-related knowledge and capabilities for participation in society has been widely proven (Gal 2000; Rammstedt 2013; Geiger et al. 2015; Yasukawa et al. 2018). Yet,

1 Numeracy practices of adults with learning difficulties (NumPuD); belongs to the Hamburg Numeracy Project, funded by Landesforschungsförderung Hamburg (2017-2020) (LFF-FV 52). 
the extant body of scholarship is based for the most part on quantitative methods and largescale studies, which so far have collected only very limited data on adults with learning difficulties (Schreiber-Barsch et al. 2020). Therefore, an exploratory qualitative study on numeracy practices has been chosen because it provides an in-depth understanding of subjective sense-making processes and numeracy-related abilities as they unfold in everyday contexts in order to analyse numeracy as a social practice (rather than as behavioural or cognitive facets of the individuals to be assessed). To this end, principles of participatory research (von Unger 2014; Walmsley/Johnson 2016) were combined with Grounded Theory Methodology (GTM) (Strauss/Corbin 1990; Strübing 2014). This research used the shadowing method (Bøe et al. 2017) for in situ explorations $(n=11)$ of everyday numeracy practices of adults with learning difficulties, conducted semi-structured interviews $(n=13)$, again with adults with learning difficulties, and, finally, implemented focus groups (Barbour 2007) with professionals working in these contexts. The choice of methods and the degree of sharing control thus also depend on the persons involved. For example, the methods used in this study required as participants persons with learning difficulties who had abilities at their command that would allow them to communicate with the researchers, move without personal assistance, and be able and willing to explain. Ethical issues, furthermore, had to be met by placing a special emphasis on the means of gaining informed consent (following Porter/Lacey 2005: 92-95; Buchner 2008: 517).

In the following, we present five insights from these research approaches with particular emphasis on the role of the persons with disabilities, the conceptualization of collaborative research, and the methods used (narrative story-telling, photo-voice and mapping, shadowing).

\subsubsection{Persons with Disabilities and Their Role in the Research}

Participatory research strands differ according to the role that is awarded to persons with disabilities. Several of the South African studies referenced involved persons with disabilities directly as co-researchers in the research process (Rule 2011; Rule/Modipa 2012; Chappell 2013). This required careful selection of co-researchers based on criteria such as educational level, embeddedness in the community, and interpersonal skills. It also required comprehensive briefing by the senior researcher; training in research skills, techniques, and ethical requirements; and continuing support of researchers through the process. One advantage of this was that it afforded persons with disabilities a voice in the process and developed their capacity as researchers, including their confidence in contributing to the process. As one co-researcher commented:

At first I thought that Paul [the senior researcher] was in charge. Then as we went on I forgot all about that because the things we discussed here were the very same things I discussed with my friends and that made me feel comfortable as if we were all on the same age group (Chappell 2013).

This comment points to the importance of creating an open and enabling learning environment within the research process, which allows co-researchers the freedom to contribute and develop.

Within the externally funded framework of the German-based numeracy study, with its a priori given conditions, the role of participants and researchers had to be construed differently, in the sense of working in alliances (Chappell 2000). It was the researchers who decided, at the project's outset, on the overall research question and the design to be 
implemented. However, the sampling strategy and the methods used (see below) equipped the persons with learning difficulties with a high degree of control over the procedures and contents; there was also emphasis placed on these persons, giving voice and evidence to their abilities, meanings, and experiences (for more detail, see Schreiber-Barsch et al. 2020; Curdt/Schreiber-Barsch 2020). As a parallel measure, a participatory research group with coresearchers with learning difficulties (these individuals had no overlap with the respondents) was formed; this group provided information and debated the steps of data collection and analysis. Within this group, for example, a co-researcher suggested the opening question for the interviews conducted with adults with learning difficulties; this suggestion was then applied.

\subsubsection{Conceptualizing Research Collaboratively}

Although, as demonstrated, it is not always possible to involve persons with disabilities in the overall conceptualization of the research because of institutional and practical limitations, doing so can assist in grounding the study in the experiences and perspectives of persons with disabilities - thus making it more meaningful to them and their lives. An example from the joint conceptualization of a research project on the experiences of persons with disabilities in the realm of education illustrates this (Rule/Modipa 2012). In this action research project, the research team consisted of both able-bodied and disabled members. The senior researcher, an able-bodied male, proposed the following conception of the study: Investigating the Needs and Responses of Adult Learners with Disabilities. In the ensuing discussion, the researchers with disabilities pointed out that this conception assumed a passive view of persons with disabilities as those with "needs" who "responded," rather than as individuals who could take an active role. From this discussion, an alternative conception arose: Investigating the Attitudes and Experiences of Adult Learners with Disabilities. This positioned persons with disabilities as agents and contributors to knowledge. It was an important moment of assertion for the researchers with disabilities, who exercised their own voices and agency as members of the disability movement; and a key learning moment for the able-bodied researcher who had not been aware of his own assumptions about disability, and how these might position persons with disabilities as passive recipients within the research process. This points to the importance of participatory research as a process of learning and consciousness-raising for all participants.

In the German case, the GTM was explicitly chosen as the research methodology in order to install an iterative process of data sampling, analysis, and theory development. In consequence, snowball sampling was used to recruit participants for the shadowing portion and the interviews. This was achieved by introducing (in easy language) the research project at various places that were frequently and commonly used by adults with learning difficulties, and asking for their participation as experts in numeracy under the conditions of impairment and disability. In this way, the research participants profoundly contributed to conceptualizing the research collaboratively, not least by demanding the researchers clearly concretize the reasons why it is beneficial for all persons involved to contribute to knowledge production related to numeracy practices. 


\subsubsection{Narrative Story-telling}

The South African studies point to the importance of narrative story-telling as a research method that enabled persons with disabilities to exercise "voice" and tell their often-unheard or neglected stories regarding education. Through narrative interviews, adult learners with disabilities articulated barriers that forced them to leave school as children:

We were isolated by both teachers and learners and that is why we failed to [achieve] with our schooling like other kids. The learners would tease us and reports to teachers proved futile. That is why I stopped schooling and now I am uneducated (Bathabile).

I could not see the board and exercise books. I was not able to continue with schooling (Zodwa).

I walked a long distance from home to school and I would be constantly punished for being late. The problem was that I could never walk fast to avoid being late (Fikile).

These adult learners expose the disabling barriers that operated at school, including both physical barriers ("could not see the board," "could never walk fast to avoid being late") and the social barriers ("isolated by both teachers and learners"). Besides affording voice to adults with disabilities, this research process generated ideas for developing an inclusive adult basic education program that could overcome such barriers (Rule/Modipa 2012).

\subsubsection{Photo-voice and Mapping}

Photo-voice and mapping are participatory methods that enable persons with disabilities to use visual and spatial techniques to present and reflect upon their experiences. Photo-voice involves providing research participants with cameras (or, more recently, asking participants to use the cameras on their mobile phones) to take photos of a particular aspect of their lives (photo-voice) and then to talk about what the photo means to them (photo-voice), thus combining the visual and verbal (Wang 2003). This enables participants both to record and to reflect on an issue in their life-worlds, engage in critical dialogue around the issue, and inform policy-making (Wang/Burris 1997: 370). In her study of the experiences of young adults with disabilities at a vocational college in South Africa, Siwela (2017) used photos to enable participants to talk about their experiences of barriers to learning. For example, one participant with a physical impairment talked about the flights of stairs that she was not able to negotiate (there were no lifts) - she remained at the bottom of the stairs while fellow students attended the course; she relied on them taking notes for her.

Adding "voice" to the photo, a student with a physical impairment reflected on how the college could improve:

I think they should just put lifts in all the buildings. Also ramps may help. Sometimes I just feel like learning from home and only come to college to collect notes and write tests and exams. Nothing is working for me (Siwela 2017: 66).

The students with disabilities at the college also used the method of mapping to identify physical barriers on the campus. 
Figure 1: Barriers to learning at a Technical \& Vocational Education and Training College
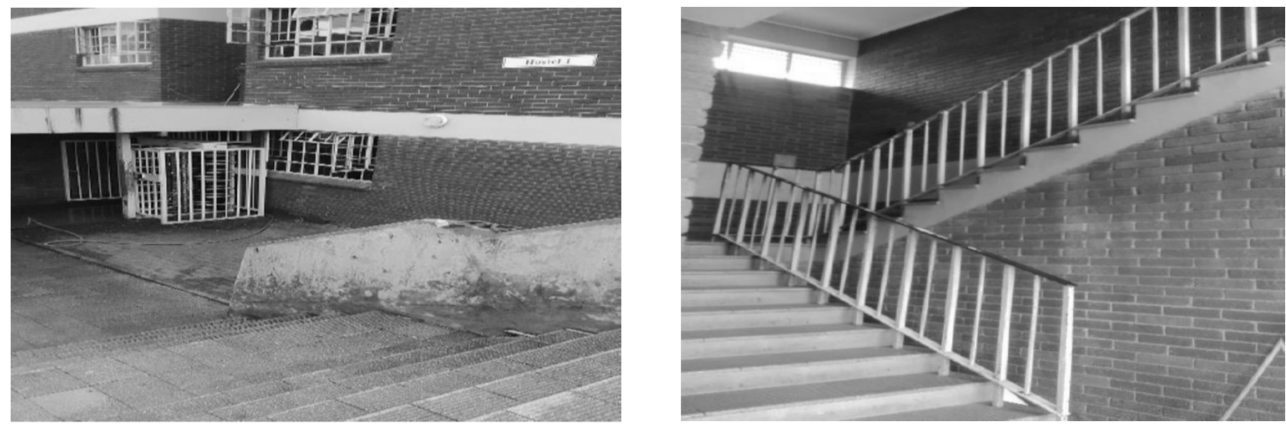

Source: own research.

Figure 2: Map of barriers on campus

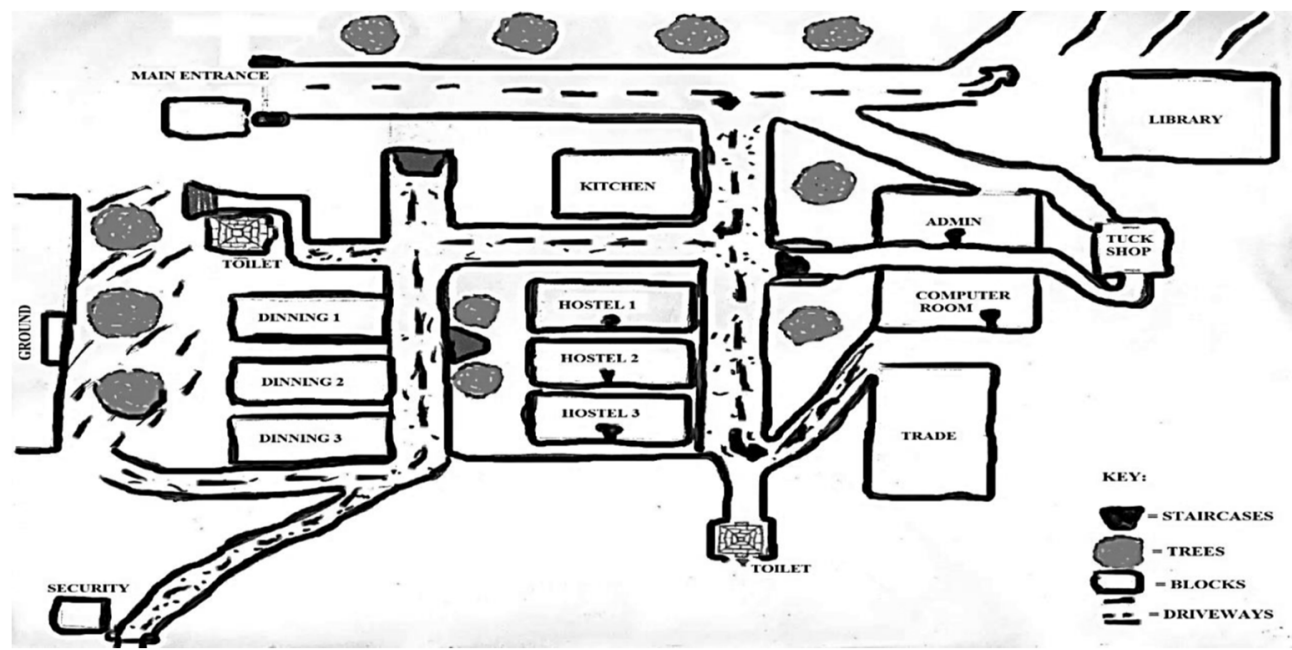

Source: own research.

This involved drawing a map of the campus and marking the particular places - such as staircases, sections of broken pavement, and driveways - that posed obstacles or dangers to the students. Photo-voice and mapping thus provided alternative ways of representing their experiences of barriers to learning, in relation to which they could both reflect and record their experiences, as well as think critically and pose alternatives to improve the situation. 


\subsubsection{Shadowing}

In the German study, the shadowing method (Bøe et al. 2017) was used for the in-situ explorations of numeracy activities. This enabled insights into the complexity of social practices, which were sometimes invisible to the persons themselves, and also allowed the study participants to share their views and sense-making as part of the data collection. The persons with learning difficulties were asked to allow the shadowing of their daily activities as they usually take place; thus, they were in full control of the procedures and circumstances (McDonald/Simpson 2014). A meeting-point was agreed upon and, from that point, the researcher followed the participant wherever he or she went (though, of course, respecting their privacy and not following into, for example, the swimming pool's changing room during swim training). A recording template (observational protocol) was used throughout the shadowing to focus the researcher's attention on general components of numeracy.

The following shadowing sequence illustrates the process of negotiating control in the process of data collection (for more detail, see Curdt/Schreiber-Barsch 2020). A researcher met Maja after she had finished her vocational training course close to a train station; the only information given was that the researcher would follow her spending free time and going shopping. The following event took place when changing trains (noted in the recording template):

Maja: "Do we want to go to the [shopping center]?"

Researcher: "You decide. I'm just following you today."

Maja: "Then we'll go to the [shopping center]" .

She is going purposively to the platform. We are standing at the station and waiting.

Maja: "We can also go to the airport. I was there the other day with my friend".

Researcher: "Yes, we can do that, too".

Maja: "Or to the [shopping center]."

Two minutes before the train arrives, Maja changes her mind: "We'll take my route to school. Going home, that's not that far. That's better".

She turns, goes back downstairs in order to change platforms.

Maja: "The train is already there!" She quickly runs up the stairs and jumps into the train.

I [the researcher] run after her.

(Sh_3_Maja: para 7_1-7)

\section{Conclusion: Challenges and Benefits of Shifting Lenses to a Participatory Ethos in Research}

Besides the advantages that participatory methods offer in conducting research with persons with disabilities, the aforementioned studies also highlight a number of challenges (For more, see Curdt/Schreiber-Barsch 2020). The first challenge concerns power dynamics and 
addresses equivalency within the process of conducting the research. While a participatory approach can reduce the power differentials within research by enabling agency and the exercise of voice, it does not eliminate them. Power differentials remain between more and less experienced, able-bodied participants and those with impairments, as well as between researchers with different kinds of impairments (Chappell et al. 2014; Nuwagaba/Rule 2015b). In addition, the intersectionality of disability with other social identities, such as gender, race, class, age, and educational level, might further accentuate such differentials. It is important to identify such differentials and address them explicitly and transparently in the research process, and to provide opportunities for reflection and learning.

A second challenge concerns positionality (of the persons involved and their roles) as well as ethical concerns and situated ethics. This includes issues such as the aforementioned positionality, as well as vulnerability, signed consent, anonymity, and research committee composition (Nuwagaba/Rule 2015a). A study on microfinance and adult learning of persons with disabilities in Uganda found that conventional ethical procedures such as signed consent and anonymity might not be appropriate for persons with disabilities. Participants were suspicious of signing consent documents, which they associated with police affidavits and a lack of trust, and preferred to give oral consent. Regarding anonymity, they preferred their names to be known, as they were proud of their educational achievements and wished to be seen as role models in the community (Nuwagaba/Rule 2015a). Ethical issues such as this have to be situated in the particular context of the research, and in relation to the needs and value systems of the participants.

Ownership of the research is a third challenge. If persons with disabilities participate actively in the research, contributing their stories and experiences, the question arises regarding who owns the research, controls its dissemination, and ultimately benefits from it. This can also be an opportunity for creative collaboration, for example in co-authoring articles (Rule/Modipa 2012; Chappell et al. 2014) and co-presenting conference papers (De Lange/Rule 2015), as well as exploring creative and popular forms of dissemination such as transforming research into easy-reading material for adult learners (Rule 2008). The issue of ownership relates to accountability: who is accountable to whom in the research process, particularly in the context of the struggles of the disability movement for rights, voice, and services? Again, these are issues that should be addressed explicitly and transparently in the research process; researchers should acknowledge the institutional and logistical limitations that often circumscribe research projects (Barnes 2003).

Finally, there is the challenge of building and sustaining capacity within the wider framework of participation in research and society at large. Persons with disabilities who participate in research projects, particularly as co-researchers, should come out of the process with skills and knowledge that could serve them in the future, and contribute to a more inclusive research community more widely. Thus, training and capacity-building should be built into the process, and pathways for sustaining participants' retention of skills and further learning should be considered. A participatory approach to research, which explicitly foregrounds and enables learning, can help to address this challenge.

In conclusion, this chapter has focused on a comparative investigation of inclusive adult education in two nation states, Germany and South Africa. Our comparative approach showed the importance of the historical and ideological context in understanding the respective frames of inclusive adult education. We found that there are many theoretical and methodological gaps in both countries regarding not only the inclusion of adults with disabilities in educational practice, but also in the respective bodies of scholarship. In both countries, a 
rights and ethics discourse is prominent in the public sphere, not least due to intergovernmental policy agendas, while an efficacy discourse is conspicuously less developed. Yet, in an age of increasing competition (Powell 2020), in which countries often pursue the promises of a global testing culture in education (Smith 2017) - and in an age of increasing neoliberalism, in which questions surrounding the financing of education are paramount - we recommend that studies should contribute to furthering the efficacy discourse in order to give evidence not only to the price of inclusive education, but also to the much higher one of sustaining exclusion and persistent segregation.

Against this backdrop, we concluded by shifting the focus to participatory research approaches: these involve persons with disabilities, but also pose particular challenges, including power dynamics, situated ethics, and ownership of the research, which should be considered seriously. Using this comparative lens, however, illuminated the fact that commonalities, similarities, and differences are, in this case, less a question of the national settings than of the approach used.

Overall, we would argue that a participatory ethos in research can shed new light on the phenomenon of disability, and on voices and data that are still missing to a substantial degree in the global knowledge production machinery. As Stepanek Lockhart has rightly emphasized, "only what is measured gets counted" (2018: 228) in research endeavours. Thus, in our view, to know how to move toward creating inclusive adult educations systems that are both sensitive to local contexts and aligned with the shared global goal of education for all, ultimately depends on broadening the methodological pathways in counting voices and perspectives.

\section{References}

Ackermann, Karl-Ernst/Ditschek, Eduard Jan (2015): Von der separierenden zur inkludierenden Erwachsenenbildung. Rückblick, Situationsanalyse und Ausblick. In: Hessische Blätter für Volksbildung 65, 4, pp. 308-316.

Ahmed, Sara (2012): On Being Included: Racism and Diversity in Institutional Life. Durham: Duke University Press.

Allan, Julie (2013): Inclusion. Patterns and possibilities. In: Zeitschrift für Inklusion 4-2012. https://www.inklusion-online.net/index.php/inklusion-online/article/view/31 [Accessed: 09.07.2019]

Armstrong, Felicity (2003): Spaced Out. Policy, Difference and the Challenge of Inclusive Education. Dordrecht: Kluwer Academic Publishers.

Artiles, Alfredo J./Kozleski, Elizabeth B./Waitoller, Federico R. (2015) (eds.): Inclusive Education. Examining Equity on Five Continents. Cambridge, MA: Harvard Education Press.

Autorengruppe Bildungsberichterstattung (eds.) (2014): Bildung in Deutschland 2014. Ein indikatorengestützter Bericht mit einer Analyse zur Bildung von Menschen mit Behinderungen. Bielefeld: W. Bertelsmann.

Banks, Lena Morgon/Polack, Sarah (2014): The economic costs of exclusion and gains of inclusion of people with disabilities. Evidence from low and middle income countries. London: International Centre for Evidence in Disability, London School of Hygiene \& Tropical Medicine.

Barbour, Rosaline (2007): Doing focus groups. Los Angeles: SAGE. 
Barnes, Colin (2003): What a Difference a Decade Makes. Reflections on Doing "Emancipatory" Disability Research. In: Disability \& Society 18, 1, pp. 3-17.

Barnes, Colin (2014): Understanding the social model of disability. Past, present and future. In: Watson, Nick/Roulstone, Alan/Thomas, Carol (eds.): Routledge Handbook of Disability Studies. New York: Routledge, pp. 12-29.

Bereday, George Z.F. (1964): Comparative Method in Education. New York: Holt, Rinehart and Winston.

Biermann, Julia (2021 in this volume): Der Einfluss der UN-BRK auf inklusive Bildung in Nigeria und Deutschland. In: Köpfer, Andreas/Powell, Justin J.W./Zahnd, Raphael (eds.): Handbuch Inklusion international. Opladen: Budrich, pp. 167-178.

Biermann, Julia/Powell, Justin J.W. (2014): Institutionelle Dimensionen inklusiver Schulbildung - Herausforderungen der UN-Behindertenrechtskonvention für Deutschland, Island und Schweden im Vergleich. In: Zeitschrift für Erziehungswissenschaft 17, pp. 679-700.

Bilger, Frauke/Behringer, Friedericke/Kuper, Harm/Schrader, Josef (2017) (eds.): Weiterbildungsverhalten in Deutschland 2016. Ergebnisse des Adult Education Survey (AES). Bielefeld: WBV.

BMAS - Bundesministerium für Arbeit und Soziales (2011): Unser Weg in eine inklusive Gesellschaft. Der Nationale Aktionsplan der Bundesregierung zur Umsetzung der UN-Behindertenrechtskonvention. Berlin: BMAS.

BMAS - Bundesministerium für Arbeit und Soziales (2016): Unser Weg in eine inklusive Gesellschaft. Nationaler Aktionsplan 2.0 der Bundesregierung zur UN-Behindertenrechtskonvention (UN-BRK). Berlin: BMAS.

Bøe, Marit/Hognestad, Karin/Waniganayake, Manjula (2017): Qualitative shadowing as a research methodology for exploring early childhood leadership in practice. In: Educational Management Administration \& Leadership 45, 4, pp. 605-620.

Buchner, Tobias (2008): Das qualitative Interview mit Menschen mit so genannter geistiger Behinderung - Ethische, methodologische und praktische Aspekte. In: Biewer, Gottfried/Luciak, Mikael/Schwinge, Mirella (eds.): Begegnung und Differenz: Menschen - Länder - Kulturen. Beiträge zur Heil- und Sonderpädagogik. Bad Heilbrunn: Klinkhardt, pp. 516-528.

Buchner, Tobias/Koenig, Oliver/Schuppener, Saskia (2011): Gemeinsames Forschen mit Menschen mit intellektueller Behinderung: Geschichte, Status quo und Möglichkeiten im Kontext der UN-Behindertenkonvention. In: Teilhabe 50, 1, pp. 4-10.

Buchner, Tobias/Pfahl, Lisa/Traue, Boris (2015): Zur Kritik der Fähigkeiten. Ableism als neue Forschungsperspektive der Disability Studies und ihrer Partner_innen. In: Zeitschrift für Inklusion, 2, 2015. https://www.inklusion-online.net/index.php/inklusion-online/article/view/273 [Accessed: 09.07.2019]

Buckup, Sebastian (2009): The price of exclusion. The economic consequences of excluding people with disabilities from the world of work. International labour office employment working paper no. 43. https://www.ilo.org/employment/Whatwedo/Publications/working-papers/WCM S_119305/lang--en/index.htm [Accessed: 09.07.2019]

Campbell, Fiona Kumari (2009): Contours of ableism. New York: Palgrave Macmillan.

Chappell, Anne Louise (2000): Emergence of participatory methodology in learning difficulty research: Understanding the context. In: British Institute of Learning Disabilities. British Journal of Learning Disabilities 28, pp. 38-43. doi.org/10.1046/j.1468-3156.2000.00004 .x.

Chappell, Paul (2013): The social construction of the sexual identities of Zulu-speaking youth with disabilities in KwaZulu-Natal, South Africa, in the context of the HIV pandemic. Unpublished PhD thesis. University of KwaZulu-Naal, Pietermaritzburg.

Chappell, Paul/Rule, Peter/Dlamini, Mfana/Nkala, Nompilo (2014): Troubling power dynamics. Youth with disabilities as co-researchers in sexuality research in South Africa. In: Childhood. 21, 3, S. 385-399. 
Christie, Pam/Krut, Orenna/Perold, Helene/Butler, Dawn (1991): The Right to Learn: The struggle for education in South Africa (2nd ed.). Johannesburg: Ravan.

Corbett, Jenny/Slee, Roger (2000): An International Conversation on Inclusive Education. In: Armstrong, Felicity/Armstrong, Derrick/Barton, Len (eds.): Inclusive Education: Policy, Contexts and Comparative Perspectives. London: David Fulton, pp. 133-146.

Curdt, Wiebke/Schreiber-Barsch, Silke (2020): Abilities in the blind spot of testing regimes: Eliciting the benefits and limitations of participatory research approaches for numeracy in adult basic education. In: International Review of Education, 66(2-3), pp. 387-413. https://link.springer.com/article/10.1007/s11159-020-09848-9

Davenport, T.R.H/Saunders, Christopher (2000): South Africa: A modern history (5th ed.). London: Macmillan.

Dederich, Markus (2014): Intersektionalität und Behinderung. Ein Problemaufriss. In: Behinderte Menschen 1, pp. 47-53.

De Lange, Michelle (2016): Experiences and challenges faced by adult learners with 'hidden' disabilities in Higher Education. Unpublished M.Ed thesis, University of KwaZulu-Natal, Pietermaritzburg.

De Lange, Michelle/Rule, Peter (2015): Adult learners with "hidden" disabilities in Higher Education. South African Educational Research Association Conference, University of the Free State, Bloemfontein, 27-30 October.

Department of Education (2001): Education White paper 6. Special needs education. Building an inclusive education and training system. Pretoria: Department of Education.

Department of Higher Education and Training (2013): White Paper on Post-School Education and Training. Pretoria: DHET.

Desjardins, Richard (2017): Political Economy of Adult Learning Systems. Comparative Study of Strategies, Policies and Constraints. London: Bloomsbury Academic.

Dieckmann, Friedrich/Giovis, Christos (2012): Der demografische Wandel bei Erwachsenen mit geistiger Behinderung. Vorausschätzung der Altersentwicklung am Beispiel von WestfalenLippe. In: Teilhabe 51, 1, pp. 12-19.

Donohue, Dana/Bornman, Juan (2014): The challenges of realising inclusive education in South Africa. In: South African Journal of Education 34, 2, 14 pages. http://www.sajournalofeducation.co.za/index.php/saje/article/viewFile/806/415

Dreyer, Lorna M. (2017): Inclusive education. In: Ramrathan, Labby/Le Grange, Lesley/Higgs, Philip (eds.): Education studies for initial teacher development. Cape Town: Juta, pp. 383-399.

Dyson, Alan (1999): Inclusion and Inclusions. Theories and Discourses in Inclusive Education. In: Daniels, H./Garner, P. (eds.): World Yearbook of Education 1999. Inclusive Education. London: Kogan Page, pp. 36-53.

Elphick, Jean/De SasKropiwnicki, Zosa/Elphick, Rosalind (2015): "Our children have the right to an education too". Strategies Employed by Orange Farm Caregivers of Children with Disabilities in Pursuit of the Right to a Basic Education. In: Disability, CBR \& Inclusive Development 26, 4, pp. 101-116.

Engelbrecht, Petra/Savolainen, Hannu/Nel, Mirna/Koskela, Teija/Okkolin, Mari-Ann (2017): Making meaning of inclusive education: classroom practices in Finnish and South African classrooms. In: Compare: A Journal of Comparative and International Education 47, 5, pp. 684-702.

EUROSTAT (2019): Adult Education Survey (AES). https://ec.europa.eu/eurostat/web/microdata/adult-education-survey [Accessed: 09.07.2019]

Evans, Jeff (2019): Adult skills surveys and transnational organisations: Globalising educational policy. In: Evans, Jeff/Ruane, Sally/Southall, Humphrey (eds.): Data in society. Challenging statistics in an age of globalisation. Bristol: Policy Press, pp. 65-77. 
Fairbrother, Gregory P. (2016): Quantitative and Qualitative Approaches to Comparative Education. In: Bray, Mark/Adamson, Bob/Mason, Mark (eds.): Comparative Education Research. Approaches and Methods. Springer: Hong Kong, pp.71-93.

Gal, Iddo (2000): The numeracy challenge. In: Gall, Iddo (ed.): Adult numeracy development: Theory, research, practice. Cresskill: Hampton Press, pp. 9-31.

Geiger, Vince/Goos, Merrilyn/Forgasz, Helen (2015): A rich interpretation of numeracy for the 21st century: A survey of the state of the field. ZDM: The International Journal on Mathematics Education 47, 4, pp. 531-548.

Goodley, Dan (2017): Disability Studies. An Interdisciplinary Introduction. London: Sage.

Grech, Shaun/Soldatic, Karen (2016) (eds.): Disability in the Global South. The Critical Handbook. Cham: Springer.

Hanass-Hancock, Jill (2009): Interweaving conceptualizations of gender and disability in the context of vulnerability to HIV/AIDS in KwaZulu-Natal, South Africa. In: Sexuality and Disability 27 , pp. 35-47.

Heimlich, Ulrich/Behr, Isabel (2018): Inklusion von Menschen mit Behinderung in der Erwachsenenbildung/Weiterbildung. In: Tippelt, Rudolf/Hippel, Aiga von (eds.): Handbuch Erwachsenenbildung/Weiterbildung. Wiesbaden: VS, pp. 1207-1223.

ISSC/IDS/UNESCO (2016): World Social Science Report. Challenging Inequalities: Pathways to a Just World. Paris: UNESCO \& ISSC.

Katzenbach, Dieter (2015): Zu den Theoriefundamenten der Inklusion - Eine Einladung zum Diskurs aus der Perspektive der kritischen Theorie. In: Schnell, Irmtraud (ed.): Herausforderung Inklusion. Bad Heilbrunn: Klinkhardt, pp. 19-32.

Kiernan, Chris (1999): Participation in Research by People with Learning Disability: Origins and Issues. In: British Journal of Learning Disabilities 27, 2, pp. 43-47.

Koscheck, Stefan/Weiland, Meike/Ditschek, Eduard Jan (2013): wbmonitor Umfrage 2012: Klima und Strukturen der Weiterbildungslandschaft. Bonn: BiBB und DIE. https://www.bibb. de/dokumente/pdf/wbmonitor_Ergebnisbericht_Umfrage_2012.pdf [Accessed: 01.03.2020].

Lindmeier, Christian/Lütje-Klose, Birgit (2015): Inklusion als Querschnittsaufgabe in der Erziehungswissenschaft. In: Erziehungswissenschaft, Mitteilungen der Deutschen Gesellschaft für Erziehungswissenschaft 26, 51, pp. 7-16.

Manzon, Maria (2016): Comparing Places. Comparing Race, Class and Gender. In: Bray, Mark/Adamson, Robert Bob/Mason, Mark (eds.): Comparative Education Research. Approaches and Methods. Hong Kong: Springer, pp. 97-137.

Mason, Mark (2016): Comparing Cultures. In: Bray, Mark/Adamson, Robert Bob/Mason, Mark (eds.): Comparative Education Research. Approaches and Methods. Hong Kong: Springer, pp. 221-257.

McDonald, Seonaidh/Simpson, Barbara (2014): Shadowing research in organizations: The methodological debates. In: Qualitative Research in Organizations and Management: An International Journal 9, 1, pp. 3-20.

McKay, Veronica I. (2018): Literacy, lifelong learning and sustainable development. In: Australian Journal of Adult Learning 58, 3, pp. 390-425.

McKay, Veronica I./Room, Norma R.A. (2015): Narratives of agency. The experiences of Braille literacy practitioners in the KhaRiGude South African Mass Literacy Campaign. In: International Journal of Inclusive Education, 19, 4, pp. 435-456.

Nuwagaba, Ephraim Lemmy/Rule, Peter (2015a): Navigating the ethical maze in disability research in an African context. In: Disability \& Society 30, 5, pp. 255-269.

Nuwagaba, Ephraim Lemmy/Rule, Peter (2015b): Power dynamics among PWDs during adult learning processes: Motivator or demotivator? In: Rwandan Journal of Education 3, 1, pp. 5265.

OECD (2018): Education at a Glance 2018: OECD Indicators. Paris: OECD Publishing. 
Office of the Deputy President (1997): Integrated National Disability Strategy. White Paper. Pretoria: Office of the President, Republic of South Africa.

Ozga, Jenny/Jones, Robert (2006): Travelling and embedded policy. The case of knowledge transfer. In: Journal of Education Policy 21, 1, pp. 1-17.

Porter, Jill/Lacey, Penny (2005): Researching Learning Difficulties. A Guide for Practitioners. London: Paul Chapman Publishing.

Poore, Carol (2009). Disability in Twentieth-Century German Culture. Ann Arbor: The University of Michigan Press.

Powell, Justin J.W. (2020): Comparative education in an age of competition and collaboration. In: Comparative Education 56, 1, pp. 57-78.

Powell, Justin J.W./Pfahl, Lisa (2012): Sonderpädagogische Fördersysteme. In: Bauer, Ullrich/ Bittlingmeyer, Uwe H./Scherr, Albert (eds.): Handbuch Bildungs- und Erziehungssoziologie. Wiesbaden: VS Verlag, pp. 721-740.

Rammstedt, Beatrice (2013) (ed.): Grundlegende Kompetenzen Erwachsener im internationalen Vergleich: Ergebnisse von PIAAC 2012. Münster: Waxmann.

Rancière, Jacques (1999): Disagreement: Politics and Philosophy. Minneapolis: University of Minnesota Press.

Republic of South Africa (1996): The Constitution of South Africa. (Act no. 108 of 1996). Pretoria: Government Printer.

Richardson, John G./Powell, Justin J.W. (2011): Comparing Special Education: Origins to Contemporary Paradoxes. Stanford, CA: Stanford University Press.

Ross-Gordon, Jovita M. (2018): Disabilities and Adult and Lifelong Education. In: Milana, Marcella/Webb, Sue/Holford, John/Waller, Richard (eds.): The Palgrave International Handbook on Adult and Lifelong Education and Learning. London: Palgrave Macmillan, pp. 879898.

Rule, Peter (2008): Shoeeees! The story of Leonard Gregory. Durban: New Readers Publishers.

Rule, Peter (2011): Cross-learning: The possibilities of a learning dialogue between the HIV \& AIDS and disability movements. In: Studies in the Education of Adults 43, 2, pp. 216-233.

Rule, Peter (2018): Engaging persons with disabilities in participatory research. Methods and perspectives. Conference paper, Numeracy as part of Adult (Basic) Education: International and Comparative Perspectives, Hamburg, Germany, 15-16 November.

Rule, Peter/Modipa, Taadi Ruth (2012): "We must believe in ourselves". Attitudes and experiences of adult learners with disabilities in KwaZulu-Natal, South Africa. In: Adult Education Quarterly 62, 2, pp.138-158.

Sayed, Yusuf/Soudien, Crain (2003): (Re)Framing Education Exclusion and Inclusion Discourses. Limits and Possibilities. In: IDS Bulletin 34,1, pp. 9-19.

Schneider, Marguerite (2006): Disability and the environment. In: Watermeyer, Brian/Swartz, Leslie/Lorenzo,Theresa/Schneider Marguerite/Priestley, Mark (eds.): Disability and social change. A South African agenda. Pretoria: HSRC Press, pp. 8-18.

Schreiber-Barsch, Silke (2018): Who counts? Disruptions to adult education's idyll and its topography of lifelong learning. Interlinking Rancière's political philosophy with adult education. In: European Journal for Research on the Education and Learning of Adults 9, 2, pp. 179-192.

Schreiber-Barsch, Silke/Fawcett, Emma (2017): Inklusionsarchitekturen. Wie wird ein Lernort zu einem inklusiven Lernort im öffentlichen Raum des Lebenslangen Lernens? In: Zeitschrift für Weiterbildungsforschung 3, pp. 295-319.

Schreiber-Barsch, Silke/Fawcett, Emma (2019): Semantiken von Inklusion: Benennungspraktiken an Volkshochschulen unter der Agenda inklusiver Erwachsenenbildung. In: Hessische Blätter für Volksbildung, pp. 18-30. 
Schreiber-Barsch, Silke/Curdt, Wiebke/Gundlach, Hanna (2020): Whose voices matter? Adults with learning difficulties and the emancipatory potential of numeracy practices. ZDM Mathematics Education, 52(3), 581-592, https://doi.org/10.1007/s11858-020-01133-1.

Shakespeare, Tom (2014): Disability rights and wrongs revisited (2. Ed.). Abingdon: Routledge.

Siwela, Sanele (2017): An exploratory case study of the experiences of students with disabilities at a TVET college. Factors that facilitate or impede their access and success. Unpublished M.Ed thesis, University of KwaZulu-Natal, Pietermaritzburg.

Smith, William C. (2017) (ed.): The Global Testing Culture: Shaping education policy, perceptions, and practice. Oxford: Symposium Books.

Smyth, Fiona/Shevlin, Michael/Buchner, Tobias/Biewer, Gottfried/Flynn, Paula/Latimer Camille/Šiška, Jan/Toboso-Martin, Mario/Rodríguez Díaz, Susana/ Ferreira, Miguel A.V. (2014): Inclusive education in progress. Policy evolution in four European countries. In: European Journal of Special Needs Education 29, 4, pp. 433-445.

Snyder, Sharon L./Mitchell, David T. (2006): Cultural Locations of Disability. Chicago: The University of Chicago Press.

Stalker, Kirsten (2014): Theorizing the position of people with learning difficulties within disability studies. Progress and pitfalls. In: Watson, Nick/Roulstone, Alan/Thomas, Carol (eds.): Routledge handbook of disability studies. New York: Routledge, pp. 122-135.

Stepanek-Lockhart, Ashley (2018): Monitoring ESD: Lessons learned and ways forward. In: UNESCO (Ed.): Issues and trends in education for sustainable development. Paris: UNESCO, pp. 215-231.

Strauss, Anselm/Corbin, Juliet (1990): Basics of qualitative research. Grounded theory procedures and techniques. London: Sage.

Strübing, Jörg (2014): Grounded theory: Zur sozialtheoretischen und epistemologischen Fundierung eines pragmatistischen Forschungsstils. Wiesbaden: Springer VS.

The World Bank (2019): Every learner matters: Unpacking the learning crisis for children with disabilities. Washington, DC: The World Bank.

UNESCO/Leonard Cheshire Disability (2019): Inclusive education for persons with disabilities Are we making progress? Background paper prepared for the International Forum on inclusion and equity in education - Every learner matters, Cali, Colombia. https://unesdoc.unesco. org/ark:/48223/pf0000370386 [Accessed: 01.03.2020].

United Nations (2006): Convention on the rights of persons with disabilities. http://www.un.org/ disabilities/documents/convention/convention_accessible_pdf.pdf [Accessed: 19.01.2019]

United Nations (2015): International Day of Persons with Disabilities, 3 December 2015. https:/www.un.org/development/desa/disabilities/international-day-of-persons-with-disabilities-3-december/international-day-of-persons-with-disabilities-3-december-2015.html\#background [Accessed: 09.07.2019]

von Unger, Hella (2014): Partizipative Forschung. Einführung in die Forschungspraxis. Wiesbaden: Springer VS.

Waldschmidt, Anne (2020): Handbuch Disability Studies. Wiesbaden: Springer VS

Walmsley, Jan/Johnson, Kelley (2016): Vorwort. In: Buchner, Tobias/Koenig, Oliver/Schuppener, Saskia (Eds.): Inklusive Forschung. Gemeinsam mit Menschen mit Lernschwierigkeiten forschen. Bad Heilbrunn: Verlag Julius Klinkhardt, pp. 9-12.

Walton, Elizabeth (2011): Getting inclusion right in South Africa. In: Intervention in School and Clinic 46, 4, pp. 240-245.

Wang, Caroline (2003): Chapter Nine: Using Photovoice as a Participatory Assessment and Issue Selection Tool: A Case Study with the Homeless in Ann Arbor. In: Wallerstein, Nina/Minkler, Meredith (Eds.): Community-based participatory research for health. San Francisco, CA: Jossey-Bass. 
Wang, Caroline/Burris Mary Ann (1997): Photovoice: Concept, methodology, and use for participatory needs assessment. In: Health Education and Behaviour 2, 3, pp. 69-387.

Wansing, Gudrun/Westphal, Manuela (Eds.) (2014): Behinderung und Migration. Inklusion, Diversität, Intersektionalität. Wiesbaden: Springer VS.

Watson, Nick/Roulstone, Alan/Thomas, Carol (2014): Routledge handbook of disability studies. New York: Routledge.

Weisser, Jan (2018): Inklusion, Fähigkeiten und Disability Studies. In: Sturm, Tanja/WagnerWilli, Monika (eds.): Handbuch schulische Inklusion. Opladen: Barbara Budrich, pp. 93-106.

WHO (World Health Organization) (2002): Towards a Common Language for Functioning, Disability and Health: ICF. Geneva: World Health Organization.

WHO (World Health Organization) (2007): Atlas. Global resources for persons with intellectual disabilities. Geneva: WHO.

Yasukawa, Keiko/Rogers, Alan/Jackson, Kara/Street, Brian V. (Eds.) (2018): Numeracy as social practice. Global and local perspectives. London: Routledge.

Zahnd, Raphael (2021 in this volume): Weltbank. In: Köpfer, Andreas/Powell, Justin J.W./Zahnd, Raphael (eds.): Handbuch Inklusion international. Opladen: Verlag Barbara Budrich, pp. 107126. 


\section{Autor*innenverzeichnis}

Mel Ainscow is Professor of Education, University of Glasgow, Emeritus Professor of Education at the University of Manchester, and Adjunct Professor at Queensland University of Technology, Australia. He is internationally recognized as an authority on the promotion of inclusion and equity in education. A long-term consultant to UNESCO, Mel is currently working on efforts to promote equity and inclusion globally. He has recently completed collaborative research projects with networks of schools in Australia, Austria, Denmark, England, Portugal and Spain.

E-Mail: mel.ainscow@manchester.ac.uk

Stefanija Ališauskienė is Professor of Special Education at Šiauliai University, Lithuania, and Visiting Professor at Canterbury Christ Church University, UK. Her research interests include early childhood intervention and special and inclusive education. For over ten years, she has been Editor-in-Chief of the scientific periodical Special Education.

E-Mail: stefanija.alisauskiene@su.lt

Cristina Allemann-Ghionda ist Professorin i. R. Dr. habil., Universität zu Köln, Humanwissenschaftliche Fakultät, Department Erziehungs- und Sozialwissenschaften, Vergleichende Bildungsforschung. Forschungsschwerpunkte: Vergleichende Untersuchungen zu interkultureller und Diversitätspädagogik, Inklusion und Schulbildung, Internationalisierung der Hochschule, Mehrsprachigkeit und Bildung, Ganztagsschule; interkulturelle Kommunikation und Sensibilisierung.

E-Mail: cristina.allemann-ghionda@uni-koeln.de

Julie Allan is Professor of Equity and Inclusion in the School of Education, University of Birmingham, UK. Key research interests include: disability and special educational needs, inclusion, children's rights, social capital and space.

E-Mail: j.allan.1@bham.ac.uk

Bettina Amrhein ist Professorin für International Perspectives on Inclusive Education, Universität Bielefeld, Deutschland. Forschungsschwerpunkte: International Perspectives on Inclusive Education, Inklusive Schul- und Unterrichtsentwicklung, Inklusion und Profession, De- \& Rekonstruktion im Förderschwerpunkt emotionale und soziale Entwicklung, Mindfulness in Education.

E-Mail: bettina.amrhein@uni-bielefeld.de

Benjamin Badstieber ist wissenschaftlicher Mitarbeiter, Universität Bielefeld, Forschungsschwerpunkte: Transformationsforschung in Schule unter Berücksichtigung von Heterogenität und Differenz, professionelle Rollen in inklusionsorientierter Schul- und Unterrichtsentwicklung, Rekonstruktionen unterrichtlicher Praktiken von Schüler*innen, De- \& Rekonstruktion im Förderschwerpunkt emotionale und soziale Entwicklung.

E-Mail: benjamin.badstieber@uni-bielefeld.de 
Julia Biermann ist Universitäts-Assistentin (Post-Doc), Lehr- und Forschungsbereich Disability Studies und Inklusive Bildung, Institut für Erziehungswissenschaft, Leopold-FranzensUniversität Innsbruck, Österreich. Forschungsschwerpunkte: International vergleichende Bildungsforschung, Inklusive Bildung, Disability Studies in Education, Diskursforschung, Menschenrechtsforschung.

E-Mail: julia.biermann@uibk.ac.at

Gottfried Biewer war Universitätsprofessor am Institut für Bildungswissenschaft der Universität Wien von 2004 bis 2020. Seit Oktober 2020 ist er Gastprofessor am Institut für Rehabilitationswissenschaften der Humboldt-Universität zu Berlin. Forschungsschwerpunkte: Schulische und außerschulische Inklusion.

E-Mail: gottfried.biewer@univie.ac.at; gottfried.biewer@hu-berlin.de

Margarita Bilgeri ist Vertretungsprofessorin, Institut für Sonderpädagogik, Universität Koblenz-Landau, Deutschland. Forschungsschwerpunkte: Inklusive Bildung, Behinderung im Globalen Süden, Behinderung, Armut und Gender, Intersektionalität, Behinderung und Migration.

E-Mail: bilgeri@uni-landau.de

Michelle Brendel is Senior Lecturer in Education at the University of Luxembourg. A trained family therapist and Gestalt therapist, she worked with children in foster care before joining the initial teacher training programs at the University of Luxembourg. Her research interests are collaborative teacher research, inclusive education, and portfolio approaches.

E-Mail: michelle.brendel@uni.lu

Tobias Buchner ist Professor für Inklusive Pädagogik, Institut für Inklusive Pädagogik, Pädagogische Hochschule Oberösterreich, Österreich. Forschungsschwerpunkte: dis*ability und Bildung, Raum und Inklusion, Partizipatorische Forschung.

E-Mail: tobias.buchner@ph-ooe.at

Gerardo Echeita Sarrionandia is Senior Lecturer in the Department of Developmental and Educational Psychology in the Faculty of Psychology at Autonomous University of Madrid, Spain. He is a well-known expert in Spain and Iberoamerican countries in the field of inclusive education, especially regarding policies and practices.

E-Mail: gerardo.echeita@uam.es

Lani Florian is Bell Chair of Education, The University of Edinburgh Moray House School of Education and Sport. Research interests focus on inclusion and achievement for all learners, inclusive pedagogy, and teacher education for inclusion.

E-Mail: lani.florian@ed.ac.uk

Bettina Fritzsche is Professor for General Education with an Emphasis on Qualitative Methods, Institute of Education, University of Education in Freiburg, Germany. Research interests: qualitative research in education, cultural-comparative educational research, inequality and diversity in educational institutions.

E-Mail: bettina.fritzsche@ph-freiburg.de 
Julia Gasterstädt ist wissenschaftliche Mitarbeiterin (post-doc), Institut für Sonderpädagogik, Goethe-Universität Frankfurt am Main, Deutschland. Forschungsschwerpunkte: Inklusion und Exklusion im Schulsystem, Herstellung und Bearbeitung von Differenz, qualitativrekonstruktive Methoden.

E-Mail: gasterstaedt@em.uni-frankfurt.de

Hafdís Guðjónsdóttir is Professor of Education in the School of Education at the University of Iceland. She was a general classroom teacher and special educator in compulsory schools for 26 years, an experience that has affected her academic focus. Her research interests are in the area of inclusive and multicultural education, pedagogy and educational practices, teacher development and professionalism, and teacher education.

E-Mail: hafdgud@hi.is

Katharina Heyer is Associate Professor of Political Science, University of Hawai'i at Mānoa, USA. Research interests: disability law and activism, comparative law, social movements, disability human rights and bioethics.

E-Mail: heyer@hawaii.edu

Marianne Hirschberg ist Professorin für Behinderung, Inklusion und Soziale Teilhabe, Institut für Sozialwesen, Universität Kassel, Deutschland. Forschungsschwerpunkte: Menschenrechte, Disability Studies, Behinderungskonstruktionen, Barrierefreiheit.

E-Mail: hirschberg@uni-kassel.de

Myriam Hummel ist Leiterin des Referats Internationalisierung, Fakultät für Erziehungswissenschaft, Universität Hamburg. Forschungsschwerpunkte: International vergleichende Bildungsforschung, Inklusive Bildung, Lehrer*innenprofessionalisierung.

E-Mail: myriam.hummel@uni-hamburg.de

Tiina Itkonen, $\mathrm{PhD}$, is Professor, Education and Political Science, California State University Channel Islands, USA. Research interests: Special education policy and politics, interest/ advocacy groups and collective action.

E-Mail: tiina.itkonen@csuci.edu

Markku Jahnukainen, PhD, is Professor, Faculty of Educational Sciences, University of Helsinki, Finland. Research interests: International and Comparative Research on Inclusive and Special Education; Education Policy.

E-Mail: markku.jahnukainen@helsinki.fi

Clara Rübner Jørgensen is Research Fellow in the Department for Disability Inclusion and Special Needs, School of Education, University of Birmingham, UK. Key research interests: migration and special educational needs, children's participation, power and empowerment, space, mobility and inclusion.

E-Mail: c.joergensen@bham.ac.uk

Michalis Kakos is a Reader (Associate Professor) in Education in Leeds Beckett University, UK and Visiting Professor at the University of Education Freiburg, Germany. His research interests include citizenship education, educational inclusion of refugee and migrant students, meta-ethnography and educational evaluation.

E-Mail: m.kakos@leedsbeckett.ac.uk 
Jóhanna Karlsdóttir is Assistant Professor in the School of Education at the University of Iceland. She completed her M.Ed at the University of Iceland in 2001. Her research focuses on inclusive pedagogy and education, diverse learners, and teacher stories about innovative inclusive practices.

E-Mail: johannak@hi.is

Swantje Köbsell ist Professorin für Disability Studies an der Alice Salomon Hochschule in Berlin, Deutschland. Forschungsschwerpunkte: Disability Studies, Disability Studies in Education, Intersektionalität im Kontext von Behinderung, Eugenik/Bioethik.

E-Mail: koebsell@ash-berlin.eu

Andreas Köpfer ist Tenure-Track-Professor für Inklusive Bildung und Lernen am Institut für Erziehungswissenschaft, Allgemeine Erziehungswissenschaft, der Pädagogischen Hochschule Freiburg, Deutschland. Forschungsschwerpunkte: Inklusive Bildung im internationalen Vergleich, Behinderungen und Unterstützung in Bildungsorganisationen, Raumproduktion/Raumaneignung, qualitativ-rekonstruktive Inklusionsforschung.

E-Mail: andreas.koepfer@ph-freiburg.de

Arthur Limbach-Reich is Associate Professor in Social and Educational Sciences at the University of Luxembourg. He is Luxembourg's national representative in the Academic Network of European Disability Experts (ANED). His recent research focuses on the implementation of the UN Convention on the Rights of Persons with Disabilities in Luxembourg and the Greater Region.

E-Mail: arthur.limbach@uni.lu

Mathias Mejeh ist Oberassistent am Institut für Erziehungswissenschaft, Abteilung Schulund Unterrichtsforschung, Universität Bern, Schweiz. Arbeits- und Forschungsschwerpunkte: Selbstreguliertes Lernen, Inklusive Bildung, Methodologie, Netzwerkanalyse, Institutionenanalyse.

E-Mail: mathias.mejeh@edu.unibe.ch

Kerstin Merz-Atalik is Professor of Education Considering Disability and Disadvantage/ Inclusion in the Department of Special Education at the University of Education Ludwigsburg, Germany. The main focus of her research is on teacher education for inclusion and inclusive school development, with an emphasis on international comparison and the diversity dimensions disability and migration.

E-Mail: merz-atalik@ph-ludwigsburg.de

Rita Meliene is Associate Professor of Education in the Department of Special Education at the Šiauliai University, Lithuania. Her research interests are teaching and learning in inclusive schooling, teaching methods, and learning native languages.

E-Mail: rita.meliene@gmail.com

Lina Miltenienè is Associate Professor of Education in the Department of Special Education at the University of Šiauliai, Lithuania. Her studies highlight the importance of creating an inclusive school culture and environment through development of collaboration and teamwork practice.

E-Mail: lina.milteniene@su.lt 
Edda Óskarsdóttir is Associate Professor at the School of Education, University of Iceland. She also holds a position as a project consultant at the European Agency for Special Needs and Inclusive Education. Her research interests are inclusive education policy, pedagogy and practice as well as teacher professional development. Her research is mainly qualitative with a focus on action research and self-study.

E-Mail: eddao@hi.is

Marcelo Parreira do Amaral ist Professor für Erziehungswissenschaft mit dem Schwerpunkt Internationale und Vergleichende Erziehungswissenschaft am Institut für Erziehungswissenschaft der Westfälischen Wilhelms-Universität Münster, Deutschland. Parreira do Amaral lehrt und forscht in den Arbeitsschwerpunkten Internationale und Vergleichende Erziehungswissenschaft, International Educational Governance und Internationale Bildungspolitik.

E-Mail: parreira@uni-muenster.de

Dame Alison Peacock, DL, Dlitt, is Professor and Chief Executive at the Chartered College of Teaching, London, England. Research interests: ,Learning without Limits ${ }^{6}$ - teaching without labelling by ability.

E-Mail: alison.m.peacock@gmail.com

Bengt Persson is Senior Professor of Special Needs Education at the University of Borås, Sweden. He holds a doctorate in Education from the University of Gothenburg. His research interests focus on the tension between special needs education and inclusive education at various levels of the educational system.

E-Mail: bengtperssontorstad@gmail.com

Elisabeth Persson is Senior Lecturer at the University of Borås, Sweden. She holds a doctorate in Mathematics Education from Stockholm University. Her research mainly concerns school development and inclusive education.

E-Mail: elisabeth.persson@hb.se

Elisabeth Plate is Postdoctoral Researcher at Humboldt-Universität zu Berlin, Germany. Her research interests are in inclusive school development internationally and inclusive teacher education and professionalism.

E-Mail: elisabeth.plate@hu-berlin.de

Justin J.W. Powell is Professor of Sociology of Education in the Institute of Education \& Society at the University of Luxembourg. His comparative institutional analyses of education systems chart persistence and change in special and inclusive education, in vocational training and higher education, and in science and research policy.

E-Mail: justin.powell@uni.lu

Michelle Proyer ist Tenure-Track-Professessorin für Inklusive Pädagogik, Zentrum für Lehrer*innenbildung, Institut für Bildungswissenschaft, Universität Wien, Österreich. Forschungsschwerpunkte: inclusive education, Kultur und Inklusion, inclusion and refugee studies, participatory research.

E-Mail: michelle.proyer@univie.ac.at 
Peter Rule is Associate Professor in the Centre for Higher and Adult Education at Stellenbosch University. He has thirty years' experience of working in adult and higher education in South Africa. He has published in the areas of adult education, dialogue and learning, reading education, disability and HIV/AIDS in education and also worked in not-for-profit organisations involved in adult literacy and disability, early childhood education and language education.

E-Mail: prule2015@sun.ac.za

Marta Sandoval Mena is Senior Lecturer in the College of Education at the Autonomous University in Madrid, Spain. Her research focuses on supports for schools to become more inclusive, especially supports for teachers and recognizing students' voices. She has specialized in collaborative research with agents of change, beginning with the adaptation into Spanish of the Index for Inclusion guidelines.

E-Mail: marta.sandoval@uam.es

Lea Schäfer ist wissenschaftliche Mitarbeiterin (Post-doc) an der Humboldt-Universität zu Berlin, Institut für Rehabilitationswissenschaften. Forschungsschwerpunkte: International vergleichende Bildungsforschung, inklusive Bildung, educational governance, qualitative Sozialforschung.

E-Mail: lea.schaefer@hu-berlin.de

Silke Schreiber-Barsch is Assistant Professor of Adult Education at the University of Hamburg (Germany), Faculty of Education, Unit of Lifelong Learning, and Associated Junior Professor at the UNESCO-Institute for Lifelong Learning, Hamburg. Her main research areas are international and comparative adult education, theory and practices of lifelong learning, issues of participation and social inclusion/exclusion, with particular emphasis on adult education and disability, and global citizenship education.

E-Mail: silke.schreiber-barsch@uni-hamburg.de

Anneli Schwartz has a Ph.D. in Educational Science and has taught professionals in teacher education programs focusing on integration and leadership. She is Head of Central Administration of Education, Culture and Integration in a Swedish municipality.

E-Mail: anneli.schwartz@alingsas.se

Cecilia Simón Rueda is Senior Lecturer in the Department of Developmental and Educational Psychology in the Faculty of Psychology at Autonomous University of Madrid, Spain, and currently its Delegate of the Rector for Attention to Diversity. Her current themes of research include equity and inclusive education (cultures, policies, and practices); school, family and community partnerships; and inclusion in higher education.

E-Mail: cecilia.simon@uam.es

Tanja Sturm ist Professorin im Arbeitsbereich Inklusion und Exklusion in Bildung, Erziehung und Sozialisation an der Philosophischen Fakultät III der Martin-Luther Universität Halle-Wittenberg, Deutschland. Forschungsschwerpunkte: Inklusion/Exklusion in Schule und Unterricht, Differenzkonstruktionen in unterrichtlichen Praktiken, Praxeologische Wissenssoziologie und Dokumentarische Methode (Gruppendiskussion, Videografie).

E-Mail: tanja.sturm@paedagogik.uni-halle.de 
Run Tan is Research Fellow and substitute for the junior professorship ,Educational Research with Focus on Heterogeneity ' in the Department of Empirical Educational Research, University of Konstanz, Germany. Key research interests include: early childhood inclusion, inclusive teacher education, inclusion in the global south, children's social and emotional development.

Email: run.tan@uni-konstanz.de

Sally Tomlinson has been teaching, writing and researching for over thirty-five years in the areas of race, ethnicity and education, special education and educational policy. She has held Professorial Chairs at the Universities of Lancaster, Wales (Swansea) and Goldsmiths, London University, where she also served as a Pro-Warden. Recent books include $A$ Sociology of Special and Inclusive Education (Routledge, 2017) and Education and Race from Empire to Brexit (Policy Press, 2019).

Email: s@stomlinson.net

Heike Tiemann is Professor of Physical Education in the Department of Sport Science at the Universität Leipzig, Germany. The main focus of her research is on inclusive physical education and teacher education for inclusion. She received her PhD from Freie Universität Berlin, Germany. Recent publications relate to concepts, models, and strategies for inclusive physical education.

E-Mail: heike.tiemann@uni-leipzig.de

Katja Weber is a PhD student in the Department of Special Education and Physical Education at the University of Education Ludwigsburg, Germany. She also teaches at a middle school in Freiburg, Germany. The main focus of her research combines inclusion, physical education as well as teacher training.

E-Mail: weber-kati@gmx.de

Raphael Zahnd ist Professor für Inklusive Didaktik und Heterogenität am Institut Spezielle Pädagogik und Psychologie der Pädagogischen Hochschule FHNW, Schweiz. Lehr- und Forschungsschwerpunkte: Inklusive Didaktik, Analyse des Diskurses zum Thema Behinderung, gesellschaftliche und schulische Ein- und Ausschlussprozesse, Globale Perspektiven auf Inklusion und Behinderung.

E-Mail: raphael.zahnd@fhnw.ch

Jozef Zelinka ist Doktor in Politischer Theorie und lehrt am Privatgymnasium mit Begabtenförderung (CENADA) in Bratislava, Slowakei. Lehr- und Forschungsschwerpunkte: Kritische Politische Theorie; Subjektivierung; Vulnerabilitätsstudien.

E-Mail: jozo.zeli@gmail.com 


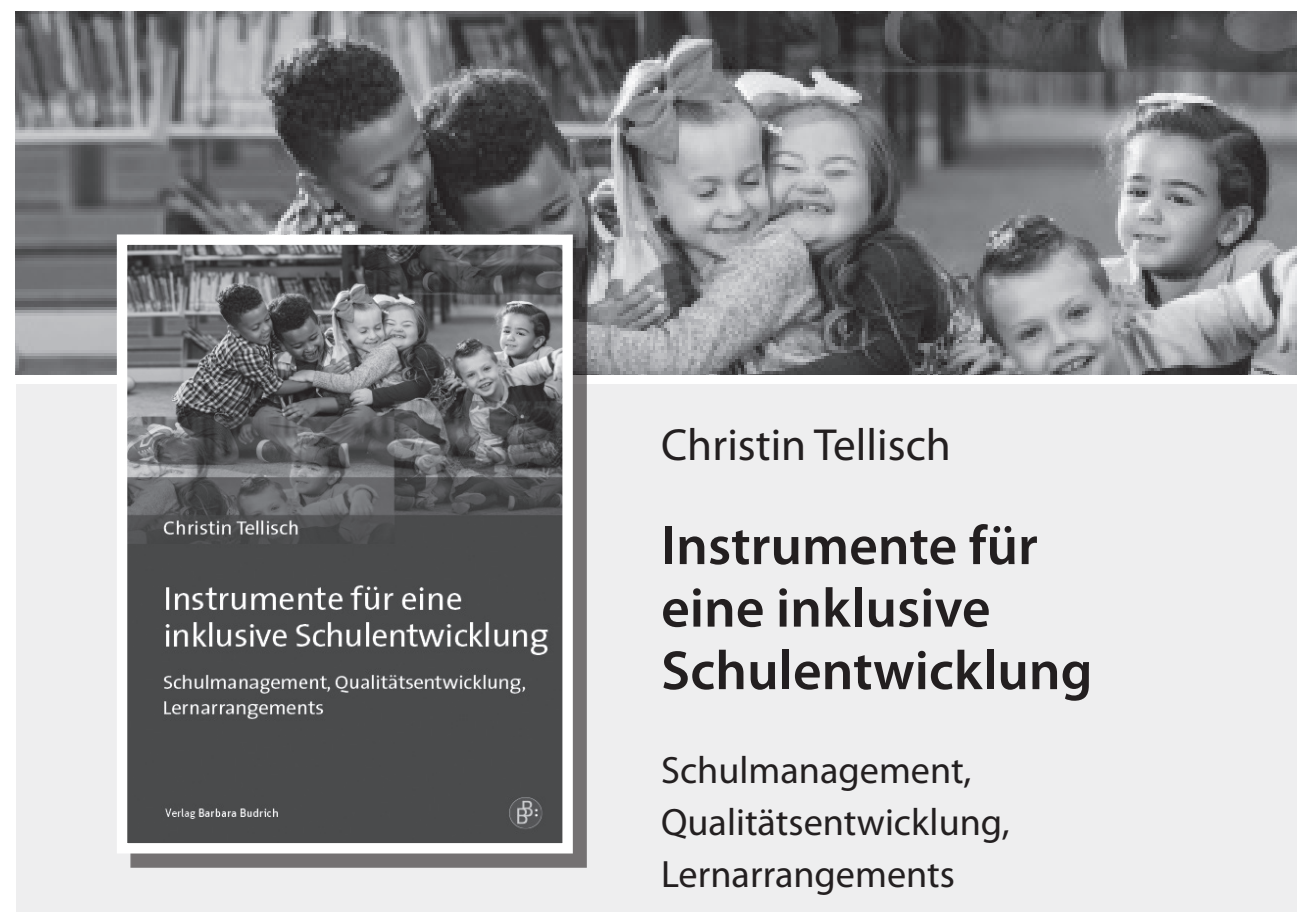

$2020 \cdot 330$ Seiten $\cdot$ Kart. $\cdot 38,00 €(D) \cdot 39,10 €(A)$

ISBN 978-3-8474-2382-9 • eISBN 978-3-8474-1537-4

Von wegen Utopie: Dieser Band gibt den Anstoß dafür, Inklusion in der Schule als eine lösbare Aufgabe auf dem Weg zu einer inklusiven Gesellschaft zu sehen und sie anzugehen. Anhand ausgewählter Elemente des Schul-, Personal- und Qualitätsmanagements, der Lernformen, Digitalisierung und Schulöffnung werden wissenschaftliche Einblicke mit praktischen Umsetzungsmöglichkeiten verbunden. Das ist sowohl für weiterführende empirische Untersuchungen, aber eben auch für die schulpraktische Umsetzung interessant. 
Globales Paradigma, nationale Normen und lokale Praxen. Das Handbuch Inklusion international verbindet theoretische Entwicklungslinien und vielfältige vergleichende Perspektiven der Inklusiven Bildung in ihrer globalen Verbreitung. Die Beiträge bieten einen umfassenden Zugang zu internationalen Diskursen, vergleichenden Forschungsergebnissen und ,inspiring practices' aus diversen Weltregionen - Europa, Afrika, Asien und Nordamerika. Besondere Aufmerksamkeit wird dabei der Relevanz komparativer Studien gewidmet.

Global paradigm, national norms and local practices. The International Handbook of Inclusive Education combines theoretical approaches and myriad comparative perspectives on inclusive education considering its global diffusion. Its contributions provide comprehensive access to international discourses, comparative research results, and inspiring practices from diverse world regions-Europe, Africa, Asia, and North America—and emphasize the relevance of comparative studies.

DIE HERAUSGEBER / THE EDITORS:

Andreas Köpfer, Juniorprofessor für Inklusive Bildung und Lernen, Institut für Erziehungswissenschaft, Allgemeine Erziehungswissenschaft, Pädagogische Hochschule Freiburg, Deutschland

Justin J.W. Powell, Professor für Bildungssoziologie, Institute of

Education \& Society, University of Luxembourg

Raphael Zahnd, Leiter Professur für Inklusive Didaktik und Heterogenität, Institut Spezielle Pädagogik und Psychologie, Pädagogische Hochschule FHNW, Muttenz, Schweiz

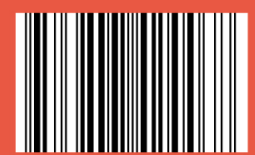

ISBN 978-3-8474-2446-8 www.budrich-verlag.de 